\title{
LISTADO SISTEMÁTICO, SINONÍMICO Y BIOGEOGRÁFICO DE LOS ÁCAROS ORIBÁTIDOS (ACARIFORMES, ORIBATIDA) DEL MUNDO (1758-2002)
}

\author{
L. S. Subías*
}

\begin{abstract}
RESUMEN
Se elabora por primera vez un listado mundial de ácaros oribátidos y en él se relacionan sistemáticamente las cerca de 9.000 especies (y subespecies) válidas hasta ahora descritas con sus correspondientes sinonimias, más de un millar, las cuales se agrupan en 1.204 géneros (y subgéneros), también con sus sinonimias reconocidas, pertenecientes a 169 familias. Para cada una de ellas se aporta su distribución geográfica actualmente conocida (alrededor de 3.200 especies paleárticas, 1.500 etiópicas, 1.500 neotropicales, 1.400 orientales, 1.200 neárticas, 1.000 australianas y 100 antárticas), y lo mismo se hace para cada género y familia. Se han realizado numerosas modificaciones sistemáticas y nomenclaturales destacando la creación de las nuevas familias Cerocepheidae y Ametroproctidae, de los nuevos géneros Multimaudheimia y Perezinigokalumma y de los nuevos subgéneros Malaconothrus (Cristonothrus) y Oribatella (Multoribatella). Además se ha dado el nuevo nombre genérico de Paschoalia para Hammeriella Paschoal, 1898, nombre ya utilizado por Balogh, 1983, así como 83 nuevos nombres específicos y subespecíficos para otros tantos casos de homonimias que han surgido en la realización del listado. Se reivindica y generaliza el uso del subgénero como una categoría taxonómica de gran utilidad y, en cambio, sólo se ha utilizado la categoría de subfamilia dentro de la extensa familia de los Oppiidae, la cual agrupa 13 subfamilias y 166 géneros (y subgéneros) válidos que incluyen unas 1.000 especies descritas. Las distribuciones geográficas conocidas de algunas especies son notablemente ampliadas.
\end{abstract}

Palabras clave: Ácaros, Oribatida, listado sistemático, taxonomía, biogeografía, nuevas familias, nuevos géneros, nuevos subgéneros, nuevos nombres, sinonimias.

\section{ABSTRACT \\ Systematic, synonimical and biogeographical check-list of the world's oribatid mites (Acariformes, Oribatida) (1758-2002)}

This is the first check-list of the world's oribatid mites in which the approximately 9,000 currently valid, described species (and subspecies) are systematically recorded. In addition, the list contains over a thousand corresponding synonomies, grouped into 1.204 genera (and subgenera) along with their recognized synonomies, belonging to 169 families. The currently known geographic distribution is given for each species (approximately 3,200 palaearctic species, 1,500 ethiopic, 1,500 neotropical, 1,400 oriental, 1,200 neoarctic, 1,000 australian and 100 antarctic) as well as for each family and genus. Numerous systematic and nomenclatorial modifications have been made. Particularly noteworthy is the creation of the new families Cerocepheidae and Ametroproctidae, the new genera Multimaudheimia and Perezinigokalumma, and the new subgenera Malaconothrus (Cristonothrus) and Oribatella (Multoribatella). A new name, Paschoalia, is given to the genus Hammeriella Paschoal, 1898 (a name already used by Balogh, 1983) along with 83 new specific and subspecific names for other homonimies that appeared during the making of this check-list. The subgenus category is re-established as a very useful category and used widely in this revision; however, the subfamily category has only been used within the extensive Oppiidae family, which includes 13 valid subfamilies, 166 genera (and subgenera), and some 1,000 described species, only the subfamily category is used. The geographic distribution of some known species is notably enlarged.

Key words: Mites, Oribatida, sistematic check-list, taxonomy, biogeography, new families, new genera, new subgenera, new names, synonymies.

* Departamento de Zoología. Facultad de Biología. Universidad Complutense. Madrid 28040. E-mail: subias@bio.ucm.es 
Índice

Introducción

Metodología

Biogeografía

Ordenación sistemática de familias y géneros

Listado sistemático de especies

Comentarios al listado

Agradecimientos

Referencias

\section{Introducción}

Los ácaros oribátidos constituyen un grupo cosmopolita de microartrópodos edáficos de los más importantes, tanto por su abundancia en los más variados tipos de suelos, hasta en los más adversos, como por su diversidad pero que, desgraciadamente, no han sido tratados con la profundidad necesaria debido a su aparente poco interés económico (son de vida libre y saprófagos o microfitófagos), a su pequeño tamaño (lo que implica mayores dificultades de manipulación) y a su complejidad taxonómica; todo esto ha hecho que hayan despertado poca atracción y hayan pasado bastante desapercibidos, motivando que su conocimiento actual sea bastante incompleto, existiendo en la actualidad extensas regiones del globo totalmente inexploradas y siendo numerosas las especies (y otros taxones de nivel superior) que quedan por describir. Además, la tendencia general que ha habido en los últimos años (lo que afortunadamente parece que ahora está cambiando) a menospreciar los trabajos de tipo taxonómico en favor de los ecológicos o aplicados, ha traído consigo que cada vez sea menor el número de investigadores que se dedican al estudio de este grupo de artrópodos.

Por otra parte, se trata de un grupo animal muy antiguo del que ya se conocen fósiles paleozoicos: del Ordovícico (BERNINI et al., 2002), del Devónico (NorTOn et al., 1988) y del Carbonífero (SuBÍAs \& ArILlo, 2002), muy similares a los actuales oribátidos primitivos pertenecientes a los Palaeosomata y Enarthronota, debido fundamentalmente a que el medio en el que viven, el edáfico, ha variado mucho menos que otros con el transcurso del tiempo (dando lugar a formas más estables debido a que su ritmo evolutivo sería mucho más lento que el de otros grupos de animales) apareciendo ya desde el Jurásico fósiles de oribátidos pertenecientes a géneros presentes en la fauna actual y considerados como muy evolucionados, lo que ha motivado que los ácaros oribátidos de hoy en día incluyan fami- lias (incluso géneros y hasta especies) con una distribución geográfica muy cosmopolita y que los taxónomos que se dedican a su estudio deban de hacerlo a un nivel mundial y no se puedan restringir a faunas más o menos locales.

La continua descripción de nuevos taxones (al ritmo de unas 150-200 especies y 10-15 géneros por año), así como las permanentes recolocaciones de los ya existentes, han complicado enormemente la sistemática del grupo, proliferando las descripciones de especies, en algunos géneros de amplia distribución, que no tienen en cuenta muchas de las ya existentes en la literatura (aunque en muchos casos es muy difícil disponer de toda la bibliografía existente por lo disperso de la misma) lo que, seguramente, implicará numerosas sinonimias cuando en el futuro se lleven a cabo las tan necesarias revisiones globales; este es el caso, por ejemplo, del género Scheloribates Berlese, 1908 (el que más especies presenta de todos los oribátidos) que incluye en la actualidad 234 especies (y subespecies) conocidas. También contribuye a esta complejidad el hecho de que se describan nuevos géneros sin que se incluyan en ellos todas las especies que por su diagnosis deberían de estarlo, o que se basen en caracteres de nueva utilización que no se sabe si los presentan los taxones próximos ni cual es su verdadero valor filogenético-sistemático, y como nuestra intención ha sido facilitar el acceso de este complejo grupo a nuevos investigadores se han considerado, por el momento, como sinónimos a una serie de taxones supraespecíficos de difícil acceso, hasta para los especialistas. Todo esto explica que, aproximadamente, la tercera parte de los géneros descritos de oribátidos sean considerados en la actualidad como sinónimos. Una de las pruebas de la complejidad nomenclatural existente dentro de los oribátidos y de la necesidad de trabajos como el presente, lo constituye el hecho de las numerosas homonimias que se han recogido y que han obligado a que se les tenga que dar un nuevo nombre (procurando tener presente en todo momento el Código Internacional de Nomenclatura Zoológica), en total 83 nuevos nombres específicos y subespecíficos, además de uno genérico.

Por lo tanto, se hacía ya necesaria la existencia de un listado mundial de especies de oribátidos (algunos autores citan un trabajo de Piffl de 1969: Katalog der Oribatiden der Welt, Zoologische Institut der Universität Wien, que nunca fue publicado) que recopilase las más de 10.000 especies descritas hasta la fecha y pertenecientes a los más de 1.500 géneros también descritos hasta ahora, que recogiese además de su actual posición siste- 
mática (se han distribuido entre 51 superfamilias y 169 familias), sus sinonimias y distribución geográfica, facilitando de esta manera el estudio de dicho grupo, y posibilitase el acceso al mismo de nuevos investigadores. No sería justo si no destacase aquí algunos trabajos recientes de gran interés en esa línea y que me han posibilitado en gran medida la realización de este listado; este es el caso de GHILYAROV \& KRIVOLUTSKY (1975) con sus claves de identificación de la fauna de la antigua Unión Soviética (cuyas publicaciones son a veces muy locales y difíciles de consultar); BALOGH \& MAHUNKA (1983) y sus claves de los oribátidos primitivos de la región Paleártica; Marshall et al. (1987) con un catálogo exhaustivo ejemplar dedicado al estudio de los oribátidos de Estados Unidos y Canadá (que posibilita el acceso a los autores norteamericanos que hasta los años 30 del siglo pasado han seguido trayectorias paralelas a los europeos, describiendo por separado las mismas especies a un lado y otro del Atlántico, muchas de las cuales serán cosideradas con toda seguridad como sinónimos en futuras revisiones); SuBÍAs \& BALOGH (1989) y su trabajo sobre los óppidos mundiales, en el que se ordenan sistemáticamente las especies descritas en la familia Oppiidae Sellnick, 1937, la que incluye mayor número de especies descritas, alrededor de un millar, distribuidas en unos 150 géneros y subgéneros válidos; BALOGH \& BALOGH (1988 y 1990) con las claves de oribátidos neotropicales; BALOGH \& BALOGH (1992) y sus claves de géneros del mundo; MAHUNKA \& MAHUNKAPAPP (1995) y la revisión de los oribátidos de la "Colección Berlese" (autor que describió a principios del siglo pasado numerosas especies, cuya auténtica identidad era en muchos casos problemática); BALOGH \& BALOGH (2002) y sus claves de oribátidos extraholárticos (que, lamentablemente, presenta numerosos errores posiblemente debido a lo precipitado de su publicación por el fallecimiento del autor senior) y, por último, también mencionar los trabajos sobre los oribátidos ibéricos publicados por PÉREZ-ÍÑIGO (1993 y 1997) y SuBíAs (2001) en la serie "Fauna Ibérica", muy utilizada fuera de nuestro país.

\section{Metodología}

Desde un principio se planteó el presente listado como un punto de partida, ambicioso pero realizable, para posteriores trabajos más elaborados (como sería el caso de un catálogo completo); en él deberían figurar los datos más básicos para cual- quiera que desee realizar un estudio taxonómico con oribátidos. Uno de ellos es la posición sistemática actualizada de todos los taxones básicos (familias, géneros y especies), hasta el año 2002 inclusive, con la perspectiva que da un estudio global de este tipo (lo que ha implicado necesariamente numerosas reorganizaciones de niveles taxonómicos para intentar tratar a todos los grupos de oribátidos con los mismos criterios), pero sin perder de vista que lo fundamental es la elaboración del listado final motivo por el cual, y al no tratarse de un catálogo exhaustivo, no se ha creído conveniente entrar a discutir las numerosas sinonimias y los centenares de nuevas combinaciones nomenclaturales que han sido necesarias llevar a cabo (y las que, con toda seguridad habrá que seguir realizando en el futuro), lo que supondría un voluminoso trabajo de discusión aparte y que no es la finalidad que nos habíamos porpuesto ni encajaría en un listado como el presente; solamente en el caso de las sinonimias realizadas en el nivel genérico se explicita a continuación "sin. nov.". Esta "filosofía" utilitarista y pragmática seguida en la elaboración de este trabajo está inspirada por BALOGH (1972), maestro de oribatólogos que elaboró unas claves de géneros de oribátidos del mundo adecuadas a los conocimientos del momento y que, aunque podrían ser objeto de numerosas críticas por los "puristas" del momento al no entrar en discusiones sobre la creación de los nuevos taxones y sinonimias que realiza así como al no ajustarse en muchos casos a las normas del Código Internacional de Nomenclatura Zoológica, han sido fundamentales e imprescindibles para la formación de todos los interesados en el estudio de los oribátidos que surgimos con posterioridad.

Algo parecido se puede decir del apartado Bibliografía ya que no se trata de incluir las más de 5.000 publicaciones conocidas que abarcan aspectos taxonómicos, sistemáticos o faunísticos sobre los ácaros oribátidos, incluyéndose sólo los listados parciales regionales más recientes, aparte de los trabajos citados en el texto, y alguna otra obra básica para nuestro estudio, si bien para la elaboración del mismo se ha tenido en cuenta, como es natural, toda la bibliografía existente.

El listado presenta una ordenación sistemática que, naturalmente, sigue las tendencias actuales, aunque es un poco ecléctica ya que no hemos adoptado exactamente la propuesta por algún autor en concreto (aunque se ha tomado como punto de partida el trabajo de BALOGH \& BALOGH, 1992) sino la que se ha creído más adecuada entre las distintas propuestas por los diferentes autores. Los 
grandes grupos sistemáticos de oribátidos se ordenan en superfamilias y dentro de cada familia, y género, se ordenan alfabéticamente los géneros, y especies, que, respectivamente, incluyen. Se ha prescindido, por los problemas de lo incompleto de nuestros conocimientos taxonómicos mencionados, de la categoría de subfamilia, salvo dentro de la familia Oppiidae debido a lo necesaria y práctica que resulta su utilización dentro de la misma por el, ya también comentado, elevado número de géneros y especies en ella descritos, y porque está bien elaborada y es adoptada de forma general a partir del trabajo de SuBías \& BALOGH (1989). Por el contrario, se ha adoptado el uso generalizado de los subgéneros lo que, dentro de los oribátidos, no se venía haciendo tal vez por comodidad de uso pero que, realmente, se ha considerado que nos están indicando que la separación de determinados géneros no es lo suficientemente clara desde el punto de vista sistemático, siendo en otros casos de gran utilidad en géneros muy amplios para la separación de grupos de especies para su identificación, aunque pudiera tratarse de una agrupación no del todo natural.

Las especies vienen relacionadas con su nombre actual y, en caso de que no coincida con la denominación original, entre paréntesis figurará abreviada a continuación dicha denominación. De cada género y especie se relacionan también todas sus sinonimias, prescindiendo de las denominaciones diferentes debidas a "lapsus". En algunas casos existen divergencias (año arriba o año abajo) entre los diferentes autores sobre la fecha de publicación de algunos taxones (fundamentalmente Koch y Berlese); en ese caso hemos adoptado el criterio seguido por MARSHALl et al. (1987). A continuación de cada taxón supraespecífico figura, entre paréntesis, el número de taxones de rango inferior que engloba. Para facilitar el acceso al voluminoso número de taxones compilados, delante del listado figura una relación de todos los taxones supraespecíficos con la ordenación sistemática seguida.

Se tiene en cuenta también la distribución geográfica actualmente conocida de las familias, géneros y especies (y sus taxones subordinados), pero como su conocimiento es todavía más incompleto que el taxonómico (además de que muchas citas realizadas por no especialistas en trabajos de tipo ecológico forzosamente han de ser puestas en cuarentena) se ha considerado opotuno no concretar demasiado la misma; en los niveles de familia y género figura su distribución siguiendo básicamente las grandes regiones biogeográficas, aunque por cuestiones prácticas se ha dado a la región
Paleártica un tratamiento algo diferente a las demás ya que, como se conoce mucho mejor que las otras, se ha subdivido más en subregiones. En el caso de las especies se ha especificado, como es natural, algo más si bien cuando existen tres o más citas de países diferentes dentro de una misma gran región biogeográfica (en distribuciones de menor entidad con dos países diferentes es suficiente) se le ha asignado directamente dicha distribución. En el caso de citas muy dudosas (o de países muy grandes sin concretar más, o pertenecientes a otros ya desaparecidos) se colocan entre comillas ("); en ciertos casos en los que ha cambiado la denominación de algunos países, se pone tambien entre corchetes [ ] el otro nombre por el que es conocido. Aunque normalmente se hace referencia a países (procurando, siempre que ha sido posible, que figuren en español o castellanizados), en casos concretos, en muchas ocasiones islas (pertenecientes a un país o, por el contrario, compartidas por más de uno), aparece el nombre del lugar geográfico y no el del país.

En este listado no se incluyen fósiles salvo que sea imprescindible (en dicho caso figura el símbolo + detrás del nombre de la especie), como es el caso de la especie tipo de géneros fósiles de los que se han descrito posteriormente especies que viven en la actualidad, o el de nombres de especies fósiles que han sido objeto de homonimias con especies actuales. En cambio, sí se han tenido en cuenta los nombre dados a inmaduros (en general, las formas juveniles de los oribátidos son muy diferentes a los adultos y, en la mayoría de los casos, todavía desconocidas por lo que la sistemática se fundamenta básicamente en adultos), en su gran mayoría considerados actualmente como "species inquiriendae".

\section{Taxonomía y nomenclatura}

En este apartado sólo se van a tener en cuenta aquellas modificaciones taxonómicas o nomenclaturales que presenten cierta trascendencia (por lo ya comentado previamente sobre la imposibilidad de comentar en un trabajo como éste todas y cada una de la multitud de dichas modificaciones llevadas a cabo: nuevas combinaciones, cambio de estatus, etc.). En general, siempre hemos procurado que prevalezca el criterio de prioridad, como indica el Código Internacional de Nomenclatura Zoológica (C.I.N.Z.), en contra del criterio de algunos otros autores que están más de acuerdo en que prevalezca el de estabilidad (BALOGH, 1987). Este es el caso de la familia Mycobatidae Grandjean, 1954, denomi- 
nación que ha sido sustituida por Punctoribatidae Thor, 1937 ya que sus respectivos géneros tipo pertenecen a la misma familia. En cambio en el caso de la familia Oppiidae Sellnick, 1937 se ha mantenido el criterio de estabilidad, como indica el C.I.N.Z. en su artículo 40-a, respecto a Dameosomidae Sellnick, 1928, familia esta última basada en un género tipo considerado sinónimo del género tipo de la primera familia con anterioridad al año 1960.

También se ha cambiado la autoría de algunas familias tradicionalmente asignadas a autores posteriores, como es el caso de Chamobatidae Thor, 1937 (en lugar de Grandjean, 1954) y Limnozetidae Thor, 1937 (en lugar de Grandjean, 1954). Asimismo, se ha sustituido la familia Chaunoproctidae Balogh, 1961 por Caloppiidae Balogh, 1960 (según el artículo 40 del C.I.N.Z.).

Para las nuevos nombres específicos y subespecíficos que nos hemos visto obligados a dar a causa de las numerosas homonimias primarias (según el artículo 59-a del C.I.N.Z. en este caso siempre debe de darse un nombre nuevo al homónimo posterior) o secundarias (originadas por las numerosas recombinaciones genéricas realizadas y que también dan lugar a una renominación, según el artículo 59-b del C.I.N.Z.), se ha tratado de buscar una denominación concreta que sirviese para todos los taxones y que hiciese referencia de una u otra manera a esa utilización ya previa del epíteto específico (neonominatus-ta, praeoccupatus-ta, iteratus-ta, etc.); tras el nombre objeto de sustitución figura, entre paréntesis, si se trata de una homonimia primaria o secundaria. También ha habido que dar un nuevo nombre genérico debido a otro caso de homonimia, Paschoalia nom. nov. para Hammeriella Paschoal, 1989, nombre ya utilizado por Balogh, 1983.

Sólo en casos muy concretos (y por coherencia en el tratamiento dado en este listado a otros grupos taxonómicos de oribátidos próximos) se han creado nuevos taxones supraespecíficos; uno de ellos es la familia Cerocepheidae fam. nov., perteneciente a la superfamilia Cepheoidea Berlese, 1896, que englobaría a los géneros Cerocepheus Trägardh, 1931, que sería el género tipo, Bornebuschia Hammer, 1966 y Dicrotegaeus Luxton, 1988, los cuales presentan los quelíceros estrechados, adaptados para la succión (en lugar de los típicos masticadores), carácter que también se ha utilizado para separar otras familias proximas, como los Ceratoppiidae Kunst, 1971 (masticadores) de los Metrioppiidae Balogh, 1943 (suctores), o superfamilias como los Oppidoidea Sellnick, 1937 (masticadores) de los Trizetoidea Ewing, 1917 (suctores). Otro caso hace referencia a la familia Ametroproctidae fam. nov. cuya creación, dentro de la superfamilia Cymbaeremaeoidea Sellnick, 1928, ha sido necesaria para agrupar los géneros Ametroproctus Higgins y Woolley, 1968 (con el subgénero Ametroproctus (Coropoculia) Aoki y Fujikawa, 1972) y Scapuleremaeus Behan-Pelletier, 1989 recientemente incluidos en la familia Cymbaeremaeidae Sellnick, 1928, pero que presentan unas lamelas muy desarrolladas y que ocupan gran parte del prodorso, contactando distalmente, en contra de los demás representantes de dicha familia que, o no presentan lamelas o están reducidas a estrechas cóstulas lamelares, motivo que creemos justifica suficientemente la creación de esta nueva familia. También se ha procedido a la erección, dentro de la familia Maudheimiidae J. y P. Balogh, 1984, del género Multimaudheimia gen. nov. para Maudheimia marshalli Coetzee, 1997, especie que presenta 15 pares de setas notogastrales en lugar de los 10 de las restantes especies del género Maudheimia Dalenius, 1958 (carácter habitualmente utilizado, dentro de la taxonomía de oribátidos, para la separación de géneros y subgéneros) así como, dentro de la familia Parakalummidae Grandjean, 1936, del género Perezinigokalumma gen. nov. (en memoria del prestigioso oribatólogo español hace pocos años fallecido), del que se designa como especie tipo a Protokalumma afrum Pérez-Íñigo, 1969 y que engloba varias especies con sáculos notogastrales caracterizadas por la presencia de anchas lamelas, carácter atípico dentro de la superfamilia Galumnoidea Jacot, 1925 a la que pertenece. El último caso hace referencia a los subgéneros Malaconothrus (Cristonothrus) subgen. nov., al que designamos como especie tipo a Malaconothrus pauciareolatus Subías y Sarkar, 1983, que engloba un numeroso grupo de especies que presentan el notogáster con tendencia al aplastamiento dorsoventral y que muestra un par de quillas longitudinales dorsales que se bifurcan posteriormente dando lugar a sendas depresiones, mientras que Malaconothrus s. str. Berlese, 1904 presenta el notogáster más o menos cilíndrico y sin quillas ni depresiones dorsales (en el género próximo Trimalaconothrus Berlese, 1914, de patas tridáctilas en lugar de monodáctilas, pasa lo mismo con el subgénero Malaconothrus (Tyrphonothrus) Knülle, 1957) y al subgénero Oribatella (Multoribatella) subgen. nov., que engloba las especies de Oribatella Banks, 1895, con 12-14 pares de setas notogastrales, quedando en el género típico las especies que presentan 10-11 pares, y del que se ha designado como especie tipo a Oribatella bromeliarum Behan-Pelletier y Paoletti, 1993. 
Ha habido una serie de familias (o superfamilias) que han sido objeto de cambios significativos en su posición sistemática y de ellas vamos a ocuparnos a continuación. En primer lugar vamos a tratar de una atípica familia de oribátidos que se ha descrito recientemente, los Aribatidae Aoki, Takaku e Ito, 1994, cuyos autores (AOKI et al., 1994) no adscriben a ninguna superfamilia conocida en concreto por no presentar botridios ni sensilos, aunque dan una serie de similitudes con algunas familias, entre ellas los Eremaeidae Oudemans, 1900, por presentar un número de setas anales superior al habitual; creemos que por las restantes características que presenta debería ser ubicada, al menos provisionalmente, dentro de la superfamilia Eremaeoidea Oudemans, 1900, asemejándose morfológicamente bastante a la familia allí incluida Kodiakellidae Hammer, 1967, a pesar de la ausencia total de cóstulas lamelares.

También se ha procedido a la erección de una superfamilia para reunir una serie de familias tradicionalmente incluidas dentro de la hipertrofiada superfamilia Oppioidea Sellnick, 1937, a saber: Eremellidae Balogh, 1961, Caleremaeidae Grandjean, 1965, Oribellidae Kunst, 1971 (familia desgajada de los Thyrisomidae Grandjean, 1953 que se mantendría dentro de los Oppioidea), Machadobelbidae Balogh, 1972, Arceremaeidae Balogh, 1972, Spinozetidae Balogh, 1972 y Platyameridae J. \& P. Balogh, 1983, ya que no presentan los epímeros $3+4$ fusionados por la ausencia del apodema 3 como ocurre en las demás familias, siendo por lo tanto necesaria la erección de la superfamilia Eremelloidea Balogh, 1961 (que toma el nombre de la familia más antigua de todas ellas) que las agrupe; además se ha traspasado a la familia Eremellidae (por la presencia, entre otros caracteres, de cóstulas lamelares y setas desarrolladas en todo el notogáster) el género Licnocepheus Woolley, 1969 que BALOGH \& BALOGH (1992) situaban dentro de la familia Licnodamaeidae Grandjean, 1954.

La familia Tegeocranellidae J. y P. Balogh, 1988 la situamos dentro de la superfamilia Tectocepheoidea Grandjean, 1954 y no en los Polypterozetoidea Grandjean, 1959, como hacen BALOGH \& BALOGH (1992) (en cambio BALOGH, 1972 incluía el género Tegeocranellus Berlese, 1913 en la familia Tectocepheidae Grandjean, 1954), debido a que sus ninfas no son euferedermas como en dicha superfamilia sino aferedermas (es decir que no portan exuvias ninfales en su dorso) y, además, dichas ninfas presentan el tegumento parcialmente plegado como en la primera de las subfamilias, ajustándose también bastante bien las características de los adultos a las de los Tectocepheoidea, al contrario que ocurre con los adultos de los Ameronothroidea Willmann, 1931, superfamilia de oribátidos acuáticos en la que BeHAN-PELletier (1997a) incluye dicha familia recientemente. En el estado actual de nuestros conocimientos, y con lo poco que como hemos dicho sabemos todavía de los estados juveniles y en aras de una identificación más asequible, pienso que la morfología de los adultos debe tener todavía un peso fundamental en la clasificación de los oribátidos, uno de los motivos por el cual hemos decidido prescindir de la familia Euzetidae Grandjean, 1954, basada fundamentalmente en la morfología de las formas juveniles, que queda englobada dentro de los Ceratozetidae Jacot, 1925, familia en la que incluimos también los Zetomimidae Shaldybina, 1966, ya que no hemos podido encontrar criterios de separación que fuesen utilizables en todos los géneros en ella considerados.

Otra familia en la que se ha introducido alguna modificación ha sido en los Otocepheidae Balogh, 1961 ya que se ha considerado conveniente elevar a la categoría de familia, Tetracondylidae Aoki, 1961 (dentro de la superfamilia Otocepheoidea Balogh, 1961), a la subfamilia que bajo dicho nombre incluía debido, fundamentalmente, a la notable característica de presentar los tectopedios 2 y 3 hiperdesarrollados y muy prominentes. Lo mismo se ha hecho (dentro de los Oripodoidea Jacot, 1925) con la familia Pseudoppiidae Mahunka, 1975, hasta ahora incluida como subfamilia dentro de los Oribatulidae Thor, 1929 y caracterizada por presentar, en visión dorsal, el carácter notable y poco habitual de mostrar los quelíceros sin cubrir completamente por el borde rostral, y con las familias Pirnodidae Grandjean, 1956 y Parapirnodidae Aoki y Ohkubo, 1974 (hasta ahora subfamilias dentro de la familia Oripodidae Jacot, 1925), la primera caracterizada por el notable, y nada habitual, dimorfismo sexual que presentan en la disposición de las placas genitales y anales (en los machos se juntan en la parte posterior del cuerpo), y la segunda por presentar áreas porosas, monodactilia $\mathrm{y}$ ausencia de pteromorfos.

Otra notable modificación introducida consiste en incluir dentro de los oribátidos superiores poronóticos la familia Micreremidae Grandjean, 1954 (dentro de la superfamilia Licneremaeoidea Grandjean, 1931), hasta ahora incluida en la superfamilia Cymbaeremaeoidea, basándonos el el trabajo de BALOGH \& BALOGH (1998) por el que la familia Fenicheliidae J. y P. Balogh, 1984 (considerada hasta entonces como perteneciente a los Oripodoidea, con áreas porosas o sáculos más o menos vestigiales) queda absorbida por la familia anterior. 
La familia Unduloribatidae Kunst, 1971, al presentar características de poronótico pero no áreas porosas o sáculos, ha sido objeto de diferentes ubicaciones figurando finalmente en el catálogo de MARShall et al. (1987) dentro de la superfamilia Phenopelopoidea Petrunkevitch, 1955. Por otra parte, SCHATZ (2001) representa las ninfas de la familia Eremaeozetidae Piffl, 1972, aferedermas (es decir que no portan exuvias en su dorso) y de tegumento plegado por lo que su situación dentro de los Polypterozetoidea Grandjean, 1959, donde habitualmente se les colocaba, ha de ser revisada. Por la morfología que presentan los adultos, donde destaca la presencia de un cuerpo de tegumento grueso y esculpido, y de unas lamelas muy anchas que contactan centralmente, han sido situados junto a los Unduloribatidae en la superfamilia de nueva erección Unduloribatoidea Kunst, 1971, en la que también vamos a situar la familia Idiozetidae Aoki, 1976, estrambóticos oribátidos cuya posción también era bastante incierta y que para BALOGH \& BALOGH (1992) constituían una superfamilia independiente.

Algo similar nos hemos planteado con la familia de oribátidos acuáticos Limnozetidae Thor, 1937, que venía siendo situada, junto a la familia Hydrozetidae Grandjean, 1954, dentro de los oribátidos superiores picnonóticos, entre otras características, por la ausencia de áreas porosas o sáculos en el notogáster; pero la presencia de pteromorfos y auténticas lamelas, así como la inclusión en dicha familia de un género con sáculos, nos ha decidido a incluirla dentro de los oribátidos poronóticos. La presencia de ninfas de tegumento plegado, junto con las características morfológicas de los adultos, induce a pensar en cierto parentesco con los miembros de la familia Austrachipteriidae Luxton, 1985 y, aunque Behan-Pelletier (2001) la considera a como sinónima de la familia Achiperiidae Thor, 1929, nosotros hemos preferido seguir manteniéndola como válida y erigir la superfamilia Limnozetoidea Thor, 1937 para englobar ambas familias, considerándola así integrada por una serie de géneros caracterizados por la ausencia de áreas porosas o sáculos desarrollados, que serían vestigiales o quedarían como simples poros, y cuyas lamelas no alcanzarían nunca el desarrollo de los géneros que integran la superfamilia Achipterioidea Thor, 1929 que presentarían, además, unos pteromorfos mucho más desarrollados.

También se ha cambiado la posición sistemática de la familia Epactozetidae Grandjean, 1930, de parentescos inciertos pero que, hasta ahora, era tra- dicionalmente incluida dentro de la superfamilia Galumnoidea Jacot, 1925 (MARSHALl et al., 1987), por presentar pteromorfos similares, y de la que no se conocen los estados juveniles (lo que arrojaría bastante luz sobre sus relaciones con otras familias). A pesar de ello, se ha creído más oportuno incluirla dentro de los Oribatelloidea Jacot, 1925 en base, sobre todo, al gran desarrollo lamelar que presentan.

Por último, en lo referente al nivel sistemático de familia, decir que dentro de los oribátidos superiores poronóticos y para la separación de las distintas familias dentro de cada superfamilia, se han tenido en cuenta caracteres asequibles para una relativamente fácil inclusión en ellas de los distintos géneros, como es el caso de presencia en el notogáster de áreas porosas o sáculos (o ausencia de ambos), presencia o ausencia de pteromorfos, pteromorfos móviles o inmóviles, y las distintas combinaciones posibles de dichos caracteres (por ejemplo dentro de los Oripodoidea la familia Oribatulidae Thor, 1929 no presentaría pteromorfos y tendría áreas porosas, la familia Liebstadiidae J. y P. Balogh, 1984 presentaría pteromorfos y áreas porosas, los Scheloribatidae tendrían pteromorfos y sáculos, mientras que los Hemileiidae J. y P. Balogh, 1984 carecerían de pteromorfos y tendrían sáculos). Como todavía no está nada claro cual sería la clasificación más natural (como también pone de manifiesto BALOGH, 1987), no se ha creído conveniente basar la clasificación en caracteres de más difícil acceso que la harían mucho más compleja.

Por lo que respecta a las modificaciones sistemático-nomenclaturales de géneros llevados a cabo en este trabajo ya se ha comentado que son tantas que la longitud del mismo sólo permite que nos centremos en las más significativas, como es el hecho de las llevadas a cabo dentro de la familia Phthiracaridae Perty, 1841, inténsamente tratada por Niedbala (1992, 1998, 2000, 2001, 2002 y otros) en los últimos años, autor que ha utilizado para la separación de géneros la disposición de las setas genitales, carácter éste difícil de observar y del que no se conoce todavía su auténtica validez sistemática (como ya ponen de manifiesto MAHUNKA, 2002 para esta familia, o BALOGH, 1987 para los Oppiidae), lo mismo que ocurre con la familia Damaeidae Berlese, 1896 y el basar la diferenciación genérica casi exclusivamente en la quetotaxia de las patas, lo que ha complicado enormemente la sistemática de estas familias y el acceso de los "no iniciados" a las mismas, motivo por el cual se ha procedido a la realización de una serie de sinonimias y cambios de 
categoría genéricos (imposible entrar en un estudio como éste en las específicas) originadas por la reconsideración de estos caracteres. También en las superfamilias Plateremaeoidea Trägardh, 1926 y Gymnodamaeoidea Grandjean, 1954 han sido necesarias la realización de bastantes sinonimias para mantener esta coherencia, sobre todo en lo que respecta a los recientes géneros erigidos por Paschoal. De todas formas esto no obsta para que en el futuro, cuando se conozcan mejor los distintos grupos, sus relaciones y el valor de sus caracteres, puedan ser reinstaurados los géneros aquí sinonimizados.

Una modificación del nivel genérico significativa que se ha llevado a cabo ha sido la de considerar a Klapperiches Mahunka, 1979 como subgénero de Carabodes Koch, 1835 (género muy frecuente en la región Holártica con más de un centenar de especies) e incluyendo en él a las especies que presentan el borde posterior del prodorso sin ningún tipo de quilla o crestas y directamente "sentado" en el borde anterior del notogáster, sin existencia de separación o espacio cervical alguno.

Respecto al género Parachipteria Hammen, 1952, que reúne especies con áreas porosas en lugar de los sáculos de Achipteria Berlese, 1908, su autor, HAMMEN (1952), designa como especie tipo a Oribata punctata Nicolet, 1855, a pesar de ser la menos típica porque, como el mismo autor pone de manifiesto, dicha especie presenta las áreas porosas muy pequeñas y difíciles de ver (¿poros de otros autores?); de hecho en la redescripción que de dicha especie hace OudEMANS (1927) o Shaldybina (en GHILYAROV \& KRIVOLUTSKY, 1975) no las representan. Por otra parte, TRAVÉ (1960) crea el género Pseudachipteria para las especies sin áreas porosas ni sáculos y designa a Notaspis magnus Sellnick, 1928 como especie tipo (descrita originalmente como con poros); sospecho que la especie tipo de Parachipteria, Oribata punctata, por las razones anteriormente comentadas tal vez debiera ser incluida entre las que no presentan áreas porosas ni sáculos bien definidos, a lo sumo poros o una mácula en la zona correspondiente a las áreas porosas A1 que, como TRAVÉ (1960) pone de manifiesto, podría ser confundida con auténticas áreas porosas. Por este motivo creemos más oportuno considerar a Pseudachipteria Travé, 1960 como sinónimo posterior de Parachipteria, debiéndose dar un nuevo nombre genérico para las especies con áreas porosas bien desarrolladas. Pero resulta que el género Campachipteria, descrtito por AOKI (1995), que en un principio habíamos considerado como sinónimo de Parachipteria por presentar áreas porosas, pasaría a ser el nombre genérico válido para dicho grupo de especies.

Como una muestra más de la complejidad de este grupo de especies decir que también hay autores que consideran a Oribata nitens Nicolet, 1855, especie que presenta sáculos, como especie tipo de Achipteria (por ejemplo BALOGH \& BALOGH, 1992), lo que descartan por falta de fundamento otros autores, como MARSHALL et al. (1987). Además, dicha especie es un paradigma del estado nomenclatorial de los oribátidos ya que, aunque se trata de una especie muy común y frecuentemente citada como Achipteria nitens (Nicolet, 1855), el nombre de Oribata nitens ya había sido utilizado con anterioridad por otros dos autores: Oribata nitens Gervais, 1844 (actualmente en el género Liacarus Michael, 1898) y Oribata nitens Johnston, 1853 (actualmente dentro de Euzetes Berlese, 1908); se trata, por lo tanto, de un caso de homonimia primaria y objeto de rechazo permanente, como indica el C.I.N.Z. en el artículo 59-a, y que tendría que haber sido objeto de una nueva denominación. En el presente trabajo hemos considerado que Achipteria acuta Berlese, 1908 es un sinónimo suyo y, en consecuencia, el nombre válido de la especie pasaría a ser el de esta última.

Una de las decisiones que más dudas nos ha planteado ha sido la revalidación de géneros y todo ello por un problema muy generalizado en los géneros de oribátidos descritos en el siglo XIX como es el conocer la auténtica identidad de su especie tipo (los medios y conocimientos de que se disponían en esa época eran insuficientes para poder discernir entre especies tan parecidas y de dimensiones tan pequeñas, $0,5 \mathrm{~mm}$ por término medio) lo que, en muchos casos y en ausencia del material original, se ha solventado siguiendo el artículo 70 del C.I.N.Z. que ofrece varias posibilidades, siendo una de ellas la sustitución por otra especie común (artículo 70-a,ii). Este es el caso de los géneros Murcia Koch, 1835 y Trichoribates Berlese, 1910 ya que, como ponen de manifiesto MARSHALl et al. (1987), la auténtica identidad de la especie tipo de Trichoribates es la de Murcia trimaculata Koch, 1835, y como ésta es la única especie válida reconocible incluida por su autor cuando describe el género Murcia, consideramos que se trata de su especie tipo y entonces Trichoribates pasaría a ser un sinónimo objetivo posterior de Murcia, reinstaurándose en el presente listado, por lo tanto, dicho género.

Otra modificación nomenclatural genérica llevada a cabo ha sido la de considerar el género Acrotritia Jacot, 1923 como válido y a Rhysotritia 
Märkel y Meyer, 1959 como sinónimo posterior suyo ya que sus respectivas especies tipo se corresponden con una misma especie (es un caso de sinonimia objetiva). Es curioso que, aunque MARSHALL et al. (1987) ponen de manifiesto este hecho, continuan considerando a Rhysotritia como el nombre válido y a Acrotritia, que es anterior, como sinónimo suyo. También, y siguiendo a PÉREZ-Í̃̃IGO (1993), se ha reintroducido el uso del género Neoliodes Berlese, 1888 (incomprensiblemente rechazado por los distintos autores desde mediados del siglo pasado) en lugar de Liodes Heyden, 1826 ya que fue rechazado en el año 1954 por la “Opinion" 218, apareciendo en el Índice Oficial de Nombres de Géneros en Zoología Rechazados e Invalidados, por su similitud y posible confusión con Leiodes Latreille, 1796.

Para terminar este apartado decir que el clásico género Oribata Latreille, 1802 (que da nombre a este grupo animal) lo seguimos considerando como dudoso, como se viene haciendo desde hace tiempo, y que al referirnos a especies en él descritas siempre hemos utilizado dicha denominación, y no la de Oribates que ha sido utilizada muchos autores, unificando así ese aspecto nomenclatural.

Realmente, hubiese sido interesante el haber acompañado este trabajo con unas claves de identificación de familias y géneros que siguiesen la ordenación sistemática aquí propuesta, donde se pudiesen explicar más detalladamente las sinonimias y cambios nomenclaturales realizados y que sirviese de complemento a las magníficas de BALOGH \& BALOGH (1992) e hiciesen todavía más asequible el acceso a la identificación de dichos taxones y al conocimiento de las características utilizadas para su separación, pero su volumen no aconsejaba su presentación conjunta con el presente listado, siendo nuestra intención presentarlas como complemento en un futuro trabajo.

\section{Biogeografía}

Aunque en la mayoría de las especies se ha sacado su distribución geográfica de la bibliografía existente, en algunas especies se ha ampliado notablemente dicha distribución con nuevas citas de material propio o al que hemos tenido acceso y que, sistemáticamente dispuestas, serían las siguientes: Canadá (Ontario) para Gehypochthonius urticinus (Berlese, 1910), especie hasta ahora sólo conocida de U.S.A. (Florida); U.S.A. (Luisiana) para la especie Liochthonius asper Chinone, 1978, hasta ahora sólo conocida de Japón y China; U.S.A. (Carolina del Norte) para las especies paleárticas Liochthonius evansi (Forsslund, 1958) y Sellnickochthonius jacoti (Evans, 1952); Grecia (Isla de Rodas) para la especie Liochthonius morit$z i$ Balogh y Mahunka, 1983, hasta ahora sólo conocida de Japón y España; India (Tripura) para Liochthonius simplex (Forsslund, 1942), especie semicosmopolita sólo citada de Filipinas para la región Oriental; Guinea Ecuatorial para las especies Acrotritia clavata (Märkel, 1964), propia de la región Neotropical, y Allonothrus russeolus Wallwork, 1960, hasta ahora sólo citada de la región Etiópica de Ghana; U.S.A. (Luisiana) para la especie paleártica Trhypochthonius japonicus Aoki, 1970; U.S.A. (Carolina del Norte) para la especie paleártica Malaconothrus egregius (Berlese, 1904); Canadá (Ontario) para la especie paleártica Malaconothrus pygmaeus Aoki, 1969; Canadá (Ontario) y U.S.A. (Carolina del Norte) para la especie paleártica Trimalaconothrus tardus (Michael, 1888); U.S.A. (Carolina del Norte) para la especie europea Nothrus perezinigoi Mahunka, 1980, para la especie paleártica Cepheus cepheiformis (Nicolet, 1855) y para la especie iberomacaronésica Liacarus mucronatus Willmann, 1939; España (Sierra de Guadarrama) para la especie descrita de Túnez Eueremaeus brevifer (Mahunka, 1980); Canadá (Quebec) para la especie europea Graptoppia (Apograptoppia) foveolata (Berlese, 1908); Hawaii para las siguientes especies de Oppiidae y Suctobelbidae: Lasiobelba kuehnelti (Csiszár, 1961) (especie oriental), Pseudoamerioppia javensis (Hammer, 1979) (especie sólo conocida de Indonesia), Multioppia insulana Pérez-Íñigo, 1972 (especie etiópica y macaronésica), Suctobelbella claviseta (Hammer, 1961) (especie conocida de Perú, Isla Mauricio y Japón), Suctobelbella longicurva (Hammer, 1966) (especie sólo conocida de Nueva Zelanda) y Suctobelbella (Flagrosuctobelba) sinuata (Hammer, 1982) (especie oriental); U.S.A. (Carolina del Norte) para la especie europea Suctobelbella alloenasuta Moritz, 1971; Canadá (Quebec) y U.S.A. (Luisiana) para la especie paleártica Suctobelbella arcana Moritz, 1970; U.S.A. (Luisiana) para la especie europea Suctobelbella hamata Moritz, 1970; Marruecos para la especie europea Suctobelbella italica (Mahunka, 1966); U.S.A. (Alaska y Carolina del Norte) para la especie paleártica Suctobelbella longirostris (Forsslund, 1941); U.S.A. (Alaska) para Suctobelbella subtrigona (Oudemans, 1900), especie paleártica sólo conocida en la región Neártica de Groenlandia; U.S.A. (Luisiana) para Suctobelbella (Flagrosuctobelba) elegantula (Hammer, 
1958), especie neotropical conocida también de la India y como paleártica; India (Tripura) para las especies de Borneo Dolicheremaeus sabahnus Mahunka, 1988 y Megalotocepheus singularis (Mahunka, 1988); Canadá (Ontario) para Carabodes palmifer Berlesle, 1904, especie holártica sólo conocida en la región Neártica del Sureste de U.S.A.; U.S.A. (Carolina del Norte) para la especie paleártica Licneremaeus licnophorus (Michael, 1882); India (Tripura) para la subespecie de Europa meridional Tectocepheus velatus clavatus Mahunka, 1983; Mozambique y Yemen para Ethiovertex sculperens (Kok, 1968), especie hasta ahora sólo conocida de Sudáfrica; Yemen para Bipassalozetes deserticus Bayartogtokh y Aoki, 1978, especie descrita de Mongolia; Guinea Ecuatorial para la especie paleártica Passalozetes africanus Grandjean, 1932; Marruecos para Passalozetes stellifer PérezÍñigo y Peña, 1997, especie hasta ahora sólo conocida de las Islas Canarias; U.S.A. (Carolina del Norte) para la especie neotropical Eupelops suramericanus (Hammer, 1961); Yemen para Hypozetes imitator Balogh, 1959, especie descrita de Tanzania y también citada de la India; Norte de Polonia para la especie mediterránea Achipteria praeoccupata nom. nov.; Francia para Scotiazetes aragonensis (Pérez-Íñigo jr., Herrero y Pérez-Íñigo, 1988), especie sólo conocida de España; Turquía para Ceratozetes lagrecai Bernini, 1973, especie hasta ahora sólo conocida del Mediterráneo occidental; Marruecos para la especie española Ceratozetes nasutus Subías, Kahwash y Ruiz, 1990; Canadá (Quebec) para la especie paleártica Fuscozetes pseudosetosus Shaldybina, 1975; España (Islas Baleares) para la especie sudeuropea Chamobates (Xiphobates) depauperatus (Berlese, 1886); Francia para la especie Minguezetes conjunctus Subías, Kahwash y Ruiz, 1990, hasta ahora sólo conocida de España; Canadá (Ontario) para Minguezetes hexagonus (Berlese, 1908), especie paleártica hasta ahora sólo conocida en Norteamérica de Alaska; U.S.A. (Tejas) para la especie neotropical y malgache Oribatula (Zygoribatula) bonairensis (Willmann, 1936); Yemen para la especie mediterránea Oribatula (Zygoribatula) lenticulata (Mínguez y Subías, 1986); Canadá (Quebec) para la especie paleártica Liebstadia longior (Berlese, 1908); U.S.A. (Carolina del Norte) para la especie paleártica Liebstadia pannonica (Willmann, 1951); India (Tripura) para la especie Scheloribates heterodactylus (Mahunka, 1988), hasta ahora sólo conocida de Borneo; Guinea Ecuatorial y U.S.A. (Carolina del Norte) para Scheloribates minifimbriatus Mínguez, Subías y Ruiz, 1986, especie mediterránea también encontrada en Panamá; Mali y Guinea Ecuatorial para la especie semicosmopolita Scheloribates pallidulus (Koch, 1841), especie hasta ahora nunca citada en la región Etiópica; U.S.A. (Tejas) para la especie pantropical Scheloribates praeincisus (Berlese, 1910), tanto "s. str." como la subespecie "sandvicensis" (que también es citada aquí por primera vez de la región Oriental, de la India: Tripura); Francia para la especie española Topobates comatus (Pérez-Íñigo jr., Herrero y Pérez-Íñigo, 1987); U.S.A. (Carolina del Norte y Luisiana) para la especie europea Lauritzenia (Incabates) elegans (Kunst, 1977); Portugal para la especie española Lauritzenia (Incabates) hispanica Morell, 1991; U.S.A. (Tejas) para la especie Peloribates pinguisetus Mahunka, 1985, descrita de las Antillas; Portugal para Peloribates tunisiensis Mahunka, 1980, especie descrita de Túnez; India (Tripura) para Pilobatella schauenbergi Mahunka, 1977, especie sólo conocida de la Península Malaya y Bali; U.S.A. (Luisiana y Carolina del Norte) para la especie pantropical Galumna flabellifera Hammer, 1958; Grecia (Isla de Rodas) para Galumna gibbula Grandjean, 1956, especie mediterránea occidental; U.S.A. (Tejas) para la especie neotropical Galumna similis Pérez-Íñigo y Baggio, 1980; Tanzania (Zanzíbar) y Guinea Ecuatorial (Bioco) para la especie paleártica Pergalumna myrmophila (Berlese, 1914) y Marruecos para Iberogalumnella alandalusica Arillo y Subías, 1993, especie descrita de España.

En fin, aunque se ha tratado de cuidar al máximo que no quedase fuera del presente listado ninguna especie ni se deslizase ningún error, espero comprensión si a la hora de consultarlo se percata el lector de alguno ya que en un trabajo de este tipo, con tal cantidad de nombres, fechas, paréntesis y otros datos, es muy fácil que así sea. También confío en la indulgencia de los especialistas en sistemática de oribátidos si aprecian divergencias con sus criterios. Todos los que publicamos algo sabemos que estamos sometidos a la crítica de los demás. 


\section{Ordenación sistemática de familias y géneros}

\section{ORIBATIDA Dugès, 1834 \\ PALAEOSOMATA Grandjean, 1969 \\ Acaronychoidea Grandjean, 1932 \\ Acaronychidae Grandjean, 1932}

Acaronychus Grandjean, 1932

Amuracarus Lange, 1975

Archeonothrus Trägardh, 1906

Loftacarus Lee, 1981

Stomacarus Grandjean, 1952 (= Andacarus Grandjean, 1958)

Zachvatkinella Lange, 1954 (= Himalacarus Sheals, 1965)

Palaeacaroidea Grandjean, 1932

Palaeacaridae Grandjean, 1932

Palaeacaroides Lange, 1972

Palaeacarus Trägardh, 1932 (= Traegardhacarus Zachvatkin, 1945)

Ctenacaridae Grandjean, 1954

Beklemishevia Zachvatkin, 1945

Ctenacarus Grandjean, 1939 (= Gradjeanacarus Zachvatkin, 1958)

Gilarovella Lange, 1974

Neoctenacarus Moritz, 1974

Aphelacaridae Grandjean, 1954

Adelphacarus Grandjean, 1932

Aphelacarus Grandjean, 1932

PARHYPOSOMATA Balogh y Mahunka, 1979

Parhypochthonioidea Grandjean, 1932

Parhypochthoniidae Grandjean, 1932

Parhypochthonius Berlese, 1904

Gehypochthoniidae Strenzke, 1963

Gehypochthonius Jacot, 1936

Elliptochthoniidae Norton, 1975

Elliptochthonius Norton, 1975

ENARTHRONOTA Grandjean, 1947

Hypochthonioidea Berlese, 1910

Hypochthoniidae Berlese, 1910

Eohypochthonius Jacot, 1938 (=Afrhypochthonius Balogh, 1958)

E. (Neoatrichosus) Fernández, 1984

Hypochthonius Koch, 1835

Malacoangelia Berlese, 1913

Nothrolohmannia Balogh, 1968

Eniochthoniidae Grandjean, 1947

Hypochthoniella Berlese, 1910 (=Arthrochthonius Ewing, 1917)

(=Eniochthonius Grandjean, 1933)

Arborichthoniidae J. y P. Balogh, 1992

Arborichthonius Norton, 1982

\section{Brachychthonioidea Thor, 1934}

Brachychthoniidae Thor, 1934

Brachychthonius Berlese, 1910 (=Brachychochthonius Jacot, 1938)

Eobrachychthonius Jacot, 1936

Liochthonius Hammen, 1959

L. (Afroliochthonius) Mahunka, 1995

Mixochthonius Niedbala, 1972

Neobrachychthonius Moritz, 1976

Neoliochthonius Lee, 1982 (=Moritzichthonius Pereda e Iturrondobeitia, 1990)

(=Paraliochthonius Moritz, 1976 nom praeoc.)

Papillochthonius Gil-Martín, Subías y Arillo, 1992

Poecilochthonius Balogh, 1943

Sellnickochthonius Krivolutsky, 1964
Synchthonius Hammen, 1952

Verachthonius Moritz, 1976

Cosmochthonioidea Grandjean, 1947

Cosmochthoniidae Grandjean, 1947

Cosmochthonius Berlese, 1910

C. (Nanochthonius) Subías y Gil-Martín, 1995

(=Microchthonius Kahwash, Subías y

Gozmanyina Balogh y Mahunka, 1983

uiz, 1989 nom. praeoc.

(=Marshallia Gordeeva, 1980 nom. praeoc.)

Krivolutskiella Gordeeva, 1980

Nipponiella Gordeeva, 1980

Phyllozetes Gordeeva, 1978

Trichthonius Hammer, 1961

Heterochthoniidae Grandjean, 1954

Heterochthonius Berlese, 1910

Neochthonius Karppinen, 1984

Ovochthonius Rjabinin, 1977

Haplochthoniidae Hammen, 1959

Amnemochthonius Grandjean, 1949

Haplochthonius Willmann, 1930 (=Tetrochthonius Hammer, 1958)

H. (Senilochthonius) Mahunka, 1992 Pediculochelidae Lavoipierre, 1946

Paralycus Womersley, 1944 (=Pediculochelus Lavoipierre, 1946) Sphaerochthoniidae Grandjean, 1947

Similochthonius Mahunka, 1985 (=Sphaerochochthonius Mahunka, 1985)

Sphaerochthonius Berlese, 1910 (=Sphaerochthoniella Mahunka, 1985)

Atopochthonioidea Grandjean, 1949

Atopochthoniidae Grandjean, 1949

Atopochthonius Grandjean, 1949

Pterochthoniidae Grandjean, 1950

Pterochthonius Berlese, 1913

$$
\text { Phyllochthoniidae Travé, } 1967
$$

Phyllochthonius Travé, 1967

Protoplophoroidea Ewing, 1917

Protoplophoridae Ewing, 1917

Arthrhoplophora Berlese, 1910 (=Neoprototritia Shereef, 1978) (=Triplophora Mahunka, 1977)

Bursoplophora Subías y Pérez-Íñigo, 1978

$$
\text { (=Csibiplophora Mahunka, 1984) }
$$

Cryptoplophora Grandjean, 1932

Grandjeanoplophora Balogh y Mahunka, 1979

G. (Tauroplophora) Gordeeva, Niemi y Petrova-Nikitina, 1998

Hauseroplophora Mahunka, 1977

Protoplophora Berlese, 1910

Prototritia Berlese, 1910

P. (Siciliophora) Bernini, 1983

MIXONOMATA Grandjean, 1969

DICHOSOMATA Balogh y Mahunka, 1967

Nehypochthonioidea Norton y Metz, 1980

Nehypochthoniidae Norton y Metz, 1980

Nehypochthonius Norton y Metz, 1980

Perlohmannioidea Grandjean, 1954

Perlohmaniidae Grandjean, 1954

Hololohmannia Kubota y Aoki, 1998

Perlohmannia Berlese, 1916 (=Neolohmannia Bulanova-Zachvatkina, 1960)

P. (Apolohmannia) Aoki, 1960 
Collohmanniidae Grandjean, 1958

Collohmannia Sellnick, 1922

Eulohmannioidea Grandjean, 1931

Eulohmanniidae Grandjean, 1931

Eulohmannia Berlese, $1910 \quad$ (=Arthronothrus Trägardh, 1910)

Epilohmannioidea Oudemans, 1923

Epilohmanniidae Oudemans, 1923

Epilohmannia Berlese, $1910 \quad$ (=Lesseria Oudemans, 1917)

(=Neoepilohmannia Bolen y McDaniel, 1989)

(=Sinolohmannia Balogh y Mahunka, 1979)

Epilohmannoides Jacot, 1936 (=Iburidania Aoki, 1959)

\section{Lohmannioidea Berlese, 1916}

Lohmanniidae Berlese, 1916

Annectacarus Grandjean, 1950

Cryptacarus Grandjean, 1950

Dendracarus Balogh, 1961

Haplacarus Wallwork, 1962

Heptacarus Piffl, 1963

(=Neotrichacarus Hammer, 1973) (=Pseudocryptacarus McDaniel, Norton

y Bolen, 1979)

Javacarus Balogh, 196

(=Tongacarus Hammer, 1973)

J. (Euryacarus) Woolley, 1966

Lepidacarus Csiszár, 1961

Lohmannia Michael, 1898

L. (Carolohmannia) Norton, Metz y Sharma, 1978

Meristacarus Grandjean, 1934 (=Javalohmannia Hammer, 1979)

Meristolohmannia Balogh y Mahunka, 1966

Mixacarus Balogh, 1958 (=Hamacarus Hammer, 1977)

M. (Phyllolohmannia) J. y P. Balogh, 1987

Nesiacarus Csiszár, 1961

Ozacarus Colloff y Halliday, 1998

(=Austracarus J. y P. Balogh, 1983

Papillacarus Kunst, 1959

nom. praeoc.)

Paulianacarus Balogh, 196

P. (Millotacarus) Balogh, 1961

Reptacarus Pérez-Íñigo y Peña, 1995

Strinatacarus Mahunka, 1974

Thamnacarus Grandjean, 1950

Torpacarus Grandjean, 1950

(=Asiacarus Krivolutsky, 1971) (=Talpacarus Grandjean, 1939 nom praeoc.)

Ululohmannia Mahunka, 1987 (=Austracarus Mahunka, 1984) Xenolohmannia Balogh y Mahunka, 1969

EUPTYCTIMA Grandjean, 1967

Mesoplophoroidea Ewing, 1917

Mesoplophoridae Ewing, 1917

Apoplophora Aoki, 1980

Archoplophora Hammen, 1959

Dudichoplophora Mahunka, 1982

Mesoplophora Berlese, 1904 (=Phthiracarulus Berlese, 1920)

M. (Parplophora) Niedbala, 1985

\section{Euphthiracaroidea Jacot, 1930}

Oribotritiidae Grandjean, 1954

Austrotritia Sellnick, 1959

(=Macarotritia Pérez-Íñigo, 1986)

Indotritia Jacot, 1929

I. (Afrotritia) Mahunka, 1988

I. (Zeaotritia) Mahunka, 1988

Maerkelotritia Hammer, 1967

Mesotritia Forsslund, 1963

M. (Perutritia) Märkel, 1964

Oribotritia Jacot, 1924

(=Entomotritia Märkel, 1964)

(=Berndotritia Mahunka, 1987)

(=Philotritia Mahunka, 1988)

(=Plesiotritia Walker, 1965)

(=Tritia Berlese, 1883 nom. praeoc.)
Paratritia Moritz, 1976

Protoribotritia Jacot, 1938

Sabacarus Ramsay y Sheals, 1969

Terratritia Ramsay y Sheals, 1969

$$
\text { Euphthiracaridae Jacot, } 1930
$$

Acrotritia Jacot, 1923

(=Rhysotritia Märkel y Meyer, 1959)

Bukitritia Mahunka, 1990

Euphthiracarus Ewing, 1917

(=Niedbalaia Mahunka, 1999)

(=Pseudotritia Willmann, 1919)

(?=Hummelia Oudemans, 1916 gen. inq.)

E. (Brasiliotritia) Märkel, 1964

E. (Pocsia) Mahunka, 1983

Microtritia Märkel, 1964

Sumatrotritia Mahunka, 1989

Synichotritiidae Walker, 1965

Apotritia Norton y Lions, 1992

Sabahtritia Mahunka, 1987

Synichotritia Walker, 1965

Temburongia Mahunka, 1990

Phthiracaroidea Perty, 1841

Phthiracaridae Perty, 1841

Atropacarus Ewing, 1917

(=Helvetacarus Mahunka, 1993)

Hoplophorella Berlese, 1923

H. (Kakophthiracarus) Mahunka, 1992

H. (Steganacarellus) Mahunka, 1986

Hoplophthiracarus Jacot, 1933

Notophthiracarus Ramsay, 1966 (=Arphthicarus Niedbala, 1994)

N. (Besuchetacarus) Mahunka, 1985

N. (Calyptophthiracarus) Aoki, 1980

(=Hauserophthiracarus Mahunka, 1982)

(Phthirarica) Mahunka, 1982

N. (Protophthiracarus) Balogh, 1972

(=Notophthiracarus Balogh y Mahunka, 1967 nom. praeoc.)

Phthiracarus Perty, 1841

(=Ginglymacarus Ewing, 1917)

(=Hoploderma Michael, 1898)

(=Hoplophora Koch, 1836 nom. praeoc.)

=Peridromotritia Jacot, 1923)

P. (Archiphthiracarus) Balogh y Mahunka, 1979

(=Archiphthiracarella Mahunka, 1996)

(=Microphthiracarus Mahunka, 1982)

P. (Metaphthiracarus) Aoki, 1980

=Paraphthiracarus Aoki, 1980)

P. (Neophthiracarus) Balogh y Csiszár, 1963

(=Austrophthiracarus Balogh y Ma-

hunka, 1978)

(=Antarctoplophora Mahunka, 1980)

(=Fuegoplophora Mahunka, 1980)

(=Neoprotophthiracarus Mahunka, 1980)

(=Sturmacarus P. Balogh, 1984)

Rhacaplacarus Niedbala, 1986 (=Plonaphacarus Niedbala, 1986)

R. (Mantigueracarus) Balogh y Mahunka, 1992

Steganacarus Ewing, $1917 \quad$ (=Trachyhoplophora Berlese, 1923)

S. (Rafacarus) Niedbala, 1982 (=Neosteganacarus Balogh y Mahunka, 1992)

(=Nortonacarus Balogh y Mahunka, 1992)

S. (Tropacarus) Ewing, 1917 (=Calhoplophora Berlese, 1923)

HOLOSOMATA Grandjean, 1969

Crotonioidea Thorell, 1876

Trhypochthoniidae Willmann, 1931

Afronothrus Wallwork, 1961

Allonothrus Hammen, 1953

A. (Pseudonothrus) Balogh, 1958

Archegozetes Grandjean, 1931 
Mainothrus Choi, 1996

Mucronothrus Trägardh, 1931

Trhypochthoniellus Willmann, 1928

(=Hydronothrus Aoki, 1964)

Trhypochthonius Berlese, 1904 (=Albonothrus Tseng, 1982)

(=Trilohmannia Willmann, 1923)

(=Tumidalvus Ewing, 1908)

Malaconothridae Berlese, 1916

Malaconothrus Berlese, 1904

M. (Cristonothrus) subgen. nov.

Trimalaconothrus Berlese, 1916 (=Zeanothrus Hammer, 1966)

T. (Tyrphonothrus) Knülle, 1957 (=Fossonothrus Hammer, 1962) Nothridae Berlese, 1896

Nothrus Koch, 1836

(=Angelia Berlese, 1885)

=Gymnonothrus Ewing, 1917)

Novonothrus Hammer, 1966

(=Vigilomicrozetes Tseng, 1982)

Trichonothrus Mahunka, 1986

$$
\text { Camisiidae Oudemans, } 1900
$$

Austronothrus Hammer, 1966

Camisia Heyden, 1826

(=Uronothrus Berlese, 1913)

C. (Ensicamisia) Kunst, 1971

Heminothrus Berlese, 1913

(=Ivarsia Karppinen y Krivolutsky, 1987)

=Paulonothrus Kunst, 1971)

H. (Capillonothrus) Kunst, 1971 (=Ovonothrus Kunst, 1971) (=Paracamisia Olszanowski y Norton, 2002)

H. (Platynothrus) Berlese, 1913 (=Neonothrus Forsslund, 1955) (=Sigmonothrus Chakrabarti y Kundu, 1978)

Crotoniidae Thorell, 1876

Crotonia Thorell, 1876

(=Acronothrus Berlese, 1916) (=Westwoodia Cambridge, 1875 nom

Holonothrus Wallwork, 1963 praeoc.)

Nanhermannioidea Sellnick, 1928

Nanhermanniidae Sellnick, 1928

Bicyrthermannia Hammer, 1979

Cosmohermannia Aoki y Yoshida, 1970

Cyrthermannia Balogh, 1958 (=Linothrus Tseng, 1982)

Dendrohermannia P. Balogh, 1985

Masthermannia Berlese, 1913 (=Phyllonothrus Sellnick, 1959)

(=Posthermannia Grandjean, 1954)

Nanhermannia Berlese, 1913

N. (Nippohermannia) P. Balogh, 1985

Notohermannia P. Balogh, 1985

\section{Hermannioidea Sellnick, 1928}

Hermanniidae Sellnick, 1928

Galapagacarus P. Balogh, 1985

Hermannia Nicolet, 1855

H. (Heterohermannia) Woas, 1992

H. (Phyllhermannia) Berlese, 1916

(=Lawrencoppia Jacot, 1936)

Neohermannia Bayoumi y Mahunka, 1979

BRACHYPYLINA Hull, 1918

PYCNONOTICAE Grandjean, 1954

Hermannielloidea Grandjean, 1934

Hermanniellidae Grandjean, 1934

Akansilvanus Fujikawa, 1993

Ampullobates Grandjean, 1962

Baloghacarus Mahunka, 1983

Dicastribates J. y P. Balogh, 1988

Hermanniella Berlese, 1908
Hermannobates Hammer, 1961

Issaniella Grandjean, 1962

Mahunkobates Calugar, 1989

Sacculobates Grandjean, 1962 (=Bruneiella Mahunka, 1997) Plasmobatidae Grandjean, 1961

Orbiculobates Grandjean, 1961

Plasmobates Grandjean, 1929

Solenozetes Grandjean, 1931

Neoliodoidea Sellnick, 1928

Neoliodidae Sellnick, 1928

Neoliodes Berlese, 1888

(=Liodes Heyden, 1826 nom. praeoc.) (=Udetaliodes Jacot, 1929)

Platyliodes Berlese, 1916

Poroliodes Grandjean, 1934

Teleioliodes Grandjean, 1934

\section{Plateremaeoidea Trägardh, 1926}

Plateremaeidae Trägardh, 1926

Allodamaeus Banks, 1947

Calipteremaeus Paschoal, 1987

Lopheremaeus Paschoal, 1988

Paralopheremaeus Paschoal, 1987

Plateremaeus Berlese, 1908

$$
\text { Pheroliodidae Paschoal, } 1987
$$

Darthvaderum Hunt, 1996

Flammaeremaeus Balogh, 1968

Licnoliodes Grandjean, 1931

Lopholiodes Passchoal, 1987

Lyrifissiella Paschoal, 1989

(=Octoliodes Paschoal, 1987)

Neonooliodes Hunt, 1996

Nooliodes Paschoal, 1989

Novazelandiella Paschoal, 1989 (=Labiogena Hunt, 1996)

Paschoalia nom. nov.

(=Hammeriella Paschoal, 1989 nom. praeoc.)

Pheroliodes Grandjean, $1931 \quad$ (=Andesperuviella Paschoal, 1989)

(=Pedrocortesia Hammer, 1958)

Licnodamaeidae Grandjean, 1954

Hexachaetoniella Paschoal, 1987

Licnodamaeolus Covarrubias, 1998

Licnodamaeus Grandjean, 1931

Nacunansella Fernández y Cleva, 1998

Pedrocortesella Hammer, 1961 (=Acupedicellus Hunt y Lee, 1995)

Licnobelbidae Grandjean, 1965

Flabellobelba Pérez-Íñigo, 1995

Licnobelba Grandjean, 1931

\section{Gymnodamaeoidea Grandjean, 1954}

Gymnodamaeidae Grandjean, 1954

Adrodamaeus Paschoal, 1984 (=Heterodamaeus Woolley, 1957 nom.

Arthrodamaeus Grandjean, 1954 praeoc.)

Austrodamaeus Balogh y Mahunka, 1981

Gymnodamaeus Kulczynski, 1902

(=Idiodamaeus Paschoal, 1984)

(=Heterodamaeus Ewing, 1917)

(=Johnstonella Paschoal, 1983)

(=Odontodamaeus Paschoal, 1982)

Jacotella Banks, 1947

(=Pleodamaeus Paschoal, 1983)

Joshuella Wallwork, 1972

Nortonella Paschoal, 1982

Plesiodamaeus Grandjean, 1954

Aleurodamaeidae Paschoal y Johnston, 1985

Aleurodamaeus Grandjean, 1954

A. (Trichodamaeus) Mahunka, 1984 
Damaeoidea Berlese, 1896

Damaeidae Berlese, 1896

Acanthobelba Enami y Aoki, 1993

Belba Heyden, 1826

B. (Caenobelba) Norton, 1980

Belbodamaeus Bulanova-Zachvatkina, 1960

B. (Lanibelba) Norton, 1980

B. (Protobelba) Norton, 1979

Damaeus Koch, 1835

(=Hypodamaeus Bulanova-Zachvatkina, 1957)

D. (Adamaeus) Norton, 1977

D. (Epidamaeus) Bulanova-Zachvatkina, 1957

D. (Eudamaeus) Pérez-Íñigo, 1987

D. (Paradamaeus) Bulanova-Zachvatkina, 1957

Dameobelba Sellnick, 1928

Metabelba Grandjean, 1936

M. (Allobelba) Kunst, 1961

M. (Neobelba) Bulanova-Zachvatkina, 1967

M. (Parametabelba) Mihelcic, 1964

Metabelbella Bulanova-Zachvatkina, 1957

M. (Akrodamaeus) Norton, 1979

Nortonbelba Bernini, 1980

Parabelbella Bulanova-Zachvatkina, 1967

(=Heterodamaeus Mihelcic, 1964 nom praeoc.)

(=Mirobelba Pérez-Íñigo y Peña, 1994)

P. (Nododamaeus) Hammer, 1977 (=Dasybelba Woolley y Higgins, 1979)

P. (Tectodamaeus) Aoki, 1984

Porobelba Grandjean, 1936

Spatiodamaeus Bulanova-Zachvatkina, 1957

Subbelba Bulanova-Zachvatkina, 1967

S. (Dyobelba) Norton, 1979

S. (Quatrobelba) Norton, 1980

\section{Polypterozetoidea Grandjean, 1959}

Polypterozetidae Grandjean, 1959

Polypterozetes Berlese, 1916

$$
\text { Podopterotegaeidae Piffl, } 1972
$$

Podopterotegaeus Aoki, 1969

\section{Cepheoidea Berlese, 1896}

Cepheidae Berlese, 1896

Cepheus Koch, 1835

(=Tegeocranus Nicolet, 1855)

Compactozetes Hammer, 1966

Conoppia Berlese, 1908

(=Balzania Jacot, 1929)

(=Phyllotegeus Berlese, 1913)

Eupterotegaeus Berlese, 1916

Hypocepheus Krivolusky, 1971

Ommatocepheus Berlese, 1913

Oribatodes Banks, 1895

Pilocepheus Pérez-Íñigo, 1992

Protocepheus Jacot, 1928

Reticulocepheus Vasiliu y Calugar, 1977

(=Cubacepheus Balogh y Mahunka, 1979)

Sadocepheus Aoli, 1965

(=Geocepheus Sitnikova, 1979)

Sphodrocepheus Woolley y Higgins, 1963

=Hamotegeus Balogh y Mahunka, 1969)

Tereticepheus S. y F. Bernini, 1990

Tikizetes Hammer, 1967

Tritegeus Berlese, 1913

$$
\text { Niphocepheidae Travé, } 1959
$$

Niphocepheus Balogh, 1943

$$
\text { Cerocepheidae fam. nov. }
$$

Bornebuschia Hammer, 1966
Cerocepheus Trägardh, 1931

Dicrotegaeus Luxton, 1988

$$
\text { Eutegaeidae Balogh, } 1965
$$

Atalotegaeus Luxton, 1988

Birotegaeus Luxton, 1988

Dudichella Balogh, 1970

Eutegaeus Berlese, 1916

Neoeutegaeus Aoki, 1964

Neseutegaeus Woolley, 1965

Pareutegaeus Woolley, 1965

Porrhotegaeus Balogh y Mahunka, 1966

Pterozetes Hammer, 1966 Pterobatidae Balogh y Mahunka, 1977

Pterobates Balogh y Mahunka, 1977

$$
\text { Nodocepheidae Piffl, } 1972
$$

Nemacepheus Aoki, 1968

Nodocepheus Hammer, 1958

Tumerozetidae Hammer, 1966

Tumerozetes Hammer, 1966

\section{Charassobatoidea Grandjean, 1958}

Microtegeidae Balogh, 1972

Microtegeus Berlese, 1916

Suctotegeus Mahunka, 1987

Charassobatidae Grandjean, 1958

Charassobates Grandjean, 1929

$$
\text { Nosybeidae Mahunka, } 1993
$$

Lamellocepheus Balogh, 1961 (=Nosybea Mahunka, 1993)

(=Tessacarus Grandjean, 1962)

Topalia Balogh y Csiszár, 1963

\section{Microzetoidea Grandjean, 1936}

Microzetidae Grandjean, 1936

Acanthozetes Balogh, 1958

Acaroceras Grandjean, 1936

A. (Malgoceras) Mahunka, 1993

A. (Trichacaroceras) Mahunka, 1991

Afrozetes Engelbrecht, 1972

Anakingia Hammer, 1961

Arenozetes Krivolutsky, 1971

Berlesezetes Mahunka, 1980

Brazilozetes Balogh y Mahunka, 1969

Calozetes Balogh y Mahunka, 1969

Caucasiozetes Shtanchaeva, 1984 (=Teraja Mahunka, 1995)

Cavernozetes Mahunka, 1984

Christovizetes Krivolutsky, 1975

Comorozetes Mahunka, 1994

Cosmozetes Balogh y Mahunka, 1969

C. (Magoebazetes) Engelbrecht, 1972

Cuspitegula Hammer, 1966

Dinozetes Balogh, 1962

Fusozetes Balogh, 1972

Hauserozetes Mahunka, 1980

Hymenozetes Balogh, 1962

Kalyptrazetes Balogh, 1972 (=Allozetes Higgins, 1965 nom. praeoc.)

Kaszabozetes Mahunka, 1988 (=Sinozetes Mahunka, 2000)

Licnozetes Balogh y Mahunka, 1969

L. (Undulozetes) Balogh y Mahunka, 1969

Microzetes Berlese, 1913 (=Nellacaroides Mahunka, 1998)

M. (Megazetes) Balogh, 1959

M. (Stylozetes) Balogh y Mahunka, 1969

Mirabilozetes Mahunka, 1977

Miracarus Kunst, 1959

Mystacozetes Balogh, 1962 
Mysterozetes Hammer, 1961

Orthozetes Balogh, 1962

Oxyzetes Balogh, 1958

Papuazetes Balogh, 1968

Phylacozetes Grandjean, 1936

Plumozetes Balogh, 1972

Protozetes Balogh, 1962

Rhabdozetes Hammer, 1962

Rhopalozetes Balogh, 1962

Rugozetes Balogh, 1960

Schalleria Balogh, 1962

Schalleriella Engelbrecht, 1972

Schizozetes Balogh, 1962

Sturmozetes J. y P. Balogh, 1992 (=Gymnozetes P. Balogh, 1984 nom. praeoc.)

Szentivanyella Balogh y Mahunka, 1969

Trichozetes Balogh y Mahunka, 1980

Vermacarus Balogh y Mahunka, 1980

\section{Zetorchestoidea Michael, 1898}

Zetorchestidae Michael, 1898

Belorchestes Grandjean, 1951

Litholestes Grandjean, 1951

Microzetorchestes Balogh, 1943 (=Diorchestes Grandjean, 1951)

Saxicolestes Grandjean, 195

Strenzkea Travé, 1967

Zetorchestes Berlese, 1888

(=Leptorchistis Canestrini y Berlese, 1885 nom. praeoc.)

(=Phyllorchestes Mahunka, 1983)

Gustavioidea Oudemans, 1900

Astegistidae Balogh, 1961

Astegistes Hull, 1916

(=Cultrozetes Sellnick, 1922)

Cultroribella Mahunka, 1985

Cultroribula Berlese, 1908

Furcoppia Balogh y Mahunka, 1966

F. (Mexicoppia) Mahunka, 1983

Furcoribula Balogh, 1943

Lamellozetes Covarrubias, 1967

Maorizetes Hammer, 1966

Monofurcoppia Pérez-Íñigo y Sarasola, 1995

Quadroribula J. y P. Balogh, 1992

Sulcoribula Hammer, 1971

$$
\text { Multoribulidae Balogh, } 1972
$$

Multoribula Balogh y Mahunka, 1966

Peloptoribula Mahunka, 1984

$$
\text { Ceratoppiidae Kunst, } 1971
$$

Amazoppia Balogh y Mahunka, 1969

Austroceratoppia Hammer, 1979

Ceratoppia Berlese, $1908 \quad(?=$ Notaspis Hermann, 1804 gen. inq.)

Ceratorchestes Balogh y Mahunka, 1969

Comeremaeus Hammer, 1962

Dendrozetes Aoki, 1970

Macquarioppia Wallwork, 1964 (=Macquariella Wallwork, 1963 nom.

Metapyroppia Woolley, 1969 praeoc.)

Negroppia Vasiliu y Calugar, 1977

Notoppia Balogh y Mahunka, 1966

Paraceratoppia Rjabinin, 1982

Parapyroppia Pérez-Íñigo y Subías, 1979

Pseudoceratoppia Hammer, 1962 (=Hauseroceratoppia Mahunka, 1980)

Pseudopyroppia Rjabinin, 1987

Pyroppia Hammer, 1955

Trichoppia Balogh, 1961

Metrioppiidae Balogh, 1943

Ceratoppiella Hammer, 1977

Metrioppia Grandjean, $1931 \quad$ (=Peloppia Sellnick, 1931)

Paenoppia Woolley y Higgins, 1965

\section{Gustaviidae Oudemans, 1900}

Gustavia Kramer, $1879 \quad$ (=Neozetes Berlese, 1885)

(=Serrarius Michael, 1883)

Liacaridae Sellnick, 1928

Adoristes Hull, 1916

Birsteinius Krivolutsky, 1965

Liacarus Michael, 1898

(=Leiosoma Nicolet, 1855 nom. praeoc.) (=Leuroxenillus Woolley y Higgins, 1966)

(=Stenoxenillus Woolley y Higgins, 1966)

L. (Dorycranosus) Woolley, 1969

L. (Procorynetes) Woolley, 1967

L. (Rhaphidosus) Woolley, 1969

Opsioristes Woolley, 1967

Planoristes Iturrondobeitia y Subías, 1978

Xenillidae Woolley y Higgins, 1966

Xenilloides Pérez-Íñigo y Baggio, 1989

Xenillus Robineau-Desvoidy, 1839 (=Banksia Oudemans y Voigts, 1905) (=Dinoxenillus Pérez-Íñigo y Baggio, 1980)

(=Kochia Oudemans, 1900)

=Pseudocepheus Jacot, 1928)

X. (Stonyxenillus) Woolley y Higgins, 1966

Tenuialidae Jacot, 1929

Ceratotenuiala Aoki y Maruyama, 1983

Hafenferrefia Jacot, 1939
Hafenrefferia Oudemans, 1906

Peltenuiala Norton, 1983

Tenuiala Ewing, 1913

Tenuialoides Woolley y Higgins, 1966

Eremaeoidea Oudemans, 1900

Kodiakellidae Hammer, 1967

Kodiakella Hammer, 1967

Megeremaeidae Higgins y Woolley, 1968

Megeremaeus Higgins y Woolley, 1965

Eremaeidae Oudemans, 1900

Asperemaeus Behan-Pelletier, 1982

Carinabella Hammer, 1977

Eremaeus Koch, 1835

Eueremaeus Mihelcic, 1963 (=Kartoeremaeus Higgins, 1979)

Tricheremaeus Berlese, 1908

Aribatidae Aoki, Takaku e Ito, 1994

Aribates Aoki, Takaku e Ito, 1994

Amerobelboidea Grandjean, 1954

Ctenobelbidae Grandjean, 1965

Ctenobelba Balogh, $1943 \quad$ (=Elapheremaeus Grandjean, 1944)

Amerobelbidae Grandjean, 1954

Amerobelba Berlese, 1908

Berndamerus Mahunka, 1977

Hellenamerus Mahunka, 1974

Mongaillardia Grandjean, 1961

Rastellobata Grandjean, 1961

Yambaramerus Aoki, 1996

$$
\text { Eremulidae Grandjean, } 1965
$$

Austroeremulus Mahunka, 1985

Caveremulus Mahunka, 1983 (=Berndbella Mahunka, 1985)

Eremulus Berlese, 1908

Fenestrella Mahunka, 1987

Pseuderemulus Balogh y Mahunka, 1968

Reteremuloides Mahunka, 1989

Reteremulus Balogh y Mahunka, 1966 
Damaeolidae Grandjean, 1965

Caudamaeolus P. Balogh, 1988

Damaeolus Paoli, 1908

Fosseremus Grandjean, 1954

Gressittolus Balogh, $1970 \quad$ (=Fijirella Hammer, 1971)

Hungarobelbidae Miko y Travé, 1996

Costeremus Aoki, 1970

Hungarobelba Balogh, 1943

Eremobelbidae Balogh, 1961

Eremobelba Berlese, 1908

Heterobelbidae Balogh, 1961

Furcodamaeus Pérez-Íñigo y Baggio, 1980

Haplobelba Balogh y Mahunka, 1969

Heterobelba Berlese, 1913

Basilobelbidae Balogh, 1961

Basilobelba Balogh, 1958

Xiphobelba Csiszár, 1961

(=Hammation Grandjean, 1959)

Ameridae Bulanova-Zachvatkina, 1957

Amerus Berlese, 1896

A. (Neamerus) Willmann, 1939

Andesamerus Hammer, 1962

Caenosamerus Higgins y Woolley, 1969

Ctenamerus J. y P. Balogh, 1992

Haplamerus J. y P. Balogh, 1992

Hymenobelba Balogh, 1962

Pteramerus Balogh, 1964

Staurobatidae Grandjean, 1966

Staurobates Grandjean, 1966

Stauroma Grandjean, 1966

Eremelloidea Balogh, 1961

Platyameridae J. y P. Balogh, 1983

Cristamerus Hammer, 1977

Defectamerus Aoki, 1984

Platyamerus J. y P. Balogh, 1983

(?=Gymnodampia Jacot, 1937 gen. inq. $)$

Caleremaeidae Grandjean, 1965

Anderemaeus Hammer, 1958

Caleremaeus Berlese, 1910

Cristeremaeus Balogh y Csiszár, 1963

Epieremulus Berlese, $1916 \quad$ (=Carabodoides Jacot, 1937)

Luxtoneremaeus J. y P. Balogh, 1992

Veloppia Hammer, 1955

Yungaseremaeus Balogh y Mahunka, 1969

Eremellidae Balogh, 1961

Archeremella Balogh y Mahunka, 1974

Eremella Berlese, 1913

(=Proteremella Balogh, 1959)

Licnocepheus Woolley, 1969

Triteremella Kunst, 1971

(=Afreremella Mahunka, 1974)

Machadobelbidae Balogh, 1972

Machadobelba Balogh, 1958

Ramogneta Karppinen, 1966

Rioppia Balogh y Mahunka, 1977

Oribellidae Kunst, 1971

Infernobates Karppinen y Poltavskaja, 1990

Kaszabobates Balogh, 1972 (=Gobiella Balogh y Mahunka, 1965

Montizetes Kunst, 1971

nom. praeoc.)

ribella Berlese, 1908

Oribellopsis Kunst, 1971

Pantelozetes Grandjean, 1953

Proteremaeus Piffl, 1965

(=Gemmazetes Fujikawa, 1979)

(=Sibiremaeus Rjabinin y Krivolutsky, 1975)
Arceremaeidae Balogh, 1972

Arceremaeus Hammer, 1961

Tecteremaeus Hammer, 1961

Spinozetidae Balogh, 1972

Grypoceramerus Suzuki y Aoki, 1970

Iberoppia Pérez-Iñigo, 1986

Spinozetes Piffl, 1966

(=Mirus Kulijev, 1967)

Oppioidea Sellnick, 1937

Autognetidae Grandjean, 1960

Austrogneta Balogh y Csiszár, 1963

Autogneta Hull, 1916

A. (Rhaphigneta) Grandjean, 1960

Conchogneta Grandjean, 1963

Cosmogneta Grandjean, 1960

Eremobodes Jacot, 1937

Parautogneta Golosova, 1974

Thyrisomidae Grandjean, 1953

Banksinoma Oudemans, 1930 (=Thyrisoma Grandjean, 1953) Oppiidae Sellnick, 1937

Antilloppiinae Mahunka, 1985

Joboppia Ruiz, Mínguez y Subías, 1988

Neoppia Bhattacharya y Banerjee, 1981

(=Antilloppia Mahunka, 1985)

Paternoppiinae Gil-Martín, Subías y Arillo, 2000

Paternoppia Gil-Martín, Subías y Arillo, 2000

Lanceoppiinae Balogh, 1983

Acutoppia Balogh, 1983

Basiloppia Balogh, 1983

Cycloppia Balogh, 1983

Drepanoppia Balogh, 1983

Geminoppia J. y P. Balogh, 1983

Globoppia Hammer, 1962

Laminoppia Hammer, 1968

Lanceoppia Hammer, 1962

L. (Baioppia) Luxton, 1985

L. (Bicristoppia) Subías, 1989

L. (Convergoppia) Balogh, 1983

L. (Hamoppia) Hammer, 1968

L. (Lancelalmoppia) Subías, 1989 (=Radamoppia Mahunka, 1994)

Loboppia Balogh, 1983

Membranoppia Hammer, 1968 (=Nesoppia Luxton, 1985)

M. (Pravoppia) Luxton, 1985

Operculoppia Hammer, 1968

Otoppia Balogh, 1983

Polyoppia Hammer, 1968

Processoppia Balogh, 1983

Pustuloppia Mahunka, 1994

Setoppia Balogh, 1983

Trematoppia Balogh, 1964

(=Rhaphoppia Balogh, 1983)

(=Tectoppiella Mahunka, 1984)

Chaviniinae Balogh, 1983

Chavinia Hammer, 1961

Oppiinae Sellnick, 1937

Aeroppia Hammer, 1961

Aethioppia Balogh, 1983

Amerioppia Hammer, 1961

Erioppia Balogh, 1983

Exanthoppia J. y P. Balogh, 1983

Fusuloppia Balogh, 1983

Goyoppia Balogh, 1983

Heteroppia Balogh, 1970

Laroppia Subías, 1989

Lasiobelba Aoki, 1959 (=Cilioppia Balogh, 1983)

L. (Antennoppia) Mahunka, 1983 (=Daedaloppia Hauser y Mahunka, 1983)

Lemuroppia Mahunka, 1944 
Neoamerioppia Subías, 1989

N. (Amerigloboppia) Subías, 1989

Niloppia Balogh, 1983

Oligoppia Balogh, 1983

Oppia Koch, 1836

Paroppia Hammer, 1968

Pluritrichoppia Subías y Arillo, 1989

Quinquoppia Tseng, 1982
Sphagnoppia J. y P. Balogh, 1986

Taiwanoppia Tseng, 1982

T. (Paragloboppia) Subías, 1989

Tanzoppia Mahunka, 1988

Tectoppia Wallwork, 1961

Xenoppia Mahunka, 1982

Multioppiinae Balogh, 1983

Anomaloppia Subías, 1978

Condyloppia Balogh, 1983

Cryptoppia Csiszár, 1961

Cubaoppia Balogh, 1983

Graptoppia Balogh, 1983

G. (Apograptoppia) Subías y Rodríguez 1985 Mahunka, 1983)

G. (Stenoppia) Balogh, 1983

Helioppia Balogh, 1983

Intermedioppia Subías y Rodríguez, 1987

Javieroppia Mínguez y Subías, 1986

Multioppia Hammer, 1961

M. (Furculoppia) Balogh, 1983

M. (Multilanceoppia) Subías, 1989

Octoppia Balogh y Mahunka, 1969

Pararamusella Mahunka y Palacios-Vargas, 1998

Pseudoamerioppia Subías, 1989 (=Minoricoppia Pérez-Íñigo Jr., 1991)

Pulchroppiella Balogh, 1983

Ramonoppia Morell, 1990

Ramusella Hammer, 1962

(=Alcioppia Balogh, 1983)

(=Amolops Hull, 1916 nom. praeoc.)

R. (Insculptoppia) Subías, 1980

(=Bioppia Mahunka y Paoletti, 1984)

(Insculptoppiella) Subías y Rodríguez, 1986

R. (Rectoppia) Subías, 1980

R. (Sabahoppia) Mahunka, 1987

Ramuselloppia Subías y Rodríguez, 1986

Uroppia Balogh, 1983

Pulchroppiinae Balogh, 1983

Multipulchroppia Subías, 1989

Pulchroppia Hammer, 1979

Varioppia Mahunka, 1985

Arcoppiinae Balogh, 1983

Arcoppia Hammer, 1977

Basidoppia Mahunka, 1983

(=Chuoppia Tseng, 1982)

Mimoppia Balogh, 1983

M. (Dysarcoppia) Mahunka y Palacios-Vargas, 1998

Porrhoppia Balogh, 1970

Similoppia Mahunka, 1983

S. (Reductoppia) P. Balogh, 1984

Wallworkoppia Subías, 1989

(=Wallworkella Balogh, 1983 nom. praeoc.)

Brachioppiinae Subías, 1989

Austroppia Balogh, 1983

Brachioppia Hammer, 1961

Brachioppiella Hammer, 1962 (=Ugandoppia Mahunka, 1988)

B. (Gressittoppia) Balogh, 1983 (=Jermyia Mahunka, 2002)

Brassoppia Balogh, 1983

B. (Plaesioppia) Balogh, 1983

Ctenoppia Balogh, 1983

Gittella Hammer, 1961

Kokoppia Balogh, 1983

Leptoppia Mahunka, 1997

Pletzenoppia Balogh, 1983
Setuloppia Balogh, 1983

Trapezoppia Balogh y Mahunka, 1969

Medioppiinae Subías y Mínguez, 1985

Congoppia Balogh, 1983

Discoppia Balogh, 1983

D. (Cylindroppia) Subías y Rodríguez, 1986

Medioppia Subías y Mínguez, 1985 (=Kunoppia Mahunka, 1987)

Medioxyoppia Subías, 1989

Microppia Balogh, 1983

Miroppia Hammer, 1968

Multimedioppia Subías, 1991

Paramedioppia Mahunka y Mahunka-Papp, 2000

Ramuloppia Balogh, 1961

Rhinoppia Balogh, 1983

Serratoppia Subías y Mínguez, 1985

Solenoppia Hammer, 1968

S. (Campbelloppia) Luxton, 1985

Oppiellinae Seniczak, 1975

Abchasiella Gordeeva y Tarba, 1990

Autoppia Golosova y Karppinen, 1983

Belloppia Hammer, 1968

Berniniella Balogh, 1983

B. (Canaloppia) Mahunka y Palacios-Vargas, 1998

Dissorhina Hull, $1916 \quad$ (=Cosmoppia Balogh, 1983)

Elaphoppia Balogh, $1983 \quad$ (=Parasynoppia Aoki, 1983)

Hypogeoppia Subías, 1981

Kulievia Vasiliu e Ivan, 1999

Lauroppia Subías y Mínguez, 1986 (=Ctenoppiella Gordeeva y Karppinen, 1988)

Liacaroppia Subías y Rodríguez, 1986

Moritzoppia Subías y Rodríguez, 1988

M. (Moritzoppiella) Gordeeva, 2000

(19iella Balogh, 1983 nom. praeoc.)

Tetroppia Gordeeva, 1999)

(=Dentoppia Gordeeva, 2001)

(=Drukoppia Gordeeva, 2001)

Neostrinatina Mahunka, 1979

Neotrichoppia Subías e Iturrondobeitia, 1980

N. (Ancestroppia) Subías y Rodríguez, 1986

N. (Confinoppia) Subías y Rodríguez, 1986

Oppiella Jacot, 1937

O. (Perspicuoppia) Pérez-Íñigo, 1971

Pseudobrachioppiella Tseng, 1982

Ptiloppia Balogh, 1983

Tripiloppia Hammer, 1968

Tuberoppia Golosova, 1974

Oxyoppiinae Subías, 1989

Acroppia Balogh, 1983

Baloghoppia Mahunka, 1983

Foraminoppia Subías y Arillo, 1998

Fossoppia Mahunka, 1994

Foveolatoppia Mahunka, 1988

Lineoppia J. y P. Balogh, 1983

Mahunkella Balogh, 1983

Oxybrachioppia Subías, 1989

Oxyoppia Balogh y Mahunka, 1969

O. (Aciculoppia) Subías y Rodríguez, 1986

O. (Dzarogneta) Kulijev, 1978 (=Fineoppia Khanbekyan y Gordeeva, 1991)

(=Pectinoppia Subías y Rodríguez, 1986)

O. (Oxyoppiella) Subías y Rodríguez, 1986

Oxyoppioides Subías y Mínguez, 1985

Sacculoppia Balogh y Mahunka, 1968

Separatoppia Mahunka, 1983

Subiasella Balogh, 1983

S. (Dividoppia) Mahunka, 1987

S. (Lalmoppia) Subías y Rodríguez, 1986

(=Cassioppia Poltavskaya, 1994)

(=Pararectoppia Mahunka, 1987) 
S. (Lucioppia) Mahunka, 1985

Mystroppiinae Balogh, 1983

Cheloppia Hammer, 1971

Corynoppia Balogh, 1983

Karenella Hammer, 1962

K. (Glabroppia) Subías y Rodríguez, 1986

K. (Stakarenoppia) Subías y Rodríguez, 1986

Mystroppia Balogh, 1959

Rugoppia Mahunka, 1986

Stachyoppia Balogh, 1961

(=Mahnertella Mahunka, 1997)

Striatoppia Balogh, 1958

$$
\text { Epimerellidae Ayyildiz y Luxton, } 1989
$$

Enisella Ayyildiz y Luxton, 1989

Epimerella Kulijev, 1967

Luxtonia Mahunka, 2001

$$
\text { Lyroppiidae Balogh, } 1983
$$

Lyroppia Balogh, 1961

Granuloppiidae Balogh, 1983

Bornemiszaella P. Balogh, 1994

Enantioppia Balogh y Mahunka, 1969

Granuloppia Balogh, $1958 \quad$ (=Pocsoppia Mahunka, 1984)

Hammerella Balogh, 1983 (=Interoppia Mahunka, 1987)

(=Woasella J. y P. Balogh, 2002)

Senectoppia Aoki, 1976

(=Macrosoma Hammer, 1979)

Teratoppiidae Balogh, 1983

Brasiloppia Pérez-Íñigo y Baggio, 1986

Granuloteratoppia P. Balogh, 1988

Leoppia Pérez-Ĩñigo, 1983

Teratoppia Balogh, $1959 \quad$ (=Cretoppia Mahunka, 1986)

T. (Teratoppiella) Balogh, 1983

Sternoppiidae Balogh y Mahunka, 1969

Sternoppia Balogh y Mahunka, 1968

(=Synoppia Balogh y Mahunka, 1969)

Machuellidae Balogh, 1983

Gredosella Gil-Martín, Arillo y Subías, 2000

Machuella Hammer, 1961

Papillonotidae Balogh, 1983

Papillonotus Wallwork, 1961

Tuparezetidae Balogh, 1972

Tuparezetes Spain, 1969

Quadroppiidae Balogh, 1983

Borhidia Balogh y Mahunka, 1974

Hexoppia Balogh, 1958

Quadroppia Jacot, 1939

Q. (Coronoquadroppia) Ohkubo, 1995

Trizetoidea Ewing, 1917

Nosybelbidae Mahunka, 1994

Nosybelba Mahunka, 1994

Cuneoppiidae Balogh, 1983

Cuneoppia Balogh y Mahunka, 1969

Suctobelbidae Jacot, 1938

Allosuctobelba Moritz, 1970

Condylobelba Mahunka, 2001

Fenestrobelba Balogh, 1970

F. (Parasuctobelba) Hammer, 1977

Helvetobelba Mahunka y Mahunka-Papp, 1999

Neosuctobelba Balogh y Mahunka, 1969

Novosuctobelba Hammer, 1977

N. (Coartobelba) Mahunka, 2001
Parisuctobelba Higgins y Woolley, 1976

Persuctobelba Mahunka, 2000

Rhinosuctobelba Woolley y Higgins, 1969

Rhynchobelba Willmann, 1953

Rhynchoppia Balogh, 1968

Serratobelba Mahunka, 1984

Sucteremaeus Golosova y Krivolutsky, 1975

Suctobelba Paoli, 1908

Suctobelbata Gordeeva, 1991 (=Unicobelba Mahunka y Mahunka-

Suctobelbella Jacot, 1937

Papp, 1999)

S. (Flagrosuctobelba) Hammer, 1979

S. (Ussuribata) Rjabinin, 1975 (=Bruneibelba Mahunka, 2001)

Suctobelbila Jacot, 1937

=Discosuctobelba Hammer, 1979)

(=Reticobella Hammer, 1962)

(=Rhynchobella Hammer, 1961)

Suctobelbiloides Mahunka, 1988

=Suctoppia Balogh, 1958)

Zeasuctobelba Hammer, 1966

Rhynchoribatidae Balogh, 1961

Rhynchoribates Grandjean, 1929

Suctoribates Balogh, 1963

$$
\text { Oxyameridae Aoki, } 1965
$$

Oxyamerus Aoki, 1965

$$
\text { Trizetidae Ewing, } 1917
$$

Trizetes Berlese, 1904

$$
\text { Otocepheoidea Balogh, } 1961
$$

Dampfiellidae Balogh, 1961

Beckiella Grandjean, 1964

Dampfiella Sellnick, 1931

$$
\text { Tetracondylidae Aoki, } 1961
$$

Afrotocepheus Mahunka, 1985

A. (Didierotocepheus) Mahunka, 1994

Borneremaeus Mahunka, 1991

Bulbocepheus Mahunka, 1988

Cavernocepheus Balogh y Mahunka, 1969

Dolicheremaeus Jacot, 1938 (=Dicondyla Aoki, 1965)

Fernandocepheus Mahunka, 1982

Fissicepheus Balogh y Mahunka, 1967

F. (Psammocepheus) Aoki, 1970

Flagellocepheus P. Balogh, 1984

Hydroecocepheus Corpuz-Raros, 1979

Leptotocepheus Balogh, 1961

Longocepheus Balogh y Mahunka, 1966

Lophotocepheus J. y P. Balogh, 1983

Neotrichocepheus Hammer, 1973

Papillocepheus Balogh y Mahunka, 1966

Paradolicheremaeus Tseng, 1982

Plenotocepheus Hammer, 1966

P. (Neotocepheus) Hammer, 1966

Pseudotocepheus Balogh, 1961 (=Nesotocepheus Hammer, 1972)

P. (Constrictocepheus) Grobler, 1998

Seboetocepheus Mahunka, 1985

Spinotocepheus Hammer, 1981

Trichocepheus Balogh y Mahunka, 1966

Trichocondyla J. y P. Balogh, 1986

Wallworkodes J. y P. Balogh, 2002

Otocepheidae Balogh, 1961

Acrotocepheus Aoki, 1965

A. (Hexatocepheus) Wen, 1993

Basiceramerus Corpuz-Raros, 1979 
Cerostocepheus Mahunka, 1973

Eurostocepheus Aoki, 1965

Ikarotocepheus Mahunka, 1988

Kalayaan Corpuz-Raros, 1998

Megalotocepheus Aoki, 1965

Ocellotocepheus Mahunka, 1989

(=Archegotocepheus Mahunka, 1988)

Otocepheus Berlese, 1905

Papuacepheus Balogh, 1968

Philippotocepheus J. y P. Balogh, 1992

Rimandocepheus Corpuz-Raros, 1998

Samarocepheus Corpuz-Raros, 1990

Trichotocepheus Aoki, 1965

Tokunocepheidae Aoki, 1966

Tokunocepheus Aoki, 1966

\section{Carabodoidea Koch, 1837}

Carabodidae Koch, 1837

Apotomocepheus Aoki, 1965

Archegocepheus Aoki, 1965

Austrocarabodes Hammer, 1966

A. (Baloghodes) Mahunka, 1986

A. (Uluguroides) Mahunka, 1983 (=Ngorongobodes J. y P. Balogh, 1992)

Bathocepheus Aoki, 1978

(=Philippobodes J. y P. Balogh, 1992)

Carabodes Koch, 1835

(=Neocepheus Willmann, 1936)

C. (Flexa) Kulijev, 1977

C. (Klapperiches) Mahunka, 1979

C. (Phyllocarabodes) Balogh y Mahunka, 1969

(=Antillobodes Mahunka, 1985)

=Pentabodes P. Balogh, 1984)

Cavernocarabodes Mahunka, 1974

Congocepheus Balogh, 1958

Cubabodes Balogh y Mahunka, 1974

Diplobodes Aoki, 1958

D. (Kalloia) Mahunka, 1985 (=Mauribodes J. y P. Balogh, 1992)

D. (Neocarabodes) Balogh y Mahunka, 1969

Gibbicepheus Balogh, 1958

G. (Gibbibodes) Mahunka, 1986

Gymnobodes Balogh, 1965

Hardybodes Balogh, 1970

Machadocepheus Balogh, 1958

(=Carabodella Mahunka, 1986)

Malgasodes Mahunka, 2000

Meriocepheus Aoki, 1973

Odontocepheus Berlese, 1913

O. (Aokiella) Balogh y Mahunka, 1967

O. (Indotocepheus) Mondal y Kundu, 1999

Pasocepheus Aoki, 1976

P. (Guineobodes) Mahunka, 1987

Pseudocarabodes Mahunka, 1991 (=Bakobodes Mahunka, 1996)

(=Yemenobodes Mahunka, 2000)

Sagittabodes J. y P. Balogh, 1992

Singabodes Mahunka, 1998

Spathulocepheus Balogh y Mahunka, 1969

Tansocepheus Mahunka, 1983

Tectocarabodes Mahunka, 1988

Trichocarabodes Balogh, 1961 (=Ceylobodes J. y P. Balogh, 1992)

Tuberocepheus Balogh y Mahunka, 1969

Yoshiobodes Mahunka, 1986

Y. (Berndobodes) Mahunka, 1986

Carabocepheidae Mahunka, 1986

Carabocepheus Berlese, 1910

Nippobodidae Aoki, 1959

Leobodes Aoki, 1965

Nippobodes Aoki, 1959

Tectocepheoidea Grandjean, 1954

Tectocepheidae Grandjean, 1954

Tectocepheus Berlese, 1896
Tegeozetes Berlese, 1913

Tegeocranellidae P. Balogh, 1987

Tegeocranellus Berlese, 1913

Hydrozetoidea Grandjean, 1954

Hydrozetidae Grandjean, 1954

Hydrozetes Berlese, 1902

H. (Argentinobates) Fernández, 1984

H. (Heloribates) Grandjean, 1966

Ameronothroidea Willmann, 1931

Ameronothridae Willmann, 1931

Alaskozetes Hammer, 1955

Ameronothrus Berlese, 1896 (=Hygroribates Jacot, 1934)

Antarcticola Wallwork, 1967

Aquanothrus Engelbrecht, 1975

Capillibates Hammer, 1966

Chudalupia Wallwork, 1981

Halozetes Berlese, 1916

(=Anarea Dalenius, 1958)

Podacarus Grandjean, 1955

(=Pertorgunia Dalenius, 1958)

Pseudantarcticola Balogh, 1970

Selenoribatidae Schuster, 1963

Arotrobates Luxton, 1992

Psednobates Luxton, 1992

Schusteria Grandjean, 1968

Selenoribates Strenzke, 1961

Thalassozetes Schuster, 1963

Fortuyniidae Hammen, 1963

Alismobates Luxton, 1992

Circellobates Luxton, 1992

Fortuynia Hammen, 1960

Adhaesozetes Hammer, 1966

Cymbaeremaeoidea Sellnick, 1928

Adhaesozetidae Hammer, 1973

Cymbaeremaeidae Sellnick, 1928

Bulleremaeus Hammer, 1966

Cymbaeremaeus Berlese, 1896

Glanderemaeus Balogh y Csiszár, 1963

Scapheremaeus Berlese, 1910

Seteremaeus Hammer, 1971

$$
\text { Ametroproctidae fam. nov. }
$$

Ametroproctus Higgins y Woolley, 1968

A. (Coropoculia) Aoki y Fujikawa, 1972

Scapuleremaeus Behan-Pelletier, 1989

PORONOTICAE Grandjean, 1954

Licneremaeoidea Grandjean, 1931

Micreremidae Grandjean, 1954

Fenichelia Balogh, 1970

(=Porofenichelia Mahunka, 1985)

Mexiceremus J. y P. Balogh, 1998

Micreremus Berlese, 1908

Phylloribatula Balogh y Mahunka, 1978

$$
\text { Lamellareidae Balogh, } 1972
$$

Lamellarea Kok, 1968

Microlamellarea Coetzee, 1987

Tenuelamellarea Subías e Iturrondobeitia, 1978

Licneremaeidae Grandjean, 1931

Huilicheremaeus Fernández, Marcangeli y Eguaras, 1997

Licneremaeus Paoli, 1908

Scutoverticidae Grandjean, 1954

Arthrovertex Balogh, 1970 (=Argentinovertex Fernández y Cleva, 2001) 
Ethiovertex Mahunka, 1982

Exochocepheus Woolley y Higgins, 1968

$$
\text { (=Latovertex Mahunka, 1987) }
$$

Hypovertex Krivolutsky, 1969

Lamellovertex Bernini, 1976

Provertex Mihelcic, 1959

Scutovertex Michael, 1879

Scutoverticosus Kok, 1968

Passalozetidae Grandjean, 1954

Bipassalozetes Mihelcic, 1957 (=Salpasozetes Mahunka, 1977)

B. (Passalobates) Pérez-Íñigo y Peña, 1996

Passalozetes Grandjean, 1932

P. (Passalomonia) Mahunka, 1987

Phenopelopoidea Petrunkevitch, 1955

Phenopelopidae Petrunkevitch, 1955

Eupelops Ewing, 1917

$$
\begin{aligned}
& \text { (=Allopelops Hammer, 1952) } \\
& \text { (=Celaeno Koch, 1835) } \\
& \text { (=Globonothrus Tseng, 1982) } \\
& \text { (=Pelops Koch, } 1835 \text { nom. praeoc.) } \\
& \text { (=Phenopelops Petrunkevitch, 1955) } \\
& \text { (=Tectopelops Jacot, 1929) }
\end{aligned}
$$

Nesopelops Hammer, 1973

Peloptulus Berlese, 1908

Propelops Jacot, 1937

(=Hammeria Sellnick, 1944)

Unduloribatoidea Kunst, 197

Unduloribatidae Kunst, 1971

Koreoribates Choi, 1994

Unduloribates Balogh, 1943

Eremaeozetidae Piffl, 1972

Eremaeozetes Berlese, 1913

Mahunkaia Schatz, 2002

Seteremaeozetes P. Balogh, 1988

Idiozetidae Aoki, 1976

Idiozetes Aoki, 1976

$$
\text { Limnozetoidea Thor, } 1937
$$

Limnozetidae Thor, 1937

Limnozetella Willmann, 1931

(=Austroceratobates Mahunka, 1985)

Limnozetes Hull, 1916

=Vietobates Mahunka, 1987)

Austrachipteriidae Luxton, 1985

Allozetes Berlese, 1913

Austrachipteria Balogh y Mahunka, 1966

(=Parahypozetes Hammer, 1967)

Ceratobates Balogh y Mahunka, 1969

Granizetes Hammer, 1961

Hypozetes Balogh, 1959

Lamellobates Hammer, 1958

L. (Paralamellobates) Bhaduri y Raychaudhuri, 1968

Palmitalia Pérez-Íñigo y Peña, 1997

Patagonozetes J. y P. Balogh, 1990

Punctizetes Hammer, 1971

Sacculozetes Behan-Pelletier y Rjabinin, 1991

Sagittazetes J. y P. Balogh, 1983

\section{Achipterioidea Thor, 1929}

Achipteriidae Thor, 1929

Achipteria Berlese, 1885

A. (Izuachipteria) Balogh y Mahunka, 1979

(=Hokkachipteria Balogh y Mahunka 1979)

Anachipteria Grandjean, 1932

Campachipteria Aoki, 1995

Cerachipteria Grandjean, 1935 (=Zygachipteria Mihelcic, 1956)

Cubachipteria Balogh y Mahunka, 1979
Dentachipteria Nevin, 1974

Hoffmanacarus Mahunka, 1995

Parachipteria Hammen, 1952 (=Pseudachipteria Travé, 1960)

Plakoribates Popp, 1960

$$
\text { Tegoribatidae Grandjean, } 1954
$$

Lemurobates Mahunka, 1997

Lepidozetes Berlese, 1910

Neophysobates Luxton, 1987

(=Onazetes Bugrov, 1991)

(=Paraphysobates Luxton, 1985 nom. praeoc.)

(=Pseudophysobates Fujikawa, 1991)

Paraphysobates Mahunka, 1985

Physobates Hammer, 1962

Scutozetes Hammer, 1952

Tegoribates Ewing, 1917

Umbellozetes Krivolutsky, 1969

Williamszetes Hammer, 1961

(=Monophysobates J. y P. Balogh, 1992)

(=Neolepidozetes Hammer, 1977)

=Lepidoribates Sellnick, 1920)

(=Williamsia Hammer, 1958 nom. praeoc.)

Oribatelloidea Jacot, 1925

Oribatellidae Jacot, 1925

Adoribatella Woolley, 1967

Cuspidozetes Hammer, 1962

Ferolocella Grabowski, 1971

Joelia Oudemans, 1906

=Gendzella Kulijev, 1977)

Kunstella Krivolutsky, 1974

Novoribatella Engelbrecht, 1986

Ophidiotrichus Grandjean, 1953

Oribatella Banks, 1895

O. (Cavernella) Bernini, 1975

O. (Fenestrobates) Balogh y Mahunka, 1969

O. (Multoribatella) subgen. nov.

Prionoribatella Aoki, 1975

Pseudotectoribates Subías, 1977

Siciliotrichus Bernini, 1973

Tectoribates Berlese, 1910 Ceratokalummidae Balogh, 1970

Achipterina Berlese, 1916

Arcozetes Hammer, 1958

Belemacarus Pérez-Íñigo y Baggio, 1997

Cultrobates Willmann, 1930

Genavensia Mahunka, 1983

Guaranozetes Balogh y Mahunka, 1981

$$
\text { Epactozetidae Grandjean, } 1930
$$

Epactozetes Grandjean, 1930

Truncozetes Balogh y Mahunka, 1969

\section{Ceratozetoidea Jacot, 1925}

Heterozetidae Kunst, 1971

Farchacarus Wallwork, 1967 (=Africacarus Wallwork, 1965 nom. praeoc.)

Heterozetes Willmann, 1917

Ceratozetidae Jacot, 1925

Ceratozetella Shaldybina, 1966 (=Ceratozetoides Shaldybina, 1966) =Ceresella Paulitchenko, 1993)

C. (Cyrtozetes) Behan-Pelletier, 1985

Ceratozetes Berlese, 1908

C. (Magellozetes) Hammer, 1962

C. (Mixozetes) J. y P. Balogh, 1990

Dentizetes Hammer, 1952

Edwardzetes Berlese, 1914

E. (Gamerozetes) J. y P. Balogh, 1990

Euzetes Berlese, 1908

Furcobates Sellnick, 1959

F. (Parafurcobates) Hammer, 1967

F. (Pseudogeminozetes) Tseng, 1984 
Fuscozetes Sellnick, 1928

Geminozetes Balogh y Csiszár, 1963

G. (Naiazetes) Behan-Pelletier, 1996

=Selvazetes Behan-Pelletier, 1998)

Ghilarovizetes Shaldybina, 1969

Jugatala Ewing, 1913

Lobozetes Hammer, 1958

Macrogena Wallwork, 1966

M. (Safrobates) Mahunka, 1989

Melanozetes Hull, 1916

Murcia Koch, 1835

Neogymnobates Ewing, 1917

N. (Koreozetes) Aoki, 1974

Onychobates Hammer, 1967

Oromurcia Thor, 1930

Pentazetes J. y P. Balogh, 1983

P. (Porallozetes) J. y P. Balogh, 1992

Scotiazetes Wallwork, 1966

S. (Guatermalozetes) Mahunka, 1979

Sphaerozetes Berlese, $1885 \quad$ (=Incudozetes J. y P. Balogh, 2002)

$(=$ Sphaerozetella Jacot, 1929)

S. (Porozetes) Hammer, 1962

Tutorozetes Hammer, 1967

Viracochiella Hammer, 1961 (=Leebates Balogh y Mahunka, 1996)

V. (Laminizetes) Behan-Pelletier, 1986

V. (Latilamellobates) Shaldybina, 1971

(=Trichoribatella Mahunka, 1983)

(=Vicinebates Paulitchenko, 1991)

Zetomimus Hull, 1916

(=Hamobates Hammer, 1962)

Z. (Protozetomimus) Pérez-Íñigo, 1990

$$
\text { Chamobatidae Thor, } 1937
$$

Chamobates Hull, 1916

C. (Xiphobates) Paulitchenko, 1993

Globozetes Sellnick, 1928

Iugoribates Sellnick, 1944

Ocesobates Aoki, 1965

(=Chamozetes Sellnick, 1974)

(=Danobates Gjelstrup, 1978)

Pedunculozetes Hammer, 1962

Humerobatidae Grandjean, 1970

Africoribates Evans, 1953

Anellozetes Hammer, 1962

Diapterobates Grandjean, 1936

Humerobates Sellnick, 1928

(=Kilimabates Mahunka, 1984)

(=Antarctozetes Balogh, 1961)

(=Baloghobates Hammer, 1967)

(=Banksinus Jacot, 1938)

(=Jeannelia Dalenius, 1958)

(=Trihumerozetes Sellnick, 1959)

H. (Cordylobates) Luxton, 1995

Ramsayellus Spain y Luxton, 1970 (=Ulugurozetes Mahunka, 1984) (=Zealandobates Hammer, 1967 nom. praeoc.)

Svalbardia Thor, 1930

Punctoribatidae Thor, 1937

Allomycobates Aoki, 1976

Alpizetes Mahunka, 2001

Cryptobothria Wallwork, 1963

Ellipsozetes Bernini, 1980

Eupunctoribates Hammer, 1977

Feiderzetes Subías, 1977

(=Allozetes Feider, Vasiliu y Calugar,

1971 nom. praeoc.)

Inigozetes Subías, 2000

Minguezetes Subías, Kahwash y Ruiz, 1990

Minunthozetes Hull, 1916 (=Jurabates Jacot, 1929)

Mycobates Hull, 1916

M. (Calyptozetes) Thor, 1930 (=Permycobates Strenzke, 1954)
Mycozetes Spain, 1968

Neomycobates Wallwork, 1963

Pelopsis Hall, 1911

(=Ewingozetes Hammer, 1952)

(=Parapelops Jacot, 1938)

Punctoribates Berlese, 1908

Semipunctoribates Mahunka, 1987

Zachvatkinibates Shaldybina, 1973 (=Schweizerzetes Mahunka, 2001)

\section{Zetomotrichoidea Grandjean, 1934}

Zetomotrichidae Grandjean, 1934

Anoplozetes Lee y Pajak, 1987

Demisalto Coetzee, 1993

D. (Saltatrichus) Coetzee, 1993

Ghilarovus Krivolutsky, 1966

Mabulatrichus Coetzee, 1993 (=Hungaromotrichus Mahunka, 1993)

Mikizetes Hammer, 1958

M. (Oglasacarus) Bernini, 1979

Pallidacarus Krivolutsky, 1975

Rohria Balogh y Mahunka, 1977

Zetomotrichus Grandjean, 1934

Z. (Keralotrichus) Mahunka, 1985

$$
\begin{aligned}
& \text { Oripodoidea Jacot, } 1925 \\
& \text { Drymobatidae J. y P. Balogh, } 1984
\end{aligned}
$$

Drymobates Grandjean, 1930

Drymobatoides Jacot, 1936 (=Pelokylla Adolph y Haq, 1982)

Rykella Balogh, 1962

Mochlozetidae Grandjean, 1960

Calugarella J. y P. Balogh, 1992

Dynatozetes Grandjean, 1960

Gephyrazetes Hirauchi, 1999

Mahunkazetes J. y P. Balogh, 1992

Mochlobates Norton, 1984

Mochloribatula Mahunka, 1978

Mochlozetes Grandjean, 1930

Nesiotizetes Jacot, 1934

Paralobozetes Tseng, 1984

Podoribates Berlese, 1908

=Cardioribates Jacot, 1934)

=Falsolobozetes Tseng, 1984)

(=Sphaerobates Sellnick, 1928)

Unguizetes Sellnick, 1925 (=Terrazetes Jacot, 1929)

Uracrobates Balogh y Mahunka, 1967

(=Urobates Hammer, 1973)

Neotrichozetidae Balogh, 1965

Neotrichozetes Travé, 1961

$$
\text { Oribatulidae Thor, } 1929
$$

Capilloppia Balogh y Mahunka, 1966

Crassoribatula Hammer, 1967

Diphauloppia J. y P. Balogh, 1984

Grandjeania Balogh, 1963 (=Gradjeanella Balogh, 1961 nom. praeoc.)

Jornadia Wallwork y Weems, 1984 (=Woolleybates J. y P. Balogh, 1984)

Lucoppia Berlese, 1908 (=Romanobates Feider, Vasiliu y Calugar, 1970)

Lunoribatula Mahunka, 1982

Megatrichobates Grobler, 2000

Neolucoppia Tseng, 1984

Oribatula Berlese, 1896

(=Ceroribatula Lee y Birchby, 1989)

O. (Zygoribatula) Berlese, 1916 (=Fovoribatula Lee y Birchby, 1991) (=Neoribatula Ewing, 1917)

(=Zetobelba Hull, 1916)

Ovobates Mahunka, 1994

Paraphauloppia Hammer, 1967

P. (Monophauloppia) P. Balogh, 1988

Phauloppia Berlese, 1908

(=Calvoppia Jacot, 1934)

(=Eporibatula Sellnick, 1928) 
(=Imaparatoppia Jacot, 1934)

(?=Oribata Latreille, 1802 gen. inq. $)$

(=Paraliodes Hall, 1911)

(=Trichoribatula Balogh, 1961)

Phauloppiella Subías, 1977

Reticuloppia Balogh y Mahunka, 1966

=Decoribatula Lee y Birchby, 1989)

Sellnickia Oudemans, 1927

Spinoppia Higgins y Woolley, 1966

Subphauloppia Hammer, 1967

$$
\text { Nesozetidae J. y P. Balogh, } 1984
$$

Nesozetes Hammer, 1971

$$
\text { Pseudoppiidae Mahunka, } 1975
$$

Ausoribula Lee, 1992

Pseudoppia Pérez-Íñigo, 1966 (=Symphauloppia Balogh, 1972)

Senoribula Mahunka, 1975

$$
\text { Parapirnodidae Aoki y Ohkubo, } 1974
$$

Gerloubia Coetzer, 1968

$$
\text { (=Fuerteventuria Pérez-Íñigo y Peña, }
$$
1996)

Parapirnodus Balogh y Mahunka, 1968

Pontiobates Luxton, 1989

$$
\text { Caloppiidae Balogh, } 1960
$$

Brassiella Balogh, 1970

Chaunoproctellus Mahunka, 1992

Chaunoproctus Pearce, $1906 \quad$ (=Caloppia Balogh, 1958) (=Pabulozetes Tseng, 1982)

(=Zetorchella Berlese, 1916)

Stelechobates Grandjean, 1965

Hemileiidae J. y P. Balogh, 1984

Balazsella Mahunka, 1983

Baobabula Mahunka, 1975

Constrictobates Balogh y Mahunka, 1966

Dometorina Grandjean, 1951 (=Cryptozetes Hammer, 1962)

Exoribatula Jacot, $1936 \quad$ (=Nesoribatula Aoki, 1964)

E. (Multoribates) Hammer, 1961

Hemileius Berlese, 1916

H. (Tuberemaeus) Sellnick, 1930 (=Anisochthodes Newell, 1957)

H. (Turcibates) Ayyildiz y Luxton, 1989

(=Setulobates Mahunka, 1984)

H. (Urubambates) Hammer, 1961

$$
\text { (=Tenuileius Lee, 1989) }
$$

Heteroleius Balogh y Mahunka, 1966

Monoschelobates Balogh y Mahunka, 1969

Mucrobates Balogh y Mahunka, 1979

Nasozetes Sellnick, $1930 \quad$ (=Kinabaluella Mahunka, 1996)

Paraleius Travé, 1960

P. (Metaleius) Travé, 1960

P. (Wallworkiella) Hammer, 1979

Plumobates Balogh y Mahunka, 1966

Siculobata Grandjean, $1953 \quad$ (=Floribates Norton y Kethley, 1989)

S. (Vesiculobates) Hammer, 1979

Simkinia Krivolutsky, 1966

Zeascheloribates Luxton, 1982

Maudheimiidae J. y P. Balogh, 1984

Maudheimia Dalenius, 1958

Multimaudheimia gen. nov

Liebstadiidae J. y P. Balogh, 1984

Angullozetes Hammer, 1967

Areozetes Hammer, 1961

Berndibula Mahunka, 2000

Cordiozetes Mahunka, 1983

Haloribatula Schuster, 1957

Liebstadia Oudemans, 1906

(=Mixoribatula Mahunka, 1987)

(=Irinobates Krivolutsky y Christov, 1970)
Maculobates Hammer, 1962 (=Subulobates Hammer, 1972)

(=Rajskibates J. y P. Balogh, 1984)

Poroscheloribates Arillo, Gil-Martín y Subías, 1994

Reductobates Balogh y Mahunka, 1966

Totobates Hammer, 1961

$$
\text { (=Ingella Hammer, 1967) }
$$

Piffliella Hammer, 1979

Symbioribatidae Aoki, 1966

Symbioribates Aoki, 1966

Scheloribatidae Jacot, 1935

Annobonzetes Pérez-Íñigo, 1983

Coronibatula Mahunka, 1988

Cosmobates Balogh, 1959

Euscheloribates Kunst, 1958 (=Corynobates J. y P. Balogh, 1992)

E. (Nanobates) J. y P. Balogh, 1984

(=Tribates Mahunka, 1978)

Fijibates Hammer, 1971

Fissurobates Balogh y Mahunka, 1969

Grandjeanobates Ramsay, 1967 (=Aellenobates Mahunka, 1978)

Hammerabates Balogh, $1970 \quad$ (=Otaheitea Hammer, 1972)

Muliercula Coetzer, 1968

(=Yoronoribates Aoki, 1987)

Nannerlia Coetzer, 1968

Pachygena Hammer, 1972

Perscheloribates Hammer, 1973 (=Ischeloribates Corpuz-Raros, 1980) (=Scheloribatella Mahunka, 1984)

P. (Makischeloribates) Corpuz-Raros, 1980

P. (Oxyscheloribates) J. y P. Balogh, 1990

Planobates Hammer, 1973

Rhabdoribates Aoki, 1967

Samoabates Hammer, 1973

Scheloribates Berlese, 1908

(=Bischeloribates Mahunka, 1988)

=Megascheloribates Lee y Pajak, 1990)

(=Paraschelobates Jacot, 1934)

(=Philoribates Corpuz-Raros, 1980)

=Protoschelobates Jacot, 1934)

(=Semischeloribates Hammer, 1973)

(=Storkania Jacot, 1929)

S. (Andeszetes) Hammer, 1961

Scheloribatoides Mahunka, 1988

Similobates Mahunka, 1982

Striatobates Hammer, 1973

Topobates Grandjean, 1958

(=Berndia Mahunka, 1988)

(=Flagellobates Mahunka, 1978)

(=Setobates Balogh, 1961)

Oripodidae Jacot, 1925

Anoripoda Sellnick, 1959

Benoibates Balogh, 1958

(=Exoripoda Woolley, 1961)

(=Haploripoda Balogh y Mahunka, 1967)

(=Reductoripoda Mahunka y Palacios-

Vargas, 1996)

Birobates Balogh, 1970

(=Trischeloribates Hammer, 1971)

Brachyoripoda Balogh, 1970

Calobates Balogh, 196

C. (Protoripoda) Balogh, 1970

Campbellobates Wallwork, 1964

Cosmopirnodus Balogh, 1970

Gymnobates Banks, 1902

Gymnobatoides Woolley, 1966

Oripoda Banks, 1904

Pseudopirnodus Baranek, 1985

Pteroripoda Balogh y Mahunka, 1974

Scriptoripoda P. Balogh, 1985

Subpirnodus Mahunka, 1988

Truncopes Grandjean, 1956

Pirnodidae Grandjean, 1956

Cryptoribatula Jacot, 1934 (=Euaella Hammer, 1973) 
Huarpescopes Fernández, Monetti y Martínez, 1995

Pirnodus Grandjean, 1956

Protoribatidae J. y P. Balogh, 1984

Cribrozetes Balogh, 1970

Perxylobates Hammer, 1972

Protoribates Berlese, 1908

(=Octodurozetes Mahunka, 1993)

(=Alloribates Banks, 1947)

(=Propeschelobates Jacot, 1936)

(=Styloribates Jacot, 1934)

(=Xylobates Jacot, 1929)

P. (Triaungius) Kulijev, 1978

(=Brasilobates Pérez-Íñigo y Baggio, 1980)

=Glaberoribates Tseng, 1984)

Setoxylobates Balogh y Mahunka, 1967

S. (Plenoxylobates) Hammer, 1979

S. (Polyxylobates) Hammer, 1973

Sicaxylobates Luxton, 1985

Transoribates Pérez-Íñigo, 1992 (=Lagenobates Weigmann y Miko, 2002)

Trixylobates Balogh y Mahunka, 1978

Tuxenia Hammer, 1958

Vilhenabates Balogh, 1963

V. (Phalacrozetes) Aoki, 1965

Haplozetidae Grandjean, 1936

Acutozetes Balogh, 1970

Afroleius Mahunka, 1984

Baloghia Mahunka, 1994

Baloghiella Bulanova-Zachvatkina, 1960

Berlesiella Hammer, 1979

B. (Comororibula) Mahunka, 1994

Bolkiah Mahunka, 1997

Borneozetes Mahunka, 1997

Cantharozetes Hammer, 1961

Conozetes Balogh y Mahunka, 1969

Indoribates Jacot, 1929

(=Nixozetes Mahunka, 1977)

=Sundazetes Hammer, 1979)

I. (Haplozetes) Willmann, 1935

I. (Mancoribates) Hammer, 1961 (=Minasbates Pérez-Íñigo y Baggio, 1996)

I. (Philippizetes) Corpuz-Raros, 1979

I. (Triungulozetes) Subías, 2001 (=Mixobates Gil-Martín y Subías, 1993 nom. praeoc.)

Lauritzenia Hammer, 1958

L. (Incabates) Hammer, 1961 (=Canaribates Pérez-Íñigo y Peña, 1994)

L. (Magnobates) Hammer, 1967 (=Gymnozetes Calugar y Vasiliu, 1983) Magyaria Balaogh, 1963

Paraxylobates Balogh y Mahunka, 1969

Peloribates Berlese, 1908

(=Capillozetes Balogh, 1943)

(=Euryparazetes Radford, 1950)

(=Indobates Pandit y Bhattacharya, 1999)

P. (Aokibates) Mahunka, 1988

(=Parazetes Willmann, 1930)

P. (Tentaculozetes) Balogh, 1970

Pilobatella Balogh y Mahunka, 1967

Pilobates Balogh, 1960

P. (Italobates) Mahunka, 1994

Setincabates Lee, 1993

Trachyoribates Berlese, 1908

T. (Rostrozetes) Sellnick, 1925 (=Carabozetes Mihelcic, 1957)

(=Zaherizetes Yousef y Nasr, 1976)

Nasobatidae Balogh, 1972

Nasobates Woolley, 1966

Tubulozetidae P. Balogh, 1989

Tubulozetes P. Balogh, 1989
Galumnoidea Jacot, 1925

Parakalummidae Grandjean, 1936

Neoribates Berlese, 1914

N. (Parakalumma) Jacot, 1929

N. (Protokalumma) Jacot, 1929

Perezinigokalumma gen. nov.

Sandenia Oudemans, 1917

S. (Porokalumma) Wallwork, 1966 (=Neokalumma Tseng, 1984)

Galumnidae Jacot, 1925

Acrogalumna Grandjean, 1956

Aegyptogalumna Al-Assiuty, Abdel-Hamid, Seif y El-Deeb, 1985

Allogalumna Grandjean, 1936 (=Ctenogalumna Balogh, 1961)

Carinogalumna Engelbrecht, 1973 (=Paracarinogalumna Mahunka, 1998) (=Pseudogalumna Pérez-Íñigo y Baggio, 1994)

Centroribates Berlese, 1914

Cryptogalumna Grandjean, 1957

Dicatozetes Grandjean, 1956

Didymonycha Mahunka, 1984

Dimidiogalumna Engelbrecht, 1972

Disparaglumna Hammer, 1973

Flagellozetes Balogh, 1970

Galumna Heyden, $1826 \quad$ (=Holokalumma Jacot, 1929)

=Holozetes Jacot, 1929)

(=Zetes Koch, 1836)

G. (Angulogalumna) Grishina, 1981

G. (Cosmogalumna) Aoki, 1988 (=Variogalumna Mahunka, 1995)

G. (Erogalumna) Grandjean, 1964

G. (Indogalumna) Balakrishnan, 1985

G. (Kabylogalumna) Bernini, 1984

Globogalumna J. y P. Balogh, 1990

Heterogalumna Balogh, 1960

Kinezogalumna Aoki y Hu, 1993

Leptogalumna Balogh, 1960

Neogalumna Hammer, 1973

Notogalumna Sellnick, 1959

Orthogalumna Balogh, 1961

Pergalumna Grandjean, 1936

(=Mirogalumna Mahunka, 1993)

Pilizetes Sellnick, 1939

P. (Neopilizetes) J. y P. Balogh, 1990

$(?=$ Kratzensteinia Oudemans, 1919

P. (Sarawakiella) Mahunka, 1996

gen. inq.)

Pilogalumna Grandjean, 1956

Psammogalumna Balogh, 1943

Rostrogalumna Engelbrecht, 1973

Sacculogalumna Engelbrecht, 1973

Setogalumna P. Balogh, 1985

Sphaerogalumna Balogh, 1961

Stictozetes Berlese, 1916

(=Strabogalumna Mahunka, 1995)

Taeniogalumna Balogh, 1962

Trichogalumna Balogh, 1960

Trichogalumnella Mahunka, 1992

Vaghia Oudemans, 1919

Xenogalumna Balogh, 1961

Galumnellidae Piffl, 1970

Galumnella Berlese, 1916

G. (Bigalumnella) Mahunka, 1994

Galumnopsis Grandjean, 1931

G. (Porogalumnella) Balogh, 1968

Iberogalumnella Arillo y Subías, 1993

Monogalumnella Mahunka, 1986

Trypogalumnella Mahunka, 1995 
Nacional de Ciencias Naturales y

Consejo Superior de Investigaciones Científicas Licencia Creative Commons 3.0 España (by-nc) 


\section{LISTADO SISTEMÁTICO DE ESPECIES}

\author{
Orden ORIBATIDA Dugès, 1834 \\ Suborden PALAEOSOMATA Grandjean, 1969 \\ Acaronychoidea Grandjean, 1932
}

Acaronychidae Grandjean, 1932 (6 gen. y 23 spp.)

DisTRIBUCIÓN: Cosmopolita (excepto Oriental).

Acaronychus Grandjean, 1932 (3 spp.)

ESPECIE TIPO: Acaronychus traegardhi Grandjean, 1932

DISTRIBUCIÓN: Neotropical y subtropical (Holártica meridional)

-Acaronychus grandjeani Lange, 1975

DisTRIBUCIÓN: Cáucaso.

-Acaronychus proximus Schubart, 1968

DisTRIBUCIÓN: Brasil.

-Acaronychus traegardhi Grandjean, 1932

DISTRIBUCIÓN: Holártica meridional (Mediterránea occidental,

Sur de Paleártica oriental y U.S.A. meridional) e I. Galápagos.

Amuracarus Lange, 1975 (1 sp.)

EsPECIE TIPO: Amuracarus voskresenskii Lange, 1975

DISTRIBUCIÓN: Paleártica.

-Amuracarus voskresenskii Lange, 1975

DistribuCIÓN: Este de Rusia asiática.

Archeonothrus Trägardh, 1906 (1 sp.)

EsPECIE TIPO: Archeonothrus natalensis Trägardh, 1906

DISTRIBUCIÓN: Etiópica.

-Archeonothrus natalensis Trägardh, 1906

DisTRIBUCIÓN: Sudáfrica.

Loftacarus Lee, 1980 (2 spp.)

EsPECIE TIPO: Loftacarus siefi Lee, 1981

DisTRIBUCIÓN: Subtropical austral (excepto Etiópica).

-Loftacarus longicaudatus (Balogh y Csiszár, 1963) (Stomacarus)

DisTRIBUCIÓN: Argentina.

-Loftacarus siefi Lee, 1981

DISTRIBUCIÓN: Australia.

Stomacarus Grandjean, 1952 (9 spp.)

(=Andacarus Grandjean, 1958)

EsPECIE TIPO: Stomacarus tristani Grandjean, 1952

DISTRIBUCIÓN: Austral.

-Stomacarus abresi Lee, 1981

Distribución: Australia.

-Stomacarus campbellensis (Wallwork, 1966) (Andacarus)

DisTRIBUCIÓN: Antártica (I. Campbell)

-Stomacarus ciliosus Luxton, 1982

DisTRIBUCión: Nueva Zelanda.

-Stomacarus leei Mahunka, 1989

DisTRIBUCión: Australia.

-Stomacarus ligamentifer (Hammer, 1967) (Andacarus)

DisTRIBUCIÓN: Nueva Zelanda.

-Stomacarus macfarlani Grandjean, 1957

DistRIBUCión: Austral (Argentina y Antártica: I. Kerguelen).

-Stomacarus setiger R. Martínez y Casanueva, 1999

DisTRIBUCIÓN: Chile.

-Stomacarus tristani Grandjean, 1952

DistRiBución: Subantártica (I. Tristán da Cunha).

-Stomacarus watsoni (Travé, 1964) (Andacarus)

DisTRIBUCIÓN: Antártica (I. Macquarie).

Zachvatkinella Lange, 1954 (7 spp.)

(=Himalacarus Sheals, 1965)

EsPECIE TIPO: Zachvatkinella belbiformis Lange, 1954

DISTRIBUCIÓN: Holártica.

-Zachvatkinella baicalica Lange, 1972

DisTRIBUCIÓN: Sur de Siberia.
-Zachvatkinella belbiformis Lange, 1954

DisTRIBUCIÓN: Paleártica oriental.

-Zachvatkinella caucasica Lange, 1972

DistriBución: Cáucaso.

-Zachvatkinella chimalae (Sheals, 1965) (Himalacarus)

DisTRIBUCIÓN: Nepal.

-Zachvatkinella doryura Lange, 1972

DISTRIBUCIÓN: Este de Rusia asiática.

-Zachvatkinella longipilis (Jacot, 1938) (Acaronychus traegardhi l.)

DisTRIBUCIÓN: U.S.A. (Carolina del Norte).

-Zachvatkinella nipponica Aoki, 1980

DisTRIBUCIÓN: Japón.

Palaeacaroidea Grandjean, 1932

Palaeacaridae Grandjean, 1932 (2 gen., 6 spp. y 1 ssp.) DisTRIBUCIÓN: Holártica.

Palaeacaroides Lange, 1972 (1 sp.)

ESPECIE TIPO: Palaeacaroides pacificus Lange, 1972

DISTRIBUCIÓN: Paleártica.

-Palaeacaroides pacificus Lange, 1972

DistriBución: Paleártica oriental (excepto Norte).

Palaeacarus Trägardh, 1932 (5 spp. y 1 ssp.)

(=Traegardhacarus Zachvatkin, 1945)

EsPECIE TIPO: Palaeacarus hystricinus Trägardh, 1932

DisTRIBUCIÓN: Holártica.

-Palaeacarus caucasicus Lange, 1972

Distribución: Paleártica (Cáucaso e Islas del Este de Rusia asiática).

-Palaeacarus hystricinus Trägardh, 1932

DisTRIBUCIÓN: Holártica.

-Palaeacarus hystricinus hystricinus Trägardh, 1932

(=Palaeacarus appalachicus Jacot, 1938)

DisTRIBUCIÓN: Holártica (menos frecuente en el Sur).

-Palaeacarus hystricinus japonicus Aoki, 1980

DisTRIBUCIÓN: Japón.

-Palaeacarus kamenskii (Zachvatkin, 1945) (Traegardhacarus)

(=Palaeacarus hystricinus vaesterbottensis Grandjean, 1954)

Distribución: Paleártica (Paleártica occidental: Norte de Europa

y Crimea, y Paleártica oriental).

-Palaeacarus lapshovi Zachvatkin, 1945

Distribución: Ucrania.

-Palaeacarus orientalis Bulanova-Zachvatkina, 1967

Distribución: Paleártica (Sur de Rusia europea, Este de Paleártica oriental)

Ctenacaridae Grandjean, 1954 (4 gen. y 10 spp.)

DISTRIBUCIÓN: Pantropical y subtropical.

Beklemishevia Zachvatkin, 1945 (6 spp.)

ESPECIE TIPO: Beklemishevia galeodula Zachvatkin, 1945

DisTRIBUCIÓN: Semicosmopolita (Holártica, Etiópica y Neotropical).

-Beklemishevia africana (Mahunka, 1974) (Ctenacarus)

Distribución: Rodesia [Zimbabue].

-Beklemishevia barbata (Schubart, 1968) (Ctenacarus)

DisTriBución: Neotropical (Brasil y Pequeñas Antillas).

-Beklemishevia demeteri Mahunka, 1984

DisTRIBUCIÓN: Etiópica.

-Beklemishevia galeodula Zachvatkin, 1945

DiSTRIBUCIÓN: Holártica meridional (Sureste de Europa y U.S.A.:

Califormia).

-Beklemishevia hispaniola Pérez-Íñigo, 1997

DisTRIBUCIÓN: Mediterránea occidental.

-Beklemishevia zachvatkini Bulanova-Zachvatkina, 1980

DistRIBUCIÓN: Sur de Rusia europea. 
Ctenacarus Grandjean, 1939 (2 spp.)

(=Grandjeanacarus Zachvatkin, 1945)

EsPeCIE TIPO: Palaeacarus araneola Grandjean, 1932

DISTRIBUCIÓN: Pantropical y subtropical.

-Ctenacarus araneola (Grandjean, 1932) (Palaeacarus)

DisTRIBUCión: Pantropical y subtropical.

-Ctenacarus foliisetosus Bulanova-Zachvatkina, 1980

DisTRIBUCIÓN: Sur de Rusia europea.

Gilarovella Lange, 1974 (1 sp.)

EsPECIE TIPO: Gilarovella demetrii Lange, 1974

DisTRIBUCIÓN: Holártica.

-Gilarovella demetrii Lange, 1974

DistRIBUCIÓN: Holártica meridional (Sur de Europa, Asia centrooccidental y U.S.A.: Nuevo Méjico).

Neoctenacarus Moritz, 1974 (1 sp.)

ESPECIE TIPO: Neoctenacarus hastilis Moritz, 1974

DisTRIBUCIÓN: Paleártica.

-Neoctenacarus hastilis Moritz, 1974

DISTRIBUCIÓN: Europa centrooccidental.

Aphelacaridae Grandjean, 1954 (2 gen., 2 spp. y 1 ssp.)

DisTRIBUCIÓN: Semicosmopolita (Holártica, Etiópica y Neotropical).

Adelphacarus Grandjean, 1952 (1 sp.)

ESPECIE TIPO: Adelphacarus sellnicki Grandjean, 1952

DisTRIBUCIÓN: Paleártica.

-Adelphacarus sellnicki Grandjean, 1952

DISTRIBUCIÓN: Europa.

Aphelacarus Grandjean, 1932 (1 sp. y 1 ssp.)

EsPeCIE TIPO: Parhypochthonius acarinus Berlese, 1910

Distribución: Semicosmopolita (Holártica, Etiópica y Neotropical).

-Aphelacarus acarinus (Berlese, 1910) (Parhypochthonius)

Distribución: Semicosmopolita (Holártica, Etiópica y

Neotropical).

-Aphelacarus acarinus acarinus (Berlese, 1910)

(=Aphelacarus rossicus Zachvatkin, 1945)

DistRIBUCIÓN: Semicosmopolita (Holártica: menos frecuente

en el Norte, Etiópica y Neotropical).

-Aphelacarus acarinus sahariensis Gil-Martín, Subías y Arillo, 1992

DisTRIBUCIÓN: Sahara occidental.

Suborden PARHYPOSOMATA Balogh y Mahunka, 1979

Parhypochthonioidea Grandjean, 1932

Parhypochthoniidae Grandjean, 1932 (1 gen. y 9 spp.) DisTRIBUCIÓN: Cosmopolita (excepto Antártica).

Parhypochthonius Berlese, 1904 (9 spp.)

EsPeCIE TIPO: Parhypochthonius aphidinus Berlese, 1904

DistRIBUCIÓN: Cosmopolita (excepto Antártica).

-Parhypochthonius africanus Balogh, 1958 "sp. inq."

DISTRIBUCIÓN: Angola.

-Parhypochthonius aphidinus Berlese, 1904

(=Parhypochthonius aphidinus germanicus Willmann, 1931)

(=Parhypochthonius aphidinus octofilamentis Jacot, 1938)

DisTRIBUCIÓN: Semicosmopolita (Holártica: frecuente, Australiana e I. Galápagos).

-Parhypochthonius asiaticus Mahunka, 1997

DisTRIBUCIÓN: Borneo.

-Parhypochthonius botschi Schweizer, 1956 "sp. inq."

DisTRIBUCIÓN: Suiza.

-Parhypochthonius dubiosus Schweizer, 1956 "sp. inq." DisTRIBUCIÓN: Suiza.

-Parhypochthonius macrorostrum Schweizer, 1956 "sp. inq." DisTriBuCIÓN: Suiza.

-Parhypochthonius nivalis Schweizer, 1956 "sp. inq."

DisTRIBUCIÓN: Suiza.
-Parhypochthonius pilosus Mahunka, 1991

DistribuCión: Macaronésica (Cabo Verde).

-Parhypochthonius stabelchodi Schweizer, 1956 "sp. inq."

DISTRIBUCIÓN: Suiza.

Gehypochthoniidae Strenzke, 1963 (1 gen. y 8 spp.)

DisTRIBUCIÓN: Cosmopolita (excepto Etiópica y Antártica).

Gehypochthonius Jacot, 1936 (8 spp.)

ESPECIE TIPO: Gehypochthonius rhadamanthus Jacot, 1936

DisTRIBUCIÓN: Cosmopolita (excepto Etiópica y Antártica).

-Gehypochthonius antonii Lombardini, 1962 "sp. inq."

DISTRIBUCIÓN: Italia.

-Gehypochthonius frondifer Aoki, 1975

DISTRIBUCIÓN: Japón.

-Gehypochthonius gracilis Pankov, 2002

DisTRIBUCión: Este de Rusia asiática.

-Gehypochthonius marianoi Martínez y Laborde, 2000

DISTRIBUCIÓN: Argentina.

-Gehypochthonius rhadamanthus Jacot, 1936

DisTRIBUCIÓN: Semicosmopolita (Holártica: Europa occidental,

Japón y Neártica, Norte de Oriental, Australia y Panamá).

-Gehypochthonius strenzkei Lee, 1982

DisTRIBUCIÓN: Australia.

-Gehypochthonius urticinus (Berlese, 1910) (Parhypochthonius)

DistRIBUCión: Neártica: U.S.A. (Florida) y Canadá.

Gehypochthonius xarifae Strenzke, 1963

DisTRIBUCIÓN: Oriental (I. Maldivas y Borneo).

Elliptochthoniidae Norton, 1975 (1 gen. y 1 sp.) DISTRIBUCIÓN: Neártica.

Elliptochthonius Norton, 1975 (1 sp.)

ESPECIE TIPO: Elliptochthonius profundus Norton, 1975

DISTRIBUCIÓN: Neártica.

-Elliptochthonius profundus Norton, 1975

DisTRIBUCIÓN: U.S.A. (Califormia).

Suborden ENARTHRONOTA Grandjean, 1947 Hypochthonioidea Berlese, 1910

Hypochthoniidae Berlese, 1910 (4 gen., 1 subg., 20 spp. y 4 sspp.) DisTRIBUCIÓN: Cosmopolita (excepto Antártica).

Eohypochthonius Jacot, 1938 (11 spp.)

ESPECIE TIPO: Hypochthonius gracilis Jacot, 1936

DISTRIBUCIÓN: Pantropical y subtropical.

Eohypochthonius (Eohypochthonius) Jacot, 1938 (10 spp.)

(=Afrhypochthonius Balogh, 1958)

DisTRIBUCIÓN: Pantropical y subtropical.

-Eohypochthonius (E.) africanus Mahunka, 1978

DistRIBUCIÓN: Malgache (I. Mauricio).

-Eohypochthonius (E.) asiaticus (Berlese, 1910) (Hypochthonius)

DisTRIBUCIÓN: Java.

-Eohypochthonius (E.) becki Balogh y Mahunka, 1979

DisTRIBUCIÓN: Cuba.

-Eohypochthonius (E.) crassisetiger Aoki, 1959 (E. gracilis c.)

DisTRIBUCIÓN: Paleártica oriental (excepto Norte) y Australianooriental.

-Eohypochthonius (E.) gracilis (Jacot, 1936) (Hypochthonius)

DisTRIBUCIÓN: Pantropical (excepto Etiópica): frecuente y subtropical.

-Eohypochthonius (E.) magnus Aoki, 1977

DisTRIBUCión: Holártica (Sureste de Paleártica oriental y U.S.A.: Minesota).

-Eohypochthonius (E.) parvus Aoki, 1977

DisTRIBUCIÓN: Paleártica meridional (Mediterránea occidental y Sureste de Paleártica oriental). 
-Eohypochthonius (E.) salicifolius Hammer, 1979

DisTRIBUCIÓN: Oriental (Java y Borneo).

-Eohypochthonius (E.) vermicularis Hammer, 1979

DISTRIBUCIÓN: Java.

-Eohypochthonius (E.) vilhenarum (Balogh, 1958) (Afrhypochthonius) DisTRIBUCIÓN: Etiópica e India (Bengala Occidental).

Eohypochthonius (Neoatrichosus) Fernández, 1984 (1 sp.) ESPECIE TIPO: Eohypochthonius (Neoatrichosus) travei Fernández, 1984 DisTRIBUCIÓN: Neotropical.

-Eohypochthonius (Neoatrichosus) travei Fernández, 1984 DISTRIBUCIÓN: Argentina.

Hypochthonius Koch, 1835 (5 spp. y 3 sspp.)

EsPECIE TIPO: Hypochthonius rufulus Koch, 1835

Distribución: Cosmopolita (excepto Etiópica y Antártica).

-Hypochthonius elegans Hammer, 1979

DISTRIBUCIÓN: Java

-Hypochthonius latirostris Schweizer, 1956 "sp. inq." DISTRIBUCIÓN: Suiza.

-Hypochthonius luteus Oudemans, 1917

(=Hypochthonius luteus rectosetosus Krivolutsky, 1965)

DISTRIBUCIÓN: Holártica (frecuente) y Nueva Zelanda.

-Hypochthonius rufulus Koch, 1835

DisTRIBUCIÓN: Semicosmopolita (Holártica, I. Seychelles y Méjico).

-Hypochthonius rufulus rufulus Koch, 1835

(=Hypochthonius rufulus brevisetosus Krivolutsky, 1965)

(=Hypochthonius pallidulus Koch, 1835)

Distribución: Semicosmopolita (Holártica: frecuente, I. Seychelles y Méjico).

-Hypochthonius rufulus carolinicus Jacot, 1936

DistriBución: U.S.A. (Carolina del Norte)

-Hypochthonius rufulus europaeus Krivolutsky, 1965

DisTRIBUCIÓN: Europa oriental (Rusia y Ucrania).

-Hypochthonius rufulus paucipectinatus Jacot, 1934 DisTRIBUCIÓN: Noreste de U.S.A.

-Hypochthonius ventricosus (Canestrini, 1898) (Hermannia) "sp. inq." DistriBUCIÓn: Nueva Guinea.

Malacoangelia Berlese, 1913 (3 spp. y 1 ssp.)

EsPECIE TIPO: Malacoangelia remigera Berlese, 1913

DISTRIBUCIÓN: Pantropical y subtropical.

-Malacoangelia assamica Talukdar y Chakrabarti, 1984

DistriBución: India (Assam).

-Malacoangelia remigera Berlese, 1913

DisTRIBUCión: Pantropical y subtropical.

-Malacoangelia remigera remigera Berlese, 1913

(=Malacoangelia remigera symmetrica Wallwork, 1960)

Distribución: Pantropical (frecuente) y subtropical.

-Malacoangelia remigera indica Chakrabarti, Bhaduri y

Raychaudhuri, 1972

DistriBución: India (Bengala Occidental).

-Malacoangelia similis Sarkar y Subías, 1982

DisTRIBUCIÓN: India (Tripura)

Nothrolohmannia Balogh, 1968 (1 sp.)

EsPECIE TIPO: Nothrolohmannia calcarata Balogh, 1968 DISTRIBUCIÓN: Australiana.

-Nothrolohmannia calcarata Balogh, 1968

DistriBución: Nueva Guinea.

Eniochthoniidae Grandjean, 1947 (1 gen. y 5 spp.)

DistRIBUCIÓN: Cosmopolita (excepto Antártica).

Hypochthoniella Berlese, 1910 (5 spp.)

(=Arthrochthonius Ewing, 1917)

(=Eniochthonius Grandjean, 1933)

EsPECIE TIPO: Hypochthonius minutissimus Berlese, 1904

DisTRIBUCIÓN: Cosmopolita (excepto Antártica).
-Hypochthoniella crosbyi (Ewing, 1909) (Hypochthonius)

(=Eniochthonius borealis Jacot, 1939)

DISTRIBUCIÓN: U.S.A. central y nororiental.

-Hypochthoniella fukushiraensis Shiraishi y Aoki, 1994

DISTRIBUCIÓN: Japón.

-Hypochthoniella minutissima (Berlese, 1904) (Hypochthonius)

(=Eniochthonius grandjeani Hammen, 1952)

DisTRIBUCIÓN: Cosmopolita (excepto Antártica).

-Hypochthoniella paludicola Fujikawa, 1994

DISTRIBUCIÓN: Japón.

-Hypochthoniella sumatrana Mahunka, 1989

DisTRIBUCIÓN: Oriental (Sumatra y Borneo) y Malgache.

Arborichthoniidae J. y P. Balogh, 1992 (1 gen. y 1 sp.) DisTRIBUCIÓN: Holártica.

Arborichthonius Norton, 1982 (1 sp.)

ESPECIE TIPO: Arborichthonius styosetosus Norton, 1982

DisTRIBUCIÓN: Holártica.

-Arborichthonius styosetosus Norton, 1982

DisTribución: Canadá y "China".

Brachychthonioidea Thor, 1934

Brachychthoniidae Thor, 1934 (11 gen., 1 subg., 158 spp. y 2 sspp.) DisTRIBUCIÓN: Cosmopolita.

Brachychthonius Berlese, 1910 (16 spp.)

=Brachychochthonius Jacot, 1938)

ESPECIE TIPO: (Hypochthonius brevis Michael "sensu” Berlese, 1910)

=Brachychthonius berlesei Willmann, 1928

DISTRIBUCIÓN: Semicosmopolita (Holártica, Etiópica y Australiana).

-Brachychthonius africanus Balogh, 1958 "sp. inq."

DISTRIBUCIÓN: Angola.

-Brachychthonius amicabilis Gil-Martín y Subías, 1997

DisTRIBUCIÓN: España.

-Brachychthonius berlesei Willmann, 1928

DisTRIBUCIÓN: Holártica (frecuente).

-Brachychthonius bimaculatus Willmann, 1936

(=Brachychthonius helveticus Schweizer, 1956)

DisTRIBUCIÓN: Holártica (Paleártica: menos frecuente en el Norte,

y Canadá).

-Brachychthonius hauserorum (Mahunka, 1979) (Brachychochthonius)

DistriBución: Mediterránea (Grecia y Túnez).

-Brachychthonius hirtus Moritz, 1976

DISTRIBUCIÓN: Europa centromeridional.

-Brachychthonius impressus Moritz, 1976

(=Brachychochthonius subcricoides Balogh y Mahunka, 1979)

DisTRIBUCIÓN: Paleártica.

-Brachychthonius jugatus (Jacot, 1938) (Brachychochthonius) "sp. inq." DisTRIBUCIÓN: Neártica.

-Brachychthonius laevis (Jacot, 1938) (Brachychochthonius) "sp. inq." DisTRIBUCión: U.S.A. (Ténesi).

-Brachychthonius lineatus (Mahunka, 1982) (Brachychochthonius)

DisTRIBUCión: Etiopía.

-Brachychthonius pacificus Hammer, 1973

DisTRIBUCIÓn: Polinesia (I. Samoa).

-Brachychthonius parahirtus Subías y Gil-Martín, 1991

DisTRIBUCIÓN: Ibérica.

-Brachychthonius pauliani Balogh y Mahunka, 1966

Distribución: Congo.

-Brachychthonius pius Moritz, 1976

DisTRIBUCIÓN: Holártica (Europa occidental y Canadá).

-Brachychthonius psammophylus Mahunka, 1987

DisTRIBUCIÓN: Hungría.

-Brachychthonius pseudoimmaculatus Subías y Gil-Martín, 1991

DisTRIBUCIÓN: Mediterránea. 
Eobrachychthonius Jacot, 1936 (7 spp. y 1 ssp.)

ESPECIE TIPO: (Eobrachychthonius sexnotatus Jacot, 1936) $=$ Brachychthonius latior Berlese, 1910

DisTRIBUCIÓN: Semicosmopolita (Holártica, Neotropical y Antártica).

-Eobrachychthonius borealis Forsslund, 1942

DisTRIBUCIÓN: Holártica (menos frecuente en el Sur).

-Eobrachychthonius latior (Berlese, 1910) (Brachychthonius)

DisTRIBUCIÓN: Holártica.

-Eobrachychthonius latior latior (Berlese, 1910)

(=Brachychthonius brevis glabra Thor, 1930)

(=Eobrachychthonius grandis Sellnick, 1944)

(=Eobrachychthonius sexnotatus Jacot, 1936)

DISTRIBUCIÓN: Holártica (frecuente).

-Eobrachychthonius latior strictior Lombardini, 1963 "ssp. inq." DISTRIBUCIÓN: Italia.

-Eobrachychthonius longisetosus Csiszár, 1960

DisTRIBUCIÓN: Hungría.

-Eobrachychthonius mooseri (Hammen, 1951) (Brachychthonius)

DisTRIBUCIÓN: Europa centromeridional.

-Eobrachychthonius oudemansi Hammen, 1952

(=Eobrachychthonius argentinensis Hammer, 1958)

Distribución: Semicosmopolita (Holártica, Neotropical y Antártica: I. Georgia del Sur).

-Eobrachychthonius setus Sergienko, 1992

DisTRIBUCIÓN: Ucrania.

-Eobrachychthonius similis Mahunka, 1979

DisTRIBUCIÓN: Grecia.

Liochthonius Hammen, 1959 (73 spp. y 1 ssp.)

EsPECIE TIPO: (Brachychthonius perpusillus Berlese, 1910) =Hypochthonius brevis Michael, 1888

DisTRIBUCIÓN: Cosmopolita.

Liochthonius (Liochthonius) Hammen, 1959 (72 spp. y 1 ssp.)

DisTRIBUCIÓN: Cosmopolita.

-Liochthonius (L.) africanus Mahunka, 1983

DisTRIBUCIÓN: Tanzania.

-Liochthonius (L.) alius Chinone, 1978 (L. plumosus a.)

(=Liochthonius plumosus Chinone y Aoki, 1972, "nom. praeoc." por Mahunka, 1969)

DistribuCión: Japón y "China”.

-Liochthonius (L.) alpestris (Forsslund, 1958) (Brachychthonius) DisTRIBUCIÓN: Paleártica.

-Liochthonius (L.) altimonticola (Hammer, 1958) (Brachychthonius) DisTRIBUCIÓN: Neotropical y Nueva Zelanda.

-Liochthonius (L.) altus (Hammer, 1958) (Brachychthonius) DisTRIBUCIÓN: Subtropical austral (Argentina y Nueva Zelanda).

-Liochthonius (L.) andinus (Hammer, 1958) (Brachychthonius) DisTRIBUCIÓN: Neotropical.

-Liochthonius (L.) andrewi Evison, 1981

DisTRIBUCIÓN: Suiza.

-Liochthonius (L.) approximatus (Jacot, 1939) (Brachychthonius) DisTRIBUCIÓN: U.S.A. (Nueva Hampshire).

-Liochthonius (L.) asotthalomensis Marshall, Reeves y Norton, 1987 (=Liochthonius forsslundi Mahunka, 1969, "nom. praeoc." por Hammer, 1952) DISTRIBUCIÓN: Europa centromeridional.

-Liochthonius (L.) asper Chinone, 1978

DisTRIBUCión: Japón, “China” y U.S.A. (Luisiana).

-Liochthonius (L.) attenuatus (Jacot, 1938) (Brachychthonius)

DisTRIBUCión: U.S.A. (Carolina del Norte).

-Liochthonius (L.) australis Covarrubias, 1968

DisTRIBUCIÓN: Antártica.

-Liochthonius (L.) bifurcatus (Jacot, 1936) (Brachychthonius) DisTRIBUCión: U.S.A. (Carolina del Norte).

-Liochthonius (L.) brevis (Michael, 1888) (Hypochthonius) (=Brachychthonius perpusillus Berlese, 1910)

DisTRIBUCIÓN: Holártica (frecuente).

-Liochthonius (L.) breviseta (Hammer, 1958) (Brachychthonius) DISTRIBUCIÓN: Argentina.
-Liochthonius (L.) clavatus (Forsslund, 1942) (Brachychthonius)

DisTRIBUCIÓN: Paleártica (menos frecuente en el Sur).

-Liochthonius (L.) crassus Chinone, 1978 (L. alpestris c.)

DisTRIBUCIÓN: Japón.

-Liochthonius (L.) dilutus Moritz, 1976

DISTRIBUCIÓN: Europa occidental.

-Liochthonius (L.) evansi (Forsslund, 1958) (Brachychthonius)

(=Liochthonius forsslundi levis Chinone, 1974)

DisTRIBUCIÓN: Holártica (Paleártica: menos frecuente en el Sur, y

U.S.A.: Carolina del Norte)

-Liochthonius (L.) fimbriatissimus Hammer, 1962

(=Brachychthonius fimbriatus Hammer, 1958, "nom. praeoc." por Jacot, 1936)

DistrIBUCIÓN: Semicosmopolita (Australiana, Neotropical: Argentina, y Antártica: I. Príncipe Eduardo).

-Liochthonius (L.) fimbriatus (Jacot, 1936) (Brachychthonius)

DistribuCión: U.S.A. (Carolina del Norte).

-Liochthonius (L.) forsslundi (Hammer, 1952) (Brachychthonius)

DisTRIBUCIÓN: Neártica septentrional.

-Liochthonius (L.) furcillatus (Willmann, 1942) (Brachychthonius) (=Brachychthonius ensifer Strenzke, 1951)

DisTRIBUCIÓN: Paleártica (menos frecuente en el Sur).

-Liochthonius (L.) galba Chinone, 1978

DisTRIBUCIÓN: Japón.

-Liochthonius (L.) gisini (Schweizer, 1948) (Brachychthonius)

DisTRIBUCIÓN: Eurosiberiana.

-Liochthonius (L.) horridus (Sellnick, 1928) (Brachychthonius)

DisTRIBUCIÓN: Paleártica (frecuente).

-Liochthonius (L.) hystricinus (Forsslund, 1942) (Brachychthonius) (=Brachychthonius ocellatus Hammer, 1952)

DISTRIBUCIÓN: Holártica (frecuente).

-Liochthonius (L.) idem Hammer, 1966

DisTRIBUCIÓN: Nueva Zelanda.

-Liochthonius (L.) intermedius Chinone y Aoki, 1972

DistriBución: Paleártica oriental (excepto Norte).

-Liochthonius (L.) khencensis Hammer, 1961

DisTRIBUCIÓN: Perú.

-Liochthonius (L.) kirghisicus Krivolutsky, 1971

DistRIBUCIÓN: Sur de Paleártica oriental.

-Liochthonius (L.) lacunosus Wang y Cui, 1992

DISTRIBUCIÓN: China suroriental.

-Liochthonius (L.) laetepictus (Berlese, 1910) (Brachychthonius)

"sp. inq."

DisTRIBUCIÓN: Italia.

-Liochthonius (L.) lapponicus (Trägardh, 1910) (Hypochthonius

brevis $l$.)

DISTRIBUCIÓN: Holártica (frecuente).

-Liochthonius (L.) latus (Jacot, 1936) (Brachychthonius)

DistribuCión: U.S.A. (Carolina del Norte).

-Liochthonius (L.) lentus Chinone, 1974

DisTRIBUCIÓN: Japón.

-Liochthonius (L.) leptaleus Moritz, 1976

DISTRIBUCIÓN: Europa centromeridional.

-Liochthonius (L.) longipilus (Womersley, 1945) (Brachychthonius)

DistRIBUCIÓN: Australia y Argentina.

-Liochthonius (L.) mollis (Hammer, 1958) (Brachychthonius)

DisTRIBUCIÓN: Argentina y Antártica.

-Liochthonius (L.) moritzi Balogh y Mahunka, 1983

(=Liochthonius hystricinus tuxeni Chinone, 1974, "nom. praeoc." por Forsslund, 1957)

DisTRIBUCIÓN: Paleártica meridional (Japón y Sur de Europa).

Liochthonius (L.) muscorum Forsslund, 1964

DistriBuCión: Paleártica.

-Liochthonius (L.) mussardi Mahunka, 1974

DisTRIBUCIÓN: Rodesia [Zimbabue].

-Liochthonius (L.) neglectus Moritz, 1976

DisTRIBUCIÓN: Europa occidental.

-Liochthonius (L.) neonominatus nom. nov.

[=Liochthonius latus Mahunka, 1982, “nom. praeoc.” por Jacot, 1936 ("hom. sec.")]

DisTRIBUCIÓN: Etiopía. 
-Liochthonius (L.) nodifer Hammer, 1962

DisTRIBUCIÓN: América austral.

-Liochthonius (L.) nortoni R. Martínez y Casanueva, 1995 DisTRIBUCIÓN: Chile.

-Liochthonius (L.) oceanicus Hammer, 1973

DisTribución: Polinesia (I. Samoa).

-Liochthonius (L.) ohnishi Chinone, 1978 DisTRIBUCIÓN: Japón.

-Liochthonius (L.) patagoniensis Hammer, 1962

DisTRIBUCIÓN: Argentina.

-Liochthonius (L.) peduncularius (Strenzke, 1951) (Brachychthonius) DISTRIBUCIÓN: Europa central.

-Liochthonius (L.) penicillus Chinone, 1978 DISTRIBUCIÓN: Japón.

-Liochthonius (L.) pepitensis Hammer, 1962 DisTRIBUCIÓN: América austral.

-Liochthonius (L.) perelegans Moritz, 1976

DISTRIBUCIÓN: Europa centrooccidental.

-Liochthonius (L.) perfusorius Moritz, 1976 DISTRIBUCIÓN: Europa.

-Liochthonius (L.) phitosi Mahunka, 1982

DISTRIBUCIÓN: Grecia.

-Liochthonius (L.) plumosus Mahunka, 1969

DisTRIBUCIÓN: Paleártica (Europa centrooriental y Corea).

-Liochthonius (L.) prior (Jacot, 1938) (Brachychthonius)

DisTRIBUCión: U.S.A. (Ténesi)

-Liochthonius (L.) propinquus Niedbala, 1972

DisTRIBUCión: Paleártica occidental (excepto Norte).

-Liochthonius (L.) pseudohystricinus Balogh y Mahunka, 1983

DisTRIBUCIÓN: Japón.

-Liochthonius (L.) pusillus Chinone, 1978

DisTRIBUCIÓN: Paleártica (Japón y Rumania).

-Liochthonius (L.) rigidisetosus Hammer, 1962

DisTRIBUCIÓN: Chile.

-Liochthonius (L.) rigidisetosus rigidisetosus Hammer, 1962 DistriBuCIÓN: Chile.

-Liochthonius (L.) rigidisetosus curtus Hammer, 1962 Distribución: Chile.

-Liochthonius (L.) saltaensis (Hammer, 1958) (Brachychthonius) DisTRIBUCIÓN: Austral (América austral, Nueva Zelanda y Antártica).

-Liochthonius (L.) sellnicki (Thor, 1930) (Brachychthonius)

(?=Brachychthonius brevis expolitus Berlese, 1910 "sp. inq.")

(=Brachychthonius nodosus Willmann, 1952)

(=Brachychthonius scalaris Forsslund, 1942)

DISTRIBUCIÓN: Holártica (frecuente).

-Liochthonius (L.) similis Mahunka, 1974

DistRIBUCIÓN: Rodesia [Zimbabue].

-Liochthonius (L.) simplex (Forsslund, 1942) (Brachychthonius)

Distribución: Semicosmopolita: Paleártica, Groenlandia, Oriental (Filipinas e India: Tripura) y Australia.

-Liochthonius (L.) strenzkei Forsslund, 1963 DisTRIBUCIÓN: Holártica.

-Liochthonius (L.) szemmelveiszi J. y P. Balogh, 1983

DisTriBución: Nueva Caledonia.

-Liochthonius (L.) tanzanicus Mahunka, 1984

DisTRIBUCIÓN: Tanzania.

-Liochthonius (L.) tuberculatus Hammer, 1961

DISTRIBUCIÓN: Perú.

-Liochthonius (L.) tuxeni (Forsslund, 1957) (Brachychthonius hystricinus $t$.)

DISTRIBUCIÓN: Europa occidental.

-Liochthonius (L.) tyrrhenicus Bernini, 1985 DisTRIBUCIÓN: Italia.

-Liochthonius (L.) unilateralis Hammer, 1962

DisTRIBUCIÓN: América austral.

Liochthonius (Afroliochthonius) Mahunka, 1995 (1 sp.)

ESPECIE TIPO: Liochthonius (Afroliochthonius) reductus Mahunka, 1995 DisTRIBUCIÓN: Etiópica.
-Liochthonius (Afroliochthonius) reductus Mahunka, 1995

Distribución: Malgache (I. Comores).

Mixochthonius Niedbala, 1972 (3 spp.)

ESPECIE TIPO: Brachychthonius pilososetosus Forsslund, 1942 DisTRIBUCIÓN: Holártica.

-Mixochthonius concavus (Chinone, 1974) (Liochthonius)

DisTRIBUCIÓN: Japón y "China".

-Mixochthonius moritzi Evison, 1981

DISTRIBUCIÓN: Suiza.

-Mixochthonius pilososetosus (Forsslund, 1942) (Brachychthonius)

DisTRIBUCIÓN: Boreal (Paleártica occidental y Canadá).

Neobrachychthonius Moritz, 1976 (2 spp.)

ESPECIE TIPO: Brachychthonius marginatus Forsslund, 1942

DisTRIBUCIÓN: Paleártica.

-Neobrachychthonius magnus Moritz, 1976 (N. marginatus m.)

DisTRIBUCIÓN: Paleártica (Europa y Japón).

-Neobrachychthonius marginatus (Forsslund, 1942) (Brachychthonius)

(=Brachychthonius neosimplex Schweizer, 1956)

DistRIBUCIÓN: Paleártica (menos frecuente en el Sur).

Neoliochthonius Lee, 1982 (3 spp.)

(=Moritzichthonius Pereda e Iturrondobeitia, 1990 "sin. nov.")

(=Paraliochthonius Moritz, 1976, "nom. praeoc." por Beier, 1956)

EsPeCIE TIPO: Brachychthonius piluliferus Forsslund, 1942

DisTRIBUCIÓN: Holártica.

-Neoliochthonius globuliferus (Strenzke, 1951) (Brachychthonius)

DISTRIBUCIÓN: Europa centromeridional.

-Neoliochthonius occultus (Niedbala, 1971) (Liochthonius)

DisTRIBUCIÓN: Europa septentrional (Suecia y Polonia).

-Neoliochthonius piluliferus (Forsslund, 1942) (Brachychthonius)

DisTRIBUCIÓN: Holártica (menos frecuente en el Sur).

Papillochthonius Gil-Martín, Subías y Arillo, 1992 (1 sp.)

ESPECIE TIPO: Papillochthonius astatus Gil-Martín, Subías y Arillo, 1992 DisTRIBUCIÓN: Paleártica.

-Papillochthonius astatus Gil-Martín, Subías y Arillo, 1992

DisTRIBUCIÓN: Sahara occidental.

Poecilochthonius Balogh, 1943 (3 spp.)

ESPECIE TIPO: Brachychthonius brevis italicus Berlese, 1910

DisTRIBUCIÓN: Semicosmopolita (Holártica, Australiana y Neotropical).

-Poecilochthonius italicus (Berlese, 1910) (Brachychthonius brevis i.) DisTRIBUCión: Holártica (menos frecuente en el Norte).

-Poecilochthonius parallelus (Womersley, 1945) (Brachychthonius)

DisTRIBUCIÓN: Australiana: Australia y Nuevas Hébridas (Vanuatu).

-Poecilochthonius spiciger (Berlese, 1910) (Brachychthonius brevis s.)

(=Brachychthonius rapoporti Balogh y Mahunka, 1968)

DISTRIBUCIÓN: Holártica y Neotropical (Argentina).

Sellnickochthonius Krivolutsky, 1964 (45 spp.)

ESPECIE TIPO: Brachychthonius zelawaiensis Sellnick, 1928

DISTRIBUCIÓN: Cosmopolita.

-Sellnickochthonius americanus (MacQuitty, 1986) (Brachychochthonius)

Distribución: U.S.A. (Nuevo Méjico).

-Sellnickochthonius anonymus Ruiz, Subías y Kahwash, 1991

DisTRIBUCión: España.

-Sellnickochthonius aokii (Chinone, 1974) (Brachychthonius)

DisTRIBUCión: Este de Paleártica oriental.

-Sellnickochthonius borealis (Krivolutsky, 1965) (Brachychthonius)

DISTRIBUCIÓN: Europa oriental.

-Sellnickochthonius comorensis Mahunka, 1995

Distribución: Malgache (I. Comores).

-Sellnickochthonius cricoides (Weis-Fogh, 1948) (Brachychthonius

(Brachychochthonius))

DisTRIBUCIÓN: Paleártica, India y Australia.

-Sellnickochthonius danubialis Mahunka, 1997

DisTRIBUCión: Hungría. 
-Sellnickochthonius dolosus Gil-Martín, Subías y Arillo, 1992

DISTRIBUCIÓN: Ibérica.

-Sellnickochthonius elisabethae Mahunka, 1974

DisTRIBUCIÓN: Rodesia [Zimbabue].

-Sellnickochthonius elsosneadensis (Hammer, 1958) (Brachy-

chochthonius)

DistRIBUCIÓN: Semicosmopolita (Paleártica oriental: excepto

Norte, Australia y Argentina).

-Sellnickochthonius foliatifer Mahunka, 1982

DisTRIBUCIÓN: Etiopía.

-Sellnickochthonius foliatus (Hammer, 1958) (Brachychochthonius)

DisTRIBUCIÓN: Neotropical.

-Sellnickochthonius formosus (Cooreman, 1947) (Eobrachychthonius)

DisTRIBUCIÓN: Europa septentrional.

-Sellnickochthonius fuentesi Ruiz, Subías y Kahwash, 1991

DisTRIBUCión: España.

-Sellnickochthonius furcatus (Weis-Fogh, 1948) (Brachychthonius

(Brachychochthonius))

DisTRIBUCIÓN: Paleártica occidental.

-Sellnickochthonius gracilis (Chinone, 1974) (Brachychthonius)

DisTRIBUCIÓN: Japón.

-Sellnickochthonius griseus (Hammer, 1958) (Brachychochthonius)

DisTRIBUCión: Argentina.

-Sellnickochthonius guanophilus (Mahunka, 1979) (Brachychoch-

thonius)

DisTRIBUCIÓN: Grecia.

-Sellnickochthonius hanyensis Mahunka y Mahunka-Papp, 2002

DisTRIBUCión: Hungría.

-Sellnickochthonius heterotrichus (Balogh, 1963) (Brachychthonius)

DisTRIBUCIÓN: Angola.

-Sellnickochthonius honestus (Moritz, 1976) (Brachychochthonius) DisTRIBUCIÓN: Europa occidental.

-Sellnickochthonius hungaricus (Balogh, 1943) (Poecilochthonius)

DistriBución: Paleártica meridional.

-Sellnickochthonius immaculatus (Forsslund, 1942) (Brachycho-

chthonius)

(=Brachychochthonius arcticus Hammer, 1952)

(=Brachychochthonius berlesei erosus Jacot, 1938)

(=Brachychthonius gygeri Bader, 1963)

(=Brachychthonius obscurus Krivolutsky, 1966)

(=Brachychthonius semiornatus Evans, 1952)

(=Brachychthonius striatus Willmann, 1956)

DisTRIBUCIÓN: Holártica (frecuente) y Norte de Neotropical.

-Sellnickochthonius jacoti (Evans, 1952) (Brachychthonius)

DistRIBUCión: Holártica (Paleártica y U.S.A.: Carolina del Norte)

-Sellnickochthonius japonicus (Chinone, 1974) (Brachychthonius)

DISTRIBUCIÓN: Japón.

-Sellnickochthonius lydiae (Jacot, 1938) (Brachychochthonius)

(=Brachychthonius hammerae Chinone y Aoki, 1972)

DisTRIBUCIÓN: Neártica y Japón.

-Sellnickochthonius maroccanus (Mahunka, 1980) (Brachychochthonius)

DISTRIBUCIÓN: Marruecos.

-Sellnickochthonius meridionalis (Bernini, 1973) (Brachychochthonius)

DisTRIBUCIÓN: Mediterránea occidental.

-Sellnickochthonius miyauchii (Chinone, 1978) (Brachychochthonius)

DisTRIBUCIÓN: Japón.

-Sellnickochthonius monticola (Hammer, 1961) (Brachychthonius)

DISTRIBUCIÓN: Perú.

-Sellnickochthonius muara Mahunka, 1995

DisTRIBUCIÓN: Borneo.

-Sellnickochthonius niliacus (Al-Assiuty, Bayoumi y Abdel-Hamid, 1984) (Brachychochthonius)

DisTRIBUCIÓN: Egipto.

-Sellnickochthonius novazealandicus (Hammer, 1966) (Brachychthonius)

DisTRIBUCIÓN: Nueva Zelanda.

-Sellnickochthonius oesziae (Balogh y Mahunka, 1979) (Brachychochthonius)

DisTRIBUCión: Hungría.
-Sellnickochthonius paraplanus (Niedbala, 1982) (Brachychochthonius) DisTRIBUCIÓN: Polonia.

-Sellnickochthonius phyllophorus (Moritz, 1976) (Brachychochthonius)

DISTRIBUCIÓN: Europa centromeridional.

-Sellnickochthonius planus (Chinone, 1974) (Brachychthonius)

DisTRIBUCIÓN: Japón y Borneo.

-Sellnickochthonius plumosus Subías y Gil-Martín, 1991

DisTRIBUCIÓN: Mediterránea occidental.

-Sellnickochthonius rostratus (Jacot, 1936) (Brachychthonius)

DisTRIBUCIÓN: Holártica.

-Sellnickochthonius rotundatus (Hammer, 1958) (Brachychochthonius)

DisTRIBUCIÓN: Neotropical (Argentina y Bolivia).

-Sellnickochthonius similis (Hammer, 1961) (Brachychthonius)

DistriBución: Neotropical (Perú y Chile) y Nueva Guinea.

-Sellnickochthonius suecicus (Forsslund, 1942) (Brachychochthonius

jugatus s.)

Distribución: Semicosmopolita (Paleártica, Neártica: Canadá,

Australiana: Nueva Zelanda, y subantártica: I. Amsterdam).

-Sellnickochthonius tropicus (Hammer, 1958) (Brachychthonius)

DisTRIBUCIÓN: Bolivia.

-Sellnickochthonius variabilis (Mahunka, 1982) (Brachychochthonius)

DistRIBUCIÓN: Mediterránea oriental.

-Sellnickochthonius zelawaiensis (Sellnick, 1928) (Brachychthonius)

DisTRIBUCIÓN: Holártica (frecuente).

Synchthonius Hammen, 1952 (2 spp.)

ESPECIE TIPO: (Synchthonius boschmai Hammen, 1952) =Brachychochthonius crenulatus Jacot, 1938

DISTRIBUCIÓN: Holártica.

-Synchthonius crenulatus (Jacot, 1938) (Brachychochthonius)

(=Synchthonius boschmai Hammen, 1952)

DisTRIBUCIÓN: Holártica (frecuente).

-Synchthonius elegans Forsslund, 1957

DisTRIBUCIÓN: Holártica (Paleártica y Neártica septentrional).

Verachthonius Moritz, 1976 (6 spp.)

EsPECIE TIPO: Brachychthonius laticeps Strenzke, 1951

DisTRIBUCIÓN: Semicosmopolita (Holártica, Australiana y Neotropical).

-Verachthonius congruus Moritz, 1976

DisTRIBUCIÓN: Alemania.

-Verachthonius diversus Moritz, 1976

DISTRIBUCIÓN: Europa centrooccidental.

-Verachthonius laticeps (Strenzke, 1951) (Brachychthonius)

DisTRIBUCión: Paleártica (Europa y Japón).

-Verachthonius montanus (Hammer, 1952) (Eobrachychthonius)

DistriBuCión: Canadá y Argentina.

-Verachthonius moritzi Lee, 1982

DisTRIBUCIÓN: Australia.

-Verachthonius pseudolaticeps (Subías, 1977) (Liochthonius)

DISTRIBUCIÓN: España.

Cosmochthonioidea Grandjean, 1947

Cosmochthoniidae Grandjean, 1947 (6 gen., 1 subg., 46 spp. y 3 sspp.) DistriBución: Cosmopolita (excepto Antártica).

Cosmochthonius Berlese, 1910 (32 spp. y 3 ssp.)

EsPeCIE TIPO: Hypochthonius lanatus Michael, 1885

DisTRIBUCIÓN: Cosmopolita (excepto Antártica).

Cosmochthonius (Cosmochthonius) Berlese, 1910 (30 spp. y 3 ssp.)

DisTRIBUCIÓN: Cosmopolita (excepto Antártica).

-Cosmochthonius (C.) asiaticus Gordeeva, 1980

DisTRIBUCión: Paleártica meridional (Asia centrooccidental y España).

-Cosmochthonius (C.) assamensis Talukdar y Chakrabarti, 1984

DisTRIBUCIÓN: India (Assam).

-Cosmochthonius (C.) australicus Womersley, 1945 (C. plumatus a.)

DISTRIBUCIÓN: Australia. 
-Cosmochthonius (C.) bengalensis Chakrabarti, Bhaduri y Raychaudhuri, 1972

DisTRIBUCIÓN: India (Bengala Occidental)

-Cosmochthonius (C.) bhutanensis Chakarbarti y Wilson, 1981

DISTRIBUCIÓN: Bután.

-Cosmochthonius (C.) concavus Aoki, 1994

DisTRIBUCIÓN: Micronesia (I. Marianas).

-Cosmochthonius (C.) desaussurei Mahunka, 1982

Distribución: Méjico.

-Cosmochthonius (C.) domesticus Grandjean, 1947 "sp. inq."

DISTRIBUCIÓN: Francia.

-Cosmochthonius (C.) foliatus Subías, 1982

DISTRIBUCIÓN: Mediterránea occidental.

-Cosmochthonius (C.) imperfectus Aoki, 2000

DisTRIBUCIÓN: Japón.

-Cosmochthonius (C.) lanatus (Michael, 1885) (Hypochthonius)

DisTRIBUCIÓn: Cosmopolita (excepto Australiana y Antártica).

-Cosmochthonius (C.) lanatus lanatus (Michael, 1885)

(=Cosmochthonius arctosus Pankov, 2002)

(=Cosmochthonius reticulatus Grandjean, 1947)

(=Cosmochthonius wallworki Lee, 1982)

Distribución: Cosmopolita (Holártica: frecuente, Etiópica,

Oriental y Neotropical).

-Cosmochthonius (C.) lanatus diversiseta Sarkar y Subías, 1982 DisTRIBUCIÓN: India (Tripura).

-Cosmochthonius (C.) lanatus foveolatus Beck, 1962

Distribución: Neotropical (Perú y Chile) y Japón.

-Cosmochthonius (C.) macrosetosus Ayyildiz y Luxton, 1990

DisTRIBUCIÓN: Turquía.

-Cosmochthonius (C.) maroccanus Gil-Martín, Subías y Arillo, 1992

DisTRIBUCión: Mediterránea occidental.

-Cosmochthonius (C.) minifoveolatus Gil-Martín, Subías y Candelas, 1991

DisTRIBUCIÓN: España.

-Cosmochthonius (C.) monegrensis Pérez-Íñigo jr., 1991

DisTRIBUCión: España.

-Cosmochthonius (C.) nayoroensis Fujikawa, 1980

DisTRIBUCIÓN: Japón.

-Cosmochthonius (C.) novus Sergienko, 1991

DisTRIBUCIÓN: Ucrania.

-Cosmochthonius (C.) pallidus Lombardini, 1961 "sp. inq."

DISTRIBUCIÓN: Italia.

-Cosmochthonius (C.) perezinigoi Morell, 1988

DisTRIBUCIÓN: España.

-Cosmochthonius (C.) plumatus Berlese, 1910

DisTRIBUCIÓN: Semicosmopolita (Holártica, Oriental y Neotropical).

-Cosmochthonius (C.) plumatus plumatus Berlese, 1910

DisTRIBUCIÓN: Holártica meridional.

-Cosmochthonius (C.) plumatus suramericanus Hammer, 1958

Distribución: Argentina, U.S.A. (Nuevo Méjico), e India (Tripura).

-Cosmochthonius (C.) ponticus Gordeeva, 1980

DistriBución: Sureste de Europa.

-Cosmochthonius (C.) semiareolatus Hammer, 1966

DistRIBUCión: Nueva Zelanda.

-Cosmochthonius (C.) semifoveolatus Subías, 1982

DisTRIBUCIÓN: España.

-Cosmochthonius (C.) signatus Pérez-Íñigo, 1990

DisTRIBUCIÓN: España.

-Cosmochthonius (C.) spinosus Gil-Martín, Subías y Candelas, 1991

DISTRIBUCIÓN: Ibérica.

-Cosmochthonius (C.) sublanatus Mahunka, 1977

DisTRIBUCIÓN: Oriental (Java y Filipinas).

-Cosmochthonius (C.) taurus Niemi, Gordeeva y Ayyildiz, 2002

DisTRIBUCIÓN: Turquía.

-Cosmochthonius (C.) tenuisetus Gordeeva, 1980

DisTRIBUCIÓN: Ucrania (Crimea).

-Cosmochthonius (C.) trivialis Sergienko, 1991

DisTRIBUCIÓN: Ucrania.

-Cosmochthonius (C.) ugamaensis Gordeeva, 1980

DisTRIBUCIÓN: Asia centrooccidental.
Cosmochthonius (Nanochthonius) Subías y Gil-Martín, 1995 (2 spp.) (=Microchthonius Kahwash, Subías y Ruiz, 1989, "nom. praeoc." por Hadzi, 1933)

EsPeCIE TIPO: Cosmochthonius (Microchthonius) ruizi Kahwash,

Subías y Ruiz, 1989

DisTRIBUCión: Holártica.

Cosmochthonius (Nanochthonius) juvenalis Kamill, 1986 (C.)

Distribución: U.S.A. (Nuevo Méjico).

-Cosmochthonius (Nanochthonius) ruizi Kahwash, Subías y Ruiz, 1989 (C. (Microchthonius))

DisTRIBUCIÓN: Mediterránea occidental.

Gozmanyina Balogh y Mahunka, 1983 (3 spp.)

(=Marshallia Gordeeva, 1980, “nom. praeoc.” por Zittel, 1887)

ESPECIE TIPO: Trichthonius majestus Marshall y Reeves, 1971

DISTRIBUCIÓN: Boreal y Neotropical austral.

-Gozmanyina golosovae (Gordeeva, 1980) (Marshallia)

DistRIBUCIÓN: Este de Paleártica oriental (I. Kuriles y Japón).

-Gozmanyina majestus (Marshall y Reeves, 1971) (Trichthonius)

DisTRIBUCIÓN: Neártica septentrional.

-Gozmanyina pehuen R. Martínez y Casanueva, 1996

DistriBuCiÓN: Chile.

Krivolutskiella Gordeeva, 1980 (1 sp.)

ESPECIE TIPO: Krivolutskiella pubescens Gordeeva, 1980

DisTRIBUCIÓN: Paleártica.

-Krivolutskiella pubescens Gordeeva, 1980

DistribuCióN: Mediterránea occidental.

Nipponiella Gordeeva, 1980 (1 sp.)

EsPECIE TIPO: Trichthonius simplex Aoki, 1966

DISTRIBUCIÓN: Paleártica.

-Nipponiella simplex (Aoki, 1966) (Trichthonius)

Distribución: Este de Paleártica oriental.

Phyllozetes Gordeeva, 1978 (8 spp.)

EsPECIE TIPO: Cosmochthonius emmae Berlese, 1910

DISTRIBUCIÓN: Cosmopolita (excepto Antártica).

-Phyllozetes alatus Mahunka, 1982

DisTRIBUCión: Etiopía.

-Phyllozetes emmae (Berlese, 1910) (Cosmochthonius)

(=Trichthonius heterotrichus Sanyal y Bhaduri, 1983)

DistriBUCIÓN: Pantropical (excepto Etiópica) y subtropical.

-Phyllozetes hypoquercus McDaniel y Bolen, 1980

DisTRIBUCIÓN: U.S.A. (Tejas).

-Phyllozetes latifolius Gordeeva, 1980

Distribución: Mediterránea e Indonesia (Java y Komodo).

-Phyllozetes longifolius Balakrishnan, 1986

DisTRIBUCIÓN: India (Kerala).

-Phyllozetes nilamburicus Balakrishnan, 1986

DisTRIBUCIÓN: India (Kerala).

-Phyllozetes osithchnjukovi Gordeeva, 1980

Distribución: Sureste de Europa.

-Phyllozetes tauricus Gordeeva, 1978

DisTRIBUCIÓN: Mediterránea.

Trichthonius Hammer, 1961 (1 sp.)

ESPECIE TIPO: Cosmochthonius pulcherrimus Hammer, 1958

DisTRIBUCIÓN: Australiana y Neotropical.

-Trichthonius pulcherrimus (Hammer, 1958) (Cosmochthonius)

DisTRIBUCIÓN: Neotropical y Australia.

Heterochthoniidae Grandjean, 1954 (3 gen. y 6 spp.) DisTRIBUCIÓN: Paleártica.

Heterochthonius Berlese, 1910 (4 spp.)

EsPECIE TIPO: Cosmochthonius (Heterochthonius) gibbus Berlese, 1910 DisTRIBUCIÓN: Paleártica.

-Heterochthonius byzovae Krivolutsky, 1977

DisTRIBUCIÓN: Asia central rusa. 
-Heterochthonius caucasicus Krivolutsky, 1977

DisTRIBUCIÓN: Cáucaso.

-Heterochthonius gibbus (Berlese, 1910) (Cosmochthonius (H.))

DisTRIBUCIÓN: Paleártica meridional.

-Heterochthonius monocerus Pankov, 2002

DisTRIBUCIÓN: Este de Rusia asiática.

Neochthonius Karppinen, 1984 (1 sp.)

EsPECIE TIPO: Neochthonius latisetosus Golosova y Karppinen, 1984 DISTRIBUCIÓN: Paleártica.

-Neochthonius latisetosus Golosova y Karppinen, 1984

DisTRIBUCIÓN: Este de Rusia asiática.

Ovochthonius Rjabinin, 1977 (1 sp.)

ESPECIE TIPO: Ovochthonius rossicus Rjabinin, 1977

DisTRIBUCIÓN: Paleártica.

-Ovochthonius rossicus Rjabinin, 1977

DisTRIBUCIÓN: Este de Paleártica oriental.

Haplochthoniidae Hammen, 1959 (2 gen., 1 subg. y 14 spp.) DISTRIBUCIÓN: Cosmopolita.

Amnemochthonius Grandjean, 1949 (1 sp.)

EsPECIE TIPO: Amnemochthonius taeniophorus Grandjean, 1949

DisTRIBUCión: Paleártica y Australiana.

-Amnemochthonius taeniophorus Grandjean, 1949

DisTRIBUCIÓN: Europa occidental y Australia.

Haplochthonius Willmann, 1930 (13 spp.)

(=Tetrochthonius Hammer, 1958)

ESPECIE TIPO: Cosmochthonius (Haplochthonius) simplex Willmann, 1930

DistriBuCión: Cosmopolita (excepto Australiana).

Haplopchthonius (Haplochthonius) Willmann, 1930 (12 spp.)

DistRIBUCión: Cosmopolita (excepto Australiana).

-Haplochthonius (H.) antarcticus Sanyal, Basak y Barman, 2002

DisTRIBUCIÓN: Antártida.

-Haplochthonius (H.) chamela Mahunka y Mejía-Recamier, 1998 DisTRIBUCIÓN: Méjico.

-Haplochthonius (H.) clavatus (Hammer, 1958) (Tetrochthonius) DISTRIBUCIÓN: Argentina, India y España.

-Haplochthonius (H.) crassisetosus Gil-Martín, Subías y Arillo, 1992 DisTRIBUCIÓN: Mediterránea occidental.

-Haplochthonius (H.) graecus Mahunka, 1992 DistriBución: Grecia.

-Haplochthonius (H.) intermedius Chakrabarti, Bhaduri y Raychaudhuri, 1977

DisTRIBUCIÓN: India (Bengala Occidental)

-Haplochthonius (H.) longisetus Sanyal, Basak y Barman, 2002 DisTRIBUCión: Antártida.

-Haplochthonius (H.) maitri Sanyal, Basak y Barman, 2002

DISTRIBUCIÓN: Antártida.

-Haplochthonius (H.) muscicola Fujikawa, 1996

DisTRIBUCIÓN: Japón.

-Haplochthonius (H.) sanctaeluciae Bernini, 1973

Distribución: Mediterránea occidental, Senegal y Chile.

-Haplochthonius (H.) simplex (Willmann, 1930) (Cosmochthonius (H.))

DisTRIBUCIÓN: Semicosmopolita (Holártica, Etiópica: Senegal, y Oriental).

-Haplochthonius (H.) variabilis Wallwork, 1972

DisTRIBUCIÓN: Neártica meridional.

Haplochthonius (Senilochthonius) Mahunka, 1992 (1 sp.)

ESPECIE TIPO: Senilochthonius baobab Mahunka, 1992

DisTRIBUCIÓN: Etiópica.

-Haplochthonius (Senilochthonius) baobab (Mahunka, 1992) (S.)

DisTRIBUCIÓN: Senegal.
Pediculochelidae Lavoipierre, 1946 (1 gen. y 4 spp.)

DisTRIBUCIÓN: Semicosmopolita (Holártica meridional, Etiópica y Australiana).

Paralycus Womersley, 1944 (4 spp.)

(=Pediculochelus Lavoipierre, 1946)

EsPeCIE TIPO: Alycus pyrigerus Berlese, 1905

DistribuCión: Semicosmopolita (Holártica meridional, Etiópica y Australiana).

-Paralycus lavoipierrei (Price, 1973) (Pediculochelus)

Distribución: U.S.A. (California) y Australia.

-Paralycus parvulus (Price, 1973) (Pediculochelus)

DistribuCIÓN: U.S.A. (California).

-Paralycus pyrigerus (Berlese, 1905) (Alycus)

DisTRIBUCIÓN: Mediterránea occidental.

-Paralycus raulti (Lavoipierre, 1946) (Pediculochelus)

DisTRIBUCIÓN: Sudáfrica.

Sphaerochthoniidae Grandjean, 1947 (2 gen. y 17 spp.) DISTRIBUCIÓN: Cosmopolita (excepto Antártica).

Similochthonius Mahunka, 1985 (2 spp.)

=Sphaerochochthonius Mahunka, 1985)

EsPECIE TIPO: Similochthonius decoratus Mahunka, 1985

DistRIBUCIÓN: Paleotropical.

-Similochthonius australis (Mahunka, 1985) (Spaerochochthonius)

DisTRIBUCIÓN: Sudáfrica.

-Similochthonius decoratus Mahunka, 1985

DisTRIBUCIÓN: India (Kerala).

Sphaerochthonius Berlese, 1910 (15 spp.)

(=Sphaerochthoniella Mahunka, 1985)

ESPECIE TIPO: Hypochthonius splendidus Berlese, 1904

DistRIBUCIÓN: Cosmopolita (excepto Antártica)

-Sphaerochthonius bengalensis Sanyal y Sengupta, 1990

DisTRIBUCIÓN: India (Bengala Occidental).

-Sphaerochthonius dilutus Sergienko, 1991

DisTRIBUCIÓN: Ucrania.

-Sphaerochthonius fungifer Mahunka, 1983

DisTRIBUCIÓN: Guatemala.

-Sphaerochthonius gemma (Oudemans, 1909) (Hypochthonius)

(=Sphaerochthonius elegans Berlese, 1910)

(=Sphaerochthonius elegans Berlese, 1916, "nom. praeoc.” por Berlese, 1910)

(=Sphaerochthonius wallworki Lee, 1982)

DisTRIBUCIÓN: Indonesia (Java y Komodo), Etiópica (Ghana y

Somalia) y Sureste de Europa (Crimea).

-Sphaerochthonius longisetus Wallwork, 1977

DisTRIBUCIÓN: Indonesia (Komodo).

-Sphaerochthonius ovatus Sergienko, 1991

DISTRIBUCIÓN: Ucrania.

-Sphaerochthonius pallidus Muñoz-Mingarro, 1987

DisTRIBUCIÓN: España.

-Sphaerochthonius phyllophorus Balogh y Mahunka, 1969

DisTRIBUCIÓN: Brasil.

-Sphaerochthonius spectabilis Gordeeva, Niemi y Petrova-Nikitina, 1996

DisTRIBUCIÓN: Asia centrooccidental.

-Sphaerochthonius splendidus (Berlese, 1904) (Hypochthonius)

DISTRIBUCIÓN: Semicosmopolita (Holártica meridional: frecuente,

Australiana: Australia y Polinesia, y Neotropical: Chile).

-Sphaerochthonius strinatii Mahunka, 1982

DistriBUCIÓN: Melanesia (I. Fiji).

-Sphaerochthonius suzukii Aoki, 1977

DisTRIBUCIÓN: Japón y Hawai.

-Sphaerochthonius transversus Wallwork, 1960

Distribución: Semicosmopolita (Paleártica meridional, Ghana, India y Cuba).

-Sphaerochthonius uruguayensis Pérez-Íñigo y Sarasola, 1998

DISTRIBUCIÓN: Uruguay. 
-Sphaerochthonius variesetosus Mahunka, 1997

DISTRIBUCIÓN: Madagascar.

Atopochthonioidea Grandjean, 1949

Atopochthoniidae Grandjean, 1949 (1 gen. y 2 spp.) DisTRIBUCIÓN: Holártica.

Atopochthonius Grandjean, 1949 (2 spp.)

ESPECIE TIPO: Atopochthonius artiodactylus Grandjean, 1949

DisTRIBUCIÓN: Holártica.

-Atopochthonius artiodactylus Grandjean, 1949

DISTRIBUCIÓN: Holártica.

-Atopochthonius maimaensis Grishina, 1971

DISTRIBUCIÓN: Asia central rusa.

Pterochthoniidae Grandjean, 1950 (1 gen. y 1 sp.) DisTRIBUCIÓN: Holártica y Australiana.

Pterochthonius Berlese, 1913 (1 sp.)

EsPeCIE TIPO: Cosmochthonius angelus Berlese, 1910

DisTRIBUCIÓN: Holártica y Australiana.

-Pterochthonius angelus (Berlese, 1910) (Cosmochthonius)

DistriBución: Holártica, Méjico y Hawai.

Phyllochthoniidae Travé, 1967 (1 gen. y 1 sp.) DisTRIBUCIÓN: Etiópica.

Phyllochthonius Travé, 1967 (1 sp.)

EsPeCIE TIPO: Phyllochthonius aoutii Travé, 1967

DISTRIBUCIÓN: Etiópica.

-Phyllochthonius aoutii Travé, 1967

Distribución: Costa de Marfil.

Protoplophoroidea Ewing, 1917

Protoplophoridae Ewing, 1917 (7 gen., 2 subg. y 28 spp.) DisTRIBUCIÓN: Cosmopolita (excepto Antártica).

Arthrhoplophora Berlese, 1910 (3 spp.)

(=Neoprototritia Shereef, 1978)

(=Triplophora Mahunka, 1977)

ESPECIE TIPO: Arthrhoplophora paradoxa Berlese, 1910

DisTRIBUCIÓN: Paleotropical.

-Arthrhoplophora paradoxa Berlese, 1910

DisTRIBUCIÓN: Java.

-Arthrhoplophora vulpes Berlese, 1916

(=Arthrhoplophora (Triplophora) berlesei Mahunka, 1977)

DisTRIBUCIÓN: África oriental.

-Arthrhoplophora zachvatkini (Shereef, 1978) (Neoprototritia)

DisTRIBUCIÓN: Egipto.

Bursoplophora Subías y Pérez-Íñigo, 1978 (9 spp.)

(=Csibiplophora Mahunka, 1984)

EsPECIE TIPO: Bursoplophora iberica Subías y Pérez-Íñigo, 1978

DistriBuCIÓN: Semicosmopolita (Paleártica meridional, Etiópica y

Neotropical).

-Bursoplophora bivaginata (Grandjean, 1932) (Protoplophora)

DisTRIBUCIÓN: Mediterránea.

-Bursoplophora ethiopica Mahunka, 1982

DisTRIBUCIÓN: Etiopía.

-Bursoplophora genavensium (Mahunka, 1984) (Csibiplophora)

DisTRIBUCión: Paraguay.

-Bursoplophora iberica Subías y Pérez-Íñigo, 1978

DisTRIBUCión: España.

-Bursoplophora insularis Kahwash, Subías y Ruiz, 1989

DisTRIBUCIÓN: Mediterránea occidental.

-Bursoplophora madagassica Mahunka, 1994

DISTRIBUCIÓN: Madagascar.
-Bursoplophora meridionalis Bernini, 1983

DisTRIBUCIÓN: Italia (Sicilia).

-Bursoplophora muraiae Mahunka y Mejía-Recamier, 1998

DistriBución: Méjico.

-Bursoplophora tyrrhenica Bernini, 1983

DISTRIBUCIÓN: Mediterránea occidental.

Cryptoplophora Grandjean, 1932 (2 spp.)

EsPECIE TIPO: Cryptoplophora abscondita Grandjean, 1932

DISTRIBUCIÓN: Cosmopolita (excepto Australiana y Antártica).

-Cryptoplophora abscondita Grandjean, 1932

DisTRIBUCIÓn: Semicosmopolita (Paleártica: Mediterránea occi-

dental y Japón,U.S.A.: Tejas, Etiópica: Nigeria, Oriental: Java, y

Neotropical)

-Cryptoplophora asiatica Gordeeva, Niemi y Petrova-Nikitina, 1998

DISTRIBUCIÓN: Asia centrooccidental.

Grandjeanoplophora Balogh y Mahunka, 1979 (2 spp.)

ESPECIE TIPO: Cryptoplophora mauritanica Grandjean, 1932

DisTRIBUCIÓN: Paleártica.

Grandjeanoplophora (Grandjeanoplophora) Balogh y Mahunka, 1979 (1 sp.)

DisTRIBUCIÓN: Paleártica.

-Grandjeanoplophora (G.) mauritanica (Grandjean, 1932) (Cryptoplophora)

DISTRIBUCIÓN: Marruecos.

Grandjeanoplophora (Tauroplophora) Gordeeva, Niemi y PetrovaNikitina, 1998 (1 sp.)

EsPeCIE TIPO: Tauroplophora aureonotata Gordeeva, Niemi y PetrovaNikitina, 1998

DisTRIBUCIÓN: Paleártica.

-Grandjeanoplophora (Tauroplophora) aureonotata (Gordeeva,

Niemi y Petrova-Nikitina, 1998) (T.)

DisTRIBUCIÓN: Ucrania.

Hauseroplophora Mahunka, 1977 (3 spp.)

EsPECIE TIPO: Hauseroplophora soniae Mahunka, 1977

DisTRIBUCIÓN: Paleártica y Etiópica.

-Hauseroplophora flagellata Mahunka, 1987

DISTRIBUCIÓN: Kenia.

-Hauseroplophora phitosi Mahunka, 1977

DisTRIBUCIÓN: Grecia.

-Hauseroplophora soniae Mahunka, 1977

DisTRIBUCIÓN: Kenia.

Protoplophora Berlese, 1910 (2 spp.)

EsPeCIE TIPO: Protoplophora palpalis Berlese, 1910

DisTRIBUCIÓN: Holártica y Australiana.

-Protoplophora oglasicola Bernini, 1983

DISTRIBUCIÓN: Italia.

-Protoplophora palpalis Berlese, 1910

(=Protoplophora grandjeani Bernini, 1983)

DisTRIBUCIÓN: Holártica meridional (Paleártica meridional y

U.S.A.: California) y Australia.

Prototritia Berlese, 1916 (7 spp.)

EsPeCIE TIPO: Arthrhoplophora (Prototritia) armadillo Berlese, 1916

DisTRIBUCIÓN: Cosmopolita (excepto Antártica).

Prototritia (Prototritia) Berlese, 1916 (6 spp.)

(=Aedoplophora Grandjean, 1932)

DisTRIBUCIÓN: Pantropical.

-Prototritia (P.) africana (Mahunka, 1977) (Aedoplophora)

DisTRIBUCIÓN: Kenia.

-Prototritia (P.) armadillo (Berlese, 1916) (Arthrhoplophora (P.))

DisTRIBUCIÓN: Somalia.

-Prototritia (P.) glomerata (Grandjean, 1932) (Aedoplophora)

DISTRIBUCIÓN: Neotropical y Filipinas. 
-Prototritia (P.) grandjeani (Mahunka, 1977) (Aedoplophora)

DisTRIBUCIÓN: Indonesia (Java y Komodo).

-Prototritia (P.) major (Jacot, 1933) (Aedoplophora)

DisTRIBUCIÓN: U.S.A. (Florida).

-Prototritia (P.) palaciosi Mahunka y Mejía-Recamier, 1998

Distribución: Méjico.

Prototritia (Siciliophora) Bernini, 1983 (1 sp.)

EsPecie Tipo: Prototritia (Siciliophora) sicula Bernini, 1983

DisTRIBUCIÓN: Paleártica.

-Prototritia (Siciliophora) sicula Bernini, 1983

DISTRIBUCIÓN: Italia (Sicilia).

Suborden MIXONOMATA Grandjean, 1969 Infraorden DICHOSOMATA Balogh y Mahunka, 1967

Nehypochthonioidea Norton y Metz, 1980

Nehypochthoniidae Norton y Metz, 1980 (1 gen. y 2 spp.) DISTRIBUCIÓN: Holártica meridional y Australiana.

Nehypochtonius Norton y Metz, 1980 (2 spp.)

EsPECIE TIPO: Nehypochthonius porosus Norton y Metz, 1980

DISTRIBUCIÓN: Holártica meridional y Australiana.

-Nehypochthonius porosus Norton y Metz, 1980

DistRIBUCIÓN: Sureste de U.S.A. y Hawai.

-Nehypochthonius yanoi Aoki, 2002

DisTRIBUCIÓN: Japón.

\section{Perlohmannioidea Grandjean, 1954}

Perlohmanniidae Grandjean, 1954 (2 gen., 1 subg. y 9 spp.) DistRIBUCIÓN: Holártica y Australiana.

Hololohmannia Kubota y Aoki, 1998 (1 sp.)

ESPECIE TIPO: Hololohmannia alaskensis Kubota y Aoki, 1998 DisTRIBUCIÓN: Neártica.

-Hololohmannia alaskensis Kubota y Aoki, 1998

DisTRIBUCIÓN: Alaska.

Perlohmannia Berlese, 1916 (8 spp.)

EsPeCIE TIPO: Lohmannia insignis Berlese, 1904

DISTRIBUCIÓN: Paleártica y Australiana.

Perlohmannia (Perlohmannia) Berlese, 1916 (7 spp.)

(=Neolohmannia Bulanova-Zachvatkina, 1960)

DisTRIBUCIÓN: Holártica y Australiana.

-Perlohmannia (P.) altaica Grishina, 1968

Distribución: Paleártica (Cáucaso, Asia centrooccidental e Islas del Este de Rusia asiática).

-Perlohmannia (P.) coiffaiti Grandjean, 1961

DisTRIBUCión: Paleártica (Europa centromeridional y Este de Asia) y "Neártica septentrional".

-Perlohmannia (P.) dissimilis (Hewitt, 1908) (Lohmannia insignis d.) DistribuCión: Paleártica (Europa centromeridional, Asia centrooccidental y Este de Rusia asiática) y Hawai.

-Perlohmannia (P.) insignis (Berlese, 1904) (Lohmannia)

DisTRIBUCión: Europa occidental (excepto Norte).

-Perlohmannia (P.) nasuta Schuster, 1960

DISTRIBUCIÓN: Europa centromeridional.

-Perlohmannia (P.) skrjabini (Bashkirova, 1958) (Eulohmannia) "sp. inq."

DisTRIBUCIÓN: Sur de Rusia europea.

-Perlohmannia (P.) zachvatkini (Bulanova-Zachvatkina, 1960)

(Neolohmannia)

Distribución: Paleártica (Cáucaso e I. Sajalín).

Perlohmannia (Apolohmannia) Aoki, 1960 (1 sp.)

ESPECIE TIPO: Apolohmannia gigantea Aoki, 1960

DisTRIBUCIÓN: Paleártica.
-Perlohmannia (Apolohmannia) gigantea (Aoki, 1960) (A.)

DistRiBución: Paleártica oriental (excepto Norte).

Collohmanniidae Grandjean, 1958 (1 gen. y 2 spp.) DisTRIBUCIÓN: Paleártica.

Collohmannia Sellnick, 1922

EsPeCIE TIPO: Collohmannia gigantea Sellnick, 1922

DistribuCIÓN: Paleártica.

-Collohmannia asiatica Krivolutsky y Christov, 1970

DistRIBUCIÓN: Asia centrooccidental.

-Collohmannia gigantea Sellnick, 1922

(=Collohmannia nova Sellnick, 1932)

DisTRIBUCIÓN: Paleártica meridional (Europa centrooriental y Asia centrooccidental).

Eulohmannioidea Grandjean, 1931

Eulohmanniidae Grandjean, 1931 (1 gen. y 1 sp.) DisTRIBUCIÓN: Holártica.

Eulohmannia Berlese, 1910 (1 sp.)

(=Arthronothrus Trägardh, 1910)

ESPECIE TIPO: Lohmannia (Eulohmannia) ribagai Berlese, 1910

Distribución: Holártica.

-Eulohmannia ribagai (Berlese, 1910) (Lohmannia (E.))

(=Arthronothrus biunguiculatus Trägardh, 1910)

DisTRIBUCIÓN: Holártica (frecuente).

Epilohmannioidea Oudemans, 1923

Epilohmanniidae Oudemans, 1923 (2 gen., 47 spp. y 9 sspp.) DisTRIBUCIÓN: Cosmopolita (excepto Antártica).

Epilohmannia Berlese, 1910 (40 spp. y 9 sspp.)

(=Lesseria Oudemans, 1917)

(=Neoepilohmannia Bolen y McDaniel, 1989 "sin. nov.")

(=Sinolohmannia Balogh y Mahunka, 1979 "sin. nov.")

ESPECIE TIPO: Lohmannia cylindrica Berlese, 1904

DistriBución: Cosmopolita (excepto Antártica).

-Epilohmannia amygdaliformis Berlese, 1916

DisTRIBUCIÓN: "África oriental".

-Epilohmannia barbatula Balogh, 1958 "sp. inq."

DisTRIBUCIÓN: Angola.

-Epilohmannia cultrata Woolley, 1966

DisTRIBUCIÓN: Guatemala.

-Epilohmannia cylindrica (Berlese, 1904) (Lohmannia)

DistriBución: Cosmopolita (excepto Antártica).

-Epilohmannia cylindrica cylindrica (Berlese, 1904)

(=Phthiracarus szanisloi Oudemans, 1915)

(=Epilohmannia verrucosa Jacot, 1934)

DisTRIBUCIÓN: Semicosmopolita (Paleártica: menos frecuente en el Norte, Neártica, Etiópica: Chad, Oriental, Australiana: Hawai, y Neotropical: Cuba).

-Epilohmannia cylindrica media Lee, 1985

DisTRIBUCIÓN: Australia.

-Epilohmannia cylindrica minima Schuster, 1960

DisTRIBUCIÓN: Europa meridional (frecuente).

-Epilohmannia daghestanica Karppinen y Shtanchaeva, 1987

DisTriBUCIÓN: Cáucaso.

-Epilohmannia dimorpha Wallwork, 1962

DisTRIBUCIÓN: Ghana.

-Epilohmannia dolosa Pérez-Íñigo y Baggio, 1985

DisTRIBUCión: Brasil.

-Epilohmannia flagellifer Mahunka, 1987

DisTRIBUCIÓN: Borneo.

-Epilohmannia flexuosa Wallwork, 1962

DisTRIBUCión: Ghana.

-Epilohmannia gigantea Berlese, 1916

DISTRIBUCIÓN: Europa meridional. 
-Epilohmannia guarani Balogh y Mahunka, 1977

DisTRIBUCIÓN: Paraguay.

-Epilohmannia heterotricha Mahunka, 1966

DisTRIBUCIÓN: Italia.

-Epilohmannia imreorum Bayoumi y Mahunka, 1976

DisTRIBUCIÓN: Rumania.

-Epilohmannia inexpectata Schuster, 1960

DistriBución: Mediterránea e I. Santa Helena.

-Epilohmannia insignipes Balogh, 1964

DisTRIBUCIÓN: Madagascar.

-Epilohmannia kulaginae Shtanchaeva, 1993

Distribución: Cáucaso.

-Epilohmannia lenkoi Balogh y Mahunka, 1977

DisTRIBUCIÓN: Brasil.

-Epilohmannia mahunkai Calugar y Vasiliu, 1983

Distribución: Cuba.

-Epilohmannia maurii Fernández, 1978

DisTRIBUCIÓN: Neotropical (Argentina y Uruguay).

-Epilohmannia minuta Berlese, 1920

(=Epilohmannia elongata Banks, 1947)

DISTRIBUCIÓN: U.S.A. centrooriental.

-Epilohmannia multisetosa Hammer, 1971

Distribución: Melanesia (I. Fiji).

-Epilohmannia neotricha Wallwork, 1962

DISTRIBUCIÓN: Ghana.

-Epilohmannia norberti Moreno y Moraza, 1994

DisTRIBUCIÓN: España.

-Epilohmannia nortoni Mahunka, 1977

DISTRIBUCIÓN: Borneo.

-Epilohmannia ornata Mahunka, 1993

DISTRIBUCIÓN: Ruanda.

-Epilohmannia ovalis Berlese, 1916

DisTRIBUCIÓN: Argentina.

-Epilohmannia ovata Aoki, 1961

DistRIBUCión: Paleártica oriental (excepto Norte).

-Epilohmannia pallida Wallwork, 1962

DisTRIBUCIÓN: Pantropical y subtropical

-Epilohmannia pallida pallida Wallwork, 1962

Distribución: Pantropical (Etiópica, Oriental: India, y Neotropical: Panamá).

-Epilohmannia pallida aegyptica Bayoumi y Mahunka, 1976

DistRIBUCIÓN: Mediterránea oriental.

-Epilohmannia pallida americana Balogh y Mahunka, 1981

DisTRIBUCIÓN: Neotropical.

-Epilohmannia pallida areolata Sarkar y Subías, 1982

DisTRIBUCIÓN: India (Tripura).

-Epilohmannia pallida australica Lee, 1985

DisTRIBUCIÓN: Australia.

-Epilohmannia pallida indica Bhattacharya y Banerjee, 1979

DisTRIBUCIÓN: India (Bengala Occidental).

-Epilohmannia pallida pacifica Aoki, 1965

Distribución: Pantropical (excepto Etiópica) y Este de Paleártica oriental.

-Epilohmannia pallida rugosa Sarkar y Subías, 1982 DistriBución: India (Tripura).

-Epilohmannia pilosa Li y Chen, 1990

DisTRIBUCIÓN: China suroriental.

-Epilohmannia praetritia Berlese, 1916

DisTRIBUCión: U.S.A. (Misuri).

-Epilohmannia puella Berlese, 1916

(=Epilohmannia nota McDaniel y Bolen, 1983)

DisTRIBUCIÓN: Sur de U.S.A.

-Epilohmannia schusteri Calugar y Vasiliu, 1976

DISTRIBUCIÓN: Rumania.

-Epilohmannia sculpturata Balogh y Mahunka, 1980

DisTRIBUCIÓN: Cuba.

-Epilohmannia shtanchaevae Bayartogtokh, 2000

DisTRIBUCIÓN: Mongolia.

-Epilohmannia spathulata Aoki, 1970

Distribución: Paleártica oriental (excepto Norte).
-Epilohmannia spathuloides Bayartogtokh, 2000

DisTRIBUCIÓN: Japón.

-Epilohmannia styriaca Schuster, 1960

DisTRIBUCIÓN: Europa meridional.

-Epilohmannia taeda (Bolen y McDaniel, 1989) (Neoepilohmannia)

DisTRIBUCIÓN: U.S.A. (Carolina del Norte).

-Epilohmannia taiwanica Tseng, 1982

DistriBución: Taiwan [Formosa].

-Epilohmannia zwarti Hammen, 1986

DisTRIBUCIÓN: Nueva Guinea.

Epilohmannoides Jacot, 1936 (7 spp.)

(=Iburidania Aoki, 1959)

ESPECIE TIPO: Epilohmannoides terrae Jacot, 1936

Distribución: Tropical (Oriental y Neotropical) y subtropical

(Holártica meridional).

-Epilohmannoides bipectinatus (Aoki, 1959) (Iburidania)

(=Epilohmannoides kishidai Ohkubo, 2002)

DisTRIBUCIÓN: Japón.

-Epilohmannoides esulcatus Ohkubo, 1979

DisTRIBUCIÓN: Japón y Borneo.

-Epilohmannoides jacoti Norton, Metz y Sharma, 1978

DisTRIBUCIÓN: Sureste de U.S.A.

-Epilohmannoides rabori (Corpuz-Raros, 1979) (Epilohmannia)

DisTRIBUCIÓN: Filipinas.

-Epilohmannoides terrae Jacot, 1936

DisTRIBUCIÓN: Este de U.S.A. y Panamá.

-Epilohmannoides wallworki Hammer, 1981

DisTRIBUCión: Java.

-Epilohmannoides xena (Mahunka, 1983) (Epilohmannia)

DisTRIBUCIÓN: Guatemala.

Lohmannioidea Berlese, 1916

Lohmanniidae Berlese, 1916 (21 gen., 4 subg., 179 spp. y 9 sspp.) DisTRIBUCIÓN: Pantropical y subtropical.

Annectacarus Grandjean, 1950 (15 spp.)

ESPECIE TIPO: Annectacarus mucronatus Grandjean, 1950

DisTRIBUCIÓN: Pantropical.

-Annectacarus africanus Balogh, 1961

Distribución: Etiópica (Tanzania y Malgache) e Indonesia (Komodo).

-Annectacarus aokii Jaikumar, Haq y Ramani, 1994

Distribución: India (Kerala).

-Annectacarus eksteeni Coetzee, 2001

DisTRIBUCIÓN: Sudáfrica.

-Annectacarus granditrichosus Sengbusch, 1984

DISTRIBUCIÓN: Micronesia.

-Annectacarus hainanensis Hu y X. Wang, 1989

Distribución: China suroriental.

-Annectacarus insculptus Wallwork, 1962

Distribución: Ghana.

-Annectacarus krachan Mahunka, 1995

DISTRIBUCIÓN: Tailandia.

-Annectacarus longisetosus Bhattacharya, Bhaduri y Raychaudhuri, 1974

DistriBUCión: India (Bengala Occidental)

-Annectacarus mahabaeus Corpuz-Raros, 1979

DisTRIBUCión: Filipinas.

-Annectacarus mucronatus Grandjean, 1950

DisTRIBUCIÓN: Neotropical (frecuente).

-Annectacarus parallelus (Berlese, 1916) (Lohmannia)

DisTRIBUCIÓN: Somalia.

-Annectacarus perezinigoi Calugar y Vasiliu, 1983

DisTRIBUCIÓN: Cuba.

-Annectacarus sejugatus Wallwork, 1962

Distribución: Ghana.

-Annectacarus trivandricus Haq, 1978

DISTRIBUCIÓN: India (Kerala). 
-Annectacarus unilateralis Hammer, 1973

DistriBución: Pantropical: Polinesia (I. Tonga), Java e I. Galápagos.

Cryptacarus Grandjean, 1950 (4 spp.)

ESPECIE TIPO: Cryptacarus promecus Grandjean, 1950

DISTRIBUCIÓN: Pantropical (excepto Neotropical) y Paleártica meridional.

-Cryptacarus dendrisetosus Bhattacharya, Bhaduri y Raychaudhuri, 1974 DistriBución: India (Bengala Occidental)

-Cryptacarus promecus Grandjean, 1950

(=Lohmannia valdemorica Mihelcic, 1956)

Distribución: Paleártica meridional (Mediterránea y Asia centrooccidental) y Etiópica.

-Cryptacarus schauenbergi Mahunka, 1977

DisTRIBUCIÓN: Oriental y Australiana (Indonesia: Lombok).

-Cryptacarus tuberculatus Csiszár, 1961

DISTRIBUCIÓN: Oriental.

Dendracarus Balogh, 1961 (1 sp.)

ESPECIE TIPO: Dendracarus pulchellus Balogh, 1961

DisTRIBUCIÓN: Etiópica.

-Dendracarus pulchellus Balogh, 1961

DisTRIBUCIÓN: Madagascar.

Haplacarus Wallwork, 1962 (9 spp. y 1 ssp.)

EsPECIE TIPO: Haplacarus foliatus Wallwork, 1962

DisTRIBUCIÓN: Pantropical.

-Haplacarus bhadurii Sanyal, 1984

DISTRIBUCIÓN: India (Maharashtra).

-Haplacarus foliatus Wallwork, 1962

DISTRIBUCIÓN: África centrooccidental y Oriental.

-Haplacarus foliatus foliatus Wallwork, 1962

DISTRIBUCIÓN: África centrooccidental y Oriental

-Haplacarus foliatus bengalensis Bhattacharya, Bhaduri y Raychaudhuri, 1974

DisTRIBUCIÓN: Noreste de India.

-Haplacarus javensis Hammer, 1979

DISTRIBUCIÓN: Oriental (Java) y Centroamérica.

-Haplacarus keralensis Haq, Mubarak y Ramani, 1983

DisTRIBUCión: India (Kerala).

-Haplacarus maharashtraensis Sanyal, 1984

DISTRIBUCIÓN: India (Maharashtra).

-Haplacarus pairathi Aoki, 1965

DisTRIBUCIÓN: Tailandia.

-Haplacarus pandanus Sengbusch, 1982

DisTRIBUCIÓN: Micronesia.

-Haplacarus porosus Jaikumar y Ramani, 1994

DisTRIBUCIÓN: India (Kerala).

-Haplacarus rugosus Mahunka, 1987

DISTRIBUCIÓN: Borneo.

Heptacarus Piffl, 1963 (10 spp.)

(=Neotrichacarus Hammer, 1973)

(=Pseudocryptacarus McDaniel, Norton y Bolen, 1979)

EsPeCIE TIPO: Heptacarus notoneotrichus Wallwork, 1963

DISTRIBUCIÓN: Pantropical y subtropical (Holártica meridinal).

-Heptacarus encantadae Schatz, 1994

Distribución: I. Galápagos.

-Heptacarus graminosus (McDaniel, Norton y Bolen, 1979) (Pseu-

docryptacarus)

Distribución: U.S.A. (Tejas).

-Heptacarus hirsutus Wallwork, 1964

DisTRIBUCIÓN: Etiópica (Chad) yOriental (India: Kerala).

-Heptacarus neotropicus Mahunka, 1985

DistriBución: Pequeñas Antillas.

-Heptacarus notoneotrichus Piffl, 1963

DisTRIBUCIÓN: África nororiental.

-Heptacarus ornatus Bayoumi y Al-Khalifa, 1985

DisTRIBUCIÓN: Mediterránea oriental.

-Heptacarus piffli Mahunka, 1977

DisTRIBUCIÓN: Indonesia (Sumbawa).
-Heptacarus plumosus (Hammer, 1973) (Neotrichacarus)

Distribución: Polinesia (I. Samoa).

-Heptacarus reticulatus Mahunka, 1977

DisTRIBUCIÓN: Indonesia (Komodo) e I. Seychelles.

-Heptacarus supertrichus Piffl, 1967

DISTRIBUCIÓN: Iraq.

Javacarus Balogh, 1961 (10 spp.)

EsPECIE TIPO: Javacarus kuehnelti Balogh, 1961

DisTRIBUCIÓN: Pantropical.

Javacarus (Javacarus) Balogh, 1961 (8 spp.)

(=Tongacarus Hammer, 1973)

DisTRIBUCIÓN: Pantropical.

-Javacarus (J.) foliatus Hammer, 1972 (J. kuehnelti f.)

DistribuCión: Australiana (Islas del Pacífico) y Oriental (India: Tripura).

-Javacarus (J.) granulatus Csiszár, 1961

DistribuCión: Java y Melanesia (I. Fiji)

-Javacarus (J.) inexpectatus Balogh, 1962

DisTRIBUCIÓN: Neotropical (Perú y Surinam).

-Javacarus (J.) jocelynae Judson, 1991

DISTRIBUCIÓN: Camerún.

-Javacarus (J.) kuehnelti Balogh, 1961

DisTRIBUCIÓN: Tropical (Oriental: frecuente y Australiana: Islas del Pacífico) y Egipto.

-Javacarus (J.) marginatus (Hammer, 1973) (Tongacarus) "sp. inq." DISTRIBUCIÓN: Polinesia (I. Tonga).

-Javacarus (J.) porosus Hammer, 1960

DistribuCión: Pantropical (excepto Australiana)

-Javacarus (J.) reticulatus Sengbusch, 1982

DistRIBUCIÓN: Micronesia.

Javacarus (Euryacarus) Woolley, 1966 (2 spp.)

ESPECIE TIPO: Euryacarus petalus Woolley, 1966

DisTRIBUCIÓN: Neotropical.

-Javacarus (Euryacarus) petalus (Woolley, 1966) (E.)

DisTRIBUCIÓN: Guatemala.

-Javacarus (Euryacarus) pilosus (Mahunka, 1982) (E.)

Distribución: Costa Rica.

Lepidacarus Csiszár, 1961 (2 spp. y 1 spp.)

EsPECIE TIPO: Lepidacarus ornatissimus Csiszár, 1961

DISTRIBUCIÓN: Australianooriental.

-Lepidacarus ennarpi Haq y Ramani, 1997

DisTRIBUCIÓN: India (Kerala).

-Lepidacarus ornatissimus Csiszár, 1961

DisTRIBUCIÓN: Oriental y Australiana (Nueva Caledonia)

-Lepidacarus ornatissimus ornatissimus Csiszár, 196 DistRIBUCIÓN: Oriental (frecuente) y Australiana (Nueva Caledonia).

-Lepidacarus ornatissimus rehmabia Haq, Mubarak y Ramani, 1983 DISTRIBUCIÓN: India (Kerala).

Lohmannia Michael, 1898 (24 spp. y 1 ssp.)

ESPECIE TIPO: Michaelia paradoxa Haller, 1884

DisTRIBUCIÓN: Pantropical y subtropical.

Lohmannia (Lohmannia) Michael, 1898 (23 spp. y 1 ssp.)

(=Michaelia Haller, 1884, "nom. praeoc." por Roemer, 1852)

DISTRIBUCIÓN: Pantropical y subtropical.

-Lohmannia (L.) banksi Norton, Metz y Sharma, 1978

DisTRIBUCIÓN: U.S.A. (Carolina del Norte y Tejas).

-Lohmannia (L.) bifoliata Willmann, 1936

Distribución: Pequeñas Antillas.

-Lohmannia (L.) corallium Nakatamari, 1982

DisTRIBUCIÓN: Japón.

-Lohmannia (L.) coreana Choi, 1985

DISTRIBUCION: Corea. 
-Lohmannia (L.) embrionalis Mahunka, 1978 DistribuCIÓN: Malgache.

-Lohmannia (L.) guzhangensis Hu y X. Wang, 1989 DisTRIBUCIÓN: China.

-Lohmannia (L.) hungarorum Mahunka, 1980 DisTRIBUCIÓN: Mediterránea occidental.

-Lohmannia (L.) javana Balogh, 1961

DistribuCión: Este de Paleárticaoriental, Oriental y Melanesia (I. Fiji).

-Lohmannia (L.) javana javana Balogh, 1961 Distribución: Oriental, Melanesia (I. Fiji) y Japón.

-Lohmannia (L.) javana interrupta Choi, 1985 DISTRIBUCIÓN: Corea.

-Lohmannia (L.) jornoti Mahunka, 1985

DisTRIBUCIÓN: Norte de Neotropical.

-Lohmannia (L.) juliae Mahunka, 1984 DisTRIBUCIÓN: Paraguay.

-Lohmannia (L.) lanceolata Grandjean, 1950

DisTRIBUCIÓN: Neotropical y China suroriental.

-Lohmannia (L.) paradoxa (Haller, 1884) (Michaelia) (=Lohmannia loebli Mahunka, 1974)

DisTRIBUCIÓN: Paleártica occidental (Mediterránea y Alemania).

-Lohmannia (L.) pinnigera Sengbusch, 1984

DISTRIBUCIÓN: Micronesia.

-Lohmannia (L.) regalis Berlese, 1923

DisTRIBUCIÓN: Mediterránea.

-Lohmannia (L.) reticulata Sellnick, 1931 (L. regalis r.) DistriBución: Grecia.

-Lohmannia (L.) semibarbulata Ruiz, Subías y Kahwash, 1991 DisTRIBUCIÓN: España.

-Lohmannia (L.) serrata Hu y Wang, 1989 DistriBuCIÓN: China suroriental.

-Lohmannia (L.) similis Balogh, 1962 Distribución: Neotropical (Perú e I. Galápagos).

-Lohmannia (L.) spinosa Hall, 1911 “sp. inq."

DisTRIBUCión: U.S.A. (California).

-Lohmannia (L.) texana (Banks, 1910) (Hypochthonius)

Distribución: U.S.A. (Tejas).

-Lohmannia (L.) turcmenica Bulanova-Zachvatkina, 1960 (L. lanceolata $t$.)

(=Lohmannia hispaniola Pérez-Íñigo, 1967)

DisTRIBUCIÓN: Paleártica meridional y Argentina.

-Lohmannia (L.) vanharteni Mahunka, 1987

DistRIBUCIÓN: Macaronésica (Cabo Verde).

-Lohmannia (L.) vulcania Schatz, 1993

DisTRIBUCIÓN: I. Galápagos.

Lohmannia (Carolohmannia) Norton, Metz y Sharma, 1978 (1 sp.) ESPECIE TIPO: Lohmannia (Carolohmannia) carolensis Norton, Metz y Sharma, 1978

DisTRIBUCIÓN: Neártica.

-Lohmannia (Carolohmannia) carolensis Norton, Metz y Sharma, 1978 DisTRIBUCIÓN: U.S.A. (Carolina del Sur).

Meristacarus Grandjean, 1934 (13 spp. y 3 sspp.)

(=Javalohmannia Hammer, 1979)

EsPECIE TIPO: Meristacarus porcula Grandjean, 1934

DisTRIBUCIÓN: Pantropical.

-Meristacarus africanus Balogh, 1958

DistRIBUCIÓN: África centrooccidental.

-Meristacarus africanus africanus Balogh, 1958 Distribución: Angola.

-Meristacarus africanus annobonensis Pérez-Íñigo, 1969 Distribución: Guinea Ecuatorial: I. Pagalu [Annobón].

-Meristacarus biroi Balogh, 1961

Distribución: Nueva Guinea.

-Meristacarus bogorensis Hammer, 1979

DisTRIBUCIÓN: Java.

-Meristacarus degradatus Haq y Jaikumar, 1993

DisTRIBUCIÓN: India (Kerala).
-Meristacarus douhereti J. y P. Balogh, 1983

DistriBución: Nueva Caledonia.

-Meristacarus heterotrichus Csiszár, 1961

DisTRIBUCIÓN: Oriental y Melanesia (I. Fiji).

-Meristacarus heterotrichus heterotrichus Csiszár, 1961

Distribución: Java, China suroriental y Melanesia (I. Fiji).

-Meristacarus heterotrichus glabrisetus Mahunka, 1988 (M. g.) Distribución: Borneo.

-Meristacarus longisetosus Mahunka, 1978

DistriBución: Centroamérica (frecuente).

-Meristacarus madagascarensis Balogh, 1964

DistriBUCión: Etiópica (Malgache) y Oriental (Tailandia).

-Meristacarus madagascarensis madagascarensis Balogh, 1964 DistriBución: Malgache.

-Meristacarus madagascarensis obscurus Aoki, 1965 DisTRIBUCIÓN: Tailandia.

-Meristacarus porcula Grandjean, 1934

Distribución: Pantropical (Neotropical: frecuente, Etiópica: Ghana, y Oriental: Filipinas).

-Meristacarus rubescens (Canestrini, 1897) (Hermannia)

Distribución: Australiana (Nueva Guinea y Polinesia: Tahití) y

Oriental (Java y Península Malaya).

-Meristacarus striatus (Hammer, 1979) (Javalohmannia) "sp. inq."

DisTRIBUCIÓN: Java.

-Meristacarus sundensis Hammer, 1979

Distribución: Java.

-Meristacarus tuloyus Corpuz-Raros, 1979

DisTRIBUCIÓN: Filipinas.

Meristolohmannia Balogh y Mahunka, 1966 (2 spp.)

ESPECIE TIPO: Meristolohmannia meristacaroides Balogh y Mahunka, 1966

DisTRIBUCión: Australiana y Paleártica meridional.

-Meristolohmannia chinensis (Bulanova-Zachvatkina, 1960)

(Meristacarus)

Distribución: Oeste de China.

-Meristolohmannia meristacaroides Balogh y Mahunka, 1966

DISTRIBUCIÓN: Australia.

Mixacarus Balogh, 1958 (16 spp. y $1 \mathrm{ssp}$.)

EsPECIE TIPO: Mixacarus integrus Balogh, 1958

Distribución: Pantropical (excepto Australiana) y subtropical (Holártica meridional).

Mixacarus (Mixacarus) Balogh, 1958 (13 spp. y 1 ssp.)

(=Hamacarus Hammer, 1977)

Distribución: Pantropical (excepto Australiana) y subtropical (Holártica meridional).

-Mixacarus (M.) brevipes (Banks, 1947) (Lohmannia)

Distribución: U.S.A. (Carolina del Norte y del Sur) y Costa Rica.

-Mixacarus (M.) chapmani Wallwork, 1962

DisTRIBUCIÓN: Ghana.

-Mixacarus (M.) exilis Aoki, 1970

Distribución: Este de Paleártica oriental.

-Mixacarus (M.) exilis exilis Aoki, 1970

Distribución: Este de Paleártica oriental.

-Mixacarus (M.) exilis foveolatus Aoki, 1987

DisTRIBUCIÓN: Japón.

-Mixacarus (M.) integrus Balogh, 1958

Distribución: Angola.

-Mixacarus (M.) lawariensis (Hammer, 1977) (Hamacarus) "sp. inq." DisTRIBUCión: Pakistán.

-Mixacarus (M.) murcioides (Berlese, 1896) (Angelia)

DisTRIBUCIÓN: Italia.

-Mixacarus (M.) neotropicus Balogh, 1962

DisTRIBUCIÓN: Neotropical.

-Mixacarus (M.) quadrifasciatus Balakrishnan, 1986

DisTRIBUCIÓn: India (Kerala).

-Mixacarus (M.) sublestus Tseng, 1982

DisTRIBUCIÓN: Taiwan [Formosa] 
-Mixacarus (M.) suoxiensis Hu y X. Wang, 1989

Distribución: China suroriental.

-Mixacarus (M.) tianmuensis Y. y L. Li, 1985

DisTRIBUCIÓN: China suroriental.

-Mixacarus (M.) vanhonggui Mahunka, 1973

DISTRIBUCIÓN: Corea.

-Mixacarus (M.) zhuzhikovi Bulanova-Zachvatkina, 1979

DisTRIBUCIÓN: Asia centrooccidental.

Mixacarus (Phyllolohmannia) J. y P. Balogh, 1987 (3 spp.)

ESPECIE TIPO: Mixacarus hamanni Balogh, 1961

DisTRIBUCIÓN: Oriental.

-Mixacarus (Phyllolohmannia) foliifer Golosova, 1984 (M.)

DISTRIBUCIÓN: Vietnam.

-Mixacarus (Phyllolohmannia) hamanni Balogh, 1961 (M.) DisTRIBUCIÓN: Java.

-Mixacarus (Phyllolohmannia) yinae (Hu y Aoki, 1993) (P.)

DisTRIBUCIÓN: China suroriental.

Nesiacarus Csiszár, 1961 (7 spp.)

EsPeCIE TIPO: Nesiacarus reticulatus Csiszár, 1961

Distribución: Pantropical (excepto Etiópica).

-Nesiacarus australis Balogh y Mahunka, 1981

DisTRIBUCión: Paraguay.

-Nesiacarus completus Hu y Wang, 1989

DisTRIBUCiÓN: China suroriental.

-Nesiacarus granulatus Hammer, 1972

DisTrIBUCión: Polinesia (Tahití), Japón y Centroamérica.

-Nesiacarus philippinensis Mahunka, 1991

DisTRIBUCIÓN: Filipinas.

-Nesiacarus reticulatus Csiszár, 1961

DISTRIBUCIÓN: Java.

-Nesiacarus schusteri Schatz, 1994

DisTRIBUCIÓN: I. Galápagos.

-Nesiacarus sinensis $\mathrm{Hu}$ y X. Wang, 1988

DISTRIBUCIÓN: China suroriental.

Ozacarus Colloff y Halliday, 1998 (2 spp.)

(=Austracarus J. y P. Balogh, 1983, “nom. praeoc.” por Lawrence, 1949)

ESPECIE TIPO: Austracarus reductus J. y P. Balogh, 1983

DISTRIBUCIÓN: Australiana.

-Ozacarus reductus (J. y P. Balogh, 1983) (Austracarus)

DisTRIBUCIÓN: Australia.

-Ozacarus tahitiensis (Hammer, 1972) (Meristacarus)

DistriBuCIÓN: Polinesia.

Papillacarus Kunst, 1959 (28 spp.)

(=Vepracarus Aoki, 1965)

ESPECIE TIPO: Lohmannia murcioides aciculata Berlese, 1905

DisTRIBUCIÓN: Pantropical y subtropical.

-Papillacarus abchasicus Tarba, 1985

Distribución: Cáucaso.

-Papillacarus aciculatus (Berlese, 1905) (Lohmannia murcioides a.)

DisTRIBUCIÓN: Paleártica occidental (excepto Norte) y Vietnam.

-Papillacarua aequalis Mahunka, 1991

DisTRIBUCIÓN: Macaronésica (Cabo Verde).

-Papillacarus akimovi Sergienko, 1992

DisTRIBUCión: Sureste de Europa.

-Papillacarus angulatus Wallwork, 1962

DisTRIBUCIÓN: África centrooccidental y Cáucaso.

-Papillacarus arboriseta Jeleva y Vu, 1987

DistriBución: Vietnam.

-Papillacarus chamartinensis Pérez-Íñigo, 1967

DisTRIBUCión: España.

-Papillacarus cornutus (Sarkar y Subías, 1984) (Vepracarus)

DisTRIBUCIÓN: India (Tripura).

-Papillacarus cruzae Corpuz-Raros, 1979

DistriBUCIÓN: Oriental (Filipinas y China suroriental).
-Papillacarus echinatus Li y Chen, 1991

DisTRIBUCIÓN: China suroriental.

-Papillacarus egypticus Elbadry y Nasr, 1977

DisTRIBUCIÓN: Egipto.

-Papillacarus gueyeae (Pérez-Íñigo, 1989) (Vepracarus)

Distribución: Senegal.

-Papillacarus hirsutus (Aoki, 1961) (Cryptacarus)

DisTRIBUCIÓN: Semicosmopolita (Paleártica oriental: excepto

Norte, Etiópica, Oriental: frecuente, y Polinesia).

-Papillacarus incompletus (Mahunka, 1985) (Vepracarus)

DisTRIBUCión: Antillas.

-Papillacarus indicus Kardar, 1972

Distribución: India (Uttar Pradesh).

-Papillacarus koreanus (Mahunka, 1973) (Vepracarus)

DisTRIBUCIÓN: Corea.

-Papillacarus lienhardi Mahunka, 1997

DisTRIBUCIÓN: Borneo.

-Papillacarus ogawai (Aoki, 1965) (Vepracarus)

DisTRIBUCIÓN: Tailandia.

-Papillacarus ondriasi Mahunka, 1974

DistRIBUCIÓN: Grecia y China suroriental.

-Papillacarus pavlovskii (Bulanova-Zachvatkina, 1960) (Thamnacarus) DisTRIBUCIÓN: Paleártica meridional (Sur de Europa y Asia centrooccidental)

-Papillacarus pseudoaciculatus Mahunka, 1980

DisTRIBUCIÓN: Mediterránea occidental y Australia.

-Papillacarus punctatus Hu y X. Wang, 1989

DisTRIBUCIÓN: China suroriental.

-Papillacarus ramirezae (Corpuz-Raros, 1979) (Vepracarus)

DisTRIBUCIÓN: Filipinas.

-Papillacarus ramosus Balogh, 1961

DistriBución: Oriental.

-Papillacarus simplirostratus Bhattacharya, Bhaduri y Raychaudhuri, 1974

Distribución: India (Bengala Occidental).

-Papillacarus spinosus Alzuet, 1972

Distribución: Argentina.

-Papillacarus undirostratus Aoki, 1965

DistRIBUCIÓN: Tailandia.

-Papillacarus vitis Elbadry y Nasr, 1977

DisTRIBUCIÓN: Egipto.

Paulianacarus Balogh, 1961 (12 spp.)

EsPecie TiPo: Paulianacarus laevis Balogh, 1961

DisTRIBUCIÓN: Paleotropical.

Paulianacarus (Paulianacarus) Balogh, 1961 (9 spp.)

Distribución: Paleotropical.

-Paulianacarus (P.) barlowi Coetzee, 2001

DistRIBUCIÓN: Sudáfrica.

-Paulianacarus (P.) foliatus Mondal y Chakrabarti, 1982

DisTRIBución: India (Bengala Occidental).

-Paulianacarus (P.) grobleri Coetzee, 2001

Distribución: Sudáfrica.

-Paulianacarus (P.) laevis Balogh, 1961

DisTRIBUCIÓN: Madagascar.

-Paulianacarus (P.) nodosus Balogh, 1961

DisTRIBUCIÓN: Madagascar.

-Paulianacarus (P.) rugosus Balogh, 1961

Distribución: Madagascar.

-Paulianacarus (P.) rugulosus Mahunka, 1995

DisTRIBUCIÓN: Tailandia.

-Paulianacarus (P.) sarbias Coetzee, 2001

(=Paulianacarus foliatus Sarkar y Subías, 1984, "nom. praeoc." por Mondal

y Chakrabarti, 1982)

(=Paulianacarus sarkari P. Balogh, 2002)

DisTRIBUCIÓN: India (Tripura)

-Paulianacarus (P.) simplisetosus Mahunka, 1985

DistRIBUCIÓN: India (Kerala). 
Paulianacarus (Millotacarus) Balogh, 1961 (3 spp.)

EsPeCIE TIPO: Millotacarus granulatus Balogh, 1961

DisTRIBUCión: Paleotropical.

-Paulianacarus (Millotacarus) granulatus (Balogh, 1961) (M.)

DisTRIBUCión: Madagascar.

-Paulianacarus (Millotacarus) indicus (Sanyal, 1992) (M.)

DisTRIBUCIÓN: India (Bengala Occidental).

-Paulianacarus (Millotacarus) orientalis (Mahunka, 1988) (M.) DISTRIBUCIÓN: Borneo.

Reptacarus Pérez-Íñigo y Peña, 1995 (1 sp.)

EsPecie TiPo: Reptacarus sagatus Pérez-Íñigo y Peña, 1995

DisTRIBUCIÓN: Paleártica.

-Reptacarus sagatus Pérez-Íñigo y Peña, 1995

DisTRIBUCIÓN: I. Canarias.

Strinatacarus Mahunka, 1977 (1 sp.)

EsPECIE TIPO: Strinatacarus aelleni Mahunka, 1977

DisTRIBUCIÓN: Etiópica.

-Strinatacarus aelleni Mahunka, 1977

DisTRIBUCIÓN: Kenia.

Thamnacarus Grandjean, 1950 (5 spp.)

(=Asiacarus Krivolutsky, 1971)

EsPecie TiPo: Lohmannia deserticola Grandjean, 1934

DistRIBUCIÓN: Subtropical (Paleártica meridional).

-Thamnacarus arnoldii (Bashkirova, 1958) (Trhypochthonius) "sp. inq." DistRIBUCIÓN: Sur de Rusia europea.

-Thamnacarus deserticola (Grandjean, 1934) (Lohmannia)

DisTRIBUCIÓN: Paleártica meridional.

-Thamnacarus elongatus (Krivolutsky, 1971) (Asiacarus)

(=Thamnacarus moribei Aoki, 1971)

DisTRIBUCIÓN: Asia centrooccidental y Etiopía.

-Thamnacarus longisetosus Bulanova-Zachvatkina, 1978

DistRIBUCIÓN: Paleártica meridional: Suroeste de Europa y Asia centrooccidental.

-Thamnacarus smirnovi Bulanova-Zachvatkina, 1978 DISTRIBUCIÓN: Asia centrooccidental.

Torpacarus Grandjean, 1950 (14 spp. y 2 sspp.)

(=Talpacarus Grandjean, 1939 nom. praeoc.)

EsPECIE TIPO: Torpacarus omittens Grandjean, 1950

Distribución: Tropical (Etiópica y Neotropical) y subtropical

(Neártica meridional).

-Torpacarus callipygus Mahunka, 1983

DisTRIBUCIÓN: Guatemala.

-Torpacarus cinctus Wallwork, 1962 DisTRIBUCIÓN: Ghana

-Torpacarus cylindricus Stary, 1998

DisTRIBUCIÓN: Cuba.

-Torpacarus elegans Stary, 1998

DisTRIBUCIÓN: Cuba.

-Torpacarus foliatus Stary, 1998 DisTRIBUCión: Cuba.

-Torpacarus foveolatus Wallwork, 1962

DISTRIBUCIÓN: Ghana.

-Torpacarus gramineus McDaniel, Norton y Bolen, 1979

DisTRIBUCIÓN: U.S.A. (Tejas).

-Torpacarus izabalensis Schatz, 1994

DISTRIBUCIÓN: Guatemala.

-Torpacarus lobatus Stary, 1998

DisTRIBUCiÓN: Cuba.

-Torpacarus magnus Wallwork, 1962

DisTRIBUCIÓN: Ghana.

-Torpacarus omittens Grandjean, 1950

DisTRIBUCIÓN: Neotropical y África occidental.

-Torpacarus omittens omittens Grandjean, 1950

DistRIBUCIÓN: Neotropical (frecuente) y África occidental.

-Torpacarus omittens galapagensis Schatz, 1994

DistriBución: I. Galápagos.
-Torpacarus omittens paraguayensis Balogh y Mahunka, 1981

DisTRIBUCión: Paraguay.

-Torpacarus pseudocallipygus Stary, 1998

DISTRIBUCIÓN: Cuba.

-Torpacarus remotus Schatz, 1994

DisTRIBUCIÓN: I. Galápagos.

-Torpacarus schatzi Stary, 1998

DisTRIBUCIÓN: Cuba.

Ululohmannia Mahunka, 1987 (1 sp.)

(=Austracarus Mahunka, 1984, "nom. praeoc.” por Lawrence, 1949)

ESPECIE TIPO: Austracarus cristatus Mahunka, 1984

DISTRIBUCIÓN: Etiópica.

-Ululohmannia cristata (Mahunka, 1984) (Austracarus)

DisTRIBUCIÓN: Tanzania.

Xenolohmannia Balogh y Mahunka, 1969 (3 spp.)

ESPECIE TIPO: Xenolohmannia discrepans Balogh y Mahunka, 1969

DisTRIBUCIÓN: Neotropical.

Xenolohmannia capillata Balogh y Mahunka, 1978

Distribución: Brasil.

-Xenolohmannia comosa P. Balogh, 1984

DisTRIBUCIÓN: Colombia.

-Xenolohmannia discrepans Balogh y Mahunka, 1969

DisTRIBUCIÓN: Brasil.

Infradorden EUPTYCTIMA Grandjean, 1967

Mesoplophoroidea Ewing, 1917

Mesoplophoridae Ewing, 1917 (4 gen., 1 subg., 37 spp. y 1 ssp.) DISTRIBUCIÓN: Cosmopolita (excepto Antártica)

Apoplophora Aoki, 1980 (10 spp.)

ESPECIE TIPO: (Apoplophora remota Aoki, 1980) = Mesoplophora pantotrema Berlese, 1913

DisTRIBUCIÓN: Australianooriental.

-Apoplophora cristata Mahunka, 1991

DistriBución: Península Malaya.

-Apoplophora heterotricha Mahunka, 1987

DISTRIBUCIÓN: Oriental.

-Apoplophora malaya Mahunka, 1991

DisTRIBUCión: Península Malaya.

-Apoplophora marcuardi Mahunka, 1991

DistriBución: Península Malaya.

-Apoplophora ornata Niedbala, 2001

DisTRIBUCIÓN: Borneo.

-Apoplophora pantotrema (Berlese, 1913) (Mesoplophora)

(=Mesoplophora discreta Berlese, 1913)

(=Mesoplophora indica Mahunka, 1985)

(=Apoplophora jaccoudi Mahunka, 1991)

(=Apoplophora lineata Mahunka, 1987)

(=Apoplophora ornatissima Mahunka, 1988)

(=Apoplophora remota Aoki, 1980)

(=Mesophophora rostrorugosa Hammer, 1979)

(=Mesoplophora striata Mahunka, 1985)

DISTRIBUCIÓN: Australianooriental: frecuente.

-Apoplophora phalerata Niedbala, 2001

DISTRIBUCIÓN: Oriental.

-Apoplophora solomonensis Niedbala, 1998

DisTRIBUCión: Melanesia (I. Salomón).

-Apoplophora spinosa Mahunka, 1987

DISTRIBUCIÓN: Borneo.

-Apoplophora triseta Mahunka, 1991

DisTRIBUCIÓN: Península Malaya.

Archoplophora Hammen, 1959 (1 sp.)

ESPECIE TIPO: (Phthiracarulus laevis Jacot, 1938) =Phthiracarulus rostralis Willmann, 1930

DISTRIBUCIÓN: Semicosmopolita (Holártica, Oriental y Neotropical). 
-Archoplophora rostralis (Willmann, 1930) (Phthiracarulus)

(=Phthiracarulus laevis Jacot, 1938)

(=Archoplophora villosa Aoki, 1980)

Distribución: Holártica (Neártica: menos frecuente en el Sur, y

Paleártica oriental: excepto Norte), Oriental y Neotropical (Centroamérica)

Dudichoplophora Mahunka, 1982 (1 sp.)

ESPECIE TIPO: Dudichoplophora reticulata Mahunka, 1982

DisTRIBUCIÓN: Paleártica.

-Dudichoplophora reticulata Mahunka, 1982

DisTRIBUCIÓN: Corea.

Mesoplophora Berlese, 1904 (25 spp. y 1 ssp.)

EsPeCIE TIPO: Mesoplophora michaeliana Berlese, 1904

DisTRIBUCIÓN: Cosmopolita (excepto Antártica).

Mesoplophora (Mesoplophora) Berlese, 1904 (15 spp. y 1 ssp.) (=Phthiracarulus Berlese, 1920)

DistriBUCión: Pantropical (excepto Australiana) y subtropical.

-Mesoplophora (M.) africana Balogh, 1958

(=Mesoplophora insularis Pérez-Íñigo, 1983)

DisTRIBUCIÓN: Etiópica (frecuente).

-Meloplophora (M.) bolivari Calugar, 1990

DisTRIBUCIÓN: Venezuela.

-Mesoplophora (M.) crassisetosa Subías y Sakar, 1984

DisTRIBUCIÓN: India (Tripura)

-Mesoplophora (M.) cubana Calugar y Vasiliu, 1977

DisTRIBUCIÓN: Centroamérica.

-Mesoplophora (M.) cubana cubana Calugar y Vasiliu, 1977 (=Mesoplophora longiseta Mahunka, 1982) DistRiBución: Centroamérica.

-Mesoplophora (M.) cubana pratensis Scull, 1985 DisTRIBUCIÓN: Cuba.

-Mesoplophora (M.) gaveae Schuster, 1962

DisTRIBUCión: Neotropical y Bután.

-Mesoplophora (M.) hauseri Mahunka, 1982

DisTRIBUCIÓN: Neotropical.

-Mesoplophora (M.) ifeana Badejo, Woas y Beck, 2001

DisTRIBUCIÓN: Nigeria.

-Mesoplophora (M.) ignota Niedbala, 1988

DisTRIBUCIÓN: Brasil.

-Mesoplophora (M.) invisitata Niedbala, 1983

DistriBución: África central e India (Goa).

-Mesoplophora (M.) longisetosa Calugar y Vasiliu, 1977

DisTRIBUCIÓN: Cuba.

-Mesoplophora (M.) michaeliana Berlese, 1904

(=Mesoplophora caucasica Krivolutsky, 1975)

(=Mesoplophora graeca Walzl, 1974)

(=Mesoplophora pectinata Mahunka, 1979)

(=Phthiracarus (Phthiracarulus) perexiguus Berlese, 1920)

DistriBución: Paleártica meridional.

-Mesoplophora (M.) paragaveae Niedbala, 2002

DISTRIBUCIÓN: I. Bermudas.

-Mesoplophora (M.) permodica Niedbala, 1985

DisTRIBUCIÓN: U.S.A. centrooriental y Venezuela.

-Mesoplophora (M.) pusilla Schuster, 1962

DisTRIBUCIÓN: Neotropical.

-Mesoplophora (M.) vesca Niedbala y Corpuz-Raros, 1998

DisTRIBUCIÓN: Filipinas.

Mesoplophora (Parplophora) Niedbala, 1985 (10 spp.)

ESPECIE TIPO: Mesoplophora pulchra Sellnick, 1928

DisTRIBUCIÓn: Cosmopolita (excepto Antártica).

-Mesoplophora (Parplophora) abscondita Niedbala, 1988

DisTRIBUCIÓN: U.S.A. (frecuente)

-Mesoplophora (Parplophora) flavida Niedbala, 1985

DisTRIBUCión: Oriental.
-Mesoplophora (Parplophora) japonica Aoki, 1970 (M.)

DistribuCiÓN: Holártica (Este de Paleártica oriental, Neártica septentrional y U.S.A.: California).

-Mesoplophora (Parplophora) leviseta Hammer, 1979 (M.)

(=Mesoplophora gibba Mahunka, 1988)

Distribución: Pantropical (Etiópica: I. Seychelles, Oriental: Java, y Australiana: Islas del Pacífico).

-Mesoplophora (Parplophora) paraleviseta Mahunka, 1991

DistriBución: Península Malaya.

-Mesoplophora (Parplophora) pertenuis Niedbala, 2001

DisTRIBUCIón: U.S.A. meridional.

-Mesoplophora (Parplophora) polita Niedbala, 1985 (M.)

(=Mesoplophora villosa Mahunka, 1987)

DistRIBUCIÓN: Borneo y Nueva Guinea.

-Mesoplophora (Parplophora) pulchra Sellnick, 1928 (M.)

DistRiBución: Paleártica occidental (excepto Norte).

-Mesoplophora (Parplophora) rostrata Mahunka, 1988

DisTRIBUCIÓN: Tanzania.

-Mesoplophora (Parplophora) subtilis Niedbala, 1981 (M.)

DisTRIBUCIÓN: Pantropical (excepto Etiópica)

Euphthiracaroidea Jacot, 1930

Oribotritiidae Grandjean, 1954 ( 9 gen., 3 subg., 129 spp. y 5 sspp.) DistriBuCIÓn: Cosmopolita.

Austrotritia Sellnick, 1959 (14 spp. y 3 sspp.)

(=Macarotritia Pérez-Íñigo, 1986 "sin. nov.")

EsPeCIE TIPO: Autrotritia quadricarinata Sellnick, 1959

DISTRIBUCIÓN: Australianooriental y Paleártica meridional.

-Austrotritia bifurca Niedbala, 2000

Distribución: Australiana (Nueva Guinea y Australia).

- Austrotritia carinata Niedbala, 2000

DistriBuCIÓN: Australiana (Nueva Guinea y Australia).

-Austrotritia dentata Aoki, 1980

DisTRIBUCIÓN: Este de Paleártica oriental

-Austrotritia gibba Bayoumi y Mahunka, 1979

DISTRIBUCIÓN: India (Cachemira).

-Austrotritia glabrata Niedbala, 2000

DisTRIBUCIÓN: Nepal.

-Austrotritia herenessica (Pérez-Íñigo, 1986) (Indotritia (Macarotritia))

DisTRIBUCIÓN: I. Canarias.

-Austrotritia kinabaluensis Ramsay y Sheals, 1969

DisTRIBUCIÓN: Borneo.

-Austrotritia lanceolata Aoki, 1988

DisTRIBUCIÓN: Japón.

-Austrotritia lebronneci (Jacot, 1934) (Indotritia)

DisTRIBUCIÓN: Australianooriental.

-Austrotritia lebronneci lebronneci (Jacot, 1934)

DisTRIBUCIÓN: Australianooriental.

-Austrotritia lebronneci crassiori Jacot, 1934

DisTRIBUCIÓN: Polinesia (I. Marquesas).

-Austrotritia lebronneci flagelloides Jacot, 1934

Distribución: Polinesia (I. Marquesas).

-Austrotritia lebronneci tahitiana (Jacot, 1934) (Indotritia)

DisTriBución: Polinesia (Tahití).

-Austrotritia quadricarinata Sellnick, 1959

DisTRIBUCIÓN: Polinesia: I. Australes ( I. Tubuai).

-Austrotritia ramsayi Mahunka, 1991

DisTRIBUCIÓN: Borneo.

-Austrotritia robusta Niedbala y Corpuz-Raros, 1998

DistribuCIÓN: Filipinas y Nueva Guinea.

-Austrotritia saraburiensis Aoki, 1965 (A. lebronneci s.)

(=Austrotritia ishigakiensis Aoki, 1980)

(=Austrotritia optabilis Niedbala, 1991)

(=Austrotritia shealsi Mahunka, 1987)

DisTRIBUCIÓn: Tropical (Oriental y Australiana: Islas del Pacífico)

y Japón.

-Austrotritia unicarinata Aoki, 1980

DisTRIBUCIÓN: Japón y Micronesia (I. Marianas). 
Indotritia Jacot, 1929 (22 spp.)

ESPECIE TIPO: Tritia krakatauensis Sellnick, 1924

DISTRIBUCIÓN: Cosmopolita.

Indotritia (Indotritia) Jacot, 1929 (18 spp.)

DisTRIBUCIÓN: Cosmopolita.

-Indotritia (I.) africana Mahunka, 1984

DisTRIBUCIÓN: Sudáfrica.

-Indotritia (I.) aspera Niedbala, 2000

DisTRIBUCIÓN: Nepal.

-Indotritia (I.) bellingeri Niedbala y Schatz, 1996

DISTRIBUCIÓN: Neotropical.

-Indotritia (I.) breviseta (Berlese, 1923) (Tritia berlesei b.)

DisTRIBUCIÓN: "África oriental".

-Indotritia (I.) bryani (Jacot, 1929) (Euphthiracarus (I.)) "sp. inq." DisTRIBUCIÓN: Hawai.

-Indotritia (I.) clavata Wallwork, 1977

DisTRIBUCIÓN: I. Santa Helena.

-Indotritia (I.) consimilis Märkel, 1964 (I. krakatauensis c.) DisTRIBUCIÓN: Europa centromeridional.

-Indotritia (I.) jacoti Niedbala, 2001

DisTRIBUCIÓN: U.S.A. oriental.

-Indotritia (I.) javensis (Sellnick, 1923) (Tritia)

(=Indotritia completa Mahunka, 1987)

(=Oribotritia mollis Aoki, 1959)

Distribución: Oriental (frecuente), Este de Paleártica oriental y subantártica (I. Amsterdam).

-Indotritia (I.) krakatauensis (Sellnick, 1923) (Tritia)

(=Indotritia acanthophora Märkel, 1964)

(=Indotritia heterotricha Mahunka, 1984)

(=Indotritia sellnicki Aoki, 1965)

(=Indotritia septentrionalis Mahunka, 1967)

(=Indotritia tropica Stary, 1993)

DisTRIBUCIÓN: Pantropical (frecuente).

-Indotritia (I.) missouri Niedbala, 2002

DISTRIBUCIÓN: U.S.A. centromeridional.

-Indotritia (I.) nuda Mahunka, 1988

(=Indotritia usambarensis Stary, 1993)

DisTRIBUCIÓN: Tanzania.

-Indotritia (I.) paulyi Niedbala, 1998

DISTRIBUCIÓN: Madagascar.

-Indotritia (I.) propinqua Niedbala, 1991

DistriBuCIÓN: Oriental: Ceilán [Sri Lanka] y Noreste de India

-Indotritia (I.) retusa Niedbala y Schatz, 1996

DisTRIBUCIÓN: Norte de Neotropical.

-Indotritia (I.) tripartita Niedbala, 1998

DISTRIBUCIÓN: Malgache (I. Comores).

-Indotritia (I.) undulata Bayoumi y Mahunka, 1979

DisTRIBUCIÓN: Himalaya (frecuente).

-Indotritia (I.) zangherii Mahunka y Paoletti, 1984

DisTRIBUCIÓN: Italia.

Indotritia (Afrotritia) Mahunka, 1988 (1 sp.)

ESPECIE TIPO: Indotritia (Afrotritia) compacta Mahunka, 1988

DisTRIBUCIÓN: Etiópica.

-Indotritia (Afrotritia) compacta Mahunka, 1988

DISTRIBUCIÓN: África centrooriental.

Indotritia (Zeaotritia) Mahunka, 1988 (3 spp.)

ESPECIE TIPO: Indotritia aotearoana Ramsay, 1966

DISTRIBUCIÓN: Australiana.

-Indotritia (Zeaotritia) aotearoana Ramsay, 1966 (I. a.)

DisTRIBUCIÓN: Nueva Zelanda.

-Indotritia (Zeaotritia) brevipilosa Niedbala, 2000

DisTRIBUCIÓN: Australia.

-Indotritia (Zeaotritia) brevisetosa Niedbala, 2000

(=Indotritia breviseta Niedbala y Colloff, 1997, "nom. praeoc." por Berlese, 1923)

(=Indotritia colloffi J. y P. Balogh, 2002)

DistriBuCión: Australiana (Australia y Tasmania).
Maerkelotritia Hammer, 1967 (3 spp. y 1 ssp.)

ESPECIE TIPO: (Maerkelotritia alaskensis Hammer, 1967) =Oribotritia

kishidai Aoki, 1958

DISTRIBUCIÓN: Holártica.

-Maerkelotritia crytopa (Banks, 1904) (Phthiracarus)

$(=$ Oribotritia gibbera Walker, 1965)

(=Phthiracarus maximus Ewing, 1913)

DiSTRIBUCIÓN: Neártica occidental (frecuente).

-Maerkelotritia kishidai (Aoki, 1958) (Oribotritia)

(=Maerkelotritia alaskensis Hammer, 1967)

(=Oribotritia sellnicki Walker, 1965)

DisTRIBUCIÓN: Holártica (Paleártica oriental: Japón y Este de Rusia asiática, y Neártica: frecuente en Oeste).

-Maerkelotritia krivolutskyi Märkel, 1968

DisTRIBUCIÓN: Sur de Paleártica oriental.

-Maerkelotritia krivolutskyi krivolutskyi Märkel, 1968

DISTRIBUCIÓN: Paleártica oriental (Asia centrooccidental y Corea).

-Maerkelotritia krivolutskyi mongolica Mahunka, 1981

DISTRIBUCIÓN: Mongolia.

Mesotritia Forsslund, 1963 (26 spp.)

EsPECIE TIPO: (Mesotritia testacea Forsslund, 1963) =Phthiracarus flagelliformis Ewing, 1909

DISTRIBUCIÓN: Cosmopolita (excepto Australiana y Antártica).

Mesotritia (Mesotritia) Forsslund, 1963 (23 spp.)

(=Entomotritia Märkel, 1964)

DisTRIBUCIÓN: Cosmopolita (excepto Australiana y Antártica).

-Mesotritia (M.) australis Mahunka, 1984

DisTRIBUCIÓN: Tanzania.

-Mesotritia (M.) brasiliensis (Sellnick, 1923) (Tritia)

DisTRIBUCIÓN: Brasil.

-Mesotritia (M.) breviseta Niedbala y Schatz, 1996

DisTRIBUCIón: Centroamérica.

-Mesotritia (M.) clara Stary, 1992

Distribución: India (Cachemira).

-Mesotritia (M.) dissimilis Hammer, 1977 (M. (Entomotritia))

DistribuCión: Pakistán.

-Mesotritia (M.) elegantula Stary, 1992

DistriBuCión: Cuba.

-Mesotritia (M.) exobothridialis Bayartogtokh y Aoki, 1998

DISTRIBUCIÓN: Mongolia.

-Mesotritia (M.) faeroensis (Sellnick, 1923) (Tritia)

DisTRIBUCIÓN: Noroeste de Europa.

-Mesotritia (M.) flagelliformis (Ewing, 1909) (Phthiracarus)

(=Mesotritia testacea Forsslund, 1963)

Distribución: Holártica (Paleártica: Norte de Europa y Este de

Rusia asiática, y Neártica: frecuente)

-Mesotritia (M.) glabra Niedbala, 2002

DISTRIBUCIÓN: Sur de U.S.A.

-Mesotritia (M.) glabrata (Say, 1821) (Oribata)

DisTRIBUCIÓN: U.S.A.

-Mesotritia (M.) grandjeani (Feider y Suciu, 1957) (Oribotritia)

Distribución: Paleártica (Europa oriental y Este de Rusia asiática) y Méjico.

-Mesotritia (M.) indica Sanyal, 1988

DistribuCIÓN: India (Himalaya).

-Mesotritia (M.) jacoti Niedbala, 2001

DisTRIBUCIÓN: U.S.A. (Florida).

-Mesotritia (M.) maerkeli Sheals, 1965

=Mesotritia flagellata Bayoumi y Mahunka, 1979)

(=Mesotritia spinosa Aoki, 1980)

DisTRIBUCIÓN: Himalaya y Japón.

-Mesotritia (M.) minima Niedbala, 2002

Distribución: U.S.A. (Tejas).

-Mesotritia (M.) nitida Hammer, 1977 (M. (Entomotritia))

DistRIBUCIÓN: Pakistán.

-Mesotritia (M.) nova Stary, 1992

DISTRIBUCION: Cuba. 
-Mesotritia (M.) nuda (Berlese, 1887) (Tritia)

(=Oribotritia brachythrix Walker, 1965)

(=Mesotritia elastica Sergienko, 1988)

(=Mesotritia (Entomotritia) piffli Märkel, 1964)

DisTRIBUCIÓN: Holártica (Paleártica: menos frecuente en el Norte, y Neártica: frecuente) y Norte de Neotropical.

-Mesotritia (M.) okuyamai Aoki, 1980

DisTRIBUCIÓN: Este de Paleártica oriental.

-Mesotritia (M.) ruwenzorii Niedbala, 1998

DisTRIBUCIÓN: África central.

-Mesotritia (M.) similis Niedbala, 2000

DisTRIBUCIÓN: India (Cachemira) y Tailandia.

-Mesotritia (M.) virginiensis (Jacot, 1930) (Oribotritia)

DisTRIBUCIÓN: U.S.A. centrooriental.

Mesotritia (Perutritia) Märkel, 1964 (3 spp.)

EsPeCIE TIPO: Perutritia amazonensis Märkel, 1964

DisTRIBUCIÓN: Neotropical.

-Mesotritia (Perutritia) amazonensis (Märkel, 1964) (P.)

DisTRIBUCión: Neotropical (Perú y Centroamérica).

-Mesotritia (Perutritia) asensillata (Ojeda, 1992) (P.)

Distribución: Méjico.

-Mesotritia (Perutritia) curviseta (Hammer, 1961) (Oribotritia)

DisTRIBUCIÓN: Neotropical.

Oribotritia Jacot, 1924 (54 spp. y 1 ssp.)

(=Berndotritia Mahunka, 1987 "sin. nov.")

(=Philotritia Mahunka, 1988)

(=Plesiotritia Walker, 1965)

(=Tritia Berlese, 1883, "nom. praeoc.” por Risso, 1826)

EsPECIE TIPO: (Hoplophora decumana Koch "sensu” Berlese, 1883)

=Phthiracarus berlesei Michael, 1898

DISTRIBUCIÓN: Cosmopolita (excepto Antártica).

-Oribotritia africana Stary, 1993

(=Oribotritia bicarinata Niedbala, 1993)

DISTRIBUCIÓN: Etiópica (frecuente)

-Oribotritia ampla Niedbala, 1991

Distribución: Australiana (Islas del Pacífico)

-Oribotritia anceps Niedbala, 2000

DisTRIBUCIÓN: Nepal.

-Oribotritia angusta Mahunka, 1982

DisTRIBUCIÓN: Corea.

-Oribotritia aokii Mahunka, 1987

DisTRIBUCIÓN: Oriental.

-Oribotritia asiatica Hammer, 1977

(=Oribotritia koreense Mahunka, 1982)

DisTRIBUCión: Paleártica oriental (excepto Norte).

-Oribotritia asiatica asiatica Hammer, 1977

DisTRIBUCIÓN: Paleártica oriental (Pakistán y Corea).

-Oribotritia asiatica norikoae Suzuoka, 1983

DisTRIBUCIÓN: Japón.

-Oribotritia attenuata Niedbala y Schatz, 1996

DisTRIBUCIÓN: Centroamérica.

-Oribotritia bagnalli (Hull, 1916) (Phthiracarus)

DisTRIBUCIÓN: I. Británicas.

-Oribotritia banksi (Oudemans, 1916) (Tritia)

DisTRIBUCIÓN: U.S.A. (frecuente)

-Oribotritia berlesei (Michael, 1898) (Phthiracarus)

DisTRIBUCIÓN: Paleártica (frecuente).

-Oribotritia bipartita Niedbala, 2000

DisTRIBUCIÓN: Nepal.

-Oribotritia brevis Niedbala y Colloff, 1997

DisTRIBUCión: Tasmania.

-Oribotritia bulbifer (Mahunka, 1987) (Berndotritia)

DisTRIBUCIÓN: Borneo.

-Oribotritia capitanea Niedbala y Corpuz-Raros, 1998

DISTRIBUCIÓN: Filipinas.

-Oribotritia carolinae Jacot, 1930

DISTRIBUCIÓN: Este de U.S.A.
-Oribotritia cherokee Niedbala, 2002

DisTRIBUCIÓN: U.S.A. (Arkansas).

-Oribotritia chichijimensis Aoki, 1980

(=Oribotritia chichijimensis ryukyuensis Nakatamari, 1985)

DISTRIBUCIÓN: Japón.

-Oribotritia contortula Niedbala, 1993

DISTRIBUCIÓN: Australiana.

-Oribotritia contraria Niedbala, 2000

DisTRIBUCIÓN: Australiana.

-Oribotritia corporaali (Oudemans, 1926) (Tritia)

DisTRIBUCIÓN: Sumatra.

-Oribotritia decumana (Koch, 1836) (Hoplophora) "sp. inq."

DisTRIBUCIÓN: Alemania.

-Oribotritia didyma Niedbala y Schatz, 1996

DistRIBUCIÓN: Norte de Neotropical.

-Oribotritia duplex Niedbala, 2000

DistRIBUCIÓN: Australia.

-Oribotritia exilis (Sellnick, 1923) (Tritia)

DisTRIBUCIÓN: Neotropical.

-Oribotritia fennica Forsslund y Märkel, 1963

DisTRIBUCIÓN: Paleártica (Europa septentrional y oriental, y Japón).

-Oribotritia gigas Bayoumi y Mahunka, 1979

DisTRIBUCIÓN: Nepal y Corea.

-Oribotritia hauseri Mahunka, 1982

DistRIBUCIÓN: Grecia.

-Oribotritia hawaiiensis (Jacot, 1929) (Euphthiracarus (Indotritia))

DisTRIBUCIÓN: Hawai.

-Oribotritia henicos Niedbala, 2002

DistriBuCión: Canadá.

-Oribotritia hermanni Grandjean, 1967

DISTRIBUCIÓN: Mediterránea.

-Oribotritia heterotricha Niedbala, 2000

DisTRIBUCIÓN: Borneo.

-Oribotritia incognita Niedbala, 2000

DisTRIBUCIÓN: Nueva Zelanda.

-Oribotritia magna (Ewing, 1907) (Phthiracarus)

DisTRIBUCIÓN: U.S.A. centromeridional.

-Oribotritia megale (Walker, 1965) (Plesiotritia)

DisTRIBUCIÓN: U.S.A. (California).

-Oribotritia nepalensis Niedbala, 2000

DisTRIBUCIÓN: Nepal.

-Oribotritia opipara Niedbala, 2002

DistRIBUCIÓN: U.S.A.

-Oribotritia oregonensis Niedbala, 2002

DisTRIBUCIÓN: U.S.A. (Oregón).

-Oribotritia paraaokii Niedbala, 2000

DISTRIBUCIÓN: Vietnam.

-Oribotritia paraspinosa Mahunka, 1999

DisTRIBUCIÓN: Malgache.

-Oribotritia pulla Niedbala, 1998

DisTRIBUCIÓN: Hawai.

-Oribotritia rafalskii Niedbala, 1997

DISTRIBUCIÓN: Argelia.

-Oribotritia samoaensis Niedbala, 1998

Distribución: Australiana (Islas del Pacífico).

-Oribotritia serrata Feider y Suciu, 1958

DisTRIBUCIÓN: Europa suroriental.

-Oribotritia serrula Niedbala y Schatz, 1996

DisTRIBUCIÓN: Centroamérica (I. Cocos).

-Oribotritia solitaria Niedbala, 1993

DisTRIBUCIÓN: Congo.

-Oribotritia spinosa (Mahunka, 1988) (Philotritia)

DISTRIBUCIÓN: Malgache (frecurente).

-Oribotritia storkani Feider y Suciu, 1957

DisTRIBUCIÓN: Europa meridional.

-Oribotritia submolesta Niedbala, 2000

DisTRIBUCIÓN: Oriental (Vietnam e India: Uttar Pradesh).

-Oribotritia succinta Niedbala, 1998

DisTRIBUCIÓN: Madagascar. 
-Oribotritia teretis Niedbala, 1993

DisTRIBUCIÓN: Nueva Zelanda.

-Oribotritia tiwi Mahunka, 1987

DisTRIBUCIÓN: África oriental (frecuente).

-Oribotritia tokukoae Aoki, 1973

DisTRIBUCión: Este de Paleártica oriental.

-Oribotritia trisetosa Niedbala y Schatz, 1996

Distribución: Centroamérica.

-Oribotritia virgulata Niedbala, 2001

DISTRIBUCIÓN: Madagascar.

Paratritia Moritz, 1966 (1 sp.)

EsPeCIE TIPO: Paratritia baloghi Moritz, 1966

DisTRIBUCIÓN: Paleártica.

-Paratritia baloghi Moritz, 1966

DISTRIBUCIÓN: Europa centromeridional.

Protoribotritia Jacot, 1938 (5 spp.)

ESPECIE TIPO: Protoribotritia canadaris Jacot, 1938

DISTRIBUCIÓN: Holártica y Etiópica.

-Protoribotritia aberrans (Märkel y Meyer, 1959) (Oribotritia)

DisTRIBUCIÓN: Alemania.

-Protoribotritia africana Mahunka, 1985

DisTRIBUCIÓN: Sudáfrica.

-Protoribotritia canadaris Jacot, 1938

DisTRIBUCIÓN: Neártica septentrional y Nuevo Méjico.

-Protoribotritia ensifer Aoki, 1969 (P. aberrans e.)

DisTRIBUCiÓn: Este de Paleártica oriental.

-Protoribotritia oligotricha Märkel, 1963

DisTRIBUCión: Holártica (Norte de Paleártica occidental: Suecia,

y Neártica: menos frecuente en el Sur).

Sabacarus Ramsay y Sheals, 1969 (2 spp.)

EsPECIE TIPO: Sabacarus corneri Ramsay y Sheals, 1969

DisTRIBUCIÓN: Semicosmopolita (Oriental, Australiana y subantártica).

-Sabacarus corneri Ramsay y Sheals, 1969

(=Sabacarus ranokaoensis Hammer, 1970)

Distribución: Tropical: Oriental, Australiana e I. de Pascua (I

Easter), y subantártica (I. Amsterdam).

-Sabacarus japonicus Shimano y Aoki, 1997

DisTRIBUCIÓN: Japón.

Terratritia Ramsay y Sheals, 1969 (2 spp.)

ESPECIE TIPO: Terratritia askewi Ramsay y Sheals, 1969

DISTRIBUCIÓN: Oriental.

-Terratritia askewi Ramsay y Sheals, 1969

DISTRIBUCIÓN: Borneo.

-Terratritia seconda Mahunka, 1991

DISTRIBUCIÓN: Borneo.

Euphthiracaridae Jacot, 1930 (5 gen., 2 subg., 108 spp. y 8 sspp.) DisTRIBUCIÓN: Cosmopolita.

Acrotritia Jacot, 1923 (36 spp. y 5 sspp.)

(=Rhysotritia Märkel y Meyer, 1959)

ESPECIE TIPO: (Phthiracarus americanus Ewing, 1909) =Hoplophora ardua Koch, 1841

DISTRIBUCIÓN: Cosmopolita.

-Acrotritia aokii (Niedbala, 2000) (Rhysotritia)

DisTRIBUCión: Oriental (frecuente) y Este de Paleártica oriental.

-Acrotritia ardua (Koch, 1841) (Hoplophora)

DISTRIBUCIÓN: Cosmopolita.

-Acrotritia ardua ardua (Koch, 1841)

(=Phthiracarus americanus Ewing, 1909)

(=Hoplophora arctata Riley, 1874)

(=Pseudotritia ardua antetriheterodactylum Jacot, 1933)

(=Phthiracarus canestrinii Michael, 1898)

(=Phthiracarus pectinatus Ewing, 1917)

DISTRIBUCIÓN: Cosmopolita (frecuente).
-Acrotritia ardua affinis (Sergienko, 1989) (Rhysotritia)

DisTRIBUCIÓN: Paleártica (Sureste de Europa y Oeste de Siberia).

-Acrotritia ardua jinyunia (Li y Chen, 1990) (Rhysotritia)

DisTRIBUCIÓN: China suroriental.

-Acrotritia ardua neotropicalis (Pérez-Íñigo y Baggio, 1993)

(Rhysotritia)

DISTRIBUCIÓN: Brasil.

-Acrotritia bipartita (Niedbala, 2000) (Rhysotritia)

DisTRIBUCIÓN: Australia.

-Acrotritia brasiliana (Mahunka, 1983) (Rhysotritia)

Distribución: Pantropical (Neotropical, Oriental: India,

Etiópica: I. Reunión, y subantártica: I. Amsterdam).

-Acrotritia clavata (Märkel, 1964) (Rhysotritia)

Distribución: Tropical (Neotropical y Etiópica: Guinea

Ecuatorial) y Oeste de Europa.

-Acrotritia clavata clavata (Märkel, 1964)

DisTRIBUCión: Neotropical (frecuente) y Etiópica (Guinea

Ecuatorial).

-Acrotritia clavata sextiana (Lions, 1966) (Rhysotritia)

$(=$ Rhysotritia riezuae Moreno y Moraza, 1994)

DisTRIBUCIÓn: Oeste de Europa.

-Acrotritia corletti (Mahunka, 2000) (Rhysotritia)

DisTRIBUCIÓn: China (Hong-Kong).

-Acrotritia curticephala (Jacot, 1938) (Pseudotritia ardua c.)

(=Rhysotritia lucida Niedbala, 1998)

Distribución: Cosmopolita (excepto Paleártica, Etiópica y Antártica): frecuente.

-Acrotritia diaphoros (Niedbala, 2002) (Rhysotritia)

DisTRIBUCIÓN: Neártica y Neotropical (Brasil y Chile).

-Acrotritia dikra (Niedbala y Schatz, 1996) (Rhysotritia)

DISTRIBUCIÓN: Neotropical y U.S.A. oriental.

-Acrotritia dinota (Niedbala y Schatz, 1996) (Rhysotritia)

DisTRIBUCIÓN: Norte de Neotropical.

-Acrotritia divida (Mahunka, 1991) (Rhysotritia)

DisTRIBUCIÓN: Oriental.

-Acrotritia dixa (Niedbala y Schatz, 1996) (Rhysotritia)

DisTRIBUCIÓN: Norte de Neotropical.

-Acrotritia duplicata (Grandjean, 1953) (Pseudotritia)

DISTRIBUCIÓN: Paleártica y Vietnam.

-Acrotritia duplicata duplicata (Grandjean, 1953)

DisTRIBUCIÓN: Paleártica (frecuente) y Vietnam.

- Acrotritia duplicata limbata (Märkel y Meyer, 1959) (Rhysotritia) DiSTRIBUCIÓN: Europa occidental (Alemania y Bulgaria).

-Acrotritia ejuncida (Niedbala y Schatz, 1996) (Rhysotritia)

DisTRIBUCIÓN: Norte de Neotropical.

-Acrotritia furcata (Bayoumi y Mahunka, 1979) (Rhysotritia)

DISTRIBUCIÓN: Oriental.

-Acrotritia gracile (Niedbala, 2000) (Rhysotritia)

DisTRIBUCIÓN: India (Uttar Pradesh).

-Acrotritia granulata (Mahunka,1999) (Rhysotritia)

DISTRIBUCIÓN: Madagascar.

-Acrotritia hallasanensis (Niedbala, 2002) (Rhysotritia)

DisTRIBUCIÓN: Corea

-Acrotritia hyeroglyphica (Berlese, 1916) (Tritia lentula h.)

(=Rhysotritia hauseri Mahunka, 1991)

(=Rhysotritia ardua penicillata Pérez-Íñigo, 1969)

(=Acrotritia sinensis triheterodactylis Jacot, 1923)

Distribución: Semicosmopolita (Holártica, Etiópica: Somalia,

Oriental y Neotropical: Panamá).

-Acrotritia koreensis Mahunka, 1997

(=Rhysotritia penicillata Mahunka, 1982, "nom. praeoc.” por Pérez-Íñigo, 1969)

DisTRIBUCIÓN: Corea.

-Acrotritia lentula (Koch, 1841) (Tritia) “sp. inq.'

DisTRIBUCIÓN: Alemania.

-Acrotritia loricata (Rathke, 1799) (Trombidium) "sp. inq."

Distribución: "Norte de Europa".

-Acrotritia monodactyla (Niedbala, 2002) (Rhysotritia)

DISTRIBUCIÓN: Neotropical. 
-Acrotritia ornata (Niedbala, 2002) (Rhysotritia)

Distribución: U.S.A. (Georgia) y Méjico.

-Acrotritia otaheitensis (Hammer, 1972) (Rhysotritia ardua o.)

DISTRIBUCIÓN: Polinesia e India (Bengala Occidental).

-Acrotritia parareticulata (Niedbala, 2002) (Rhysotritia)

DisTRIBUCión: Canadá.

-Acrotritia peruensis (Hammer, 1961) (Oribotritia)

DistRIBUCIÓN: Neotropical (frecuente) e India (Bengala Occidental).

-Acrotritia refracta (Niedbala, 1998) (Rhysotritia)

DistriBUCIÓN: Pantropical (Oriental: Borneo y Filipinas,

Australiana: Islas del Pacífico, y Norte de Neotropical.

-Acrotritia reticulata (Mahunka, 1988) (Rhysotritia)

DisTRIBUCIÓN: Tanzania.

-Acrotritia rustica (Niedbala, 1991) (Rhysotritia)

DISTRIBUCIÓN: Etiópica.

-Acrotritia scotti (Walker, 1965) (Rhysotritia)

DistribuCión: U.S.A. (California).

-Acrotritia simile (Mahunka, 1982) (Rhysotritia)

DisTRIBUCIÓN: Corea.

-Acrotritia sinensis Jacot, 1923

(=Rhysotritia clavata spiculifera Mahunka, 1991)

(=Rhysotritia rasile Mahunka, 1982)

DisTRIBUCIÓN: Pantropical (excepto Neotropical) y subtropical (Paleártica meridional)

-Acrotritia sterigma (Niedbala, 1998) (Rhysotritia)

Distribución: Polinesia (Tahití).

-Acrotritia vestita (Berlese, 1913) (Hoploderma)

(=Rhysotritia anchiseta Niedbala, 1998)

(=Rhysotritia bifurcata Niedbala, 1993)

(=Rhysotritia comteae Mahunka, 1983)

(=Rhysotritia niedbalai J. y P. Balogh, 2002)

DisTRIBUCIÓN: Pantropical (frecuente) y Canadá.

-Acrotritia wallworki (Lee, 1981) (Rhysotritia)

DistribuCión: Australia.

Bukitritia Mahunka, 1990 (1 sp.)

ESPECIE TIPO: Bukitritia timah Mahunka, 1990

DisTRIBUCIÓN: Paleotropical.

-Bukitritia timah Mahunka, 1990

DisTRIBUCIÓN: Península Malaya y Madagascar.

Eupththiracarus Ewing, 1917 (54 spp. y 2 sspp.)

EsPECIE TIPO: Phthiracarus flavus Ewing, 1908

DisTRIBUCIÓN: Cosmopolita (excepto Antártica).

Eupththiracarus (Euphthiracarus) Ewing, 1917 (40 spp. y 1 ssp.)

(?=Hummelia Oudemans, 1916 "gen. inq.")

(=Niedbalaia Mahunka, 1999 "sin. nov.")

(=Pseudotritia Willmann, 1919)

DisTRIBUCIÓN: Cosmopolita (excepto Antártica).

-Euphthiracarus (E.) aggenitalis Aoki, 1984

DisTRIBUCIÓN: Japón.

-Euphthiracarus (E.) alazon Walker, 1965

DisTRIBUCión: U.S.A. (California).

-Euphthiracarus (E.) antipai Feider y Calugar, 1969

DisTRIBUCIÓN: Rumania.

-Euphthiracarus (E.) bhutanicus Reddy, 1988

DisTRIBUCIÓN: Bután.

-Euphthiracarus (E.) cathayanus Mahunka, 2000

Distribución: China (Hong-Kong).

-Euphthiracarus (E.) cernuus Walker, 1965

DisTRIBUCIÓN: Neártica occidental.

-Euphthiracarus (E.) comteae Mahunka, 1982

DisTRIBUCIÓN: Centroamérica.

-Euphthiracarus (E.) crassisetae Jacot, 1938

(=Euphthiracarus polytretos Walker, 1965)

DisTRIBUCIÓN: Neártica.

-Euphthiracarus (E.) cribrarius (Berlese, 1904) (Phthiracarus)

(=Phthiracarus punctulatus Berlese, 1913)

DISTRIBUCIÓN: Holártica (frecuente).
-Euphthiracarus (E.) depressculus Jacot, 1924

DisTRIBUCIÓN: Neártica centrooriental.

-Euphthiracarus (E.) excultus Pérez-Íñigo, 1987

DisTRIBUCIÓN: I. Azores.

-Euphthiracarus (E.) flavus (Ewing, 1908) (Phthiracarus)

DisTRIBUCIÓN: Neártica.

-Euphthiracarus (E.) foveolatus Aoki, 1980

DistriBución: Este de Paleártica oriental.

-Euphthiracarus (E.) fulvus (Ewing, 1909) (Phthiracarus)

DisTRIBUCIÓN: Neártica (menos frecuente en el Sur).

-Euphthiracarus (E.) fusticulus Niedbala y Schatz, 1996

DisTRIBUCión: Centroamérica (I. Cocos).

-Euphthiracarus (E.) fusulus Niedbala, 200

DisTRIBUCIÓN: U.S.A. (excepto Oeste)

-Euphthiracarus (E.) humeralis Norton y Metz, 1977

DisTRIBUCIÓN: Sureste de U.S.A.

-Euphthiracarus (E.) incredibilis Mahunka, 1999

DisTRIBUCIÓN: Madagascar.

-Euphthiracarus (E.) inglisi Sheals, 1965

DisTRIBUCIÓN: Nepal.

-Euphthiracarus (E.) karpellesi (Oudemans, 1916) (Hummelia)

"sp. inq."

DisTRIBUCIÓN: Hungría.

-Euphthiracarus (E.) klabati Niedbala, 2000

DistRiBución: Borneo e I. Célebes [Sulawesi].

-Euphthiracarus (E.) labyrinthicus Stary, 1993

DisTRIBUCIÓN: Vietnam.

-Euphthiracarus (E.) longirostralis Walker, 1965

DistriBUCión: Neártica (U.S.A.: California, y Canadá).

-Euphthiracarus (E.) meghalayensis Sanyal, 1988

DistRiBución: India (Himalaya).

-Euphthiracarus (E.) monodactylus (Willmann, 1919) (Tritia (Pseudotritia))

(=Tritia aequipunctata Berlese, 1923)

(=Euphthiracarus mixtus Mahunka, 1979)

DisTRIBUCIÓN: Holártica (Paleártica occidental: menos frecuente

en el Norte, y Neártica: excepto Sur) y Nueva Guinea.

-Euphthiracarus (E.) monyx Walker, 1965

DisTRIBUCIÓN: U.S.A.

-Euphthiracarus (E.) nasutus Niedbala, 1988

DisTRIBUCIÓN: Hawai.

-Euphthiracarus (E.) pakistanensis Hammer, 1977

DisTRIBUCIÓN: Pakistán.

-Euphthiracarus (E.) parafusulus Niedbala, 2002

DisTRIBUCIÓN: U.S.A. nororiental.

-Euphthiracarus (E.) parareticulatus Niedbala, 2000

DisTRIBUCIÓN: Vietnam.

-Euphthiracarus (E.) pulchrum Jacot, 1930 (E. flavum p.)

(=Euphthiracarus pulchrum pisgahi Jacot, 1939)

DisTRIBUCIÓN: Neártica centrooriental (frecuente).

-Euphthiracarus (E.) punctulatus Jacot, 1930

("non" Phthiracarus punctulatus Berlese, 1913, =Euphthiracarus cribrarius)

DisTRIBUCIÓN: Neártica oriental.

-Euphthiracarus (E.) reticulatus (Berlese, 1913) (Phthiracarus)

DisTRIBUCIÓN: Paleártica (excepto Norte)

-Euphthiracarus (E.) reticulatus reticulatus (Berlese, 1913)

(Phthiracarus)

(=Pseudotritia intermedia Feider y Suciu, 1958)

DisTRIBUCIÓN: Paleártica meridional.

-Euphthiracarus (E.) reticulatus alpinus Märkel, 1964

DisTRIBUCIÓN: Paleártica (Europa centromeridional y Este de Rusia asiática).

-Euphthiracarus (E.) shogranensis Hammer, 1967

DisTRIBUCIÓN: Pakistán.

-Euphthiracarus (E.) spinus Niedbala, 2002

DisTRIBUCIÓN: U.S.A. nororiental.

-Euphthiracarus (E.) takahashii Aoki, 1980

DisTRIBUCIÓN: Japón.

-Euphthiracarus (E.) tanythrix Walker, 1965

(=Euphthiracarus tanythrix sierriensis Walker, 1965)

DistRIBUCIÓN: U.S.A. (California y Ténesi) 
-Euphthiracarus (E.) vicinus Niedbala, 2002 DisTRIBUCIÓN: U.S.A. (Dakota del Sur).

-Euphthiracarus (E.) vietnamicus Stary, 1993 DISTRIBUCIÓN: Vietnam.

-Euphthriacarus (E.) virgatus Niedbala, 2002 Distribución: U.S.A. (Misisipí).

Euphthiracarus (Brasiliotritia) Märkel, 1964 (3 spp. y 1 ssp.) ESPECIE TIPO: Euphthiracarus (Brasiliotritia) brasiliensis Märkel, 1964 DISTRIBUCIÓN: Neotropical.

-Euphthiracarus (Brasiliotritia) brasiliensis Märkel, 1964 DisTRIBUCIÓN: Brasil.

-Euphthiracarus (Brasiliotritia) dlouhyorum Mahunka, 1984 DisTRIBUCión: Neotropical (Paraguay y Brasil). -Euphthiracarus (Brasiliotritia) dlouhyorum dlouhyorum Mahunka, 1984

DisTRIBUCIÓN: Paraguay.

-Euphthiracarus (Brasiliotritia) dlouhyorum sanctipauli (PérezÍnigo y Baggio, 1993) (B.) DisTRIBUCIÓN: Brasil.

-Euphthiracarus (Brasiliotritia) similis (Sellnick, 1925) (Pseudotritia) DistriBuCIÓn: Neotropical (Brasil y Panamá).

Euphthiracarus (Pocsia) Mahunka, 1983 (11 spp.)

ESPECIE TIPO: Pocsia africana Mahunka, 1983

DISTRIBUCIÓN: Tropical (Etiópica y Neotropical).

-Euphthiracarus (Pocsia) africanus (Mahunka, 1983) (P.) Distribución: Tanzania.

-Euphthiracarus (Pocsia) bicarinatus (Stary, 1991) (P.) DistriBución: Kenia.

-Euphthiracarus (Pocsia) disparilis Niedbala, 1998

DISTRIBUCIÓN: África centrooriental.

-Euphthiracarus (Pocsia) dubius (Niedbala y Schatz, 1996) (P.) DisTRIBUCIÓN: Guatemala.

-Euphthiracarus (Pocsia) heterotrichus (Mahunka, 1988) (P.) DISTRIBUCIÓN: África centrooriental.

-Euphthiracarus (Pocsia) inopinatus (Niedbala, 1993) (P.) DisTRIBUCIÓn: Congo.

-Euphthiracarus (Pocsia) kunsti (Stary, 1988) (P.) DisTRIBUCIÓN: Tanzania.

-Euphthiracarus (Pocsia) microseta (Stary, 1993) (P.) DisTRIBUCIÓN: Cuba.

-Euphthiracarus (Pocsia) sarawaki Niedbala, 2000 DISTRIBUCIÓN: Borneo.

-Euphthiracarus (Pocsia) secundus (Mahunka, 1983) (P.) DisTRIBUCIÓN: Tanzania.

-Euphthiracarus (Pocsia) trentus (Mahunka, 1983) (P.) DISTRIBUCIÓN: Tanzania

Microtritia Märkel, 1964 (14 spp. y 1 ssp.)

EsPECIE TIPO: Phthiracarus minimus Berlese, 1904

DISTRIBUCIÓN: Cosmopolita (excepto Antártica).

-Microtritia contraria Niedbala, 1993 DISTRIBUCIÓN: Australiana.

-Microtritia fissurata Märkel, 1968 Distribución: Oeste de Siberia.

-Microtritia fusa Niedbala, 2000

DisTRIBUCIÓN: Australiana (Australia y Nueva Zelanda).

- Microtritia glabrata Niedbala, 1993

DisTRIBUCIÓN: Australiana.

-Microtritia hauseri Mahunka, 1993

(=Microtritia altissima Mahunka, 1994)

DisTRIBUCIÓN: Malgache.

-Microtritia hawaiiensis Niedbala, 1994

DisTRIBUCIÓN: Hawai.

-Microtritia incisa Märkel, 1964

DISTRIBUCIÓN: Neotropical.
-Microtritia minima (Berlese, 1904) (Phthiracarus)

(=Tritia (Pseudotritia) minuta Willmann, 1919)

(=Microtritia xilofila Subías, 1975)

DisTRIBUCIÓN: Semicosmopolita (Paleártica: frecuente, Neártica oriental, Oriental: Vietnam, Australiana: Hawai, y Neotropical: Centroamérica).

-Microtritia neonominata nom. nov.

[=Microtritia glabrata Stary, 1993, "nom. praeoc." por Niedbala, 1993 ("hom. prim.")]

DisTRIBUCIÓN: Cuba.

-Microtritia schusteri Märkel, 1964

DisTRIBUCIÓN: Neotropical (Brasil y Venezuela).

-Microtritia simplex (Jacot, 1930) (Pseudotritia)

(=Rhysotritia paeneminima Walker, 1965)

DisTRIBUCIÓN: Neártica (frecuente) y Centroamérica.

-Microtritia striatissima Mahunka, 1999

DISTRIBUCIÓN: Madagascar.

-Microtritia tropica Märkel, 1964

DisTRIBUCIÓN: Pantropical.

-Microtritia tropica tropica Märkel, 1964

(=Microtritia ethiopica Mahunka, 1988)

DisTRIBUCIÓN: Pantropical.

-Microtritia tropica dusan Ramsay y Sheals, 1969

DISTRIBUCIÓN: Borneo.

-Microtritia tumida Niedbala, 1988

DisTRIBUCIÓN: Australiana (Islas del Pacífico).

Sumatrotritia Mahunka, 1989 (3 spp.)

EsPECIE TIPO: Sumatrotritia inusitata Mahunka, 1989

DisTRIBUCIÓN: Oriental.

-Sumatrotritia elegans Mahunka, 1991

DistriBución: Península Malaya.

-Sumatrotritia inusitata Mahunka, 1989

DISTRIBUCIÓN: Sumatra.

-Sumatrotritia murphyi Mahunka, 1999

DisTRIBUCión: Península Malaya.

Synichotritiidae Walker, 1965 (4 gen. y 13 spp.)

DISTRIBUCIÓN: Oriental y subtropical (Neártica meridional).

Apotritia Norton y Lions, 1992 (1 sp.)

ESPECIE TIPO: Apotritia walkeri Norton y Lions, 1992

DISTRIBUCIÓN: Neártica meridional.

-Apotritia walkeri Norton y Lions, 1992

DisTRIBUCIÓN: Oeste de U.S.A.

Sabahtritia Mahunka, 1987 (7 spp.)

ESPECIE TIPO: Sabahtritia hauseri Mahunka, 1987

DisTRIBUCIÓN: Oriental.

-Sabahtritia foveolata (Hu, X. Wang y Aoki, 1991) (Synichotritia)

DisTRIBUCIÓN: China suroriental.

-Sabahtritia furcata (Hu y X. Wang, 1992) (Synichotritia)

DisTRIBUCIÓN: China suroriental.

-Sabahtritia hauseri Mahunka, 1987

DisTRIBUCIÓN: Borneo.

Sabahtritia lienhardi Mahunka, 1995

DISTRIBUCIÓN: Borneo.

-Sabahtritia mirabilis Mahunka, 1991

DisTRIBUCIÓN: Península Malaya.

-Sabahtritia sarawak Mahunka, 1996

DisTRIBUCIÓN: Borneo.

-Sabahtritia tianmuensis (Hu, X. Wang y Aoki, 1991) (Synichotritia)

DISTRIBUCIÓN: China suroriental.

Synichotritia Walker, 1965 (4 spp.)

EsPECIE TIPO: Synichotritia caroli Walker, 1965

DISTRIBUCIÓN: Neártica meridional.

Synichotritia caroli Walker, 1965

DisTRIBUCIÓN: U.S.A. (excepto Nororiental). 
-Synichotritia longipila Niedbala, 2002

DisTRIBUCIÓN: U.S.A. centromeridional.

-Synichotritia parvipilosa Niedbala, 2002

DisTRIBUCIÓN: U.S.A. (Misisipí).

-Synichotritia spinulosa Walker, 1965

DistriBUCIÓN: Neártica occidental y Misisipí.

Temburongia Mahunka, 1990 (1 sp.)

EsPeCIE TIPO: Temburongia patoi Mahunka, 1990

DisTRIBUCIÓN: Oriental.

-Temburongia patoi Mahunka, 1990

DISTRIBUCIÓN: Borneo.

\section{Phthiracaroidea Perty, 184}

Phthiracaridae Perty, 1841 (7 gen., 12 subg., 561 spp. y 5 sspp.) DISTRIBUCIÓN: Cosmopolita.

Atropacarus Ewing, 1917 (32 spp.)

(=Helvetacarus Mahunka, 1993)

ESPECIE TIPO: Hoplophora stricula Koch, 1835

Distribución: Cosmopolita (excepto Etiópica y Antártica).

-Atropacarus absimilis (Niedbala, 1982) (Steganacarus (A.))

DisTRIBUCIÓN: Sureste de U.S.A.

-Atropacarus bichei Niedbala, 1986

DistriBución: Mediterránea occidental.

-Atropacarus ciliosus Niedbala, 2002

DisTRIBUCIÓN: U.S.A. suroccidental.

-Atropacarus clavatus Aoki, 1980 (A. sticulus c.)

DisTRIBUCión: Japón y Méjico.

-Atropacarus clavigerus (Berlese, 1904) (Hoploderma)

(=Phthiracarus lutulentus Sellnick, 1920)

DisTRIBUCión: Mediterránea.

-Atropacarus controversus Niedbala, 2000

DisTRIBUCIÓN: Nueva Zelanda.

-Atropacarus csiszarae (Balogh y Mahunka, 1979) (Steganacarus)

DisTRIBUCIÓN: Europa centrooriental.

-Atropacarus decipiens (Niedbala, 1984) (Steganacarus (A.))

DISTRIBUCIÓN: Mediterránea oriental.

-Atropacarus echinodiscus (Mahunka, 1982) (Steganacarus)

DisTRIBUCIÓN: Grecia.

-Atropacarus genavensis (Mahunka, 1993) (Helvetacarus)

DisTRIBUCIÓN: Suiza.

-Atropacarus griseus (Niedbala, 1984) (Steganacarus (A.))

DISTRIBUCIÓN: Australiana.

-Atropacarus immundus (Niedbala, 1983) (Steganacarus (A.))

DisTRIBUCIÓN: Cáucaso.

-Atropacarus inconditus Mahunka, 1991

DisTRIBUCión: Hungría.

-Atropacarus inculpatus (Niedbala, 1984) (Steganacarus (A.))

DISTRIBUCIÓN: Ibérica.

-Atropacarus macrosculpturatus (Mahunka y Mahunka-Papp, 1999)

(Steganacarus)

DisTRIBUCión: Hungría.

-Atropacarus maculosus (Niedbala, 1983) (Steganacarus (A.))

Distribución: Cáucaso.

-Atropacarus mirabilis (Mahunka, 1979) (Steganacarus)

DISTRIBUCIÓN: Mediterránea.

-Atropacarus multisetosus Niedbala, 2002

Distribución: U.S.A. (Nuevo Méjico).

-Atropacarus murciensis Niedbala, 1986

Distribución: España.

-Atropacarus obesus (Niedbala, 1983) (Steganacarus (A.))

DistriBución: Cáucaso.

-Atropacarus ochraceus (Niedbala, 1983) (Steganacarus (A.))

DisTRIBUCIÓN: Mediterránea oriental.

-Atropacarus paraclavatus Niedbala, 2002

DistriBución: I. Bermudas.

-Atropacarus parvulus (Niedbala, 1983) (Steganacarus (A.))

DisTRIBUCIÓN: Cáucaso.
-Atropacarus perversus (Niedbala, 1983) (Steganacarus (A.))

DisTRIBUCIÓN: Cáucaso.

-Atropacarus phyllophorus (Berlese, 1904) (Hoploderma)

(=Steganacarus ropalus Feider y Suciu, 1957)

DISTRIBUCIÓN: Europa centromeridional y Vietnam.

-Atropacarus platakisi (Mahunka, 1979) (Steganacarus)

(=Atropacarus papillatus Gil-Martín, Subías y Arillo, 1992)

DisTRIBUCIÓN: Mediterránea.

-Atropacarus punctulatus Moraza, 1985

DisTRIBUCIÓN: España.

-Atropacarus serratus (Feider y Suciu, 1957) (Steganacarus)

Distribución: Mediterránea.

-Atropacarus striculus (Koch, 1835) (Hoplophora)

DISTRIBUCIÓN: Semicosmopolita (Holártica, Oriental y Australiana: Hawai).

-Atropacarus striculus striculus (Koch, 1835)

(=Steganacarus diaphanus Jacot, 1930)

(=Steganacarus senex Aoki, 1958)

(=Steganacarus trichosus Mahunka, 1982)

DistRIBUCIÓN: Semicosmopolita (Holártica: frecuente, Oriental, Méjico y Australiana: Hawai).

-Atropacarus striculus insularis (Weigmann, 1976) (Steganacarus) DISTRIBUCIÓN: I. Azores.

-Atropacarus substrictus (Niedbala, 1983) (Steganacarus (A.))

DisTRIBUCión: Cáucaso.

-Atropacarus terrapene (Jacot, 1937) (Steganacarus)

DisTRIBUCión: Este de U.S.A.

-Atropacarus wandae (Niedbala, 1981) (Steganacarus)

(=Steganacarus navarrensis Moraza, 1985)

DisTRIBUCIÓN: Europa occidental.

Hoplophorella Berlese, 1923 (68 spp. y 1 ssp.)

EsPECIE TIPO: Hoploderma cucullatum Ewing, 1909

DisTRIBUCIÓN: Pantropical y subtropical

Hoplophorella (Hoplophorella) Berlese, 1923 (55 spp. y 1 ssp.)

DISTRIBUCIÓN: Pantropical y subtropical.

-Hoplophorella (H.) angolensis Mahunka, 1984

DisTRIBUCIÓN: Angola.

-Hoplophorella (H.) berninii (Niedbala, 1991) (Protophthiracarus)

DisTRIBUCión: Centroamérica.

-Hoplophorella (H.) brasiliensis (Niedbala, 1988) (Austrophthiracarus)

DisTRIBUCIÓN: Neotropical (Centroamérica y Brasil).

-Hoplophorella (H.) brevipilis (Balogh, 1958) (Steganacarus)

DisTRIBUCIÓN: África centrooriental.

-Hoplophorella (H.) collaris (Balogh, 1958) (Steganacarus)

DisTRIBUCIÓN: Angola.

-Hoplophorella (H.) craterifer (Hammer, 1971) (Steganacarus)

Distribución: Melanesia (I. Fiji)

-Hoplophorella (H.) cucullata (Ewing, 1909) (Hoploderma)

DisTRIBUCIÓN: Pantropical y subtropical.

-Hoplophorella (H.) cucullata cucullata (Ewing, 1909)

(=Hoplophorella cucullata cuculloides Jacot, 1933)

(=Hoploderma licnophorum Berlese, 1913)

DISTRIBUCIÓN: Pantropical (frecuente) y subtropical (frecuente).

-Hoplophorella (H.) cucullata curassensis Willmann, 1936 DISTRIBUCIÓN: Pequeñas Antillas.

-Hoplophorella (H.) diaphoros (Niedbala, 2000) (Atropacarus (H.)) DISTRIBUCIÓN: Australia

-Hoplophorella (H.) digna Niedbala, 1983

DISTRIBUCIÓN: Africa central.

-Hoplophorella (H.) dissimilis (Niedbala, 1998) (Atropacarus (H.))

DisTRIBUCIÓN: Polinesia.

-Hoplophorella (H.) dubia (Niedbala, 1992) (Hoplophthiracarus)

DisTRIBUCIÓN: Neotropical (Brasil y Venezuela).

-Hoplophorella (H.) ensifera Mahunka, 1985

DisTRIBUCIÓN: Angola.

-Hoplophorella (H.) ephippigera (Balogh y Mahunka, 1977) (Steganacarus)

DisTRIBUCIÓN: Brasil. 
-Hoplophorella (H.) evergladensis (Niedbala, 2001) (Protophthiracarus)

DisTRIBUCIÓN: U.S.A. (Florida)

-Hoplophorella (H.) finitima (Niedbala, 2002) (Protophthiracarus) DisTRIBUCIÓN: Neotropical.

-Hoplophorella (H.) fonseciai (Pérez-Íñigo y Baggio, 1980) (Steganacarus)

DisTRIBUCIÓN: Neotropical (Brasil y Venezuela).

-Hoplophorella (H.) grandjeani Niedbala, 1981

DisTRIBUCIÓN: Neotropical (Brasil y Venezuela).

-Hoplophorella (H.) hamata (Ewing, 1909) (Hoploderma)

(=Hoplophorella cochlearia Pérez-Íñigo y Baggio, 1993)

(=Hoplophorella cucullata floridae Jacot, 1933)

(=Phthiracarus (Hoplophorella) cucullatus obsoletior Berlese, 1923)

(=Hoplophorella cuneiseta Mahunka, 1988)

(=Hoplophorella glauca Hammer, 1972)

(=Hoplophorella ligulifera Mahunka, 1987)

(=Hoplophorella schauenbergi Mahunka, 1978)

(=Hoplophorella spatulata Parry, 1980)

DisTRIBUCIÓN: Pantropical (frecuente) y subtropical.

-Hoplophorella (H.) heterotricha Mahunka, 1984

DisTRIBUCIÓN: Sudáfrica.

-Hoplophorella (H.) insolens Niedbala, 1988

DisTRIBUCIÓN: Congo.

-Hoplophorella (H.) kulczynskii Niedbala, 1981

DISTRIBUCIÓN: Brasil.

-Hoplophorella (H.) lanceoseta (Balogh y Mahunka, 1981) (Steganacarus)

(=Hoplophorella neglecta Niedbala, 1984)

DisTRIBUCIÓN: Neotropical.

-Hoplophorella (H.) lanceosetoides Mahunka, 1985

DisTRIBUCIÓN: Centroamérica.

-Hoplophorella (H.) lemuria Mahunka, 1993

DisTRIBUCIÓN: Madagascar.

-Hoplophorella (H.) manipurensis Misra, Bhaduri y Raychaudhuri, 1982 DisTRIBUCIÓN: India (Assam).

-Hoplophorella (H.) minisetosa Mahunka, 1978

DisTRIBUCIÓN: Etiópica oriental (Malgache y Malawi).

-Hoplophorella (H.) neonominata nom. nov.

[=Protophthiracarus cavernosus Niedbala, 2002, "nom. praeoc." por Mahunka, 1987 ("hom. sec.")]

DisTRIBUCIÓN: U.S.A. (Arizona).

-Hoplophorella (H.) nigeriensis (Badejo, Woas y Beck, 2001) (Atropacarus (H.))

DISTRIBUCIÓN: Nigeria.

-Hoplophorella (H.) oblonga Niedbala, 1983

DisTRIBUCIÓN: Kenia.

-Hoplophorella (H.) pallens Niedbala, 1983

Distribución: Pantropical (Etiópica, Australiana: Australia, y Neotropical).

-Hoplophorella (H.) paraensis (Pérez-Íñigo y Baggio, 1996) (Protophthiracarus)

DisTRIBUCIÓN: Brasil.

-Hoplophorella (H.) pergrata (Niedbala, 1998) (Atropacarus) DISTRIBUCIÓN: Polinesia.

-Hoplophorella (H.) perisi Subías y Sarkar, 1984

DisTRIBUCIÓN: India (Tripura).

-Hoplophorella (H.) permirus (Niedbala, 1991) (Protophthiracarus) DisTRIBUCIÓN: Centroamérica.

-Hoplophorella (H.) pervicax (Niedbala, 1984) (Hoplophthiracarus) DisTRIBUCIÓN: Jamaica.

-Hoplophorella (H.) praeoccupata nom. nov.

[=Atropacarus (Hoplophorella) tuberosus Niedbala y Schatz, 1996, "nom. praeoc." por Mahunka, 1988 ("hom. sec.")]

DistriBución: Guatemala.

-Hoplophorella (H.) prominens (Balogh, 1958) (Steganacarus)

DisTRIBUCIÓN: Etiópica (Congo y Angola).

-Hoplophorella (H.) pustulata Mahunka, 1993 DISTRIBUCIÓN: Ruanda.
-Hoplophorella (H.) rafalskii (Niedbala, 1997) (Notophthiracarus) DisTRIBUCIÓN: Madagascar.

-Hoplophorella (H.) rangiroaensis Hammer, 1972

DisTRIBUCión: Australiana (Islas del Pacífico).

-Hoplophorella (H.) remigera (Berlese, 1923) (Phthiracarus

(Trachyhoplophora))

DISTRIBUCIÓN: Somalia.

-Hoplophorella $(H$.$) repetita nom. nov.$

[=Protophthiracarus reductus Niedbala, 1991, "nom. praeoc." por Mahunka, 1986 ("hom. sec.")]

DistriBución: Centroamérica.

-Hoplophorella (H.) sabahna Mahunka, 1991

DISTRIBUCIÓN: Borneo.

-Hoplophorella (H.) sculptilis (Niedbala, 1988) (Hoplophthiracarus) (=Hoplophthiracarus magnus Mahunka, 1988)

DisTRIBUCIÓN: Malgache.

-Hoplophorella (H.) singularis Sellnick, 1959

(=Hoplophorella queenslandica Balogh y Mahunka, 1978)

(=Hoplophorella regalis Mahunka, 1978)

(=Hoplophorella sundarbanensis Sanyal y Bhaduri, 1982)

DISTRIBUCIÓN: Pantropical.

-Hoplophorella (H.) stilifera (Hammer, 1961) (Steganacarus)

(=Hoplophorella benoiti Mahunka, 1984)

DisTRIBUCIÓN: Pantropical.

-Hoplophorella (H.) subciliata Mahunka, 1983

DisTRIBUCIÓN: Tanzania.

-Hoplophorella (H.) subita Niedbala, 1983

DisTRIBUCIÓN: Kenia.

-Hoplophorella (H.) subsellata Balogh y Mahunka, 1977

(=Steganacarus galeatus Balogh y Mahunka, 1978)

DisTRIBUCIÓN: Brasil.

-Hoplophorella (H.) tripartita (Niedbala y Schatz, 1996) (Protophthiracarus)

DisTRIBUCIÓN: Belice.

-Hoplophorella (H.) unqus (Niedbala, 1991) (Notophthiracarus) DISTRIBUCIÓN: Borneo.

-Hoplophorella (H.) varia (Niedbala y Schatz, 1996) (Protophthiracarus)

DisTRIBUCIÓN: Centroamérica.

-Hoplophorella (H.) varians Jacot, 1933

DisTRIBUCIÓN: U.S.A. (Florida) y Antillas.

-Hoplophorella (H.) venusta Niedbala, 1983

DisTRIBUCIÓN: Uganda.

-Hoplophorella (H.) vitrina (Berlese, 1913) (Hoploderma)

(=Hoplophorella africana Wallwork, 1967)

(=Steganacarus andrei Balogh, 1958)

(=Hoplophorella lienhardi Mahunka, 1987)

(=Hoplophorella raychaudhurii Subías y Sarkar, 1984)

(=Hoplophorella scapellata Aoki, 1965)

DisTRIBUCIÓN: Pantropical (frecuente).

Hoplophorella (Kakophthiracarus) Mahunka, 1992 (7 spp.)

EsPECIE TIPO: (Hoplophorella tuberosa Mahunka, 1988) =Steganacarus tuberculosissimus Mahunka, 1978

DISTRIBUCIÓN: Paleotropical.

-Hoplophorella (Kakophthiracarus) balazsi Mahunka, 1983 (H.) DisTRIBUCIÓN: Madagascar.

-Hoplophorella (Kakophthiracarus) cavernosa Mahunka, 1987 (H.) DisTRIBUCIÓN: Tanzania.

-Hoplophorella (Kakophthiracarus) enigmatica (Mahunka, 1996) (K.) DISTRIBUCIÓN: Borneo.

-Hoplophorella (Kakophthiracarus) multirugosa (Mahunka, 1978)

(Steganacarus)

(=Steganacarus cornutus Mahunka, 1978)

DisTRIBUCIÓN: África centrooriental.

-Hoplophorella (Kakophthiracarus) multituberculata (Balogh y

Mahunka, 1966) (Steganacarus)

DistRIBUCIÓN: Sudáfrica.

-Hoplophorella (Kakophthiracarus) mwali Mahunka, 1994 (K.)

DisTRIBUCIÓN: Malgache (I. Comores). 
-Hoplophorella (Kakophthiracarus) tuberculosissima (Mahunka, 1978) (Steganacarus)

(=Hoplophorella horrida Mahunka, 1984)

(=Hoplophorella meszarosi Mahunka, 1988)

(=Hoplophorella tuberosa Mahunka, 1988)

(=Hoplophorella verrucosa Mahunka, 1987)

DISTRIBUCIÓN: Etiópica.

Hoplophorella (Steganacarellus) Mahunka, 1986 (6 spp.)

EsPECIE TIPO: Steganacarellus reductus Mahunka, 1986

DisTRIBUCIÓN: Tropical (Etiópica y Australiana).

-Hoplophorella (Steganacarellus) heterosetosa (Niedbala, 1988)

(Notophthiracarus)

DisTRIBUCión: Melanesia (I. Salomón).

-Hoplophorella (Steganacarellus) heterotricha (Niedbala, 2000)

(Arphthicarus)

DistriBUCIÓN: Nueva Zelanda.

-Hoplophorella (Steganacarellus) paraparvula (Niedbala, 2000)

(Notophthiracarus)

DisTRIBUCión: Nueva Caledonia.

-Hoplophorella (Steganacarellus) parvula (Niedbala, 1998)

(Notophthiracarus)

DisTRIBUCión: Nueva Caledonia.

-Hoplophorella (Steganacarellus) perlucunda (Niedbala, 2000)

(Notophthiracarus)

DisTRIBUCIÓN: Nueva Zelanda.

-Hoplophorella (Steganacarellus) reducta (Mahunka, 1986) (S.) DISTRIBUCIÓN: Sudáfrica.

Hoplophthiracarus Jacot, 1933 (33 spp.)

EsPecie TIPO: Hoploderma histricinum Berlese, 1908

DisTRIBUCIÓn: Cosmopolita (excepto Antártica).

-Hoplophthiracarus angustatus Niedbala, 1984 DisTRIBUCIÓN: Nepal.

-Hoplophthiracarus bengalensis Sanyal, 1992

DisTRIBUCIÓN: India (Bengala Occidental)

-Hoplophthiracarus bisulcus Niedbala, 1993

DisTRIBUCión: Nueva Zelanda.

-Hoplophthiracarus cazanicus Feider y Calugar, 1970

DISTRIBUCIÓN: Rumania.

-Hoplophthiracarus clavellatus Niedbala y Corpuz-Raros, 1998 DISTRIBUCIÓN: Filipinas.

-Hoplophthiracarus concinuus Niedbala, 1982

DisTRIBUCión: Nepal y Noreste de India.

-Hoplophthiracarus cristatus Aoki, 1980 (Hoplophorella)

DisTRIBUCIÓN: Japón.

-Hoplophthiracarus foveolatus Aoki, 1980

DISTRIBUCIÓN: Japón.

-Hoplophthiracarus frater (Balogh, 1958) (Steganacarus)

DisTRIBUCIÓN: Etiópica (Angola y Congo).

-Hoplophthiracarus grossmani Jacot, 1933 "sp. inq."

DisTRIBUCIÓN: U.S.A. (Florida).

-Hoplophthiracarus hamatus (Hammer, 1973) (Phthiracarus)

DistriBución: Polinesia, Japón y Malgache (I. Reunión).

-Hoplophthiracarus histricinus (Berlese, 1908) (Hoploderma)

(=Phthiracarus (Trachyhoplophora) histricinus nitidior Berlese, 1923)

(=Hoplophthiracarus minus Krivolutsky, 1966)

(=Hoplophthiracarus robustior Jacot, 1933)

DisTRIBUCIÓN: Holártica (menos frecuente en el Norte).

-Hoplophthiracarus hulli Niedbala, 1987

DisTRIBUCIÓN: Australia.

-Hoplophthiracarus illinoisensis (Ewing, 1909) (Hoploderma)

(=Hoplophthiracarus paludis Jacot, 1938)

(=Hoplophthiracarus vanderhammeni Niedbala, 1991)

DisTRIBUCIÓN: Holártica (frecuente) y Norte de Neotropical.

-Hoplophthiracarus inoueae Aoki, 1994

DISTRIBUCIÓN: Japón.

-Hoplophthiracarus ishikawai Aoki, 1980

DisTRIBUCIÓN: Este de Paleártica oriental.
-Hoplophthiracarus kugohi Aoki, 1959

(=Hoplophthiracarus africanus Mahunka, 1984)

(=Hoplophthiracarus minor Mahunka, 1991)

(=Hoplophthiracarus rimosus Mahunka, 1985)

(=Hoplophthiracarus siamensis Aoki, 1965)

(=Hoplophthiracarus wittmeri Bayoumi y Mahunka, 1979)

(=Hoplophthiracarus (Plonaphacarus) yoshii Mahunka, 1991)

DisTRIBUCIÓN: Pantropical (frecuente) y Paleártica meridional.

-Hoplophthiracarus marianus Aoki, 1994

DisTRIBUCIÓN: Micronesia (I. Marianas).

-Hoplophthiracarus maritimus (C. y C., jr. Pérez-Íñigo, 1996)

(Calyptophthiracarus)

DisTRIBUCIÓN: I. Azores.

-Hoplophthiracarus mirandus (Niedbala, 1988) (Calyptophthiracarus)

DISTRIBUCIÓN: Ecuador.

-Hoplophthiracarus mutabilis Niedbala y Schatz, 1996

DisTRIBUCIÓN: Guatemala.

-Hoplophthiracarus nasalis Mahunka, 1991

DistriBución: Península Malaya.

-Hoplophthiracarus nepalensis Sheals, 1965

DisTRIBUCIÓN: Nepal.

-Hoplophthiracarus pakistanensis Hammer, 1977

DisTRIBUCIÓN: Himalaya.

-Hoplophthiracarus parafoveolatus Niedbala, 2000

DisTRIBUCIÓN: Sumatra.

-Hoplophthiracarus proximus Niedbala, 1984

Distribución: Pantropical (Australiana, Oriental: Península

Malaya, y Neotropical: Venezuela).

-Hoplophthiracarus punctatus Mondal y Kundu, 1988

DisTRIBUCIÓN: India (Bengala Occidental)

-Hoplophthiracarus rafalskii Niedbala, 1997

DisTRIBUCión: Nueva Caledonia.

-Hoplophthiracarus repetitus nom. nov.

[=Hoplophthiracarus indicus Sanyal, 1990, "nom. praeoc." por Bayoumi y Mahunka, 1979 (“hom. prim.")]

DisTRIBUCIÓN: India (Kerala)

-Hoplophthiracarus similis Niedbala, 2000

DISTRIBUCIÓN: India (Cachemira).

-Hoplophthiracarus stigmosus Niedbala, 2000

DisTRIBUCIÓN: Oriental (Vietnam y Borneo).

-Hoplophthiracarus tenuiseta (J. y P. Balogh, 1986) (Steganacarus)

DisTRIBUCIÓN: Nueva Guinea.

-Hoplophthiracarus tropicus Mondal y Kundu, 1988

DisTRIBUCIÓN: India (Bengala Occidental).

Notophthiracarus Ramsay, 1966 (145 spp. y 3 sspp.)

EsPECIE TIPO: Phthiracarus maculatus Trägardh, 1931

DISTRIBUCIÓN: Cosmopolita.

Notophthiracarus (Notophthiracarus) Ramsay, 1966 (115 spp.)

(=Arphthicarus Niedbala, 1994 "sin. nov.")

DisTRIBUCIÓN: Pantropical y subtropical.

-Notophthiracarus (N.) abstemius Niedbala y Colloff, 1997

DISTRIBUCIÓN: Tasmania.

-Notophthiracarus (N.) aculeatus Niedbala, 1988

DisTRIBUCIÓN: Neotropical (Ecuador y Panamá).

-Notophthiracarus (N.) alienus Niedbala, 1989

DistRiBUCión: Australiana (Australia y Tasmania).

-Notophthiracarus (N.) alpinus Balogh y Mahunka, 1997

DistriBución: Nueva Guinea.

-Notophthiracarus (N.) aokii (Mahunka, 1983) (Hoplophthiracarus)

DisTRIBUCIÓN: África oriental.

-Notophthiracarus (N.) aquilus Niedbala, 2000

DisTRIBUCIÓN: Nueva Zelanda.

-Notophthiracarus (N.) araios (Niedbala, 2001) (Protophthiracarus)

DisTRIBUCIÓN: Madagascar.

-Notophthiracarus (N.) ater Niedbala, 2000

DisTRIBUCIÓN: Nueva Zelanda. 
-Notophthiracarus (N.) australis Ramsay, 1966

(=Notophthiracarus caliginosus Niedbala, 1989)

DisTRIBUCión: Nueva Zelanda.

-Notophthiracarus (N.) austroafricanus (Mahunka, 1984) (Hoplophorella)

DisTRIBUCIÓN: Sudáfrica.

-Notophthiracarus (N.) bentoni Niedbala, 1998

DisTRIBUCIÓN: Polinesia (I. Pitcairn).

-Notophthiracarus (N.) calugari Niedbala, 1987

DISTRIBUCIÓN: Australia.

-Notophthiracarus (N.) capillatus Niedbala, 1989

DisTRIBUCIÓN: Australia.

-Notophthiracarus (N.) caudatus (Balogh y Mahunka, 1977) (Phthi-

racarus)

DISTRIBUCIÓN: Neotropical (Centroamérica y Bolivia).

-Notophthiracarus (N.) claviger Niedbala, 1993

DisTRIBUCIÓN: Nueva Zelanda.

-Notophthiracarus (N.) comparativus Niedbala y Colloff, 1997

DISTRIBUCIÓN: Tasmania.

-Notophthiracarus (N.) consimilis Niedbala y Colloff, 1997

DisTRIBUCIÓN: Australiana (Australia y Tasmania).

-Notophthiracarus (N.) conspicuus Niedbala, 1989

DisTRIBUCIÓN: Nueva Zelanda.

- Notophthiracarus (N.) contortulus Niedbala, 1992

DISTRIBUCIÓN: Sudáfrica.

-Notophthiracarus (N.) curiosus Niedbala, 1998

DisTRIBUCión: Polinesia (Tahití).

-Notophthiracarus (N.) dactyloscopicus Mahunka, 1978

DisTRIBUCión: Malgache (I. Mauricio).

-Notophthiracarus (N.) distinctus Niedbala, 1989

DisTRIBUCIÓN: Australia.

-Notophthiracarus (N.) echinus (Balogh, 1964) (Hoplophorella)

DiSTRIBUCIÓN: Madagascar.

-Notophthiracarus (N.) endroedryyoungai (Mahunka, 1984) (Hoplophorella)

DisTRIBUCIÓN: Sudáfrica.

-Notophthiracarus (N.) evexus (Niedbala, 2000) (Arphthicarus)

DISTRIBUCIÓN: Vietnam.

-Notophthiracarus (N.) fatidicus (Niedbala, 1982) (Hoplophthiracarus)

DisTRIBUCión: Australiana (Nueva Guinea y Australia).

-Notophthiracarus (N.) fecundus Niedbala, 2000

DisTRIBUCión: Nueva Zelanda.

-Notophthiracarus (N.) feideri (Balogh y Ciszár, 1963) (Phthiracarus)

DISTRIBUCIÓN: Argentina.

-Notophthiracarus (N.) flagrus Niedbala, 2000

DisTRIBUCIÓN: Australia.

-Notophthiracarus (N.) flexiloquus Niedbala, 1989

DisTRIBUCIÓN: Tasmania.

-Notophthiracarus (N.) fornicarius (Niedbala, 1982) (Phthiracarus)

DisTRIBUCIÓN: Neotropical (Perú y Venezuela).

-Notophthiracarus (N.) fulvus (Niedbala, 1985) (Hoplophthiracarus)

DisTRIBUCIÓN: Nueva Guinea.

-Notophthiracarus (N.) furcatus (Niedbala y Corpuz-Raros, 1998)

(Arphthicarus)

DISTRIBUCIÓN: Filipinas.

-Notophthiracarus (N.) fusticulus (Niedbala, 2000) (Austrophthiracarus)

DisTRIBUCIÓN: Australia.

-Notophthiracarus (N.) gressitti Balogh y Mahunka, 1997

DisTRIBUCIÓN: Nueva Guinea.

-Notophthiracarus (N.) hamidi (Mahunka, 1987) (Hoplophthiracarus) DisTRIBUCIÓN: Kenia.

-Notophthiracarus (N.) hammeni Niedbala, 1987

DisTRIBUCión: Australia.

-Notophthiracarus (N.) hammerae Niedbala, 1987

DisTRIBUCIÓN: Australia.

-Notophthiracarus (N.) ignobilis Niedbala, 2000

DistriBución: Ceilán [Sri Lanka].

-Notophthiracarus (N.) improvisus (Niedbala, 1982) (Phthiracarus)

DisTRIBUCIÓN: Perú.
-Notophthiracarus (N.) inaequus (Niedbala, 1984) (Hoplophthiracarus)

DisTRIBUCIÓN: África oriental.

-Notophthiracarus (N.) inauditus (Niedbala, 1982) (Phthiracarus)

DisTRIBUCIÓN: Perú.

-Notophthiracarus (N.) incomparabilis Niedbala, 2000

DisTRIBUCión: Nueva Zelanda.

-Notophthiracarus (N.) indicus (Bayoumi y Mahunka, 1979)

(Hoplophthiracarus)

DISTRIBUCIÓN: Himalaya.

-Notophthiracarus (N.) indiligens (Niedbala, 1984) (Hoplophthiracarus)

DISTRIBUCIÓN: Malawi.

-Notophthiracarus (N.) indubiatus Niedbala y Colloff, 1997

DISTRIBUCIÓN: Tasmania.

-Notophthiracarus (N.) inelegans (Niedbala, 1986) (Hoplophthiracarus) (=Hoplophthiracarus atypicus Mahunka, 1988)

DisTRIBUCIÓN: Neotropical y Etiópica (Malgache).

-Notophthiracarus (N.) inenarrabilis (Niedbala, 1982) (Hoplophthiracarus)

DisTRIBUCIÓN: India (Cachemira)

-Notophthiracarus (N.) ineptus (Niedbala, 1984) (Hoplophthiracarus)

DisTRIBUCión: India (Cachemira) y Melanesia (I. Salomón).

-Notophthiracarus (N.) kamillae Niedbala, 1987

Distribución: Australia.

-Notophthiracarus (N.) latebrosus (Niedbala, 1982) (Hoplophthi-

racarus)

DISTRIBUCIÓN: Neotropical.

-Notophthiracarus (N.) latior (Niedbala, 1982) (Hoplophthiracarus)

Distribución: Australiana (Hawai y Nueva Caledonia), India (Himalaya) y Este de Rusia asiática.

-Notophthiracarus (N.) leei Niedbala, 1987

DisTRIBUCIÓN: Australia.

-Notophthiracarus (N.) lineolatus Mahunka, 1993

DistriBución: Tanzania.

-Notophthiracarus (N.) lionsi Niedbala, 1987

DisTRIBUCIÓN: Australia.

-Notophthiracarus (N.) longisetus Niedbala, 2000

DisTRIBUCIÓN: Australia.

-Notophthiracarus (N.) maculatus (Trägardh, 1931) (Phthiracarus)

DISTRIBUCIÓN: Chile.

-Notophthiracarus (N.) mahunkai Niedbala, 1987

DisTRIBUCIÓN: Australia.

-Notophthiracarus (N.) marginatus (Mahunka, 1984) (Hoplophthi-

racarus)

DistRiBución: Tanzania.

-Notophthiracarus (N.) maurus Niedbala, 2000

DisTRIBUCIÓN: Nueva Zelanda.

-Notophthiracarus (N.) mayottei (Niedbala, 2001) (Protophthiracarus)

DISTRIBUCIÓN: África oriental.

-Notophthiracarus (N.) michaeli (Niedbala, 1987) (Calyptophthiracarus)

DisTRIBUCIÓN: Australia.

-Notophthiracarus (N.) mirus Niedbala, 2000

DisTRIBUCIÓN: Ceilán [Sri Lanka].

-Notophthiracarus (N.) modicus Niedbala, 2000

DISTRIBUCIÓN: Australia.

-Notophthiracarus (N.) nicoleti (Niedbala, 1987) (Calyptophthiracarus) DisTRIBUCión: Australia.

-Notophthiracarus (N.) nigerrimus (Berlese, 1920) (Phthiracarus)

DISTRIBUCIÓN: Argentina.

-Notophthiracarus (N.) notatus (Niedbala, 2001) (Arphthicarus)

DisTRIBUCión: Gabón.

-Notophthiracarus (N.) obsessus nom. nov.

[=Notophthiracarus admirabilis Niedbala y Colloff, 1997, "nom. praeoc." por Niedbala, 1982 ("hom. sec.”)]

DISTRIBUCIÓN: Tasmania.

-Notophthiracarus (N.) paracuriosus Niedbala, 2000

Distribución: Polinesia (Tahití). 
-Notophthiracarus (N.) parareductus Mahunka, 1992 (=Hoplophorella reducta Mahunka, 1988, “nom. praeoc.” por Mahunka, 1986) DistRIBUCIÓN: Malgache (I. Reunión).

-Notophthiracarus (N.) parasentus (Niedbala, 2000) (Arphthicarus) DisTRIBUCIÓN: Vietnam.

-Notophthiracarus (N.) parasomalicus Niedbala, 2001 DisTRIBUCIÓN: Madagascar.

-Notophthiracarus (N.) parasummersi Niedbala, 2001 DisTRIBUCIÓN: Madagascar.

-Notophthiracarus (N.) paravariolosus Niedbala, 1994 DisTRIBUCión: Chile.

-Notophthiracarus (N.) pardinus (Berlese, 1916) (Hoploderma) DisTRIBUCIÓN: "África oriental".

-Notophthiracarus (N.) parilloi Niedbala, 2001

DisTRIBUCIÓN: Madagascar.

-Notophthiracarus (N.) pauliani (Balogh, 1964) (Hoplophorella) DISTRIBUCIÓN: Madagascar.

-Notophthiracarus (N.) perezinigoi Niedbala, 1987 DisTRIBUCión: Australia.

-Notophthiracarus (N.) perti (Niedbala, 1987) (Calyptophthiracarus) DisTRIBUCIÓN: Australia.

-Notophthiracarus (N.) procerus Niedbala, 2001

DISTRIBUCIÓN: Madagascar.

-Notophthiracarus (N.) quietus Niedbala, 1989

DisTRIBUCión: Australia.

-Notophthiracarus (N.) rafalskii (Niedbala, 1997) (Arphthicarus) DisTRIBUCIÓN: Méjico.

-Notophthiracarus (N.) ramsayi Niedbala, 1987

DisTRIBUCIÓN: Australia.

-Notophthiracarus (N.) remotus (Niedbala, 1989) (Arphthicarus) DISTRIBUCIÓN: Australia.

-Notophthiracarus (N.) repostus Niedbala, 1989

DisTRIBUCión: Australia.

-Notophthiracarus (N.) sacyae (Mahunka, 1983) (Steganacarus) DisTRIBUCIÓN: Tanzania.

-Notophthiracarus (N.) schatzi Niedbala, 1989

DisTRIBUCIÓN: I. Galápagos.

-Notophthiracarus (N.) schizocomus (Hammer, 1962) (Steganacarus) (=Notophthiracarus niger Mahunka, 1980)

DisTRIBUCIÓN: América austral.

-Notophthiracarus (N.) schusteri Niedbala, 1987

DisTRIBUCión: Australiana (Australia y Tasmania).

-Notophthiracarus (N.) sentus (Niedbala, 1989) (Austrophthiracarus) DisTRIBUCIÓN: Oriental (Vietnam y Sumatra).

-Notophthiracarus (N.) shealsi (Lee, 1981) (Hoplophthiracarus) DisTriBución: Australia.

-Notophthiracarus (N.) sicilicomus (Hammer, 1962) (Phthiracarus) DisTRIBUCIÓN: Chile.

-Notophthiracarus (N.) similis Niedbala, 2001

DisTRIBUCIÓN: Madagascar.

-Notophthiracarus (N.) sinuosus (Niedbala, 1982) (Phthiracarus) DisTRIBUCIÓN: Nueva Guinea.

-Notophthiracarus (N.) solitarius Niedbala y Colloff, 1997 DisTRIBUCIÓN: Tasmania.

-Notophthiracarus (N.) solomonensis Niedbala, 1998

DistRiBución: Melanesia (I. Salomón).

-Notophthiracarus (N.) somalicus (Berlese, 1923) (Phthiracarus

(Trachyhoplophora))

DisTRIBUCIÓN: Somalia.

-Notophthiracarus (N.) sordidus Niedbala y Colloff, 1997 DISTRIBUCIÓN: Tasmania.

-Notophthiracarus (N.) sororius (Niedbala, 1982) (Hoplophthiracarus) DisTRIBUCIÓN: Norte de Neotropical.

-Notophthiracarus (N.) spurcus Niedbala y Colloff, 1997 DisTriBución: Tasmania.

-Notophthiracarus (N.) stenotus Niedbala y Corpuz-Raros, 1998 DisTRIBUCIÓN: Filipinas.

-Notophthiracarus (N.) sumatranus (Niedbala, 2000) (Arphthicarus) DISTRIBUCIÓN: Sumatra.
-Notophthiracarus (N.) summersi Niedbala, 2001

DISTRIBUCIÓN: Madagascar.

-Notophthiracarus (N.) tinctus (Niedbala, 2000) (Arphthicarus)

DistRiBuCión: Nueva Caledonia.

-Notophthiracarus (N.) tohivea Niedbala, 1998

Distribución: Polinesia (I. Sociedad).

-Notophthiracarus (N.) trichosus (Mahunka, 1988) (Hoplophthiracarus)

DisTRIBUCIÓN: Malgache (I. Mauricio).

-Notophthiracarus (N.) tripartitus Niedbala, 1989

DisTRIBUCión: Nueva Zelanda.

-Notophthiracarus (N.) uncinatus Niedbala y Colloff, 1997

DisTRIBUCIÓN: Australiana (Australia y Tasmania).

-Notophthiracarus (N.) uncinulus Niedbala, 2000

DisTRIBUCión: Nueva Zelanda.

-Notophthiracarus (N.) unicarinatus Niedbala, 2000

DisTRIBUCIÓN: Nueva Zelanda.

-Notophthiracarus (N.) usitatus Niedbala, 1989

DisTRIBUCIÓN: Australiana (Australia y Tasmania).

-Notophthiracarus (N.) variolosus (Berlese, 1888) (Hoplophora)

DisTRIBUCIÓN: Brasil.

-Notophthiracarus (N.) veteratorius (Niedbala, 1988) (Hoplophthiracarus)

DisTRIBUCIÓN: Malgache (I. Comores).

-Notophthiracarus (N.) weigmanni Niedbala, 1987

DistriBuCión: Australia.

-Notophthiracarus (N.) zebra (Balogh, 1964) (Hoplophorella)

DISTRIBUCIÓN: Madagascar.

Notophthiracarus (Besuchetacarus) Mahunka, 1985 (5 spp.)

EsPeCIE TIPO: Besuchetacarus orientalis Mahunka, 1985

DisTRIBUCIÓN: Pantropical (excepto Australiana).

-Notophthiracarus (Besuchetacarus) bicarinatus Niedbala, 2001 (N.)

DisTRIBUCIÓN: Madagascar.

-Notophthiracarus (Besuchetacarus) hauseri Mahunka, 1995 (N.)

DisTRIBUCIÓN: Borneo.

-Notophthiracarus (Besuchetacarus) lienhardi Mahunka, 1996 (N.) DISTRIBUCIÓN: Borneo.

-Notophthiracarus (Besuchetacarus) orientalis (Mahunka, 1985) (B.)

DisTRIBUCIÓN: India (Kerala).

-Notophthiracarus (Besuchetacarus) tricarinatus (Niedbala, 1988)

(Calyptophthiracarus)

DisTRIBUCIÓN: Brasil.

Notophthiracarus (Calyptophthiracarus) Aoki, 1980 (15 spp. y 3 sspp.)

(=Hauserophthiracarus Mahunka, 1982)

(=Phrathicarus Niedbala, 1994 "sin. nov.")

EsPECIE TIPO: Calyptophthiracarus mitratus Aoki, 1980

DisTRIBUCIÓN: Cosmopolita (excepto Oriental y Antártica)

-Notophthiracarus (Calyptophthiracarus) canariensis (Pérez-Íñigo

y Peña, 1996) $(C$.

DistRiBUCIÓN: I. Canarias

-Notophthiracarus (Calyptophthiracarus) candidulus (Niedbala,

1983) (Hoplophthiracarus)

DistribuCión: Cáucaso.

-Notophthiracarus (Calyptophthiracarus) comatus Niedbala, 2000 (N.)

DisTRIBUCión: Nueva Zelanda.

-Notophthiracarus (Calyptophthiracarus) concolor (Sergienko, 2000) (C.)

DisTRIBUCIÓN: Ucrania.

-Notophthiracarus (Calyptophthiracarus) costai (Macfarlane y

Sheals, 1965) (Hoplophthiracarus)

DISTRIBUCIÓN: Israel.

-Notophthiracarus (Calyptophthiracarus) excellens (Niedbala,

1982) (Neophthiracarus)

DisTRIBUCIÓN: Perú.

-Notophthiracarus (Calyptophthiracarus) heterotrichus (Mahunka,

1979) (Hoplophthiracarus)

DistriBuCiÓN: Paleártica meridional (Mediterránea, Asia centrooccidental y Este de Rusia asiática). 
-Notophthiracarus (Calyptophthiracarus) inflatus (Niedbala, 1994) (Phrathicarus)

DisTRIBUCIÓN: Nueva Zelanda.

-Notophthiracarus (Calyptophthiracarus) inusitatus (Niedbala, 1983) (Hoplophthiracarus)

DisTRIBUCIÓN: Paleártica oriental (Este de Rusia asiática y Corea) -Notophthiracarus (Calyptophthiracarus) meridionalis (Sergienko, 1992) (Hoplophthiracarus)

DISTRIBUCIÓN: Ucrania.

-Notophthiracarus (Calyptophthiracarus) mitratus (Aoki, 1980) (C.)

DisTRIBUCIÓN: Japón.

-Notophthiracarus (Calyptophthiracarus) nitidus (Pérez-Íñigo y

Baggio, 1988) (C.)

DisTRIBUCIÓN: Brasil.

-Notophthiracarus (Calyptophthiracarus) oenipontanus (Mahunka,

1982) (Hauserophthiracarus)

DisTRIBUCIÓN: Grecia.

-Notophthiracarus (Calyptophthiracarus) olivaceus (Jacot, 1929)

(Phthiracarus)

(=Phthiracarus erinaceus Jacot, 1930)

DisTRIBUCIÓN: Neártica (frecuente).

-Notophthiracarus (Calyptophthiracarus) pavidus (Berlese, 1913)

(Hoploderma)

DISTRIBUCIÓN: Paleártica meridional

-Notophthiracarus (Calyptophthiracarus) pavidus pavidus (Berlese, 1913)

(=Hoplophthiracarus cretensis Mahunka, 1979)

DisTRIBUCIÓN: Paleártica meridional.

-Notophthiracarus (Calyptophthiracarus) pavidus inopinatus

(Mahunka, 1991) (C. cretensis $i$.)

DisTRIBUCión: Hungría.

-Notophthiracarus (Calyptophthiracarus) texanus (Niedbala, 2002) (Protophthiracarus)

DisTRIBUCIÓN: U.S.A. (Tejas).

-Notophthiracarus (Calyptophthiracarus) vicinus (Niedbala, 1984)

(Hoplophthiracarus)

DISTRIBUCIÓN: Mediterránea oriental.

Notophthiracarus (Phthirarica) Mahunka, 1982 (2 spp.)

EsPECIE TIPO: Phthirarica ridicula Mahunka, 1982

DISTRIBUCIÓN: Tropical (Neotropical y Australiana).

-Notophthiracarus (Phthirarica) riduculus (Mahunka, 1982) (P.)

DisTRIBUCIÓN: Centroamérica.

-Notophthiracarus (Phthirarica) traegardhi (Niedbala, 1987)

(Calyptophthiracarus)

DISTRIBUCIÓN: Australia.

Notophthiracarus (Protophthiracarus) Balogh, 1972 (8 spp.)

(=Notophthiracarus Balogh y Mahunka, 1967, "nom. praeoc." por Ramsay, 1966) EsPeCIE TIPO: Notophthiracarus chilensis Balogh y Mahunka, 1967

Distribución: Semicosmopolita (Oriental, Australiana, Neotropical y Antártica).

-Notophthiracarus (Protophthiracarus) admirabilis (Niedbala, 1982) (Neophthiracarus)

DisTRIBUCIÓN: Costa Rica.

-Notophthiracarus (Protophthiracarus) andinus (P. Balogh, 1984)

(Phthirarica)

DisTRIBUCIÓN: Colombia.

-Notophthiracarus (Protophthiracarus) brasiliensis (Pérez-Íñigo y Baggio, 1980) (P.)

DISTRIBUCIÓN: Brasil.

-Notophthiracarus (Protophthiracarus) chilensis Balogh y Mahunka, 1967 (N.)

DisTRIBUCIÓN: Chile.

-Notophthiracarus (Protophthiracarus) daimonios (Niedbala, 2000) (Austrophthiracarus)

DisTRIBUCión: Nueva Zelanda.

-Notophthiracarus (Protophthiracarus) neotrichus (Wallwork, 1966)

(Neophthiracarus)

DisTRIBUCIÓN: Antártica (I. Campbell).
-Notophthiracarus (Protophthiracarus) strigosus (Niedbala, 1984)

(Neophthiracarus)

DisTRIBUCIÓN: Argentina.

-Notophthiracarus (Protophthiracarus) ventosus (Hammer, 1961)

(Steganacarus)

DisTRIBUCIÓN: Neotropical (Perú y Argentina) y Oriental (India: Bengala Occidental).

Phthiracarus Perty, 1841 (181 spp.)

EsPeCIE TIPO: (Phthiracarus contractilis Perty, 1841) =Hoplophora laevigata Koch, 1841

DISTRIBUCIÓN: Cosmopolita.

Phthiracarus (Phthiracarus) Perty, 1841 (60 spp.)

(=Ginglymacarus Ewing, 1917)

(=Hoploderma Michael, 1898)

(=Hoplophora Koch, 1835, "nom. praeoc." por Germar, 1833)

(=Peridromotritia Jacot, 1923)

DISTRIBUCIÓN: Cosmopolita (excepto Antártica).

-Phthiracarus (P.) abstemius Niedbala, 1989

DisTRIBUCIÓN: Vietnam.

-Phthiracarus (P.) affinis (Hull, 1914) (Hoploderma)

DisTRIBUCIÓN: Europa occidental.

-Phthiracarus (P.) banksi Niedbala, 1987

DisTRIBUCIÓN: Australia.

-Phthiracarus (P.) benoiti Balogh, 1958 “sp. inq.”

DistriBución: Angola.

-Phthiracarus (P.) boresetosus Jacot, 1930

(=Phthiracarus dudichi Mahunka, 1982)

(=Phthiracarus kaszabi Mahunka, 1981)

(=Phthiracarus minimarginatus Woolley, 1954)

(=Phthiracarus tenuis Hammer, 1977)

DistriBUCIÓN: Holártica (frecuente en Neártica), Neotropical e India (Cachemira).

-Phthiracarus (P.) capitatus (Hall, 1911) (Hoploderma) "sp. inq."

DisTRIBUCIÓN: U.S.A. (Connecticut).

-Phthiracarus (P.) clavifer Mahunka, 1988

DisTRIBUCión: Malgache (I. Reunión).

-Phthiracarus (P.) claviger (Pearce, 1906) (Hoploderma) "sp. inq." DisTRIBUCión: India (Sikkim).

-Phthiracarus (P.) clemens Aoki, 1963

(=Phthiracarus clemens kyushuensis Aoki, 1980)

DistribuCión: Paleártica oriental (excepto Norte) y Norte de Oriental.

-Phthiracarus (P.) commutabilis Niedbala, 1983

DisTRIBUCIÓN: Asia centrooccidental.

-Phthiracarus (P.) compressus Jacot, 1930

(=Phthiracarus pallidus Feider y Suciu, 1958)

DisTRIBUCIÓN: Holártica.

-Phthiracarus (P.) crenophilus Willmann, 1951

Distribución: Europa central.

-Phthiracarus (P.) crinitus (Koch, 1841) (Hoplophora)

DisTRIBUCIÓN: Europa centromeridional.

-Phthiracarus (P.) danubianus Feider y Calugar, 1970

DisTRIBUCIÓN: Rumania.

-Phthiracarus (P.) dasypus (Dugès, 1834) (Oribata) "sp. inq."

DisTRIBUCIÓn: Francia.

-Phthiracarus (P.) dubinini Feider y Suciu, 1958

DisTRIBUCIÓN: Europa suroriental.

-Phthiracarus (P.) extraordinarius (Niedbala, 2000) (Notophthiracarus)

DisTRIBUCión: Ceilán [Sri Lanka].

-Phthiracarus (P.) ferrugineus (Koch, 1841) (Hoplophora)

(=Phthiracarus juvenalis Parry, 1979)

(=Phthiracarus paraligneus Iturrondobeitia y Saloña, 1989)

DisTRIBUCIÓN: Paleártica occidental.

-Phthiracarus (P.) furcatus (Kramer, 1898) (Hoplophora) "sp. inq."

Distribución: América austral (Tierra del Fuego).

-Phthiracarus (P.) heterotrichosus (Niedbala, 2000) (Hoplophthiracarus)

DisTRIBUCión: Península Malaya. 
-Phthiracarus (P.) incertus Niedbala, 1983

(=Phthiracarus conformis Sergienko, 1987)

DisTRIBUCIÓN: Holártica (Europa centrooriental y Canadá).

-Phthiracarus (P.) incredibilis Niedbala, 1983

DisTRIBUCIÓN: Paleártica meridional (Cáucaso e Irán).

-Phthiracarus (P.) inexpectatus Niedbala, 1983

Distribución: Asia centrooccidental.

-Phthiracarus (P.) insularis Jacot, 1934

DisTribución: Polinesia.

-Phthiracarus (P.) irreprehensus Niedbala, 1988

Distribución: Oeste de Neártica.

-Phthiracarus (P.) italicus (Oudemans, 1900) (Hoploderma)

DisTRIBUCIÓN: Holártica (Paleártica: frecuente, y U.S.A.: Michigan)

-Phthiracarus (P.) jacoti Feider y Suciu, 1958

DisTRIBUCIÓN: Europa suroriental.

-Phthiracarus (P.) japonicus Aoki, 1958

Distribución: Holártica (Paleártica oriental: excepto Norte, y

Neártica: frecuente) y Norte de Oriental.

-Phthiracarus (P.) koumantanosi Niedbala, 1983

DistriBución: Grecia.

-Phthiracarus (P.) laevigatus (Koch, 1841) (Hoplophora)

(=Phthiracarus contractilis Perty, 1841)

DistRIBUCIÓN: Paleártica (menos frecuente en el Norte).

-Phthiracarus (P.) lautus Niedbala, 1981

DisTRIBUCIÓN: Mediterránea oriental.

-Phthiracarus (P.) lentulus (Koch, 1841) (Hoplophora)

(=Phthiracarus angolensis Mahunka, 1985)

(=Phthiracarus rotundus Berlese, 1923, "nom. praeoc." por Ewing, 1908)

DisTRIBUCión: Holártica (Paleártica: menos frecuente en el Norte, y Neártica) y Angola.

-Phthiracarus (P.) longulus (Koch, 1841) (Hoplophora)

(=Phthiracarus apiculatus Jacot, 1939)

(=Phthiracarus flexisetosus Parry, 1979)

(=Phthiracarus hortobagyensis Mahunka, 1982)

(=Phthiracarus montium Jacot, 1937)

(=Phthiracarus mundus Niedbala, 1983)

(=Phthiracarus prior Jacot, 1933)

(=Phthiracarus setosellus Jacot, 1929)

(=Phthiracarus tardus Forssluns, 1943)

DisTRIBUCIÓN: Holártica.

-Phthiracarus (P.) luridus (Ewing, 1909) (Hoploderma)

DisTRIBUCIÓN: Neártica.

-Phthiracarus (P.) machadoi Balogh, 1958 "sp. inq."

DisTRIBUCIÓN: Angola.

-Phthiracarus (P.) malagensis Niedbala, 1986

Distribución: España.

-Phthiracarus (P.) membranifer Parry, 1979

DistriBución: Paleártica (Paleártica occidental y Corea) y Ceilán [Sri Lanka].

-Phthiracarus (P.) nitens (Nicolet, 1855) (Hoplophora)

(=Phthiracarus roubali Berlese, 1923)

Distribución: Paleártica (menos frecuente en el Norte), Perú e I. Santa Helena.

-Phthiracarus (P.) perlucidus Niedbala, 1994

DisTRIBUCIÓN: Hawai.

-Phthiracarus (P.) perparvus (Niedbala, 1989) (Notophthiracarus)

DisTRIBUCIÓN: Vietnam.

-Phthiracarus (P.) persimplex Mahunka, 1982

Distribución: Este de Paleártica oriental.

-Phthiracarus (P.) piger (Scopoli, 1763) (Acarus)

(=Hoplophora lucida Koch, 1841)

DisTRIBUCIÓN: Holártica (frecuente en Paleártica).

-Phthiracarus (P.) planus (Mahunka, 1985) (Besuchetacarus) DISTRIBUCIÓN: India meridional.

-Phthiracarus (P.) probus Niedbala y Colloff, 1977 DistribuCión: Tasmania.

-Phthiracarus (P.) propinquus Niedbala, 1983

DisTRIBUCIÓN: Paleártica (Mediterránea oriental y Asia centrooccidental).
-Phthiracarus (P.) pudicus Berlese, 1923

DistriBución: Sudáfrica.

-Phthiracarus (P.) pusillus Niedbala, 2001

DisTRIBUCIÓN: U.S.A. suroriental.

-Phthiracarus (P.) rapax (Berlese, 1916) (Hoploderma)

DistribuCIÓN: "África oriental".

-Phthiracarus (P.) reductus Niedbala, 1998

DistribuCIÓN: Hawai.

-Phthiracarus (P.) retalticus Stoll, 1893 "sp. inq."

DisTRIBUCión: U.S.A. (Connecticut) y Guatemala.

-Phthiracarus (P.) sarahae Jacot, 1930

(=Phthiracarus validus Niedbala, 1986)

DisTRIBUCIÓN: Neártica (frecuente)

-Phthiracarus (P.) serrulatus Parry, 1979

DisTRIBUCIÓN: Europa occidental.

-Phthiracarus (P.) setanus Jacot, 1939

Distribución: U.S.A. (excepto Noroeste) y Chile.

-Phthiracarus (P.) spadix Niedbala, 1983

DistRIBUCIÓN: Europa oriental.

-Phthiracarus (P.) stenotus Niedbala, 2002

Distribución: Norte de Neotropical.

-Phthiracarus (P.) swiftae Niedbala, 1998

DisTRIBUCIÓN: Hawai.

-Phthiracarus (P.) testudineus (Koch, 1841) (Hoplophora) "sp. inq." DisTRIBUCIÓN: Europa central.

-Phthiracarus (P.) tristis Mahunka y Paoletti, 1984

DisTRIBUCIÓN: Italia.

-Phthiracarus (P.) turgidus (Niedbala, 1991) (Notophthiracarus)

DistriBuCión: Ceilán [Sri Lanka].

-Phthiracarus (P.) zicmani Balogh y Mahunka, 1983

(=Phthiracarus feideri Balogh y Mahunka, 1979, "nom. praeoc." por Balogh y Csiszár, 1963)

DISTRIBUCIÓN: Europa centrooriental (Hungría y Rumania).

Phthiracarus (Archiphthiracarus) Balogh y Mahunka, 1979 (78 spp.)

(=Archiphthiracarella Mahunka, 1996 "sin. nov.")

(=Microphthiracarus Mahunka, 1982)

(=Paraphthiracarus Aoki, 1980)

ESPECIE TIPO: Archiphthiracarus variabilis Balogh y Mahunka, 1979

DisTRIBUCIÓN: Cosmopolita (excepto Antártica).

-Phthiracarus (Archiphthiracarus) aegypticus (Al-Assiuty, Bayoumi

y Abdel-Hamid, 1984) (A.)

DisTRIBUCIÓN: Egipto.

-Phthiracarus (Archiphthiracarus) aliquantus Niedbala, 1988 (P.)

DisTRIBUCIÓN: Neártica occidental (frecuente) y Kentucky.

-Phthiracarus (Archiphthiracarus) anonymus Grandjean, $1933(P$.

(=Phthiracarus anonymus amicus Jacot, 1938)

(=Archiphthiracarus hauseri Mahunka, 1988)

(=Archiphthiracarus pocsi Mahunka, 1983)

DisTRIBUCIÓn: Pantropical y subtropical.

-Phthiracarus (Archiphthiracarus) armatus (Mahunka, 1986)

(Hoplophorella)

DisTRIBUCIÓN: Tanzania.

-Phthiracarus (Archiphthiracarus) assimilis Niedbala, $1983(P$.

DisTRIBUCIÓN: Cáucaso.

-Phthiracarus (Archiphthiracarus) atlanticus (Pérez-Íñigo, 1987) (A.)

DisTRIBUCIÓN: I. Azores.

-Phthiracarus (Archiphthiracarus) atratus (Niedbala, 2000)

(Notophthiracarus)

DistRiBución: Nueva Zelanda.

-Phthiracarus (Archiphthiracarus) australis (Aoki, 1980)

(Paraphthiracarus)

DisTRIBUCIÓN: Japón.

-Phthiracarus (Archiphthiracarus) baloghi Feider y Suciu, 1957 (P.)

DistriBUCIÓN: Mediterránea oriental.

-Phthiracarus (Archiphthiracarus) borealis (Trägardh, 1910)

(Hoploderma)

DisTRIBUCIÓN: Holártica (menos frecuente en el Sur). 
-Phthiracarus (Archiphthiracarus) brevisetae Jacot, $1930(P$.

(=Phthiracarus restrictus Jacot, 1937)

DisTRIBUCIÓN: Neártica (frecuente) y Méjico.

-Phthiracarus (Archiphthiracarus) bryobius Jacot, 1930 (P. setose-

llum b.)

(=Phthiracarus crinitosimilis Willmann, 1939)

(=Archiphthiracarus gobiensis Mahunka, 1981)

(=Archiphthiracarus hungaricus Balogh y Mahunka, 1979)

(=Phthiracarus lanatus Feider y Suciu, 1957)

(=Archiphthiracarus pilosus Sergienko, 1987)

DisTRIBUCión: Holártica (Paleártica: menos frecuente en el Norte, y Neártica: excepto Sureste).

-Phthiracarus (Archiphthiracarus) bulbiferus (Mahunka, 1996)

(Archiphthiracarella)

(=Archiphthiracarella baloghorum Mahunka, 1997)

(=Phthiracarus insularis Balogh, 1964, "nom. praeoc." por Jacot, 1935)

(=Phthiracarus minor Niedbala, 2001)

DisTRIBUCIÓN: Madagascar.

-Phthiracarus (Archiphthiracarus) cadizi Niedbala, $1986(P$. DisTRIBUCIÓN: España.

-Phthiracarus (Archiphthiracarus) cavernosus (Wallwork, 1977)

(Hoplophthiracarus)

DisTRIBUCIÓN: I. Santa Helena.

-Phthiracarus (Archiphthiracarus) clavatus Parry, $1979(P$.

DisTRIBUCIÓN: Holártica (Paleártica occidental y Canadá).

-Phthiracarus (Archiphthiracarus) cognatus Niedbala, 1988 (P.)

(=Phthiracarus rafalskii Niedbala, 1997)

DisTRIBUCión: Neártica (excepto Sureste): frecuente.

-Phthiracarus (Archiphthiracarus) comatus Niedbala, 1983 (P.)

DisTRIBUCión: Este de Rusia asiática.

-Phthiracarus (Archiphthiracarus) crassus Niedbala, 1983 (P.)

DisTRIBUCIÓN: Mediterránea oriental.

-Phthiracarus (Archiphthiracarus) crispus Hammer, 1972 (P.)

DistriBuCión: Australiana (Islas del Pacífico) y Oriental (Vietnam).

-Phthiracarus (Archiphthiracarus) curiosus Niedbala, 1998 (P.)

DisTRIBUCIÓN: Hawai.

-Phthiracarus (Archiphthiracarus) curtulus Berlese, 1923 (P.)

DisTRIBUCIÓN: Sureste de U.S.A.

-Phthiracarus (Archiphthiracarus) dissonus Niedbala, 1983 (P.)

DisTRIBUCIÓN: Cáucaso.

-Phthiracarus (Archiphthiracarus) dominiaki Niedbala, 1984 (P.)

DisTRIBUCIÓN: Turquía.

-Phthiracarus (Archiphthiracarus) endroedrii (Mahunka, 1984) (A.) DistriBución: Sudáfrica.

-Phthiracarus (Archiphthiracarus) eupalineus Mahunka, 2001 DisTRIBUCIÓN: Grecia.

-Phthiracarus (Archiphthiracarus) falciformis Morell y Subías, 1991 DisTRiBución: I. Azores.

-Phthiracarus (Archiphthiracarus) flagellatus Wallwork, 1977 (P.) Distribución: I. Santa Helena.

-Phthiracarus (Archiphthiracarus) flexipilus Calugar y Vasiliu, 1981 (P.) DisTRIBUCIÓN: Rumania.

-Phthiracarus (Archiphthiracarus) fraternus Niedbala, 1998 (P.) DisTRIBUCIÓN: Hawai.

-Phthiracarus (Archiphthiracarus) furvus Niedbala, $1983(P$.

DisTRIBUCIÓN: Mediterránea oriental.

-Phthiracarus (Archiphthiracarus) gibber (Aoki, 1980) (Paraphthiracarus)

DisTRIBUCIÓN: Este de Paleártica oriental.

-Phthiracarus (Archiphthiracarus) globifer Hammer, 1962 (P.)

DisTRIBUCIÓN: Chile.

-Phthiracarus (Archiphthiracarus) globosus (Koch, 1841) (Hoplophora)

(=Phthiracarus globus Parry, 1979)

(=Phthiracarus rotundus Ewing, 1908)

(=Hoplophora sphaerula Banks, 1895)

(=Phthiracarus subglobosus Berlese, 1923)

DisTRIBUCIÓN: Holártica (frecuente).

-Phthiracarus (Archiphthiracarus) imbecillis (Mahunka, 1989) (A.)

DISTRIBUCIÓN: Sudáfrica.
-Phthiracarus (Archiphthiracarus) inacessus Niedbala, 1998 (P.)

DisTRIBUCIÓN: Hawai.

-Phthiracarus (Archiphthiracarus) inornatus Niedbala, 1984 (P.)

DisTRIBUCIÓN: Turquía.

-Phthiracarus (Archiphthiracarus) invenustus Niedbala, 2000 (P.)

DistriBución: Oriental.

-Phthiracarus (Archiphthiracarus) largus Niedbala, 1984 (P.)

Distribución: Paleártica oriental (Asia central rusa e I. Sajalín).

-Phthiracarus (Archiphthiracarus)ligneus Willmann, 1931 (P.)

(=Phthiracarus sellnicki Feider y Suciu, 1957)

DisTRIBUCIÓN: Holártica.

-Phthiracarus (Archiphthiracarus) liparus Niedbala, $1984(P$.

DisTRIBUCIÓn: Mediterránea (Turquía y Argelia).

-Phthiracarus (Archiphthiracarus) mediocris Niedbala, 1984 (P.)

DisTRIBUCIÓN: Asia central rusa.

-Phthiracarus (Archiphthiracarus) modestus Niedbala, 1988 (P.)

Distribución: Neártica (excepto Sureste).

-Phthiracarus (Archiphthiracarus) montanus (Pérez-Íñigo, 1969) (P.)

(=Phthiracarus murphyi Harding, 1976)

(=Phthiracarus rectisetosus Parry, 1979)

(=Archiphthiracarus tzanoudakisi Mahunka, 1979)

DisTRIBUCIÓN: Paleártica occidental.

-Phthiracarus (Archiphthiracarus) neonominatus nom. nov.

[=Microphthiracarus baloghi Mahunka, 1982, "nom. praeoc." por Feider y Suciu, 1957 ("hom. sec.")]

DISTRIBUCIÓN: Etiopía.

-Phthiracarus (Archiphthiracarus) nitidus Niedbala, 1986 (P.)

DisTRIBUCIÓN: Neártica (Canadá y U.S.A.: Utah).

-Phthiracarus (Archiphthiracarus) obscurus Niedbala, $1986(P$.

DisTRIBUCIÓN: Oriental y Australia.

-Phthiracarus (Archiphthiracarus) occultus Niedbala, 1981 (P.)

DisTRIBUCIÓN: Polonia.

-Phthiracarus (Archipthtiracarus) ochthus Niedbala, 2001 (P.)

DistriBuCIÓN: Etiópica.

-Phthiracarus (Archiphthiracarus) opacus Niedbala, $1986(P$.)

DisTRIBUCIÓN: Paleártica.

-Phthiracarus (Archiphthiracarus) ornatus Mahunka, 1991

DisTRIBUCIÓN: Borneo.

-Phthiracarus (Archiphthiracarus) papillosus Parry, 1979 (P.)

DisTRIBUCIÓN: Austria.

-Phthiracarus (Archiphthiracarus) parabaloghi Niedbala, 1983 (P.)

DisTRIBUCIÓN: Etiópica (Uganda y Camerún).

-Phthiracarus (Archiphthiracarus) parabotrichus Feider y Suciu, 1957 (P.)

DisTRIBUCIÓN: Europa centrooriental.

-Phthiracarus (Archiphthiracarus) paraglobosus Niedbala, 1982 (P.)

DisTRIBUCIÓN: India (Cachemira).

-Phthiracarus (Archiphthiracarus) parapocsi Niedbala, 2001 (P.)

DisTRIBUCIÓN: Madagascar.

-Phthiracarus (Archiphthiracarus) paratubulus Niedbala, 1991 (P.)

Distribución: Ceilán [Sri Lanka].

-Phthiracarus (Archiphthiracarus) parmatus (Nakatamari, 1985)

(Paraphthiracarus)

DisTRIBUCIÓN: Japón.

-Phthiracarus (Archiphthiracarus) paucus Niedbala, 1991 (P.)

Distribución: Australiana, I. de Pascua (I. Easter) y Vietnam.

-Phthiracarus (Archiphthiracarus) pellucidus Ramsay, 1966 (P.)

DisTRIBUCión: Nueva Zelanda.

-Phthiracarus (Archiphthiracarus) peristomaticus Willmann, 1951 (P.)

(=Archiphthiracarus hammeni Balogh y Mahunka, 1979)

(=Archiphthiracarus variabilis Balogh y Mahunka, 1979)

DisTRIBUCIÓN: Paleártica occidental (excepto Norte).

-Phthiracarus (Archiphthiracarus) persimilis Niedbala, 1998 (P.)

DisTRIBUCIÓN: Hawai.

-Phthiracarus (Archiphthiracarus) plenus Niedbala, 1998 (P.)

DisTRIBUCión: Hawai.

-Phthiracarus (Archiphthiracarus) puylaerti (Niedbala, 2001)

(Notophthiracarus)

DisTRIBUCIÓN: Congo. 
-Phthiracarus (Archiphthiracarus) pygmaeus Balogh, 1958 (P.)

(=Archiphthiracarus foveolatus Mahunka, 1988)

(=Archiphthiracarus minutissimus Balogh y Mahunka, 1980)

(=Phthiracarus passimpunctatus $\mathrm{P}$. Balogh, 1988)

(=Phthiracarus serrula Balogh y Mahunka, 1977)

DisTRIBUCIÓN: Pantropical y subtropical.

-Phthiracarus (Archiphthiracarus)robertsi Sheals, 1965 (P.)

DistriBución: Nepal e India (Bengala Occidental).

-Phthiracarus (Archiphthiracarus) sangumburiensis (Niedbala, 2002)

(Hoplophthiracarus)

DISTRIBUCIÓN: Corea.

-Phthiracarus (Archiphthiracarus) sanvicensis Subías y Gil-Martín,

$1990(P$.

Distribución: Portugal.

-Phthiracarus (Archiphthiracarus) schauenbergi (Mahunka, 1988) (A.)

DisTRIBUCIÓN: Malgache.

-Phthiracarus (Archiphthiracarus) scitus Niedbala, 1983 (P.)

DisTRIBUCión: Cáucaso.

-Phthiracarus (Archiphthiracarus) similis Niedbala, 1981 (P.)

DisTRIBUCIÓN: Asia suroccidental.

-Phthiracarus (Archiphthiracarus) stramineus (Koch, 1841) (Hoplophora)

DisTRIBUCIÓN: Boreal (Europa: excepto Sur, y Noreste de U.S.A.)

-Phthiracarus (Archiphthiracarus) subdolus Niedbala, 1983 (P.)

DisTRIBUCIÓN: Mediterránea oriental.

-Phthiracarus (Archiphthiracarus) subiasi Niedbala, 1986 (P.)

DistribuCión: España.

-Phthiracarus (Archiphthiracarus) sudamericanus (Pérez-Íñigo y

Baggio, 1993) (A.)

DisTRIBUCIÓN: Neotropical (Brasil y Argentina).

-Phthiracarus (Archiphthiracarus) torosus Willmann, $1939(P$.

Distribución: Macaronésica (Madeira).

-Phthiracarus (Archiphthiracarus) tubulus $(P$.

Distribución: Polinesia (Tahití).

-Phthiracarus (Archiphthiracarus) wallworki Niedbala, $1984(P$.

DisTRIBUCIÓN: I. Santa Helena.

Phthiracarus (Metaphthiracarus) Aoki, 1980 (2 spp.)

ESPECIE TIPO: (Metaphthiracarus bacillatus Aoki, 1980) =Hoplophora setosa Banks, 1895

DisTRIBUCIÓN: Holártica.

-Phthiracarus (Metaphthiracarus) jumbongiensis (Niedbala, 2002)

(Austrophthiracarus)

DisTRIBUCión: Corea.

-Phthiracarus (Metaphthiracarus) setosus (Banks, 1895) (Hoplo-

phora)

(=Metaphthiracarus bacillatus Aoki, 1980)

(=Phthiracarus falcatus Hammer, 1977)

(=Archiphthiracarus filiferus Mahunka, 1982)

DisTRIBUCIÓN: Holártica (Neártica: frecuente, y Paleártica oriental: excepto Norte).

Phthiracarus (Neophthiracarus) Balogh y Csiszár, 1963 (41 spp.)

(=Antarctoplophora Mahunka, 1980)

(=Austrophthiracarus Balogh y Mahunka, 1978)

(=Fuegoplophora Mahunka, 1980)

(=Neoprotophthiracarus Mahunka, 1980)

(=Sturmacarus P. Balogh, 1984)

EsPeCIE TIPO: Neophthiracarus insignis Balogh y Csiszár, 1963

DisTRIBUCIÓN: Pantropical y subtropical.

-Phthiracarus (Neophthiracarus) aculeatus (Niedbala y Colloff,

1997) (Austrophthiracarus)

DisTRIBUCIÓN: Tasmania.

-Phthiracarus (Neophthiracarus) aenus (Niedbala, 2000) (Austroph-

thiracarus)

Distribución: Nueva Caledonia.

-Phthiracarus (Neophthiracarus) anosculpturatus (Mahunka, 1987)

(Hoplophthiracarus)

DisTRIBUCIÓN: Kenia.
-Phthiracarus (Neophthiracarus) aokii (Niedbala, 1987) (Austrophthiracarus)

DistriBución: Australia

-Phthiracarus (Neophthiracarus) aureus (Niedbala, 2000) (Austrophthiracarus)

DisTRIBUCIÓN: Nueva Zelanda.

-Phthiracarus (Neophthiracarus) baloghi (Niedbala, 1987) (Austrophthiracarus)

DisTRIBUCIÓN: Australia.

-Phthiracarus (Neophthiracarus) comosus (Aoki, 1980) (N.)

DisTRIBUCIÓN: Japón.

-Phthiracarus (Neophthiracarus) darwini (Mahunka, 1980) (Antarctoplophora)

DistriBución: América austral (Tierra del Fuego).

-Phthiracarus (Neophthiracarus) diazae (Ojeda, 1985) (Protophthiracarus)

(=Calyptophthiracarus cucundus Niedbala, 1988)

DISTRIBUCIÓN: Neotropical.

-Phthiracarus (Neophthiracarus) dilucidus (Niedbala, 1988) (Calyptophthiracarus)

DisTRIBUCIÓN: Neotropical (Ecuador y Chile).

-Phthiracarus (Neophthiracarus) egregius (Niedbala y Colloff, 1997) (Austrophthiracarus)

DISTRIBUCIÓN: Tasmania.

-Phthiracarus (Neophthiracarus) elizabethiensis (Niedbala, 1988) (Calyptophthiracarus)

DisTRIBUCIÓN: Brasil.

-Phthiracarus (Neophthiracarus) equisetosus (Mahunka, 1980)

(Neoprotophthiracarus)

DisTRIBUCIÓN: Neotropical.

-Phthiracarus (Neophthiracarus) espeletiae (P. Balogh, 1984) (Sturmacarus)

Distribución: Colombia.

-Phthiracarus (Neophthiracarus) facetus (Niedbala y Colloff, 1997)

(Austrophthiracarus)

DisTRIBUCIÓN: Tasmania.

-Phthiracarus (Neophthiracarus) foveoreticulatus (Mahunka, 1980)

(Fuegoplophora)

DisTRIBUCIÓN: América austral.

-Phthiracarus (Neophthiracarus) hallidayi (Niedbala y Colloff,

1997) (Austrophthiracarus)

DISTRIBUCIÓN: Tasmania.

-Phthiracarus (Neophthiracarus) helluonis (Niedbala, 1982) (N.)

DisTRIBUCIÓN: Perú.

-Phthiracarus (Neophthiracarus) hirtus (P. Balogh, 1984) (Sturmacarus)

DisTRIBUCIÓN: Neotropical (Colombia y Chile)

-Phthiracarus (Neophthiracarus) incrassatus (Niedbala, 1984) (N.)

DisTRIBUCIÓN: América austral.

-Phthiracarus (Neophthiracarus) insignis (Balogh y Csiszár, 1963) (N.)

DisTRIBUCIÓN: Argentina.

-Phthiracarus (Neophthiracarus) kochi (Niedbala, 1987) (Calyptophthiracarus)

DistriBuCión: Australia.

-Phthiracarus (Neophthiracarus) lamingtoni (Niedbala, 2000) (Austrophthiracarus)

DisTRIBUCIÓN: Australia.

-Phthiracarus (Neophthiracarus) multisetosus (J. y P. Balogh, 1983)

(Austrophthiracarus)

DistriBución: Australia.

-Phthiracarus (Neophthiracarus) mutabilis (Niedbala y Colloff,

1997) (Austrophthiracarus)

DISTRIBUCIÓN: Tasmania.

-Phthiracarus (Neophthiracarus) obsessus nom. nov.

[=Calyptophthiracarus sellnicki Niedbala, 1987, "nom. praeoc." por Feider y Suciu, 1957 ("hom. sec.")]

Distribución: Australiana (Australia y Tasmania).

-Phthiracarus (Neophthiracarus) perpropinqus (Niedbala y Colloff,

1997) (Austrophthiracarus)

DISTRIBUCIÓN: Tasmania. 
-Phthiracarus (Neophthiracarus) phaleratus (Niedbala, 1982) (N.) Distribución: Costa Rica.

-Phthiracarus (Neophthiracarus) pilosus (Niedbala y Colloff, 1997)

(Austrophthiracarus)

DisTRIBUCIÓN: Tasmania.

-Phthiracarus (Neophthiracarus) portentosus (Niedbala, 1988)

(Calyptophthiracarus)

DISTRIBUCIÓN: Ecuador.

-Phthiracarus (Neophthiracarus) praeoccupatus nom. nov.

[=Austrophthiracarus dissonus Niedbala y Colloff, 1997, "nom. praeoc." por Niedbala, 1983 ("hom. sec.")]

DisTRIBUCIÓN: Tasmania.

-Phthiracarus (Neophthiracarus) pulchellus (Niedbala, 1993)

(Austrophthiracarus)

DisTRIBUCIÓN: Nueva Zelanda.

-Phthiracarus (Neophthiracarus) pullus (Niedbala, 1989) (Calyptophthiracarus)

DisTRIBUCIÓN: Oriental (Borneo e India: Kerala).

-Phthiracarus (Neophthiracarus) radiatus (Balogh y Mahunka, 1978)

(Austrophthiracarus)

(=Austrophthiracarus similis J. y P. Balogh, 1983)

DistribuCIÓN: Australia.

-Phthiracarus (Neophthiracarus) repetitus nom. nov.

[=Austrophthiracarus largus Niedbala, 2000, “nom. praeoc.” por Niedbala, 1984 ("hom. sec.")]

DistriBución: Nueva Caledonia.

-Phthiracarus (Neophthiracarus) scopoli (Niedbala, 1987) (Calyptophthiracarus)

DistriBución: Australiana (Australia y Tasmania).

-Phthiracarus (Neophthiracarus) shiptoni (Sheals y Macfarlane,

1966) $(N$.

(=Neoprotophthiracarus flagellatus Mahunka, 1980)

(=Phthiracarus mahunkai Niedbala, 1994)

DisTRIBUCIÓN: América austral.

-Phthiracarus (Neophthiracarus) tuberculatus (Niedbala y Corpuz-

Raros, 1998) (Austrophthiracarus)

DisTRIBUCIÓN: Oriental.

-Phthiracarus (Neophthiracarus) villosus (Niedbala, 1982) (N.)

DisTRIBUCIÓN: Norte de India.

-Phthiracarus (Neophthiracarus) wallworki (J. y P. Balogh, 1983)

(Austrophthiracarus)

DisTRIBUCIÓN: Australia.

-Phthiracarus (Neophthiracarus) willmanni (Niedbala, 1987)

(Austrophthiracarus)

DisTRIBUCIÓN: Australia.

Rhacaplacarus Niedbala, 1986 (49 spp.)

EsPECIE TIPO: Hoplophorella amoena Niedbala, 1983

DisTRIBUCIÓN: Cosmopolita (excepto Antártica).

Rhacaplacarus (Rhacaplacarus) Niedbala, 1986 (44 spp.)

(=Plonaphacarus Niedbala, 1986 "sin. nov.")

DISTRIBUCIÓN: Cosmopolita (excepto Antártica).

-Rhacaplacarus (R.) aduncus (Niedbala y Colloff, 1997) (Plonaphacarus)

DisTRIBUCión: Tasmania.

-Rhacaplacarus (R.) ambiguus (Niedbala, 1982) (Hoplophthiracarus) DISTRIBUCIÓN: Etiópica.

-Rhacaplacarus (R.) amoenus (Niedbala, 1983) (Hoplophorella) DisTRIBUCIÓN: Uganda.

-Rhacaplacarus (R.) berlesei (Niedbala, 1987) (Plonaphacarus) DisTRIBUCIÓN: Australia.

-Rhacaplacarus (R.) brevisetus (Mahunka, 1984) (Hoplophthiracarus) DisTRIBUCión: Tanzania.

-Rhacaplacarus (R.) contrarius (Niedbala, 1984) (Hoplophthiracarus) DisTRIBUCIÓN: Iraq.

-Rhacaplacarus (R.) diaphoros (Niedbala, 2000) (Steganacarus (R.))

DisTRIBUCIÓN: Australia.

-Rhacaplacarus (R.) dikros (Niedbala, 2000) (Plonaphacarus)

DisTRIBUCIÓN: Nueva Zelanda.
-Rhacaplacarus (R.) discrepus (Niedbala, 1982) (Hoplophthiracarus)

DisTRIBUCIÓN: África centrooriental.

-Rhacaplacarus (R.) dispar (Niedbala, 2000) (Plonaphacarus)

DISTRIBUCIÓN: Borneo.

-Rhacaplacarus (R.) ecphylus (Niedbala, 2001) (Plonaphacarus)

DisTRIBUCIÓN: Kenia.

-Rhacaplacarus (R.) eximius (Niedbala, 1982) (Hoplophthiracarus)

(=Steganacarus politus Mahunka, 1983)

DisTRIBUCIÓN: Méjico.

-Rhacaplacarus (R.) feideri (Niedbala, 1987) (Plonaphacarus)

Distribución: Australia.

-Rhacaplacarus (R.) forsslundi (Niedbala, 1987) (Plonaphacarus)

DISTRIBUCIÓN: Australiana.

-Rhacaplacarus (R.) granulatus (Banks, 1902) (Hoploderma)

DisTRIBUCIÓN: Neártica septentrional.

-Rhacaplacarus (R.) inflatus (Niedbala, 1984) (Hoplophthiracarus)

DisTRIBUCIÓN: África central.

-Rhacaplacarus (R.) insignitus (Niedbala, 1989) (Plonaphacarus)

DISTRIBUCIÓN: Vietnam.

-Rhacaplacarus (R.) insolens (Niedbala, 2000) (Plonaphacarus)

Distribución: Nueva Zelanda.

-Rhacaplacarus (R.) jacoti (Niedbala, 1987) (Steganacarus (R.))

Distribución: Australia.

-Rhacaplacarus (R.) kaszabi (P. Balogh, 1988) (Hoplophthiracarus) DistRIBUCIÓN: Ceilán [Sri Lanka].

-Rhacaplacarus (R.) laetabilis (Niedbala, 1988) (Hoplophorella)

DisTRIBUCIÓN: Congo.

-Rhacaplacarus (R.) laterospinosus Mahunka, 1993

DISTRIBUCIÓN: Ruanda.

-Rhacaplacarus (R.) lividus (Niedbala, 2000) (Hoplophthiracarus)

Distribución: Nueva Zelanda.

-Rhacaplacarus (R.) loebli (Mahunka, 1985) (Hoplophthiracarus)

DisTRIBUCIÓN: India (Kerala).

-Rhacaplacarus (R.) machadoi (Balogh, 1958) (Steganacarus)

DISTRIBUCIÓN: Etiópica (frecuente).

-Rhacaplacarus (R.) mirandus (Niedbala, 1988) (Austrophthiracarus)

Distribución: Costa Rica.

-Rhacaplacarus (R.) montigenus (Niedbala, 1981) (Hoplophthiracarus)

DisTRIBUCIÓN: Australiana.

-Rhacaplacarus (R.) ngongi (Niedbala, 2001) (Plonaphacarus)

DisTRIBUCIÓN: Kenia.

-Rhacaplacarus (R.) optivus (Niedbala, 1994) (Plonaphacarus)

DisTRIBUCIÓN: Somalia.

-Rhacaplacarus (R.) ortizi (Pérez-Íñigo, 1970) (Steganacarus)

DisTRIBUCIÓN: Europa occidental.

-Rhacaplacarus (R.) persimilis (Niedbala, 1994) (Plonaphacarus)

DISTRIBUCIÓN: Somalia.

-Rhacaplacarus (R.) pervigens (Niedbala, 1988) (Hoplophorella)

DistriBución: Congo.

-Rhacaplacarus (R.) rafalskii (Niedbala, 1997) (Plonaphacarus)

DistriBución: Oriental (India: Bengala Occidental, y Borneo).

-Rhacaplacarus (R.) sarawaki (Niedbala, 2000) (Austrophthiracarus)

DISTRIBUCIÓN: Borneo.

-Rhacaplacarus (R.) saucius (Niedbala, 1988) (Austrophthiracarus)

DisTRIBUCIÓN: Norte de Neotropical.

-Rhacaplacarus (R.) scrupeus (Niedbala, 1989) (Plonaphacarus)

DisTRIBUCión: Vietnam.

-Rhacaplacarus (R.) spinatus (Niedbala, 2001) (Steganacarus (R.)) DisTRIBUCIÓN: Congo.

-Rhacaplacarus (R.) spiniferus Mahunka, 1993

DisTRIBUCIÓN: Tanzania.

-Rhacaplacarus (R.) spiniger (Aoki, 1980) (Hoplophorella)

DisTRIBUCIÓN: Japón.

-Rhacaplacarus (R.) styphelos (Niedbala, 2001) (Plonaphacarus)

DisTRIBUCIÓN: Tanzania.

-Rhacaplacarus (R.) succinctus (Niedbala, 2001) (Steganacarus (R.))

Distribución: Congo.

-Rhacaplacarus (R.) tanzicus (Mahunka, 1993) (Hoplophthiracarus

(Plonaphacarus))

DisTRIBUCión: Tanzania. 
-Rhacaplacarus (R.) thoreaui (Jacot, 1930) (Steganacarus)

DistriBución: Neártica (frecuente).

-Rhacaplacarus (R.) zicsii (Mahunka, 1988) (Steganacarus (R.))

DisTRIBUCIÓN: Tanzania.

Rhacaplacarus (Mantigueracarus) Balogh y Mahunka, 1992 (5 spp.) EsPECIE TIPO: Mantigueracarus baggioi Balogh y Mahunka, 1992 DisTRIBUCIÓN: Pantropical (excepto Etiópica).

-Rhacaplacarus (Mantigueracarus) aculeatus (Mahunka, 1995)

(Hoplophthiracarus (Plonaphacarus))

DisTRIBUCIÓN: Borneo.

-Rhacaplacarus (Mantigueracarus) baggioi (Balogh y Mahunka, 1992) (M.)

DisTRIBUCIÓN: Brasil.

-Rhacaplacarus (Mantigueracarus) grandjeani (Niedbala, 1987)

(Plonaphacarus)

DistriBUCIÓN: Australiana (Australia y Melanesia: I. Fiji).

-Rhacaplacarus (Mantigueracarus) incredibilis (Niedbala, 1982)

(Neophthiracarus)

DisTRIBUCIÓN: Neotropical.

-Rhacaplacarus (Mantigueracarus) perezinigoi (Balogh y Mahunka, 1992) (M.)

DISTRIBUCIÓN: Brasil.

Steganacarus Ewing, 1917 (52 spp. y 1 ssp.)

EsPECIE TIPO: (Hoplophora anomala Berlese, 1883) =Hoplophora magna Nicolet, 1855

DisTRIBUCIÓN: Semicosmopolita (Holártica, Etiópica y Neotropical).

Steganacarus (Steganacarus) Ewing, 1917 (36 spp. y 1 ssp.)

(=Trachyhoplophora Berlese, 1923)

DisTRIBUCIÓN: Holártica y Etiópica.

-Steganacarus (S.) applicatus (Sellnick, 1920) (Phthiracarus)

(=Phthiracarus berlesei Oudemans, 1915)

(=Hoplophora quadriseriata Haupt, 1882)

DisTRIBUCIÓN: Paleártica occidental.

-Steganacarus (S.) balearicus Pérez-Íñigo, 1969

Distribución: Mediterránea occidental.

-Steganacarus (S.) bicarinatus Jeleva, 1970

DisTRIBUCIÓN: Bulgaria.

-Steganacarus (S.) caelestis Niedbala, 1984

DisTRIBUCIÓN: Irán.

-Steganacarus (S.) carlosi Niedbala, 1984

Distribución: I. Canarias.

-Steganacarus (S.) carusoi Bernini y Avanzati, 1989

(?=Steganacarus applicatus crassisetosus Willmann, 1939 "sp. inq.") DisTRIBUCIÓN: Mediterránea occidental.

-Steganacarus (S.) coniunctus Niedbala, 1983 (=Steganacarus danae Mahunka y Miko, 1989) DisTRIBUCIÓN: Mediterránea oriental.

-Steganacarus (S.) donatoi Avanzati, Baratti y Bernini, 1994 DisTRIBUCIÓN: Italia.

-Steganacarus (S.) doumandji Niedbala, 1986 DistRIBUCIÓN: Mediterránea occidental.

-Steganacarus (S.) flagellatissimus Mahunka, 1979

DisTRIBUCIÓN: Mediterránea oriental.

-Steganacarus (S.) grandjeani Mahunka, 1977

(=Steganacarus sacculiferus Parry, 1978)

DisTRIBUCIÓN: Israel.

-Steganacarus (S.) guanarteme Pérez-Íñigo y Peña, 1996 DisTRIBUCIÓN: I Canarias.

-Steganacarus (S.) herculeanus Willmann, 1953

(=Steganacarus longisetosus Moraza, 1985)

DisTRIBUCIÓN: Europa centrooccidental.

-Steganacarus (S.) hirsutus Pérez-Íñigo, 1974

Distribución: Mediterránea occidental.

-Steganacarus (S.) hirsutus hirsutus Pérez-Íñigo, 1974

(=Steganacarus perezinigoi Balogh y Mahunka, 1979)

DISTRIBUCIÓN: Mediterránea occidental.
-Steganacarus (S.) hirsutus azorensis Pérez-Iñigo, 1992

DisTRIBUCIÓN: I. Azores.

-Steganacarus (S.) inaestimabilis Niedbala, 1984

DISTRIBUCIÓN: Irán.

-Steganacarus (S.) incognitus Niedbala, 1984

DisTRIBUCión: España.

-Steganacarus (S.) incomptus Niedbala, 1983

Distribución: Cáucaso.

-Steganacarus (S.) insulanus C. y C., jr. Pérez-Íñigo, 1996

DistRIBUCIÓN: I. Azores.

-Steganacarus (S.) inurbanus Niedbala, 1983

DisTRIBUCIÓN: Uganda.

-Steganacarus (S.) magnus (Nicolet, 1855) (Hoplophora)

(=Hoplophora anomala Berlese, 1883)

(=Steganacarus karamani Tarman, 1962)

(=Phthiracarus magnus anomaloides Oudemans, 1915)

(=Phthiracarus magnus oblongus Oudemans, 1915)

(=Phthiracarus magnus rotundus Oudemans, 1915)

(=Phthiracarus magnus transitans Oudemans, 1915)

DisTRIBUCIÓN: Holártica (Paleártica occidental: frecuente, y U.S.A.)

e I. Santa Helena.

-Steganacarus (S.) manganellii Avanzati, Baratti y Bernini, 1994

DISTRIBUCIÓN: Italia.

-Steganacarus (S.) maximus (Haupt, 1882) (Hoplophora) "sp. inq." Distribución: Alemania.

-Steganacarus (S.) michaeli Bernini y Avanzati, 1987

DisTRIBUCIÓN: Europa occidental.

-Steganacarus (S.) nemorosus Gordeeva, 1991

DisTRIBUCIÓN: Rusia europea central.

-Steganacarus (S.) patruelis Niedbala, 1983

(=Steganacarus lazitanicus Mahunka y Miko, 1989)

DISTRIBUCIÓN: Mediterránea oriental.

-Steganacarus (S.) personatus Niedbala, 1983

Distribución: Sureste de Europa.

-Steganacarus (S.) similis Willmann, 1939

DisTRIBUCiÓN: Macaronésica (Madeira).

-Steganacarus (S.) simonettae Bernini y Avanzati, 1987

DisTRIBUCIÓN: Mediterránea occidental.

-Steganacarus (S.) sol Balogh, 1958

(=Hoplophthiracarus peracutus Mahunka, 1983)

DisTRIBUCIÓN: Africa centrooriental.

-Steganacarus (S.) spinosus (Sellnick, 1920) (Phthiracarus)

(=Steganacarus barborae Mahunka y Miko, 1989)

(=Steganacarus punctulatus Sergienko, 1985)

DisTRIBUCIÓN: Europa.

-Steganacarus (S.) tenerifensis Pérez-Íñigo, 1972

DistRIBUCión: I. Canarias.

-Steganacarus (S.) travei Lions, 1968

DisTRIBUCIÓN: Mediterránea occidental.

-Steganacarus (S.) vernaculus Niedbala, 1982

Distribución: Polonia.

-Steganacarus (S.) vestitus Niedbala, 1983

(=Steganacarus complicatus Mahunka, 1993)

DisTRIBUCIÓN: África centrooriental.

-Steganacarus (S.) wallworki Mahunka, 1984

DisTRIBUCIÓN: Tanzania.

-Steganacarus (S.) werneri Mahunka, 1993

DISTRIBUCIÓN: Ruanda.

Steganacarus (Rafacarus) Niedbala, 1982 (5 spp.)

(=Neosteganacarus Balogh y Mahunka, 1992 "sin. nov.")

(=Nortonacarus Balogh y Mahunka, 1992 "sin. nov.")

ESPECIE TIPO: Rafacarus rafalskii Niedbala, 1982

DisTRIBUCIÓN: Neotropical.

-Steganacarus (Rafacarus) angulatus (Balogh y Mahunka, 1992)

(Neosteganacarus)

DisTRIBUCIÓN: Brasil.

-Steganacarus (Rafacarus) niedbalai (Pérez-Íñigo y Baggio, 1996)

(Protophthiracarus)

DistRIBUCIÓN: Brasil. 
-Steganacarus (Rafacarus) rafalskii (Niedbala, 1992) (R.)

(=Neosteganacarus cataracta Balogh y Mahunka, 1992)

DisTRIBUCIÓN: Brasil.

-Steganacarus (Rafacarus) relictus (Balogh y Mahunka, 1992)

(Neosteganacarus)

DisTRIBUCIÓN: Brasil.

-Steganacarus (Rafacarus) valeriae (Balogh y Mahunka, 1992)

(Nortonacarus)

DISTRIBUCIÓN: Brasil.

Steganacarus (Tropacarus) Ewing, 1917 (11 spp.)

(=Calhoplophora Berlese, 1923)

EsPECIE TIPO: Hoplophora carinata Koch, 1841

DisTRIBUCIÓN: Paleártica.

-Steganacarus (Tropacarus) balcanicus Berninni y Avanzati, 1989 Distribución: Grecia.

-Steganacarus (Tropacarus) boulfekhari Niedbala, 1986 (S.) DisTRIBUCIÓn: Argelia.

-Steganacarus (Tropacarus) brevipilus (Berlese, 1923) (Phthiracarus (Trachyhoplophora))

(=Hoploderma brevipilus perfecta Sellnick, 1931)

DisTRIBUCIÓN: Paleártica occidental (excepto Norte).

-Steganacarus (Tropacarus) callainii Bernini, S. Bernini y Avanzati, 1989

DisTRIBUCIÓN: Norte de África.

-Steganacarus (Tropacarus) carinatus (Koch, 1841) (Hoplophora) (=Hoplophora pulcherrima Berlese, 1887)

DisTRIBUCión: Paleártica (menos frecuente en el Norte).

-Steganacarus (Tropacarus) desmeti Niedbala, 1988 (S.)

(=Steganacarus (Tropacarus) altitudinis Bernini, S. Bernini y Avanzati, 1989) DisTRIBUCIÓN: Argelia.

-Steganacarus (Tropacarus) excavatus (Niedbala, 1981) (T.) DISTRIBUCIÓN: Turquía.

-Steganacarus (Tropacarus) lasithiensis Mahunka, 1979 (S.) DistriBución: Grecia.

-Steganacarus (Tropacarus) maghrebinus Bernini, S. Bernini y Avanzati, 1989)

DisTRIBUCIÓN: Argelia.

-Steganacarus (Tropacarus) malatestai Avanzati, Baratti y Bernini, 1994

DisTRIBUCión: Italia.

-Steganacarus (Tropacarus) pseudocarinatus Bernini y Avanzati, 1989 Distribución: Paleártica meridional (Mediterránea y Asia central rusa).

\section{Suborden HOLOSOMATA Grandjean, 1960} Crotonioidea Thorell, 1876

Trhypochthoniidae Willmann, 1931 (7 gen., 1 subg., 51 spp. y 6 sspp.) DISTRIBUCIÓN: Cosmopolita.

Afronothrus Wallwork, 1961 (2 spp.)

ESPECIE TIPO: Afronothrus incisivus Wallwork, 1961

DisTRIBUCIÓN: Pantropical.

-Afronothrus arboreus Ramani y Haq, 1992

DISTRIBUCIÓN: India (Kerala).

-Afronothrus incisivus Wallwork, 1961

(=Afronothrus incisivus maheensis Mahunka, 1978)

(=Afronothrus incisivus neotropicus Balogh y Mahunka, 1974)

(=Afronothrus incisivus paraguayensis Mahunka, 1984)

(=Afronothrus sulcatus Hammer, 1972)

DISTRIBUCIÓN: Pantropical.

Allonothrus Hammen, 1953 (15 spp. y 1 ssp.)

EsPECIE TIPO: Allonothrus schuilingi Hammen, 1953

DISTRIBUCIÓN: Pantropical.

Allonothrus (Allonothrus) Hammer, 1953 (12 spp. y 1 ssp.) DisTRIBUCIÓN: Pantropical.
-Allonothrus (A.) foveolatus Pérez-Iñigo y Baggio, 1988

DisTRIBUCIÓN: Brasil.

-Allonothrus (A.) giganticus Haq, 1978

DisTRIBUCIÓN: India (Kerala).

-Allonothrus (A.) indicus Bhaduri y Raychaudhuri, 1968

DisTRIBUCIÓN: India (Bengala Occidental).

-Allonothrus (A.) monensis Ghosh y Bhaduri, 1978

DisTRIBUCIÓN: India (Nagaland).

-Allonothrus (A.) monodactylus Wallwork, 1960

DISTRIBUCIÓN: África occidental y Oriental.

-Allonothrus (A.) neotropicus Balogh y Mahunka, 1969

DisTRIBUCIÓN: Neotropical (frecuente).

-Allonothrus (A.) pararusseolus Subías y Sarkar, 1982

DistribuCión: India (Tripura).

-Allonothrus (A.) pyriformis (Berlese, 1913) (Angelia)

DisTRIBUCIÓN: Java.

-Allonothrus (A.) russeolus Wallwork, 1960

DisTRIBUCIÓN: Pantropical.

-Allonothrus (A.) russeolus russeolus Wallwork, 1960

Distribución: Pantropical (Etiópica: Ghana, Oriental y Norte de Neotropical).

-Allonothrus (A.) russeolus reticulatus Hammer, 1972

DisTRIBUCIÓN: Australiana (Polinesia) y Java.

-Allonothrus (A.) schuilingi Hammen, 1953

DISTRIBUCIÓN: Pantropical (frecuente en Australiana) y subtropical.

-Allonothrus (A.) sinicus Wang y Norton, 1988

DisTRIBUCIÓN: China suroriental.

-Allonothrus (A.) tuxtlasensis Palacios-Vargas e Iglesias, 1997

Distribución: Méjico.

Allonothrus (Pseudonothrus) Balogh, 1958 (1 spp.)

EsPeCIE TIPO: Pseudonothrus hirtus Balogh, 1958

DISTRIBUCIÓN: Etiópica.

-Allonothrus (Pseudonothrus) hirtus (Balogh, 1958) (P.)

=Allonothrus ghanensis Wallwork, 1961)

DisTRIBUCIÓN: Etiópica.

Archegozetes Grandjean, 1931 (2 spp. y 1 ssp.)

EsPeCIE TIPO: Epilohmannia magna Sellnick, 1925

DisTRIBUCIÓN: Pantropical.

-Archegozetes longisetosus Aoki, 1965

(=Archegozetes chamelensis Palacios-Vargas e Iglesias, 1997)

(=Archegozetes magnus indicus Bhaduri y Raychaudhuri, 1968)

DISTRIBUCIÓN: Pantropical (excepto Etiópica): frecuente en

Oriental y Neotropical.

-Archegozetes magnus (Sellnick, 1925) (Epilohmannia)

DisTRIBUCIÓN: Pantropical.

-Archegozetes magnus magnus (Sellnick, 1925)

(=Archegozetes tuberculatus Sarkar, 1985)

(=Archegozetes veracruzensis Palacios-Vargas e Iglesias, 1997)

Distribución: Pantropical (Oriental: frecuente, Australiana:

Polinesia, Etiópica: Senegal y Neotropical: Méjico).

-Archegozetes magnus mediosetosus Mahunka, 1978

DisTRIBUCIÓN: Etiópica (I. Seychelles) y Norte de Neotropical.

Mainothrus Choi, 1996 (3 spp.)

(=Altrhypochthonius Weigmann, 1997)

ESPECIE TIPO: Mainothrus aquaticus Choi, 1996

DISTRIBUCIóN: Holártica y Neotropical austral.

-Mainothrus aquaticus Choi, 1996

DISTRIBUCIÓN: Corea.

-Mainothrus badius (Berlese, 1905) (Trhypochthonius)

DISTRIBUCIÓN: Holártica (menos frecuente en el Sur).

-Mainothrus breviclava (Hammer, 1958) (Trhypochthonius)

DISTRIBUCIÓN: Argentina

Mucronothrus Trägardh, 1931 (2 spp.)

ESPECIE TIPO: (Mucronothrus rostratus Trägardh, 1931) = Malaconothrus nasalis Willmann, 1929

DISTRIBUCIÓN: Semicosmopolita (Holártica, Australiana y Neotropical). 
-Mucronothrus nasalis (Willmann, 1929) (Malaconothrus)

(=Mucronothrus rostratus Trägardh, 1931)

DistRIBUCión: Semicosmopolita (Boreal: frecuente, Australiana y Neotropical).

-Mucronothrus willmanni Norton, Behan-Pelletier y Wang, 1996 DisTRIBUCIÓN: Oeste de U.S.A.

Trhypochthoniellus Willmann, 1928 (5 spp. y 2 sspp.)

(=Hydronothrus Aoki, 1964)

ESPECIE TIPO: (Trhypochthonius (Trhypochthoniellus) setosus Willmann, 1928) =Trhypochthonius longisetus Berlese, 1904

DISTRIBUCIÓN: Cosmopolita (excepto Antártica).

-Trhypochthoniellus ashoroensis Fujikawa, 2000

DISTRIBUCIÓN: Japón.

-Trhypochthoniellus longisetus (Berlese, 1904) (Trhypochthonius)

DistribuCión: Semicosmopolita (Holártica, Etiópica: I.Santa

Helena, Australiana y Neotropical).

-Trhypochthoniellus longisetus longisetus (Berlese, 1904)

(=Hydronothrus aquariorum Fain y Lambrechts, 1987)

(=Nothrus crassus Warburton y Pearce, 1905)

(=Hydronothrus crispus Aoki, 1964)

(=Camisia excavata Willmann, 1919)

(=Hydronothrus puniceus Habeeb, 1981)

(=Trhypochthonius (Trhypochthoniellus) setosus Willmann, 1929)

(=Trhypochthonius trichosus Schweizer, 1922)

DistribuCIÓn: Semicosmopolita (Holártica, Etiópica: I. Santa Helena, Australiana y Neotropical).

-Trhypochthoniellus longisetus brasilensis Pérez-Íñigo y Baggio, 1988 (T. setosus b.)

DisTRIBUCIÓN: Brasil.

-Trypochthoniellus longisetus canadensis Hammer, 1952 (T. setosus c.)

DISTRIBUCIÓN: Neártica septentrional.

-Trhypochthoniellus porticus Fujikawa, 2000

DisTRIBUCIÓN: Japón.

-Trhypochthoniellus ramosus Hammer, 1982

DISTRIBUCIÓN: Bali.

-Trhypochthoniellus reticulatus Willmann, 1931

DISTRIBUCIÓN: Sumatra.

Trhypochthonius Berlese, 1904 (24 spp. y 2 sspp.)

(=Albonothrus Tseng, 1982 "sin. nov.")

(=Trilohmannia Willmann, 1919)

(=Tumidalvus Ewing, 1908)

ESPECIE TIPO: Hypochthonius tectorum Berlese, 1896

DisTRIBUCIÓn: Cosmopolita (excepto Antártica).

-Trhypochthonius africanus Balogh, 1958 "sp. inq."

DisTRIBUCión: Angola.

-Trhypochthonius americanus (Ewing, 1908) (Tumidalvus)

DisTRIBUCIÓN: U.S.A

-Trhypochthonius cladonicola (Willmann, 1919) (Camisia)

DisTRIBUCIÓN: Paleártica (menos frecuente en el Sur).

-Trhypochthonius conspectus Sergienko, 1991

DisTRIBUCión: Sureste de Europa.

-Trhypochthonius elegantulus Schweizer, 1956 "sp. inq."

DISTRIBUCIÓN: Suiza.

-Thypochthonius fujinitaensis Fujikawa, 2000

DISTRIBUCIÓN: Japón.

-Trhypochthonius hammerae Schweizer, 1956 "sp. inq."

DisTRIBUCIÓN: Suiza.

-Trhypochthonius japonicus Aoki, 1970

Distribución: Holártica meridional (Paleártica: Ucrania y

Paleártica oriental: excepto Norte, y U.S.A.: Luisiana).

-Trhypochthonius javanus Csiszár, 1961

DisTRIBUCIÓN: Java.

-Trhypochthonius longipes Warburton, 1912 (T. tectorum l.) "sp. inq."

DisTRIBUCIÓN: I. Seychelles.

-Trhypochthonius misumaiensis Fujikawa, 2000

DISTRIBUCIÓN: Japón.
-Thrypochthonius montanus Hammen, 1955

DisTRIBUCIÓN: Nueva Guinea.

-Trhypochthonius multisulcatus (Tseng, 1982) (Albonothrus) "sp. inq."

DistriBUCióN: Taiwan [Formosa].

-Trhypochthonius nigricans Willmann, 1928

DisTRIBUCIÓN: Holártica (Paleártica: menos frecuente en el Sur y

Neártica septentrional).

-Trhypochthonius ruttneri Willmann, 1931

DISTRIBUCIÓN: Java.

-Trhypochthonius sclerotrichus Willmann, 1923 (T. cladonicola s.)

"sp. inq."

DisTRIBUCIÓN: Alemania.

-Trhypochthonius septentrionalis Fujikawa, 1995

DisTRIBUCIÓN: Japón.

-Trhypochthonius setantis Golosova, 1983

DisTRIBUCIÓN: Mongolia.

-Trhypochthonius silvestris Jacot, 1937

DisTRIBUCIÓN: U.S.A. (menos frecuente en el Norte).

-Trhypochthonius sphagnicola Weigmann, 1997

DISTRIBUCIÓN: Alemania.

-Trhypochthonius stercus Fujikawa, 2000

DISTRIBUCIÓN: Japón.

-Trhypochthonius tablasotus Schweizer, 1956 "sp. inq."

DisTRIBUCIÓN: Suiza.

-Trhypochthonius tectorum (Berlese, 1896) (Hypochthonius)

(=Tumidalvus mutsalpina Kishida, 1931)

DisTRIBUCIÓN: Holártica y Neotropical.

-Trhypochthonius tectorum tectorum (Berlese, 1896) (=Trhypochthonius monodactylus Mihelcic, 1957) DisTRIBUCión: Holártica (frecuente) y Perú.

-Trhypochthonius tectorum congregator Grandjean, 1940 DisTRIBUCIÓN: Europa meridional.

-Trhypochthonius tectorum spinosus Kulijev, 1968 Distribución: Cáucaso.

-Trhypochthonius tepoztecus Palacios-Vargas e Iglesias, 1997

DisTRIBUCión: Méjico.

Malaconothridae Berlese, 1916 (2 gen., 2 subg., 137 spp. y 3 sspp.) Distribución: Cosmopolita.

Malaconothrus Berlese, 1904 (59 spp.)

EsPECIE TIPO: Nothrus monodactylus Michael, 1888

DISTRIBUCIÓN: Cosmopolita.

Malaconothrus (Malaconothrus) Berlese, 1904 (26 spp.)

DisTRIBUCIÓN: Cosmopolita (excepto Antártica).

-Malaconothrus (M.) angulatus Hammer, 1958

DisTRIBUCIÓN: América austral.

-Malaconothrus (M.) atuelanus Hammer, 1958

DisTRIBUCIÓN: Argentina.

-Malaconothrus (M.) calcehtokensis Palacios-Vargas e Iglesias, 1997 Distribución: Méjico.

-Malaconothrus (M.) chajulensis Palacios-Vargas e Iglesias, 1997 Distribución: Méjico.

-Malaconothrus (M.) conicus Hammer, 1958

DisTRIBUCIÓN: Argentina.

-Malaconothrus (M.) crassisetosus Subías y Sarkar, 1982

DisTRIBUCión: India (Tripura).

-Malaconothrus (M.) dipankari Saha y Sanyal, 1996

DisTRIBUCIÓN: India (Tripura).

-Malaconothrus (M.) dorsofoveolatus Hammer, 1979

DisTRIBUCIÓN: Oriental (Indonesia).

-Malaconothrus (M.) egregius (Berlese, 1904) (Lohmannia (M.))

(=Malaconothrus pulcher Mihelcic, 1957)

DisTRIBUCión: Holártica (Paleártica: frecuente en Paleártica occidental, y U.S.A.: Carolina del Norte).

-Malaconothrus (M.) globiger Trägardh, 1910

Distribución: Paleártica occidental (excepto Suroeste) 
-Malaconothrus (M.) hexasetosus Hammer, 1971

Distribución: Australiana (Islas del Pacífico) y Oriental (Indonesia).

-Malaconothrus (M.) japonicus Aoki, 1966 DistriBución: Este de Paleártica oriental.

-Malaconothrus (M.) laetus Sergienko y Melamud, 1993 DisTRIBUCIÓN: Ucrania.

-Malaconothrus (M.) marginatus Yamamoto, 1998 DisTRIBUCIÓN: China suroriental.

-Malaconothrus (M.) monodactylus (Michael, 1888) (Nothrus) (=Malaconothrus gracilis Hammen, 1952)

(=Malaconothrus mollisetosus Hammer, 1952)

DisTRIBUCIÓN: Holártica (frecuente) y Neotropical.

-Malaconothrus (M.) neonominatus nom. nov.

[=Malaconothrus pulcher Hammer, 1961, “nom. praeoc.” por Mihelcic, 1957 (“hom. prim.")]

DisTRIBUCIÓN: Perú.

-Malaconothrus (M.) pilosellus Balogh y Mahunka, 1969 DisTRIBUCIÓN: Brasil.

-Malaconothrus (M.) praeoccupatus nom. nov.

[=Malaconothrus punctulatus Balogh, 1958 "nom. praeoc.", por Hammen, 1952 ("hom. prim.")]

DisTRIBUCIÓN: Angola.

-Malaconothrus (M.) processus Hammen, 1952

DISTRIBUCIÓN: Europa centromeridional.

-Malaconothrus (M.) pseudolamellatus Willmann, 1931

DisTRIBUCIÓN: Oriental (Java e India: Tripura).

-Malaconothrus (M.) punctulatus Hammen, 1952

DisTRIBUCIÓN: Euroatlántica.

-Malaconothrus (M.) purvisi Luxton, 1987

DisTRIBUCIÓN: I. Británicas.

-Malaconothrus (M.) pygmaeus Aoki, 1969

DisTRIBUCión: Holártica: Paleártica (excepto Norte) y Canadá

-Malaconothrus (M.) scutatus Luxton, 1987

DisTRIBUCIÓN: I. Británicas.

-Malaconothrus (M.) silvaticus Pérez-Íñigo y Baggio, 1985 DisTRIBUCIÓN: Brasil.

-Malaconothrus (M.) yinae Yamamoto, Aoki, Wang y Hu, 1993 DistriBuCIÓN: China suroriental.

Malaconothrus (Cristonothrus) subgen. nov. (30 spp.)

EsPeCIE TIPO: Malaconothrus pauciareolatus Subías y Sarkar, 1982

DisTRIBUCIÓN: Cosmopolita (excepto Boreal).

-Malaconothrus (Cristonothrus) asiaticus Aoki, 1967 (M. robustus a.) DistRIBUCIÓN: Oriental (Tailandia e India: Tripura).

-Malaconothrus (Cristonothrus) assamensis Chakrabarti y Talukdar,

1979 (M.)

DISTRIBUCIÓN: Noreste de India.

-Malaconothrus (Cristonothrus) cordisetus Mahunka, 1993 (M.) DisTRIBUCIÓN: Ruanda.

-Malaconothrus (Cristonothrus) cornutus Hammer, 1973 (M.) Distribución: Polinesia (I. Samoa).

-Malaconothrus (Cristonothrus) ensifer Mahunka, 1982 (M.) DisTRIBUCIÓN: Etiopía.

-Malaconothrus (Cristonothrus) foliatus Aoki, 1994 (M.)

DisTRIBUCIÓN: Micronesia (I. Marianas).

-Malaconothrus (Cristonothrus) geminus Hammer, 1972 (M.)

Distribución: Polinesia (Tahití) y Oriental (India: Bengala Occidental, y Java).

-Malaconothrus (Cristonothrus) granulosus Palacios-Vargas e Iglesias, 1997 (M.)

DisTRIBUCión: Méjico.

-Malaconothrus (Cristonothrus) hauseri Mahunka, 1983 (M.) DISTRIBUCIÓN: Neotropical y Etiópica (I. Seychelles).

-Malaconothrus (Cristonothrus) heterotrichus Mahunka, 1992 (M.) DisTRIBUCIÓn: Senegal.

-Malaconothrus (Cristonothrus) indifferens Hammer, 1966 (M.) DisTRIBUCIÓN: Nueva Zelanda.
-Malaconothrus (Cristonothrus) iriomotensis Yamamoto y Aoki, 1997 (M.)

DisTRIBUCIÓN: Japón.

-Malaconothrus (Cristonothrus) keriensis Hammer, 1966 (M.)

DistriBución: Nueva Zelanda y Bali.

-Malaconothrus (Cristonothrus) kiiensis Yamamoto, 1996 (M.)

DisTRIBUCIÓN: Japón.

-Malaconothrus (Cristonothrus) neoplumosus Balogh y Mahunka, 1969 (M.)

DisTRIBUCIÓN: Bolivia.

-Malaconothrus (Cristonothrus) pachypilus Hammer, 1972 (M.)

DisTRIBUCIÓN: Polinesia.

-Malaconothrus (Cristonothrus) pauciareolatus Subías y Sarkar,

$1982(M$.

DISTRIBUCIÓN: India (Tripura).

-Malaconothrus (Cristonothrus) peruensis Hammer, 1961 (M.)

DisTRIBUCión: Perú e India (Tripura).

-Malaconothrus (Cristonothrus) plumosus Willmann, 1929 (M.)

DisTRIBUCión: Java.

-Malaconothrus(Cristonothrus) prismaticus Warburton, 1912 (M.)

DisTRIBUCIÓN: I. Seychelles.

-Malaconothrus (Cristonothrus) ramensis Hammer, 1966 (M.)

DistriBución: Paleártica meridional (Mediterránea y Pakistán).

-Malaconothrus (Cristonothrus) robustus Hammer, 1958 (M.)

DistriBución: Neotropical (Bolivia y Brasil).

-Malaconothrus (Cristonothrus) rohri P. Balogh, 1997 (M.)

(=Malaconothrus rohri J. y P. Balogh, 2002, "nom. praeoc." por P. Balogh, 1997)

DisTRIBUCIÓN: Brasil.

-Malaconothrus (Cristonothrus) rostropilosus Saha y Sanyal, 1996 (M.)

DisTRIBUCIÓN: India (Tripura).

-Malaconothrus (Cristonothrus) subrasus Balogh, 1964 (M.)

DisTRIBUCIÓN: Madagascar.

-Malaconothrus (Cristonothrus) translamellatus Hammer, 1958 (M.)

DisTRIBUCIÓN: Neotropical y subantártica (I. Amsterdam).

-Malaconothrus (Cristonothrus) variosetosus Hammer, 1971 (M.)

Distribución: Melanesia (I. Fiji).

-Malaconothrus (Cristonothrus) vilhenarum Balogh, 1958 (M.) "sp. inq."

DisTRIBUCión: Angola.

-Malaconothrus (Cristonothrus) yamamotoi Aoki, 1994 (M.)

DisTRIBUCIÓN: Japón.

-Malaconothrus(Cristonothrus) zealandicus Hammer, 1966 (M.)

DisTRIBUCión: Nueva Zelanda.

Trimalaconothrus Berlese, 1916 (78 spp. y 3 sspp.)

ESPECIE TIPO: Malaconothrus (Trimalaconothrus) indusiatus Berlese, 1916 DisTRIBUCIÓN: Cosmopolita.

Trimalaconothrus (Trimalaconothrus) Berlese, 1916 (54 spp. y 1 ssp.) (=Zeanothrus Hammer, 1966 "sin. nov.")

DISTRIBUCIÓN: Cosmopolita.

-Trimalaconothrus (T.) africanus Balogh, 1958 "sp. inq."

DisTRIBUCión: Angola.

-Trimalaconothrus (T.) almagrensis Iglesias, Palacios-Vargas y

Mahunka, 2001

DistribuCión: Méjico.

-Trimalaconothrus (T.) altissimus Piffl, 1971

DisTRIBUCIÓN: Nepal.

-Trimalaconothrus (T.) angulatus Willmann, 1931

DisTRIBUCIÓN: Holártica (Euroatlántica y U.S.A.: Nueva York).

-Trimalaconothrus (T.) aureopunctatus (Hammer, 1979) (Malaconothrus)

DISTRIBUCIÓN: Oriental (Indonesia).

-Trimalaconothrus (T.) azumaensis Yamamoto, Kuriki y Aoki, 1993 DisTRIBUCIÓN: Japón.

-Trimalaconothrus (T.) barbatus Yamamoto, 1977

DisTRIBUCIÓN: Japón.

-Trimalaconothrus (T.) brevisetiger Yamamoto y Aoki, 1998

DistriBuCión: China suroriental. 
-Trimalaconothrus (T.) crinitus (Berlese, 1908) (Malaconothrus)

DisTRIBUCIÓN: U.S.A. (Misuri).

-Trimalaconothrus (T.) crispus Hammer, 1962

DisTRIBUCIÓN: Chile y Nueva Zelanda.

-Trimalaconothrus (T.) eichhornicus Iglesias, Palacios-Vargas y Mahunka, 2001

DisTRIBUCIÓN: Méjico.

-Trimalaconothrus (T.) elegans (Hammer, 1966) (Zeanothrus)

DistriBución: Nueva Zelanda.

-Trimalaconothrus (T.) flagelliformis Wallwork, 1970

Distribución: Antártica (I. Georgia del Sur).

-Trimalaconothrus (T.) foveolatus Willmann, 1931

DisTRIBUCIÓN: Boreal y Oriental (Indonesia).

-Trimalaconothrus (T.) foveolatus foveolatus Willmann, 1931 DistRIBUCIÓN: Boreal (Europa: menos frecuente en el Sur, Pakistán y Neártica septentrional).

-Trimalaconothrus (T.) foveolatus sundaicus Willmann, 1931 DisTRIBUCIÓN: Oriental (Indonesia).

-Trimalaconothrus (T.) grandis Hammen, 1952

DisTRIBUCIÓN: Paleártica (Europa: Holanda, y Japón).

-Trimalaconothrus (T.) granulatus Tseng, 1982

DisTRIBUCIÓN: Taiwan [Formosa].

-Trimalaconothrus (T.) hakonensis Yamamoto, 1977

DisTRIBUCIÓN: Este de Paleártica oriental.

-Trimalaconothrus (T.) heterotrichus Wallwork, 1973

DisTRIBUCión: Camerún.

-Trimalaconothrus (T.) indusiatus (Berlese, 1916) (Malaconothrus (T.)) DisTRIBUCIÓN: Europa.

-Trimalaconothrus (T.) iteratus nom. nov.

[=Trimalaconothrus longirostris Hammer, 1966, "nom. praeoc." por Willmann, 1929 ("hom. prim.")]

DisTRIBUCión: Nueva Zelanda e India (Orissa).

-Trimalaconothrus (T.) joonsooi Choi, 1986

DisTRIBUCión: Corea.

-Trimalaconothrus (T.) kurikii Yamamoto, 1997

DisTRIBUCIÓN: Japón.

-Trimalaconothrus (T.) lineolatus J. y P. Balogh, 1986

DisTRIBUCIÓN: Nueva Guinea.

-Trimalaconothrus (T.) longirostris Willmann, 1929

Distribución: Oriental (Indonesia).

-Trimalaconothrus (T.) longisetosus Yamamoto y Aoki, 2000

DisTRIBUCIÓN: China suroriental.

-Trimalaconothrus (T.) maniculatus Fain y Lambrechts, 1987

DisTRIBUCIÓN: Europa central.

-Trimalaconothrus (T.) mirabilis Sergienko y Melamud, 1993

DisTRIBUCIÓN: Ucrania.

-Trimalaconothrus (T.) montanus Hammer, 1958

DisTRIBUCIÓN: Bolivia.

-Trimalaconothrus (T.) multipilosus J. y P. Balogh, 2002

DistriBución: Costa Rica.

-Trimalaconothrus (T.) nipponicus Yamamoto y Aoki, 1971

DisTRIBUCión: Este de Paleártica oriental.

-Trimalaconothrus (T.) nodosus Yamamoto, 1997

DisTRIBUCIÓN: Japón.

-Trimalaconothrus (T.) obsessus nom. nov.

[=Trimalaconothrus albulus Tseng, 1982, “nom. praeoc.” por Hammer, 1972 (“hom. prim.")]

DISTRIBUCIÓN: Taiwan [Formosa].

-Trimalaconothrus (T.) opisthoseta Hammer, 1966

Distribución: Nueva Zelanda.

-Trimalaconothrus (T.) oppositus P. Balogh, 1997

(=Trimalaconothrus oppositus J. y P. Balogh, 2002, "nom. praeoc." por P. Balogh, 1997)

DisTRIBUCIÓN: Brasil

-Trimalaconothrus (T.) oxyrhinus Hammer, 1962

DisTRIBUCIÓN: Chile y Nueva Zelanda.

-Trimalaconothrus (T.) pallidus Wallwork, 1977

Distribución: I. Santa Helena.

-Trimalaconothrus (T.) pilipes Willmann, 1933

DistriBución: Pequeñas Antillas.
-Trimalaconothrus (T.) planus Balogh, 1958 “sp. inq."

DisTRIBUCIÓN: Angola.

-Trimalaconothrus (T.) platyrhinus Hammer, 1962

DisTRIBUCIÓN: Neotropical (Chile y Venezuela) y Nueva Zelanda.

-Trimalaconothrus (T.) prahuensis Hammer, 1979

DISTRIBUCIÓN: Java.

-Trimalaconothrus (T.) rafalskii Olszanowski, 1997

Distribución: Polonia.

-Trimalaconothrus (T.) repetitus nom. nov.

[=Trimalaconothrus reticulatus Yamamoto, 1977, "nom. praeoc." por

Balogh, 1958 (“hom. prim.")]

DisTRIBUCIÓN: Japón.

-Trimalaconothrus (T.) reticulatus Balogh, 1958 "sp. inq."

DisTRIBUCión: Angola.

-Trimalaconothrus (T.) sacculus Hammer, 1966

DisTRIBUCIÓN: Nueva Zelanda.

-Trimalaconothrus (T.) scimitarum Tarras-Wahlberg, 1985

DisTRIBUCIÓN: Kenia.

-Trimalaconothrus (T.) scutatus Mihelcic, 1959

DisTRIBUCión: Austria.

-Trimalaconothrus (T.) similis Balogh, 1958 “sp. inq."

DistriBuCión: Angola.

-Trimalaconothrus (T.) simplex (Banks, 1895) (Nothrus)

DisTRIBUCIÓN: U.S.A. (Nueva York).

-Trimalaconothrus (T.) soror Balogh, 1958 "sp. inq."

DisTRIBUCIÓN: Angola.

-Trimalaconothrus (T.) tardus (Michael, 1888) (Nothrus)

Distribución: Paleártica (excepto Norte) y Neártica (U.S.A.:

Carolina del Norte, y Canadá).

-Trimalaconothrus (T.) tonkini Luxton, 1982

DisTRIBUCión: Nueva Zelanda.

-Trimalaconothrus (T.) vietsi Willmann, 1925

DistRIBUCión: Paleártica occidental (Euroatlántica y Cáucaso).

-Trimalaconothrus (T.) wallworki (Stary y Block, 1995) (Fossonothrus)

DisTRIBUCIÓN: Subantártica: I. Malvinas [I. Falkland].

-Trimalaconothrus (T.) yachidairaensis Yamamoto, Kuriki y Aoki, 1993

DISTRIBUCIÓN: Japón.

Trimalaconothrus (Tyrphonothrus) Knülle, 1957 (24 spp. y 2 sspp.)

(=Fossonothrus Hammer, 1962)

EsPeCIE TIPO: (Malaconothrus novus Sellnick, 1921) =Malaconothrus maior Berlese, 1910

DisTRIBUCIÓN: Cosmopolita (excepto Antártica).

-Trimalaconothrus (Tyrphonothrus) albulus Hammer, 1972 (Trim.)

DistriBución: Polinesia (Tahití).

-Trimalaconothrus (Tyrphonothrus) angustirostrum Hammer, 1966 (Trim.)

DisTRIBUCIÓN: Nueva Zelanda

-Trimalaconothrus (Tyrphonothrus) australis Hammer, 1958 (Trim.)

DisTRIBUCIÓN: Neotropical.

-Trimalaconothrus (Tyrphonothrus) barrancensis Hammer, 1961

(Trim.)

DisTRIBUCIÓN: Perú.

-Trimalaconothrus (Tyrphonothrus) blancus Hammer, 1961 (Trim.)

DistriBución: Perú.

-Trimalaconothrus (Tyrphonothrus) buresi Kunst, 1959 (Trim.)

DisTRIBUCión: Sur de Europa.

-Trimalaconothrus (Tyrphonothrus) cajamarcensis Hammer, 1961

(Trim.)

DistRIBUCIÓN: Perú e India nororiental.

-Trimalaconothrus (Tyrphonothrus) canopeus Iglesias, Palacios-Vargas

y Mahunka, 2001 (Trim.)

DistriBución: Méjico.

-Trimalaconothrus (Tyrphonothrus) crassisetosus Willmann, 1931

(Trim.)

DistriBUCión: Oriental (Indonesia) y Australiana (Islas del

Pacífico).

-Trimalaconothrus (Tyrphonothrus) crassisetosus crassisetosus

Willmann, 1931

DisTRIBUCIÓN: Oriental (Indonesia). 
-Trimalaconothrus (Tyrphonothrus) crassisetosus fijiensis Hammer 1971 (Trim.)

Distribución: Australiana (Islas del Pacífico)

-Trimalaconothrus (Tyrphonothrus) glaber (Michael, 1888) (Nothrus)

DisTRIBUCión: Holártica (Paleártica y U.S.A.) y Java.

-Trimalaconothrus (Tyrphonothrus) glaber glaber (Michael, 1898)

DisTRIBUCión: Holártica (Paleártica: frecuente, y U.S.A.).

-Trimalaconothrus (Tyrphonothrus) glaber javensis Willmann, 1931 (Trim.)

DisTRIBUCIÓN: Java.

-Trimalaconothrus (Tyrphonothrus) humeratus P. Balogh, 1997 (Trim.) (=Trimalaconothrus humeratus J. y P. Balogh, 2002, "nom. praeoc." por P. Balogh, 1997)

DisTRIBUCIÓN: Brasil.

-Trimalaconothrus (Tyrphonothrus) itatiaiae P. Balogh, 1997 (Trim.) (=Trimalaconothrus itatiaiae J. y P. Balogh, 2002, “nom. praeoc." por P. Balogh, 1997)

DisTRIBUCIÓN: Brasil.

-Trimalaconothrus (Tyrphonothrus) latus (Hammer, 1962) (Fossonothrus)

DISTRIBUCIÓN: Chile.

-Trimalaconothrus (Tyrphonothrus) lisosetosus Iglesias, PalaciosVargas y Mahunka, 2001 (Trim.)

DisTRIBUCIÓN: Méjico.

-Trimalaconothrus (Tyrphonothrus) magnilamellatus Yamamoto, 1996 (Trim.)

DisTRIBUCIÓN: Japón.

-Trimalaconothrus (Tyrphonothrus) magnisetosus Iglesias,

Palacios-Vargas y Mahunka, 2001 (Trim.)

DisTRIBUCión: Méjico.

-Trimalaconothrus (Tyrphonothrus) maior (Berlese, 1910) (Malaconothrus)

(=Trimalaconothrus intermedius Cooreman, 1941)

(=Malaconothrus novus Sellnick, 1921)

(=Malaconothrus sphagnicola Trägardh, 1910)

Distribución: Semicosmopolita (Holártica: frecuente en

Paleártica occidental, América austral, I. Santa Helena y Nueva Zelanda).

-Trimalaconothrus (Tyrphonothrus) novaezealandiae (Hammer, 1966) (Fossonothrus)

DisTRIBUCión: Nueva Zelanda.

-Trimalaconothrus (Tyrphonothrus) pitentzin Iglesias, Palacios-

Vargas y Mahunka, 2001 (Trim.)

DistriBución: Méjico.

-Trimalaconothrus (Tyrphonothrus) saxosus Knülle, 1957 (Trim.)

(?=Malaconothrus optatus Berlese, 1908 "sp. inq.")

DisTRIBUCIÓN: Paleártica (Europa meridional y Este de Rusia asiática).

-Trimalaconothrus (Tyrphonothrus) sculptus Knülle, 1957 (Trim.)

DisTRIBUCión: Alemania.

-Trimalaconothrus (Tyrphonothrus) undulatus Yamamoto, Kuriki y Aoki, 1993 (Trim.)

DisTRIBUCIÓN: Japón.

-Trimalaconothrus (Tyrphonothrus) wuyanensis Yamamoto, Aoki, Wang y Hu, 1993 (Trim.)

DISTRIBUCIÓN: China suroriental.

-Trimalaconothrus (Tyrphonothrus) yunnanensis Yamamoto y Aoki, 1998 (Trim.)

DistRIBUCIÓN: China suroriental.

Nothridae Berlese, 1896 (3 gen., 73 spp. y 6 sspp.)

DisTRIBUCIÓN: Cosmopolita (excepto Antártica).

Nothrus Koch, 1836 (67 spp. y 6 sspp.)

(=Angelia Berlese, 1885)

(=Gymnonothrus Ewing, 1917)

(=Vigilomicrozetes Tseng, 1982 "sin. nov.")

EsPECIE TIPO: Nothrus palustris Koch, 1839

DisTRIBUCión: Cosmopolita (excepto Antártica)
-Nothrus akitaensis Fujikawa, 1999

DisTRIBUCIÓN: Japón.

-Nothrus anauniensis Canestrini y Fanzago, 1876

(=Nothrus pseudoborussicus Mahunka 1978)

DistriBución: Cosmopolita (excepto Antártica): frecuente en Paleártica.

-Nothrus angolensis Balogh, 1958 "sp. inq."

DisTRIBUCIÓN: Angola.

-Nothrus ashoroensis Fujikawa, 1999

DisTRIBUCIÓN: Japón.

-Nothrus asiaticus Aoki y Ohnishi, 1974 (N. palustris a.)

DISTRIBUCIÓN: Japón y China suroriental.

-Nothrus basilewskyi Balogh, 1958

DisTRIBUCIÓN: Tanzania.

-Nothrus becki Balogh y Mahunka, 1981

DISTRIBUCIÓN: Neotropical.

-Nothrus biciliatus Koch, 1841 "sp. inq."

DistriBUCIÓN: Alemania.

-Nothrus bicolor Koch, 1844 "sp. inq."

DisTRIBUCIÓN: Alemania.

-Nothrus bispinosus Koch, 1839 "sp. inq."

DisTRIBUCIÓN: Alemania.

-Nothrus borussicus Sellnick, 1928

DisTRIBUCIÓN: Holártica.

-Nothrus borussicus borussicus Sellnick, 1928

DistriBuCión: Holártica (frecuente en Paleártica).

-Nothrus borussicus neonominatus nom. nov.

[=Nothrus borussicus longipilus Mihelcic, 1959, "nom. praeoc." por Berlese, 1910 (“hom. sec.")]

DisTRIBUCIÓN: Austria.

-Nothrus brasilensis Pérez-Íñigo y Baggio, 1988

DISTRIBUCIÓN: Brasil.

-Nothrus brevirostris (Ewing, 1910) (Notaspis) "sp. inq."

DISTRIBUCIÓN: India (Orissa).

-Nothrus coniferus Gordeeva y Karppinen, 1984

DISTRIBUCIÓN: Este de Rusia asiática.

-Nothrus corticalis Karpelles, 1893 "sp. inq."

DISTRIBUCIÓN: Hungría.

-Nothrus crassisetus Mahunka, 1982

DisTRIBUCión: Etiopía.

-Nothrus crinitus (Berlese, 1916) (Angelia pulchella c.)

Distribución: "África oriental".

-Nothrus discifer Hammer, 1961

DisTRIBUCIÓN: Perú.

-Nothrus espinarensis Beck, 1962

DistRiBución: Perú.

-Nothrus ezoensis Fujikawa, 1999

DisTRIBUCIÓN: Japón.

-Nothrus flagellum Csiszár, 1961

DisTRIBUCión: Java.

-Nothrus gracilis Hammer, 1961

DistRIBUCIÓN: Neotropical e India (Bengala Occidental).

-Nothrus hatanoensis Fujikawa, 1999

DISTRIBUCIÓN: Japón.

-Nothrus hauseri Mahunka, 1974

DistriBUCión: Rodesia [Zimbabue].

-Nothrus ishikariensis Fujikawa, 1999

DisTRIBUCIÓN: Japón.

-Nothrus jaliscoensis Palacios-Vargas e Iglesias, 1997

DistriBuCión: Méjico.

-Nothrus leleupi Balogh, 1958

DisTRIBUCIÓN: Tanzania.

-Nothrus longipilus (Berlese, 1910) (Angelia anauniensis l.)

DISTRIBUCIÓN: Europa centromeridional.

-Nothrus lucunosus Sergienko y Melamud, 1993

DISTRIBUCIÓN: Ucrania.

-Nothrus lugubris Canestrini, 1898 "sp. inq."

DisTRIBUCIÓN: Nueva Guinea.

-Nothrus macedi Beck, 1962

DisTRIBUCIÓN: Neotropical (Perú y Argentina). 
-Nothrus madagascarensis Mahunka, 2000

DisTRIBUCIÓN: Madagascar.

-Nothrus magnus Palacios-Vargas e Iglesias, 1997

DistriBución: Méjico.

-Nothrus meakanensis Fujikawa, 1999

DisTRIBUCIÓN: Japón.

-Nothrus minimus Koch, 1844 "sp. inq."

DisTRIBUCión: Alemania.

-Nothrus mirabilis Sergienko y Melamud, 1993

DISTRIBUCIÓN: Ucrania.

-Nothrus monodactylus (Berlese, 1910) (Angelia anauniensis m.)

(=Nothrus terminalis Banks, 1910)

(=Nothrus terminalis carolinae Jacot, 1937)

DisTRIBUCIÓN: U.S.A. (menos frecuente en el Norte).

-Nothrus monticola Hammer, 1961

DisTRIBUCIÓN: Neotropical y Bután.

-Nothrus mystax Mahunka, 1986

DISTRIBUCIÓN: Tanzania.

-Nothrus oblongus Hammer, 1961

DisTRIBUCIÓN: Perú e India (Bengala Occidental).

-Nothrus obsoletus (Koch, 1841) (Celaeno) "sp. inq."

DisTRIBUCIÓN: Alemania.

-Nothrus oceanicus Sellnick, 1959

DisTRIBUCión: Islas del Pacífico y Oriental (Indonesia).

-Nothrus ovivorus Packard, 1868 "sp. inq."

(=Oribata aspidioti Ashmead, 1879)

DISTRIBUCIÓN: U.S.A. oriental.

-Nothrus palustris Koch, 1839

DisTRIBUción: Holártica e I. Santa Helena.

-Nothrus palustris palustris Koch, 1839

(=Nothrus pallens Koch, 1844)

DistriBución: Holártica (frecuente en Paleártica) e I. Santa Helena.

-Nothrus palustris azorensis Pérez-Íñigo, 1987

DISTRIBUCIÓN: I. Azores.

-Nothrus palustris bipilis Banks, 1895 (N. b.)

DISTRIBUCIÓN: Neártica septentrional.

-Nothrus parvus Sitnikova, 1975

DisTRIBUCión: Paleártica: Cáucaso y Paleártica oriental (excepto Sur).

-Nothrus perezinigoi Mahunka, 1980

DisTRIBUCIÓN: Holártica (Europa centooccidental y U.S.A.

Carolina del Norte)

-Nothrus peruensis Hammer, 1961

DISTRIBUCIÓN: Neotropical.

-Nothrus pileiformis Karpelles, 1884 "sp. inq."

Distribución: U.S.A. (Pensilvania).

-Nothrus posticus Koch, 1839 “sp. inq."

DistriBución: Alemania.

-Nothrus praeoccupatus nom. nov.

[=Vigilomicrozetes pulchellus Tseng, 1982,"nom. praeoc.” por Berlese, 1910 ("hom. sec.")]

DISTRIBUCIÓN: Taiwan [Formosa].

-Nothrus pratensis Sellnick, 1928

DisTRIBUCIÓN: Holártica (menos frecuente en el Sur).

-Nothrus pulchellus (Berlese, 1910) (Angelia)

DisTRIBUCIÓN: Europa centromeridional.

-Nothrus pumilatus Golosova y Karppinen, 1985

DistriBUCión: I. Kuriles.

-Nothrus quadripilus Ewing, 1909

DisTRIBUCión: U.S.A. oriental.

-Nothrus reticulatus Sitnikova, 1975

DisTRIBUCIÓN: Islas del Este de Rusia asiática

-Nothrus reunionensis Mahunka, 1978

DisTRIBUCióN: Malgache (I. Reunión)

-Nothrus sadoensis Fujikawa, 1999

DisTRIBUCIÓN: Japón.

-Nothrus senegalensis Mahunka, 1992

DisTRIBUCión: Senegal.

-Nothrus septatus Golosova y Karppinen, 1985

DistriBUCIÓN: Norte de Rusia europea.
-Nothrus seychellensis Warburton, 1912

(=Nothrus scotti Warburton, 1912)

DisTRIBUCIÓN: I. Seychelles.

-Nothrus silvestris Nicolet, 1855

DisTRIBUCIÓN: Holártica y Australiana.

-Nothrus silvestris silvestris Nicolet, 1855

DisTRIBUCIÓN: Holártica (frecuente) y Nueva Zelanda.

-Nothrus silvestris bistilus Jacot, 1937

DISTRIBUCIÓN: Este de U.S.A.

-Nothrus silvestris exilior Jacot, 1937

Distribución: U.S.A. (Kentucky).

-Nothrus silvicus Jacot, 1937 (N. truncatus s.)

DisTRIBUCIÓN: U.S.A. oriental.

-Nothrus springsmythi Sheals, 1965

DisTRIBUCIÓN: Nepal.

-Nothrus suramericanus Hammer, 1958

DisTRIBUCIÓN: Neotropical.

-Nothrus taisetsuensis Fujikawa, 1999

DISTRIBUCIÓN: Japón.

-Nothrus truncatus Banks, 1895

DisTRIBUCIÓN: U.S.A.

-Nothrus truncatus truncatus Banks, 1895

DisTRIBUCIÓN: U.S.A. oriental.

-Nothrus truncatus robustus Jacot, 1937

DisTRIBUCIÓN: Noreste de U.S.A.

-Nothrus willmanni Mahunka, 1983

DisTRIBUCIÓN: Centroamérica.

Novonothrus Hammer, 1966 (5 spp.)

ESPECIE TIPO: Novonothrus flagellatus Hammer, 1966

DisTRIBUCIÓN: Subtropical austral (excepto Etiópica).

-Novonothrus covarrubiasi Casanueva y Norton, 1997

DisTRIBUCIÓN: Chile.

-Novonothrus flagellatus Hammer, 1966

DisTRIBUCIÓN: Australiana (Nueva Zelanda y Australia).

-Novonothrus kethleyi Casanueva y Norton, 1998

DisTRIBUCión: Chile.

-Novonothrus papuensis Hammer, 1966

DisTRIBUCIÓN: Nueva Zelanda.

-Novonothrus puyehue Casanueva y Norton, 1997

DistribuCIÓN: Chile.

Trichonothrus Mahunka, 1986 (1 sp.)

ESPECIE TIPO: Trichonothrus austroafricanus Mahunka, 1986

DISTRIBUCIÓN: Etiópica.

-Trichonothrus austroafricanus Mahunka, 1986

DisTRIBUCIÓN: Sudáfrica.

Camisiidae Oudemans, 1900 (3 gen., 3 subg., 78 spp. y 5 sspp.) DISTRIBUCIÓN: Cosmopolita.

Austronothrus Hammer, 1966 (1 sp.)

ESPECIE TIPO: Austronothrus curviseta Hammer, 1966

DISTRIBUCIÓN: Australiana.

-Austronothrus curviseta Hammer, 1966

DisTRIBUCIÓN: Nueva Zelanda.

Camisia Heyden, 1826 (33 spp. y 1 ssp.)

EsPeCIE TiPo: Notaspis segnis Hermann, 1804

DisTRIBUCIÓN: Cosmopolita.

Camisia (Camisia) Heyden, 1826 (29 spp. y 1 ssp.)

(=Uronothrus Berlese, 1913)

DisTRIBUCIÓN: Cosmopolita.

-Camisia (C.) abdosensilla Olszanowski y Clayton, 2002

DistriBuCión: Canadá.

-Camisia (C.) amictus (Sowerby, 1806) (Acarus) "sp. inq."

(=Nothrus angulatus Koch, 1839)

DISTRIBUCIÓN: Euroatlántica (I. Británicas y Alemania). 
-Camisia (C.) anomia Colloff, 1993

DistRIBUCIÓn: Suecia.

-Camisia (C.) arcuata Hammer, 1961

DISTRIBUCIÓN: Neotropical.

-Camisia (C.) australis Hammer, 1958

DistribuCión: América austral.

-Camisia (C.) biurus (Koch, 1839) (Nothrus)

DisTRIBUCIÓN: Holártica.

-Camisia (C.) biurus biurus (Koch, 1839)

(=Camisia exuvialis Grandjean, 1939)

(=Nothrus furcatus Koch, 1844)

(=Uronothrus kochi Willmann, 1943)

(=Nothrus pigerrimus Koch, 1844)

DisTRIBUCIÓN: Holártica.

-Camisia (C.) biurus amicus Jacot, 1938 (C. segnis a.) Distribución: U.S.A. (Carolina del Norte).

-Camisia (C.) biverrucata (Koch, 1839) (Nothrus)

(=Camisia berlesei Oudemans, 1900)

(=Camisia fischeri Oudemans, 1900)

(=Camisia nicoleti Oudemans, 1900)

DisTRIBUCIÓN: Holártica (frecuente en la Paleártica).

-Camisia (C.) carrolli André, 1980

DisTRIBUCIÓN: U.S.A. (Oregón).

-Camisia (C.) dictyna Colloff, 1993

Distribución: Holártica (Canadá, U.S.A.: California, y Este de Rusia asiática)

-Camisia (C.) flagellata (Berlese, 1888) (Nothrus)

DisTRIBUCIÓN: Argentina.

-Camisia (C.) foveolata Hammer, 1955

Distribución: Boreal (Alaska, Canadá, Groenlandia y Norte de Europa).

-Camisia (C.) grymae Colloff, 1993

DisTRIBUCIÓN: Australia.

-Camisia (C.) hamulifera Hammer, 1961

DistRIBUCIÓN: Neotropical, Sudáfrica y subantártica (I. Tristán da Cunha).

-Camisia (C.) heterospinifer Fujikawa, 1998

Distribución: Japón.

-Camisia (C.) horrida (Hermann, 1804) (Notaspis)

(=Nothrus bistriatus Koch, 1839)

(=Nothrus borealis Thorell, 1871)

(=Nothrus mutilus Koch, 1839)

(=Nothrus rugulosus Banks, 1895)

(=Nothrus runcinatus Koch, 1839)

(=Nothrus sinuatus Koch, 1839)

DISTRIBUCIÓN: Holártica (frecuente).

-Camisia (C.) invenusta (Michael, 1888) (Nothrus)

DisTRIBUCIÓN: Paleártica.

-Camisia (C.) khencensis Hammer, 1961

Distribución: Perú.

-Camisia (C.) nortoni Colloff, 1993

DistriBuCión: Pequeñas Antillas.

-Camisia (C.) nova (Hammer, 1966) (C. segnis n.)

Distribución: Australiana (Nueva Zelanda y Hawai) y Neotropical (frecuente).

-Camisia (C.) oregonae Colloff, 1993

DisTRIBUCIÓN: Neártica (U.S.A.: Oregón, y Canadá).

-Camisia (C.) orthogonia Olszanowski, Szywilewska y Norton, 2001

Distribución: Oeste de Neártica.

-Camisia (C.) polytricha Piffl, 1971

DisTRIBUCIÓN: Nepal.

-Camisia (C.) segnis (Hermann, 1804) (Notaspis)

(=Nothrus bicarinatus Koch, 1839)

(=Nothrus excisus Banks, 1895)

(=Nothrus rostratus Koch, 1839)

(=Camisia segnis segnoides André, Lebrun y Leroy, 1984)

( $=$ Nothrus ventricosus Koch, 1844)

DistRIBUCIÓN: Semicosmopolita (Holártica: frecuente, Oriental, y Antártica).
-Camisia (C.) spinifer (Koch, 1835) (Nothrus)

(=Nothrus ansatus Haupt, 1882)

(=Nothrus concavus Haupt, 1882)

(=Nothrus echinatus Koch, 1835)

(=Nothrus quadracanthus Haupt, 1882)

(=Nothrus sordidus Koch, 1839)

(=Nothrus superbus Berlese, 1910$)$

(=Nothrus taurinus Banks, 1906)

DistRIBUCIÓN: Semicosmopolita (Holártica: frecuente, Norte de Oriental y Brasil).

-Camisia (C.) tatrica Olszanowski, 1994

DisTRIBUCIÓN: Polonia.

-Camisia (C.) tryphosa Colloff, 1993

Distribución: Kenia.

-Camisia (C.) umbratilis (Hull, 1915) (Nothrus) "sp. inq."

DistribuCión: I. Británicas.

-Camisia (C.) valeriae Colloff, 1993

DisTRIBUCIÓN: Borneo.

-Camisia (C.) zangherii Lombardini, 1963 "sp. inq."

DISTRIBUCIÓN: Italia.

Camisia (Ensicamisia) Kunst, 1971 (4 spp.)

(=Ivarsia Karppinen y Krivolutsky, 1987 "sin. nov.”)

EsPeCIE TIPO: Nothrus lapponicus Trägardh, 1910

DisTRIBUCIÓN: Holártica.

-Camisia (Ensicamisia) lapponica (Trägardh, 1910) (Nothrus)

(=Camisia labradorica Behan-Pelletier, 1978)

DisTRIBUCIÓN: Holártica (menos frecuente en el Sur)

-Camisia (Ensicamisia) presbytis Colloff, 1993 (C.)

DisTRIBUCIÓN: U.S.A. (Washington).

-Camisia (Ensicamisia) sibirica Karppinen y Krivolutsky, 1987 (C.

(Ivarsia))

Distribución: Sur de Siberia.

- Camisia (Ensicamisia) solhoeyi Colloff, 1993 (C.)

(=Camisia islandica Gjelstrup y Solhoy, 1994)

DisTRIBUCIÓN: Holártica (Europa occidental, Japón y Canadá).

Heminothrus Berlese, 1913 (44 spp. y 4 sspp.)

EsPECIE TIPO: Nothrus targionii Berlese, 1885

DISTRIBUCIÓN: Cosmopolita.

Heminothrus (Heminothrus) Berlese, 1913 (13 spp.)

(=Paulonothrus Kunst, 1971 "sin. nov.")

DISTRIBUCIÓN: Cosmopolita (excepto Neotropical y Antártica).

-Heminothrus (H.) apophysiger Hammer, 1979

DistRIBUCIÓN: Java.

-Heminothrus (H.) exaggeratus Hammer, 1979

DisTRIBUCIÓN: Java.

-Heminothrus (H.) glaber Mahunka, 1984

DisTRIBUCIÓN: Tanzania.

-Heminothrus (H.) interlamellaris Jacot, 1939 (H. longisetosus i.)

DistRibuCión: U.S.A. (Nueva Hampshire).

-Heminothrus (H.) leleupi (Balogh, 1958) (Platynothrus)

DisTRIBUCIÓN: África central.

-Heminothrus (H.) longisetosus Willmann, 1925 (H. paolianus l.)

DisTRIBUCIÓN: Boreal (frecuente).

-Heminothrus (H.) microclava Hammer, 1966

DisTRIBUCIÓN: Nueva Zelanda.

-Heminothrus (H.) minor Aoki, 1969

Distribución: Este de Paleártica oriental.

-Heminothrus (H.) ornatissimus (Berlese, 1910) (Angelia)

DISTRIBUCIÓN: U.S.A. oriental.

-Heminothrus (H.) oromii Morell y Subías, 1991

DisTRIBUCIÓN: I. Azores.

-Heminothrus (H.) paolianus (Berlese, 1913) (Nothrus (H.))

DisTRIBUCióN: Holártica (Europa, Asia central, U.S.A.: Michigan, y Groenlandia).

-Heminothrus (H.) similis Fujikawa, 1998

DisTRIBUCIÓN: Japón. 
-Heminothrus (H.) targionii (Berlese, 1885) (Nothrus)

(=Nothrus (Heminothrus) princeps Berlese, 1916)

DisTRIBUCIÓN: Holártica (frecuente en Paleártica).

Heminothrus (Capillonothrus) Kunst, 1971 (8 spp. y 2 sspp.)

(=Ovonothrus Kunst, 1971 "sin. nov.")

(=Paracamisia Olszanowski y Norton, 2002 "sin. nov.")

ESPECIE TIPO: Angelia thori Berlese, 1904

DisTRIBUCIÓN: Holártica y Neotropical.

-Heminothrus (Capillonothrus) capillatus (Berlese, 1914) (Angelia)

DistRIBUCIÓN: Holártica (Paleártica, U.S.A.: California, y

Groenlandia)

-Heminothrus (Capillonothrus) capillatus capillatus (Berlese, 1914)

(=Platynothrus capillatus septentrionalis Sellnick, 1944)

(=Platynothrus maior Willmann, 1956)

DistribuCión: Holártica (Paleártica, U.S.A.: California, y Groenlandia).

-Heminothrus (Capillonothrus) capillatus kikonaiensis (Fujikawa, 1982) (Platynothrus)

DISTRIBUCIÓN: Japón.

-Heminothrus (Capillonothrus) capillatus peltiferinus (Winkler, 1957) (Platynothrus)

DisTRIBUCIÓN: Chequia.

-Heminothrus (Capillonothrus) fluviatilis (Hull, 1913) (Hermannia)

DisTRIBUCIÓN: I. Británicas.

-Heminothrus (Capillonothrus) meakanensis (Fujikawa, 1982) (Pla-

tynothrus)

DisTRIBUCIÓN: Japón.

-Heminothrus (Capillonothrus) nevadensis (Pérez-Íñigo, 1969)

(Platynothrus)

DisTRIBUCIÓN: España.

-Heminothrus (Capillonothrus) numatai (Aoki, 1965) (Platynothrus)

DisTRIBUCIÓN: Himalaya.

-Heminothrus (Capillonothrus) osornensis (Olszanowski y Norton 2002) (Paracamisia)

Distribución: Chile.

-Heminothrus (Capillonothrus) thori (Berlese, 1904) (Angelia)

(=Nothrus crinitus Warburton y Pearce, 1905)

DisTRIBUCIÓN: Holártica (Paleártica: frecuente, y Neártica: menos frecuente en el Sur).

-Heminothrus (Capillonothrus) yamasakii Aoki, 1958 (H.)

DisTRIBUCIÓN: Paleártica oriental (excepto Siberia): frecuente.

Heminothrus (Platynothrus) Berlese, 1913 (23 spp. y 2 sspp.)

(=Neonothrus Forsslund, 1955)

(=Sigmonothrus Chakrabarti y Kundu, 1978 "sin. nov.")

EsPECIE TIPO: Nothrus peltifer Koch, 1839

DISTRIBUCIÓN: Cosmopolita.

-Heminothrus (Platynothrus) altimontanus (Hammer, 1958) (P.)

DistRIBUCIÓN: Neotropical.

-Heminothrus (Platynothrus) banksi (Michael, 1898) (Nothrus)

(=Nothrus furcatus Banks, 1895, "nom. praeoc." por Koch, 1844)

DisTRIBUCIÓN: Neártica occidental y China suroriental.

-Heminothrus (Platynothrus) biangulatus (Hammer, 1962) (P.)

DistriBuCión: América austral.

-Heminothrus (Platynothrus) bicarinatus (Jacot, 1938) (P.)

DisTRIBUCIÓN: Neártica y Panamá.

-Heminothrus (Platynothrus) bistriatus (Chakrabarti y Mondal, 1978)

(Sigmonothrus)

DisTRIBUCIÓN: India (Bengala Occidental)

-Heminothrus (Platynothrus) brevisetosus (Lee, 1985) (P.)

DisTriBución: Australia.

-Heminothrus (Platynothrus) carinatus (Banks, 1910) (Nothrus)

("non" Hermannia carinata Kramer, 1897, =Heminothrus (Platynothrus)

punctatus)

DisTRIBUCIÓN: U.S.A. oriental.

-Heminothrus (Platynothrus) castaneus (Hammer, 1961) (P.)

Distribución: Neotropical (Perú y Chile).

-Heminothrus (Platynothrus) fossatus (Kramer, 1898) (Nothrus)

"sp. inq."

DisTRIBUCión: "Neotropical".
-Heminothrus (Platynothrus) hooki (Piffl, 1966) (P.)

DisTRIBUCIÓN: Kenia.

-Heminothrus (Platynothrus) humicola (Forsslund, 1955) (Neonothrus)

DistRIBUCIÓN: Boreal.

-Heminothrus (Platynothrus) novaezealandicus (J. y P. Balogh, 2002) (P.)

(=Platynothrus maior Hammer, 1966, “nom. praeoc." por Willmann, 1956) DistRIBUCión: Nueva Zelanda.

-Heminothrus (Platynothrus) ovatus (Kundu y Mondal, 1978)

(Sigmonothrus)

DisTRIBUCIÓN: India (Bengala Occidental).

-Heminothrus (Platynothrus) peltifer (Koch, 1839) (Nothrus)

Distribución: Semicosmopolita (Holártica, Norte de Oriental,

Australiana: Nueva Zelanda, Norte de Neotropical e I. Santa

Helena).

-Heminothrus (Platynothrus) peltifer peltifer (Koch, 1839)

(=Heminothrus abchasicus Tarba, 1990)

(=Nothrus bicristatus Haupt, 1882)

(=Nothrus cirrosus Canestini y Fanzago, 1876)

(=Platynothrus grandjeani Sitnikova, 1975)

(?=Acarus infusionem Schrank, 1803 “sp. inq.")

(=Nothrus palliatus Koch, 1839)

(=Nothrus spirofilus Haupt, 1882)

DistRIBUCIÓN: Semicosmopolita (Holártica: frecuente, Norte de

Oriental, Australiana: Nueva Zelanda, Norte de Neotropical e I.

Santa Helena).

-Heminothrus (Platynothrus) peltifer japonensis (Fujikawa, 1972) (P.)

DisTRIBUCIÓN: Japón.

-Heminothrus (Platynothrus) praeoccupatus nom. nov.

[=Sigmonothrus quadristriatus Chakrabarti y Kundu, 1978, "nom. praeoc." por Hammer, 1958 ("hom. sec.")]

DistRIBUCIÓN: India (Bengala Occidental).

-Heminothrus (Platynothrus) punctatus (L. Koch, 1879) (Nothrus)

(=Hermannia carinata Kramer, 1897)

(=Heminothrus valentianus Hull, 1916)

DISTRIBUCIÓN: Boreal.

-Heminothrus (Platynothrus) quadristriatus (Hammer, 1958) (P.)

DisTRIBUCIÓN: Argentina.

-Heminothrus (Platynothrus) reductus (J. y P. Balogh, 1986) (P.)

DisTRIBUCIÓN: Nueva Guinea.

-Heminothrus (Platynothrus) robustior Berlese, 1916 (H.)

DisTRIBUCIÓN: Neotropical (Argentina y Uruguay).

-Heminothrus (Platynothrus) sibiricus (Sitnikova, 1975) (P.)

DistRIBUCIÓN: Holártica (Paleártica oriental y Neártica).

-Heminothrus (Platynothrus) skoettsbergi (Trägardh, 1931) (P.)

Distribución: Austral (América austral, Nueva Zelanda y Antártica: I. Georgia del Sur).

-Heminothrus (Platynothrus) skoettsbergi skoettsbergi (Trägardh, 1931)

DisTRIBUCIÓN: Neotropical y Nueva Zelanda.

-Heminothrus (Platynothrus) skoettsbergi expansus (Wallwork, 1966) (P.)

DistRIBUCIÓN: Antártica (I. Georgia del Sur)

-Heminothrus (Platynothrus) tenuiclava (Hammer, 1966) (P.)

DisTRIBUCión: Nueva Zelanda.

-Heminothrus (Platynothrus) traversus Hammer, $1966(H$.

DistriBuCión: Australiana (Nueva Zelanda y Australia).

Crotoniidae Thorell, 1876 (2 gen. y 45 spp.)

DistribuCión: Cosmopolita (excepto Holártica y Oriental).

Crotonia Thorell, 1876 (32 spp.)

(=Acronothrus Berlese, 1916)

(=Westwoodia Cambridge, 1875, "nom. praeoc." por Brullé, 1846)

EsPeCIE TIPO: Westwoodia obtecta Cambridge, 1875

Distribución: Cosmopolita (excepto Holártica y Oriental)

-Crotonia allaudi (Berlese, 1916) (Nothrus (Acronothrus))

DisTRIBUCIÓN: "África oriental". 
-Crotonia americana (Berlese, 1901) (Neoliodes)

DisTRIBUCIÓN: América austral.

-Crotonia ardala Luxton, 1987

DisTRIBUCIÓN: Australia.

-Crotonia borbora Luxton, 1987

DisTRIBUCIÓN: Australia.

-Crotonia brassicae Wallwork, 1977

Distribución: I. Santa Helena.

-Crotonia brevicornuta (Wallwork, 1966) (Acronothrus)

DisTRIBUCIÓN: Antártica (I. Clampbell).

-Crotonia camelus (Berlese, 1910) (Nothrus)

DisTRIBUCIÓN: Nueva Caledonia.

-Crotonia capistrata Luxton, 1987

DistriBución: Australia.

-Crotonia caudalis (Hammer, 1966) (Acronothrus)

DisTRIBUCIÓN: Nueva Zelanda.

-Crotonia cervicorna Luxton, 1982

DisTRIBUCIÓN: Nueva Zelanda.

-Crotonia chiloensis Wallwork, 1977

DisTRIBUCIÓN: Chile.

-Crotonia cophinaria (Michael, 1908) (Nothrus)

(=Acronothrus brachyrostrum Hammer, 1966)

DisTRIBUCIÓN: Nueva Zelanda y Argentina.

-Crotonia cupulata Luxton, 1982

DisTRIBUCIÓN: Nueva Zelanda.

-Crotonia dicella Colloff, 1990

DisTRIBUCIÓN: Sudáfrica.

-Crotonia ecphyla Colloff, 1990

DisTRIBUCIÓN: Sudáfrica.

-Crotonia flagellata (Balogh y Csiszár, 1966) (Acronothrus)

DISTRIBUCIÓN: América austral.

-Crotonia jethurmerae Lee, 1985

DisTRIBUCIÓN: Australia.

-Crotonia lanceolata Wallwork, 1977

DistriBuCión: I. Santa Helena.

-Crotonia longibulbula Luxton, 1982

DisTRIBUCIÓN: Nueva Zelanda.

-Crotonia lyrata Colloff, 1990

DisTRIBUCIÓN: Sudáfrica.

-Crotonia marlenae Olszanowski, 1977

DisTRIBUCIÓN: Brasil.

-Crotonia nukuhivae (Jacot, 1934) (Acronothrus)

(=Crotonia melanesiae Wallwork, 1977)

(=Acronothrus nukuhivae hivaoae Jacot, 1934)

DistRIBUCIÓN: Australiana (Islas del Pacífico).

-Crotonia obtecta (Cambridge, 1875) (Westwoodia)

DisTRIBUCión: Nueva Zelanda y Chile.

-Crotonia ovata Olszanowki, 2000

DISTRIBUCIÓN: Tasmania.

-Crotonia pauropelor Colloff, 1990

DisTRIBUCiÓN: Sudáfrica.

-Crotonia perforata Wallwork, 1977

DISTRIBUCIÓN: I. Santa Helena.

-Crotonia pulchra (Beck, 1962) (Acronothrus)

DisTRIBUCIÓN: Neotropical (Perú y Ecuador).

-Crotonia reticulata Luxton, 1982

DisTRIBUCIÓN: Nueva Zelanda.

-Crotonia rothschildi (Berlese, 1916) (Nothrus (Acronothrus))

DISTRIBUCIÓN: Etiopía.

-Crotonia tryjanowskii Olszanowski, 2000

DisTRIBUCIÓN: Australia.

-Crotonia tuberculata Luxton, 1982

DisTRIBUCIÓN: Nueva Zelanda.

-Crotonia unguifera (Michael, 1908) (Nothrus)

DisTRIBUCIÓN: Nueva Zelanda.

Holonothrus Wallwork, 1963 (13 spp.)

EsPECIE TIPO: Holonothrus foliatus Wallwork, 1963

DisTRIBUCIÓN: Cosmopolita (excepto Holártica y Oriental).
-Holonothrus artus Olszanowski, 1999

DisTRIBUCión: Chile y Melanesia (I. Salomón).

-Holonothrus concavus Wallwork, 1966

DisTRIBUCIÓN: Antártica (I. Campbell).

-Holonothrus foliatus Wallwork, 1963

DISTRIBUCIÓN: Antártica (I. Macquarie).

-Holonothrus gracilis Olszanowski, 1997

DisTRIBUCIÓN: Nueva Zelanda.

-Holonothrus mitis Olszanowski, 1991

DisTRIBUCIón: Australiana (Tasmania y Australia).

-Holonothrus naskreckii Olszanowski, 1997

DistriBUCIÓN: Nueva Zelanda.

-Holonothrus nortoni Olszanowski, 1999

DistRIBUCión: Pequeñas Antillas.

-Holonothrus novaecaledoniae Olszanowski, 1997

DistRIBUCIÓn: Nueva Caledonia.

-Holonothrus papua J. y P. Balogh, 1986

DistRIBUCIÓN: Nueva Guinea.

-Holonothrus pulcher Hammer, 1966

DISTRIBUCIÓN: Nueva Zelanda.

-Holonothrus robustus Olszanowski, 1991

DistriBuCión: Australiana (Tasmania y Australia).

-Holonothrus venetiolanus Olszanowski, 1999

DisTRIBUCIÓN: Neotropical (Venezuela y Chile).

-Holonothrus virungensis Norton y Olszanowski, 1989

DisTRIBUCión: Congo.

\section{Nanhermannioidea Sellnick, 1928}

Nanhermanniidae Sellnick, 1928 ( 7 gen., 1 subg. y 58 spp.) DisTRIBUCIÓN: Cosmopolita (excepto Antártica).

Bicyrthermannia Hammer, 1979 (4 spp.)

EsPECIE TIPO: Bicyrthermannia duodentata Hammer, 1979

DISTRIBUCIÓN: Tropical (Oriental y Neotropical).

-Bicyrthermannia bicornuta (Haq, 1978) (Cyrthermannia)

DISTRIBUCIÓN: India.

-Bicyrthermannia cagayana (Corpuz-Raros, 1979) (Cyrthermannia)

DistRIBUCIÓN: Filipinas.

-Bicyrthermannia duodentata Hammer, 1979

DISTRIBUCIÓN: Oriental (Indonesia).

-Bicyrthermannia foliata (Mahunka, 1985) (Cyrthermannia)

DistriBuCión: Pequeñas Antillas.

Cosmohermannia Aoki y Yoshida, 1970 (2 spp.)

EsPeCIE TIPO: Cosmohermannia frondosa Aoki y Yoshida, 1970

DISTRIBUCIÓN: Paleártica meridional y Australiana.

Cosmohermannia frondosa Aoki y Yoshida, 1970

DisTRIBUCión: Este de Paleártica oriental.

-Cosmohermannia robusta (Aoki, 1994) (Nippohermannia)

DisTRIBUCIÓN: Micronesia (I. Marianas).

Cyrthermannia Balogh, 1958 (13 spp.)

(=Linothrus Tseng, 1982 "sin. nov.")

ESPECIE TIPO: Cyrthermannia tuberculata Balogh, 1958

DisTRIBUCIÓN: Pantropical.

-Cyrthermannia baloghorum Pérez-Íñigo y Baggio, 1988

DISTRIBUCIÓN: Brasil.

-Cyrthermannia bicornicula Tseng, 1982

DisTRIBUCión: Taiwan [Formosa].

-Cyrthermannia ezzati Bayoumi y Mahunka, 1977

DistribuCión: Egipto.

-Cyrthermannia florens Balogh y Mahunka, 1980

DistriBución: Cuba.

-Cyrthermannia formosana Tseng, 1982

DistriBUCIÓN: Taiwan [Formosa].

-Cyrthermannia guadeloupensis Mahunka, 1985

DisTRIBUCión: Centroamérica.

-Cyrthermannia luminosa Hammer, 1971

Distribución: Australiana (Islas del Pacífico). 
-Cyrthermannia perinfamis (Tseng, 1982) (Linothrus) "sp. inq." DisTRIBUCIóN: Taiwan [Formosa].

-Cyrthermannia quadricornuta Chakrabarti y Bhaduri, 1978

DisTRIBUCIÓN: India (Bengala Occidental).

-Cyrthermannia simplex Mahunka, 1985

DisTRIBUCIÓN: Centroamérica.

-Cyrthermannia stellata Balogh, 1970

Distribución: Nueva Guinea y Ceilán [Sri Lanka].

-Cyrthermannia tuberculata Balogh, 1958

DisTRIBUCión: Congo, Egipto y Java.

-Cyrthermannia vicinicornuta Aoki, 1965

DISTRIBUCIÓN: Oriental.

Dendrohermannia P. Balogh, 1985 (1 sp.)

EsPecie TIPo: Cosmohermannia monstruosa Aoki, 1977

DISTRIBUCIÓN: Australiana.

-Dendrohermannia monstruosa (Aoki, 1977) (Cosmohermannia)

DisTRIBUCIÓN: Nueva Guinea.

Masthermannia Berlese, 1913 (6 spp.)

(=Phyllonothrus Sellnick, 1959)

(=Posthermannia Grandjean, 1954)

EsPECIE TIPO: Angelia mammillaris Berlese, 1904

DisTRIBUCIÓN: Pantropical y subtropical.

-Masthermannia extrema (Balogh, 1958) (Posthermannia) "sp. inq." DisTRIBUCIÓN: Congo.

-Masthermannia grandjeani (Balogh, 1958) (Posthermannia) “sp. inq."

Distribución: Congo.

-Masthermannia hirsuta (Hartman, 1949) (Nanhermannia)

DisTRIBUCión: Este de Paleártica oriental y U.S.A. (Tejas)

-Masthermannia mammillaris (Berlese, 1904) (Angelia)

(=Posthermannia nematophora Grandjean, 1954)

Distribución: Pantropical (excepto Etiópica) y subtropical.

-Masthermannia ornatissima Pérez-Íñigo y Baggio, 1988

DISTRIBUCIÓN: Brasil.

-Masthermannia runcifer (Sellnick, 1959) (Phyllonothrus)

DisTRIBUCIÓN: Polinesia: I. Australes [I. Tubuai].

Nanhermannia Berlese, 1913 (31 spp.)

EsPECIE TIPO: Nothrus nanus Nicolet, 1855

DisTRIBUCIÓN: Cosmopolita (excepto Antártica).

Nanhermannia (Nanhermannia) Berlese, 1913 (30 spp.)

DisTRIBUCIÓN: Cosmopolita (excepto Antártica).

-Nanhermannia (N.) acutisetosa Hammer, 1966

DistRIBUCIÓN: Nueva Zelanda.

-Nanhermannia (N.) bifurcata Fujikawa, 1990

DisTRIBUCIÓN: Japón.

-Nanhermannia (N.) comitalis Berlese, 1916

(=Nanhermannia ussurica Golosova, 1969)

DisTRIBUCIÓN: Holártica (Paleártica: menos frecuente en el Norte y Noreste de U.S.A.)

-Nanhermannia (N.) continua Golosova y Karppinen, 1985

DisTRIBUCión: Mongolia.

-Nanhermannia (N.) domrowi Balogh y Mahunka, 1978

DistRIBUCIÓN: Australia.

-Nanhermannia (N.) dorsalis (Banks, 1896) (Carabodes)

(=Nanhermannia coronata Berlese, 1913)

DistRIBUCIÓN: Holártica y Neotropical.

-Nanhermannia (N.) elegantissima Hammer, 1958

DisTRIBUCIÓN: Neotropical.

-Nanhermannia (N.) elegantula Berlese, 1913

(=Nanhermannia areolata Strenzke, 1953)

DistRIBUCión: Holártica e I. Santa Helena.

-Nanhermannia (N.) fenneri Balogh, 1970

DisTRIBUCIÓN: Nueva Guinea y Filipinas.

-Nanhermannia (N.) forsslundi Karppinen, 1958

Distribución: Paleártica (Norte de Europa y Sur de Siberia)

-Nanhermannia (N.) gladiata P. Balogh, 1985

Distribución: Australia.
-Nanhermannia (N.) gorodkovi Sitnikova, 1975

Distribución: Paleártica oriental (Asia central rusa e I. Kuriles).

-Nanhermannia (N.) grandjeani Lee, 1985

DistRIBUCIÓN: Australia.

-Nanhermannia (N.) himalayensis Chakrabarti y Raychaudhuri, 1978

DistRIBUCIÓN: India (Bengala Occidental).

-Nanhermannia (N.) komareki Kunst, 1956

DISTRIBUCIÓN: Europa centrooriental.

-Nanhermannia (N.) laevis Sitnikova, 1975

DisTRIBUCIÓN: I. Sajalín.

-Nanhermannia (N.) milloti Balogh, 1961

DisTRIBUCIÓN: Malgache.

-Nanhermannia (N.) nana (Nicolet, 1855) (Nothrus)

DistriBUCIÓN: Cosmopolita (Neotropical, Norte de Oriental, Nueva

Zelanda, I. Santa Helena, Antártica y frecuente en Holártica).

-Nanhermannia (N.) nasata (Warburton, 1912) (Hermannia)

DisTRIBUCIÓN: I. Seychelles.

-Nanhermannia (N.) pectinata Strenzke, 1953

Distribución: Paleártica centromeridional.

-Nanhermannia (N.) pluriseta Mahunka, 1984

DisTRIBUCIÓN: Tanzania.

-Nanhermannia (N.) quadridentata Balogh, 1958

DISTRIBUCIÓN: Etiópica.

-Nanhermannia (N.) sabahensis Fujikawa, 1990

(=Nanhermannia pluriseta Mahunka, 1988, "nom. praeoc." por Mahunka, 1984)

DISTRIBUCIÓN: Borneo.

-Nanhermannia (N.) sellnicki Forsslund, 1958

DisTRIBUCión: Paleártica (Eurosiberiana, China: Tíbet, y Cáucaso).

-Nanhermannia (N.) tenuicoma Hammer, 1966

DisTRIBUCIÓN: Nueva Zelanda.

-Nanhermannia (N.) tenuisetosa P. Balogh, 1985

DisTRIBUCIÓN: Australia.

-Nanhermannia (N.) thaiensis Aoki, 1965

DisTRIBUCIÓN: Oriental.

-Nanhermannia (N.) tokara Aoki, 1987 (N. nana t.)

DisTRIBUCIÓN: Japón.

-Nanhermannia (N.) transversaria Hammer, 1972

DisTRIBUCIÓN: Polinesia (Tahití).

-Nanhermannia (N.) triangula Fujikawa, 1990

DISTRIBUCIÓN: Japón.

Nanhermannia (Nippohermannia) P. Balogh, 1985 (1 sp.)

ESPECIE TIPO: Nanhermannia parallela Aoki, 1961

DISTRIBUCIÓN: Paleártica meridional y Oriental.

-Nanhermannia (Nippohermannia) parallela Aoki, 1961 (Nan.)

DisTRIBUCIÓN: Este de Paleártica oriental y Oriental.

Notohermannia P. Balogh, 1985 (1 sp.)

EsPecie TIPO: Notohermannia obtusa P. Balogh, 1985

DisTRIBUCIÓN: Australiana.

-Notohermannia obtusa P. Balogh, 1985

DistRIBUCIÓN: Australia.

\section{Hermannioidea Sellnick, 1928}

Hermanniidae Sellnick, 1928 (3 gen., 2 subg. y 80 spp.) DISTRIBUCIÓN: Cosmopolita.

Galapagacarus P. Balogh, 1985 (1 sp.)

EsPeCIE TIPO: Galapagacarus schatzi P. Balogh, 1985

DISTRIBUCIÓN: Neotropical.

-Galapagacarus schatzi P. Balogh, 1985

DisTRIBUCIÓN: I. Galápagos.

Hermannia Nicolet, 1855 (78 spp.)

ESPECIE TIPO: (Hermannia crassipes Nicolet, 1855) =Nothrus gibbus Koch, 1839

DISTRIBUCIÓN: Cosmopolita. 
Hermannia (Hermannia) Nicolet, 1855 (14 spp.)

DisTRIBUCIÓN: Holártica, Paleotropical y "Australiana". -Hermannia (H.) canariensis Pérez-Íñigo y Peña, 1996 Distribución: I. Canarias.

-Hermannia (H.) clara Sitnikova, 1975

DisTRIBUCIÓN: Noreste de Paleártica oriental.

-Hermannia (H.) convexa (Koch, 1839) (Nothrus)

(=Hermannia grandis Berlese, 1910)

(=Claviceps rugosus $\mathrm{L}$. Koch, 1879)

DisTRIBUCión: Holártica (Europa: menos frecuente en el Sur, Paleártica oriental y U.S.A.: Virginia) e I. Seychelles.

-Hermannia (H.) dentipes Canestrini, 1898 "sp. inq." DisTRIBUCIÓN: Nueva Guinea.

-Hermannia (H.) doliaris (Koch, 1839) (Nothrus) "sp. inq." DisTRIBUCIÓN: Alemania.

-Hermannia (H.) exilis Canestrini, 1897 "sp. inq." DistRIBUCIÓN: Nueva Guinea.

-Hermannia (H.) fungifer Mahunka, 1988

DISTRIBUCIÓN: Borneo.

-Hermannia (H.) gibba (Koch, 1839) (Nothrus)

(=Hermannia crassipes Nicolet, 1855)

DisTRIBUCión: Holártica e I. Seychelles.

-Hermannia (H.) gigantea Sitnikova, 1975

DisTRIBUCIÓN: Sur de Siberia.

-Hermannia (H.) heterotricha Mahunka, 1988 DISTRIBUCIÓN: Borneo.

-Hermannia (H.) hokkaidensis Aoki y Ohnishi, 1974 DisTRIBUCióN: Japón y Canadá.

-Hermannia (H.) jesti Travé, 1977

DisTRIBUCIÓN: Nepal.

-Hermannia (H.) neotricha Choi, 1994 DISTRIBUCIÓN: Corea.

-Hermannia (H.) trinebulosa Riley, 1885 "sp. inq." DisTRIBUCIÓN: "U.S.A."

Hermannia (Heterohermannia) Woas, 1992 (13 spp.)

ESPECIE TIPO: Hermannia reticulata Thörell, 1871

DISTRIBUCIÓN: Holártica.

-Hermannia (Heterohermannia) evidens Pérez-Íñigo, 1992 (Herm.) DisTRIBUCión: I. Azores.

-Hermannia (Heterohermannia) gracilis Woas, 1978 (Herm.) DisTRIBUCIÓN: Francia.

-Hermannia (Heterohermannia) intermedia Woas, 1980 (Herm.) DISTRIBUCIÓN: Francia.

-Hermannia (Heterohermannia) minuta Woas, 1980 (Herm.) DisTRIBUCIÓN: Europa meridional.

-Hermannia (Heterohermannia) nodosa Michael, 1888 (Herm.) DisTRIBUCIÓN: Europa occidental.

-Hermannia (Heterohermannia) polystriata Woas, 1978 (Herm.) DistRibución: U.S.A. (California).

-Hermannia (Heterohermannia) pseudonodosa Woas, 1981 (Herm.) DISTRIBUCIÓN: Neártica.

-Hermannia (Heterohermannia) pulchella Willmann, 1952 (Herm.) DisTRIBUCIÓN: Euroatlántica.

-Hermannia (Heterohermannia) reticulata Thörell, 1871 (Herm.) (=Hermannia quadriseriata Banks, 1899) DISTRIBUCIÓN: Boreal.

-Hermannia (Heterohermannia) scabra (L. Koch, 1879) (Nothrus) DisTRIBUCIÓN: Holártica (menos frecuente en el Sur).

-Hermannia (Heterohermannia) schusteri Woas, 1981 (Herm.) DisTRIBUCIÓN: Sur de Europa (excepto Oeste).

-Hermannia (Heterohermannia) subglabra Berlese, 1910 (Herm.) DisTRIBUCIÓN: Holártica (Europa y Neártica septentrional).

-Hermannia (Heterohermannia) woasi Pérez-Íñigo, 1992 (Herm.) DisTRIBUCIÓN: I. Azores.

Hermannia (Phyllhermannia) Berlese, 1916 (51 spp.)

(=Lawrencoppia Jacot, 1936)

EsPECIE TIPO: Hermannia phyllophora Michael, 1908

DisTRIBUCIÓN: Pantropical, subtropical y subantártica.
-Hermannia (Phyllhermannia) africana (Balogh, 1958) (P.)

Distribución: Tanzania.

-Hermannia (Phyllhermannia) angulata (Balogh y Mahunka, 1966) (P.)

DisTRIBUCIÓN: Sudáfrica.

-Hermannia (Phyllhermannia) areolata (Aoki, 1970) (P.)

DISTRIBUCIÓN: Japón.

-Hermannia (Phyllhermannia) becki (Pérez-Íñigo y Baggio, 1988) (P.) DisTRIBUCIÓN: Brasil.

-Hermannia (Phyllhermannia) berlesei (Mondal, 1984) (P.) DisTRIBUCIÓN: India (Bengala Occidental).

-Hermannia (Phyllhermannia) bimaculata (Hammer, 1979) (P.)

DisTRIBUCIÓN: Java.

-Hermannia (Phyllhermannia) clavata Mahunka, 1985 (H.)

DisTRIBUCIÓN: Sudáfrica.

-Hermannia (Phyllhermannia) colloffi (Luxton, 1991) (P.)

Distribución: Filipinas.

-Hermannia (Phyllhermannia) comparabilis Wet, 1993 (H.)

DisTRIBUCIÓN: Sudáfrica.

-Hermannia (Phyllhermannia) coronata (Mahunka, 1991) (P.)

DISTRIBUCIÓN: Borneo.

-Hermannia (Phyllhermannia) dentata Trägardh, 1931 (P.)

(=Phyllhermannia dentata glabra Hammer, 1962)

DisTRIBUCión: Chile.

-Hermannia (Phyllhermannia) dinghuensis Lu y Wang, 1995 DISTRIBUCIÓN: China suroriental.

-Hermannia (Phyllhermannia) engelbrechti Wet, 1993 (H.) Distribución: Sudáfrica.

-Hermannia (Phyllhermannia) eusetosa (Lee, 1985) (P.)

DISTRIBUCIÓN: Australia.

-Hermannia (Phyllhermannia) exobothridialis Wet, 1993 (H.) DisTRIBUCIÓN: Sudáfrica.

-Hermannia (Phyllhermannia) exornata (Balogh, 1964) (P.)

DisTRIBUCIÓN: Madagascar.

-Hermannia (Phyllhermannia) falklandica (P. Balogh, 1988) (P.)

DistriBuCión: Subantártica: I. Malvinas [I. Falkland].

-Hermannia (Phyllhermannia) foliata (Hammer, 1966) (P.)

DisTRIBUCIÓN: Nueva Zelanda.

-Hermannia (Phyllhermannia) forsteri (P. Balogh, 1985) (P.)

DisTRIBUCión: Nueva Zelanda.

-Hermannia (Phyllhermannia) foveolata (Sanyal, 1990) (P.)

DisTRIBUCIÓN: India (Kerala).

-Hermannia (Phyllhermannia) gladiata (Aoki, 1965) (P.)

DisTRIBUCIÓN: Tailandia.

-Hermannia (Phyllhermannia) javensis (Hammer, 1979) (P.) DisTRIBUCIÓN: Java.

-Hermannia (Phyllhermannia) kanoi Aoki, 1959 (H.)

DisTRIBUCIÓN: Oriental y Japón.

-Hermannia (Phyllhermannia) louisiae Wet, 1993 (H.)

DisTRIBUCIÓN: Sufáfrica.

-Hermannia (Phyllhermannia) mauritii (Jacot, 1936) (Lawrencoppia) DistRIBUCIÓN: Malgache (I. Mauricio).

-Hermannia (Phyllhermannia) modesta (Mahunka, 1978) (P.)

Distribución: Malgache (I. Reunión).

-Hermannia (Phyllhermannia) mollis (Hammer, 1966) $(P$.

DisTRIBUCIÓN: Nueva Zelanda.

-Hermannia (Phyllhermannia) natalensis Wet, 1993 (H.)

DISTRIBUCIÓN: Sudáfrica.

-Hermannia (Phyllhermannia) nathanaeli Wet, 1993 (H.)

DisTRIBUCIÓN: Sudáfrica.

-Hermannia (Phyllhermannia) neonominata nom. nov.

[=Phyllhermannia mauritii Mahunka, 1978, “nom. praeoc.” por Jacot, 1936 ("hom. sec.")]

DisTRIBUCIÓN: Malgache (I. Mauricio)

-Hermannia (Phyllhermannia) neotropica Woas, 1992 DisTRIBUCIÓN: Brasil.

-Hermannia (Phyllhermannia) notogastralis Wet, 1993 (H.)

DisTRIBUCIÓN: Sudáfrica.

-Hermannia (Phyllhermannia) pacifica (Hammer, 1972) (P.)

Distribución: Polinesia. 
-Hermannia (Phyllhermannia) pauliani (Balogh, 1964) (P.)

DisTRIBUCIÓN: Madagascar.

-Hermannia (Phyllhermannia) phylliformis Wet, 1993 (H.) DISTRIBUCIÓN: Sudáfrica.

-Hermannia (Phyllhermannia) phyllophora Michael, 1908 (H.) Distribución: Nueva Zelanda.

-Hermannia (Phyllhermannia) pocsi Mahunka, 1985 (H.) "sp. inq." DisTRIBUCIÓN: Tanzania.

-Hermannia (Phyllhermannia) pulchra (Aoki, 1973) (P.)

DisTRIBUCIÓN: Japón.

-Hermannia (Phyllhermannia) punctata (Sanyal, 1990) (P.)

DisTRIBUCIÓN: India (Kerala).

-Hermannia (Phyllhermannia) quadrirotunda (Hammer, 1979) (P.) DisTRIBUCIÓN: Java.

-Hermannia (Phyllhermannia) rubra (Hammer, 1966) (P.)

DisTRIBUCIÓN: Nueva Zelanda.

-Hermannia (Phyllhermannia) sculpturata Mahunka, 1983 (H.) DisTRIBUCIÓN: Tanzania.

-Hermannia (Phyllhermannia) serrata (Balogh y Mahunka, 1966) (P.) DisTRIBUCIÓN: Sudáfrica.

-Hermannia (Phyllhermannia) setiformis Wet, 1993 (H.) DisTRIBUCIÓN: Sudáfrica.

-Hermannia (Phyllhermannia) similis (Balogh y Mahunka,1967) (P.) DisTRIBUCIÓN: Vietnam.

-Hermannia (Phyllhermannia) spathulata Mahunka, 1985 (H.) DistRIBUCión: Sudáfrica.

-Hermannia (Phyllhermannia) tenuiseta (P. Balogh, 1988) (P.) DisTRIBUCIÓN: Ceilán [Sri Lanka].

-Hermannia (Phyllhermannia) transvaalensis Wet, 1993 (H.) DisTRIBUCión: Sudáfrica.

-Hermannia (Phyllhermannia) tremicta (Mahunka, 1978) (P.)

Distribución: Malgache (I. Mauricio).

-Hermannia (Phyllhermannia) truncata (Wang, 1993) (P.)

DisTRIBUCIÓN: China suroriental.

-Hermannia (Phyllhermannia) tuberculata (Covarrubias, 1967) (P.) DisTRIBUCIÓN: Neotropical (Chile y Uruguay).

Neohermannia Bayoumi y Mahunka, 1979 (1 sp.)

ESPECIE TIPO: Neohermannia trichosa Bayoumi y Mahunka, 1979 DisTRIBUCIÓN: Oriental.

-Neohermannia trichosa Bayoumi y Mahunka, 1979

DISTRIBUCIÓN: Bután.

Suborden BRACHYPYLINA Hull, 1918 Infraorden PYCNONOTICAE Grandjean, 1954

Hermannielloidea Grandjean, 1934

Hermanniellidae Grandjean, 1934 (9 gen., 51 spp. y 3 sspp.) DisTRIBUCIÓN: Cosmopolita (excepto Antártica).

Akansilvanus Fujikawa, 1993 (1 sp.)

EsPECIE TIPO: Akansilvanus parvus Fujikawa, 1993

DISTRIBUCIÓN: Paleártica.

-Akansilvanus parvus Fujikawa, 1993

DISTRIBUCIÓN: Japón.

Ampullobates Grandjean, 1962 (1 sp.)

EsPECIE TIPO: Ampullobates nigriclavatus Grandjean, 1962

DISTRIBUCIÓN: Neotropical.

-Ampullobates nigriclavatus Grandjean, 1962

DisTRIBUCIÓN: Centroamérica.

Baloghacarus Mahunka, 1983 (2 spp.)

EsPECIE TIPO: Baloghacarus hauseri Mahunka, 1983

DISTRIBUCIÓN: Neotropical.

-Baloghacarus australis Balogh y Mahunka, 1981

DisTRIBUCión: Paraguay.

-Baloghacarus hauseri Mahunka, 1983

DISTRIBUCIÓN: Norte de Neotropical.
Dicastribates J. y P. Balogh, 1988 (2 spp.)

EsPECIE TIPO: Sacculobates heterotrichus Mahunka, 1984

DisTRIBUCIÓN: Neotropical y Paleártica meridional.

-Dicastribates heterotrichus (Mahunka, 1984) (Sacculobates)

DisTRIBUCIÓN: Neotropical (Paraguay y Panamá).

-Dicastribates tenuisetosus Choi, Bayartogtokh y Aoki, 2001

DisTRIBUCIÓN: Corea.

Hermanniella Berlese, 1908 (33 spp. y 2 sspp.)

ESPECIE TIPO: Hermannia granulata Nicolet, 1855

DisTRIBUCIÓN: Cosmopolita (excepto Neotropical y Antártica).

-Hermanniella aristosa Aoki, 1965

DisTRIBUCIÓN: Este de Paleártica oriental y Micronesia (I. Marianas).

-Hermanniella barbata Moraza, 1990

DisTRIBUCIÓN: España.

-Hermanniella canestrinii (Tietze, 1899) (Hypochthonius) "sp. inq." DisTRIBUCIÓN: Italia.

-Hermanniella clavasetosa Hammer, 1966

DisTRIBUCIÓN: Nueva Zelanda.

-Hermanniella clavigera Berlese, 1908

Distribución: U.S.A. (Misuri) y Cáucaso.

-Hermanniella congoensis Balogh, 1958

DisTRIBUCIÓN: África centrooriental.

-Hermanniella diversisetosa Hammer, 1966

Distribución: Australiana (Nueva Zelanda y Micronesia: I.

Marianas).

-Hermanniella dolosa Grandjean, 1931

DisTRIBUCIÓN: Paleártica (menos frecuente en el Norte).

-Hermanniella dubinini Sitnikova, 1973

DistriBución: Sur de Paleártica oriental y Cáucaso.

-Hermanniella gibber Kulijev, 1979

DISTRIBUCIÓN: Cáucaso.

-Hermanniella grandis Sitnikova, 1973

DistRIBUCIÓN: Este de Rusia asiática.

-Hermanniella granulata (Nicolet, 1855) (Hermannia)

DisTRIBUCIÓN: Holártica (Paleártica: menos frecuente en el Norte,

y Noreste de U.S.A.).

-Hermanniella humilis Balogh, 1962

DISTRIBUCIÓN: Tanzania.

-Hermanniella incondita Pérez-Íñigo, 1987

DisTRIBUCIÓN: I. Azores.

-Hermanniella issanielloides Gil-Martín y Subías, 1997

DisTRIBUCión: España.

-Hermanniella laurisilvae Pérez-Íñigo, 1972

DisTRIBUCIÓN: I. Canarias.

-Hermanniella longisetosa Hammer, 1966

DisTRIBUCIÓN: Nueva Zelanda.

-Hermanniella madagascarensis Balogh, 1962

DisTRIBUCIÓN: Madagascar.

-Hermanniella mastyx Mahunka, 1983

DistRIBUCIÓN: Tanzania.

-Hermanniella microsetosa Hammer, 1966

DISTRIBUCIÓN: Nueva Zelanda.

-Hermanniella multipora Sitnikova, 1973

Distribución: Paleártica (Cáucaso, Sur de Siberia y Este de Rusia asiática)

-Hermanniella oblitera Sitnikova, 1973

DisTRIBUCIÓN: Paleártica (Cáucaso y Asia centrooccidental).

-Hermanniella occidentalis Ewing, 1918 (H. punctulata o.)

DisTRIBUCIÓN: U.S.A. (Oregón).

-Hermanniella orbiculata Hammer, 1979

DisTRIBUCIÓN: Java.

-Hermanniella picea (Koch, 1839) (Nothrus)

$(=$ Hermannia arrecta Nicolet, 1855)

(=Hermanniella punctulata Berlese, 1908)

DisTRIBUCIÓN: Holártica (Paleártica: menos frecuente en el Norte,

y U.S.A.: Virginia) y Filipinas.

-Hermanniella reticulata Sitnikova, 1973

DISTRIBUCIÓN: Asia centrooccidental. 
-Hermanniella robusta Ewing, 1918 (H. punctulata r.) DisTRIBUCIÓN: Neártica.

-Hermanniella septentrionalis Berlese, 1910 (H. punctulata s.) DisTRIBUCión: Holártica (Paleártica occidental: excepto Norte, y U.S.A.: Oregón).

-Hermanniella serrata Sitnikova, 1973

DisTRIBUCIÓN: Sureste de Europa.

-Hermanniella similis Sitnikova, 1973

DISTRIBUCIÓN: Asia centrooccidental.

-Hermanniella subnigra (Ewing, 1909) (Hermannia)

DISTRIBUCIÓN: Neártica y Australiana.

-Hermanniella subnigra subnigra (Ewing, 1909)

(=Hermanniella punctulata columbiana Berlese, 1910)

DistRIBUCión: Neártica y Australiana (Islas del Pacífico).

-Hermanniella subnigra rigida Jacot, 1938

DISTRIBUCIÓN: U.S.A.: Ilinois.

-Hermanniella subnigra setacea Jacot, 1937

Distribución: U.S.A.: Kentucky.

-Hermanniella thani Mahunka, 1987

DisTRIBUCIÓN: Vietnam.

-Hermanniella yasumai Aoki, 1973

DISTRIBUCIÓN: Japón.

Hermannobates Hammer, 1961 (8 spp.)

EsPECIE TIPO: Hermannobates monstruosus Hammer, 1961 DISTRIBUCIÓN: Neotropical.

-Hermannobates cubanus Stary, 1998

DisTRIBUCIÓN: Cuba.

-Hermannobates flagelliseta Balogh y Mahunka, 1981 DisTRIBUCIÓN: Paraguay.

-Hermannobates hammerae Stary, 1998

DISTRIBUCIÓN: Cuba.

-Hermannobates horridus Pérez-Íñigo y Baggio, 1988 DiSTRIBUCIÓN: Brasil.

-Hermannobates intermedius Calugar, 1990 DisTRIBUCIÓN: Venezuela.

-Hermannobates monstruosus Hammer, 1961 DisTRIBUCIÓN: Neotropical.

-Hermannobates ruseki Stary, 1998 DisTRIBUCión: Cuba.

-Hermannobates scoparius Pérez-Íñigo y Baggio, 1993 DISTRIBUCIÓN: Brasil.

Issaniella Grandjean, 1962 (1 sp. y 1 ssp.)

EsPECIE TIPO: Issaniella mograbin Grandjean, 1962

DISTRIBUCIÓN: Paleártica y Neotropical.

-Issaniella mograbin Grandjean, 1962

DisTRIBUCIÓN: Mediterránea e I. Galápagos.

-Issaniella mograbin mograbin Grandjean, 1962 DisTRIBUCIÓN: Mediterránea e I. Galápagos.

-Issaniella mograbin hauseri Mahunka, 2001

DisTRIBUCIÓN: Grecia.

Mahunkobates Calugar, 1989 (1 sp.)

EsPECIE TIPO: Mahunkobates singularis Calugar, 1989 DISTRIBUCIÓN: Neotropical.

-Mahunkobates singularis Calugar, 1989

DisTRIBUCIÓN: Venezuela.

Sacculobates Grandjean, 1962 (2 spp.)

=Bruneiella Mahunka, 1997 "sin. nov.")

EsPeCIE TIPO: Sacculobates horologiorum Grandjean, 1962

DisTRIBUCIÓN: Tropical (Oriental y Neotropical).

-Sacculobates horologiorum Grandjean, 1962 DisTRIBUCIÓN: Neotropical (frecuente).

-Sacculobates sultan (Mahunka, 1997) (Bruneiella)

DISTRIBUCIÓN: Borneo.
Plasmobatidae Grandjean, 1961 (3 gen. y 18 spp.)

DistribuCión: Pantropical.

Orbiculobates Grandjean, 1961 (2 spp.)

EsPECIE TIPO: Plasmobates orbiculus Grandjean, 1929

DISTRIBUCIÓN: Neotropical.

Orbiculobates orbiculus (Grandjean, 1929) (Plasmobates)

DisTRIBUCIÓN: Pequeñas Antillas.

-Orbiculobates transvectus (Grandjean, 1929) (Plasmobates)

DistriBución: Pequeñas Antillas.

Plasmobates Grandjean, 1929 (10 spp.)

EsPeCIE TIPO: Plasmobates pagoda Grandjean, 1929

Distribución: Pantropical.

-Plasmobates acutirostrum Hammer, 1973

Distribución: Polinesia (I. Samoa).

-Plasmobates africanus Balogh, 1958 "sp. inq."

DisTRIBUCIÓN: Congo.

-Plasmobates asiaticus Aoki, 1973

DisTRIBUCIÓN: Japón y Filipinas.

-Plasmobates carboneli Pérez-Íñigo y Sarasola, 1998

Distribución: Uruguay.

-Plasmobates hyalinus Hammer, 1971

Distribución: Melanesia (I. Fiji).

-Plasmobates javensis Hammer, 1979

DistRIBUCIÓN: Java.

-Plasmobates machadoi Balogh, 1958 "sp. inq."

DISTRIBUCIÓN: Congo.

-Plasmobates minor Balogh, 1958 "sp. inq."

DistriBución: Congo.

-Plasmobates pagoda Grandjean, 1929

DistriBución: Pequeñas Antillas e India (Bengala Occidental).

-Plasmobates schubarti Pérez-Íñigo y Baggio, 1998

DistriBución: Brasil.

Solenozetes Grandjean, 1931 (6 spp.)

EsPeCIE TIPO: Plasmobates cribratus Grandjean, 1929

DisTRIBUCIÓN: Tropical (Australiana y Neotropical).

-Solenozetes australis (Balogh y Csiszár, 1963) (Orbiculobates)

DisTRIBUCIÓN: Argentina.

-Solenozetes carinatus (Hammer, 1961) (Plasmobates)

DistribuCión: Perú.

-Solenozetes cribratus (Grandjean, 1929) (Plasmobates)

DisTRIBUCIÓN: Norte de Neotropical.

-Solenozetes flagellatus Balogh y Mahunka, 1969

DisTRIBUCIÓN: Brasil.

-Solenozetes flagellifer Mahunka, 1983

Distribución: Méjico.

-Solenozetes gallonae Hunt, 1994

DISTRIBUCIÓN: Australia.

Neoliodoidea Sellnick, 1928

Neoliodidae Sellnick, 1928 (4 gen., 50 spp. y 1 ssp.) DISTRIBUCIÓN: Cosmopolita (excepto Antártica).

Neoliodes Berlese, 1888 (39 spp. y 1 ssp.)

(=Liodes Heyden, 1826, "nom. praeoc." por Leiodes Latreille, 1796)

$=$ Udetaliodes Jacot, 1929)

EsPECIE TIPO: Notaspis theleproctus Hermann, 1804

DISTRIBUCIÓN: Cosmopolita (excepto Antártica).

-Neoliodes alatus (Hammer, 1979) (Liodes)

DisTRIBUCIÓN: Java.

-Neoliodes backstroemi Trägardh, 1931

DistriBuCión: Chile (I. Juan Fernández).

-Neoliodes bataviensis Sellnick, 1925

DistRIBUCión: Java y Australiana (Islas del Pacífico)

-Neoliodes canaliculatus (Koch, 1839) (Nothrus) "sp. inq."

DISTRIBUCIÓN: Alemania. 
-Neoliodes capensis Berlese, 1910 "sp. inq."

DisTRIBUCIÓN: Sudáfrica.

-Neoliodes concentricus (Say, 1821) (Oribata)

(=Nothrus malleolus Karpelles, 1884)

DisTRIBUCIÓN: U.S.A

-Neoliodes elongatus (Fernández, Marcangeli y Martínez, 1995)

(Liodes)

DisTRIBUCIÓN: Argentina

-Neoliodes eques (Canestrini, 1897) (Nothrus) "sp. inq."

DISTRIBUCIÓN: Nueva Guinea.

-Neoliodes floridensis Banks, 1906

DisTRIBUCIÓN: U.S.A. (Florida).

-Neoliodes funafutiensis (Jacot, 1929) (Udetaliodes)

DisTRIBUCIÓN: Melanesia: I. Ellice [Tuvalu].

-Neoliodes globosus (Subías y Gil-Martín, 1990) (Liodes)

DisTRIBUCIÓN: Mediterránea occidental.

-Neoliodes hawaiiensis (Jacot, 1929) (Udetaliodes)

Distribución: Australiana (Islas del Pacífico).

-Neoliodes hawaiiensis hawaiiensis (Jacot, 1929)

Distribución: Australiana (Islas del Pacífico).

-Neoliodes hawaiiensis aculeatisetae (Jacot, 1934) (Udetaliodes)

DistRIBUCIÓN: Polinesia (I. Marquesas).

-Neoliodes ionicus Sellnick, 1931

DisTRIBUCión: Mediterránea.

-Neoliodes kornhuberi (Karpelles, 1884) (Nothrus)

DistriBución: Este de Paleártica oriental.

-Neoliodes lamellatus (Rainbow, 1897) (Oribata)

DistRIBUCión: Melanesia: I. Ellice [Tuvalu]

-Neoliodes lanceosetosus (Wallwork, 1977) (Liodes)

DisTRIBUCIÓN: I. Santa Helena.

-Neoliodes lawrencei Jacot, 1940

DisTRIBUCIÓN: Sudáfrica.

-Neoliodes longipes Berlese, 1916 "sp. inq."

DisTRIBUCIÓN: "África oriental".

-Neoliodes marplatensis (Fernández, Marcangeli y Martínez, 1995)

(Liodes)

DisTRIBUCIÓN: Argentina.

-Neoliodes mauritius (Jacot, 1936) (Udetaliodes)

Distribución: Malgache (I. Mauricio).

-Neoliodes modestior Berlese, 1916 (N. squamiger m.) "sp. inq."

DistriBUCIÓN: "África oriental".

-Neoliodes nigricans (Hammer, 1967) (Liodes)

DisTRIBUCIÓN: Nueva Zelanda.

-Neoliodes notactis (Gervais, 1849) (Oribata) "sp. inq."

DisTRIBUCIÓN: Chile.

-Neoliodes ocellatus Pearce, 1906

DisTRIBUCIÓN: India (Sikkim)

-Neoliodes polysetosus (Sitnikova, 1975) (Liodes)

DisTRIBUCIÓN: Este de Rusia asiática.

-Neoliodes porcellus Berlese, 1888 (N. theleproctus p.) "sp. inq." DISTRIBUCIÓN: Paraguay.

-Neoliodes pyramidalis (Wallwork, 1963) (Liodes) DisTRIBUCIÓN: Ghana.

-Neoliodes ramosus (Hammer, 1971) (Liodes)

Distribución: Melanesia (I. Fiji).

-Neoliodes rugosus Warburton, 1912

DisTRIBUCIÓN: I. Seychelles.

-Neoliodes segestris (Wallwork, 1963) (Liodes)

DISTRIBUCIÓN: Ghana.

-Neoliodes silvestris (Hammer, 1977) (Liodes)

DisTRIBUCIÓN: Pakistán.

-Neoliodes squamiger Berlese, 1916 "sp. inq."

DisTRIBUCIÓN: Somalia.

-Neoliodes striatus Warburton, 1912

(=Neoliodes femoralis Warburton, 1912)

DisTRIBUCIÓN: I. Seychelles.

-Neoliodes swezeyi (Jacot, 1929) (Udetaliodes)

DisTRIBUCIÓN: Hawai.
-Neoliodes terrestris (Wallwork, 1963) (Liodes)

Distribución: Semicosmopolita (Paleártica: Cáucaso y Asia centrooccidental, Etiópica: Ghana, Oriental: India, y Neotropical: Venezuela).

-Neoliodes theleproctus (Hermann, 1804) (Notaspis)

(=Nothrus circumvallatus Haupt, 1882)

$(=$ Nothrus dimera Haupt, 1882)

(=Nothrus dorsatus Canestrini y Fanzago, 1876)

DisTRIBUCIÓN: Semicosmopolita (Paleártica: menos frecuente en el

Norte, Oriental: Vietnam, y Australiana: Nueva Guinea y Hawai).

-Neoliodes vermiculatus Jacot, 1924

DisTRIBUCIÓN: Noreste de China.

-Neoliodes wakensis (Jacot, 1929) (Udetaliodes hawaiiensis w.)

DisTRIBUCIÓN: Islas del Pacífico.

-Neoliodes zimmermanni (Sellnick, 1959) (Liodes)

DISTRIBUCIÓN: Polinesia: I. Sureste, y Japón.

Platyliodes Berlese, 1916 (7 spp.)

EsPeCIE TIPO: Nothrus doderleini Berlese, 1883

DisTRIBUCIÓN: Holártica.

-Platyliodes doderleini (Berlese, 1883) (Nothrus)

(?=Nothrus venosus Grube, 1859 "sp. inq.")

DisTRIBUCIÓN: Mediterránea.

-Platyliodes graecus Sellnick, 1927

DisTRIBUCIÓN: Grecia.

-Platyliodes japonicus Aoki, 1979

DistribuCIÓN: Este de Paleártica oriental.

-Platyliodes longisetosus Sitnikova, 1975

Distribución: Paleártica meridional (Sureste de Europa y Asia centrooccidental).

-Platyliodes macroprionus Woolley y Higgins, 1969

DisTRIBUCiÓN: Neártica (U.S.A.: Washington, y Canadá) y Japón.

-Platyliodes montanus Fujikawa, 2001

DISTRIBUCIÓN: Japón.

-Platyliodes scaliger (Koch, 1839) (Nothrus)

DisTRIBUCIÓN: Holártica (frecuente en Mediterránea).

Poroliodes Grandjean, 1934 (1 sp.)

EsPECIE TIPO: (Notaspis theleproctus Hermann “sensu” Michael, 1888)

=Nothrus farinosus Koch, 1839

DISTRIBUCIÓN: Paleártica.

-Poroliodes farinosus (Koch, 1839) (Nothrus)

(=Neoliodes caudatus Berlese, 1914)

DisTRIBUCIÓN: Paleártica (frecuente en Paleártica occidental).

Teleioliodes Grandjean, 1934 (3 spp.)

EsPeCIE TIPO: Teleioliodes madininensis Grandjean, 1934

DISTRIBUCIÓN: Tropical (Etiópica y Neotropical).

-Teleioliodes ghanensis Wallwork, 1963

DisTRIBUCIÓN: Ghana.

-Teleioliodes madininensis Grandjean, 1934

DisTRIBUCIÓN: Norte de Neotropical.

-Teleioliodes zikani (Sellnick, 1930) (Neoliodes)

DisTRIBUCIÓN: Neotropical (frecuente).

Plateremaeoidea Trägardh, 1926

Plateremaeidae Trägardh, 1926 (5 gen. y 17 spp.)

DISTRIBUCIÓN: Pantropical y subtropical.

Allodamaeus Banks, 1947 (2 spp.)

EsPeCIE TIPO: Allodamaeus ewingi Banks, 1947

DISTRIBUCIÓN: Holártica.

- Allodamaeus coralgablensis Paschoal, 1987

DisTRIBUCIÓN: U.S.A.: Florida.

-Allodamaeus ewingi Banks, 1947

DisTRIBUCIÓN: U.S.A. (Carolina del Norte) y Asia centrooccidental. 
Calipteremaeus Paschoal, 1987 (1 sp.)

EsPeCIE TIPO: Plateremaeus yaginumai Aoki, 1977

DisTRIBUCIÓN: Paleártica.

-Calipteremaeus yaginumai (Aoki, 1977) (Plateremaeus) Distribución: Este de Paleártica oriental.

Lopheremaeus Paschoal, 1987 (3 spp.)

EsPECIE TIPO: Plateremaeus mirabilis Csiszár, 1962

DisTRIBUCIÓN: Subtropical (Paleártica meridional) y Etiópica.

-Lopheremaeus laminipes (Berlese, 1916) (Plateremaeus) DisTRIBUCIÓN: Europa meridional.

-Lopheremaeus mirabilis (Csiszár, 1962) (Plateremaeus) DISTRIBUCIÓN: Bulgaria.

-Lopheremaeus tunicatus (Balogh, 1958) (Gymnodamaeus) "sp. inq." DisTRIBUCIÓN: Congo.

Paralopheremaeus Paschoal, 1987 (2 spp.)

EsPeCIE TIPO: Plateremaeus legendrei Balogh, 1962

DisTRIBUCIÓn: Paleotropical y subtropical (Paleártica meridional).

-Paralopheremaeus hispanicus (Ruiz, Kahwash y Subías, 1990)

(Plateremaeus)

DisTRIBUCIÓN: España.

-Paralopheremaeus legendrei (Balogh, 1962) (Plateremaeus)

(=Plateremaeus elongatus Mahunka, 1987)

DistriBuCión: Etiópica (Madagascar y Kenia) y Ceilán [Sri Lanka].

Plateremaeus Berlese, 1908 (9 spp.)

EsPeCIE TIPO: Damaeus ornatissimus Berlese, 1888

DisTRIBUCIÓN: Pantropical.

-Plateremaeus anteriosetosus Woas, 1992

Distribución: Perú.

-Plateremaeus berlesei Balogh y Mahunka, 1978

DisTRIBUCIÓN: Neotropical (Brasil y Panamá).

-Plateremaeus callosus Hammer, 1979

DisTRIBUCIÓN: Java.

-Plateremaeus complanatus (Warburton, 1912) (Damaeus) Distribución: I. Seychelles.

-Plateremaeus costulatus Balogh y Mahunka, 1978 Distribución: Brasil.

-Plateremaeus excavatus Hammer, 1979

DisTRIBUCIÓN: Java.

-Plateremaeus latus P. Balogh, 1988

DisTRIBUCiÓN: Ceilán [Sri Lanka].

-Plateremaeus novemsetosus J. y P. Balogh, 1983 DisTriBución: Australia.

-Plateremaeus ornatissimus (Berlese, 1888) (Damaeus) DisTRIBUCIÓN: Brasil.

Pheroliodidae Paschoal, 1987 (9 gen. y 55 spp.) DisTRIBUCIÓN: Cosmopolita.

Darthvaderum Hunt, 1996 (1 sp.)

ESPECIE TIPO: Darthvaderum greensladeae Hunt, 1996

DISTRIBUCIÓN: Australiana.

-Darthvaderum greensladeae Hunt, 1996

DISTRIBUCIÓN: Tasmania.

Flammaeremaeus Balogh, 1968 (1 sp.)

EsPECIE TIPO: Flammaeremaeus gressitti Balogh, 1968

DISTRIBUCIÓN: Australiana.

-Flammaeremaeus gressitti Balogh, 1968

DisTRIBUCIÓN: Nueva Guinea.

Licnoliodes Grandjean, 1931 (3 spp.)

EsPeCIE TIPO: Licnoliodes andrei Grandjean, 1931 DisTRIBUCIÓN: Paleártica.

-Licnoliodes adminensis Grandjean, 1933

DISTRIBUCIÓN: Mediterránea occidental.
-Licnoliodes andrei Grandjean, 1931

DisTRIBUCIÓN: Paleártica occidental (menos frecuente en el Norte) y Asia centrooccidental

-Licnoliodes apunctatus Mahunka, 1977

DisTRIBUCIÓN: Grecia.

Lopholiodes Paschoal, 1987 (6 spp.)

(=Octoliodes Paschoal, 1987 "sin. nov.")

ESPECIE TIPO: Lopholiodes micropunctinatum Paschoal, 1987

DISTRIBUCIÓN: Tropical (Neotropical y Australiana).

-Lopholiodes diamantei Fernández y Cleva, 1999

DISTRIBUCIÓN: Argentina.

-Lopholiodes luteomarginatus (Hammer, 1966) (Pedrocortesia)

DisTRIBUCIÓN: Nueva Zelanda.

-Lopholiodes macropunctinatum Paschoal, 1987

DisTRIBUCIÓN: Brasil.

-Lopholiodes micropunctinatum Paschoal, 1987

DisTRIBUCIÓN: Brasil.

-Lopholiodes robustus (Hunt y Lee, 1995) (Pheroliodes)

DISTRIBUCIÓN: Australia.

-Lopholiodes rotoruensis (Hammer, 1966) (Pedrocortesia)

DistRIBUCIÓN: Nueva Zelanda.

Lyrifissiella Paschoal, 1989 (3 spp.)

ESPECIE TIPO: Pedrocortesella latoclava Hammer, 1966

DisTRIBUCIÓN: Subtropical austral (excepto Etiópica).

- Lyrifissiella cryptonota (Hammer, 1966) (Pedrocortesella)

DisTRIBUCIÓN: Nueva Zelanda.

-Lyrifissiella latoclava (Hammer, 1966) (Pedrocortesella)

DisTRIBUCIÓN: Nueva Zelanda.

-Lyrifissiella vestita (Trägardh, 1931) (Plateremaeus)

DistriBuCión: Chile (I. Juan Fernández).

Neonooliodes Hunt, 1996 (1 sp.)

ESPECIE TIPO: Neonooliodes ceroplastes Hunt, 1996

DISTRIBUCIÓN: Australiana.

-Neonooliodes ceroplastes Hunt, 1996

DistRIBUCIÓN: Australia.

Nooliodes Paschoal, 1989 (2 spp.)

EsPeCIE TIPO: Plateremaeus glaber Balogh, 1962

DISTRIBUCIÓN: Etiópica.

-Nooliodes franzi (Balogh, 1966) (Pedrocortesia)

DISTRIBUCIÓN: Chad.

-Nooliodes glaber (Balogh, 1962) (Plateremaeus)

DISTRIBUCIÓN: Madagascar.

Paschoalia nom. nov. (2 spp.)

(=Hammeriella Paschoal, 1989, "nom. praeoc." por Hammerella Balogh, 1983)

ESPECIE TIPO: Pedrocortesia grandis Hammer, 1961

DisTRIBUCIÓN: Neotropical y Antártica.

-Paschoalia australis (Hammer, 1962) (Pedrocortesia)

DisTRIBUCIÓN: América austral y Antártica.

-Paschoalia grandis (Hammer, 1961) (Pedrocortesia)

DISTRIBUCION: Peru.

Pheroliodes Grandjean, 1931 (36 spp.)

(=Andesperuviella Paschoal, 1989 "sin. nov.")

(=Pedrocortesia Hammer, 1958)

EsPecIE TIPO: Cymbaeremaeus wehnckei Willmann, 1930

DISTRIBUCIÓN: Pantropical.

-Pheroliodes achalensis Baranek, 1986

DISTRIBUCIÓN: Argentina.

-Pheroliodes africanus (Balogh, 1966) (Pedrocortesia)

(=Pedrocortesella pletzenae J. y P. Balogh, 2002)

DistRIBUCIÓN: Kenia.

-Pheroliodes barringtonensis Hunt, 1996

Distribución: Australia.

-Pheroliodes carinulatus (Berlese, 1888) (Eremaeus)

DistriBución: Brasil. 
-Pheroliodes casabranquensis Paschoal, 1987

DisTRIBUCIÓN: Brasil.

-Pheroliodes complanatus (Berlese, 1901) (Eremaeus)

DisTRIBUCIÓN: Chile.

-Pheroliodes concavus Hunt, 1996

DisTRIBUCión: Australia.

-Pheroliodes copiosus Hunt y Lee, 1995

DisTRIBUCión: Australia.

-Pheroliodes cordobensis Baranek, 1986

DisTRIBUCIÓN: Argentina.

-Pheroliodes delticus Baranek, 1984

DisTriBución: Argentina.

-Pheroliodes dentatus (Hammer, 1961) (Pedrocortesia)

DisTRIBUCIÓN: Perú.

-Pheroliodes elegans (Hammer, 1961) (Pedrocortesia)

DisTRIBUCión: Neotropical.

-Pheroliodes enigma Hunt, 1996) (Pedrocortesella)

DistribuCión: Tasmania.

-Pheroliodes humeratus (Mahunka, 1978) (Pedrocortesia)

DisTRIBUCIÓN: Malgache (I. Mauricio).

-Pheroliodes inca Fernández, Martínez y Eguaras, 1991

DisTRIBUCión: Argentina.

-Pheroliodes intermedius (Hammer, 1961) (Pedrocortesia)

DistRIBUCIÓN: Neotropical (Perú y Argentina).

-Pheroliodes lindsayae Hunt, 1996

DisTRIBUCIÓN: Tasmania.

-Pheroliodes longiceps Balogh y Mahunka, 1966

Distribución: Sudáfrica.

-Pheroliodes longisetus Baranek, 1986

DisTRIBUCIÓN: Argentina.

-Pheroliodes lordhowensis Hunt, 1996

DisTRIBUCIÓN: Australia (I. Lord Howe).

-Pheroliodes minutus Baranek, 1984

DisTRIBUCIÓN: Argentina.

-Pheroliodes mirabilis (Hammer, 1958) (Pedrocortesia)

DisTRIBUCIÓN: Argentina.

-Pheroliodes monteithi Hunt, 1996

DistribuCIÓN: Australia.

-Pheroliodes nemoricultricis Paschoal, 1987

DisTRIBUCIÓN: Brasil.

-Pheroliodes neuquinus Baranek, 1986

DistriBUCIÓN: Argentina.

-Pheroliodes pellitus Paschoal, 1987

DisTRIBUCIÓN: Brasil.

-Pheroliodes roblensis Covarrubias, 1968

Distribución: Chile.

-Pheroliodes rotundatus (Berlese, 1913) (Plateremaeus)

DisTRIBUCIÓN: Oriental.

-Pheroliodes rusticus Woas, 1992

DisTRIBUCión: Brasil.

-Pheroliodes sicarius Hunt, 1996

DisTRIBUCIÓN: Australia.

-Pheroliodes springthorpei Hunt, 1996

DisTRIBUCIÓN: Australia.

-Pheroliodes transversus Hunt, 1996

DistriBución: Australia.

-Pheroliodes uruguayensis Pérez-Íñigo y Sarasola, 1995

DisTRIBUCión: Uruguay.

-Pheroliodes vermicularis (Balogh, 1970) (Pedrocortesia)

DisTRIBUCión: Nueva Guinea.

-Pheroliodes vulgaris Baranek, 1984

DisTRIBUCIÓN: Argentina.

-Pheroliodes wehnckei (Willmann, 1930) (Cymbaeremaeus)

DisTRIBUCIÓN: Neotropical.

Licnodamaeidae Grandjean, 1954 (6 gen. y 58 spp.) DISTRIBUCIÓN: Cosmopolita (excepto Antártica).

Hexachaetoniella Paschoal, 1987 (5 spp.)

EsPeCIE TIPO: Pedrocortesella sexpilosa Hammer, 1966

DISTRIBUCIÓN: Australiana.
-Hexachaetoniella bunya Hunt, 1996

DistriBuCIÓN: Australia.

-Hexachaetoniella contigua Hunt, 1996

DisTRIBUCIÓN: Tasmania.

-Hexachaetoniella dispersa (P. Balogh, 1985) (Pedrocortesella)

DisTRIBUCIÓN: Australia.

-Hexachaetoniella norfolkensis Hunt, 1996

Distribución: Australia (I. Norfolk).

-Hexachaetoniella sexpilosa (Hammer, 1966) (Pedrocortesella)

DisTRIBUCIÓN: Nueva Zelanda.

Licnodamaeolus Covarrubias, 1998 (1 sp.)

ESPECIE TIPO: Licnodamaeolus travei Covarrubias, 1998

DISTRIBUCIÓN: Neotropical.

-Licnodamaeolus travei Covarrubias, 1998

DistriBución: Chile.

Licnodamaeus Grandjean, 1931 (9 spp.)

ESPECIE TIPO: Licneremaeus undulatus Paoli, 1908

DisTRIBUCIÓN: Paleártica, Oriental y Neotropical.

-Licnodamaeus baccettii Bernini, 1972

DisTRIBUCión: Italia.

-Licnodamaeus costula Grandjean, 1931

DISTRIBUCIÓN: Mediterránea.

-Licnodamaeus fissuratus (Balogh y Mahunka, 1965) (Pedrocortesia)

DisTRIBUCIÓN: Paleártica oriental (Mongolia y Asia centrooccidental).

-Licnodamaeus granulatus Balogh y Csiszár, 1963

DistRIBUCiÓN: Argentina.

-Licnodamaeus inaequalis (Balogh y Mahunka, 1965) (Pedrocortesia)

Distribución: Paleártica (Sureste de Europa: Crimea, y

Paleártica oriental central).

-Licnodamaeus pulcherrimus (Paoli, 1908) (Licneremaeus)

Distribución: Paleártica (excepto Norte).

-Licnodamaeus stebaevae (Grishina, 1981) (Pedrocortesia)

DistRIBUCión: Asia centrooccidental.

-Licnodamaeus triangulatus (Bayartogtokh, 2001) (Pedrocortesella)

Distribución: Mongolia.

-Licnodamaeus undulatus (Paoli, 1908) (Licneremaeus)

Distribución: Paleártica (excepto Norte) y Taiwan [Formosa].

Nacunansella Fernández y Cleva, 1998 (1 sp.)

EsPeCIE Tipo: Nacunansella diminuta Fernández y Cleva, 1998

DisTRIBUCIÓN: Neotropical.

-Nacunansella diminuta Fernández y Cleva, 1998

DISTRIBUCIÓN: Argentina

Novazelandiella Paschoal, 1989 (5 spp.)

(=Labiogena Hunt, 1996 "sin. nov.")

EsPeCIE TIPO: Pedrocortesella nigroclava Hammer, 1966

DisTRIBUCión: Australiana.

-Novazelandiella convexa (Hunt, 1996) (Labiogena)

DISTRIBUCIÓN: Australia.

-Novazelandiella kellyi Hunt, 1996

DisTRIBUCIÓN: Australia

-Novazelandiella nigroclava (Hammer, 1966) (Pedrocrotesella)

DistriBUCIÓN: Nueva Zelanda.

-Novazelandiella queenslandica (P. Balogh, 1985) (Pedrocortesella)

DISTRIBUCIÓN: Australia.

-Novazelandiella walteri (Hunt, 1996) (Labiogena)

DisTRIBUCIÓN: Australia.

Pedrocortesella Hammer, 1961 (37 spp.)

(=Acupedicellus Hunt y Lee, 1995 "sin. nov.")

ESPECIE TIPO: Pedrocortesella pulchra Hammer,1961

Distribución: Semicosmopolita (Paleártica, Etiópica, Australiana y Neotropical).

-Pedrocortesella africana Pletzen, 1963

DistRIBUCión: Sudáfrica.

-Pedrocortesella anica Hunt, 1996

DistriBuCión: Australia. 
-Pedrocortesella augusta Hunt, 1996

DisTRIBUCIÓN: Australia

-Pedrocortesella bannisteri Hunt, 1996

DISTRIBUCIÓN: Australia.

-Pedrocortesella bithongabela Hunt, 1996

Distribución: Australia.

-Pedrocortesella callitarsus Hunt, 1996

Distribución: Australia.

-Pedrocortesella calmorum Hunt, 1996

Distribución: Australia.

-Pedrocortesella conundrum Hunt, 1996

Distribución: Australia.

-Pedrocortesella cornuta (Hunt y Lee, 1995) (Acupedicellus)

Distribución: Australia.

-Pedrocortesella cryptoreticulata Hunt y Lee, 1995

DisTRIBUCIÓN: Australia.

-Pedrocortesella fusca (Rjabinin, 1986) (Pedrocortesia)

DisTRIBUCIÓN: Asia centrooccidental.

-Pedrocortesella gunjina Hunt, 1996

DistriBuCIÓN: Australia.

-Pedrocortesella gymnonota Hammer, 1966

DisTRIBUCIÓN: Nueva Zelanda.

-Pedrocortesella hangayi Hunt, 1996

DistriBución: Australia.

-Pedrocortesella hardyi Balogh, 1968

DisTRIBUCIÓn: Nueva Guinea y Japón.

-Pedrocortesella impedita Hunt, 1996

DisTRIBUCIÓN: Australia.

-Pedrocortesella japonica Aoki y Suzuki, 1970

DisTRIBUCión: Este de Paleártica oriental.

-Pedrocortesella kanangra Hunt, 1996

Distribución: Australia.

-Pedrocortesella leei Hunt, 1996

DisTribución: Australia.

-Pedrocortesella monicai Eguaras, Martínez y Fernández, 1990 DisTRIBUCIÓN: Argentina.

-Pedrocortesella montis Fernández, 1990 DISTRIBUCIÓN: Argentina.

-Pedrocortesella neonominata nom. nov. [=Pedrocortesia reticulata Rjabinin, 1986, "nom. praeoc." por Warburton, 1912 ("hom. sec.")]

DisTRIBUCIÓN: I. Kuriles.

-Pedrocortesella nortoni Hunt, 1996

DisTRIBUCión: Tasmania.

-Pedrocortesella obesa Hunt, 1996

DisTRIBUCIÓN: Australia.

-Pedrocortesella parva Pletzen, 1963

DISTRIBUCIÓN: Sudáfrica.

-Pedrocortesella propinqua P. Balogh, 1985

DisTRIBUCIÓN: Australia.

-Pedrocortesella pulchra Hammer, 1961

DisTRIBUCión: Neotropical (Perú y Chile) y Este de Rusia asiática.

-Pedrocortesella rarisetosa Bayartogtokh y Smelyansky, 2002

DisTRIBUCIÓN: Asia centrooccidental.

-Pedrocortesella reticulata (Warburton, 1912) (Notaspis)

DisTRIBUCIÓN: I. Seychelles.

-Pedrocortesella rjabinini Golosova, 1980

DistRIBUCIÓn: Este de Rusia asiática.

-Pedrocortesella sculptrata (Aoki, 1974) (Pedrocortesia)

Distribución: Corea y China.

-Pedrocortesella semireticulata Hunt y Lee, 1995

DisTRIBUCIÓN: Australia.

-Pedrocortesella stellata (Rjabinin, 1986) (Pedrocortesia)

DisTRIBUCIÓN: Asia centrooccidental.

-Pedrocortesella subula Hunt, 1996

DistRIBUCIÓN: Australia.

-Pedrocortesella temperata P. Balogh, 1985

DisTRIBUCIÓn: Australia.
-Pedrocortesella tristius Eguaras, Martínez y Fernández, 1990

DisTRIBUCIÓN: Argentina.

-Pedrocortesella truncata Hunt, 1996

DISTRIBUCIÓN: Australia.

Licnobelbidae Grandjean, 1965 (2 gen. y 3 spp.)

DisTRIBUCIÓN: Paleártica.

Flabellobelba Pérez-Íñigo, 1994 (1 sp.)

EsPeCIE TIPO: Licnobelba almeriensis Ruiz, Kahwash y Subías, 1990 DisTRIBUCIÓN: Paleártica.

-Flabellobelba almeriensis (Ruiz, Kahwash y Subías, 1990) (Licnobelba)

DISTRIBUCIÓN: España.

Licnobelba Grandjean, 1931 (2 spp.)

EsPECIE TIPO: (Licnobelba alestensis Grandjean, 1931) =Licneremaeus

latiflabellatus Paoli, 1908

DisTRIBUCIÓN: Paleártica.

-Licnobelba caesarea (Berlese, 1910) (Licneremaeus)

DisTRIBUCión: Mediterránea.

-Licnobelba latiflabellata (Paoli, 1908) (Licneremaeus)

(=Licnobelba alestensis Grandjean, 1931)

(=Licnobelba montana Mihelcic, 1957)

DistRIBUCIÓN: Paleártica occidental (excepto Norte).

Gymnodamaeoidea Grandjean, 1954

Gymnodamaeidae Grandjean, 1954 (8 gen. y 62 spp.)

DistRIBUCIÓN: Cosmopolita (excepto Australiana y Antártica).

Adrodamaeus Paschoal, 1984 (13 spp.)

(=Heterodamaeus Woolley, 1957, "nom. praeoc." por Ewing, 1917)

EsPeCIE TIPO: Damaeus magnisetosus Ewing, 1909

DISTRIBUCIÓN: Holártica.

-Adrodamaeus decemsetiger (Choi y Aoki, 1985) (Allodamaeus)

DISTRIBUCIÓN: Corea.

-Adrodamaeus femoratus (Koch, 1839) (Damaeus)

(=Damaeus dugesi Michael, 1890)

(=Belba gibba Canestrini y Fanzago, 1877)

Distribución: Paleártica (Paleártica occidental: excepto Norte, y Pakistán).

-Adrodamaeus haradai (Aoki, 1984) (Allodamaeus)

DisTRIBUCIÓN: Japón.

-Adrodamaeus hispanicus (Grandjean, 1928) (Gymnodamaeus)

(=Gymnodamaeus noster Mihelcic, 1957)

(=Gymnodamaeus sculptus Mihelcic, 1957)

DisTRIBUCIÓN: Paleártica meridional.

-Adrodamaeus italicus (Berlese, 1916) (Gymnodamaeus)

(=Arthrodamaeus parvulus Kunst, 1958)

DisTRIBUCIÓN: Europa centromeridional.

-Adrodamaeus magnisetosus (Ewing, 1909) (Damaeus)

DISTRIBUCIÓN: U.S.A. (Ilinois).

-Adrodamaeus musci Paschoal, 1984

DisTRIBUCión: U.S.A. (Misuri).

-Adrodamaeus pusillus (Berlese, 1910) (Gymnodamaeus)

DisTRIBUCIÓN: Europa centromeridional.

-Adrodamaeus rossicus (Bulanova-Zachvatkina, 1967) (Allodamaeus)

DistRIBUCIÓN: Rusia europea central.

-Adrodamaeus siculus (Berlese, 1910) (Gymnodamaeus reticulatus s.) (=Arthrodamaeus ignotus Paschoal, 1984)

DISTRIBUCIÓN: Europa centromeridional.

-Adrodamaeus starki (Bulanova-Zachvatkina, 1967) (Allodamaeus)

DisTRIBUCIÓN: Paleártica (Mediterránea oriental y Este de Rusia asiática).

-Adrodamaeus striatus (Aoki, 1984) (Allodamaeus)

Distribución: Este de Paleártica oriental.

-Adrodameus woonhahi (Choi y Aoki, 1985) (Allodamaeus)

DISTRIBUCION: Corea. 
Arthrodamaeus Grandjean, 1954 (6 spp.)

EsPeCIE TIPO: Gymnodamaeus reticulatus Berlese, 1910

DISTRIBUCIÓN: Paleártica.

-Arthrodamaeus bicristatus Subías, Arillo y J. Subías, 1997

DisTRIBución: Mediterránea occidental.

-Arthrodamaeus cereus Subías, Arillo y J. Subías, 1997

DisTRIBUCIÓN: Italia.

-Arthrodamaeus mediterraneus Subías, Arillo y J. Subías, 1997

DistribuCión: Mediterránea occidental.

-Arthrodamaeus octosetosus Subías, Arillo y J. Subías, 1997

DisTRIBUCIÓN: España.

-Arthrodamaeus reticulatus (Berlese, 1910) (Gymnodamaeus)

DisTRIBUCIÓN: Europa occidental.

-Arthrodamaeus rosarius Subías, Arillo y J. Subías, 1997

(?=Arthrodamaeus reticulatus tenuis Mihelcic, 1958 "sp. inq.")

DISTRIBUCIÓN: España.

Austrodamaeus Balogh y Mahunka, 1981 (2 spp.)

(=Idiodamaeus Paschoal, 1984)

EsPECIE TIPO: Austrodamaeus rimosus Balogh y Mahunka, 1981

DISTRIBUCIÓN: Etiópica y Neotropical.

-Austrodamaeus australis (Woas, 1992) (Aleurodamaeus) DisTribución: Sudáfrica.

-Austrodamaeus elegantulus (Hammer, 1958) (Gymnodamaeus)

(=Idiodamaeus illecebrosus Paschoal, 1984)

(=Austrodamaeus rimosus Balogh y Mahunka, 1981)

(=Allodamaeus trisetosus Balogh y Mahunka, 1969)

DisTRIBUCIÓN: Neotropical.

Gymnodamaeus Kulczynski, 1902 (22 spp.)

(=Heterodamaeus Ewing, 1917)

(=Johnstonella Paschoal, 1983 "sin. nov.")

(=Odontodamaeus Paschoal, 1982 "sin. nov.")

(=Pleodamaeus Paschoal, 1983 "sin. nov.")

ESPECIE TIPO: Damaeus bicostatus Koch, 1935

DisTRIBUCIÓN: Holártica y Oriental.

-Gymnodamaeus adpressus (Aoki y Fujikawa, 1971) (Allodamaeus)

DisTRIBUCión: Japón e India (Bengala Occidental).

-Gymnodamaeus bicostatus (Koch, 1835) (Damaeus)

(=Eremaeus asperulus Berlese, 1882)

DISTRIBUCIÓN: Holártica.

-Gymnodamaeus chalazionus Woolley, 1972

DisTRIBUCión: U.S.A. (Colorado).

-Gymnodamaeus gregarius Paschoal, 1982

DisTRIBUCIÓN: U.S.A. (Washington).

-Gymnodamaeus guilfordidei Paschoal, 1982

DisTRIBUCión: U.S.A. (Utah).

-Gymnodamaeus kazakhstanicus (Bayartogtokh y Smelyansky, 2002)

(Pleodamaeus)

DisTRIBUCIÓN: Asia centrooccidental.

-Gymnodamaeus knowltoni Paschoal, 1982

DisTRIBUCión: Oeste de U.S.A.

-Gymnodamaeus leurolomasus Woolley y Higgins, 1973

Distribución: Holártica (U.S.A.: Colorado, y Este de Rusia asiática).

-Gymnodamaeus notoapodematus Paschoal, 1982

DisTRIBUCIÓN: U.S.A. (Washington).

-Gymnodamaeus orbicularius Paschoal, 1982

DisTRIBUCIÓN: U.S.A. (Utah).

-Gymnodamaeus ornatus Hammer, 1952

DisTRIBUCIÓN: Canadá.

-Gymnodamaeus pearsei Banks, 1947

Distribución: U.S.A. (Carolina del Norte).

-Gymnodamaeus plokosus Woolley y Higgins, 1973

DisTRIBUCIÓN: Neártica.

-Gymnodamaeus quadriseta Ruiz, Kahwash y Subías, 1990

DisTRIBUCión: Mediterránea occidental.

-Gymnodamaeus rotundigranulatus (Bayartogtokh, 2001) (Pleodamaeus)

DisTRIBUCIÓN: Mongolia.
-Gymnodamaeus saltuensis Paschoal, 1982

DisTRIBUCIÓN: Canadá.

-Gymnodamaeus subalpinus (Paschoal, 1983) (Johnstonella)

DistRIBUCIÓN: U.S.A. (Washington).

-Gymnodamaeus taedaceus Paschoal, 1982

DisTRIBUCIÓN: Canadá.

-Gymnodamaeus theliis Woolley y Higgins, 1973

DisTRIBUCIÓN: U.S.A. occidental

-Gymnodamaeus umbraticus Paschoal, 1982

DisTRIBUCIÓN: U.S.A. (Utah)

-Gymnodamaeus veriornatus Higgins, 1961

DisTRIBUCIÓN: Oeste de U.S.A.

-Gymnodamaeus victoriae Paschoal, 1982

DISTRIBUCIÓN: Canadá.

Jacotella Banks, 1947 (9 spp.)

ESPECIE TIPO: Gymnodamaeus quadricaudiculus Jacot, 1937

DisTRIBUCIÓN: Holártica, Oriental y Neotropical.

-Jacotella austriaca (Willmann, 1935) (Gymnodamaeus)

(=Gymnodamaeus austriacus stepposus Bashkirova, 1958)

DistriBuCIÓN: Paleártica (Europa centromeridional y Asia centrooccidental).

-Jacotella enoplura Paschoal, 1983

DisTRIBUCIÓN: Holártica: Noroeste de U.S.A. y Sur de Europa (Bulgaria).

-Jacotella frondeus (Kulijev, 1979) (Plesiodamaeus)

(=Plesiodamaeus ornatus Mahunka, 1979)

(=Plesiodamaeus perezinigoi Mahunka, 1986)

DistRIBUCIÓN: Mediterránea oriental.

-Jacotella glabra (Mihelcic, 1957) (Plesiodamaeus)

(?=Gymnodamaeus aprofundatus Mihelcic, 1956 "sp. inq.")

DisTRIBUCIÓN: Paleártica meridional.

- Jacotella neonominata nom. nov.

[=Plesiodamaeus ornatus Pérez-Íñígo, 1972, “nom. praeoc.” por Balogh y Csiszár, 1963 ("hom. sec.”)]

DisTRIBUCIÓN: Mediterránea.

-Jacotella ornata (Balogh y Csiszár, 1963) (Allodamaeus)

(=Allodamaeus tuberculatus Aoki y Fujikawa, 1971)

DistRIBUCIÓN: Argentina e India (Himalaya).

-Jacotella quadricaudicula (Jacot, 1937) (Gymnodamaeus)

(=Gymnodamaeus minor Banks, 1947)

Distribución: Neártica (U.S.A.: Carolina del Norte, y Canadá).

- Jacotella reticulata Ruiz, Kahwash y Subías, 1990

DisTRIBUCión: Suroeste de Europa.

-Jacotella whartoni Paschoal, 1983

DisTRIBUCIÓN: U.S.A. (Washington).

Joshuella Wallwork, 1972 (3 spp.)

EsPECIE TIPO: Joshuella striata Wallwork, 1972

DisTRIBUCIÓN: Neártica.

- Joshuella agrosticula Paschoal, 1983

Distribución: U.S.A. (Colorado).

-Joshuella bicentenaria Paschoal, 1983

DisTRIBUCIÓN: Oeste de U.S.A.

-Joshuella striata Wallwork, 1972

DisTRIBUCIÓN: Suroeste de U.S.A. y Méjico.

Nortonella Paschoal, 1982 (6 spp.)

ESPECIE TIPO: Gymnodamaeus gildersleeveae Hammer, 1952

DisTRIBUCIÓN: Holártica.

-Nortonella gildersleeveae Hammer, 1952

DisTRIBUCIÓN: Neártica.

-Nortonella helvetica (Woas, 1992) (Gymnodamaeus)

DisTRIBUCIÓN: Suroeste de Europa.

-Nortonella mongolica Bayartogtokh y Aoki, 1997

DisTRIBUCIÓN: Mongolia.

-Nortonella polygrammus (Wen y Chen, 1992) (Allodamaeus)

DisTRIBUCIÓN: China suroriental. 
-Nortonella tectoria (Wen y Chen, 1992) (Allodamaeus)

DisTRIBUCIÓN: China suroriental.

-Nortonella transitus (Aoki, 1984) (Allodamaeus)

DisTRIBUCIÓN: Este de Paleártica oriental.

Plesiodamaeus Grandjean, 1954 (1 sp.)

EsPECIE TIPO: Damaeus craterifer Haller, 1884

DISTRIBUCIÓN: Paleártica.

-Plesiodamaeus craterifer (Haller, 1884) (Damaeus)

Distribución: Paleártica (Europa centromeridional y Sur de Siberia).

Aleurodamaeidae Paschoal y Johnston, 1985 (1 gen., 1 subg. y 5 spp.) DISTRIBUCIÓN: Paleártica meridional y Etiópica.

Aleurodamaeus Grandjean, 1954 (5 spp.)

EsPeCIE TIPO: Damaeus setosus Bersese, 1883

DISTRIBUCIÓN: Paleártica meridional y Etiópica.

Aleurodamaeus (Aleurodamaeus) Grandjean, 1954 (4 spp.)

DISTRIBUCIÓN: Paleártica meridional y Etiópica.

-Aleurodamaeus (A.) cephalotes (Berlese, 1916) (Gymnodamaeus)

DisTRIBUCIÓN: "África oriental".

-Aleurodamaeus (A.) hungaricus Paschoal y Johnston, 1985 DisTRIBUCIÓN: Hungría.

-Aleurodamaeus (A.) setosus (Berlese, 1883) (Damaeus)

(=Gymnodamaeus gibbus Mihelcic, 1963)

(=Gymnodamaeus nitidus Mihelcic, 1956)

DisTRIBUCIÓN: Paleártica meridional.

-Aleurodamaeus (A.) trichosus (Kulijev, 1979) (Allodamaeus)

DisTRIBUCIÓN: Cáucaso.

Aleurodamaeus (Trichodamaeus) Mahunka, 1984 (1 sp.)

ESPECIE TIPO: Aleurodamaeus (Trichodamaeus) africanus Mahunka, 1984 DisTRIBUCIÓN: Etiópica.

-Aleurodamaeus (Trichodamaeus) africanus Mahunka, 1984

DISTRIBUCIÓN: Tanzania.

\section{Damaeoidea Berlese, 1896}

Damaeidae Berlese, 1896 (12 gen, 15 subg., 250 spp. y 7 sspp.) DistriBUCión: Cosmopolita (excepto Antártica).

Acanthobelba Enami y Aoki, 1993 (2 spp.)

ESPECIE TIPO: Acanthobelba tortuosa Enami y Aoki, 1993

DisTRIBUCIÓN: Paleártica.

-Acanthobelba heterosetosa Choi, 1997

DISTRIBUCIÓN: Corea.

-Acanthobelba tortuosa Enami y Aoki, 1993

DISTRIBUCIÓN: Japón.

Belba Heyden, 1826 (41 spp. y 1 ssp.)

EsPECIE TIPO: Notaspis corynopus Hermann, 1804

DisTRIBUCIÓN: Holártica, Oriental y “Australiana”.

Belba (Belba) Heyden, 1826 (40 spp. y 1 ssp.)

DistriBuCión: Holártica, Oriental y "Australiana".

-Belba (B.) aegrota (Kulczynski, 1902) (Oribata)

DisTRIBUCIÓN: Europa central.

-Belba (B.) alpina Schwizer, 1956

DisTRIBUCIÓN: Suiza.

-Belba (B.) aurata Kulijev, 1967

DisTRIBUCión: Cáucaso.

-Belba (B.) barbata Fujita y Fujikawa, 1986

DisTRIBUCIÓN: Japón.

-Belba (B.) clavasensilla Norton y Palacios-Vargas, 1982

Distribución: Méjico.

-Belba (B.) compta (Kulczynski, 1902) (Oribata)

DisTRIBUCIÓN: Euroatlántica.
-Belba (B.) cornuta Wang y Norton, 1995

DisTRIBUCIÓN: China suroriental.

-Belba (B.) corynopus (Hermann, 1804) (Notaspis)

(=Belba bulbipes Karpelles, 1893)

(=Damaeus sufflexus Michael, 1885)

DisTRIBUCIÓN: Holártica y Norte de Oriental.

-Belba (B.) crassisetosa Bayartogtokh, 2000

DistriBución: Mongolia.

-Belba (B.) daghestanica Bulanova-Zachvatkina, 1962

DistriBución: Sureste de Europa.

-Belba (B.) dubinini Bulanova-Zachvatkina, 1962

DistriBUCión: Paleártica (Europa oriental: excepto Norte, y Oeste de Siberia).

-Belba (B.) flagellata Tseng, 1982

DisTRIBUCIÓN: Taiwan [Formosa].

-Belba (B.) flammeisetosa Tolstikov, 1995

DisTRIBUCIÓN: Asia centrooccidental.

-Belba (B.) globiceps (Canestrini y Fanzago, 1876) (Oribata) "sp. inq." DisTRIBUCIÓN: Italia.

-Belba (B.) helvetica Schweizer, 1956

DisTRIBUCIÓN: Suiza.

-Belba (B.) interlamellaris Willmann, 1939

DisTRIBUCIÓN: Sur de Paleártica occidental (Macaronésica: Madeira, y Ucrania).

-Belba (B.) jacoti Wilson, 1936

DisTRIBUCIÓN: Sureste de U.S.A.

-Belba (B.) limasetosa Bulanova-Zachvatkina, 1962

DisTRIBUCIÓN: Sureste de Europa.

-Belba (B.) macropoda Berlese, 1904

DisTRIBUCIÓN: Java.

-Belba (B.) meridionalis Bulanova-Zachvatkina, 1962

Distribución: Paleártica meridional (Sur de Europa y Sur de Paleártica oriental).

-Belba (B.) minor Mihelcic, 1953 "sp. inq."

DisTRIBUCIÓN: Austria.

-Belba (B.) minuta Bulanova-Zachvatkina, 1962

DISTRIBUCIÓN: Paleártica meridional (Rumania y Asia centrooccidental).

-Belba (B.) mongolica Bayartogtokh, 2000

DisTRIBUCIÓN: Mongolia.

-Belba (B.) paracorynopus Bulanova-Zachvatkina, 1962

DisTRIBUCIÓN: Paleártica (Rumania y Este de Paleártica oriental).

-Belba (B.) patelloides (Michael, 1888) (Notaspis)

(=Belba bartosi Winkler, 1955)

(=Belba pseudocorynopus Märkel, 1960)

DisTRIBUCIÓN: Paleártica occidental (excepto Norte).

-Belba (B.) pectinifera Canestrini, 1897 "sp. inq."

DistribuCión: Nueva Guinea.

-Belba (B.) piriformis Mihelcic, 1964

DISTRIBUCIÓN: Austria.

-Belba (B.) prasadi Bayartogtokh, 2000

DisTRIBUCIÓN: Mongolia.

-Belba (B.) pulchra Mihelcic, 1964

Distribución: Austria.

-Belba (B.) rossica Bulanova-Zachvatkina, 1962

DistriBución: Paleártica (Europa oriental y Paleártica oriental).

-Belba (B.) sarvari Tolstikov, 1995

DisTRIBUCIÓN: Asia centrooccidental.

-Belba (B.) sasakawai Enami, 1989

(=Belba notialis Wang y Norton, 1995)

DisTRIBUCIÓN: Japón y China.

-Belba (B.) sculpta Mihelcic, 1957

(=Belba aberrans Mihelcic, 1957)

(=Belba ignota Mihelcic, 1957)

DISTRIBUCIÓN: Mediterránea occidental.

-Belba (B.) sellnicki Bulanova-Zachvatkina, 1962

DisTRIBUCIÓN: Paleártica (Ucrania y Este de Paleártica oriental).

-Belba (B.) servadeii Lombardini, 1963 "sp. inq."

DISTRIBUCIÓN: Italia. 
-Belba (B.) subtilis Canestrini, 1897 "sp. inq."

DisTRIBUCIÓN: Nueva Guinea.

-Belba (B.) tenuisetosa Bulanova-Zachvatkina, 1962

DisTRIBUCIÓN: Asia centrooccidental.

-Belba (B.) unicornis Enami,1994

Distribución: Este de Paleártica oriental.

-Belba (B.) ursina Thor, 1930

DisTRIBUCIÓN: Noruega.

-Belba (B.) verrucosa Bulanova-Zachvatkina, 1962

DisTRIBUCIÓN: Paleártica (Europa: excepto Oeste y Paleártica orien-

tal) y Taiwan [Formosa].

-Belba (B.) verrucosa verrucosa Bulanova-Zachvatkina, 1962

DisTRIBUCIÓN: Paleártica (Europa: excepto Oeste y Paleártica oriental).

-Belba (B.) verrucosa japonica Aoki, 1984

DisTRIBUCIÓN: Japón y Taiwan [Formosa].

Belba (Caenobelba) Norton, 1980 (1 sp.)

EsPeCIE TIPO: Caenobelba alleganiensis Norton, 1980

DisTRIBUCIÓN: Neártica.

-Belba (Caenobelba) alleganiensis (Norton, 1980) (C.)

DisTRIBUCIÓN: Neártica septentrional.

Belbodamaeus Bulanova-Zachvatkina, 1960 (4 spp.)

EsPECIE TIPO: Belbodamaeus tuberculatus Bulanova-Zachvatkina, 1960 DisTRIBUCIÓN: Holártica.

Belbodamaeus (Belbodamaeus) Bulanova-Zachvatkina, 1960 (2 spp.) DisTRIBUCIÓN: Paleártica.

-Belbodamaeus (B.) marginatus Kulijev, 1967

Distribución: Cáucaso.

-Belbodamaeus (B.) tuberculatus Bulanova-Zachvatkina, 1960

DistRIBUCIÓN: Paleártica (Europa oriental y Paleártica oriental).

Belbodamaeus (Lanibelba) Norton, 1980 (1 sp.)

EsPECIE TIPO: Lanibelba pini Norton, 1980

DisTRIBUCIÓN: Neártica.

-Belbodamaeus (Lanibelba) pini (Norton, 1980) (L.)

DisTRIBUCIÓN: Neártica.

Belbodamaeus (Protobelba) Norton, 1979 (1 sp.)

ESPECIE TIPO: Oribata californica Banks, 1904

DisTRIBUCIÓN: Neártica.

-Belbodamaeus (Protobelba) californica (Banks, 1904) (Oribata) DisTRIBUCIÓN: U.S.A. occidental.

Damaeus Koch, 1835 (116 spp. y 3 sspp.)

EsPeCIE TIPO: Damaeus auritus Koch, 1835

DisTRIBUCIÓN: Holártica y Paleotropical.

Damaeus (Damaeus) Koch, 1835 (33 spp. y 1 ssp.)

(=Hypodamaeus Bulanova-Zachvatkina, 1957)

DisTRIBUCIÓN: Holártica.

-Damaeus (D.) ainu Enami y Aoki, 1998

DisTRIBUCIÓN: Japón.

-Damaeus (D.) angustipes (Banks, 1905) (Oribata)

(=Belba kingi Hartenstein, 1962)

DisTRIBUCIÓN: U.S.A. oriental.

-Damaeus (D.) appalachicus Norton, 1978

DisTRIBUCIÓN: Este de U.S.A.

-Damaeus (D.) arvernensis Grandjean, 1960

DistribuCión: Oeste de Europa.

-Damaeus (D.) auritus Koch, 1835

(=Oribata gracilipes Kulczynski, 1902)

DisTRIBUCIÓN: Holártica (Paleártica: frecuente, y Groenlandia).

-Damaeus (D.) australis (Banks, 1895) (Belba)

(=Damaeus atlanticus Norton, 1978)

DisTRIBUCIÓN: U.S.A.

-Damaeus (D.) bulbipedata Packard, 1887 "sp. inq."

DisTRIBUCIÓN: U.S.A. (Kentucky).
-Damaeus (D.) bulbofemoralis Bulanova-Zachvatkina, 1957 (D.

(Hypodamaeus))

DisTRIBUCIÓN: Asia centrooccidental.

-Damaeus (D.) costanotus Wang y Norton, 1989

DistribuCIÓN: China.

-Damaeus (D.) crispatus (Kulczynski, 1902) (Oribata)

DisTRIBUCIÓN: Paleártica (Europa y Pakistán).

-Damaeus (D.) echinopus Bulanova-Zachvatkina, 1957 (D. (Hypodamaeus))

DisTRIBUCIÓN: Sureste de Europa.

-Damaeus (D.) flagellatus Wang, 1994

DisTRIBUCIÓN: China suroriental.

-Damaeus (D.) flagellifer Michael, 1890

(=Damaeus kulczynskii Grandjean, 1943)

(=Damaeus longipes Mihelcic, 1957, "nom. praeoc." por Willmann, 1941)

(=Belba pachytrichosa Mihelcic, 1956)

DisTRIBUCIÓN: Paleártica occidental (excepto Norte).

-Damaeus (D.) formica (Gervais, 1849) (Oribata) "sp. inq."

Distribución: Chile.

-Damaeus (D.) foroliviensis (Lombardini, 1943) (Neophyllobius) "sp. inq."

DisTRIBUCIÓN: Italia.

-Damaeus (D.) glycyphagoides Bulanova-Zachvatkina, 1957 (D.

(Hypodamaeus))

Distribución: Cáucaso.

-Damaeus (D.) granulatus (Willmann, 1951) (Belba)

DisTRIBUCIÓN: Austria.

-Damaeus (D.) grossmani (Wilson, 1936) (Oribata)

DisTRIBUCIÓN: U.S.A. suroriental.

-Damaeus (D.) lengersdorfi (Willmann, 1932) (Belba)

DisTRIBUCIÓN: Europa central.

-Damaeus (D.) lengersdorfi lengersdorfi (Willmann, 1932)

DistriBUCión: Europa central.

-Damaeus (D.) lengersdorfi moraviae (Willmann, 1954) (Belba) DisTRIBUCIÓN: Chequia.

-Damaeus (D.) longipes (Willmann, 1941) (Belba)

Distribución: "Yugoslavia".

-Damaeus (D.) maximus Mihelcic, 1957

DisTRIBUCIÓN: Europa meridional (España y Rumania).

-Damaeus (D.) nidicola (Willmann, 1936) (Belba)

DistRIBUCIÓN: Euroatlantica.

-Damaeus (D.) nivalis (Kulczynski, 1902) (Oribata)

Distribución: Polonia y U.S.A. (Ténesi).

-Damaeus (D.) opilioides Norton, 1978

Distribución: U.S.A. (Carolina del Norte).

-Damaeus (D.) ortizi Pérez-Íñigo, 1995

DisTRIBUCIÓN: España.

-Damaeus (D.) pseudoauritus Bulanova-Zachvatkina, 1957 (D.

(Hypodamaeus))

DistriBuCión: Cáucaso.

-Damaeus(D.) recasensi Capilla, 1971

DisTRIBUCIÓN: Mediterránea occidental.

-Damaeus (D.) riparius Nicolet, 1855

(=Damaeus alienus Hull, 1915)

DistRIBUCIÓN: Paleártica (frecuente).

-Damaeus (D.) selgae Pérez-Íñigo, 1966

DisTRIBUCión: España.

-Damaeus (D.) smirnovi Bulanova-Zachvatkina, 1957 (D. (Hypo-

damaeus))

DistriBUCIÓN: Paleártica (Europa oriental: excepto Norte, y Este de Paleártica oriental)

-Damaeus (D.) tauricus Bulanova-Zachvatkina, 1957 (D. (Hypodamaeus))

DisTRIBUCIÓN: Ucrania.

-Damaeus (D.) tenuitibialis Bulanova-Zachvatkina, 1957 (D.

(Hypodamaeus))

DisTRIBUCIÓN: Paleártica oriental (excepto Norte).

-Damaeus (D.) torquisetosus (Mihelcic, 1955) (Belba)

(=Damaeus ornatus Mihelcic, 1957)

DisTRIBUCIÓN: España. 
Damaeus (Adamaeus) Norton, 1977 (2 spp.)

ESPECIE TIPO: Damaeus onustus Koch, 1841

DisTRIBUCIÓN: Paleártica y Etiópica.

-Damaeus (Adamaeus) firmus Kunst, 1958 (D.)

DisTRIBUCión: Europa meridional (Bulgaria y Rumania).

-Damaeus (Adamaeus) onustus Koch, 1841 (D.)

(=Belba geniculosa Oudemans, 1929)

DisTRIBUCIÓN: Paleártica occidental (frecuente), Etiópica (Tanzania) e I. Santa Helena.

Damaeus (Epidamaeus) Bulanova-Zachvatkina, 1957 (79 spp. y 2 sspp.) EsPECIE TIPO: Oribata bituberculata Kulczynski, 1902

DisTRIBUCIÓN: Holártica y tropical (Oriental y Neotropical).

-Damaeus (Epidamaeus) aborigensis (Behan-Pelletier y Norton, 1985)

(E.)

DistriBución: Este de Rusia asiática.

-Damaeus (Epidamaeus) affinis (Bulanova-Zachvatkina, 1957) (E.)

Distribución: Paleártica (Noreste de Paleártica occidental y Centrooeste de Paleártica oriental).

-Damaeus (Epidamaeus) aleinikovae (Bulanova-Zachvatkina, 1964)

(E.)

DisTRIBUCión: Este de Rusia europea.

-Damaeus (Epidamaeus) angulatus (Fujikawa y Fujita, 1985) (E.) DisTRIBUCIÓN: Japón.

-Damaeus (Epidamaeus) angustirostratus (Bayartogtokh, 2001) (E.) DisTRIBUCIÓN: Mongolia.

-Damaeus (Epidamaeus) aokii (Bayartogtokh, 2001) (E.)

DISTRIBUCIÓN: Mongolia.

-Damaeus (Epidamaeus) arcticola (Hammer, 1952) (Belba) DisTRIBUCIÓn: Boreal.

-Damaeus (Epidamaeus) bacillum (Fujikawa y Fujita, 1985) (E.) DisTRIBUCIÓN: Japón.

-Damaeus (Epidamaeus) bakeri (Hammer, 1952) (Belba) DisTRIBUCIÓN: Boreal.

-Damaeus (Epidamaeus) berlesei Michael, 1908 (D.)

DisTRIBUCión: Paleártica occidental (excepto Norte).

-Damaeus (Epidamaeus) bituberculatus (Kulczynski, 1902) (Oribata) Distribución: Paleártica.

-Damaeus (Epidamaeus) brevisetosus (Bayartogtokh, 2000) (E.)

DisTRIBUCIÓN: Mongolia.

-Damaeus (Epidamaeus) brevitibialis Bulanova-Zachvatkina, 1957

(D. (Hypodamaeus))

DisTRIBUCIÓN: Norte de Europa

-Damaeus (Epidamaeus) canadensis (Banks, 1909) (Oribata)

DisTRIBUCIÓN: Neártica (U.S.A.: Ilinois, y Canadá).

-Damaeus (Epidamaeus) chukchi (Behan-Pelletier y Norton, 1985) (E.) DisTRIBUCIÓN: Este de Rusia asiática.

-Damaeus (Epidamaeus) cincinnatus (Wang y Norton, 1993) (E.) DistRIBUCión: Noreste de China.

-Damaeus (Epidamaeus) coreanus Aoki, 1966 (D. (Hypodamaeus)) DisTRIBUCIÓN: Este de Paleártica oriental.

-Damaeus (Epidamaeus) coxalis (Hammer, 1952) (Belba) DisTRIBUCIÓN: Boreal.

-Damaeus (Epidamaeus) craigheadi (Jacot, 1939) (Metabelba) DisTRIBUCIÓn: U.S.A. (Nueva Hampshire).

-Damaeus (Epidamaeus) crassisensillatus (Bayartogtokh, 2000) (E.) DistriBución: Mongolia.

-Damaeus (Epidamaeus) culterisetosus (Bayartogtokh, 2000) (E.) DISTRIBUCIÓN: Mongolia.

-Damaeus (Epidamaeus) elegantis (Wang y Norton, 1993) (E.) DisTRIBUCIÓN: China suroriental.

-Damaeus (Epidamaeus) farinosus (Trägardh, 1902) (Oribata) DisTRIBUCIÓN: Suecia.

-Damaeus (Epidamaeus) flagelloides (Norton, 1979) (E.) Distribución: Chile.

-Damaeus (Epidamaeus) flexispinosus (Kunst, 1961) (E.) DisTRIBUCIÓN: Sureste de Europa.

-Damaeus (Epidamaeus) flexus (Fujikawa y Fujita, 1985) (E.) DISTRIBUCIÓN: Japón.
-Damaeus (Epidamaeus) floccosus (Behan-Pelletier y Norton, 1985)

(E.)

DisTRIBUCIÓN: Neártica septentrional.

-Damaeus (Epidamaeus) floridus (Wilson, 1936) (Belba globifer $f$.)

(=Belba alachua Wilson, 1936)

DisTRIBUCIÓN: Sureste de U.S.A.

-Damaeus (Epidamaeus) folium (Fujikawa y Fujita, 1985) (E.)

DISTRIBUCIÓN: Japón.

-Damaeus (Epidamaeus) fortisensillus (Enami y Aoki, 2001) (E.)

DisTRIBUCIÓN: Japón.

-Damaeus (Epidamaeus) fortispinosus (Hammer, 1967) (E.)

DisTRIBUCIÓN: Boreal.

-Damaeus (Epidamaeus) fragilis (Enami y Fujikawa, 1989) (E.)

DisTRIBUCIÓN: Japón.

-Damaeus (Epidamaeus) gibbofemoratus (Hammer, 1955) (Belba)

DistriBución: Alaska.

-Damaeus (Epidamaeus) gilyarovi (Behan-Pelletier y Norton, 1985) (E.)

DistRIBUCIÓN: Este de Rusia asiática.

-Damaeus (Epidamaeus) globifer Ewing, 1913 (D.)

DisTRIBUCIÓN: U.S.A. nororiental.

-Damaeus (Epidamaeus) golosovae (Ljaschev y Tolstikov, 1993) (E.)

DisTRIBUCIÓN: Asia centrooccidental.

-Damaeus (Epidamaeus) granulatus (Bayartogtokh, 2000) (E.)

DisTRIBUCIÓn: Mongolia.

-Damaeus (Epidamaeus) groenlandicus (Hammer, 1953) (Belba)

DisTRIBUCIÓN: Boreal (Groenlandia y Oeste de Siberia).

-Damaeus (Epidamaeus) hammerae (Behan-Pelletier y Norton, 1983)

(E.)

DistRibución: Este de Rusia asiática.

-Damaeus (Epidamaeus) hastatus (Hammer, 1967) (E.)

DisTRIBUCIÓN: Alaska.

-Damaeus (Epidamaeus) inornatus Strenzke, 1952 (D.)

DistribuCión: Groenlandia.

-Damaeus (Epidamaeus) johnstoni (Tolstikov, 1997) (E.)

DISTRIBUCIÓN: Asia centrooccidental.

-Damaeus (Epidamaeus) kamaensis (Sellnick, 1925) (Oribata)

DisTRIBUCIÓN: Paleártica.

-Damaeus (Epidamaeus) karelicus (Bulanova-Zachvatkina, 1957) (E.)

DisTRIBUCIÓN: Noreste de Europa.

-Damaeus (Epidamaeus) khustaiensis (Bayartogtokh, 2000) (E.)

DisTRIBUCIÓN: Mongolia.

-Damaeus (Epidamaeus) kodiakensis (Hammer, 1967) (E.)

DistribuCión: Boreal (Neártica septentrional, Este de Paleártica oriental).

-Damaeus (Epidamaeus) koyukon (Behan-Pelletier y Norton, 1985) (E.)

DisTRIBUCIÓN: Neártica septentrional.

-Damaeus (Epidamaeus) longipedus (Bulanova-Zachvatkina, 1974) (E.)

DistRIBUCIÓn: Cáucaso.

-Damaeus (Epidamaeus) longisetosus (Willmann, 1953) (Belba)

DISTRIBUCIÓN: Europa centromeridional.

-Damaeus (Epidamaeus) longispinosus (Wang y Norton, 1993) (E.)

DisTRIBUCIÓN: China suroriental.

-Damaeus (Epidamaeus) longitarsalis (Hammer, 1952) (Belba)

DisTRIBUCIÓN: Boreal.

-Damaeus (Epidamaeus) mackenziensis (Hammer, 1952) (Belba)

DisTRIBUCIÓN: Neártica septentrional.

-Damaeus (Epidamaeus) michaeli Ewing, 1909 (D.)

DISTRIBUCIÓN: U.S.A. oriental.

-Damaeus (Epidamaeus) microspinus (Bulanova-Zachvatkina, 1957)

(E.)

DistriBución: Este de Paleártica oriental

-Damaeus (Epidamaeus) mitlsensillus (Palacios-Vargas, 1984) (E.)

DisTRIBUCión: Méjico.

-Damaeus (Epidamaeus) mongolicus (Bayatortogkh, 2000) (E.)

DisTRIBUCIÓN: Mongolia.

-Damaeus (Epidamaeus) nasutus (Behan-Pelletier y Norton, 1985) (E.)

DisTRIBUCIÓN: Neártica septentrional.

-Damaeus (Epidamaeus) olitor (Jacot, 1937) (Belba)

DistriBUCIÓN: U.S.A. (Carolina del Norte). 
-Damaeus (Epidamaeus) paraspinosus (Bulanova-Zachvatkina, 1974)

(E)

DisTRIBUCión: Paleártica oriental (Noroeste y Sur de Siberia).

-Damaeus (Epidamaeus) pavlovskii (Bulanova-Zachvatkina, 1957) (E)

DisTRIBUCIÓN: Paleártica (Sureste de Europa y Asia centroocidental).

-Damaeus (Epidamaeus) pinguis (Kulijev, 1967) (E.)

DisTRIBUCIÓN: Cáucaso.

-Damaeus (Epidamaeus) plumosus (Bulanova-Zachvatkina, 1974) (E.)

DisTRIBUCIÓN: Europa oriental (centromeridional).

-Damaeus (Epidamaeus) pseudotatricus (Bulanova-Zachvatkina,

1957) $(E$.

Distribución: Este de Paleártica occidental.

-Damaeus (Epidamaeus) puritanicus (Banks, 1906) (Oribata)

(=Epidamaeus grandjeani Bulanova-Zachvatkina, 1957)

DisTRIBUCIÓN: Holártica.

-Damaeus (Epidamaeus) pyrenaicus (Pérez-Íñigo jr., 1991) (E.)

(=Epidamaeus ibericus Moraza, Moreno y Saloña, 1991)

DisTRIBUCIÓN: España.

-Damaeus (Epidamaeus) quadrituberculatus (Bulanova-Zachvatkina

1957) $(E$.)

DISTRIBUCIÓN: Paleártica (Europa centromeridional: Rumania, y Asia centrooccidental).

-Damaeus (Epidamaeus) setiger (Kulczynski, 1902) (Oribata)

DistRibución: Europa oriental (excepto Norte).

-Damaeus (Epidamaeus) subiasi (Pérez-Íñigo, 1995) (E.)

DisTRIBUCIÓN: España.

-Damaeus (Epidamaeus) tatricus (Kulczynski, 1902) (Oribata)

DisTRIBUCIÓN: Holártica.

-Damaeus (Epidamaeus) tatricus tatricus (Kulczynski, 1902) DisTRIBUCIÓN: Holártica.

-Damaeus (Epidamaeus) tatricus diversus (Mihelcic, 1952) (Belba) DISTRIBUCIÓN: Austria.

-Damaeus (Epidamaeus) tecticola Michael, 1888 (D.)

DisTRIBUCIÓN: Europa.

-Damaeus (Epidamaeus) tecticola tecticola Michael, 1888 DisTRIBUCIÓN: Europa.

-Damaeus (Epidamaeus) tecticola romaniae Cooreman, 1951 (D.) DisTRIBUCIÓN: Rumania.

-Damaeus (Epidamaeus) tenuipes Michael, 1885 (D.)

DistRIBUCIÓN: Holártica (Europa occidental: I. Británicas, Oeste de Paleártica oriental, I. Kuriles y Groenlandia).

-Damaeus (Epidamaeus) tenuisetosus (Bayartogtokh, 2001) (E.)

Distribución: Mongolia.

-Damaeus (Epidamaeus) tenuissimus (Hammer, 1967) (E.)

DisTRIBUCIÓN: Neártica septentrional.

-Damaeus (Epidamaeus) trigonalis Strenzke, 1952 (D.)

DisTRIBUCIÓN: Groenlandia.

-Damaeus (Epidamaeus) tritylos (Behan-Pelletier y Norton, 1983) (E.) Distribución: Boreal (Neártica septentrional y Este de Rusia asiática).

-Damaeus (Epidamaeus) uenoi (Aoki, 1966) (E.)

DisTRIBUCIÓN: Corea.

-Damaeus (Epidamaeus) variabilis (Fujikawa y Fujita, 1985) (E.)

DisTRIBUCIÓN: Japón.

-Damaeus (Epidamaeus) verrucatus (Enami y Fujikawa, 1989) (E.) DisTRIBUCIÓN: Japón.

-Damaeus (Epidamaeus) yunnanensis (Enami, Aoki y Hu, 1994) (E.)

DISTRIBUCIÓN: China suroriental.

Damaeus (Eudamaeus) Pérez-Íñigo, 1987 (1 sp.)

EsPeCIE TIPO: Damaeus (Eudamaeus) pomboi Pérez-Íñigo, 1987

DisTRIBUCIÓN: Paleártica.

-Damaeus (Eudamaeus) pomboi Pérez-Íñigo, 1987

DistriBución: I. Azores.

Damaeus (Paradamaeus) Bulanova-Zachvatkina, 1957 (1 sp.)

EsPECIE TIPO: Notaspis clavipes Hermann, 1804

Distribución: Paleártica.

-Damaeus (Paradamaeus) clavipes (Hermann, 1804) (Notaspis)

(=Damaeus nodipes Koch, 1839)

DiSTRIBUCIÓN: Paleártica occidental (frecuente).
Dameobelba Sellnick, 1928 (1 sp.)

ESPECIE TIPO: Oribata minutissima Sellnick, 1920

DisTRIBUCIÓN: Paleártica.

-Dameobelba minutissima (Sellnick, 1929) (Oribata)

Distribución: Paleártica (Paleártica occidental y Este de Rusia asiática).

Metabelba Grandjean, 1936 (31 spp.)

EsPECIE TIPO: Damaeus papillipes Nicolet, 1855

DisTRIBUCIÓN: Cosmopolita (excepto Neotropical y Antártica).

Metabelba (Metabelba) Grandjean, 1936 (22 spp.)

DisTRIBUCIÓN: Cosmopolita (excepto Neotropical y Antártica).

-Metabelba (M.) aphelesa (Woolley y Higgins, 1979) (Dasybelba)

DisTRIBUCIÓN: U.S.A. (Colorado).

-Metabelba (M.) benoiti Balogh, 1958 “sp. inq."

DisTRIBUCIÓN: Tanzania.

-Metabelba (M.) filippovae Bulanova-Zachvatkina, 1965

DisTRIBUCIÓN: Cáucaso.

-Metabelba (M.) flagelliseta Bulanova-Zachvatkina, 1965

DisTRIBUCIÓN: Sureste de Europa.

-Metabelba (M.) glabriseta Mahunka, 1982

(?=Metabelba angolensis Balogh, 1958 "sp. inq.")

DisTRIBUCIÓN: Etiópica (Etiopía y Angola).

-Metabelba (M.) heteropoda Balogh, 1958 “sp. inq."

DisTRIBUCIÓN: Congo.

-Metabelba (M.) horrida Balogh, 1958 “sp. inq."

DistribuCIÓn: Congo.

-Metabelba (M.) machadoi Pérez-Íñigo, 1986

DistribuCión: I. Canarias.

-Metabelba (M.) monilipeda Bulanova-Zachvatkina, 1965

DisTRIBUCIÓN: Cáucaso.

-Metabelba (M.) obtusa Hammer, 1966

DistriBución: Nueva Zelanda e India (Bengala Occidental).

-Metabelba (M.) orientalis Balogh y Mahunka, 1967

DISTRIBUCIÓN: Vietnam.

-Metabelba (M.) papillipes (Nicolet, 1855) (Damaeus)

(=Metabelba cremersi Hammen, 1952)

$(=$ Belba globipes Canestrini y Berlese, 1884$)$

(=Belba michaeli Oudemans, 1900)

(=Belba obscura Mihelcic, 1956)

DisTRIBUCIÓN: Holártica (Paleártica occidental: menos frecuente en el Norte, Oeste de Siberia y U.S.A.).

-Metabelba (M.) paraitalica Kulijev, 1967

Distribución: Cáucaso.

-Metabelba (M.) parapulverosa Moritz, 1966

DISTRIBUCIÓN: Europa centromeridional.

-Metabelba (M.) propexa (Kulczynski, 1902) (Oribata)

DisTRIBUCIÓN: Europa central.

-Metabelba (M.) propinqua (Sellnick, 1943) (Damaeus)

DisTRIBUCIÓN: Italia.

-Metabelba (M.) pseudoitalica Bulanova-Zachvatkina, 1965

DistriBución: Cáucaso.

-Metabelba (M.) pulverulenta (Koch, 1839) (Nothrus)

(=Metabelba pulverosa Strenzke, 1953)

DisTRIBUCIÓN: Holártica (Paleártica: frecuente, y U.S.A.: Virginia).

-Metabelba (M.) rara Bulanova-Zachvatkina, 1965

DisTRIBUCIÓN: Paleártica (Sureste de Europa y Oeste de Siberia).

-Metabelba (M.) rohdendorfi Bulanova-Zachvatkina, 1965

DistriBUCIÓN: Europa centrooriental.

- Metabelba (M.) singularis Mihelcic, 1964

DistriBuCión: Austria.

-Metabelba (M.) tanganyikensis Balogh, 1958 "sp. inq."

DisTRIBUCIÓN: Tanzania.

Metabelba (Allobelba) Kunst, 1961 (1 sp.)

ESPECIE TIPO: Allobelba aculeata Kunst, 1961

DisTRIBUCIÓN: Paleártica.

- Metabelba (Allobelba) aculeata (Kunst, 1961) (A.)

DISTRIBUCIÓN: Europa meridional (Bulgaria y Rumania). 
Metabelba (Neobelba) Bulanova-Zachvatkina, 1967 (1 sp.)

ESPECIE TIPO: Neobelba pseudopapillipes Bulanova-Zachvatkina, 1967 DisTRIBUCIÓN: Paleártica.

-Metabelba (Neobelba) pseudopapillipes (Bulanova-Zachvatkina, 1967) $(N$.)

DISTRIBUCIÓN: Europa meridional.

Metabelba (Parametabelba) Mihelcic, 1964 (7 spp.)

EsPeCIE TIPO: (Metabelba gladiator Mihelcic, 1963) =Damaeus romandiolae Sellnick, 1943

DisTRIBUCIÓN: Paleártica.

-Metabelba (Parametabelba) ericius Kunst, 1958 (M.)

DisTRIBUCIÓN: Sureste de Europa.

-Metabelba (Parametabelba) italica (Sellnick, 1931) (Damaeus)

DISTRIBUCIÓN: Europa centromeridional.

-Metabelba (Parametabelba) lanceolata Hammen, 1952 (M.) DisTRIBUCIÓN: Holanda.

-Metabelba (Parametabelba) platynotus Grandjean, 1954 (M.)

DisTRIBUCIÓN: Mediterránea occidental.

- Metabelba (Parametabelba) rhodopeia Kunst, 1961 (M.)

DisTRIBUCIÓn: Sureste de Europa.

-Metabelba (Parametabelba) romandiolae (Sellnick, 1943) (Damaeus)

(=Metabelba gladiator Mihelcic, 1963)

DisTRIBUCIÓN: Suroeste de Europa.

-Metabelba (Parametabelba) sphagni Strenzke, 1950 (M.)

DISTRIBUCIÓN: Euroatlántica.

Metabelbella Bulanova-Zachvatkina, 1957 (14 spp.)

ESPECIE TIPO: Metabelbella zachvatkini Bulanova-Zachvatkina, 1957

DisTRIBUCIÓN: Holártica y Neotropical.

Metabelbella (Metabelbella) Bulanova-Zachvatkina, 1957 (10 spp.)

DisTRIBUCIÓN: Paleártica.

-Metabelbella (M.) clavigera (Willmann, 1954) (Belba)

DisTRIBUCIÓN: Chequia.

-Metabelbella (M.) gratiosa (Willmann, 1941) (Belba)

DistriBución: Europa centrooriental (excepto Norte).

-Metabelbella (M.) interlamellaris Pérez-Iñigo, 1987

DISTRIBUCIÓN: Mediterránea occidental.

-Metabelbella (M.) janae Pérez-Íñigo jr., 1991

DisTRIBUCIÓN: España.

-Metabelbella (M.) kosarovi Jeleva, 1970

DisTRIBUCIÓN: Bulgaria.

-Metabelbella (M.) macerochaeta Bulanova-Zachvatkina, 1965 DisTRIBUCIÓN: Sureste de Europa.

-Metabelbella (M.) phalangioides (Michael, 1890) (Damaeus)

DisTRIBUCIÓN: Mediterránea occidental.

-Metabelbella (M.) soror Bulanova-Zachvatkina, 1965

DisTRIBUCIÓN: Europa oriental central.

-Metabelbella (M.) tichonravovi Bulanova-Zachvatkina, 1965

DisTRIBUCIÓN: Ucrania (Crimea).

-Metabelbella (M.) zachvatkini Bulanova-Zachvatkina, 1957

DisTRIBUCIÓN: Cáucaso.

Metabelbella (Akrodamaeus) Norton, 1979 (4 spp.)

EsPECIE TIPO: Oribata longiseta Banks, 1906

DisTRIBUCIÓN: Neártica y Neotropical.

-Metabelbella (Akrodamaeus) flagellata (Balogh y Mahunka, 1969)

(Metabelba)

DisTRIBUCión: Neotropical (Bolivia y Chile).

-Metabelbella (Akrodamaeus) inaequipes (Banks, 1947) (Belba)

DistriBución: U.S.A. (Carolina del Norte).

-Metabelbella (Akrodamaeus) longiseta (Banks, 1906) (Oribata)

DisTRIBUCIÓN: Neártica.

-Metabelbella (Akrodamaeus) meridiana (Norton, 1979) (Epidamaeus (A.))

DisTRIBUCIÓN: Chile.

Nortonbelba Bernini, 1980 (1 sp. y 1 ssp.)

EsPECIE TIPO: Nortonbelba italica Bernini, 1980
DiSTRIBUCIÓN: Paleártica.

- Nortonbelba italica Bernini, 1980

DisTRIBUCIÓN: Mediterránea occidental.

-Nortonbelba italica italica Bernini, 1980

DisTRIBUCIÓN: Mediterránea occidental.

-Nortonbelba italica plesiomorphica (Saloña e Iturrondobeitia,

1989) (Epidamaeus p.)

DisTRIBUCióN: España.

Parabelbella Bulanova-Zachvatkina, 1967 (16 spp.)

EsPECIE TIPO: Metabelbella crenatosetosa Bulanova-Zachvatkina, 1957

DISTRIBUCIÓN: Holártica y Oriental.

Parabelbella (Parabelbella) Bulanova-Zachvatkina, 1967 (4 spp.)

(=Heterodamaeus Mihelcic, 1964, "nom. praeoc." por Ewing, 1917)

(=Mirobelba Pérez-Íñigo y Peña, 1994 "sin. nov.")

DisTRIBUCIÓN: Paleártica.

-Parabelbella (P.) crenatosetosa (Bulanova-Zachvatkina, 1957)

(Metabelbella)

DistriBución: Centro de Paleártica oriental.

-Parabelbella (P.) elisabethae Bulanova-Zachvatkina, 1967

DistribuCión: Paleártica (Centro de Rusia europea y Asia centrooccidental).

-Parabelbella (P.) exilior (Mihelcic, 1964) (Damaeus (Heterodamaeus))

DistRIBUCIÓN: Italia.

-Parabelbella (P.) grancanariae (Pérez-Íñigo y Peña, 1994) (Mirobelba) DISTRIBUCIÓN: I. Canarias.

Parabelbella (Nododamaeus) Hammer, 1977 (2 spp.)

(=Dasybelba Woolley y Higgins, 1979 "sin. nov.")

ESPECIE TIPO: Nododamaeus monticola Hammer, 1977

DisTRIBUCIÓN: Holártica.

-Parabelbella (Nododamaeus) monticola (Hammer, 1977) (N.)

Distribución: Pakistán.

-Parabelbella (Nododamaeus) perona (Woolley y Higgins, 1979)

(Dasybelba)

Distribución: U.S.A. (Colorado).

Parabelbella (Tectodamaeus) Aoki, 1984 (10 spp.)

ESPECIE TIPO: Tectodamaeus armata Aoki, 1984

DISTRIBUCIÓN: Paleártica y Oriental.

-Parabelbella (Tectodamaeus) armatus (Aoki, 1984) (T.)

DisTRIBUCión: Este de Paleártica oriental.

-Parabelbella (Tectodamaeus) breviseta (Wang, 1994) (Damaeus)

DisTRIBUCIÓN: China suroriental.

-Parabelbella (Tectodamaeus) cuii (Wang y Lu, 1995) (Damaeus (T.))

DisTRIBUCIÓN: China suroriental.

-Parabelbella (Tectodamaeus) exserta (Wang, 1994) (Damaeus)

DistRIBUCIÓN: China suroriental.

-Parabelbella (Tectodamaeus) exspinosa (Wang y Norton, 1989)

(Damaeus)

DisTRIBUCIÓN: China suroriental.

-Parabelbella (Tectodamaeus) furcata (Wang y Lu, 1995) (Damaeus (T.))

DisTRIBUCIÓN: China suroriental.

-Parabelbella (Tectodamaeus) spiniger (Wang, 1994) (Damaeus)

DistriBuCión: China suroriental.

-Parabelbella (Tectodamaeus) striata (Enami y Aoki, 1988) (T.)

DisTRIBUCIÓN: Este de Paleártica oriental.

-Parabelbella (Tectodamaeus) wulongensis (Wang y Cui, 1992)

(Damaeus)

DISTRIBUCIÓN: China suroriental.

-Parabelbella (Tectodamaeus) yaoi (Wang, 1994) (Damaeus)

DistribuCión: China suroriental.

Porobelba Grandjean, 1936 (4 spp.)

EsPecie Tipo: Oribata spinosa Sellnick, 1920

DISTRIBUCIÓN: Holártica. 
-Porobelba grandjeanica Subías, 1977

DisTRIBUCIÓN: España.

-Porobelba parki Jacot, 1937

DisTRIBUCIÓN: U.S.A. centrooriental.

-Porobelba robusta Mihelcic, 1955

DisTRIBUCIÓN: Austria.

-Porobelba spinosa (Sellnick, 1920) (Oribata)

(=Belba parvula Mihelcic, 1955)

(=Belba parvula Mihelcic, 1956, "nom. praeoc." por Mihelcic, 1955)

DisTRIBUCIÓN: Paleártica (frecuente).

Spatiodamaeus Bulanova-Zachvatkina, 1957 (8 spp.)

EsPeCIE TIPO: Damaeus verticillipes Nicolet, 1855

DISTRIBUCIÓN: Holártica.

-Spatiodamaeus boreus (Bulanova-Zachvatkina, 1957) (Damaeus

(S.))

DisTRIBUCIÓN: Europa oriental.

-Spatiodamaeus crassispinosus (Mihelcic, 1964) (Damaeus (S.))

DisTRIBUCión: Austria.

-Spatiodamaeus diversipilis (Willmann, 1951) (Belba)

DisTRIBUCIÓN: Europa central.

-Spatiodamaeus fageti (Bulanova-Zachvatkina, 1957) (Damaeus (S.))

DISTRIBUCIÓN: Europa oriental.

-Spatiodamaeus glabrisetus (Willmann, 1930) (Belba)

DisTRIBUCIÓN: Holanda.

-Spatiodamaeus similis (Willmann, 1951) (Belba verticillipes s.)

DisTRIBUCIÓN: Europa central.

-Spatiodamaeus subverticillipes (Bulanova-Zachvatkina, 1957)

(Damaeus (S.))

DistRIBUCIÓN: Paleártica (Europa oriental y Paleártica oriental).

-Spatiodamaeus verticillipes (Nicolet, 1855) (Damaeus)

(=Belba mirabilis Karpelles, 1893)

(=Damaeus quadrihastatus Märkel y Meyer, 1960)

(?=Damaeus torvus Koch, 1835 "sp. inq.")

DisTRIBUCIÓN: Holártica (Paleártica occidental: frecuente, y

U.S.A.: New Hampshire)

Subbelba Bulanova-Zachvatkina, 1967 (11 spp. y 2 sspp.)

EsPECIE TIPO: Metabelba partiocrispa Bulanova-Zachvatkina, 1957

DisTRIBUCIÓN: Holártica y Neotropical.

Subbelba (Subbelba) Bulanova-Zachvatkina, 1967 (3 spp. y 2 sspp.)

DisTRIBUCIÓN: Paleártica.

-Subbelba (S.) elisae Pérez-Íñigo, 1972

DisTRIBUCIÓN: I. Canarias.

-Subbelba (S.) elisae elisae Pérez-Íñigo, 1972

DisTRIBUCIÓN: I. Canarias.

-Subbelba (S.) elisae fuerteventurae Pérez-Íñigo y Peña, 1996

DisTRIBUCIÓN: I. Canarias.

-Subbelba (S.) elisae grancanariae Pérez-Íñigo y Peña, 1996

DisTRIBUCión: I. Canarias.

-Subbelba (S.) montana (Kulczynski, 1902) (Oribata)

DistRIBUCIÓN: Paleártica (Euroatlántica y Asia centrooccidental).

-Subbelba (S.) partiocrispa (Bulanova-Zachvatkina, 1957) (Metabelba)

DisTRIBUCIÓN: Eurosiberiana (Europa oriental: excepto Sur, y Siberia).

Subbelba (Dyobelba) Norton, 1979 (7 spp.)

ESPECIE TIPO: Oribata carolinensis Banks, 1947

DisTRIBUCIÓN: Holártica y Neotropical.

-Subbelba (Dyobelba) armata (Norton, 1979) (D.)

DisTRIBUCIÓN: Chile.

-Subbelba (Dyobelba) biclavata (Wang y Norton, 1993) (D.)

DisTRIBUCIÓN: Paleártica oriental (centrooriental).

-Subbelba (Dyobelba) carolinensis (Banks, 1947) (Oribata)

(=Oribata diversipes Banks, 1947)

DisTRIBUCIÓN: Sureste de U.S.A.
-Subbelba (Dyobelba) kushiroensis (Enami y Aoki, 2001) (D.)

DisTRIBUCIÓN: Japón.

-Subbelba (Dyobelba) paucituberculata (Bayartogtokh, Choi y Aoki, 2000) (D.)

DISTRIBUCIÓN: Corea.

-Subbelba (Dyobelba) reevesi (Norton y Rjabinin, 1994) (D.)

DisTRIBUCIÓN: U.S.A. (New Hampshire).

-Subbelba (Dyobelba) tectopediosa (Jacot, 1938) (Oribata)

DisTRIBUCIÓN: U.S.A. (Carolina del Norte).

Subbelba (Quatrobelba) Norton, 1980 (1 sp.)

EsPeCIE TIPO: (Quatrobelba montana Norton, 1980, "nom. praeoc.” por

Kulczynski, 1902) =Subbelba (Quatrobelba) neonominata nom. nov.

DisTRIBUCIÓN: Neártica

-Subbelba (Quatrobelba) neonominata nom. nov.

[=Quatrobelba montana Norton, 1980, "nom. praeoc." por Kulczynski, 1902 ("hom. sec.")]

DISTRIBUCIÓN: Neártica.

\section{Polypterozetoidea Grandjean, 1959}

Polypterozetidae Grandjean, 1959 (1 gen. y 1 sp.) DistRIBUCIÓN: Paleártica.

Polypterozetes Berlese, 1916 (1 sp.)

EsPeCIE TIPO: Polypterozetes cherubin Berlese, 1916

DisTRIBUCIÓN: Paleártica.

-Polypterozetes cherubin Berlese, 1916

DISTRIBUCIÓN: Italia.

Podopterotegaeidae Piffl, 1972 (1 gen. y 3 spp.) DisTRIBUCIÓN: Holártica.

Podopterotegaeus Aoki, 1969 (3 spp.)

EsPeCIE TIPO: Podopterotegaeus tectus Aoki, 1969

DisTRIBUCIÓN: Holártica.

-Podopterotegaeus altimonticola Piffl, 1972

DISTRIBUCIÓN: Nepal.

-Podopterotegaeus bisetus Pankov, 1984

DistriBución: Este de Rusia asiática.

-Podopterotegaeus tectus Aoki, 1969

DisTRIBUCIÓN: Holártica (Paleártica oriental: frecuente, y Canadá).

Cepheoidea Berlese, 1896

Cepheidae Berlese, 1896 (15 gen., 79 spp. y 2 sspp.)

DistRIBUCIÓN: Cosmopolita (excepto Etiópica y Antártica).

Cepheus Koch, 1835 (24 spp.)

(=Tegeocranus Nicolet, 1855)

EsPeCIE TiPO: Cepheus latus Koch, 1835

DISTRIBUCIÓN: Semicosmopolita (Holártica y Australianooriental).

-Cepheus brachiatus Sitnikova, 1975

DistriBución: Paleártica (Polonia, y Paleártica oriental).

-Cepheus caucasicus Sitnikova, 1975

DistriBución: Cáucaso.

-Cepheus cepheiformis (Nicolet, 1855) (Tegeocranus)

(=Cepheus michaeli Jacot, 1928)

Distribución: Holártica (Paleártica: frecuente, y U.S.A.:

Carolina del Norte).

-Cepheus corae Jacot, 1928

DisTRIBUCióN: Neártica (frecuente)

-Cepheus dentatus (Michael, 1888) (Tegeocranus)

(=Cepheus grandis Sitnikova, 1975)

Distribución: Paleártica: Europa (frecuente) y Este de Rusia asiática.

-Cepheus feideri Suciu y Panu, 1972

DistribuCIÓN: Rumania

-Cepheus gracilipes (Canestrini, 1897) (Tegeocranus) "sp. inq."

DistRIBUCIÓN: Nueva Guinea. 
-Cepheus granulosus Mihelcic, 1958 DisTRIBUCIÓN: Austria.

-Cepheus heterosetosus (Sitnikova, 1975) (Oribatodes) DisTRIBUCIÓN: Europa oriental: excepto Norte.

-Cepheus hokkaiensis Fujikawa, 1992 DisTRIBUCIÓN: Japón.

-Cepheus incisus Mihelcic, 1958

DisTRIBUCIÓn: Austria.

-Cepheus jindingensis Wen y Sun, 1990 Distribución: China occidental.

-Cepheus kurosawai Aoki, 1986

DisTRIBUCIÓN: Japón.

-Cepheus latus Koch, 1835

DisTRIBUCIÓN: Holártica (frecuente en Paleártica).

-Cepheus lobatus Mihelcic, 1953 "sp. inq." DisTRIBUCIÓn: Austria.

-Cepheus mirabiloides Jacot, 1938

Distribución: U.S.A. (Carolina del Norte).

-Cepheus pegazzanoae Bernini y Nannelli, 1982

DisTRIBUCión: Mediterránea occidental.

-Cepheus pertusus Haupt, 1882 "sp. inq."

DisTRIBUCión: Alemania.

-Cepheus pustulatus (Pearce, 1910) (Tegeocranus)

DisTRIBUCIÓN: Hawai.

-Cepheus takasago Aoki, 1991

DistriBuCIÓN: Taiwan [Formosa].

-Cepheus transversalis Balogh, 1958 "sp. inq."

DisTRIBUCIÓN: Tanzania.

-Cepheus transylvanicus Cooreman, 1951

DisTRIBUCIÓN: Rumania.

-Cepheus tuberculosus Strenzke, 1951

DistRIBUCIÓn: Paleártica occidental: excepto Norte.

-Cepheus verrucosus Bernini, 1971

DISTRIBUCIÓN: Mediterránea occidental.

Compactozetes Hammer, 1966 (5 spp.)

ESPECIE TIPO: Compactozetes rotoruensis Hammer, 1966

DisTRIBUCIÓN: Australiana.

-Compactozetes hastatus Hammer, 1973

DistriBución: Australiana (Islas del Pacífico).

-Compactozetes niger Hammer, 1966

DisTRIBUCIÓN: Nueva Zelanda.

-Compactozetes rotoruensis Hammer, 1966

DisTRIBUCIÓN: Nueva Zelanda.

-Compactozetes serratus Balogh, 1970

DistriBución: Nueva Guinea.

-Compactozetes zeugus Luxton, 1988

DisTRIBUCIÓN: Nueva Zelanda.

Conoppia Berlese, 1908 (2 spp.)

(=Balzania Jacot, 1929)

(=Phyllotegeus Berlese, 1913)

EsPECIE TIPO: (Oppia microptera Berlese, 1885) =Leiosoma palmicinctum Michael, 1884

DisTRIBUCIÓN: Semicosmopolita (Holártica, Oriental y Neotropical).

-Conoppia palmicincta (Michael, 1884) (Leiosoma)

(=Oppia microptera Berlese, 1885)

(=Oribata microptera Canestrini, 1896, "nom. praeoc." por Berlese, 1885)

DisTRIBUCIÓN: Holártica (Paleártica occidental centromeridional

frecuente, Este de Paleártica oriental y U.S.A.), Taiwan [Formosa] y Bolivia.

-Conoppia setiformis Golosova y Karppinen, 1985

DISTRIBUCIÓN: Mongolia.

Eupterotegaeus Berlese, 1916 (10 spp.)

(=Diodontocepheus Mihelcic, 1958)

EsPeCIE TIPO: Tegeocranus ornatissimus Berlese, 1908

DisTRIBUCIÓN: Holártica y Neotropical.

-Eupterotegaeus armatus Aoki, 1969

DistRIBUCIÓN: Este de Paleártica oriental.

-Eupterotegaeus dentatus Sitnikova, 1979

DISTRIBUCIÓN: Bolivia.
-Eupterotegaeus flavus (Ewing, 1918) (Cepheus)

DisTRIBUCión: U.S.A. (Oregón).

-Eupterotegaeus nasalis Sitnikova, 1979

DisTRIBUCIÓN: Ecuador.

-Eupterotegaeus ornatissimus (Berlese, 1908) (Tegeocranus)

(=Diodontocepheus steinboecki Mihelcic, 1958)

DISTRIBUCIÓN: Holártica (Paleártica meridional, I. Sajalín y Canadá).

-Eupterotegaeus pseudosculptus (Coggi, 1900) (Cepheus) "sp. inq." DisTRIBUCIÓN: Italia.

-Eupterotegaeus rhamphosus Higgins y Woolley, 1968

DisTRIBUCión: Neártica (Noroeste de U.S.A. y Canadá).

-Eupterotegaeus rostratus Higgins y Woolley, 1963

DisTRIBUCIÓN: Neártica (U.S.A. occidenal y Canadá)

-Eupterotegaeus spinatus Higgins y Woolley, 1963

DisTRIBUCIÓN: U.S.A. (Utah).

-Eupterotegaeus xizangensis Wang y Solhoy, 2001

DisTRIBUCión: China (Tíbet).

Hypocepheus Krivolutsky, 1971 (3 spp.)

ESPECIE TIPO: Hypocepheus mirabilis Krivolutsky, 1971

DisTRIBUCIÓN: Paleártica.

-Hypocepheus helveticus Mahunka y Mahunka-Papp, 2002

DISTRIBUCIÓN: Suiza.

-Hypocepheus krivolutskyi Calugar y Vasiliu, 1976

DisTRIBUCIÓN: Rumania.

-Hypocepheus mirabilis Krivolutsky, 1971

DISTRIBUCIÓN: Sureste de Europa.

Ommatocepheus Berlese, 1913 (5 spp. y 1 ssp.)

EsPECIE TIPO: Cepheus ocellatus Michael, 1882

DisTRIBUCIÓN: Holártica.

-Ommatocepheus clavatus Woolley y Higgins, 1964

Distribución: Holártica (Este de Paleártica oriental: Japón e I.

Kuriles, y U.S.A.: Carolina del Norte).

-Ommatocepheus clavatus clavatus Woolley y Higgins, 1964

Distribución: U.S.A. (Carolina del Norte).

-Ommatocepheus clavatus japonicus Aoki, 1974

DistribuCión: Este de Paleártica oriental (Japón e I. Kuriles).

-Ommatocepheus crassisetosus Pérez-Íñigo y Peña, 1996

Distribución: I. Canarias.

-Ommatocepheus ocellatus (Michael, 1882) (Cepheus)

(=Scutovertex pulcherrimus Berlese, 1913)

Distribución: Paleártica (Paleártica occidental: excepto Norte, e India: Himalaya).

-Ommatocepheus parvilamellatus C. y C., jr. Pérez-Íñigo, 1996

DisTriBución: I. Azores.

-Ommatocepheus reticulatus Pérez-Íñigo y Peña, 1997

DisTRIBUCIÓN: I. Canarias.

Oribatodes Banks, 1895 (2 spp.)

EsPECIE TIPO: Oribatodes mirabilis Banks, 1895

DISTRIBUCIÓN: Holártica.

-Oribatodes crenulatus Csiszár, 1962

DisTRIBUCión: Sureste de Europa.

-Oribatodes mirabilis Banks, 1895

(=Cepheus lamellatus Banks, 1906)

(=Tegeocranus longisetus Berlese, 1910)

DISTRIBUCIÓN: Noreste de Neártica.

Pilocepheus Pérez-Íñigo, 1992 (1 sp.)

EsPecie Tipo: Pilocepheus azoricus Pérez-Íñigo, 1992

DisTRIBUCIÓN: Paleártica.

-Pilocepheus azoricus Pérez-Íñigo, 1992

DisTRIBUCIÓN: I. Azores.

Protocepheus Jacot, 1928 (1 sp.)

EsPecIE TIPO: Tegeocranus hericius Michael, 1887

Distribución: Paleártica.

-Protocepheus hericius (Michael, 1887) (Tegeocranus)

DisTRIBUCIÓN: Europa central. 
Reticulocepheus Vasiliu y Calugar, 1977 (4 spp.)

(=Cubacepheus Balogh y Mahunka, 1979 "sin. nov.")

(=Geocepheus Sitnikova, 1979)

EsPeCIE TIPO: Reticulocepheus decoui Vasiliu y Calugar, 1977

DisTRIBUCIÓN: Neotropical.

-Reticulocepheus areolatus (Sitnikova, 1979) (Geocepheus)

DISTRIBUCIÓN: Ecuador.

-Reticulocepheus decoui Vasiliu y Calugar, 1977

(=Cepheus (Cubacepheus) lobatus Balogh y Mahunka, 1979)

DisTRIBUCIÓN: Norte de Neotropical.

-Reticulocepheus grandis (Sitnikova, 1979) (Geocepheus)

DISTRIBUCIÓN: Ecuador.

-Reticulocepheus reticulatus (Sitnikova, 1979) (Geocepheus)

DISTRIBUCIÓN: Ecuador.

Sadocepheus Aoki, 1965 (10 spp. y 1 ssp.)

(=Hamotegeus Balogh y Mahunka, 1969 "sin. nov.")

EsPECIE TIPO: Sadocepheus undulatus Aoki, 1965

DisTRIBUCIÓN: Semicosmopolita (Holártica y Pantropical: excepto Etiópica).

-Sadocepheus breviseta (P. Balogh, 1986) (Hamotegeus)

DisTRIBUCIÓN: Colombia.

-Sadocepheus dubius Hammer, 1979

DisTRIBUCIÓN: Java.

-Sadocepheus elevatus Mahunka, 1987

DisTRIBUCIÓN: Borneo.

-Sadocepheus foveolatus Luxton, 1988

DistRiBución: Nueva Zelanda.

-Sadocepheus franzi (P. Balogh, 1986) (Hamotegeus)

DisTriBución: Chile.

-Sadocepheus granulatus (Balogh y Mahunka, 1969) (Hamotegeus)

DisTRIBUCIÓN: Neotropical (Brasil y Paraguay).

-Sadocepheus longiseta (P. Balogh, 1986) (Hamotegeus)

DistriBución: Colombia.

-Sadocepheus makarcevae Sitnikova, 1975

DisTRIBUCIÓN: Este de Rusia asiática.

-Sadocepheus subniger (Ewing, 1917) (Tegeocranus)

DisTRIBUCIÓN: U.S.A. (Iowa).

-Sadocepheus undulatus Aoki, 1965

DisTRIBUCión: Este de Paleártica oriental.

-Sadocepheus undulatus undulatus Aoki, 1965

DistRIBUCIÓN: Este de Paleártica oriental.

-Sadocepheus undulatus setiger Fujita y Fujikawa, 1986

DisTRIBUCIÓN: Japón.

Sphodrocepheus Woolley y Higgins, 1963 (5 spp.)

ESPECIE TIPO: Sphodrocepheus tridactylus Woolley y Higgins, 1963

DISTRIBUCIÓN: Holártica y Oriental.

-Sphodrocepheus anthelionus Woolley y Higgins, 1968

DisTRIBUCIÓN: Neártica (Noroeste de U.S.A. y Canadá).

-Sphodrocepheus dentatus Fujikawa, 1972

DISTRIBUCIÓN: Este de Paleartica oriental.

-Sphodrocepheus mitratus Aoki, 1967

Distribución: Este de Paleártica oriental.

-Sphodrocepheus tridactylus Woolley y Higgins, 1963

DisTRIBUCIÓN: U.S.A. occidental.

-Sphodrocepheus tuberculatus Mahunka, 1988

DISTRIBUCIÓN: Vietnam.

Tereticepheus S. y F. Bernini, 1990 (2 spp.)

EsPeCIE TIPO: Tereticepheus annamariae S. y F. Bernini, 1990

DisTRIBUCIÓN: Paleártica.

-Tereticepheus annamariae S. y F. Bernini, 1990

DISTRIBUCIÓN: Argelia.

-Tereticepheus undulatus (Willmann, 1939) (Cepheus)

DISTRIBUCIÓN: Macaronésica (Madeira).

Tikizetes Hammer, 1967 (1 sp.)

EsPECIE TIPO: Tikizetes spinipes Hammer, 1967

DisTRIBUCIÓN: Australiana.
-Tikizetes spinipes Hammer, 1967

DistRIBUCIÓN: Nueva Zelanda.

Tritegeus Berlese, 1913 (4 spp.)

EsPeCIE TIPO: (Cepheus bifidatus Nicolet "sensu" Michael, 1880)

=Tritegeus bisulcatus Grandjean, 1953

DisTRIBUCIÓN: Paleártica.

-Tritegeus bisulcatus Grandjean, 1953

DisTRIBUCIÓN: Paleártica occidental (menos fecuente en el Norte).

-Tritegeus brevisetus Sitnikova, 1975

DisTRIBUCIÓN: Paleártica (Sureste de Europa y Paleártica oriental).

-Tritegeus major Golosova y Karppinen, 1984

Distribución: Sur de Siberia.

-Tritegeus sculptus S. y F. Bernini, 1990

DisTRIBUCIÓN: Mediterránea occidental.

Niphocepheidae Travé, 1959 (1 gen., 4 spp. y 3 sspp.) DisTRIBUCIÓN: Paleártica y Oriental.

Niphocepheus Balogh, 1943 (4 spp. y 3 sspp.)

ESPECIE TIPO: Cepheus nivalis Schweizer, 1922

DisTRIBUCIÓN: Paleártica y Oriental.

-Niphocepheus aborigensis Behan-Pelletier, 1982

DisTRIBUCIÓN: Este de Rusia asiática.

-Niphocepheus guadarramicus Subías, 1977

DISTRIBUCIÓN: España.

-Niphocepheus nivalis (Schweizer, 1922) (Cepheus)

DisTRIBUCIÓN: Paleártica (Paleártica occidental: excepto Norte, y

Japón) y Taiwan [Formosa].

-Niphocepheus nivalis nivalis (Schweizer, 1922)

DistRIBUCión: Paleártica (Europa central y Japón) y Taiwan [Formosa].

-Niphocepheus nivalis baloghi Travé, 1959 DISTRIBUCIÓN: Mediterránea.

-Niphocepheus nivalis delamarei Travé, 1959 DisTRIBUCIÓN: Mediterránea occidental.

-Niphocepheus nivalis grandjeani Travé, 1959

DisTRIBUCIÓN: Europa occidental (Francia y Suiza).

-Niphocepheus travei Bulanova-Zachvatkina, 1967

DistriBución: Este de Rusia asiática.

Cerocepheidae fam. nov. (3 gen. y 4 spp.)

DisTRIBUCIÓN: Tropical (Australiana y Neotropical)

Bornebuschia Hammer, 1966 (2 spp.)

ESPECIE TIPO: Bornebuschia peculiaris Hammer, 1966

DISTRIBUCIÓN: Australiana.

-Bornebuschia binodosa Luxton, 1988

Distribución: Nueva Zelanda.

-Bornebuschia peculiaris Hammer, 1966

DistRIBUCión: Nueva Zelanda.

Cerocepheus Trägardh, 1931 (1 sp.)

EsPECIE TIPO: Cerocepheus mirabilis Trägardh, 1931

DISTRIBUCIÓN: Neotropical.

-Cerocepheus mirabilis Trägardh, 1931

Distribución: Chile (I. Juan Fernández).

Dicrotegaeus Luxton, 1988 (1 sp.)

ESPECIE TIPO: Dicrotegaeus mirabilis Luxton, 1988

DisTRIBUCIÓN: Australiana.

-Dicrotegaeus mirabilis Luxton, 1988

DisTRIBUCIÓN: Nueva Zelanda.

Eutegaeidae Balogh, 1965 (9 gen. y 28 spp.)

DisTRIBUCIÓN: Cosmopolita (excepto Holártica).

Atalotegaeus Luxton, 1988 (1 sp.)

EsPECIE TIPO: Eutegaeus mensarosi J. y P. Balogh, 1983 
DISTRIBUCIÓN: Australiana.

-Atalotegaeus mensarosi (J. y P. Balogh, 1983) (Eutegaeus) DisTRIBUCIÓN: Australia.

Birotegaeus Luxton, 1988 (1 sp.)

ESPECIE TIPO: Eutegaeus biroi Balogh, 1970

DISTRIBUCIÓN: Australiana.

-Birotegaeus biroi (Balogh, 1970) (Eutegaeus)

DisTRIBUCIÓN: Nueva Guinea.

Dudichella Balogh, 1970 (1 sp.)

ESPECIE TIPO: Dudichella membranigera Balogh, 1970

DISTRIBUCIÓN: Australianooriental.

-Dudichella membranigera Balogh, 1970

Distribución: Ceilán [Sri Lanka] y Melanesia (I. Fiji).

Eutegaeus Berlese, 1916 (10 spp.)

EsPECIE TIPO: Oribata bostocki Michael, 1908

DisTRIBUCión: Australiana, Neotropical y Antártica.

-Eutegaeus biovatus Hammer, 1972

DisTRIBUCIÓn: Polinesia (Tahití).

-Eutegaeus bostocki (Michael, 1908) (Oribata)

(=Eutegaeus curviseta Hammer, 1966)

DisTRIBUCIÓN: Nueva Zelanda y Antártica (I. Campbell)

-Eutegaeus fueginus Arcidiacono, 1993

DisTRIBUCIÓN: América austral (Tierra del Fuego).

-Eutegaeus lagrecai Arcidiacono, 1993

DisTRIBUCIÓN: América austral (Tierra del Fuego).

-Eutegaeus membraniger Hammer, 1966

DisTRIBUCIÓN: Nueva Zelanda.

-Eutegaeus papuensis Aoki, 1965

DISTRIBUCIÓN: Nueva Guinea.

-Eutegaeus pinnatus Hammer, 1966

DisTRIBUCIÓN: Nueva Zelanda.

-Eutegaeus radiatus Hammer, 1966

DisTRIBUCIÓN: Nueva Zelanda.

-Eutegaeus soror P. Balogh, 1985

DISTRIBUCIÓN: Australia.

-Eutegaeus stylesi Hammer, 1966

DisTRIBUCIÓN: Nueva Zelanda.

Neoeutegaeus Aoki, 1965 (3 spp.)

ESPECIE TIPO: Eutegaeus silvicola Aoki, 1965

DistriBuCIÓN: Semicosmopolita (Etiópica, Australiana y Neotropical).

-Neoeutegaeus africanus Mahunka, 1974

DisTRIBUCIÓN: Camerún.

-Neoeutegaeus phyllophorus J. y P. Balogh, 1983

DISTRIBUCIÓN: Australia.

-Neoeutegaeus silvicola (Hammer, 1962) (Eutegaeus)

DisTRIBUCIÓN: América austral.

Neseutegaeus Woolley, 1965 (7 spp.)

ESPECIE TIPO: Neseutegaeus spinatus Woolley, 1965

DISTRIBUCIÓN: Australianooriental.

-Neseutegaeus angustus Hammer, 1966

DisTRIBUCIÓN: Nueva Zelanda.

-Neseutegaeus consimilis Hammer, 1966

DisTRIBUCión: Nueva Zelanda.

-Neseutegaeus denticulatus P. Balogh, 1988

DisTRIBUCIÓN: Ceilán [Sri Lanka].

-Neseutegaeus distentus Hammer, 1966

Distribución: Nueva Zelanda.

-Neseutegaeus latus Hammer, 1966

DisTRIBUCIÓN: Nueva Zelanda.

-Neseutegaeus monteithi J. y P. Balogh, 1983

Distribución: Australia.

-Neseutegaeus spinatus Woolley, 1965

DisTRIBUCión: Nueva Zelanda.
Pareutegaeus Woolley, 1965 (2 spp.)

EsPeCIE TIPO: Eutegaeus similis Trägardh, 1931

DISTRIBUCIÓN: Neotropical austral.

-Pareutegaeus pulcher (Balogh y Csiszár, 1963) (Eutegaeus)

DisTRIBUCIÓN: Argentina.

-Pareutegaeus similis (Trägardh, 1931) (Eutegaeus)

Distribución: Chile (I. Juan Fernández).

Porrhotegaeus Balogh y Mahunka, 1966 (2 spp.)

EsPECIE TIPO: Porrhotegaeus ornatus Balogh y Mahunka, 1966

DisTRIBUCIÓN: Australiana.

-Porrhotegaeus herminae J. y P. Balogh, 1983

Distribución: Nueva Caledonia.

-Porrhotegaeus ornatus Balogh y Mahunka, 1966

DisTRIBUCIÓN: Australia.

Pterozetes Hammer, 1966 (1 sp.)

EsPeCIE TIPO: Pterozetes novazealandicus Hammer, 1966

DisTRIBUCIÓN: Australiana.

-Pterozetes novazealandicus Hammer, 1966

Distribución: Nueva Zelanda.

Pterobatidae Balogh y Mahunka, 1977 (1 gen. y 1 sp.) DISTRIBUCIÓN: Neotropical.

Pterobates Balogh y Mahunka, 1977 (1 sp.)

EsPeCIE TIPO: Pterobates incertus Balogh y Mahunka, 1977

DISTRIBUCIÓN: Neotropical.

-Pterobates incertus Balogh y Mahunka, 1977

DistRIBUCIÓN: Brasil.

Nodocepheidae Piffl, 1972 (2 gen., 7 spp. y 1 ssp.)

DisTRIBUCIÓN: Cosmopolita (excepto Oriental).

Nemacepheus Aoki, 1968 (1 sp.)

EsPeCIE TIPO: Nemacepheus dentatus Aoki, 1968

DisTRIBUCIÓN: Paleártica.

-Nemacepheus dentatus Aoki, 1968

DisTRIBUCIÓN: Este de Paleártica oriental (Japón e I. Kuriles).

Nodocepheus Hammer, 1958 (6 spp. y 1 ssp.)

EsPeCIE TIPO: Nodocepheus dentatus Hammer, 1958

DisTRIBUCión: Pantropical (excepto Oriental) y austral.

-Nodocepheus baloghi Mahunka, 1983

DisTRIBUCIÓN: Tanzania.

-Nodocepheus cerebralis Mahunka, 1980

DisTRIBUCIÓN: América austral (Tierra del Fuego).

-Nodocepheus dentatus Hammer, 1958

DisTRIBUCIÓN: Neotropical, Australiana (Nueva Zelanda)y subantártica.

-Nodocepheus dentatus dentatus Hammer, 1958

DistRIBUCIÓN: Neotropical y subantártica: I. Malvinas [I. Falkland].

-Nodocepheus dentatus barbatus Hammer, 1966

DistribuCión: Australiana (Nueva Zelanda) y subantártica (I. Amsterdam).

-Nodocepheus hammerae Balogh, 1961

DISTRIBUCIÓN: Etiópica.

-Nodocepheus laterodentatus Piffl, 1972

Distribución: Costa Rica.

-Nodocepheus minimus Mahunka, 1985

DISTRIBUCIÓN: Sudáfrica.

Tumerozetidae Hammer, 1966 (1 gen. y 5 spp.) DisTRIBUCIÓN: Australiana.

Tumerozetes Hammer, 1966 (5 spp.)

EsPECIE TIPO: Tumerozetes bifurcatus Hammer, 1966

DISTRIBUCIÓN: Australiana. 
-Tumerozetes bifurcatus Hammer, 1966 DisTRIBUCión: Nueva Zelanda.

-Tumerozetes circularis Hammer, 1966 DistRIBUCIÓN: Nueva Zelanda.

-Tumerozetes indistinctus Hammer, 1966 DisTRIBUCIÓN: Nueva Zelanda.

-Tumerozetes parallelus Hammer, 1966 Distribución: Nueva Zelanda.

-Tumerozetes pumilis Hammer, 1966 DisTRIBUCIÓN: Nueva Zelanda.

\section{Charassobatoidea Grandjean, 1958}

Microtegeidae Balogh, 1972 (2 gen. y 31 spp.) DistRIBUCIÓN: Pantropical.

Microtegeus Berlese, 1916 (29 spp.)

EsPeCIE Tipo: Tegeocranus (Microtegeus) undulatus Berlese, 1916 DisTRIBUCIÓN: Pantropical.

-Microtegeus alvarezi Pérez-Íñigo, 1969

DisTRIBUCión: Guinea Ecuatorial: I. Pagalu [Annobón].

-Microtegeus asiaticus Aoki y Yamamoto, 2000

DistribuCIÓN: China suroriental.

-Microtegeus borhidii Balogh y Mahunka, 1974

DistRIBUCIÓN: Norte de Neotropical, Filipinas y Japón.

-Microtegeus cardosensis Pérez-Íñigo y Baggio, 1985

DistriBución: Neotropical (Brasil y Venezuela)

-Microtegeus cervus Mahunka, 1983

DisTRIBUCión: Tanzania.

-Microtegeus ceylonicus Balogh, 1970

DisTRIBUCión: Ceilán [Sri Lanka].

-Microtegeus cornutus Balogh, 1970

Distribución: Ceilán [Sri Lanka].

-Microtegeus curvisetosus Wen, 1997

DisTRIBUCIÓN: China suroriental.

-Microtegeus foveolatus Balogh, 1968

DistRIBUCión: Australiana (Nueva Guinea) y Oriental (Malaya y Filipinas).

-Microtegeus globifer Mahunka, 1985

DistriBuCión: Pequeñas Antillas.

-Microtegeus granulatus Wang y Shen, 2001

Distribución: China (Tíbet).

-Microtegeus hauseri Mahunka, 1988

DistriBución: Pequeñas Antillas

-Microtegeus hirashimai Balogh, 1970

DisTRIBUCIÓN: Nueva Guinea.

-Microtegeus humeratus Balogh y Mahunka, 1974

DistriBución: Cuba y Filipinas.

-Microtegeus labyrinthicus Balogh, 1968

DistriBución: Nueva Guinea.

-Microtegeus lucianus Mahunka, 1998

DisTRIBUCión: Pequeñas Antillas.

-Microtegeus mexicanus Mahunka, 1983

DisTRIBUCIÓN: Méjico.

-Microtegeus modestus Aoki, 1976

Distribución: Península Malaya

-Microtegeus papillosus Mahunka, 1984

DisTRIBUCIÓN: Tanzania.

-Microtegeus paracervus Mahunka, 1997

(=Microtegeus cervus Mahunka, 1996, "nom. praeoc.” por Mahunka, 1983) DisTRIBUCIÓN: Madagascar.

-Microtegeus quadrisetosus Balogh y Mahunka, 1977

DisTRIBUCIÓN: Neotropical (Bolivia y Brasil) y Ceilán [Sri Lanka]

-Microtegeus quadristriatus Mahunka, 1984

DisTRIBUCIÓN: Paraguay.

-Microtegeus reticulatus Aoki, 1965

DisTRIBUCIÓN: Oriental (frecuente).
-Microtegeus rimosus Hammer, 1982

DisTRIBUCIÓN: Bali.

-Microtegeus rugosus Mahunka, 1982

DisTRIBUCIÓN: Etiopía.

-Microtegeus sabahnus Mahunka, 1987

DisTRIBUCIÓN: Borneo.

-Microtegeus similis Balogh y Mahunka, 1980

DisTRIBUCIÓN: Cuba.

-Microtegeus undulatus (Berlese, 1916) (Tegeocranus (M.))

DisTRIBUCión: Etiópica (Somalia y Tanzania).

-Microtegeus variabilis Mahunka, 1988

DISTRIBUCIÓN: Tanzania.

Suctotegeus Mahunka, 1987 (2 spp.)

EsPeCIE TIPO: Suctotegeus tumescitus Mahunka, 1987

DISTRIBUCIÓN: Oriental.

-Suctotegeus philippinensis Corpuz-Raros, 1991

DisTRIBUCIÓN: Filipinas.

-Suctotegeus tumescitus Mahunka, 1987

DISTRIBUCIÓN: Borneo.

Charassobatidae Grandjean, 1958 (1 gen. y 7 spp.) DISTRIBUCIÓN: Neotropical.

Charassobates Grandjean, 1929 (7 spp.)

EsPeCIE TIPO: Charassobates cavernosus Grandjean, 1929

DISTRIBUCIÓN: Neotropical.

-Charassobates baudi Mahunka, 1984

DisTRIBUCIÓN: Paraguay.

-Charassobates cavernosus Grandjean, 1929

DisTRIBUCIÓN: Neotropical (Venezuela y Perú).

-Charassobates incipatus Balogh y Mahunka, 1974

Distribución: Cuba.

-Charassobates minimus Balogh y Mahunka, 1981

DisTRIBUCIÓN: Paraguay.

-Charassobates ornatus Balogh y Mahunka, 1969

DistrIBUCIÓN: Brasil.

-Charassobates simplex Balogh y Mahunka, 1969

DisTRIBUCIÓN: Neotropical (Brasil y Paraguay).

-Charassobates tuberosus Balogh y Mahunka, 1981

DisTRIBUCIÓN: Paraguay.

Nosybeidae Mahunka, 1993 (2 gen. y 7 spp.) DISTRIBUCIÓN: Subtropical.

Lamellocepheus Balogh, 1961 (2 spp.)

=Nosybea Mahunka, 1993 "sin. nov")

(=Tessacarus Grandjean, 1962)

EsPeCIE TIPO: Tectocepheus personatus Berlese, 1910

DISTRIBUCIÓN: Paleártica meridional y Etiópica.

-Lamellocepheus genavensis (Mahunka, 1993) (Nosybea)

DisTRIBUCIÓN: Madagascar.

-Lamellocepheus personatus (Berlese, 1910) (Tectocepheus)

(=Lamellocepheus ambitus Kulijev, 1966)

DisTRIBUCIÓN: Europa meridional.

Topalia Balogh y Csiszár, 1963 (5 spp.)

EsPeCIE TIPO: Topalia problematica Balogh y Csiszár, 1963

DISTRIBUCIÓN: Subtropical austral.

-Topalia africana Mahunka, 1985

DisTRIBUCIÓN: Sudáfrica.

-Topalia clavata Hammer, 1966

DisTRIBUCIÓN: Nueva Zelanda.

-Topalia granulata Hammer, 1966

DisTRIBUCión: Nueva Zelanda.

-Topalia problematica Balogh y Csiszár, 1963

DISTRIBUCIÓN: Argentina.

-Topalia velata Hammer, 1966

DisTRIBUCIÓN. Nueva Zelanda. 


\section{Microzetoidea Grandjean, 1936}

Microzetidae Grandjean, 1936 (42 gen., 6 subg., 180 spp. y 5 sspp.) DISTRIBUCIÓN: Cosmopolita.

Acanthozetes Balogh, 1958 (1 sp.)

EsPECIE TIPO: Acanthozetes platypterus Balogh, 1958

DisTRIBUCIÓN: Etiópica.

-Acanthozetes platypterus Balogh, 1958

DisTRIBUCIÓN: Congo.

Acaroceras Grandjean, 1936 (19 spp.)

EsPECIE TIPO: Acaroceras odontotus Grandjean, 1936

DisTRIBUCIÓN: Tropical (Etiópica y Neotropical).

Acaroceras (Acaroceras) Grandjean, 1936 (17 spp.)

DisTRIBUCIÓN: Neotropical.

-Acaroceras (A.) becki Balogh, 1962

DisTRIBUCIÓN: Perú.

-Acaroceras (A.) cavernosus Balogh, 1962

Distribución: Perú.

-Acaroceras (A.) dechambrieri Mahunka, 1983

DisTRIBUCIÓN: Norte de Neotropical.

-Acaroceras (A.) feideri Calugar y Vasiliu, 1977

Distribución: Cuba.

-Acaroceras (A.) furcatus Balogh, 1962

DisTRIBUCiÓn: Perú.

-Acaroceras (A.) galapagoensis Schatz y Palacios-Vargas, 1999 DisTRIBUCIÓN: I. Galápagos.

-Acaroceras (A.) hamifer Balogh y Mahunka, 1977

DisTRIBUCión: Neotropical (Bolivia y Méjico).

-Acaroceras (A.) index Balogh y Mahunka, 1977 Distribución: Brasil.

-Acaroceras (A.) interiunctus Schatz y Palacios-Vargas, 1999 DisTRIBUCIÓN: I. Galápagos.

-Acaroceras (A.) oaxacanus Mahunka y Palacios-Vargas, 1996 DisTRIBUCiÓN: Méjico.

-Acaroceras (A.) odontotus Grandjean, 1936

DisTRIBUCIÓN: Venezuela.

-Acaroceras (A.) porosus Balogh y Mahunka, 1977

DisTRIBUCión: Bolivia.

-Acaroceras (A.) pseudofurcatus Balogh y Mahunka, 1969 DisTRIBUCIÓN: Bolivia.

-Acaroceras (A.) pugio Balogh, 1962

DISTRIBUCIÓN: Perú.

-Acaroceras (A.) schalleri Balogh, 1962

DISTRIBUCIÓN: Perú.

-Acaroceras (A.) similis Balogh, 1962

Distribución: Perú.

-Acaroceras (A.) taurus Schatz y Palacios-Vargas, 1999

DisTRIBUCIÓN: I. Galápagos.

Acaroceras (Malgoceras) Mahunka, 1993 (1 sp.)

ESPECIE TIPO: Acaroceras (Malgoceras) helleri Mahunka, 1993 DisTRIBUCIÓN: Etiópica.

-Acaroceras (Malgoceras) helleri Mahunka, 1993

DISTRIBUCIÓN: Madagascar.

Acaroceras (Trichacaroceras) Mahunka, 1991 (1 sp.)

EsPECIE TIPO: Acaroceras (Trichacaroceras) africanus Mahunka, 1991

DisTRIBUCIÓN: Paleártica.

-Acaroceras (Trichacaroceras) africanus Mahunka, 1991

Distribución: Macaronésica (Cabo Verde).

Afrozetes Engelbrecht, 1972 (3 spp. y 1 ssp.)

ESPECIE TIPO: Afrozetes magoebaensis Engelbrecht, 1972

DisTRIBUCIÓN: Etiópica.

-Afrozetes champaignensis Engelbrecht, 1972

DiSTRIBUCIÓN: Sudáfrica.
-Afrozetes magoebaensis Engelbrecht, 1972

DisTRIBUCIÓN: Sudáfrica.

-Afrozetes magoebaensis magoebaensis Engelbrecht, 1972 DisTRIBUCIÓN: Sudáfrica.

-Afrozetes magoebaensis capensis Engelbrecht, 1972 DISTRIBUCIÓN: Sudáfrica.

-Afrozetes natalensis Engelbrecht, 1972

DisTRIBUCIÓN: Sudáfrica.

Anakingia Hammer, 1961 (3 spp.)

EsPECIE TIPO: Anakingia williamsae Hammer, 1961

DISTRIBUCIÓN: Tropical (Oriental y Neotropical).

-Anakingia borneensis Mahunka, 1997

DISTRIBUCIÓN: Borneo.

-Anakingia reticulata Balogh y Mahunka, 1969

DistRIBUCIÓN: Neotropical (Bolivia y Perú).

Anakingia williamsae Hammer, 1961

DistriBución: Perú.

Arenozetes Krivolutsky, 1971 (1 sp.)

EsPECIE TIPO: Arenozetes christovi Krivolutsky, 197

DisTRIBUCIÓN: Paleártica.

-Arenozetes christovi Krivolutsky, 1971

Distribución: Paleártica meridional (Sureste de Europa y Asia centroocidental).

Berlesezetes Mahunka, 1980 (12 spp. y 3 sspp.)

ESPECIE TIPO: (Microzetes auxiliaris Grandjean, 1936) =Microzetes ornatissimus Berlese, 1913

DISTRIBUCIÓN: Cosmopolita.

-Berlesezetes aegypticus (Bayoumi, 1977) (Microzetes)

DistRIBUCIÓN: Egipto.

-Berlesezetes alces (Piffl, 1961) (Microzetes)

Distribución: Mediterránea.

-Berlesezetes arenarius (Krivolutsky, 1966) (Microzetes)

DisTRIBUCIÓN: Asia centroocidental.

-Berlesezetes brazilozetoides Balogh y Mahunka, 1981

DisTRIBUCIÓN: Neotropical.

-Berlesezetes brevis (Warburton, 1912) (Oribata)

DisTRIBUCIÓN: Etiópica: I. Seychelles, y Neotropical: Guyana

[Guayana Británica].

-Berlesezetes cuspidatus Mahunka, 1982

Distribución: Grecia.

-Berlesezetes glaber Mahunka, 1982

DisTRIBUCión: Etiopía.

-Berlesezetes grandjeani (Mahunka, 1977) (Microzetes)

DistRIBUCIÓN: Mediterránea oriental.

-Berlesezetes kingi Bayoumi y Al-Khalifa, 1986

Distribución: Arabia Saudita.

-Berlesezetes natalensis (Engelbrecht, 1972) (Microzetes)

DisTRIBUCIÓN: Sudáfrica.

-Berlesezetes ornatissimus (Berlese, 1913) (Microzetes)

DistriBución: Cosmopolita.

-Berlesezetes ornatissimus ornatissimus (Berlese, 1913)

(=Microzetes africanus Balogh, 1958)

(=Microzetes auxiliaris Grandjean, 1936)

(=Microzetes auxiliaris pachyseta Hammer, 1971)

DisTRIBUCIÓN: Pantropical y subtropical (frecuente).

-Berlesezetes ornatissimus appalachicola (Jacot, 1938) (Microzetes

auxiliaris a.)

DisTRIBUCIÓN: U.S.A. oriental.

-Berlesezetes ornatissimus mirus (Mihelcic, 1956) (Microzetes m.) DISTRIBUCIÓN: España.

-Berlesezetes ornatissimus orkneyensis (Engelbrecht, 1972)

(Microzetes auxiliaris o.)

DisTRIBUCIÓN: Antártica: I. Orcadas del Sur (I. Orkney).

-Berlesezetes peruensis (Hammer, 1961) (Microzetes)

DistriBución: Perú e India (Bengala Occidental). 
Brazilozetes Balogh y Mahunka, 1969 (3 spp.)

EsPECIE TIPO: Brazilozetes flagellatus Balogh y Mahunka, 1969

DISTRIBUCIÓN: Neotropical.

-Brazilozetes flagellatus Balogh y Mahunka, 1969

DisTRIBUCIÓN: Brasil.

-Brazilozetes fusiger Balogh y Mahunka, 1981

DisTRIBUCIÓN: Paraguay.

-Brazilozetes phaseolus Balogh y Mahunka, 1969

DisTRIBUCIÓN: Neotropical (Brasil y Paraguay).

Calozetes Balogh y Mahunka, 1969 (1 sp.)

EsPECIE TIPO: Calozetes monticola Balogh y Mahunka, 1969

DistriBuCión: Neotropical.

-Calozetes monticola Balogh y Mahunka, 1969

DisTRIBUCIÓN: Bolivia.

Caucasiozetes Shtanchaeva, 1984 (7 spp.)

(=Teraja Mahunka, 1995 "sin. nov.")

EsPecIE TIPO: Caucasiozetes lamellatus Shtanchaeva, 1984

DisTRIBUCIÓN: Oriental y Paleártica meridional.

-Caucasiozetes asymmetricus (Mahunka, 2001) (Teraja)

DisTRIBUCIÓN: Borneo.

-Caucasiozetes fimbriatus (Mahunka, 1988) (Microzetes)

DisTRIBUCIÓN: Borneo.

-Caucaiozetes flagellifer (Mahunka, 1989) (Microzetes)

DisTRIBUCión: Península Malaya.

-Caucasiozetes lamellatus Shtanchaeva, 1984

DisTRIBUCIÓN: Cáucaso.

-Caucasiozetes sungai Mahunka, 1997 (Teraja)

DisTRIBUCión: Borneo.

-Caucasiozetes tuberculatus (Mahunka, 1987) (Microzetes)

DisTRIBUCIÓN: Borneo.

-Caucasiozetes wongi Mahunka, 1995 (Teraja)

DisTRIBUCIÓN: Borneo.

Cavernozetes Mahunka, 1984 (3 spp.)

EsPeCIE TIPO: Cavernozetes excavatus Mahunka, 1984

DisTRIBUCIÓN: Etiópica.

-Cavernozetes excavatus Mahunka, 1984

DisTRIBUCIÓN: Tanzania.

-Cavernozetes interruptus Mahunka, 1988

DISTRIBUCIÓN: Tanzania.

-Cavernozetes latus Mahunka, 1988

DisTRIBUCIÓN: Tanzania.

Christovizetes Krivolutsky, 1975 (2 spp.)

ESPECIE TIPO: Christovizetes ovatus Krivolutsky, 1975

DisTRIBUCIÓN: Paleártica y Oriental.

-Christovizetes ovatus Krivolutsky, 1975

DisTRIBUCIÓN: Asia centroocidental.

-Christovizetes prasadi Mahunka, 1995

DisTRIBUCIÓN: Tailandia.

Comorozetes Mahunka, 1994 (3 spp.)

EsPecIE TIPO: Comorozetes atavisticus Mahunka, 1994

DISTRIBUCIÓN: Etiópica.

-Comorozetes atavisticus Mahunka, 1994

Distribución: Malgache (I. Comores).

-Comorozetes concurvatus Mahunka, 1999

DisTRIBUCIÓN: Madagascar.

-Comorozetes corrugatus Mahunka,1997

DisTRIBUCIÓN: Madagascar.

Cosmozetes Balogh y Mahunka, 1969 (14 spp.)

EsPECIE TIPO: Cosmozetes striatissimus Balogh y Mahunka, 1969

DisTRIBUCIÓN: Tropical (Etiópica y Neotropical).

Cosmozetes (Cosmozetes) Balogh y Mahunka, 1969 (7 spp.)

DISTRIBUCIÓN: Neotropical.
-Cosmozetes (C.) cubanus Balogh y Mahunka, 1974

Distribución: Cuba.

-Cosmozetes (C.) ecuadoriensis P. Balogh, 1989

DISTRIBUCIÓN: Ecuador.

-Cosmozetes (C.) jaccoudi Mahunka, 1998

Distribución: Pequeñas Antillas.

-Cosmozetes (C.) negroi Balogh y Mahunka, 1977

DisTRIBUCIÓN: Brasil.

-Cosmozetes (C.) rohri Balogh y Mahunka, 1969

DisTRIBUCIÓN: Brasil.

-Cosmozetes (C.) striatissimus Balogh y Mahunka, 1969

DisTRIBUCIÓN: Bolivia.

-Cosmozetes (C.) vermiculatus (Balogh y Mahunka, 1980) (Rhopalozetes)

DisTRIBUCIÓN: Cuba.

Cosmozetes (Magoebazetes) Engelbrecht, 1973 (7 spp.)

EsPECIE TIPO: Magoebazetes magoebaensis Engelbrecht, 1973

DisTRIBUCIÓN: Tropical (Etiópica y Neotropical)

-Cosmozetes (Magoebazetes) cornutus (Mahunka, 1999) (M.)

DistRIBUCIÓN: Madagascar.

-Cosmozetes (Magoebazetes) engelbrechti (Mahunka, 1985) (M.)

DisTRIBUCIÓN: Sudáfrica.

-Cosmozetes (Magoebazetes) magoebaensis (Engelbrecht, 1973) (M.)

DisTRIBUCIÓN: Sudáfrica.

-Cosmozetes (Magoebazetes) natalensis (Engelbrecht, 1973) (M.)

DisTRIBUCIÓN: Sudáfrica.

-Cosmozetes (Magoebazetes) nosybe (Mahunka, 1993) (Megazetes)

DisTRIBUCIÓN: Madagascar.

-Cosmozetes (Magoebazetes) pentasetarum (Engelbrecht, 1973) (M.)

DistribuCión: Sudáfrica.

-Cosmozetes (Magoebazetes) reticulatus (Balogh, 1962) (Rhopalozetes)

DisTRIBUCión: Perú.

Cuspitegula Hammer, 1966 (1 sp.)

EsPeCIE TIPO: Cuspitegula stellifer Hammer, 1966

DISTRIBUCIÓN: Australiana.

-Cuspitegula stellifer Hammer, 1966

DISTRIBUCIÓN: Nueva Zelanda.

Dinozetes Balogh, 1962 (2 spp.)

EsPeCIE TIPO: Dinozetes mirabilis Balogh, 1962

DISTRIBUCIÓN: Neotropical.

-Dinozetes latissimus Balogh, 1962

DisTRIBUCIÓN: Perú.

-Dinozetes mirabilis Balogh, 1962

DisTRIBUCIÓN: Perú.

Fusozetes Balogh, 1972 (1 sp.)

EsPECIE TIPO: Rhopalozetes fusiger Balogh, 1962

DisTRIBUCIÓN: Neotropical.

-Fusozetes fusiger (Balogh, 1962) (Rhopalozetes)

DISTRIBUCIÓN: Perú.

Hauserozetes Mahunka, 1980 (1 sp.)

ESPECIE TIPO: Hauserozetes mausiae Mahunka, 1980

DisTRIBUCIÓN: Neotropical.

-Hauserozetes mausiae Mahunka, 1979

DisTRIBUCIÓN: Guatemala.

Hymenozetes Balogh, 1962 (5 spp.)

EsPECIE TIPO: Hymenozetes mirabilis Balogh, 1962

DISTRIBUCIÓN: Etiópica.

-Hymenozetes mirabilisBalogh, 1962

DisTRIBUCIÓN: Madagascar.

-Hymenozetes quadricornutus Mahunka, 1993

DisTRIBUCIÓN: Madagascar.

-Hymenozetes reticulatus Mahunka, 1996

DISTRIBUCIÓN: Madagascar. 
-Hymenozetes stellifer Mahunka, 1999

DISTRIBUCIÓN: Madagascar.

-Hymenozetes verticillatus Mahunka, 1997

DISTRIBUCIÓN: Madagascar.

Kalyptrazetes Balogh, 1972 (4 spp.)

(=Allozetes Higgins, 1965, "nom. praeoc." por Berlese, 1914)

EsPECIE TIPO: Allozetes harpezus Higgins, 1965

DISTRIBUCIÓN: Neártica meridional y Neotropical.

-Kalyptrazetes americanus Mahunka, 1995

Distribución: U.S.A. (Virginia).

-Kalyptrazetes desaussurei Mahunka, 1983

DisTRIBUCIÓN: Méjico.

-Kalyptrazetes harpezus (Higgins, 1965) (Allozetes)

DisTRIBUCión: U.S.A. (Carolina del Norte).

-Kalyptrazetes lupitae Mahunka y Palacios-Vargas, 1996 DisTRIBUCIÓN: Méjico.

Kaszabozetes Mahunka, 1988 (2 spp.)

(=Sinozetes Mahunka, 2000 "sin. nov.")

EsPECIE TIPO: Kaszabozetes velatus Mahunka, 1988

DistriBución: Oriental.

-Kaszabozetes botulus (Mahunka, 2000) (Sinozetes) DistriBución: China (Hong-Kong).

-Kaszabozetes velatus Mahunka, 1988

DISTRIBUCIÓN: Vietnam.

Licnozetes Balogh y Mahunka, 1969 (5 spp.)

ESPECIE TIPO: Licnozetes flabellatus Balogh y Mahunka, 1969

DISTRIBUCIÓN: Neotropical.

Licnozetes (Licnozetes) Balogh y Mahunka, 1969 (2 spp.)

DisTRIBUCIÓN: Neotropical.

-Licnozetes (L.) flabellatus Balogh y Mahunka, 1969 DISTRIBUCIÓN: Neotropical.

-Licnozetes (L.) multiareolatus Balogh y Mahunka, 1969 DISTRIBUCIÓN: Brasil.

Licnozetes (Undulozetes) Balogh y Mahunka, 1969 (3 spp.)

EsPeCIE TIPO: Undulozetes granulatus Balogh y Mahunka, 1969

DisTRIBUCIÓN: Neotropical.

-Licnozetes (Undulozetes) granulatus (Balogh y Mahunka, 1969) (U.) DisTRIBUCIÓN: Neotropical.

-Licnozetes (Undulozetes) margaritatus (Balogh y Mahunka, 1969) (U.) DisTRIBUCIÓN: Bolivia.

-Licnozetes (Undulozetes) topali (Balogh y Csiszár, 1963) (Rhopalozetes)

DISTRIBUCIÓN: Argentina.

Microzetes Berlese, 1913 (31 spp.)

EsPECIE TIPO: Sphaerozetes (Tectoribates) mirandus Berlese, 1908

DisTRIBUCIÓN: Paleártica meridional y Etiópica.

Microzetes (Microzetes) Berlese, 1913 (17 spp.)

(=Nellacaroides Mahunka, 1998 "sin. nov.")

(=Nellacarus Grandjean, 1936)

DisTRIBUCIÓN: Paleártica meridional y Etiópica.

-Microzetes (M.) adansoni (Lions, 1966) (Nellacarus)

Distribución: Suroeste de Europa.

-Microzetes (M.) asiaticus (Krivolutsky, 1975) (Nellacarus)

DisTRIBUCIÓN: Paleártica (Ucrania, y Asia centroocidental).

-Microzetes (M.) baloghi (Jeleva, 1962) (Nellacarus)

DisTRIBUCIÓN: Europa meridional (Bulgaria y Rumania).

-Microzetes (M.) castrii (Mahunka, 1966) (Nellacarus) DisTRIBUCIÓN: Italia.

-Microzetes (M.) caucasicus (Krivolutsky, 1967) (Nellacarus) DisTRIBUCIÓN: Cáucaso.

-Microzetes (M.) costulatus (Travé, 1956) (Nellacarus)

DISTRIBUCIÓN: Europa meridional.
-Microzetes (M.) hellenicus (Mahunka, 1977) (Nellacarus)

DistRIBUCIÓN: Grecia.

-Microzetes (M.) lunaris (Aoki, 1984) (Nellacarus)

DISTRIBUCIÓN: Japón.

-Microzetes (M.) mirandus (Berlese, 1908) (Sphaerozetes (Tectori-

bates))

DISTRIBUCIÓN: Mediterránea occidental.

-Microzetes (M.) petrocoriensis (Grandjean, 1936) (Nellacarus)

DisTRIBUCIÓn: Europa centromeridional.

-Microzetes (M.) phitosi (Mahunka, 1979) (Nellacarus)

DisTRIBUCIÓN: Grecia.

-Microzetes (M.) pyrenaicus (Travé, 1956) (Nellacarus)

DisTRIBUCIÓN: Suroeste de Europa.

-Microzetes (M.) raczi Mahunka, 1991

DisTRIBUCIÓN: Hungría.

-Microzetes (M.) septentrionalis (Kunst, 1963) (Nellacarus)

(=Nellacarus latens Moritz, 1964)

DISTRIBUCIÓN: Europa central.

-Microzetes (M.) sestai Mahunka, 1982

DisTRIBUCIÓN: Grecia.

-Microzetes (M.) simplisetus (Mahunka, 1998) (Nellacaroides)

DisTRIBUCIÓN: Uganda.

-Microzetes (M.) viedmai Subías, Ruiz y Kahwash, 1990

DISTRIBUCIÓN: España.

Microzetes (Megazetes) Balogh, 1959 (7 spp.)

EsPECIE TIPO: Megazetes micropterus Balogh, 1959

DisTRIBUCIÓN: Etiópica

-Microzetes (Megazetes) eckeri (Mahunka, 1982) (Meg.)

DisTRIBUCIÓN: Etiopía.

-Microzetes (Megazetes) flagellifer (Mahunka, 1988) (Meg.)

DisTRIBUCIÓN: Tanzania.

-Microzetes (Megazetes) micropterus (Balogh, 1959) (Meg.)

DisTRIBUCIÓN: Angola.

-Microzetes (Megazetes) pocsi (Mahunka, 1998) (Meg.)

DisTRIBUCIÓN: Uganda.

-Microzetes (Megazetes) rugosus (Mahunka, 1986) (Meg.)

DistRIBUCIÓN: Tanzania.

-Microzetes (Megazetes) scriptus (Mahunka, 1988) (Meg.)

DisTRIBUCión: Tanzania.

-Microzetes (Megazetes) tanzicus (Mahunka, 1983) (Meg.)

DisTRIBUCIÓN: Tanzania.

Microzetes (Stylozetes) Balogh y Mahunka, 1969 (4 spp.)

EsPECIE TIPO: Stylozetes physoseta Balogh y Mahunka, 1969

DISTRIBUCIÓN: Neotropical.

-Microzetes (Stylozetes) discrepans (Balogh, 1962) (Nellacarus)

Distribución: Perú.

-Microzetes (Stylozetes) physoseta (Balogh y Mahunka, 1969) (S.)

DISTRIBUCIÓN: Brasil.

-Microzetes (Stylozetes) processus (Balogh y Mahunka, 1977) (S.) DisTRIBUCIÓN: Brasil.

-Microzetes (Stylozetes) spinosus (Balogh y Mahunka, 1981) (S.)

DisTRIBUCIÓN: Paraguay.

Mirabilozetes Mahunka, 1977 (1 sp.)

EsPeCIE TIPO: Mirabilozetes dentatus Mahunka, 1977

DISTRIBUCIÓN: Etiópica.

-Mirabilozetes dentatus Mahunka, 1977

DisTRIBUCIÓN: Kenia.

Miracarus Kunst, 1959 (4 spp.)

EsPeCIE TIPO: Miracarus hurkai Kunst, 1959

DisTRIBUCión: Paleártica.

-Miracarus discrepans Mahunka, 1966

DisTRIBUCIÓN: Mediterránea occidental.

-Miracarus hurkai Kunst, 1959

DistriBuCión: Bulgaría.

-Miracarus senensis Bernini, 1975

DisTRIBUCIÓN: Mediterránea occidental. 
-Miracarus similis Subías e Iturrondobeitia, 1978

(=Miracarus abeloosi Lions, 1979)

DisTRIBUCIÓN: Mediterránea occidental.

Mystacozetes Balogh, 1962 (2 spp.)

EsPECIE TIPO: Mystacozetes ornatus Balogh, 1962

DISTRIBUCIÓN: Neotropical.

-Mystacozetes nervosus (Balogh y Mahunka, 1969) (Acaroceras) DISTRIBUCIÓN: Brasil.

-Mystacozetes ornatus Balogh, 1962

DisTRIBUCIÓN: Perú

Mysterozetes Hammer, 1961 (1 sp.)

EsPecIE TIPO: Mysterozetes scapulatus Hammer, 1961

DISTRIBUCIÓN: Neotropical.

-Mysterozetes scapulatus Hammer, 1961

Distribución: Perú.

Orthozetes Balogh, 1962 (3 spp.)

EsPeCIE TIPO: Orthozetes dispar Balogh, 1962

DISTRIBUCIÓN: Tropical (Australiana y Neotropical)

-Orthozetes depilatus Balogh, 1968

DisTRIBUCIÓN: Nueva Guinea.

-Orthozetes dispar Balogh, 1962

DisTRIBUCIÓN: Perú.

-Orthozetes papuanus Balogh, 1968

DisTRIBUCIÓN: Nueva Guinea.

Oxyzetes Balogh, 1958 (1 sp.)

EsPECIE TIPO: Oxyzetes pectiniger Balogh, 1958

DISTRIBUCIÓN: Etiópica.

-Oxyzetes pectiniger Balogh, 1958

DisTRIBUCIÓN: Congo.

Papuazetes Balogh, 1968 (1 sp.)

EsPeCIE TIPO: Papuazetes insignis Balogh, 1968

DISTRIBUCIÓN: Australiana.

-Papuazetes insignis Balogh, 1968

DISTRIBUCION: Nueva Guinea.

Phylacozetes Grandjean, 1936 (3 spp.)

EsPECIE TIPO: Phylacozetes membranulifer Grandjean, 1936

DISTRIBUCIÓN: Tropical (Etiópica y Neotropical).

-Phylacozetes ensifer Mahunka, 1984

DisTRIBUCIÓN: Tanzania.

-Phylacozetes membranulifer Grandjean, 1936

DisTRIBUCIÓN: Venezuela.

-Phylacozetes quadridentatus Mahunka, 1985

Distribución: Pequeñas Antillas.

Plumozetes Balogh, 1972 (1 sp.)

EsPECIE TIPO: Rhopalozetes plumifer Balogh y Mahunka, 1968

DISTRIBUCIÓN: Neotropical

-Plumozetes plumifer (Balogh y Mahunka, 1968) (Rhopalozetes)

DisTRIBUCIÓN: Argentina.

Protozetes Balogh, 1962 (4 spp. y 1 ssp.)

EsPecIE TIPO: Protozetes capitulum Balogh, 1962

DISTRIBUCIÓN: Neotropical.

-Protozetes capitulum Balogh, 1962

Distribución: Perú.

-Protozetes clavatus Mahunka y Palacios-Vargas, 1996

DisTRIBUCIÓN: Méjico.

-Protozetes digitifer Mahunka, 1985

DistribuCIÓN: Neotropical.

-Protozetes digitifer digitifer Mahunka, 1985

DistriBución: Norte de Neotropical.

-Protozetes digitifer alticola P. Balogh, 1989

DISTRIBUCIÓN: Ecuador.
-Protozetes longicornis P. Balogh, 1989

DisTRIBUCIÓN: Ecuador.

Rhabdozetes Hammer, 1962 (1 sp.)

EsPECIE TIPO: Rhabdozetes pennatus Hammer, 1962

DISTRIBUCIÓN: Neotropical.

-Rhabdozetes pennatus Hammer, 1962

DistribuCIÓN: Chile.

Rhopalozetes Balogh, 1962 (10 spp.)

(=Austrozetes Balogh y Mahunka, 1969 "sin. nov.")

EsPeCIE TIPO: Rhopalozetes milloti Balogh, 1962

DisTRIBUCIÓN: Pantropical (excepto Australiana).

-Rhopalozetes brazilianus (Balogh y Mahunka, 1969) (Austrozetes)

DISTRIBUCIÓN: Neotropical (Brasil y Paraguay).

-Rhopalozetes canagaratnami Balogh, 1970

DistriBución: Ceilán [Sri Lanka].

-Rhopalozetes capensis Mahunka, 1985

DisTRIBUCIÓN: Sudáfrica.

-Rhopalozetes georgensis Engelbrecht, 1972

DISTRIBUCIÓN: Sudáfrica.

-Rhopalozetes lokobensis Mahunka, 1993

DisTRIBUCIÓN: Madagascar.

-Rhopalozetes madecassus Mahunka, 1993

DisTRIBUCIÓN: Madagascar.

-Rhopalozetes maximus (Pérez-Íñigo y Baggio, 1994) (Austrozetes)

DISTRIBUCIÓN: Brasil.

-Rhopalozetes milloti Balogh, 1962

DISTRIBUCIÓN: Madagascar.

-Rhopalozetes panabokkei Balogh, 1970

DisTRIBUCIÓN: Ceilán [Sri Lanka].

-Rhopalozetes witfonteinensis Engelbrecht, 1972

DistriBución: Sudáfrica.

Rugozetes Balogh, 1960 (2 spp.)

EsPeCIE TIPO: Microzetes grandjeani Balogh, 1959

DisTRIBUCIÓN: Tropical (Etiópica y Neotropical).

-Rugozetes gladiator Balogh, 1962

DisTRIBUCIÓN: Neotropical (Perú y Brasil)

-Rugozetes grandjeani (Balogh, 1959) (Microzetes)

DisTRIBUCIÓN: Congo.

Schalleria Balogh, 1962 (11 spp.)

ESPECIE TIPO: Schalleria sexcornuta Balogh, 1962

DisTRIBUCIÓN: Neotropical.

-Schalleria bacillifera Balogh, 1962

DistRIBUCIÓn: Perú.

-Schalleria cruciata Balogh y Mahunka, 1969

DisTRIBUCIÓN: Bolivia.

-Schalleria forceps Balogh y Mahunka, 1980

DisTRIBUCIÓN: Cuba.

-Schalleria incurvata Balogh y Mahunka, 1969

DisTRIBUCIÓN: Brasil.

-Schalleria latilamellata Balogh y Mahunka, 1980

DisTRIBUCión: Cuba.

-Schalleria martii Balogh y Mahunka, 1974

Distribución: Cuba.

-Schalleria mexicana Mahunka y Palacios-Vargas, 1996

Distribución: Méjico.

-Schalleria monoceros Balogh, 1962

DisTRIBUCIÓN: Perú.

-Schalleria ramosa Balogh y Mahunka, 1969

DisTRIBUCIÓN: Neotropical.

-Schalleria sexcornuta Balogh, 1962

DisTRIBUCIÓN: Perú.

-Schalleria trifurcata Balogh y Mahunka, 1979

DisTRIBUCión: Cuba.

Schalleriella Engelbrecht, 1972 (1 sp.)

ESPECIE TIPO: Schalleriella gravouwensis Engelbrecht, 1972 
DISTRIBUCIÓN: Etiópica.

-Schalleriella gravouwensis Engelbrecht, 1972 DISTRIBUCIÓN: Sudáfrica.

Schizozetes Balogh, 1962 (2 spp.)

EsPeCIE TIPO: Schizozetes quadrilineatus Balogh, 1962

DISTRIBUCIÓN: Tropical (Etiópica y Neotropical).

-Schizozetes quadrilineatus Balogh, 1962

DisTRIBUCIÓN: Perú.

-Schizozetes similis Mahunka, 1966

Distribución: Congo.

Sturmozetes J. y P. Balogh, 1992 (2 spp.)

(=Gymnozetes P. Balogh, 1984, "nom. praeoc." por Calugar y Vasiliu, 1983)

ESPECIE TIPO: Gymnozetes marginatus P. Balogh, 1984

DisTRIBUCIÓN: Neotropical.

-Sturmozetes longisetus (P. Balogh, 1989) (Gymnozetes)

DisTRIBUCIÓN: Ecuador.

-Sturmozetes marginatus (P. Balogh, 1984) (Gymnozetes)

DISTRIBUCIÓN: Colombia.

Szentivanyella Balogh y Mahunka, 1969 (3 spp.)

ESPECIE TIPO: Szentivanyella latilamellata Balogh y Mahunka, 1969

DisTRIBUCIÓN: Pantropical (excepto Australiana).

-Szentivanyella africana Mahunka, 1984

DisTRIBUCIÓN: Tanzania.

-Szentivanyella latilamellata Balogh y Mahunka, 1969

DISTRIBUCIÓN. Bolivia.

-Szentivanyella szentivanyi Hammer, 1979

DISTRIBUCIÓN: Java

Trichozetes Balogh y Mahunka, 1980 (1 sp.)

EsPECIE TIPO: Trichozetes neotrichus Balogh y Mahunka, 1980

DISTRIBUCIÓN: Neotropical.

-Trichozetes neotrichus Balogh y Mahunka, 1980

DisTRIBUCIÓN: Cuba.

Vermacarus Balogh y Mahunka, 1980 (2 spp.)

EsPECIE TIPO: Vermacarus longissimus Balogh y Mahunka, 1980

DISTRIBUCIÓN: Tropical (Etiópica y Neotropical).

-Vermacarus armatus Mahunka, 1997

DisTRIBUCIÓN: Madagascar.

-Vermacarus longissimus Balogh y Mahunka, 1980

DisTRIBUCIÓN: Cuba.

\section{Zetorchestoidea Michael, 1898}

Zetorchestidae Michael, 1898 (6 gen., 26 spp. y 1 ssp.) DisTRIBUCIÓN: Cosmopolita (excepto Antártica).

Belorchestes Grandjean, 1951 (3 spp.)

ESPECIE TIPO: Belorchestes planatus Grandjean, 1951

DISTRIBUCIÓN: Paleártica.

-Belorchestes gebennicus Grandjean, 1957

DisTRIBUCIÓN: Mediterránea occidental.

-Belorchestes planatus Grandjean, 1951

DisTRIBUCIÓN: Europa meridional.

-Belorchestes sectus Pérez-Íñigo y Peña, 1996

DisTRIBUCIÓN: I. Canarias.

Litholestes Grandjean, 1951 (1 sp.)

ESPECIE TIPO: Litholestes altitudinis Grandjean, 1951

DisTRIBUCIÓN: Paleártica.

-Litholestes altitudinis Grandjean, 1951

DisTRIBUCIÓN: Europa central.

Microzetorchestes Balogh, 1943 (2 spp.)

(=Diorchestes Grandjean, 1951)

EsPECIE TIPO: Zetorchestes emeryi Coggi, 1898
DiSTRIBUCIÓN: Paleártica.

-Microzetorchestes emeryi (Coggi, 1898) (Zetorchestes)

(=Zetorchestes consanguineus Oudemans, 1902)

DisTRIBUCIÓN: Paleártica meridional.

-Microzetorchestes italicus (Eyndhoven, 1942) (Zetorchestes)

DisTRIBUCIÓN: Euroatlántica.

Saxicolestes Grandjean, 1951 (3 spp.)

EsPecie TIPO: Saxicolestes auratus Grandjean, 1951

DISTRIBUCIÓN: Paleártica.

-Saxicolestes auratus Grandjean, 195

DISTRIBUCIÓN: Europa meridional (Francia y Rumania).

-Saxicolestes corsicanus Grandjean, 1956

DisTRIBUCIÓN: Mediterránea occidental.

Saxicolestes pollinivorus Travé, 1963

DISTRIBUCIÓN: Francia.

Strenzkea Travé, 1967 (1 sp.)

EsPECIE TIPO: Strenzkea depilata Travé, 1967

DISTRIBUCIÓN: Paleártica.

-Strenzkea depilata Travé, 1967

DISTRIBUCIÓN: Francia.

Zetorchestes Berlese, 1888 (16 spp. y 1 ssp.)

(=Leptorchistis Canestrini y Berlese, 1885, "nom. praeoc." por Leptorchestes

Thorell, 1870)

(=Phyllorchestes Mahunka, 1983)

EsPECIE TIPO: Carabodes micronychus Berlese, 1883

DisTRIBUCIÓN: Cosmopolita (excepto Antártica).

-Zetorchestes aokii Krisper, 1987

DisTRIBUCIÓN: Este de Paleártica oriental y Nueva Guinea.

-Zetorchestes equestris Berlese, 1908

DisTRIBUCIÓN: U.S.A. centrooriental.

-Zetorchestes flabrarius Grandjean, 1951

DisTRIBUCIÓN: Mediterránea.

-Zetorchestes grandjeani Krisper, 1987

DISTRIBUCIÓN: Europa meridional.

-Zetorchestes micronychus (Berlese, 1883) (Carabodes)

DisTRIBUCIÓN: Paleártica (Paleártica occidental y Este de Rusia asiática).

-Zetorchestes micronychus micronychus (Berlese, 1883) (=Zetorchestes falzonii Coggi, 1898)

DistRIBUCIÓN: Paleártica (Paleártica occidental: menos frecuente en el Norte, y Este de Rusia asiática).

-Zetorchestes micronychus unicorniculatus Lombardini, 1961 "ssp. inq."

DISTRIBUCIÓN: Italia.

-Zetorchestes novaguineanus Krisper, 1987

Distribución: Nueva Guinea.

-Zetorchestes ornatus Mahunka, 1985

DisTRIBUCIÓN: Sudáfrica.

-Zetorchestes pacificus Sellnick, 1959 (Z. micronychus p.)

DisTRIBUCIÓN: Polinesia: I. Australes [I. Tubuai] y Java.

-Zetorchestes phylliferus Mahunka, 1983 (Z. (Phyllorchestes))

DISTRIBUCIÓN: Madagascar.

-Zetorchestes phyllosetus Mahunka, 1977

DisTRIBUCIÓN: Mediterránea oriental.

-Zetorchestes reticulatus Karppinen y Poltavskaja, 1990

Distribución: Cáucaso.

-Zetorchestes saltator Oudemans, 1915

DisTRIBUCIÓN: Oriental.

-Zetorchestes schusteri Krisper, 1984

DisTRIBUCión: Neotropical (Brasil y Pequeñas Antillas).

-Zetorchestes transvaalensis Coetzee, 1989

DisTRIBUCIÓN: Sudáfrica.

-Zetorchestes trituberculatus Berlese, 1916 "sp. inq."

DisTRIBUCIÓN: "África oriental".

-Zetorchestes vanderhammeni Krisper, 1987

DISTRIBUCION: Nueva Guinea. 
Gustavioidea Oudemans, 1900

Astegistidae Balogh, 1961 (10 gen., 1 subg. y 49 spp.) DISTRIBUCIÓN: Cosmopolita (excepto Antártica).

\author{
Astegistes Hull, 1916 (2 spp.) \\ (=Cultrozetes Sellnick, 1922) \\ EsPeCIE TIPO: (Liacarus bicornis Warburton y Pearce, 1905) =Zetes \\ pilosus Koch, 1841 \\ DisTRIBUCión: Paleártica. \\ -Astegistes hallaensis Choi, 1998 \\ DisTRIBUCIÓN: Corea. \\ -Astegistes pilosus (Koch, 1841) (Zetes) \\ (=Liacarus bicornis Warburton y Pearce, 1905) \\ DisTRIBUCIÓN: Europa (menos frecuente en el Sur).
}

Cultroribella Mahunka, 1985 (1 sp.)

ESPECIE TIPO: Cultroribella lineata Mahunka, 1985

DisTRIBUCIÓN: Etiópica.

-Cultroribella lineata Mahunka, 1985

DisTriBuCión: Sudáfrica.

Cultroribula Berlese, 1908 (26 spp.)

EsPeCIE TIPO: Notaspis juncta Michael, 1885

DisTRIBUCIÓn: Cosmopolita (excepto Antártica).

-Cultroribula almagulae Poltavskaja, 1994

DistRIBUCIÓN: Asia centrooccidental.

-Cultroribula angulata Aoki, 1984

DisTRIBUCIÓN: Japón.

-Cultroribula arctica Poltavskaja, 1994

DisTRIBUCIÓN: Siberia central.

-Cultroribula argentinensis Balogh y Csiszár, 1963

DISTRIBUCIÓN: Argentina.

-Cultroribula bicultrata (Berlese, 1905) (Dameosoma)

(=Cultroribula falcata Evans, 1952)

(=Cultroribula szentivanyi Balogh, 1943)

(=Cultroribula trifurcata Jacot, 1939)

(=Cultroribula trifurcata rotundata Krivolutsky, 1962)

DisTRIBUCIÓN: Holártica (frecuente en Paleártica) y Java.

-Cultroribula bicuspidata Mahunka, 1978

DisTRIBUCIÓN: Malgache (I. Mauricio).

-Cultroribula brasilensis Pérez-Íñigo y Baggio, 1997

DisTRIBUCIÓN: Brasil.

-Cultroribula breviclavata Aoki, 1984

Distribución: Este de Paleártica oriental.

-Cultroribula castriensis Mahunka, 1985

Distribución: Pequeñas Antillas.

-Cultroribula confinis Berlese, 1908

DisTRIBUCIÓn: Europa meridional.

-Cultroribula divergens Jacot, 1939

DisTRIBUCIÓN: Neártica oriental.

-Cultroribula diversa Oudemans, 1915

DisTRIBUCión: Ceilán [Sri Lanka].

-Cultroribula elongata Fujikawa, 1972

DisTRIBUCión: Japón.

-Cultroribula humerata Balogh y Mahunka, 1966

DisTRIBUCión: Congo.

-Cultroribula juncta (Michael, 1885) (Notaspis)

DisTRIBUCIÓN: Holártica (Europa y U.S.A. oriental).

-Cultroribula lata Aoki, 1961

Distribución: Paleártica oriental, Oriental y Australiana

(Australia y Nueva Zelanada).

-Cultroribula laticuspis Balogh, 1970

DisTRIBUCIÓN: Nueva Guinea.

-Cultroribula ligulata Grishina, 1980

Distribución: Sur de Siberia.

-Cultroribula magnifica Berlese, 1908

Distribución: U.S.A. (Washington D.C.).
-Cultroribula neonominata nom. nov.

[=Cultroribula tridentata Aoki, 1965, “nom. praeoc." por Mihelcic, 1958 ("hom. prim.")]

DistRIBUCIÓN: Este de Paleártica oriental (frecuente).

-Cultroribula quinquesetosa J. y P. Balogh, 1983

DisTRIBUCIÓN: Australia.

-Cultroribula shukuminensis Nakatamari, 1982

DisTRIBUCIÓN: Japón.

-Cultroribula tridentata Mihelcic, 1958

DisTRIBUCIÓN: Austria.

-Cultroribula tropica Balogh, 1958

DisTRIBUCión: Etiópica (Congo y Angola).

-Cultroribula variolosa Fujikawa, 1991

DisTRIBUCIÓN: Japón.

Cultroribula zicsii Balogh y Mahunka, 1981

DistRIBUCIÓN: Neotropical (Paraguay y Uruguay).

Furcoppia Balogh y Mahunka, 1969 (10 spp.)

EsPeCIE TIPO: Furcoppia imitans Balogh y Mahunka, 1966

DistriBUCIÓN: Cosmopolita (excepto Antártica).

Furcoppia (Furcoppia) Balogh y Mahunka, 1969 (6 spp.)

DISTRIBUCIÓN: Pantropical.

-Furcoppia (F.) americana Pérez-Íñigo y Baggio, 1994

DisTRIBUCIÓN: Brasil.

-Furcoppia (F.) cornuta Hammer, 1972

Distribución: Polinesia (Tahití) e India (Bengala Occidental). -Furcoppia (F.) horakae Mahunka, 1987

DisTRIBUCIÓN: Borneo.

-Furcoppia (F.) imitans Balogh y Mahunka, 1966

DisTRIBUCIÓN: Sudáfrica.

-Furcoppia (F.) parva Balogh y Mahunka, 1967

DisTRIBUCIÓN: Vietnam.

-Furcoppia (F.) tricornuta Mahunka, 1978

DisTRIBUCIÓN: Malgache.

Furcoppia (Mexicoppia) Mahunka, 1983 (4 spp.)

EsPeCIE TIPO: Furcoppia (Mexicoppia) hauseri Mahunka, 1983

DISTRIBUCIÓN: Holártica.

-Furcoppia (Mexicoppia) dentata (Willmann, 1950) (Cultroribula)

DistriBución: Boreal (Eurosiberiana, China: Tíbet, y Neártica septentrional).

-Furcoppia (Mexicoppia) hauseri Mahunka, 1983

Distribución: Méjico.

-Furcoppia (Mexicoppia) microdentata (Krivolutsky, 1962) (Cultroribula)

DisTRIBUCIÓN: Paleártica (Ucrania, y Este de Rusia asiática).

-Furcoppia (Mexicoppia) vtorovi (Krivolutsky, 1971) (Cultroribula)

Distribución: Holártica (Oeste de Paleártica oriental y Neártica).

Furcoribula Balogh, 1943 (4 spp.)

EsPECIE TIPO: Notaspis furcillata Nordenskiöld, 1901

DISTRIBUCIÓN: Holártica.

-Furcoribula bilamellata (Hall, 1911) (Notaspis)

DisTRIBUCIÓN: U.S.A. (California).

-Furcoribula furcillata (Nordenskiöld, 1901) (Notaspis)

(=Notaspis bicuspidata Storkan, 1925)

(=Eremaeus copulatus Oudemans, 1906)

(=Cultroribula confinis magna Ewing, 1917)

DisTRIBUCIÓN: Holártica (Europa centrooriental, Paleártica oriental y U.S.A.: Ilinois)

-Furcoribula pacifica Krivolutsky, 1975

DisTRIBUCIÓN: Paleártica (Cáucaso e Islas del Este de Rusia asiática).

-Furcoribula tridentata Wen, 1991

DistriBución: Noreste de China.

Lamellozetes Covarrubias, 1967 (2 spp.)

EsPECIE TIPO: Lamellozetes pseudoareolatus Covarrubias, 1967

DISTRIBUCIÓN: Neotropical. 


\author{
-Lamellozetes chilensis Covarrubias, 1967 \\ Distribución: Chile. \\ -Lamellozetes pseudoareolatus Covarrubias, 1967 \\ DisTRIBUCIÓN: Chile.
}

Maorizetes Hammer, 1966 (1 sp.)

ESPECIE TIPO: Maorizetes ferox Hammer, 1966

DisTRIBUCIÓN: Australiana.

-Maorizetes ferox Hammer, 1966

DisTRIBUCIÓN: Nueva Zelanda.

Monofurcoppia Pérez-Íñigo y Sarasola, 1995 (1 sp.)

EsPECIE TIPO: Furcoppia (Monofurcoppia) austroamericana PérezÍnigo y Sarasola, 1995

DisTRIBUCIÓN: Neotropical.

-Monofurcoppia austroamericana (Pérez-Íñigo y Sarasola, 1995)

(Furcoppia (M.))

DISTRIBUCIÓN: Uruguay.

Quadroribula J. y P. Balogh, 1992 (1 sp.)

EsPECIE TIPO: Cultroribula quadrisetosa J. y P. Balogh, 1983

DISTRIBUCIÓN: Paleártica y Australiana.

-Quadroribula quadrisetosa (J. y P. Balogh, 1983) (Cultroribula)

DisTRIBUCIÓN: Australia y Japón.

Sulcoribula Hammer, 1971 (1 sp.)

ESPECIE TIPO: Sulcoribula laticuspidata Hammer, 1971

DISTRIBUCIÓN: Australiana.

-Sulcoribula laticuspidata Hammer, 1971

DistriBUCIÓN: Melanesia (I. Fiji).

Multoribulidae Balogh, 1972 (2 gen. y 3 spp.)

DISTRIBUCIÓN: Subtropical austral (excepto Australiana).

Multoribula Balogh y Mahunka, 1966 (2 spp.)

ESPECIE TIPO: Multoribula multipunctata Balogh y Mahunka, 1966

DISTRIBUCIÓN: Subtropical austral (excepto Australiana).

-Multoribula multipunctata Balogh y Mahunka, 1966

DisTRIBUCión: Sudáfrica.

-Multoribula suramericana Balogh y Mahunka, 1977

DisTriBución: Chile.

Peloptoribula Mahunka, 1984 (1 sp.)

EsPECIE TIPO: Peloptoribula spinulosa Mahunka, 1984

DisTRIBUCIÓN: Etiópica.

-Peloptoribula spinulosa Mahunka, 1984

DisTRIBUCIÓN: Sudáfrica.

Ceratoppiidae Kunst, 1971 (16 gen., 51 spp. y 2 sspp.) DISTRIBUCIÓN: Cosmopolita.

Amazoppia Balogh y Mahunka, 1969 (1 sp.)

ESPECIE TIPO: Amazoppia tricuspidata Balogh y Mahunka, 1969

DISTRIBUCIÓN: Neotropical.

-Amazoppia tricuspidata Balogh y Mahunka, 1969

DisTRIBUCIÓN: Neotropical.

Austroceratoppia Hammer, 1979 (4 spp.)

ESPECIE TIPO: Austroceratoppia dentata Hammer, 1979

DistriBución: Oriental y Paleártica.

-Austroceratoppia dentata Hammer, 1979

DisTRIBUCIÓN: Java.

-Austroceratoppia japonica Aoki, 1984

DistRIBUCIÓN: Este de Paleártica oriental y Noreste de Oriental.

-Austroceratoppia sabahna Mahunka, 1996

DisTRIBUCIÓN: Borneo.

-Austroceratoppia serapi Mahunka, 1996

DISTRIBUCIÓN: Borneo.
Ceratoppia Berlese, 1908 (13 spp. y 2 sspp.)

(?=Notaspis Hermann, 1804 "gen. inq.")

EsPECIE TIPO: Notaspis bipilis Hermann, 1804

DisTRIBUCIÓN: Holártica, Oriental y "Australiana".

-Ceratoppia abchasica Krivolutsky y Tarba, 1971

Distribución: Cáucaso.

-Ceratoppia acuminata Golosova, 1981

("non" Murcia acuminata Koch, 1841, =Ceratoppia bipilis)

DistRIBUCIÓN: Sureste de Paleártica oriental.

-Ceratoppia bipilis (Hermann, 1804) (Notaspis)

DisTRIBUCIÓN: Holártica (frecuente).

-Ceratoppia bipilis bipilis (Hermann, 1804)

(=Murcia acuminata Koch, 1841)

(=Oribata badius Koch, 1839)

(=Oppia cornuta Koch, 1844)

(=Oribata distincta Karpelles, 1893)

(=Ceratoppia herculeana Berlese, 1908)

DisTRIBUCIÓN: Holártica (frecuente).

-Ceratoppia bipilis curtipilis Hammer, 1977

DisTRIBUCIÓN: Pakistán.

-Ceratoppia bipilis spinipes (Banks, 1906) (Oppia s.)

(=Ceratoppia bipilis brevicuspis Jacot, 1934)

(=Oppia canadensis Banks, 1906)

(=Oppia montana Banks, 1906)

DISTRIBUCIÓN: Neártica centrooriental (frecuente).

-Ceratoppia clavisensillata Choi, 1998

DisTRIBUCIÓn: Corea.

-Ceratoppia crassiseta Balogh y Mahunka, 1967

DistRIBUCIÓN: Oriental (Vietnam y Filipinas).

-Ceratoppia hoeli Thor, 1930

DistRiBUCión: Boreal (Europa septentrional, Siberia y Groenlandia).

-Ceratoppia incisa Kaneko y Aoki, 1982

DisTRIBUCIÓN: Japón.

-Ceratoppia longifilis (Canestrini, 1897) (Oribata) "sp. inq."

DistRIBUCIÓN: Nueva Guinea.

-Ceratoppia oblectatoria Tseng, 1982

DISTRIBUCIÓN: Taiwan [Formosa].

Ceratoppia quadridentata (Haller, 1882) (Notaspis bipilis q.)

(=Ceratoppia quadridentata arctica Hammer, 1955)

DisTRIBUCIÓN: Holártica (Paleártica: frecuente, y Neártica septentrional).

-Ceratoppia sexpilosa Willmann, 1938

Distribución: Holártica (Europa centromeridional, Este de

Paleártica oriental y Neártica septentrional).

-Ceratoppia sphaerica (L. Koch, 1879) (Oppia)

DisTRIBUCIÓN: Boreal.

-Ceratoppia violabilis Tseng, 1982

DisTRIBUCIÓN: Taiwan [Formosa].

Ceratorchestes Balogh y Mahunka, 1969 (4 spp.)

EsPeCIE TIPO: Ceratorchestes setosus Balogh y Mahunka, 1969

DISTRIBUCIÓN: Neotropical.

-Ceratorchestes baloghi Mahunka, 1982

DisTRIBUCión: Méjico.

-Ceratorchestes cornutus Mahunka, 1982

Distribución: Costa Rica.

-Ceratorchestes globosus Balogh y Mahunka, 1969

DISTRIBUCIÓN: Bolivia.

-Ceratorchestes setosus Balogh y Mahunka, 1969

DisTRIBUCIÓN: Neotropical (Brasil y Venezuela).

Comeremaeus Hammer, 1962 (1 sp.)

EsPeCIE TIPO: Comeremaeus castaneus Hammer, 1962

DisTRIBUCIÓN: Neotropical.

-Comeremaeus castaneus Hammer, 1962

DISTRIBUCIÓN: Argentina.

Dendrozetes Aoki, 1970 (1 sp.)

ESPECIE TIPO: Dendrozetes caudatus Aoki, 1970 
DisTRIBUCIÓN: Paleártica.

-Dendrozetes caudatus Aoki, 1970

DisTRIBUCIÓN: Japón.

Macquarioppia Wallwork, 1964 (1 sp.)

(=Macquariella Wallwork, 1963, “nom. praeoc.” por Finlay, 1927)

ESPECIE TIPO: Macquariella striata Wallwork, 1963

DISTRIBUCIÓN: Antártica.

-Macquarioppia striata (Wallwork, 1963) (Macquariella)

DisTRIBUCIÓN: Antártica (frecuente).

Metapyroppia Woolley, 1969 (1 sp.)

EsPECIE TIPO: Metapyroppia doratosa Woolley, 1969

DISTRIBUCIÓN: Neártica.

-Metapyroppia doratosa Woolley, 1969

DisTRIBUCIÓN: U.S.A. (Ténesi).

Negroppia Vasiliu y Calugar, 1977 (1 sp.)

EsPECIE TIPO: Negroppia cornuta Vasiliu y Calugar, 1977

DisTRIBUCIÓN: Neotropical.

-Negroppia cornuta Vasiliu y Calugar, 1977

DISTRIBUCIÓN: Cuba.

Notoppia Balogh y Mahunka, 1966 (1 sp.)

EsPECIE TIPO: Notoppia humerata Balogh y Mahunka, 1966

DisTRIBUCIÓN: Australiana.

-Notoppia humerata Balogh y Mahunka, 1966

DisTRIBUCIÓN: Australia.

Paraceratoppia Rjabinin, 1982 (2 spp.)

ESPECIE TIPO: Paraceratoppia meridionalis Rjabinin, 1982 DISTRIBUCIÓN: Paleártica.

-Paraceratoppia asiatica (Krivolutsky, 1965) (Pyroppia)

DistribuCión: Paleártica (Norte de Rusia europea, Asia centra rusa e I. Sajalín).

-Paraceratoppia meridionalis Rjabinin, 1982

DISTRIBUCIÓN: I. Kuriles.

Parapyroppia Pérez-Íñigo y Subías, 1979 (3 spp.)

EsPeCIE TIPO: (Parapyroppia monodactyla Pérez-Íñigo y Subías, 1979)

=Oribella cornuta Berlese, 1910

DisTRIBUCIÓN: Holártica.

-Parapyroppia cornuta (Berlese, 1910) (Oribella)

(=Oribella magna Mihelcic, 1957)

(=Parapyroppia monodactyla Pérez-Íñigo y Subías, 1979)

DistRiBución: Mediterránea occidental.

-Parapyroppia filiformis Hirauchi, 1998

DisTRIBUCiÓN: Japón.

-Parapyroppia transitoria (Berlese, 1908) (Protoribates)

(=Notaspis lamellata Ewing, 1909)

DistriBUCIÓN: U.S.A. (Misuri).

Pseudoceratoppia Hammer, 1967 (7 spp.)

(=Hauseroceratoppia Mahunka, 1980)

EsPeCIE TIPO: Pseudoceratoppia sexsetosa Hammer, 1967

DisTRIBUCIÓN: Australiana.

-Pseudoceratoppia asetosa Hammer, 1967

DisTRIBUCión: Nueva Zelanda.

-Pseudoceratoppia clavasetosa Hammer, 1967

Distribución: Nueva Zelanda.

-Pseudoceratoppia diversa Hammer, 1967

DistRIBUCIÓN: Nueva Zelanda.

-Pseudoceratoppia horaki (Mahunka, 1980) (Hauseroceratoppia)

DisTRIBUCIÓN: América austral (Tierra del Fuego)

-Pseudoceratoppia mariannae J. y P. Balogh, 1983

DisTRIBUCIÓN: Australia.

-Pseudoceratoppia microsetosa Hammer, 1967

Distribución: Nueva Zelanda.

-Pseudoceratoppia sexsetosa Hammer, 1967

Distribución: Nueva Zelanda.
Pseudopyroppia Rjabinin, 1987 (2 spp.)

ESPECIE TIPO: Pseudopyroppia orientalis Rjabinin, 1987

DisTRIBUCIÓN: Paleártica.

-Pseudopyroppia orientalis Rjabinin, 1987

DisTRIBUCIÓN: Este de Rusia asiática.

-Pseudopyroppia rotunda Hirauchi, 1998

DisTRIBUCIÓN: Japón.

Pyroppia Hammer, 1955 (7 spp.)

EsPeCIE TIPO: Pyroppia lanceolata Hammer, 1955

DISTRIBUCIÓN: Holártica.

-Pyroppia arctica Krivolutsky, 1974

Distribución: Paleártica oriental.

-Pyroppia dentata Krivolutsky, 1974

DisTRIBUCIÓN: Neártica septentrional (I. Aleutianas y Alaska).

-Pyroppia lanceolata Hammer, 1955

Distribución: Holártica (Paleártica occidental: Cáucaso,

Paleártica oriental: Kamchatka, y Neártica septentrional).

-Pyroppia serrifrons (Banks, 1923) (Notaspis)

DisTRIBUCIÓN: Alaska.

-Pyroppia sibirica Golosova y Karppinen, 1984

DistRIBUCIÓN: Sur de Siberia.

-Pyroppia tajikistanica Krivolutsky y Christov, 1970

DisTRIBUCIÓN: Paleártica meridional (Cáucaso y Asia centrooccidental).

-Pyroppia ulneungensis Choi, 1997

DisTRIBUCIÓN: Corea.

Trichoppia Balogh, 1961 (2 spp.)

ESPECIE TIPO: Trichoppia longiseta Balogh, 1961

DISTRIBUCIÓN: Etiópica.

-Trichoppia longiseta Balogh, 1961

DISTRIBUCIÓN: Madagascar.

-Trichoppia piriformis (Warburton, 1912) (Liacarus)

DisTRIBUCIÓN: I. Seychelles.

Metrioppiidae Balogh, 1943 (3 gen. y 10 spp.) DisTRIBUCIÓN: Holártica.

Ceratoppiella Hammer, 1977 (1 sp.)

EsPeCIE TIPO: Ceratoppiella lutea Hammer, 1977

DISTRIBUCIÓN: Paleártica.

-Ceratoppiella lutea Hammer, 1977

DISTRIBUCIÓN: Pakistán.

Metrioppia Grandjean, 1931 (8 spp.)

(=Peloppia Sellnick, 1931)

EsPeCIE TIPO: Metrioppia helvetica Grandjean, 1931

DisTRIBUCIÓN: Holártica.

-Metrioppia atlantica Jacot, 1938 (M. serrata a.)

Distribución: U.S.A. (Carolina del Norte).

-Metrioppia helvetica Grandjean, 1931

(=Ceratoppia microseta Hammer, 1952)

DistRIBUCIÓN: Holártica (Europa centromeridional, Paleártica oriental y Neártica).

-Metrioppia krivolutskyi Bayartogtokh, 2000

DisTRIBUCIÓN: Mongolia.

-Metrioppia oregonensis Woolley y Higgins, 1969

DisTRIBUCIÓN: U.S.A. (Oregón).

-Metrioppia quadrisetosa Fujita y Fujikawa, 1986 DisTRIBUCIÓN: Japón.

-Metrioppia serrata (Sellnick, 1931) (Peloppia)

Distribución: Méjico y U.S.A. (Virginia).

-Metrioppia tricuspidata Aoki y Wen, 1983

DistRIBUCIÓN: Este de Paleártica oriental.

-Metrioppia zlotini Krivolutsky, 1971

DistRIBUCIÓN: Asia centrooccidental. 
Paenoppia Woolley y Higgins, 1965 (1 sp.)

EsPeCIE TIPO: Paenoppia forficula Woolley y Higgins, 1965

DisTRIBUCIÓN: Neártica.

-Paenoppia forficula Woolley y Higgins, 1965

DisTRIBUCIÓN: U.S.A. (Colorado).

Gustaviidae Oudemans, 1900 (1 gen. y 12 spp.) DisTRIBUCIÓN: Holártica y Etiópica.

Gustavia Kramer., 1879 (12 spp.)

(=Neozetes Berlese, 1885)

(=Serrarius Michael, 1883)

ESPECIE TIPO: (Gustavia sol Kramer, 1879) =Leiosoma microcephala

Nicolet, 1855

DISTRIBUCIÓN: Holártica y Etiópica.

-Gustavia aethiopica Mahunka, 1982

DisTRIBUCIÓN: Etiopía.

-Gustavia fusifer (Koch, 1841) (Oribata)

DisTRIBUCIÓN: Paleártica occidental (menos frecuente en el Norte).

-Gustavia latolamellata Hammer, 1977

DisTRIBUCIÓN: Pakistán.

-Gustavia longicornis (Berlese, 1904) (Serrarius)

DistRIBUCIÓN: Mediterránea occidental y Taiwan [Formosa]

-Gustavia longiseta Mahunka, 1984

DisTRIBUCIÓN: Tanzania.

-Gustavia magnifica Golosova y Karppinen, 1984

DisTRIBUCIÓN: Este de Rusia asiática.

-Gustavia magnilamellata (Ewing, 1909) (Liacarus)

(=Serrarius connexus Berlesle, 1916)

DisTRIBUCIÓN: U.S.A. (Misuri).

-Gustavia maior (Berlese, 1904) (Serrarius)

(=Gustavia microcephala longirostris Mihelcic, 1957)

DISTRIBUCIÓN: Europa meridional.

-Gustavia microcephala (Nicolet, 1855) (Leiosoma)

(=Neozetes bicornis Berlese, 1885)

(=Gustavia sol Kramer, 1879)

Distribución: Paleártica (frecuente) y Méjico.

-Gustavia oceanica Pérez-Íñigo, 1987

DisTRIBUCIÓN: Mediterránea occidental.

- Gustavia parvula (Banks, 1909) (Liacarus)

DisTRIBUCión: U.S.A. (Minesota).

-Gustavia rugosa Balogh, 1958 "sp. inq."

DisTRIBUCión: Etiópica (Congo y Tanzania).

Liacaridae Sellnick, 1928 (5 gen., 3 subg., 125 spp. y 2 sspp.) Distribución: Cosmopolita (excepto Antártica).

Adoristes Hull, 1916 (3 spp.)

EsPecie Tipo: Oribata ovata Koch, 1839

DisTRIBUCIÓN: Holártica.

-Adoristes ammonoosuci Jacot, 1938 (A. ovatus a.)

DISTRIBUCIÓN: Neártica oriental.

-Adoristes ovatus (Koch, 1839) (Oribata)

(=Adoristes extraneus Mihelcic, 1955)

(=Conoppia grandis Berlese, 1908)

(=Leiosoma pyrigerum Berlese, 1888)

(=Leiosoma truncatum Canestrini y Fanzago, 1876)

DisTRIBUCIÓN: Paleártica (Paleártica occidental: frecuente, y Asia centrooccidental)

-Adoristes poppei (Oudemans, 1906) (Liacarus)

DisTRIBUCión: Holártica (Paleártica occidental: frecuente, Oeste de Siberia y U.S.A.: Minesota).

Birsteinius Krivolutsky, 1965 (8 spp.)

ESPECIE TIPO: Birsteinius clavatus Krivolutsky, 1965

DisTRIBUCIÓN: Paleártica.

-Birsteinius clavatus Krivolutsky, 1965

DisTRIBUCIÓN: Europa oriental.

-Birsteinius krivolutskyi Rjabinin, 1979

DisTRIBUCIÓN: Este de Rusia asiática.
-Birsteinius microchaetus Krivolutsky, 1967

Distribución: Paleártica (Sureste de Europa, Sur de Siberia e Islas del Este de Rusia asiática).

-Birsteinius mongolicus (Mahunka, 1964) (Liacarus)

DisTRIBUCión: Paleártica meridional (Rumania, Asia centrooccidental y Mongolia).

-Birsteinius monticolus Wang y Shen, 2001

DisTRIBUCIÓN: China (Tíbet)

-Birsteinius perlongoides Hammer, 1977

DisTRIBUCIÓN: Pakistán.

-Birsteinius perlongus Krivolutsky, 1965

Distribución: Paleártica meridional (Sureste de Europa, Asia centrooccidental y Mongolia).

-Birsteinius trimucronatus Yamamoto y Aoki, 2000

DisTRIBUCIÓN: China suroriental.

Liacarus Michael, 1898 (112 spp. y 2 sspp.)

ESPECIE TIPO: Oribata nitens Gervais, 1844

DisTRIBUCIÓn: Cosmopolita (excepto Antártica).

Liacarus (Liacarus) Michael, 1898 (71 spp. y 2 sspp.)

(=Leiosoma Nicolet, 1855, "nom. praeoc." por Stephens, 1829)

(=Leuroxenillus Woollely y Higgins, 1966)

(=Stenoxenillus Woolley y Higgins, 1966 "sin. nov.")

DisTRIBUCIÓN: Cosmopolita (excepto Neotropical y Antártica).

-Liacarus (L.) arduus Fujikawa, 1989

DISTRIBUCIÓN: Japón.

-Liacarus (L.) atraktus (Woolley y Higgins, 1966) (Stenoxenillus) Distribución: Sureste de U.S.A.

-Liacarus (L.) bidentatus Ewing, 1918

DisTRIBUCión: Neártica.

-Liacarus (L.) borealis Wen, 1991

DisTRIBUCIÓN: China suroriental.

-Liacarus (L.) brevilamellatus Mihelcic, 1955

(=Liacarus cartalinicus Djaparidze, 1979)

(=Liacarus internodentatus Kulijev, 1962)

(=Liacarus major Mihelcic, 1955)

(=Liacarus ovalis Mihelcic, 1956)

(=Liacarus vastus Mihelcic, 1956)

Distribución: Mediterránea.

-Liacarus (L.) castaneus (Banks, 1906) (Notaspis)

DisTRIBUCión: U.S.A. (California).

-Liacarus (L.) celisi Balogh, 1958 "sp. inq."

DisTRIBUCIÓN: Africa central.

-Liacarus (L.) chiebunensis Fujita y Fujikawa, 1984

DisTRIBUCIÓN: Japón.

-Liacarus (L.) chroniosus Woolley, 1968

(=Liacarus robustus Mihelcic, 1954, "nom. praeoc.” por Ewing, 1918)

DISTRIBUCIÓN: Europa centromeridional.

-Liacarus (L.) cidarus Woolley, 1968

DisTRIBUCIÓN: U.S.A. occidental

-Liacarus (L.) conjunctus Mihelcic, 1954

DisTRIBUCIÓN: Austria.

-Liacarus (L.) contiguus Aoki, 1969

DisTRIBUCIÓN: Japón.

-Liacarus (L.) coracinus (Koch, 1841) (Oribata)

Distribución: Paleártica e I. Santa Helena.

-Liacarus (L.) coracinus coracinus (Koch, 1841)

(=Oribata politus Koch y Berendt, 1854)

(=Leiosoma similis Nicolet, 1855)

(=Liacarus vombi Dalenius, 1950)

Distribución: Paleártica (frecuente) e I. Santa Helena.

-Liacarus (L.) coracinus simplex Mihelcic, 1952

DISTRIBUCIÓN: Austria.

-Liacarus (L.) curvidentatus Balogh, 1958 "sp. inq."

DisTRIBUCIÓN: Congo.

-Liacarus (L.) detosus Woolley, 1968

Distribución: Este de U.S.A.

-Liacarus (L.) emeiensis Wen, 1991

DistribuCión: China suroriental. 
-Liacarus (L.) externus Aoki, 1987

DisTRIBUCiÓN: Japón.

-Liacarus (L.) flammeus Aoki, 1967

DisTRIBUCIÓN: Este de Paleártica oriental.

-Liacarus (L.) gammatus Aoki, 1967

DisTRIBUCIÓN: Este de Paleártica oriental.

-Liacarus (L.) gammatus gammatus Aoki, 1967 DISTRIBUCIÓN: Japón.

-Liacarus (L.) gammatus coreanus Choi, 1994 DISTRIBUCIÓN: Corea.

-Liacarus (L.) granulatus Willmann, 1940 "sp. inq."

DisTRIBUCión: "Yugoslavia".

-Liacarus (L.) inermis Aoki, 1965

DisTRIBUCión: Sur de Paleártica oriental.

-Liacarus (L.) janetscheki Mihelcic, 1957

DisTribuCIÓN: Austria.

-Liacarus (L.) jordanai Moraza y Pérez-Íñigo, 1979

DisTRIBUCIÓN: España.

-Liacarus (L.) kanekoi Bayartogtokh y Aoki, 2002

DISTRIBUCIÓN: Japón.

-Liacarus (L.) kilchini Choi, 1985

DisTRIBUCIÓN: Corea.

-Liacarus (L.) koeszegiensis Balogh, 1943

(=Liacarus sejunctus Mihelcic, 1954)

DisTRIBUCIÓN: Europa centromeridional.

-Liacarus (L.) laterostris Mihelcic, 1954

DisTRIBUCIÓN: Europa centromeridional.

-Liacarus (L.) latilamellatus Kaneko y Aoki, 1982

DisTRIBUCIÓN: Japón.

-Liacarus (L.) latus Ewing, 1909

DisTRIBUCIÓN: U.S.A

-Liacarus (L.) lectronus Higgins y Woolley, 1969

DistribuCIÓN: U.S.A. (California).

-Liacarus (L.) leleupi Balogh, 1958 "sp. inq.".

Distribución: Congo.

-Liacarus (L.) lencoranicus Krivolutsky, 1967

Distribución: Paleártica (Sureste de Europa y Sur de Siberia).

-Liacarus (L.) longilamellatus Mihelcic, 1954

DisTRIBUCIÓN: Austria.

-Liacarus (L.) logipilis (Moniez, 1894) (Leiosoma)

DisTRIBUCión: Nueva Zelanda.

-Liacarus (L.) lucidus Ewing, 1909

DistribuCión: U.S.A. (Ilinois).

-Liacarus (L.) luscus Hirauchi, 1998

DisTRIBUCIÓN: Japón.

-Liacarus (L.) madeirensis Willmann, 1939

DisTRIBUCIÓN: Mediterránea occidental.

-Liacarus (L.) marginatus (Nicolet, 1855) (Leiosoma) "sp. inq." DisTRIBUCIÓN: Francia.

-Liacarus (L.) matshabelii Djaparidze, 1980

DisTRIBUCIÓN: Cáucaso.

-Liacarus (L.) medialis Banks, 1910

DisTRIBUCIÓN: Este de U.S.A.

-Liacarus (L.) matshabelii Djaparidze, 1980

Distribución: Cáucaso.

-Liacarus (L.) medialis Banks, 1910

DisTRIBUCión: Este de U.S.A

-Liacarus (L.) montanus Aoki, 1984

DISTRIBUCIÓN: Japón.

-Liacarus (L.) mucronatus Willmann, 1939

DisTRIBUCIÓN: Holártica meridional (Mediterránea occidental y

U.S.A.: Carolina del Norte)

-Liacarus (L.) murotensis Aoki, 1988

DisTRIBUCIÓN: Japón.

-Liacarus (L.) nigrescens Pearce, 1906

DisTRIBUCIÓN: India (Sikkim).

-Liacarus (L.) nitens (Gervais, 1844) (Oribata)

Distribución: Holártica (Paleártica occidental, Este de Paleártica oriental y U.S.A.: Virginia).
-Liacarus (L.) nitidulus Krivolutsky, 1967

DisTRIBUCIÓn: Sureste de Europa.

-Liacarus (L.) ocellatus Aoki, 1987

DisTRIBUCIÓN: Japón.

-Liacarus (L.) olivaceus (Grube, 1859) (Leiosoma) "sp. inq."

DISTRIBUCIÓN: Alemania.

-Liacarus (L.) oribatelloides Winkler, 1956

DisTRIBUCIÓN: Europa central.

-Liacarus (L.) orthogonios Aoki, 1959

Distribución: Este de Paleártica oriental y Noreste de Oriental.

-Liacarus (L.) ovatus Mihelcic, 1954

(=Liacarus mihelcici Woolley, 1968)

DisTRIBUCIÓN: Austria.

-Liacarus (L.) parvus Mihelcic, 1954

DisTRIBUCIÓN: Europa centromeridional.

-Liacarus (L.) perezinigoi Capilla, 1972

DistRIBUCIÓN: Mediterránea occidental.

-Liacarus (L.) pseudocontiguus Fujikawa, 1991

DisTRIBUCIÓN: Japón.

-Liacarus (L.) regeli Djaparidze, 1983

Distribución: Cáucaso.

-Liacarus (L.) reticulatus Mahunka, 1966

DISTRIBUCIÓN: Italia.

-Liacarus (L.) robustus Ewing, 1918

DisTRIBUCIÓN: Noroeste de U.S.A.

-Liacarus (L.) rotundatus Mihelcic, 1954

DISTRIBUCIÓN: Austria.

-Liacarus (L.) schweizeri Forsslund, 1963

(=Liacarus latus Schweizer, 1956, "nom. praeoc." por Ewing, 1909)

(=Liacarus schweizeri Woolley, 1968, "nom. praeoc.” por Forsslund, 1963)

DisTRIBUCIÓN: Suiza.

-Liacarus (L.) sphaericus Djaparidze, 1980

Distribución: Paleártica (Cáucaso y Este de Rusia asiática).

-Liacarus (L.) spiniger Jacot, 1937

DisTRIBUCIÓN: Sureste de U.S.A.

-Liacarus (L.) subterraneus (Koch, 1844) (Oribata)

(=Liacarus gracilis Mihelcic, 1954)

(=Leiosoma lativentris Nicolet, 1855 )

DistriBuCión: Holártica (Paleártica occidental: frecuente,

Suroeste de Siberia y U.S.A.: Virginia)

-Liacarus (L.) tanzicus Mahunka, 1983

DisTRIBUCIÓN: Tanzania

-Liacarus (L.) tremellae (Linnaeus, 1761) (Acarus) "sp. inq."

(=Oribata globosa Koch, 1844 )

DisTRIBUCIÓN: Suecia.

-Liacarus (L.) trichionus (Woolley y Higgins, 1966) (Leuroxenillus)

DisTRIBUCIÓN: U.S.A. (Oregón).

-Liacarus (L.) tubifer Djaparidze y Melamud, 1990

DisTRIBUCión: Sureste de Europa.

-Liacarus (L.) unjangensis Choi, Bayartogtokh y Aoki, 2001

DisTRIBUCIÓN: Corea.

-Liacarus (L.) willmanni Pschorn-Walcher, 1951

DisTRIBUCIÓN: Austria.

-Liacarus (L.) xylariae (Schrank. 1803) (Acarus)

(=Liacarus cuspidatus Mihelcic, 1954)

(=Oribata fusca Koch, 1841)

(=Liacarus translamellatus Kulijev, 1962)

DisTRIBUCIÓN: Paleártica (frecuente).

-Liacarus (L.) yayeyamensis Aoki, 1973

DisTRIBUCIÓN: Este de Paleártica oriental.

Liacarus (Dorycranosus) Woolley, 1969 (29 spp.)

EsPeCIE TIPO: Liacarus abdominalis Banks, 1906

DisTRIBUCIÓN: Holártica.

-Liacarus (Dorycranosus) abdominalis Banks, 1906 (L.)

DistRIBUCIÓN: Neártica (U.S.A.: California y Alaska).

-Liacarus (Dorycranosus) acutidens Aoki, 1965 (L.)

DisTRIBUCIÓN: Holártica (Este de Paleártica oriental y Alaska). 
-Liacarus (Dorycranosus) acutus Pschorn-Walcher, 1951 (L.) (=Liacarus claviger Mihelcic, 1956) (=Cultroribula grandis Mihelcic, 1956) (=Liacarus infissus Gunhold, 1953) DisTRIBUCIÓN: Paleártica occidental (menos frecuente en el Norte)

-Liacarus (Dorycranosus) aequidentatus Ewing, 1918 (L.) DisTRIBUCIÓN: U.S.A.

-Liacarus (Dorycranosus) altaicus (Krivolutsky, 1974) (D.) DisTRIBUCIÓN: Holártica (Asia central rusa y Neártica septentrional).

-Liacarus (Dorycranosus) angustatus (Weigmann, 1976) (D.) DisTRIBUCIÓN: I. Azores.

-Liacarus (Dorycranosus) arcticus (Banks, 1899) (Oppia) “sp. inq." DisTRIBUCIÓN: Alaska.

-Liacarus (Dorycranosus) badghysi Krivolutsky, 1966 (L.) DisTRIBUCIÓN: Paleártica meridional (Crimea, y Asia centrooccidental).

-Liacarus (Dorycranosus) columbianus Berlese, 1908 (L.) Distribución: Holártica (Este de Paleártica oriental: I. Kuriles, y U.S.A.: Misuri)

-Liacarus (Dorycranosus) coronatus (Golosova y Ljashchev, 1984) (D.) Distribución: I. Sajalín.

-Liacarus (Dorycranosus) dickersoni (Moraza, 1990) (D.) DisTRIBUCIÓN: España.

-Liacarus (Dorycranosus) floridensis Berlese, 1908 (L.) DisTRIBUCIÓN: U.S.A. (Florida).

-Liacarus (Dorycranosus) frontinalis Banks, 1906 (L.) DisTRIBUCIÓN: U.S.A. (Virginia).

-Liacarus (Dorycranosus) fusiformis Ewing, 1917 (L.) DisTRIBUCIÓN: U.S.A. (Iowa y Utah).

-Liacarus (Dorycranosus) indentatus (Aoki, 1973) (D.) DISTRIBUCIÓN: Japón.

-Liacarus (Dorycranosus) keretinus Nordenskiöld, 1901 (L.) DisTRIBUCIÓN: Norte de Paleártica occidental.

-Liacarus (Dorycranosus) latiusculus Wen, 1991 (L.) DisTRIBUCIÓN: China suroriental.

-Liacarus (Dorycranosus) lingulatus (Golosova y Ljashchev, 1984) (D.) DisTRIBUCIÓN: I. Sajalín.

-Liacarus (Dorycranosus) neonominatus nom. nov. [=Dorycranosus arcticus Grishina, 1984, "nom. praeoc." por Banks, 1899 ("hom. sec.")] DisTRIBUCIÓN: Rusia (Urales y Siberia).

-Liacarus (Dorycranosus) nitidus (Banks, 1895) (Cepheus) (=Liacarus glaber Ewing, 1909) (=Liacarus nitidus fissuratus Jacot, 1937) DisTRIBUCIÓN: U.S.A.

-Liacarus (Dorycranosus) paoliensis (Woolley, 1969) (D.) DisTRIBUCIÓN: U.S.A. oriental.

-Liacarus (Dorycranosus) parallelus Hammer, 1967 (L.) Distribución: Boreal (Islas del Este de Rusia asiática y Neártica septentrional).

-Liacarus (Dorycranosus) polychothomus Wen, 1991 (L.) Distribución: Este de Paleártica oriental.

-Liacarus (Dorycranosus) pulcher (Sergienko y Djaparidze, 1981) (D.) DisTRIBUCIÓN: Ucrania.

-Liacarus (Dorycranosus) splendens (Coggi, 1898) (Cepheus)

(=Liacarus alatus Berlese, 1904)

(=Liacarus alatus curtipilis Willmann, 1935)

(=Liacarus diversus Mihelcic, 1956)

(=Liacarus ghilarovi Bashkirova, 1958)

(=Liacarus moraviacus Willmann, 1954)

(=Dorycranosus ovatus Djaparidze, 1973)

(=Liacarus punctulatus Mihelcic, 1956)

DisTRIBUCIÓN: Paleártica centromeridional (frecuente).

-Liacarus (Dorycranosus) tenuilamellatus Hirauchi, 1998 (L.) DisTRIBUCIÓN: Japón.

-Liacarus (Dorycranosus) triapicini (Djaparidze, 1983) (D.) DisTRIBUCIÓN: Cáucaso.

-Liacarus (Dorycranosus) yezoensis Fujikawa y Aoki, 1970 (L.) DistRiBUCIÓN: Este de Paleártica oriental (Japón e I. Kuriles).
-Liacarus (Dorycranosus) zachvatkini Kulijev, 1962 (L.)

(=Dorycranosus ibericus Djaparidze, 1973)

DisTRIBUCIÓN: Sureste de Europa.

Liacarus (Procorynetes) Woolley, 1969 (7 spp.)

EsPECIE TIPO: Liacarus nigerrimus Berlese, 1916

DisTRIBUCIÓN: Semicosmopolita (Holártica, Etiópica y Neotropical).

-Liacarus (Procorynetes) andinus (P. Balogh, 1984) (P.)

Distribución: Colombia.

-Liacarus (Procorynetes) breviclavatus Aoki, 1970 (L.)

Distribución: Este de Paleártica oriental.

-Liacarus (Procorynetes) clavatus Fujikawa y Aoki, 1970 (L.)

DistRIBUCión: Este de Paleártica oriental.

-Liacarus (Procorynetes) espeletiae (P. Balogh, 1984) (P.)

DisTRIBUCIÓn: Colombia.

-Liacarus (Procorynetes) globifer (Kramer, 1897) (Leiosoma)

DistRIBUCIÓn: Boreal (Groenlandia y Finlandia).

-Liacarus (Procorynetes) nigerrimus Berlese, 1916 (L.)

DISTRIBUCIÓN: “África oriental”.

-Liacarus (Procorynetes) tsendsureni (Bayartogtokh, 1998) (P.)

DistriBUCIÓN: Mongolia.

Liacarus (Rhaphidosus) Woolley, 1969 (5 spp.)

ESPECIE TIPO: Liacarus carolinensis Banks, 1906

DisTRIBUCIÓN: Holártica y Neotropical.

-Liacarus (Rhaphidosus) acuminatus (Woolley, 1969) (R.)

DisTRIBUCIÓN: Holártica (U.S.A.: Colorado, y Norte de Rusia europea).

-Liacarus (Rhaphidosus) alticola (P. Balogh, 1984) (R.)

DisTRIBUCIÓN: Colombia.

-Liacarus (Rhaphidosus) bacillatus Fujikawa y Aoki, 1970 (L.) DISTRIBUCIÓN: Japón.

-Liacarus (Rhaphidosus) carbonarius (Banks, 1906) (Notaspis) Distribución: U.S.A. (California).

-Liacarus (Rhaphidosus) carolinensis Banks, 1906 (L.)

DisTRIBUCIÓN: Este de U.S.A.

Opsioristes Woolley, 1967 (1 sp.)

ESPECIE TIPO: Opsioristes eurhostus Woolley, 1967

DisTRIBUCIÓN: Neártica.

-Opsioristes eurhostus Woolley, 1967

DISTRIBUCIÓN: U.S.A. centrooccidental.

Planoristes Iturrondobeitia y Subías, 1978 (1 sp.)

EsPeCIE TIPO: Planoristes acuspidatus Iturrondobeitia y Subías, 1978 DisTRIBUCIÓN: Paleártica.

-Planoristes acuspidatus Iturrondobeitia y Subías, 1978 DisTRIBUCIÓN: España.

Xenillidae Woolley y Higgins, 1966 (2 gen., 1 subg., 75 spp. y 2 sspp.) DISTRIBUCIÓN: Holártica y Neotropical.

Xenilloides Pérez-Íñigo y Baggio, 1989 (1 sp.)

EsPecie TiPo: Xenilloides aenigmaticus Pérez-Íñigo y Baggio, 1989

DISTRIBUCIÓN: Neotropical.

-Xenilloides aenigmaticus Pérez-Íñigo y Baggio, 1989

DisTRIBUCIÓN: Brasil.

Xenillus Robineau-Desvoidy, 1839 (74 spp. y 2 sspp.)

EsPeCIE TIPO: Xenillus clypeator Robineau-Desvoidy, 1839

DiSTRIBUCIÓN: Holártica y Neotropical.

Xenillus (Xenillus) Robineau-Desvoidy, 1839 (70 spp. y 2 sspp.)

(=Banksia Oudemans y Voigts, 1905)

(=Dinoxenillus Pérez-Íñigo y Baggio, 1980)

(=Kochia Oudemans, 1900)

(=Pseudocepheus Jacot, 1928)

DisTRIBUCIÓN: Holártica y Neotropical.

-Xenillus (X.) adelae Pérez-Íñigo y Peña, 1996

DisTRIBUCIÓN: I. Canarias. 
-Xenillus (X.) amazonensis Pérez-Iñigo y Baggio, 1997 DistribuCión: Brasil.

-Xenillus (X.) amazonicus J. y P. Balogh, 1985 DISTRIBUCIÓN: Brasil.

-Xenillus (X.) anasillus Woolley y Higgins, 1966 DistRIBUCIÓN: Líbano.

-Xenillus (X.) argentinensis J. y P. Balogh, 1985 DISTRIBUCIÓN: Argentina.

-Xenillus (X.) arilloi Gil-Martín y Subías, 1997 DisTRIBUCIÓN: España.

-Xenillus (X.) baggioi P. Balogh, 1995 DisTRIBUCIÓN: Brasil.

-Xenillus (X.) bolivianus J. y P. Balogh, 1985 Distribución: Bolivia.

-Xenillus (X.) brasilianus Balogh y Mahunka, 1969 DistriBución: Neotropical (Brasil y Venezuela).

-Xenillus (X.) butantaniensis Pérez-Íñigo y Baggio, 1980 DistriBuCión: Brasil.

-Xenillus (X.) capitatus Balogh y Mahunka, 1977 DisTRIBUCIÓN: Neotropical.

-Xenillus (X.) clavatopilus Mihelcic, 1966 DistribuCión: Mediterránea occidental.

-Xenillus (X.) clypeator Robineau-Desvoidy, 1839 (=Cepheus latus Nicolet, 1855)

(=Cepheus permixtus André, 1925)

DisTRIBUCIÓN: Holártica (Paleártica occidental: frecuente, Paleártica oriental: excepto Norte, y U.S.A.: Michigan).

-Xenillus (X.) columbianus P. Balogh, 1984

Distribución: Colombia.

-Xenillus (X.) confusus Mahunka, 1979 DisTRIBUCIÓN: Grecia.

-Xenillus (X.) davisorum J. y P. Balogh, 1985 DistribuCión: Brasil.

-Xenillus (X.) deformatus P. Balogh, 1986 Distribución: Perú.

-Xenillus (X.) discrepans Grandjean, 1936 (X. permixtus d.) DistRIBUCión: Paleártica meridional (Mediterránea y Asia centrooccidental).

-Xenillus (X.) discrepans discrepans Grandjean, 1936

(=Xenillus baderi Mahunka, 1996)

(=Xenillus sculptrus Kulijev, 1963)

DisTRIBUCIÓN: Paleártica meridional (Mediterránea y Asia centrooccidental).

-Xenillus (X.) discrepans azorensis Pérez-Ínigo, 1987 DisTRIBUCión: I. Azores.

-Xenillus (X.) discrepans canariensis Pérez-Íñigo, 1976 DisTRIBUCIÓN: I. Canarias.

-Xenillus (X.) disjunctus Balogh y Mahunka, 1977 Distribución: Chile.

-Xenillus (X.) diversisetosus P. Balogh, 1986 DistribuCión: Chile.

-Xenillus (X.) ecuadorensis J. y P. Balogh, 1985 DISTRIBUCIÓN: Ecuador.

-Xenillus (X.) erbanensis Pérez-Íñigo y Peña, 1996 Distribución: I. Canarias.

-Xenillus (X.) fazendae J. y P. Balogh, 1985 Distribución: Neotropical (Brasil y Venezuela)

-Xenillus (X.) fecundus P. Balogh, 1986 DisTRIBUCIÓN: Brasil.

-Xenillus (X.) fernandoi Morell, 1989 DISTRIBUCIÓN: Mediterránea occidental.

-Xenillus (X.) forceps J. y P. Balogh, 1985 DisTRIBUCIÓN: Brasil.

-Xenillus (X.) fusifer Balogh y Mahunka, 1977 DisTriBuCIÓN: Brasil.

-Xenillus (X.) gelasinus Woolley y Higgins, 1966 Distribución: U.S.A. (Utah).

-Xenillus (X.) hammerae J. y P. Balogh, 1985 DISTRIBUCIÓN: Brasil.
-Xenillus (X.) heterosetiger Aoki, 1967

DistribuCión: Este de Paleártica oriental.

-Xenillus (X.) heterotrichus J. y P. Balogh, 1985

DisTRIBUCIÓN: Brasil.

-Xenillus (X.) ibericus Djaparidze, 1974

Distribución: Cáucaso.

-Xenillus (X.) imitator Pérez-Íñigo y Baggio, 1993

DistriBuCión: Brasil.

-Xenillus (X.) ionthadosus Woolley y Higgins, 1966

DistribuCión: U.S.A. meridional.

-Xenillus (X.) irregularis P. Balogh, 1986

DistRIBUCIÓN: Ecuador.

-Xenillus (X.) lamellatus Rjabinin, 1975

Distribución: Este de Rusia asiática.

-Xenillus (X.) latilamellatus Willmann, 1939

DisTRIBUCIÓN: Macaronésica (Madeira).

-Xenillus (X.) lawrencei Balogh y Mahunka, 1968

Distribución: Argentina.

-Xenillus (X.) lebanonensis Woolley, 1970

DisTRIBUCIÓN: Líbano.

-Xenillus (X.) longipes Mahunka, 1984

DistribuCIÓn: Paraguay.

-Xenillus (X.) longipilus Pérez-Íñigo y Peña, 1996 DistribuCión: I. Canarias.

-Xenillus (X.) longisetosus Balogh y Mahunka, 1969 DISTRIBUCIÓN: Bolivia.

-Xenillus (X.) matskasii Mahunka, 1996

DistribuCión: Hungría.

-Xenillus (X.) moonsani Choi y Aoki, 1993

DisTRIBUCión: Corea.

-Xenillus (X.) moyae Pérez-Íñigo y Peña, 1994

Distribución: I. Canarias.

-Xenillus (X.) multisetosus Choi, 1996

DisTRIBUCión: Corea.

-Xenillus (X.) mutabilis Mahunka y Mahunka-Papp, 1999 DisTRIBUCIÓN: Hungría.

-Xenillus $(X$.) neonominatus nom. nov.

[=Xenillus punctulatus J. y P. Balogh, 1985, “nom. praeoc.” por Banks, 1895 ("hom. sec.")]

DistriBuCión: Bolivia.

-Xenillus (X.) niger (Ewing, 1909) (Liacarus)

DisTRIBUCIÓN: U.S.A. (Ilinois).

-Xenillus (X.) occultus Banks, 1947

(=Xenillus phryxothrixus Woolley y Higgins, 1966)

DistRIBUCión: Sureste de U.S.A.

-Xenillus (X.) ornatus (Covarrubias, 1967) (Liacarus)

Distribución: Chile.

-Xenillus (X.) penicilliger Csiszár, 1962

DistribuCIÓN: Sureste de Europa.

-Xenillus (X.) peruensis J. y P. Balogh, 1985

Distribución: Perú.

-Xenillus (X.) pulvillus Pérez-Íñigo y Peña, 1996

DistriBUCIÓN: I. Canarias.

-Xenillus (X.) punctulatus (Banks, 1895) (Cepheus)

DisTRIBUCIÓN: U.S.A. (Nueva York)

-Xenillus (X.) rohri J. y P. Balogh, 1985

DisTRIBUCIÓN: Neotropical (Brasil y Venezuela).

-Xenillus (X.) salamoni Mahunka, 1996

DisTRIBUCIÓN: Hungría.

-Xenillus (X.) sanctipauli Pérez-Íñigo y Baggio, 1980

DistribuCión: Brasil.

-Xenillus (X.) selgae Morell, 1987

DisTRIBUCIÓN: Mediterránea occidental.

-Xenillus (X.) setiger J. y P. Balogh, 1985

DistribuCión: Perú.

-Xenillus (X.) singularis Golosova y Ljashchev, 1984

DistribuCión: I. Sajalín.

-Xenillus (X.) stepensis Djaparidze, 1974

DISTRIBUCION: Caucaso. 
-Xenillus (X.) subnudus J. y P. Balogh, 1985

DistribuCIÓN: Brasil.

-Xenillus (X.) superbus (Pérez-Íñigo y Baggio, 1980) (Dinoxenillus) DisTRIBUCIÓN: Brasil.

-Xenillus (X.) tegeocranus (Hermann, 1804) (Notaspis) (=Cepheus bifidatus Nicolet, 1855)

(=Cepheus vulgaris Nicolet, 1855)

DisTRIBUCIÓN: Paleártica (frecuente).

-Xenillus (X.) tuberculatus Subías y Arillo, 2000

DisTRIBUCión: España.

-Xenillus (X.) variabilis Balogh y Mahunka, 1981

DisTRIBUCIÓN: Paraguay.

-Xenillus (X.) variolosus (Berlese, 1888) (Cepheus) DistribuCIÓN: Brasil.

-Xenillus (X.) venezuelanus J. y P. Balogh, 1985 DistriBución: Venezuela.

-Xenillus (X.) ybarrai Morell, 1987

DisTRIBUCIÓN: España.

Xenillus (Stonyxenillus) Woolley y Higgins, 1966 (4 spp.)

EsPeCIE TIPO: Stonyxenillus spilotus Woolley y Higgins, 1966

DisTRIBUCIÓN: Holártica

-Xenillus (Stonyxenillus) akidosus (Woolley y Higgins, 1966) (S.) Distribución: U.S.A. (Virginia).

-Xenillus (Stonyxenillus) anakolosus (Woolley y Higgins, 1966) (S.) DisTRIBUCIÓn: Sureste de U.S.A.

-Xenillus (Stonyxenillus) gephyrus (Woolley, 1970) (S.) DISTRIBUCIÓN: Líbano.

-Xenillus (Stonyxenillus) spilotus (Woolley y Higgins, 1966) (S.) Distribución: U.S.A. (Carolina del Norte).

Tenuialidae Jacot, 1929 (6 gen. y 15 spp.) DisTRIBUCIÓN: Holártica.

Ceratotenuiala Aoki y Maruyama, 1983 (1 sp.)

EsPeCIE TIPO: Ceratotenuiala echigoensis Aoki y Maruyama, 1983 DisTRIBUCIÓN: Paleártica.

-Ceratotenuiala echigoensis Aoki y Maruyama, 1983 DisTRIBUCIÓN: Japón.

Hafenferrefia Jacot, 1939 (5 spp.)

$(=$ Hafenrefferiella Sellnick, 1952)

EsPeCIE TIPO: Galumna nitidula Banks, 1906

DisTRIBUCIÓN: Holártica.

-Hafenferrefia hyrcanica (Krivolutsky, 1967) (Hafenrefferiella) DisTRIBUCIÓN: Europa meridional.

-Hafenferrefia imperialis (Berlese, 1910) (Achipteria) "sp. inq." DISTRIBUCIÓN: Italia (Sicilia).

-Hafenferrefia lata Golosova, 1980

DisTRIBUCIÓN: Este de Rusia asiática.

-Hafenferrefia nevesi (Sellnick, 1952) (Hafenrefferiella) DISTRIBUCIÓN: Ibérica.

-Hafenferrefia nitidula (Banks, 1906) (Galumna)

DisTRIBUCIÓN: Neártica septentrional.

Hafenrefferia Oudemans, 1906 (2 spp.)

EsPECIE TIPO: Oribata gilvipes Koch, 1839

DisTRIBUCIÓN: Paleártica.

-Hafenrefferia acuta Aoki, 1966

DisTRIBUCión: Este de Paleártica oriental (frecuente).

-Hafenrefferia gilvipes (Koch, 1839) (Oribata)

(=Liacarus auritus Nordenskiöld, 1901)

(=Liacarus pterotus Coggi, 1900)

DisTRIBUCión: Paleártica (Paleártica occidental: menos frecuente en el Sur, y Paleártica oriental: excepto Norte).

Peltenuiala Norton, 1983 (2 spp.)

EsPECIE TIPO: Peltenuiala pacifica Norton, 1983

DisTRIBUCIÓN: Holártica.
-Peltenuiala orbiculata (Aoki y Ohnishi, 1974) (Hafenferrefia)

Distribución: Este de Paleártica oriental.

-Peltenuiala pacifica Norton, 1983

DisTRIBUCIÓN: Noroeste de U.S.A.

Tenuiala Ewing, 1913 (3 spp.)

EsPeCIE TIPO: Tenuiala nuda Ewing, 1913

DisTRIBUCIÓN: Holártica.

-Tenuiala crenulata Woolley y Higgins, 1966

DisTRIBUCIÓN: Líbano.

-Tenuiala kurti Woolley y Higgins, 1955

DISTRIBUCIÓN: U.S.A. centrooccidental.

-Tenuiala nuda Ewing, 1913

DISTRIBUCIÓN: U.S.A. centrooccidental.

Tenuialoides Woolley y Higgins, 1966 (2 spp.)

EsPECIE TIPO: Tenuialoides medialis Woolley y Higgins, 1966

DISTRIBUCIÓN: Holártica.

-Tenuialoides fusiformis Aoki, 1969

(=Hafenferrefia translamellata Aoki y Fujikawa, 1969)

DistRIBUCIÓN: Este de Paleártica oriental

-Tenuialoides medialis Woolley y Higgins, 1966

DisTRIBUCión: U.S.A. (Carolina del Norte) y Japón.

Eremaeoidea Oudemans, 1900

Kodiakellidae Hammer, 1967 (1 gen. y 2 spp.)

DisTRIBUCIÓN: Holártica.

Kodiakella Hammer, 1967 (2 spp.)

EsPECIE TIPO: Kodiakella lutea Hammer, 1967

DisTRIBUCIÓN: Holártica.

-Kodiakella dimorpha Pérez-Íñigo y Subías, 1978

DisTRIBUCIÓN: Mediterránea occidental.

-Kodiakella lutea Hammer, 1967

DisTRIBUCión: Alaska.

Megeremaeidae Higgins y Woolley, 1968 (1 gen. y 8 spp.) DISTRIBUCIÓN: Holártica.

Megeremaeus Higgins y Woolley, 1965 (8 spp.)

EsPECIE TIPO: Megeremaeus montanus Higgins y Woolley, 1965 DISTRIBUCIÓN: Holártica.

-Megeremaeus ditrichosus Woolley y Higgins, 1968

DisTRIBUCIÓN: U.S.A. (Oregón).

-Megeremaeus expansus Aoki y Fujikawa, 1971

Distribución: Este de Paleártica oriental.

-Megeremaeus hylaius Behan-Pelletier, 1990

DisTRIBUCIÓN: Neártica septentrional.

-Megeremaeus keewatin Behan-Pelletier, 1990

DisTRIBUCIÓN: Neártica septentrional.

-Megeremaeus kootenai Behan-Pelletier, 1990

DisTRIBUCIÓN: Canadá.

-Megeremaeus montanus Higgins y Woolley, 1965

DisTRIBUCIÓN: Neártica (Noroeste de U.S.A. y Canadá)

-Megeremaeus rameus Wen y Zhao, 1993

DisTRIBUCIÓN: China suroriental.

-Megeremaeus spinosus Aoki y Yamamoto, 2000

DisTRIBUCIÓN: China suroriental.

Eremaeidae Oudemans, 1900 (5 gen. y 66 spp.) DisTRIBUCIÓN: Holártica.

Asperemaeus Behan-Pelletier, 1982 (1 sp.)

EsPECIE TIPO: Asperemaeus longipilus Behan-Pelletier, 1982 DisTRIBUCIÓN: Paleártica.

-Asperemaeus longipilus Behan-Pelletier, 1982

DistRIBUCión: Este de Rusia asiática. 
Carinabella Hammer, 1977 (2 spp.)

EsPECIE TIPO: Carinabella pulchra Hammer, 1977

DISTRIBUCIÓN: Paleártica.

-Carinabella pulchra Hammer, 1977

DisTRIBUCIÓN: Pakistán.

-Carinabella tuberculata Bayoumi y Mahunka, 1979

DisTRIBUCIÓN: Norte de India.

Eremaeus Koch, 1835 (26 spp,)

EsPeCIE TIPO: Eremaeus hepaticus Koch, 1835

DisTRIBUCIÓN: Holártica.

-Eremaeus appalachicus Behan-Pelletier, 1993

Distribución: Este de U.S.A.

-Eremaeus boreomontanus Behan-Pelletier, 1993

DisTRIBUCIÓN: Canadá

-Eremaeus brevitarsus (Ewing, 1917) (Damaeus)

DisTRIBUCIÓN: Neártica.

-Eremaeus californiensis Behan-Pelletier, 1993

DistRibuCión: U.S.A. (California).

-Eremaeus gracilis Behan-Pelletier, 1993

DisTRIBUCIÓN: U.S.A. (Oregón).

-Eremaeus grandis Hammer, 1952

Distribución: Boreal (Neártica septentrional y Este de Rusia asiática).

-Eremaeus hepaticus Koch, 1835

(=Eremaeus cordiformis Grandjean, 1934)

(=Notaspis dentilamellatus Storkán, 1925)

(=Eremaeus hepaticus acruciata Mihelcic, 1952)

(=Eremaeus magnus Mihelcic, 1957)

(=Eremaeus ovalis Mihelcic, 1955)

(=Eremaeus setiger Mihelcic, 1957)

DistriBuCión: Holártica (Paleártica: frecuente, y U.S.A.: Virginia).

-Eremaeus insertus Grishina, 1980

DisTRIBUCIÓN: Asia centrooccidental.

-Eremaeus kananaskis Behan-Pelletier, 1993

DisTRIBUCIÓN: Canadá.

-Eremaeus kevani Behan-Pelletier, 1993

DisTRIBUCIÓN: Neártica septentrional.

-Eremaeus longiseta Djaparidze, 1990

DisTRIBUCIÓN: Cáucaso.

-Eremaeus megistos Behan-Pelletier, 1993

DisTRIBUCIÓN: Oeste de U.S.A.

-Eremaeus monticolus Behan-Pelletier, 1993

Distribución: Suroeste de U.S.A.

-Eremaeus neonominatus nom. nov.

[=Eremaeus borealis Wen, 1988, "nom. praeoc." por Strenzke, 1952 ("hom. prim.")]

DisTRIBUCIÓN: China.

-Eremaeus nortoni Behan-Pelletier, 1993

DisTRIBUCIÓN: Noreste de U.S.A.

-Eremaeus occidentalis Behan-Pelletier, 1993

DisTRIBUCIÓN: Neártica septentrional.

-Eremaeus oresbios Behan-Pelletier, 1993

DisTRIBUCIÓN: Suroeste de U.S.A.

-Eremaeus orientalis Golosova, 1970

DisTRIBUCIÓN: Este de Paleártica oriental.

-Eremaeus plumosus Woolley, 1964

Distribución: Neártica (Oeste de U.S.A. y Canadá)

-Eremaeus porosus Behan-Pelletier, 1993

DisTRIBUCión: U.S.A. (Colorado)

-Eremaeus roissi Piffl, 1966

DisTRIBUCIÓN: Pakistán.

-Eremaeus salish Behan-Pelletier, 1993

DisTRIBUCIÓN: Canadá.

-Eremaeus tenuisetiger Aoki, 1970

Distribución: Paleártica (Cáucaso y Este de Paleártica oriental)

-Eremaeus translamellatus Hammer, 1952

(=Eremaeus oblongus borealis Strenzke, 1952)

DisTRIBUCIÓN: Holártica.
-Eremaeus tuberosus Gordeeva, 1970

DisTRIBUCIÓN: Europa oriental.

-Eremaeus walteri Behan-Pelletier, 1993

DisTRIBUCIÓN: U.S.A. centrooccidental.

Eueremaeus Mihelcic, 1963 (32 spp.)

(=Kartoeremaeus Higgins, 1979)

ESPECIE TIPO: Eremaeus oblongus Koch, 1835

DISTRIBUCIÓN: Holártica.

-Eueremaeus acostulatus Behan-Pelletier, 1993

DisTRIBUCIÓN: Neártica septentrional.

-Eueremaeus alvordensis Behan-Pelletier, 1993

Distribución: Oeste de U.S.A.

-Eueremaeus aridulus Behan-Pelletier, 1993

DISTRIBUCIÓN: Canadá.

-Eueremaeus aysineep Behan-Pelletier, 1993

DisTRIBUCIÓN: Neártica (U.S.A: Colorado, y Canadá).

-Eueremaeus brevifer (Mahunka, 1980) (Eremaeus)

DisTRIBUCIÓN: Mediterránea occidental.

-Eueremaeus carinatus Behan-Pelletier, 1993

DisTRIBUCIÓN: U.S.A. (Nevada).

-Eueremaeus chiatous (Higgins, 1979) (Kartoeremaeus)

Distribución: Neártica (Oeste de U.S.A. y Canadá).

-Eueremaeus columbianus (Berlese, 1916) (Eremaeus)

(=Eremaeus politus Banks, 1947)

(=Kartoeremaeus reevesi Higgins, 1979)

DistRIBUCIÓN: Neártica.

-Eueremaeus cornutus (Lombardini, 1943) (Eremaeus) "sp. inq."

DistriBución: Italia.

-Eueremaeus danos Behan-Pelletier, 1993

DisTRIBUCIÓN: Oeste de U.S.A.

-Eueremaeus elongatus (Fujikawa, 1972) (Eremaeus)

DISTRIBUCIÓN: Japón.

-Eueremaeus foveolatus (Hammer, 1952) (Eremaeus)

DisTRIBUCIÓN: Holártica.

-Eueremaeus higginsi Behan-Pelletier, 1993

DisTRIBUCión: U.S.A. (California).

-Eueremaeus hokkaiensis Fujikawa, 1991

DisTRIBUCIÓN: Japón.

-Eueremaeus lindquisti Behan-Pelletier, 1993

Distribución: Oeste de U.S.A.

-Eueremaeus magniporus (Wallwork, 1972) (Eremaeus)

DisTRIBUCIÓN: U.S.A. (California).

-Eueremaeus marshalli Behan-Pelletier, 1993

DisTRIBUCIÓN: Neártica.

-Eueremaeus masinasin Behan-Pelletier, 1993

DistRIBUCIÓN: Neártica (U.S.A.: Colorado, y Canadá).

-Eurermaeus michaeli Behan-Pelletier, 1993

DistribuCión: Canadá.

-Eueremaeus nahani Behan-Pelletier, 1993

DisTRIBUCIÓN: Neártica (U.S.A.: Colorado, y Canadá).

-Eueremaeus nemoralis Behan-Pelletier, 1993

DisTRIBUCIÓN: Noreste de U.S.A.

-Eueremaeus oblongus (Koch, 1835) (Eremaeus)

(=Eremaeus areolatus Kunst, 1959)

(=Eremaeus figuratus Winkler, 1956)

(=Eremaeus granulatus Mihelcic, 1955)

(=Eremaeus intermedius Mihelcic, 1955)

(=Eueremaeus kuehnelti Mihelcic, 1963)

(=Eremaeus major Mihelcic, 1953)

(=Eremaeus ornatus Mihelcic, 1957)

(=Eremaeus silvestris Forsslund, 1956)

(=Eremaeus valkanovi Kunst, 1957)

DisTRIBUCIÓN: Holártica (frecuente).

-Eueremaeus osoyoosensis Behan-Pelletier, 1993

Distribución: Neártica (Oeste de U.S.A. y Canadá).

-Eueremaeus proximus (Berlese, 1916) (Eremaeus)

DisTRIBUCIÓN: U.S.A. centrooriental. 
-Eueremaeus quadrilamellatus (Hammer, 1952) (Eremaeus) (=Eremaeus fossulatus Kunst, 1959)

(=Eueremaeus valkanovi debilis Mihelcic, 1963)

DisTRIBUCIÓN: Holártica (Neártica septentrional y Europa centromeridional)

-Eueremaeus stiktos (Higgins, 1962) (Eremaeus)

DisTRIBUCión: Neártica (U.S.A. occidental y Canadá).

-Eueremaeus tetrosus (Higgins, 1979) (Kartoeremaeus)

DisTRIBUCIÓN: Neártica (frecuente en U.S.A.).

-Eueremaeus travei Mihelcic, 1963

(=Eremaeus oblongus major Mihelcic, 1957, "nom. praeoc." por Mihelcic, 1953)

Distribución: Oeste de Europa.

-Eueremaeus triglavensis (Tarman, 1958) (Eremaeus)

DisTRIBUCIÓN: Europa meridional.

-Eueremaeus trionus (Higgins, 1979) (Kartoeremaeus)

Distribución: Neártica (U.S.A.: Colorado, y Canadá).

-Eueremaeus woolleyi (Higgins, 1979) (Kartoeremaeus)

DisTRIBUCIÓN: U.S.A. occidental.

-Eueremaeus yukonensis Behan-Pelletier, 1993

DisTRIBUCIÓN: Canadá.

Tricheremaeus Berlese, 1908 (5 spp.)

ESPECIE TIPO: Notaspis serrata Michael, 1885

DisTRIBUCIÓN: Paleártica.

-Tricheremaeus conspicuus Berlese, 1916

DISTRIBUCIÓN: Europa centromeridional.

-Tricheremaeus grandjeani Bernini, 1970

DisTRIBUCIÓN: Italia.

-Tricheremaeus nemossensis Grandjean, 1963

DisTRIBUCIÓN: Europa meridional.

-Tricheremaeus serratus (Michael, 1885) (Notaspis)

DisTRIBUCIÓN: Europa occidental.

-Tricheremaeus travei Miko, 1993

DisTRIBUCIÓN: Eslovaquia.

Aribatidae Aoki, Takaku e Ito, 1994 (1 gen. y 1 sp.) DisTRIBUCIÓN: Oriental.

Aribates Aoki, Takaku e Ito, 1994 (1 sp.)

EsPECIE TIPO: Aribates javensis Aoki, Takaku e Ito, 1994

DISTRIBUCIÓN: Oriental.

-Aribates javensis Aoki, Takaku e Ito, 1994

DisTRIBUCIÓN: Java.

\section{Amerobelboidea Grandjean, 1954}

Ctenobelbidae Grandjean, 1965 (1 gen. y 19 spp.) DisTRIBUCIÓN: Paleártica.

Ctenobelba Balogh, 1943 (19 spp.)

(=Elapheremaeus Grandjean, 1944)

ESPECIE TIPO: Eremobelba pectinigera Berlese, 1908

DisTRIBUCIÓN: Paleártica.

-Ctenobelba apatomorpha Iturrondobeitia, Saloña, Andrés y Caballero, 1998

DisTRIBUCIÓN: España.

-Ctenobelba brevipilosa Mahunka, 1965

DisTRIBUCIÓn: Hungría.

-Ctenobelba csiszarae Mahunka, 1977

DisTRIBUCIÓN: Hungría.

-Ctenobelba foliata Hammer, 1961

DisTRIBUCIÓN: Mediterránea occidental.

-Ctenobelba grancanariae Pérez-Íñigo y Peña, 1997

DisTRIBUCIÓN: I. Canarias.

-Ctenobelba longisetosa Suzuoka y Aoki, 1980

DISTRIBUCIÓN: Japón.

-Ctenobelba mahnerti Mahunka, 1974

DISTRIBUCIÓN: Europa meridional.
-Ctenobelba marcuzzii Mahunka, 1974

DisTRIBUCIÓN: Italia.

-Ctenobelba obsoleta (Koch, 1841) (Murcia) "sp. inq."

DisTRIBUCIÓN: Alemania.

-Ctenobelba parafoliata Pérez-Íñigo jr., 1991

DisTRIBUCIÓN: España (I. Baleares).

-Ctenobelba pectinigera (Berlese, 1908) (Eremobelba)

(=Amerobelba bicarinata Berlese, 1910)

(=Ctenobelba fenestrata Pérez-Íñigo jr., 1990)

(?=Notaspis maculosa Warburton y Pearce, 1905 "sp. inq.")

DISTRIBUCIÓN: Europa centromeridional (frecuente).

-Ctenobelba perezinigoi Moraza, 1985

DISTRIBUCIÓN: Mediterránea occidental.

-Ctenobelba pilosella Jeleva, 1962

(=Ctenobelba tuberculata Kulijev, 1966)

DistRIBUCIÓN: Europa meridional.

-Ctenobelba polysetosa Aoki y Yamamoto, 2000

DisTRIBUCIÓN: Japón.

-Ctenobelba pulchellula Gil-Martín y Subías, 1997

DisTRIBUCIÓN: España.

-Ctenobelba serrata Mahunka, 1964

DisTRIBUCIÓN: Europa meridional.

-Ctenobelba simplex (Willmann, 1940) (Eremobelba)

Distribución: "Yugoslavia".

-Ctenobelba soloduchi Pankov, 1988

DisTRIBUCIÓN: Este de Rusia asiática.

-Ctenobelba translamellata Iorkansky, 1990

DisTRIBUCIÓn: Sureste de Europa.

Amerobelbidae Grandjean, 1954 (6 gen. y 13 spp.) DisTRIBUCIÓN: Holártica.

Amerobelba Berlese, 1908 (1 sp.)

EsPecie Tipo: Amerobelba decedens Berlese, 1908

DisTRIBUCIÓN: Paleártica.

-Amerobelba decedens Berlese, 1908

(=Eremobelba maxima Willmann, 1951)

DisTRIBUCIÓN: Mediterránea.

Berndamerus Mahunka, 1977 (3 spp.)

EsPECIE TIPO: Berndamerus hellenicus Mahunka, 1977

DisTRIBUCIÓN: Paleártica.

-Berndamerus bicostatus (Berlese, 1910) (Amerobelba)

DisTRIBUCIÓN: Europa meridional.

-Berndamerus eremuloides (Berlese, 1910) (Amerobelba)

DistRIBUCIÓN: Europa meridional.

-Berndamerus hellenicus Mahunka, 1977

DISTRIBUCIÓN: Grecia.

Hellenamerus Mahunka, 1974 (1 sp.)

ESPECIE TIPO: Hellenamerus ionicus Mahunka, 1974

DisTRIBUCIÓN: Paleártica.

-Hellenamerus ionicus Mahunka, 1974

DistRIBUCión: Mediterránea.

Mongaillardia Grandjean, 1961 (5 spp.)

EsPeCIE TIPO: Mongaillardia callitoca Grandjean, 1961

DisTRIBUCIÓN: Paleártica y Etiópica.

-Mongaillardia aeoliana (Bernini, 1979) (Amerobelba)

DisTRIBUCIÓN: Italia (Sicilia).

-Mongaillardia callitoca Grandjean, 1961

DistriBución: Europa (Francia y Hungría).

-Mongaillardia eveana Grandjean, 1961

DISTRIBUCIÓN: Europa occidental.

-Mongaillardia grandjeani Calugar y Vasiliu, 1984

DISTRIBUCIÓN: Rumania.

-Mongaillardia magna Wallwork, 1977

DistribuCIÓN: I. Santa Helena. 
Rastellobata Grandjean, 1961 (1 sp.)

ESPECIE TIPO: Amerobelba rastelligera Berlese, 1908

DISTRIBUCIÓN: Paleártica.

-Rastellobata rastelligera (Berlese, 1908) (Amerobelba) DisTRIBUCIÓN: Mediterránea.

Yambaramerus Aoki, 1996 (2 spp.)

EsPeCIE TIPO: Yambaramerus itoi Aoki, 1996

DISTRIBUCIÓN: Paleártica.

-Yambaramerus arcuatus Aoki y Yamamoto, 2000

DisTRIBUCIÓN: China suroriental.

-Yambaramerus itoi Aoki, 1996

DisTRIBUCIÓN: Japón.

Eremulidae Grandjean, 1965 (7 gen., 38 spp. y 1 ssp.) DistRIBUCIÓN: Cosmopolita.

Austroeremulus Mahunka, 1985 (1 sp.)

ESPECIE TIPO: Austroeremulus glabrus Mahunka, 1985

DISTRIBUCIÓN: Etiópica.

-Austroeremulus glabrus Mahunka, 1985

DisTRIBUCIÓN: Sudáfrica.

Caveremulus Mahunka, 1983 (2 spp.)

(=Berndbella Mahunka, 1985)

ESPECIE TIPO: Caveremulus cordisetus Mahunka, 1983

DisTRIBUCIÓN: Paleotropical.

-Caveremulus cordisetus Mahunka, 1983

DISTRIBUCIÓN: Madagascar.

-Caveremulus serratus (Mahunka, 1985) (Berndbella)

DISTRIBUCIÓN: India (Kerala).

Eremulus Berlese, 1908 (30 spp.)

ESPECIE TIPO: Eremulus flagellifer Berlese, 1908

DisTRIBUCIÓN: Cosmopolita.

-Eremulus adami Balogh y Mahunka, 1966

DistriBución: Congo.

-Eremulus africanus Balogh, 1958 "sp. inq."

DisTRIBUCIÓN: África centrooriental.

-Eremulus antis Poltavskaja, 1987

DisTRIBUCión: Sureste de Europa.

-Eremulus australis Wen, 1988

DistriBuCión: China suroriental.

-Eremulus avenifer Berlese, 1913

DisTRIBUCión: Paleártica meridional (Italia y Paleártica oriental: excepto Norte), Oriental (frecuente) y Polinesia (Tahití).

-Eremulus baliensis Hammer, 1982

DisTRIBUCIÓN: Bali.

-Eremulus berlesei Mahunka, 1977

Distribución: Indonesia (Komodo)

-Eremulus brasiliensis Pére-Íñigo y Baggio, 1985

DisTRIBUCIÓN: Brasil.

-Eremulus cingulatus Jacot, 1937

DistriBución: U.S.A. (Carolina del Norte).

-Eremulus clavatoflagellatus Lombardini, 1963 "sp. inq."

DisTRIBUCIÓN: Italia.

-Eremulus crispus Hammer, 1958

DisTRIBUCIÓN: Neotropical.

-Eremulus curviseta Hammer, 1971

Distribución: Australiana (Islas del Pacífico)

-Eremulus densus Hammer, 1979

DisTRIBUCIÓN: Java.

-Eremulus elegans Gordeeva, 1987

DisTRIBUCIÓN: Ucrania (Crimea).

-Eremulus flagellifer Berlese, 1908

(=Eremulus modestus Berlese, 1908)

DisTRIBUCIÓN: Semicosmopolita (Paleártica: menos frecuente en

el Norte, U.S.A.: Washington D.C., India: Bengala Occidental, Australiana y subantártica: I. St. Paul).
-Eremulus foveolaltus Hammer, 1962

DistriBuCión: Chile.

-Eremulus hastatus Hammer, 1961

DistRIBUCIÓN: Perú.

-Eremulus lanceocrinus Balogh, 1958 "sp. inq."

DistriBución: Congo.

-Eremulus monstrosus Hammer, 1972

DistriBución: Polinesia (Tahití).

-Eremulus nigrisetosus Hammer, 1958

DisTRIBUCIÓN: India (Tripura) y Neotropical.

-Eremulus pectinatus Jacot, 1937

Distribución: U.S.A. (Carolina del Norte).

-Eremulus pilipinus Corpuz-Raros, 1979

DISTRIBUCIÓN: Filipinas.

-Eremulus renukae Sanyal, 1992

DisTRIBUCIÓN: India (Bengala Occidental).

-Eremulus rigidisetus Balogh y Mahunka, 1969

DISTRIBUCIÓN: Neotropical.

-Eremulus rimosus Tseng, 1982

DistRIBUCIÓN: Taiwan (Formosa).

-Eremulus serratus Hammer, 1966

DistRIBUCIÓN: Nueva Zelanda.

-Eremulus spinifer Woolley y Higgins, 1963

DisTRIBUCIÓN: U.S.A. (Misuri).

-Eremulus tenuis Hammer, 1979

DistRIBUCIÓN: Java.

-Eremulus translamellatus Balogh y Mahunka, 1969

DisTRIBUCIÓN: Neotropical.

-Eremulus truncatus Hammer, 1971

DisTRIBUCIÓN: Oriental (Filipinas y Java) y Australiana (Islas del Pacífico).

Fenestrella Mahunka, 1987 (2 spp.)

EsPeCIE TIPO: Fenestrella bifurcata Mahunka, 1987

DISTRIBUCIÓN: Oriental.

-Fenestrella bifurcata Mahunka, 1987

DisTRIBUCIÓN: Vietnam.

-Fenestrella sinica Zhao y Wen, 1994

DistriBUCIÓN: China suroriental.

Pseuderemulus Balogh y Mahunka, 1968 (1 sp.)

ESPECIE TIPO: Pseuderemulus gladiator Balogh y Mahunka, 1968 DisTRIBUCIÓN: Neotropical.

-Pseuderemulus gladiator Balogh y Mahunka, 1968

DisTRIBUCIÓN: Argentina.

Reteremuloides Mahunka, 1989 (1 sp.)

EsPeCIE TIPO: Reteremuloides bifurcatus Mahunka, 1989

DISTRIBUCIÓN: Oriental.

-Reteremuloides bifurcatus Mahunka, 1989

DISTRIBUCIÓN: Sumatra.

Reteremulus Balogh y Mahunka, 1966 (1 sp.y 1 ssp.)

EsPECIE TIPO: Reteremulus aciculatus Balogh y Mahunka, 1966

DISTRIBUCIÓN: Etiópica y Australiana.

-Reteremulus aciculatus Balogh y Mahunka, 1966

DistRIBUCión: Sudáfrica y Nueva Guinea.

-Reteremulus aciculatus aciculatus Balogh y Mahunka, 1966 DisTRIBUCIÓN: Sudáfrica.

-Reteremulus aciculatus papuanus Balogh 1968

DiSTRIBUCIÓN: Nueva Guinea.

Damaeolidae Grandjean, 1965 (4 gen. y 11 spp.)

DisTRIBUCIÓN: Cosmopolita (excepto Antártica).

Caudamaeolus P. Balogh, 1988 (1 sp.)

EsPECIE TIPO: Caudamaeolus petalus P. Balogh, 1988

DisTRIBUCIÓN: Ecuador.

-Caudamaeolus petalus P. Balogh, 1988

DisTRIBUCIÓN: Ecuador. 
Damaeolus Paoli, 1908 (6 spp.)

EsPeCIE TiPo: Dameosoma asperatum Berlese, 1904

DisTRIBUCIÓN: Holártica y Etiópica.

-Damaeolus asperatus (Berlese, 1904) (Dameosoma)

DistriBUCión: Holártica (Paleártica: excepto Norte, y U.S.A Columbia).

-Damaeolus bregetovae Csiszár, 1962

DisTRIBUCIÓN: Mediterránea.

-Damaeolus cellulatus Subías, Ruiz y Kahwash, 1990 DisTRIBución: España.

-Damaeolus magnus Mahunka, 1979

DISTRIBUCIÓN: Grecia.

-Damaeolus ocellatus Mahunka, 2000

DISTRIBUCIÓN: Madagascar.

-Damaeolus ornatissimus Csiszár, 1962

Distribución: Paleártica meridional (Sur de Europa y Asia centrooccidental)

Fosseremus Grandjean, 1954 (3 spp.)

EsPeCIE TIPo: Dameosoma laciniatum Berlese, 1905

DisTRIBUCIÓn: Cosmopolita (excepto Antártica).

-Fosseremus americanus (Jacot, 1938) (Damaeolus laciniatus a.) Distribución: U.S.A. (Carolina del Norte).

-Fosseremus laciniatus (Berlese, 1905) (Dameosoma)

(=Fosseremus africanus Balogh, 1958)

(=Damaeolus ornatus Mihelcic, 1956)

(=Damaeolus pistillifer Berlese, 1913)

(=Fosseremus quadripertitus Grandjean, 1965)

(=Damaeolus saltaensis Hammer, 1958)

DisTRIBUCIÓN: Cosmopolita (excepto Antártica): frecuente.

-Fosseremus sculpturatus Mahunka, 1982

DisTRIBUCIÓN: Etiopía.

Gressittolus Balogh, 1970 (1 spp.)

(=Fijirella Hammer, 1971)

EsPECIE TIPO: Gressittolus marginatus Balogh, 1970

DISTRIBUCIÓN: Australianooriental.

-Gressittolus marginatus Balogh, 1970

(=Fijirella mollis Hammer, 1971)

DISTRIBUCIÓN: Australianooriental.

Hungarobelbidae Miko y Travé, 1996 (2 gen. y 7 spp.) DISTRIBUCIÓN: Paleártica.

Costeremus Aoki, 1970 (4 spp,)

ESPECIE TIPO: Costeremus ornatus Aoki, 1970

DisTRIBUCIÓN: Paleártica.

-Costeremus barbatus Choi, 1997

DisTRIBUCión: Corea.

-Costeremus cornutus Wang, 1996

DisTRIBUCIÓN: China suroriental.

-Costeremus ornatus Aoki, 1970

DisTRIBUCión: Este de Paleártica oriental.

-Costeremus yezoensis Fujikawa y Fujita, 1985

DisTRIBUCIÓN: Japón.

Hungarobelba Balogh, 1943 (3 spp.)

ESPECIE TIPO: Belba visnyai Balogh, 1938

DisTRIBUCIÓN: Paleártica.

-Hungarobelba baloghi Bulanova-Zachvatkina, 1967

DisTriBución: Este de Paleártica oriental.

-Hungarobelba pyrenaica Miko y Travé, 1996

DISTRIBUCIÓN: Oeste de Europa.

-Hungarobelba visnyai (Balogh, 1938) (Belba)

DISTRIBUCIÓN: Eruropa centromeridional.

Eremobelbidae Balogh, 1961 (1 gen. y 42 spp.) DisTRIBUCIÓN: Pantropical y subtropical.

Eremobelba Berlese, 1908 (42 spp.)

EsPECIE TIPO: Eremaeus leporosus Haller, 1884

DisTRIBUCIÓN: Pantropical y subtropical.
-Eremobelba balazsi Mahunka, 1983

DisTRIBUCIÓN: Brasil.

-Eremobelba bella Hammer, 1982

DisTRIBUCIÓN: Bali.

-Eremobelba bellicosa Balogh y Mahunka, 1967

DistriBución: Vietnam.

-Eremobelba bisulcata Balogh, 1958 "sp. inq."

DistriBución: Congo.

-Eremobelba breviseta Balogh, 1958

DistriBuCIÓn: Nueva Guinea.

-Eremobelba brevispathulata Balogh y Mahunka, 1969

DISTRIBUCIÓN: Bolivia.

-Eremobelba capitata Berlese, 1913

DisTRIBUCIÓN: Oriental y Nueva Guinea.

-Eremobelba cellulosa Mahunka, 1997

DisTRIBUCIÓN: Madagascar.

-Eremobelba comteae Mahunka, 1988

DisTRIBUCIÓN: Borneo.

-Eremobelba coronata Pérez-Íñigo y Baggio, 1989

DISTRIBUCIÓN: Brasil.

-Eremobelba curtipetata Wen, 1996

DISTRIBUCIÓN: China suroriental.

-Eremobelba esposi Balogh y Mahunka, 1969

DISTRIBUCIÓN: Bolivia.

-Eremobelba flexuosa Hammer, 1979

DisTRIBUCIÓN: Java.

-Eremobelba foliata Hammer, 1958

DISTRIBUCIÓN: Neotropical.

-Eremobelba geographica Berlese, 1908

DISTRIBUCIÓN: Europa centromeridional.

-Eremobelba gracilior Berlese, 1908

(=Eremobelba leporosa flagellaris Jacot, 1938)

(=Oribata neosota Banks, 1909)

(=Eremobelba nervosa Hartenstein, 1962)

Distribución: Holártica (Neártica oriental y Cáucaso) y Méjico.

-Eremobelba graciosa Mahunka, 1984

DisTRIBUCIÓN: Tanzania.

-Eremobelba hamata Hammer, 1961

DISTRIBUCIÓN: Neotropical.

-Eremobelba heterotricha Mahunka, 1977

DisTRIBUCIÓN: Oriental (Malaya y Borneo).

-Eremobelba himalayensis Mondal y Kundu, 1984

DistRIBUCión: India (Bengala Occidental).

-Eremobelba indica Ghosh y Bhaduri, 1978

DISTRIBUCIÓN: India (Nagaland).

-Eremobelba japonica Aoki, 1959

DistRIBUCIÓN: Este de Paleártica oriental y Oriental.

-Eremobelba leporoides Jacot, 1938 (E. leporosus l.)

Distribución: U.S.A. (Carolina del Norte).

-Eremobelba leporosa (Haller, 1884) (Eremaeus)

DisTRIBUCIÓN: "Neotropical".

-Eremobelba longisetosa Subías, Ruiz y Kahwash, 1990

DISTRIBUCIÓN: España.

-Eremobelba mahunkai Balogh, 1968

DisTRIBUCIÓn: Nueva Guinea.

-Eremobelba miliae Sanyal, 1992

DisTRIBUCIÓN: India (Bengala Occidental).

-Eremobelba minuta Aoki y Wen, 1983

DisTRIBUCIÓN: Japón

-Eremobelba nagaroorica Haq, 1978

DISTRIBUCIÓN: India (Kerala)

-Eremobelba obscura Balogh, 1958 "sp. inq."

DisTRIBUCIÓn: Congo.

-Eremobelba okinawa Aoki, 1987

DisTRIBUCIÓN: Japón.

-Eremobelba ornata Balogh y Mahunka, 1969

DISTRIBUCIÓN: Bolivia.

-Eremobelba perrugosa Balogh y Mahunka, 1968

DISTRIBUCION: Java. 
-Eremobelba piffli Mahunka, 1985

Distribución: Norte de Neotropical.

-Eremobelba porcella Mahunka, 2001

DISTRIBUCIÓN: Borneo.

-Eremobelba pulchella Balogh y Mahunka, 1969

DisTRIBUCIÓN: Neotropical (Bolivia y Brasil).

-Eremobelba shillongensis Sanyal, 1988

DisTRIBUCión: India (Himalaya).

-Eremobelba truncata Wen, 1996

DisTRIBUCión: China suroriental.

-Eremobelba tuberculata Mahunka, 1982

Distribución: Etiopía.

-Eremobelba wittmeri Bayoumi y Mahunka, 1979

DisTRIBUCIÓN: Norte de India.

-Eremobelba yunnanensis Aoki y Yamamoto, 2000

DisTRIBUCIÓN: China suroriental.

-Eremobelba zicsii Balogh y Mahunka, 1969

DisTRIBUCIÓN: Neotropical.

Heterobelbidae Balogh, 1961 (3 gen., 15 spp. y 1 ssp.) DisTRIBUCIÓN: Pantropical (excepto Australiana).

Furcodamaeus Pérez-Íñigo y Baggio, 1980 (1 sp.)

ESPECIE TIPO: Furcodamaeus bifurcatus Pérez-Íñigo y Baggio, 1980

DISTRIBUCIÓN: Neotropical.

-Furcodamaeus bifurcatus Pérez-Íñigo y Baggio, 1980

DISTRIBUCIÓN: Brasil.

Haplobelba Balogh y Mahunka, 1969 (1 sp.)

EsPECIE TIPO: Haplobelba simplex Balogh y Mahunka, 1969 DISTRIBUCIÓN: Neotropical.

-Haplobelba simplex Balogh y Mahunka, 1969

DisTRIBUCIÓN: Neotropical (Bolivia y Perú)

Heterobelba Berlese, 1913 (13 spp. y 1 ssp.)

EsPeCIE TIPO: Heterobelba galerulata Berlese, 1913

DISTRIBUCIÓN: Pantropical (excepto Australiana).

-Heterobelba africana Balogh, 1958

DISTRIBUCIÓN: Etiópica.

-Heterobelba barbata Beck, 1962

DisTRIBUCIÓN: Perú.

-Heterobelba crassisetosa Beck, 1962

DisTRIBUCión: Perú.

-Heterobelba furcata Mahunka, 1978

Distribución: Antillas (La Española: R. Dominicana)

-Heterobelba galerulata Berlese, 1913

DisTRIBUCIÓN: Oriental.

-Heterobelba magna Beck, 1962

DisTRIBUCIÓN: Perú.

-Heterobelba oxapampensis Beck, 1962

DisTRIBUCIÓN: Neotropical.

-Heterobelba rostrata Mondal y Kundu, 1984

DisTRIBUCIÓN: India (Bengala Occidental).

-Heterobelba spinitecta Mahunka, 1983

DisTRIBUCIÓN: Tanzania.

-Heterobelba spinosissima Balogh y Mahunka, 1981

DisTRIBUCIÓN: Paraguay.

-Heterobelba spumosa Mahunka, 1983

DisTRIBUCIÓN: Tanzania.

-Heterobelba stellifera Okayama, 1980

DisTRIBUCión: Japón y Noreste de Orietal.

-Heterobelba stellifera stellifera Okayama, 1980

DisTRIBUCIÓN: Japón y Noreste de Orietal.

- Heterobelba stellifera formosana Aoki, 1990

DisTRIBUCIÓN: Noreste de Orietal.

-Heterobelba zikani Sellnick, 1922

DISTRIBUCIÓN: Brasil.
Basilobelbidae Balogh, 1961 (2 gen., 19 spp. y 1 ssp.)

DisTRIBUCIÓN: Pantropical.

Basilobelba Balogh, 1958 (14 spp. y 1 ssp.)

(=Hammation Grandjean, 1959)

EsPeCIE TIPO: Damaeus retiarius Warburton, 1912

DISTRIBUCIÓN: Pantropical.

-Basilobelba aethiopica Bernini, 1988

DisTRIBUCIÓN: Etiopía.

-Basilobelba africana Wallwork, 1961

DisTRIBUCIÓN: Ghana.

-Basilobelba atimonensis Corpuz-Raros, 1980

DisTRIBUCIÓN: Filipinas.

-Basilobelba baltazarae Corpuz-Raros, 1979

DISTRIBUCIÓN: Filipinas.

-Basilobelba barbata Mondal y Kundu, 1984

DistriBuCIÓN: India (Bengala Occidental).

-Basilobelba cendanai Corpuz-Raros, 1980

DisTRIBUCIÓN: Filipinas.

- Basilobelba foliata Hammer, 1982

DISTRIBUCIÓN: Bali.

-Basilobelba indica Bhaduri, Chakrabarti y Raychaudhuri, 1974

DistriBución: India (Bengala Occidental).

-Basilobelba insularis Mahunka, 1985

Distribución: Pantropical (Sudáfrica, Micronesia: I. Marianas, y

Centroamérica).

- Basilobelba monstruosetosa Mahunka, 1974

DisTRIBUCIÓN: Camerún.

-Basilobelba pacifica Hammer, 1971

Distribución: Melanesia (I. Fiji).

-Basilobelba parmata Okayama, 1980

DisTRIBUCIÓN: Japón.

-Basilobelba retiaria (Warburton, 1912) (Damaeus)

DistriBUCIón: Pantropical y Japón.

-Basilobelba retiaria retiaria (Warburton, 1912)

(=Hammation sollertius Grandjean, 1959)

DISTRIBUCIÓN: Pantropical y Japón.

-Basilobelba retiaria symmetrica Wallwork, 1962

DisTRIBUCIÓN: Ghana.

-Basilobelba werneri Mahunka, 1982

Distribución: Costa Rica.

Xiphobelba Csiszár, 1961 (5 spp.)

ESPECIE TIPO: Xiphobelba hamanni Csiszár, 196

DisTRIBUCIÓN: Australianooriental.

-Xiphobelba barbata Talukdar y Chakrabarti, 1984

DisTRIBUCIÓN: India (Assam).

-Xiphobelba hamanni Csiszár, 1961

(=Xiphobelba margosetosa Hammer, 1979)

DistRIBUCión: Java.

-Xiphobelba ismalia Haq, 1980

DistRIBUCión: India (Kerala).

-Xiphobelba mindanensis Corpuz-Raros, 1980

DisTRIBUCIÓN: Filipinas.

-Xiphobelba setosa Aoki, 1968

Distribución: Melanesia (Nueva Bretaña).

Ameridae Bulanova-Zachvatkina, 1957 (7 gen., 1 subg., 17 spp. y 1 ssp.) DisTRIBUCIÓN: Tropical y subtropical.

Amerus Berlese, 1896 (3 spp.)

EsPECIE TIPO: Belba troisi Berlese, 1883

DISTRIBUCIÓN: Paleártica.

Amerus (Amerus) Berlese, 1896 (2 spp.)

DISTRIBUCIÓN: Paleártica.

-Amerus (A.) polonicus Kulczynski, 1902

DisTRIBUCIÓN: Europa centromeridional.

-Amerus (A.) troisi (Berlese, 1883) (Belba)

DistRIBUCIÓN: Mediterránea. 
Amerus (Neamerus) Willmann, 1939 (1 sp.)

EsPeCIE TIPO: Amerus (Neamerus) lundbladi Willmann, 1939

DisTRIBUCIÓN: Paleártica.

-Amerus (Neamerus) lundbladi Willmann, 1939

(?=Amerus laticephalus Berlese, 1915 “sp. inq.")

DisTRIBUCIÓN: Mediterránea occidental.

Andesamerus Hammer, 1962 (2 spp.)

ESPECIE TIPO: Andesamerus peculiaris Hammer, 1962

DISTRIBUCIÓN: Subtropical austral (excepto Etiópica).

-Andesamerus novaezealandiae J. y P. Balogh, 1986

DistriBución: Nueva Zelanda.

-Andesamerus peculiaris Hammer, 1962

DisTRIBUCIÓN: América austral.

Caenosamerus Higgins y Woolley, 1969 (3 spp. y 1 ssp.) EsPeCie Tipo: Caenosamerus litosus Higgins y Woolley, 1969 DISTRIBUCIÓN: Holárica.

-Caenosamerus litosus Higgins y Woolley, 1969

Distribución: U.S.A. (Carolina del Norte).

-Caenosamerus shirakamiensis Fijikawa, 2002

DisTRIBUCIÓN: Japón.

-Caenosamerus spatiosus Aoki, 1977

Distribución: Este de Paleártica oriental.

-Caenosamerus spatiosus spatiosus Aoki, 1977

Distribución: Este de Paleártica oriental.

-Caenosamerus spatiosus maoershanensis Wen, 1992 DisTRIBUCión: Noreste de China.

Ctenamerus J. y P. Balogh, 1992 (1 sp.)

EsPECIE TIPO: Hymenobelba flagellata Covarrubias, 1967

DisTRIBUCIÓN: Neotropical austral.

-Ctenamerus flagellatus (Covarrubias, 1967) (Hymenobelba)

DisTRIBUCIÓN: Chile.

Haplamerus J. y P. Balogh, 1992 (1 sp.)

ESPECIE TIPO: Hymenobelba annulus Balogh y Mahunka, 1966 Distribución: Etiópica.

-Haplamerus annulus (Balogh y Mahunka, 1966) (Hymenobelba) DisTRIBUCIÓN: Sudáfrica.

Hymenobelba Balogh, 1962 (6 spp.)

ESPECIE TIPO: Hymenobelba ypsilon Balogh, 1962

DisTRIBUCIÓN: Pantropical y subtropical austral.

-Hymenobelba coarctata Balogh y Mahunka, 1966 DiSTRIBUCIÓN: Sudáfrica.

-Hymenobelba diversisetosa Luxton, 1988

DisTRIBUCIÓN: Nueva Zelanda.

-Hymenobelba domahidyi J. y P. Balogh, 1983

DISTRIBUCIÓN: Australia.

-Hymenobelba flexisetosa Luxton, 1988

DisTRIBUCIÓN: Nueva Zelanda.

-Hymenobelba ramulosa Covarrubias, 1967

DistriBución: Chile.

-Hymenobelba ypsilon Balogh, 1962

DisTRIBUCIÓN: Madagascar e India (Bengala Occidental).

Pteramerus Balogh, 1964 (1 sp.)

EsPecie Tipo: Pteramerus draco Balogh, 1964

DisTRIBUCIÓN: Etiópica.

-Pteramerus draco Balogh, 1964

DisTRIBUCIÓN: Madagascar.

Staurobatidae Grandjean, 1966 (2 gen., 2 spp. y 1 ssp.) DisTRIBUCIÓN: Neotropical.

Staurobates Grandjean, 1966 (1 sp. y 1 ssp.)

EsPECIE TIPO: Staurobates schusteri Grandjean, 1966

DISTRIBUCIÓN: Neotropical.
-Staurobates schusteri Grandjean, 1966

DisTRIBUCIÓN: Neotropical.

-Staurobates schusteri schusteri Grandjean, 1966 DisTRIBUCIÓN: Brasil.

-Staurobates schusteri cordobensis Balogh y Mahunka, 1968 DisTRIBUCIÓN: Neotropical (Argentina y Paraguay).

Stauroma Grandjean, 1966 (1 sp.)

EsPecie TiPo: Stauroma cephalotum Grandjean, 1966

DISTRIBUCIÓN: Neotropical.

-Stauroma cephalotum Grandjean, 1966

DisTRIBUCIÓN: Brasil.

Eremelloidea Balogh, 1961

Platyameridae J. y P. Balogh, 1983 (3 gen., 9 spp. y 2 sspp.) DisTRIBUCIÓN: Holártica y Australianooriental

Cristamerus Hammer, 1977 (2 spp.)

EsPecie TIPO: Cristamerus spinosus Hammer, 1977

DistRIBUCIÓN: Paleártica.

-Cristamerus spinosus Hammer, 1977

DisTRIBUCIÓN: Pakistán.

-Cristamerus yunnanensis Aoki y Yamamoto, 2000

DISTRIBUCIÓN: China suroriental.

Defectamerus Aoki, 1984 (6 spp. y 2 sspp.)

(?=Gymnodampia Jacot, 1937 "gen. inq.")

EsPeCIE TIPO: Defectamerus crassisetiger Aoki, 1984

DisTRIBUCIÓN: Holártica y Oriental.

-Defectamerus conformis Fujikawa, 2002

DisTRIBUCIÓN: Japón.

-Defectamerus crassisetiger Aoki, 1984

DisTRIBUCIÓN: Este de Paleártica oriental y Taiwan [Formosa].

-Defectamerus crassisetiger crassisetiger Aoki, 1984 Distribución: Este de Paleártica oriental.

-Defectamerus crassisetiger australis Aoki, 1991 Distribución: Taiwan [Formosa].

-Defectamerus crassisetiger coreanus Choi y Aoki, 1985

DisTRIBUCIÓN: Corea.

-Defectamerus fuscus Fujikawa, 2002

DisTRIBUCIÓN: Japón.

-Defectamerus setatus (Berlese, 1916) (Amerobelba)

DisTRIBUCIÓN: U.S.A. centrooriental.

-Defectamerus soonkii Choi y Aoki, 1985

DisTRIBUCIÓN: Corea.

-Defectamerus sungohi Choi, 1994

DisTRIBUCIÓN: Corea.

Platyamerus J. y P. Balogh, 1983 (1 sp.)

EsPeCIE TIPO: Platyamerus peculiaris J. y P. Balogh, 1983

DISTRIBUCIÓN: Australiana.

-Platyamerus peculiaris J. y P. Balogh, 1983

DISTRIBUCIÓN: Australia.

Caleremaeidae Grandjean, 1965 (7 gen., 27 spp. y 1 ssp.) DistriBuCión: Cosmopolita (excepto Antártica)

Anderemaeus Hammer, 1958 (8 spp.)

ESPECIE TIPO: Anderemaeus monticola Hammer, 1958

DisTRIBUCIÓN: Tropical (Australiana y Neotropical) y Neotropical austral.

-Anderemaeus australiensis J. y P. Balogh, 1983

DisTRIBUCión: Australia.

-Anderemaeus capitatus J. y P. Balogh, 1985

Distribución: Colombia.

-Anderemaeus chilensis Hammer, 1962

DisTRIBUCIÓN: América austral.

-Anderemaeus hammerae Mahunka, 1980

DisTRIBUCIÓN: América austral (Tierra del Fuego). 
-Anderemaeus hidasii P. Balogh, 1995

DistriBución: Brasil.

-Anderemaeus magellanis Hammer, 1962

DisTRIBUCIÓN: América austral.

-Anderemaeus monticola Hammer, 1958

DistribuCión: Neotropical.

-Anderemaeus sturmi J. y P. Balogh, 1985

Distribución: Colombia.

Caleremaeus Berlese, 1910 (3 spp.)

EsPECIE TIPO: Damaeus monilipes Michael, 1882

DISTRIBUCIÓN: Holártica.

-Caleremaeus divisus Mihelcic, 1952

Distribución: Austria.

-Caleremaeus monilipes (Michael, 1882) (Damaeus)

Distribución: Paleártica occidental (frecuente).

-Caleremaeus retractus (Banks, 1947) (Carabodoides)

DistribuCIÓN: U.S.A. (Carolina del Norte).

Cristeremaeus Balogh y Csiszár, 1963 (2 spp.)

EsPECIE TIPO: Cristeremaeus humeratus Balogh y Csiszár, 1963

DisTRIBUCIÓN: Neotropical austral.

-Cristeremaeus clavatus Mahunka, 1980

Distribución: América austral (Tierra del Fuego).

-Cristeremaeus humeratus Balogh y Csiszár, 1963

DISTRIBUCIÓN: Argentina.

Epieremulus Berlese, 1916 (10 spp. y 1 ssp.)

(=Carabodoides Jacot, 1937)

ESPECIE TIPO: Eremulus (Epieremulus) geometricus Berlese, 1916

DisTRIBUCIÓN: Cosmopolita (excepto Australiana y Antártica).

-Epieremulus apicalis (Banks, 1895) (Carabodes)

Distribución: U.S.A. (Nueva York).

-Epieremulus braziliensis (Balogh y Mahunka, 1969) (Carabodoides)

Distribución: Brasil y Filipinas.

-Epieremulus circulus Yamamoto y Aoki, 2000

DisTRIBUCIÓN: China suroriental.

-Epieremulus frontatus (Warburton, 1912) (Notaspis)

DisTRIBUCIÓN: I. Seychelles.

-Epieremulus geometricus (Berlese, 1916) (Eremulus (Epi.))

(=Carabodoides saccharomycetoides Jacot, 1937)

DisTRIBUCIÓN: U.S.A. oriental.

-Epieremulus granulatus (Balogh y Mahunka, 1979) (Carabodoides) DisTRIBUCIÓN: Neotropical.

-Epieremulus granulatus granulatus (Balogh y Mahunka, 1979) DisTRIBUCIÓN: Norte de Neotropical.

-Epieremulus granulaltus andicola (P. Balogh, 1988) (Carabodoides) DISTRIBUCIÓN: Ecuador.

-Epieremulus humeratus (Aoki, 1987) (Carabodoides)

DisTRIBUCIÓN: Japón.

-Epieremulus laticeps Balogh, 1963

DisTRIBUCIÓN: Angola.

-Epieremulus longicarinatus (Balogh y Mahunka, 1978) (Carabodoides)

DisTRIBUCIÓN: Brasil.

-Epieremulus longiseta (P. Balogh, 1988) (Carabodoides)

DISTRIBUCIÓN: Ecuador

Luxtoneremaeus J. y P. Balogh, 1992 (1 sp.)

EsPECIE TIPO: Anderemaeus forsteri J. y P. Balogh, 1985

DisTRIBUCIÓN: Australiana.

-Luxtoneremaeus forsteri (J. y P. Balogh, 1985) (Anderemaeus)

DisTRIBUCión: Nueva Zelanda.

Veloppia Hammer, 1955 (2 spp.)

ESPECIE TIPO: Veloppia pulchra Hammer, 1955

DisTRIBUCIÓN: Neártica septentrional.

-Veloppia kananaskis Norton, 1978

DISTRIBUCIÓN: Canadá.
-Veloppia pulchra Hammer, 1955

DISTRIBUCIÓN: Neártica septentrional.

Yungaseremaeus Balogh y Mahunka, 1969 (1 sp.)

EsPeCIE TIPO: Yungaseremaeus longisetosus Balogh y Mahunka, 1969 DisTRIBUCIÓN: Neotropical.

-Yungaseremaeus longisetosus Balogh y Mahunka, 1969

DISTRIBUCIÓN: Bolivia.

Eremellidae Balogh, 1961 (4 gen. y 11 spp.) DisTRIBUCIÓN: Tropical y subtropical.

Archeremella Balogh y Mahunka, 1974 (2 spp.)

ESPECIE TIPO: Eremella (Archeremella) leowae Balogh y Mahunka, 1974 DISTRIBUCIÓN: Paleotropical.

-Archeremella africana Pérez-Íñigo, 1982

Distribución: Guinea Ecuatorial: I. Pagalu [Annobón].

-Archeremella leowae (Balogh y Mahunka, 1974) (Eremella (A.))

Distribución: Península Malaya.

Eremella Berlese, 1913 (6 spp.)

(=Proteremella Balogh, 1959)

EsPECIE TIPO: Eremella vestita Berlese, 1913

DISTRIBUCIÓN: Pantropical y Paleártica meridional.

-Eremella africana (Balogh, 1966) (Proteremella)

DisTRIBUCIÓN: Chad.

-Eremella ensifera Balogh y Mahunka, 1968

DisTRIBUCIÓN: Argentina.

-Eremella induta Berlese, 1913

Distribución: Pantropical y subtropical (Oriental, Japón,

Australiana: I. Tonga, y Etiópica: Congo).

-Eremella matildebellae Mahunka y Palacios-Vargas, 1995

DisTRIBUCióN: Méjico.

-Eremella pulchella (Balogh, 1959) (Proteremella)

DisTRIBUCIÓN: Europa central.

Eremella vestita Berlese, 1913

DisTRIBUCIÓN: Oriental (Java y Filipinas).

Licnocepheus Woolley, 1969 (1 sp.)

ESPECIE TIPO: Licnocepheus reticulatus Woolley, 1969

DISTRIBUCIÓN: Neártica.

-Licnocepheus reticulatus Woolley, 1969

DisTRIBUCIÓN: U.S.A. (Luisiana)

Triteremella Kunst, 1971 (2 spp.)

(=Afreremella Mahunka, 1974 "sin. nov.")

EsPeCIE TIPO: Eremella kaszabi Csiszár, 1962

DisTRIBUCIÓN: Paleártica y Etiópica.

-Triteremella kaszabi (Csiszár, 1962) (Eremella)

(?=Licneremaeus tuberculatus Paoli, 1908 "sp. inq.")

DisTRIBUCIÓN: Europa centromeridional.

-Triteremella luisiae (Mahunka, 1974) (Afreremella)

DisTRIBUCIÓN: Rodesia [Zimbabue].

Machadobelbidae Balogh, 1972 (3 gen. y 17 spp.)

DisTRIBUCión: Pantropical (excepto Australiana).

Machadobelba Balogh, 1958 (14 spp.)

ESPECIE TIPO: Machadobelba symmetrica Balogh, 1958

DisTRIBUCIÓN: Pantropical (excepto Australiana).

-Machadobelba baloghi Mondal y Kundu, 1999

DisTRIBUCIÓN: India (Bengala Occidental).

-Machadobelba bifurcata Wen, 1998

DISTRIBUCIÓN: China suroriental.

-Machadobelba ceylonica Balogh, 1970

DisTRIBUCión: Ceilán [Sri Lanka].

-Machadobelba descombesi Mahunka, 1988

DISTRIBUCIÓN: Borneo.

-Machadobelba dispar Balogh, 1958

DisTRIBUCIÓN: Etiópica (Congo y Ghana). 
-Machadobelba foliata Hammer, 1982

DISTRIBUCIÓN: Bali.

-Machadobelba neotropica P. Balogh, 1988

DISTRIBUCIÓN: Ecuador.

-Machadobelba serrata Hammer, 1979

DisTRIBUCIÓN: Java.

-Machadobelba similis Mahunka, 1988

DisTRIBUCIÓN: Borneo.

-Machadobelba simplex Csiszár, 1961

DISTRIBUCIÓN: Java.

-Machadobelba spathulifer Mahunka, 1987

DISTRIBUCIÓN: Borneo.

-Machadobelba symmetrica Balogh, 1958

DisTRIBUCIÓN: Etiópica e India (Tripura).

-Machadobelba tanzica Mahunka, 1988

DisTRIBUCIÓN: Tanzania.

-Machadobelba tuberculata Csiszár, 1961

DISTRIBUCIÓN: Java.

Ramogneta Karppinen, 1966 (1 sp.)

EsPECIE TIPO: Ramogneta lamottei Karppinen, 1966

Distribución: Etiópica.

-Ramogneta lamottei Karppinen, 1966

DISTRIBUCIÓN: Guinea.

Rioppia Balogh y Mahunka, 1977 (2 spp.)

EsPECIE TIPO: Rioppia nodulifera Balogh y Mahunka, 1977

DisTRIBUCIÓN: Neotropical.

-Rioppia comteae Mahunka, 1985

Distribución: Pequeñas Antillas.

-Rioppia nodulifera Balogh y Mahunka, 1977

DisTRIBUCIÓN: Neotropical.

Oribellidae Kunst, 1971 (7 gen., 36 spp. y 1 ssp.)

DisTRIBUCIÓN: Holártica, Etiópica y "Oriental”.

Infernobates Karppinen y Poltavskaja, 1990 (1 sp.)

ESPECIE TIPO: Infernobates citelli Karppinen y Poltavskaja, 1990 DISTRIBUCIÓN: Paleártica.

-Infernobates citelli Karppinen y Poltavskaja, 1990

DisTRIBUCIÓn: Sureste de Europa.

Kaszabobates Balogh, 1972 (3 spp.)

(=Gobiella Balogh y Mahunka, 1965, “nom. praeoc."por Cienkowsky, 1882)

EsPeCIE TIPO: Gobiella kaszabi Balogh y Mahunka, 1965

DISTRIBUCIÓN: Paleártica.

-Kaszabobates helveticus Mahunka y Mahunka-Papp, 2000

DisTRIBUCIÓN: Suiza.

-Kaszabobates kaszabi (Balogh y Mahunka, 1965) (Gobiella)

DisTRIBUCIÓN: Sur de Paleártica oriental.

-Kaszabobates olbiopolitanus Sergienko, 1980

DisTRIBUCIÓN: Sureste de Europa.

Montizetes Kunst, 1971 (6 spp.)

ESPECIE TIPO: Xenillus alpestris Willmann, 1929

DISTRIBUCIÓN: Holártica.

-Montizetes abulensis Pérez-Íñigo, 1984

DISTRIBUCIÓN: Ibérica.

-Montizetes alpestris (Willmann, 1929) (Xenillus)

DisTRIBUCIÓN: Holártica (Paleártica y Neártica septentrional).

-Montizetes arcticus (Pankov, 1993) (Gemmazetes)

DisTRIBUCIÓN: Este de Rusia asiática.

-Montizetes delamellatus Pérez-Íñigo jr., 1991

DisTRIBUCIÓN: España.

-Montizetes mongolicus (Balogh y Mahunka, 1965) (Oribella)

DISTRIBUCIÓN: Mongolia.

-Montizetes rarisetosus Bayartogtokh, 1998

DisTRIBUCIÓN: Mongolia.
Oribella Berlese, 1908 (5 spp.)

EsPeCIE TIPO: Notaspis pectinata Michael, 1885

DISTRIBUCIÓN: Holártica.

-Oribella adelaidae Golosova y Karppinen, 1985

DisTRIBUCIÓN: Mongolia.

-Oribella canariensis Pérez-Íñigo, 1986

DisTRIBUCión: I. Canarias.

-Oribella fujikawae Mahunka, 1982

DisTRIBUCIÓN: Grecia.

-Oribella matritensis Arillo, Bordel y Subías, 1988

DisTRIBUCIÓN: España.

-Oribella pectinata (Michael, 1885) (Notaspis)

(=Dameosoma crinitum Berlese, 1916)

(=Xenillus limburgiensis Oudemans, 1912)

DisTRIBUCIÓN: Holártica (Paleártica occidental: frecuente, Asia centrooccidental y U.S.A.: Nuevo Méjico).

Oribellopsis Kunst, 1971 (8 spp. y 1 ssp)

(=Gemmazetes Fujikawa, 1979 "sin. nov.")

EsPeCIE TIPO: Oribella cavatica Kunst, 1962

DisTRIBUCIÓN: Holártica.

-Oribellopsis cavaticus (Kunst, 1962) (Oribella)

DisTRIBUCIÓN: Holártica (Paleártica: Europa central y Noreste de China, y Norte de U.S.A.).

-Oribellopsis clavatus (Jacot, 1937) (Oribella crosbyi c.)

DisTRIBUCIÓN: U.S.A. (Kentucky).

-Oribellopsis clavigerus (Mihelcic, 1958) (Oribella)

DISTRIBUCIÓN: Austria.

-Oribellopsis crosbyi (Berlese, 1908) (Oribella)

DistribuCión: Holártica (Paleártica: Europa central y Noreste de

China, y U.S.A. centrooriental).

-Oribellopsis crosbyi crosbyi (Berlese, 1908)

DisTRIBUCIÓN: Holártica (Europa central y U.S.A. centrooriental).

-Oribellopsis crosbyi maoershanensis Wen, 1992

DisTRIBUCión: Noreste de China.

-Oribellopsis etruscus (Bernini, 1980) (Gemmazetes)

DISTRIBUCIÓN: Italia.

-Oribellopsis forsslundi (Moritz, 1965) (Oribella)

DisTRIBUCIÓN: Alemania.

-Oribellopsis kushiroensis (Aoki, 1992) (Gemmazetes)

DisTRIBUCIÓN: Japón.

-Oribellopsis tianshanensis (Wen, 1989) (Gemmazetes)

DisTRIBUCIÓN: Noroeste de China.

Pantelozetes Grandjean, 1953 (4 spp.)

EsPecie TIPO: Xenillus paolii Oudemans, 1913

DisTRIBUCIÓN: Holártica y "Oriental”.

-Pantelozetes berlesei Fujikawa, 1979

DisTRIBUCIÓN: Europa suroccidental.

-Pantelozetes pannonicus (Mahunka, 1969) (Oribella)

DisTRIBUCiÓN: Paleártica (Sur de Europa oriental y Asia centrooccidental).

-Pantelozetes paolii (Oudemans, 1913) (Xenillus)

(=Amazoppia caucasica Djaparidze, 1986)

(=Oribella dentata Mihelcic, 1956)

(=Oribella paolii multidentata Evans, 1954)

(=Pantelozetes paolii tuxeni Fujikawa, 1979)

DisTRIBUCIÓN: Holártica (Paleártica occidental: frecuente, y

Neártica septentrional) y Java.

-Pantelozetes tricuspidatus (Djaparidze, 1986) (Amazoppia)

DISTRIBUCIÓN: Cáucaso.

Proteremaeus Piffl, 1965 (9 spp.)

(=Sibiremaeus Krivolutsky, 1975)

EsPecie TiPo: Proteremaeus jonasi Piffl, 1965

DisTRIBUCIÓN: Paleártica.

-Proteremaeus angarensis (Rjabinin y Krivolutsky, 1975) (Sibiremaeus)

DISTRIBUCIÓN: Siberia centrooccidental. 
-Proteremaeus chadaevae Golosova, 1983

Distribución: Mongolia.

-Proteremaeus elongatus (Rjabinin y Krivolutsky, 1975) (Sibiremaeus)

Distribución: Sur de Paleártica oriental.

-Proteremaeus jonasi Piffl, 1965

DistRIBUCión: Sur de Paleártica oriental.

-Proteremaeus lawariensis Hammer, 1977

DisTRIBUCIÓN: Pakistán.

-Proteremaeus macleani Behan-Pelletier, 1982

Distribución: Este de Rusia asiática.

-Proteremaeus mongolicus (Golosova, 1983) (Sibiremaeus)

DistriBución: Mongolia.

-Proteremaeus nebaikini Behan-Pelletier y Rjabinin, 1991

Distribución: Este de Rusia asiática.

-Proteremaeus punctulatus Bayartogtokh, 2000

DisTRIBUCión: Mongolia.

Arceremaeidae Balogh, 1972 (2 gen. y 10 spp.) DistRIBUCIÓN: Tropical (Oriental y Neotropical).

Arceremaeus Hammer, 1961 (3 spp.)

EsPECIE TIPO: Arceremaeus incaensis Hammer, 1961

DISTRIBUCIÓN: Neotropical.

-Arceremaeus cubanus Balogh y Mahunka, 1980

Distribución: Cuba.

-Arceremaeus incaensis Hammer, 1961

DisTRIBUCión: Neotropical (Perú y Ecuador).

-Arceremaeus jimenezi Calugar y Vasiliu, 1977

DisTribución: Cuba.

Tecteremaeus Hammer, 1961 (7 spp.)

ESPECIE TIPO: Tecteremaeus cornutus Hammer, 1961

DisTRIBUCIÓN: Tropical (Oriental y Neotropical).

-Tecteremaeus anoporosus Balogh y Mahunka, 1969

DisTRIBUCIÓN: Brasil.

-Tecteremaeus bogorensis Hammer, 1979

DisTRIBUCIÓN: Oriental (Indonesia).

-Tecteremaeus cachoeirensis Franklin y Woas, 1992

Distribución: Brasil.

-Tecteremaeus cornutus Hammer, 1961

DisTRIBUCIÓN: Notropical (Perú y Ecuador) y Filipinas.

-Tecteremaeus cristatus Balogh y Mahunka, 1969

DisTRIBUCIÓN: Brasil.

-Tecteremaeus hauseri Mahunka, 1982

DisTRIBUCIÓN: Filipinas.

-Tecteremaeus incompletus Mahunka, 1988

DISTRIBUCIÓN: Borneo.

Spinozetidae Balogh, 1972 (3 gen. y 4 spp.) DisTRIBUCIÓN: Paleártica.

Grypoceramerus Suzuki y Aoki, 1970 (1 sp.)

EsPECIE TIPO: Grypoceramerus acutus Suzuki y Aoki, 1970

DISTRIBUCIÓN: Paleártica.

-Grypoceramerus acutus Suzuki y Aoki, 1970

DISTRIBUCIÓN: Japón.

Iberoppia Pérez-Íñigo, 1986 (1 sp.)

EsPECIE TIPO: Iberoppia paradoxa Pérez-Íñigo, 1986

DisTRIBUCIÓN: Paleártica.

-Iberoppia paradoxa Pérez-Íñigo, 1986

DISTRIBUCIÓN: España.

Spinozetes Piffl, 1966 (2 spp.)

(=Mirus Kulijev, 1967)

EsPecie TIPO: Spinozetes inexspectatus Piffl, 1966

DisTRIBUCIÓN: Paleártica.

-Spinozetes inexspectatus Piffl, 1966

DisTRIBUCIÓN: Sureste de Europa.
-Spinozetes pectinatus (Kulijev, 1967) (Mirus)

Distribución: Cáucaso.

Oppioidea Sellnick, 1937

(=Dameosomoidea Sellnick, 1928)

Autognetidae Grandjean, 1960 (6 gen., 1 subg., 22 spp. y 2 sspp.) DistriBución: Holártica y subtropical austral (excepto Etiópica).

Austrogneta Balogh y Csiszár, 1963 (2 spp.)

EsPECIE TIPO: Austrogneta multipilosa Balogh y Csiszár, 1963

DisTRIBUCIÓN: Subtropical austral (excepto Etiópica).

-Austrogneta multipilosa Balogh y Csiszár, 1963

DisTRIBUCIÓN: Australiana (Australia y Nueva Zelanda) y Argentina. -Austrogneta quadridentata Hammer, 1966

DisTRIBUCIÓN: Nueva Zelanda.

Autogneta Hull, 1916 (12 spp. y 2 sspp.)

ESPECIE TIPO: Notaspis longilamellata Michael, 1885

DISTRIBUCIÓN: Holártica.

Autogneta (Autogneta) Hull, 1916 (10 spp. y 2 sspp.)

DISTRIBUCIÓN: Holártica.

-Autogneta (A.) amica Jacot, 1938 (A. longilamellata a.)

DisTRIBUCIÓN: U.S.A. (Carolina del Norte).

-Autogneta (A.) flumengalei Jacot, 1939 (A. longilamellata f.)

DisTRIBUCIÓN: U.S.A.(Nueva Hampshire).

-Autogneta (A.) inundata Winkler, 1957

DISTRIBUCIÓN: Chequia.

-Autogneta (A.) japonica Fujikawa, 1972

DisTRIBUCIÓN: Japón.

-Autogneta (A.) kaisilai Karppinen, 1967

DistribuCión: I. Spitsberg (Svalvard).

-Autogneta (A.) longilamellata (Michael, 1885) (Notaspis)

DisTRIBUCIÓN: Holártica (Paleártica, y Neártica septentrional).

-Autogneta (A.) longilamellata longilamellata (Michael, 1885)

DisTRIBUCIÓN: Holártica (Paleártica: frecuente, y Neártica septentrional).

-Autogneta (A.) longilamellata intermedia (Mihelcic, 1952)(Oppia) DisTRIBUCIÓN: Austria.

-Autogneta (A.) masahitoi Aoki, 1963

DisTRIBUCIÓN: Este de Paleártica oriental.

-Autogneta (A.) parva Forsslund, 1947

(=Autogneta rugosa Mihelcic, 1956)

DisTRIBUCIÓN: Paleártica.

-Autogneta (A.) penicillum Grandjean, 1960

DisTRIBUCIÓN: Oeste de Europa.

-Autogneta (A.) willmanni (Dyrdowska, 1929) (Dameosoma)

DisTRIBUCIÓN: Europa.

-Autogneta (A.) willmanni willmanni (Dyrdowska, 1929) DISTRIBUCIÓN: Europa central

-Autogneta (A.) willmanni herzegowinensis (Willmann, 1941) (Oppia)

DisTRIBUCIÓN: Bosnia.

Autogneta (Rhaphigneta) Grandjean, 1960 (2 spp.)

EsPECIE TIPO: Rhaphigneta numidiana Grandjean, 1960

DISTRIBUCIÓN: Paleártica.

-Autogneta (Rhaphigneta) flagellata (Mahunka, 1977) (R.)

DISTRIBUCIÓN: Grecia.

-Autogneta (Rhaphigneta) numidiana (Grandjean, 1960) (R.)

DisTRIBUCIÓN: Mediterránea.

Conchogneta Grandjean, 1963 (2 spp.)

EsPECIE TIPO: Autogneta dalecarlica Forsslund, 1947

DISTRIBUCIÓN: Holártica.

-Conchogneta dalecarlica (Forsslund, 1947) (Autogneta)

(=Oppia talischica Kulijev, 1962)

DISTRIBUCION: Paleartica. 
-Conchogneta traegardhi (Forsslund, 1947) (Autogneta) Distribución: Holártica (Paleártica y Canadá).

Cosmogneta Grandjean, 1960 (3 spp.)

EsPeCIE TIPO: Cosmogneta impedita Grandjean, 1960 DISTRIBUCIÓN: Paleártica.

-Cosmogneta cassolai Bernini, Baratti y Avanzati, 1991 DisTRIBUCIÓN: Suroeste de Europa.

-Cosmogneta impedita Grandjean, 1960 DisTRIBUCIÓN: Francia.

-Cosmogneta kargi Grandjean, 1963

DISTRIBUCIÓN: Europa centrooccidental.

Eremobodes Jacot, 1937 (1 sp.)

EsPECIE TIPO: Eremobodes pectinatus Jacot, 1937

DISTRIBUCIÓN: Neártica.

-Eremobodes pectinatus Jacot, 1937

DistriBuCión: U.S.A. (Carolina del Norte).

Parautogneta Golosova, 1974 (2 spp.)

EsPECIE TIPO: Parautogneta silvatica Golosova, 1974

DisTRIBUCIÓN: Paleártica.

-Parautogneta golosovae Rjabinin, 1975

DisTRIBUCIÓN: Paleártica oriental.

-Parautogneta silvatica Golosova, 1974

DisTRIBUCIÓn: Este de Rusia asiática.

Thyrisomidae Grandjean, 1953 (1 gen., 17 spp. y 5 sspp.)

DistRIBUCIÓN: Cosmopolita (excepto Etiópica y Antártica).

Banksinoma Oudemans, 1930 (17 spp. y 5 sspp.)

(=Thyrisoma Grandjean, 1953)

EsPECIE TIPO: (Notaspis lanceolata Michael "sensu" Oudemans, 1930)

=Banksinoma lanceolata oudemansi Fujikawa, 1979

DisTRIBUCión: Cosmopolita (excepto Etiópica y Antártica).

-Banksinoma akhtyamovi Rjabinin, 1993

Distribución: Este de Rusia asiática.

-Banksinoma arcuatum (Hammer, 1958) (Oribella)

DisTRIBUCIÓN: Argentina.

-Banksinoma borealis (Willmann, 1943) (Oribella)

DisTRIBUCIÓN: Suecia.

-Banksinoma castanea (Hermann, 1804) (Notaspis) "sp. inq." DisTRIBUCIÓN: Francia.

-Banksinoma cincta Zhao y Wen, 1993

DistriBución: Noreste de China.

-Banksinoma exobothridialis Bayartogtokh, 1997

DistriBución: Mongolia.

-Banksinoma insignis Balogh y Mahunka, 1965

Distribución: Mongolia.

-Banksinoma japonica Fujikawa, 1979

DISTRIBUCIÓN: Japón.

-Banksinoma lanceolata (Michael, 1885) (Notaspis)

DisTRIBUCIÓN: Holártica y Hawai.

-Banksinoma lanceolata lanceolata (Michael, 1885)

(?=Zetes dorsatus Koch, 1841 "sp. inq.")

(?=Zetes flavipes Koch, 1841 "sp. inq.")

(=Banksinoma lanceolata islandica Fujikawa, 1979)

DistriBución: Paleártica (excepto Este): frecuente, y Hawai.

-Banksinoma lanceolata bifurcata (Jacot, 1938) (Oribella) DisTRIBUCIÓN: U.S.A. (Ilinois).

-Banksinoma lanceolata canadensis Fujikawa, 1979 DisTRIBUCIÓN: Canadá.

-Banksinoma lanceolata lunare (Hull, 1914) (Dameosoma) DisTRIBUCIÓN: Islas Británicas.

-Banksinoma lanceolata oudemansi Fujikawa, 1979 DisTRIBUCión: Holanda.

-Banksinoma longisetosa Bayartogtokh y Aoki, 1998

DisTRIBUCIÓN: Mongolia.
-Banksinoma monoceros (Balogh y Mahunka, 1968) (Oribella spinifera $\mathrm{m}$.)

DisTRIBUCIÓn: Argentina.

-Banksinoma ovata (Wallwork, 1962) (Thyrisoma)

Distribución: U.S.A. (Michigan).

-Banksinoma pretiosa Tseng, 1982

DisTRIBUCIÓN: Taiwan [Formosa]

-Banksinoma setosa Rjabinin, 1974

DistRIBUCIÓN: Holártica (Sur de Siberia, I. Kuriles y Canadá).

-Banksinoma sinica Wen, 1990

Distribución: Noreste de China.

-Banksinoma spinifera (Hammer, 1952) (Oribella)

Distribución: Neártica (Suroeste de U.S.A. y Canadá) y

Neotropical (Bolivia y Argentina).

-Banksinoma spinifera spinifera (Hammer, 1952)

DistribUCIÓN: Neártica (Suroeste de U.S.A. y Canadá).

-Banksinoma spinifera fissurata (Hammer, 1952) (Oribella)

DisTRIBUCIÓN: Neotropical (Bolivia y Argentina).

-Banksinoma watanabei Aoki, 2002

DISTRIBUCIÓN: Japón.

Oppiidae Sellnick, 1937 (130 gen., 36 subg., 907 spp. y 42 sspp.)

(=Dameosomidae Sellnick, 1928)

DisTRIBUCIÓN: Cosmopolita.

[Antilloppiinae] Mahunka, 1985 (2 gen. y 6 spp.)

DistRIBUCIÓN: Neotropical y subtropical (Holártica meridional).

Joboppia Ruiz, Mínguez y Subías, 1988 (2 spp.)

EsPeCIE TIPO: Neoppia (Joboppia) dichosa Ruiz, Mínguez y Subías, 1988 DISTRIBUCIÓN: Holártica.

-Joboppia dichosa (Ruiz, Mínguez y Subías, 1988) (Neoppia (J.))

DisTRIBUCIÓN: España y "U.S.A."

-Joboppia expansum (Paoli, 1908) (Dameosoma)

Distribución: Sur de Europa.

Neoppia Bhattacharya y Banerjee, 1981 (4 spp.)

(=Antilloppia Mahunka, 1985)

EsPECIE TIPO: Neoppia minuta Bhattacharya y Banerjee, 1981

DISTRIBUCIÓN: Neotropical y subtropical (Paleártica meridional).

-Neoppia bayoumii (Al-Assiuty y El-Deeb, 1983) (Multioppia)

DISTRIBUCIÓN: Egipto.

-Neoppia discreta Ruiz, Mínguez y Subías, 1988

DISTRIBUCIÓN: Europa meridional.

-Neoppia minuta Bhattacharya y Banerjee, 1981

DisTRIBUCIÓN: India (Bengala Occidental).

-Neoppia schauenbergi (Mahunka, 1985) (Antilloppia)

DistriBución: Neotropical (Pequeñas Antillas e I. Galápagos).

[Paternoppiinae] Gil-Martín, Subías y Arillo, 2000 (1 gen. y 1 sp.) DisTRIBUCIÓN: Paleártica.

Paternoppia Gil-Martín, Subías y Arillo, 2000 (1 sp.)

EsPECIE TIPO: Paternoppia andalusicabulensis Gil-Martín, Subías y Arillo, 2000

DISTRIBUCIÓN: Paleártica.

-Paternoppia andalusicabulensis Gil-Martín, Subías y Arillo, 2000

DISTRIBUCIÓN: España.

[Lanceoppiinae] Balogh, 1983 (17 gen., 6 subg., 126 spp. y 1 ssp.) DistRIBUCIÓN: Cosmopolita (excepto Boreal).

Acutoppia Balogh, 1983 (2 spp.)

EsPECIE TIPO: Operculoppia crassiseta Hammer, 1968

DisTRIBUCIÓN: Australiana.

-Acutoppia crassiseta (Hammer, 1968) (Operculoppia)

DistRIBUCión: Nueva Zelanda.

-Acutoppia jelevae (Hammer, 1968) (Operculoppia)

DISTRIBUCION: Nueva Zelanda. 
Basiloppia Balogh, 1983 (1 sp.)

ESPECIE TIPO: Oppia hexatricha Balogh y Mahunka, 1975

DisTRIBUCIÓN: Australiana.

-Basiloppia hexatricha (Balogh y Mahunka, 1975) (Oppia)

DISTRIBUCIÓN: Australia.

Cycloppia Balogh, 1983 (3 spp.)

EsPeCIE TIPO: (Lanceoppia simplex Suzuki, 1973) =Oppia restata Aoki, 1963

DisTRIBUCIÓN: Australiana y Paleártica meridional.

-Cycloppia latisternum J. y P. Balogh, 1986

DisTRIBUCIÓN: Nueva Guinea.

-Cycloppia restata (Aoki, 1963) (Oppia)

(=Lanceoppia simplex Suzuki, 1973)

DISTRIBUCIÓN: Japón.

-Cycloppia szentirmayi (Balogh, 1970) (Oppia)

DisTRIBUCIÓN: Nueva Guinea.

Drepanoppia Balogh, 1983 (2 spp.)

EsPECIE TIPO: Oppia falxa Kok, 1967

DisTRIBUCIÓN: Etiópica.

-Drepanoppia falxa (Kok, 1967) (Oppia)

DisTRIBUCión: Sudáfrica.

-Drepanoppia koki Mahunka, 1993

DisTRIBUCIÓN: Tanzania.

Geminoppia J. y P. Balogh, 1983 (3 spp.)

EsPeCIE TIPO: Geminoppia papineaui J. y P. Balogh, 1983

DisTRIBUCIÓN: Pantropical (excepto Oriental).

-Geminoppia ansifera (Mahunka, 1985) (Tectoppiella)

DisTRIBUCión: Sudáfrica.

-Geminoppia papineaui J. y P. Balogh, 1983

DistriBución: Nueva Caledonia.

-Geminoppia velata (Franklin y Woas, 1992) (Globoppia) DistRIBUCIóN: Brasil.

Globoppia Hammer, 1962 (13 spp. y 1 ssp.)

ESPECIE TIPO: Globoppia intermedia Hammer, 1962

DisTRIBUCIÓn: Pantropical y austral.

-Globoppia brinoni J. y P. Balogh, 1983

DisTRIBUCión: Nueva Caledonia.

-Globoppia cochlearium (Paoli, 1908) (Dameosoma)

DisTRIBUCIÓN: "Neotropical".

-Globoppia curviclavata (Mahunka, 1985) (Pletzenoppia)

DisTRIBUCIÓN: Sudáfrica.

-Globoppia gibba Mahunka, 1984

DisTRIBUCIÓN: Sudáfrica.

-Globoppia globifera (Mahunka, 1989) (Membranoppia)

DisTRIBUCIÓN: Sudáfrica.

-Globoppia hamiltoni (Mahunka, 1988) (Pletzenoppia)

DisTRIBUCIÓN: Tanzania.

-Globoppia heterotricha (Balogh y Mahunka, 1969) (Oppia)

DISTRIBUCIÓN: Bolivia.

-Globoppia intermedia Hammer, 1962

DisTRIBUCIÓN: Neotropical (Austral y Antártica).

-Globoppia intermedia intermedia Hammer, 1962

DisTRIBUCIÓN: América austral y Antártica.

-Globoppia intermedia longiseta Wallwork, 1970

DisTRIBUCIÓN: Antártica (frecuente).

-Globoppia latifasciata (Willmann, 1931) (Oppia)

DISTRIBUCIÓN: Sumatra.

-Globoppia maior Hammer, 1962

DistRIBUCIÓN: América austral y subantártica: I. Malvinas [I.

Falkland].

-Globoppia minor Hammer, 1962

DisTRIBUCIÓN: América austral.

-Globoppia nidicola Hammer, 1968

DistRIBUCión: Nueva Zelanda.

-Globoppia simplex (Warburton, 1912) (Notaspis)

DistRIBUCión: I. Seychelles.
Laminoppia Hammer, 1968 (1 sp.)

ESPECIE TIPO: Laminoppia blocki Hammer, 1968

DisTRIBUCIÓN: Australiana.

-Laminoppia blocki Hammer, 1968

DistRIBUCIÓN: Nueva Zelanda.

Lanceoppia Hammer, 1962 (59 spp.)

EsPecie TIPO: Lanceoppia hexapili Hammer, 1962

DISTRIBUCIÓN: Pantropical, austral y "Paleártica".

Lanceoppia (Lanceoppia) Hammer, 1962 (26 spp.)

DisTRIBUCIÓN: Austral.

-Lanceoppia (L.) adjuncta Mahunka, 1989

DISTRIBUCIÓN: Tasmania.

-Lanceoppia (L.) bertheti Hammer, 1968

DistriBución: Nueva Zelanda.

-Lanceoppia (L.) csiszarae Hammer, 1968

DisTRIBUCIÓN: Nueva Zelanda.

-Lanceoppia (L.) elegantula Stary y Block, 1995

DistRIBUCión: Antártica (I. Georgia del Sur).

-Lanceoppia (L.) haarlovi (Hammer, 1968) (Oppia)

DistRIBUCIÓN: Nueva Zelanda.

-Lanceoppia (L.) hexapili Hammer, 1962

DisTRIBUCIón: América austral (Tierra del Fuego).

-Lanceoppia (L.) jacoti Hammer, 1968

DisTRIBUCIÓN: Nueva Zelanda.

-Lanceoppia (L.) knuellei Hammer, 1968

DistRIBUCIÓN: Nueva Zelanda.

-Lanceoppia (L.) kovacsi (Balogh y Csiszár, 1963) (Oppia)

DisTRIBUCIÓN: Argentina.

-Lanceoppia (L.) lancearia (Balogh y Mahunka, 1975) (Oppia)

DISTRIBUCIÓN: Australia.

-Lanceoppia (L.) madagascarensis Mahunka, 2002

DisTRIBUCIÓN: Madagascar.

-Lanceoppia (L.) maerkeli Hammer, 1968

DistRIBUCión: Nueva Zelanda.

-Lanceoppia (L.) microlancearia (Balogh y Mahunka, 1975) (Oppia) DISTRIBUCIÓN: Australia.

-Lanceoppia (L.) microtricha (Balogh y Mahunka, 1975) (Oppia)

DistriBución: Australia.

-Lanceoppia (L.) microtrichoides (Balogh y Mahunka, 1975) (Oppia)

DISTRIBUCIÓN: Australia.

-Lanceoppia (L.) piffli Hammer, 1968

DistrIBUCión: Nueva Zelanda.

-Lanceoppia (L.) poppi Hammer, 1968

DisTRIBUCIÓN: Nueva Zelanda.

-Lanceoppia (L.) ramsayi Hammer, 1968

DisTRIBUCión: Nueva Zelanda.

-Lanceoppia (L.) rigidiseta Hammer, 1968

DisTRIBUCIÓN: Nueva Zelanda.

-Lanceoppia (L.) seydi Hammer, 1968

DisTRIBUCIÓN: Nueva Zelanda.

-Lanceoppia (L.) strenzkei Hammer, 1968

DisTRIBUCIÓN: Nueva Zelanda.

-Lanceoppia (L.) tortile Mahunka, 1989

DISTRIBUCIÓN: Tasmania.

-Lanceoppia (L.) translucens (Mahunka, 1985) (Pletzenoppia)

DisTRIBUCIÓN: Sudáfrica.

-Lanceoppia (L.) vanderhammeni Hammer, 1968

Distribución: Nueva Zelanda.

-Lanceoppia (L.) willmanni Hammer, 1968

DisTRIBUCIÓN: Nueva Zelanda.

-Lanceoppia (L.) woodringi Hammer, 1968

DistRIBUCIÓN: Nueva Zelanda.

Lanceoppia (Baioppia) Luxton, 1985 (4 spp.)

ESPECIE TIPO: Lanceoppia moritzi Hammer, 1968

Distribución: Australiana y "Paleártica".

-Lanceoppia (Baioppia) luxtoni Hammer, 1968 (L.)

DisTRIBUCIÓN: Nueva Zelanda. 
-Lanceoppia (Baioppia) menkei Hammer, 1968 (L.) DISTRIBUCIÓN: Nueva Zelanda.

-Lanceoppia (Baioppia) moritzi Hammer, 1968 (L.) DISTRIBUCIÓN: Nueva Zelanda.

-Lanceoppia (Baioppia) talacrensis Monson, 2000 DisTRIBUCIÓN: I. Británicas.

Lanceoppia (Bicristoppia) Subías, 1989 (5 spp.)

EsPeCIE TIPO: Oppia bicristata Hammer, 1962

DISTRIBUCIÓN: Subtropical austral.

-Lanceoppia (Bicristoppia) bicristata (Hammer, 1962) (Oppia) DisTRIBUCIÓN: Argentina.

-Lanceoppia (Bicristoppia) binodosa (Hammer, 1962) (Oppia) DistriBución: Chile.

-Lanceoppia (Bicristoppia) cucheana Mahunka, 1994 (L.) DISTRIBUCIÓN: Madagascar.

-Lanceoppia (Bicristoppia) feideri (Hammer, 1968) (Oppia) DISTRIBUCIÓN: Nueva Zelanda.

-Lanceoppia (Bicristoppia) kalalao Mahunka, 1977 DISTRIBUCIÓN: Madagascar.

Lanceoppia (Convergoppia) Balogh, 1983 (5 spp.)

EsPeCIE TIPO: (Oppia pletzenae Hammer, 1968, “nom. praeoc.” por Kok, 1967) =Lanceoppia (Convergoppia) neonominata nom. nov.

DisTRIBUCIÓN: Australiana.

-Lanceoppia (Convergoppia) australis (Balogh, 1982) (Teratoppia) DisTRIBUCIÓN: Australia.

-Lanceoppia (Convergoppia) ewingi Hammer, 1968 (L.) DisTRIBUCIÓN: Nueva Zelanda.

-Lanceoppia (Convergoppia) neonominata nom. nov. [=Oppia pletzenae Hammer, 1968, "nom. praeoc.” por Kok, 1967 ("hom. prim.")]

DistriBución: Nueva Zelanda.

-Lanceoppia (Convergoppia) schusteri Hammer, 1968 (L.) DisTRIBUCIÓN: Nueva Zelanda.

-Lanceoppia (Convergoppia) sellnicki Hammer, 968 (L.) DisTRIBUCIÓN: Nueva Zelanda.

Lanceoppia (Hamoppia) Hammer, 1968 (5 spp.)

EsPeCIE TIPO: Hamoppia lionsi Hammer, 1968

DisTRIBUCIÓN: Australiana.

-Lanceoppia (Hamoppia) lionsi (Hammer, 1968) (H.) DistRIBUCión: Nueva Zelanda.

-Lanceoppia (Hamoppia) schweizeri Hammer, 1968 (L.) DisTRIBUCIÓN: Nueva Zelanda.

-Lanceoppia (Hamoppia) soosi (Balogh, 1982) (Oppia) DisTRIBUCIÓN: Australia.

-Lanceoppia (Hamoppia) thamdrupi (Hammer, 1968) (H.) DisTRIBUCIÓN: Nueva Zelanda.

-Lanceoppia (Hamoppia) turki (Hammer, 1968) (Oppia) DisTRIBUCIÓN: Nueva Zelanda.

Lanceoppia (Lancelalmoppia) Subías, 1989 (14 spp.)

(=Radamoppia Mahunka, 1994 "sin. nov.")

ESPECIE TIPO: Oppia perezinigoi Hammer, 1968

DISTRIBUCIÓN: Paleotropical y austral.

-Lanceoppia (Lancelalmoppia) banksi Hammer, 1968 (Lanceop.) DISTRIBUCIÓN: Nueva Zelanda.

-Lanceoppia (Lancelalmoppia) becki Hammer, 1968 (Lanceop.) DisTRIBUCIÓN: Nueva Zelanda.

-Lanceoppia (Lancelalmoppia) berlesei Hammer, 1968 (Lanceop.) DisTRIBUCión: Nueva Zelanda.

-Lanceoppia (Lancelalmoppia) consimilis Mahunka, 1989 (Lanceop.) DisTRIBUCIÓN: Tasmania.

-Lanceoppia (Lancelalmoppia) gressitti (Wallwork, 1964) (Globoppia) DisTRIBUCIÓN: Antártica (I. Campbell).

-Lanceoppia (Lancelalmoppia) nodosa (Hammer, 1958) (Oppia) DisTRIBUCIÓN: Argentina e India (Bengala Occidental).

-Lanceoppia (Lancelalmoppia) perezinigoi (Hammer, 1968) (Oppia) DisTRIBUCIÓN: Nueva Zelanda.
-Lanceoppia (Lancelalmoppia) pertineata Mahunka, 1989 (Lanceop.) DisTRIBUCIÓN: Tasmania.

-Lanceoppia (Lancelalmoppia) rattura (Mahunka, 1985) (Pletzenoppia) DISTRIBUCIÓN: Sudáfrica.

-Lanceoppia (Lancelalmoppia) ravenala (Mahunka, 1994) (Radamoppia)

DISTRIBUCIÓN: Madagascar.

-Lanceoppia (Lancelalmoppia) tasmanica Mahunka, 1989 (Lanceop.) DISTRIBUCIÓN: Tasmania.

-Lanceoppia (Lancelalmoppia) thori Hammer, 1968 (Lanceop.) DisTRIBUCión: Nueva Zelanda.

-Lanceoppia (Lancelalmoppia) vaneki Hammer, 1968 (Lanceop.) DistRIBUCIÓN: Nueva Zelanda.

-Lanceoppia (Lancelalmoppia) zicsica (Mahunka, 1988) (Pletzenoppia)

DISTRIBUCIÓN: Tanzania

Loboppia Balogh, 1983 (1 sp.)

EsPeCIE TIPO: Oppia covarrubiasi Hammer, 1968

DISTRIBUCIÓN: Australiana.

-Loboppia covarrubiasi (Hammer, 1968) (Oppia)

DisTRIBUCIÓN: Nueva Zelanda.

Membranoppia Hammer, 1968 (14 spp.)

ESPECIE TIPO: Membranoppia krivoluzkyi Hammer, 1968

DisTRIBUCIÓn: Neotropical y austral (excepto Etiópica).

Membranoppia (Membranoppia) Hammer, 1968 (5 spp.)

(=Nesoppia Luxton, 1985)

DisTRIBUCIÓN: Neotropical y subtropical austral (excepto Etiópica)

-Membranoppia (M.) breviclava (Hammer, 1958) (Oppia)

DisTRIBUCIÓN: Argentina.

-Membranoppia (M.) krivoluzkyi Hammer, 1968

(=Membranoppia karppineni Hammer, 1968)

DistRIBUCIÓN: Nueva Zelanda.

-Membranoppia (M.) sitnikovae Hammer, 1968

DistriBUCión: Nueva Zelanda.

-Membranoppia (M.) truncata (Hammer, 1961) (Oppia)

DisTRIBUCIÓN: Perú.

-Membranoppia (M.) tuxeni Hammer, 1968

DisTRIBUCIÓN: Nueva Zelanda.

Membranoppia (Pravoppia) Luxton, 1985 (9 spp.)

EsPECIE TIPO: Oppia disjuncta Wallwork, 1964

DISTRIBUCIÓN: Neotropical austral y Antártica.

-Membranoppia (Pravoppia) argentinensis (Balogh y Csiszár, 1963)

(Oppia)

DisTRIBUCIÓN: Argentina.

-Membranoppia (Pravoppia) campbellensis (Wallwork, 1964) (Oppia)

DistRIBUCión: Antártica (I. Campbell).

-Membranoppia (Pravoppia) disjuncta (Wallwork, 1964) (Oppia)

Distribución: Antártica (I. Campbell).

-Membranoppia (Pravoppia) loxolineata (Wallwork, 1965) (Oppia)

(=Oppia loxolineata longipilosa Covarrubias, 1968)

DISTRIBUCIÓN: Antártica (frecuente).

-Membranoppia (Pravoppia) patagonica (Mahunka, 1980) (Oppia) Distribución: América austral (Tierra del Fuego)

-Membranoppia (Pravoppia) pseudocorrugata (Mahunka, 1980) (Oppia)

DISTRIBUCIÓN: América austral (Tierra del Fuego).

-Membranoppia (Pravoppia) scotiae (Wallwork, 1970) (Oppia)

Distribución: Antártica (I. Georgia del Sur).

-Membranoppia (Pravoppia) ventrolaminata (Hammer, 1962) (Oppia) DISTRIBUCIÓN: Argentina.

-Membranoppia (Pravoppia) wallworki (Mahunka, 1980) (Globoppia)

DistribuCión: América austral (Tierra del Fuego).

Operculoppia Hammer, 1968 (1 sp.)

EsPECIE TIPO: Operculoppia kunsti Hammer, 1968 
DISTRIBUCIÓN: Australiana.

-Operculoppia kunsti Hammer, 1968

DisTRIBUCIÓN: Nueva Zelanda.

Otoppia Balogh, 1983 (2 spp.)

EsPECIE TIPO: Oppia midas Balogh, 1964

DisTRIBUCIÓN: Etiópica.

-Otoppia midas (Balogh, 1964) (Oppia)

Distribución: Madagascar.

-Otoppia spinipes (Mahunka, 1994) (Lanceoppia)

DISTRIBUCIÓN: Madagascar.

Polyoppia Hammer, 1968 (2 sp.)

EsPeCIE TIPO: Polyoppia baloghi Hammer, 1968

DISTRIBUCIÓN: Tropical (Australiana y Neotropical).

-Polyoppia baloghi Hammer, 1968

DistRibución: Nueva Zelanda.

-Polyoppia magnum (Sellnick, 1924) (Dameosoma)

DistribuCión: Brasil.

Processoppia Balogh, 1983 (3 spp.)

(=Rhaphoppia Balogh, 1983)

EsPECIE TIPO: Oppia oudemansi Hammer, 1968

DISTRIBUCIÓN: Australiana.

-Processoppia mihelcicana (Ohkubo, 2003) (Rhaphoppia) (=Oppia mihelcici Hammer, 1968, “nom. praeoc.” por Pérez-Íñigo, 1965) DisTRIBUCIÓN: Nueva Zelanda.

-Processoppia oudemansi (Hammer, 1968) (Oppia)

Distribución: Nueva Zelanda.

-Processoppia sphagnicola (J. y P. Balogh, 1986) (Rhaphoppia) DisTRIBUCIÓN: Nueva Guinea.

Pustuloppia Mahunka, 1994 (1 sp.)

EsPeCIE TIPO: Pustuloppia madagassica Mahunka, 1994 DISTRIBUCIÓN: Etiópica.

-Pustuloppia madagassica Mahunka, 1994

DisTRIBUCIÓN: Madagascar.

Setoppia Balogh, 1983 (17 spp.)

(=Tectoppiella Mahunka, 1984)

ESPECIE TIPO: Oppia toeroeki Balogh, 1982

DistRIBUCIÓN: Pantropical (excepto Oriental) y austral.

-Setoppia angustopili (Hammer, 1962) (Lanceoppia)

DisTRIBUCIÓN: América austral.

-Setoppia antennata (Balogh y Mahunka, 1966) (Oppia)

DisTRIBUCIÓN: Sudáfrica.

-Setoppia bornemisszai (Balogh, 1982) (Oppia)

DisTRIBUCIÓN: Australia.

-Setoppia clavimera (Mahunka, 1985) (Tectoppiella)

DisTRIBUCión: Sudáfrica.

-Setoppia compressa (Balogh y Mahunka, 1975) (Oppia)

DisTRIBUCIÓN: Australia.

-Setoppia fortis (Balogh y Mahunka, 1966) (Oppia)

DisTRIBUCión: Sudáfrica.

-Setoppia karinae (Mahunka, 1974) (Tectoppia)

DisTRIBUCIÓN: Rodesia [Zimbabue].

-Setoppia longisetosa (Balogh y Mahunka, 1975) (Oppia)

DISTRIBUCIÓN: Australia.

-Setoppia mahunkai (Hammer, 1968) (Lanceoppia)

DisTRIBUCIÓN: Nueva Zelanda.

-Setoppia quattuor Kok, 1967 (Oppia)

DisTRIBUCIÓN: Sudáfrica.

-Setoppia strinovichi (Balogh, 1972) (Oppia)

DisTRIBUCIÓN: Australia.

-Setoppia szaboi (Mahunka, 1988) (Antennoppia)

DisTRIBUCIÓN: Tanzania.

-Setoppia toeroeki (Balogh, 1982) (Oppia)

DistriBución: Australia.

-Setoppia toxotes (Balogh, 1982) (Oppia)

DISTRIBUCIÓN: Australia.
-Setoppia tuberosa (Mahunka, 1984) (Tectoppiella)

DisTRIBUCIÓN: Sudáfrica.

-Setoppia vanga (Mahunka, 1994) (Radamoppia)

DISTRIBUCIÓN: Madagascar.

-Setoppia verrucosa (Mahunka, 1985) (Tectoppiella)

DISTRIBUCIÓN: Sudáfrica.

Trematoppia Balogh, 1964 (1 sp.)

ESPECIE TIPO: Trematoppia cristipes Balogh, 1964

DISTRIBUCIÓN: Etiópica.

-Trematoppia cristipes Balogh, 1964

DISTRIBUCIÓN: Madagascar.

[Chaviniinae] Balogh, 1983 (1 gen. y 2 spp.) DisTRIBUCIÓN: Neotropical.

Chavinia Hammer, 1961 (2 spp.)

EsPeCIE TIPO: Chavinia paradoxa Hammer, 1961

DisTRIBUCIÓN: Neotropical.

-Chavinia paradoxa Hammer, 1961

DisTRIBUCIÓN: Neotropical (Perú y Ecuador)

-Chavinia similis P. Balogh, 1984

DistriBución: Colombia.

[Oppiinae] Sellnick, 1937 (23 gén., 3 subg., 150 spp. y 3 sspp.) (=[Dameosominae $]$ Sellnick, 1928) DistribuCión: Cosmopolita.

Aeroppia Hammer, 1961 (13 spp.)

EsPECIE TIPO: Aeroppia peruensis Hammer, 1961

DisTRIBUCIÓN: Neotropical y Neártica.

-Aeroppia abdita Pérez-Íñigo y Baggio, 1997

DistriBuCión: Brasil.

-Aeroppia adjacens Mahunka, 1985

DistRiBuCiÓN: Neotropical (Pequeñas Antillas e I. Galápagos). -Aeroppia asymmetrica Mahunka, 1985

Distribución: Pequeñas Antillas.

-Aeroppia clavatum Higgins, 1966

DisTRIBUCIÓN: Guyana [Guayana Británica].

-Aeroppia floridana (Banks, 1896) (Belba)

(=Oribata consimilis Banks, 1910)

DisTRIBUCIÓN: Sureste de U.S.A.

-Aeroppia hammerae Mahunka, 1985

DistriBución: Pequeñas Antillas.

-Aeroppia insularis Higgins, 1966

Distribución: Antillas (La Española: R. Dominicana).

-Aeroppia magnipilosa (Ewing, 1909) (Damaeus)

(=Aeroppia columbiana Hammer, 1961)

DistRIBUCIÓN: U.S.A. (excepto Sur).

-Aeroppia mariehammerae Subías et al., 2004

DisTRIBUCIÓN: Neotropical (Chile y Panamá).

-Aeroppia nasalis Mahunka, 1984

DisTRIBUCIÓN: Paraguay.

-Aeroppia peruensis Hammer, 1961

DisTRIBUCIÓN: Perú.

-Aeroppia sculpturata Mahunka, 1985

DistRIBUCIÓN: Pequeñas Antillas.

-Aeroppia vacua (Berlese, 1888) (Belba concolor v.)

DisTRIBUCIÓN: Neotropical.

Aethioppia Balogh, 1983 (3 spp.)

EsPeCIE Tipo: Oppia bacilligera Balogh, 1962

DISTRIBUCIÓN: Etiópica.

-Aethioppia bacilligera (Balogh, 1962) (Oppia)

DisTRIBUCIÓN: Tanzania.

-Aethioppia oligochaeta (Mahunka, 1984) (Xenoppia)

DisTRIBUCIÓN: Tanzania.

-Aethioppia spinipes (Balogh, 1964) (Oppia)

DISTRIBUCIÓN: Madagascar. 
Amerioppia Hammer, 1961 (2 spp.)

ESPECIE TIPO: Amerioppia rudentigera Hammer, 1961

DISTRIBUCIÓN: Tropical (Etiópica y Neotropical).

-Amerioppia meruensis (Balogh, 1961) (Oppia)

DISTRIBUCIÓN: Tanzania.

-Amerioppia rudentigera Hammer, 1961

DISTRIBUCIÓN: Perú.

Erioppia Balogh, 1983 (1 sp. y 1 ssp.)

EsPeCIE TIPO: Multioppia problematica Balogh, 1966

DisTRIBUCIÓN: Tropical (Etiópica y Australiana).

-Erioppia problematica (Balogh, 1966) (Multioppia)

DisTRIBUCIÓN: África oriental y Polinesia (I. Samoa)

-Erioppia problematica problematica (Balogh, 1966)

DisTRIBUCIÓN: Africa oriental.

-Erioppia problematica pacifica J. y P. Balogh, 1986

Distribución: Polinesia (I. Samoa).

Exanthoppia J. y P. Balogh, 1983 (1 sp.)

EsPECIE TIPO: Exanthoppia ornatissima J. y P. Balogh, 1983

DISTRIBUCIÓN: Australiana.

-Exanthoppia ornatissima J. y P. Balogh, 1983

DISTRIBUCIÓN: Hawai.

Fusuloppia Balogh, 1983 (2 spp.)

EsPECIE TIPO: (Oppia simplex Balogh, 1962, “nom. praeoc.” por Jacot, 1938) =Fusuloppia neonominata nom. nov.

DISTRIBUCIÓN: Etiópica.

-Fusuloppia fusuligera (Balogh, 1962) (Oppia)

DisTRIBUCión: Tanzania.

-Fusuloppia neonominata nom. nov.

[=Oppia simplex Balogh, 1964, "nom. praeoc." por Jacot, 1938 ("hom. prim.”)] DISTRIBUCIÓN: Madagascar.

Goyoppia Balogh, 1983 (3 spp.)

ESPECIE TIPO: Oppia sexpilosa Balogh, 1961

DisTRIBUCIÓN: Etiópica y Paleártica meridional

-Goyoppia longissima (Wen, 1987) (Oppia)

DISTRIBUCIÓN: China suroriental.

-Goyoppia sagami (Aoki, 1984) (Oppia)

Distribución: Este de Paleártica oriental.

-Goyoppia sexpilosa (Balogh, 1961) (Oppia)

DISTRIBUCIÓN: Madagascar.

Heteroppia Balogh, 1970 (3 spp.)

ESPECIE TIPO: Heteroppia globigera Balogh, 1970

DisTRIBUCIÓN: Australianooriental.

-Heteroppia globigera Balogh, 1970

DistRIBUCIÓN: Ceilán [Sri Lanka].

-Heteroppia orthodactyla (Willmann, 1931) (Oppia)

DISTRIBUCIÓN: Java.

-Heteroppia pauciseta (Hammer, 1971) (Globoppia (Aeroppia))

DisTRIBUCIÓN: Melanesia (I. Fiji) y Filipinas.

Laroppia Subías, 1989 (1 sp.)

EsPECIE TIPO: Oppia petiolata Wallwork, 1977

Distribución: Atlántico Sur.

-Laroppia petiolata (Wallwork, 1977) (Oppia)

DisTRIBUCIÓN: I. Santa Helena.

Lasiobelba Aoki, 1959 (31 spp.)

EsPeCIE TIPO: Lasiobelba remota Aoki, 1959

DisTRIBUCIÓN: Cosmopolita (excepto Antártica).

Lasiobelba (Lasiobelba) Aoki, 1959 (17 spp.)

(=Cilioppia Balogh, 1983)

DisTRIBUCIÓN: Pantropical y subtropical.

-Lasiobelba (L.) arabica Mahunka, 2000

DisTRIBUCIÓN: Yemen.
-Lasiobelba (L.) arcidiaconoae (Bernini, 1973) (Oppia)

DisTRIBUCIÓN: Mediterránea e India (Tripura).

-Lasiobelba (L.) decui (Vasiliu e Ivan, 1995) (Cilioppia)

DISTRIBUCIÓN: Israel.

-Lasiobelba (L.) gibbosa (Mahunka, 1985) (Oppia)

DisTRIBUCión: Etiópica (Angola y Malawi).

-Lasiobelba (L.) hesperidiana (Pérez-Íñigo, 1986) (Cilioppia)

DisTRIBUCIÓN: I. Canarias.

-Lasiobelba (L.) insulata Ohkubo, 2001

DISTRIBUCIÓN: Japón.

-Lasiobelba (L.) kuehnelti (Csiszár, 1961) (Oppia)

(=Oppia yodai Aoki, 1965)

DisTRIBUCIÓN: Oriental (frecuente) y Australiana (Indonesia: Komodo, y Hawai).

-Lasiobelba (L.) lemuria Mahunka, 1997

DisTRIBUCIÓN: Madagascar.

-Lasiobelba (L.) neonominata nom. nov.

[=Oppia yodai africana Kok, 1967, "nom. praeoc." por Evans, 1953) ("hom. prim.")]

Distribución: Etiópica (Sudáfrica y Malgache), Hawai y Mediterránea occidental.

-Lasiobelba (L.) pori (Vasiliu e Ivan, 1995) (Cilioppia)

DISTRIBUCIÓN: Israel.

-Lasiobelba (L.) remota Aoki, 1959

DisTRIBUCIÓN: Este de Paleártica oriental y Noreste de Oriental.

-Lasiobelba (L.) rubida (Wallwork, 1977) (Oppia)

DisTRIBUCióN: I. Santa Helena.

-Lasiobelba (L.) sculpta Wang, 1993

DISTRIBUCIÓN: China suroriental.

-Lasiobelba (L.) subuligera (Berlese, 1916) (Dameosoma denticulatum s.)

DisTRIBUCIÓN: Argentina.

-Lasiobelba (L.) suchetae Sanyal, 1992

Distribución: India (Bengala Occidental).

-Lasiobelba (L.) vietnamica Balogh, 1983

DisTRIBUCIÓN: Vietnam.

-Lasiobelba (L.) yunnanensis Wen, 1999

DISTRIBUCIÓN: China suroriental.

Lasiobelba (Antennoppia) Mahunka, 1983 (14 spp.)

=Daedaloppia Hauser y Mahunka, 1983)

EsPECIE TIPO: Antennoppia minor Mahunka, 1983

DisTRIBUCIÓN: Pantropical, subtropical (Paleártica meridional) y Neártica.

-Lasiobelba (Antennoppia) abchasica (Golosova y Tarba, 1974) (Oppia) DisTRIBUCIÓN: Sureste de Europa.

-Lasiobelba (Antennoppia) capilligera (Berlese, 1916) (Dameosoma) DisTRIBUCIÓN: África oriental.

-Lasiobelba (Antennoppia) granulata (Mahunka, 1986) (A.)

DISTRIBUCIÓN: Tanzania.

-Lasiobelba (Antennoppia) heterosa (Wallwork, 1964) (Oppia) DISTRIBUCIÓN: Chad.

-Lasiobelba (Antennoppia) insignis Balogh, 1970 (L.)

DisTRIBUCIÓN: Nueva Guinea.

-Lasiobelba (Antennoppia) izquierdoae Arillo, Gil-Martín y Subías, 1994 (L.)

DistriBución: I. Canarias.

-Lasiobelba (Antennoppia) major (Mahunka, 1983) (A.) DisTRIBUCIÓN: Tanzania.

-Lasiobelba (Antennoppia) minor (Mahunka, 1983) (A.) DisTRIBUCIÓN: Tanzania.

-Lasiobelba (Antennoppia) quadriseta Subías, 1989 (L.) (=Lasiobelba icaria Mahunka, 2001)

DISTRIBUCIÓN: Grecia.

-Lasiobelba (Antennoppia) rigida (Ewing, 1909) (Damaeus)

DisTRIBUCIÓN: Noreste de U.S.A.

-Lasiobelba (Antennoppia) subnitida (Sellnick, 1924) (Dameosoma) DisTRIBUCIÓN: Brasil

-Lasiobelba (Antennoppia) trichoseta (Mahunka, 1983) (A.)

DisTRIBUCIÓN: Tanzania. 
-Lasiobelba (Antennoppia) ultraciliata (Jacot, 1934) (Dameosoma) DistriBución: Australiana (Islas del Pacífico).

-Lasiobelba (Antennoppia) yoshii (Mahunka, 1987) (A.)

DisTRIBUCIÓN: Borneo.

Lemuroppia Mahunka, 1994 (1 sp.)

EsPECIE TIPO: Lemuroppia helleri Mahunka, 1994

DISTRIBUCIÓN: Etiópica.

-Lemuroppia helleri Mahunka, 1994

DISTRIBUCIÓN: Madagascar.

Neoamerioppia Subías, 1989 (42 spp. y 1 ssp.)

EsPeCIE TIPO: Amerioppia decemsetosa Hammer, 1973

DisTRIBUCIÓN: Pantropical y subtropical.

Neoamerioppia (Neoamerioppia) Subías, 1989 (35 spp. y 1 ssp.) DisTRIBUCIÓN: Pantropical y subtropical.

-Neoamerioppia (N.) aelleni (Mahunka, 1982) (Amerioppia)

Distribución: Melanesia (I. Fiji).

-Neoamerioppia (N.) africana (Kok, 1967) (Amerioppia)

DisTRIBUCIÓN: Sudáfrica y subantártica (I. San Pablo y Amsterdam)

-Neoamerioppia (N.) asiatica (Hammer, 1977) (Amerioppia) DisTRIBUCIón: Himalaya.

-Neoamerioppia (N.) chavinensis (Hammer, 1961) (Amerioppia) Distribución: Perú e India (Bengala Occidental).

-Neoamerioppia (N.) chilensis (Hammer, 1962) (Amerioppia) DisTRIBUCión: Chile.

-Neoamerioppia (N.) cocuyana (P. Balogh, 1984) (Amerioppia) DistriBUCIÓN: Neotropical (Colombia y Venezuela).

-Neoamerioppia (N.) decemsetosa (Hammer, 1973) (Amerioppia) Distribución: Polinesia (I. Tonga).

-Neoamerioppia (N.) deficiens (Balogh, 1959) (Oppia)

DisTRIBUCIÓn: Congo.

-Neoamerioppia (N.) extrusa (Mahunka, 1983) (Amerioppia) DisTRIBUCIÓN: Tanzania.

-Neoamerioppia (N.) flagellata (Hammer, 1975) (Amerioppia) DisTRIBUCIÓN: Sahara.

-Neoamerioppia (N.) flagelliapex Ohkubo y Aoki, 1995

Distribución: Micronesia (I. Marianas).

-Neoamerioppia (N.) foveolata (Mahunka, 1984) (Amerioppia) DISTRIBUCIÓN: Tanzania.

-Neoamerioppia (N.) hamidi (Al-Assiuty y El-Deeb, 1983) (Amerioppia)

DisTRIBUCIÓN: Egipto.

-Neoamerioppia (N.) hexapilis (Hammer, 1961) (Amerioppia) DisTRIBUCIÓN: Perú.

-Neoamerioppia (N.) interrrogata (Mahunka, 1976) (Amerioppia) Distribución: Oriental (China: Hong-Kong, y Filipinas).

-Neoamerioppia (N.) lanceolata (Hammer, 1958) (Oppia)

DisTRIBUCIÓN: Neotropical.

-Neoamerioppia (N.) longiclava (Hammer, 1962) (Amerioppia) DISTRIBUCIÓN: Neotropical y Australiana (Nueva Zelanda y Nueva Guinea).

-Neoamerioppia (N.) longiclava longiclava (Hammer, 1962) DisTRIBUCIÓN: Neotropical y Nueva Zelanda.

-Neoamerioppia (N.) longiclava microseta (J. y P. Balogh, 1986) (Amerioppia)

DisTRIBUCIÓN: Nueva Guinea.

-Neoamerioppia (N.) longicoma (Hammer, 1958) (Oppia) Distribución: Bolivia.

-Neoamerioppia (N.) minima (Hammer, 1961) (Amerioppia) DisTRIBUCIÓN: Perú.

-Neoamerioppia (N.) nagyi (Mahunka, 1969) (Oppia) DisTRIBUCIÓN: Tanzania.

-Neoamerioppia (N.) notata (Hammer, 1958) (Oppia) DISTRIBUCIÓN: Bolivia.

-Neoamerioppia (N.) papuana (J. y P. Balogh, 1986) (Amerioppia) DistRIBUCIÓn: Nueva Guinea.

-Neoamerioppia (N.) paripilis (Hammer, 1961) (Amerioppia) DisTRIBUCIÓN: Perú.
-Neoamerioppia (N.) pectigera (Hammer, 1961) (Amerioppia)

Distribución: Neotropical (Perú y Chile).

-Neoamerioppia (N.) phoretica (Franklin y Woas, 1992) (Oppia) DISTRIBUCIÓN: Brasil.

-Neoamerioppia (N.) polygonata (Mahunka, 1982) (Amerioppia)

DisTRIBUCIÓN: Etiopía.

-Neoamerioppia (N.) rotunda (Hammer, 1958) (Oppia)

DISTRIBUCIÓN: Neotropical (Bolivia y Chile).

-Neoamerioppia (N.) similis (Covarrubias, 1967) (Amerioppia)

DisTRIBUCIÓN: Chile.

-Neoamerioppia (N.) sturmi (P. Balogh, 1984) (Amerioppia)

DisTRIBUCIÓN: Neotropical (Colombia y Venezuela).

-Neoamerioppia (N.) trichosa (Hammer, 1958) (Oppia)

DistriBuCión: Neotropical (Bolivia y Perú).

-Neoamerioppia (N.) trichosoides (Hammer, 1961) (Amerioppia) DistRIBUCIÓN: Perú.

-Neoamerioppia (N.) ventrosquamosa (Hammer, 1979) (Amerioppia)

DisTRIBUCIÓN: Oriental (Indonesia), Australiana (Micronesia: I.

Marianas) y Japón

-Neoamerioppia (N.) vicina (Hammer, 1971) (Amerioppia)

DistribuCIÓn: Melanesia (I. Fiji) y Java.

-Neoamerioppia (N.) vietnamica (Mahunka, 1988) (Amerioppia)

DISTRIBUCIÓN: Vietnam.

-Neoamerioppia (N.) woolleyi (Hammer, 1968) (Amerioppia)

DISTRIBUCIÓN: Australiana.

Neoamerioppia (Amerigloboppia) Subías, 1989 (7 spp.)

EsPECIE TIPO: Amerioppia espeletiarum P. Balogh, 1984

DisTRIBUCIÓN: Tropical (Neotropical y Etiópica) y "Paleártica".

-Neoamerioppia (Amerigloboppia) badensis (Woas, 1986) (Oppia)

DisTRIBUCIÓN: Alemania.

-Neoamerioppia (Amerigloboppia) centraliamericana (Mahunka, 1983) (Globoppia)

DisTRIBUCIÓN: Norte de Neotropical.

-Neoamerioppia (Amerigloboppia) costulifera Mahunka, 1997 (N.)

DisTRIBUCIÓN: Kenia.

-Neoamerioppia (Amerigloboppia) espeletiarum (P. Balogh, 1984)

(Amerioppia)

DisTRIBUCIÓN: Neotropical (Colombia e I. Galápagos).

-Neoamerioppia (Amerigloboppia) extrema (Mahunka, 1985)

(Amerioppia)

DistRiBUCión: Neotropical (Pequeñas Antillas e I. Galápagos).

-Neoamerioppia (Amerigloboppia) salvadoriensis (Woas, 1986)

(Oppia)

DisTRIBUCIÓN: Centroamérica.

-Neoamerioppia (Amerigloboppia) senecionis (P. Balogh, 1984)

(Amerioppia)

DistribuCIÓN: Neotropical (Colombia e I. Galápagos).

Niloppia Balogh, 1983 (1 sp.)

EsPeCIE TiPo: Oppia sticta Popp, 1960

DisTRIBUCIÓN: Paleártica.

-Niloppia sticta (Popp, 1960) (Oppia)

DistriBUCIÓN: Egipto.

Oligoppia Balogh, 1983 (1 sp.)

ESPECIE TIPO: Amerioppia octocoma Hammer, 1973

DISTRIBUCIÓN: Australiana.

-Oligoppia octocoma (Hammer, 1973) (Amerioppia)

Distribución: Polinesia (I. Samoa).

Oppia Koch, 1836 (21 spp. y 1 ssp.)

(=Dameosoma Berlese, 1892)

ESPECIE TIPO: Oppia nitens Koch, 1836

DistriBución: Cosmopolita (excepto Australiana).

-Oppia capense (Paoli, 1908) (Dameosoma)

DistRIBUCión: Sudáfrica y "Alemania".

-Oppia cephalota (Hall, 1911) (Damaeus) "sp. inq."

DISTRIBUCIÓN: U.S.A. (Connecticut) 
-Oppia coloradensis Woolley, 1969

DisTRIBUCIÓN: U.S.A. (Colorado).

-Oppia concolor Koch, 1839 "sp. inq."

DISTRIBUCIÓN: Alemania.

-Oppia denticulata (G. y R. Canestrini, 1882) (Belba)

(=Oppia cyclosoma Mihelcic, 1955)

(=Oppia grandis Mihelcic, 1955)

(=Oppia willmanni Oudemans, 1937)

DisTRIBUCIÓN: Paleártica (Paleártica occidental: menos frecuente en

el Norte, y Sur de Siberia) y Antillas (La Española: R. Dominicana).

-Oppia elegans (Trägardh, 1931) (Dameosoma) "sp. inq."

Distribución: Chile (I. Juan Fernández).

-Oppia elongata Jacot, 1938 "sp. inq."

(=Oppia parviaures Jacot, 1939)

DisTRIBUCión: U.S.A. (Carolina del Norte).

-Oppia ewingi (Berlese, 1916) (Dameosoma denticulatum e.)

DisTRIBUCIÓN: U.S.A. (Ilinois y Florida).

-Oppia flagellifera Wang, 1993

DistriBución: China suroriental.

-Oppia huangshanensis Wen y Chen, 1991

DisTRIBUCIÓN: Este de China.

-Oppia hungarica Karpelles, 1893 "sp. inq."

DisTRIBUCIÓN: Hungría.

-Oppia incisirostra Woas, 1986

DisTRIBUCIÓN: El Salvador.

-Oppia leleupi Balogh, 1958 "sp. inq."

DisTRIBUCIÓN: Congo.

-Oppia neonominata nom. nov.

[=Oppia concolor tridentata Pérez-Íñigo, 1976, “nom. praeoc." por Forsslund, 1942 ("hom. prim.")]

DistriBución: I. Canarias.

-Oppia nitens Koch, 1836

DisTRIBUCIÓN: Holártica (Paleártica occidental, Asia centroocci-

dental y Neártica oriental) y Antártica.

-Oppia nitens nitens Koch, 1836

(=Belba minuta Banks, 1895)

(=Dameosoma myrmecophila Sellnick, 1928)

(=Oribata perolata Banks, 1909)

DistRIBUCIÓN: Holártica (Paleártica occidental: menos frecuente en el Norte, Asia centrooccidental y Neártica oriental).

-Oppia nitens brachytrichinus Dalenius, 1958

DistribuCión: Antártica (I. Crozet).

-Oppia orientalis Sanyal y Bhaduri, 1989

DisTRIBUCIÓN: India (Bengala Occidental).

-Oppia quadrisetosa (Kramer, 1898) (Notaspis) "sp. inq."

DistriBución: América austral (Tierra del Fuego).

-Oppia samadi Kardar, 1976

Distribución: India (Uttar Pradesh).

-Oppia seminuda Scull, Jeleva y Cruz, 1984

DisTRIBUCIÓN: Cuba.

-Oppia speciosa Golosova, 1981

DisTRIBUCIÓN: I. Kuriles.

-Oppia vitrea (Tseng, 1982) (Brachioppia)

DisTRIBUCIÓN: Taiwan [Formosa].

Paroppia Hammer, 1968 (4 spp.)

EsPecie Tipo: Paroppia lebruni Hammer, 1968

DisTRIBUCIÓN: Australiana y Etiópica.

-Paroppia breviseta (Balogh, 1962) (Oppia)

DistriBución: Tanzania.

-Paroppia flagellata J. y P. Balogh, 1983

DisTRIBUCIÓN: Hawai.

-Paroppia hawaiiensis J. y P. Balogh, 1983

DisTRIBUCIÓN: Hawai.

-Paroppia lebruni Hammer, 1968

DisTRIBUCIÓN: Nueva Zelanda.

Pluritrichoppia Subías y Arillo, 1989 (1 sp.)

EsPeCIE TIPO: Pluritrichoppia insolita Subías y Arillo, 1989

DisTRIBUCIÓN: Paleártica.
-Pluritrichoppia insolita Subías y Arillo, 1989

DisTRIBUCIÓN: España.

Quinquoppia Tseng, 1982 (2 spp.)

(=Damaeoppia Ohkubo, 1995 "sin. nov.")

ESPECIE TIPO: Quinquoppia nobilis Tseng, 1982

DISTRIBUCIÓN: Oriental.

-Quinquoppia formosana (Ohkubo, 1995) (Damaeoppia)

DistRIBUCIÓN: Taiwan [Formosa].

-Quinquoppia nobilis Tseng, 1982

DisTRIBUCIÓN: Taiwan [Formosa].

Sphagnoppia J. y P. Balogh, 1986 (2 spp.)

EsPECIE TIPO: Sphagnoppia biflagellata J. y P. Balogh, 1986

DisTRIBUCIÓN: Tropical (Australiana) y subtropical (Neártica meridional).

-Sphagnoppia biflagellata J. y P. Balogh, 1986

Distribución: Nueva Guinea.

-Sphagnoppia durhamensis (Metz y Sharma, 1975) (Oppia)

DistribuCión: U.S.A. (Carolina del Norte).

Taiwanoppia Tseng, 1982 (10 spp.)

EsPECIE TIPO: Taiwanoppia subtropica Tseng, 1982

DISTRIBUCIÓN: Pantropical y subtropical.

Taiwanoppia (Taiwanoppia) Tseng, 1982 (4 spp.)

(=Vietoppia Mahunka, 1988)

DisTRIBUCIÓN: Oriental.

-Taiwanoppia (T.) fujianensis (Wang, 1993) (Vietoppia)

DISTRIBUCIÓN: China suroriental.

-Taiwanoppia (T.) hungarorum (Mahunka, 1988) (Vietoppia)

DisTRIBUCIÓN: Vietnam.

-Taiwanoppia (T.) papillaris Tseng, 1982

DisTRIBUCIÓN: Taiwan [Formosa].

-Taiwanoppia (T.) subtropica Tseng, 1982

DisTRIBUCIÓN: Taiwan [Formosa].

Taiwanoppia (Paragloboppia) Subías, 1989 (6 spp.)

EsPECIE TIPO: Oppia diversiseta Mahunka, 1985

DisTRIBUCIÓN: Pantropical (excepto Oriental) y subtropical.

-Taiwanoppia (Paragloboppia) calva (Warburton, 1912) (Notaspis clavipectinata c.)

DisTRIBUCIÓN: I. Seychelles.

-Taiwanoppia (Paragloboppia) diversiseta (Mahunka, 1985) (Oppia) DisTRIBUCIÓN: Sudáfrica.

-Taiwanoppia (Paragloboppia) mercedesae (Arillo y Subías, 1997)

(Vietoppia (P.))

DisTRIBUCIÓN: España.

-Taiwanoppia (Paragloboppia) pacifica (Ohkubo y Aoki, 1995)

(Vietoppia (P.))

DistRIBUCIÓN: Micronesia (I. Marianas).

-Taiwanoppia (Paragloboppia) senegalensis (Mahunka, 1975) (Oppia) DisTRIBUCIÓn: Senegal.

-Taiwanoppia (Paragloboppia) trichotos (Balogh y Mahunka, 1977)

(Oppia)

DISTRIBUCIÓN: Brasil.

Tanzoppia Mahunka, 1988 (1 sp.)

ESPECIE TIPO: Tanzoppia triseta Mahunka, 1988

DISTRIBUCIÓN: Etiópica.

-Tanzoppia triseta Mahunka, 1988

DISTRIBUCIÓN: Tanzania.

Tectoppia Wallwork, 1961 (3 spp.)

ESPECIE TIPO: Tectoppia nigricans Wallwork, 1961

DISTRIBUCIÓN: Etiópica.

-Tectoppia costulata Mahunka, 1988

DisTRIBUCIÓN: Tanzania.

-Tectoppia longisetosa Mahunka, 1974

DisTRIBUCIÓN: Camerún. 
-Tectoppia nigricans Wallwork, 1961

DisTRIBUCIÓN: Ghana.

Xenoppia Mahunka, 1982 (1 sp.)

ESPECIE TIPO: Xenoppia brevipila Mahunka, 1982

DISTRIBUCIÓN: Etiópica.

-Xenoppia brevipila Mahunka, 1982

DisTRIBUCIÓN: Etiopía.

[Multioppiinae] Balogh, 1983 (17 gen., 8 subg., 165 spp. y 7 sspp.) DISTRIBUCIÓN: Cosmopolita.

Anomaloppia Subías, 1978 (9 spp.)

ESPECIE TIPO: Anomaloppia canariensis Subías, 1978

Distribución: Semicosmopolita (Holártica, Etiópica, Neotropical y Antártica).

-Anomaloppia canariensis Subías, 1978

DistriBución: I. Canarias.

-Anomaloppia chitinofincta (Kulijev, 1962) (Oppia)

DisTRIBUCIÓN: Paleártica meridional (Mediterránea oriental y Asia central rusa.

-Anomaloppia differens Mahunka y Topercer, 1983

DISTRIBUCIÓN: Europa centromeridional.

-Anomaloppia dispariseta (Hammer, 1958) (Oppia)

DisTRIBUCión: América austral y subantártica: I. Malvinas [I Falkland].

-Anomaloppia iranica Bayartogtokh y Akrami, 2000

DisTRIBUCIÓN: Irán.

-Anomaloppia madeirensis Arillo y Subías, 1990

DisTRIBUCIÓN: Macaronésica (Madeira).

-Anomaloppia manifera (Hammer, 1955) (Oppia)

DistriBución: Boreal (Paleártica: excepto Sur, y Alaska).

-Anomaloppia ozkani Ayyildiz, 1989

DisTRIBUCIÓN: Turquía.

-Anomaloppia peregovitsi (Mahunka, 1986) (Insculptoppia)

DisTRIBUCIÓN: Tanzania.

Condyloppia Balogh, 1983 (2 spp. y 1 ssp.)

EsPECIE TIPO: Oppia condylifer Hammer, 1979

DISTRIBUCIÓN: Paleotropical.

-Condyloppia condylifer (Hammer, 1979) (Oppia)

DisTRIBUCIÓN: Oriental.

-Condyloppia pilosella (Balogh, 1959) (Oppia)

DisTRIBUCIÓN: Etiópica (Congo y Chad).

-Condyloppia pilosella pilosella (Balogh, 1959) Distribución: Congo.

-Condyloppia pilosella longiseta (Wallwork, 1964) (Oppia)

DISTRIBUCIÓN: Chad.

Cryptoppia Csiszár, 1961 (3 spp.)

EsPECIE TIPO: Cryptoppia elongata Csiszár, 196

DisTRIBUCIÓN: Oriental.

-Cryptoppia brevisetiger Wen, Aoki y Wang, 1984

DISTRIBUCIÓN: China suroriental.

-Cryptoppia elongata Csiszár, 1961

DISTRIBUCIÓN: Oriental (Java y Vietnam).

-Cryptoppia mahunkai (Wang y Li, 1997) (Pulchroppia)

(=Pulchroppia elegans Mahunka, 1988, "nom. praeoc.” por Hammer, 1979)

(=Pulchroppia mahunkarum J. y P. Balogh, 2002)

DISTRIBUCIÓN: Borneo.

Cubaoppia Balogh, 1983 (1 sp.)

EsPECIE TIPO: Oppia fusisetosa Balogh y Mahunka, 1980

DISTRIBUCIÓN: Neotropical.

-Cubaoppia fusisetosa (Balogh y Mahunka, 1980) (Oppia)

DisTRIBUCIÓN: Cuba.

Graptoppia Balogh, 1983 (16 spp. y 1 ssp.)

ESPECIE TIPO: (Dameosoma foveolatum Paoli "sensu" Bernini, 1973)

=Graptoppia paraanalis Subías y Rodríguez, 1985

DisTRIBUCIÓN: Pantropical (excepto Australiana), subtropical y austral.
Graptoppia (Graptoppia) Balogh, 1983 (9 spp. y 1 ssp.)

(=Frondoppia Mahunka, 1983)

DisTRIBUCIÓN: Pantropical (excepto Australiana) y subtropical.

-Graptoppia (G.) arenaria Ohkubo, 1993

DisTRIBUCIÓN: Japón.

-Graptoppia (G.) exigua (Mahunka, 1983) (Frondoppia)

DisTRIBUCión: Neotropical (Brasil e I. Galápagos).

-Graptoppia (G.) mussardi Mahunka, 1992

DisTRIBUCIÓN: Senegal.

-Graptoppia (G.) neonominata nom. nov.

[=Oppia parva Kok, 1967, "nom. praeoc." por Lombardini, 1952 ("hom. prim.")]

DisTRIBUCiÓN: Sudáfrica y Sur de Europa.

-Graptoppia (G.) nukusia (Shtanchaeva, 1984) (Oppia)

DisTRIBUCIÓN: Asia centrooccidental.

-Graptoppia (G.) paraanalis Subías y Rodríguez, 1985

(=Stenoppia italica quinquepilosa Morell, 1987)

DistriBUCión: Paleártica occidental (menos frecuente en el Norte).

-Graptoppia (G.) paradoxa Ivan y Vasiliu, 1997

Distribución: Rumania.

-Graptoppia (G.) sundensis (Hammer, 1979) (Oppia)

DisTRIBUCIÓN: Oriental y Turquía.

-Graptoppia (G.) sundensis sundensis (Hammer, 1979) DisTRIBUCIÓN: Oriental.

-Graptoppia (G.) sundensis acuta Ayyildiz, 1989 DISTRIBUCIÓN: Turquía.

-Graptoppia (G.) tanaitica Karppinen y Poltavskaja, 1990

DistRIBUCIÓN: Sureste de Europa.

Graptoppia (Apograptoppia) Subías y Rodríguez, 1985 (1 sp.)

EsPeCIE TIPO: Dameosoma foveolatum Paoli, 1908

DISTRIBUCIÓN: Holártica.

-Graptoppia (Apograptoppia) foveolata (Paoli, 1908) (Dameosoma) (=Graptoppia (Apograptoppia) paolii Ohkubo, 1993)

DistribuCión: Sur de Europa y Canadá.

Graptoppia (Stenoppia) Balogh, 1983 (6 spp.)

EsPECIE TIPO: (Oppia heterotricha Bernini, 1969) =Oppia italica Bernini, 1973

DistribuCIÓn: Semicosmopolita (Etiópica, Neotropical, subtropical y austral).

-Graptoppia (Stenoppia) africana Mahunka, 1987 (G.)

DISTRIBUCIÓN: Nigeria.

-Graptoppia (Stenoppia) angusta (Hammer, 1962) (Oppia)

DistriBución: América austral.

-Graptoppia (Stenoppia) cristata Ohkubo, 1996

DisTRIBUCIÓN: Japón.

-Graptoppia (Stenoppia) italica (Bernini, 1973) (Oppia)

(=Oppia heterotricha Bernini, 1969, “nom. praeoc." por Balogh y

Mahunka, 1969)

DisTRIBUCIÓN: Mediterránea occidental.

-Graptoppia (Stenoppia) multicorrugata (Hammer, 1962) (Oppia)

DistriBución: Chile.

-Graptoppia (Stenoppia) quathlambae (Kok, 1967) (Brachioppia)

DisTRIBUCIÓN: Sudáfrica.

Helioppia Balogh, 1983 (1 sp.)

EsPeCIE TiPo: Oppia sol Balogh, 1959

DISTRIBUCIÓN: Etiópica.

-Helioppia sol (Balogh, 1959) (Oppia)

DISTRIBUCIÓN: Etiópica.

Intermedioppia Subías y Rodríguez, 1987 (1sp.)

EsPECIE TIPO: Oppia alvarezi Pérez-Iñigo, 1982

DISTRIBUCIÓN: Etiópica.

-Intermedioppia alvarezi (Pérez-Íñigo, 1982) (Oppia)

DistribuCión: Guinea Ecuatorial: I. Pagalu [Annobón]. 
Javieroppia Mínguez y Subías, 1986 (1sp.)

EsPecie Tipo: Javieroppia cervus Mínguez y Subías, 1986

DISTRIBUCIÓN: Paleártica meridional y Australiana.

-Javieroppia cervus Mínguez y Subías, 1986

DisTRIBUCIÓN: España y Australia.

Multioppia Hammer, 1961 (41 spp. y 3 sspp.)

ESPECIE TIPO: Multioppia radiata Hammer, 1961

DISTRIBUCIÓN: Cosmopolita (excepto Antártica).

Multioppia (Multioppia) Hammer, 1961 (36 spp. y 2 sspp.)

DisTRIBUCIÓN: Cosmopolita (excepto Antártica).

-Multioppia (M.) australis Hammer, 1962

DisTRIBUCIÓN: Chile.

-Multioppia (M.) bacilliseta Ohkubo, 1996

DisTRIBUCIÓN: Japón.

-Multioppia (M.) canariensis Pérez-Íñigo y Peña, 1996

DistRIBUCIÓN: I. Canarias.

-Multioppia (M.) engelmanni (Calugar y Vasiliu, 1983) (Oppia)

DisTRIBUCIÓN: Cuba.

-Multioppia (M.) formosana Tseng, 1982

DisTRIBUCIÓN: Taiwan [Formosa].

-Multioppia (M.) furugelma Rjabinin, 1987

DisTRIBUCIÓN: Este de Rusia asiática.

-Multioppia (M.) gapsaensis Choi, 1986

DisTRIBUCIÓN: Corea.

-Multioppia (M.) ghiljarovi (Kulijev, 1962) (Oppia)

DisTRIBUCIÓN: Sureste de Europa

-Multioppia (M.) gilmartinoi Subías y Arillo, 1996

DisTRIBUCIÓN: España.

-Multioppia (M.) glabra (Mihelcic, 1955) (Oppia)

DisTRIBUCIÓN: Europa centrooriental y Oeste de Siberia.

-Multioppia (M.) gracilis Hammer, 1972

Distribución: Polinesia (Tahití), India (Tripura) y "China".

-Multioppia (M.) indica Haq, 1978

DisTRIBUCIÓN: India (Kerala)

-Multioppia (M.) insolita Ivan y Vasiliu, 1999

("non" Oppia insolita Mihelcic, 1956, ?=Multioppia wilsoni laniseta)

DisTRIBUCIÓN: Rumania.

-Multioppia (M.) insulana Pérez-Íñigo, 1982

DisTRIBUCIÓN: Guinea Ecuatorial: I. Pagalu [Annobón], Hawai y

Macaronésica (Madeira).

-Multioppia (M.) integra Pérez-Íñigo jr., 1990

DisTRIBUCIÓN: España.

-Multioppia (M.) jandiae Pérez-Íñigo y Peña, 1996

DisTRIBUCIÓN: I. Canarias.

-Multioppia (M.) longisetosa Mahunka, 1986

DisTRIBUCIÓN: Kenia.

-Multioppia (M.) moritzi Mahunka y Topercer, 1983

DisTRIBUCIÓN: Europa central.

-Multioppia (M.) neglecta Pérez-Íñigo, 1969

(=Multioppia excisa Moritz, 1971)

DisTRIBUCIÓN: Holártica (Europa: excepto Este, y U.S.A.: Nuevo

Méjico) y Hawai.

-Multioppia (M.) pakistanensis Hammer, 1977

DisTRIBUCIÓN: Pakistán.

-Multioppia (M.) pauciramosa J. y P. Balogh, 1986

DistriBución: Nueva Guinea.

-Multioppia (M.) perfecta Mahunka y Topercer, 1983

DisTRIBUCIÓN: Eslovaquia.

-Multioppia (M.) pulchra Littlewood y Wallwork, 1972

DISTRIBUCIÓN: I. Británicas.

-Multioppia (M.) radiata Hammer, 1961

DisTRIBUCión: Neotropical e India (Bengala Occidental)

-Multioppia (M.) rangifera Ivan y Vasiliu, 1999

DISTRIBUCIÓN: Rumania.

-Multioppia (M.) shinanoensis Fujita, 1989

DisTRIBUCIÓN: Japón.
-Multioppia (M.) similis Mahunka, 1982

DistrIBUCIÓN: Melanesia (I. Fiji)

-Multioppia (M.) simplitricha Sanyal y Bhaduri, 1989

DISTRIBUCIÓN: India (Bengala Occidental).

-Multioppia (M.) spinifera Mahunka, 1982

Distribución: Polinesia (Tahití).

-Multioppia (M.) stellifera Hammer, 1961

DistriBución: Perú e India (Bengala Occidental).

-Multioppia (M.) tamdao Mahunka, 1988

DistRIBUCIÓN: Vietnam.

-Multioppia (M.) tenue Mahunka y Mahunka-Papp, 1999

DisTRIBUCIÓN: Hungría.

-Multioppia (M.) translamellaris J. y P. Balogh, 1986

Distribución: Nueva Guinea.

-Multioppia (M.) trembleyi Mahunka, 1977

(=Multioppia calcarata Mahunka, 1992)

DISTRIBUCIÓN: Etiópica.

-Multioppia (M.) wilsoni Aoki, 1964

DisTRIBUCIÓN: Cosmopolita (excepto Antártica)

-Multioppia (M.) wilsoni wilsoni Aoki, 1964

(?=Oppia carolinae Jacot, 1938 "sp. inq.")

(?=Oppia carolinae barbatis Jacot, 1938 "sp. inq.")

DistRIBUCIÓN: Cosmopolita (excepto Neotropical y Antártica).

-Multioppia (M.) wilsoni hungarica Mahunka, 1983 (M. laniseta h.) DisTRIBUCIÓN: Hungría.

-Multioppia (M.) wilsoni laniseta Moritz, 1966 (M. l.)

(?=Oppia caporiacci Valle, 1955 “sp. inq.")

(?=Dameosoma clavipectinatum lamellatum Thamdrup, 1932 "sp. inq.")

(?=Oppia insolita Mihelcic, 1956 "sp. inq.")

(?=Oppia sexmaculata Dalenius, 1950 "sp. inq.")

DistriBuCióN: Paleártica occidental (frecuente), Paleártica oriental (excepto Este), "U.S.A." y Venezuela.

-Multioppia (M.) xinjiangensis L. Wang, Zheng, X. Wang, X. Zhang

y Wen, 1990

DisTRIBUCIÓN: Oeste de China.

Multioppia (Furculoppia) Balogh, 1983 (1 sp.)

ESPECIE TIPO: Oppia furcata Kunst, 1958

DisTRIBUCIÓN: Paleártica.

-Multioppia (Furculoppia) furcata (Kunst, 1958) (Oppia)

(=Oppia bulgarica Mahunka, 1987)

(=Oppia ramulifera Kunst, 1959)

DisTRIBUCIÓN: Europa meridional.

Multioppia (Multilanceoppia) Subías, 1989 (4 spp. y 1ssp.) ESPECIE TIPO: Multioppia ramulifera carpatica Schalk, 1966 DisTRIBUCIÓN: Paleártica, Oriental y Neotropical.

-Multioppia (Multilanceoppia) brevipectinata Suzuki, 1976 (Multiop.)

DisTRIBUCIÓN: Este de Paleártica oriental y Noreste de Oriental.

-Multioppia (Multilanceoppia) brevipectinata brevipectinata Suzuki, 1976

DisTRIBUCIÓN: Este de Paleártica oriental y Noreste de Oriental.

-Multioppia (Multilanceoppia) brevipectinata lenis Fujita y Fujikawa, 1986 (Multiop.)

DisTRIBUCIÓN: Japón.

-Multioppia (Multilanceoppia) carpatica Schalk, 1966 (Multiop. ramulifera $c$.)

DisTRIBUCIÓN: Rumania.

-Multioppia (Multilanceoppia) insularis Mahunka, 1985 (Multiop.) DISTRIBUCIÓN: Neotropical.

-Multioppia (Multilanceoppia) pankovi Rjabinin, 1987 (Multiop.)

DisTRIBUCIÓN: Este de Rusia asiática.

Octoppia Balogh y Mahunka, 1969 (1 sp.)

EsPeCIE TIPO: Octoppia irmayi Balogh y Mahunka, 1969

DisTRIBUCIÓN: Neotropical.

Octoppia irmayi Balogh y Mahunka, 1969

DISTRIBUCION: Brasi 
Pararamusella Mahunka y Palacios-Vargas, 1998 (1 sp.)

EsPeCIE TIPO: Pararamusella disjuncta Mahunka y Palacios-Vargas, 1998 DISTRIBUCIÓN: Neotropical.

-Pararamusella disjuncta Mahunka y Palacios-Vargas, 1998 DisTRIBUCIÓN: Méjico.

Pseudoamerioppia Subías, 1989 (9 spp.)

(=Minoricoppia Pérez-Íñigo jr., 1991)

ESPECIE TIPO: Oppia barrancensis paraguayensis Balogh y Mahunka, 1981 DisTRIBUCIÓN: Pantropical, subtropical (Paleártica meridional) y Neártica.

-Pseudoamerioppia ankae (Mahunka, 1974) (Amerioppia)

DistriBUCión: Rodesia [Zimbabue].

-Pseudoamerioppia balearica (Pérez-Iñigo jr., 1991) (Minoricoppia) DisTRIBUCión: España.

-Pseudoamerioppia barrancensis (Hammer, 1961) (Oppia)

DistRIBUCIÓN: Neotropical y Filipinas.

-Pseudoamerioppia circumciliata (Balogh, 1959) (Oppia deficiens c.) DisTRIBUCIÓN: Etiópica (Angola y Camerún).

-Pseudoamerioppia floralis (Ohkubo, 1990) (Ramusella) DisTRIBUCIÓN: Japón.

-Pseudoamerioppia javensis (Hammer, 1979) (Amerioppia)

DisTRIBUCión: Oriental (Indonesia) y Australiana (Hawai).

-Pseudoamerioppia lamellata (Wallwork, 1961) (Oppia deficiens l.) DisTRIBUCIÓN: Ghana.

-Pseudoamerioppia minuta (Ewing, 1917) (Damaeus)

Distribución: Noreste de U.S.A.

-Pseudoamerioppia paraguayensis (Balogh y Mahunka, 1981) (Oppia

barrancensis $p$.)

DisTRIBUCIÓN: Neotropical e I. Canarias.

Pulchroppiella Balogh, 1983 (2 spp.)

EsPeCIE TIPO: Oppia plurisetosa Mihelcic, 1956

DisTRIBUCIÓN: Paleártica.

-Pulchroppiella littlewoodi Subías, 1989

DisTRIBUCIÓN: I. Británicas.

-Pulchroppiella plurisetosa (Mihelcic, 1956) (Oppia)

DisTRIBUCIÓN: Europa meridional

Ramonoppia Morell, 1990 (2 spp.)

EsPECIE TIPO: Ramonoppia amparoae Morell, 1990

DisTRIBUCIÓN: Paleártica.

-Ramonoppia aequiseta (Pérez-Íñigo jr., 1990) (Multioppia)

DisTRIBUCión: España.

-Ramonoppia amparoae Morell, 1990

DisTRIBUCIÓN: España.

Ramusella Hammer, 1962 (72 spp. y 2 sspp.)

ESPECIE TIPO: Ramusella puertomonttensis Hammer, 1962

DISTRIBUCIÓN: Cosmopolita.

Ramusella (Ramusella) Hammer, 1962 (20 spp.)

(=Alcioppia Balogh, 1983)

(=Amolops Hull, 1916, "nom. praeoc." por Cope, 1865)

(=Bioppia Mahunka y Paoletti, 1984)

DistRIBUCIÓN: Cosmopolita (excepto Antártica).

-Ramusella (R.) assimiloides Subías y Rodríguez, 1987

DistRIBUCIÓN: Mediterránea occidental.

-Ramusella (R.) chulumaniensis (Hammer, 1958) (Oppia)

DisTRIBUCIÓN: Neotropical y Oriental (Java e India: Tripura).

-Ramusella (R.) clavipectinata (Michael, 1885) (Notaspis)

(=Oppia alamellata Kardar, 1976)

(=Dameosoma alces Jacot, 1934)

$(=$ Oppia assimilis Mihelcic, 1956)

(=Oppia taminae Rjabinin, 1975)

DistRIBUCIÓN: Semicosmopolita (Paleártica: frecuente, U.S.A.

California, I. Seychelles, Oriental y Hawai)

-Ramusella (R.) confusa Arillo y Subías, 1990

Distribución: Macaronésica (Madeira).

-Ramusella (R.) cordobensis (Balogh y Mahunka, 1968) (Oppia)

DisTRIBUCIÓN: Neotropical.
-Ramusella (R.) curtipilus (Hammer, 1971) (R. chulumaniensis c.) (=Oppia nana Woas, 1986)

DistriBuCión: Pantropical (Australiana: Islas del Pacífico, Norte de Neotropical y Oriental: Bali).

-Ramusella (R.) defectuosa Subías y Rodríguez, 1987

DistriBuCión: España.

-Ramusella (R.) filigera (Mahunka, 1985) (Brachioppia)

Distribución: Sudáfrica.

-Ramusella (R.) gyrata (Mahunka y Paoletti, 1984) (Bioppia)

DistRIBUCIÓN: Italia.

-Ramusella (R.) hainardorum (Mahunka, 1992) (Uroppia)

DisTRIBUCIÓN: Senegal.

-Ramusella (R.) hippy (Mahunka, 1983) (Oppia)

Distribución: Méjico.

-Ramusella (R.) junonis Pérez-Íñigo, 1986

DistRIBUCIÓN: Mediterránea occidental y Norte de Neotropical.

-Ramusella (R.) lyroseta (Wallwork, 1964) (Oppia fusiformis l.)

DistriBución: Chad.

-Ramusella (R.) philippinensis (Mahunka, 1982) (Oppia)

DISTRIBUCIÓN: Filipinas.

-Ramusella (R.) pinifera Mahunka, 1988

DisTRIBUCIÓN: Borneo.

-Ramusella (R.) puertomonttensis Hammer, 1962

DistribuCión: Semicosmopolita (Mediterránea, Este de Rusia asiática, India: Bengala Occidental, Melanesia: I. Fiji, y Chile).

-Ramusella (R.) remyi (Karppinen, 1966) (Oppia)

DisTRIBUCIÓN: Guinea.

-Ramusella (R.) sengbuschi Hammer, 1968

(=Oppia tokyoensis Aoki, 1974)

(=Ramusella tuberculata Mahunka y Topercer, 1983)

DisTRIBUCIÓN: Pantropical (excepto Etiópica) y subtropical.

-Ramusella (R.) tasetata Subías, 1980

DisTRIBUCIÓN: Pakistán.

-Ramusella (R.) translamellata Subías, 1980

DistriBUCIÓN: Mediterránea.

Ramusella (Insculptoppia) Subías, 1980 (29 spp.)

EsPeCIE TIPO: Dameosoma insculptum Paoli, 1908

DistRIBUCIÓN: Cosmopolita (excepto Antártica).

-Ramusella (Insculptoppia) alata (Mahunka, 1997) (Sphagnoppia)

DisTRIBUCIÓN: Madagascar.

-Ramusella (Insculptoppia) ananthakrishni (Sanyal y Bhaduri, 1989)

(Brachioppia)

DistRIBUCión: India (Bengala Occidental).

-Ramusella (Insculptoppia) anuncata Subías y Rodríguez, 1986

DisTRIBUCIÓN: Mediterránea occidental.

-Ramusella (Insculptoppia) bagnalli (Hull, 1916) (Amolops)

DisTRIBUCIÓN: I. Británicas.

-Ramusella (Insculptoppia) berninii (Pérez-Íñigo, 1975) (Oppia)

DistriBuCión: España.

-Ramusella (Insculptoppia) cavernalis (Ohkubo y Cokendolpher, 2002) (I.)

DisTRIBUCIÓN: Suroeste de U.S.A.

-Ramusella (Insculptoppia) claudelionsi (Calugar y Vasiliu, 1983)

(Oppia)

DisTRIBUCIÓN: Cuba.

-Ramusella (Insculptoppia) corniculata Pérez-Íñigo y Peña, 1997

DistriBUCión: I. Canarias.

-Ramusella (Insculptoppia) crenata (Mahunka, 1992) (I.)

DistriBuCión: Senegal.

-Ramusella (Insculptoppia) elliptica (Berlese, 1908) (Lohmannia)

(=Ramusella abarkouhiensis Bayartogtokh y Akrami, 2000)

(=Insculptoppia lamellata Pérez-Íñigo jr., 1991)

DisTRIBUCIÓN: Holártica meridional (Sur de Europa: frecuente, Irán y U.S.A.: Nuevo Méjico)y Costa Rica.

-Ramusella (Insculptoppia) elmela Subías y Rodríguez, 1986

DisTRIBUCIÓN: Pakistán.

-Ramusella (Insculptoppia) furcata (Willmann, 1928) (Dameosoma) DISTRIBUCIÓN: Europa. 
-Ramusella (Insculptoppia) fusiformis (Wallwork, 1961) (Oppia soror $f$.)

DisTRIBUCión: Etiópica (Ghana y Chad).

-Ramusella (Insculptoppia) golosovae (Rjabinin, 1987) (Oppia)

DisTRIBUCIÓN: Este de Rusia asiática.

-Ramusella (Insculptoppia) humicola Wang y Li, 1997

DisTRIBUCión: Noreste de China.

-Ramusella (Insculptoppia) insculpta (Paoli, 1908) (Dameosoma) (=Oppia shaldybinae Kulijev, 1962)

(=Ramusella insularis Rjabinin, 1987)

Distribución: Paleártica occidental (excepto Norte): frecuente,

Este de Rusia asiática y Vietnam.

-Ramusella (Insculptoppia) japonica (Aoki, 1983) (Brachioppia) DisTRIBUCIÓN: Japón.

-Ramusella (Insculptoppia) krivolutskyi (Kulijev, 1966) (Oppia)

Distribución: Paleártica (Sureste de Europa y Asia centrooccidental).

-Ramusella (Insculptoppia) luxtoni (Ayyildiz, 1989) (Gressittoppia) DisTRIBUCIÓN: Turquía.

-Ramusella (Insculptoppia) merimna (Balogh y Mahunka, 1977)

(Oppia)

DistriBuCIÓN: Brasil.

-Ramusella (Insculptoppia) neonominata nom. nov.

[=Ramusella (Insculptoppia) corniculata Ivan y Vasiliu, 1999, "nom. praeoc" por Pérez-Íñigo y Peña, 1997 (“hom. prim.")]

DISTRIBUCIÓN: Rumania.

-Ramusella (Insculptoppia) paolii Ivan y Vasiliu, 1999

DisTRIBUCIÓN: Rumania.

-Ramusella (Insculptoppia) remota (Ohkubo y Aoki, 1995) (I.) DisTRIBUCIÓN: Micronesia (I. Marianas).

-Ramusella (Insculptoppia) sensiclavata (Kardar, 1976) (Oppia) DisTRIBUCIÓN: India (Uttar Pradesh).

-Ramusella (Insculptoppia) sheshanensis (Wen, Aoki y Wang, 1984)

(Brachioppiella)

DistriBuCión: China suroriental.

-Ramusella (Insculptoppia) soror (Balogh, 1958) (Oppia)

DisTRIBUCión: Etiópica (Congo y Tanzania) y Cáucaso.

-Ramusella (Insculptoppia) subiasi (Pérez-Íñigo jr., 1990) (Oxyoppia) DisTRIBUCIÓN: España.

-Ramusella (Insculptoppia) suciui (Hammer, 1968) (Brachioppia) DisTRIBUCIÓN: Nueva Zelanda.

-Ramusella (Insculptoppia) terricola Subías y Rodríguez, 1986 DisTRIBUCIÓN: Mediterránea.

Ramusella (Insculptoppiella) Subías y Rodríguez, 1986 (12 spp.)

EsPeCIE TIPO: Oppia alfonsii Bernini, 1980

DisTRIBUCión: Pantropical (excepto Australiana) y subtropical.

-Ramusella (Insculptoppiella) aepyornis Mahunka, 1994 (R.) DisTRIBUCIÓN: Madagascar.

-Ramusella (Insculptoppiella) alejnicovae (Krivolutsky y Gatilova,

1974) (Oppia)

DistRIBUCIÓN: Paleártica centromeridional (Sureste de Europa,

Sur de Siberia y Mongolia).

-Ramusella (Insculptoppiella) alfonsii (Bernini, 1980) (Oppia)

DISTRIBUCIÓN: Italia.

-Ramusella (Insculptoppiella) baichengensis (Zhao y Wen, 1992)

(Oppia)

DISTRIBUCIÓN: Noreste de China.

-Ramusella (Insculptoppiella) bicillata Ohkubo, 1996 (R. (Insculptoppia))

DISTRIBUCIÓN: Japón.

-Ramusella (Insculptoppiella) elongata (Paoli, 1908) (Dameosoma) DisTRIBUCIÓN: Europa meridional.

-Ramusella (Insculptoppiella) filamentosa (Mahunka, 1985) (Brachioppia)

DisTRIBUCIÓN: Sudáfrica.

-Ramusella (Insculptoppiella) flagellaris Ohkubo, 1996 (R. (Insculptoppia))

DISTRIBUCIÓN: Japón.
-Ramusella (Insculptoppiella) paillei (Mahunka, 19980) (Oppia)

DisTRIBUCIÓN: Marruecos.

-Ramusella (Insculptoppiella) pocsi (Balogh y Mahunka, 1967)

(Oppia)

DistRIBUCIÓN: Vietnam.

-Ramusella (Insculptoppiella) triancantha (Mahunka, 1963) (Oppia)

DistribuCión: Méjico.

-Ramusella (Insculptoppiella) varians (Wallwork, 1961) (Oppia)

Distribución: Ghana, I. Santa Helena y Arabia Saudita.

Ramusella (Rectoppia) Subías, 1980 (10 spp. y 2 sspp.)

EsPeCie TiPo: Oppia mihelcici Pérez-Iñigo, 1965

DisTRIBUCIÓN: Cosmopolita (excepto Australiana).

-Ramusella (Rectoppia) debililamellata (Kulijev, 1962) (Oppia)

Distribución: Cáucaso.

-Ramusella (Rectoppia) eduardoi Arillo y Subías, 1996

DISTRIBUCIÓN: España.

-Ramusella (Rectoppia) fasciata (Paoli, 1908) (Dameosoma)

DisTRIBUCIÓN: Semicosmopolita (Paleártica occidental: excepto

Norte, "U.S.A.: Florida", Somalia, India: Tripura, y subantártica:

I. Amsterdam).

-Ramusella (Rectoppia) fasciata fasciata (Paoli, 1908)

DistribuCión: Paleártica (Europa centromeridional y Corea),

"U.S.A. (Florida)", Somalia, India (Tripura) e "I. Amsterdam".

-Ramusella (Rectoppia) fasciata sahariensis (Hammer, 1975)

(Oppia s.)

DisTRIBUCIÓN: Mediterránea (frecuente).

-Ramusella (Rectoppia) fasciata sudamericana Pérez-Iñigo y

Sarasola, 1998

DisTRIBUCIÓN: Uruguay.

-Ramusella (Rectoppia) incisiva (Balogh y Mahunka, 1980) (Oppia)

DisTRIBUCIÓN: Cuba.

-Ramusella (Rectoppia) mihelcici (Pérez-Íñigo, 1965) (Oppia)

(=Oppia guelticola Hammer, 1975)

DisTRIBUCIÓN: Paleártica (menos frecuente en el Norte) y Venezuela.

-Ramusella (Rectoppia) radiata (Balogh, 1961) (Oppia)

DisTRIBUCIÓN: Tanzania y subantártica (I. San Pablo y Amsterdam).

-Ramusella (Rectoppia) rhinina Subías y Mínguez, 1981

DisTRIBUCIÓN: España.

-Ramusella (Rectoppia) strinatii (Mahunka, 1980) (Oppia)

DisTRIBUCIÓN: Mediterránea occidental.

-Ramusella (Rectoppia) strinatii strinatii (Mahunka, 1980)

DISTRIBUCIÓN: Mediterránea occidental.

-Ramusella (Rectoppia) strinatii curtiramosa Subías y Rodríguez, 1987

DisTRIBUCIÓN: España.

Ramusella (Sabahoppia) Mahunka, 1987 (1 sp.)

EsPeCIE TIPO: (Sabahoppia hauseri Mahunka, 1987) =Xenillus blattarum Oudemans, 1911

DISTRIBUCIÓN: Oriental.

-Ramusella (Sabahoppia) blattarum (Oudemans, 1911) (Xenillus)

(=Sabahoppia hauseri Mahunka, 1987)

DisTRIBUCIÓN: Oriental (Java y Borneo).

Ramuselloppia Subías y Rodríguez, 1986 (1 sp.)

ESPECIE TIPO: Ramuselloppia anomala Subías y Rodríguez, 1986 DisTRIBUCIÓN: Paleártica.

-Ramuselloppia anomala Subías y Rodríguez, 1986

DISTRIBUCIÓN: Ibérica.

Uroppia Balogh, 1983 (2 spp)

EsPECIE TIPO: Oppia akusiensis Wallwork, 1961

DisTRIBUCIÓN: Paleotropical.

-Uroppia akusiensis (Wallwork, 1961) (Oppia)

DISTRIBUCIÓN: Ghana e India (Tripura).

-Uroppia kenyaensis Mahunka, 1986

DISTRIBUCIÓN: Kenia 
[Pulchroppiinae] Balogh, 1983 (3 gen., 15 spp. y 1 ssp.)

Distribución: Pantropical (excepto Australiana) y subtropical.

Multipulchroppia Subías, 1989 (8 spp. y 1 ssp.)

EsPECIE TIPO: Multioppia berndhauseri Mahunka, 1978

DISTRIBUCIÓN: Pantropical (excepto Australiana) y subtropical (Paleártica meridional).

-Multipulchroppia amazonica (Balogh y Mahunka, 1969) (Multioppia) DisTRIBUCIÓN: Neotropical (Brasil y Venezuela).

-Multipulchroppia berndhauseri (Mahunka, 1978) (Multioppia)

DisTRIBUCIÓN: Malgache (I. Reunión) y Japón.

-Multipulchroppia graeca (Mahunka, 1977) (Multioppia)

DisTRIBUCIÓN: Grecia.

-Multipulchroppia gyoergyi (Balogh y Mahunka, 1969) (Multioppia) DisTRIBUCIÓN: Bolivia.

-Multipulchroppia pectinata (Balogh y Mahunka, 1967) (Multioppia) (=Multioppia vietnamica Balogh, 1983)

DisTRIBUCIÓN: Vietnam.

-Multipulchroppia schauenbergi (Mahunka, 1978) (Multioppia)

DISTRIBUCIÓN: Malgache (I. Reunión) y Japón.

-Multipulchroppia schauenbergi schauenbergi (Mahunka, 1978) DisTRIBUCIÓN: Malgache (I. Reunión).

-Multipulchroppia schauenbergi punctulata Ohkubo, 1992 DisTRIBUCIÓN: Japón.

-Multipulchroppia siamensis (Suzuki, 1976) (Multioppia)

(=Multioppia pectinata Aoki, 1967, “nom. praeoc.” por Balogh y

Mahunka, 1967)

DISTRIBUCIÓN: Tailandia.

-Multipulchroppia similis (Hammer, 1979) (Pulchroppia)

DisTRIBUCIÓN: Java.

Pulchroppia Hammer, 1979 (6 spp.)

EsPecie TIPO: Pulchroppia elegans Hammer, 1979

DISTRIBUCIÓN: Oriental.

-Pulchroppia burckhardti Mahunka, 1987

DisTRIBUCIÓN: Borneo.

-Pulchroppia elegans Hammer, 1979

DisTriBución: Java.

-Pulchroppia granulata Mahunka, 1988

DisTRIBUCIÓN: Oriental (Vietnam y China suroriental).

-Pulchroppia malapectinata (Corpuz-Raros, 1979) (Brachioppiella) DistriBUCIÓN: Filipinas.

-Pulchroppia pendula (Balogh, 1970) (Brachioppia)

DisTRIBución: Ceilán [Sri Lanka].

-Pulchroppia ramifera Wang y Li, 1997

DistriBución: Noreste de China.

Varioppia Mahunka, 1985 (1 sp.)

EsPECIE TIPO: Varioppia radiata Mahunka, 1985

DISTRIBUCIÓN: Neotropical.

-Varioppia radiata Mahunka, 1985

(=Pulchroppia curarii Franklin y Woas, 1992)

DISTRIBUCIÓN: Neotropical.

[Arcoppiinae] Balogh, 1983 (6 gen., 2 subg., 69 spp. y 8 sspp.) DisTRIBUCIÓN: Pantropical y subtropical.

Arcoppia Hammer, 1977 (52 spp. y 8 sspp.)

(=Chuoppia Tseng, 1982 "sin. nov.")

ESPECIE TIPO: Arcoppia brachyramosa Hammer, 1977

DISTRIBUCIÓN: Pantropical y subtropical.

-Arcoppia aequivoca Subías, 1989

(=Arcoppia sabahensis Mahunka, 1988, “nom. praeoc.” por Mahunka, 1987)

(=Arcoppia kalimantanensis Ohkubo y Aoki, 1995)

DisTRIBUCIÓN: Borneo.

-Arcoppia arcualis (Berlese, 1913) (Dameosoma)

Distribución: Pantropical (excepto Etiópica).

-Arcoppia arcualis arcualis (Berlese, 1913)

DISTRIBUCIÓN: Oriental (frecuente).
-Arcoppia arcualis curtiseta Rodríguez y Subías, 1984 (=Arcoppia arcualis novaezealandiae J. y P. Balogh, 1986) DisTRIBUCIÓN: Australiana.

-Arcoppia arcualis enghoffi Rodríguez y Subías, 1984 DISTRIBUCIÓN: Brasil.

-Arcoppia arcualis novaeguineae J. y P. Balogh, 1986

DISTRIBUCIÓN: Nueva Guinea.

-Arcoppia bacilligera Mahunka, 1983

DisTRIBUCIÓN: Tanzania.

-Arcoppia bidentata Hammer, 1979

DisTRIBUCIÓN: Oriental.

-Arcoppia bidentata bidentata Hammer, 1979

DISTRIBUCIÓN: Java.

-Arcoppia bidentata sabahensis Mahunka, 1987

DisTRIBUCIÓN: Oriental (Borneo y Filipinas).

-Arcoppia bifilis (Canestrini, 1898) (Belba) "sp. inq."

DisTRIBUCIÓN: Nueva Guinea.

-Arcoppia biflagellata J. y P. Balogh, 1986

DistribuCión: Melanesia (I. Fiji).

-Arcoppia brachyramosa Hammer, 1977

DisTRIBUCIÓN: Pakistán.

-Arcoppia campinaranensis (Franklin y Woas, 1992) (Oppia (A.))

DISTRIBUCIÓN: Brasil.

-Arcoppia corniculifera (Mahunka, 1978) (Oppia)

DisTRIBUCIÓN: Etiópica (Malgache: I. Mauricio, y Uganda).

-Arcoppia cronus (Jacot, 1934) (Oppia)

DistriBución: Australiana.

-Arcoppia cronus cronus (Jacot, 1934)

DisTRIBUCIÓN: Hawai.

-Arcoppia cronus papua J. y P. Balogh, 1986

DisTRIBUCión: Nueva Guinea.

-Arcoppia cronus winkleri (Hammer, 1968) (Oppia w.)

DistriBuCión: Australiana (Nueva Zelanda y Polinesia: Tahití).

-Arcoppia cryptomeriae (Mondal y Kundu, 1985) (Oppia)

DisTRIBUCIón: India (Bengala Occidental).

-Arcoppia curtipila J. y P. Balogh, 1986

DisTRIBUCIÓN: Nueva Guinea.

-Arcoppia curvirostrata Ohkubo y Aoki, 1995

DisTRIBUCIÓN: Micronesia (I. Marianas).

-Arcoppia dechambrierorum (Mahunka, 1983) (Oppia)

DisTRIBUCión: Méjico.

-Arcoppia dissimilis (Berlese, 1905) (Dameosoma)

DisTRIBUCIÓN: Java.

-Arcoppia dissimiloides (Sellnick, 1925) (Dameosoma)

DISTRIBUCIÓN: Java.

-Arcoppia fenestralis (Wallwork, 1961) (Oppia)

DisTRIBUCIÓN: Pantropical (excepto Neotropical).

-Arcoppia fenestralis fenestralis (Wallwork, 1961) DisTRIBUCIÓN: África occidental.

-Arcoppia fenestralis orientalis J. y P. Balogh, 1986 DisTRIBUCIÓN: Nueva Guinea e India nororiental.

-Arcoppia fenestralis sinensis (Mahunka, 1976) (Oppia arcualis s.) DISTRIBUCIÓN: China suroriental.

-Arcoppia flagellata (Warburton, 1912) (Notaspis)

DisTRIBUCIÓN: I. Seychelles.

-Arcoppia grucheti (Mahunka, 1978) (Oppia)

DistRIBUCIÓN: Malgache (I. Reunión).

-Arcoppia guineana Pérez-Íñigo, 1982

DistRIBUCIÓN: Guinea Ecuatorial: I. Pagalu [Annobón].

-Arcoppia hammerae Rodríguez y Subías, 1984

DisTRIBUCIÓN: Oriental.

-Arcoppia inaequirostris Mahunka, 1999

DisTRIBUCIÓN: Uganda.

-Arcoppia incerta J. y P. Balogh, 1983

DISTRIBUCIÓN: Australia.

-Arcoppia interrupta Ohkubo, 1996

DISTRIBUCIÓN: Japón.

-Arcoppia kaindicola J. y P. Balogh, 1986

DistrIBUCIÓN: Nueva Guinea. 
-Arcoppia longisetosa Balogh, 1982

DisTRIBUCIÓN: Australia y Vietnam.

-Arcoppia mahunkai Rodríguez y Subías, 1984

DisTRIBUCIÓN: I. Canarias.

-Arcoppia mcadami J. y P. Balogh, 1986

Distribución: Nueva Guinea e India (Tripura).

-Arcoppia obtusa Mahunka, 1999

DisTRIBUCIÓN: Uganda.

-Arcoppia palmaria (Tseng, 1982) (Chuoppia)

DisTRIBUCIÓn: Taiwan [Formosa].

-Arcoppia perezinigoi Rodríguez y Subías, 1984

DisTRIBUCIÓN: I. Canarias.

-Arcoppia perisi Rodríguez y Subías, 1984

DisTRIBUCIÓN: I. Canarias.

-Arcoppia piffli Mahunka, 1999

DisTRIBUCIÓn: Uganda.

-Arcoppia porifera (Franklin y Woas, 1992) (Oppia (A.))

DistRIBUCIÓN: Brasil.

-Arcoppia praearcuata J. y P. Balogh, 1986

DisTRIBUCIÓN: Nueva Guinea.

-Arcoppia radiata (Wallwork, 1961) (Oppia angolensis $r$ )

DisTRIBUCIÓN: Ghana

-Arcoppia rangifer J. y P. Balogh, 1986

DisTRIBUCIÓN: Nueva Guinea.

-Arcoppia robusta Mahunka, 1988

(=Arcoppia confusa Subías, 1989)

DisTRIBUCIÓN: Borneo.

-Arcoppia robustia (Berlese, 1913) (Dameosoma arcuale r.)

DisTRIBUCIÓN: Oriental.

-Arcoppia rotunda Hammer, 1979

DISTRIBUCIÓN: Java.

-Arcoppia rugosa (Mahunka, 1974) (Oppia)

(=Arcoppia pergeli Mahunka, 1982)

Distribución: Etiópica: Rodesia [Zimbabue] y Etiopía.

-Arcoppia secata Mahunka, 1999

DisTRIBUCIÓn: Uganda.

-Arcoppia semicostulata (Mahunka, 1985) (Pletzenoppia)

DistriBución: Sudáfrica.

-Arcoppia serrulata (Balogh y Mahunka, 1980) (Oppia)

DisTRIBUCIÓn: Norte de Neotropical.

-Arcoppia teraja Mahunka, 2001

DISTRIBUCIÓN: Borneo.

-Arcoppia tripartita (Hammer, 1961) (Oppia)

(=Oppia gilva Wallwork, 1961)

DisTRIBUCIÓN: Neotropical y África occidental.

-Arcoppia triramosa (Tseng, 1982) (Chuoppia)

DISTRIBUCIÓN: Taiwan [Formosa].

-Arcoppia tuberosa Mahunka, 1988

DisTRIBUCIÓN: Tanzania.

-Arcoppia varia Hammer, 1979

DisTRIBUCión: Brasil y Oriental (Indonesia)

-Arcoppia viperea (Aoki, 1959) (Oppia)

(=Arcoppia baloghi Rodríguez y Subías, 1984)

Distribución: Este de Paleártica oriental (frecuente), Oriental y Sudáfrica.

-Arcoppia vittata Hammer, 1979

DISTRIBUCIÓN: Java

-Arcoppia waterhousei J. y P. Balogh, 1983

DisTRIBUCIÓN: Australia.

Basidoppia Mahunka, 1983 (6 spp.)

EsPeCIE TIPO: Basidoppia basidii Mahunka, 1983

DisTRIBUCIÓN: Etiópica y Paleártica meridional.

-Basidoppia angolensis (Balogh, 1958) (Oppia)

DisTRIBUCión: Etiópica (Angola y Uganda).

-Basidoppia basidii Mahunka, 1983

DisTRIBUCIÓN: Tanzania.

-Basidoppia curtispinosa (Ohkubo, 1996) (Arcoppia) DisTRIBUCIÓN: Japón.
-Basidoppia demeteri (Mahunka, 1982) (Oppia)

DisTRIBUCIÓN: Etiopía.

-Basidoppia pocsorum Mahunka, 1993

DisTRIBUCIÓN: Tanzania.

-Basidoppia psyla Mahunka, 1983

DISTRIBUCIÓN: Tanzania.

Mimoppia Balogh, 1983 (3 spp.)

EsPECIE TIPO: Oppia tenuiseta Wallwork, 1961

DISTRIBUCIÓN: Tropical (Etiópica y Neotropical).

Mimoppia (Mimoppia) Balogh, 1983 (2 spp.)

DISTRIBUCIÓN: Tropical (Etiópica y Neotropical).

-Mimoppia (M.) dendropectinata (Woas, 1986) (Arcoppia)

DisTRIBUCIÓN: El Salvador.

-Mimoppia (M.) tenuiseta (Wallwork, 1961) (Oppia)

DISTRIBUCIÓN: Ghana.

Mimoppia (Dysarcoppia) Mahunka y Palacios-Vargas, 1998 (1 sp.)

EsPeCIE TIPO: Arcoppia (Dysarcoppia) mexicana Mahunka y PalaciosVargas, 1998

DisTRIBUCIÓN: Neotropical.

-Mimoppia (Dysarcoppia) mexicana (Mahunka y Palacios-Vargas,

1998) (Arcoppia (D.))

DistribuCIÓN: Méjico.

Porrhoppia Balogh, 1970 (1 sp.)

EsPecIE TIPO: Porrhoppia crux Balogh, 1970

DISTRIBUCIÓN: Oriental.

-Porrhoppia crux Balogh, 1970

DisTRIBUCión: Ceilán [Sri Lanka].

Similoppia Mahunka, 1983 (2 spp.)

EsPECIE TIPO: Similoppia halterata Mahunka, 1983

DISTRIBUCIÓN: Etiópica y Neotropical.

Similoppia (Similoppia) Mahunka, 1983 (1 sp.)

DISTRIBUCIÓN: Etiópica.

-Similoppia (S.) halterata Mahunka, 1983

DistRIBUCIÓN: Tanzania.

Similoppia (Reductoppia) P. Balogh, 1984 (1 sp.)

EsPeCIE TIPO: Reductoppia espeletiae P. Balogh, 1984

DISTRIBUCIÓN: Neotropical.

-Similoppia (Reductoppia) espeletiae (P. Balogh, 1984) (R.)

DisTRIBUCIÓN: Colombia.

Wallworkoppia Subías, 1989 (5 spp.)

(=Wallworkella Balogh, 1983, “nom. praeoc.” por Wallworkiella Hammer, 1979)

EsPeCIE TIPO: Oppia trimucronata Wallwork, 1961

DISTRIBUCIÓN: Etiópica y Neotropical.

-Wallworkoppia cervifer (Mahunka, 1983) (Oppia)

(=Arcoppia longiramosa Woas, 1986)

DisTRIBUCIÓN: Norte de Neotropical.

-Wallworkoppia machadoi (Balogh, 1958) (Oppia)

(=Arcoppia granulata Mahunka, 1986)

DISTRIBUCIÓN: África centrooriental (frecuente).

-Wallworkoppia parasensillus (Mahunka, 1999) (Arcoppia)

DisTRIBUCIÓN: Uganda.

-Wallworkoppia trimucronata (Wallwork, 1961) (Oppia)

DisTRIBUCIÓN: Etiópica (Ghana y Uganda).

-Wallworkoppia vibrissa (Mahunka, 1983) (Wallworkella)

DisTRIBUCIÓN: Tanzania.

[Brachioppiinae] Subías, 1989 (11 gen., 2 subg., 60 spp. y 1 ssp.) DISTRIBUCIÓN: Pantropical, subtropical y austral.

Austroppia Balogh, 1983 (2 spp.)

ESPECIE TIPO: (Oppia magellanis Hammer, 1962) $=$ Notaspis crozetensis

Richters, 1908

DisTRIBUCIÓN: Austral (Antártica y Neotropical) 
-Austroppia crozetensis (Richters, 1908) (Notaspis)

(=Oppia crozetensis anareensis Dalenius, 1958)

(=Oppia magellanis Hammer, 1962)

DisTRIBUCIÓN: Antártica (frecuente) y América austral (Tierra del Fuego)

-Austroppia petrohuensis (Hammer, 1962) (Brachioppiella)

DisTRIBUCIÓN: América austral.

Brachioppia Hammer, 1961 (10 spp.)

ESPECIE TIPO: Brachioppia cuscensis Hammer, 1961

DistRiBución: Pantropical (excepto Australiana) y subtropical.

-Brachioppia cajamarcensis Hammer, 1961

DisTRIBUCIÓN: Neotropical (Perú y Brasil).

-Brachioppia cuscensis Hammer, 1961

DistRIBUCIóN: Neotropical (frecuente), India (Bengala Occidental) y Japón.

-Brachioppia deliciosa Hammer, 1961

(=Oppia guarani Balogh y Mahunka, 1981)

(=Oppia pseudocostulata Balogh y Mahunka, 1969)

DiSTRIBUCIÓN: Neotropical (excepto austral): frecuente.

-Brachioppia excrescens Mahunka, 1985

DisTRIBUCIón: Sudáfrica.

-Brachioppia glabra Franklin y Woas, 1992

DisTRIBUCIÓN: Brasil.

-Brachioppia koki Mahunka, 1985

DisTRIBUCIÓN: Sudáfrica.

-Brachioppia palmata Mahunka, 1985

Distribución: Sudáfrica.

-Brachioppia triglochin (Balogh y Mahunka, 1977) (Oppia)

DisTRIBUCIÓN: Brasil.

-Brachioppia triramosa (Sellnick, 1924) (Dameosoma)

DISTRIBUCIÓN: Brasil.

-Brachioppia tropicalis Pérez-Íñigo y Baggio, 1980

DisTRIBUCIÓN: Brasil.

Brachioppiella Hammer, 1962 (24 spp. y 1 ssp.)

EsPECIE TIPO: Brachioppiella periculosa Hammer, 1962

DisTRIBUCIÓN: Pantropical (excepto Oriental) y austral.

Brachioppiella (Brachioppiella) Hammer, 1962 (12 spp.)

(=Ugandoppia Mahunka, 1998 "sin. nov.")

DisTRIBUCIÓN: Pantropical (excepto Oriental).

-Brachioppiella (B.) bifurcata (Mahunka, 1998) (Ugandoppia)

DisTRIBUCIÓN: Uganda.

-Brachioppiella (B.) biseriata (Balogh y Mahunka, 1975) (Oppia)

DistriBución: Australia.

-Brachioppiella (B.) hannecarti J. y P. Balogh, 1983

DisTRIBUCIÓN: Nueva Caledonia.

-Brachioppiella (B.) higginsi (Hammer, 1968) (Brachioppia)

Distribución: Australiana (Nueva Zelanda y Australia).

-Brachioppiella (B.) nasalis (Evans, 1953) (Oppia)

DisTRIBUCIÓN: Tanzania.

-Brachioppiella (B.) paranasalis Lee y Subías, 1991

DisTRIBUCIÓN: Australia.

-Brachioppiella (B.) periculosa Hammer, 1962

DisTRIBUCIÓN: Neotropical.

-Brachioppiella (B.) rajskii Hammer, 1968

DisTRIBUCIÓN: Nueva Zelanda.

-Brachioppiella (B.) ramosa (Karppinen, 1966) (Oppia)

DisTRIBUCIÓN: Guinea.

-Brachioppiella (B.) tenuicoma (Hammer, 1958) (Oppia)

Distribución: Neotropical (Bolivia y Perú).

-Brachioppiella (B.) triramosa Hammer, 1962

DistriBución: Chile.

-Brachioppiella (B.) walkeri (Hammer, 1968) (Brachioppia)

DISTRIBUCIÓN: Nueva Zelanda.

Brachioppiella (Gressittoppia) Balogh, 1983 (12 spp. y 1 ssp.)

(=Jermyia Mahunka, 2002 "sin. nov.")

ESPECIE TIPO: Brachioppia moresonensis Hammer, 1967

DisTRIBUCIÓN: Austral.
-Brachioppiella (Gressittoppia) baderi (Hammer, 1968) (Oppia)

Distribución: Nueva Zelanda.

-Brachioppiella (Gressittoppia) corallifera (Mahunka, 1985) (Oppia)

DISTRIBUCIÓN: Sudáfrica.

-Brachioppiella (Gressittoppia) hartensteini (Hammer, 1968) (Brachioppia)

DISTRIBUCIÓN: Nueva Zelanda.

-Brachioppiella (Gressittoppia) magna Lee y Subías, 1991

DISTRIBUCIÓN: Australia.

-Brachioppiella (Gressittoppia) minima Lee y Subías, 1991

Distribución: Australia.

-Brachioppiella (Gressittoppia) moresonensis (Kok, 1967) (Bra-

chioppia)

DisTRIBUCióN: Sudáfrica.

-Brachioppiella (Gressittoppia) orkneyensis (Kok, 1967) (Brachioppia)

DistriBución: Antártica: I. Orcadas del Sur (I. Orkney)

-Brachioppiella (Gressittoppia) pepitensis (Hammer, 1962) (Oppia)

DisTRIBUCIÓN: América austral (Tierra del Fuego) y Antártica (I.

Shetland del Sur).

-Brachioppiella (Gressittoppia) pepitensis pepitensis (Hammer, 1962)

DisTRIBUCión: América austral (Tierra del Fuego).

-Brachioppiella (Gressittoppia) pepitensis brevipectinata (Covarrubias, 1968) (Oppia)

DisTRIBUCión: Antártica (I. Shetland del Sur).

-Brachioppiella (Gressittoppia) pocsi (Mahunka, 2002) (G.)

DisTRIBUCIÓN: Madagascar.

-Brachioppiella (Gressittoppia) pseudohigginsi Lee y Subías, 1991

DisTRIBUCIÓN: Australia.

-Brachioppiella (Gressittoppia) sensilla (Mahunka, 2002) (Jermyia)

DisTRIBUCIÓN: Madagascar.

-Brachioppiella (Gressittoppia) usheri (P. Balogh, 1988) (Solenoppia)

DisTRIBUCIÓN: Subantártica: I. Malvinas [I. Falkland].

Brassoppia Balogh, 1983 (3 spp.)

ESPECIE TIPO: Oppia brassi Balogh, 1981

DisTRIBUCIÓN: Australiana y Neotropical austral.

Brassoppia (Brassoppia) Balogh, 1983 (2 spp.)

DisTRIBUCIÓN: Australiana.

-Brassoppia (B.) brassi (Balogh, 1982) (Oppia)

DistriBuCIÓN: Australia.

-Brassoppia (B.) lamellata J. y P. Balogh, 1986

DistRIBUCIÓN: Nueva Guinea.

Brassoppia (Plaesioppia) Balogh, 1983 (1 sp.)

ESPECIE TIPO: Brachioppiella peullaensis Hammer, 1962

DisTRIBUCIÓN: Neotropical austral

-Brassoppia (Plaesioppia) peullaensis (Hammer, 1962) (Brachioppiella)

DistriBuCiÓN: Chile.

Ctenoppia Balogh, 1983 (2 spp.)

EsPECIE TIPO: Oppia variopectinata Balogh y Mahunka, 1975

DISTRIBUCIÓN: Australiana.

Ctenoppia eupectinata (Balogh y Mahunka, 1975) (Oppia)

DisTRIBUCIÓN: Australiana.

-Ctenoppia variopectinata (Balogh y Mahunka, 1975) (Oppia)

DisTRIBUCIÓN: Australiana.

Gittella Hammer, 1961 (4 spp.)

EsPECIE TIPO: Gittella punctata Hammer, 1961

DisTRIBUCIÓN: Neotropical.

-Gittella flagellata (Mahunka, 1983) (Pulchroppiella)

DisTRIBUCIÓN: Brasil.

-Gittella insularis Mahunka, 1998

DisTRIBUCIÓN: Pequeñas Antillas.

-Gittella maxima (Balogh y Mahunka, 1981) (Multioppia)

DistRIBUCIÓN: Neotropical (Paraguay y Panamá). 
-Gittella punctata Hammer, 1961

DisTRIBUCIÓN: Perú.

Kokoppia Balogh, 1983 (7 spp.)

ESPECIE TIPO: Brachioppia longisetosa Kok, 1967

DisTRIBUCIÓN: Pantropical.

-Kokoppia dendricola (Jeleva y Vu, 1987) (Cryptoppia) DISTRIBUCIÓN: Vietnam.

-Kokoppia dudichi (Balogh, 1982) (Oppia)

DisTRIBUCión: Australia.

-Kokoppia euramosa (Balogh y Mahunka, 1969) (Oppia) DISTRIBUCIÓN: Brasil.

-Kokoppia gracilis (Woas, 1986) (Arcoppia)

DisTRIBUCIÓN: El Salvador.

-Kokoppia longisetosa (Kok, 1967) (Brachioppia) DisTRIBUCIÓN: Sudáfrica.

-Kokoppia pectinata (Kok, 1967) (Brachioppia)

DisTRIBUCIÓN: Sudáfrica e India (Tripura)

-Kokoppia rafalskii (Hammer, 1968) (Brachioppiella) DisTRIBUCIÓN: Nueva Zelanda.

Leptoppia Mahunka, 1967 (2 spp.)

ESPECIE TIPO: Leptoppia procera Mahunka, 1997

DISTRIBUCIÓN: Etiópica.

-Leptoppia benyovszkyi Mahunka, 1996

DisTRIBUCIÓN: Madagascar.

-Leptoppia procera Mahunka, 1997

DISTRIBUCIÓN: Madagascar.

Pletzenoppia Balogh, 1983 (3 spp.)

EsPeCIE TIPO: Oppia pletzenae Kok, 1967

DISTRIBUCIÓN:

-Pletzenoppia aseta Mahunka, 1986 DisTRIBUCIÓN: Sudáfrica.

-Pletzenoppia inclinata (Hammer, 1962) (Oppia) Distribución: Neotropical (Argentina y Cuba).

-Pletzenoppia pletzenae (Kok, 1967) (Oppia) DisTRIBUCIÓN: Sudáfrica.

Setuloppia Balogh, 1983 (1 sp.)

EsPeCIE TIPO: Oppia newelli Hammer, 1968

DisTRIBUCIÓN: Australiana.

-Setuloppia newelli (Hammer, 1968) (Oppia)

DisTRIBUCIÓN: Nueva Zelanda.

Trapezoppia Balogh y Mahunka, 1969 (25 spp.)

EsPECIE TIPO: Tanzoppia longipectinata Balogh y Mahunka, 1969

DISTRIBUCIÓN: Neotropical.

-Trapezoppia longipectinata Balogh y Mahunka, 1969 DISTRIBUCIÓN: Brasil.

-Trapezoppia nova Franklin y Woas, 1992

DISTRIBUCIÓN: Brasil.

[Medioppiinae] Subías y Mínguez, 1985 (12 gen., 2 subg., 55 spp. y 3 sspp.)

DISTRIBUCIÓN: Cosmopolita.

Congoppia Balogh, 1983 (2 spp.)

EsPECIE TIPO: Oppia deboissezoni Balogh y Mahunka, 1966

DistriBución: Paleotropical.

-Congoppia deboissezoni (Balogh y Mahunka, 1966) (Oppia)

(=Congoppia extrema Mahunka, 1987)

DisTRIBUCIÓN: Etiópica (Congo y Nigeria).

-Congoppia ramisetosa (Sanyal y Bhaduri, 1989) (Oppia)

DistriBución: India (Bengala Occidental)

Discoppia Balogh, 1983 (4 spp. y 1 ssp.)

EsPeCIE TIPO: Oppia limae Balogh y Mahunka, 1974

DisTRIBUCIÓN: Tropical (Oriental y Neotropical) y subtropical.
Discoppia (Discoppia) Balogh, 1983 (1 sp.)

DisTRIBUCIÓN: Oriental.

-Discoppia (D.) limae (Balogh y Mahunka, 1974) (Oppia)

DistriBuCión: Península Malaya.

Discoppia (Cylindroppia) Subías y Rodríguez, 1986 (3 spp. y 1 ssp.)

ESPECIE TIPO: Oppia minus cylindrica Pérez-Iñigo, 1965

DISTRIBUCIÓN: Tropical (Oriental y Neotropical) y subtropical.

-Discoppia (Cylindroppia) cylindrica (Pérez-Íñigo, 1965) (Oppia minus c.)

DisTRIBUCIÓN: Paleártica meridional y Centroamérica

-Discoppia (Cylindroppia) cylindrica cylindrica (Pérez-Íñigo, 1965) (=Oppia agricola Fujikawa, 1982)

(=Oppia bifidus Bayoumi y Al-Khalifa, 1985)

(=Oppia casuarina Abdel-Hamid, Hussein, Bayoumi y Al-Assiuty, 1983)

(=Oppia sitnikovae Shereef, 1976, "nom. praeoc." por Kulijev, 1962)

DisTRIBUCIÓN: Paleártica (menos frecuente en el Norte) y Panamá.

-Discoppia (Cylindroppia) cylindrica rostroincisa Subías y Rodríguez, 1986

DISTRIBUCIÓN: Europa centrooccidental y Antillas (La Española: R. Dominicana)

-Discoppia (Cylindroppia) pentasetata Subías y Rodríguez, 1986 DisTRIBUCIÓN: Java.

-Discoppia (Cylindroppia) tenuis (Hammer, 1958) (Oppia)

DISTRIBUCIÓN: Argentina.

Medioppia Subías y Mínguez, 1985 (26 spp.)

(=Kunoppia Mahunka, 1987)

EsPeCIE TIPO: Oppia media Mihelcic, 1956

DISTRIBUCIÓN: Cosmopolita (excepto Oriental y Antártica).

-Medioppia beskidyensis Niemi y Skubala, 1993

DisTRIBUCIÓN: Polonia.

-Medioppia centrodentata Gordeeva y Niemi, 1990

DisTRIBUCIÓN: Sureste de Europa.

-Medioppia gomozovae (Gordeeva y Karppinen, 1988) (Ctenoppiella)

DisTRIBUCIÓN: Ucrania (Crimea).

-Medioppia heterotricha Ivan y Vasiliu, 1997

DISTRIBUCIÓN: Rumania.

-Medioppia hygrophila (Mahunka, 1987) (Kunoppia)

DisTRIBUCIÓN: Europa central.

-Medioppia lamellata (Golosova y Karppinen, 1985) (Oppia)

DisTRIBUCIÓN: Mongolia.

-Medioppia loksai (Schalk, 1966) (Oppia)

DistriBuCión: Rumania.

-Medioppia media (Mihelcic, 1956) (Oppia)

(=Medioppia templadoi Pérez-Íñigo, 1988)

DISTRIBUCIÓN: Mediterránea (frecuente) y Perú.

-Medioppia melisi (Valle, 1949) (Oppia)

DISTRIBUCIÓN: Italia.

-Medioppia minidentata Subías y Rodríguez, 1988

DisTRIBUCIÓN: Mediterránea occidental.

-Medioppia minimedia Arillo y Subías, 1990

DisTRIBUCIÓN: Macaronésica (Madeira).

-Medioppia oblongata (Gordeeva y Melamud, 1991) (Ctenoppiella) DISTRIBUCIÓN: Ucrania.

-Medioppia obsoleta (Paoli, 1908) (Dameosoma fallax o.)

(=Dameosoma vitrinum Hull, 1914)

Distribución: Paleártica (excepto Este de Paleártica oriental): frecuente, Groenlandia y Australiana (Nueva Zelanda y Hawai).

-Medioppia ordunensis (Iturrondobeitia y Saloña, 1988) (Lauroppia)

(=Oppiella acutirostris Pérez-Íñigo jr., 1990)

DISTRIBUCIÓN: Europa centrooccidental.

-Medioppia parapectinata (Rjabinin, 1987) (Oppia)

DISTRIBUCIÓN: I. Kuriles.

-Medioppia parva (Lombardini, 1952) (Oppia) "sp. inq."

DisTRIBUCIÓN: Italia.

-Medioppia pinsapi Arillo y Subías, 1996

Distribución: España.

-Medioppia plumata (Gordeeva y Karppinen, 1988) (Ctenoppiella) Distribución: Cáucaso. 
-Medioppia producta Iturrondobeitia y Arillo, 1997

DisTRIBUCIÓN: España.

-Medioppia samaina Mahunka, 2001

DISTRIBUCIÓN: Grecia.

-Medioppia subpectinata (Oudemans, 1900) (Eremaeus)

(=Dameosoma subpectinatum aberratum Thamdrup, 1932)

(=Oppia bulanovae Kulijev, 1962)

(=Oppia globosa Mihelcic, 1956)

(=Oppia paoliana Cooreman, 1941)

(=Oppia zachvatkini Kulijev, 1962)

Distribución: Holártica (excepto Este de Paleártica oriental):

frecuente en Paleártica occidental, y Senegal.

-Medioppia tridentata Subías y Mínguez, 1985

DISTRIBUCIÓN: España.

-Medioppia trilobata Khanbekjan y Gordeeva, 1991

DisTRIBUCIÓN: Cáucaso.

-Medioppia truncata Iturrondobeitia y Saloña, 1988

DistRIBUCIÓN: España.

-Medioppia tuberculata (Bulanova-Zachvatkina, 1964) (Oppia)

DisTRIBUCIÓN: Europa oriental.

-Medioppia vera (Mihelcic, 1956) (Oppia)

DisTRIBUCIÓN: Sur de Europa.

Medioxyoppia Subías, 1989 (5 spp.)

EsPeCIE TIPO: Oppia yuwana Aoki, 1983

DisTRIBUCIÓN: Paleártica.

-Medioxyoppia actirostrata (Aoki, 1983) (Oppia)

DisTRIBUCIÓN: Japón.

-Medioxyoppia acuta (Aoki, 1984) (Oxyoppia)

DISTRIBUCIÓN: Japón.

-Medioxyoppia mastigophora (Golosova, 1970) (Oppia)

DisTRIBUCión: Este de Paleártica oriental.

-Medioxyoppia nagoyae Ohkubo, 1991

DisTRIBUCIÓN: Japón.

-Medioxyoppia yuwana (Aoki, 1983) (Oppia)

DisTRIBUCIÓN: Japón.

Microppia Balogh, 1983 (2 spp. y 1 ssp.)

EsPecie Tipo: Dameosoma minus Paoli, 1908

DISTRIBUCIÓN: Cosmopolita.

-Microppia arcuata Gordeeva y Tarba, 1990

DisTRIBUCión: Cáucaso.

-Microppia minus (Paoli, 1908) (Dameosoma)

DisTRIBUCIÓN: Cosmopolita.

-Microppia minus minus (Paoli, 1908)

(=Oppia minus simplex Jacot, 1938)

(=Oppia minutissima Sellnick, 1950)

DisTRIBUCIÓN: Cosmopolita (frecuente en Paleártica).

-Microppia minus longisetosa Subías y Rodríguez, 1988

DISTRIBUCIÓN: Sur de Europa occidental y Argentina.

Miroppia Hammer, 1968 (1 sp.)

ESPECIE TIPO: Miroppia zealandica Hammer, 1968

DISTRIBUCIÓN: Australiana.

-Miroppia zealandica Hammer, 1968

DISTRIBUCIÓN: Nueva Zelanda.

Multimedioppia Subías, 1991 (1 sp.)

EsPECIE TIPO: Multimedioppia mirena Subías, 1991

DISTRIBUCIÓN: Paleártica.

-Multimedioppia mirena Subías, 1991

DisTRIBUCIÓN: Ibérica.

Paramedioppia Mahunka y Mahunka-Papp, 2000 (1 sp.)

ESPECIE TIPO: Paramedioppia helvetica Mahunka y Mahunka-Papp, 2000 DisTRIBUCIÓN: Paleártica.

-Paramedioppia helvetica Mahunka y Mahunka-Papp, 2000

DisTRIBUCIÓN: Suiza.
Ramuloppia Balogh, 1961 (1 sp. y 1 ssp.)

EsPecie TIPO: Oppia ramiseta Balogh, 1959

DISTRIBUCIÓN: Etiópica.

-Ramuloppia ramiseta (Balogh, 1959) (Oppia)

DisTRIBución: Etiópica (Angola y Ghana).

-Ramuloppia ramiseta ramiseta (Balogh, 1959)

DisTRIBUCión: Angola.

-Ramuloppia ramiseta atypica (Wallwork, 1961) (Oppia) DISTRIBUCIÓN: Ghana.

Rhinoppia Balogh, 1983 (1 sp.)

EsPecie TIPO: Oppia nasuta Moritz, 1965

DisTRIBUCIÓN: Paleártica.

-Rhinoppia nasuta (Moritz, 1965) (Oppia)

DISTRIBUCIÓN: Europa centrooriental.

Serratoppia Subías y Mínguez, 1985 (6 spp.)

EsPECIE TIPO: Oppia serrata Mihelcic, 1956

DistriBUCión: Paleártica y Neotropical.

-Serratoppia duffyi (Evans, 1954) (Oppia)

DisTRIBUCIÓN: I. Británicas.

-Serratoppia guanicola Subías y Arillo, 1996

DisTRIBUCIÓN: España.

-Serratoppia intermedia Subías y Rodríguez, 1988

(?=Serratoppia toletana Muñoz-Mingarro, 1987 “sp. inq.")

DISTRIBUCIÓN: Ibérica.

-Serratoppia minima Subías y Rodríguez, 1988

DisTRIBUCIÓN: Ibérica y Costa Rica.

-Serratoppia mitrofanovi (Gordeeva y Karppinen, 1988) (Berniniella)

DisTRIBUCIÓN: Ucrania (Crimea).

-Serratoppia serrata (Mihelcic, 1956) (Oppia)

DISTRIBUCIÓN: Mediterránea y Venezuela.

Solenoppia Hammer, 1968 (5 spp.)

EsPECIE TIPO: Solenoppia grandjeani Hammer, 1968

DistRIBUCIÓN: Austral.

Solenoppia (Solenoppia) Hammer, 1968 (3 spp.)

DisTRIBUCIÓN: Australiana austral.

-Solenoppia (S.) grandjeani Hammer, 1968

DisTRIBUCióN: Nueva Zelanda.

-Solenoppia (S.) taberlyi Hammer, 1968

DistRIBUCión: Nueva Zelanda.

-Solenoppia (S.) travei Hammer, 1968

DistrIBUCIÓN: Nueva Zelanda.

Solenoppia (Campbelloppia) Luxton, 1985 (2 spp.)

ESPECIE TIPO: Oppia diaphora Wallwork, 1964

DistRIBUCIÓN: Antártica.

-Solenoppia (Campbelloppia) diaphora (Wallwork, 1964) (Oppia)

DisTRIBUCIÓN: Antártica (I. Campbell).

-Solenoppia (Campbelloppia) pernettyae J. y P. Balogh, 1988 (S.)

Distribución: Subantártica: I. Malvinas [I. Falkland].

[Oppiellinae] Seniczak, 1975 (18 gen., 5 subg., 142 spp. y 17 sspp.) DisTRIBUCIÓN: Cosmopolita.

Abchasiella Gordeeva y Tarba, 1990 (1 sp.)

ESPECIE TIPO: Abchasiella dentata Gordeeva y Tarba, 1990

DISTRIBUCIÓN: Paleártica.

-Abchasiella dentata Gordeeva y Tarba, 1990

Distribución: Cáucaso.

Autoppia Golosova y Karppinen, 1983 (1 sp.)

EsPECIE TIPO: Autoppia algicola Golosova y Karppinen, 1983

DisTRIBUCIÓN: Paleártica.

-Autoppia algicola Golosova y Karppinen, 1983

DisTRIBUCIÓN: I. Sajalín. 
Belloppia Hammer, 1968 (4 spp.)

EsPECIE TIPO: Belloppia wallworki Hammer, 1968

DisTRIBUCIÓN: Austral (Australiana y Antártica).

-Belloppia beemanensis (Wallwork, 1964) (Oppia)

DistriBución: Antártica (I. Campbell).

-Belloppia evansi Hammer, 1968

DisTRIBUCIÓN: Nueva Zelanda.

-Belloppia shealsi Hammer, 1968

DisTRIBUCIÓN: Nueva Zelanda.

-Belloppia wallworki Hammer, 1968

DisTRIBUCIÓN: Australiana austral

Berniniella Balogh, 1983 (31 spp. y 2 sspp.)

EsPeCIE TIPO: Oppia aeoliana Bernini, 1973

DisTRIBUCIÓN: Semicosmopolita (Holártica, Oriental y Neotropical).

Berniniella (Berniniella) Balogh, 1983 (30 spp. y 2 sspp.)

DisTRIBUCIÓN: Semicosmopolita (Holártica, Oriental y Neotropical).

-Berniniella (B.) aeoliana (Bernini, 1973) (Oppia)

DisTRIBUCIÓN: Mediterránea.

-Berniniella (B.) azerbeidjanica (Kulijev, 1962) (Oppia)

Distribución: Sur de Europa (excepto Oeste).

-Berniniella (B.) berninii Ivan y Vasiliu, 1997

DISTRIBUCIÓN: Rumania.

-Berniniella (B.) bicarinata (Paoli, 1908) (Dameosoma)

(=Oppia triconica Mihelcic, 1956)

DisTRIBUCIÓN: Paleártica (frecuente) y Vietnam.

-Berniniella (B.) carinatissima Subías, Rodríguez y Mínguez, 1987

DisTRIBUCIÓN: Sur de Europa occidental.

-Berniniella (B.) conjuncta (Strenzke, 1951) (Oppia sigma c.)

DisTRIBUCIÓN: Euroatlántica.

-Berniniella (B.) extrudens Subías, Rodríguez y Mínguez, 1987 DisTRIBUCIÓN: España.

-Berniniella (B.) fissurata Ivan y Vasiliu, 1997

DISTRIBUCIÓN: Rumania.

-Berniniella (B.) formosa Gordeeva y Karppinen, 1988

DisTRIBUCIÓN: Ucrania (Crimea).

-Berniniella (B.) grandis Gordeeva, 1991

Distribución: Centro de Rusia europea.

-Berniniella (B.) hungarica (Bayoumi, 1979) (Oppia)

DisTRIBUCión: Hungría.

-Berniniella (B.) inornata (Mihelcic, 1957) (Oppia)

(=Oppia simplex Mihelcic, 1956, "nom. praeoc." por Jacot, 1938)

DisTRIBUCIÓN: Mediterránea (frecuente).

-Berniniella (B.) intrudens Subías, Rodríguez y Mínguez, 1987 DisTRIBUCIÓN: España y Cuba.

-Berniniella (B.) jahnae (Sellnick, 1961) (Oppia)

DisTRIBUCIÓN: Sur de Europa.

-Berniniella (B.) kazakovi Gordeeva y Melamud, 1991

DISTRIBUCIÓN: Ucrania.

-Berniniella (B.) latidens Subías, Rodríguez y Mínguez, 1987 DISTRIBUCIÓN: Ibérica.

-Berniniella (B.) lunaris (Evans, 1952) (Oppia)

DisTRIBUCiÓN: I. Británicas.

-Berniniella (B.) minuta (Bulanova-Zachvatkina, 1964) (Oppia)

DISTRIBUCIÓN: Europa oriental.

-Berniniella (B.) nana Gordeeva, 1991

DisTRIBUCIÓN: Centro de Rusia europea.

-Berniniella (B.) parasigma Iturrodobeitia, 1987

DisTRIBUCión: España.

-Berniniella (B.) robusta Ivan y Vasiliu, 1997

DISTRIBUCIÓN: Rumania.

-Berniniella (B.) rossica Gordeeva, 1991

DisTRIBUCión: Centro de Rusia europea.

-Berniniella (B.) sakeni Gordeeva y Tarba, 1990

DisTRIBUCIÓN: Cáucaso.

-Berniniella (B.) serratirostris (Golosova, 1970) (Oppia)

DisTRIBUCión: Holártica (Europa, Este de Rusia asiática y U.S.A.: Carolina del Norte).
-Berniniella (B.) serratirostris serratirostris (Golosova, 1970)

DisTRIBUCIÓN: Holártica (Europa central, Este de Rusia asiática y U.S.A.: Carolina del Norte).

-Berniniella (B.) serratirostris hauseri (Mahunka, 1974) (Oppia) (=Berniniella coronata Mahunka y Paoletti, 1984)

(=Oppiella rafalskii Oplotna y Rajski, 1983)

DISTRIBUCIÓN: Europa.

-Berniniella (B.) serratirostris oscensis Pérez-Íñigo jr., 1990 (B. coronata o.)

DISTRIBUCIÓN: España.

-Berniniella (B.) setilonga Iturrondobeitia y Saloña, 1988

DISTRIBUCIÓN: Europa centrooccidental.

-Berniniella (B.) sigma (Strenzke, 1951) (Oppia)

DistRiBución: Paleártica (Europa y Asia centrooccidental).

-Berniniella (B.) silvatica (Vasiliu y Calugar, 1976) (Oppia)

DistribuCión: Rumania.

-Berniniella (B.) tenuis Gordeeva y Melamud, 1991

DISTRIBUCIÓN: Ucrania.

-Berniniella (B.) tequila (Mahunka, 1983) (Oppia)

Distribución: Méjico.

-Berniniella (B.) tichomirovae (Rjabinin, 1974) (Oppia)

DisTRIBUCIÓN: Noreste de Rusia asiática.

Berniniella (Canaloppia) Mahunka y Palacios-Vargas, 1998 (1 sp.) EsPeCIE TIPO: Berniniella (Canaloppia) borhidii Mahunka y PalaciosVargas, 1998

DisTRIBUCIÓN: Neotropical.

-Berniniella (Canaloppia) borhidii Mahunka y Palacios-Vargas, 1998 DistribuCión: Méjico.

Dissorhina Hull, 1916 (7 spp. y 5 sspp.)

(=Cosmoppia Balogh, 1983)

EsPECIE TIPO: (Notaspis splendens Koch "sensu” Michael, 1888)

=Eremaeus ornatus Oudemans, 1900

DisTRIBUCIÓn: Semicosmopolita (Holártica, Etiópica y Neotropical).

-Dissorhina bolei (Tarman, 1958) (Oppia)

DisTRIBUCIÓN: "Yugoslavia".

-Dissorhina bulganensis Bayartogtokh, 1999

DISTRIBUCIÓN: Mongolia.

-Dissorhina longipilosa (Kunst, 1958) (Oppia ornata l.)

DisTRIBUCIÓN: Europa meridional.

-Dissorhina longipilosa longipilosa (Kunst, 1958) DisTRIBUCIÓN: Sur de Europa.

-Dissorhina longipilosa carpatica (Gordeeva, 1991) (Cosmoppia) DisTRIBUCIÓN: Ucrania.

-Dissorhina neotropicalis Mahunka, 1998

DisTRIBUCIÓN: Pequeñas Antillas.

-Dissorhina ornata (Oudemans, 1900) (Eremaeus)

DisTRIBUCIÓN: Holártica e I. Seychelles.

-Dissorhina ornata ornata (Oudemans, 1900)

(=Dameosoma captator Hull, 1915)

(=Oppia lignivora Jacot, 1939)

(=Dameosoma tricarinatum Paoli, 1908)

(=Dameosoma vetula Hull, 1914)

DistRIBUCIÓN: Holártica: frecuente en Paleártica (excepto Este de Paleártica oriental) e I. Seychelles.

-Dissorhina ornata corniculata (Paoli, 1908) (Dameosoma tricarinatum $c$.)

DisTRIBUCIÓN: Europa occidental.

-Dissorhina ornata globosa (Paoli, 1908) (Dameosoma tricarinatum g.)

DisTRIBUCIÓN: Holártica meridional (Sur de Europa y U.S.A.: Florida).

-Dissorhina ornata peloponnesiaca (Mahunka, 1974) (Oppia)

DisTRIBUCIÓN: Sur de Europa.

-Dissorhina ornata tunisica (Mahunka, 1980) (Oppia)

DisTRIBUCIÓN: Túnez.

-Dissorhina signata (Schwalbe, 1989) (Oppiella)

DISTRIBUCIÓN: Europa central. 
-Dissorhina tricarinatoides (Dubinina, Sosnina, Vysotskaya, Markov

y Atanasov, 1966) (Oppia)

DISTRIBUCIÓN: Europa centromeridional.

Elaphoppia Balogh, 1983 (3 spp.)

(=Parasynoppia Aoki, 1983)

EsPECIE TIPO: Oppia quadripilosa Balogh, 1961

DISTRIBUCIÓN: Tropical (Etiópica y Australiana) y Paleártica meridional. -Elaphoppia lapelerii J. y P. Balogh, 1983

DisTRIBUCIón: Nueva Caledonia.

-Elaphoppia longisensillata (Aoki, 1983) (Parasynoppia)

Distribución: Este de Paleártica oriental.

-Elaphoppia quadripilosa (Balogh, 1961) (Oppia)

DISTRIBUCIÓN: Madagascar.

Hypogeoppia Subías, 1981 (5 spp. y 1 ssp.)

EsPeCIE TIPO: Hypogeoppia terricola Subías, 1981

DisTRIBUCIÓN: Europa centromeridional, "Java” y Cuba.

-Hypogeoppia dungeri Schwalbe, 1995

DistriBución: Alemania.

-Hypogeoppia exempta (Mihelcic, 1958) (Oppia)

DisTRIBUCIÓN: Oeste de Europa.

-Hypogeoppia hypogea (Paoli, 1908) (Dameosoma)

DisTRIBUCIÓN: Oeste de Europa.

-Hypogeoppia perezinigoi Subías y Arillo, 1996

DisTRIBUCIÓN: España.

-Hypogeoppia terricola Subías, 1981

DisTRIBUCIÓn: Ibérica, "Java” y Cuba.

-Hypogeoppia terricola terricola Subías, 1981

(=Hypogeoppia festoneata Moraza y Moreno, 1988)

DisTRIBUCIÓN: Ibérica, “Java” y Cuba.

-Hypogeoppia terricola salmanticensis Morell, 1987 (H. s.) DisTRIBUCIÓN: España.

Kulievia Vasiliu e Ivan, 1999 (1 sp.)

EsPecie TiPo: Oppia paradecipiens Kulijev, 1962

DISTRIBUCIÓN: Paleártica.

-Kulievia paradecipiens (Kulijev, 1962) (Oppia)

DistRIBUCIÓN: Europa centromeridional (excepto Oeste).

Lauroppia Subías y Mínguez, 1986 (26 spp. y 3 sspp.)

(=Ctenoppiella Gordeeva y Karppinen, 1988)

EsPeCIE TIPO: Dameosoma fallax Paoli, 1908

DistRIBUCIÓN: Cosmopolita (excepto Etiópica y Antártica).

-Lauroppia articristata (Aoki, 1988) (Oppiella)

DisTRIBUCIÓN: Japón.

-Lauroppia baetica Arillo y Subías, 1996

DisTRIBUCIÓN: España.

-Lauroppia breviseta Vasiliu e Ivan, 1999

DisTRIBUCIÓN: Rumania.

-Lauroppia carniolica (Tarman, 1958) (Oppia)

DisTRIBUCIÓN: "Yugoslavia".

-Lauroppia commutata (Gordeeva y Grishina, 1991) (Moritziella) DisTRIBUCión: "Siberia".

-Lauroppia compositocarinata (Mihelcic, 1958) (Oppia)

DisTRIBUCIÓN: Austria.

-Lauroppia decempectinata (Fujikawa, 1986) (Mahunkella)

DISTRIBUCIÓN: Japón.

-Lauroppia dentata (Golosova y Karppinen, 1985) (Oppia)

DistriBución: Mongolia.

-Lauroppia denticulata (Grishina, 1980) (Oppia)

DisTRIBUCIÓN: Sur de Siberia.

-Lauroppia distincta (Vasiliu y Calugar, 1981) (Oppiella)

DISTRIBUCIÓN: Rumania.

-Lauroppia doris (E. Pérez-Iñigo, 1978) (Oppia)

DisTRIBUCIÓN: España.

-Lauroppia editae (Mahunka y Mahunka-Papp, 2002) (Oppiella)

DisTRIBUCión: Hungría.

-Lauroppia falcata (Paoli, 1908) (Dameosoma)

DisTRIBUCIÓN: Paleártica occidental y Este de Rusia asiática.
-Lauroppia falcata falcata (Paoli, 1908)

DistribuCIÓN: Paleártica (Paleártica occidental: menos frecuente en el Sur, y Este de Rusia asiática).

-Lauroppia falcata marginedentata (Strenzke, 1951) (Oppia) DISTRIBUCIÓN: Europa central.

-Lauroppia fallax (Paoli, 1908) (Dameosoma)

(=Oppia aligarhiensis Kardar, 1976)

(=Oppiella dubia Hammer, 1962)

DisTRIBUCIÓN: Semicosmopolita (Holártica: frecuente en Paleártica

occidental, India: Uttar Pradesh, Nueva Zelanda y Chile).

-Lauroppia feideri Vasiliu e Ivan, 1999

DISTRIBUCIÓN: Rumania.

-Lauroppia getica (Vasiliu y Calugar, 1981) (Oppia)

DistRIBUCIÓN: Rumania.

-Lauroppia lebedevi (Rjabinin, 1975) (Oppia)

Distribución: Este de Rusia asiática.

-Lauroppia maritima (Willmann, 1928) (Dameosoma falcatum m.)

DisTRIBUCIÓN: Holártica (Paleártica y Neártica septentrional).

-Lauroppia maritima maritima (Willmann, 1928) (=Oppia fissurata Hammer, 1952)

DistRIBUCIÓN: Holártica (Paleártica: menos frecuente en el Sur, y Neártica septentrional).

-Lauroppia maritima acuminata (Strenzke, 1951) (Oppia)

DISTRIBUCIÓN: Holártica (Europa occidental y Neártica septentrional).

-Lauroppia maritima carinthiaca (Mihelcic, 1963) (Oppia) DISTRIBUCIÓN: Austria.

-Lauroppia motasi Vasiliu e Ivan, 1999

DISTRIBUCIÓN: Rumania.

-Lauroppia notabilis (Golosova y Karppinen, 1983) (Oppia)

DistribuCIÓN: Este de Rusia asiática.

-Lauroppia obscura Mahunka y Mahunka-Papp, 2000

DisTRIBUCIÓN: Suiza.

-Lauroppia orientalis (Wen y Bu, 1988) (Belloppia)

Distribución: Oeste de China.

-Lauroppia quadrituberculata (Mahunka, 1987) (Hypogeoppia)

DisTRIBUCIÓN: Hungría.

-Lauroppia similifallax Subías y Mínguez, 1986

DisTRIBUCión: Sur de Europa (España y Rumania).

-Lauroppia tenuipectinata Subías y Rodríguez, 1988

DISTRIBUCIÓN: Mediterránea occidental.

-Lauroppia trapezoida (Grishina, 1981) (Oppiella)

DisTRIBUCIÓN: Paleártica meridional (Sur de Rusia europea y Sur de Paleártica oriental).

Liacaroppia Subías y Rodríguez, 1986 (1 sp.)

EsPeCIE TIPO: Oppiella doryphoros J. y P. Balogh, 1983

DISTRIBUCIÓN: Australiana.

-Liacaroppia doryphoros (J. y P. Balogh, 1983) (Oppiella)

DisTRIBUCIÓN: Hawai.

Moritzoppia Subías y Rodríguez, 1988 (33 spp. y 5 sspp.)

EsPeCIE TIPO: Oppia keilbachi Moritz, 1969

DisTRIBUCIÓN: Holártica, Neotropical y "Australiana".

Moritzoppia (Moritzoppia) Subías y Rodríguez, 1988 (19 spp. y 5 sspp.) (=Moritziella Balogh, 1983, "nom. praeoc." por Börner, 1908)

(=Tetroppia Gordeeva, 1999 "sin. nov.")

DisTRIBUCIÓN: Holártica y "Australiana”.

-Moritzoppia (M.) cuneocostulata Bayartogtokh, 1997

DisTRIBUCIÓN: Mongolia.

-Moritzoppia (M.) escotata (Subías y Rodríguez, 1986) (Moritziella) DisTRIBUCIÓN: España.

-Moritzoppia (M.) hamata (Pearce, 1906) (Notaspis) "sp. inq."

DisTRIBUCIÓN: India (Sikkim).

-Moritzoppia (M.) jamalica (Gordeeva y Grishina, 1991) (Moritziella) DistRIBUCIÓN: "Siberia".

-Moritzoppia (M.) longilamellata (Subías y Rodríguez, 1986)

(Moritziella)

DisTRIBUCIÓN: España. 
-Moritzoppia (M.) longohisterosoma (Kulijev, 1962) (Oppia)

DisTRIBUCIÓN: Cáucaso.

-Moritzoppia (M.) myrmophila (Gordeeva y Grishina, 1991) (Moritziella)

DistRIBUCión: Paleártica oriental

-Moritzoppia (M.) myrmophila myrmophila (Gordeeva y Grishina, 1991)

Distribución: "Siberia".

-Moritzoppia (M.) myrmophila altaica (Gordeeva y Grishina, 1991) (Moritziella)

DisTRIBUCIÓN: Asia central rusa.

-Moritzoppia (M.) oreia Colloff y Seyd, 1991

DISTRIBUCIÓN: Euroatlántica.

-Moritzoppia (M.) problematica Mahunka y Mahunka-Papp, 2002

DisTRIBUCIÓN: Hungría.

-Moritzoppia (M.) punctata (Mihelcic, 1958) (Oppia)

DisTRIBUCIÓn: Austria.

-Moritzoppia (M.) sharipovi Niemi y Skubala, 1993

DisTRIBUCIÓN: Polonia.

-Moritzoppia (M.) similis (Gordeeva y Grishina, 1991) (Moritziella)

DisTRIBUCIÓN: "Siberia".

-Moritzoppia (M.) sitnikovae (Kulijev, 1962) (Oppia)

Distribución: Cáucaso.

-Moritzoppia (M.) splendens (Koch, 1841) (Oppia) "sp. inq."

DisTRIBUCIÓN: Alemania.

-Moritzoppia (M.) tridentata (Forsslund, 1942) (Oppia)

DisTRIBUCIÓN: Euroatlántica.

-Moritzoppia (M.) uherkovichi (Mahunka, 1985) (Moritziella)

DisTRIBUCIÓN: Europa centrooriental.

-Moritzoppia (M.) unicarinata (Paoli, 1908) (Dameosoma)

DisTRIBUCIÓN: Holártica y "Australiana".

-Moritzoppia (M.) unicarinata unicarinata (Paoli, 1908)

(=Oppia fixa Mihelcic, 1956)

(=Dameosoma formosum Hull, 1914)

(=Dameosoma minus lamellata Halbert, 1923)

DisTRIBUCIÓN: Holártica (frecuente en Paleártica) y "Australiana”.

-Moritzoppia (M.) unicarinata clavigera (Hammer, 1952) (Oppia c.) DisTRIBUCIÓN: Boreal.

-Moritzoppia (M.) unicarinata cristata (Subías y Rodríguez, 1986)

(Moritziella)

DisTRIBUCIÓN: España

-Moritzoppia (M.) unicarinata keilbachi (Moritz, 1969) (Oppia k.) Distribución: Europa y Oeste de Siberia.

-Moritzoppia (M.) unicarinata unicarinatoides (Subías y Rodríguez,

1986) (Moritziella)

DISTRIBUCIÓN: Europa centromeridional.

Moritzoppia (Moritzoppiella) Gordeeva, 2000 (14 spp.)

(=Dentoppia Gordeeva, 2001 "sin. nov.")

(=Drukoppia Gordeeva, 2001 "sin. nov.")

EsPecIE TIPO: Moritziella microdentata Gordeeva y Grishina, 1991

DISTRIBUCIÓN: Holártica y Neotropical.

-Moritzoppia (Moritzoppiella) dentifera (Bayartogtokh y Gordeeva,

2001) (Tetroppia (Drukoppia))

DisTRIBUCIÓN: Mongolia.

-Moritzoppia (Moritzoppiella) incisa Mahunka y Mahunka-Papp, 2000 (Moritzoppia)

DisTRIBUCIÓN: Suiza.

-Moritzoppia (Moritzoppiella) metulifera (Bayartogtokh y Gordeeva, 2001) (Tetroppia (Dentoppia))

DISTRIBUCIÓN: Mongolia.

-Moritzoppia (Moritzoppiella) microdentata (Gordeeva y Grishina, 1991) (Moritziella)

DisTRIBUCIÓN: "Siberia".

-Moritzoppia (Moritzoppiella) minuta (Gordeeva y Grishina, 1991) (Moritziella)

DisTRIBUCIÓN: "Siberia".

-Moritzoppia (Moritzoppiella) montana (Gordeeva y Bayartogtokh, 2001) (Tetroppia (Drukoppia))

DisTRIBUCIÓN: Mongolia.
-Moritzoppia (Moritzoppiella) nana (Gordeeva y Bayartogtokh, 2001)

(Tetroppia (Drukoppia))

DistriBuCión: Mongolia.

-Moritzoppia (Moritzoppiella) neerlandica (Oudemans, 1900)

(Eremaeus longilamellatus $n$.)

(=Dameosoma translamellatum Willmann, 1923)

DisTRIBUCIÓN: Holártica (menos frecuente en el Sur).

-Moritzoppia (Moritzoppiella) nikolskii (Gordeeva y Grishina, 1991)

(Moritziella)

DisTRIBUCIÓN: "Siberia".

-Moritzoppia (Moritzoppiella) nitens (Gordeeva y Grishina, 1991)

(Moritziella)

DisTRIBUCIÓN: "Siberia".

-Moritzoppia (Moritzoppiella) pinea (Gordeeva y Grishina, 1991)

(Moritziella)

DISTRIBUCIÓN: "Siberia".

-Moritzoppia (Moritzoppiella) praestans (Gordeeva y Grishina, 1991)

(Moritziella)

DistriBución: "Siberia".

-Moritzoppia (Moritzoppiella) subfallax Mahunka, 1998 (Moritzoppia)

DistRIBUCiÓN: Pequeñas Antillas.

-Moritzoppia (Moritzoppiella) volcanensis (Hammer, 1962) (Oppiella)

DistriBución: Chile.

Neostrinatina Mahunka, 1979 (1 sp.)

ESPECIE TIPO: Neostrinatina mixoppia Mahunka, 1979

DisTRIBUCIÓN: Neotropical.

-Neostrinatina mixoppia Mahunka, 1979

DISTRIBUCIÓN: Guatemala.

Neotrichoppia Subías e Iturrondobeitia, 1980 (8 spp. y 1 ssp.)

ESPECIE TIPO: Neotrichoppia pseudoconfinis Subías e Iturrondobeitia, 1980 DisTRIBUCIÓN: Paleártica.

Neotrichoppia (Neotrichoppia) Subías e Iturrondobeitia, 1980 (1 sp.) DisTRIBUCIÓN: Paleártica.

-Neotrichoppia (N.) pseudoconfinis Subías e Iturrondobeitia, 1980 DISTRIBUCIÓN: Ibérica.

Neotrichoppia (Ancestroppia) Subías y Rodríguez, 1986 (1 sp.)

ESPECIE TIPO: Neotrichoppia (Ancestroppia) berninii Subías y Rodríguez, 1986

DisTRIBUCIÓN: Paleártica.

-Neotrichoppia (Ancestroppia) berninii Subías y Rodríguez, 1986

DisTRIBUCIÓN: Mediterránea occidental.

Neotrichoppia (Confinoppia) Subías y Rodríguez, 1986 (6 spp. y 1 ssp.) EsPecie TIPO: Dameosoma confine Paoli, 1908

DisTRIBUCIÓN: Paleártica.

-Neotrichoppia (Confinoppia) adanata Iturrondobeitia y Saloña, 1988 DisTRIBUCIÓN: España.

-Neotrichoppia (Confinoppia) confinis (Paoli, 1908) (Dameosoma)

DisTRIBUCión: Paleártica occidental (excepto Norte).

-Neotrichoppia (Confinoppia) confinis confinis (Paoli, 1908) DisTRIBUCIÓN: Europa centromeridional.

-Neotrichoppia (Confinoppia) confinis tenuiseta Subías y Rodríguez, 1986

DisTRIBUCión: Mediterránea

-Neotrichoppia (Confinoppia) gibber (Mahunka, 1982) (Oppia)

DisTRIBUCIÓN: Grecia.

-Neotrichoppia (Confinoppia) hauseri (Mahunka y Mahunka-Papp, 2000) (Lauroppia)

DisTRIBUCIÓN: Suiza.

-Neotrichoppia (Confinoppia) variabilis Iturrondobeitia y Subías, 1981 DistribuCión: España.

-Neotrichoppia (Confinoppia) zushi (Aoki, 1984) (Oppiella)

DisTRIBUCIÓN: Japón. 
Oppiella Jacot, 1937 (9 spp.)

EsPeCIE TIPO: (Dameosoma corrugatum Berlese, 1904) =Eremaeu novus Oudemans, 1902

DISTRIBUCIÓN: Cosmopolita.

Oppiella (Oppiella) Jacot, 1937 (6 spp.)

DisTRIBUCIÓN: Cosmopolita.

-Oppiella (O.) baburini Rjabinin, 1979

Distribución: Este de Rusia asiática.

-Oppiella (O.) besucheti Mahunka y Mahunka-Papp, 2000

DisTRIBUCIÓN: Suiza.

-Oppiella (O.) neonominata nom. nov.

[=Oppiella distincta Rjabinin, 1989, "nom. praeoc. por Vasiliu y Calugar,

1981 (“hom. prim.")]

DisTRIBUCIÓN: Este de Rusia asiática.

-Oppiella (O.) nova (Oudemans, 1902) (Eremaeus)

(=Oppiella aegyptiaca Elbadry y Nasr, 1974)

(=Oppiella chistyakovi Rjabinin, 1975)

(=Dameosoma corrugatum Berlese, 1904)

(=Oppiella corrugata apicalis Jacot, 1937)

(=Dameosoma corrugatum intralamellatum Thamdrup, 1932)

(=Oppiella corrugata squarrosa Jacot, 1937)

(=Dameosoma krygeri Trägardh, 1931)

(=Oppia neerlandica sumatrensis Willmann, 1931)

(=Oppiella nova palustris Laskova, 1980)

(=Oppiella orientata Rjabinin, 1975)

(=Oppia rossica Bulanova-Zachvatkina, 1964)

(=Notaspis sculptilis Warburton y Pearce, 1905)

(=Dameosoma uliginosum Willmann, 1919)

(=Oppia washburni Hammer, 1952)

DisTRIBUCIÓN: Cosmopolita (frecuente).

-Oppiella (O.) primorica (Golosova, 1969) (Oppia)

Distribución: Paleártica (Europa centrooriental y Este de Paleártica oriental).

-Oppiella (O.) propinqua Mahunka y Mahunka-Papp, 2000

DISTRIBUCIÓN: Suiza.

Oppiella (Perspicuoppia) Pérez-Íñigo, 1971 (3 spp.)

ESPECIE TIPO: Oppia perspicua Mihelcic, 1956

Distribución: Paleártica.

-Oppiella (Perspicuoppia) minidentata (Subías, 1977) (P.)

DisTRIBUCión: Europa.

-Oppiella (Perspicuoppia) perspicua (Mihelcic, 1956) (Oppia)

Distribución: Sur de Europa.

-Oppiella (Perspicuoppia) rara Ivan y Vasiliu, 1997

DISTRIBUCIÓN: Rumania.

Pseudobrachioppiella Tseng, 1982 (1 sp.)

EsPeCIE TIPO: Pseudobrachioppiella ramosa Tseng, 1982

DISTRIBUCIÓN: Oriental.

-Pseudobrachioppiella ramosa Tseng, 1982

DisTRIBUCIÓN: Taiwan [Formosa].

Ptiloppia Balogh, 1983 (1 sp.)

EsPeCIE TIPO: Oppiella bulanovae Hammer, 1968

DisTRIBUCIÓN: Australianooriental

-Ptiloppia bulanovae (Hammer, 1968) (Oppiella)

DistriBuCión: Nueva Zelanda.

-Ptiloppia lienhardi Mahunka, 2001

DISTRIBUCIÓN: Borneo.

Tripiloppia Hammer, 1968 (6 spp.)

EsPeCIE TIPO: Tripiloppia aokii Hammer, 1968

DISTRIBUCIÓN: Australiana.

-Tripiloppia aokii Hammer, 1968

DisTRIBUCIÓN: Nueva Zelanda.

-Tripiloppia dalenii Hammer, 1968

DisTRIBUCión: Nueva Zelanda.

-Tripiloppia forsslundi Hammer, 1968

DISTRIBUCIÓN: Nueva Zelanda.
-Tripiloppia subiasi Balogh, 1982

DistRIBUCIÓN: Australia.

-Tripiloppia tarraswahlbergi Hammer, 1968

DisTRIBUCión: Nueva Zelanda.

-Tripiloppia traegardhi Hammer, 1968

DisTRIBUCIÓN: Nueva Zelanda.

Tuberoppia Golosova, 1974 (2 spp.)

EsPeCIE TIPO: Oppia rotundata Golosova, 1970

DISTRIBUCIÓN: Paleártica.

-Tuberoppia paradoxa Golosova, 1980

DisTRIBUCIÓN: Este de Rusia asiática.

-Tuberoppia rotundata (Golosova, 1970) (Oppia)

DisTRIBUCIÓN: Este de Paleártica oriental.

[Oxyoppiinae] Subías, 1989 (13 gen., 6 subg., 68 spp. y 1 ssp.) Distribución: Cosmopolita.

Acroppia Balogh, 1983 (6 spp.)

EsPECIE TIPO: Stachyoppia processigera Balogh y Mahunka, 1967

DISTRIBUCIÓN: Pantropical.

-Acroppia amazonica (Balogh y Mahunka, 1969) (Stachyoppia)

DISTRIBUCIÓN: Neotropical.

-Acroppia antillensis Mahunka, 1985

DisTRIBUCIÓN: Pequeñas Antillas.

-Acroppia curvispina (Mahunka, 1983) (Stachyoppia)

DistRiBución: Neotropical (Brasil y Panamá).

-Acroppia monstruosa (Mahunka, 1989) (Stachyoppia)

DISTRIBUCIÓN: Nigeria.

-Acroppia processigera (Balogh y Mahunka, 1967) (Stachyoppia)

(=Acroppia papua J. y P. Balogh, 2002)

Distribución: Etiópica (Congo), Oriental (Filipinas e Indonesia:

Krakatoa) y Australiana (Nueva Guinea).

-Acroppia translamellata (Balogh y Mahunka, 1966) (Stachyoppia)

DisTRIBUCIÓN: Congo.

Baloghoppia Mahunka, 1983 (2 spp.)

EsPECIE TIPO: Baloghoppia dentata Mahunka, 1983

DISTRIBUCIÓN: Neotropical.

-Baloghoppia dentata Mahunka, 1983

DisTRIBUCIÓN: Brasil.

-Baloghoppia inornata Franklin y Woas, 1992

DisTRIBUCIÓN: Brasil.

Foraminoppia Subías y Arillo, 1998 (2 spp.)

EsPeCIE TIPO: Foraminoppia iturrondobeitiai Subías y Arillo, 1998 DisTRIBUCIÓN: Paleártica.

-Foraminoppia iturrondobeitiai Subías y Arillo, 1998

DisTRIBUCión: España.

-Foraminoppia salonae Subías y Arillo, 1998

DisTRIBUCIÓN: España.

Fossoppia Mahunka, 1994 (2 spp.)

EsPeCIE TIPO: Fossoppia calcarata Mahunka, 1994

DISTRIBUCIÓN: Etiópica.

-Fossoppia calcarata Mahunka, 1994

DisTRIBUCIÓN: Madagascar.

-Fossoppia pirata Mahunka, 1994

DisTRIBUCIÓN: Madagascar.

Foveolatoppia Mahunka, 1988 (1 sp.)

ESPECIE TIPO: Foveolatoppia foveolata Mahunka, 1988

DISTRIBUCIÓN: Oriental.

-Foveolatoppia foveolata Mahunka, 1988

DisTRIBUCIÓN: Borneo.

Lineoppia J. y P. Balogh, 1983 (2 spp.)

EsPECIE TIPO: Lineoppia frouini J. y P. Balogh, 1983

DISTRIBUCIÓN: Australiana y Neotropical. 


\author{
-Lineoppia frouini J. y P. Balogh, 1983 \\ DisTRIBUCIÓN: Nueva Caledonia. \\ -Lineoppia mastax (Balogh y Mahunka, 1977) (Oxyoppia) \\ DISTRIBUCIÓN: Neotropical (Bolivia y Paraguay).
}

Mahunkella Balogh, 1983 (1 sp.)

ESPECIE TIPO: Oppiella transitoria Balogh y Mahunka, 1978

DISTRIBUCIÓN: Neotropical.

-Mahunkella transitoria (Balogh y Mahunka, 1978) (Oppiella)

DistriBución: Brasil.

Oxybrachioppia Subías, 1989 (3 spp.)

EsPECIE TIPO: Brachioppiella ctenifera barbata Choi, 1986

DisTRIBUCIÓN: Paleártica.

-Oxybrachioppia barbata (Choi, 1986) (Brachioppiella ctenifera b.) DisTRIBUCIÓN: Corea.

-Oxybrachioppia ctenifera (Golosova, 1970) (Oppia)

Distribución: Paleártica: Europa centromeridional (excepto Oeste) y Este de Paleártica oriental.

-Oxybrachioppia glabriseta Wang y Shen, 1999

DISTRIBUCIÓN: China (Tíbet).

Oxyoppia Balogh y Mahunka, 1969 (28 spp. y 1 ssp.)

EsPeCIE TiPo: Oppia spinosa Hammer, 1958

DisTRIBUCIÓN: Cosmopolita.

Oxyoppia (Oxyoppia) Balogh y Mahunka, 1969 (3 spp.)

DisTRIBUCIÓN: Tropical (Etiópica y Neotropical).

-Oxyoppia (O.) complicata Mahunka, 1986

Distribución: Kenia.

-Oxyoppia (O.) polita P. Balogh, 1984

DisTRIBUCIÓN: Colombia.

-Oxyoppia(O.) spinosa (Hammer, 1958) (Oppia)

(=Oxyoppia espozi J. y P. Balogh, 2002)

DISTRIBUCIÓN: Bolivia.

Oxyoppia (Aciculoppia) Subías y Rodríguez, 1986 (2 spp.)

ESPECIE TIPO: Oxyoppia genavensium Mahunka, 1982

DisTRIBUCIÓN: Neotropical y Paleártica.

Oxyoppia (Aciculoppia) clavata Aoki, 1983 (O.)

DisTRIBUCIÓN: Japón (frecuente).

-Oxyoppia (Aciculoppia) genavensium Mahunka, 1982 (O.) DisTRIBUCIÓN: Méjico.

Oxyoppia (Dzarogneta) Kulijev, 1978 (11 spp.)

(=Fineoppia Khanbekyan y Gordeeva, 1991 "sin. nov.")

(=Pectinoppia Subías y Rodríguez, 1986)

EsPecie TIPO: Oppia dubia Kulijev, 1966

DisTRIBUCIÓN: Tropical (Etiópica y Neotropical) y subtropical (Paleártica meridional).

-Oxyoppia (Dzarogneta) cristata Hammer, 1977 (O.)

DISTRIBUCIÓN: Pakistán.

-Oxyoppia (Dzarogneta) dubia (Kulijev, 1966) (Oppia)

Distribución: Cáucaso.

-Oxyoppia (Dzarogneta) intermedia Subías y Rodríguez, 1986 (O.

(Pectinoppia)

DisTRIBUCIÓN: España.

-Oxyoppia (Dzarogneta) khosrovica (Khanbekyan y Gordeeva, 1991)

(Fineoppia)

DisTRIBUCIÓN: Cáucaso

-Oxyoppia (Dzarogneta) latisternalis (Balogh y Mahunka, 1974)

(Oppia)

DisTRIBUCIÓN: Cuba.

-Oxyoppia (Dzarogneta) pluripectinata (Balogh, 1958) (Oppia)

DisTRIBUCión: Angola.

-Oxyoppia (Dzarogneta) pustulata Mahunka, 1997 (O.)

DISTRIBUCIÓN: Madagascar.

-Oxyoppia (Dzarogneta) sadbinia (Shtanchaeva, 1984) (Oppia)

DisTRIBUCIÓN: Asia centrooccidental.
-Oxyoppia (Dzarogneta) taurus Pérez-Iñigo y Baggio, 1997 (O.

(Oxyoppiella)

DISTRIBUCIÓN: Brasil.

-Oxyoppia (Dzarogneta) uncinata Wen, 1993 (O.)

Distribución: Noreste de China.

-Oxyoppia (Dzarogneta) yepesensis Muñoz-Mingarro, 1987 (O.)

DisTRIBUCIÓN: Suroeste de Europa.

Oxyoppia (Oxyoppiella) Subías y Rodríguez, 1986 (12 spp. y 1 ssp.)

EsPECIE TIPO: Oppiella polynesia Hammer, 1972

DisTRIBUCIÓN: Cosmopolita (excepto Boreal).

-Oxyoppia (Oxyoppiella) antillensis Mahunka, 1998 (Oxyoppia)

DisTRIBUCIÓN: Pequeñas Antillas.

-Oxyoppia (Oxyoppiella) baliensis (Hammer, 1982) (Oppia)

DistRIBUCIÓN: Bali.

-Oxyoppia (Oxyoppiella) bituberculata (Balogh, 1958) (Oppia)

DisTRIBUCIÓN: Etiópica y subantártica (I. San Pablo y Amsterdam).

-Oxyoppia (Oxyoppiella) bituberculata bituberculata (Balogh, 1958)

DisTRIBUCIÓN: Etiópica y subantártica (I. San Pablo y Amsterdam).

-Oxyoppia (Oxyoppiella) bituberculata cognata (Wallwork, 1961)

(Oppia)

DisTRIBUCIÓN: Ghana.

-Oxyoppia (Oxyoppiella) cubana Balogh y Mahunka, 1980 (Oxyoppia)

DisTRIBUCIÓN: Cuba.

-Oxyoppia (Oxyoppiella) polynesia (Hammer, 1972) (Oppiella)

(=Oxyoppia pilosa Balogh y Mahunka, 1981)

DistriBución: Australiana (Polinesia: Tahití), Oriental (India:

Tripura) y Neotropical (Paraguay y Panamá).

-Oxyoppia (Oxyoppiella) saskai (Balogh, 1961) (Oppia)

DisTRIBUCIÓN: Tanzania.

-Oxyoppia (Oxyoppiella) scalifera (Hammer, 1958) (Oppia)

DISTRIBUCIÓN: Neotropical (Argentina y Perú) y Filipinas.

-Oxyoppia (Oxyoppiella) spiculifera Mahunka, 1985 (Oxyoppia)

DisTRIBUCIÓN: Sudáfrica.

-Oxyoppia (Oxyoppiella) struthio Mahunka, 1983 (Oxyoppia)

DisTRIBUCIÓN: Tanzania.

-Oxyoppia (Oxyoppiella) suramericana (Hammer, 1958) (Oppia)

DISTRIBUCIÓN: Neotropical (frecuente), India (Orissa), Australiana

(Nueva Zelanda y Australia).

-Oxyoppia (Oxyoppiella) vtorovi (Rjabinin, 1987) (Oppia)

DisTRIBUCIÓN: Asia centrooccidental.

-Oxyoppia (Oxyoppiella) zsuzsankae (Mahunka, 2002) (Oxyoppiella)

DISTRIBUCIÓN: Madagascar.

Oxyoppioides Subías y Mínguez, 1985 (1 sp.)

EsPeCIE TIPO: Dameosoma decipiens Paoli, 1908

DISTRIBUCIÓN: Paleártica.

-Oxyoppioides decipiens (Paoli, 1908) (Dameosoma)

DisTRIBUCIÓN: Paleártica meridional (Paleártica occidental: excepto

Norte, y Sur de Siberia).

Sacculoppia Balogh y Mahunka, 1968 (1 sp.)

ESPECIE TIPO: Sacculoppia singularis Balogh y Mahunka, 1968

DisTRIBUCIÓN: Neotropical.

-Sacculoppia singularis Balogh y Mahunka, 1968

DISTRIBUCIÓN: Argentina.

Separatoppia Mahunka, 1983 (4 spp.)

ESPECIE TIPO: Oppia africana Evans, 1953

DISTRIBUCIÓN: Etiópica.

-Separatoppia acutipes (Warburton, 1912) (Notaspis)

DisTRIBUCIÓN: I. Seychelles.

-Separatoppia africana (Evans, 1953) (Oppia)

DisTRIBUCIÓN: Tanzania.

-Separatoppia gracilis Mahunka, 1997

DisTRIBUCIÓN: Kenia.

-Separatoppia robusta Mahunka, 1997

DISTRIBUCIÓN: Kenia. 
Subiasella Balogh, 1983 (15 spp.)

EsPECIE TIPO: Oppia exigua Hammer, 197

DisTRIBUCIÓN: Semicosmopolita (Holártica, Australiana y Neotropical)

Subiasella (Subiasella) Balogh, 1983 (2 spp.)

DisTRIBUCIÓN: Tropical (Australiana y Neotropical) y subtropical

-Subiasella (S.) exigua (Hammer, 1971) (Oppia)

DisTRIBUCIÓN: Australiana e I. Galápagos.

-Subiasella (S.) sigmella (Golosova, 1970) (Oppia)

Distribución: Paleártica (Sur de Europa oriental y Este de

Paleártica oriental).

Subiasella (Dividoppia) Mahunka, 1987 (1 sp.)

ESPECIE TIPO: Dividoppia aperta Mahunka, 1987

DisTRIBUCiÓN: Paleártica.

-Subiasella (Dividoppia) aperta (Mahunka, 1987) (D.)

DisTRIBUCIÓN: Hungría.

Subiasella (Lalmoppia) Subías y Rodríguez, 1986 (11 spp.)

(=Cassioppia Poltavskaja, 1994 "sin. nov.")

(=Pararectoppia Mahunka, 1987)

EsPECIE TIPO: (Oppia ventronodosa Hammer, 1962) =Oppia maculata Hammer, 1952

DisTRIBUCIÓN: Holártica y Neotropical

-Subiasella (Lalmoppia) arcuata (Hammer, 1958) (Oppia)

DisTRIBUCIÓN: Argentina.

-Subiasella (Lalmoppia) boninensis Ohkubo, 1996

DisTRIBUCIÓN: Japón.

-Subiasella (Lalmoppia) europaea (Mahunka, 1982) (Oxyoppia)

DISTRIBUCIÓN: Europa central.

-Subiasella (Lalmoppia) gracilis (Paoli, 1908) (Dameosoma)

DisTRIBUCIÓN: Holártica (Paleártica occidental: Bulgaria, y U.S.A.

Misuri)

-Subiasella (Lalmoppia) incurva (Aoki, 1983) (Oxyoppia)

DisTRIBUCIÓN: Este de Paleártica oriental.

-Subiasella (Lalmoppia) krivolutskyi (Poltavskaja, 1994) (Cassioppia) DistribuCión: Paleártica (Sureste de Europa y "Siberia”).

-Subiasella (Lalmoppia) maculata (Hammer, 1952) (Oppia)

(=Oppia ventronodosa Hammer, 1962)

DistRIBUCIÓN: Holártica (Europa oriental, Paleártica oriental excepto Este, y Neártica septentrional ) y Chile.

-Subiasella (Lalmoppia) quadrimaculata (Evans, 1952) (Oppia)

DistRIBUCión: Paleártica (Europa y Este de Rusia asiática).

-Subiasella (Lalmoppia) simplissima (Jacot, 1938) (Oppia minus s.)

DisTRIBUCIÓN: Neártica (U.S.A.: Ilinois, y Canadá).

-Subiasella (Lalmoppia) subiasi (Mahunka, 1987) (Pararectoppia) DisTRIBUCIÓN: Europa central.

-Subiasella (Lalmoppia) zeyensis (Rjabinin, 1975) (Oppia)

DisTRIBUCIÓN: Este de Rusia asiática.

Subiasella (Lucioppia) Mahunka, 1985 (1 sp.)

EsPECIE TIPO: Lucioppia hauseri Mahunka, 1985

DisTRIBUCIÓN: Neotropical.

-Subiasella (Lucioppia) hauseri (Mahunka, 1985) (L.)

DisTRIBUCión: Pequeñas Antillas.

[Mystroppiinae] Balogh, 1983 (7 gen., 2 subg., 48 spp. y 2 sspp.) DisTRIBUCIÓN: Pantropical y subtropical.

Cheloppia Hammer, 1971 (2 spp.)

ESPECIE TIPO: Cheloppia hyalina Hammer, 1971

DisTRIBUCIÓN: Tropical (Australiana y Neotropical).

-Cheloppia americana Mahunka, 1985

DistRIBUCión: Pequeñas Antillas.

-Cheloppia hyalina Hammer, 1971

DistRIBUCIÓN: Melanesia (I. Fiji).

Corynoppia Balogh, 1983 (6 spp. y 1 ssp.)

EsPECIE TIPO: Stachyoppia kosarovi Jeleva, 1962

Distribución: Tropical (Oriental y Neotropical) y subtropical (Paleártica meridional).
-Corynoppia andulau Mahunka, 2001

DisTRIBUCIÓN: Borneo.

-Corynoppia foliata (Mihelcic, 1957) (Damaeolus)

DisTRIBUCIÓN: España.

-Corynoppia foliatoides Subías y Rodríguez, 1986

Distribución: España.

-Corynoppia kosarovi (Jeleva, 1962) (Stachyoppia)

DisTRIBUCIÓN: Sur de Europa y Panamá.

-Corynoppia kosarovi kosarovi (Jeleva, 1962)

Distribución: Sur de Europa y Panamá

-Corynoppia kosarovi matritensis (Pérez-Íñigo, 1967) (Stachyoppia) DisTRIBUCIÓn: Sur de Europa.

-Corynoppia maritima Pérez-Íñigo jr., 1991 (C. kosarovi m.)

DisTRIBUCIÓN: España (I. Baleares).

-Corynoppia papillisetigera Iturrondobeitia y Saloña, 1998

DISTRIBUCIÓN: España.

Karenella Hammer, 1962 (10 spp. y 1 ssp.)

ESPECIE TIPO: Karenella lobata Hammer, 1962

DISTRIBUCIÓN: Pantropical.

Karenella (Karenella) Hammer, 1962 (6 spp. y 1 ssp.)

DISTRIBUCIÓN: Pantropical.

-Karenella (K.) acuta (Csiszár, 1961) (Oppia)

DisTRIBUCIÓN: Oriental (Java y Vietnam)

-Karenella (K.) bruneiana Mahunka, 2001

DISTRIBUCIÓN: Borneo.

-Karenella (K.) lanceoseta (Balogh, 1959) (Oppia)

DisTRIBUCIÓN: Etiópica (Congo y Ghana).

-Karenella (K.) lanceoseta lanceoseta (Balogh, 1959) DisTRIBUCión: Congo.

-Karenella (K.) lanceoseta occidentalis (Wallwork, 1961) (Oppia) DistriBución: Ghana.

-Karenella (K.) lanceosetoides (Hammer, 1971) (Oppia)

DistRIBUCIÓN: Australiana (Islas del Pacífico) y Oriental (Java).

-Karenella (K.) lobata Hammer, 1962

DisTRIBUCIÓN: América austral.

-Karenella (K.) turgiseta (Mahunka, 1985) (Corynoppia)

DisTRIBUCIÓN: Norte de Neotropical.

Karenella (Glabroppia) Subías y Rodríguez, 1986 (2 spp.)

EsPeCIE TIPO: Oppia minutisetosa Hammer, 1982

Distribución: Paleotropical.

-Karenella (Glabroppia) cohici (Balogh y Mahunka, 1966) (Oppia)

DisTRIBUCión: Congo.

-Karenella (Glabroppia) minutisetosa (Hammer, 1982) (Oppia)

DISTRIBUCIÓN: Bali.

Karenella (Stakarenoppia) Subías y Rodríguez, 1986 (2 spp.)

EsPeCIE TIPO: Stachyoppia granulosa Subías y Sarkar, 1983

DisTRIBUCIÓN: Paleotropical.

-Karenella (Stakarenoppia) foveolata Mahunka, 1992 (K.)

DisTRIBUCIÓN: Senegal.

- Karenella (Stakarenoppia) granulosa (Subías y Sarkar, 1983) (Sta-

chyoppia)

DISTRIBUCIÓN: India (Tripura).

Mystroppia Balogh, 1959 (3 spp.)

EsPECIE TIPO: Mystroppia sellnicki Balogh, 1959

DisTRIBUCIÓN: Paleártica.

-Mystroppia dallaii Bernini, 1973

DisTRIBUCIÓN: Mediterránea occidental

-Mystroppia rethejumi Krivolutsky, 1971

DISTRIBUCIÓN: Asia centrooccidental.

-Mystroppia sellnicki Balogh, 1959

DISTRIBUCIÓN: Europa centromeridional.

Rugoppia Mahunka, 1986 (5 spp.)

=Mahnertella Mahunka, 1997 "sin. nov.")

EsPECIE TIPO: Rugoppia luisiae Mahunka, 1986

DISTRIBUCIÓN: Etiópica. 
-Rugoppia boraha (Mahunka, 1994) (Brachioppiella) DisTRIBUCIÓN: Madagascar.

-Rugoppia lamellicornis (Warburton, 1912) (Notaspis) DisTRIBUCIÓN: I. Seychelles.

-Rugoppia luisiae Mahunka, 1986

DISTRIBUCIÓN: Tanzania.

-Rugoppia quadrituberculata (Mahunka, 1997) (Mahnertella) DisTRIBUCIÓN: Kenia.

-Rugoppia vilagiorum Mahunka, 1998 DISTRIBUCIÓN: Uganda.

Stachyoppia Balogh, 1961 (1 sp.)

EsPECIE TIPO: Stachyoppia muscicola Balogh, 1961

DISTRIBUCIÓN: Etiópica y Paleártica meridional.

-Stachyoppia muscicola Balogh, 1961

DisTRIBución: Congo y Cáucaso.

Striatoppia Balogh, 1958 (21 spp. y 1 ssp.)

EsPECIE TIPO: Stiatoppia machadoi Balogh, 1958

DisTRIBUCIÓN: Pantropical y subtropical.

-Striatoppia baloghi Mahunka, 1974

DISTRIBUCIÓN: Camermún.

-Striatoppia breviclava Mahunka, 1982

DisTRIBUCIÓN: Etiopía.

-Striatoppia foliosa (Jacot, 1937) (Oppiella)

DISTRIBUCIÓN: Este de U.S.A.

-Striatoppia hammeni Mahunka, 1977

DistRIBUCIÓN: Oriental y Australiana (Indonesia: Lombok).

-Striatoppia lanceolata Hammer, 1972

Distribución: Australiana (Polinesia: Tahití), Oriental (India:

Bengala Occidental) y Neotropical (I. Galápagos).

-Striatoppia luisae Mahunka, 1994

DisTRIBUCIÓN: Madagascar.

-Striatoppia machadoi Balogh, 1958

DisTRIBUCión: Angola e India (Bengala Occidental).

-Striatoppia madagascarensis Balogh, 1961

DISTRIBUCIÓN: Madagascar.

-Striatoppia margaritata Mahunka, 1969

DISTRIBUCIÓN: Tanzania

-Striatoppia margaritifera Balogh y Mahunka, 1966

DisTRIBUCIÓN: Congo.

-Striatoppia modesta Mahunka, 1988

DisTRIBUCIÓn: Borneo.

-Striatoppia multilineata Corpuz-Raros, 1979

DisTRIBUCIÓN: Filipinas.

-Striatoppia niliaca (Popp, 1960) (Oppiella)

DisTRIBUCión: Mediterránea oriental e India (Bengala Occidental).

-Striatoppia opuntiseta Balogh y Mahunka, 1968

DisTRIBUCIÓN: Pantropical (excepto Australiana) y Japón.

-Striatoppia papillata Balogh y Mahunka, 1966

DisTRIBUCIÓN: Congo, Egipto y Vietnam.

-Striatoppia quadrilineata Hammer, 1982

DisTRIBUCión: Bali.

-Striatoppia silvicola Franklin y Woas, 1992

DisTRIBUCIÓN: Brasil.

-Striatoppia similis Subías y Sarkar, 1983

DistriBución: India (Tripura) y Polinesia (Tahití)

-Striatoppia similis similis Subías y Sarkar, 1983

DisTRIBUCIÓN: India (Tripura).

-Striatoppia similis polynesica Subías y Sarkar, 1983 Distribución: Polinesia (Tahití).

-Striatoppia stipularis (Jacot, 1937) (Oppiella)

DisTRIBUCión: U.S.A. (Carolina del Norte).

-Striatoppia tribuliformis Balogh y Mahunka, 1981

DistriBución: Neotropical (Paraguay y Pequeñas Antillas).

-Striatoppia tripurensis Subías y Sarkar, 1983

DisTRIBUCIÓN: India (Tripura).
Epimerellidae Ayyildiz y Luxton, 1989 (3 gen., 6 spp. y 1 ssp.)

DisTRIBUCIÓN: Oriental y subtropical (Paleártica meridional).

Enisella Ayyildiz y Luxton, 1989 (1 sp.)

ESPECIE TIPO: Enisella turcica Ayyildiz y Luxton, 1989

DisTRIBUCIÓN: Paleártica meridional

-Enisella turcica Ayyildiz y Luxton, 1989

DisTRIBUCIÓN: Turquía.

Epimerella Kulijev, 1967 (4 spp. y 1 ssp.)

EsPeCIE TIPO: Oppia smirnovi Kulijev, 1962

DisTRIBUCIÓN: Paleártica meridional.

-Epimerella distenta Ayyildiz y Luxton, 1989

DISTRIBUCIÓN: Turquía.

-Epimerella puzanovi Gordeeva y Karppinen, 1988

Distribución: Ucrania (Crimea).

-Epimerella rubeni Khanbekjan y Gordeeva, 1991

DisTRIBUCIÓN: Cáucaso.

-Epimerella smirnovi (Kulijev, 1962) (Oppia)

DISTRIBUCIÓN: Sureste de Europa.

-Epimerella smirnovi smirnovi (Kulijev, 1962)

DisTRIBUCIÓN: Sureste de Europa.

-Epimerella smirnovi longisetosa Kulijev, 1967

DisTRIBUCIÓN: Sureste de Europa.

Luxtonia Mahunka, 2001 (1 sp.)

ESPECIE TIPO: Luxtonia hauseri Mahunka, 2001

DisTRIBUCIÓN: Oriental.

-Luxtonia hauseri Mahunka, 2001

DisTRIBUCIÓN: Borneo.

Lyroppiidae Balogh, 1983 (1 gen. y 5 spp.)

DisTRIBUCIÓN: Pantropical (excepto Australiana).

Lyroppia Balogh, 1961 (5 spp.)

ESPECIE TIPO: Lyroppia scutigera Balogh, 196

DisTRIBUCIÓn: Pantropical (excepto Australiana).

-Lyroppia anareolata Balogh y Mahunka, 1981

DisTRIBUCIÓN: Paraguay.

-Lyroppia delicata Wang y Lu, 1995

DisTRIBUCIÓN: China suroriental.

-Lyroppia neotropica Balogh y Mahunka, 1974

DisTRIBUCIÓN: Norte de Neotropical.

-Lyroppia scutigera Balogh, 1961

DisTRIBUCIÓN: Etiópica (Cogo y Camerún) y Neotropical (Perú).

-Lyroppia similis Balogh y Mahunka, 1977

DISTRIBUCIÓN: Bolivia.

Granuloppiidae Balogh, 1983 (5 gen. y 23 spp.)

DistriBución: Pantropical y subtropical (Paleártica meridional).

Bornemiszaella P. Balogh, 1994 (3 spp.)

ESPECIE TIPO: Bornemiszaella fournieri P. Balogh, 1994

DisTRIBUCIÓN: Neotropical.

-Bornemiszaella fournieri P. Balogh, 1994

Distribución: Costa Rica.

-Bornemiszaella ramirezi P. Balogh, 1994

Distribución: Costa Rica.

-Bornemiszaella salasi P. Balogh, 1994

DISTRIBUCION: Costa Rica.

Enantioppia Balogh y Mahunka, 1969 (1 sp.)

ESPECIE TIPO: Enantioppia multituberculata Balogh y Mahunka, 1969 DISTRIBUCIÓN: Neotropical.

Enantioppia multituberculata Balogh y Mahunka, 1969

DISTRIBUCIÓN: Bolivia.

Granuloppia Balogh, 1958 (9 spp.)

(=Pocsoppia Mahunka, 1984)

EsPeCIE TIPO: Granuloppia congoensis Balogh, 1958

DISTRIBUCIÓN: Paleotropical. 
-Granuloppia conflata Mahunka, 1974

Distribución: Camerún.

-Granuloppia congoensis Balogh, 1958

DisTRIBUCIÓN: Etiópica (Congo y Camerún).

-Granuloppia extrema (Mahunka, 1984) (Pocsoppia)

DisTRIBUCión: Tanzania.

-Granuloppia ghanensis Wallwork, 1961 (G. congoensis g.)

DisTRIBUCIÓN: Ghana.

-Granuloppia kamerunensis Mahunka, 1974

DistribuCión: Camerún.

-Granuloppia major Balogh, 1958

DisTRIBUCIÓN: Congo.

-Granuloppia neonominata nom. nov.

[=Dameosoma megacephalum Berlese, 1905, "nom. praeoc." por Berlese,

1901 ("hom. prim.")]

DisTRIBUCIÓN: Java.

-Granuloppia nuda Wallwork, 1961 (G. major n.)

DISTRIBUCIÓN: Ghana.

-Granuloppia stigmata (Hammer, 1979) (Oppia)

DISTRIBUCIÓN: Java.

Hammerella Balogh, 1983 (5 spp.)

(=Interoppia Mahunka, 1987)

(=Woasella J. y P. Balogh, 2002 "sin. nov.")

EsPECIE TIPO: Brachioppiella gracilis Hammer, 1977

DisTRIBUCIÓN: Pantropical (Oriental, Neotropical y "Australiana”) y subtropical (Paleártica meridional)

-Hammerella gracilis (Hammer, 1977) (Brachioppiella)

DisTRIBUCIÓN: Pakistán.

-Hammerella mirabilis (Mahunka, 1987) (Interoppia)

DISTRIBUCIÓN: Borneo.

-Hammerella pinnatifilis (Canestrini, 1898) (Belba) "sp. inq." DistribuCIÓN: Nueva Guinea.

-Hammerella rostroreticulata (Ohkubo, Aoki y Hu, 1993) (Interoppia) DisTRIBUCión: China suroriental.

-Hammerella sufflata (Franklin y Woas, 1992) (Pulchroppia)

DisTRIBUCIÓN: Brasil.

Senectoppia Aoki, 1976 (5 spp.)

(=Macrosoma Hammer, 1979)

EsPecie Tipo: Senectoppia rugosa Aoki, 1976

DisTRIBUCIÓN: Oriental y Paleártica meridional.

-Senectoppia complicatum (Paoli, 1908) (Dameosoma)

(=Ptiloppia castagnoliae Mahunka y Mahunka-Papp, 1995)

(=Dameosoma corrugatum Berlese, 1905, "nom. praeoc." por Berlese, 1904)

DisTRIBUCión: Java.

-Senectoppia kerangas Mahunka, 2001

DistRIBUCIÓN: Borneo.

-Senectoppia multisulcatum (Berlese, 1913) (Dameosoma)

(=Senectoppia hammerae Aoki, 1983)

(=Macrosoma rugosa Hammer, 1979)

DISTRIBUCIÓN: Java.

-Senectoppia pectinata Aoki, 1983

Distribución: Japón y China: Taiwan [Formosa].

-Senectoppia rugosa Aoki, 1976

DistriBución: Península Malaya.

Teratoppiidae Balogh, 1983 (4 gen., 1 subg. y 17 spp.) DisTRIBUCIÓn: Pantropical (excepto Australiana).

Brasiloppia Pérez-Íñigo y Baggio, 1986 (1 sp.)

EsPecIE TIPO: Brasiloppia flechtmanni Pérez-Íñigo y Baggio, 1986 DisTRIBUCIÓN: Neotropical.

-Brasiloppia flechtmanni Pérez-Íñigo y Baggio, 1986

DisTRIBUCIÓN: Brasil.

Granuloteratoppia P. Balogh, 1988 (1 sp.)

EsPECIE TIPO: Granuloteratoppia annulata P. Balogh, 1988

DisTRIBUCIÓN: Oriental.
Granuloteratoppia annulata P. Balogh, 1988

DisTRIBUCIÓN: Ceilán [Sri Lanka].

Leoppia Pérez-Íñigo, 1983 (1 sp.)

ESPECIE TIPO: Leoppia longicoma Pérez-Íñigo, 1983

DISTRIBUCIÓN: Etiópica.

-Leoppia longicoma Pérez-Íñigo, 1983

DisTRIBUCIÓN: Guinea Ecuatorial: I. Pagalu [Annobón].

Teratoppia Balogh, 1959 (14 spp.)

ESPECIE TIPO: Teratoppia calcarata Balogh, 1959

DisTRIBUCIÓn: Tropical (Etiópica y Neotropical).

Teratoppia (Teratoppia) Balogh, 1959 (7 spp.)

(=Cretoppia Mahunka, 1986)

DISTRIBUCIÓN: Tropical (Etiópica y Neotropical).

-Teratoppia (T.) brasiliensis Pérez-Íñigo y Baggio, 1996

DisTRIBUCIÓN: Brasil.

-Teratoppia (T.) calcarata Balogh, 1959

DisTRIBUCIÓN: Angola.

-Teratoppia (T.) ciliata Wallwork, 1961

DisTRIBUCIÓn: Etiópica (Ghana y Tanzania).

-Teratoppia (T.) creta (Mahunka, 1986) (Cretoppia)

DisTRIBUCIÓN: Tanzania.

-Teratoppia (T.) minor Wallwork, 1961

DISTRIBUCIÓN: Ghana.

-Teratoppia (T.) reducta Balogh y Mahunka, 1969

DisTRIBUCIÓN: Neotropical.

-Teratoppia (T.) translamellata Mahunka, 1983

DisTRIBUCIÓN: Tanzania.

-Teratoppia (T.) uspiensis Pérez-Íñigo y Baggio, 1980

DistriBUCiÓN: Neotropical (Brasil y Venezuela).

Teratoppia (Teratoppiella) Balogh, 1983 (7 spp.)

ESPECIE TIPO: Teratoppia brevipectinata Balogh y Mahunka, 1978

DISTRIBUCIÓN: Tropical (Etiópica y Neotropical).

-Teratoppia (Teratoppiella) ardua (Pérez-Íñigo y Baggio, 1997)

(Teratoppiella)

DistRIBUCIÓN: Brasil.

-Teratoppia (Teratoppiella) brevipectinata Balogh y Mahunka, 1978

(Teratoppia)

DisTRIBUCión: Chile.

-Teratoppia (Teratoppiella) centroamericana Woas, 1986 (Teratoppia) DisTRIBUCIÓN: El Salvador.

-Teratoppia (Teratoppiella) fimbriata (Mahunka, 1983) (Teratoppiella) DisTRIBUCIÓN: Tanzania.

-Teratoppia (Teratoppiella) pectinata Balogh, 1961 (Teratoppia)

DisTRIBUCión: Tanzania.

-Teratoppia (Teratoppiella) pluripectinata Balogh y Mahunka, 1978

(Teratoppia)

(=Teratoppia baloghi Pérez-Íñigo y Baggio, 1980)

(=Teratoppia pectinata Balogh y Mahunka, 1969, "nom. praeoc." por Balogh, 1961)

DisTRIBUCIÓN: Brasil (frecuente).

-Teratoppia (Teratoppiella) regalis Mahunka, 1983 (Teratoppia)

DistRIBUCIÓN: Méjico.

Sternoppiidae Balogh y Mahunka, 1969 (1 gen. y 10 spp.) DisTRIBUCIÓN: Neotropical.

Sternoppia Balogh y Mahunka,1968 (10 spp.)

(=Synoppia Balogh y Mahunka, 1969)

EsPECIE TIPO: Sternoppia mirabilis Balogh y Mahunka, 1968

DisTRIBUCIÓN: Neotropical.

-Sternoppia boliviana Balogh y Mahunka, 1969

DisTRIBUCIÓN: Bolivia.

-Sternoppia brasiliensis Franklin y Woas, 1992

DisTRIBUCIÓN: Brasil.

-Sternoppia incisa Balogh y Mahunka, 1977

DisTRIBUCIÓN: Bolivia. 
-Sternoppia minor Balogh y Mahunka, 1980

DisTRIBUCión: Cuba.

-Sternoppia mirabilis Balogh y Mahunka, 1968

DISTRIBUCIÓN: Argentina.

-Sternoppia quadriseta (Balogh y Mahunka, 1969) (Synoppia)

DisTRIBUCIÓN: Bolivia.

-Sternoppia reticulata Balogh y Mahunka, 1969

DisTRIBUCIÓN: Brasil.

-Sternoppia sphaerodendron Balogh y Mahunka, 1979

DisTRIBUCIÓN: Cuba.

-Sternoppia striata Mahunka, 1983

DistRIBUCIÓN: Guatemala.

-Sternoppia vicina Balogh y Mahunka, 1980

DisTRIBUCIÓN: Norte de Neotropical.

Machuellidae Balogh, 1983 (2 gen., 5 spp. y 5 sspp.) DisTRIBUCIÓN: Pantropical y Paleártica.

Gredosella Gil-Martín, Arillo y Subías, 2000 (2 spp.)

EsPECIE TIPO: Gredosella fraternalis Gil-Martín, Subías y Arillo, 2000) DISTRIBUCIÓN: Paleártica.

-Gredosella capitata (Kulijev, 1967) (Machuella)

DisTRIBUCIÓn: Sureste de Europa.

-Gredosella fraternalis Gil-Martín, Arillo y Subías, 2000

DISTRIBUCIÓN: España.

Machuella Hammer, 1961 (3 spp. y 5 sspp.)

ESPECIE TIPO: Machuella ventrisetosa Hammer, 1961

DisTRIBUCIÓN: Pantropical y Paleártica.

-Machuella draconis Hammer, 1961

(=Machuella africana Mahunka, 1978)

DistRIBUCIÓN: Paleártica occidental (menos frecuente en el

Norte) y Malgache (I. Reunión)

-Machuella lineata Hammer, 1973

DisTRIBUCIÓN: Polinesia (I. Samoa).

-Machuella ventrisetosa Hammer, 196

DisTRIBUCIÓN: Pantropical y Paleártica.

-Machuella ventrisetosa ventrisetosa Hammer, 1961

(=Machuella hellenica Mahunka, 1982)

(=Machuella hippy Niemi y Gordeeva, 1991)

Distribución: Pantropical (excepto Etiópica) y Paleártica meridional.

-Machuella ventrisetosa bilineata Weigmann, 1976 (M. b.) DisTRIBUCIÓN: Euroatlántica.

-Machuella ventrisetosa plicata Hammer, 1979 DISTRIBUCIÓN: Oriental.

-Machuella ventrisetosa pyriformis Hammer, 1968 (M. p.) DistriBUCión: Nueva Zelanda.

- Machuella ventrisetosa robusta Hammer, 1971 DistriBuCIÓN: Melanesia (I. Fiji)

-Machuella ventrisetosa zehntneri Mahunka, 1977 (M. z.) DISTRIBUCIÓN: Oriental y Malgache (I. Reunión).

Papillonotidae Balogh, 1983 (1 gen. y 4 spp.) DisTRIBUCIÓN: Paleotropical.

Papillonotus Wallwork, 1961 (4 spp.)

ESPECIE TIPO: Papillonotus maculatus Wallwork, 1961

DistRIBUCIÓN: Paleotropical.

-Papillonotus granulosus Wallwork, 1961

DisTRIBUCIÓN: Ghana.

-Papillonotus hauseri Mahunka, 1988

DisTRIBUCIÓn: Borneo.

-Papillonotus maculatus Wallwork, 1961

DisTRIBUCIÓN: Ghana.

-Papillonotus tricarinatus Sarkar y Subías, 1983

DISTRIBUCIÓN: India (Tripura).
Tuparezetidae Balogh, 1972 (1 gen. y 2 spp.)

DisTRIBUCIÓN: Australiana.

Tuparezetes Spain, 1969 (2 spp.)

EsPeCIE TIPO: Tuparezetes christineae Spain, 1969

DisTRIBUCIÓN: Australiana.

-Tuparezetes christineae Spain, 1969

DisTRIBUCIÓN: Nueva Zelanda.

-Tuparezetes philodendrus Spain, 1969

DisTRIBUCIÓN: Nueva Zelanda.

Quadroppiidae Balogh, 1983 (3 gen., 1 subg. y 22 spp.)

DisTRIBUCIÓn: Cosmopolita (excepto Antártica)

Borhidia Balogh y Mahunka, 1974 (2 spp.)

EsPeCIE TIPO: Borhidia cubana Balogh y Mahunka, 1974

DISTRIBUCIÓN: Neotropical.

Borhidia andina P. Balogh, 1988

DisTRIBUCIÓN: Ecuador.

-Borhidia cubana Balogh y Mahunka, 1974

DisTRIBUCIÓN: Cuba.

Hexoppia Balogh, 1958 (1 sp.)

EsPeCIE TIPO: Hexoppia heterotricha Balogh, 1958

DISTRIBUCIÓN: Etiópica.

Hexoppia heterotricha Balogh, 1958

DisTRIBUCIÓN: Etiópica (Congo y Ghana)

Quadroppia Jacot, 1939 (19 spp.)

ESPECIE TIPO: Notaspis quadricarinata Michael, 1885

DisTRIBUCIÓn: Cosmopolita (excepto Antártica).

Quadroppia (Quadroppia) Jacot, 1939 (7 spp.)

DISTRIBUCIÓN: Cosmopolita (excepto Antártica).

-Quadroppia (Q.) cristata Balogh y Mahunka, 1980

DisTRIBUCIÓN: Cuba.

-Quadroppia (Q.) hammerae Mínguez, Ruiz y Subías, 1985

Distribución: Semicosmopolita (Paleártica centromeridional,

U.S.A.: Carolina del Norte, Australiana: Nueva Zelanda y Hawai, y Costa Rica).

-Quadroppia (Q.) Ilinoisensis (Jacot, 1938) (Oppia quadricarinata i.) DisTRIBUCióN: U.S.A. (Ilinois).

-Quadroppia (Q.) longisetosa Mínguez, Ruiz y Subías, 1985

DistRIBUCIÓN: Mediterránea occidental.

-Quadroppia (Q.) maritalis Lions, 1982 (Q. quadricarinata m.)

DistriBución: Europa centromeridional.

-Quadroppia (Q.) obsoleta Mínguez, Ruiz y Subías, 1985 (Q. longisetosa o.)

DisTRIBUCIÓN: España.

-Quadroppia (Q.) quadricarinata (Michael, 1885) (Notaspis)

(=Quadroppia skookumchucki Jacot, 1939)

(=Qudroppia quadricarinata virginalis Lions, 1982)

DISTRIBUCIÓN: Holártica (frecuente), China suroriental y Malgache (I. Mauricio)

Quadroppia (Coronoquadroppia) Ohkubo, 1995 (12 spp.)

EsPECIE TIPO: (Coronoquadroppia parallela Ohkubo, 1995) =Oppia circumita Hammer, 1961

DisTRIBUCIÓn: Cosmopolita (excepto Antártica).

-Quadroppia (Coronoquadroppia) abchasica Gordeeva y Tarba, 1990 (Q. a.)

Distribución: Cáucaso.

-Quadroppia (Coronoquadroppia) circumita (Hammer, 1961)

(Oppia c.)

(?=Oppia quadricarinata ferrumequina Jacot, 1938 “sp. inq.")

(=Coronoquadroppia parallela Ohkubo, 1995)

DistribuCiÓN: Pantropical (excepto Etiópica) y subtropical.

-Quadroppia (Coronoquadroppia) crenata Mahunka, 1984 (Q.c.)

DISTRIBUCION: Tanzania. 
-Quadroppia (Coronoquadroppia) expansa (Ohkubo, 1995) (C. e.) DisTRIBUCIÓN: Japón.

-Quadroppia (Coronoquadroppia) galaica Mínguez, Ruiz y Subías,

1985 (Q. pseudocircumita g.)

(=Quadroppia bellula Luxton, 1987)

DisTRIBUCIÓN: Europa occidental y Cuba.

-Quadroppia (Coronoquadroppia) media Gordeeva, 1983 (Q. m.)

DisTRIBUCIÓN: Europa oriental.

-Quadroppia (Coronoquadroppia) michaeli Mahunka, 1977 (Q. m.)

(=Quadroppia gumista Gordeeva y Tarba, 1990)

(=Quadroppia lesleyae Monson, 2000)

DisTRIBUCIÓN: Paleártica occidental (menos frecuente en el Norte).

-Quadroppia (Coronoquadroppia) monstruosa Hammer, 1979 (Q. m.)

(=Quadroppia mahunkai Mínguez, 1981)

(=Quadroppia paolii Woas, 1986)

(=Quadroppia ritza Gordeeva y Tarba, 1990)

DisTRIBUCIÓN: Holártica (Europa centromeridional y U.S.A.

Carolina del Norte) y Java.

-Quadroppia (Coronoquadroppia) nana Gordeeva, 1983 (Q.n.)

DistRIBUCIÓN: Cáucaso.

-Quadroppia (Coronoquadroppia) nasalis Gordeeva, 1983 (Q.n.)

DisTRIBUCIÓN: Sureste de Europa.

-Quadroppia (Coronoquadroppia) omodeoi Mahunka y Paoletti, 1984

(Q. o.)

DISTRIBUCIÓN: Europa central.

-Quadroppia (Coronoquadroppia) pseudocircumita Mínguez, Ruiz y Subías, 1985 (Q.p.)

Distribución: Holártica (Oeste de Paleártica occidental y Canadá) y Perú.

Trizetoidea Ewing, 1917

Nosibelbidae Mahunka, 1994 (1 gen. y 1 sp.) DisTRIBUCIÓN: Etiópica.

Nosybelba Mahunka, 1994 (1 sp.)

ESPECIE TIPO: Nosybelba oppiana Mahunka, 1994

DISTRIBUCIÓN: Etiópica.

-Nosybelba oppiana Mahunka, 1994

DISTRIBUCIÓN: Madagascar.

Cuneoppiidae Balogh, 1983 (1 gen. y 2 spp.)

DisTRIBUCIÓN: Tropical (Oriental y Neotropical).

Cuneoppia Balogh y Mahunka, 1969 (2 spp.)

ESPECIE TIPO: Cuneoppia laticeps Balogh y Mahunka, 1969

DISTRIBUCIÓN: Tropical (Oriental y Neotropical).

-Cuneoppia dogmai Corpuz-Raros, 1979

DisTRIBUCIÓN: Filipinas.

-Cuneoppia laticeps Balogh y Mahunka, 1969

DisTRIBUCIÓN: Neotropical.

Suctobelbidae Jacot, 1938 (19 gen., 4 subg., 269 spp. y 12 sspp.) DISTRIBUCIÓN: Cosmopolita.

Allosuctobelba Moritz, 1970 (11 spp. y 3 sspp.)

ESPECIE TIPO: Suctobelba grandis Paoli, 1908

DisTRIBUCIÓN: Holártica, Oriental y Neotropical.

-Allosuctobelba bicuspidata Aoki, 1984

DisTRIBUCIÓN: Japón.

-Allosuctobelba bidentata Wen, 1993

DisTRIBUCIÓN: Este de China.

-Allosuctobelba centroamericana (Woas, 1986) (Suctobelba)

DisTRIBUCIÓN: El Salvador.

-Allosuctobelba gigantea (Hammer, 1955) (Suctobelba)

DisTRIBUCIÓN: Alaska.

-Allosuctobelba grandis (Paoli, 1908) (Suctobelba)

Distribución: Holártica y Filipinas.

-Allosuctobelba grandis grandis (Paoli, 1908)

DisTRIBUCIÓN: Holártica.
-Allosuctobelba grandis europaea (Willmann, 1933) (Suctobelba) DisTRIBUCIÓN: Europa (excepto Sur).

-Allosuctobelba grandis philippinensis Corpuz-Raros, 1979

DISTRIBUCIÓN: Filipinas.

-Allosuctobelba hauseri Mahunka y Mahunka-Papp, 2001

DisTRIBUCIÓN: Suiza.

-Allosuctobelba huangshanensis Wen, 1993

DisTRIBUCIÓN: Este de China

-Allosuctobelba menglunensis Wen, 1997

DisTRIBUCIÓN: China suroriental.

-Allosuctobelba nova (Krivolustky, 1971) (Rhynchobelba)

DisTRIBUCIÓN: Paleártica oriental.

-Allosuctobelba obtusa (Jacot, 1938) (Suctobelba grandis o.)

DisTRIBUCión: Este de U.S.A.

-Allosuctobelba tricuspidata Aoki, 1984

DisTRIBUCIÓN: Japón.

-Allosuctobelba tricuspidata tricuspidata Aoki, 1984 DisTRIBUCIÓN: Japón.

-Allosuctobelba tricuspidata tokara Aoki, 1987 DISTRIBUCIÓN: Japón.

Condylobelba Mahunka, 2001 (3 spp.)

ESPECIE TIPO: Condylobelba agathis Mahunka, 2001

DISTRIBUCIÓN: Oriental.

-Condylobelba agathis Mahunka, 2001

DisTRIBUCIÓN: Oriental.

-Condylobelba bruneiensis Mahunka, 2001

DisTRIBUCIÓN: Borneo.

-Condylobelba sculpturata Mahunka, 2001

DisTRIBUCIÓN: Borneo.

Fenestrobelba Balogh, 1970 (12 spp.)

EsPECIE TIPO: Fenestrobelba annulata Balogh, 1970

DisTRIBUCIÓN: Pantropical (excepto Etiópica).

Fenestrobelba (Fenestrobelba) Balogh, 1970 (7 spp.)

DisTRIBUCIÓN: Pantropical (excepto Etiópica).

-Fenestrobelba (F.) annulata Balogh, 1970

DisTRIBUCIÓN: Ceilán [Sri Lanka].

-Fenestrobelba (F.) inenodabilis (Hammer, 1979) (Suctobelbella)

DisTRIBUCIÓN: Java.

-Fenestrobelba (F.) nodosa (Hammer, 1966) (Zeasuctobelba)

DisTRIBUCIÓN: Nueva Zelanda.

-Fenestrobelba (F.) nondivisa (Hammer, 1966) (Suctobelba)

DisTRIBUCIÓN: Australiana (Nueva Zelanda y Australia) e India

(Bengala Occidental).

-Fenestrobelba (F.) octo Balogh y Mahunka, 1974

Distribución: Península Malaya.

-Fenestrobelba (F.) quatuour Balogh y Mahunka, 1974

DisTRIBUCión: Oriental (Península Malaya y Filipinas).

-Fenestrobelba (F.) transitoria (Balogh y Mahunka, 1974) (Suctobelba)

DistribuCión: Oriental (Península Malaya y Filipinas).

Fenestrobelba (Parasuctobelba) Hammer, 1977 (5 spp.)

EsPECIE TIPO: Suctobelba fijiensis Hammer, 1971

DistriBución: Pantropical (excepto Etiópica).

-Fenestrobelba (Parasuctobelba) compacta (Woas, 1986) (Suctobelba)

DisTRIBUCiÓn: Perú.

-Fenestrobelba (Parasuctobelba) fijiensis (Hammer, 1971) (Suctobelba)

Distribución: Melanesia (I. Fiji).

-Fenestrobelba (Parasuctobelba) fragilis (Woas, 1986) (Suctobelba)

DisTRIBUCión: Perú.

-Fenestrobelba (Parasuctobelba) monstruosa (Balogh y Mahunka, 1980) (Suctobelba)

DisTRIBUCIÓN: Cuba.

-Fenestrobelba (Parasuctobelba) subcomplexa (Balogh y Mahunka, 1968) (Suctobelba)

DistribuCión: Neotropical (Argentina y Panamá) y Oriental (Borneo). 
Helvetobelba Mahunka y Mahunka-Papp, 1999 (1 sp.)

ESPECIE TIPO: Helvetobelba dichotoma Mahunka y Mahunka-Papp, 1999 DisTRIBUCIÓN: Paleártica.

-Helvetobelba dichotoma Mahunka y Mahunka-Papp, 1999 DisTRIBUCIÓN: Suiza.

Neosuctobelba Balogh y Mahunka, 1969 (2 spp.)

ESPECIE TIPO: Neosuctobelba transitoria Balogh y Mahunka, 1969 DisTRIBUCIÓN: Tropical (Etiópica y Neotropical).

-Neosuctobelba transitoria Balogh y Mahunka, 1969 DisTRIBUCIÓN: Neotropical (Bolivia y Brasil).

-Neosuctobelba xena Mahunka, 1978

DISTRIBUCIÓN: Malgache (I. Reunión).

Novosuctobelba Hammer, 1977 (8 spp.)

ESPECIE TIPO: Novosuctobelba dentissima Hammer, 1977

DisTRIBUCIÓN: Tropical (Oriental y Neotropical) y subtropical (Paleártica meridional).

Novosuctobelba (Novosuctobelba) Hammer, 1977 (3 spp.)

DISTRIBUCIÓN: Oriental y Paleártica meridional.

-Novosuctobelba (N.) dentissima Hammer, 1977

DistRIBUCIÓN: Pakistán e India (Tripura).

-Novosuctobelba (N.) shogranensis Hammer, 1977

DisTRIBUCIÓN: Pakistán.

-Novosuctobelba (N.) vietnamica (Balogh y Mahunka, 1967)

(Suctobelba)

DISTRIBUCIÓN: Vietnam

Novosuctobelba (Coartobelba) Mahunka, 2001 (5 spp.)

ESPECIE TIPO: Coartobelba pauper Mahunka, 2001

DISTRIBUCIÓN: Tropical (Oriental y Neotropical).

-Novosuctobelba (Coartobelba) campestris (Balogh y Mahunka, 1981) (Suctobelba pontigera c.)

DisTRIBUCIÓN: Paraguay.

-Novosuctobelba (Coartobelba) microclava (Hammer, 1958)

(Suctobelba)

DisTRIBUCIÓN: Neotropical.

-Novosuctobelba (Coartobelba) pauper (Mahunka, 2001) (C.)

DisTRIBUCIÓn: Borneo.

-Novosuctobelba (Coartobelba) pontigera (Hammer, 1961)

(Suctobelba)

DistriBución: Perú.

-Novosuctobelba (Coartobelba) transversalis (Hammer, 1958)

(Suctobelba)

DISTRIBUCIÓN: Bolivia.

Parisuctobelba Higgins y Woolley, 1976 (1 sp.)

EsPeCIE TIPO: Parisuctobelba septenia Higgins y Woolley, 1976

DisTRIBUCIÓN: Neártica.

-Parisuctobelba septenia Higgins y Woolley, 1976

DisTRIBUCIÓN: U.S.A. (Colorado).

Persuctobelba Mahunka, 2000 (2 spp.)

EsPeCIE TIPO: Persuctobelba divisa Mahunka, 2000

Distribución: Etiópica.

-Persuctobelba divisa Mahunka, 2000

DisTRIBUCIÓN: Madagascar.

-Persuctobelba monster Mahunka, 2000

DisTRIBUCIÓN: Madagascar.

Rhinosuctobelba Woolley y Higgins, 1969 (1 sp.)

EsPeCIE TIPO: Rhinosuctobelba dicerosa Woolley y Higgins, 1969

DISTRIBUCIÓN: Neártica.

-Rhinosuctobelba dicerosa Woolley y Higgins, 1969

DisTRIBUCIÓN: U.S.A. (Washington).

Rhynchobelba Willmann, 1953 (5 spp.)

ESPECIE TIPO: Rhynchobelba inexpectata Willmann, 1953

DISTRIBUCIÓN: Paleártica.
-Rhynchobelba altaica Krivolutsky, 1971

DisTRIBUCIÓN: Asia central rusa.

-Rhynchobelba inexpectata Willmann, 1953

DISTRIBUCIÓN: Europa centrooriental.

-Rhynchobelba machadoi Pérez-Íñigo, 1976

Distribución: I. Canarias.

-Rhynchobelba ornithorhyncha (Willmann, 1953) (Suctobelba)

DISTRIBUCIÓN: Europa central.

-Rhynchobelba simplex (Fujikawa, 1972) (Allosuctobelba)

DisTRIBUCión: Este de Paleártica oriental.

Rhynchoppia Balogh, 1968 (6 spp.)

ESPECIE TIPO: Rhynchoppia sedlaceki Balogh, 1968

DisTRIBUCIÓN: Pantropical (excepto Neotropical).

-Rhynchoppia azaisi J. y P. Balogh, 1983

DisTRIBUCIÓN: Nueva Caledonia.

-Rhynchoppia capillata (Balogh, 1963) (Suctobelbila)

DisTRIBUCIÓN: Etiópica (Angola y Ruanda).

-Rhynchoppia hauseri (Mahunka, 1976) (Suctobelba)

DistriBución: China (Hong-Kong).

-Rhynchoppia malakipinae (Corpuz-Raros, 1979) (Suctobelba)

DistRIBUCIÓN: Filipinas.

-Rhynchoppia sedlaceki Balogh, 1968

DisTRIBUCIÓN: Nueva Guinea.

-Rhynchoppia widagdoi Mahunka, 1989

DISTRIBUCIÓN: Sumatra.

Serratobelba Mahunka, 1984 (2 spp.)

EsPECIE TIPO: Suctobelbila multidentata Mahunka, 1983

DISTRIBUCIÓN: Etiópica.

-Serratobelba multidentata (Mahunka, 1983) (Suctobelbila)

DisTRIBUCión: Tanzania.

-Serratobelba rugosa Mahunka, 1984

Distribución: Tanzania.

Sucteremaeus Golosova y Krivolutsky, 1975 (1 sp.)

ESPECIE TIPO: Rhinosuctobelba makartzevi Krivolutsky y Golosova, 1974

DisTRIBUCIÓN: Paleártica.

-Sucteremaeus makartzevi (Krivolutsky y Golosova, 1974) (Rhinosuctobelba)

DistRIBUCIÓN: Este de Rusia asiática.

Suctobelba Paoli, 1908 (17 spp.)

EsPeCIE TIPO: Notaspis trigona Michael, 1888

DisTRIBUCIÓN: Holártica.

-Suctobelba aliena Moritz, 1970

DisTRIBUCIÓN: Europa.

-Suctobelba altvateri Moritz, 1970

DisTRIBUCIÓN: Europa central.

-Suctobelba atomaria Moritz, 1970

DISTRIBUCIÓN: Europa centromeridional.

-Suctobelba beringiana Krivolutsky, 1974

Distribución: Paleártica (I. Kuriles y Polonia).

-Suctobelba consimilis Mahunka y Mahunka-Papp, 2001

DisTRIBUCIÓN: Suiza

-Suctobelba discrepans Moritz, 1970

DisTRIBUCIÓN: Europa central.

-Suctobelba granulata Hammen, 1952 (S. trigona g.)

(=Suctobelba saetosa Kulijev, 1965)

DISTRIBUCIÓN: Europa centromeridional.

-Suctobelba lapidaria Moritz, 1970

DisTRIBUCIÓN: Europa centromeridional.

-Suctobelba lobodentata Mihelcic, 1957

DisTRIBUCIÓN: Austria.

-Suctobelba media Mihelcic, 1953

DisTRIBUCIÓN: Austria

-Suctobelba regia Moritz, 1970

DisTRIBUCIÓN: Europa. 
-Suctobelba reticulata Moritz, 1970

DisTRIBUCIÓN: Europa central.

-Suctobelba scalpellata Moritz, 1970

DISTRIBUCIÓN: Europa centromeridional.

-Suctobelba secta Moritz, 1970

DisTRIBUCIÓN: Europa centromeridional.

-Suctobelba sorrentensis Hammer, 1961

DisTRIBUCIÓN: Europa centromeridional.

-Suctobelba trigona (Michael, 1888) (Notaspis)

Distribución: Paleártica (Paleártica occidental: frecuente, Siberia y Asia centrooccidental).

-Suctobelba tumulata Higgins y Woolley, 1976

DisTRIBUCIÓN: U.S.A. (Colorado).

Suctobelbata Gordeeva, 1991 (4 spp.)

(=Unicobelba Mahunka y Mahunka-Papp, 1999 "sin. nov.")

ESPECIE TIPO: Suctobelbata nova Gordeeva, 1991

DISTRIBUCIÓN: Holártica.

-Suctobelbata nova Gordeeva, 1991

DisTRIBUCIÓN: Rusia europea central.

-Suctobelbata prelli (Märkel y Meyer, 1958) (Suctobelba)

Distribución: Holártica (Europa centrooccidental y Canadá).

-Suctobelbata truncicola (Forsslund, 1941) (Suctobelba)

DisTRIBUCIÓN: Paleártica (Suecia y “Japón”).

-Suctobelbata ypsilonsignata (Mahunka y Mahunka-Papp, 1999)

(Unicobelba)

DisTRIBUCIÓN: Suiza.

Suctobelbella Jacot, 1937 (155 spp. y 7 sspp.)

ESPECIE TIPO: Suctobelbella serratirostrum Jacot, 1937

DISTRIBUCIÓN: Cosmopolita.

Suctobelbella (Suctobelbella) Jacot, 1937 (101 spp. y 4 sspp.)

DisTRIBUCIÓN: Cosmopolita.

-Suctobelbella (S.) acutidens (Forsslund, 1941) (Suctobelba)

DisTRIBUCIÓN: Holártica y Argentina.

-Suctobelbella (S.) acutidens acutidens (Forsslund, 1941)

(=Suctobelba brachyodon Mihelcic, 1958)

(?=Dameosoma cornigerum Berlese, 1902 "sp. inq.")

(=Suctobelba perpendiculata Forsslund, 1958)

(=Suctobelba tuberosa Mihelcic, 1956)

DisTRIBUCIÓN: Holártica (frecuente) y Argentina.

-Suctobelbella (S.) acutidens lobata (Strenzke, 1950) (Suctobelba) DisTRIBUCIÓN: Europa.

-Suctobelbella (S.) acutissima (Balogh, 1958) (Suctobelba) "sp. inq." DisTribución: Angola.

-Suctobelbella (S.) alloenasuta Moritz, 1971

DISTRIBUCIÓN: Holártica meridional (Europa centromeridional y U.S.A.: Carolina del Norte).

-Suctobelbella (S.) amurica (Krivolutsky, 1966) (Suctobelba)

Distribución: Paleártica (excepto Norte) e India (Tripura)

-Suctobelbella (S.) arcana Moritz, 1970

DistRiBución: Holárica: Paleártica (Europa centromeridional y

Este de Rusia asiática) y Neártica (U.S.A.: Luisiana, y Canadá).

-Suctobelbella (S.) armata Hammer, 1982

DisTRIBUCIÓN: Bali.

-Suctobelbella (S.) asinus (Balogh y Mahunka, 1980) (Suctobelba)

DisTRIBUCIÓN: Cuba.

-Suctobelbella (S.) baculifera (Balogh y Mahunka, 1981) (Suctobelba perdentata $b$.)

DisTRIBUCIÓN: Neotropical (Paraguay y Pequeñas Antillas).

-Suctobelbella (S.) biangulata Hammer, 1979

DisTRIBUCIÓN: Java.

-Suctobelbella (S.) bullocki (Balogh y Mahunka, 1974) (Suctobelba) Distribución: Oriental (Península Malaya y Filipinas)

-Suctobelbella (S.) carcharodon (Moritz, 1966) (Suctobelba)

DisTRIBUCIÓN: Europa centromeridional e India (Tripura).

-Suctobelbella (S.) carinata (Balogh y Mahunka, 1969) (Suctobelba) DisTRIBUCIÓN: Bolivia.
-Suctobelbella (S.) chabarica Rjabinin, 1975

DisTRIBUCIÓN: Este de Rusia asiática.

-Suctobelbella (S.) claviseta (Hammer, 1961) (Suctobelba)

Distribución: Perú, Malgache (I. Mauricio), Hawai y Japón.

-Suctobelbella (S.) claviseta claviseta (Hammer, 1961) DisTribución: Perú, Hawai y Japón.

-Suctobelbella (S.) claviseta mauritii (Mahunka, 1978) (Suctobelba) DistriBución: Malgache (I. Mauricio).

-Suctobelbella (S.) claviseta nipponica Fujikawa, 1986 DisTRIBUCIÓN: Japón.

-Suctobelbella (S.) conica Zhao y Wen, 1993

DisTRIBUCión: Noreste de China.

-Suctobelbella (S.) crisposetosa Hammer, 1979

DisTRIBUCIÓN: Java.

-Suctobelbella (S.) dargoltsiana (Krivolutsky, 1966) (Suctobelba)

DisTRIBUCión: Paleártica meridional (Sureste de Europa y Asia centrooccidental).

-Suctobelbella (S.) decorata (P. Balogh, 1984) (Suctobelba)

Distribución: Colombia.

-Suctobelbella (S.) delessei (Strenzke, 1955) (Suctobelba)

DisTRIBUCIÓN: Groenlandia.

-Suctobelbella (S.) delicata (Krivolutsky, 1966) (Suctobelba)

DistriBución: Este de Paleártica oriental.

-Suctobelbella (S.) dispersosetosa Hammer, 1979

DisTRIBUCIÓN: Oriental.

-Suctobelbella (S.) duplex (Strenzke, 1950) (Suctobelba)

(=Suctobelba glabroclava Kulijev, 1965)

DisTRIBUCIÓN: Paleártica (Europa centromeridional y Asia centrooccidental).

-Suctobelbella (S.) elegantissima (Hammer, 1979) (Parasuctobelba) DisTRIBUCión: Java.

-Suctobelbella (S.) finlayi (Balogh y Mahunka, 1980) (Suctobelba) DisTRIBUCIÓN: Cuba.

-Suctobelbella (S.) flabellifera (Balogh, 1958) (Suctobelba) “sp. inq."

DisTRIBUCIÓN: Angola.

-Suctobelbella (S.) flagellata (Balogh, 1959) (Suctobelba)

DisTRIBUCIÓN: África centrooccidental.

-Suctobelbella (S.) frondosa Aoki y Fukuyama, 1976

DisTRIBUCIÓN: Este de Paleártica oriental y China suroriental.

-Suctobelbella (S.) frothinghami Jacot, 1937

DisTRIBUCIÓN: Neártica oriental.

-Suctobelbella (S.) geometrica (Balogh y Mahunka, 1974) (Suctobelba)

DistribuCión: Oriental (Península Malaya y Filipinas).

-Suctobelbella (S.) hamata Moritz, 1970

DisTRIBUCIÓN: Holártica (Europa centrooccidental y U.S.A.: Luisiana).

-Suctobelbella (S.) hamifera (Balogh, 1958) (Suctobelba)

DisTRIBUCiÓN: Angola.

-Suctobelbella (S.) hammerae (Krivolutsky, 1965) (Suctobelba)

DisTRIBUCIÓN: Paleártica (excepto Europa occidental): frecuente, y Canadá.

-Suctobelbella (S.) harteni Mahunka, 1991

DisTRIBUCIÓN: Macaronésica (Cabo Verde).

-Suctobelbella (S.) hastata Pankov, 1986

DistRIBUCIÓN: I. Kuriles.

-Suctobelbella (S.) horrida (Balogh y Mahunka, 1966) (Suctobelba) DisTRIBUCIÓN: Congo.

-Suctobelbella (S.) hurshi Jacot, 1937

Distribución: Neártica (U.S.A.: Carolina del Norte, y Canadá).

-Suctobelbella (S.) italica (Mahunka, 1966) (Suctobelba)

(=Suctobelbella diffissa Moritz, 1974)

DisTRIBUCIÓN: Paleártica occidental (excepto Norte).

-Suctobelbella (S.) kurilica Golosova, 1980

DistRIBUCIÓN: I. Kuriles.

-Suctobelbella (S.) laevis Jacot, 1938

DisTRIBUCIÓN: Holártica (U.S.A.: Carolina del Norte, e I. Kuriles).

-Suctobelbella (S.) laiae Tseng, 1982

Distribución: Taiwan [Formosa].

-Suctobelbella (S.) latirostris (Strenzke, 1950) (Suctobelba)

Distribución: Paleártica (Europa y Oeste de Siberia) y Vietnam. 
-Suctobelbella (S.) lienhardi Mahunka, 1983

DisTRIBUCIÓN: Méjico.

-Suctobelbella (S.) loksai (Balogh y Mahunka, 1981) (Suctobelba)

DISTRIBUCIÓN: Paraguay.

-Suctobelbella (S.) longicurva (Hammer, 1966) (Suctobelba)

DisTRIBUCión: Australiana (Nueva Zelanda y Hawai).

-Suctobelbella (S.) longicuspis Jacot, 1937

Distribución: Semicosmopolita: Holártica (Neártica oriental,

Europa e I. Kuriles), El Salvador y Nueva Zelanda.

-Suctobelbella (S.) longicuspis longicuspis Jacot, 1937

(=Suctobela falcata Forsslund, 1941)

DistRiBUCión: Semicosmopolita: Holártica (Neártica oriental,

Europa: frecuente, e I. Kuriles), El Salvador y Nueva Zelanda.

-Suctobelbella (S.) longicuspis lanceolata Jacot, 1939

DistRiBUCión: U.S.A. (Nueva Hampshire).

-Suctobelbella (S.) longirostris (Forsslund, 1941) (Suctobelba)

DisTRIBUCIÓN: Holártica: Paleártica (Europa: menos frecuente en

el Sur, y Este de Paleártica oriental) y Neártica (U.S.A.: Alaska y

Carolina del Norte)

-Suctobelbella (S.) longisensillata Fujita y Fujikawa, 1987

DisTRIBUCIÓN: Japón.

-Suctobelbella (S.) longisetosa (Hammer, 1961) (Suctobelba)

DisTRIBUCión: Perú.

-Suctobelbella (S.) macrodentata (Hammer, 1962) (Suctobelba) DisTRIBUCIÓN: Chile.

-Suctobelbella (S.) messneri Moritz, 1971

DISTRIBUCIÓN: Europa centromeridional

-Suctobelbella (S.) microdentata (Hammer, 1962) (Suctobelba) DisTRIBUCIÓN: Chile.

-Suctobelbella (S.) minor (Mihelcic, 1958) (Suctobelba) DisTRIBUCIÓN: Austria.

-Suctobelbella (S.) mirabilis (Balogh, 1958) (Suctobelba)

DisTRIBUCIÓn: Angola.

-Suctobelbella (S.) naranensis Hammer, 1967

DisTRIBUCIÓN: Pakistán.

-Suctobelbella (S.) nayoroensis Fujita y Fujikawa, 1987

DisTRIBUCIÓN: Japón.

-Suctobelbella (S.) neonominata nom. nov.

[=Suctobelbila ornata Balogh y Mahunka, 1969, "nom. praeoc." por Krivolutsky, 1966 ("hom. sec.)]

DISTRIBUCIÓN: Bolivia.

-Suctobelbella (S.) opistodentata (Golosova, 1970) (Suctobelba) Distribución: Paleártica (Europa meridional y Este de Paleártica oriental).

-Suctobelbella (S.) ornata (Krivolutsky, 1966) (Suctobelba) DisTRIBUCIÓN: Paleártica oriental.

-Suctobelbella (S.) ornatissima (Hammer, 1958) (Suctobelba) DisTRIBUCIÓN: Neotropical.

-Suctobelbella (S.) palustris (Forsslund, 1953) (Suctobelba) DisTRIBUCIÓN: Holártica.

-Suctobelbella (S.) paracutidens Mahunka, 1983

DISTRIBUCIÓN: Europa central.

-Suctobelbella (S.) parapinnata Mahunka, 1986 DisTRIBUCIÓN: Tanzania.

-Suctobelbella (S.) perarmata (Mahunka, 1978) (Suctobelba) DisTRIBUCión: Malgache (I. Mauricio).

-Suctobelbella (S.) perdentata (Hammer, 1961) (Suctobelba) DisTRIBUCIÓN: Neotropical (Perú y Argentina).

-Suctobelbella (S.) perforata (Strenzke, 1950) (Suctobelba) DisTRIBUCIÓN: Paleártica.

-Suctobelbella (S.) pinnigera (Mahunka, 1978) (Suctobelba) Distribución: Malgache (I. Mauricio).

-Suctobelbella (S.) plicata Pankov, 1986

DisTRIBUCIÓN: I. Kuriles.

-Suctobelbella (S.) plumata (Hammer, 1966) (Suctobelba) DisTRIBUCIÓN: Nueva Zelanda.

-Suctobelbella (S.) prominens (Moritz, 1966) (Suctobelba) DistriBuCión: Europa central.

-Suctobelbella (S.) pseudornata (Woas, 1986) (Suctobelba) DisTRIBUCIÓN: El Salvador.
-Suctobelbella (S.) pseudornatissima (Balogh y Mahunka, 1981)

(Suctobelba)

DisTRIBUCiÓn: Paraguay.

-Suctobelbella (S.) pulchra (Mihelcic, 1958) (Suctobelba)

DisTRIBUCIÓN: Austria.

-Suctobelbella (S.) punctata (Hammer, 1955) (Suctobelba)

DisTRIBUCión: Alaska.

-Suctobelbella (S.) ramosa (Mahunka, 1974) (Suctobelba)

DisTRIBUCIÓN: Camerún.

-Suctobelbella (S.) roigi (Balogh y Mahunka, 1980) (Suctobelba)

DisTRIBUCIÓN: Cuba.

-Suctobelbella (S.) ruzsinszkyi Mahunka, 1983

DisTriBución: Tanzania.

-Suctobelbella (S.) sabahensis Mahunka, 1988

DISTRIBUCIÓN: Borneo.

-Suctobelbella (S.) sarekensis (Forsslund, 1941) (Suctobelba)

DistRiBUCión: Holártica (Paleártica occidental y Neártica).

-Suctobelbella (S.) scrofa (Balogh y Mahunka, 1968) (Suctobelba)

DisTRIBUCión: Neotropical (Argentina y Paraguay).

-Suctobelbella (S.) semidentata Hammer, 1982

Distribución: Bali.

-Suctobelbella (S.) serratirostrum Jacot, 1937

DisTRIBUCIÓN: U.S.A. (Carolina del Norte).

-Suctobelbella (S.) setosoclavata (Hammer, 1952) (Suctobelba)

DiSTRIBUCIÓN: Neártica septentrional.

-Suctobelbella (S.) sexdentata (Mihelcic, 1958) (Suctobelba)

DisTRIBUCión: Austria.

-Suctobelbella (S.) similidentata Mahunka, 1983

DisTRIBUCiÓN: Méjico.

-Suctobelbella (S.) similis (Forsslund, 1941) (Suctobelba)

DISTRIBUCIÓN: Europa.

-Suctobelbella (S.) singularis (Strenzke, 1950) (Suctobelba)

Distribución: Paleártica (Europa: excepto Oeste, y Este de Paleártica oriental) y Taiwan [Formosa].

-Suctobelbella (S.) sinica Wen, 1987

DISTRIBUCIÓN: China suroriental.

-Suctobelbella (S.) spiculigera (Berlese, 1913) (Suctobelba cornigera s.) "sp. inq."

DISTRIBUCIÓN: Java.

-Suctobelbella (S.) spirochaeta Mahunka, 1983

DistriBución: Tanzania.

-Suctobelbella (S.) squamiseta (Mahunka, 1978) (Suctobelba) DistriBución: Malgache (I. Mauricio).

-Suctobelbella (S.) subcornigera (Forsslund, 1941) (Suctobelba)

(?=Suctobelbella laxtoni Jacot, 1937 "sp. inq.")

(=Suctobelba macrodon Mihelcic, 1956)

DisTRIBUCión: Semicosmopolita (Holártica: frecuente en Paleártica, Oriental y Nueva Zelanda).

-Suctobelbella (S.) subtrigona (Oudemans, 1900) (Eremaeus)

(=Suctobelba intermedia Willmann, 1939)

DistRIBUCión: Holártica (Paleártica: frecuente en Paleártica occidental, y Neártica septentrional) y "Sudamérica".

-Suctobelbella (S.) tatarica (Krivolutsky, 1968) (Suctobelba)

DisTRIBUCIÓN: Paleártica (excepto Europa occidental).

-Suctobelbella (S.) toeglyesii Mahunka, 1987

DisTRIBUCión: Hungría.

-Suctobelbella (S.) tohokuensis Enami y Chinone, 1997

DisTRIBUCIÓN: Japón.

-Suctobelbella (S.) trichosa Bayoumi,1979

DisTRIBUCIÓN: Hungría.

-Suctobelbella (S.) tschabovskyi (Krivolutsky, 1966) (Suctobelba) DisTRIBUCIÓN: Paleártica (Europa centromeridional y Asia centrooccidental).

-Suctobelbella (S.) tuberculata (Strenzke, 1950) (Suctobelba) DisTRIBUCIÓN: Europa.

-Suctobelbella (S.) variabilis (Hammer, 1962) (Suctobelba)

DisTRIBUCión: Chile.

-Suctobelbella (S.) vera (Moritz, 1964) (Suctobelba)

DistriBución: Paleártica (Europa y Oeste de Siberia). 
-Suctobelbella (S.) yezoensis Fujita y Fujikawa, 1987

DISTRIBUCIÓN: Japón.

Suctobelbella (Flagrosuctobelba) Hammer, 1979 (32 spp. y 3 sspp.) ESPECIE TIPO: Flagrosuctobelba multiplumosa Hammer, 1979 DISTRIBUCIÓN: Cosmopolita.

-Suctobelbella (Flagrosuctobelba) affinis Hammer, 1977 (S.)

DisTRIBUCIÓN: Pakistán.

-Suctobelbella (Flagrosuctobelba) arcuata Hammer, 1977 (S.)

DisTRIBUCIÓN: Pakistán.

-Suctobelbella (Flagrosuctobelba) baloghi (Forsslund, 1958) (Suctobelba)

DisTRIBUCIÓN: Paleártica occidental.

-Suctobelbella (Flagrosuctobelba) besucheti Mahunka y MahunkaPapp, 2001 (S.)

DISTRIBUCIÓN: Suiza.

-Suctobelbella (Flagrosuctobelba) biarcuata Hammer, 1979 (S.)

DisTRIBUCIÓN: Java.

-Suctobelbella (Flagrosuctobelba) chitralensis Hammer, 1977 (S.)

DisTRIBUCIÓN: Pakistán.

-Suctobelbella (Flagrosuctobelba) diversosetosa (Hammer, 1979) (F.)

DisTRIBUCIÓN: Oriental (Indonesia).

-Suctobelbella (Flagrosuctobelba) elegantula (Hammer, 1958)

(Suctobelba)

(=Suctobelba naginata Aoki, 1961)

(=Suctobelba quadricarina Hammer, 1958)

DistribuCIÓN: Neotropical, India (Bengala Occidental) y Holártica meridional (España, Paleártica oriental: excepto Norte, y U.S.A.: Luisiana)

-Suctobelbella (Flagrosuctobelba) flabella (Mondal, 1984) (F.

DisTRIBUCión: India (Bengala Occidental).

-Suctobelbella (Flagrosuctobelba) forsslundi (Strenzke, 1950)

(Suctobelba)

DistRIBUCIÓN: Paleártica (Paleártica occidental: frecuente, y Siberia centrooccidental).

-Suctobelbella (Flagrosuctobelba) insulana (Hammer, 1972)

(Suctobelba)

Distribución: Polinesia y Filipinas.

-Suctobelbella (Flagrosuctobelba) kaliurangensis Hammer, 1979 (S.) DisTRIBUCIÓN: Oriental (Indonesia).

-Suctobelbella (Flagrosuctobelba) lineata (Balogh, 1970) (Suctobelba)

DistriBUCIÓN: Nueva Guinea.

-Suctobelbella (Flagrosuctobelba) macroseta Mahunka, 1996 (S.)

DisTRIBUCIÓN: Hungría.

-Suctobelbella (Flagrosuctobelba) magnifera (Mahunka, 1978)

(Suctobelba)

DisTRIBUCIÓN: Malgache (I. Mauricio).

-Suctobelbella (Flagrosuctobelba) memorabilis (Hammer, 1979) (F.)

DISTRIBUCIÓN: Java.

-Suctobelbella (Flagrosuctobelba) meridionalis (Kahwash, Subías y Ruiz, 1991) (F.)

DisTRIBUCIÓN: España.

-Suctobelbella (Flagrosuctobelba) moritzi Mahunka, 1987 (S.)

DisTRIBUCión: Hungría.

-Suctobelbella (Flagrosuctobelba) multiplumosa (Hammer, 1979) (F.)

DisTRIBUCIÓN: Oriental (Indonesia).

-Suctobelbella (Flagrosuctobelba) nasalis (Forsslund, 1941) (Suctobelba)

(?=Dameosoma bellum Berlese, 1904 "sp. inq.")

DistRibución: Paleártica (frecuente en Paleártica occidental) y Nueva Zelanda.

-Suctobelbella (Flagrosuctobelba) parallelodentata Hammer, 1979 (S.) DisTRIBUCIÓN: Oriental (Indonesia).

-Suctobelbella (Flagrosuctobelba) penicillata (Balogh y Mahunka 1966) (Suctobelba)

DisTRIBUCIÓN: Etiópica (Congo y Nigeria) y Panamá.

-Suctobelbella (Flagrosuctobelba) peracuta (Balogh y Mahunka, 1980) (Suctobelba)

DisTRIBUCión: Cuba.

-Suctobelbella (Flagrosuctobelba) pilifera (Mahunka, 1978) (Suctobelba)

DisTRIBUCIÓN: Malgache (I. Mauricio).
-Suctobelbella (Flagrosuctobelba) ponticula (Hammer, 1971) (Suctobelba)

DistribuCión: Melanesia (I. Fiji) e India (Bengala Occidental).

-Suctobelbella (Flagrosuctobelba) praeoccupata nom. nov.

[=Flagrosuctobelba plumata Hammer, 1979, "nom. praeoc." por Hammer, 1966 ("hom. sec.")]

DISTRIBUCIÓN: Nueva Zelanda.

-Suctobelbella (Flagrosuctobelba) quadrata (Mahunka, 1978) (Suctobelba)

DisTRIBUCIÓN: Malgache (I. Mauricio).

-Suctobelbella (Flagrosuctobelba) quinquedentata (Hammer, 1970) (Suctobelba)

DistRIBUCIÓN: Paleártica meridional (Sureste de Europa y Asia centrooccidental).

-Suctobelbella (Flagrosuctobelba) semiplumosa (Balogh y Mahunka, 1967) (Suctobelba)

DisTRIBUCIÓN: Oriental y Nueva Guinea.

-Suctobelbella (Flagrosuctobelba) semiplumosa semiplumosa (Balogh y Mahunka, 1967)

(=Flagrosuctobelba simillima J. y P. Balogh, 2002)

DistRIBUCIÓN: Nueva Guinea y Oriental (Vietnam y Filipinas).

-Suctobelbella (Flagrosuctobelba) semiplumosa indica (Haq, 1978)

(Suctobelba)

DisTRIBUCIÓN: India (Kerala).

-Suctobelbella (Flagrosuctobelba) semiplumosa simplex (Balogh, 1968) (Suctobelba)

DisTRIBUCión: Nueva Guinea.

-Suctobelbella (Flagrosuctobelba) semiplumosa tahitiensis (Hammer, 1972) (Suctobelba)

DISTRIBUCIÓN: Australiana.

-Suctobelbella (Flagrosuctobelba) setosa (Hammer, 1979) (F.)

DisTRIBUCIÓN: Java.

-Suctobelbella (Flagrosuctobelba) sicilifera (Hammer, 1961)

(Suctobelba)

DistRIBUCIÓN: Perú.

-Suctobelbella (Flagrosuctobelba) sinuata (Hammer, 1982) (F.)

DisTRIBUCIÓN: Oriental (Bali), Australiana (Hawai) y subantártica (I. Amsterdam)

Suctobelbella (Ussuribata) Rjabinin, 1975 (22 spp.)

(=Bruneibelba Mahunka, 2001 "sin. nov.")

(=Discosuctobelba Hammer, 1979 "sin. nov.")

EsPECIE TIPO: Ussuribata clavata Rjabinin, 1975

DISTRIBUCIÓN: Pantropical y subtropical

-Suctobelbella (Ussuribata) acutodentata (Hammer, 1979) (Discosuctbelba)

DisTRIBUCIÓN: Java.

-Suctobelbella (Ussuribata) andrassyi (Balogh y Mahunka, 1981) (Suctobelba)

DisTRIBUCIÓN: Paraguay.

-Suctobelbella (Ussuribata) baliensis Hammer, 1982 (S.)

DisTRIBUCIÓN: Bali.

-Suctobelbella (Ussuribata) bifoveolata (Hammer, 1958) (Suctobelba)

DisTRIBUCIÓN: Bolivia.

-Suctobelbella (Ussuribata) bivittata (Hammer, 1979) (Discosuctobelba)

DisTRIBUCIÓN: Java.

-Suctobelbella (Ussuribata) clavata (Rjabinin, 1975) (U.)

DistRIBUCIÓN: Este de Rusia asiática.

-Suctobelbella (Ussuribata) complexa (Hammer, 1958) (Suctobelba)

DisTRIBUCIÓN: Tropical (Neotropical: frecuente, y Oriental).

-Suctobelbella (Ussuribata) cornuta (Hammer, 1962) (Suctobelba)

DistriBución: Chile.

-Suctobelbella (Ussuribata) foliosa Mahunka, 1988 (S.)

DisTRIBUCIÓN: Borneo.

-Suctobelbella (Ussuribata) latodentata (Hammer, 1979) (Discosuctobelba)

DisTRIBUCión: Java.

-Suctobelbella (Ussuribata) longiclava (Hammer, 1958) (Suctobelba)

DisTRIBUCIÓN: Neotropical (Argentina y Panamá). 
-Suctobelbella (Ussuribata) magnodentata Hammer, 1982 (S.) DisTRIBUCIÓN: Bali.

-Suctobelbella (Ussuribata) medialis (Balogh y Mahunka, 1974)

(Suctobelba)

DistriBución: Oriental (Península Malaya y Filipinas).

-Suctobelbella (Ussuribata) multituberculata (Balogh y Mahunka, 1967) (Suctobelba)

DisTRIBUCIÓN: Oriental (Vietnam y Filipinas).

-Suctobelbella (Ussuribata) papuana (Balogh, 1968) (Suctobelba)

(=Suctobelbella permixta J. y P. Balogh, 2002)

DistRIBUCIÓN: Nueva Guinea.

-Suctobelbella (Ussuribata) reticulata (Hammer, 1982) (Discosuctobelba)

DisTRIBUCIÓN: Bali.

-Suctobelbella (Ussuribata) separata (Mahunka, 2001) (Bruneibelba) DisTRIBUCIÓN: Borneo.

-Suctobelbella (Ussuribata) sexsetosa (Hammer, 1979) (Discosuctobelba)

DisTRIBUCIÓN: Oriental (Indonesia).

-Suctobelbella (Ussuribata) tricornuta Mahunka, 1989 (S.)

DisTRIBUCIÓN: Nigeria.

-Suctobelbella (Ussuribata) tuberosa (Mahunka, 2001) (Bruneibelba)

("non" Suctobelba tuberosa Mihelcic, 1956, =Suctobelbella acutidens)

DISTRIBUCIÓN: Borneo.

-Suctobelbella (Ussuribata) variosetosa (Hammer, 1961) (Suctobelba) DISTRIBUCIÓN: Pantropical (frecuente).

-Suctobelbella (Ussuribata) womersleyi (Balogh, 1968) (Suctobelba) Distribución: Nueva Guinea y Filipinas.

Suctobelbila Jacot, 1937 (31 spp. y 2 sspp.)

(=Reticobella Hammer, 1962)

(=Rhynchobella Hammer, 1961)

(=Suctoppia Balogh, 1958)

EsPECIE TIPO: Suctobelbila punctillata Jacot, 1937

DISTRIBUCIÓN: Pantropical y subtropical.

-Suctobelbila approximata Balogh, 1968

DisTRIBUCIÓN: Nueva Guinea.

-Suctobelbila baderi Mahunka, 1988

DisTRIBUCIÓN: Borneo.

-Suctobelbila cornuta Balogh y Mahunka, 1974

DisTRIBUCIÓN: Cuba.

-Suctobelbila dentata (Hammer, 1961) (Rhynchobella)

DistRiBuCión: Tropical (Neotropical: Perú y Panamá, Australiana,

e India: Bengala Occidental) y Paleártica (Alemania y Pakistán).

-Suctobelbila dentata dentata (Hammer, 1961)

DisTRIBUCIÓN: Tropical (Neotropical: Perú y Panamá, Australiana, e India: Bengala Occidental) y subtropical (Pakistán).

-Suctobelbila dentata europaea Moritz, 1974

DisTRIBUCIÓN: Alemania.

-Suctobelbila elizabethae Jacot, 1938

DisTRIBUCión: U.S.A. (Ilinois).

-Suctobelbila endroedyyoungai Mahunka, 1985

DisTRIBUCIÓN: Sudáfrica.

-Suctobelbila excavata Mahunka, 1974

DisTRIBUCIÓN: Camerún.

-Suctobelbila fissurata Hammer, 1979

DisTRIBUCIÓN: Oriental (Indonesia).

-Suctobelbila fonticula Hammer, 1975

DisTRIBUCIÓn: Argelia.

-Suctobelbila globulifera (Balogh, 1958) (Suctoppia)

DisTRIBUCIÓN: Angola.

-Suctobelbila hauseri Mahunka, 1974

DISTRIBUCIÓN: Camerún.

-Suctobelbila longitudinalis Balogh y Mahunka, 1974

DisTRIBUCIÓN: Antillas.

-Suctobelbila margaritata Balogh y Mahunka, 1980

DISTRIBUCIÓN: Cuba.

-Suctobelbila minima Hammer, 1979

DisTRIBUCIÓN: Java.
-Suctobelbila multituberculata Hammer, 1979

DisTRIBUCIÓN: Oriental (Indonesia).

-Suctobelbila neonominata nom. nov.

[=Suctobelbila ornata Hammer, 1979, "nom. praeoc." por Balogh y

Mahunka, 1969 ("hom. prim.")]

DisTRIBUCIÓN: Oriental (Indonesia).

-Suctobelbila peruensis Woas, 1986

DisTRIBUCIÓN: Perú.

-Suctobelbila pocsi Balogh y Mahunka, 1980

DisTRIBUCión: Centroamérica.

-Suctobelbila pulchella (Hammer, 1962) (Rhynchobella)

DisTRIBUCIÓN: Chile.

-Suctobelbila punctillata Jacot, 1937

DisTRIBUCIón: U.S.A. (Carolina del Norte).

-Suctobelbila quinquenodosa Balogh, 1968

DistriBuCIÓN: Nueva Guinea y Filipinas.

-Suctobelbila scutata Hammer, 1972 (S. squamosa s.)

Distribución: Polinesia (Tahití) y Borneo.

-Suctobelbila sexnodosa Balogh, 1968

DisTRIBUCIÓN: Nueva Guinea y Filipinas.

-Suctobelbila spicata Jacot, 1938

DisTRIBUCIÓN: U.S.A. oriental.

-Suctobelbila spicata spicata Jacot, 1938 DisTRIBUCIÓN: U.S.A. (Ilinois)

-Suctobelbila spicata lanceolata Jacot, 1938

DistriBución: U.S.A. (Carolina del Norte).

-Suctobelbila squamosa (Hammer, 1961) (Rhynchobella)

DisTRIBUCIÓN: Perú y Melanesia (I. Fiji).

-Suctobelbila suctobelboides Balogh, 1970

DisTRIBUCión: Nueva Guinea.

-Suctobelbila transrugosa Mahunka, 1986

DISTRIBUCIÓN: Tanzania.

-Suctobelbila tripartita Balogh y Mahunka, 1974

DISTRIBUCIÓN: Cuba.

-Suctobelbila tuberculata Aoki, 1970

DisTRIBUCIÓN: Este de Paleártica oriental.

-Suctobelbila undulata Hammer, 1979

DisTRIBUCIÓN: Java.

-Suctobelbila waterhouseri Balogh, 1970

Distribución: Nueva Guinea.

Suctobelbiloides Mahunka, 1988 (1 sp.)

ESPECIE TIPO: Suctobelbiloides armatus Mahunka, 1988

DISTRIBUCIÓN: Oriental.

-Suctobelbiloides armatus Mahunka, 1988

DisTRIBUCIÓN: Borneo.

Zeasuctobelba Hammer, 1966 (4 spp.)

ESPECIE TIPO: Zeasuctobelba quinquenodosa Hammer, 1966

DisTRIBUCIÓN: Austral (Australiana y Neotropical).

-Zeasuctobelba arcuata Hammer, 1968

DisTRIBUCIÓN: Nueva Zelanda.

-Zeasuctobelba diceros (Mahunka, 1980) (Suctobelba)

Distribución: América austral (Tierra del Fuego).

-Zeasuctobelba quinquenodosa Hammer, 1966

DisTRIBUCIÓN: Nueva Zelanda.

-Zeasuctobelba trinodosa Hammer, 1966

DisTRIBUCIÓN: Nueva Zelanda.

Rhynchoribatidae Balogh, 1961 (2 gen. y 29 spp.)

DisTRIBUCIÓN: Pantropical (excepto Australiana).

Rhynchoribates Grandjean, 1929 (24 spp.)

ESPECIE TIPO: Rhynchoribates rostratus Grandjean, 1969

DISTRIBUCIÓN: Pantropical (excepto Australiana)

-Rhynchoribates acutus Balogh, 1958

DisTRIBUCIÓN: Angola.

-Rhynchoribates amazonicus Woas, 1986

DISTRIBUCIÓN: Brasil. 
-Rhynchoribates borhidii Mahunka, 1986

DISTRIBUCIÓN: Tanzania.

-Rhynchoribates brasiliensis Woas, 1986 DisTRIBUCIÓN: Brasil.

-Rhynchoribates dilatatus Balogh y Mahunka, 1969

DisTRIBUCIÓN: Neotropical (Brasil y Venezuela).

-Rhynchoribates ecuadoriensis P. Balogh, 1988

DISTRIBUCIÓN: Ecuador.

-Rhynchoribates edentatus Balogh y Mahunka, 1969

DisTRIBUCIÓN: Neotropical (Bolivia y Panamá).

-Rhynchoribates excelsior Mahunka, 1985

DisTRIBUCIÓN: Angola.

-Rhynchoribates fabulosus Beck, 1961

DisTRIBUCIÓN: Perú.

-Rhynchoribates genavensium Mahunka, 1977

DisTRIBUCIÓN: Madagascar.

-Rhynchoribates grandis Hammer, 1961

DisTRIBUCIÓN: Perú.

-Rhynchoribates insignis Balogh y Mahunka, 1969

DISTRIBUCIÓN: Bolivia.

-Rhynchoribates mirus Beck, 1961

DisTRIBUCIÓN: Perú

-Rhynchoribates montanus Balogh, 1962

DisTRIBUCIÓN: Tanzania.

-Rhynchoribates obtusus Mahunka, 1985

DisTRIBUCIÓN: Angola.

-Rhynchoribates orientalis Balogh, 1970

DisTRIBUCIÓN: Ceilán [Sri Lanka].

-Rhynchoribates pocsi Mahunka, 1986

DisTRIBUCión: Tanzania.

-Rhynchoribates radula Mahunka, 1983

DISTRIBUCIÓN: Tanzania.

-Rhynchoribates robinsoni Balogh, 1962

DisTRIBUCIÓN: Madagascar.

-Rhynchoribates rostratus Grandjean, 1929

DisTRIBUCIÓN: Venezuela.

-Rhynchoribates serratus Balogh, 1958

DisTRIBUCIÓN: Congo.

-Rhynchoribates spathulatus Balogh y Mahunka, 1969

DisTRIBUCIÓN: Neotropical (Bolivia y Panamá).

-Rhynchoribates spectabilis Balogh y Mahunka, 1969

DisTRIBUCIÓN: Bolivia.

-Rhynchoribates subaequalis Balogh, 1962

DISTRIBUCIÓN: Madagascar.

Suctoribates Balogh, 1963 (5 spp.)

EsPeCIE TIPO: Suctoribates suctorius Balogh, 1963

DisTRIBUCIÓN: Pantropical (excepto Australiana).

-Suctoribates carinatus Hammer, 1979

DISTRIBUCIÓN: Java.

-Suctoribates crassisetosus Franklin y Woas, 1992

DisTRIBUCIÓN: Brasil.

-Suctoribates foliatus Mahunka, 1997

DisTRIBUCIÓN: Borneo.

-Suctoribates neotropicus Balogh y Mahunka, 1969

DisTRIBUCIÓN: Neotropical (Bolivia y Paraguay).

-Suctoribates suctorius Balogh, 1963

DisTRIBUCIÓN: Angola.

Oxyameridae Aoki, 1965 (1 gen. y 6 spp.)

DISTRIBUCIÓN: Australianooriental y subtropical (Paleártica meridional).

Oxyamerus Aoki, 1965 (6 spp.)

ESPECIE TIPO: Oxyamerus spathulatus Aoki, 1965

DisTRIBUCIÓN: Australianooriental y subtropical (Paleártica meridional)

-Oxyamerus aokii Balogh, 1968

DisTRIBUCIÓN: Nueva Guinea y Filipinas.

-Oxyamerus hauserorum Mahunka, 1987

DISTRIBUCIÓN: Borneo.
-Oxyamerus hyalinus Hammer, 1979

DisTRIBUCIÓN: Java.

-Oxyamerus latirostris Balogh, 1968

DistriBución: Nueva Guinea.

-Oxyamerus spathulatus Aoki, 1965

DisTRIBUCIÓN: Tailandia y Japón.

-Oxyamerus truncatus Hammer, 1979

DisTRIBUCIÓN: Java.

Trizetidae Ewing, 1917 (1 gen. y 1 sp.)

DisTRIBUCIÓN: Subtropical (Paleártica meridional).

Trizetes Berlese, 1904 (1 sp.)

EsPECIE TIPO: Trizetes pyramidalis Berlese, 1904

DISTRIBUCIÓN: Subtropical (Paleártica meridional).

-Trizetes pyramidalis Berlese, 1904

DisTRIBUCIÓN: Mediterránea occidental.

Otocepheoidea Balogh, 1961

Dampfiellidae Balogh, 1961 (2 gen. y 56 spp.)

DisTRIBUCIÓN: Pantropical.

Beckiella Grandjean, 1964 (31 spp.)

EsPeCIE TIPO: Dampfiella cejansis Beck, 1962

DISTRIBUCIÓN: Tropical (Etiópica y Neotropical).

-Beckiella acuta Balogh y Mahunka, 1978

DistriBución: Cuba.

-Beckiella africana (Balogh, 1958) (Dampfiella)

DisTRIBUCIÓN: Angola.

-Beckiella arcta Pérez-Íñigo y Baggio, 1986

DistRIBUCIÓN: Brasil.

-Beckiella bloszyki Stary, 1989

DisTRIBUCIÓN: Cuba.

-Beckiella borhidii Balogh y Mahunka, 1978

DistriBución: Cuba.

-Beckiella bucephala Balogh y Mahunka, 1978

DisTRIBUCión: Brasil.

-Beckiella capitulum Balogh y Mahunka, 1978

Distribución: Cuba.

-Beckiella carinata (Beck, 1962) (Dampfiella)

DisTRIBUCIÓN: Perú.

-Beckiella cejansis (Beck, 1962) (Dampfiella)

DisTRIBUCIÓN: Perú.

-Beckiella clavata Mahunka, 1988

DisTRIBUCIÓN: Tanzania.

-Beckiella cubana Stary, 1989

Distribución: Cuba.

-Beckiella deficiens Balogh y Mahunka, 1978

Distribución: Cuba.

-Beckiella discoidalis Balogh y Mahunka, 1978

DisTRIBUCIÓN: Cuba.

-Beckiella duplicata Balogh y Mahunka, 1978

DistriBución: Cuba.

-Beckiella elongata Balogh y Mahunka, 1969

DistriBuCión: Bolivia.

-Beckiella foveolata Balogh y Mahunka, 1969

DisTRIBUCIÓN: Neotropical (Brasil y Panamá).

-Beckiella fratercula Balogh y Mahunka, 1978

DistriBución: Cuba.

-Beckiella garciai Balogh y Mahunka, 1979

DistriBuCión: Cuba.

-Beckiella inca Stary, 1992

DisTRIBUCIÓN: Perú.

-Beckiella interlamellaris Balogh y Mahunka, 1978

DistriBución: Cuba.

-Beckiella irmayi Balogh y Mahunka, 1969

DistriBuCIÓN: Bolivia.

-Beckiella lamellata Balogh y Mahunka, 1969

DistRIBUCIÓN: Bolivia. 
-Beckiella latirostris Stary, 1992

DisTRIBUCIÓN: Perú.

-Beckiella microseta Balogh y Mahunka, 1979

DistriBución: Cuba.

-Beckiella opposita Mahunka, 1982

DisTRIBUCIÓN: Etiopía.

-Beckiella recta Balogh y Mahunka, 1978

DistriBución: Cuba.

-Beckiella reticulofemorata Balogh y Mahunka, 1979

DisTRIBUCIón: Cuba.

-Beckiella sellnicki (Hammer, 1961) (Dampfiella)

DisTRIBUCIÓN: Perú.

-Beckiella silvai Balogh y Mahunka, 1979

DistriBución: Cuba.

-Beckiella synlamellata Balogh y Mahunka, 1974

DistriBución: Cuba.

-Beckiella vitiosa Mahunka, 1985

DistriBución: Pequeñas Antillas.

Dampfiella Sellnick, 1931 (25 spp.)

EsPECIE TIPO: Dampfiella procera Sellnick, 1931

DisTRIBUCIÓN: Pantropical.

-Dampfiella ambigua Pérez-Íñigo, 1976

DisTRIBUCIÓN: I. Canarias.

-Dampfiella angusta Hammer, 1979

DisTRIBUCIÓN: Java.

-Dampfiella dubia Hammer, 1971

Distribución: Melanesia (I. Fiji) y Polinesia (I. Tonga).

-Dampfiella euaensis Hammer, 1973

DisTRIBUCIÓN: Polinesia (I. Tonga).

-Dampfiella foliata Mahunka, 1974

DisTRIBUCióN: Oriental (Península Malaya y Filipinas).

-Dampfiella jandai Stary, 1993

DisTRIBUCIÓN: Uganda.

-Dampfiella kinabalu Mahunka, 2000

DisTRIBUCIÓN: Borneo.

-Dampfiella luzonica Corpuz-Raros, 1997

DISTRIBUCIÓN: Filipinas.

-Dampfiella mahunkai Stary, 1993

DisTRIBUCIÓN: Uganda.

-Dampfiella nebulosa Mahunka, 2000

DisTRIBUCIÓN: Borneo.

-Dampfiella ovalis Corpuz-Raros, 1997

DISTRIBUCIÓN: Filipinas.

-Dampfiella papuana J. y P. Balogh, 1986

DisTRIBUCIÓn: Nueva Guinea.

-Dampfiella paratina (Mahunka, 1983) (Beckiella)

DisTRIBUCIÓN: Tanzania.

-Dampfiella peseki Stary, 1993

DisTRIBUCIÓN: Uganda.

-Dampfiella philippica Corpuz-Raros, 1997

DisTRIBUCIÓN: Filipinas.

-Dampfiella procera Sellnick, 1931

DisTRIBUCión: Méjico.

-Dampfiella prostrata Aoki, 1965

DisTRIBUCIÓN: Tailandia.

-Dampfiella putinglupa Corpuz-Raros, 1997

DisTRIBUCIÓN: Filipinas.

-Dampfiella sepilok Mahunka, 2000

DisTRIBUCIÓn: Borneo.

-Dampfiella setosa Mahunka, 1984

DisTRIBUCIÓN: Tanzania.

-Dampfiella similis Hammer, 1971

DistRIBUCIÓN: Melanesia (I. Fiji) y Filipinas.

-Dampfiella similoides Corpuz-Raros, 1997

DisTRIBUCIÓN: Filipinas.

-Dampfiella tupi Pérez-Íñigo y Baggio, 1989

DisTRIBUCIÓN: Brasil.

-Dampfiella vanalinearis Corpuz-Raros, 1997

DISTRIBUCIÓN: Filipinas.
-Dampfiella zellwegeri Mahunka, 1997

DisTRIBUCIÓN: Borneo.

Tetracondylidae Aoki, 1961 (22 gen., 4 subg., 234 spp. y 10 sspp.) DisTRIBUCIÓN: Pantropical y subtropical.

Afrotocepheus Mahunka, 1985 (2 spp.)

EsPECIE TIPO: Afrotocepheus sinarmatus Mahunka, 1985

DISTRIBUCIÓN: Etiópica.

Afrotocepheus (Afrotocepheus) Mahunka, 1985 (1 sp.)

DISTRIBUCIÓN: Etiópica.

-Afrotocepheus sinarmatus Mahunka, 1985

DisTRIBUCIÓN: Sudáfrica.

Afrotocepheus (Didierotocepheus) Mahunka, 1994 (1 sp.)

ESPECIE TIPO: Didierotocepheus berndi Mahunka, 1994

DISTRIBUCIÓN: Etiópica.

-Afrotocepheus (Didierotocepheus) berndi (Mahunka, 1994) (D.)

DISTRIBUCIÓN: Madagascar.

Borneremaeus Mahunka, 1991 (1 sp.)

ESPECIE TIPO: Borneremaeus hauseri Mahunka, 1991

DISTRIBUCIÓN: Oriental.

-Borneremaeus hauseri Mahunka, 1991

DISTRIBUCIÓN: Borneo.

Bulbocepheus Mahunka, 1988 (1 sp.)

ESPECIE TIPO: Bulbocepheus hauserorum Mahunka, 1988

DISTRIBUCIÓN: Oriental.

-Bulbocepheus hauserorum Mahunka, 1988

DISTRIBUCIÓN: Borneo.

Cavernocepheus Balogh y Mahunka, 1969 (7 spp.)

ESPECIE TIPO: Cavernocepheus monstruosus Balogh y Mahunka, 1969

DISTRIBUCIÓN: Neotropical.

-Cavernocepheus acutus P. Balogh y Palacios-Vargas, 1997

DistRIBUCIÓN: Méjico.

-Cavernocepheus furcatus P. Balogh y Palacios-Vargas, 1997

Distribución: Méjico.

-Cavernocepheus fusifer P. Balogh y Palacios-Vargas, 1997

DisTRIBUCión: Méjico.

-Cavernocepheus monstruosus Balogh y Mahunka, 1969

DisTRIBUCIÓN: Neotropical.

-Cavernocepheus monticola P. Balogh, 1989

DistRiBución: Ecuador.

-Cavernocepheus obliquus P. Balogh, 2002

Distribución: Costa Rica.

-Cavernocepheus undulatus P. Balogh, 2002

Distribución: Costa Rica.

Dolicheremaeus Jacot, 1938 (124 spp. y 9 sspp.)

(=Dicondyla Aoki, 1965)

(=Tetracondyla Newell, 1956)

EsPeCIE TIPO: Dolicheremaeus rubripedes Jacot, 1938

DisTRIBUCIÓN: Pantropical y subtropical.

-Dolicheremaeus absolon (Balogh y Csiszár, 1963) (Tetracondyla)

DisTRIBUCIÓN: Argentina.

-Dolicheremaeus africanus (Wallwork, 1962) (Tetracondyla)

DisTRIBUCión: Ghana.

-Dolicheremaeus almerodai Corpuz-Raros, 1991

DisTRIBUCIÓN: Filipinas.

-Dolicheremaeus alticola J. y P. Balogh, 1986

DisTRIBUCIÓN: Nueva Guinea.

-Dolicheremaeus alveolatus (Oudemans, 1915) (Carabodes)

Distribución: Ceilán [Sri Lanka].

-Dolicheremaeus amazonicus Balogh y Mahunka, 1969

DisTRIBUCIÓN: Neotropical (Brasil y Panamá).

-Dolicheremaeus andulauensis Mahunka, 1997

DisTRIBUCIÓN: Borneo. 
-Dolicheremaeus angustus Hammer, 1981

DisTRIBUCIÓN: Java.

-Dolicheremaeus aokii Balogh y Mahunka, 1967

DISTRIBUCIÓN: Vietnam.

-Dolicheremaeus auritus (Aoki, 1965) (Dicondyla)

DistriBución: Oriental (Tailandia e India: Kerala).

-Dolicheremaeus baloghi Aoki, 1967

DisTRIBUCIÓN: Este de Paleártica oriental y Taiwan [Formosa]

-Dolicheremaeus bartkei Rajski y Szudrowicz, 1974

DisTRIBUCIÓN: Vietnam.

-Dolicheremaeus bengalensis Sanyal, 1992

DistriBución: India (Bengala Occidental).

-Dolicheremaeus bifidus (Csiszár, 1961) (Tetracondyla)

DisTRIBUCIÓN: Java.

-Dolicheremaeus bolivianus Balogh y Mahunka, 1969

Distribución: Bolivia.

-Dolicheremaeus brasilianus Pérez-Íñigo y Baggio, 1993

DisTRIBUCIÓN: Brasil.

-Dolicheremaeus bruneiensis Aoki, 1967

DisTRIBUCIÓN: Oriental (Borneo e India: Assam).

-Dolicheremaeus capillatus (Balogh, 1959) (Tetracondyla)

DisTRIBUCIÓN: Etiópica.

-Dolicheremaeus capillatus capillatus (Balogh, 1959)

DisTRIBUCIÓN: Etiópica (Tanzania y Congo).

-Dolicheremaeus capillatus neonominatus nom. nov.

[=Tetracondyla capillata minor Wallwork, 1962, "nom. praeoc." por Berlese, 1913 ("hom. sec.")] DisTRIBUCIÓN: Ghana.

-Dolicheremaeus capreolatus (Wallwork, 1962) (Tetracondyla)

DisTRIBUCIÓN: Ghana.

-Dolicheremaeus carinatus Aoki, 1995

DisTRIBUCión: Taiwan [Formosa].

-Dolicheremaeus ceylonicus Balogh, 1970

DistriBUCióN: Ceilán [Sri Lanka].

-Dolicheremaeus cicatricosus Mahunka, 1991

DisTRIBUCIÓN: Borneo.

-Dolicheremaeus claviger Mahunka, 2000

Distribución: Borneo.

-Dolicheremaeus conjunctus Mahunka, 1973

DisTRIBUCióN: Ceilán [Sri Lanka].

-Dolicheremaeus coronarius Chakrabarti, Bhaduri y Kundu, 1981

DisTRIBUCIÓN: India (Bengala Occidental).

-Dolicheremaeus crispus (Balogh, 1962) (Tetracondyla)

Distribución: Tanzania.

-Dolicheremaeus curvisetus Mahunka, 1974

DisTRIBUCIÓN: Camerún.

-Dolicheremaeus cuspidatus (Wallwork, 1962) (Tetracondyla)

DisTRIBUCIÓN: Ghana.

-Dolicheremaeus damoeoides (Berlese, 1913) (Otocepheus)

(=Dolicheremaeus berlesei J. y P. Balogh, 2002)

(=Tetracondyla pallida Newell, 1956)

Distribución: Pantropical (Oriental: Java, Australiana: Nueva

Guinea y Hawai, y Neotropical: I. Juan Fernández).

-Dolicheremaeus densefoveolatus P. Balogh, 1988

DistriBución: Ceilán [Sri Lanka].

-Dolicheremaeus distinctus Aoki, 1982

DisTRIBUCIÓN: Japón.

-Dolicheremaeus dorni (Balogh, 1937) (Oppia)

DISTRIBUCIÓN: Europa meridional.

-Dolicheremaeus duplicarinatus Hammer, 1981

DisTRIBUCIÓN: Java.

-Dolicheremaeus duplicatus Mahunka, 1989

DisTRIBUCIÓN: Sumatra.

-Dolicheremaeus elisabethae Balogh, 1970

DistriBución: Ceilán [Sri Lanka].

-Dolicheremaeus elongatus Aoki, 1967

Distribución: Este de Paleártica oriental y Noreste de Oriental.

-Dolicheremaeus euaensis Hammer, 1973

Distribución: Polinesia (I. Tonga).
-Dolicheremaeus fijiensis Hammer, 1971

Distribución: Melanesia (I. Fiji).

-Dolicheremaeus fujikawae Mahunka, 2000

DISTRIBUCIÓN: Borneo.

-Dolicheremaeus furcatus (Balogh, 1961) (Tetracondyla)

DisTRIBUCIÓN: Etiópica.

-Dolicheremaeus furcillatus Mahunka, 1977

DisTRIBUCIÓN: Borneo.

-Dolicheremaeus furcula Balogh, 1970

DisTRIBUCIÓN: Ceilán [Sri Lanka].

-Dolicheremaeus geminus Mondal y Kundu, 1986

DisTRIBUCIÓN: India (Bengala Occidental).

-Dolicheremaeus georgii Bulanova-Zachvatkina, 1967

DisTRIBUCIÓN: Ucrania.

-Dolicheremaeus giganticus (Wallwork, 1962) (Tetracondyla)

DisTRIBUCIÓN: Ghana.

-Dolicheremaeus grafatus Mahunka, 1988

DisTRIBUCIÓN: Tanzania.

-Dolicheremaeus granulatus Hammer, 1981

DisTRIBUCIÓN: Java.

-Dolicheremaeus heterotrichus J. y P. Balogh, 1986

DistriBuCión: Nueva Guinea.

-Dolicheremaeus himalayensis Chakrabarti, Bhaduri y Kundu, 1981

DisTRIBUCIÓN: India (Bengala Occidental).

-Dolicheremaeus hirsutus (Wallwork, 1962) (Tetracondyla)

DisTRIBUCIÓN: Ghana.

-Dolicheremaeus inaequalis Balogh y Mahunka, 1967

DisTRIBUCIÓN: Vietnam.

-Dolicheremaeus indicus Haq, 1978

DisTRIBUCIÓN: India (Kerala)

-Dolicheremaeus infrequens Aoki, 1967

DisTRIBUCión: Este de Paleártica oriental y Noreste de Oriental.

-Dolicheremaeus infrequens infrequens Aoki, 1967 DisTRIBUCIÓN: Japón.

-Dolicheremaeus infrequens amamiensis Aoki, 1982 DisTRIBUCIÓN: Japón.

-Dolicheremaeus infrequens coreanus Choi, 1986 DistriBuCión: Corea.

-Dolicheremaeus infrequens hachijoensis Aoki, 1967 DisTRIBUCIÓN: Japón y China suroriental.

-Dolicheremaeus infrequens taiwanus Aoki, 1991 DisTRIBUCIÓN: Taiwan [Formosa].

-Dolicheremaeus inopinatus Aoki, 1967

DisTRIBUCIÓN: Nepal.

-Dolicheremaeus keralensis Sanyal, 1990

DisTRIBUCIÓN: India (Kerala).

-Dolicheremaeus krantzi Mahunka, 2000

DistRIBUCIÓN: Borneo.

-Dolicheremaeus kummeri Balogh, 1970

DisTRIBUCIÓN: Nueva Guinea.

-Dolicheremaeus lineatus Balogh, 1970

DistriBuCión: Ceilán [Sri Lanka].

-Dolicheremaeus lineolatus Balogh y Mahunka, 1967

DisTRIBUCIÓN: Vietnam.

-Dolicheremaeus longipilus (Higgins y Woolley, 1963) (Autogneta)

DistRIBUCIÓN: U.S.A.

-Dolicheremaeus lucidus (Wallwork, 1962) (Tetracondyla)

DisTRIBUCIÓN: Ghana.

-Dolicheremaeus luxtoni Mahunka, 2000

DisTRIBUCIÓN: Borneo.

-Dolicheremaeus machadoi (Balogh, 1958) (Tetracondyla)

DistriBución: Congo.

-Dolicheremaeus magnus (Balogh, 1958) (Tetracondyla)

DisTRIBUCIÓN: Etiópica (Tanzania y Ghana).

-Dolicheremaeus magnus magnus (Balogh, 1958) DistriBución: Tanzania.

-Dolicheremaeus magnus ghanensis (Wallwork, 1962) (Tetracondyla)

DISTRIBUCIÓN: Ghana 
-Dolicheremaeus magnus iteratus nom. nov.

[=Tetracondyla magna minor Wallwork, 1962, "nom. praeoc." por Berlese, 1913 ("hom. sec.")]

DISTRIBUCIÓN: Ghana.

-Dolicheremaeus magnus longiseta (Wallwork, 1962) (Tetracondyla)

DisTRIBUCIÓN: Ghana.

-Dolicheremaeus malangensis Hammer, 198

DisTRIBUCIÓN: Oriental (Java y Filipinas)

-Dolicheremaeus manifera Hammer, 1981

DisTRIBUCIÓN: Java.

-Dolicheremaeus mariehammerae Corpuz-Raros, 1991

DisTRIBUCIÓN: Filipinas.

-Dolicheremaeus markusi Balogh, 1970

DisTRIBUCIÓN: Ceilán [Sri Lanka].

-Dolicheremaeus mauritii Mahunka, 1978

DistriBUCIÓn: Malgache (I. Reunión).

-Dolicheremaeus minor (Berlese, 1913) (Otocepheus longior m.)

DISTRIBUCIÓN: Java.

-Dolicheremaeus montanus Krivolutsky, 1971

DisTRIBUCión: Paleártica meridional.

-Dolicheremaeus murphyi Mahunka, 1989

DistriBución: Península Malaya.

-Dolicheremaeus mutabilis Aoki, 1967

DistriBución: Nueva Guinea.

-Dolicheremaeus nasalis Hammer, 1981

DisTRIBUCiÓN: Java.

-Dolicheremaeus nepalensis Aoki, 1967

DisTRIBUCión: Himalalya.

-Dolicheremaeus nimbus Karppinen, 1966

DisTRIBUCIÓN: Guinea.

-Dolicheremaeus obsessus nom. nov.

[=Dolicheremaeus lineolatus Hammer, 1981, "nom. praeoc." por Balogh y Mahunka, 1967 (“hom. prim.")]

DisTRIBUCIÓN: Oriental (Java e India: Tripura).

-Dolicheremaeus obtusisetus Mahunka, 1988

DisTRIBUCIÓN: Tanzania.

-Dolicheremaeus oginoi (Aoki, 1965) (Dicondyla)

DISTRIBUCIÓN: Tailandia.

-Dolicheremaeus orientalis (Aoki, 1965) (Tetracondyla)

DisTRIBUCIÓN: Tailandia.

-Dolicheremaeus ornatus Balogh y Mahunka, 1967

DisTRIBUCIÓN: Vietnam.

-Dolicheremaeus pannosus Hammer, 1981

DisTRIBUCIÓN: Java.

-Dolicheremaeus papuensis Aoki, 1967

DisTRIBUCIÓN: Nueva Guinea.

-Dolicheremaeus pectinatus Balogh, 1970

Distribución: Ceilán [Sri Lanka].

-Dolicheremaeus perisi Pérez-Íñigo, 1969

Distribución: Guinea Ecuatorial: I. Pagalu [Annobón].

-Dolicheremaeus perreti Mahunka, 1974

DistriBución: Camerún.

-Dolicheremaeus philippinensis Aoki, 1967

DisTRIBUCIÓN: Filipinas.

-Dolicheremaeus phyllotrichus Mahunka, 1988

DisTRIBUCIÓn: Tanzania.

-Dolicheremaeus plurisetus Mahunka, 1988

DisTRIBUCIÓN: Malgache (I. Reunión).

-Dolicheremaeus porcinolus Aoki, 1967

DisTRIBUCIÓN: Nepal.

-Dolicheremaeus praeoccupatus nom. nov.

[=Dolicheremaeus curvisetus Hammer, 1981, “nom. praeoc." por Mahunka, 1974 ("hom. prim.")]

DisTRIBUCIÓN: Java.

-Dolicheremaeus pseudofurcatus Mahunka, 1988

DistriBución: Tanzania.

-Dolicheremaeus punctatus Mahunka, 2000

DISTRIBUCIÓN: Borneo.
-Dolicheremaeus pustulatus Mahunka, 1989

DisTRIBUCión: Península Malaya.

-Dolicheremaeus renukae Sanyal, 1990

DisTRIBUCIÓN: India (Kerala).

-Dolicheremaeus repetitus nom. nov.

[=Dolicheremaeus elongatus Balogh, 1968, “nom. praeoc.” por Aoki, 1967 ("hom. prim.")]

DistriBución: Nueva Guinea.

-Dolicheremaeus robustus Mahunka, 1973

DisTRIBUCión: Ceilán [Sri Lanka].

-Dolicheremaeus rubripedes Jacot, 1938

DisTRIBUCIÓN: U.S.A. (Florida).

-Dolicheremaeus sabahnus Mahunka, 1988

DistriBución: Oriental: Borneo e India (Tripura)

-Dolicheremaeus samarensis Corpuz-Raros, 1990

DisTribuCión: Filipinas.

-Dolicheremaeus semicapillatus (Balogh, 1962) (Tetracondyla)

DisTRIBUCión: Tanzania.

-Dolicheremaeus semilunatus Hammer, 1981

DisTRIBUCIÓN: Java.

-Dolicheremaeus seychellensis (Warburton, 1912) (Amerus)

Distribución: I. Seychelles.

-Dolicheremaeus siamensis Aoki, 1967

DistRIBUCión: Tailandia.

-Dolicheremaeus simplex (Balogh, 1962) (Tetracondyla)

Distribución: Tanzania.

-Dolicheremaeus singaporensis Mahunka, 1989

DisTRIBUCión: Península Malaya.

-Dolicheremaeus speciosus (Pearce, 1906) (Amerus) "sp. inq."

DisTRIBUCión: India (Sikkim).

-Dolicheremaeus subsimilis Balogh, 1968

DistriBución: Nueva Guinea.

-Dolicheremaeus sulcatus Mahunka, 2000

DisTRIBUCIÓN: Borneo.

-Dolicheremaeus sumatranus Mahunka, 1989

DisTRIBUCIÓN: Sumatra.

-Dolicheremaeus szentivanyi Balogh, 1968

DisTRIBUCIÓN: Nueva Guinea.

-Dolicheremaeus taidinchani Mahunka, 1976

DisTRIBUCiÓN: China (Hong-Kong)

-Dolicheremaeus tamurai Aoki, 1990

DisTRIBUCIÓN: Taiwan [Formosa].

-Dolicheremaeus tricornutus Mahunka, 1982

DisTRIBUCIÓN: Etiopía.

-Dolicheremaeus trimucronatus Mahunka, 1973

DisTRIBUCión: Ceilán [Sri Lanka].

-Dolicheremaeus variolatus Mahunka, 1989

DisTRIBUCión: Península Malaya.

-Dolicheremaeus varilobatus Hammer, 1981

DistRIBUCIÓN: Java.

-Dolicheremaeus vilhenarum (Balogh, 1958) (Tetracondyla)

DisTRIBUCIÓN: Etiópica (Congo y Ghana).

-Dolicheremaeus vilhenarum vilhenaorum (Balogh, 1958)

DisTriBución: Congo.

-Dolicheremaeus vilhenarum barbatula (Wallwork, 1962) (Tetracondyla)

DistriBución: Ghana.

-Dolicheremaeus vitraeus (Balogh, 1958) (Tetracondyla)

DisTRIBUCIÓN: Congo.

-Dolicheremaeus wallacei Mahunka, 1997

DISTRIBUCIÓN: Borneo.

-Dolicheremaeus wallworki Aoki, 1967

DisTRIBUCIÓN: Nueva Guinea.

-Dolicheremaeus wangi Aoki y Hu, 1993

Distribución: China suroriental.

-Dolicheremaeus yoshii Mahunka, 2000

DISTRIBUCIÓN: Borneo.

-Dolicheremaeus yunnanensis (Wen, 1999) (Dicondyla)

DISTRIBUCIÓN: China suroriental. 
Fernandocepheus Mahunka, 1982 (1 sp.)

EsPECIE TIPO: Fernandocepheus franzi Mahunka, 1982

DISTRIBUCIÓN: Neotropical.

-Fernandocepheus franzi Mahunka, 1982

Distribución: Chile (I. Juan Fernández).

Fissicepheus Balogh y Mahunka, 1967 (16 spp. y 1 ssp.) ESPECIE TIPO: Fissicepheus elegans Balogh y Mahunka, 1967 DistRIBUCIÓN: Paleártica y Oriental.

Fissicepheus (Fissicepheus) Balogh y Mahunka, 1967 (14 spp. y 1 ssp.) DisTRIBUCIÓN: Paleártica y Oriental.

-Fissicepheus (F.) chinensis Wen, 1993

DisTRIBUCIÓN: China suroriental.

-Fissicepheus (F.) clavatus (Aoki, 1959) (Tetracondyla) Distribución: Este de Paleártica oriental (frecuente) y China suroriental.

-Fissicepheus (F.) claviopsis Mahunka, 1971

DisTRIBUCIÓN: Corea.

-Fissicepheus (F.) coronarius Aoki, 1967

DistRIBUCIÓN: Este de Paleártica oriental y Taiwan [Formosa]

-Fissicepheus (F.) coronarius coronarius Aoki, 1967

DisTRIBUCIÓN: Japón y Taiwan [Formosa].

-Fissicepheus (F.) coronarius koreensis Mahunka, 1971 DisTRIBUCIÓN: Corea.

-Fissicepheus (F.) curvisetosus Kubota, 2001

DisTRIBUCión: Japón.

-Fissicepheus (F.) elegans Balogh y Mahunka, 1967

DisTRIBUCIÓN: Vietnam.

-Fissicepheus (F.) mitis Aoki, 1970

DisTRIBUCIÓN: Japón y China suroriental.

-Fissicepheus (F.) mitratus Mahunka, 1971

DisTRIBUCIÓN: Corea.

-Fissicepheus (F.) nakanei Aoki, 1986

DisTRIBUCIÓN: Japón.

-Fissicepheus (F.) ornithorrhynchus Wen, 1993

DISTRIBUCIÓN: China suroriental.

-Fissicepheus (F.) sitnikovae Sergienko, 1976

DISTRIBUCIÓN: Ucrania.

-Fissicepheus (F.) steinmanni Mahunka, 1971

DisTRIBUCIÓN: Corea.

-Fissicepheus (F.) subclavatus Mahunka, 1971

DisTRIBUCIÓN: Corea.

-Fissicepheus (F.) vicinus Aoki, 1986

DisTRIBUCIÓN: Japón.

Fissicepheus (Psammocepheus) Aoki, 1970 (2 spp.)

ESPECIE TIPO: Fissicepheus (Psammocepheus) amabilis Aoki, 1970

DisTRIBUCIÓN: Paleártica.

-Fissicepheus (Psammocepheus) amabilis Aoki, 1970

DisTRIBUCIÓN: Japón.

-Fissicepheus (Psammocepheus) haradai Choi, 1986

DISTRIBUCIÓN: Corea.

Flagellocepheus P. Balogh, 1984 (1 sp.)

ESPECIE TIPO: Flagellocepheus sagittatus P. Balogh,1984

DISTRIBUCIÓN: Neotropical.

-Flagellocepheus sagittatus P. Balogh, 1984

DISTRIBUCIÓN: Colombia.

Hydroecocepheus Corpuz-Raros, 1979 (1 sp.)

ESPECIE TIPO: Hydroecocepheus romeroi Corpuz-Raros, 1979

DisTRIBUCIÓN: Oriental.

-Hydroecocepheus romeroi Corpuz-Raros, 1979

DISTRIBUCIÓN: Filipinas.

Leptotocepheus Balogh, 1961 (4 spp.)

ESPECIE TIPO: Leptotocepheus trimucronatus Balogh, 1961

DistRiBución: Paleotropical.
-Leptotocepheus flagellifer Mahunka, 1988

DisTRIBUCIÓN: Tanzania.

-Leptotocepheus macromucronatus Mahunka, 1988

DISTRIBUCIÓN: Tanzania.

-Leptotocepheus orientalis Mahunka, 1988

DisTRIBUCIÓN: Borneo.

-Leptotocepheus trimucronatus Balogh, 1961

DistRIBUCIÓN: Tanzania.

Longocepheus Balogh y Mahunka, 1966 (4 spp.)

ESPECIE TIPO: Longocepheus australis Balogh y Mahunka, 1966

DISTRIBUCIÓN: Etiópica.

-Longocepheus australis Balogh y Mahunka, 1966

DisTRIBUCIÓN: Sudáfrica.

-Longocepheus globosus Grobler, 1995

DisTRIBUCIÓN: Sudáfrica.

-Longocepheus longus (Balogh, 1961) (Pseudotocepheus)

DisTRIBUCIÓN: Madagascar.

-Longocepheus youngai (Mahunka, 1984) (Nesotocepheus)

DISTRIBUCIÓN: Sudáfrica.

Lophotocepheus J. y P. Balogh, 1983 (1 sp.)

EsPECIE TIPO: Lophotocepheus simplex J. y P. Balogh, 1983

DISTRIBUCIÓN: Australiana.

-Lophotocepheus simplex J. y P. Balogh, 1983

DISTRIBUCIÓN: Australia.

Neotrichocepheus Hammer, 1973 (1 sp.)

EsPeCIE TIPO: Dolicheremaeus (Neotrichocepheus) tongaensis Hammer, 1973

DisTRIBUCIÓN: Australiana.

-Neotrichocepheus tongaensis (Hammer, 1973) (Dolicheremaeus (N.))

Distribución: Polinesia (I. Tonga)

Papillocepheus Balogh y Mahunka, 1966 (8 spp.)

=Clavazetes Hammer, 1966 "sin. nov.")

ESPECIE TIPO: Papillocepheus heterotrichus Balogh y Mahunka, 1966

DisTRIBUCIÓN: Etiópica y subtropical austral.

-Papillocepheus areolatus Mahunka, 1987

Distribución: Kenia.

-Papillocepheus decoratus Mahunka, 1994

DisTRIBUCIÓN: Madagascar.

-Papillocepheus decorus (Hammer, 1966) (Clavazetes)

DistriBución: Nueva Zelanda.

-Papillocepheus deficiens J. y P. Balogh, 1983

DistriBución: Australia.

-Papillocepheus heterotrichus Balogh y Mahunka, 1966

DistriBución: Sudáfrica.

-Papillocepheus neotropicus (P. Balogh, 1988) (Clavazetes)

DisTRIBUCión: Chile.

-Papillocepheus reductus Mahunka, 1994

DistriBución: Madagascar.

-Papillocepheus tuberculatus (Mahunka, 1978) (Clavazetes)

DisTRIBUCIÓN: Malgache.

Paradolicheremaeus Tseng, 1982 (1 sp.)

EsPeCIE TIPO: Paradolicheremaeus tabulatus Tseng, 1982

DisTRIBUCIÓN: Oriental.

-Paradolicheremaeus tabulatus Tseng, 1982

DisTRIBUCIÓN: Taiwan [Formosa].

Plenotocepheus Hammer, 1966 (10 spp.)

EsPECIE TIPO: Plenotocepheus mollicoma Hammer, 1966

DisTRIBUCIÓN: Subtropical austral.

Plenotocepheus (Plenotocepheus) Hammer, 1966 (8 spp.)

DisTRIBUCIÓN: Subtropical austral (excepto Neotropical).

-Plenotocepheus (P.) africanus Mahunka, 1984

DISTRIBUCION: Sudafrica. 
-Plenotocepheus (P.) crinitus Grobler, 1995

DisTRIBUCIÓN: Sudáfrica.

-Plenotocepheus (P.) delicatissimus Hammer, 1966

DISTRIBUCIÓN: Nueva Zelanda.

-Plenotocepheus (P.) dentatus Grobler, 1995

DistriBución: Sudáfrica.

-Plenotocepheus (P.) discrepans Grobler, 1995 Distribución: Sudáfrica.

-Plenotocepheus (P.) mollicoma Hammer, 1966 DistRiBución: Nueva Zelanda.

-Plenotocepheus (P.) undatus Mahunka, 1974 DistriBución: Rodesia [Zimbabue].

-Plenotocepheus (P.) verrucosus Grobler, 1995 DisTRIBUCIÓN: Sudáfrica.

Plenotocepheus (Neotocepheus) Hammer, 1966 (2 spp.)

EsPECIE TIPO: Neotocepheus colliger Hammer, 1961

DisTRIBUCIÓN: Subtropical austral (excepto Etiópica).

-Plenotocepheus (Neotocepheus) colliger (Hammer, 1966) (N.)

DisTRIBUCión: Nueva Zelanda.

-Plenotocepheus (Neotocepheus) longipilus (Trägardh, 1931) (Otocepheus)

Distribución: Chile (I. Juan Fernández).

Pseudotocepheus Balogh, 1961 (41 spp.)

EsPeCIE TIPO: Pseudotocepheus pauliani Balogh, 1961

DisTRIBUCIÓN: Pantropical y subtropical austral.

Pseudotocepheus (Pseudotocepheus) Balogh, 1961 (33 spp.)

(=Nesotocepheus Hammer, 1972)

DISTRIBUCIÓN: Pantropical y subtropical austral.

-Pseudotocepheus (P.) amonstruosus Mahunka, 1973

(=Pseudotocepheus septemtuberculatus Balogh y Mahunka, 1978)

(=Pseudotocepheus simplex Pérez-Íñigo y Baggio, 1980)

(=Pseudotocepheus transversalis Mahunka, 1978)

Distribución: Pantropical: Etiópica (Sudáfrica y Malgache),

Ceilán [Sri Lanka] y Neotropical.

-Pseudotocepheus (P.) andinus (Fernández, Martínez y Eguaras, 1990)

(Nesotocepheus)

DisTRIBUCIÓN: Argentina.

-Pseudotocepheus (P.) aokii Grobler, 1997

DisTRIBUCIÓN: Sudáfrica.

-Pseudotocepheus (P.) asymmetricus J. y P. Balogh, 1983

DisTRIBUCIÓN: Australia.

-Pseudotocepheus (P.) australis (Mahunka, 1980) (Nesotocepheus)

Distribución: América austral (Tierra del Fuego).

-Pseudotocepheus (P.) bacilliger J. y P. Balogh, 1983

DisTRIBUCIÓn: Australia.

-Pseudotocepheus (P.) clavigerus (Mahunka 1978) (Nesotocepheus)

DisTRIBUCIÓN: Malgache.

-Pseudotocepheus (P.) contractus Grobler, 1997

DisTRIBUCIÓN: Sudáfrica.

-Pseudotocepheus (P.) curtipilus (Trägardh, 1931) (Odontocepheus)

"sp. inq."

Distribución: Chile (I. Juan Fernánadez).

-Pseudotocepheus (P.) dentatus Grobler, 1998

DisTRIBUCIÓN: Sudáfrica.

-Pseudotocepheus (P.) flagellatus (Mahunka, 1987) (Plenotocepheus) DisTRIBUCIÓN: Kenia.

-Pseudotocepheus (P.) foveolatus Hammer, 1966

DistriBUCión: Nueva Zelanda.

-Pseudotocepheus (P.) granulatus Mahunka, 1985 DisTRIBUCión: Sudáfrica.

-Pseudotocepheus (P.) hammerae Chakrabarti y Kundu, 1978 Distribución: India (Bengala Occidental).

-Pseudotocepheus (P.) hauseri (Mahunka, 1980) (Nesotocepheus)

DisTRIBUCión: América austral (Tierra del Fuego).

-Pseudotocepheus (P.) lanceolatus Grobler, 1998

DisTRIBUCIÓN: Sudáfrica.
-Pseudotocepheus (P.) lienhardi Mahunka, 1993

DisTRIBUCIÓN: Madagascar.

-Pseudotocepheus (P.) medius Balogh y Mahunka, 1966

DisTRIBUCIÓN: Sudáfrica.

-Pseudotocepheus (P.) neonominatus nom. nov.

[=Pseudotocepheus longus Mahunka, 1973, "nom. praeoc." por Balogh,

1961 ("hom. prim.")]

Distribución: Ceilán [Sri Lanka].

-Pseudotocepheus (P.) parallelus J. y P. Balogh, 1983

DistribuCión: Australia.

-Pseudotocepheus (P.) pauliani Balogh, 1961

DISTRIBUCIÓN: Madagascar.

-Pseudotocepheus (P.) pauliensis Pérez-Íñigo y Baggio, 1993

DisTRIBUCIÓN: Neotropical (Brasil y Uruguay).

-Pseudotocepheus (P.) promissus Grobler, 1998

DisTRIBUCIÓN: Sudáfrica.

-Pseudotocepheus (P.) pygmaeus Balogh, 1962

DisTRIBUCIÓN: Madagascar.

-Pseudotocepheus (P.) radiatus Hammer, 1973

Distribución: Polinesia (I. Tonga).

-Pseudotocepheus (P.) setiger (Hammer, 1972) (Nesotocepheus)

Distribución: Polinesia (Tahití)

-Pseudotocepheus (P.) sexdentatus (Trägardh, 1931) (Odontocepheus)

"sp. inq."

DistriBución: Chile (I. Juan Fernández).

-Pseudotocepheus (P.) sexidimorphus (Vasiliu y Calugar, 1977)

(Nesotocepheus)

DisTRIBUCIÓN: Cuba.

-Pseudotocepheus (P.) shauni Grobler, 1997

DisTRIBUCIÓN: Sudáfrica.

-Pseudotocepheus (P.) sturmi P. Balogh, 1984

Distribución: Colombia.

-Pseudotocepheus (P.) szentivanyorum Balogh y Mahunka, 1978

Distribución: Australia.

-Pseudotocepheus (P.) tolanaro Mahunka, 1997

DisTRIBUCIÓN: Madagascar.

-Pseudotocepheus (P.) vicarius Balogh y Mahunka, 1978

DistRIBUCIÓN: Australia.

Pseudotocepheus (Constrictocepheus) Grobler, 1998 (8 spp.)

EsPECIE TIPO: Pseudotocepheus geminatus Balogh y Mahunka, 1969

DisTRIBUCIÓN: Pantropical (excepto Etiópica).

-Pseudotocepheus (Constrictocepheus) coarctatus P. Balogh, 1985 (P.) Distribución: Australia.

-Pseudotocepheus (Constrictocepheus) curtiseta Hammer, 1966 (P.) DisTRIBUCión: Nueva Zelanda.

-Pseudotocepheus (Constrictocepheus) geminatus Balogh y Mahunka, $1969(P$.

DisTRIBUCIÓN: Brasil.

-Pseudotocepheus (Constrictocepheus) gobletus Chakrabarti y Mondal, $1978(P$.

DistRIBUCión: India (Bengala Occidental).

-Pseudotocepheus (Constrictocepheus) monteithi J. y P. Balogh, 1983 (P.)

Distribución: Australia.

-Pseudotocepheus (Constrictocepheus) orientalis Mondal y Kundu, $1983(P$.

DisTRIBUCIÓN: India (Bengala Occidental).

-Pseudotocepheus (Constrictocepheus) punctatus Hammer, 1966 (P.)

Distribución: Nueva Zelanda y Chile.

-Pseudotoceheus (Constrictocepheus) tenuiseta Hammer, 1966 (P.)

DisTRIBUCIÓN: Nueva Zelanda.

Seboetocepheus Mahunka, 1985 (1 sp.)

EsPECIE TIPO: Seboetocepheus coronatus Mahunka, 1985

DISTRIBUCIÓN: Etiópica.

-Seboetocepheus coronatus Mahunka, 1985

DisTRIBUCIÓN: Sudáfrica. 
Spinotocepheus Hammer, 1981 (4 spp.)

ESPECIE TIPO: Spinotocepheus javensis Hammer, 1981

DISTRIBUCIÓN: Oriental.

-Spinotocepheus foveolatus Hammer, 1981

DisTRIBUCIÓN: Java.

-Spinotocepheus javensis Hammer, 1981

DISTRIBUCIÓN: Java.

-Spinotocepheus nigromaculatus Hammer, 1981

DisTRIBUCIÓN: Java.

-Spinotocepheus tjibodensis Hammer, 1981

DisTRIBUCIÓN: Java.

Trichocepheus Balogh y Mahunka, 1966 (2 spp.)

EsPECIE TIPO: Trichocepheus lamellatus Balogh y Mahunka, 1966 DISTRIBUCIÓN: Etiópica.

-Trichocepheus auriculus Grobler, 1995

DisTRIBUCiÓN: Sudáfrica.

-Trichocepheus lamellatus Balogh y Mahunka, 1966

DisTRIBUCIÓN: Sudáfrica.

Trichocondyla J. y P. Balogh, 1986 (1 sp.)

ESPECIE TIPO: Trichocondyla multisetosa J. y P. Balogh, 1986

DisTRIBUCIÓN: Australiana.

-Trichocondyla multisetosa J. y P. Balogh, 1986

DistribuCión: Nueva Guinea.

Wallworkodes J. y P. Balogh, 2002 (2 spp.)

EsPECIE TIPO: Carabodes carinatus Wallwork, 1977

DISTRIBUCIÓN: Atlántico Sur.

-Wallworkodes carinatus (Wallwork, 1977) (Carabodes)

DisTRIBUCIÓN: I. Santa Helena.

-Wallworkodes hyalinus (Wallwork, 1977) (Carabodes)

DisTRIBUCión: I. Santa Helena.

Otocepheidae Balogh, 1961 (14 gen., 1 subg., 80 spp. y 3 sspp.) DISTRIBUCIÓN: Australianooriental y subtropical (Paleártica meridional).

Acrotocepheus Aoki, 1965 (25 spp. y 1 ssp.)

EsPeCIE TIPO: Otocepheus (Acrotocepheus) quateorum Aoki, 1965

DisTRIBUCIÓN: Australianooriental y subtropical (Paleártica meridional)

Acrotocepheus (Acrotocepheus) Aoki, 1965 (24 spp. y 1 ssp.)

DisTRIBUCIÓN: Australianooriental y subtropical (Paleártica meridional)

-Acrotocepheus (A.) besucheti Mahunka, 1973

DistriBución: Ceilán [Sri Lanka].

-Acrotocepheus (A.) bucephalus Balogh, 1970

DisTRIBUCIÓN: Ceilán [Sri Lanka].

-Acrotocepheus (A.) burckhardti Mahunka, 1987

DISTRIBUCIÓN: Borneo.

-Acrotocepheus (A.) caudatus (Hammer, 1981) (Otocepheus (A.)) DisTRIBUCIÓN: Java

-Acrotocepheus (A.) consimilis Balogh, 1970

DisTRIBUCiÓN: Ceilán [Sri Lanka].

-Acrotocepheus (A.) curvisetiger Aoki, 1984

DISTRIBUCIÓN: Japón.

-Acrotocepheus (A.) diehli Mahunka, 1989

DisTRIBUCIÓN: Sumatra.

-Acrotocepheus (A.) duplicornutus (Aoki, 1965) (Otocepheus (A.))

DisTRIBUCIÓN: Oriental (Tailandia y Vietnam)

-Acrotocepheus (A.) duplicornutus duplicornutus (Aoki, 1965) DistRIBUCión: Tailandia.

-Acrotocepheus (A.) duplicornutus discrepans Balogh y Mahunka, 1967

DisTRIBUCIÓN: Vietnam.

-Acrotocepheus (A.) excelsus (Aoki, 1965) (Otocepheus (A.))

DisTRIBUCIÓN: Oriental (Tailandia y Sumatra).

-Acrotocepheus (A.) gracilis Aoki, 1973

DisTRIBUCIÓN: Japón.

-Acrotocepheus (A.) grandis Mahunka, 1973

DistriBución: Ceilán [Sri Lanka].
-Acrotocepheus (A.) heterotrichus (Balogh y Mahunka, 1968) (Oto-

cepheus (A.))

DisTRIBUCIÓN: Java.

-Acrotocepheus (A.) holtmanni (Aoki, 1965) (Otocepheus (A.))

DistriBuCión: Nueva Guinea y Filipinas.

-Acrotocepheus (A.) horakae Mahunka, 1987

DISTRIBUCIÓN: Borneo.

-Acrotocepheus (A.) lienhardi Mahunka, 1989

Distribución: Península Malaya.

-Acrotocepheus (A.) loebli Mahunka, 1973

DisTRIBUCión: Ceilán [Sri Lanka].

-Acrotocepheus (A.) macrodentatus (Hammer, 1981) (Otocepheus (A.))

DisTRIBUCIÓN: Java.

-Acrotocepheus (A.) pangasuganensis Corpuz-Raros, 1990

DISTRIBUCIÓN: Filipinas.

-Acrotocepheus (A.) philippinensis (Aoki, 1965) (Otocepheus (A.))

DistrIBUCIÓN: Filipinas.

-Acrotocepheus (A.) quateorum (Aoki, 1965) (Otocepheus (A.))

DistriBución: Nueva Guinea.

-Acrotocepheus (A.) surigaoensis Corpuz-Raros, 1979

DISTRIBUCIÓN: Filipinas.

-Acrotocepheus (A.) triplicicornutus Balogh y Mahunka, 1967

DisTRIBUCIÓN: Vietnam.

-Acrotocepheus (A.) tupasae Corpuz-Raros, 1990

DisTRIBUCIÓN: Filipinas.

-Acrotocepheus (A.) wallacei Mahunka, 1989

Distribución: Península Malaya.

Acrotocepheus (Hexatocepheus) Wen, 1993 (1 sp.)

EsPecie TIPO: Acrotocepheus (Hexatocepheus) emeiensis Wen, 1993 DISTRIBUCIÓN: Oriental.

-Acrotocepheus (Hexatocepheus) emeiensis Wen, 1993

DistRIBUCIÓN: China suroriental.

Basiceramerus Corpuz-Raros, 1979 (3 spp.)

ESPECIE TIPO: Basiceramerus upelbensis Corpuz-Raros, 1979

DisTRIBUCIÓN: Oriental.

-Basiceramerus babalus Corpuz-Raros, 1979

DISTRIBUCIÓN: Filipinas.

-Basiceramerus makilingensis Corpuz-Raros, 1979

DISTRIBUCIÓN: Filipinas.

-Basiceramerus upelbensis Corpuz-Raros, 1989

DISTRIBUCIÓN: Filipinas.

Cerostocepheus Mahunka, 1973 (1 sp.)

EsPECIE TIPO: Eurostocepheus trisetosus Balogh, 1970

DISTRIBUCIÓN: Oriental.

-Cerostocepheus trisetosus (Balogh, 1970) (Eurostocepheus)

DisTRIBUCIÓN: Oriental: Ceilán [Sri Lanka] y Filipinas.

Eurostocepheus Aoki, 1965 (3 spp.)

ESPECIE TIPO: Eurostocepheus aquilinus Aoki, 1965

DISTRIBUCIÓN: Oriental.

-Eurostocepheus aquilinus Aoki, 1965

DistriBUCIÓN: Tailandia.

-Eurostocepheus heterotrichus Wen, 1999

DistRIBUCIÓN: China suroriental.

-Eurostocepheus sajisei Corpuz-Raros, 1979

DISTRIBUCIÓN: Filipinas.

Ikarotocepheus Mahunka, 1988 (1 sp.)

ESPECIE TIPO: Ikarotocepheus alatus Mahunka, 1988

DisTRIBUCIÓN: Oriental.

-Ikarotocepheus alatus Mahunka, 1988

DisTRIBUCIÓN: Borneo.

Kalayaan Corpuz-Raros, 1998 (3 spp.)

EsPeCIE TIPO: Kalayaan bonifacioi Corpuz-Raros, 1998

DISTRIBUCIÓN: Oriental. 
-Kalayaan bonifacioi Corpuz-Raros, 1998 DisTRIBUCIÓN: Filipinas.

-Kalayaan centenarius Corpuz-Raros, 1998 DisTRIBUCIÓN: Filipinas.

-Kalayaan rizali Corpuz-Raros, 1998 DISTRIBUCIÓN: Filipinas.

Megalotocepheus Aoki, 1965 (15 spp. y 1 ssp.)

(=Archegotocepheus Mahunka, 1988 "sin. nov.")

EsPeCIE TIPO: Megalotocepheus japonicus Aoki, 1965

DisTRIBUCIÓN: Oriental y Paleártica.

-Megalotocepheus brevisetus (Mahunka, 1989) (Archegotocepheus) Distribución: Oriental: Península Malaya e India (Tripura).

-Megalotocepheus ceylonicus Balogh, 1970 DisTRIBUCIÓN: Ceilán [Sri Lanka].

-Megalotocepheus crinitus (Berlese, 1905) (Carabodes (Otocepheus)) Distribución: Oriental (Java y Filipinas) y Este de Paleártica oriental.

-Megalotocepheus fornicatus Wen, 1999

DisTRIBUCIÓN: China suroriental.

-Megalotocepheus hainanensis Wen y Zhao, 1993 DisTRIBUCIÓN: China suroriental.

-Megalotocepheus himalayensis Aoki, 1965 DisTRIBUCIÓN: Nepal.

-Megalotocepheus japonicus Aoki, 1965

DisTRIBUCIÓN: Este de Paleártica oriental.

-Megalotocepheus japonicus japonicus Aoki, 1965 DisTRIBUCión: Este de Paleártica oriental.

-Megalotocepheus japonicus setosus Aoki, 1987 DisTRIBUCIÓN: Japón.

-Megalotocepheus latus Aoki, 1965

DisTRIBUCIÓN: Oriental (Tailandia y Filipinas)

-Megalotocepheus loksai Balogh, 1970

DisTRIBUCIÓN: Ceilán [Sri Lanka].

-Megalotocepheus mahunkarum J. y P. Balogh, 2002

(=Megalotocepheus ceylonicus Mahunka, 1973, "nom. praeoc.” por Balogh, 1970)

DistriBución: Ceilán [Sri Lanka].

-Megalotocepheus nigrolobatus Hammer, 1981

DISTRIBUCIÓN: Java.

-Megalotocepheus polyptychus Wen, 1999

DisTRIBUCIÓN: China suroriental.

-Megalotocepheus singularis (Mahunka, 1988) (Archegotocepheus) DISTRIBUCIÓN: Borneo.

-Megalotocepheus tianschanicus Krivolutsky, 1969

DISTRIBUCIÓN: Asia centrooccidental.

-Megalotocepheus undulatus Hammer, 1981

DisTRIBUCIÓN: Java.

Ocellotocepheus Mahunka, 1989 (1 sp.)

ESPECIE TIPO: Ocellotocepheus tuberculatus Mahunka, 1989

DisTRIBUCIÓN: Oriental.

-Ocellotocepheus tuberculatus Mahunka, 1989

DistriBUCIÓn: Península Malaya.

Otocepheus Berlese, 1905 (19 spp.)

EsPECIE TIPO: Carabodes (Otocepheus) longior Berlese, 1905

DisTRIBUCión: Pantropical (Oriental, Australiana y "Neotropical") y

subtropical (Paleártica meridional).

-Otocepheus bajau Mahunka, 2000

DisTRIBUCIÓN: Borneo.

-Otoceheus berndhauseri Mahunka, 2000

DisTRIBUCIÓN: Borneo.

-Otocepheus cristatus (Canestrini, 1897) (Eremaeus)

DisTRIBUCIÓn: Nueva Guinea y Java.

-Otocepheus durian Mahunka, 1997

DisTRIBUCIÓN: Borneo.

-Otocepheus hauseri Mahunka, 1989

DisTRIBUCIÓn: Península Malaya.
-Otocepheus heterosetiger Aoki, 1965

DisTRIBUCIÓN: Tailandia.

-Otocepheus kadazan Mahunka, 2000

DISTRIBUCIÓN: Borneo.

-Otoceheus keningau Mahunka, 2000

DisTRIBUCIÓN: Borneo.

-Otoceheus lienhardorum Mahunka, 2000

DisTRIBUCIÓN: Borneo.

-Otocepheus longior (Berlese, 1905) (Carabodes (O.))

DisTRIBUCIÓN: Oriental (Java y Tailandia) y Cáucaso.

-Otocepheus negrosensis Corpuz-Raros, 1991

DistRIBUCIÓN: Filipinas.

-Otocepheus nepenthes Mahunka, 2000

Distribución: Borneo.

-Otocepheus orangutan Mahunka, 2000

DisTRIBUCIÓN: Borneo.

-Otocepheus pacificus Trägardh, 1931 "sp. inq."

DistriBución: Chile (I. Juan Fernández).

-Otocepheus plumosus Mahunka, 1989

DisTRIBUCIÓN: Sumatra.

-Otocepheus rafflesiae Mahunka, 2000

DISTRIBUCIÓN: Borneo.

-Otocepheus reniformis Mahunka, 2000

DisTRIBUCIÓN: Borneo,

-Otocepheus spatulatus Mahunka, 2000

DISTRIBUCIÓN: Borneo.

-Otocepheus verrucosus Mahunka, 2000

DISTRIBUCIÓN: Borneo.

Papuacepheus Balogh, 1968 (2 spp.)

EsPeCIE TIPO: Megalotocepheus (Papuacepheus) geminatus Balogh, 1968 DisTRIBUCIÓN: Australianooriental

-Papuacepheus geminatus (Balogh, 1968) (Megalotocepheus (P.))

DistriBución: Nueva Guinea.

-Papuacepheus vagorum Corpuz-Raros, 1990

DisTRIBUCIÓN: Filipinas.

Philippotocepheus J. y P. Balogh, 1992 (1 sp.)

EsPeCIE TIPO: Papuacepheus siniloanensis Corpuz-Raros, 1979

DisTRIBUCIÓN: Oriental.

-Philippotocepheus siniloanensis (Corpuz-Raros, 1979) (Papuacepheus)

DisTRIBUCIÓN: Filipinas.

Rimandocepheus Corpuz-Raros, 1998 (3 spp.)

EsPECIE TIPO: Rimandocepheus leoi Corpuz-Raros, 1998

DISTRIBUCIÓN: Oriental.

-Rimandocepheus brevisetosus Corpuz-Raros, 1998

DisTRIBUCIÓN: Filipinas.

-Rimandocepheus leoi Corpuz-Raros, 1998

DisTRIBUCIÓN: Filipinas.

-Rimandocepheus longisetosus Corpuz-Raros, 1998

DisTRIBUCIÓN: Filipinas.

Samarocepheus Corpuz-Raros, 1990 (1 sp.)

EsPECIE TIPO: Samarocepheus cavernicolus Corpuz-Raros, 1990

DisTRIBUCIÓN: Oriental.

-Samarocepheus cavernicolus Corpuz-Raros, 1990

DISTRIBUCIÓN: Filipinas.

Trichotocepheus Aoki, 1965 (2 spp. y 1 ssp.)

ESPECIE TIPO: Trichotocepheus erabuensis Aoki, 1965

DisTRIBUCIÓN: Paleártica oriental.

-Trichotocepheus amamiensis Aoki, 1965

DisTRIBUCIÓN: Japón.

-Trichotocepheus erabuensis Aoki, 1965

DistRIBUCIÓN: Este de Paleártica oriental y China suroriental.

-Trichotocepheus erabuensis erabuensis Aoki, 1965

DisTRIBUCIÓN: Este de Paleártica oriental y China suroriental. 
-Trichotocepheus erabuensis modestus Aoki, 1973 DISTRIBUCIÓN: Japón.

Tokunocepheidae Aoki, 1966 (1 gen. y 1 sp.) DisTRIBUCIÓN: Paleártica.

Tokunocepheus Aoki, 1966 (1 sp.)

EsPeCIE TIPO: Tokunocepheus mizusawai Aoki, 1966 DisTRIBUCIÓN: Paleártica.

-Tokunocepheus mizusawai Aoki, 1966

DISTRIBUCIÓN: Este de Paleárica oriental (frecuente)

Carabodoidea Koch, 1837

Carabodidae Koch, 1837 (26 gen., 12 subg., 300 spp. y 5 sspp.) DISTRIBUCIÓN: Cosmopolita.

Apotomocepheus Aoki, 1965 (1 sp.)

EsPECIE TIPO: Apotomocepheus gressitti Aoki, 1965

DISTRIBUCIÓN: Australiana.

-Apotomocepheus gressitti Aoki, 1965

DistribuCIÓN: Nueva Guinea.

Archegocepheus Aoki, 1965 (2 spp.)

EsPECIE TIPO: Archegocepheus imadatei Aoki, 1965

DistRIBUCIÓN: Oriental y Paleártica oriental.

-Archegocepheus imadatei Aoki, 1965

DISTRIBUCIÓN: Tailandia.

-Archegocepheus macrofoliatus Wen, 1997

DisTRIBUCión: China suroriental.

Austrocarabodes Hammer, 1966 (68 spp. y 2 sspp.)

EsPECIE TIPO: Carabodes ensifer Sellnick, 193

DISTRIBUCIÓN: Cosmopolita (excepto Boreal).

Austrocarabodes (Austrocarabodes) Hammer, 1966 (61 spp. y 1 ssp.)

DISTRIBUCIÓN: Cosmopolita (excepto Boreal).

-Austrocacabodes (A.) agalawatta P. Balogh, 1988

DisTRIBUCIÓN: Ceilán [Sri Lanka].

-Austrocarabodes (A.) agressor Balogh y Mahunka, 1978

Distribución: Australia.

-Austrocarabodes (A.) albidus (Balogh, 1961) (Carabodes)

DisTRIBUCión: Madagascar.

-Austrocarabodes (A.) alveolatus Hammer, 1973

DisTRIBUCIÓN: Polinesia (I. Tonga).

-Austrocarabodes (A.) angulatus (Balogh, 1958) (Carabodes) "sp. inq." DisTRIBUCIÓN: Angola.

-Austrocarabodes (A.) arrogans Pérez-Íñigo, 1967

DisTRIBUCIÓN: Mediterránea.

-Austrocarabodes (A.) australis (Balogh y Csiszár, 1963) (Carabodes)

DisTRIBUCIÓN: Argentina.

-Austrocarabodes (A.) bacilliger Mahunka, 1978

DisTRIBUCIÓN: Malgache (I. Mauricio).

-Austrocarabodes (A.) bellicosus P. Balogh, 1988

DistriBución: Ceilán [Sri Lanka].

-Austrocarabodes (A.) boninensis (Aoki, 1978) (Carabodes australis b.) DisTRIBUCIÓN: Japón.

-Austrocarabodes (A.) butiae Pérez-Íñigo y Sarasola, 1998

DisTRIBUCIÓN: Uruguay.

-Austrocarabodes (A.) cadeti Mahunka, 1978

DisTRIBUCIÓN: Malgache (I. Reunión).

-Austrocarabodes (A.) cellularis (Balogh, 1962) (Carabodes) DisTRIBUCIÓN: Madagascar.

-Austrocarabodes (A.) corpulentus (Trägardh, 1931) (Cepheus) "sp. inq." Distribución: Chile (I. Juan Fernández).

-Austrocarabodes (A.) costulatus (Balogh, 1958) (Carabodes)

DisTRIBUCIÓN: Angola.

-Austrocarabodes (A.) crenellatus Mahunka, 1983

DISTRIBUCIÓN: Tanzania.
-Austrocarabodes (A.) crinitus (Trägardh, 1931) (Cepheus) "sp. inq."

Distribución: Chile (I. Juan Fernández).

-Austrocarabodes (A.) curvisetiger Aoki, 1982

DISTRIBUCIÓN: Japón.

-Austrocarabodes (A.) davisi (Balogh y Mahunka, 1969) (Carabodes)

DisTRIBUCIÓN: Brasil.

-Austrocarabodes (A.) elegans Hammer, 1966

DistriBución: Nueva Zelanda.

-Austrocarabodes (A.) ensifer (Sellnick, 1931) (Carabodes)

DisTRIBUCIÓN: Paleártica meridional (Mediterránea y Asia centrooccidental).

-Austrocarabodes (A.) erectus Mahunka, 1984

DistRIBUCIÓN: Tanzania.

-Austrocarabodes (A.) falcatus Hammer, 1973

Distribución: Polinesia (I. Tonga).

-Austrocarabodes (A.) fenestratus (Wallwork, 1977) (Carabodes)

DistriBución: I. Santa Helena.

-Austrocarabodes (A.) flabellifer Mahunka, 1986

DisTRIBUCión: Angola.

-Austrocarabodes (A.) foliaceisetus Krivolutsky, 1971

DisTRIBUCIÓn: Paleártica meridional (Sur de Europa oriental y

Asia centrooccidental)

-Austrocarabodes (A.) glabrus Mahunka 1982

DisTRIBUCIÓN: Etiopía.

-Austrocarabodes (A.) gressitti Balogh y Mahunka, 1978

DisTRIBUCIÓN: Australia.

-Austrocarabodes (A.) haradai (Aoki, 1978) (Carabodes)

DisTRIBUCIÓN: Japón.

-Austrocarabodes (A.) horridus (Wallwork, 1977) (Carabodes)

DistriBución: I. Santa Helena.

-Austrocarabodes (A.) imperfectus (Sellnick, 1959) (Carabodes)

DisTRIBUCIÓN: Islas del Pacífico.

-Austrocarabodes (A.) imperfectus imperfectus (Sellnick, 1959) Distribución: Australiana (Islas del Pacífico)

-Austrocarabodes (A.) imperfectus squamosus Hammer, 1970 DisTRIBUCIÓN: I. de Pascua (I. Easter).

-Austrocarabodes (A.) intermedius Ruiz, Subías y Kahwash, 1989

DisTRIBUCión: España.

-Austrocarabodes (A.) latissimus Mahunka, 2000

DisTRIBUCIÓN: Yemen.

-Austrocarabodes (A.) lepidus Aoki, 1978

DisTRIBUCIÓN: Japón.

-Austrocarabodes (A.) longulus (Balogh, 1958) (Carabodes) "sp. inq." DisTRIBUCIÓN: Tanzania.

-Austrocarabodes (A.) lunaris (Balogh, 1962) (Carabodes)

DisTRIBUCIÓN: Madagascar.

-Austrocarabodes (A.) maculatus Hammer, 1966

Distribución: Australiana (Nueva Zelanda y Australia).

-Austrocarabodes (A.) microlaminatus Mahunka, 1983

DisTRIBUCIÓN: Tanzania.

-Austrocarabodes (A.) mixtus Mahunka, 1996

DISTRIBUCIÓN: Madagascar.

-Austrocarabodes (A.) nodosus Hammer, 1966

DisTRIBUCIÓn: Nueva Zelanda y Antártica (I. Auckland).

-Austrocarabodes (A.) obsoletus (Berlese, 1916) (Carabodes)

Distribución: Somalia.

-Austrocarabodes (A.) ocellatus Mahunka, 1987

DisTRIBUCIÓN: Kenia.

-Austrocarabodes (A.) picturatus Mahunka, 1987

DistriBUCIÓN: Kenia.

-Austrocarabodes (A.) pinnatus Mahunka, 1986

Distribución: Sudáfrica.

-Austrocarabodes (A.) polytrichus Balogh y Mahunka, 1978

DistRIBUCIÓN: Australia.

-Austrocarabodes (A.) pseudoreticulatus Covarrubias, 1967

DisTRIBUCIÓN: Chile.

-Austrocarabodes (A.) rimosus Mahunka, 1987

Distribución: Angola.

-Austrocarabodes (A.) rugosus Mahunka, 1969

DistRibución: Tanzania. 
-Austrocarabodes (A.) schauenbergi Mahunka, 1978

DistriBución: Malgache (I. Reunión)

-Austrocarabodes (A.) schwartzi (Balogh y Mahunka, 1969) (Cara-

bodes)

DisTRIBUCIÓN: Brasil.

-Austrocarabodes (A.) similis Mahunka, 1978

DisTRIBUCiÓN: Malgache.

-Austrocarabodes (A.) sinuosociliatus Mahunka, 1983

DisTRIBUCIÓN: Tanzania.

-Austrocarabodes (A.) sordidus (Balogh, 1958) (Carabodes) "sp. inq." DisTRIBUCIÓN: "África oriental".

-Austrocarabodes (A.) spathulatus Mahunka, 1978

DisTRIBUCIÓN: Malgache (I. Reunión).

-Austrocarabodes (A.) sphaeroideus Mahunka, 1978

DisTRIBUCIÓN: Malgache.

-Austrocarabodes (A.) sphaerula Balogh, 1970

DisTRIBUCIÓN: Ceilán [Sri Lanka].

-Austrocarabodes (A.) szentivanyi (Balogh y Mahunka, 1967)

(Carabodes)

DISTRIBUCIÓN: Vietnam y Japón.

-Austrocarabodes (A.) tarandus Mahunka, 1986

Distribución: Angola.

-Austrocarabodes (A.) travei (Balogh y Csiszár, 1963) (Carabodes) DisTRIBUCIÓN: Argentina.

-Austrocarabodes (A.) vaucheri Mahunka, 1984

DisTRIBUCIÓN: Paraguay.

-Austrocarabodes (A.) verrucatus (Trägardh, 1931) (Carabodes)

Distribución: Chile (I. Juan Fernández).

Austrocarabodes (Baloghodes) Mahunka, 1986 (2 spp.)

EsPECIE TIPO: Baloghodes heterotrichosus Mahunka, 1986

DisTRIBUCIÓN: Etiópica.

-Austrocarabodes (Baloghodes) heterotrichosus (Mahunka, 1986) (B.) DisTRIBUCión: Tanzania.

-Austrocarabodes (Baloghodes) secundus (Mahunka, 1993) (B.) DisTRIBUCIÓN: Kenia.

Austrocarabodes (Uluguroides) Mahunka, 1983 (5 spp. y 1 ssp.)

(=Ngorongobodes J. y P. Balogh, 1992 "sin. nov.")

EsPECIE TIPO: Uluguroides trichosus Mahunka, 1983

DisTRIBUCIÓN: Etiópica.

-Austrocarabodes (Uluguroides) grandis (Mahunka, 1984) (“Ulugurozetes")

DisTRIBUCIÓN: Tanzania.

-Austrocarabodes (Uluguroides) incrustatus (Wallwork, 1977)

(Carabodes)

DisTRIBUCión: I. Santa Helena.

-Austrocarabodes (Uluguroides) minitricha (Mahunka, 1993) (U.) DisTRIBUCIÓN: Tanzania.

-Austrocarabodes (Uluguroides) pentatrichus (Balogh, 1962)

(Carabodes)

Distribución: Etiópica (Tanzania e I. Santa Helena).

-Austrocarabodes (Uluguroides) pentatrichus pentatrichus (Balogh, 1962)

DisTRIBUCIÓN: Tanzania.

-Austrocarabodes (Uluguroides) pentatrichus clavatus (Wallwork, 1977) (Carabodes)

DisTRIBUCión: I. Santa Helena.

-Austrocarabodes (Uluguroides) trichosus (Mahunka, 1983) (U.)

DisTRIBUCIÓN: Tanzania.

Bathocepheus Aoki, 1978 (2 spp.)

(=Philippobodes J. y P. Balogh, 1992 "sin. nov.")

ESPECIE TIPO: Bathoceheus concavus Aoki, 1978

Distribución: Oriental y Paleártica oriental.

-Bathocepheus concavus Aoki, 1978

DisTRIBUCIÓN: Japón.

-Bathocepheus manguiati (Corpuz-Raros, 1979) (Machadocepheus) DisTRIBUCIÓN: Filipinas.
Carabodes Koch, 1835 (121 spp. y 3 sspp.)

EsPeCIE TIPO: Carabodes coriaceus Koch, 1835

DistriBuCión: Cosmopolita (excepto Antártica)

Carabodes (Carabodes) Koch, 1835 (70 spp. y 2 sspp.)

(=Neocepheus Willmann, 1936)

DistriBUCIÓN: Semicosmopolita: Holártica y pantropical (excepto Australiana).

-Carabodes (C.) affinis Berlese, 1913

DistRIBUCIÓN: Mediterránea.

-Carabodes (C.) apuanicus Bernini, 1979

DISTRIBUCIÓN: Italia.

-Carabodes (C.) arduinii Valle, 1955

DisTRIBUCIÓN: Mediterránea occidental.

-Carabodes (C.) areolatus Berlese, 1916

Distribución: Paleártica (frecuente).

-Carabodes (C.) auriculatus Mahunka, 1987

DistriBuCión: Cáucaso.

-Carabodes (C.) azoricus C. y C., jr. Pérez-Íñigo, 1996

DisTRIBUCIÓN: I. Azores.

-Carabodes (C.) basilewskyi Balogh, 1958 "sp. inq."

Distribución: Congo.

-Carabodes (C.) bellus Aoki, 1959

DistRIBUCIÓN: Este de Paleártica oriental.

-Carabodes (C.) berninii Mahunka, 1983

DisTRIBUCión: Hungría.

-Carabodes (C.) bicolor Balogh, 1958 “sp. inq."

DisTRIBUCIÓN: Angola.

-Carabodes (C.) brevis Banks, 1896

Distribución: U.S.A. (Nueva York).

-Carabodes (C.) cephalotes Koch, 1836 "sp. inq."

(=Carabodes cynocephalus Koch, 1839)

DistriBución: Alemania.

-Carabodes (C.) cerebrum Fujikawa, 1993

DisTRIBUCIÓN: Japón.

-Carabodes (C.) cherokee Reeves, 1993

DisTRIBUCIÓN: U.S.A. oriental.

-Carabodes (C.) chirstlus Mahunka, 1987

(=Carabodes foliatus Morell, 1990)

DisTRIBUCIÓN: Mediterránea occidental

-Carabodes (C.) clavatus Warburton, 1912 (C. lalbyrinthicus c.)

"sp. inq."

Distribución: I. Seychelles.

-Carabodes (C.) cochleaformis Reeves, 1990

DistRIBUCIÓN: Neártica oriental.

-Carabodes (C.) colorado Reeves y Behan-Pelletier, 1998

DisTRIBUCIÓN: Neártica occidental.

-Carabodes (C.) comas Kulijev, 1979

DisTRIBUCIÓN: Cáucaso.

-Carabodes (C.) coriaceus Koch, 1835

(=Carabodes nepos Hull, 1914)

(=Carabodes papillosus Lucas, 1849 )

DisTRIBUCIÓN: Paleártica occidental (frecuente) y "U.S.A. (Virginia)".

-Carabodes (C.) coweetaensis Reeves, 1995

DisTRIBUCIÓN: U.S.A.

-Carabodes (C.) dudichi Mahunka y Mahunka-Papp, 1999

DisTRIBUCIÓN: Hungría.

-Carabodes (C.) egregius Djaparidze, 1990

Distribución: Cáucaso.

-Carabodes (C.) erectus Reeves, 1992

DisTRIBUCIÓN: Neártica.

-Carabodes (C.) falcatus Jacot, 1937

DisTRIBUCIÓN: U.S.A. (Carolina del Norte).

-Carabodes (C.) femoralis (Nicolet, 1855) (Tegeocranus)

DisTRIBUCIÓN: Paleártica (Paleártica occidental: frecuente, y Asia centrooccidental).

-Carabodes (C.) floridus Berlese, 1913

DistriBUCIÓN: U.S.A. oriental.

-Carabodes (C.) gibbiceps Berlese, 1916

Distribución: U.S.A. (excepto Sur). 
-Carabodes (C.) grandjeani Bernini, 1977

DisTRIBUCIÓN: Italia.

-Carabodes (C.) granulatus Banks, 1895

(=Carabodes omo Jacot, 1937)

DISTRIBUCIÓN: Neártica oriental

-Carabodes (C.) gregorioi Gil-Martín y Subías, 1997

DisTRIBUCIÓN: España.

-Carabodes (C.) guadarramicus Subías y Arillo, 2001

DisTRIBUCIÓN: España.

-Carabodes (C.) hispanicus Pérez-Íñigo, 1966

DisTRIBUCIÓN: España.

-Carabodes (C.) hoh Reeves y Behan-Pelletier, 1998

DisTRIBUCIÓN: Neártica septentrional.

-Carabodes (C.) hummelincki (Willmann,1936) (Neocepheus)

DisTRIBUCión: Pequeñas Antillas.

-Carabodes (C.) hungaricus Balogh, 1943

DistriBución: Sureste de Europa.

-Carabodes (C.) ikeharai Aoki, 1988

DisTRIBUCIÓN: Japón

-Carabodes (C.) interruptus Reeves, 1992

DisTRIBUCIÓN: Este de U.S.A.

-Carabodes (C.) labyrinthicus (Michael, 1879) (Tegeocranus)

(=Cepheus heimi Oudemans, 1903)

(=Carabodes marginepunctatus Trägardh, 1902)

(=Carabodes scymnus Hull, 1914)

(=Carabodes vermiculatus Berlese, 1916)

DisTRIBUCIÓN: Holártica (frecuente)

-Carabodes (C.) magnus Kunst, 1961

DisTRIBUCión: Sureste de Europa.

-Carabodes (C.) marginatus (Michael, 1884) (Tegeocranus)

(=Carabodes pontiger Berlese, 1913)

Distribución: Paleártica (Paleártica occidental: frecuente, y

Noroeste de Siberia).

-Carabodes (C.) montanus Bernini, 1979

DisTRIBUCIÓN: Suroeste de Europa.

-Carabodes (C.) nantahalaensis Reeves, 1993

DisTRIBUCión: Este de U.S.A.

-Carabodes (C.) neonominatus nom. nov.

[=Carabodes gibbiceps clavatus Jacot, 1938, “nom. praeoc." por Warburton, 1912 ("hom. prim.")]

DisTRIBUCIÓN: Sureste de U.S.A.

-Carabodes (C.) niger Banks, 1895

DisTRIBUCIÓN: Neártica.

-Carabodes (C.) nitens Johnston, 1847 "sp. inq."

Distribución: I. Británicas.

-Carabodes (C.) ornatus Storkan, 1925

(=Carabodes forsslundi Sellnick, 1953)

Distribución: Paleártica (excepto Este de Paleártica oriental) frecuente.

-Carabodes (C.) palmifer Berlese, 1904

(=Carabodes peniculatus Aoki, 1970)

DisTRIBUCIÓN: Holártica (Paleártica: Mediterránea occidental y

Este de Paleártica oriental, y Neártica oriental) e India (Bengala Occidental).

-Carabodes (C.) paraspinosus Kulijev, 1968

Distribución: Cáucaso.

-Carabodes (C.) pentasetosus Reeves, 1992

DisTRIBUCIÓN: U.S.A.

-Carabodes (C.) perezinigoi Salinas, 1971

DistribuCIÓN: España.

-Carabodes (C.) phylliformis Reeves, 1993

DisTRIBUCIÓN: U.S.A

-Carabodes (C.) pirenaicus Subías y Arillo, 2001

DisTRIBUCIÓN: España.

-Carabodes (C.) pirinensis Kunst, 1961

DisTRIBUCIÓN: Bulgaria

-Carabodes (C.) polyporetes Reeves, 1991

DisTRIBUCIÓN: Neártica.

-Carabodes (C.) prunum Fujikawa, 1993

DisTRIBUCIÓN: Japón.
-Carabodes (C.) quadrangulus Bernini, 1979

(?=Carabodes flagellifer Mihelcic, 1966 "sp. inq.")

DistriBuCión: Mediterránea (excepto Este).

Carabodes (C.) reticulatus Berlese, 1913 (C. coriaceus $r$ )

DisTRIBUCIÓN: Paleártica occidental.

-Carabodes (C.) rimosus Aoki, 1959

DisTRIBUCIÓN: Este de Paleártica oriental y Taiwan [Formosa].

-Carabodes (C.) rugosior Berlese, 1916 (C. femoralis $r$.)

DisTRIBUCIÓN: Holártica (Paleártica y Neártica septentrional).

-Carabodes (C.) spiniformis Reeves, 1995

DisTRIBUCIÓN: U.S.A.

-Carabodes (C.) spinosus Storkan, 1925

DisTRIBUCión: Chequia.

-Carabodes (C.) subalpinus Thor, 1937

DISTRIBUCIÓN: Noruega.

-Carabodes (C.) subarcticus Trägardh, 1902 (C. elongatus s.)

DistribuCión: Paleártica (Europa: menos frecuente en el Sur, y Paleártica oriental).

-Carabodes (C.) subcarinatus Mahunka y Mahunka-Papp, 2000

(=Carabodes carinatus Mahunka y Mahunka-Papp, 1999, "nom. praeoc."

por Wallwork, 1977)

DisTRIBUCIÓN: Hungría.

Carabodes (C.) tenuis Forsslund, 1953

Distribución: Paleártica (Europa y Pakistán)

-Carabodes (C.) tenuis tenuis Forsslund, 1953

DisTRIBUCIÓN: Paleártica: Europa (menos frecuente en el Sur) y Pakistán.

-Carabodes (C.) tenuis longisetosus Kulijev, 1968 DistriBución: Cáucaso.

-Carabodes (C.) transversarius Choi y Aoki, 1986

DisTRIBUCIÓN: Este de Paleártica oriental.

-Carabodes (C.) tsushimaensis Aoki, 1970 (C. rimosus t.)

Distribución: Este de Paleártica oriental.

-Carabodes (C.) tsushimaensis tsushimaensis Aoki, 1970 DistribuCIÓN: Este de Paleártica oriental.

-Carabodes (C.) tsushimaensis dorsalis Choi y Aoki, 1986 DISTRIBUCIÓN: Corea.

-Carabodes (C.) wettsteini Mahunka, 1996

DisTRIBUCIÓN: Suiza.

-Carabodes (C.) wonalancetanus Reeves, 1990

DisTRIBUCIÓN: Neártica septetrional.

Carabodes (Flexa) Kulijev, 1977 (6 spp.)

EsPeCIE TIPO: Carabodes dubius Kulijev, 1968

DisTRIBUCIÓN: Paleártica.

-Carabodes (Flexa) bidens (Djaparidze, 1990) (F.)

DistRIBUCIÓN: Cáucaso.

-Carabodes (Flexa) breviclava Aoki, 1970 (C.)

DisTRIBUCIÓN: Japón.

-Carabodes (Flexa) dubius Kulijev, 1968 (C.)

DisTRIBUCIÓN: Cáucaso.

-Carabodes (Flexa) horeo (Djaparidze, 1990) (F.)

DisTRIBUCIÓN: Cáucaso.

-Carabodes (Flexa) intermedius Willmann, 1951 (C.)

DISTRIBUCIÓN: Europa centromeridional.

-Carabodes (Flexa) scopulae Kulijev, 1968 (C.)

DisTRIBUCIÓN: Cáucaso.

Carabodes (Klapperiches) Mahunka, 1979 (40 spp. y 1 ssp.)

EsPECIE TIPO: Klapperiches nigrosetosus Mahunka, 1979

DistRIBUCión: Cosmopolita (excepto Antártica).

-Carabodes (Klapperiches) agenjoi Pérez-Íñigo, 1969 (C.)

DisTRIBUCIÓN: Guinea Ecuatorial: I. Pagalu [Annobón].

-Carabodes (Klapperiches) andasibe Mahunka, 1993 (C.)

DisTRIBUCIÓn: Madagascar.

-Carabodes (Klapperiches) atrichosus Mahunka, 1984 (C.)

DisTRIBUCIÓN: Neotropical.

-Carabodes (Klapperiches) borhidii Balogh y Mahunka, 1979 (C.)

DISTRIBUCIÓN: Cuba. 
-Carabodes (Klapperiches) california Reeves y Behan-Pelletier, 1998

(C.)

DisTRIBUCIÓN: Suroeste de U.S.A.

-Carabodes (Klapperiches) chandleri Reeves, 1992 (C.)

DisTRIBUCIÓN: Neártica oriental.

-Carabodes (Klapperiches) coronatus Mahunka, 1986 (C.)

DisTRIBUCIÓN: Sudáfrica.

-Carabodes (Klapperiches) depilatus Balogh y Mahunka, 1969 (C.)

DisTRIBUCIÓN: Bolivia.

-Carabodes (Klapperiches) dickinsoni Reeves y Behan-Pelletier, 1998 (C.)

DisTRIBUCIÓN: Neártica occidental.

-Carabodes (Klapperiches) dissimilis Bernini, 1976 (C.)

DisTRIBUCión: Suroeste de Europa.

-Carabodes (Klapperiches) ecuadoriensis P. Balogh, 1988 (C.) DisTRIBUCIÓN: Ecuador.

-Carabodes (Klapperiches) excellens Balogh y Mahunka, 1969 (C.)

DisTRIBUCIÓN: Neotropical.

-Carabodes (Klapperiches) globiger Balogh, 1970 (C.)

DisTRIBUCIÓN: Ceilán [Sri Lanka].

-Carabodes (Klapperiches) granosus Sellnick, 1959 (C.)

Distribución: Sureste de Polinesia.

-Carabodes (Klapperiches) higginsi Reeves, 1988 (C.)

DisTRIBUCIÓN: Neártica septentrional.

-Carabodes (Klapperiches) jamaicaensis Woolley, 1967 (C.)

Distribución: Norte de Neotropical.

-Carabodes (Klapperiches) kusseri J. y P. Balogh, 1983 (C.)

DisTRIBUCión: Nueva Caledonia.

-Carabodes (Klapperiches) littoristicus (Reeves, 1997) (K.)

DisTRIBUCIÓN: U.S.A. (Florida).

-Carabodes (Klapperiches) luteoauratus Hammer, 1972 (C.)

DisTRIBUCión: Polinesia.

-Carabodes (Klapperiches) manganoi Bernini, 1976 (C.) DISTRIBUCIÓN: Italia.

-Carabodes (Klapperiches) manifera Hammer, 1977 (C.) DisTRIBUCIÓN: Pakistán.

-Carabodes (Klapperiches) microtrichus Mahunka, 1984 (C.) DistriBución: Sudáfrica.

-Carabodes (Klapperiches) minusculus Berlese, 1923 (C.)

(=Carabodes castrii Mahunka, 1966)

DisTRIBUCión: Paleártica (Paleártica occidental: frecuente, y Este de Paleártica oriental) y "U.S.A.".

-Carabodes (Klapperiches) nigrosetosus (Mahunka, 1979) (K.)

DisTRIBUCión: Antillas (La Española: R. Dominicana).

-Carabodes (Klapperiches) ornatissimus Hammer, 1966 (C.) Distribución: Nueva Zelanda.

-Carabodes (Klapperiches) penicillatus Berlese, 1916 (C.)

DisTRIBUCIÓN: Somalia.

-Carabodes (Klapperiches) penicillus (Mahunka, 1998) (K.)

DisTRIBUCIÓN: Pequeñas Antillas.

-Carabodes (Klapperiches) pocsi Mahunka, 1983 (C.)

DisTRIBUCión: Tanzania.

-Carabodes (Klapperiches) poggii Bernini, 1976 (C.)

DisTRIBUCIÓN: Mediterránea occidental.

-Carabodes (Klapperiches) problematicus Mahunka, 1985 (C.) DisTRIBUCIÓN: Sudáfrica.

-Carabodes (Klapperiches) pulcher Bernini,1976 (C.)

DisTRIBUCIÓN: Mediterránea.

-Carabodes (Klapperiches) pulcher pulcher Bernini, 1976

DISTRIBUCIÓN: Europa centromeridional.

-Carabodes (Klapperiches) pulcher occidentalis Pérez-Íñigo y Peña, 1996 (C.)

DistRiBuCión: I. Canarias.

-Carabodes (Klapperiches) purpurarius Pérez-Íñigo y Peña, 1996

(C.)

DisTRIBUCIÓN: I. Canarias.

-Carabodes (Klapperiches) radiatus Berlese, 1916 (C.)

(=Carabodes dendroetus Reeves, 1987)

DISTRIBUCIÓN: Este de U.S.A.
-Carabodes (Klapperiches) samoensis J. y P. Balogh, 1986 (C.)

Distribución: Polinesia (I. Samoa).

-Carabodes (Klapperiches) schatzi Bernini, 1976 (C.)

DisTRIBUCIÓN: Europa centooccidental.

-Carabodes (Klapperiches) similis Ruiz, Subías y Kahwash, 1989 (C.)

(=Carabodes translamellatus Pérez-Íñigo jr., 1990)

DISTRIBUCIÓN: España.

-Carabodes (Klapperiches) strinovichi Balogh y Mahunka, 1978 (C.)

DistriBución: Australia.

-Carabodes (Klapperiches) tenerifensis Pérez-Íñigo, 1976 (C.)

DistriBuCIÓn: Mediterránea occidental.

-Carabodes (Klapperiches) variabilis Hammer, 1966 (C.)

DistRIBUCión: Nueva Zelanda.

-Carabodes (Klapperiches) willmanni Bernini, 1975 (C.)

DisTRIBUCIÓN: Holártica (Paleártica occidental y Neártica septentrional).

Carabodes (Phyllocarabodes) Balogh y Mahunka, 1969 (5 spp.)

(=Antillobodes Mahunka, 1985)

(=Pentabodes P. Balogh, 1984 "sin. nov.")

EsPeCIE TIPO: Phyllocarabodes octogonalis Balogh y Mahunka, 1969

DisTRIBUCIÓn: Neotropical.

-Carabodes (Phyllocarabodes) calcaratus (Mahunka, 1985) (Anti-

llobodes)

DistRIBUCIÓN: Pequeñas Antillas.

-Carabodes (Phyllocarabodes) inopinatus (Mahunka, 1985) (Anti-

llobodes)

Distribución: Pequeñas Antillas

-Carabodes (Phyllocarabodes) insolitus (P. Balogh, 1984) (Pentabodes)

DistRIBUCIÓN: Colombia

Carabodes (Phyllocarabodes) octogonalis (Balogh y Mahunka, 1969) (P.)

DisTRIBUCIÓn: Neotropical (Bolivia y Perú).

-Carabodes (Phyllocarabodes) ornatus (P. Balogh, 1986) (P.)

DisTRIBUCIÓN: Neotropical (Colombia y Venezuela).

Cavernocarabodes Mahunka, 1974 (2 spp.)

EsPECIE TIPO: Cavernocarabodes perreti Mahunka, 1974

DisTRIBUCIÓN: Etiópica y Paleártica meridional.

- Cavernocarabodes perreti Mahunka, 1974

DisTRIBUCIÓN: Camerún.

-Cavernocarabodes trigonosternum (Pérez-Íñigo, 1976) (Carabodes)

DisTRIBUCIÓN: I. Canarias.

Congocepheus Balogh, 1958 (8 spp.)

EsPeCIE TIPO: Congocepheus heterotrichus Balogh, 1958

DistriBución: Paleotropical.

-Congocepheus hauseri Mahunka, 1989

DISTRIBUCIÓN: Sumatra.

-Congocepheus heterotrichus Balogh, 1958

Distribución: Etiópica (Congo y Ghana).

-Congocepheus involutus Mahunka, 1997

DISTRIBUCIÓN: Madagascar.

-Congocepheus latilamellatus Mahunka, 1984

DisTRIBUCIÓN: Tanzania.

-Congocepheus orientalis Mahunka, 1989

DISTRIBUCIÓN: Borneo.

-Congocepheus ornatus Mahunka, 1983

DistriBución: Tanzania.

-Congocepheus taurus Balogh, 1961

DisTRIBUCión: Tanzania.

-Congocepheus velatus Mahunka, 1986

DISTRIBUCIÓN: Tanzania.

Cubabodes Balogh y Mahunka, 1974 (5 spp.)

ESPECIE TIPO: Cubabodes hexagonalisBalogh y Mahunka, 1974

DisTRIBUCIÓN: Neotropical.

-Cubabodes confertus Balogh y Mahunka, 1980

DisTRIBUCIÓN: Cuba. 
-Cubabodes hexagonalis Balogh y Mahunka, 1974

DisTriBuCIÓN: Cuba.

-Cubabodes radiatus Balogh y Mahunka, 1974

DisTRIBUCIÓN: Cuba.

-Cubabodes spathulatus Balogh y Mahunka, 1980

DistriBución: Cuba.

-Cubabodes verrucatus Balogh y Mahunka, 1980

DisTRIBUCIÓN: Centroamérica.

Diplobodes Aoki, 1958 (10 spp.)

EsPeCIE TIPO: Diplobodes kanekoi Aoki, 1958

DisTRIBUCIÓN: Pantropical (excepto Australiana) y subtropical (Paleártica meridional).

Diplobodes (Diplobodes) Aoki, 1958 (6 spp.)

DisTRIBUCIÓN: Etiópica y Paleártica meridional

-Diplobodes (D.) africanus Mahunka, 1987

DistriBución: Kenia.

-Diplobodes (D.) aokii Mahunka, 1989

DisTRIBUCIÓN: Kenia.

-Diplobodes (D.) floridus Mahunka, 1978

DistRIBUCIÓN: Malgache (I. Mauricio).

-Diplobodes (D.) kanekoi Aoki, 1958

DISTRIBUCIÓN: Japón (frecuente).

-Diplobodes (D.) karubei Aoki, 2002

DisTRIBUCIÓN: Japón.

-Diplobodes (D.) superbus Mahunka, 1978

DisTRIBUCIÓN: Malgache (I. Reunión).

Diplobodes (Kalloia) Mahunka, 1985 (3 spp.)

(=Mauribodes J. y P. Balogh, 1992 "sin. nov.")

EsPECIE TIPO: Kalloia simpliseta Mahunka, 1985

DisTRIBUCIÓN: Neotropical y Etiópica.

-Diplobodes (Kalloia) foveolatus (Mahunka, 1978) (Machadocepheus) Distribución: Malgache (I. Mauricio).

-Diplobodes (Kalloia) mahunkai (Pérez-Íñigo y Baggio, 1989) (K.) DisTRIBUCIÓN: Brasil.

-Diplobodes (Kalloia) simpliseta (Mahunka, 1985) (K.)

DistriBUCIÓN: Norte de Neotropical.

Diplobodes (Neocarabodes) Balogh y Mahunka, 1969 (1 sp.)

EsPECIE TIPO: Neocarabodes sexpilosus Balogh y Mahunka, 1969 DisTRIBUCIÓN: Tropical (Neotropical y Oriental).

-Diplobodes (Neocarabodes) sexpilosus (Balogh y Mahunka, 1969) (N.)

DistRIBUCIÓN: Neotropical (Brasil y Panamá) y Oriental (Filipinas e India: Bengala Occidental).

Gibbicepheus Aoki, 1958 (17 spp.)

ESPECIE TIPO: Gibbicepheus elevatus Balogh, 1958

DisTRIBUCIÓN: Pantropical y subtropical (Paleártica meridional).

Gibbicepheus (Gibbicepheus) Aoki, 1958 (16 spp.)

DisTRIBUCIÓN: Pantropical y subtropical (Paleártica meridional). -Gibbicepheus (G.) austroamericanus Mahunka, 1984

DisTRIBUCIÓN: Neotropical (Paraguay y Uruguay).

-Gibbicepheus (G.) baccanensis Jeleva y Vu, 1987

DisTRIBUCIÓN: Vietnam.

-Gibbicepheus (G.) chinensis Wen, 1992

DisTRIBUCión: China suroriental.

-Gibbicepheus (G.) elevatus Balogh, 1958

DistribuCión: Angola.

-Gibbicepheus (G.) ensifer Balogh, 1958 "sp. inq."

DisTRIBUCIÓN: Angola.

-Gibbicepheus (G.) fenestralis Hammer, 1979

DisTRIBUCIÓN: Java.

-Gibbicepheus (G.) frondosus (Aoki, 1959) (Diplobodes)

DistRibución: Tropical (Oriental y Australiana: I. del Pacífico) y

Este de Paleártica oriental).
-Gibbicepheus (G.) kanekoi (Aoki, 1958) (Diplobodes )

DISTRIBUCIÓN: Tailandia.

-Gibbicepheus (G.) latohumeralis Hammer, 1982

DISTRIBUCIÓN: Bali.

-Gibbicepheus (G.) micheli Mahunka, 1978

DisTRIBUCIÓN: I. Seychelles.

-Gibbicepheus (G.) novus Hammer, 1973

Distribución: Australiana (Islas del Pacífico).

-Gibbicepheus (G.) rugosus Mahunka, 1978

DisTRIBUCión: I. Seychelles.

-Gibbicepheus (G.) sisiri Sanyal, 1990

DisTRIBUCIÓN: India (Kerala)

-Gibbicepheus (G.) tuberculatus Balogh, 1970

DistriBuCión: Nueva Guinea y Península Malaya.

-Gibbicepheus (G.) ventrostriatus Hammer, 1979

DisTRIBUCIÓN: Java.

-Gibbicepheus (G.) waterhousei Balogh y Mahunka, 1978

DistriBUCión: Australia.

Gibbicepheus (Gibbibodes) Mahunka, 1986 (1 sp.)

ESPECIE TIPO: Gibbibodes similis Mahunka, 1986

DISTRIBUCIÓN: Etiópica.

-Gibbicepheus (Gibbibodes) similis (Mahunka, 1986) (Gibbibodes)

DisTRIBUCIÓN: Angola.

Gymnobodes Balogh, 1965 (3 spp.)

ESPECIE TIPO: Carabodes fraterculus Balogh, 1963

DISTRIBUCIÓN: Paleotropical.

-Gymnobodes fraterculus (Balogh, 1963) (Carabodes)

DisTRIBUCIÓN: África centrooccidental.

-Gymnobodes semengok Mahunka, 1996

DISTRIBUCIÓN: Borneo.

-Gymnobodes subnudus (Balogh, 1963) (Carabodes)

DisTRIBUCIÓN: Congo.

Hardybodes Balogh, 1970 (4 spp.)

(=Carabodella Mahunka, 1986 "sin. nov.")

EsPECIE TIPO: Hardybodes mirabilis Balogh, 1970

DISTRIBUCIÓN: Pantropical (excepto Neotropical).

-Hardybodes calcaratus (Mahunka, 1986) (Carabodella)

DisTRIBUCIÓN: Tanzania.

-Hardybodes flabellatus Mahunka, 1995

DISTRIBUCIÓN: Borneo.

-Hardybodes mirabilis Balogh, 1970

DISTRIBUCIÓN: Nueva Guinea y Oriental (Filipinas y Borneo).

-Hardybodes penicillatus Mahunka, 1995

DISTRIBUCIÓN: Borneo.

Machadocepheus Balogh, 1958 (6 spp.)

ESPECIE TIPO: Machadocepheus excavatus Balogh, 1958

DistriBución: Pantropical (excepto Neotropical).

-Machadocepheus excavatus Balogh, 1958

DISTRIBUCIÓN: Etiópica.

-Machadocepheus filiferus Mahunka, 1986

DisTRIBUCIÓN: Angola.

-Machadocepheus pauliani Balogh, 1962

DisTRIBUCIÓN: Madagascar.

-Machadocepheus phyllophorus Balogh, 1970

DisTRIBUCIÓN: Nueva Guinea.

-Machadocepheus taprobanicus Balogh, 1970

DisTRIBUCIÓN: Ceilán [Sri Lanka].

-Machadocepheus tuberculosus Mahunka, 1987

DisTRIBUCIÓN: Kenia.

Malgasodes Mahunka, 2000 (2 spp.)

ESPECIE TIPO: Malgasodes curvisetus Mahunka, 2000

DisTRIBUCIÓN: Etiópica.

-Malgasodes curvisetus Mahunka, 2000

DISTRIBUCIÓN: Madagascar. 
-Malgasodes hungarorum Mahunka, 2000 DISTRIBUCIÓN: Madagascar.

Meriocepheus Aoki, 1973 (1 sp.)

EsPeCIE TIPO: Meriocepheus peregrinus Aoki, 1973

DISTRIBUCIÓN: Paleártica.

-Meriocepheus peregrinus Aoki, 1973

DISTRIBUCIÓN: Japón.

Odontocepheus Berlese, 1913 (18 spp.)

ESPECIE TIPO: Tegeocranus elongatus Michael, 1879

DISTRIBUCIÓN: Holártica y Australianooriental.

Odontocepheus (Odontocepheus) Berlese, 1913 (15 spp.)

DisTRIBUCIÓN: Holártica y Australiana.

-Odontocepheus (O.) bandae Subías y Arillo, 2001

DisTRIBUCIÓN: Marruecos.

-Odontocepheus (O.) beijingensis Wang, 1997

DisTRIBUCIÓN: Noreste de China.

-Odontocepheus (O.) curtiseta Ruiz, Subías y Kahwash, 1989 DiSTRIBUCIÓN: España.

-Odontocepheus (O.) elongatus (Michael, 1879) (Tegeocranus) (=Hermannia oblonga Karpelles, 1893)

DisTRIBUCIÓN: Holártica (Paleártica occidental: menos frecuente en el Norte, y Sureste de U.S.A.).

-Odontocepheus (O.) espatulatus Saloña e Iturrondobeitia, 1989 DISTRIBUCIÓN: España.

-Odontocepheus (O.) hungaricus Mahunka, 1991

DistriBUCIÓn: Sur de Europa.

-Odontocepheus (O.) immarginatus Jacot, 1934

DisTRIBUCIÓN: Hawai.

-Odontocepheus (O.) laeviusculus (Canestrini y Fanzago, 1877)

(Claviceps) "sp. inq." DISTRIBUCIÓN: Italia.

-Odontocepheus (O.) oblongus (Banks, 1895) (Carabodes) ("non" Hermannia oblonga Karpelles, 1893 =Odontocepheus elongatus) DisTRIBUCIÓN: Neártica.

-Odontocepheus (O.) oglasae Baratti y Bernini, 1994 DisTRIBUCión: Italia.

-Odontocepheus (O.) piramidalis Mahunka, 1987 DisTRIBUCión: Mediterránea occidental.

-Odontocepheus (O.) ruber (Canestrini y Fanzago, 1877) (Claviceps) "sp. inq."

DISTRIBUCIÓN: Italia.

-Odontocepheus (O.) rumbleseatus Reeves, 1995 DisTRIBUCIÓN: U.S.A.

-Odontocepheus (O.) villosus Mahunka, 1987

DisTRIBUCión: Mediterránea occidental.

-Odontocepheus (O.) zaballosi Subías y Arillo, 2001

DisTRIBUCIÓN: Marruecos.

Odontocepheus (Aokiella) Balogh y Mahunka, 1967 (2 spp.)

ESPECIE TIPO: Aokiella florens Balogh y Mahunka, 1967

DisTRIBUCIÓN: Oriental.

-Odontocepheus (Aokiella) florens (Balogh y Mahunka, 1967) (A.) DisTRIBUCIÓN: Vietnam.

-Odontocepheus (Aokiella) rotundus (Hammer, 1979) (A.)

DisTRIBUCIÓN: Oriental: Java y Ceilán [Sri Lanka].

Odontocepheus (Indotocepheus) Mondal y Kundu, 1999 (1 sp.) ESPECIE TIPO: Indotocepheus himalayensis Mondal y Kundu, 1999 DisTRIBUCIÓN: Oriental.

-Odontocepheus (Indotocepheus) himalayensis (Mondal y Kundu, 1999) (I.)

DisTRIBUCIÓN: India (Bengala Occidental).

Pasocepheus Aoki, 1976 (5 spp.)

EsPecie Tipo: Pasocepheus triarcuatus Aoki, 1976

DISTRIBUCIÓN: Australianooriental.
Pasocepheus (Pasocepheus) Aoki, 1976 (4 spp.)

(=Opisthocepheus Aoki, 1976 "sin. nov.")

DisTRIBUCIÓN: Oriental.

-Pasocepheus (P.) bako Mahunka, 1996

DisTRIBUCIÓN: Borneo.

-Pasocepheus (P.) eremaeozetoides Mahunka, 1995

DisTRIBUCIÓN: Borneo.

-Pasocepheus (P.) kirai (Aoki, 1976) (Opisthocepheus)

Distribución: Península Malaya.

-Pasocepheus (P.) triarcuatus Aoki, 1976

Distribución: Península Malaya.

Pasocepheus (Guineobodes) Mahunka, 1987 (1 sp.)

EsPECIE TIPO: Machadocepheus papuanus Balogh, 1970

DISTRIBUCIÓN: Australiana.

-Pasocepheus (Guineobodes) papuanus (Balogh, 1970) (Machadocepheus)

DisTRIBUCIÓN: Nueva Guinea.

Pseudocarabodes Mahunka, 1991 (3 spp.)

(=Bakobodes Mahunka, 1996 "sin. nov.")

(=Yemenobodes Mahunka, 2000 "sin. nov.")

EsPecie TIPO: Pseudocarabodes xenus Mahunka, 1991

DisTRIBUCIÓN: Paleotropical.

-Pseudocarabodes improvisus (Mahunka, 2000) (Yemenobodes)

DisTRIBUCIÓN: Yemen.

-Pseudocarabodes orangutan (Mahunka, 1996) (Bakobodes)

DISTRIBUCIÓN: Borneo.

-Pseudocarabodes xenus Mahunka, 1991

DisTRIBUCIÓN: Borneo.

Sagittabodes J. y P. Balogh, 1992 (1 sp.)

EsPECIE TIPO: Machadocepheus sagitta Balogh y Mahunka, 1966

DISTRIBUCIÓN: Etiópica.

-Sagittabodes sagitta (Balogh y Mahunka, 1966) (Machadocepheus) DisTRIBUCIÓN: Sudáfrica.

Singabodes Mahunka, 1998 (1 sp.)

EsPeCIE TIPO: Singabodes rarus Mahunka, 1998

DISTRIBUCIÓN: Oriental.

-Singabodes rarus Mahunka, 1998

DistriBución: Península Malaya.

Spathulocepheus Balogh y Mahunka, 1969 (2 spp.)

ESPECIE TIPO: Spathulocepheus amazonicus Balogh y Mahunka, 1969

DISTRIBUCIÓN: Neotropical.

-Spathulocepheus amazonicus Balogh y Mahunka, 1969

DISTRIBUCIÓN: Brasil.

-Spathulocepheus plumiger Balogh y Mahunka, 1977

DISTRIBUCIÓN: Brasil.

Tansocepheus Mahunka, 1983 (1 sp.)

ESPECIE TIPO: Tansocepheus serratus Mahunka, 1983

DISTRIBUCIÓN: Etiópica.

-Tansocepheus serratus Mahunka, 1983

DisTRIBUCIÓN: Tanzania.

Tectocarabodes Mahunka, 1988 (1 sp.)

EsPECIE TIPO: Tectocarabodes monstruosus Mahunka, 1988

DISTRIBUCIÓN: Etiópica.

-Tectocarabodes monstruosus Mahunka, 1988

DisTRIBUCión: Tanzania.

Trichocarabodes Balogh, 1961 (4 spp.)

(=Ceylobodes J. y P. Balogh, 1992 "sin. nov.")

EsPecie TIPO: Carabodes celisi Balogh, 1958

DisTRIBUCIÓN: Paleotropical.

-Trichocarabodes capillatus Balogh, 1970

DisTRIBUCIÓN: Ceilán [Sri Lanka]. 
-Trichocarabodes celisi (Balogh, 1958) (Carabodes)

DistriBución: Congo.

-Trichocarabodes costulatus Mahunka, 1984

DISTRIBUCIÓN: Tanzania.

-Trichocarabodes hettigei Balogh, 1970

DistriBución: Ceilán [Sri Lanka].

Tuberocepheus Balogh y Mahunka, 1969 (1 sp.)

ESPECIE TIPO: Machadocepheus longus Balogh, 1964

DISTRIBUCIÓN: Etiópica.

-Tuberocepheus longus (Balogh, 1964) (Machadocepheus)

DisTRIBUCIÓN: Malgache.

Yoshiobodes Balogh y Mahunka, 1986 (11 spp.)

EsPECIE TIPO: Carabodes irmayi Balogh y Mahunka, 1969

DisTRIBUCión: Pantropical (excepto Etiópica) y subtropical (Holártica meridional)

Yoshiobodes (Yoshiobodes) Balogh y Mahunka, 1986 (9 spp.) DisTRIBUCIÓN: Pantropical (excepto Etiópica) y subtropical (Holártica meridional).

-Yoshiobodes (Y.) aokii Mahunka, 1987

DISTRIBUCIÓN: Borneo.

-Yoshiobodes (Y.) arcuatus Mahunka, 1987

DISTRIBUCIÓN: Borneo.

-Yoshiobodes (Y.) humidus Mahunka, 1996

DisTRIBUCIÓN: Borneo.

-Yoshiobodes (Y.) irmayi (Balogh y Mahunka, 1969) (Carabodes)

DISTRIBUCIÓN: Neotropical (frecuente) y U.S.A. meridional.

-Yoshiobodes (Y.) nakatamarii (Aoki, 1973) (Austrocarabodes)

(?=Carabodes humeratus Berlese, 1913 "sp. inq.")

DistriBUCión: Oriental y Este de Paleártica oriental.

-Yoshiobodes (Y.) ornatus Mahunka, 1987

DISTRIBUCIÓN: Borneo.

-Yoshiobodes (Y.) papuanus (Balogh, 1970) (Austrocarabodes humeratus $p$.)

DISTRIBUCIÓN: Nueva Guinea.

-Yoshiobodes (Y.) plumosulus (Balogh, 1970) (Austrocarabodes)

DisTRIBUCIÓN: Ceilán [Sri Lanka].

-Yoshiobodes (Y.) plumosus (Balogh, 1970) (Austrocarabodes)

DistriBución: Ceilán [Sri Lanka].

Yoshiobodes (Berndobodes) Mahunka, 1986 (2 spp.)

EsPECIE TIPO: Berndobodes spiculifer Mahunka, 1986

DISTRIBUCIÓN: Oriental.

-Yoshiobodes (Berndobodes) hauseri (Mahunka, 1996) (B.)

DISTRIBUCIÓN: Borneo.

-Yoshiobbodes (Berndobodes) spiculifer (Mahunka, 1986) (B.)

DISTRIBUCIÓN: Borneo.

Carabocepheidae Mahunka, 1986 (1 gen., 1 sp. y 1 ssp.) DisTRIBUCIÓN: Etiópica.

Carabocepheus Berlese, 1910 (1 sp. y 1 ssp.)

EsPECIE TIPO: Carabodes (Carabocepheus) lounsburyi Berlese, 1910 DISTRIBUCIÓN: Etiópica.

-Carabocepheus lounsburyi (Berlese, 1910) (Carabodes (Caraboceph.))

DisTRIBUCIÓN: Etiópica: Sudáfrica y Rodesia [Zimbabue].

-Carabocepheus lounsburyi lounsburyi (Berlese, 1910)

DisTRIBUCión: Etiópica: Sudáfrica y Rodesia [Zimbabue].

-Carabocepheus lounsburyi latior Balogh y Mahunka, 1966

DisTRIBUCIÓN: Sudáfrica.

Nippobodidae Aoki, 1959 (2 gen. y 12 spp.)

DisTRIBUCIÓN: Oriental y Paleártica meridional.

Leobodes Aoki, 1965 (5 spp.)

ESPECIE TIPO: Leobodes mirabilis Aoki, 1965

DisTRIBUCIÓN: Oriental.
-Leobodes anulatus Aoki, 1965

DisTRIBUCIÓN: "Himalaya".

-Leobodes ligiangensis Aoki, 2000

DISTRIBUCIÓN: China suroriental.

-Leobodes mirabilis Aoki, 1965

DistRIBUCIÓN: Oriental (Tailandia e India: Bengala Occidental)

-Leobodes monstruosus Jeleva y Vu, 1987

DISTRIBUCIÓN: Vietnam.

-Leobodes yinae Aoki, 2000

DISTRIBUCIÓN: China suroriental.

Nippobodes Aoki, 1959 (7 spp.)

EsPecIE TIPO: Nippobodes insolitus Aoki, 1959

DISTRIBUCIÓN: Paleártica.

-Nippobodes brevisetiger Aoki, 1981

DISTRIBUCIÓN: Japón.

-Nippobodes chejuensis Choi, 1996

DISTRIBUCIÓN: Corea.

-Nippobodes insolitus Aoki, 1959

DisTRIBUCIÓN: Japón.

-Nippobodes latus Aoki, 1970

DistriBUCión: Este de Paleártica oriental.

-Nippobodes tamlaensis Choi, 1996

DisTRIBUCIÓN: Corea.

-Nippobodes tokaraensis Aoki, 1989

DisTRIBUCIÓN: Japón.

-Nippobodes yuwanensis Aoki, 1984 (N. brevisetiger y.)

DisTRIBUCIÓN: Japón.

\section{Tectocepheoidea Grandjean, 1954}

Tectocepheidae Grandjean, 1954 (2 gen., 22 spp. y 6 sspp.) DistriBución: Cosmopolita.

Tectocepheus Berlese, 1896 (20 spp. y 5 sspp.)

ESPECIE TIPO: Tegeocranus velatus Michael, 1880

DisTRIBUCIÓN: Cosmopolita.

-Tectocepheus alatus Berlese, 1913

DistRIBUCión: Paleártica (excepto Norte)

-Tectocepheus americanus Pérez-Iñigo y Baggio, 1989

DisTRIBUCIÓN: Brasil.

-Tectocepheus cervus Balogh y Mahunka, 1969 "sp. inq."

DisTRIBUCIÓN: Bolivia.

-Tectocepheus concurvatus Knülle, 1954

DisTRIBUCIÓN: Europa central.

-Tectocepheus coniunctus Knülle, 1954

DisTRIBUCIÓN: Alemania.

-Tectocepheus cuspidentatus Knülle, 1954

DisTRIBUCIÓN: Paleártica occidental (menos frecuente en el

Norte), Japón y Oriental (China suroriental y Vietnam)

-Tectocepheus depressus Pérez-Íñigo y Baggio, 1989

DISTRIBUCIÓN: Brasil.

-Tectocepheus elegans Ohkubo, 1981

DistRIBUCión: Este de Paleártica oriental.

-Tectocepheus knuellei Vanek, 1960

DistriBUCiÓN: Paleártica.

-Tectocepheus latilamellaris Kardar, 1974

DISTRIBUCIÓN: India (Uttar Pradesh).

-Tectocepheus minor Berlese, 1903

(=Tectocepheus bisignatus Moritz, 1968)

(=Tectocepheus velatus novus Hammer, 1967)

DisTRIBUCIÓN: Paleártica occidental (menos frecuente en el Norte),

U.S.A. (Virginia), Oriental (Filipinas y China: Hong-Kong) y Nueva Zelanda.

-Tectocepheus punctulatus Djaparidze, 1985

DISTRIBUCIÓN: Cáucaso.

-Tectocepheus shirakamiensis Fujikawa, 2001

DisTRIBUCIÓN: Japón.

-Tectocepheus spinosus Mahunka, 1984

DisTRIBUCión: Tanzania. 
-Tectocepheus tenuis Knülle, 1954

DisTRIBUCIÓN: Alemania.

-Tectocepheus titanius Ohkubo, 1982

DISTRIBUCIÓN: Este de Paleártica oriental.

-Tectocepheus translamellaris Kardar, 1974

DistRIBUCión: India (Uttar Paradesh)

-Tectocepheus translamellatus Knülle, 1954

DisTRIBUCIÓN: Alemania.

-Tectocepheus velatus (Michael, 1880) (Tegeocranus)

DisTRIBUCIÓN: Cosmopolita.

-Tectocepheus velatus velatus (Michael, 1880)

DisTRIBUCIÓN: Cosmopolita (frecuente).

-Tectocepheus velatus clavatus Mahunka, 1983 (T. c.)

DisTRIBUCión: Europa meridional e India (Tripura).

-Tectocepheus velatus expansus Berlese, 1914 (T. minor e.) DisTRIBUCiÓN: U.S.A

-Tectocepheus velatus granulatus Mihelcic, 1957 DisTRIBUCIÓN: Austria.

-Tectocepheus velatus sarekensis Trägardh, 1910 (=Tectocepheus velatus angulatus Mihelcic, 1957)

(=Tectocepheus velatus ibericus Mihelcic, 1957)

(=Tectocepheus velatus inflexus Mihelcic, 1957)

DISTRIBUCIÓN: Cosmopolita (frecuente).

-Tectocepheus velatus universitatum Abdel-Hamid, 1964 DisTRIBUCIÓN: Egipto.

-Tectocepheus vicarius Balogh, 1958

DistRIBUCión: Etiópica (Angola y Chad).

Tegeozetes Berlese, 1913 (2 spp. y 1 ssp.)

ESPECIE TIPO: Tegeozetes tunicatus Berlese, 1913

DisTRIBUCIÓN: Pantropical y subtropical (Paleártica meridional).

-Tegeozetes angolensis Balogh, 1958 "sp. inq." DisTRIBUCIÓN: Angola.

-Tegeozetes tunicatus Berlese, 1913

DistribuCión: Pantropical y Paleártica meridional (Hungría y Japón).

-Tegeozetes tunicatus tunicatus Berlese, 1913

DistriBuCión: Pantropical y Sur de Europa (Hungría).

-Tegeozetes tunicatus breviclava Aoki, 1970

DISTRIBUCIÓN: Japón.

Tegeocranellidae P. Balogh, 1987 (1 gen. y 14 spp.) DisTRIBUCIÓN: Cosmopolita (excepto Antártica).

Tegeocranellus Berlese, 1913 (14 spp.)

EsPECIE TIPO: Tegeocranus laevis Berlese, 1905

DistRIBUCIÓn: Cosmopolita (excepto Antártica).

-Tegeocranellus alas Behan-Pelletier, 1997

Distribución: Costa Rica.

-Tegeocranellus barbarae Behan-Pelletier, 1997

DisTRIBUCIÓN: Sureste de U.S.A.

-Tegeocranellus bolivianus Balogh y Mahunka, 1969

DisTRIBUCIÓN: Neotropical (Bolivia y Paraguay).

-Tegeocranellus bosniae (Frank, 1961) (Carabodes) DISTRIBUCIÓN: Bosnia.

-Tegeocranellus concavus J. y P. Balogh, 1983

Distribución: Australia.

-Tegeocranellus convexus J. y P. Balogh, 1983

DisTRIBUCión: Australia.

-Tegeocranellus kethleyi Behan-Pelletier, 1997

DisTRIBUCIÓN: Sureste de U.S.A.

-Tegeocranellus knysnaensis Kok, 1968

(=Tegeocranellus africanus Mahunka, 1985)

DisTRIBUCIÓN: Sudáfrica.

-Tegeocranellus laevis (Berlese, 1905) (Tegeocranus) Distribución: Sur de Europa, Oriental: Filipinas y Ceilán [Sri Lanka], y Melanesia (I. Fiji).

-Tegeocranellus mediolamellatus Behan-Pelletier, 1997 DISTRIBUCIÓN: Guatemala.
-Tegeocranellus mississippii Behan-Pelletier, 1997

DisTRIBUCIÓN: Sur de U.S.A.

-Tegeocranellus muscorum Behan-Pelletier, 1997

DistRIBUCIÓN: Neártica.

-Tegeocranellus opcus Tseng, 1982

DISTRIBUCIÓN: Taiwan [Formosa]

-Tegeocranellus sacchareus Kok, 1968

DisTRIBUCIÓN: Sudáfrica.

Hydrozetoidea Grandejan, 1954

Hydrozetidae Grandjean, 1954 (1 gen., 2 subg., 27 spp. y 1 ssp.) DISTRIBUCIÓN: Cosmopolita (excepto Antártica).

Hydrozetes Berlese, 1902 (27 spp. y 1 ssp.)

ESPECIE TIPO: Notaspis lacustris Michael, 1882

DisTRIBUCIÓN: Cosmopolita (excepto Antártica).

Hydrozetes (Hydrozetes) Berlese, 1902 (24 spp. y 1 ssp.)

DisTRIBUCIÓN: Cosmopolita (excepto Antártica).

-Hydrozetes (H.) amudariensis Koshchanova y Krivolutsky, 1984

DISTRIBUCIÓN: Asia centrooccidental.

-Hydrozetes (H.) californiensis Habeeb, 1974

DistRIBUCIÓN: U.S.A. (California).

-Hydrozetes (H.) capensis Engelbrecht, 1974

DisTRIBUCIÓN: Sudáfrica.

-Hydrozetes (H.) confervae (Schrank, 1781) (Acarus)

(=Hydrozetes terrestris Berlese, 1910)

DisTRIBUCIÓN: Holártica.

-Hydrozetes (H.) dimorphus Hammer, 1962

DistRIBUCIÓN: Neotropical (Argentina y Brasil).

-Hydrozetes (H.) dimorphus dimorphus Hammer, 1962 DisTRIBUCIÓN: Argentina.

-Hydrozetes (H.) dimorphus virginalis Fernández y Travé, 1984 DisTRIBUCIÓN: Neotropical (Argentina y Brasil).

-Hydrozetes (H.) edentulus Willmann, 1931

DisTRIBUCIÓN: Oriental (Indonesia).

-Hydrozetes (H.) escobari Fernández y Travé, 1984

DisTRIBUCIÓN: Argentina.

-Hydrozetes (H.) gueyeae Mahunka, 1990

DISTRIBUCIÓN: Senegal.

-Hydrozetes (H.) indicator Habeeb, 1974

U.S.A. (Connecticut).

-Hydrozetes (H.) laccosis Woolley, 1969

Distribución: U.S.A. (Colorado).

-Hydrozetes (H.) lacustris (Michael, 1882) (Notaspis)

(=Notaspis speciosus Chinaglia, 1917)

DisTRIBUCIÓN: Boreal (Paleártica occidental: menos frecuente en

el Sur, Siberia centrooccidenal, Japón y Canadá).

-Hydrozetes (H.) lemnae (Coggi, 1897) (Notaspis)

(=Hydrozetes mollicoma Hammer, 1958)

DisTRIBUCIÓN: Semicosmopolita (Paleártica occidental: frecuente,

Oriental: Bali, Australiana: frecuente, y Neotropical: frecuente).

-Hydrozetes (H.) megacephalum (Berlese, 1901) (Dameosoma) “sp. inq."

DISTRIBUCIÓN: América austral.

-Hydrozetes (H.) niloticus (Trägardh, 1905) (Notaspis) "sp. inq." DisTRIBUCIÓN: Sudán.

-Hydrozetes (H.) octosetosus Willmann, 1931 (H. lacustris o.)

DistRIBUCIÓN: Europa central.

-Hydrozetes (H.) parisiensis Grandjean, 1948

DisTRIBUCIÓN: Europa meridional.

-Hydrozetes (H.) paulista Pérez-Íñigo y Baggio, 1989

DisTRIBUCIÓN: Brasil.

-Hydrozetes (H.) petrunkevitchi Newel, 1945

DisTRIBUCIÓN: U.S.A. (Florida).

-Hydrozetes (H.) platensis Berlese, 1902 "sp. inq."

Distribución: Chile.

-Hydrozetes (H.) tobaicus Willmann, 1931

DistRibución: Sumatra. 
-Hydrozetes (H.) tridactylus Abdel-Hamid, 1964

DisTRIBUCIÓN: Egipto.

-Hydrozetes (H.) uberabensis Pérez-Íñigo y Baggio, 1996

DisTRIBUCIÓN: Brasil.

-Hydrozetes (H.) vicarius Balogh, 1958 "sp. inq."

DistRIBUCIÓN: Angola.

-Hydrozetes (H.) videor Habeeb, 1974

DisTRIBUCIÓN: U.S.A. (Nueva York)

Hydrozetes (Argentinobates) Fernández, 1984 (1 sp.) ESPECIE TIPO: Hydrozetes (Argentinobates) ringueleti Fernández, 1984 DISTRIBUCIÓN: Neotropical.

-Hydrozetes (Argentinobates) ringueleti Fernández, 1984

DISTRIBUCIÓN: Argentina.

Hydrozetes (Heloribates) Grandjean, 1966 (2 spp.)

ESPECIE TIPO: Hydrozetes thienemanni Strenzke, 1943

DisTRIBUCIÓN: Holártica.

-Hydrozetes (Heloribates) tamarae Toslstikov, 1996

DisTRIBUCIÓN: Norte de Siberia.

-Hydrozetes (Heloribates) thienemanni Strenzke, 1943 (Hydr.)

(=Hydrozetes incisus Grandjean, 1948)

DisTRIBUCIÓN: Boreal (Paleártica occidental: excepto Sur, y Alaska).

Ameronothroidea Willmann, 1931

Ameronothridae Willmann, 1931 (9 gen., 38 spp. y 8 sspp.) DisTRIBUCIÓN: Cosmopolita.

Alaskozetes Hammer, 1955 (3 spp. y 2 sspp.)

ESPECIE TIPO: Alaskozetes coriaceus Hammer, 1955

DisTRIBUCIÓN: Anfipolar.

-Alaskozetes antarcticus (Michael, 1903) (Notaspis)

DisTRIBUCIÓN: Antártica y Nueva Zelanda.

-Alaskozetes antarcticus antarcticus (Michael, 1903)

DisTRIBUCIÓN: Antártica (frecuente) y Nueva Zelanda.

-Alaskozetes antarcticus grandjeani (Dalenius, 1958) (Halozetes)

DisTRIBUCión: Antártica.

-Alaskozetes antarcticus intermedius Wallork, 1967

DistriBución: Antártica.

-Alaskozetes bouvetoyaensis Pletzen y Kok, 1971

DisTRIBUCIÓN: Antártica.

-Alaskozetes coriaceus Hammer, 1955

DisTRIBUCión: Alaska y Nueva Zelanda subantártica.

Ameronothrus Berlese, 1896 (13 spp.)

(=Hygroribates Jacot, 1934)

EsPECIE TIPO: Eremaeus lineatus Thorell, 1871

DisTRIBUCIÓN: Semicosmopolita (Holártica, Etiópica y Neotropical)

-Ameronothrus bilineatus (Michael, 1888) (Scutovertex)

DisTRIBUCIÓN: Norte de Europa y Sudáfrica.

-Ameronothrus dubinini Sitnikova, 1975

DisTRIBUCIÓN: Erosiberiana (Norte de Europa y Oeste de Siberia).

-Ameronothrus harioti (Michael, 1891) (Scutovertex) "sp. inq."

DistriBución: América austral (Tierra del Fuego).

-Ameronothrus lapponicus Dalenius, 1963

DisTRIBUCIÓN: Suecia.

-Ameronothrus lineatus (Thorell, 1871) (Eremaeus)

(=Scutovertex corrugatus Michael, 1888)

(=Scutovertex occidentalis Hull, 1918)

DistriBución: Boreal (excepto Paleártica oriental).

-Ameronothrus maculatus (Michael, 1882) (Scutovertex)

(=Scutovertex maculatus groenlandicus Trägardh, 1904)

(=Scutovertex pseudomaculaltus Hull, 1914)

(=Scutovertex pseudomaculatus insularis Hull, 1914)

DisTRIBUCión: Boreal (excepto Paleártica oriental) y Pequeñas

Antillas.
-Ameronothrus marinus (Banks, 1896) (Nothrus)

(=Ameronothrus fucicolum Brady, 1875)

(=Scutovertex spoofi Oudemans, 1900)

DisTRIBUCIÓN: Boreal (excepto Paleártica oriental).

-Ameronothrus nidicola Sitnikova, 1975

Distribución: Erosiberiana (Norte de Rusia europea y Este de Paleártica oriental).

-Ameronothrus nigrofemoratus (L. Koch, 1879) (Nothrus)

(=Ameronothrus lineatus brevipes Willmann, 1937)

DisTRIBUCión: Boreal (excepto Paleártica oriental)

-Ameronothrus oblongus Sitnikova, 1975

DistriBUCIÓN: Paleártica (Europa oriental y Este de Paleártica oriental)

-Ameronothrus schneideri (Oudemans, 1905) (Scutovertex)

DistRIBUCIÓN: Alemania.

-Ameronothrus schubarti Weigmann y Schulte, 1975

DisTRIBUCIÓN: U.S.A. (California).

-Ameronthrus schusteri Schubart, 1970

DisTRIBUCIÓN: Croacia.

Antarcticola Wallwork, 1967 (2 spp.)

(=Petrozetes Sitnikova, 1968)

ESPECIE TIPO: Ameronothrus meyeri Wallwork, 1967

DisTRIBUCIÓN: Antártica.

-Antarcticola georgiae Wallwork, 1970

DisTRIBUCIÓN: Antártica.

-Antarcticola meyeri Wallwork, 1967

(=Petrozetes oblongus Sitnikova, 1968)

DistribuCIÓN: Antártida

Aquanothrus Engelbrecht, 1975 (1 sp.)

ESPECIE TIPO: Aquanothrus montanus Engelbrecht, 1975

DisTRIBUCIÓN: Etiópica.

-Aquanothrus montanus Engelbrecht, 1975

DisTRIBUCIÓN: Sudáfrica.

Capillibates Hammer, 1966 (1 sp.)

EsPECIE TIPO: Capillibates stagaardi Hammer, 1966

DISTRIBUCIÓN: Australiana.

-Capillibates stagaardi Hammer, 1966

DistrIBUCIÓN: Nueva Zelanda.

Chudalupia Wallwork, 1981 (1 sp.)

ESPECIE TIPO: Chudalupia meridionalis Wallwork, 1981

DISTRIBUCIÓN: Australiana.

-Chudalupia meridionalis Wallwork, 1981

DistriBuCIÓN: Australia.

Halozetes Berlese, 1916 (15 spp. y 5 sspp.)

(=Anarea Dalenius, 1958)

=Pertorgunia Dalenius, 1958)

ESPECIE TIPO: Notaspis marina Lohmann, 1907

DISTRIBUCIÓN: Antártica y subantártica.

-Halozetes bathamae Luxton, 1984

DisTRIBUCIÓN: Nueva Zelanda.

-Halozetes belgicae (Michael, 1903) (Notaspis)

DistriBución: Antártica.

-Halozetes belgicae belgicae (Michael, 1903)

DISTRIBUCIÓN: Antártica (frecuente).

-Halozetes belgicae brevipilis Wallwork, 1963

DISTRIBUCIÓN: Antártica (I. Macquarie).

-Halozetes belgicae longisetae Wallwork, 1967

DisTRIBUCIÓN: Antártica.

-Halozetes belgicae mickii Coetzee, 1999

DisTRIBUCIÓN: Antártica (I. Príncipe Eduardo).

-Halozetes crozetensis (Richters, 1908) (Scutovertex)

(=Pertorgunia colobanthi Dalenius, 1958)

DisTRIBUCIÓN: Antártica (frecuente). 
-Halozetes edwardensis Pletzen y Kok, 1971

DisTRIBUCIÓN: Antártica (I. Príncipe Eduardo).

-Halozetes fulvus Engelbrecht, 1975

DistriBución: Antártica (I. Príncipe Eduardo).

-Halozetes impeditus Niedbala, 1986

Distribución: Antártica (I. Shetland del Sur).

-Halozetes intermedius Wallwork, 1963

DisTRIBUCIÓN: Antártica.

-Halozetes littoralis Wallwork, 1970

DistRIBUCIÓN: Antártica (I. Georgia del Sur).

-Halozetes macquariensis (Dalenius, 1958) (Pertorgunia)

DisTRIBUCIÓN: Antártica.

-Halozetes marinus (Lohmann, 1907) (Notaspis)

DisTRIBUCIÓN: Austral (Antártica y Nueva Zelanda).

-Halozetes marinus marinus (Lohmann, 1907)

(=Anarea macquariensis Dalenius, 1958)

DISTRIBUCIÓN: Antártica (frecuente) y Nueva Zelanda.

-Halozetes marinus devilliersi Engelbrecht, 1974

DisTRIBUCIÓN: Antártica.

-Halozetes marinus minor Wallwork, 1966

DisTRIBUCIÓN: Antártica.

-Halozetes marionensis Engelbrecht, 1974

DISTRIBUCIÓN: Antártica.

-Halozetes necrophagus Wallwork, 1967

Distribución: Antártica (I. Shetland del Sur).

-Halozetes otagoensis Hammer, 1966

DisTRIBUCIÓN: Nueva Zelanda.

-Halozetes plumosus Wallwork, 1966

DisTRIBUCIÓN: Antártica (I. Campbell).

-Halozetes scotiae (Trouessart, 1912) (Notaspis) “sp. inq."

DistRIBUCión: Antártica: I. Orcadas del Sur (I. Orkney).

Podacarus Grandjean, 1955 (1 sp. y 1 ssp.)

EsPecie TIPO: Podacarus auberti Grandejan, 1955

DisTRIBUCIÓN: Antártica.

-Podacarus auberti Grandjean, 1955

DISTRIBUCIÓN: Antártica.

-Podacarus auberti auberti Grandjean, 1955

DistRIBUCIÓN: Antártica (frecuente).

-Podacarus auberti occidentalis Wallwork, 1966

DISTRIBUCIÓN: Antártica.

Pseudantarcticola Balogh, 1970 (1 sp.)

EsPeCIE TIPO: Pseudantarcticola tropica Balogh, 1970

DisTRIBUCIÓN: Australiana.

-Pseudantarcticola tropica Balogh, 1970

DisTRIBUCIÓN: Nueva Guinea.

Selenoribatidae Schuster, 1963 (5 gen. y 10 spp.)

DISTRIBUCIÓN: Pantropical (excepto Australiana) y Paleártica meridional.

Arotrobates Luxton, 1992 (2 spp.)

ESPECIE TIPO: Arotrobates lanceolatus Luxton, 1992

DisTRIBUCIÓN: Oriental.

-Arotrobates granulatus Luxton, 1992

Distribución: China (Hong-Kong).

-Arotrobates lanceolatus Luxton, 1992

DISTRIBUCIÓN: China (Hong-Kong).

Psednobates Luxton, 1992 (1 sp.)

ESPECIE TIPO: Psednobates uncunguis Luxton, 1992

DISTRIBUCIÓN: Oriental.

-Psednobates uncunguis Luxton, 1992

DisTRIBUCIÓN: China (Hong-Kong).

Schusteria Grandjean, 1968 (3 spp.)

EsPECIE TIPO: Schusteria littorea Grandjean, 1968

DisTRIBUCIÓN: Tropical (Etiópica y Neotropical).

-Schusteria littorea Grandjean, 1968

DisTRIBUCIÓN: Brasil.
-Schusteria melanomerus Marshall y Pugh, 2000

DisTRIBUCIÓN: Sudáfrica.

-Schusteria ugraseni Marshall y Pugh, 2000

DisTRIBUCIÓN: Sudáfrica.

Selenoribates Strenzke, 1961 (3 spp.)

ESPECIE TIPO: Selenoribates foveiventris Strenzke, 1961

DisTRIBUCIÓN: Paleártica.

-Selenoribates foveiventris Strenzke, 1961

DisTRIBUCIÓN: Egipto.

-Selenoribates ghardaqensis Abdel-Hamid, 1973

DISTRIBUCIÓN: Egipto.

Selenoribates mediterraneus Grandjean, 1966

DistRIBUCIÓN: Sur de Europa.

Thalassozetes Schuster, 1963 (1 sp.)

EsPECIE TIPO: Thalassozetes riparius Schuster, 1963

DisTRIBUCIÓN: Paleártica.

-Thalassozetes riparius Schuster, 1963

DISTRIBUCIÓN: Croacia.

Fortuyniidae Hammen, 1963 (3 gen., 10 spp. y 2 sspp.) DisTRIBUCIÓN: Tropical y subtropical.

Alismobates Luxton, 1992 (2 spp.)

ESPECIE TIPO: Alismobates reticulatus Luxton, 1992

DISTRIBUCIÓN: Oriental.

-Alismobates reticulaltus Luxton, 1992

DisTRIBUCIÓN: China (Hong-Kong).

-Alismobates rotundus Luxton, 1992

Distribución: China (Hong-Kong).

Circellobates Luxton, 1992 (1 sp.)

EsPECIE TIPO: Circellobates venustus Luxton, 1992

DisTRIBUCIÓN: Oriental.

Circellobates venustus Luxton, 1992

DisTRIBUCIÓN: China (Hong-Kong).

Fortuynia Hammen, 1960 (7 spp. y 2 sspp.)

EsPeCIE TIPO: Fortuynia marina Hammen, 1960

DISTRIBUCIÓN: Tropical y subtropical.

-Fortuynia elamellata Luxton, 1967

DisTRIBUCión: Nueva Zelanda, Sudáfrica y Japón.

-Fortuynia elamellata elamellata Luxton, 1967 DisTRIBUCIÓN: Nueva Zelanda.

-Fortuynia elamellata micromorpha Marshall y Pugh, 2002

DISTRIBUCIÓN: Sudáfrica.

-Fortuynia elamellata shibai Aoki, 1974

DISTRIBUCIÓN: Japón.

-Fortuynia inhambanensis Marshall y Pugh, 2002

DisTRIBUCIÓN: Sudáfrica.

-Fortuynia maculata Luxton, 1986

DistriBución: Kenia.

-Fortuynia marina Hammen, 1960

DISTRIBUCIÓN: Nueva Guinea.

-Fortuynia rotunda Marshall y Pugh, 2002

DisTRIBUCIÓN: Sudáfrica.

-Fortuynia sinensis Luxton, 1992

DisTRIBUCIÓN: China (Hong-Kong).

-Fortuynia yunkeri Hammen, 1963

DISTRIBUCIÓN: Panamá.

Cymbaeremaeoidea Sellnick, 1928

Adhaesozetidae Hammer, 1973 (1 gen. y 2 spp.) DISTRIBUCIÓN: Australiana.

Adhaesozetes Hammer, 1966 (2 spp.)

EsPeCIE TIPO: Adhaesozetes barbarae Hammer, 1966

DISTRIBUCIÓN: Australiana. 
-Adhaesozetes barbarae Hammer, 1966

DisTRIBUCIÓN: Nueva Zelanda.

-Adhaesozetes polyphyllos Walter y Behan-Pelletier, 1993

DisTRIBUCIÓn: Australiana (Australia y Tasmania).

Cymbaeremaeidae Sellnick, 1928 (5 gen. y 89 spp.) DISTRIBUCIÓN: Cosmopolita (excepto Antártica).

Bulleremaeus Hammer, 1966 (2 spp.)

ESPECIE TIPO: Bulleremaeus reticulatus Hammer, 1966

DisTRIBUCIÓN: Australiana.

-Bulleremaeus reticulatus Hammer, 1966

DisTRIBUCIÓN: Nueva Zelanda.

-Bulleremaeus tuberculatus Hammer, 1966

DisTRIBUCIÓN: Nueva Zelanda.

Cymbaeremaeus Berlese, 1896 (3 spp.)

EsPeCIE TiPO: Eremaeus cymba Nicolet, 1855

DistRIBUCIÓN: Paleártica y Neotropical.

-Cymbaeremaeus cymba (Nicolet, 1855) (Eremaeus)

(=Cymbaeremaeus pulvillifer Willmann, 1931)

Distribución: Paleártica (Paleártica occidental: frecuente, y

Paleártica oriental: excepto Este) e India (Sikkim).

-Cymbaeremaeus foliatus (Balogh y Csiszár, 1963) (Scutovertex)

DisTRIBUCión: Argentina.

-Cymbaeremaeus silva Fujikawa, 2002

DISTRIBUCIÓN: Japón.

Glanderemaeus Balogh y Csiszár, 1963 (1 sp.)

EsPECIE TIPO: Glanderemaeus hammerae Balogh y Csiszár, 1963

DisTRIBUCIÓN: Neotropical.

-Glanderemaeus hammerae Balogh y Csiszár, 1963

DISTRIBUCIÓN: América austral.

Scapheremaeus Berlese, 1910 (82 spp.)

EsPeCIE TIPO: Eremaeus patella Berlese, 1886

DisTRIBUCIÓn: Cosmopolita (excepto Antártica).

-Scapheremaeus alvarezius Ríos y Palacios-Vargas, 1998 DisTribución: Méjico.

-Scapheremaeus alveolatus Hammer, 1961

DisTRIBUCIÓN: Neotropical (Perú y Argentina).

-Scapheremaeus arboreus Corpuz-Raros, 1979 DisTRIBUCIÓN: Filipinas.

-Scapheremaeus arcuatus Hammer, 1971 Distribución: Melanesia (I. Fiji).

-Scapheremaeus argentinensis Travé y Fernández, 1986 DISTRIBUCIÓN: Argentina.

-Scapheremaeus balazsi Mahunka, 1983 DisTRIBUCIÓN: Brasil.

-Scapheremaeus baloghius Ríos y Palacios-Vargas, 1998 DisTRIBUCIÓN: Méjico.

-Scapheremaeus bicornutus Hammer, 1971

DisTRIBUCIÓN: Islas del Pacífico.

-Scapheremaeus bisculpturatus Mahunka, 1984

DisTRIBUCIÓN: Paraguay.

-Scapheremaeus carinatus Willmann, 1936

DistriBución: Pequeñas Antillas.

-Scapheremaeus cellulatifer Mahunka, 1987 DisTRIBUCIÓN: Vietnam.

-Scapheremaeus chaac Ríos y Palacios-Vargas, 1998 DisTRIBUCIÓN: Méjico.

-Scapheremaeus clavifer Hammer, 1958 DISTRIBUCIÓN: Argentina.

-Scapheremaeus clavisetus Mahunka, 1978 Distribución: Malgache (I. Mauricio).

-Scapheremaeus convexus Hammer, 1979

Distribución: Oriental (Indonesia).

-Scapheremaeus corniger (Berlese, 1908) (Cymbaeremaeus)

(=Scapheremaeus madeirensis Willmann, 1939)

DisTRIBUCIÓN: Mediterránea occidental.
-Scapheremaeus cornutus Balogh, 1958

Distribución: Angola.

-Scapheremaeus crassus Mahunka, 1988

DISTRIBUCIÓN: Vietnam.

-Scapheremaeus cuspidatus Pérez-Íñigo, 1982

Distribución: Guinea Ecuatorial: I. Pagalu [Annobón].

-Scapheremaeus cyclops (Oudemans, 1915) (Cymbaeremaeus)

DisTRIBUCIÓN: Ceilán [Sri Lanka].

-Scapheremaeus demeteri Mahunka, 1983

DisTRIBUCión: Etiopía.

-Scapheremaeus emarginatus Hammer, 1966

DistRIBUCión: Nueva Zelanda.

-Scapheremaeus eugenius Ríos y Palacios-Vargas, 1998

DisTRIBUCIÓN: Méjico.

-Scapheremaeus fimbriatus (Michael, 1890) (Eremaeus)

DistriBuCIÓN: Argelia.

-Scapheremaeus fisheri Aoki, 1966

Distribución: Islas del Pacífico e India (Bengala Occidental).

-Scapheremaeus flamiferus Palacios-Vargas y Ríos, 1998

DisTRIBUCIÓN: Norte de Neotropical.

-Scapheremaeus foveolatus Mahunka, 1987

DISTRIBUCIÓN: Vietnam.

-Scapheremaeus fungisetosus Ríos y Palacios-Vargas, 1998

Distribución: Méjico.

-Scapheremaeus glaber Hammer, 1958

DisTRIBUCIÓN: Bolivia.

-Scapheremaeus grahamius Ríos y Palacios-Vargas, 1998

Distribución: Méjico.

-Scapheremaeus guerini (Berlese, 1908) (Cymbaeremaeus)

(=Cymbaeremaeus (Scapheremaeus) reticulatus Berlese, 1910)

DISTRIBUCIÓN: Europa centromeridional.

-Scapheremaeus hectorperezius Ríos y Palacios-Vargas, 1998

Distribución: Méjico.

-Scapheremaeus hieroglyphicus (Hall, 1911) (Hermannia)

DistriBUCIÓN: U.S.A. (California).

-Scapheremaeus humeratus Balogh y Mahunka, 1967

DisTRIBUCIÓN: Congo.

-Scapheremaeus hungarorum Mahunka, 1986

DisTRIBUCIÓN: Tanzania.

-Scapheremaeus insularis Hammer, 1966

DisTRIBUCión: Nueva Zelanda.

-Scapheremaeus johnsi Balogh, 1970

DisTRIBUCIÓN: Nueva Guinea.

-Scapheremaeus latirostris Willmann, 1936

DisTRIBUCIÓN: Neotropical (Pequeñas Antillas y Argentina).

-Scapheremaeus latus Mahunka, 1985

DistriBUCIÓN: Pequeñas Antillas.

-Scapheremaeus longicuspis Mahunka, 1984

DistriBuCión: Paraguay.

-Scapheremaeus longilamellatus Mahunka, 1985

DistriBución: Pequeñas Antillas.

-Scapheremaeus magdalenae Ríos y Palacios-Vargas, 1998

DisTRIBUCIÓN: Méjico.

-Scapheremaeus mahunkaius Ríos y Palacios-Vargas, 1998

DistribuCión: Méjico.

-Scapheremaeus marginalis (Banks, 1896) (Eremaeus)

DisTRIBUCIÓN: U.S.A. (Nueva York).

-Scapheremaeus marmoratus (Berlese, 1910) (Cymbaeremaeus (S.)) DisTRIBUCIÓN: U.S.A. (Florida).

-Scapheremaeus morenoi (Balogh y Mahunka, 1974) (Scutovertex) DisTRIBUCIÓN: Cuba.

-Scapheremaeus morulisensillatus Ríos y Palacios-Vargas, 1998

DisTRIBUCIÓN: Méjico.

-Scapheremaeus nashiroi Nakatamari, 1989

DisTRIBUCIÓN: Japón.

-Scapheremaeus nogueraius Ríos y Palacios-Vargas, 1998

DisTRIBUCIÓN: Méjico.

-Scapheremaeus nuciferosa Ramani y Haq, 1998

DisTRIBUCIÓN: India (Kerala). 
-Scapheremaeus obliteratus Hammer, 1961

DisTRIBUCIÓN: Perú.

-Scapheremaeus ornatus Balogh y Mahunka, 1968

DISTRIBUCIÓN: Argentina.

-Scapheremaeus palaciosi Ríos, 1996

Distribución: Méjico.

-Scapheremaeus palustris (Sellnick, 1924) (Cymbaeremaeus (S.))

DisTRIBUCIÓN: Holártica (Paleártica occidental y Neártica) e I. Santa Helena.

-Scapheremaeus parvulus (Banks, 1909) (Cymbaeremaeus) DisTriBución: Canadá.

-Scapheremaeus patella (Berlese, 1886) (Eremaeus)

(=Scapheremaeus hammerae J. y P. Balogh, 2002)

DisTRIBUCIÓN: Mediterránea y Nueva Zelanda.

-Scapheremaeus petrophagus (Banks, 1906) (Scutovertex)

DisTRIBUCIÓN: U.S.A.

-Scapheremaeus petrosus Sitnikova, 1975

DistriBución: Este de Paleártica oriental.

-Scapheremaeus pisacensis Hammer, 1961

Distribución: Perú.

-Scapheremaeus polysetosus Sitnikova, 1975

DistriBución: Paleártica oriental.

-Scapheremaeus pseudoreticulatus Mahunka, 1984

DisTRIBUCIÓN: I. Seychelles.

-Scapheremaeus pulchellus (Berlese, 1910) (Cymbaeremaeus (S.))

DisTRIBUCión: U.S.A. (Florida)

-Scapheremaeus pundamiliaensis Engelbrecht, 1975

DisTRIBUCIÓN: Sudáfrica.

-Scapheremaeus quadrilineatus Mahunka, 1978

DisTRIBUCIÓN: Malgache (I. Mauricio).

-Scapheremaeus rustenburgensis Engelbrecht, 1975

DISTRIBUCIÓN: Sudáfrica.

-Scapheremaeus schatzi Ríos y Palacios-Vargas, 1998

Distribución: Méjico.

-Scapheremaeus semiconvexus Hammer, 1982

DISTRIBUCIÓN: Bali.

-Scapheremaeus semiornatus Hammer, 1979

DisTRIBUCIÓN: Java

-Scapheremaeus simplex Ríos y Palacios-Vargas, 1998

Distribución: Méjico.

-Scapheremaeus sinuosus Aoki, 1964

DisTRIBUCIÓN: Hawai.

-Scapheremaeus stratus Hammer, 1958

DisTRIBUCIÓN: Bolivia.

-Scapheremaeus striatomarginatus Hammer, 1979

DISTRIBUCIÓN: Java.

-Scapheremaeus subcorniger Pérez-Íñigo y Peña, 1996

DistRIBUCIÓN: I. Canarias.

-Scapheremaeus subglaber Balogh y Mahunka, 1978

DisTRIBUCIÓN: Paraguay.

-Scapheremaeus taurus Balogh, 1970

DisTRIBUCIÓN: Nueva Guinea y Filipinas.

-Scapheremaeus tillandsiae Fernández y Cleva, 1997

DisTRIBUCIÓN: Argentina.

-Scapheremaeus tillandsiophilus Ríos y Palacios-Vargas, 1998 DisTRIBUCión: Méjico.

-Scapheremaeus tonatiuh Palacios-Vargas, Ríos y Vázquez, 1998 DisTRIBUCIÓN: Méjico.

-Scapheremaeus tricarinatus Sitnikova, 1975

DisTRIBUCIÓN: Mediterránea.

-Scapheremaeus trirugis Hammer, 1958

DisTRIBUCIÓN: Argentina.

-Scapheremaeus volcanicus Ríos y Palacios-Vargas, 1998 DistriBución: Méjico.

-Scapheremaeus yamashitai Aoki, 1970

DisTRIBUCIÓN: Este de Paleártica oriental.

Seteremaeus Hammer, 1971 (1 sp.)

EsPECIE TIPO: Setermaeus spinosus Hammer, 1971

DisTRIBUCIÓN: Australiana.
-Seteremaeus spinosus Hammer, 1971

Distribución: Melanesia (I. Fiji)

Ametroproctidae fam. nov. (2 gen., 1 subg. y 8 spp.) DisTRIBUCIÓN: Holártica.

Ametroproctus Higgins y Woolley, 1968 (7 spp.)

ESPECIE TIPO: Ametroproctus oresbios Higgins y Woolley, 1968 DisTRIBUCIÓN: Holártica.

Ametroproctus (Ametroproctus) Higgins y Woolley, 1968 (3 spp.) DISTRIBUCIÓN: Neártica.

-Ametroproctus (A.) aridus Behan-Pelletier, 1987

DisTRIBUCIÓN: U.S.A. (Oregón).

-Ametroproctus (A.) oresbios Higgins y Woolley, 1968

DistRIBUCIÓN: Neártica.

-Ametroproctus (A.) tuberculosus Behan-Pelletier, 1987

DISTRIBUCIÓN: Canada.

Ametroproctus (Coropoculia) Aoki y Fujikawa, 1972 (4 spp.)

EsPECIE TIPO: Coropoculia reticulata Aoki y Fujikawa, 1972

DisTRIBUCIÓN: Holártica.

-Ametroproctus (Coropoculia) beringianus Behan-Pelletier, 1987

DisTRIBUCIÓN: Canadá.

-Ametroproctus (Coropoculia) canningsi Behan-Pelletier, 1987

DistriBución: Canadá.

-Ametroproctus (Coropoculia) lamellatus (Schweizer, 1956) (Cym-

baeremaeus)

DISTRIBUCIÓN: Europa centrooccidental.

-Ametroproctus (Coropoculia) reticulatus (Aoki y Fujikawa, 1972) (C.)

DisTRIBUCIÓN: Holártica (Neártica y Japón)

Scapuleremaeus Behan-Pelletier, 1989 (1 sp.)

EsPECIE TIPO: Scapuleremaeus kobauensis Behan-Pelletier, 1989

DisTRIBUCIÓN: Neártica.

-Scapuleremaeus kobauensis Behan-Pelletier, 1989

DisTRIBUCIÓN: Canadá

Infraorden PORONOTICAE Grandjean, 1954

Licneremaeoidea Grandjean, 1931

Micreremidae Grandjean, 1954 (4 gen. y 14 spp.)

DisTRIBUCión: Cosmopolita (excepto Antártica).

Fenichelia Balogh, 1970 (3 spp.)

(=Porofenichelia Mahunka, 1985 "sin. nov.")

ESPECIE TIPO: Fenichelia biroi Balogh, 1970

DISTRIBUCIÓN: Tropical (Etiópica y Australiana).

-Fenichelia biroi Balogh, 1970

Distribución: Nueva Guinea.

-Fenichelia latipilosa Mahunka, 1982

DisTRIBUCIÓN: Etiopía.

-Fenichelia porosa (Mahunka, 1985) (Porofenichelia)

DisTRIBUCIÓN: Sudáfrica.

Mexiceremus J. y P. Balogh, 1998 (1 sp.)

EsPECIE TIPO: Micreremus rafaelinus Mahunka y Palacios-Vargas, 1995 DISTRIBUCIÓN: Neotropical.

-Mexiceremus rafaelinus (Mahunka y Palacios-Vargas, 1995)

(Micreremus)

DistribuCiÓN: Méjico

Micreremus Berlese, 1908 (8 spp.)

EsPECIE TIPO: Eremaeus brevipes Michael, 1888

DisTRIBUCión: Semicosmopolita (Paleártica y Paleotropical).

-Micreremus africanus Balogh, 1963

DistriBUCIÓN: Etiópica (Angola y Malgache: I. Reunión)

-Micreremus brevipes (Michael, 1888) (Eremaeus)

(=Micreremus gracilior Willmann, 1931) 
(=Eremaeus minimus Berlese, 1891)

(=Eremaeus minutipes Berlese, 1902)

Distribución: Paleártica (frecuente en Paleártica occidental).

-Micreremus faviger Mahunka, 1983

DisTRIBUCIÓN: Tanzania.

-Micreremus florens Mahunka, 1983

DisTRIBUCIÓN: Etiopía.

-Micreremus granulatus Balogh, 1970

DisTRIBUCión: Ceilán [Sri Lanka]

-Micreremus longisetus Mahunka, 1986

DisTRIBUCIÓN: Sudáfrica.

-Micreremus macrofissura Hammer, 1979

DisTRIBUCIÓN: Java.

-Micreremus subglaber Ito, 1982

DisTRIBUCIÓN: Japón.

Phylloribatula Balogh y Mahunka, 1978 (2 spp.)

ESPECIE TIPO: Phylloribatula pulchella Balogh y Mahunka, 1978

DisTRIBUCIÓN: Neotropical.

-Phylloribatula pulchella Balogh y Mahunka, 1978

DisTRIBUCión: Paraguay.

-Phylloribatula xanthoparmeliae Martínez y Palacios-Vargas, 1998

DisTRIBUCIÓN: Argentina.

Lamellareidae Balogh, 1972 (3 gen. y 9 spp.) DISTRIBUCIÓN: Cosmopolita.

Lamellarea Kok, 1968 (4 spp.)

ESPECIE TIPO: Lamellarea ardua Kok, 1968

DISTRIBUCIÓN: Etiópica.

-Lamellarea ardua Kok, 1968

DisTRIBUCIÓN: Sudáfrica.

-Lamellarea digitata Kok, 1968

DisTRIBUCión: Sudáfrica.

-Lamellarea forceps Kok, 1968

DisTRIBUCIÓN: Sudáfrica.

-Lamellarea quadrata Coetzee, 1987

DISTRIBUCIÓN: Sudáfrica.

Microlamellarea Coetzee, 1987 (1 sp.)

EsPECIE TIPO: Microlamellarea engelbrechti Coetzee, 1987

DISTRIBUCIÓN: Etiópica.

-Microlamellarea engelbrechti Coetzee, 1987

DisTRIBUCIÓN: Sudáfrica.

Tenuelamellarea Subías e Iturrondobeitia, 1978 (4 spp.)

ESPECIE TIPO: Tenuelamellarea hispanica Subías e Iturrondobeitia, 1978 DisTRIBUCIÓN: Cosmopolita.

-Tenuelamellarea argentinensis Martínez, Velis, Eguaras y Fernández, 1995

DisTriBuCión: Argentina.

-Tenuelamellarea hawaiiensis P. Balogh, 1985

DISTRIBUCIÓN: Hawai.

-Tenuelamellara hispanica Subías e Iturrondobeitia, 1978

DisTRIBUCión: España, Sudáfrica y subantártica (I. San Pablo y

Amsterdam).

-Tenuelamellarea taprobanae (Oudemans, 1915) (Carabodes)

DISTRIBUCIÓN: Ceilán [Sri Lanka].

Licneremaeidae Grandjean, 1931 (2 gen. y 18 spp.)

DisTRIBUCIÓN: Cosmopolita (excepto Antártica).

Huilicheremaeus Fernández, Marcangeli y Eguaras, 1997 (2 spp.) ESPECIE TIPO: Huilicheremaeus michaii Fernández, Marcangeli y Eguaras, 1997

DISTRIBUCIÓN: Neotropical.

-Huilicheremaeus atypicus (Mahunka, 1984) (Licneremaeus)

DisTRIBUCIÓN: Paraguay.

-Huilicheremaeus michaii Fernández, Marcangeli y Eguaras, 1997

DisTRIBUCIÓN: Argentina.
Licneremaeus Paoli, 1908 (16 spp.)

EsPeCIE TIPO: Notaspis licnophorus Michael, 1882

DISTRIBUCIÓN: Cosmopolita (excepto Antártica).

-Licneremaeus costulatus Mahunka, 1982

DisTRIBUCión: Etiopía.

-Licneremaeus cristatus Mahunka, 1984

DisTRIBUCIÓN: Tanzania.

-Licneremaeus cubanus Balogh y Mahunka, 1980

DistriBución: Cuba.

-Licneremaeus discoidalis Willmann, 1930

(=Licneremaeus antillensis Mahunka, 1985)

DisTRIBUCIÓN: Centroamérica.

-Licneremaeus embeyisztini Mahunka, 1980

DisTRIBUCIÓN: Mediterránea occidental.

-Licneremaeus exornatus Grandjean, 1931

DisTRIBUCIÓN: Venezuela.

-Licneremaeus giustii Bernini, 1973

DISTRIBUCIÓN: Italia (Sicilia).

-Licneremaeus licnophorus (Michael, 1882) (Notaspis)

DisTRIBUCIÓN: Holártica (Paleártica: frecuente, y U.S.A.: Carolina del Norte).

-Licneremaeus linieatus Hammer, 1979

DisTRIBUCIÓN: Java.

-Licneremaeus novaeguineae Balogh, 1968

Distribución: Pantropical (Oriental, Australiana: Nueva Guinea y Komodo, y Perú) y Japón.

-Licneremaeus novus Karppinen y Shtanchaeva, 1987

Distribución: Sureste de Europa.

-Licneremaeus polygonalis Hammer, 1971

DistriBución: Islas del Pacífico.

-Licneremaeus prodigiosus Schuster, 1958

DISTRIBUCIÓN: Europa centomeridional y Asia centrooccidental.

-Licneremaeus propinquus Balogh, 1958 "sp. inq."

DistRIBUCIÓN: Angola.

-Licneremaeus semiareolatus Mahunka, 1978

DistriBución: Malgache.

-Licneremaeus similis Pérez-Íñigo jr., 1990

DisTRIBUCIÓN: Mediterránea occidental.

Scutoverticidae Grandjean, 1954 (8 gen. y 57 spp.)

DISTRIBUCIÓN: Cosmopolita (excepto Antártica).

Arthrovertex Balogh, 1970 (8 spp.)

(=Argentinovertex Fernández y Cleva, 2001 "sin. nov.")

EsPECIE TIPO: Arthrovertex segmentatus Balogh, 1970

DISTRIBUCIÓN: Pantropical.

-Arthrovertex baloghi Mahunka, 1978

Distribución: Antillas (La Española: R. Dominicana).

-Arthrovertex bispinatus (Hammer, 1961) (Scutovertex)

DisTRIBución: Perú.

-Arthrovertex coineaui (Fernández y Cleva, 2001) (Argentinovertex)

DISTRIBUCIÓN: Argentina.

-Arthrovertex hauseri Mahunka, 1985

DistriBución: Pequeñas Antillas.

-Arthrovertex ilocanus Corpuz-Raros, 1979

DisTRIBUCIÓN: Filipinas.

-Arthrovertex leucaena Corpuz-Raros, 1991

DisTRIBUCIÓN: Filipinas.

-Arthrovertex segmentatus Balogh, 1970

Distribución: Nueva Guinea.

-Arthrovertex xena Mahunka, 1988

DistRIBUCIÓN: Malgache.

Ethiovertex Mahunka, 1982 (4 spp.)

EsPECIE TIPO: Ethiovertex macrosetosus Mahunka, 1982

DisTRIBUCIÓN: Etiópica y Paleártica meridional.

-Ethiovertex bidactylus (Mahunka, 1989) (Scutovertex)

DisTRIBUCIÓN: Kenia.

-Ethiovertex elisae Pérez-Íñigo y Peña, 1996

DistribuCIÓN: I. Canarias. 
-Ethiovertex macrosetosus Mahunka, 1982 DisTRIBUCIÓn: Etiopía.

-Ethiovertex sculperens (Kok, 1968) (Scutovertex) DISTRIBUCIÓN: Etiópica.

Exochocepheus Woolley y Higgins, 1968 (7 spp.)

(=Latovertex Mahunka, 1987 "sin. nov.")

ESPECIE TIPO: Exochocepheus eremitus Woolley y Higgins, 1968

DISTRIBUCIÓN: Holártica y Neotropical.

-Exochocepheus borealis (Rjabinin, 1984) (Hypovertex)

DisTRIBUCIÓN: Paleártica Oriental (excepto Oeste).

-Exochocepheus contiguus (Balogh y Csiszár, 1963) (Scutovertex) DISTRIBUCIÓN: Argentina.

-Exochocepheus eremitus Woolley y Higgins, 1968 DisTRIBUCIÓN: Neártica occidental.

-Exochocepheus hungaricus (Mahunka, 1987) (Latovertex) DisTRIBUCIÓN: Hungría.

-Exochocepheus laticuspis (Balogh y Mahunka, 1965) (Scutovertex) Distribución: Paleártica meridional (Sureste de Rusia y Sur de Paleártica Oriental).

-Exochocepheus stenolamellatus (Golosova, 1984) (Hypovertex) DisTRIBUCión: Mongolia.

-Exochocepheus tuberculatus (Golosova, 1984) (Hypovertex) DisTRIBUCIÓN: Mongolia.

Hypovertex Krivolutsky, 1969 (6 spp.)

ESPECIE TIPO: Hypovertex mirabilis Krivolutsky, 1969

DisTRIBUCIÓN: Paleártica meridional, Etiópica y Neotropical.

-Hypovertex africanus (Evans, 1953) (Scutovertex) DisTRIBUCIÓN: Tanzania.

-Hypovertex arcualis Hammer, 1977 DisTRIBUCIÓN: Himalaya.

-Hypovertex lenticulatus Kahwash, Ruiz y Subías, 1990 DISTRIBUCIÓN: España.

-Hypovertex mirabilis Krivolutsky, 1969

DistriBuCión: Paleártica meridional (Cáucaso y Asia centrooccidental).

-Hypovertex saxicola Sitnikova, 1975

DisTRIBUCión: Paleártica meridional (Cáucaso y Sur de Paleártica oriental).

-Hypovertex transversalis (Balogh y Csiszár, 1963) (Scutovertex) DISTRIBUCIÓN: Argentina.

Lamellovertex Bernini, 1976 (1 sp.)

EsPeCIE TIPO: Scutovertex caelatus Berlese, 1895

DisTRIBUCIÓN: Paleártica.

-Lamellovertex caelatus (Berlese, 1895) (Scutovertex)

DisTRIBUCIÓN: Europa meridional.

Provertex Mihelcic, 1959 (5 spp.)

EsPeCIE TIPO: Provertex kuehnelti Mihelcic, 1959

DISTRIBUCIÓN: Paleártica.

-Provertex delamarei Travé, 1963

DisTRIBUCIÓN: Europa suroccidental.

-Provertex forsslundi Krivolutsky, 1969

DisTRIBUCIÓN: Asia centrooccidental.

-Provertex kuehnelti Mihelcic, 1959

DISTRIBUCIÓN: Europa centromeridional.

-Provertex mailloli Travé, 1965

DisTRIBUCIÓN: Francia.

-Provertex nepalensis Piffl, 1971

DisTRIBUCIÓN: Nepal.

Scutovertex Michael, 1879 (24 spp.)

(=Neoscutovertex Mihelcic, 1957)

EsPeCIE TIPO: Scutovertex sculptus Michael, 1879

DisTRIBUCIÓN: Cosmopolita (excepto Antártica).

-Scutovertex alpinus Willmann, 1953

DISTRIBUCIÓN: Austria.

-Scutovertex bulgaricus Kunst, 1961

DisTRIBUCIÓN: Bulgaria.
-Scutovertex clypeatus (Nicolet, 1855) (Tegeocranus) "sp. inq."

DisTRIBUCIÓN: Francia.

-Scutovertex evansi Mahunka, 1984

DISTRIBUCIÓN: Tanzania.

-Scutovertex fossatus Wallwork, 1967

DisTRIBUCIÓN: Chad.

-Scutovertex glaber Mihelcic, 1957 (S. (Neoscutovertex))

DistRIBUCIÓN: Sur de Europa.

-Scutovertex glandulosus Balogh y Mahunka, 1965

DistRIBUCIÓN: Paleártica (Rusia europea y Mongolia).

-Scutovertex inlenticulatus Sitnikova, 1975

DisTRIBUCIÓN: Cáucaso y Asia centrooccidental.

-Scutovertex jindianensis Wen, 1985

DisTRIBUCIÓN: China suroriental.

-Scutovertex laminipes Hammer, 1961

DisTRIBUCIÓN: Neotropical (Perú y Argentina).

-Scutovertex marginatus Mahunka, 1983

DISTRIBUCIÓN: Tanzania.

-Scutovertex minutus (Koch, 1836) (Cepheus)

(=Eremaeus ovalis siculus Berlese, 1887)

DistRIBUCIÓN: Paleártica (frecuente).

-Scutovertex neonominatus nom. nov.

[=Scutovertex perforatus Sitnikova, 1975, "nom. praeoc." por Berlese, 1910 ("hom. prim.")]

DisTRIBUCIÓN: Europa oriental.

-Scutovertex niger Moskacheva, 1964

DistriBuCión: Bielorrusia.

-Scutovertex ornatus Bulanova-Zachvatkina, 1964

Distribución: Rusia (Este de Europa y Asia central).

-Scutovertex pannonicus Schuster, 1958

DisTRIBUCIÓN: Paleártica meridional (Europa centromeridional y Sur de Paleártica Oriental).

-Scutovertex perforatulus Mihelcic, 1958

DisTRIBUCIÓN: Sur de Europa (excepto Este).

-Scutovertex pictus Kunst, 1959

DistriBuCIÓN: Mediterránea.

-Scutovertex pilosetosus Polderman, 1977

DistRIBUCIÓN: Holanda.

-Scutovertex punctatus Sitnikova, 1975

DisTRIBUCIÓN: Paleártica meridional (Sur de Europa y Asia centrooccidental)

-Scutovertex sculptus Michael, 1879

(=Scutovertex (Neoscutovertex) granulatus Mihelcic, 1957)

(?=Eremaeus ovalis Koch, 1841 "sp. inq.")

(=Scutovertex (Neoscutovertex) rugosus Mihelcic, 1957)

(=Scutovertex steineri Mihelcic, 1956)

Distribución: Paleártica (menos frecuente en el Norte) y Nueva Zelanda.

-Scutovertex serratus Sitnikova, 1975

Distribución: Paleártica (Europa oriental y Sur de Paleártica Oriental).

-Scutovertex spinipes Berlese, 1916 "sp. inq."

DISTRIBUCIÓN: Somalia.

-Scutovertex subspinipes Balogh, 1959

DisTRIBUCIÓN: Tanzania.

Scutoverticosus Kok, 1968 (2 spp.)

EsPECIE TIPO: Scutoverticosus arcanus Kok, 1968

DISTRIBUCIÓN: Etiópica y Paleártica meridional.

-Scutoverticosus arcanus Kok, 1968

DisTRIBUCIÓN: Sudáfrica.

-Scutoverticosus insperatus Pérez-Íñigo y Peña, 1996

DISTRIBUCIÓN: I. Canarias.

Passalozetidae Grandjean, 1954 (2 gen., 2 subg., 45 spp. y 2 sspp.) DISTRIBUCIÓN: Semicosmopolita (Holártica, Etiópica y Neotropical).

Bipassalozetes Mihelcic, 1957 (24 spp.)

EsPeCIE TIPO: Passalozetes (Bipassalozetes) permixtus Mihelcic, 1957

DISTRIBUCIÓN: Holártica y Etiópica. 
Bipassalozetes (Bipassalozetes) Mihelcic, 1957 (21 spp.)

(=Salpasozetes Mahunka, 1977)

DisTRIBUCióN: Holártica y Etiópica.

-Bipassalozetes (B.) berndhauseri (Mahunka, 1997) (Passalozetes (B.)) DisTRIBUCIÓN: Madagascar.

-Bipassalozetes (B.) bidactylus (Coggi, 1900) (Scutovertex) DistRIBUCión: Paleártica (Paleártica occidental: menos frecuente en el Norte, y Asia centrooccidental) y Etiópica (Kenia).

-Bipassalozetes (B.) deserticus Bayartogtokh y Aoki, 1997 Distribución: Mongolia y Yemen.

-Bipassalozetes (B.) elegans (Sitnikova, 1975) (Passalozetes) Distribución: Sur de Paleártica oriental.

-Bipassalozetes (B.) gobiensis (Mahunka, 1964) (Passalozetes) DisTRIBUCIÓN: Sur de Paleártica oriental.

-Bipassalozetes (B.) intermedius (Mihelcic, 1954) (Passalozetes) (=Passalozetes variatepictus Mihelcic, 1956)

DisTRIBUCión: Paleártica (excepto Norte)

-Bipassalozetes (B.) lineolatus (Sitnikova, 1975) (Passalozetes) DistriBución: Asia centrooccidental.

-Bipassalozetes (B.) mahunkai Bayartogtokh y Aoki, 1997 Distribución: Mongolia.

-Bipassalozetes (B.) microsculptratus Bayartogtokh y Aoki, 1997 DisTRIBUCión: Mongolia.

-Bipassalozetes (B.) mongolicus Bayartogtokh y Aoki, 1997 DistriBución: Mongolia.

-Bipassalozetes (B.) moniles (Higgins y Woolley, 1975) (Passalozetes) Distribución: U.S.A. (Colorado)

-Bipassalozetes (B.) nitidus (Bayoumi y Al-Khalifa, 1984) (Passalozetes)

DisTRIBUCIÓN: Arabia Saudita.

-Bipassalozetes (B.) pectinatus (Wallwork, 1964) (Passalozetes) DisTRIBUCIÓN: Chad.

-Bipassalozetes (B.) perforatus (Berlese, 1910) (Scutovertex) (=Passalozetes granulatus Mihelcic, 1955)

(=Passalozetes propinquus Mihelcic, 1956)

DisTRIBUCIÓN: Paleártica centromeridional.

-Bipassalozetes (B.) permixtus (Mihelcic, 1957) (Passalozetes (B.)) DISTRIBUCIÓN: Austria.

-Bipassalozetes (B.) reticulatus (Mihelcic, 1957) (Passalozetes) Distribución: Mediterránea.

-Bipassalozetes (B.) rugosus (Sitnikova, 1975) (Passalozetes)

DisTRIBUCIÓN: Paleártica meridional (Sur de Rusia europea y Asia centrooccidental).

-Bipassalozetes (B.) striatus (Mihelcic, 1955) (Passalozetes)

(=Passalozetes kaszabi Mahunka, 1964)

DistRIBUCIÓN: Paleártica meridional (Suroeste de Europa y Mongolia).

-Bipassalozetes (B.) tibialis (Nicolet, 1855) (Eremaeus) "sp. inq." DisTrIBUCIÓN: Francia.

-Bipassalozetes (B.) vicinus (Mihelcic, 1957) (Passalozetes) DistRIBUCIÓN: Paleártica centromeridional (Europa centromeridional y Asia centrooccidental).

-Bipassalozetes (B.) wolwekransensis (Engelbrecht, 1974) (Passalozetes)

DisTRIBUCIÓN: Sudáfrica.

Bipassalozetes (Passalobates) Pérez-Iñigo y Peña, 1996 (3 spp.)

EsPeCie TIPO: Passalobates asper Pérez-Íñigo y Peña, 1996

DisTRIBUCiÓN: Holártica.

-Bipassalozetes (Passalobates) asper (Pérez-Íñigo y Peña, 1996) $(P$.) DisTRIBUCIÓN: I. Canarias.

-Bipassalozetes (Passalobates) linearis (Higgins y Woolley, 1962) (Passalozetes)

DISTRIBUCIÓN: Holártica meridional (U.S.A.: Utah, y Mediterránea). -Bipassalozete (Passalobates) sabulosus (Shtanchaeva, 1986) (Passalozetes)

DisTRIBUCIÓN: Cáucaso.

Passalozetes Grandjean, 1932 (21 spp. y 2 sspp.)

EsPECIE TIPO: Passalozetes africanus Grandjean, 1932

DistriBuCión: Semicosmopolita (Holártica, Etiópica y Neotropical).
Passalozetes (Passalozetes) Grandjean, 1932 (20 spp. y 2 sspp.)

DisTRIBUCIÓN: Semicosmopolita (Holártica, Etiópica y Neotropical).

-Passalozetes (P.) africanus Grandjean, 1932

DisTRIBUCIÓN: Paleártica centromeridional (frecuente) y Guinea

Ecuatorial.

-Passalozetes (P.) agricola Mínguez y Subías, 1983

DisTRIBUCióN: España.

-Passalozetes (P.) californicus Wallwork, 1972

Distribución: Sur de U.S.A. y Méjico.

-Passalozetes (P.) californicus californicus Wallwork, 1972 Distribución: Sur de U.S.A. y Méjico.

-Passalozetes (P.) californicus orientalis Wallwork, Weems y Kamill, 1984

DistribuCión: U.S.A. (Nuevo Méjico)

-Passalozetes (P.) douglasensis Engelbrecht, 1974

Distribución: Sudáfrica.

Passalozetes (P.) hauseri Mahunka, 1977

Distribución: Sureste de Europa.

-Passalozetes (P.) hispanicus Mihelcic, 1955

Distribución: Sur de Europa.

-Passalozetes (P.) imperfectus Pérez-Íñigo jr., 1991

DisTRIBUCIÓN: España.

-Passalozetes (P.) inlenticulatus Mihelcic, 1959

DistribuCIÓN: Mediterráneo occidental

-Passalozetes (P.) karppineni Mikhaltzova, 1982

DisTRIBUCIÓN: Norte de Europa.

-Passalozetes (P.) lienhardi Mahunka, 1997

Distribución: Madagascar.

-Passalozetes (P.) macedonicus Tarman, 1962

DisTRIBUCión: Macedonia.

-Passalozetes (P.) neomexicanus Wallwork, Weems y Kamill, 1984

Distribución: U.S.A. (Nuevo Méjico) y Méjico.

-Passalozetes (P) neomexicanus neomexicanus Wallwork, Weems

y Kamill, 1984

Distribución: U.S.A. (Nuevo Méjico) y Méjico.

-Passalozetes (P.) neomexicanus neonominatus nom. nov. [=Passalozetes neomexicanus granulatus Wallwork, Weems y Kamill, 1984, "nom. praeoc." por Mihelcic, 1955 ("hom. prim.")]

Distribución: U.S.A. (Nuevo Méjico).

-Passalozetes (P.) nesebarensis Vanek, 1966

Distribución: Bulgaria.

-Passalozetes (P.) niger Mahunka, 1987

DistribuCión: Nigeria.

-Passalozetes (P.) onubensis Subías, Ruiz y Kahwash, 1990

DisTRIBUCIÓN: España.

-Passalozetes (P.) paucesculptus Bernini, 1973

DisTRIBUCIÓN: Italia (Sicilia).

-Passalozetes (P.) prominens Balogh y Mahunka, 1968

DistRIBUCIÓN: Argentina

-Passalozetes (P.) ruderalis Mínguez y Subías, 1983

DistRIBUCIÓN: España.

-Passalozetes (P.) scholzi Pérez-Íñigo y Peña, 1996

DisTRIBUCIÓN: I. Canarias.

-Passalozetes (P.) stellifer Pérez-Íñigo y Peña, 1997

DisTRIBUCIÓN: Mediterránea occidental.

Passalozetes (Passalomonia) Mahunka, 1987 (1 sp.)

EsPECIE TIPO: Passalozetes (Passalomonia) demeteri Mahunka, 1987 DisTRIBUCIÓN: Etiópica.

-Passalozetes (Passalomonia) demeteri Mahunka, 1987

DisTRIBUCIÓN: Nigeria.

Phenopelopoidea Petrunkevitch, 1955

Phenopelopidae Petrunkevitch, 1955 (4 gen., 96 spp. y 6 sspp.) DisTRIBUCIÓN: Cosmopolita (excepto Antártica).

Eupelops Ewing, 1917 (64 spp. y 6 sspp.)

(=Allopelops Hammer, 1952)

(=Celaeno Koch, 1835, "nom. praeoc." por Leach, 1821)

(=Globonothrus Tseng, 1982 "sin. nov.") 
(=Pelops Koch, 1836, “nom. praeoc.” por Gistl, 1834)

(=Phenopelops Petrunkevitch, 1955)

(=Tectopelops Jacot, 1929)

ESPECIE TIPO: (Pelops hirsutus Koch, 1844) =Notaspis acromios Hermann, 1804

DisTRIBUCIÓn: Cosmopolita (excepto Antártica)

-Eupelops absalom (Berlese, 1916) (Pelops) "sp. inq."

DISTRIBUCIÓN: "África oriental".

-Eupelops acromios (Hermann, 1804) (Notaspis)

DistRIBUCión: Paleártica, Norte de Oriental y Sudáfrica.

-Eupelops acromios acromios (Hermann, 1804)

(=Pelops hirsutus Koch, 1844)

(=Pelops kochi Jacot, 1929

(=Pelops phytophilus Berlese, 1916)

(=Celaeno spinosa Koch, 1835)

DistribuCión: Paleártica (frecuente), Norte de Oriental y Sudáfrica.

-Eupelops acromios minor Chakrabarti, Bhaduri y Raychaudhuri, 1973

DistRIBUCIÓN: India (Bengala Occidental).

-Eupelops apicalis (Hammer, 1962) (Pelops)

DISTRIBUCIÓN: Argentina.

-Eupelops berlesei (Oudemans, 1929) (Peloptulus) "sp. inq."

DISTRIBUCIÓN: Italia.

-Eupelops bilobus (Sellnick, 1928) (Pelops)

DistRIBUCIÓN: Paleártica (excepto Este) y Groenlandia.

-Eupelops brevicuspis (Knülle, 1954) (Pelops)

DisTRIBUCIÓN: Alemania.

-Eupelops brevipilus (Jacot, 1937) (Pelops)

DistriBuCión: U.S.A. (Carolina del Norte).

-Eupelops capensis Grobler, 1989

DisTRIBUCIÓN: Sudáfrica.

-Eupelops caucasicus Sitnikova, 1975

DisTRIBUCIÓn: Sureste de Europa.

-Eupelops claviger (Berlese, 1916) (Pelops)

Distribución: Paleártica (Paleártica occidental y Este de

Paleártica oriental).

-Eupelops claviger claviger (Berlese, 1916)

DistriBución: Paleártica (Europa y Este de Paleártica oriental).

-Eupelops claviger fuerteventurae Pérez-Íñigo y Peña, 1996

DisTRIBUCión: I. Canarias.

-Eupelops contaminatus Choi, 1986

DisTRIBUCIÓN: Corea.

-Eupelops curtipilus (Berlese, 1916) (Pelops)

DISTRIBUCIÓN: Europa centromeridional y Sudáfrica.

-Eupelops curtipilus curtipilus (Berlese, 1916)

(=Pelops depilatus Berlese, 1916)

DisTRIBUCIÓN: Europa centromeridional.

-Eupelops curtipilus thabazimbiensis Engelbrecht, 1975 DisTRIBUCIÓN: Sudáfrica.

-Eupelops differens (Mihelcic, 1953) (Pelops)

DISTRIBUCIÓN: Austria.

-Eupelops distinctus Grobler, 1989

DisTRIBUCIÓN: Sudáfrica.

-Eupelops ecuadoriensis P. Balogh, 1988

DISTRIBUCIÓN: Ecuador.

-Eupelops engelbrechti Grobler, 1989

DISTRIBUCIÓN: Sudáfrica.

-Eupelops erinaceus Balogh y Mahunka, 1979

DisTRIBUCIÓN: Norte de Neotropical.

-Eupelops eximius Sitnikova, 1967

DistriBUCIÓN: Paleártica oriental.

-Eupelops eximius eximius Sitnikova, 1967

DISTRIBUCIÓN: Paleártica oriental.

-Eupelops eximius dicornus Sitnikova, 1967

DistRIBUCIÓN: Este de Rusia asiática.

-Eupelops farinosus (Nicolet, 1855) (Pelops)

DISTRIBUCIÓN: Europa septentrional.

-Eupelops forsslundi (Balogh, 1959) (Pelops)

DisTRIBUCIÓn: Etiópica (Tanzania y Sudáfrica) y Vietnam.
-Eupelops foveolatus Engelbrecht, 1975

DisTRIBUCIÓN: Sudáfrica.

-Eupelops geminus (Berlese, 1916) (Pelops)

Distribución: Paleártica (Paleártica occidental y Este de Rusia asiática).

-Eupelops gibbus (Mihelcic, 1957) (Pelops)

DisTRIBUCIÓN: España.

-Eupelops grandis (Mihelcic, 1958) (Pelops)

DISTRIBUCIÓN: Austria.

-Eupelops halophilus Pérez-Íñigo, 1969

Distribución: Sur de Europa.

-Eupelops heegi (Balogh, 1958) (Pelops) "sp. inq."

DistriBución: Congo.

-Eupelops hygrophilus (Knülle, 1954) (Pelops)

DISTRIBUCIÓN: Europa central.

-Eupelops incompletus Mahunka, 1978

Distribución: Malgache (I. Mauricio).

-Eupelops japonensis Fujikawa, 1990

DisTRIBUCIÓN: Japón.

-Eupelops kivuensis (Balogh, 1958) (Pelops) "sp. inq."

DistriBución: Congo.

-Eupelops latipilosus (Ewing, 1909) (Pelops)

Distribución: Holártica (U.S.A.: Ilinois, Cáucaso y Asia centrooccidental).

-Eupelops limbulatus (Tseng, 1982) (Globonothrus) “sp. inq."

DisTRIBUCIÓN: Taiwan [Formosa].

-Eupelops longisetosus Chakrabarti y Mondal, 1981

DistriBución: India (Bengala Occidental).

-Eupelops major (Hull, 1914) (Pelops)

DisTRIBUCIÓN: Holártica (Europa centromeridional, Pakistán y

Neártica septentrional)

-Eupelops major major (Hull, 1914)

$(=$ Pelops hirtus Berlese, 1916)

(=Pelops perarmatus Hull, 1916)

=Pelops simplex Berlese, 1916)

DISTRIBUCIÓN: Holártica (Europa centromeridional: frecuente, Pakistán y Canadá).

-Eupelops major franconia (Jacot, 1939) (Pelops simplex $f$.)

DisTRIBUCión: U.S.A. (Nueva Hampshire).

-Eupelops margatensis Engelbrecht, 1975

DISTRIBUCIÓN: Sudáfrica.

-Eupelops meridionalis Pérez-Íñigo, 1969 (E. torulosus m.)

DisTRIBUCIÓN: Mediterránea occidental.

-Eupelops minutus Grobler, 1989

DisTRIBUCIÓN: Sudáfrica.

-Eupelops mongolicus Bayartogtokh, 1999

DISTRIBUCIÓN: Mongolia.

-Eupelops nepotulus (Berlese, 1916) (Pelops)

(=Pelops pulchellus Berlese, 1916)

DistRIBUCIÓN: Paleártica centromeridional (excepto Este).

-Eupelops occultus (Koch, 1835) (Pelops)

DisTRIBUCIÓN: Paleártica (excepto Este): frecuente.

-Eupelops oudemansi (Hammen, 1952) (Pelops)

DisTRIBUCión: Holanda.

-Eupelops parvus (Mihelcic, 1953) (Pelops)

DisTRIBUCIÓN: Austria.

-Eupelops planicornis (Schrank, 1803) (Acarus) “sp. inq."

DisTRIBUCIÓN: Alemania.

-Eupelops plicatus (Koch, 1835) (Celaeno)

(=Pelops acromios diversipilus Mihelcic, 1957)

(=Pelops affinis Berlese, 1916)

(=Pelops approximatus Hull, 1916)

(=Pelops auritus Koch, 1839)

(=Pelops diversus Mihelcic, 1957)

(=Pelops fuligineus Koch, 1839)

(=Pelops fusiger Mihelcic, 1957)

(=Pelops laevigatus Nicolet, 1855)

(=Pelops siculus Berlese, 1916)

DistriBuCión: Holártica (Paleártica: frecuente, y U.S.A.: Alaska y Virginia). 
-Eupelops pocsi Mahunka, 1983

DisTRIBUCIÓN: Tanzania.

-Eupelops rapaensis (Sellnick, 1959) (Phenopelops)

DISTRIBUCIÓN: Polinesia: I. Australes [I. Tubuai]

-Eupelops reticulatus (Willmann, 1939) (Pelops)

DistRIBUCIÓN: Macaronesica (Madeira).

-Eupelops septentrionalis (Trägardh, 1910) (Pelops)

DisTRIBUCIÓN: Boreal (Norte de Europa y Neártica septentrional).

-Eupelops shagdarsureni Bayartogtokh, 1999

DISTRIBUCIÓN: Mongolia.

-Eupelops silvestris (Jacot, 1937) (Pelops)

DisTRIBUCIÓN: U.S.A. (Carolina del Norte).

-Eupelops somalicus (Berlese, 1916) (Pelops curtipilus s.)

DisTRIBUCión: Somalia.

-Eupelops spongiosus Mahunka, 1998

DisTRIBUCión: Pequeñas Antillas.

-Eupelops strenzkei (Knülle, 1954) (Pelops)

DisTRIBUCIÓN: Alemania.

-Eupelops subexutus (Berlese, 1916) (Pelops)

(?=Pelops glaber G. y R. Canestrini, 1882 "sp. inq.")

DisTRIBUCIÓN: Europa centromeridional.

-Eupelops subulatus (Berlese, 1916) (Pelops) "sp. inq."

DisTRIBUCIÓN: Noruega.

-Eupelops subuliger (Berlese, 1916) (Pelops)

(=Pelops longifissus Willmann, 1951)

DisTRIBUCIÓN: Europa centromeridional.

-Eupelops suramericanus (Hammer, 1961) (Pelops)

DisTRIBUCIÓN: Neotropical y U.S.A. (Carolina del Norte).

-Eupelops tahitiensis Hammer, 1972

DisTRIBUCIÓN: Polinesia.

-Eupelops tardus (Koch, 1835) (Pelops)

DisTRIBUCIÓN: Paleártica (excepto Este)

-Eupelops terminalis (Banks, 1909) (Pelops)

Distribución: Neártica (U.S.A.: Carolina del Norte, y Canadá).

-Eupelops tibialis (Banks, 1910) (Pelops)

Distribución: U.S.A. (Virginia).

-Eupelops torulosus (Koch, 1839) (Pelops)

DistriBución: Paleártica (excepto Este).

-Eupelops torulosus torulosus (Koch, 1839)

(=Pelops duplex Berlese, 1916)

(=Pelops variolosus Nicolet, 1855)

Distribución: Paleártica (excepto Este): frecuente.

-Eupelops torulosus macroporosus Pérez-Íñigo jr., 1991 DisTRIBUCIÓN: España.

-Eupelops ureaceus (Koch, 1839) (Pelops)

(=Pelops similis Berlese, 1916)

Distribución: Paleártica (Europa y Asia centrooccidental) y Groenlandia.

-Eupelops variatus (Mihelcic, 1957) (Pelops)

DisTRIBUCión: Sur de Europa.

Nesopelops Hammer, 1973 (11 spp.)

EsPECIE TIPO: Nesopelops caudatus Hammer, 1973

DisTRIBUCIÓN: Australianooriental.

-Nesopelops caudatus Hammer, 1973

Distribución: Polinesia (I. Tonga).

-Nesopelops crassus (Hammer, 1972) (Eupelops)

DisTRIBUCIÓN: Polinesia (Tahití).

-Nesopelops higginsi (Balogh, 1970) (Eupelops)

DisTRIBUCIÓN: Nueva Guinea.

-Nesopelops intermedius Hammer, 1979

DisTRIBUCiÓN: Java.

-Nesopelops laensis (Balogh, 1970) (Eupelops)

DisTRIBUCIÓN: Nueva Guinea.

-Nesopelops monodactylus (Hammer, 1966) (Pelops) DisTRIBUCIÓN: Nueva Zelanda.

-Nesopelops polynesius (Hammer, 1972) (Eupelops)

DisTribución: Polinesia (Tahití).

-Nesopelops punctatus Hammer, 1966

DisTRIBUCIÓN: Nueva Zelanda.
-Nesopelops samoaensis Hammer, 1973

Distribución: Polinesia (I. Samoa).

-Nesopelops tongatapuensis Hammer, 1973

DISTRIBUCIÓN: Polinesia (I. Tonga).

-Nesopelops transversus Balogh, 1970

DistriBUCIÓN: Nueva Guinea.

Peloptulus Berlese, 1908 (13 spp.)

EsPeCIE TIPO: Pelops phaeonotus Koch, 1844

DisTRIBUCIÓN: Holártica y Neotropical.

-Peloptulus americanus (Ewing, 1907) (Pelops)

DisTRIBUCIÓN: Holártica (U.S.A. oriental y Este de Paleártica oriental).

-Peloptulus borgesi C. y C., jr. Pérez-Íñigo, 1996

DisTRIBUCión: I. Azores.

-Peloptulus denticuspidatus Bayartogtokh, 1999

DisTRIBUCIÓN: Mongolia.

-Peloptulus foveolatus Hammer, 1961

DisTRIBUCIÓN: Neotropical (Perú y Ecuador).

-Peloptulus gibbus Mihelcic, 1957

DisTRIBUCIÓN: Paleártica meridional (Mediterránea y Sur de Siberia).

-Peloptulus laticuspidatus (Ewing, 1909) (Pelops)

DisTRIBUCIÓN: U.S.A. (Ilinois).

-Peloptulus latilamellatus Bayartogtokh y Aoki, 1997

DisTRIBUCión: Mongolia.

-Peloptulus latirostris Pérez-Íñigo, 1969

DisTRIBUCión: España.

-Peloptulus montanus Hull, 1914

DisTRIBUCIÓN: Paleártica (Europa y Asia centrooccidental).

-Peloptulus phaeonotus (Koch, 1844) (Pelops)

Distribución: Paleártica (frecuente).

-Peloptulus reticulatus Mihelcic, 1957

Distribución: Paleártica occidental (excepto Norte).

-Peloptulus tanytrichosus Higgins y Woolley, 1972

DistribuCión: U.S.A. (Colorado).

-Peloptulus trinacriae Arcidiacono, 1975

DisTRIBUCIÓN: Suroeste de Europa.

Propelops Jacot, 1937 (8 spp.)

(=Hammeria Sellnick, 1944)

EsPecie TIPO: Propelops pinicus Jacot, 1937

DisTRIBUCIÓN: Holártica.

-Propelops alaskensis (Hammer, 1955) (Hammeria)

Distribución: Alaska.

-Propelops canadensis (Hammer, 1952) (Hammeria)

DisTRIBUCIÓN: Holártica (Neártica y Paleártica oriental).

-Propelops groenlandicus (Sellnick, 1944) (Hammeria)

Distribución: Boreal (Neártica septentrional y Este de Rusia asiática).

-Propelops minnesotensis (Ewing, 1913) (Pelops)

DISTRIBUCIÓN: Norte de U.S.A.

-Propelops monticolus (Ewing, 1918) (Eupelops)

(=Eupelops monticolus subborealis Ewing, 1918)

DistRIBUCIÓN: U.S.A. (Oregón).

-Propelops pacificus Krivolutsky, 1971

DistrIBUCIÓN: I Sajalín.

-Propelops pinicus Jacot, 1937

DISTRIBUCIÓN: Neártica.

-Propelops rossicus (Shaldybina, 1971) (Hammeria)

DisTRIBUCIÓN: Este de Paleártica oriental.

Unduloribatoidea Kunst, 1971

Unduloribatidae Kunst, 1971 (2 gen. y 5 spp.) DisTRIBUCIÓN: Paleártica meridional.

Koreoribates Choi, 1994 (1 sp.)

EsPECIE TIPO: Koreoribates foliatus Choi, 1994

DISTRIBUCIÓN: Paleártica. 
-Koreoribates foliatus Choi, 1994

DisTRIBUCIÓN: Corea.

Unduloribates Balogh, 1943 (4 spp.)

EsPeCIE TIPO: Sphaerozetes (Tectoribates) undulatus Berlese, 1914

DISTRIBUCIÓN: Paleártica meridional.

-Unduloribates brevisetosus Nübel-Reidelbach y Woas, 1992 DisTRIBUCIÓN: Nepal.

-Unduloribates hebes Aoki, 1965

DISTRIBUCIÓN: Himalaya y Asia centrooccidental.

-Unduloribates medusa Piffl, 1972

DisTRIBUCIÓN: Nepal.

-Unduloribates undulatus (Berlese, 1914) (Sphaerozetes (Tectoribates)) DisTRIBUCIÓN: Paleártica (Europa central e Himalaya).

\section{Eremaeozetidae Piffl, 1972 (3 gen. y 33 spp.)} DisTRIBUCIÓN: Pantropical y subtropical.

Eremaeozetes Berlese, 1913 (29 spp.)

EsPECIE TIPO: Eremaeozetes tuberculatus Berlese, 1913

DisTRIBUCIÓN: Pantropical y subtropical.

-Eremaeozetes acutus Covarrubias, 1967

Distribución: Chile.

-Eremaeozetes araucana Monetti, Oppedisano y Fernándes, 1994 DisTRIBUCIÓN: Argentina.

-Eremaeozetes arboreus Nübel-Reidelbach y Woas, 1992

DISTRIBUCIÓN: Brasil.

-Eremaeozetes bilunatifer Balogh y Mahunka, 1981

DISTRIBUCIÓN: Paraguay.

-Eremaeozetes capensis Schatz, 2001

DistriBución: Sudáfrica.

-Eremaeozetes chancanii Fernández y Cleva, 2000

DISTRIBUCIÓN: Argentina.

-Eremaeozetes costulatus Mahunka, 1977

DisTRIBUCIÓN: Oriental (Bali y Filipinas).

-Eremaeozetes darwini Schatz, 2000

DisTRIBUCIÓN: I. Galápagos.

-Eremaeozetes dividipeltatus Mahunka, 1985

DistriBución: Pequeñas Antillas.

-Eremaeozetes ephippiger Balogh, 1968

DisTRIBUCIÓN: Nueva Guinea y Filipinas.

-Eremaeozetes hanswursti Mahunka, 1999

DisTriBución: Península Malaya.

-Eremaeozetes himalayensis Sanyal, 1992

DisTRIBUCIÓN: India (Himalaya).

-Eremaeozetes irenae Schatz, 2000

DISTRIBUCIÓN: I. Galápagos.

-Eremaeozetes kurozumii Aoki, 1994

DisTRIBUCIÓN: Micronesia (I. Marianas).

-Eremaeozetes lineatus Mahunka, 1985

DisTRIBUCIÓN: Norte de Neotropical e India (Himalaya).

-Eremaeozetes machadoi Mahunka, 1989

DisTRIBUCIÓN: Etiópica (Angola y Sudáfrica).

-Eremaeozetes maculosus Mahunka, 1995

DisTRIBUCIÓN: Borneo.

-Eremaeozetes octomaculatus Hammer, 1973

DisTRIBUCIÓn: Polinesia (Samoa), Filipinas y Japón.

-Eremaeozetes reticulatus Balogh, 1958

Distribución: Etiópica: Angola y Guinea Ecuatorial: I. Pagalu [Annobón].

-Eremaeozetes roguini Mahunka, 1998

Distribución: Pequeñas Antillas.

-Eremaeozetes sabinae Schatz, 2000

DISTRIBUCIÓN: Hawai.

-Eremaeozetes spathulatus Balogh, 1968

DistriBución: Nueva Guinea y Filipinas.

-Eremaeozetes trifurcus Wen, 1994

Distribución: "China".

-Eremaeozetes tsavoensis Mahunka, 1987

DISTRIBUCIÓN: Kenia.
-Eremaeozetes tuberculatus Berlese, 1913

Distribución: Java, Polinesia (Tahití) y Méjico.

-Eremaeozetes undulatus Mahunka, 1985

DistriBUCión: Pequeñas Antillas.

-Eremaeozetes ursulae Mahunka, 1985

Distribución: Pequeñas Antillas.

-Eremaeozetes verai Pérez-Íñigo y Sarasola, 1995

DistriBUCIÓN: Uruguay.

-Eremaeozetes woelkei Piffl, 1972

DistriBución: Brasil.

Mahunkaia Schatz, 2002 (3 spp.)

ESPECIE TIPO: Eremaeozetes bituberculatus Mahunka, 1983

DISTRIBUCIÓN: Etiópica.

-Mahunkaia bituberculata (Mahunka, 1983) (Eremaeozetes)

DistriBuCión: Tanzania.

-Mahunkaia gracilis (Mahunka, 1985) (Eremaeozetes)

DisTRIBUCIÓN: Sudáfrica.

-Mahunkaia tricornis Schatz, 2002

DisTRIBUCIÓN: Sudáfrica.

Seteremaeozetes P. Balogh, 1988 (1 sp.)

EsPeCIE TIPO: Eremaeozetes (Seteremaeozetes) obtectus P. Balogh, 1988 DISTRIBUCIÓN: Oriental.

-Seteremaeozetes obtectus (P. Balogh, 1988) (Eremaeozetes (S.))

DistriBuCIÓN: Ceilán [Sri Lanka].

Idiozetidae Aoki, 1976 (1 gen. y 2 spp.)

DisTRIBUCIÓN: Oriental y Paleártica.

Idiozetes Aoki, 1976 (2 spp.)

ESPECIE TIPO: Idiozetes erectus Aoki, 1976

DisTRIBUCIÓN: Oriental y Paleártica.

-Idiozetes erectus Aoki, 1976

DisTRIBUCión: Japón y Península Malaya.

-Idiozetes javensis Hammer, 1979

DISTRIBUCIÓN: Java.

Limnozetoidea Thor, 1937

Limnozetidae Thor, 1937 (2 gen., 16 spp. y 1 ssp.)

DisTRIBUCIÓN: Cosmopolita (excepto Australiana y Antártica).

Limnozetella Willmann, 1931 (2 spp.)

(=Austroceratobates Mahunka, 1985 "sin. nov.")

EsPECIE TIPO: Limnozetella lamellata Willmann, 1931

DisTRIBUCIÓN: Paleotropical.

-Limnozetella endroedyi (Mahunka, 1985) (Austroceratobates)

DISTRIBUCIÓN: Sudáfrica.

-Limnozetella lamellata Willmann, 1931

DisTRIBUCIÓN: Java.

Limnozetes Hull, 1916 (14 spp. y 1 ssp.)

(=Vietobates Mahunka, 1987)

EsPeCIE TIPO: (Oribata sphagni Michael, 1880) =Acarus ciliatus Schrank, 1803

DISTRIBUCIÓN: Semicosmopolita (Holártica, Oriental y Neotropical).

-Limnozetes amnicus Behan-Pelletier, 1989

DistRIBUCIÓN: Holártica (Neártica septentrional e Ibérica).

-Limnozetes atmetos Behan-Pelletier, 1989

DISTRIBUCIÓN: Canadá

-Limnozetes borealis Behan-Pelletier, 1989

DisTRIBUCIÓN: Neártica septentrional.

-Limnozetes ciliatus (Schrank, 1803) (Acarus)

DisTRIBUCIÓN: Holártica.

-Limnozetes ciliatus ciliatus (Schrank, 1803)

(=Limnozetes canadensis Hammer, 1952)

(=Oribata sphagni Michael, 1880)

DISTRIBUCIÓN: Holártica. 
-Limnozetes ciliatus foveolatus Willmann, 1939

DISTRIBUCIÓN: Europa septentrional.

-Limnozetes feuerborni Willmann, 1931

DISTRIBUCIÓN: Oriental (Indonesia).

-Limnozetes guyi Behan-Pelletier, 1989

DisTRIBUCIÓN: Canadá.

-Limnozetes latilamellatus Behan-Pelletier, 1989

DisTRIBUCIÓN: Canadá.

-Limnozetes lustrum Behan-Pelletier, 1989

DISTRIBUCIÓN: Neártica septentrional.

-Limnozetes onondaga Behan-Pelletier, 1989

DisTRIBUCIÓN: Holártica (Neártica septentrional y España).

-Limnozetes palmerae Behan-Pelletier, 1989

DisTRIBUCIÓN: Neártica septentrional.

-Limnozetes pustulatus Mahunka, 1987 (L. (Vietobates))

DisTRIBUCIÓN: Vietnam.

-Limnozetes rugosus (Sellnick, 1923) (Ceratozetes)

DISTRIBUCIÓN: Europa septentrional.

-Limnozetes silvicola Hammer, 1961

DisTRIBUCión: Perú y Japón.

-Limnozetes similis Pérez-Íñigo y Baggio, 1989

DisTRIBUCIÓN: Brasil.

Austrachipteriidae Luxton, 1985 (11 gen., 1 subg., 49 spp. y 2 sspp.) DISTRIBUCIÓN: Cosmopolita.

Allozetes Berlese, 1913 (7 spp.)

EsPecie Tipo: Ceratozetes (Allozetes) pusillus Berlese, 1913

DistriBución: Pantropical y Paleártica meridional.

-Allozetes africanus Balogh, 1958

DisTRIBUCión: Paleotropical.

-Allozetes alas Behan-Pelletier, 1998

DisTRIBUCión: Costa Rica.

-Allozetes lacandonicus Mahunka y Palacios-Vargas, 1996

DisTRIBUCIÓN: Norte de Neotropical.

-Allozetes latus Tseng, 1984

DisTRIBUCIÓN: Taiwan [Formosa]

-Allozetes levis Ohkubo, 1981

DisTRIBUCIÓN: Japón.

-Allozetes pusillus (Berlese, 1913) (Ceratozetes (A.))

DisTRIBUCIÓN: Oriental.

-Allozetes translamellatus Hammer, 1973

Distribución: Pantropical (Oriental: Java e "India", Polinesia: I.

Samoa, y Venezuela).

Austrachipteria Balogh y Mahunka, 1966 (13 spp.)

(=Parahypozetes Hammer, 1967)

EsPECIE TIPO: Austrachipteria lamellata Balogh y Mahunka, 1966

DisTRIBUCIÓN: Australiana y Paleártica meridional.

-Austrachipteria bidactyla (J. y P. Balogh, 1983) (Parahypozetes) DisTRIBUCIÓN: Australia.

-Austrachipteria bidentata (Hammer, 1967) (Parahypozetes)

DisTRIBUCIÓN: Nueva Zelanda.

-Austrachipteria breviseta (J. y P. Balogh, 1983) (Parahypozetes)

DisTRIBUCión: Australia.

-Austrachipteria furcata (Hammer, 1967) (Parahypozetes)

DisTRIBUCión: Nueva Zelanda.

-Austrachipteria gigantea (Hammer, 1967) (Parahypozetes)

DisTRIBUCIÓN: Nueva Zelanda.

-Austrachipteria grandis (Hammer, 1967) (Parahypozetes)

DisTRIBUCIÓN: Nueva Zelanda.

-Austrachipteria hammerae J. y P. Balogh, 1992

(=Parahypozetes hammerae Mahunka, 1997, "nom. praeoc." por J. y P. Balogh, 1992)

(=Parahypozetes lobatus J. y P. Balogh, 1983, “nom. praeoc.” por Hammer, 1967)

(=Austrachipteria marieae Colloff y Halliday, 1998)

DistriBuCión: Australia.

-Austrachipteria lamellata Balogh y Mahunka, 1966

DistriBUCIÓN: Australia.
-Austrachipteria lobata (Hammer, 1967) (Parahypozetes)

DistriBUCIÓN: Nueva Zelanda.

-Austrachipteria macrodentata (Hammer, 1967) (Parahypozetes)

DisTRIBUCIÓN: Nueva Zelanda.

-Austrachipteria maxima (Hammer, 1967) (Parahypozetes)

Distribución: Nueva Zelanda.

-Austrachipteria pulla Aoki y Honda, 1985

DisTRIBUCIÓN: Japón.

-Austrachipteria quadridentata (Hammer, 1967) (Parahypozetes)

DisTRIBUCIÓN: Nueva Zelanda.

Ceratobates Balogh y Mahunka, 1969 (3 spp.)

EsPECIE TIPO: Ceratobates pontiger Balogh y Mahunka, 1969

DisTRIBUCIÓN: Neotropical.

-Ceratobates fornerisae Pérez-Íñigo y Baggio, 1985

DisTRIBUCIÓN: Brasil.

-Ceratobates pontiger Balogh y Mahunka, 1969

DisTRIBUCIÓN: Brasil.

-Ceratobates spathulatus Balogh y Mahunka, 1981

DisTRIBUCIÓN: Neotropical (Paraguay y Uruguay).

Granizetes Hammer, 1961 (1 sp.)

EsPECIE TIPO: Granizetes curvatus Hammer, 1961

DISTRIBUCIÓN: Neotropical y subantártica.

-Granizetes curvatus Hammer, 1961

DistRIBUCión: Neotropical (Perú y Tierra del Fuego) y subantártica: I. Malvinas [I. Falkland].

Hypozetes Balogh, 1959 (7 spp. y 1 ssp.)

ESPECIE TIPO: Hypozetes imitator Balogh, 1959

DisTRIBUCIÓN: Pantropical (excepto Neotropical) y subtropical (Paleártica meridional).

-Hypozetes aviger Hammer, 1982

DistriBuCIÓN: Bali.

-Hypozetes brevisetus Mahunka, 1987

DISTRIBUCIÓN: Nigeria.

-Hypozetes bulgaricus Jeleva, 1962

DistribuCIÓN: Sur de Europa e India (Orissa).

-Hypozetes imitator Balogh, 1959

DisTRIBUCIÓN: Etiópica (Tanzania y Yemen) e India (Bengala Occidental)

-Hypozetes laysanensis Aoki, 1964

DistriBución: Hawai e India (Bengala Occidental).

-Hypozetes rostralis Tseng, 1984

DisTRIBUCIÓN: Taiwan [Formosa].

-Hypozetes translamellatus Wallwork, 1965

DisTRIBUCIÓN: Chad y Arabia Saudita.

-Hypozetes translamellatus translamellatus Wallwork, 1965 DisTRIBUCIÓN: Chad.

-Hypozetes translamellatus saudicus Bayoumi y Al-Khalifa, 1984 DistRIBUCión: Arabia Saudita.

Lamellobates Hammer, 1958 (13 spp. y 1 ssp.)

ESPECIE TIPO: (Lamellobates palustris Hammer, 1958) =Achipteria molecula Berlese, 1916

DISTRIBUCIÓN: Pantropical y subtropical.

Lamellobates (Lamellobates) Hammer, 1958 (10 spp. y 1 ssp.)

DISTRIBUCIÓN: Pantropical y subtropical.

-Lamellobates (L.) botari Balogh y Mahunka, 1977

DISTRIBUCIÓN: Brasil.

-Lamellobates (L.) engelbrechti Mahunka, 1989

DisTRIBUCIÓN: Nigeria.

-Lamellobates (L.) gyoergyi Balogh y Mahunka, 1977

Distribución: Neotropical (Brasil y Panamá).

-Lamellobates (L.) intermedius Nübel-Reidelbach y Woas, 1992

DisTRIBUCIÓN: Neotropical (Brasil y Costa Rica).

-Lamellobates (L.) molecula (Berlese, 1916) (Achiperia)

DisTRIBUCIÓN: Pantropical y subtropical. 
-Lamellobates (L.) molecula molecula (Berlese, 1916) (=Lamellobates angolensis Balogh, 1958) (=Lamellobates hauseri Mahunka 1977) (=Lamellobates palustris Hammer, 1958) (=Lamellobates rotundatus Scull, Jeleva y Cruz, 1984) DisTRIBUCIÓN: Pantropical (frecuente) y subtropical. -Lamellobates (L.) molecula aegypticus Bayoumi, 1979 (L. hauseri a.)

DisTRIBUCIÓN: Mediterránea oriental.

-Lamellobates (L.) nitidus Calugar, 1987

DisTRIBUCIÓN: Venezuela.

-Lamellobates (L.) ocularis Jeleva y Vu, 1987

DisTRIBUCIÓN: Vietnam.

-Lamellobates (L.) orientalis Csiszár, 1961

DisTRIBUCIÓN: Oriental (frecuente) y Egipto.

-Lamellobates (L.) quadricornis Pérez-Íñigo y Baggio, 1985 DisTRIBUCIÓN: Brasil.

-Lamellobates (L.) reticulatus Behan-Pelletier, 1998

Distribución: Costa Rica.

Lamellobates (Paralamellobates) Bhaduri y Raychaudhuri, 1968 (3 spp.) ESPECIE TIPO: Lamellobates (Paralamellobates) bengalensis Bhaduri y Raychaudhuri, 1968

DisTRIBUCIÓN: Pantropical y Paleártica meridional.

-Lamellobates (Paralamellobates) bengalensis Bhaduri y Raychaudhuri, 1968

DisTRIBUCIÓN: Oriental: Noreste de India y Taiwan [Formosa].

-Lamellobates (Paralamellobates) misella (Berlese, 1910) (Oribatella)

(=Oribatella ceylanica Oudemans, 1915)

(=Oribatella schoutedeni Balogh, 1959)

DisTRIBUCIÓN: Pantropical (excepto Neotropical): frecuente, y Paleártica meridional.

-Lamellobates (Paralamellobates) striatus (Behan-Pelletier, 1998) (P.) DisTRIBUCIÓN: Costa Rica.

Palmitalia Pérez-Íñigo y Peña, 1997 (1 sp.)

EsPeCIE TIPO: Palmitalia latilamellata Pérez-Íñigo y Peña, 1997

DISTRIBUCIÓN: Paleártica meridional.

-Palmitalia latilamellata Pérez-Íñigo y Peña, 1997

DisTRIBUCIÓN: I. Canarias.

Patagonozetes J. y P. Balogh, 1990 (1 sp.)

EsPECIE TIPO: Sphaerozetes exornatus Balogh y Csiszár, 1963

DisTRIBUCIÓN: Neotropical.

-Patagonozetes exornatus (Balogh y Csiszár, 1963) (Sphaerozetes) DisTRIBUCIÓN: Argentina.

Punctizetes Hammer, 1971 (1 sp.)

EsPECIE TIPO: Punctizetes penicillifer Hammer, 1971

DisTRIBUCión: Australianooriental.

-Punctizetes penicillifer Hammer, 1971

Distribución: Melanesia (I. Fiji) y Filipinas.

Sacculozetes Behan-Pelletier y Rjabinin, 1991 (1 sp.)

EsPECIE TIPO: Sacculozetes filosus Behan-Pelletier y Rjabinin, 1991 DisTRIBUCIÓN: Paleártica.

-Sacculozetes filosus Behan-Pelletier y Rjabinin, 1991

Distribución: Este de Paleártica oriental.

Sagittazetes J. y P. Balogh, 1983 (1 sp.)

EsPeCIE TIPO: Sagittazetes agressor J. y P. Balogh, 1983

DISTRIBUCIÓN: Australiana

-Sagittazetes agressor J. y P. Balogh, 1983

DISTRIBUCIÓN: Australia.

\section{Achipterioidea Thor, 1929}

Achipteriidae Thor, 1929 (9 gen., 1 subg., 83 spp. y 5 sspp.) DisTRIBUCIÓN: Holártica, Oriental y Neotropical.
Achipteria Berlese, 1885 (33 spp. y 2 sspp.)

EsPECIE TIPO: (Oribata nicoleti Berlesle, 1883) =Acarus coleoptratus Linnaeus, 1758

DISTRIBUCIÓN: Semicosmopolita (Holártica, Oriental y Neotropical).

Achipteria (Achipteria) Berlese, 1885 (31 spp. y 2 sspp.)

DISTRIBUCIÓN: Semicosmopolita (Holártica, Oriental y Neotropical))

-Achipteria (A.) acuta Berlese, 1908

(=Notaspis anglicus Oudemans, 1914)

(=Notaspis mixtus Sellnick, 1943)

(=Oribata nitens Nicolet, 1855, "nom. praeoc.” por Gervais, 1844)

(=Oribata nitens L. Koch, 1879, "nom. praeoc." por Gervais, 1844)

(=Achipteria nitens Kishida, 1930, "nom. praeoc." por Nicolet, 1855)

DistriBUCIÓN: Holártica (Paleártica: frecuente, y U.S.A.: Virginia).

-Achipteria (A.) armata (Banks, 1895) (Oribatella)

DisTRIBUCIÓN: U.S.A. oriental.

-Achipteria (A.) armata armata (Banks, 1895)

DisTRIBUCIÓN: U.S.A nororiental.

-Achipteria (A.) armata spinosa Jacot, 1938

DisTRIBUCIÓN: U.S.A. (Carolina del Norte)

-Achipteria (A.) bentonensis Marshall, Reeves y Norton, 1987

(=Achipteria oregonensis borealis Ewing, 1918, "nom. praeoc." por Banks, 1899)

DisTRIBUCIÓN: U.S.A. (Oregón).

-Achipteria (A.) bicarinata Moskacheva, 1973

DistriBución: Bielorrusia.

-Achipteria (A.) borealis (Banks, 1899) (Oribatella) "sp. inq."

Distribución: Alaska.

-Achipteria (A.) catskillensis Nevin, 1977

Distribución: U.S.A. (Nueva York).

-Achipteria (A.) clarencei Nevin, 1977

DisTRIBUCIÓN: U.S.A. (Nueva York).

-Achipteria (A.) coleoptrata (Linnaeus, 1758) (Acarus)

DisTRIBUCIÓN: Holártica (Paleártica y Neártica septentrional) e I. Santa Helena.

-Achipteria (A.) coleoptrata coleoptrata (Linnaeus, 1758)

(=Oribata intermedia Michael, 1898)

(=Oribata nicoleti Berlese, 1883)

(=Oribata ovalis Nicolet, 1855, "nom. praeoc." por Koch, 1835)

(=Oribata redux Hull, 1914)

DisTRIBUCión: Holártica (Paleártica: frecuente, y Neártica septentrional) e I. Santa Helena.

-Achipteria (A.) coleoptrata major Mihelcic, 1963

DisTRIBUCIÓN: Austria.

-Achipteria (A.) cucullata Moskacheva, 1973

Distribución: Bielorrusia.

-Achipteria (A.) curta Aoki, 1970

DISTRIBUCIÓN: Holártica (Japón y Neártica septentrional) y Vietnam.

-Achipteria (A.) dubia Weigmann, 2001

DISTRIBUCIÓN: Alemania.

-Achipteria (A.) elegans Schweizer, 1956

DisTRIBUCIÓN: Suiza.

-Achipteria (A.) hasticeps (Hull, 1914) (Oribata) "sp. inq."

DISTRIBUCIÓN: I. Británicas.

-Achipteria (A.) holomonensis Cancela da Fonseca y Stamou, 1987

DisTRIBUCIÓN: Grecia.

-Achipteria (A.) italica (Oudemans, 1913) (Notaspis)

DISTRIBUCIÓN: Europa.

-Achipteria (A.) languida (Nicolet, 1855) (Oribata) "sp. inq."

DisTRIBUCIÓN: Francia.

-Achipteria (A.) longesensillus Schweizer, 1956

DisTRIBUCIÓN: Europa centromeridional.

-Achipteria (A.) mayeriana Palacios-Vargas y Socarrás, 2001

DisTRIBUCión: Cuba.

-Achipteria (A.) minuta (Ewing, 1909) (Notaspis) "sp. inq."

DisTRIBUCIÓN: U.S.A. (Ilinois).

-Achipteria (A.) moderatior Berlese, 1923 (A. nitens m.) "sp. inq."

DistriBUCIÓN: Oeste de Europa.

-Achipteria (A.) nuda (Hall, 1911) (Notaspis) "sp. inq."

DisTRIBUCIÓN: U.S.A. (California). 
-Achipteria (A.) oregonensis Ewing, 1918

Distribución: U.S.A. (Oregón).

-Achipteria (A.) oudemansi Jacot, 1929 “sp. inq."

DisTRIBUCIÓN: Suiza.

-Achipteria (A.) praeoccupata nom. nov.

[=Achipteria oudemansi Hammen, 1952, "nom. praeoc." por Jacot, 1929

("hom. prim.")]

DisTRIBUCIÓN: Mediterránea.

-Achipteria (A.) quadridentata (Willmann, 1951) (Notaspis)

DisTRIBUCIÓN: Europa central.

-Achipteria (A.) regalis Berlese, 1908

DistriBuCión: Oeste de Europa.

-Achipteria (A.) sellnicki Hammen, 1952

DisTRIBUCión: Europa.

-Achipteria (A.) serrata Hirauchi y Aoki, 1997

DisTRIBUCIÓN: Japón.

-Achipteria (A.) setulosa Golosova, 1981

Distribución: I. Kuriles.

-Achipteria (A.) sumatrensis Willmann, 1931

DisTRIBUCIÓN: Oriental (Indonesia).

-Achipteria (A.) verrucosa Rjabinin, 1974

Distribución: Sur de Paleártica oriental.

Achipteria (Izuachipteria) Balogh y Mahunka, 1979 (2 spp.)

(=Hokkachipteria Balogh y Mahunka, 1979 "sin. nov.")

ESPECIE TIPO: Achipteria imperfecta Suzuki, 1972

DisTRIBUCIÓN: Paleártica oriental y Oriental.

-Achipteria (Izuachipteria) alpestris (Aoki, 1973) (A.)

DisTRIBUCIÓN: Japón.

-Achipteria (Izuachipteria) imperfecta (Suzuki, 1972) (A.)

DISTRIBUCIÓN: Japón y Taiwan [Formosa]

Anachipteria Grandjean, 1932 (14 spp.)

ESPECIE TIPO: Anachipteria deficiens Grandjean, 1932

DisTRIBUCIÓN: Holártica y Neotropical.

-Anachipteria achipteroides (Ewing, 1913) (Oribatella)

DisTRIBUCIÓN: Holártica (U.S.A. septentrional y Japón).

-Anachipteria acuta (Ewing, 1918) (Oribatella)

DisTRIBUCIÓN: U.S.A. (Oregón).

-Anachipteria aegyptiaca Abdel-Hamid, 1964

DisTRIBUCIÓN: Egipto.

-Anachipteria australoides Jacot, 1938 (A. achipteroides austr)

DistRibución: U.S.A. (Carolina del Norte).

-Anachipteria deficiens Grandjean, 1932

(?=Oribata tecta alpina Schweizer, 1922 "sp. inq.")

Distribución: Paleártica (Europa centromeridional y Este de Rusia asiática).

-Anachipteria grandis Aoki, 1961

Distribución: Paleártica (Cáucaso y Este de Paleártica oriental)

-Anachipteria howardi (Berlese, 1908) (Sphaerozetes)

(=Anachipteria achipteroides milleri Jacot, 1936)

(=Oribata corpuscula Ewing, 1908)

(=Sphaerozetes latitectus Berlese, 1908)

DISTRIBUCIÓN: Holártica (Europa, Asia centrooccidental y Neártica).

-Anachipteria magnilamellata (Ewing, 1909) (Oribatella)

DisTRIBUCIÓN: U.S.A. (Ilinois).

-Anachipteria mahunkai Aoki, 1974

DistRIBUCIÓN: Este de Paleártica oriental.

-Anachipteria major Mihelcic, 1957

("non" Oribata major Mihelcic, 1956 =Anachipteria perisi)

DisTRIBUCIÓN: Austria.

-Anachipteria perisi (Mihelcic, 1956) (Oribata)

(=Oribata major Mihelcic, 1956)

DisTRIBUCión: España.

-Anachipteria pratensis Scull, Jeleva y Cruz, 1984

DisTRIBUCIÓN: Cuba.

-Anachipteria signata (Banks, 1895) (Oribatella)

(=Oribatella achipteroides australis Ewing, 1917)

DisTRIBUCIÓN: U.S.A.
-Anachipteria valida (Banks, 1910) (Oribatella)

DisTRIBUCIÓN: U.S.A. (Virginia).

Campachipteria Aoki, 1995 (18 spp. y 1 ssp.)

ESPECIE TIPO: Campachipteria uenoi Aoki, 1995

DisTRIBUCIÓN: Holártica.

-Campachipteria barguzini (Golosova y Karppinen, 1984) (Parachipteria)

DisTRIBUCIÓN: Sur de Siberia.

-Campachipteria bella (Sellnick, 1928) (Notaspis)

DisTRIBUCIÓN: Holártica.

-Campachipteria distincta (Aoki, 1959) (Achipteria)

DisTRIBUCIÓN: Este de Paleártica oriental y Noreste de Oriental.

-Campachipteria distincta distincta (Aoki, 1959)

DisTRIBUCIÓn: Este de Paleártica oriental y Sureste de China.

-Campachipteria distincta incurva (Aoki, 1991) (Parachipteria) DisTRIBUCIÓn: Taiwan [Formosa].

-Campachipteria ewingi (Berlese, 1910) (Achiperia nicoleti e.)

Distribución: Sureste de U.S.A.

-Campachipteria fanzagoi (Jacot, 1929) (Achipteria)

(=Parachipteria willmanni Hammen, 1952)

DisTRIBUCIÓN: Holártica (Paleártica: frecuente, y U.S.A.: Virginia).

-Campachipteria heintoogensis (Nevin, 1979) (Parachipteria)

DistriBuCión: U.S.A. (Carolina del Norte).

-Campachipteria neotropicalis (Mahunka, 1983) (Parachipteria)

DISTRIBUCIÓN: Méjico.

-Campachipteria nivalis (Hammer, 1952) (Achipteria)

DisTRIBUCIÓN: Holártica (Europa oriental, Paleártica oriental y

Neártica septentrional)

-Campachipteria patavina (Oudemans, 1913) (Notaspis)

DISTRIBUCIÓN: Paleártica (Europa centromeridional y Rusia asiática).

-Campachipteria perproxima (Sellnick, 1931) (Notaspis)

DISTRIBUCIÓN: Europa meridional.

-Campachipteria petiti (Travé, 1960) (Parachipteria)

DisTRIBUCIÓN: Europa meridional.

-Campachipteria savagei (Nevin, 1976) (Parachipteria)

DistriBUCión: U.S.A. (Carolina del Norte).

-Campachipteria sibirica (Krivolutsky y Grishina, 1970) (Parachipteria)

DisTRIBUCIÓN: Siberia occidental.

-Campachipteria snowdonensis (Colloff y Seyd, 1987) (Parachipteria)

DisTRIBUCIÓN: I. Británicas.

-Campachipteria travei (Nevin, 1977) (Parachipteria)

DisTRIBUCIÓN: Neártica septentrional.

-Campachipteria truncata (Aoki, 1976) (Parachipteria)

DisTRIBUCIÓN: Japón.

-Campachipteria uenoi Aoki, 1995

DisTRIBUCIÓN: Taiwan [Formosa].

-Campachipteria weigmanni (Pérez-Íñigo, 1987) (Parachipteria)

DISTRIBUCIÓN: I. Azores.

Cerachipteria Grandjean, 1935 (3 spp. y 2 sspp.)

(=Zygachipteria Mihelcic, 1956)

EsPECIE TIPO: Cerachipteria digita Grandjean, 1935

DISTRIBUCIÓN: Paleártica.

-Cerachipteria digita Grandjean, 1935

DisTRIBUCIÓN: Oeste de Europa.

-Cerachipteria digita digita Grandjean, 1935

DisTRIBUCIÓN: Oeste de Europa.

-Cerachipteria digita jugata Mihelcic, 1956 (C. (Zygachipteria) j.) DisTRIBUCIÓN: España.

-Cerachipteria digita pyrenaica Pérez-Íñigo jr., 1991

(?=Cerachipteria fusiger Mihelcic, 1957 "sp. inq.")

DisTRIBUCIÓN: España.

-Cerachipteria franzi Willmann, 1953

DisTRIBUCIÓN: Austria.

-Cerachipteria minuscula (Berlese, 1902) (Oribata)

DISTRIBUCION: Italia. 
Cubachipteria Balogh y Mahunka, 1979 (2 spp.)

ESPECIE TIPO: Achipteria (Cubachipteria) remota Balogh y Mahunka, 1979 DISTRIBUCIÓN: Neotropical.

-Cubachipteria bispina (Calugar, 1990) (Achipteria (C.))

DisTRIBUCIÓN: Venezuela.

-Cubachipteria remota (Balogh y Mahunka, 1979) (Achipteria (C.))

DisTRIBUCIÓN: Cuba.

Dentachipteria Nevin, 1974 (2 spp.)

EsPeCIE TIPO: Dentachipteria ringwoodensis Nevin, 1974

DISTRIBUCIÓN: Neártica.

-Dentachipteria highlandensis Nevin, 1974

DisTRIBUCIÓN: Neártica septentrional.

-Dentachipteria ringwoodensis Nevin, 1974

Distribución: U.S.A. (Nueva York).

Hoffmanacarus Mahunka, 1995 (1 sp.)

ESPECIE TIPO: Hoffmanacarus virginianus Mahunka, 1995

DISTRIBUCIÓN: Neártica.

-Hoffmanacarus virginianus Mahunka, 1995

DisTRIBUCIÓN: U.S.A. (Virginia).

Parachipteria Hammen, 1952 (6 spp.)

(=Pseudachipteria Travé, 1960 "sin. nov.")

EsPECIE TIPO: Oribata punctata Nicolet, 1855

DisTRIBUCIÓN: Holártica.

-Parachipteria agenjoi (Pérez-Íñigo, 1976) (Pseudachipteria)

DisTRIBUCión: I. Canarias.

-Parachipteria floresiana (Pérez-Íñigo, 1992) (Pseudachipteria) DisTRIBUCIÓN: I. Azores.

-Parachipteria insularis (Pérez-Iñigo, 1992) (Pseudachipteria) DisTRIBUCIÓN: I. Azores.

-Parachipteria magna (Sellnick, 1928) (Notaspis)

DisTRIBUCIÓN: Europa.

-Parachipteria ovalis (Koch, 1835) (Oribata) “sp. inq." DisTRIBUCIÓN: Alemania.

-Parachipteria punctata (Nicolet, 1855) (Oribata)

DisTRIBUCIÓN: Holártica (frecuente en Paleártica) e I. Santa Helena.

Plakoribates Popp, 1960 (4 spp.)

ESPECIE TIPO: Plakoribates multicuspidatus Popp, 1960

DisTRIBUCIÓN: Tropical (Oriental y Neotropical) y subtropical

(Paleártica meridional)

-Plakoribates confluens Balogh, 1970

DisTRIBUCIÓN: Ceilán [Sri Lanka].

-Plakoribates multicuspidatus Popp, 1960

DisTRIBUCIÓN: Egipto.

-Plakoribates neotropicus Balogh y Mahunka, 1978

DisTRIBUCIÓN: Bolivia.

-Plakoribates scutatus Hammer, 1979

DisTRIBUCIÓN: Oriental (Indonesia).

Tegoribatidae Grandjean, 1954 (9 gen. y 24 spp.)

Distribución: Cosmopolita (excepto Antártica).

Lemurobates Mahunka, 1997 (1 sp.)

EsPECIE TIPO: Lemurobates antsiranana Mahunka, 1997

DISTRIBUCIÓN: Etiópica.

-Lemurobates antsiranana Mahunka, 1997

DisTRIBUCIÓN: Madagascar.

Lepidozetes Berlese, 1910 (6 spp.)

(=Onazetes Bugrov, 1991 "sin. nov.")

EsPECIE TIPO: Lepidozetes singularis Berlese, 1910

DISTRIBUCIÓN: Holártica.

-Lepidozetes conjunctus Schweizer, 1922

(=Lepidozetes chernovi Rjabinin, 1974)

DistriBución: Paleártica (Euroatlántica y Paleártica oriental: frecuente).
-Lepidozetes dashidorzsi Balogh y Mahunka, 1965

DistriBución: Sureste de Paleártica oriental.

-Lepidozetes latipilosus Hammer, 1952

DisTRIBUCIÓN: Holártica (Neártica septentrional, Sureste de Europa y Paleártica oriental: excepto Este).

-Lepidozetes sigularis Berlese, 1910

DisTRIBUCIÓN: Holártica (Paleártica y Neártica septentrional): frecuente.

-Lepidozetes trifolius (Fujikawa, 1972) (Tegoribates)

Distribución: Holártica (Este de Paleártica oriental y U.S.A.: California).

-Lepidozetes umbellatus (Bugrov, 1991) (Onazetes)

DISTRIBUCIÓN: "Rusia".

Neophysobates Luxton, 1987 (3 spp.)

(=Paraphysobates Luxton, 1985, “nom. praeoc." por Mahunka, 1985)

(=Pseudophysobates Fujikawa, 1991 "sin. nov.")

EsPECIE TIPO: Physobates monodactylus Hammer, 1966

DisTRIBUCIÓN: Subtropical austral (excepto Neotropical).

-Neophysobates monodactylus (Hammer, 1966) (Physobates)

DisTRIBUCIÓN: Nueva Zelanda.

-Neophysobates montanus (Engelbrecht, 1986) (Tegoribates)

DISTRIBUCIÓN: Sudáfrica.

-Neophysobates nudus (Engelbrecht, 1986) (Tegoribates)

DisTRIBUCIÓN: Sudáfrica.

Paraphysobates Mahunka, 1985 (2 spp.)

(=Monophysobates J. y P. Balogh, 1992 "sin. nov.")

EsPeCIE TIPO: Paraphysobates abditus Mahunka, 1985

DisTRIBUCIÓN: Etiópica.

-Paraphysobates abditus Mahunka, 1985

DisTRIBUCIÓN: Sudáfrica.

-Paraphysobates natalensis (Engelbrecht, 1986) (Tegoribates)

DisTRIBUCIÓN: Sudáfrica.

Physobates Hammer, 1962 (1 sp.)

EsPecie TIPO: Physobates spinipes Hammer, 1962

DISTRIBUCIÓN: Neotropical.

-Physobates spinipes Hammer, 1962

DisTRIBUCIÓN: América austral.

Scutozetes Hammer, 1952 (2 spp.)

(=Neolepidozetes Hammer, 1977 "sin. nov.")

EsPECIE TIPO: Scutozetes lanceolatus Hammer, 1952

DISTRIBUCIÓN: Holártica y Neotropical.

-Scutozetes lanceolatus Hammer, 1952

DisTRIBUCIÓN: Holártica (Neártica septentrional: frecuente,

Paleártica occidental: Sureste de Europa, y Paleártica oriental: frecuente) y Surinám.

-Scutozetes ovalis (Hammer, 1977) (Neolepidozetes)

DisTRIBUCión: Pakistán.

Tegoribates Ewing, 1917 (4 spp.)

(=Lepidoribates Sellnick, 1920)

EsPECIE TIPO: Tegoribates subniger Ewing, 1917

DISTRIBUCIÓN: Holártica y Neotropical.

-Tegoribates americanus Hammer, 1958

DisTRIBUCIÓN: Neártica septentrional (frecuente) y Argentina.

-Tegoribates bryophilus Woolley, 1965

DisTRIBUCIÓN: U.S.A. (Colorado).

-Tegoribates latirostris (Koch, 1844) (Zetes)

(=Oribata alzanii Coggi, 1900)

DistRIBUCIÓN: Paleártica (menos frecuente en el Sur)

-Tegoribates subniger Ewing, 1917

DisTRIBUCIÓN: U.S.A. (Indiana).

Umbellozetes Krivolutsky, 1969 (4 spp.)

EsPECIE TIPO: Umbellozetes fuscus Krivolutsky, 1969

DISTRIBUCIÓN: Paleártica. 
-Umbellozetes fuscus Krivolutsky, 1969

DistriBución: Paleártica meridional (Cáucaso y Sur de Paleártica oriental).

-Umbellozetes ombinatus Wang y Solhoy, 1999

Distribución: China (Tíbet).

-Umbellozetes petrovae (Golosova y Karppinen, 1984) (Tutoridens [+])

DisTRIBUCIÓN: Sur de Siberia.

-Umbellozetes squamaceus Wang y Shen, 1999

Distribución: China (Tíbet).

Williamszetes Hammer, 1961 (1 sp.)

(=Williamsia Hammer, 1958, “nom. praeoc.” por Carus, 1890)

EsPeCIE TIPO: Williamsia elsosneadensis Hammer, 1958

DISTRIBUCIÓN: Neotropical.

-Williamszetes elsosneadensis (Hammer, 1958) (Williamsia)

DisTRIBUCIÓN: Neotropical.

Oribatelloidea Jacot, 1925

Oribatellidae Jacot, 1925 (12 gen., 3 subg., 125 spp. y 2 sspp.) DISTRIBUCIÓN: Cosmopolita.

Adoribatella Woolley, 1967 (1 sp.)

ESPECIE TIPO: Adoribatella punctata Woolley, 1967

DISTRIBUCIÓN: Holártica.

-Adoribatella punctata Woolley, 1967

DisTRIBUCIÓN: Holártica (U.S.A.: Colorado, y Este de Rusia asiática).

Cuspidozetes Hammer, 1962 (1 sp.)

ESPECIE TIPO: Cuspidozetes armatus Hammer, 1962

DISTRIBUCIÓN: Neotropical.

-Cuspidozetes armatus Hammer, 1962

DisTRIBUCIÓN: Argentina.

Ferolocella Grabowski, 1971 (2 spp.)

(=Gendzella Kulijev, 1977 "sin. nov.")

ESPECIE TIPO: (Oribatella carolina Banks, 1947) =Oribatella tessella-

ta Berlese, 1908

DisTRIBUCIÓN: Holártica.

-Ferolocella cribraria (Kulijev, 1977) (Gendzella) Distribución: Cáucaso.

-Ferolocella tessellata (Berlese, 1908) (Oribatella)

(=Oribatella carolina Banks, 1947)

DisTRIBUCIÓN: U.S.A. centrooriental.

Joelia Oudemans, 1906 (4 spp.)

$(=$ Coggiella Berlese, 1916)

EsPECIE TIPO: Oribata fiorii Coggi, 1898

DisTRIBUCIÓN: "Holártica".

-Joelia americana (Haller, 1884) (Oribata) "sp. inq."

DISTRIBUCIÓN: "Neártica".

-Joelia dubia (Kulijev, 1967) (Oribatella)

DisTRIBUCión: Cáucaso.

-Joelia fiorii (Coggi, 1898) (Oribata)

DISTRIBUCIÓN: Mediterránea.

-Joelia spina Kulijev, 1979

DistRIBUCIÓN: Cáucaso.

Kunstella Krivolutsky, 1974 (1 sp.)

ESPECIE TIPO: Kunstella foveolata Krivolutsky, 1974

DisTRIBUCIÓN: Paleártica.

-Kunstella foveolata Krivolutsky, 1974

DisTRIBUCIÓN: Asia centrooccidental.

Novoribatella Engelbrecht, 1986 (3 spp.)

EsPECIE TIPO: Tectoribates transcriptus Mahunka, 1985

DisTRIBUCIÓN: Etiópica.

-Novoribatella minutisetarum Engelbrecht, 1986

DisTRIBUCIÓN: Sudáfrica.
-Novoribatella pentasetarum Engelbrecht, 1986

DisTRIBUCIÓN: Sudáfrica.

-Novoribatella transcripta (Mahunka, 1985) (Tectoribates)

DisTRIBUCIÓN: Sudáfrica.

Ophidiotrichus Grandjean, 1953 (6 spp.)

ESPECIE TIPO: (Oribata connexa Berleser, 1904)=Oribata tecta Michael, 1884

DISTRIBUCIÓN: Holártica.

-Ophidiotrichus corsicanus Bernini y Avanzati, 1983

DisTRIBUCIÓN: Francia (Córcega).

-Ophidiotrichus exastus Higgins, 1965

Distribución: U.S.A. (Carolina del Norte).

-Ophidiotrichus oglasae Bernini, 1975

DISTRIBUCIÓN: Italia (I. Montecristo).

-Ophidiotrichus tectus (Michael, 1884) (Oribata)

(=Notaspis borussicus Sellnick, 1908)

(=Oribata connexa Berlese, 1904)

DisTRIBUCIÓN: Paleártica occidental (menos frecuente en el Norte).

-Ophidiotrichus ussuricus Krivolutsky, 1971

DisTRIBUCIÓN: Sureste de Paleártica oriental.

-Ophidiotrichus vindobonensis Piffl, 1961 (O. connexus v.)

DISTRIBUCIÓN: Europa centromeridional.

Oribatella Banks, 1895 (99 spp. y 2 sspp.)

ESPECIE TIPO: Oribatella quadridentata Banks, 1895

DISTRIBUCIÓN: Cosmopolita.

Oribatella (Oribatella) Banks, 1895 (92 spp. y 2 sspp.)

DisTRIBUCIÓn: Cosmopolita.

-Oribatella (O.) angulosa Csiszár, 1962

Distribución: Sur de Europa oriental.

-Oribatella (O.) anomola Grabowski, 1970

Distribución: U.S.A. (Dakota del Sur).

-Oribatella (O.) arctica Thor, 1930

DisTRIBUCIÓN: Boreal.

-Oribatella (O.) arctica arctica Thor, 1930

Distribución: Boreal (Norte de Europa, Noroeste de Paleártica oriental y Neártica septentrional: frecuente).

-Oribatella (O.) arctica litoralis Strenzke, 1950

DisTRIBUCIÓN: Paleártica (Europa septentrional y Japón).

-Oribatella (O.) asiatica Krivolutsky, 1974

DisTRIBUCIÓN: Paleártica meridional (Cáucaso y Asia centrooccidental).

-Oribatella (O.) aviculus P. Balogh, 1989

DisTRIBUCIÓN: Ecuador.

-Oribatella (O.) barbata Choi, 1986

DISTRIBUCIÓN: Corea.

-Oribatella (O.) berlesei (Michael, 1898) (Oribata)

DisTRIBUCIÓN: Paleártica occidental.

-Oribatella (O.) berninii Pérez-Íñigo, 1989

DisTRIBUCIÓN: España.

-Oribatella (O.) blocki Stary, 1995

Distribución: Subantártica: I. Malvinas [I. Falkland].

-Oribatella (O.) brevicornuta Jacot, 1934

DisTRIBUCIÓN: Holártica (U.S.A. oriental y Japón).

-Oribatella (O.) brevicuspidata Hammer, 1961

DistriBuCión: Perú.

-Oribatella (O.) brevicuspidis Bernini, 1972

DisTRIBUCIÓN: Suroeste de Europa.

-Oribatella (O.) brevilamellata Balogh, 1958 "sp. inq."

DisTRIBUCIÓN: Etiópica (Angola y Ghana).

-Oribatella (O.) brevipila Bernini, 1977

DisTRIBUCIÓN: Paleártica meridional (Europa centromeridional y

Este de Rusia oriental).

-Oribatella (O.) bulanovae Kulijev, 1967

DISTRIBUCIÓN: Europa centrooriental.

-Oribatella (O.) byzovae Krivolutsky, 1974

DISTRIBUCIÓN: Suroeste de Siberia. 
-Oribatella (O.) calcarata (Koch, 1835) (Oribata)

(=Oribatella decumana Berlese, 1910)

DisTRIBUCIÓN: Holártica (Paleártica, Canadá y "U.S.A")

-Oribatella (O.) cespitum (Koch, 1841) (Zetes) "sp. inq."

(=Zetes morticinus Koch, 1841)

DistriBución: Alemania.

-Oribatella (O.) colchica Krivolutsky, 1974

DisTRIBUCIÓN: Sureste de Europa.

-Oribatella (O.) crassipilosa Bernini, 1975

DisTRIBUCIÓN: Suroeste de Europa.

-Oribatella (O.) dechambrieri Mahunka, 1983

DisTRIBUCIÓN: Méjico.

-Oribatella (O.) dentaticuspis Ewing, 1910

DisTRIBUCIÓN: U.S.A. (Ilinois).

-Oribatella (O.) dudichi Willmann, 1938

DisTRIBUCIÓN: Europa centrooriental.

-Oribatella (O.) eutricha Berlese, 1908

(=Oribatella producta quadrimucronata Berlese, 1910)

DisTRIBUCIÓN: Europa centromeridional.

-Oribatella (O.) exilicornis Berlese, 1910

Distribución: Sur de Europa.

-Oribatella (O.) extensa Jacot, 1934 (O. brevicornuta e.)

DisTRIBUCIÓN: U.S.A.

-Oribatella (O.) foliata Krivolutsky, 1974

DisTRIBUCión: Cáucaso.

-Oribatella (O.) gigantea Berlese, 1916

Distribución: U.S.A. (Misuri).

-Oribatella (O.) gomerae Pérez-Íñigo, 1986

DisTRIBUCIÓN: I. Canarias.

-Oribatella (O.) heterodentata Karppinen y Shtanchaeva, 1987

DisTRIBUCIÓN: Cáucaso.

-Oribatella (O.) hungarica Balogh, 1943

DisTRIBUCIÓN: Europa meridional.

-Oribatella (O.) ichnusae Bernini y Avanzati, 1983

DisTRIBUCIÓN: Italia (Cerdeña).

-Oribatella (O.) illuminata Hammer, 1961

DISTRIBUCIÓN: Perú.

-Oribatella (O.) incisa Dalenius, 1963

DISTRIBUCIÓN: Suecia.

-Oribatella (O.) incurvata Balogh y Mahunka, 1969

DisTRIBUCIÓN: Bolivia.

-Oribatella (O.) inflexa Mahunka, 1957

DisTRIBUCIÓN: Sur de Europa.

-Oribatella (O.) jucunda Calugar, 1987

DisTRIBUCIÓN: Venezuela.

-Oribatella (O.) krivolutskyi Karppinen y Shtanchaeva, 1987

DisTRIBUCIÓN: Cáucaso.

-Oribatella (O.) kunsti Bernini, 1972

DistRIBUCIÓN: Suroeste de Europa.

-Oribatella (O.) linjiangensis Gao y Wen, 1992

DistriBuCión: Noreste de China.

-Oribatella (O.) longispina Berlese, 1914

DisTRIBUCIÓN: Oeste de Europa.

-Oribatella (O.) luisae Bernini, 1979

DistRIBUCIÓN: Mediterránea occidental.

-Oribatella (O.) madagascarensis Mahunka, 1997

DisTRIBUCIÓN: Madagascar.

-Oribatella (O.) mahani Pérez-Íñigo y Peña, 1996

DisTRIBUCIÓN: I. Canarias.

-Oribatella (O.) malaya Balogh y Mahunka, 1974

DisTRIBUCIÓN: Oriental.

-Oribatella (O.) mediocris Berlese, 1916

DisTRIBUCión: U.S.A. (Misuri).

-Oribatella (O.) microfoveolata Hammer, 1977

Distribución: Pakistán e India (Himalaya).

-Oribatella (O.) minuta Banks, 1896

DisTRIBUCIÓN: U.S.A

-Oribatella (O.) molodovi Krivolutsky, 1971

DistRIBUCión: Este de Paleártica oriental.
-Oribatella (O.) monospicus Wharton, 1938

Distribución: Méjico.

-Oribatella (O.) neonominata nom. nov.

[=Oribatella longisetosa Fernández y Alzuet, 1978, "nom. praeoc." por Hammer, 1977 ("hom. prim.")]

DisTRIBUCIÓN: Argentina.

-Oribatella (O.) nugarica Bernini y Avanzati, 1983

DISTRIBUCIÓN: Mediterránea occidental.

-Oribatella (O.) orientalis Choi y Aoki, 1993

DisTRIBUCIÓN: Corea.

-Oribatella (O.) ornata (Coggi, 1900) (Oribata)

(=Oribatella producta Berlese, 1908)

DistRIBUCIÓN: Paleártica occidental (excepto Norte).

-Oribatella (O.) palustris Hammer, 1962

DisTRIBUCIÓN: América austral.

-Oribatella (O.) parvula Bernini, 1974

DisTRIBUCIÓN: Italia.

-Oribatella (O.) phyllophora Jeleva, 1962

DisTRIBUCión: Sureste de Europa.

-Oribatella (O.) plummeri Jacot, 1934 (O. brevicornuta p.)

DisTRIBUCIÓN: U.S.A. (Maryland).

-Oribatella (O.) prolongata Hammer, 1961

DisTRIBUCIÓN: Neotropical (Perú y Argentina).

-Oribatella (O.) puertomonttensis Hammer, 1962

DistriBución: Chile.

-Oribatella (O.) pulchra Bernini, 1974

DistRIBUCión: Suroeste de Europa.

-Oribatella (O.) punctata Hammer, 1958

DisTRIBUCIÓN: Argentina.

-Oribatella (O.) punctulata Balogh, 1958 “sp. inq."

DisTRIBUCIÓN: Angola.

-Oribatella (O.) pusilla Berlese, 1916

DisTRIBUCIÓN: U.S.A. (Misuri).

-Oribatella (O.) quadricornuta (Michael, 1880) (Oribata)

(?=Oribata flammula Koch, 1839 "sp. inq.")

(?=Oribata quadricuspis Grube, 1859 "sp. inq.")

DisTRIBUCIÓN: Holártica (Paleártica y Neártica septentrional):

frecuente.

-Oribatella (O.) quadridentata Banks, 1895

(=Oribatella magniseta Ewing, 1909)

DisTRIBUCIÓN: Holártica (U.S.A. oriental y Europa: Rumania).

-Oribatella (O.) quadrispinata Hammer, 1962

DisTRIBUCIÓN: Argentina.

-Oribatella (O.) reducta P. Balogh, 1985

DistriBución: Australia.

-Oribatella (O.) reticulata Berlese, 1916

DistRIBUCIÓN: Holártica meridional (Europa centromeridional,

Asia centooccidental y U.S.A.: Misuri).

-Oribatella (O.) reticuloides Hammer, 1955

DisTRIBUCIÓN: Neártica septentrional.

-Oribatella (O.) rugosula Mahunka, 1987

DisTRIBUCIÓN: Hungría.

-Oribatella (O.) sardoa Bernini, 1979

DisTRIBUCIÓN: Mediterránea occidental.

-Oribatella (O.) sculpturata Mahunka, 1987

DisTRIBUCIÓN: Vietnam.

-Oribatella (O.) serrata Balogh y Mahunka, 1969

DisTRIBUCIÓN: Neotropical.

-Oribatella (O.) serrula Pérez-Íñigo y Baggio, 1985

DistRIBUCIÓN: Brasil.

-Oribatella (O.) sexdentata Berlese, 1916

DisTRIBUCIÓN: Holártica (Europa, Este de Rusia asiática y U.S.A.: Virginia).

-Oribatella (O.) shaldybinae Rjabinin, 1974

Distribución: Sureste de Paleártica oriental.

-Oribatella (O.) similis Fujikawa, 1990

DisTRIBUCIÓN: Japón.

-Oribatella (O.) sitnikovae Djaparidze, 1989

DistRIBUCión: Cáucaso. 
-Oribatella (O.) strinatii Mahunka, 1979

DistriBución: Guatemala.

-Oribatella (O.) superbula (Berlese, 1904) (Oribata)

DisTRIBUCión: Paleártica meridional y Norte de Oriental

-Oribatella (O.) superbula superbula (Berlese, 1904) (=Oribatella meridionalis Berlese, 1908)

DistRIBUCIÓN: Paleártica meridional (Paleártica occidental: menos frecuente en el Norte, y Sur de Paleártica oriental) y Norte de Oriental.

-Oribatella (O.) superbula longisetosa Hammer, 1977 (O. meridionalis l.)

DisTRIBUCIÓN: Pakistán.

-Oribatella (O.) szaboi Balogh y Mahunka, 1979

DisTRIBUCiÓN: Norte de Neotropical.

-Oribatella (O.) szunyoghyi Balogh, 1961

DisTRIBUCIÓN: Tanzania.

-Oribatella (O.) tenerifensis Pérez-Íñigo, 1986

DisTRIBUCIÓN: I. Canarias.

-Oribatella (O.) tenuis Csiszár, 1962

DisTRIBUCIÓN: Europa suroriental.

-Oribatella (O.) triangulata Pérez-Íñigo jr., 1990

DisTRIBUCIÓN: España.

-Oribatella (O.) trichoptera Berlese, 1916

DisTRIBUCIÓN: Argentina.

-Oribatella (O.) tridactyla Ruiz, Subías y Kahwash, 1991

DisTRIBUCión: Suroeste de Europa.

-Oribatella (O.) tyrrhenica Bernini, 1975

Distribución: Suroeste de Europa.

-Oribatella (O.) undulata Choi, 1986

DisTRIBUCIÓN: Corea.

-Oribatella (O.) unispinata Hammer, 1958

DisTRIBUCIÓN: Bolivia e India (Tripura)

-Oribatella (O.) willmanni Subías y Gil-Martín, 1995

(=Oribatella similesuperbula Weigmann, 2001)

DISTRIBUCIÓN: Europa centromeridional.

Oribatella (Cavernella) Bernini, 1975 (1 sp.)

EsPECIE TIPO: Cavernella helenae Bernini, 1975

DISTRIBUCIÓN: Paleártica.

-Oribatella (Cavernella) helenae (Bernini, 1975) (C.)

DisTRIBUCIÓN: Suroeste de Europa.

Oribatella (Fenestrobates) Balogh y Mahunka, 1969 (3 spp.)

ESPECIE TIPO: Fenestrobates capucinus Balogh y Mahunka, 1969

DisTRIBUCIÓN: Neotropical y Paleártica.

-Oribatella (Fenestrobates) capucinus (Balogh y Mahunka, 1969) (F.)

DisTRIBUCIÓN: Bolivia.

-Oribatella (Fenestrobates) rossicus (Krivolutsky, 1974) (F.)

DisTRIBUCIÓN: Este de Rusia asiática.

-Oribatella (Fenestrobates) vicinus (Rjabinin, 1975) (F.)

DisTRIBUCIÓN: Este de Rusia asiática.

Oribatella (Multoribatella) subgen. nov. (3 spp.)

ESPECIE TIPO: Oribatella bromeliarum Behan-Pelletier y Paoletti, 1993

DisTRIBUCIÓN: Neotropical y Paleártica meridional.

-Oribatella (Multoribatella) bromeliarum Behan-Pelletier y Paoletti, 1993 (O.)

DisTRIBUCIÓN: Venezuela.

-Oribatella (Multoribatella) kurchevi Krivolutsky, 1974 (O.)

DisTRIBUCIÓN: Sureste de Europa.

-Oribatella (Multoribatella) nigra Kulijev, 1967 (O.)

Distribución: Cáucaso.

Prionoribatella Aoki, 1975 (2 spp.)

EsPECIE TIPO: Anachipteria dentilamellata Aoki, 1965

DisTRIBUCIÓN: Paleártica.

-Prionoribatella dentilamellata (Aoki, 1965) (Anachipteria)

DisTRIBUCIÓN: Japón.

-Prionoribatella impar Aoki, 1976

DISTRIBUCIÓN: Japón.
Pseudotectoribates Subías, 1977 (3 spp.)

ESPECIE TIPO: (Pseudotectoribates bellus Subías, 1977)=Anachipteria subsimilis Mihelcic, 1956

DisTRIBUCIÓN: Paleártica y Etiópica.

-Pseudotectoribates kittenbergeri (Balogh, 1959) (Anachipteria)

DisTRIBUCión: Etiópica (Tanzania y Sudáfrica) y Mediterránea occidental.

-Pseudotectoribates minidentatus Ruiz, Subías y Kahwash, 1991

DisTRIBUCIÓN: Mediterránea occidental.

-Pseudotectoribates subsimilis (Mihelcic, 1956) (Anachipteria)

(=Pseudotectoribates bellus Subías, 1977)

DISTRIBUCIÓN: España.

Siciliotrichus Bernini, 1983 (1 sp.)

ESPECIE TIPO: Siciliotrichus siculus Bernini, 1983

DISTRIBUCIÓN: Paleártica.

-Siciliotrichus siculus Bernini, 1983

DISTRIBUCIÓN: Mediterránea occidental.

Tectoribates Berlese, 1910 (5 spp.)

=Anoribatella Kunst, 1962)

EsPeCIE TIPO: Sphaerozetes (Tectoribates) proximus Berlese, 1910

DistriBuCión: Paleártica y tropical (Etiópica y Neotropical).

-Tectoribates africanus Balogh, 1959

DisTRIBUCIÓN: Angola.

-Tectoribates deserticola (Balogh y Mahunka, 1965) (Anoribatella) DisTRIBUCIÓN: Mongolia.

-Tectoribates mongolicus Bayartogtokh, 1997

DisTRIBUCIÓN: Mongolia.

-Tectoribates ornatus (Schuster, 1958) (Anachipteria)

DisTRIBUCIÓN: Paleártica y Neotropical (Argentina y Uruguay).

-Tectoribates proximus (Berlese, 1910) (Spaerozetes (T.))

(=Tectoribates punctatus Sellnick, 1943)

DisTRIBUCIÓN: Italia

Ceratokalummidae Balogh, 1970 (6 gen. y 12 spp.) DistriBución: Pantropical y subtropical.

Achipterina Berlese, 1916 (4 spp.)

(=Ceratokalumma Balogh, 1970)

EsPECIE TIPO: Achipteria (Achipterina) oribatelloides Berlese, 1916

DisTRIBUCIÓN: Pantropical (excepto Etiópica).

-Achipterina insignis (Balogh, 1970) (Ceratokalumma)

DISTRIBUCIÓN: Nueva Guinea y Filipinas.

-Achipterina kaszabi (J. y P. Balogh, 1988) (Ceratokalumma)

DisTRIBUCIÓN: Hawai.

-Achipterina naisselinei (J. y P. Balogh, 1988) (Ceratokalumma)

Distribución: Melanesia (I. Fiji).

-Achipterina oribatelloides (Berlese, 1916) (Achipteria (Achipterina))

DisTRIBUCIÓN: Somalia.

Arcozetes Hammer, 1958 (1 sp.)

EsPECIE TIPO: Arcozetes bicuspidatus Hammer, 1958

DisTRIBUCIÓN: Neotropical.

-Arcozetes bicuspidatus Hammer, 1958

DisTRIBUCIÓN: Neotropical.

Belemacarus Pérez-Iñigo y Baggio, 1997 (1 sp.)

EsPeCie TiPo: Belemacarus amazonicus Pérez-Íñigo y Baggio, 1997

DisTRIBUCIÓN: Neotropical.

-Belemacarus amazonicus Pérez-Iñigo y Balogh, 1997

DisTRIBUCIÓN: Brasil.

Cultrobates Willmann, 1930 (3 spp.)

EsPECIE TIPO: Cultrobates heterodactylus Willmann, 1930

DisTRIBUCIÓN: Neotropical y subtropical (Holártica meridional).

-Cultrobates heterodactylus Willmann, 1930

DisTRIBUCIÓN: Norte de Neotropical.

-Cultrobates niponicus Aoki, 1982

DisTRIBUCIÓN: Japón. 
-Cultrobates quadricuspidatus (Ewing, 1909) (Oribata) DisTRIBUCIÓN: U.S.A. centrooriental.

Genavensia Mahunka, 1983 (2 spp.)

EsPECIE TIPO: Genavensia hungarorum Mahunka, 1983 DisTRIBUCIÓN: Neotropical.

-Genavensia hungarorum Mahunka, 1983

DisTRIBUCIÓN: Guatemala.

-Genavensia longiseta Mahunka, 1985

DistriBución: Pequeñas Antillas.

Guaranozetes Balogh y Mahunka, 1981 (1 sp.)

EsPECIE TIPO: Guaranozetes nudus Balogh y Mahunka, 1981 DISTRIBUCIÓN: Neotropical.

-Guaranozetes nudus Balogh y Mahunka, 1981

DisTRIBUCIÓN: Neotropical (Paraguay y Uruguay).

Epactozetidae Grandjean, 1930 (2 gen. y 5 spp.) DISTRIBUCIÓN: Neotropical.

Epactozetes Grandjean, 1930 (2 spp.)

EsPECIE TIPO: Epactozetes imitator Grandjean, 1930

DisTRIBUCIÓN: Neotropical.

-Epactozetes imitator Grandjean, 1930

DisTRIBUCIÓN: Centroamérica.

-Epactozetes setosus Balogh y Mahunka, 1969

DISTRIBUCIÓN: Neotropical.

Truncozetes Balogh y Mahunka, 1969 (3 spp.)

EsPECIE TIPO: Truncozetes mucronatus Balogh y Mahunka, 1969

DisTRIBUCIÓN: Neotropical.

-Truncozetes mucronatus Balogh y Mahunka, 1969

DisTRIBUCIÓN: Neotropical.

-Truncozetes rugosus Mahunka, 1998

DisTRIBUCIÓN: Pequeñas Antillas.

-Truncozetes sturmi P. Balogh, 1984

DisTRIBUCIÓN: Neotropical (Colombia y Ecuador).

\section{Ceratozetoidea Jacot, 1925}

Heterozetidae Kunst, 1971 (2 gen. y 7 spp.)

DisTRIBUCIÓN: Cosmopolita (excepto Australiana y Antártica).

Farchacarus Wallwork, 1967 (2 spp.)

(=Africacarus Wallwork, 1965)

EsPeCIE TiPO: Africacarus calcaratus Wallwork, 1965

DistRIBUCIÓN: Paleotropical.

-Farchacarus calcaratus (Wallwork, 1965) (Africacarus)

Distribución: Etiópica (Chad y Senegal) y Macaronésica (Cabo Verde).

-Farchacarus philippinensis (Corpuz-Raros, 1979) (Hypozetes) DISTRIBUCIÓN: Filipinas.

Heterozetes Willmann, 1917 (5 spp.)

EsPECIE TIPO: Ceratozetes (Heterozetes) palustris Willmann, 1917

DisTRIBUCIÓN: Holártica, Oriental y Neotropical.

-Heterozetes aquaticus (Banks, 1895) (Oribatella)

(=Heterozetes patchlorum Habeeb, 1974)

DISTRIBUCIÓN: Neártica (frecuente).

-Heterozetes cuspidatus Willmann, 1931

DisTRIBUCIÓN: Oriental (Indonesia).

-Heterozetes heleios Behan-Pelletier, 1998

DistriBución: Costa Rica.

-Heterozetes minnesotensis (Ewing, 1913) (Oribata)

(=Heterozetes cayugensis Habeeb, 1974)

DisTRIBUCIÓN: Neártica septentrional.

-Heterozetes palustris (Willmann, 1917) (Ceratozetes (H.)) DisTRIBUCIÓN: Paleártica.
Ceratozetidae Jacot, 1925 (23 gen., 15 subg., 260 spp. y 10 sspp.) Distribución: Cosmopolita.

Ceratozetella Shaldybina, 1966 (24 spp. y 2 sspp.)

ESPECIE TIPO: Ceratozetes sellnicki Rajski, 1958

DisTRIBUCIÓn: Holártica, Neotropical y Australiana.

Ceratozetella (Ceratozetella) Shaldybina, 1966 (21 spp. y 1 ssp.)

(=Ceratozetoides Shaldybina, 1966)

(=Ceresella Paulitchenko, 1993 "sin. nov.")

DisTRIBUCIÓn: Holártica, Neotropical y Australiana.

-Ceratozetella (C.) aokii (Bayartogtokh, 1999) (Ceratozetoides)

DisTRIBUCIÓN: Mongolia.

-Ceratozetella (C.) bregetovae Shaldybina, 1970

DisTRIBUCIÓN: Paleártica meridional (Sureste de Europa y Sur de Siberia).

-Ceratozetella (C.) cisalpina (Berlese, 1908) (Ceratozetes)

DisTRIBUCión: Holártica (Europa: menos frecuente en el Oeste,

Paleártica oriental: excepto Norte, y U.S.A.: Minesota).

-Ceratozetella (C.) cuspidodenticulata (Kulijev, 1962) (Ceratozetes)

Distribución: Sur de Europa.

-Ceratozetella (C.) djaparidzae Shaldybina, 1979

Distribución: Cáucaso.

-Ceratozetella (C.) fjellbergi (Behan-Pelletier, 1986) (Ceratozetes)

DisTRIBUCIÓN: Holártica (Neártica septentrional y Europa: Ucrania).

-Ceratozetella (C.) helenae Paulitchenko, 1993

Distribución: Paleártica meridional (Ucrania y Este de Rusia asiática).

-Ceratozetella (C.) heterocuspis (Balogh y Mahunka, 1965) (Ceratozetes)

DISTRIBUCIÓN: Mongolia.

-Ceratozetella (C.) imperatoria (Aoki, 1963) (Ceratozetes)

DisTRIBUCIÓN: Paleártica oriental (excepto Norte).

-Ceratozetella (C.) imperatoria imperatoria Aoki, 1963 DistribuCión: Este de Paleártica oriental.

-Ceratozetella (C.) imperatoria magna Hammer, 1977 DisTRIBUCIÓN: Pakistán.

-Ceratozetella (C.) incurva (Aoki, 1964) (Ceratozetes)

DisTRIBUCIÓN: Hawai.

-Ceratozetella (C.) longispina Mahunka y Topercer, 1983

DisTRIBUCIÓN: Eslovaquia.

-Ceratozetella (C.) maxima (Berlese, 1908) (Ceratozetes)

(=Sphaerozetes major Irk, 1939)

(=Sphaerozetes maximus Willmann, 1953, "nom. praeoc." por Berlese, 1908)

(=Ceratozetes maximus jeannelli Trägardh, 1925)

(?=Acarus spinipes Müller, 1776 "sp. inq.")

(?=Sphaerobates subseminulum Oudemans, 1902 "sp. inq.")

DisTRIBUCIÓN: Paleártica occidental (excepto Norte).

-Ceratozetella (C.) minima (Sellnick, 1928) (Ceratozetes)

DisTRIBUCIÓN: Paleártica (Europa: menos frecuente en el Norte, y Asia centrooccidental)

-Ceratozetella (C.) redempta (Caroli y Maffia, 1934) (Ceratozetes) DisTRIBUCIÓN: Italia.

-Ceratozetella (C.) rotunda (Schweizer, 1956) (Ceratozetes)

DisTRIBUCIÓn: Suiza.

-Ceratozetella (C.) sellnicki (Rajski, 1958) (Ceratozetes)

Distribución: Paleártica (Europa: excepto Oeste, y Paleártica oriental): frecuente.

-Ceratozetella (C.) similis (Schweizer, 1956) (Ceratozetes)

DisTRIBUCIÓN: Suiza.

-Ceratozetella (C.) thienemanni (Willmann, 1943) (Ceratozetes)

(=Ceratozetes aelleni Mahunka, 1983)

DisTRIBUCIÓN: Boreal (frecuente)y Neotropical.

-Ceratozetella (C.) translamellata (Shaldybina, 1970) (Ceratozetoides)

Distribución: Paleártica oriental (excepto Este).

-Ceratozetella (C.) venusta (Paulitchenko, 1993) (Ceresella)

DisTRIBUCIÓN: Ucrania.

-Ceratozetella (C.) yezoensis Fujita y Fujikawa, 1987

DISTRIBUCIÓN: Japón. 
Ceratozetella (Cyrtozetes) Behan-Pelletier, 1985 (3 spp. y 1 ssp.)

ESPECIE TIPO: Ceratozetes shiranensis Aoki, 1976

DisTRIBUCIÓN: Holártica.

-Ceratozetella (Cyrtozetes) denaliensis (Behan-Pelletier, 1985) (Cyr.)

Distribución: Holártica (Neártica septentrional y Este de Paleártica oriental).

-Ceratozetella (Cyrtozetes) denaliensis denaliensis (Behan-

Pelletier, 1985) (Cyr.)

DistriBUCIÓN: Boreal (Neártica septentrional y Noreste de Siberia).

-Ceratozetella (Cyrtozetes) denaliensis minor (Hirauchi, 1999) (Cyr.) DisTRIBUCIÓN: Japón.

-Ceratozetella (Cyrtozetes) rectangularis (Paulitchenko, 1993) (Cyr.) Distribución: Este de Rusia asiática.

-Ceratozetella (Cyrtozetes) shiranensis (Aoki, 1976) (Ceratozetes) DISTRIBUCIÓN: Japón.

Ceratozetes Berlese, 1908 (60 spp. y 1 ssp.)

EsPeCIE TIPO: Oribata gracilis Michael, 1884

DISTRIBUCIÓN: Cosmopolita.

Ceratozetes (Ceratozetes) Berlese, 1908 (58 spp. y 1 ssp.)

DisTRIBUCIÓN: Cosmopolita.

-Ceratozetes (C.) ambiguus Behan-Pelletier, 1998

Distribución: Costa Rica.

-Ceratozetes (C.) angustus (Banks, 1947) (Minunthozetes)

DisTRIBUCión: U.S.A. (Carolina del Norte).

-Ceratozetes (C.) armatus Mihelcic, 1956

Distribución: Paleártica (Europa centromeridional y Asia centrooccidental).

-Ceratozetes (C.) bicornis Hammer, 1967

DisTRIBUCIÓN: Nueva Zelanda.

-Ceratozetes (C.) borealis Behan-Pelletier, 1984

DisTRIBUCIÓN: Neártica septentrional.

-Ceratozetes (C.) campestris Mihelcic, 1956

(=Ceratozetes obtusus Mihelcic, 1956)

DistribuCión: Mediterránea occidental.

-Ceratozetes (C.) catarinensis C. y C.,jr. Pérez-Iñigo, 1993

DisTRIBUCIÓN: Brasil.

-Ceratozetes (C.) clypeatus (Nicolet, 1855) (Oribata) "sp. inq."

DisTRIBUCIÓN: Francia.

-Ceratozetes (C.) conjunctus Mihelcic, 1956

(=Ceratozetes contiguus Jeleva, 1962)

DisTRIBUCIÓN: Mediterránea (frecuente).

-Ceratozetes (C.) cuspidatus Jacot, 1939 (C. gracilis c.)

DisTRIBUCIÓN: Neártica septentrional (U.S.A.: Nueva Hampshire, y Canadá: frecuente).

-Ceratozetes (C.) enodis (Ewing, 1909) (Oribata)

DisTRIBUCIÓN: U.S.A. centrooriental.

-Ceratozetes (C.) figuratus (Ewing, 1909) (Oribata)

DisTRIBUCIÓN: U.S.A. (Ilinois).

-Ceratozetes (C.) gaussi (Richters, 1908) (Oribata)

DisTRIBUCIÓN: Antártica.

-Ceratozetes (C.) gemmula Pérez-Íñigo jr., 1990

DisTRIBUCIÓN: España.

-Ceratozetes (C.) gracilis (Michael, 1884) (Oribata)

Distribución: Cosmopolita: Holártica, I. Santa Helena, Oriental,

Nueva Zelanda, Chile y Antártica (I. Shetland del Sur).

-Ceratozetes (C.) gracilis gracilis (Michael, 1884)

(=Ceratozetes fusiger Mihelcic, 1956)

(=Ceratozetes grandis Berlese, 1908)

Distribución: Cosmopolita: Holártica (frecuente), I. Santa

Helena, Oriental, Nueva Zelanda, Chile y Antártica (I Shetland del Sur).

-Ceratozetes (C.) gracilis minor (Schweizer, 1922) (Oribata)

DisTRIBUCIÓN: Europa centrooccidental.

-Ceratozetes (C.) guadarramicus Pérez-Íñigo, 1991

Distribución: España.

-Ceratozetes (C.) hamobatoides Hammer, 1967

DisTRIBUCIÓN: Nueva Zelanda.
-Ceratozetes (C.) insignis Balogh, 1966

Distribución: Camerún.

-Ceratozetes (C.) inupiaq Behan-Pelletier, 1986

DisTRIBUCión: Canadá.

-Ceratozetes (C.) kananaskis Mitchell, 1976

DisTRIBUCIÓN: Canadá

Ceratozetes (C.) kutchin Behan-Pelletier, 1986

DistriBución: Canadá.

-Ceratozetes (C.) lagrecai Bernini, 1973

(?=Sphaerozetes elongatissimus Berlese, 1908 "sp. inq.")

DisTRIBUCIÓN: Mediterránea.

-Ceratozetes (C.) laticuspidatus Menke, 1964

(?=Oribata myrmobates Berlese, 1903 "sp. inq.")

DISTRIBUCIÓN: Europa centromeridional (frecuente)

-Ceratozetes (C.) longispina Jacot, 1936 (C. subaquila l.)

Distribución: Este de U.S.A.

-Ceratozetes (C.) longocuspidatus Kulijev, 1962

DisTRIBUCIÓN: Sureste de Europa.

-Ceratozetes (C.) macromediocris Shaldybina, 1970

DisTRIBUCIÓN: Europa oriental.

-Ceratozetes (C.) mediocris Berlese, 1908

(=Ceratozetes dubius Mihelcic, 1956)

(=Ceratozetes japonicus Aoki, 1961)

DistribuCIÓN: Semicosmopolita: Holártica (frecuente en

Paleártica), Oriental (Vietnam) y Australiana (Nueva Zelanda).

-Ceratozetes (C.) microsetosus Ayyildiz y Luxton, 1989

DisTRIBUCIÓN: Turquía.

-Ceratozetes (C.) minutissimus Willmann, 1951

DisTRIBUCIÓN: Paleártica meridional (Europa centromeridional y

Asia centrooccidental)

-Ceratozetes (C.) monticola Hammer, 1961

DisTRIBUCIÓN: Perú.

-Ceratozetes (C.) nanus Subías, 2001

(=Ceratozetes minutissimus Subías y Arillo, 2000, "nom. praeoc." por Willmann, 1951)

DisTRIBUCión: España.

-Ceratozetes (C.) nasutus Subías, Kahwash y Ruiz, 1990

DisTRIBUCIÓN: España.

-Ceratozetes (C.) neonominatus nom. nov.

[=Ceratozetes similis Fujikawa, 1996, "nom. praeoc.” por Schweizer, 1956 ("hom. prim.")]

DisTRIBUCIÓN: Japón.

-Ceratozetes (C.) nigrisetosus Hammer, 1958

DisTRIBUCIÓN: Bolivia.

-Ceratozetes (C.) oresbios Behan-Pelletier, 1984

DisTRIBUCIÓN: Canadá.

-Ceratozetes (C.) ovidianus Vasiliu y Calugar, 1981

DisTRIBUCIÓN: Rumania.

-Ceratozetes (C.) pacificus Behan-Pelletier, 1984

Distribución: Canadá.

-Ceratozetes (C.) paritractus Hammer, 1977

DistRIBUCIÓN: Pakistán.

-Ceratozetes (C.) parvulus Sellnick, 1922 (C. (Allozetes))

DisTRIBUCIÓN: Boreal (Euroatlántica, Cáucaso y Neártica septentrional).

-Ceratozetes (C.) peritus Grandjean, 1951

DisTRIBUCIÓN: Holártica (Europa y Canadá).

-Ceratozetes (C.) petrovi Kulijev, 1962

DistribuCión: Cáucaso.

-Ceratozetes (C.) platyrhinoides Hammer, 1961

(=Magellozetes mahnerti Mahunka, 1984)

DisTRIBUCIÓN: Neotropical.

-Ceratozetes (C.) platyrhinus Hammer, 1958

DisTRIBUCIÓn: Neotropical (Bolivia y Perú).

-Ceratozetes (C.) problematicus Mahunka, 1982

DisTRIBUCIÓN: Etiopía.

-Ceratozetes (C.) psammophilus Horak, 2000

DisTRIBUCIÓN: Alemania

-Ceratozetes (C.) rostroserratus Wallwork, 1965

DistribuCión: Chad. 
-Ceratozetes (C.) rostroundulatus Paulitchenko, 1991 DISTRIBUCIÓN: Ucrania.

-Ceratozetes (C.) simulator Pérez-Íñigo, 1970 (=Ceratozetes petri Weigmann, 1976) DISTRIBUCIÓN: Mediterránea.

-Ceratozetes (C.) spitsbergensis Thor, 1934 DisTRIBUCIÓN: Boreal (Norte de Europa y Neártica septentrional.

-Ceratozetes (C.) striatus Hammer, 1958 DISTRIBUCIÓN: Argentina.

-Ceratozetes (C.) subaquila (Ewing, 1909) (Oribata) DisTRIBUCIÓN: U.S.A. nororiental.

-Ceratozetes (C.) subinconspicuus (Berlese, 1908) (Punctoribates) Distribución: U.S.A. (Washington D.C.).

-Ceratozetes (C.) undulatus Hammer, 1958 DisTRIBUCIÓN: América austral.

-Ceratozetes (C.) virginicus (Banks, 1906) (Galumna) (=Ceratozetes jeweli Rockett y Woodring, 1966) DistriBuCIÓN: Neártica.

-Ceratozetes (C.) volgini Shaldybina, 1970 DISTRIBUCIÓN: Este de Rusia asiática.

-Ceratozetes (C.) watertonensis Behan-Pelletier, 1984 DisTRIBUCIÓN: Canadá.

-Ceratozetes (C.) xinjiangensis Wen y $\mathrm{Bu}, 1988$ DisTRIBUCIÓN: Oeste de China.

-Ceratozetes (C.) zeteki (Ewing, 1917) (Trachyoribates) DisTRIBUCIÓN: U.S.A. (Ilinois).

Ceratozetes (Magellozetes) Hammer, 1962 (2 spp.)

EsPeCIE TIPO: Magellozetes processus Hammer, 1962

DisTRIBUCIÓN: América austral y Antártica.

-Ceratozetes (Magellozetes) antarcticus (Michael, 1895) (Oribata) DisTRIBUCIÓN: Antártica y América austral.

-Ceratozetes (Magellozetes) processus (Hammer, 1962) (M.)

DisTRIBUCIÓN: Antártica y América austral.

Ceratozetes (Mixozetes) J. y P. Balogh, 1990 (1 sp.)

EsPECIE TIPO: Arcozetes intermedius Covarrubias, 1967

DisTRIBUCIÓN: Neotropical.

-Ceratozetes (Mixozetes) intermedius (Covarrubias, 1967) (Arcozetes) DisTRIBUCIÓN: Chile.

Dentizetes Hammer, 1952 (2 spp.)

ESPECIE TIPO: Dentizetes rudentiger Hammer, 1952

DisTRIBUCIÓN: Neártica.

-Dentizetes ledensis Behan-Pelletier, 2000

Distribución: Canadá.

-Dentizetes rudentiger Hammer, 1952

DISTRIBUCIÓN: Canadá.

Edwardzetes Berlese, 1914 (10 spp.)

ESPECIE TIPO: Oribata edwardsi Nicolet, 1855

DISTRIBUCIÓN: Semicosmopolita (excepto paleotropical).

Edwardzetes (Edwardzetes) Berlese, 1914 (9 spp.)

DISTRIBUCIÓN: Semicosmopolita (excepto paleotropical).

-Edwardzetes (E.) andicola Hammer, 1958

DisTRIBUCIÓN: Neotropical (Argentina y Bolivia).

-Edwardzetes (E.) armatus (Hammer, 1958) (Jugatala)

DISTRIBUCIÓN: Bolivia.

-Edwardzetes (E.) australis Stary y Block, 1995

DisTRIBUCIÓN: Antártica (I. Georgia del Sur).

-Edwardzetes (E.) dentifer Hammer, 1962

DisTRIBUCIÓN: América austral.

-Edwardzetes (E.) edwardsi (Nicolet, 1855) (Oribata)

(=Notaspis edwardsi lapponicus Trägardh, 1902)

Distribución: Boreal: Euroatlántica (frecuente), Cáucaso y Groenlandia.

-Edwardzetes (E.) elongatus Wallwork, 1966

DisTRIBUCIÓN: Antártica (I. Georgia del Sur).
-Edwardzetes (E.) novazealandicus Hammer, 1967

DistribuCIÓN: Nueva Zelanda.

-Edwardzetes (E.) trilobus Mihelcic, 1957

DISTRIBUCIÓN: Austria.

-Edwardzetes (E.) ubali Iturrondobeitia y Caballero, 2000

DisTRIBUCIÓN: España.

Edwardzetes (Gamerozetes) J. y P. Balogh, 1990 (1 sp.)

ESPECIE TIPO: Magellozetes brevitutorium Covarrubias, 1967

DISTRIBUCIÓN: Neotropical austral.

-Edwardzetes (Gamerozetes) brevitutorium (Covarrubias, 1967)

(Magellozetes)

DISTRIBUCIÓN: Chile.

Euzetes Berlese, 1908 (3 spp.)

EsPECIE TIPO: Oribata globula Nicolet, 1855

DisTRIBUCIÓN: Holártica.

-Euzetes aterrimus (Koch, 1844) (Oribata) "sp. inq."

DisTRIBUCIÓN: Alemania.

-Euzetes globulus (Nicolet, 1855) (Oribata)

(=Oribata nitens Johnston, 1853, "nom. praeoc." por Gervais, 1844)

Distribución: Paleártica (Paleártica occidental: frecuente, y Este de Paleártica oriental).

-Euzetes seminulum (Müller, 1776) (Acarus) “sp. inq.”

DisTRIBUCIÓN: Alemania.

Furcobates Sellnick, 1959 (4 spp.)

ESPECIE TIPO: Oribata hastata Kramer, 1898

DisTRIBUCIÓn: Austral (excepto Etiópica) y Oriental.

Furcobates (Furcobates) Sellnick, 1959 (2 spp.)

DISTRIBUCIÓN: Neotropical austral.

-Furcobates (F.) hastatus (Kramer, 1898) (Oribata)

(=Oribates longicornutus Berlese, 1901)

DisTRIBUCIÓN: América austral.

-Furcobates (F.) lewissmithi Stary, 1995

DistRIBUCiÓN: I. Malvinas [I. Falkland].

Furcobates (Parafurcobates) Hammer, 1967 (1 sp.)

EsPeCIE TIPO: Parafurcobates cuspidatus Hammer 1967

DisTRIBUCIÓN: Australiana.

-Furcobates (Parafurcobates) cuspidatus (Hammer, 1967) (P.)

DisTRIBUCIÓN: Nueva Zelanda.

Furcobates (Pseudogeminozetes) Tseng, 1984 (1 sp.)

EsPecie TIPO: Pseudogeminozetes obliqus Tseng, 1984 DISTRIBUCIÓN: Oriental.

-Furcobates (Pseudogeminozetes) obliqus (Tseng, 1984) (P.)

DisTRIBUCIÓN: Taiwan [Formosa].

Fuscozetes Sellnick, 1928 (12 spp.)

EsPECIE TIPO: Oribata fuscipes Koch, 1844

DISTRIBUCIÓN: Holártica y Oriental.

-Fuscozetes angustus (Banks, 1910) (Oribatella)

Distribución: U.S.A. (Tejas).

-Fuscozetes bidentatus (Banks, 1895) (Oribatella)

DisTRIBUCIÓN: Neártica.

-Fuscozetes cuauhtemoctzini Palacios-Vargas y Martínez-Crespo, 1990 DisTRIBUCIÓN: Méjico.

-Fuscozetes floridae Jacot, 1935 (F. bidentatus $f$.)

DisTRIBUCIÓN: U.S.A. (Florida).

-Fuscozetes fuscipes (Koch, 1844) (Oribata)

(=Galumna slossonae Banks, 1906)

DISTRIBUCIÓN: Holártica (menos frecuente en el Sur) y Vietnam.

-Fuscozetes intermedius Caroli y Maffia, 1934

(?=Oribata rubens Koch, 1839 "sp. inq.")

DistRIBUCIÓN: Paleártica occidental (excepto Este).

-Fuscozetes novus Shaldybina, 1969

Distribucion: Sureste de Europa. 
-Fuscozetes pini Dalenius, 1964

DisTRIBUCIÓN: Suecia.

-Fuscozetes pseudosetosus Shaldybina, 1975

DisTRIBUCión: Holártica: Paleártica (Europa centrooriental y Suroeste de Siberia) y Canadá.

-Fuscozetes rotundatus Willmann, 1930

DISTRIBUCIÓN: Holanda.

-Fuscozetes setosus (Koch, 1839) (Oribata)

Distribución: Holártica (Paleártica, China: Tíbet, y Neártica nororiental).

-Fuscozetes tatricus Seniczak, 1993

DistribuCIÓN: Polonia.

Geminozetes Balogh y Csiszár, 1963 (4 spp.)

EsPeCIE TIPO: Geminozetes lineatus Balogh y Csiszár, 1963

DistribuCión: Tropical (Neotropical y Etiópica) y Neártica.

Geminozetes (Geminozetes) Balogh y Csiszár, 1963 (2 spp.)

DISTRIBUCIÓN: Tropical (Etiópica y Neotropical).

-Geminozetes (G.) lamellatus Balogh, 1966

DisTRIBUCIÓN: Chad.

-Geminozetes (G.) lineatus Balogh y Csiszár, 1963

DisTRIBUCIÓN: Argentina.

Geminozetes (Naiazetes) Behan-Pelletier, 1996 (2 spp.)

(=Selvazetes Behan-Pelletier, 1998)

EsPECIE TIPO: Naiazetes reevesi Behan-Pelletier, 1996

DISTRIBUCIÓN: Neártica y Neotropical.

-Geminozetes (Naiazetes) reevesi (Behan-Pelletier, 1996) (N.)

DisTRIBUCIÓN: Neártica.

-Geminozetes (Naiazetes) silvanus (Behan-Pelletier, 1998) (Selvazetes)

DISTRIBUCIÓN: Costa Rica.

Ghilarovizetes Shaldybina, 1969 (5 spp.)

EsPECIE TIPO: Ghilarovizetes obtusus Shaldybina, 1969

DisTRIBUCIÓN: Holártica y Etiópica.

-Ghilarovizetes africanus Mahunka, 1984

DisTRIBUCIÓN: Tanzania.

-Ghilarovizetes longisetosus (Hammer, 1952) (Melanozetes)

DisTRIBUCIÓN: Neártica septentrional (frecuente).

-Ghilarovizetes maruyamai Hirauchi, 1999

DisTRIBUCión: Japón.

-Ghilarovizetes obtusus Shaldybina, 1969

Distribución: Paleártica (Cáucaso y Paleártica oriental).

-Ghilarovizetes rostralis Shaldybina, 1969

DISTRIBUCIÓN: Asia centrooccidental.

Jugatala Ewing, 1913 (1 sp.)

ESPECIE TIPO: Jugatala tuberosa Ewing, 1913

DisTRIBUCIÓN: Holártica.

-Jugatala tuberosa Ewing, 1913

Distribución: Holártica (Este de Paleártica oriental y Noroeste de Neártica).

Lobozetes Hammer, 1958 (1 sp.)

EsPECIE TIPO: Lobozetes bilobatus Hammer, 1958

DISTRIBUCIÓN: Neotropical austral.

-Lobozetes bilobatus Hammer, 1958

DisTRIBUCIÓN: Argentina.

Macrogena Wallwork, 1966 (5 spp.)

ESPECIE TIPO: Macrogena monodactyla Wallwork, 1966

DISTRIBUCIÓN: Antártica y Australiana austral.

Macrogena (Macrogena) Wallwork, 1966 (4 spp.)

(=Lophozetes P. Balogh, 1985 "sin. nov.")

DistRIBUCIÓN: Antártica y Australiana austral.

-Macrogena (M.) crassa Hammer, 1967

DisTRIBUCIÓN: Nueva Zelanda.
-Macrogena (M.) monodactyla Wallwork, 1966

DistriBuCión: Antártica (I. Campbell)

-Macrogena (M.) rudentiger Hammer, 1967

DisTRIBUCIÓN: Nueva Zelanda.

-Macrogena (M.) truncata (P. Balogh, 1985) (Lophozetes)

DistRIBUCIÓN: Australia.

Macrogena (Safrobates) Mahunka, 1989 (1 sp.)

ESPECIE TIPO: Safrobates miniporus Mahunka, 1989

DisTRIBUCIÓN: Australiana austral.

-Macrogena (Safrobates) miniporus (Mahunka, 1989) (S.)

DISTRIBUCIÓN: Tasmania.

Melanozetes Hull, 1916 (23 spp. y 2 sspp.)

(=Alphypochthonius Schweizer, 1956)

(=Sculptozetes Mahunka, 1984)

EsPECIE TIPO: Oribata mollicomus Koch, 1839

DISTRIBUCIÓN: Holártica y Etiópica.

-Melanozetes aequalis (Schweizer, 1956) (Alphypochthonius) "sp. inq." DisTRIBUCión: Suiza.

-Melanozetes alpinus (Schweizer, 1956) (Alphypochthonius) "sp. inq." Distribución: Suiza.

-Melanozetes altaicus Shaldybina, 1969

DISTRIBUCIÓN: Asia centrooccidental.

-Melanozetes azoricus Weigmann, 1976

Distribución: I. Azores.

-Melanozetes azoricus azoricus Weigmann, 1976 DistriBución: I. Azores.

-Melanozetes azoricus floresianus Pérez-Íñigo, 1992 DisTRIBUCión: I. Azores.

-Melanozetes azoricus sanctaemariae Pérez-Íñigo, 1992 Distribución: I. Azores.

-Melanozetes crossleyi Behan-Pelletier, 2000

DistriBución: Canadá

-Melanozetes curtipilis (Schweizer, 1956) (Alphypochthonius) "sp. inq." DistriBución: Suiza.

-Melanozetes exobothridialis Bayartogtokh, 1998

DisTRIBUCIÓN: Mongolia.

-Melanozetes hermannioides (Schweizer, 1956) (Alphypochthonius)

"sp. inq."

DISTRIBUCIÓN: Suiza.

-Melanozetes interruptus Willmann, 1953

DistribuCión: Paleártica (Europa central y Noreste de Siberia).

-Melanozetes juradae (Schweizer, 1956) (Alphypochthonius) "sp. inq." DistriBución: Suiza.

-Melanozetes lischanni (Schweizer, 1956) (Alphypochthonius) "sp. inq." DisTRIBUCIÓN: Suiza.

-Melanozetes longesensillus Schweizer, 1956

DisTRIBUCIÓN: Centroeuropa.

-Melanozetes longisetosus (Mahunka, 1984) (Sculptozetes)

("non" Melanozetes longisetosus Hammer, 1952, ahora en Ghilarovizetes) DisTRIBUCIÓN: Tanzania.

-Melanozetes medius (Schweizer, 1956) (Alphypochthonius) "sp. inq." DisTRIBUCIÓN: Suiza.

-Melanzetes meridianus Sellnick, 1928

DisTRIBUCIÓN: Holártica (menos frecuente en el Sur).

-Melanozetes mollicomus (Koch, 1839) (Oribata)

DisTRIBUCIÓN: Boreal (Paleártica occidental: menos frecuente en

el Sur, Paleártica oriental: excepto Este, y Neártica septentrional).

-Melanozetes mollisimilis Schweizer, 1956

DISTRIBUCIÓN: Europa centrooriental.

-Melanozetes nivalis (Schweizer, 1956) (Alphypochthonius) "sp. inq." DisTRIBUCIÓN: Suiza.

-Melanozetes orientalis Shaldybina, 1969

DisTRIBUCIÓN: Siberia septentrional.

-Melanozetes sellnicki (Hammer, 1952) (Fuscozetes)

DisTRIBUCIÓN: Boreal (frecuente en Neártica septentrional).

-Melanozetes stagnatilis (Hull, 1914) (Oribata)

(=Oribata alpina Halbert, 1915)

DisTRIBUCIÓN: Europa noroccidental. 
- Melanozetes tanana Behan-Pelletier, 1986

DisTRIBUCIÓN: Neártica septentrional.

-Melanozetes trupchumi (Schweizer, 1956) (Alphypochthonius) “sp. inq."

DISTRIBUCIÓN: Suiza.

Murcia Koch, 1835 (47 spp. y 2 sspp.)

(=Claviceps Canestrini y Fanzago, 1877 “sin. nov.")

(=Trichoribates Berlese, 1910)

EsPECIE TIPO: Murcia trimaculata Koch, 1835

DisTRIBUCIÓN: Holártica, Neotropical y "Oriental".

-Murcia acaroides Koch, 1835 "sp. inq."

DISTRIBUCIÓN: Alemania.

-Murcia alpina (Aoki, 1982) (Trichoribates)

DisTRIBUCIÓN: Japón.

-Murcia angustata (Mihelcic, 1957) (Trichoribates)

(?=Sphaerozetes prudens Berlese, 1913 "sp. inq.")

DisTRIBUCIÓN: Mediterránea.

-Murcia austroamericana (Berlese, 1910) (Sphaerozetes (Trichori-

bates) setosus a.)

DistRIBUCIÓN: U.S.A. (Washington D.C.)

-Murcia biarea (Gjelstrup y Solhoy, 1994) (Trichoribates)

DisTRIBUCIÓN: Islandia.

-Murcia boletorum (Ewing, 1909) (Oribata)

DisTRIBUCIÓN: U.S.A. (Minesota).

-Murcia brevicuspis (Mihelcic, 1958) (Trichoribates)

(=Trichoribates longipilis Mihelcic, 1956, "nom. praeoc." por Willmann, 1951)

(=Trichoribates pilosus Mihelcic, 1966)

DisTRIBUCIÓN: Mediterránea occidental.

-Murcia caucasica (Shaldybina, 1971) (Trichoribates)

Distribución: Cáucaso.

-Murcia copperminensis Hammer, 1952

DisTRIBUCIÓN: Boreal (Paleártica oriental: excepto Este, y Neártica septentrional).

-Murcia deliensis Oudemans, 1922 "sp. inq."

DisTRIBUCIÓN: Java.

-Murcia ephippiata Koch, 1841 “sp. inq."

DisTRIBUCIÓN: Alemania.

-Murcia formosa (Banks, 1909) (Oribatella)

DisTRIBUCión: Neártica nororiental.

-Murcia fumigata Koch, 1841 "sp. inq."

DisTRIBUCión: Alemania.

-Murcia furcata (Schweizer, 1956) (Trichoribates)

DistRIBUCión: Paleártica (Europa: Suiza, y Este de Rusia asiática).

-Murcia heteroporosa (Wen y Bu, 1988) (Trichoribates)

Distribución: Oeste de China.

-Murcia hirtus (Canestrini y Fanzago, 1877) (Claviceps) "sp. inq." DisTRIBUCIÓN: Italia.

-Murcia latincisa (Ewing, 1909) (Oribata)

DisTRIBUCIÓN: U.S.A. nororiental.

-Murcia latincisa latincisa (Ewing, 1909) DisTRIBUCIÓN: U.S.A. (Ilinois).

-Murcia latincisa gigantea (Hall, 1911) (Oribata) DisTRIBUCión: U.S.A. (Connecticut).

-Murcia latirostris (Bayartogtok y Aoki, 1998) (Trichoribates) DisTRIBUCIÓN: Mongolia.

-Murcia longipilis Willmann, 1951

DISTRIBUCIÓN: Europa meridional.

-Murcia montana (Hammer, 1961) (Jugatala)

("non" Trichoribates montanus Irk, $1939=$ Murcia monticola)

DisTRIBUCIÓN: Perú.

-Murcia monticola (Trägardh, 1902) (Notaspis)

(=Trichoribates montanus Irk, 1939)

DisTRIBUCIÓN: Euroatlántica.

-Murcia myrica (Gjelstrup y Solhoy, 1994) (Trichoribates) DISTRIBUCIÓN: Islandia.

-Murcia neglecta (Kulijev, 1979) (Trichoribates)

Distribución: Cáucaso.

-Murcia nova Sellnick, 1928

Distribución: Holártica (Paleártica y U.S.A.: Virginia).
-Murcia nova nova Sellnick, 1928

DisTRIBUCión: Holártica (Paleártica: frecuente, y U.S.A.: Virginia).

-Murcia nova dentata (Mihelcic, 1957) (Trichoribates)

DisTRIBUCIÓN: España.

-Murcia obesa (Banks, 1895) (Oribatella)

(=Jugatala lamellata Ewing, 1918)

DisTRIBUCIÓN: Boreal (Finlandia y Neártica septentrional).

-Murcia oblongarea (Gjelstrup y Solhoy, 1994) (Trichoribates)

DISTRIBUCIÓN: Islandia.

-Murcia ocotlicus (Palacios-Vargas y Norton, 1984) (Trichoribates)

DisTRIBUCIÓN: Méjico.

-Murcia ogilviensis (Behan-Pelletier, 1986) (Trichoribates)

DisTRIBUCión: Canadá.

-Murcia perfecta (Banks, 1896) (Oribatella)

DisTRIBUCIÓN: Noreste de U.S.A.

-Murcia perlongum (Schweizer, 1956) (Trichoribates)

DisTRIBUCIÓN: Suiza.

-Murcia persimilis (Banks, 1906) (Galumna)

DisTRIBUCIÓN: U.S.A. (Nueva Hampshire).

-Murcia polaris (Hammer, 1953) (Trichoribates)

DISTRIBUCIÓN: Neártica noroccidental (frecuente).

-Murcia punctata (Shaldybina, 1971) (Trichoribates)

DISTRIBUCIÓN: Paleártica meridional (Europa suroriental y Sur de Paleártica oriental)

-Murcia rausensis (Aoki, 1962) (Trichoribates)

DisTRIBUCIÓN: Japón.

-Murcia rotunda (Willmann, 1953) (Jugatala)

DISTRIBUCIÓN: Europa centrooccidental.

-Murcia rotundicuspidata (Bayartogtokh y Aoki, 1998) (Trichoribates)

DisTRIBUCIÓN: Mongolia.

-Murcia ruber (Canestrini y Fanzago, 1877) (Claviceps) "sp. inq."

DISTRIBUCIÓN: Italia.

-Murcia serrata (C. y C., jr. Pérez-Íñigo, 1993) (Trichoribates)

DISTRIBUCIÓN: Brasil.

-Murcia setiger (Trägardh, 1910) (Oribata piriformis s.)

DisTRIBUCIÓN: Norte de Europa.

-Murcia spatulasetosa (Reeves, 1967) (Trichoribates)

DisTRIBUCIÓN: U.S.A. (Nueva York).

-Murcia spinogenuala (Ewing, 1909) (Oribata)

DisTRIBUCIÓN: U.S.A. (Ilinois).

-Murcia striata (Hammer, 1952) (Trichoribates)

DisTRIBUCIÓN: Neártica septentrional.

-Murcia tectopedacuta Thor, 1937 “sp. inq."

DistriBución: Noruega.

-Murcia tepetlensis (Palacios-Vargas y Norton, 1984) (Trichoribates) Distribución: Méjico.

-Murcia tianshanensis (Wen y $\mathrm{Bu}, 1988$ ) (Trichoribates)

Distribución: Oeste de China.

-Murcia tjanshanica (Shaldybina, 1971) (Trichoribates)

DISTRIBUCIÓN: Asia centrooccidental.

-Murcia trimaculata Koch, 1835

(=Sphaerozetes (Trichoribates) berlesei Jacot, 1929)

DisTRIBUCIÓN: Holártica (frecuente en Paleártica).

Neogymnobates Ewing, 1917 (6 spp. y 1 ssp.)

EsPECIE TIPO: Oribata multipilosa Ewing, 1907

DISTRIBUCIÓN: Holártica.

Neogymnobates (Neogymnobates) Ewing, 1917 (5 spp. y 1 ssp.)

(=Boreozetes Hammer, 1955)

DisTRIBUCIÓN: Holártica.

-Neogymnobates (N.) capitatus Wang y Solhoy, 2001

DisTRIBUCiÓN: China (Tíbet).

-Neogymnobates (N.) donghaksaensis (Choi, 1986) (Boreozetes)

DisTRIBUCIÓN: Corea.

-Neogymnobates (N.) luteus (Hammer, 1955) (Boreozetes)

DisTRIBUCIÓN: Holártica (Corea y Neártica septentrional).

-Neogymnobates (N.) luteus luteus (Hammer, 1955) DisTRIBUCIÓN: Neártica septentrional. 
-Neogymnobates (N.) luteus asiaticus Aoki, 1974 DISTRIBUCIÓN: Corea.

-Neogymnobates (N.) marilynae Behan-Pelletier, 2000

DisTRIBUCIÓN: Canadá

-Neogymnobates (N.) multipilosus (Ewing, 1907) (Oribata)

DisTRIBUCIÓN: U.S.A. (Ilinois).

Neogymnobates (Koreozetes) Aoki, 1974 (1 sp.)

EsPECIE TIPO: Koreozetes parvisetiger Aoki, 1974

DISTRIBUCIÓN: Paleártica.

-Neogymnobates (Koreozetes) parvisetiger (Aoki, 1974) (K.)

DisTRIBUCIÓN: Corea.

Onychobates Hammer, 1967 (1 sp.)

ESPECIE TIPO: Onychobates nidicola Hammer, 1967

DISTRIBUCIÓN: Australiana.

-Onychobates nidicola Hammer, 1967

DistriBUCIón: Nueva Zelanda.

Oromurcia Thor,1930 (3 spp.)

EsPecIE TIPO: Oromurcia bicuspidata Thor, 1930

DisTRIBUCIÓN: Holártica.

-Oromurcia bicuspidata Thor, 1930

DistRiBución: Boreal (Norte de Europa: frecuente, Noroeste de Siberia y Groenlandia).

-Oromurcia lucens (Koch, 1879) (Oribata)

(=Notaspis setosa curta Trägardh, 1902)

(=Claviceps trimaculatus L. Koch, 1879)

DisTRIBUCIÓN: Boreal.

-Oromurcia sudetica Willmann, 1939

(?=Trichoribates lucens distinguendus Berleser, 1923 "sp. inq.")

DISTRIBUCIÓN: Europa centromeridional.

Pentazetes J. y P. Balogh, 1983 (2 spp.)

EsPecie TiPo: Pentazetes mandjeliae J. y P. Balogh, 1983

DISTRIBUCIÓN: Australiana.

Pentazetes (Pentazetes) J. y P. Balogh, 1983 (1 sp.)

DisTRIBUCIÓN: Australiana.

-Pentazetes (P.) mandjeliae J. y P. Balogh, 1983

DisTRIBUCIÓN: Nueva Caledonia.

Pentazetes (Porallozetes) J. y P. Balogh, 1992 (1 sp.)

EsPecIE TIPO: Allozetes dispar Hammer, 1973

DisTRIBUCIÓN: Australiana.

-Pentazetes (Porallozetes) dispar (Hammer, 1973) (Allozetes)

DisTRIBUCIÓN: Polinesia (I. Tonga).

Scotiazetes Wallwork, 1966 (5 spp.)

ESPECIE TIPO: Scotiazetes bidens Wallwork, 1964

DisTRIBUCIÓN: Holártica, Neotropical y Antártica.

Scotiazetes (Scotiazetes) Wallwork, 1966 (3 spp.)

DistriBuCión: Paleártica, Neotropical y Antártica

-Scotiazetes (S.) aragonensis (Pérez-Íñigo jr., Herrero y Pérez-Íñigo,

1988) (Ceratozetes)

DistRIBUCión: Suroeste de Europa.

-Scotiazetes (S.) bidens Wallwork, 1966

DisTRIBUCión: Antártica (I. Georgia del Sur).

-Scotiazetes (S.) cuspidatus (Covarrubias, 1967) (Porozetes)

DisTRIBUCIÓN: Chile.

Scotiazetes (Guatemalozetes) Mahunka, 1979 (2 spp.)

EsPECIE TIPO: Guatemalozetes aelleni Mahunka, 1979

DisTRIBUCIÓN: Neártica y Neotropical.

-Scotiazetes (Guatemalozetes) aelleni (Mahunka, 1979) (G.)

DistriBución: Guatemala.

-Scotiazetes (Guatemalozetes) danos (Behan-Pelletier y Rjabinin,

1991) $(G$.)

DISTRIBUCIÓN: Neártica.
Sphaerozetes Berlese, 1885 (18 spp. y 1 ssp.)

ESPECIE TIPO: Oribata orbicularis Koch, 1835

DistribuCión: Semicosmopolita (Holártica, Neotropical, Australiana y

Antártica).

Sphaerozetes (Sphaerozetes) Berlese, 1885 (16 spp. y 1 ssp.)

(=Incudozetes J. y P. Balogh, 2002)

(=Sphaerozetella Jacot, 1929)

DISTRIBUCIÓN: Holártica, Neotropical y Australiana.

-Sphaerozetes (S.) arcticus Hammer, 1952

Distribución: Boreal (Norte de Rusia europea, Siberia centrooccidental: frecuente, y Neártica septentrional: frecuente).

-Sphaerozetes (S.) castaneus Hammer, 1955

DisTRIBUCión: Alaska.

-Sphaerozetes (S.) chavinensis (Hammer, 1961) (Jugatala)

Distribución: Perú.

-Sphaerozetes (S.) clathratus (Hammer, 1967) (Magellozetes)

DistriBUCión: Nueva Zelanda.

-Sphaerozetes (S.) comelicensis Lombardini, 1963

DisTRIBUCIÓN: Italia.

-Sphaerozetes (S.) firthensis Behan-Pelletier, 1986

Distribución: Canadá.

-Sphaerozetes (S.) globularis Lombardini, 1963 "sp. inq."

DisTRIBUCIÓN: Italia.

-Sphaerozetes (S.) globularis globularis Lombardini, 1963 DisTRIBUCIÓN: Italia.

-Sphaerozetes (S.) globularis neonominatus nom. nov.

[=Sphaerozetes globularis maior Lombardini, 1963, "nom. praeoc." por Irk, 1939 (“hom. prim.")]

DISTRIBUCIÓN: Italia.

-Sphaerozetes (S.) maior Irk, 1939

DisTRIBUCIÓN: Austria.

-Sphaerozetes (S.) orbicularis (Koch, 1835) (Oribata)

(=Sphaerozetes italiensis Jacot, 1939)

DisTRIBUCIÓN: Paleártica (excepto Este de Paleártica oriental): frecuente.

-Sphaerozetes (S.) perezinigoi Gómez-Llusá, 1988

DisTRIBUCIÓN: España.

-Sphaerozetes (S.) piriformis (Nicolet, 1855) (Oribata)

Distribución: Paleártica (Europa: frecuente, y Noroeste de Siberia).

-Sphaerozetes (S.) rostratus (Covarrubias, 1967) (Porozetes)

Distribución: Chile.

-Sphaerozetes (S.) shogranensis Hammer, 1977

DistriBuCIÓN: Pakistán.

-Sphaerozetes (S.) subintectus Sellnick, 1931 "sp. inq."

DisTRIBUCIÓN: Grecia.

-Sphaerozetes (S.) tricuspidatus Willmann, 1923

DistRIBUCIÓN: Paleártica (Europa y Asia central rusa).

-Sphaerozetes (S.) winchesteri Behan-Pelletier, 2000

DisTRIBUCIÓN: Canadá

Sphaerozetes (Porozetes) Hammer, 1962 (2 spp.)

EsPECIE TIPO: Porozetes polygonalis Hammer, 1962

DisTRIBUCIÓN: América austral y Antártica.

-Sphaerozetes (Porozetes) polygonalis (Hammer, 1962) (P.)

Distribución: Antártica y América austral (Tierra del Fuego).

-Sphaerozetes (Porozetes) quadrilobatus (Wallwork, 1966) (P. polygonalis $q$.)

DistRIBUCión: Antártica y América austral (Chile).

Tutorozetes Hammer, 1967 (1 sp.)

EsPeCIE TIPO: Tutorozetes termophilus Hammer, 1967

DisTRIBUCIÓN: Australiana austral.

-Tutorozetes termophilus Hammer, 1967

DistriBUCIÓN: Australiana (Nueva Zelanda y Tasmania).

Viracochiella Hammer, 1961 (11 spp. y 1 ssp.)

ESPECIE TIPO: Viracochiella tuberculata Hammer, 196

DisTRIBUCIÓN: Semicosmopolita (excepto paleotropical). 
Viracochiella (Viracochiella) Hammer, 1961 (2 spp.)

(=Leebates Balogh y Mahunka, 1996 "sin. nov.")

DisTRIBUCIÓN: Tropical (Neotropical y Australiana)

-Viracochiella (V.) bornemisszai (Balogh y Mahunka 1996) (Leebates) DisTRIBUCIÓN: Australia.

-Viracochiella (V.) tuberculata Hammer, 1961

DisTRIBUCIÓN: Perú.

Viracochiella (Laminizetes) Behan-Pelletier, 1986 (1 sp.)

EsPECIE TIPO: Laminizetes fortispinosus Behan-Pelletier, 1986 DisTRIBUCIÓN: Neártica.

-Viracochiella (Laminizetes) fortispinosus (Behan-Pelletier, 1986) (L.) DisTRIBUCIÓN: Canadá.

Viracochiella (Latilamellobates) Shaldybina, 1971 (8 spp. y 1 ssp.)

(=Trichoribatella Mahunka, 1983)

(=Vicinebates Paulitchenko, 1991 "sin. nov.")

EsPECIE TIPO: Oribata incisella Kramer, 1897

DisTRIBUCIÓN: Holártica.

-Viracochiella (Latilamellobates) algarvensis (Subías y Gil-Martín, 1990) (L.)

DISTRIBUCIÓN: Suroeste de Europa.

-Viracochiella (Latilamellobates) baloghi (Mahunka, 1983) (Trichoribatella)

DisTRIBUCión: Holártica (Europa: Hungría, y Canadá).

-Viracochiella (Latilamellobates) clavata (Mihelcic, 1956) (Trichoribates)

DisTRIBUCIÓN: Mediterránea.

-Viracochiella (Latilamellobates) columbreti (Mínguez y Subías, 1986) (L.)

DisTRIBUCIÓN: España (I. Columbretes).

-Viracochiella (Latilamellobates) incisella (Kramer, 1897) (Oribata)

DisTRIBUCIÓN: Paleártica

-Viracochiella (Latilamellobates) incisella incisella (Kramer, 1897)

(=Sphaerozetes (Trichoribates) oxypterus Berlese, 1910)

(=Sphaerozetes (Trichoribates) oxypterus meridionalis Berlese, 1910)

(=Trichoribates setosus apenninicus Berlese, 1923)

DisTRIBUCIÓN: Paleártica (frecuente).

-Viracochiella (Latilamellobates) incisella rotundata (Willmann, 1939) (Trichoribates)

DISTRIBUCIÓN: Europa central.

-Viracochiella (Latilamellobates) latilamellata (Mihelcic, 1956)

(Trichoribates)

(=Trichoribates latilamellatus angustissima Mihelcic, 1957)

DisTRIBUCIÓN: Mediterránea (frecuete).

-Viracochiella (Latilamellobates) naltschicki (Shaldybina, 1971) (L.) DistriBución: Sureste de Europa.

-Viracochiella (Latilamellobates) sergienkoae (Paulitchenko, 1991)

(Vicinebates)

DISTRIBUCIÓN: Ucrania.

Zetomimus Hull, 1916 (12 spp.)

EsPECIE TIPO: Oribata furcata Warburton y Pearce, 1905

DISTRIBUCIÓN: Holártica y Neotropical.

Zetomimus (Zetomimus) Hull, 1916 (8 spp.)

(=Hamobates Hammer, 1962)

DisTRIBUCIÓN: Holártica y Neotropical.

-Zetomimus (Z.) boothianus (Hull, 1915) (Oribata)

DISTRIBUCIÓN: I. Británicas.

-Zetomimus (Z.) brevis Ohkubo, 1987

DISTRIBUCIÓN: Japón.

-Zetomimus (Z.) cristatus (Hammer, 1962) (Hamobates)

DistriBución: Chile.

-Zetomimus (Z.) francisi (Habeeb, 1974) (Ceratozetes)

DisTRIBUCIÓN: Neártica septentrional.
-Zetomimus (Z.) furcatus (Warburton y Pearce, 1905) (Oribata)

(=Ceratozetes argentinensis Hammer, 1958)

(?=Oribata avenifera Michael, 1884 "sp. inq.")

(=Ceratozetes magnificus Berlese, 1910)

Distribución: Paleártica occidental (menos frecuente en el

Norte) y Argentina.

-Zetomimus (Z.) naias Behan-Pelletier, 1998

DisTRIBUCIÓN: Norte de Neotropical.

-Zetomimus (Z.) setosus (Banks, 1895) (Oribatella)

DisTRIBUCIÓN: Neártica septentrional.

-Zetomimus (Z.) spinosus (Hammer, 1962) (Hamobates)

DISTRIBUCION: Chile.

Zetomimus (Protozetomimus) Pérez-Íñigo, 1990 (4 spp.)

EsPeCIE TIPO: Ceratozetes acutirostris Mihelcic, 1957

DISTRIBUCIÓN: Paleártica y Neotropical.

-Zetomimus (Protozetomimus) acutirostris (Mihelcic, 1957) (Ceratozetes)

Distribución: Sur de Europa.

-Zetomimus (Protozetomimus) bulanovae (Kulijev, 1962) (Ceratozetes)

DisTRIBUCIÓN: Sureste de Europa.

-Zetomimus (Protozetomimus) kirgisicus (Shaldybina, 1970) (Ceratozetella)

DisTRIBUCIÓN: Paleártica oriental.

-Zetomimus (Protozetomimus) polpaicoensis (Hammer, 1962) (Ceratozetes)

Distribución: Chile.

Chamobatidae Thor, 1937 (5 gen., 1 subg. y 42 spp.) DisTRIBUCIÓN: Cosmopolita (excepto Antártica).

Chamobates Hull, 1916 (31 spp.)

ESPECIE TIPO: Oribata cuspidata Michael, 1884

DISTRIBUCIÓN: Holártica y Paleotropical.

Chamobates (Chamobates) Hull, 1916 (24 spp.)

DisTRIBUCIÓN: Holártica y Paleotropical.

-Chamobates (C.) alpinus Schweizer, 1956 (C. cuspidatus a.)

DISTRIBUCIÓN: Europa centromeridional.

-Chamobates (C.) apathyi (Tafner, 1905) (Oribata) "sp. inq."

DisTRIBUCIÓN: Hungría.

-Chamobates (C.) bispinosus Mahunka, 1987

DisTRIBUCIÓN: Hungría.

-Chamobates (C.) caucasicus Shaldybina, 1969

DisTRIBUCión: Sureste de Europa.

-Chamobates (C.) confusus Subías, 2000

(?=Chamobates angulatus Mihelcic, 1957 "sp. inq.")

(?=Chamobates triangularis Mihelcic, 1957 "sp. inq.")

DisTRIBUCIÓn: Paleártica occidental (excepto Norte): frecuente, Este de Paleártica oriental y Alaska.

-Chamobates (C.) cuspidatiformis (Trägardh, 1904) (Notaspis)

DisTRIBUCIÓN: Europa.

-Chamobates (C.) cuspidatus (Michael, 1884) (Oribata)

(=Oribata dominae Hull, 1914)

(=Oribata lucifer Hull, 1914)

DisTRIBUCiÓN: Holártica (frecuente en Paleártica) e I. Seychelles.

-Chamobates (C.) dentatus Mihelcic, 1956

DisTRIBUCIÓN: Mediterránea.

-Chamobates (C.) dentotutorii Shaldybina, 1969

Distribución: Paleártica meridional (Sur de Europa oriental y Asia centrooccidental)

-Chamobates (C.) egenus (Berlese, 1910) (Euzetes)

DisTRIBUCIÓN: U.S.A. (Florida).

-Chamobates (C.) geminus Fujikawa, 1997

DisTRIBUCIÓN: Japón.

-Chamobates (C.) hauseri Mahunka, 1980

DisTRIBUCIÓN: Marruecos.

-Chamobates (C.) Ilinoisensis (Ewing, 1909) (Oribata) "sp. inq." DisTRIBUCIÓN: U.S.A. (Ilinois). 
-Chamobates (C.) interpositus Pschorn-Walcher, 1953

DisTRIBUCIÓN: Europa central.

-Chamobates (C.) javensis (Hammer, 1979) (Sphaerozetes)

(=Unguizetes hammerae J. y P. Balogh, 2002)

DisTRIBUCIÓN: Java.

-Chamobates (C.) lapidarius (Lucas, 1849) (Oribata) "sp. inq."

DisTRIBUCIÓN: Argelia.

-Chamobates (C.) longipilis Willmann, 1953

DisTRIBUCIÓN: Austria.

-Chamobates (C.) pedemontanus (Berlese, 1910) (Sphaerozetes) “sp. inq."

DISTRIBUCIÓN: Italia.

-Chamobates (C.) perezinigoi Subías, 1977

DisTRIBUCIÓN: Suroeste de Europa.

-Chamobates (C.) pusillus (Berlese, 1895) (Oribata)

(=Notaspis cuspidatus borealis Trägardh, 1902)

(=Chamobates incisus Hammen, 1952)

DisTRIBUCIÓN: Paleártica (frecuente).

-Chamobates (C.) schuetzi (Oudemans, 1902) (Notaspis)

DisTRIBUCIÓN: Holártica (Paleártica occidental: frecuente, Este de Rusia asiática y U.S.A.: Virginia y Alaska).

-Chamobates (C.) subglobulus (Oudemans, 1900) (Notaspis)

(?=Oribata facula Koch, 1839 "sp. inq.")

(=Chamobates nasutus Mihelcic, 1957)

(=Euzetes nigerrimus Berlese, 1908)

(=Oribata omissa Warburton y Pearce, 1905)

Distribución: Paleártica (Paleártica occidental: frecuente, y Este de Rusia asiática)

-Chamobates (C.) tumidisetosus Monson, 2002

DisTRIBUCIÓN: I. Británicas.

-Chamobates (C.) vartismithi Hull, 1916 "sp. inq."

DISTRIBUCIÓN: I. Británicas.

Chamobates (Xiphobates) Paulitchenko, 1993 (7 spp.)

ESPECIE TIPO: Notaspis voigtsi Oudemans, 1902

DisTRIBUCIÓN: Paleártica.

-Chamobates (Xiphobates) callipygis Paulitchenko, 1991 (C.)

DISTRIBUCIÓN: Ucrania.

-Chamobates (Xiphobates) dactyloscopicus Bernini y Mahunka, 1982

(C.)

DisTRIBUCión: Hungría.

-Chamobates (Xiphobates) depauperatus (Berlese, 1886) (Oribata)

DisTRIBUCIÓN: Mediterránea.

-Chamobates (Xiphobates) kieviensis Shaldybina, 1980 (C.)

DisTRIBUCIÓN: Sur de Europa.

-Chamobates (Xiphobates) rastratus (Hull, 1914) (Oribata)

(=Chamobates spinosus Sellnick, 1928) (C.)

DisTRIBUCIÓN: Paleártica (Europa: frecuente, y Siberia centrooccidental).

-Chamobates (Xiphobates) sergienkae Shaldybina, $1980(C$.) DisTRIBUCIÓN: Ucrania.

-Chamobates (Xiphobates) voigtsi (Oudemans, 1902) (Notaspis) Distribución: Paleártica (Europa: frecuente, y Siberia centrooccidental).

Globozetes Sellnick, 1928 (4 spp.)

ESPECIE TIPO: Globozetes longipilus Sellnick, 1928

DisTRIBUCIÓN: Paleártica.

-Globozetes birulai (Kulczynski, 1902) (Notaspis)

(=Chamobates tricuspidatus Willmann, 1953)

DisTRIBUCIÓN: Paleártica (Europa y Noroeste de Siberia).

-Globozetes longipilus Sellnick, 1928

(?=Euzetes paolii Berlese, 1910 "sp. inq.")

DISTRIBUCIÓN: Europa centromeridional.

-Globozetes microtus Shaldybina, 1969

DistriBución: Cáucaso.

-Globozetes petrinjensis (Willmann, 1940) (Chamobates)

DisTRIBUCIÓN: Sur de Europa
Iugoribates Sellnick, 1944 (2 spp.)

EsPECIE TIPO: Iugoribates gracilis Sellnick, 1944

DisTRIBUCIÓN: Holártica.

-Iugoribates cornutus Mínguez, 1981

(=Chamobates incisus Mihelcic, 1957, "nom. praeoc." por Hammen, 1952)

(=Chamobates mihelcici Paulitchenko, 1992)

DISTRIBUCIÓN: Sur de Europa.

-Iugoribates gracilis Sellnick, 1944

DisTRIBUCIÓN: Neártica septentrional (frecuente).

Ocesobates Aoki, 1965 (3 spp.)

(=Chamozetes Sellnick, 1974)

(=Danobates Gjelstrup, 1978)

EsPecie TiPo: Ocesobates kumadai Aoki, 1965

DisTRIBUCIÓN: Paleártica.

-Ocesobates boedvarssoni (Sellnick, 1974) (Chamozetes)

(=Danobates insignitus Gjelstrup, 1978)

DISTRIBUCIÓN: Norte de Europa.

-Ocesobates bregetovae Shaldybina, 1974

Distribución: Paleártica meridional (España y Este de Rusia asiática).

-Ocesobates kumadai Aoki, 1965

DISTRIBUCIÓN: Japón.

Pedunculozetes Hammer, 1962 (2 spp.)

EsPeCIE TIPO: Pedunculozetes andinus Hammer, 1962

DisTRIBUCIÓN: Subtropical austral (excepto Etiópica).

-Pedunculozetes andinus Hammer, 1962

DistribUCión: Subtropical austral (Nueva Zelanda y América austral)

-Pedunculozetes minutus Hammer, 1967

DisTRIBUCIÓN: Nueva Zelanda.

Humerobatidae Grandjean, 1970 (6 gen., 1 subg., 64 spp. y 7 sspp.) DISTRIBUCIÓN: Cosmopolita.

Africoribates Evans, 1953 (9 spp.)

ESPECIE TIPO: Africoribates ornatus Evans, 1952

DISTRIBUCIÓN: Etiópica.

-Africoribates australis Mahunka, 1985

DisTRIBUCIÓN: Sudáfrica.

-Africoribates depilatus (Berlese, 1910) (Peloribates)

DisTRIBUCIÓN: Sudáfrica.

-Africoribates evansi Balogh, 1959

DistriBución: Angola.

-Africoribates glabratus (Berlese, 1908) (Peloribates)

DisTRIBUCIÓN: Sudáfrica.

-Africoribates macfarlanei Balogh, 1959

DisTRIBUCIÓN: Angola.

-Africoribates maximus Mahunka, 1984

DistRIBUCIÓN: Tanzania.

-Africoribates ornatus Evans, 1953

DisTRIBUCión: Tanzania.

-Africoribates pilosus (Mahunka, 1985) (Kilimabates)

DisTRIBUCIÓN: Sudáfrica.

-Africoribates polygonatus (Mahunka, 1985) (Kilimabates)

DisTRIBUCIÓN: Sudáfrica.

-Africoribates undulatus Balogh, 1959

DisTRIBUCIÓN: Tanzania.

Anellozetes Hammer, 1962 (11 spp.)

(=Kilimabates Mahunka, 1984)

EsPeCIE TIPO: Anellozetes muscicola Hammer, 1962

DistRIBUCIÓN: Pantropical (excepto Oriental) y subtropical.

-Anellozetes auriculatus (Mahunka, 1984) (Kilimabates) DisTRIBUCIÓN: Tanzania.

-Anellozetes bicuspidatus (Mahunka, 1985) (Kilimabates)

DisTRIBUCIÓN: Sudáfrica.

-Anellozetes discifer Hammer, 1973

Distribución: Polinesia (I. Samoa). 
-Anellozetes fusiformis Hammer, 1970

DisTRIBución: I. de Pascua (I. Easter).

-Anellozetes intermedius Hammer, 1967

DisTRIBUCIÓN: Nueva Zelanda.

-Anellozetes longicaulis Hammer, 1967

DisTRIBUCIÓN: Nueva Zelanda.

-Anellozetes luteus Hammer, 1967

DisTRIBUCIÓN: Nueva Zelanda.

-Anellozetes muscicola Hammer, 1962

DisTRIBUCIÓN: Argentina.

-Anellozetes neonominatus nom. nov.

[=Kilimabates translamellatus Mahunka, 1985, "nom. praeoc." po

Shaldybina, 1971 ("hom. sec.")]

DisTRIBUCIÓN: Sudáfrica.

-Anellozetes processus (Mahunka, 1984) (Kilimabates)

DisTRIBUCIÓN: Tanzania.

-Anellozetes translamellatus (Shaldybina, 1971) (Humerobates)

DISTRIBUCIÓN: Asia centrooccidental.

Diapterobates Grandjean, 1936 (22 spp. y 4 sspp.)

EsPECIE TIPO: (Sphaerozetes (Trichoribates) numerosus Sellnick, 1924)

=Notaspis humeralis Hermann, 1804

DISTRIBUCIÓN: Holártica.

-Diapterobates altimontanus Hammer, 1977

DISTRIBUCIÓN: Pakistán.

-Diapterobates arnoldii Krivolutsky, 1966

DISTRIBUCIÓN: Paleártica meridional (Cáucaso y Asia centrooccidental).

-Diapterobates bogduulensis Bayartogtokh y Aoki, 1998 DisTRIBUCIÓN: Mongolia.

-Diapterobates crinitus Pankov, 1982

DisTRIBUCIÓN: I. Kuriles.

-Diapterobates dubinini Shaldybina, 1971

DisTRIBUCIÓN: Europa Oriental.

-Diapterobates humeralis (Hermann, 1804) (Notaspis)

(=Sphaerozetes (Trichoribates) numerosus Sellnick, 1924)

(=Oribata picipes Koch, 1839)

DistriBución: Holártica (frecuente en Paleártica).

-Diapterobates izuensis Suzuki, 1971

DistRIBUCión: Este de Paleártica oriental.

-Diapterobates japonicus Aoki, 1982

DisTRIBUCIÓN: Japón.

-Diapterobates montivagus (Hull, 1914) (Oribata) "sp. inq." DISTRIBUCIÓN: I. Británicas.

-Diapterobates nayoroensis Fujikawa, 1984 DisTRIBUCIÓN: Japón.

-Diapterobates notatus (Thorell, 1871) (Oribata)

(=Claviceps bimaculatus L. Koch, 1879)

(=Sphaerozetes strandi Berlese, 1910)

DISTRIBUCIÓN: Boreal (frecuente).

-Diapterobates oblongus (L. Koch, 1879) (Oppia)

DistRIBUCión: Paleártica: Europa (excepto Oeste) y Paleártica oriental.

-Diapterobates oromurcii Shaldybina, 1970

DisTRIBUCIÓN: Asia centooccidental.

-Diapterobates pusillus Aoki, 1969

DistriBución: Este de Paleártica oriental.

-Diapterobates pusillus pusillus Aoki, 1969

Distribución: Este de Paleártica oriental.

-Diapterobates pusillus cuspidatus Choi, 1986 DISTRIBUCIÓN: Corea.

-Diapterobates reticulatus (L. Koch, 1879) (Oribata)

(=Sphaerozetes (Trichoribates) principalis Berlese, 1914)

DisTRIBUCIÓN: Holártica (Europa meridional, "Siberia” y Canadá).

-Diapterobates rostralis Shaldybina, 1971

DisTRIBUCión: Paleártica (Sureste de Europa y Este de Paleártica oriental).

-Diapterobates rostrotuberculatus Shaldybina, 1971

DisTRIBUCIÓN: Paleártica (Ucrania y Asia centrooccidental).
-Diapterobates rotundocuspidatus Shaldybina, 1970

(=Diapterobates siccatus Behan-Pelletier, 1986)

DisTRIBUCIÓN: Boreal (Noreste de Siberia y Neártica septentrional).

-Diapterobates sitnikovae Shaldybina, 1970

DisTRIBUCIÓn: Paleártica oriental (excepto Este) y "Alaska".

-Diapterobates songliensis Choi, 1986

DISTRIBUCIÓN: Corea.

-Diapterobates unimaculatus (Banks, 1906) (Galumna)

DisTRIBUCIÓN: U.S.A. nororiental.

-Diapterobates variabilis Hammer, 1955

DistriBUCión: Boreal (excepto Europa occidental) y Nepal.

-Diapterobates variabilis variabilis Hammer, 1955

DISTRIBUCIÓN: Boreal (excepto Europa occidental): frecuente.

-Diapterobates variabilis altissimus Piffl, 1971 DisTRIBUCIÓN: Nepal.

-Diapterobates variabilis honshuensis Aoki, 1982 DisTRIBUCIÓN: Japón.

-Diapterobates variabilis yezoensis Aoki, 1982 DISTRIBUCIÓN: Japón.

Humerobates Sellnick, 1928 (16 spp. y 3 sspp.)

ESPECIE TIPO: (Notaspis humeralis Hermann "sensu" Sellnick, 1928)

=Humerobates rostrolamellatus Grandjean, 1936

DISTRIBUCIÓN: Cosmopolita.

Humerobates (Humerobates) Sellnick, 1928 (15 spp. y 3 sspp.)

(=Antarctozetes Balogh, 1961)

(=Baloghobates Hammer, 1967)

(=Banksinus Jacot, 1938)

(=Jeannelia Dalenius, 1958, "nom. praeoc." por Raffray, 1913)

(=Trihumerozetes Sellnick, 1959 "sin. nov.")

DISTRIBUCIÓN: Cosmopolita.

-Humerobates (H.) africanus (Mahunka, 1984) (Baloghobates)

Distribución: Tanzania.

-Humerobates (H.) cornutus (Sellnick, 1959) (Trihumerozetes)

DistriBución: Polinesia: I. Australes [I. Tubuai].

-Humerobates (H.) crozetensis (Richters, 1908) (Oribata)

DISTRIBUCIÓN: Antártica.

-Humerobates (H.) flechtmanni C. y C., jr. Pérez-Íñigo, 1993

DisTRIBUCIÓN: Brasil.

-Humerobates (H.) fungorum (Linnaeus, 1758) (Acarus) "sp. inq."

DisTRIBUCIÓN: Suecia.

-Humerobates (H.) nudus (Hammer, 1967) (Baloghobates)

DistRiBución: Nueva Zelanda, Corea y Taiwan [Formosa].

-Humerobates (H.) papuanus Balogh, 1970

DistriBución: Nueva Guinea.

-Humerobates (H.) parvoglobosus (Hammer, 1967) (Baloghobates)

DisTRIBUCIÓN: Nueva Zelanda.

-Humerobates (H.) perkinsi Jacot, 1934 (H. humeralis p.)

Distribución: Australiana (Islas del Pacífico).

-Humerobates (H.) pomboi Pérez-Íñigo, 1992

DisTRIBUCIÓN: I. Azores.

-Humerobates (H.) rostrolamellatus Grandjean, 1936

(?=Oribata arborea Banks, 1895 "sp. inq.")

(=Sphaerobates dentatus Mihelcic, 1957)

(?=Murcia rubra Koch, 1841 "sp. inq.")

DistribuCIÓn: Semicosmopolita (Holártica: excepto Paleártica oriental, Etiópica, Islas del Pacífico y Méjico).

-Humerobates (H.) rostrolamellatus rostrolamellatus Grandjean, 1936

DistribUCión: Semicosmopolita (Paleártica occidental: menos frecuente en el Norte, Neártica, Etiópica, Islas de Pacífico y Méjico).

-Humerobates (H.) rostrolamellatus giganteus Willmann, 1939 (H.

fungorum $g$.)

DisTRIBUCIÓN: Macaronésica (Madeira).

-Humerobates (H.) rostrolamellatus guadarramicus Pérez-Íñigo, 1972

(?=Chamobates magnus Mihelcic, 1957 "sp. inq.")

DistriBUCIÓN: Mediterránea occidental. 
-Humerobates (H.) rostrolamellatus mauritius Jacot, $1936(H$ humeralis $m$.)

DisTRIBUCIÓN: Malgache (I. Mauricio).

-Humerobates (H.) setosus Behan-Pelletier y Mahunka, 1993

DisTRIBUCión: Sudáfrica.

-Humerobates (H.) taiwanensis Tseng, 1984

DisTRIBUCIÓN: Taiwan [Formosa]

-Humerobates (H.) varius Ohkubo, 1982

DisTRIBUCIÓN: Japón.

-Humerobates (H.) zumpti Sellnick, 1957

DISTRIBUCIÓN: Namibia.

Humerobates (Cordylobates) Luxton, 1995 (1 sp.)

ESPECIE TIPO: Cordylobates fragilis Luxton, 1995

DisTRIBUCIÓN: Atlántico Sur.

-Humerobates (Cordylobates) fragilis (Luxton, 1995) (C.)

DISTRIBUCIÓN: I. Ascensión.

Ramsayellus Spain y Luxton, 1970 (5 spp.)

(=Ulugurozetes Mahunka, 1984)

(=Zealandobates Hammer, 1967, "nom. praeoc." por Hopkins, 1966)

EsPECIE TIPO: Zealandobates grandis Hammer, 1967

DisTRIBUCión: Pantropical (excepto Neotropical).

-Ramsayellus conspicuus (Berlese, 1916) (Peloribates)

DistriBuCión: "África oriental".

-Ramsayellus fallax (Pearce, 1906) (Oribata)

DisTRIBUCIÓN: India (Sikkim).

-Ramsayellus grandis (Hammer, 1967) (Zealandobates)

DisTRIBUCión: Nueva Zelanda.

-Ramsayellus seychellensis (Warburton, 1912) (Oribata)

DisTRIBUCIÓN: I. Seychelles.

-Ramsayellus turbulentus (Mahunka, 1984) (Ulugurozetes)

DisTRIBUCión: Tanzania.

Svalbardia Thor, 1930 (1 sp.)

ESPECIE TIPO: Svalbardia paludicola Thor, 1930

DISTRIBUCIÓN: Boreal.

-Svalbardia paludicola Thor, 1930

DisTRIBUCIÓN: Boreal (Norte de Europa, Sur de Siberia y Neártica septentrional: frecuente).

Punctoribatidae Thor, 1937 (15 gen., 1 subg., 90 spp. y 1 ssp.) DISTRIBUCIÓN: Cosmopolita.

Allomycobates Aoki, 1976 (1 sp.)

EsPECIE TIPO: Allomycobates lichensis Aoki, 1976

DISTRIBUCIÓN: Paleártica.

-Allomycobates lichensis Aoki, 1976

DisTRIBUCIÓN: Japón.

Alpizetes Mahunka, 2001 (1 sp.)

EsPECIE TIPO: Alpizetes behenae Mahunka, 2001

DisTRIBUCIÓN: Paleártica.

-Alpizetes behenae Mahunka, 2001

DISTRIBUCIÓN: Suiza.

Cryptobothria Wallwork, 1963 (2 spp.)

ESPECIE TIPO: Cryptobothria monodactyla Wallwork, 1963

DisTRIBUCIÓN: Australiana y Antártica.

-Cryptobothria monodactyla Wallwork, 1963

DisTRIBUCión: Antártica (I. Macquarie).

-Cryptobothria papuana Balogh, 1970

DISTRIBUCIÓN: Nueva Guinea.

Ellipsozetes Bernini, 1980 (1 sp.)

EsPECIE TIPO: Ellipsozetes sphaerulus Bernini, 1980

DISTRIBUCIÓN: Paleártica.

-Ellipsozetes sphaerulus Bernini, 1980

DISTRIBUCIÓN: Italia.
Feiderzetes Subías, 1977 (1 sp.)

(=Allozetes Feider, Vasiliu y Calugar, 1971, “nom. praeoc." por Berlese, 1913)

EsPECIE TIPO: Punctoribates (Minunthozetes) latus Schweizer, 1956

DISTRIBUCIÓN: Paleártica.

-Feiderzetes latus (Schweizer, 1956) (Punctoribates (Minunthozetes))

DisTRIBUCIÓN: Sur de Europa.

Inigozetes Subías, 2000 (1 sp.)

EsPecie Tipo: Minunthozetes reticulatus Pérez-Íñigo, 1969

DisTRIBUCIÓN: Paleártica.

-Inigozetes reticulatus (Pérez-Íñigo, 1969) (Minunthozetes)

DISTRIBUCIÓN: España.

Minguezetes Subías, Kahwash y Ruiz, 1990 (4 spp.)

EsPeCIE TIPO: Minguezetes conjunctus Subías, Kahwash y Ruiz, 1990

DisTRIBUCIÓN: Cosmopolita (excepto Antártica).

-Minguezetes conjunctus Subías, Kahwash y Ruiz, 1990

DisTRIBUCIÓN: Suroeste de Europa.

-Minguezetes hexagonus (Berlese, 1908) (Punctoribates)

(=Punctoribates hygrophilus Mihelcic, 1957)

DisTRIBUCión: Holártica (Paleártica occidental: frecuente, Sur de Paleártica oriental y Neártica septentrional) y Vietnam.

-Minguezetes insignis (Berlese, 1910) (Punctoribates)

(=Punctoribates manzanoensis Hammer, 1958)

DisTRIBUCIÓN: Semicosmopolita (Mediterránea, Este de Paleártica oriental, Nueva Zelanda y Neotropical).

-Minguezetes longiporosus (Balogh, 1963) (Punctoribates)

DisTRIBUCIÓN: Angola y Noreste de Oriental.

Minunthozetes Hull, 1916 (5 spp.)

(=Jurabates Jacot, 1929)

EsPECIE TIPO: (Oribata fusigera Michael, 1884) =Zetes semirufus Koch, 1841

DisTRIBUCIÓN: Paleártica.

-Minunthozetes humectus (Hull, 1915) (Oribata)

DisTRIBUCIÓN: Islas Británicas.

-Minunthozetes pseudofusiger (Schweizer, 1922) (Oribata)

DISTRIBUCIÓN: Paleártica (frecuente).

-Minunthozetes quadriareatus Mínguez, Subías y Ruiz, 1986

(?=Punctoribates atomus Berlese, 1908 "sp. inq.")

DISTRIBUCIÓN: Mediterránea occidental.

-Minunthozetes semirufus (Koch, 1841) (Zetes)

(=Punctoribates bicornis Berlese, 1908)

(=Oribata fusigera Michael, 1884)

(=Minunthozetes major Mihelcic, 1957)

DistRIBUCIÓN: Paleártica (Paleártica occidental: frecuente, y Asia centrooccidental).

-Minunthozetes tarmani Feider, Vasiliu y Calugar, 1971

DISTRIBUCIÓN: Sur de Europa oriental.

Mycobates Hull, 1916 (37 spp.)

ESPECIE TIPO: Oribata parmeliae Michael, 1884

DisTRIBUCIÓN: Holártica y Neotropical.

Mycobates (Mycobates) Hull, 1916 (19 spp.)

DisTRIBUCIÓN: Holártica y Neotropical.

-Mycobates (M.) acuspidatus Behan-Pelletier, Eamer y Clayton, 2001

DISTRIBUCIÓN: Noroeste de Neártica.

-Mycobates (M.) austroamericanus Hammer, 1958

DisTRIBUCIÓN: Bolivia.

-Mycobates (M.) cambricus (Hull, 1916) (Melanozetes)

DisTRIBUCión: Islas Británicas.

-Mycobates (M.) carli (Schweizer, 1922) (Oribata)

DisTRIBUCIÓN: Europa meridional.

-Mycobates (M.) corticeus Bvehan-Pelletier, Eamer y Clayton, 2001 DisTRIBUCIÓN: Canadá.

-Mycobates (M.) debilis Mihelcic, 1957

DisTRIBUCIÓN: Austria.

-Mycobates (M.) exigualis Behan-Pelletier, 1994

DisTRIBUCIÓN: Boreal (Noruega y Canadá). 
-Mycobates (M.) ezoensis Fujikawa, 1982

DISTRIBUCIÓN: Japón.

-Mycobates (M.) flabelliger (Berlese, 1908) (Sphaerozetes)

DisTRIBUCIÓN: U.S.A. (Washington D.C.).

-Mycobates (M.) hylaeus Behan-Pelletier, 1994

DisTRIBUCIÓN: Neártica (U.S.A. oriental y Canadá).

-Mycobates (M.) integer Mihelcic, 1957

DisTRIBUCIÓn: Sur de Europa.

-Mycobates (M.) minor Subías, Kahwash y Ruiz, 1990

DisTRIBUCIÓN: Mediterránea occidental.

-Mycobates (M.) monodactylus Shaldybina, 1970

DistRIBUCIÓN: Paleártica (Rusia europea septentrional y Paleártica oriental: excepto Este).

-Mycobates (M.) parmeliae (Michael, 1884) (Oribata)

(=Sphaerozetes (Trichoribates) lenicomus Berlese, 1910)

(=Mycobates nadiji Paulitchenko, 1991)

DisTRIBUCIÓN: Holártica (Europa: frecuente, Paleártica oriental y Canadá).

-Mycobates (M.) perates Behan-Pelletier, 1994

DisTRIBUCIÓN: Neártica.

-Mycobates (M.) rileyi (Haller, 1884) (Oribata) "sp. inq."

DisTRIBUCIÓN: "Neotropical".

-Mycobates (M.) royi Palacios-Vargas y Vázquez, 1988

DisTRIBUCIÓN: Méjico.

-Mycobates (M.) tridentatus Weigmann, 1976

DisTRIBUCIÓN: I. Azores.

-Mycobates (M.) yukonensis Behan-Pelletier, 1994

DisTRIBUCIÓN: Canadá.

Mycobates (Calyptozetes) Thor, 1930 (18 spp.)

(=Permycobates Strenzke, 1954)

ESPECIE TIPO: Oribata sarekensis Trägardh, 1910

DisTRIBUCIÓN: Holártica.

-Mycobates (Calyptozetes) alpinus (Willmann, 1951) (C.)

DisTRIBUCIÓN: Suroeste de Europa.

-Mycobates (Calyptozetes) altus Behan-Pelletier, 1994 (M.) DisTRIBUCIÓN: Neártica.

-Mycobates (Calyptozetes) angulatus (Koch, 1839) (Oribata) DisTRIBUCIÓN: Centroeuropa.

-Mycobates (Calyptozetes) azaleos Behan-Pelletier, 1994 (M.) DISTRIBUCIÓN: Neártica.

-Mycobates (Calyptozetes) beringianus Behan-Pelletier, 1994 (M.) DisTRIBUCIÓN: Neártica septentrional.

-Mycobates (Calyptozetes) bicornis (Strenzke, 1954) (Permycobates) Distribución: Paleártica centromeridional (excepto Este).

-Mycobates (Calyptozetes) brevilamellatus Behan-Pelletier, 1994 (M.) DisTRIBUCIÓN: Canadá.

-Mycobates (Calyptozetes) conitus Hammer, 1952 (M.) DisTRIBUCIÓN: Neártica septentrional (frecuente).

-Mycobates (Calyptozetes) cribelliger (Berlese, 1904) (Oribata) DISTRIBUCIÓN: Europa suroccidental.

-Mycobates (Calyptozetes) dryas Behan-Pelletier, 1994 (M.) DisTRIBUCIÓN: Neártica septentrional.

-Mycobates (Calyptozetes) hammerae Behan-Pelletier, 1994 (M.) DISTRIBUCIÓN: Neártica septentrional.

-Mycobates (Calyptozetes) incurvatus Hammer, 1952 (M.) DisTRIBUCión: Holártica (Neártica y Japón).

-Mycobates (Calyptozetes) monocornis Aoki, 1973 (M.) DisTRIBUCIÓN: Japón.

-Mycobates (Calyptozetes) occidentalis Behan-Pelletier, 1994 (M.) DISTRIBUCIÓN: Neártica septentrional.

-Mycobates (Calyptozetes) patrius Shaldybina, 1970 (M.) DisTRIBUCión: Paleártica (Ucrania y Noreste de Siberia).

-Mycobates (Calyptozetes) punctatus Hammer, 1955 (M.) DisTRIBUCIÓN: Holártica (Asia centrooccidental y Neártica septentrional).

-Mycobates (Calyptozetes) sarekensis (Trägardh, 1910) (Oribata) (=Mycobates consimilis Hammer, 1952)

DisTRIBUCIÓN: Boreal (Euroatlántica: frecuente, Asia central rusa y Neártica septentrional: frecuente).
-Mycobates (Calyptozetes) tridactylus Willmann, 1929 (M.)

DisTRIBUCIÓN: Paleártica.

Mycozetes Spain, 1968 (1 sp.)

ESPECIE TIPO: Mycozetes oleariae Spain, 1968

DISTRIBUCIÓN: Australiana.

-Mycozetes oleariae Spain, 1968

DisTRIBUCIÓN: Nueva Zelanda.

Neomycobates Wallwork, 1963 (1 sp.)

ESPECIE TIPO: Neomycobates tridentatus Wallwork, 1963

DISTRIBUCIÓN: Antártica.

-Neomycobates tridentatus Wallwork, 1963

DisTRIBUCIÓN: Antártica (I. Macquarie).

Pelopsis Hall, 1911 (2 spp.)

(=Ewingozetes Hammer, 1952)

(=Parapelops Jacot, 1938)

EsPECIE TIPO: (Pelopsis nudiuscula Hall, 1911) =Pelops bifurcatus

Ewing, 1909

DisTRIBUCIÓN: Neártica y Neotropical.

-Pelopsis bidentatus (Hammer, 1961) (Parapelops)

DistribuCión: Perú.

-Pelopsis bifurcatus (Ewing, 1909) (Pelops)

(=Pelopsis nudiuscula Hall, 1911)

$(=$ Galumna partita Banks, 1910)

DisTRIBUCIÓN: U.S.A. (frecuente).

Punctoribates Berlese, 1908 (20 spp. y 1 ssp.)

ESPECIE TIPO: Oribata punctum Koch, 1839

DISTRIBUCIÓN: Semicosmopolita (Holártica y Australianooriental).

-Punctoribates angulatus Bayartogtokh, Grobler y Cobanoglu, 2000 Distribución: Turquía.

-Punctoribates armipes (Banks, 1906) (Galumna)

DisTRIBUCIÓN: Noreste de U.S.A.

-Punctoribates brevicuspidatus Bayartogtokh y Aoki, 1998

DISTRIBUCIÓN: Mongolia.

-Punctoribates eoeryi Mahunka, 1972

DisTRIBUCIÓN: Croacia.

-Punctoribates ghilarovi Shaldybina, 1969

DisTRIBUCIÓN: Europa oriental.

-Punctoribates grishinae Shaldybina, 1977

DisTRIBUCIÓN: Paleártica (Cáucaso y Siberia central).

-Punctoribates latilobatus Kunst, 1958

DisTRIBUCIÓN: Paleártica (Europa centromeridional y Asia centrooccidental) y Noreste de Oriental.

-Punctoribates liber Paulitchenko, 1991

DisTRIBUCIÓN: Ucrania.

-Punctoribates meridianus Shaldybina, 1973

DisTRIBUCIÓN: Sureste de Europa.

-Punctoribates minimus Shaldybina, 1969

DistriBuCión: Paleártica (Europa centromeridional: excepto Oeste, y Asia centrooccidental).

-Punctoribates moldavicus Vasiliu y Calugar, 1976

Distribución: Rumania.

-Punctoribates mundus Shaldybina, 1973

DistribuCión: Paleártica (Sureste de Europa y Paleártica oriental: excepto Norte).

-Punctoribates palustris (Banks, 1895) (Oribata)

DisTRIBUCIÓN: U.S.A. (Nueva York).

-Punctoribates pannonicus Mahunka, 1987

DisTRIBUCIÓN: Hungría

-Punctoribates pragensis Winkler, 1957

Distribución: Chequia.

-Punctoribates punctum (Koch, 1839) (Oribata)

DistriBUCión: Semicosmopolita (Paleártica: frecuente, U.S.A.:

Virginia, Oriental y Nueva Zelanda).

-Punctoribates selgae (Pérez-Íñigo, 1976) (Minunthozetes)

DISTRIBUCION: I. Canarias. 
-Punctoribates sellnicki Willmann, 1928

DisTRIBUCIÓN: Europa.

-Punctoribates sellnicki sellnicki Willmann, 1928

DISTRIBUCIÓN: Europa (frecuente).

-Punctoribates sellnicki crassirostris Strenzke, 1952

DisTRIBUCIÓN: Europa central.

-Punctoribates sibiricus Grishina, 1987

DisTRIBUCIÓN: Asia centrooccidental.

-Punctoribates sphaericus Shaldybina, 1987

DisTRIBUCIÓN: Paleártica meridional (Ucrania y Asia central rusa).

Semipunctoribates Mahunka, 1987 (3 spp.)

EsPECIE TIPO: Semipunctoribates hungaricus Mahunka, 1987

DISTRIBUCIÓN: Paleártica.

-Semipunctoribates astrachanicus (Shaldybina, 1973) (Punctoribates)

DistriBución: Sur de Rusia europea.

-Semipunctoribates hungaricus Mahunka, 1987

DisTRIBUCIÓN: Hungría.

-Semipunctoribates zachvatkini (Shaldybina, 1969) (Punctoribates)

DisTRIBUCIÓN: Paleártica occidental (Sur de Europa).

Zachvatkinibates Shaldybina, 1973 (10 spp.)

(=Eupunctoribates Hammer, 1977 "sin. nov.")

(=Schweizerzetes Mahunka, 2001 "sin. nov.")

EsPECIE TIPO: Zachvatkinibates nemoralis Shaldybina, 1973

DisTRIBUCIÓN: Holártica.

-Zachvatkinibates conjunctus Shaldybina, 1987

DisTRIBUCIÓN: Asia centrooccidental.

-Zachvatkinibates epiphytos Behan-Pelletier, Eamer y Clayton, 2001

DisTRIBUCIÓN: Noroeste de Neártica.

-Zachvatkinibates latilamellatus Bayartogtokh y Aoki, 1998

DISTRIBUCIÓN: Mongolia.

-Zachvatkinibates lobatus (Hammer, 1977) (Eupunctoribates)

DisTRIBUCIÓN: Pakistán.

-Zachvatkinibates maritimus Shaldybina, 1973

DistRiBución: Boreal (Este de Paleártica oriental y Neártica septentrional).

-Zachvatkinibates nemoralis Shaldybina, 1973

DISTRIBUCIÓN: Asia central rusa.

-Zachvatkinibates perlongus (Balogh, 1959) (Punctoribates)

DisTRIBUCIÓN: Europa meridional.

-Zachvatkinibates quadrivertex (Halbert, 1920) (Oribata)

DisTRIBUCIÓN: Boreal (Norte de Europa y Neártica septentrional).

-Zachvatkinibates tetrasklerosis Behan-Pelletier, 1988

DisTRIBUCIÓN: Canadá.

-Zachvatkinibates volgini Shaldybina, 1973

DistriBución: Este de Paleártica oriental.

Zetomotrichoidea Grandjean, 1934

Zetomotrichidae Grandjean, 1934 (8 gen., 3 subg., 27 spp. y 1 ssp.) DisTRIBUCIÓN: Tropical y subtropical.

Anoplozetes Lee y Pajak, 1987 (1 sp.)

EsPeCIE TIPO: Anoplozetes jamiesoni Lee y Pajak, 1987

DisTRIBUCIÓN: Australiana.

-Anoplozetes jamiesoni Lee y Pajak, 1987

DISTRIBUCIÓN: Australiana.

Demisalto Coetzee, 1993 (5 spp.)

EsPeCIE TIPO: Demisalto engelbrechti Coetzee, 1993

DisTRIBUCIÓN: Etiópica.

Demisalto (Demisalto) Coetzee, 1983 (1 sp.)

DisTRIBUCIÓN: Etiópica.

-Demisalto (D.) engelbrechti Coetzee, 1993

DisTRIBUCión: Sudáfrica.

Demisalto (Saltatrichus) Coetzee, 1993 (4 spp.)

EsPeCIE TIPO: Saltatrichus magnus Coetzee, 1993

DisTRIBUCIÓN: Etiópica.
-Demisalto (Saltatrichus) magnus (Coetzee, 1993) (S.)

DisTRIBUCIÓN: Sudáfrica.

-Demisalto (Saltatrichus) marshalli (Coetzee, 1995) (S.)

DisTRIBUCIÓN: Sudáfrica.

-Demisalto (Saltatrichus) minus (Coetzee, 1993) (S.)

DisTRIBUCión: Sudáfrica.

-Demisalto (Saltatrichus) robustus (Coetzee, 1993) (S.)

DisTRIBUCIÓN: Sudáfrica.

Ghilarovus Krivolutsky, 1966 (8 spp. y 1 ssp.)

EsPeCIE TIPO: Ghilarovus humeridens Krivolutsky, 1966

DISTRIBUCIÓN: Holártica subtropical.

-Ghilarovus changlingensis Wen, 1990

DisTRIBUCIÓN: Noreste de China.

-Ghilarovus daliensis Yamamoto y Aoki, 2000

DisTRIBUCIÓN: China suroriental.

-Ghilarovus elegans Mahunka, 1983

Distribución: Méjico.

-Ghilarovus hispanicus Subías y Pérez-Íñigo, 1977

DisTRIBUCIÓN: Mediterránea occidental.

-Ghilarovus hispanicus hispanicus Subías y Pérez-Íñigo, 1977 DisTRIBUCión: España.

-Ghilarovus hispanicus guadarramicus Subías, 1977

DisTRIBUCIÓN: Mediterránea occidental.

-Ghilarovus humeridens Krivolutsky, 1966

DistriBuCión: Paleártica meridional (Grecia y Sur de Paleártica oriental).

-Ghilarovus saxicola Aoki e Hirauchi, 2000

DisTRIBUCióN: Japón

-Ghilarovus stipatus Krivolutsky y Smelyanski, 1997

DISTRIBUCIÓN: Asia centrooccidental.

-Ghilarovus turcmenicus Krivolutsky, 1974

DISTRIBUCIÓN: Asia centrooccidental.

Mabulatrichus Coetzee, 1993 (3 spp.)

(=Hungaromotrichus Mahunka, 1993 "sin. nov.")

EsPeCIE TIPO: Mabulatrichus dentatus Coetzee, 1993

DISTRIBUCIÓN: Subtropical (Paleártica y Etiópica).

-Mabulatrichus baloghi (Mahunka, 1993) (Hungaromotrichus)

DiSTRIBUCIÓN: Rumania.

-Mabulatrichus dentatus Coetzee, 1993

DisTRIBUCIÓN: Sudáfrica.

-Mabulatrichus litoralis Aoki e Hirauchi, 2000

DisTRIBUCIÓN: Japón.

Mikizetes Hammer, 1958 (3 spp.)

EsPECIE TIPO: Mikizetes diamantensis Hammer, 1958

DistRIBUCIÓN: Neotropical y Paleártica meridional.

Mikizetes (Mikizetes) Hammer, 1958 (2 spp.)

DISTRIBUCIÓN: Neotropical.

-Mikizetes (M.) diamantensis Hammer, 1958

DISTRIBUCIÓN: América austral.

-Mikizetes (M.) flagellifer Hammer, 1961

DisTRIBUCIÓN: Perú.

Mikizetes (Oglasacarus) Bernini, 1979 (1 sp.)

EsPeCIE Tipo: Oglasacarus oglasae Bernini, 1979

DisTRIBUCIÓN: Paleártica subtropical.

-Mikizetes (Oglasacarus) oglasae (Bernini, 1979) (M.)

DisTRIBUCIÓN: Suroeste de Europa.

Pallidacarus Krivolutsky, 1975 (1 sp.)

EsPeCIE TIPO: Pallidacarus tichomirovi Krivolutsky, 1975

DisTRIBUCIÓN: Paleártica subtropical.

-Pallidacarus tichomirovi Krivolutsky, 1975

Distribución: Paleártica meridional (Sur de Rusia europea y

Asia centrooccidental) 
Rohria Balogh y Mahunka, 1977 (1 sp.)

ESPECIE TIPO: Rohria pulchella Balogh y Mahunka, 1977

DISTRIBUCIÓN: Neotropical.

-Rohria pulchella Balogh y Mahunka, 1977

DisTRIBUCIÓN: Brasil

Zetomotrichus Grandjean, 1934 (5 spp.)

EsPECIE TIPO: Zetomotrichus lacrimans Grandjean, 1934

DisTRIBUCIÓN: Paleotropical y Paleártica meridional.

Zetomotrichus (Zetomotrichus) Grandjean, 1934 (4 spp.)

DISTRIBUCIÓN: Paleotropical y subtropical (Paleártica meridonal).

-Zetomotrichus (Z.) bidentatus Hammer, 1977 (Z. lacrimans b.)

DISTRIBUCIÓN: Pakistán.

-Zetomotrichus (Z.) lacrimans Grandjean, 1934

DisTRIBUCIÓN: Mediterránea, Sudáfrica y Filipinas.

-Zetomotrichus (Z.) lienhardi Mahunka, 1989

DISTRIBUCIÓN: Sumatra.

-Zetomotrichus (Z.) linearis Tseng, 1982

DisTRIBUCIÓN: Taiwan [Formosa].

Zetomotrichus (Keralotrichus) Mahunka, 1985 (1 sp.)

EsPECIE TIPO: Keralotrichus plumosus Mahunka, 1985

DISTRIBUCIÓN: Oriental.

-Zetomotrichus (Keralotrichus) plumosus (Mahunka, 1985) (K.) DisTRIBUCIÓN: India (Kerala).

\section{Oripodoidea Jacot, 1925}

Drymobatidae J. y P. Balogh, 1984 (3 gen. y 7 spp.) DistriBuCión: Pantropical (excepto Australiana).

Drymobates Grandjean, 1930 (1 sp.)

EsPECIE TIPO: Drimobates silvicola Grandjean, 1930

DISTRIBUCIÓN: Neotropical.

-Drymobates silvicola Grandjean, 1930

Distribución: Pequeñas Antillas.

Drymobatoides Jacot, 1936 (3 spp.)

(=Pelokylla Adolph y Haq, 1982)

(=Seychellozetes Mahunka, 1984)

EsPECIE TIPO: Drymobatoides mauritius Jacot, 1936

DISTRIBUCIÓN: Paleotropical.

-Drymobatoides benoiti (Mahunka, 1984) (Seychellozetes)

DisTRIBUCIÓN: I. Seychelles.

-Drymobatoides malabaricus (Adolph y Haq, 1982) (Pelokylla)

DistriBución: India (Kerala).

-Drymobatoides mauritius Jacot, 1936

DistRIBUCIÓN: Malgache (I. Mauricio).

Rykella Balogh, 1962 (3 spp.)

EsPeCIE TIPO: Rykella insignis Balogh, 1962

DisTRIBUCIÓN: Paleotropical.

-Rykella asiatica Yamamoto y Aoki, 2000

DistRIBUCión: Sureste de China.

-Rykella elamellata (Berlese, 1916) (Podoribates)

DisTRIBUCIÓN: "África oriental".

-Rykella insignis Balogh, 1962

DISTRIBUCIÓN: Etiópica.

Mochlozetidae Grandjean, 1960 (12 gen., 56 spp. y 1 ssp.) DISTRIBUCIÓN: Tropical y subtropical.

Calugarella J. y P. Balogh, 1992 (1 sp.)

ESPECIE TIPO: Unguizetes sabahnus Mahunka, 1987 DisTRIBUCIÓN: Oriental.

-Calugarella sabahna (Mahunka, 1987) (Unguizetes)

DiSTRIBUCIÓN: Borneo.
Dynatozetes Grandjean, 1960 (3 spp.)

EsPeCIE TIPo: Dynatozetes amplus Grandjean, 1960

DISTRIBUCIÓN: Neártica y Neotropical.

-Dynatozetes amplus Grandjean, 1960

DisTRIBUCIÓN: Neotropical.

-Dynatozetes magnus (Banks, 1895) (Oribata)

DISTRIBUCIÓN: Neártica nororiental.

-Dynatozetes obesus Grandjean, 1960

DisTRIBUCIÓN: Neotropical (Venezuela y Brasil).

Gephyrazetes Hirauchi, 1999 (1 sp.)

EsPeCIE TIPO: Gephyrazetes fasciatus Hirauchi, 1999

DisTRIBUCIÓN: Paleártica.

-Gephyrazetes fasciatus Hirauchi, 1999

DISTRIBUCIÓN: Japón.

Mahunkazetes J. y P. Balogh, 1992 (2 spp.)

ESPECIE TIPO: Mochlozetes africanus Mahunka, 1988

DisTRIBUCIÓN: Etiópica.

-Mahunkazetes africanus (Mahunka, 1988) (Mochlozetes)

DistriBución: Tanzania.

-Mahunkazetes longicuspis (Balogh y Mahunka, 1967) (Podoribates)

DISTRIBUCIÓN: Congo.

Mochlobates Norton, 1984 (1 sp.)

EsPeCIE TIPO: Oribata affinis Banks, 1895

DISTRIBUCIÓN: Neártica.

-Mochlobates affinis (Banks,1895) (Oribata)

Distribución: Este de U.S.A.

Mochloribatula Mahunka, 1978 (8 spp.)

ESPECIE TIPO: Mochloribatula multiporosa Mahunka, 1978

DISTRIBUCIÓN: Neártica y Neotropical.

-Mochloribatula bahamensis Norton, 1983

Distribución: Antillas (Bahamas).

-Mochloribatula calycifera Mahunka, 1985

DisTRIBUCIÓN: Pequeñas Antillas.

-Mochloribatula depilis (Ewing, 1909) (Notaspis)

DISTRIBUCIÓN: U.S.A. (Ilinois).

-Mochloribatula floridana (Banks, 1904) (Eremaeus)

DisTRIBUCIÓN: U.S.A. (Florida).

-Mochloribatula grandjeani Mahunka, 1978

Distribución: Antillas (La Española: R. Dominicana).

-Mochloribatula metzi Norton, 1983

DisTRIBUCIÓN: U.S.A. (Carolina del Norte).

-Mochloribatula multiporosa Mahunka, 1978

Distribución: Antillas (La Española: R. Dominicana).

-Mochloribatula texana (Ewing, 1909) (Notaspis)

Distribución: U.S.A. (Tejas).

Mochlozetes Grandjean, 1930 (8 spp.)

EsPECIE TIPO: Mochlozetes penetrabilis Grandjean, 1930

DISTRIBUCIÓN: Pantropical (excepto Oriental) y subtropical.

-Mochlozetes asculpturatus Mahunka, 1985

DisTRIBUCIÓN: Neotropical.

-Mochlozetes atypicus Mahunka, 1982

DisTRIBUCión: Etiopía.

-Mochlozetes chambrieri Mahunka, 1991

DisTRIBUCIÓN: Australiana (Indonesia: Lombok).

-Mochlozetes flatus Grandjean, 1930

DISTRIBUCIÓN: Panamá

-Mochlozetes maximus (Ewing, 1908) (Oribata)

DisTRIBUCIÓN: U.S.A. central.

-Mochlozetes officiosus Grandjean, 1930

DisTRIBUCIÓN: Colombia.

-Mochlozetes penetrabilis Grandjean, 1930

DISTRIBUCIÓN: Neotropical y Japón.

-Mochlozetes saltaensis Alzuet, 1989

DISTRIBUCIÓN: Argentina. 
Nesiotizetes Jacot, 1934 (1 sp.)

EsPECIE TIPO: Nesiotizetes adamsoni Jacot, 1934

DisTRIBUCIÓN: Australiana.

-Nesiotizetes adamsoni Jacot, 1934

Distribución: Polinesia (I. Marquesas).

Paralobozetes Tseng, 1984 (1 sp.)

ESPECIE TIPO: Paralobozetes longirostralis Tseng, 1984 DISTRIBUCIÓN: Oriental.

-Paralobozetes longirostralis Tseng, 1984

DisTRIBUCIÓN: Taiwan [Formosa].

Podoribates Berlese, 1908 (14 spp. y 1 ssp.)

(=Cardioribates Jacot, 1934)

(=Falsolobozetes Tseng,1984 "sin. nov.")

(=Sphaerobates Sellnick, 1928)

EsPeCIE TIPO: Oribata longipes Berlese, 1887

DISTRIBUCIÓN: Tropical y subtropical.

-Podoribates artilamellatus (Ewing, 1909) (Oribata)

DistribuCión: U.S.A. (Ilinois).

-Podoribates cuscensis (Hammer, 1961) (Sphaerobates)

Distribución: Perú.

-Podoribates cuspidatus Sakakibara y Aoki, 1966

Distribución: Este de Paleártica oriental.

-Podoribates foveolatus (Hammer, 1958) (Sphaerobates)

DistribuCión: América austral.

-Podoribates glaber (Berlese, 1916) (Sphaerozetes (Trichoribates))

DisTribución: Nueva Caledonia.

-Podoribates javensis (Willmann,1932) (Sphaerobates)

DisTRIBUCión: Java y Sudáfrica.

-Podoribates javensis javensis (Willmann, 1932)

DisTRIBUCIÓN: Java.

-Podoribates javensis africanus Jacot, 1940

DisTRIBUCIÓN: Sudáfrica.

-Podoribates laneus (Tseng, 1984) (Falsolobozetes)

DisTRIBUCIÓN: Taiwan [Formosa].

-Podoribates longipes (Berlese, 1887) (Oribata)

(=Sphaerozetes gratus Sellnick, 1921)

DisTRIBUCIÓN: Holártica (Paleártica occidental: menos frecuente

en el Norte, Asia central rusa y Canadá)

-Podoribates minusculus (Banks, 1906) (Galumna)

DisTRIBUCIÓN: U.S.A. centrooriental.

-Podoribates oriformis (Pearce, 1910) (Oribata)

Distribución: Hawai.

-Podoribates platensis Berlese, 1916 (P. longipes p.)

DistribuCión: Argentina.

-Podoribates pratensis (Banks, 1895) (Oribata)

DisTRIBUCIÓN: Noreste de U.S.A.

-Podoribates rectus Berlese, 1916

DISTRIBUCIÓN: Uruguay.

-Podoribates turgidus (Banks, 1906) (Galumna)

DisTRIBUCIÓN: U.S.A. (excepto Norte).

Unguizetes Sellnick, 1925 (12 spp.)

(=Terrazetes Jacot, 1929)

ESPECIE TIPO: Oribata sphaerula Berlese, 1905

DisTRIBUCIÓN: Pantropical y subtropical.

-Unguizetes clavatus Aoki, 1967

Distribución: Oriental y Este de Paleártica oriental.

-Unguizetes curypterus Wen y Zhao, 1994

DISTRIBUCIÓN: China suroriental.

-Unguizetes incertus (Balogh y Mahunka, 1969) (Uracrobates)

DisTRIBUCIÓN: Neotropical (Bolivia y Brasil).

-Unguizetes inermis Mahunka, 1991

Distribución: Australiana (Indonesia: Lombok).

-Unguizetes longiporosus (Balogh y Mahunka, 1966) (Terrazetes)

DisTRIBUCIÓN: Sudáfrica.

-Unguizetes mauritius (Jacot, 1936) (Terrazetes)

DistRIBUCIÓN: Malgache (I. Mauricio).
-Unguizetes reticulatus Wallwork, 1965

DisTRIBUCIÓN: Chad y Arabia Saudita.

-Unguizetes sabahnus Mahunka, 1987

DISTRIBUCIÓN: Borneo.

-Unguizetes setiger (Balogh y Mahunka, 1978) (Uracrobates)

(=Podoribates brasiliensis Mahunka, 1983)

DisTRIBUCIÓN: Brasil.

-Unguizetes similis Mahunka, 1998

DisTRIBUCIÓN: Pequeñas Antillas.

-Unguizetes sphaerula (Berlese, 1905) (Oribata)

(=Unguizetes triplicatulus Grandjean, 1960)

DisTRIBUCIÓN: Oriental.

-Unguizetes yemenitica Mahunka, 2000

DISTRIBUCIÓN: Yemen.

Uracrobates Balogh y Mahunka, 1967 (4 spp.)

(=Urobates Hammer, 1973)

EsPECIE TIPO: Uracrobates magniporosus Balogh y Mahunka, 1967

DistRiBución: Pantropical (excepto Neotropical).

-Uracrobates africanus Mahunka, 1988

DISTRIBUCIÓN: Tanzania.

-Uracrobates indicus Ramani y Haq, 1990

DisTRIBUCIÓN: India (Kerala).

-Uracrobates magniporosus Balogh y Mahunka, 1967

DISTRIBUCIÓN: Vietnam.

-Uracrobates pygiseta (Hammer, 1973) (Urobates)

Distribución: Polinesia (I. Tonga).

Neotrichozetidae Balogh, 1965 (1 gen. y 1 spp.)

DisTRIBUCIÓN: Subtropical austral (excepto Etiópica).

Neotrichozetes Travé, 1961 (1 spp. y 2 ssp.)

EsPECIE TIPO: Notaspis spinulosa Michael, 1908

DistriBUCIÓN: Subtropical austral (excepto Etiópica).

-Neotrichozetes spinulosus (Michael, 1908) (Notaspis)

Distribución: Australiana (Nueva Zelanda y Tasmania) y

Argentina.

-Neotrichozetes spinulosus spinulosus (Michael, 1908)

DisTRIBUCIÓN: Australiana (Nueva Zelanda y Tasmania).

-Neotrichozetes spinulosus germaineae Travé, 1961

DISTRIBUCIÓN: Argentina.

Oribatulidae Thor, 1929 (18 gen., 2 subg., 201 spp. y 7 sspp.) DisTRIBUCIÓN: Cosmopolita.

Capilloppia Balogh y Mahunka, 1966 (3 spp.)

ESPECIE TIPO: Capilloppia capillata Balogh y Mahunka, 1966

DISTRIBUCIÓN: Etiópica.

-Capilloppia capillata Balogh y Mahunka, 1966

DisTRIBUCión: Sudáfrica.

-Capilloppia hessei (Jacot, 1940) (Oribatula)

DisTRIBUCIÓN: Sudáfrica.

-Capilloppia smithersi (Jacot, 1940) (Oribatula)

DISTRIBUCIÓN: Sudáfrica.

Crassoribatula Hammer, 1967 (1 sp.)

EsPeCIE TIPO: Crassoribatula maculosa Hammer, 1967

DISTRIBUCIÓN: Australiana.

-Crassoribatula maculosa Hammer, 1967

DisTRIBUCIón: Nueva Zelanda.

Diphauloppia J. y P. Balogh, 1984 (1 sp.)

ESPECIE TIPO: Subphauloppia luminosa Hammer, 1973

DISTRIBUCIÓN: Australiana.

-Diphauloppia luminosa (Hammer, 1973) (Subphauloppia)

DistriBución: Polinesia (I. Tonga).

Grandjeania Balogh, 1963 (1 sp.)

(=Grandjeanella Balogh, 1961, "nom. praeoc." por Southcott, 1961)

EsPeCIE TIPO: Grandjeanella bicaudata Balogh, 1961

DisTRIBUCIÓN: Etiópica. 
-Grandjeania bicaudata (Balogh, 1961) (Grandjeanella) DisTRIBUCIÓn: Angola.

Jornadia Wallwork y Weems, 1984 (3 spp.)

(=Woolleybates J. y P. Balogh, 1984 "sin. nov.")

ESPECIE TIPO: Jornadia larreae Wallwork y Weems, 1984

DisTRIBUCIÓN: Neártica subtropical y Neotropical.

-Jornadia dactyloscopica (Balogh y Mahunka, 1968) (Oribatula)

DISTRIBUCIÓN: Argentina.

-Jornadia larreae Wallwork y Weems, 1984

DisTRIBUCión: U.S.A. (Nuevo Méjico).

-Jornadia longipilis Pérez-Íñigo y Baggio, 1991

DISTRIBUCIÓN: Brasil.

Lucoppia Berlese, 1908 (5 spp.)

(=Romanobates Feider, Vasiliu y Calugar, 1970 "sin. nov.")

EsPECIE TIPO: (Zetes lucorum Koch "sensu” Berlese, 1892) =Notaspis burrowsi Michael, 1890

Distribución: Cosmopolita (excepto Oriental y Antártica).

-Lucoppia burrowsi (Michael, 1890) (Notaspis)

(=Zygoribatula apletosa Higgins y Woolley, 1975)

(=Romanobates maiensis Choi, 1995)

(=Notaspis pectinata Hall, 1912, "nom. praeoc.” por Michael, 1885)

(=Eremaeus sanremensis Oudemans, 1900)

(=Zygoribatula spinosissima Mihelcic, 1956)

DisTRIBUCIÓN: Holártica (Paleártica occidental: menos frecuente en el Norte, Corea y Neártica) y Hawai.

-Lucoppia nicora Djaparidze, 1986

DisTRIBUCIÓN: Cáucaso.

-Lucoppia orientalis Djaparidze, 1985

DisTRIBUCIÓN: Cáucaso.

-Lucoppia ornata Berlese, 1916

DISTRIBUCIÓN: "África oriental".

-Lucoppia reticulata (Feider, Vasiliu y Calugar, 1970) (Romanobates) ("non" Lucoppia reticulata Willmann, 1933, ahora en Chaunoproctus) DisTRIBUCIÓN: Sureste de Europa y Chile.

Lunoribatula Mahunka, 1982 (1 sp.)

EsPECIE TIPO: Lunoribatula polygonata Mahunka, 1982

DISTRIBUCIÓN: Etiópica y Neotropical.

-Lunoribatula polygonata Mahunka, 1982

DisTRIBUCIÓN: Etiopía y Chile.

Megatrichobates Grobler, 2000 (2 spp.)

ESPECIE TIPO: Megatrichobates striatus Grobler, 2000

DisTRIBUCIÓN: Etiópica.

-Megatrichobates longisetosus Grobler, 2000

DisTRIBUCIÓN: Sudáfrica.

-Megatrichobates striatus Grobler, 2000

DISTRIBUCIÓN: Sudáfrica.

Neolucoppia Tseng, 1984 (1 sp.)

ESPECIE TIPO: Neolucoppia luculenta Tseng, 1984

DISTRIBUCIÓN: Oriental.

-Neolucoppia luculenta Tseng, 1984

DISTRIBUCIÓN: Taiwan [Formosa].

Oribatula Berlese, 1896 (132 spp. y 7 sspp.)

EsPECIE TIPO: Notaspis tibialis Nicolet, 1855

DISTRIBUCIÓN: Cosmopolita.

Oribatula (Oribatula) Berlese, 1896 (46 spp. y 3 sspp.)

(=Ceroribatula Lee y Birchby, 1989 "sin. nov.")

DISTRIBUCIÓN: Cosmopolita (excepto Antártica).

-Oribatula (O.) alpina Schweizer, 1956

DisTRIBUCIÓN: Europa central.

-Oribatula (O.) angustolamellata Iordansky, 1991

Distribución: Paleártica meridional (Sureste de Europa y Asia centrooccidental)
-Oribatula (O.) arboricola Mahunka y Mahunka-Papp, 1999

DisTRIBUCIÓN: Hungría.

-Oribatula (O.) beccus Djaparidze, 1990

DistRiBución: Cáucaso.

-Oribatula (O.) bizygata (Lee y Birchby, 1991) (Ceroribatula)

DisTRIBUCIÓN: Australia.

-Oribatula (O.) commutata Iordansky, 1991

DisTRIBUCión: Sur de Siberia

-Oribatula (O.) divida Mahunka, 1987

DisTRIBUCIÓN: Hungría.

-Oribatula (O.) elegantissima Balogh y Mahunka, 1965

DISTRIBUCIÓN: Mongolia.

-Oribatula (O.) exsudans Travé, 1961

Distribución: Suroeste de Europa.

-Oribatula (O.) fraenzlei Balogh y Mahunka, 1968

DisTRIBUCIÓN: Argentina.

-Oribatula (O.) incerta Pérez-Íñigo y Peña, 1996

DISTRIBUCIÓN: I. Canarias.

-Oribatula (O.) incompleta Mahunka, 1987

DisTRIBUCIÓN: Nigeria.

-Oribatula (O.) incrustata (Lee y Birchby, 1991) (Ceroribatula)

DisTRIBUCIÓN: Australia.

-Oribatula (O.) intermedia Mihelcic, 1969

DisTRIBUCIÓN: Suroeste de Europa.

-Oribatula (O.) interrupta (Willmann, 1939) (Z.)

DisTRIBUCIÓN: Holártica (Paleártica y Neártica septentrional).

-Oribatula (O.) interrupta interrupta (Willmann, 1939) (=Zygoribatula bulanovae Kulijev, 1961)

DisTRIBUCIÓN: Holártica (Paleártica y Neártica septentrional).

-Oribatula (O.) interrupta saxicola (Kunst, 1959) (Zygoribatula s.) ("non" Oribatula saxicola Halbert, 1920, ahora en Scheloribates) (=Oribatula interrupta major Mihelcic, 1963)

DistribuCIÓN: Paleártica meridional (Sur de Europa y Asia centrooccidental)

-Oribatula (O.) latirostris Willmann, 1951

DisTRIBUCIÓN: Europa central.

-Oribatula (O.) longilamellata Schweizer, 1956

DisTRIBUCIÓN: Europa central.

-Oribatula (O.) macrostega Iturrondobeitia, 1985

(=Oribatula longilamellata Subías, 1977, "nom. praeoc." por Schweizer, 1956) (?=Eporibatula nodifer Mihelcic, 1956 "sp. inq.")

DisTRIBUCIÓN: España.

-Oribatula (O.) megaforamina (Lee y Birchby, 1991) (Ceroribatula)

DistriBuCión: Australia.

-Oribatula (O.) mesosetosa (Lee y Birchby, 1991) (Fovoribatula)

Distribución: Australia.

-Oribatula (O.) monozygata (Lee y Birchby, 1991) (Ceroribatula)

DisTRIBUCIÓN: Australia.

-Oribatula (O.) nativa Tseng, 1984

DisTRIBUCIÓN: Taiwan [Formosa]

-Oribatula (O.) nayoroensis Fujita y Fujikawa, 1987

DISTRIBUCIÓN: Japón.

-Oribatula $(O$.) neonominata nom. nov.

[=Oribatula dentata Mihelcic, 1969, "nom. praeoc." por Balogh, 1958 ("hom. sec.")]

DISTRIBUCIÓN: Austria.

-Oribatula (O.) pannonica Willmann, 1949

(=Oribatula tenella Mihelcic, 1956)

=Oribatula tenella longipilus Mihelcic, 1966)

DisTRIBUCIÓN: Paleártica.

-Oribatula parisi Travé, 1961

DisTRIBUCIÓN: Suroeste de Europa.

-Oribatula (O.) pisacensis Hammer, 1961

Distribución: Perú.

-Oribatula (O.) praeoccupata nom. nov.

[=Oribatula dentata Grobler y Skubala, 2000, "nom. praeoc." por

Mihelcic, 1969 (“hom. prim.”)]

Distribución: España

-Oribatula (O.) prominens (Bayartogtokh y Aoki, 1998) (Eporibatula)

DistribuCIÓN: Mongolia. 
-Oribatula (O.) quadrisetosa Hammer, 1961

DisTRIBUCIÓN: Perú.

-Oribatula (O.) repetita nom. nov.

[=Oribatula lineata Bayartogtokh y Aoki, 1997, "nom. praeoc." por

Hammer, 1979 ("hom. sec.")

DistribuCIÓN: Mongolia.

-Oribatula (O.) robusta Iordansky, 1991

DisTRIBUCIÓN: Sureste de Europa.

-Oribatula (O.) rotundata Thor, 1937 “sp. inq."

DisTRIBUCIÓN: Noruega.

-Oribatula (O.) runcinata Lee, 1992

DisTRIBUCIÓN: Australia.

-Oribatula (O.) sakamorii Aoki, 1970

Distribución: Este de Paleártica oriental.

-Oribatula (O.) salijanica Kulijev, 1962

DisTRIBUCIÓN: Cáucaso.

-Oribatula (O.) sitnikovae Iordansky, 1991

DisTRIBUCIÓN: Norte de Rusia europea.

-Oribatula (O.) tibialis (Nicolet, 1855) (Notaspis)

DisTRIBUCIÓN: Holártica.

-Oribatula (O.) tibialis tibialis (Nicolet, 1855)

(=Oribatula affinis Hull, 1914)

(=Oribatula amblyptera Berlese, 1916)

(=Oribatula coggii Jacot, 1929)

(=Oribata crassipes L. Koch, 1879)

=Zygoribatula incurva Mihelcic, 1969$)$

(=Liacarus minutus Ewing, 1909)

=Oribatula pallida Banks, 1906)

(=Oribatula tibialis sardoa Berlese, 1916)

(=Oribatula venusta Berlese, 1908)

DISTRIBUCIÓN: Holártica (frecuente).

-Oribatula (O.) tibialis allifera Subías, 2000

(?=Oribatula caliptera Berlese, 1902 "sp. inq.")

(=Oribatula tibialis alata Iordansky, 1991, "nom. praeoc." por Mihelcic, 1956)

Distribución: Sur de Europa.

-Oribatula (O.) tibialis azerbeidjanica Kulijev, 1962 DisTRIBUCión: Cáucaso.

-Oribatula (O.) torrijosi Subías, Ruiz y Kahwash, 1990

DisTRIBUCIÓN: Mediterránea occidental.

-Oribatula (O.) transitans Berlese, 1916 (O. (Zygoribatula))

DisTRIBUCIÓN: U.S.A. oriental.

-Oribatula (O.) translineata (Mahunka, 1985) (Zygoribatula)

Distribución: Neotropical (Pequeñas Antillas y Uruguay).

-Oribatula (O.) trirostrata (Lee y Birchby, 1991) (Ceroribatula)

DisTRIBUCIÓN: Australia.

-Oribatula (O.) unica Golosova y Karppinen, 1985

DisTRIBUCIÓN: Mongolia.

-Oribatula (O.) variabilis (Bayartogtokh y Aoki, 2000) (Eporibatula)

DisTRIBUCIÓN: Japón.

-Oribatula (O.) variaporosa Iordansky, 1991

DisTRIBUCIÓN: Ucrania.

-Oribatula (O.) vera (Bulanova-Zachvatkina, 1967) (Zygoribatula)

DisTRIBUCIÓN: Holártica (Sureste de Europa y Neártica septentrional)

Oribatula (Zygoribatula) Berlese, 1916 (87 spp. y 4 sspp.)

(=Fovoribatula Lee y Birchby, 1991 "sin. nov.")

(=Neoribatula Ewing, 1917)

(=Zetobelba Hull, 1916)

EsPECIE TIPO: Oribatula connexa Berlese, 1904

DisTRIBUCIÓN: Cosmopolita.

-Oribatula (Zygoribatula) acuminata Wallwork, 1964 (O.)

DistriBución: Etiópica (Chad) y Micronesia (I. Marianas).

-Oribatula (Zygoribatula) acuminata acuminata Wallwork, 1964 DisTRIBUCIÓN: Chad.

-Oribatula (Zygoribatula) acuminata mariana Aoki, 1994 (O.) DISTRIBUCIÓN: Micronesia (I. Marianas).

-Oribatula (Zygoribatula) agaveae (Aoki y Wang, 1986) (Z.)

DisTRIBUCIóN: China suroriental.
-Oribatula (Zygoribatula) andrianovae (Bulanova-Zachvatkina, 1967)

(Z.)

DisTRIBUCIÓN: Sur de Rusia europea

-Oribatula (Zygoribatula) arcuata (Hammer, 1977) (Z.)

DisTRIBUCIÓN: Pakistán.

-Oribatula (Zygoribatula) arcuatissima Berlese, 1916

(?=Notaspis aequalis Michael, 1890 "sp. inq.")

(=Zygoribatula gozmanyi Mahunka, 1980)

DisTRIBUCIÓN: Holártica (Mediterránea occidental y "U.S.A.:

Ilinois")

-Oribatula (Zygoribatula) baloghi (Mahunka, 1986) (Z.)

(=Zygoribatula undulata Balogh, 1966, "nom. praeoc." por Berlese, 1916)

DisTRIBUCIÓN: Tanzania.

-Oribatula (Zygoribatula) bonairensis (Willmann, 1936) (Z.)

DISTRIBUCIÓN: Neotropical, Malgache (I. Mauricio) y U.S.A. (Tejas).

-Oribatula (Zygoribatula) bonairensis bonairensis (Willmann, 1936) (=Zygoribatula heterotricha Mahunka, 1979)

DISTRIBUCIÓN: Neotropical (Antillas), Malgache (I. Mauricio) y U.S.A. (Tejas).

-Oribatula (Zygoribatula) bonairensis chilensis (Covarrubias y

Toro, 1997) $(Z$.

DisTRIBUCIÓN: Chile.

-Oribatula (Zygoribatula) brevisetosa (Ewing, 1909) (Notaspis)

DisTRIBUCIÓN: U.S.A. (Ilinois).

-Oribatula (Zygoribatula) capitata (Banks, 1910) (Liacarus)

DisTRIBUCIÓN: U.S.A. central.

-Oribatula (Zygoribatula) clavata Ewing, 1917 (O.)

DisTRIBUCIÓN: U.S.A. nororiental.

-Oribatula (Zygoribatula) cognata (Oudemans, 1902) (Eremaeus)

(=Zygoribatula diversa Mihelcic, 1956)

(=Oribatula (Zygoribatula) socia Berlese, 1916)

DistRIBUCIÓN: Paleártica centromeridional.

-Oribatula (Zygoribatula) concolor (Banks, 1895) (Scutovertex)

DisTRIBUCIÓN: U.S.A. oriental.

-Oribatula (Zygoribatula) connexa Berlese, 1904 (O.)

DistriBución: Subtropical (austral y Paleártica meridional).

-Oribatula (Zygoribatula) connexa connexa Berlese, 1904

(=Zygoribatula concinna Iordansky, 1990)

(=Oribatula (Zygoribatula) excavata Berlese, 1916)

(=Zygoribatula magna Ramsay, 1966)

(=Zygoribatula striatissima Hammer, 1962)

(=Zygoribatula terricola Hammen, 1952)

(=Zygoribatula trichosa Mihelcic, 1956)

DISTRIBUCIÓN: Subtropical (Paleártica meridional y austral: América austral, Australia y Nueva Zelanda).

-Oribatula (Zygoribatula) connexa substriata (Grobler y Kok,

1993) (Z.)

DISTRIBUCIÓN: Sudáfrica.

-Oribatula (Zygoribatula) connexa ucrainica (Iordansky, 1990) (Z. terricola $u$.)

DisTRIBUCIÓN: Sureste de Europa.

-Oribatula (Zygoribatula) contracta (Grobler, 1994) (Z.)

DisTRIBUCIÓN: Sudáfrica.

-Oribatula (Zygoribatula) cyclosporosa (Lee, 1992) (Z.)

DISTRIBUCIÓN: Australia.

-Oribatula (Zygoribatula) dactylaris (Subías, Ruiz y Kahwash, 1990)

(Z.)

DisTRIBUCIÓN: Mediterránea occidental.

-Oribatula (Zygoribatula) debilitranslamellata (Kulijev, 1962) (Z.)

DisTRIBUCIÓN: Cáucaso.

-Oribatula (Zygoribatula) dentata (Balogh, 1958) (Z.) "sp. inq."

DisTRIBUCIÓN: Angola.

-Oribatula (Zygoribatula) egelida (Tseng, 1984) (Z.)

DisTRIBUCIÓN: Taiwan [Formosa].

-Oribatula (Zygoribatula) endroedyi (Mahunka, 1986) (Z.)

DISTRIBUCIÓN: Sudáfrica.

-Oribatula (Zygoribatula) eucalla (Wen y Zhang, 1988) (Z.)

DisTRIBUCIÓN: Noreste de China. 
-Oribatula (Zygoribatula) exarata Berlese, 1916

(=Oribatula rugifrons Sellnick, 1943)

(=Oribatula rugifrons striata Mihelcic, 1955)

(=Oribatula rugosa Kulijev, 1979)

DISTRIBUCIÓN: Paleártica meridional (frecuente)

-Oribatula (Zygoribatula) exilis (Nicolet, 1855) (Notaspis)

(=Zygoribatula clavatotrichobothria Kulijev, 1962)

(=Zygoribatula extremodentata Kulijev, 1962)

(=Dameosoma jugorum Hull, 1914)

$(=$ Oribatula (Zygoribatula) lineola Berlese, 1916)

DisTRIBUCIÓN: Holártica (frecuente) e I. Santa Helena.

-Oribatula (Zygoribatula) floridana (Fritz, 1982) (Z.)

DisTRIBUCIÓN: U.S.A. (Florida) y Méjico.

-Oribatula (Zygoribatula) frisiae (Oudemans, 1900) (Eremaeus)

(=Zygoribatula frisiae insularis Travé, 1961)

(=Notaspis pyrostigmata Ewing, 1909)

(=Zygoribatula tenuelamellata Mihelcic, 1956)

(=Oribatula variabilis Berlese, 1908)

DisTRIBUCIÓN: Holártica (frecuente en Paleártica meridional).

-Oribatula (Zygoribatula) fusca (Ewing, 1913) (Notaspis pyrostig-

mata f.)

(=Zygoribatula rostrata Jacot, 1938)

DisTRIBUCIÓN: U.S.A. nororiental.

-Oribatula (Zygoribatula) galula (Mahunka, 2001) (Z.)

DisTRIBUCIÓN: Kenia.

-Oribatula (Zygoribatula) glabra (Michael, 1890) (Notaspis)

(=Oribatula (Zygoribatula) angulata Berlese, 1916)

(=Eremaeus brauni Sellnick, 1908)

(=Zygoribatula hortobagyensis Mahunka, 1983)

(=Zygoribatula laubieri Travé, 1961)

(=Zygoribatula laubieri canariensis Pérez-Íñigo y Peña, 1997)

(=Zygoribatula laubieri meridionalis Travé, 1961)

(=Zygoribatula matritensis Mihelcic, 1966)

(=Eremaeus propinquus Oudemans, 1902)

DisTRIBUCIÓN: Paleártica (menos frecuente en el Norte).

-Oribatula (Zygoribatula) globosa (Mahunka, 2000) (Z.)

DisTRIBUCIÓN: Yemen.

-Oribatula (Zygoribatula) gracilata (Grobler y Kok, 1993) (Z.)

DisTRIBUCIÓN: Sudáfrica.

-Oribatula (Zygoribatula) grandjeani (Nawar y Borolossy, 1990) (Z.)

DisTRIBUCIÓN: Egipto.

-Oribatula (Zygoribatula) granulata (Kunst, 1958) (Z.)

DisTRIBUCIÓN: Sureste de Europa.

-Oribatula (Zygroribatula) gratiosa (Tseng, 1984) (Z.)

DisTRIBUCIÓN: Taiwan [Formosa].

-Oribatula (Zygoribatula) guadarramica (E. Pérez-Íñigo, 1978) (Z.)

DISTRIBUCIÓN: España.

-Oribatula (Zygoribatula) hailongensis (Wen y Zhang, 1988) (Z.)

DisTRIBUCIÓN: Noreste de China.

-Oribatula (Zygoribatula) heterochaeta (Feider, Vasiliu y Calugar,

1970) (Z.)

DISTRIBUCIÓN: Rumania

-Oribatula (Zygoribatula) hispanica Subías y Arillo, 1998

DisTRIBUCIÓN: España.

-Oribatula (Zygoribatula) kelbadjarica (Kulijev, 1962) (Z.)

DisTRIBUCIÓN: Cáucaso.

-Oribatula (Zygoribatula) keralensis (Ramani y Haq, 1999) (Z.)

DISTRIBUCIÓN: India (Kerala).

-Oribatula (Zygoribatula) knighti (Luxton, 1987) (Z.)

DisTRIBUCIÓN: I. Británicas.

-Oribatula (Zygoribatula) koki J. y P. Balogh, 2002 (Z.)

DisTRIBUCIÓN: Chad.

-Oribatula (Zygoribatula) lata (Hammer, 1961) (Z.)

(=Zygoribatula elongata Hammer, 1961)

(=Zygoribatula oblonga Hammer, 1961)

DisTRIBUCIÓN: Neotropical (Perú y Argentina) y Corea.

-Oribatula (Zygoribatula) lenticulata (Mínguez y Subías, 1986) (Z.)

DisTRIBUCIÓN: Mediterránea.

-Oribatula (Zygoribatula) levigata (Wen y Bu, 1988) (Z.)

DistribuCión: Oeste de China.
-Oribatula (Zygoribatula) lineaporosa (Grobler, 1993) (Z.)

DisTRIBUCIÓN: Sudáfrica.

-Oribatula (Zygoribatula) lineata (Hammer, 1979) (Z.)

DISTRIBUCIÓN: Oriental (Indonesia).

-Oribatula (Zygoribatula) longicuspis (Balogh, 1966) (Z.)

DisTRIBUCión: Chad.

-Oribatula (Zygoribatula) longilinea (Wang y Li, 1997) (Z.)

DisTRIBUCIÓN: Noreste de China.

-Oribatula (Zygoribatula) longisensilla (Djaparidze, 1985) (Z.)

DisTRIBUCIÓN: Cáucaso.

-Oribatula (Zygoribatula) longiseta (Golosova, 1970) (Z.)

DistRIBUCión: Este de Paleártica oriental.

-Oribatula (Zygoribatula) mabar (Mahunka, 2000) (Z.)

DisTRIBUCIÓN: Yemen.

-Oribatula (Zygoribatula) mariehammerae (Feider, Vasiliu y Calugar,

1970) (Z.)

DISTRIBUCIÓN: Rumania.

-Oribatula (Zygoribatula) marina (Fujikawa, 1972) (Z.)

DisTRIBUCIÓN: Japón.

-Oribatula (Zygoribatula) meruensis (Mahunka, 1969) (Z.)

DISTRIBUCIÓN: Tanzania.

-Oribatula (Zygoribatula) microporosa (Bulanova-Zachvatkina, 1967)

(Z.)

DisTRIBUCIÓN: Paleártica (Europa oriental y Este de Rusia asiática).

-Oribatula (Zygoribatula) niliaca (Bayoumi, 1980) (Z.)

DisTRIBUCIÓN: Egipto.

-Oribatula (Zygoribatula) novazealandica (Hammer, 1967) (Z.)

DisTRIBUCIÓN: Nueva Zelanda.

-Oribatula (Zygoribatula) obsessa nom. nov.

[=Fovoribatula brevisetosa Lee y Birchby, 1991, "nom. praeoc." por Ewing, 1909 (“hom. sec.")]

DisTRIBUCIÓN: Australia.

-Oribatula (Zygoribatula) oceana (Hammer, 1972) (Z.)

DistRIBUCIÓN: Polinesia (Tahití).

-Oribatula (Zygoribatula) pennata (Grobler, 1993) (Z.)

DisTRIBUCIÓN: Sudáfrica.

-Oribatula (Zygoribatula) prodorsissima (Feider, Vasiliu y Calugar,

1970) (Z.)

DISTRIBUCIÓN: Rumania

-Oribatula (Zygoribatula) robusta (Grobler, 1993) (Z.)

DisTRIBUCIÓN: Sudáfrica.

-Oribatula (Zygoribatula) ruchljadevi (Bulanova-Zachvatkina, 1967)

(Z.)

DisTRIBUCIÓN: Sureste de Europa.

-Oribatula (Zygoribatula) sabulosa (Balogh, 1966) (Z.)

DisTRIBUCIÓN: Chad.

-Oribatula (Zygoribatula) salina (Balogh, 1966) (Z.)

DisTRIBUCIÓN: Chad.

-Oribatula (Zygoribatula) sayedi (Elbadry y Nasr, 1974) (Z.)

DisTRIBUCIÓN: Egipto.

-Oribatula (Zygoribatula) schauenbergi (Mahunka, 1978) (Z.)

Distribución: Malgache (I. Mauricio).

-Oribatula (Zygoribatula) sculpturata (Mahunka, 1989) (Phauloppia)

DisTRIBUCIÓN: Kenia.

-Oribatula (Zygoribatula) setosa Evans, 1953

DISTRIBUCIÓN: Etiópica.

-Oribatula (Zygoribatula) skrjabini (Bulanova-Zachvatkina, 1967) (Z.)

DisTRIBUCIÓN: Paleártica meridional (Sureste de Europa y Asia centrooccidental: frecuente).

-Oribatula (Zygoribatula) smirnovi (Bulanova-Zachvatkina, 1978) (Z.)

DisTRIBUCIÓN: Sureste de Europa.

-Oribatula (Zygoribatula) socotrensis (Mahunka, 2000) (Z.)

DisTRIBUCIÓN: Yemen.

-Oribatula (Zygoribatula) spherisensilla (Djaparidze, 1985) (Z.)

DistribuCión: Cáucaso.

-Oribatula (Zygoribatula) subantarctica (Pletzen y Kok, 1971) (Z.)

DisTRIBUCIÓN: Antártica (I. Marion).

-Oribatula (Zygoribatula) talis (Tseng, 1984) (Z.)

DisTRIBUCIÓN: Taiwan [Formosa]. 
-Oribatula (Zygoribatula) tameyei (Elbadry y Nasr, 1974) (Z.)

DisTRIBUCIÓN: Egipto.

-Oribatula (Zygoribatula) tenuiseta (Hammer, 1977) (Z.)

DISTRIBUCIÓN: Himalaya.

-Oribatula (Zygoribatula) thalassophila Grandjean, 1935

(=Oribatula travei Bernini, 1969)

DISTRIBUCIÓN: Europa centromeridional.

-Oribatula (Zygoribatula) tortilis (Hammer, 1977) (Z.)

DisTRIBUCIÓN: Himalaya.

-Oribatula (Zygoribatula) trigonella (Bulanova-Zachvatkina, 1967) (Z.)

DisTRIBUCIÓN: Asia centrooccidental.

-Oribatula (Zygoribatula) tritici (Elbadry y Nasr, 1974) (Z.)

DisTRIBUCIÓN: Egipto.

-Oribatula (Zygoribatula) truncata (Aoki, 1961) (Z.)

DistribuCión: Paleártica (Finlandia y Paleártica oriental) y

Noreste de Oriental.

-Oribatula (Zygoribatula) undulata Berlese, 1916

(=Zygoribatula dubita Coetzer, 1968)

(=Zygoribatula heteroporosa Wallwork, 1972)

(=Zygoribatula longiporosa Hammer, 1953)

(=Zygoribatula tadrosi Popp, 1960)

DisTRIBUCIÓN: Pantropical (excepto Neotropical) y subtropical.

-Oribatula (Zygoribatula) vanharteni (Mahunka, 2000) (Z.)

DisTRIBUCIÓN: Yemen.

-Oribatula (Zygoribatula) vegeta (Tseng, 1984) (Z.)

DisTRIBUCIÓN: Taiwan [Formosa].

-Oribatula (Zygoribatula) vulgaris (Bulanova-Zachvatkina, 1967) (Z.)

DisTRIBUCIÓN: Paleártica meridional (Sureste de Europa y Asia central rusa)

-Oribatula (Zygoribatula) zicsii (Bayoumi, 1979) (Z.)

DisTRIBUCIÓN: Paleártica (Hungría y Corea).

Ovobates Mahunka, 1994 (1 sp.)

EsPECIE TIPO: Micreremus subnitidus Berlese, 1913

DistriBuCIÓN: Oriental.

-Ovobates subnitidus (Berlese, 1913) (Micreremus)

DISTRIBUCIÓN: Oriental (Indonesia).

Paraphauloppia Hammer, 1967 (13 spp.)

ESPECIE TIPO: Paraphauloppia novaezealandica Hammer, 1967

DisTRIBUCIÓN: Tropical (Australiana y Neotropical) y austral (excepto Etiópica).

Paraphauloppia (Paraphauloppia) Hammer, 1967 (12 spp.)

DisTRIBUCIÓN: Tropical (Australiana y Neotropical) y austral (excepto Etiópica).

-Paraphauloppia (P.) acutinotata Lee y Birchby, 1991

DistriBuCIÓN: Australia.

-Paraphauloppia (P.) altimontana (Hammer, 1958) (Oribatula)

DisTRIBUCIÓN: Neotropical.

-Paraphauloppia (P.) altimontanoides (Hammer, 1958) (Oribatula) DISTRIBUCIÓN: Argentina.

-Paraphauloppia (P.) australis (Hammer, 1962) (Eporibatula)

DISTRIBUCIÓN: Neotropical austral y subantártica: I. Malvinas [I. Falkland].

-Paraphauloppia (P.) cordylinosa Higgins y Woolley, 1975

Distribución: U.S.A. (Colorado).

-Paraphauloppia (P.) globata Lee y Birchby, 1991

Distribución: Australia.

-Paraphauloppia (P.) gracilis (Hammer, 1958) (Eporibatula)

DisTRIBUCIÓN: Neotropical (Bolivia y Argentina).

-Paraphauloppia (P.) magniporosa (Hammer, 1958) (Oribatula) DisTRIBUCIÓN: Argentina.

-Paraphauloppia (P.) morenoi (Hammer, 1962) (Oribatula) DistribuCIÓN: Argentina.

-Paraphauloppia (P.) novazealandica Hammer, 1967

DistriBuCIÓN: Australiana (Nueva Zelanda y Australia).

-Paraphauloppia (P.) obtusinotata Lee y Birchby, 1991

DisTRIBUCIÓN: Australia.
-Paraphauloppia (P.) triforata Lee y Birchby, 1991

DistriBuCIÓN: Australia.

Paraphauloppia (Monophauloppia) P. Balogh, 1988 (1 sp.)

EsPECIE TIPO: Paraphauloppia (Monophauloppia) planissima P. Balogh, 1988

DISTRIBUCIÓN: Neotropical.

-Paraphauloppia (Monophauloppia) planissima P. Balogh, 1988 DISTRIBUCIÓN: Ecuador.

Phauloppia Berlese, 1908 (27 spp.)

(=Calvoppia Jacot, 1934)

(=Eporibatula Sellnick, 1928)

(=Imparatoppia Jacot, 1934)

(?=Oribata Latreille, 1802 "gen. inq.")

(=Paraliodes Hall, 1911)

(=Trichoribatula Balogh, 1961)

EsPECIE TIPO: (Oppia conformis Berlese, 1895) =Zetes lucorum Koch, 1841

DisTRIBUCIÓN: Semicosmopolita (Holártica, Australiana y Neotropical).

-Phauloppia adjecta Aoki y Ohkubo, 1974

DisTRIBUCIÓN: Japón.

-Phauloppia banksi Marshall, Reeves y Norton, 1987

(=Scutovertex pilosus Banks, 1895, "nom. praeoc." por Koch, 1841)

DisTRIBUCIÓN: U.S.A. oriental

-Phauloppia boletorum (Ewing, 1913) (Lucoppia)

DISTRIBUCIÓN: Neártica septentrional.

-Phauloppia bryani Jacot, 1934

DisTRIBUCIÓN: Hawai.

-Phauloppia caudata Hammer, 1973

Distribución: Polinesia (I. Tonga)

-Phauloppia coineaui Travé, 1961

(?=Lucoppia (Phauloppia) nemoralis Berlese, 1916 "sp. inq.")

DistribuCIÓN: Europa (frecuente).

-Phauloppia curviseta (Ewing, 1910) (Notaspis)

DisTRIBUCIÓN: U.S.A. centroseptentrional.

-Phauloppia cyclonota (Gervais, 1849) (Oribata) "sp. inq."

DistriBución: Chile.

-Phauloppia distincta (Karpelles, 1893) (Oribata) "sp. inq."

DisTRIBUCIÓN: Hungría.

-Phauloppia gessneri (Willmann, 1932) (Eporibatula)

DisTRIBUCIÓN: Europa central.

-Phauloppia gracilis Sellnick, 1952

DisTRIBUCIÓN: I. Bermudas.

-Phauloppia imparata (Jacot, 1934) (Imparatoppia)

DisTRIBUCIÓN: Hawai.

-Phauloppia incomperta (Pérez-Íñigo y Peña, 1996) (Zygoribatula) DisTRIBUCión: I. Canarias.

-Phauloppia knoepffleri Travé, 1961

DistRiBución: Francia (Córcega).

-Phauloppia lucorum (Koch, 1841) (Zetes)

(=Lucoppia (Phauloppia) asperula Berlese, 1916)

(=Oppia berlesei Leonardi, 1896)

(=Oppia conformis Berlese, 1895)

(=Eremaeus conjunctus Oudemans, 1902)

(?=Acarus corticalis De Geer, 1778 "sp. inq.")

(=Phauloppia dzaparidzei Mahunka, 1997)

(?=Acarus geniculatus Linnaeus, 1758 "sp. inq.")

(=Paraliodes incurvata Hall, 1911)

(=Phauloppia longiporosa Kulijev, 1968)

(=Eporibatula longiporosa Pérez-Íñigo, 1976)

(=Phauloppia longiporosa Mahunka, 1982, "nom. praeoc.” por Kulijev, 1968)

(=Phauloppia longiporosa Djaparidze, 1985, “nom. praeoc." por Kulijev, 1968)

(=Liacarus modestus Banks, 1904)

(=Eremaeus modestus Banks, 1910)

(?=Acarus musci Schrank, 1781 "sp. inq.")

(=Phauloppia ohat Mahunka, 1997)

(=Phauloppia saakadzei Djaparidze, 1985)

(=Eremaeus schneideri Oudemans, 1896)

DisTRIBUCIÓN: Holártica (frecuente en Paleártica occidental). 
-Phauloppia mitakensis Suzuki, 1979

DISTRIBUCIÓN: Japón.

-Phauloppia parvilamellata (Ewing, 1909) (Oribata) "sp. inq." DISTRIBUCIÓN: U.S.A. (Ilinois).

-Phauloppia perkinsi (Jacot, 1934) (Calvoppia) DisTRIBUCIÓN: Hawai

-Phauloppia pilosa (Koch, 1841) (Zetes)

DiSTRIBUCIÓN: Holártica (Europa centromeridional y U.S.A.: Virginia).

-Phauloppia pinnata (Gervais, 1849) (Oribata) "sp. inq." DistriBución: Chile.

-Phauloppia rauschenensis (Sellnick, 1908) (Eremaeus)

(=Oribatula lucida Sellnick, 1921)

(=Phauloppia paspalevi Csiszár, 1962)

DistRIBUCIÓN: Paleártica (Europa y Asia central rusa).

-Phauloppia saxicola Travé, 1961

DisTRIBUCión: Suroeste de Europa.

-Phauloppia tavruei (Schweizer, 1956) (Eporibatula)

Distribución: Suiza.

-Phauloppia topali Balogh y Csiszár, 1963

DisTRIBUCIÓN: Argentina.

-Phauloppia tuberosa (Fujikawa, 1972) (Eporibatula)

Distribución: Este de Paleártica oriental.

-Phauloppia vallei Bernini, 1973

DisTRIBUCión: Italia (Sicilia).

-Phauloppia xinjiangensis L. Wang, Zheng, X. Wang, X. Zhang y

Wen, 1990

DistriBución: Oeste de China.

Phauloppiella Subías, 1977 (2 spp.)

EsPECIE TIPO: Phauloppiella striata Subías, 1977

DisTRIBUCIÓN: Paleártica y Etiópica.

-Phauloppiella glabra Mahunka, 1987

DisTRIBUCIÓN: Nigeria.

-Phauloppiella striata Subías, 1977

DISTRIBUCIÓN: España.

Reticuloppia Balogh y Mahunka, 1966 (2 spp.)

(=Decoribatula Lee y Birchby, 1989 "sin. nov.")

EsPecie TiPo: Reticuloppia reticulata Balogh y Mahunka, 1966

DISTRIBUCIÓN: Australianooriental.

-Reticuloppia pustulata (Lee y Birchby, 1989) (Decoribatula) Distribución: Península Malaya.

-Reticuloppia reticulata Balogh y Mahunka, 1966 DisTRIBUCIÓN: Australia.

Sellnickia Oudemans, 1927 (1 sp.)

ESPECIE TIPO: (Sellnickia heveae Oudemans, 1927) =Notaspis caudata Michael, 1898

DISTRIBUCIÓN: Australianooriental.

-Sellnickia caudata (Michael, 1898) (Notaspis)

(=Oribatula caudata Berlese, 1910, "nom. praeoc." por Michael, 1898)

(=Sellnickia heveae Oudemans, 1927)

DisTRIBUCIÓN: Australiana (Nueva Zelanda y Australia) y Sumatra.

Spinoppia Higgins y Woolley, 1966 (1 sp.)

EsPeCIE TIPO: Spinoppia magniserrata Higgins y Woolley, 1966

DISTRIBUCIÓN: Neártica subtropical.

-Spinoppia magniserrata Higgins y Woolley, 1966

DisTRIBUCIÓN: U.S.A. (Florida).

Subphauloppia Hammer, 1967 (4 spp.)

EsPeCIE TIPO: Subphauloppia dentonyx Hammer, 1967

DisTRIBUCIÓN: Etiópica y Australiana.

-Subphauloppia dentonyx Hammer, 1967

DISTRIBUCIÓN: Nueva Zelanda.

-Subphauloppia endroedyi Mahunka, 1985

DISTRIBUCIÓN: Sudáfrica.

-Subphauloppia glabra Hammer, 1973

Distribución: Polinesia (I. Tonga).
-Subphauloppia lamellata Mahunka, 1985

DisTRIBUCIÓN: Sudáfrica.

Nesozetidae J. y P. Balogh, 1984 (1 gen. y 1 sp.) DisTRIBUCIÓN: Australiana.

Nesozetes Hammer, 1971 (1 sp.)

EsPeCIE TIPO: Nesozetes rostropterus Hammer, 1971

DISTRIBUCIÓN: Australiana.

-Nesozetes rostropterus Hammer, 1971

Distribución: Melanesia (I. Fiji)

Pseudoppiidae Mahunka, 1975 (3 gen. y 4 spp.)

DisTRIBUCIÓN: Tropical (Etiópica y Australiana) y subtropical (Paleártica meridional).

Ausoribula Lee, 1992 (1 sp.)

ESPECIE TIPO: Ausoribula quagesetosa Lee, 1992

DisTRIBUCIÓN: Australiana.

-Ausoribula quagesetosa Lee, 1992

DisTRIBUCIÓN: Australia.

Pseudoppia Pérez-Íñigo, 1966 (2 spp.)

(=Symphauloppia Balogh, 1972)

EsPeCIE TIPO: Lucoppia mediocris Mihelcic, 1957

DISTRIBUCIÓN: Paleártica meridional.

-Pseudoppia interrupta (Jeleva, 1962) (Phauloppia)

DistRIBUCIÓN: Mediterránea.

-Pseudoppia mediocris (Mihelcic, 1957) (Lucoppia)

DisTRIBUCIÓN: Mediterránea.

Senoribula Mahunka, 1975 (1 sp.)

EsPECIE TIPO: Senoribula africana Mahunka, 1975

DISTRIBUCIÓN: Etiópica.

-Senoribula africana Mahunka, 1975

DisTRIBUCIÓN: Senegal.

Parapirnodidae Aoki y Ohkubo, 1974 (3 gen. y 8 spp.)

DisTRIBUCIÓN: Semicosmopolita (Pantropical: excepto Etiópica, y Holártica).

Gerloubia Coetzer, 1968 (3 spp.)

(=Fuerteventuria Pérez-Íñigo y Peña, 1996 "sin. nov.")

EsPECIE TIPO: Eporibatula bicuspidata Hammer, 1958

DISTRIBUCIÓN: Paleártica meridional, Oriental y Neotropical.

-Gerloubia bicuspidata (Hammer, 1958) (Eporibatula)

DISTRIBUCIÓN: Argentina.

-Gerloubia chinensis Aoki y Yamamoto, 2000

DisTRIBUCIÓN: China suroriental.

- Gerloubia hispanica Subías, 1977

(=Liebstadia ahumerata Mahunka, 1987)

(=Fuerteventuria mirabilis Pérez-Íñigo y Peña, 1996)

DISTRIBUCIÓN: Mediterránea.

Parapirnodus Balogh y Mahunka, 1968 (4 spp.)

EsPeCIE TIPO: Parapirnodus longus Balogh y Mahunka, 1968

DisTRIBUCIÓN: Neotropical y Neártica.

-Parapirnodus coniferinus Behan-Pelletier, Clayton y Humble, 2001

DisTRIBUCIÓN: Canadá.

-Parapirnodus hexaporosus Behan-Pelletier, Clayton y Humble, 2001

DISTRIBUCIÓN: Canadá.

-Parapirnodus longus Balogh y Mahunka, 1968

DistriBución: Argentina.

-Parapirnodus prosopis Martínez, Fernández y Monetti, 1996

DisTRIBUCIÓN: Argentina.

Pontiobates Luxton, 1989 (1 sp.)

EsPecie TIPO: Pontiobates denigratus Luxton, 1989

DISTRIBUCIÓN: Australiana. 
-Pontiobates denigratus Luxton, 1989

DisTRIBUCIÓN: Nueva Zelanda.

Caloppiidae Balogh, 1960 (4 gen. y 22 spp.) DisTRIBUCIÓN: Pantropical.

Brassiella Balogh, 1970 (3 spp.)

EsPECIE TIPO: (Carabodes reticulatus Oudemans, 1915) =Brassiella neonominata nom. nov.

DisTRIBUCIÓN: Australianooriental.

-Brassiella arboricola J. y P. Balogh, 1983

DistriBución: Nueva Caledonia.

-Brassiella neonominata nom. nov.

[=Carabodes reticulatus Oudemans, 1915, "nom. praeoc." por Berlese,

1913 ("hom. prim.")]

DistribuCIÓn: Oriental y Australiana (Nueva Guinea)

-Brassiella penicillifer Hammer, 1973

DisTRIBUCIÓN: Polinesia (I. Tonga).

Chaunoproctellus Mahunka, 1992 (1 sp.)

ESPECIE TIPO: Chaunoproctellus rugosus Mahunka, 1992

Distribución: Etiópica.

-Chaunoproctellus rugosus Mahunka, 1992

DisTRIBUCIÓN: Senegal.

Chaunoproctus Pearce, 1906 (16 spp.)

(=Caloppia Balogh, 1958)

(=Pabulozetes Tseng, 1982 "sin. nov.")

(=Zetorchella Berlese, 1916)

EsPECIE TIPO: Chaunoproctus cancellatus Pearce, 1906

DisTRIBUCIÓN: Pantropical (excepto Australiana).

-Chaunoproctus abalai Bhaduri, Bhattacharya y Chakrabarti, 1975

DistriBución: India (Bengala Occidental).

-Chaunoproctus asperulus Pearce, 1906

DisTRIBUCIÓN: India (Sikkim).

-Chaunoproctus basilewskyi (Balogh, 1958) (Caloppia)

DistriBución: Congo.

-Chaunoproctus cancellatus Pearce, 1906

DisTRIBUCIÓN: India (Sikkim).

-Chaunoproctus deleoni Higgins, 1966

DisTRIBUCión: Guyana [Guayana Británica].

-Chaunoproctus latior (Berlese, 1913) (Oppia)

(=Chaunoproctus clavisetosus Bhaduri, Bhattacharya y Chakrabarti, 1975) DISTRIBUCIÓN: Oriental.

-Chaunoproctus longipilosus (Mahunka, 1974) (Caloppia)

DisTRIBUCIÓN: Rodesia [Zimbabue].

-Chaunoproctus longisetosus Dhali y Bhaduri, 1981

DistriBución: India (Sikkim).

-Chaunoproctus minor (Balogh, 1958) (Caloppia)

DisTRIBUCión: Angola e India (Bengala Occidental).

-Chaunoproctus orbiculatus Wen y Zhao, 1994

DisTRIBUCIón: China suroriental.

-Chaunoproctus pedestris (Berlese, 1916) (Zetorchella)

(=Chaunoproctus crinitus Karppinen, 1966)

(=Caloppia papillata Balogh, 1958)

DisTRIBUCIÓN: Etiópica (frecuente)

-Chaunoproctus plumosus (Tseng, 1982) (Pabulozetes)

DISTRIBUCIÓN: Taiwan [Formosa].

-Chaunoproctus reticulatus (Willmann, 1933) (Lucoppia)

DisTRIBUCIÓN: Sumatra.

-Chaunoproctus sejugatus (Ramani y Haq, 1997) (Caloppia)

DisTRIBUCIÓN: India (Kerala).

-Chaunoproctus sottoetgarciai (Corpuz-Raros, 1979) (Caloppia)

DisTRIBUCIÓN: Filipinas.

-Chaunoproctus vargai (Balogh, 1959) (Caloppia)

DisTRIBUCIón: Tanzania.

Stelechobates Grandjean, 1965 (2 spp.)

EsPECIE TIPO: Stelechobates megalotrichus Grandjean, 1965

DISTRIBUCIÓN: Neotropical.
-Stelechobates brazilianus P. Balogh, 1995

DisTRIBUCIÓN: Brasil.

-Stelechobates megalotrichus Grandjean, 1965

DisTRIBUCIÓN: Norte de Neotropical.

Hemileiidae J. y P. Balogh, 1984 (15 gen., 7 subg., 118 spp. y 5 sspp.) DisTRIBUCIÓN: Cosmopolita.

Balazsella Mahunka, 1983 (3 spp.)

EsPECIE TIPO: Balazsella pilososetosa Mahunka, 1983

DisTRIBUCIÓN: Neotropical.

-Balazsella ilhabellae Balogh y Palacios-Vargas, 1996

DistriBución: Brasil.

-Balazsella mexicana Balogh y Palacios-Vargas, 1996

Distribución: Méjico.

-Balazsella pilososetosa Mahunka, 1983

DisTRIBUCIÓN: Brasil.

Baobabula Mahunka, 1975 (2 spp.)

EsPeCIE TIPO: Baobabula mussardi Mahunka, 1975

DISTRIBUCIÓN: Etiópica.

-Baobabula impolita (Warburton, 1912) (Notaspis)

DisTRIBUCión: I. Seychelles.

-Baobabula mussardi Mahunka, 1975

DISTRIBUCIÓN: Senegal.

Constrictobates Balogh y Mahunka, 1966 (1 sp.)

EsPECIE TIPO: Constrictobates lineolatus Balogh y Mahunka, 1966 DISTRIBUCIÓN: Australiana.

-Constrictobates lineolatus Balogh y Mahunka, 1966

DistriBuCIÓN: Australia.

Dometorina Grandjean, 1951 (15 spp. y 3 sspp.)

(=Cryptozetes Hammer, 1962 "sin. nov.")

EsPeCIE TIPO: Oribatula plantivaga Berleser, 1895

DISTRIBUCIÓN: Cosmopolita.

-Dometorina chilensis (Hammer, 1962) (Cryptozetes)

DisTRIBUCIÓN: Neotropical austral.

-Dometorina crystallina Tseng, 1984

Distribución: Taiwan [Formosa].

-Dometorina elongata Balogh y Csiszár, 1963

DisTRIBUCIÓN: Argentina.

-Dometorina inflata (Berlese, 1916) (Oribatula (Hemileius))

DisTRIBUCIÓN: Argentina.

-Dometorina lata Mahunka, 1986

DisTRIBUCión: Sudáfrica.

-Dometorina limpida Tseng, 1984

DisTRIBUCIÓN: Taiwan [Formosa].

-Dometorina marionensis Pletzen y Kok, 1971

DisTRIBUCIÓN: Antártica.

-Dometorina plantivaga (Berlese, 1895) (Oribatula)

DistRIBUCIÓN: Cosmopolita (excepto Australiana y Antártica).

-Dometorina plantivaga plantivaga (Berlese, 1895)

DisTRIBUCIÓn: Cosmopolita (Neártica, Paleártica occidental: menos frecuente en el Norte, Paleártica oriental, Etiópica: Yemen, Oriental y Neotropical: frecuente).

-Dometorina plantivaga brasiliana C. y C., jr. Pérez-Íñigo, 1993 DISTRIBUCIÓN: Brasil.

-Dometorina plantivaga insularis Travé, 1969

Distribución: Macaronésica (I. Salvajes).

-Dometorina plantivaga saxicola Grandjean, 1951 DisTRIBUCIÓn: Sur de Europa.

-Dometorina praedatoria Wu, Xin y Aoki, 1986

DistRIBUCIÓN: China suroriental.

-Dometorina rostrata Luxton, 1993

Distribución: China (Hong-Kong).

-Dometorina sicata Mahunka, 1983

DisTRIBUCIÓN: Tanzania.

-Dometorina taiwanica Tseng, 1984

Distribución: Taiwan [Formosa]. 
-Dometorina tropica (Balogh, 1958) (Hemileius)

DistriBución: Angola.

-Dometorina tuberculata Aoki, 1984

DisTRIBUCIÓN: Este de Paleártica oriental.

-Dometorina usnea (Norton y Palacios-Vargas, 1987) (Cryptozetes) Distribución: Méjico.

Exoribatula Jacot, 1936 (8 spp.)

EsPECIE TIPO: Exoribatula biundata Jacot, 1936

DisTRIBUCIÓN: Pantropical y subtropical (Neártica meridional).

Exoribatula (Exoribatula) Jacot, 1936 (4 spp.)

(=Nesoribatula Aoki, 1964)

Distribución: Tropical (Etiópica y Australiana) y subtropica (Neártica meridional).

-Exoribatula (E.) biundata Jacot, 1936

DistribuCión: U.S.A. (Carolina del Norte).

-Exoribatula (E.) ensifer (Mahunka, 1983) (Nesoribatula)

DisTRIBUCIÓN: Tanzania.

-Exoribatula (E.) pacifica (Aoki, 1964) (Nesoribatula)

DISTRIBUCIÓN: Hawai.

-Exoribatula (E.) rotunda (Mahunka, 1988) (Nesoribatula)

DisTRIBUCIÓN: Tanzania

Exoribatula (Multoribates) Hammer, 1961 (4 spp.)

ESPECIE TIPO: Multoribates chavinensis Hammer, 1961

DISTRIBUCIÓN: Tropical (Neotropical y Oriental).

-Exoribatula (Multoribates) chavinensis (Hammer, 1961) (M.)

DisTRIBUCIÓN: Perú.

-Exoribatula (Multoribates) longior (Hammer, 1958) (Scheloribates) DisTRIBUCIÓN: Bolivia.

-Exoribatula (Multoribates) multisetosus (Balogh, 1970) (Tuberemaeus)

DisTRIBUCiÓN: Ceilán [Sri Lanka].

-Exoribatula (Multoribates) parvialatus (Hammer, 1958) (Scheloribates)

DISTRIBUCIÓN: Argentina.

Hemileius Berlese, 1916 (65 spp. y 1 ssp.)

EsPeCIE TIPO: Protoribates (Scheloribates) initialis Berlese, 1908

DisTRIBUCIÓn: Cosmopolita (excepto Antártica).

Hemileius (Hemileius) Berlese, 1916 (24 spp.)

DisTRIBUCIÓN: Cosmopolita (excepto Etiópica y Antártica).

-Hemileius (H.) biclavulus Lee, 1989

DistriBuCIÓN: Australia.

-Hemileius (H.) brevilamellatus (Willmann, 1931) (Liebstadia)

DisTRIBUCIÓN: Sumatra.

-Hemileius (H.) clavatus Aoki, 1992

DISTRIBUCIÓN: Japón.

-Hemileius (H.) copectus Lee, 1989

DisTRIBUCIÓN: Australia.

-Hemileius (H.) elongatus E. Pérez-Íñigo, 1978

Distribución: Sur de Europa.

-Hemileius (H.) gressitti J. y P. Balogh, 1983

DisTRIBUCIÓN: Hawai.

-Hemileius (H.) haydeni (Higgins y Woolley, 1975) (Multoribates)

DisTRIBUCIÓN: U.S.A. (Colorado).

-Hemileius (H.) hierrensis Pérez-Íñigo, 1984

DisTRIBUCIÓN: Mediterránea.

-Hemileius (H.) humeralis Pérez-Íñigo jr., 1991

DisTRIBUCIÓN: España.

-Hemileius (H.) initialis (Berlese, 1908) (Protoribates (Scheloribates))

(=Scheloribates confundatus Sellnick, 1928)

DisTRIBUCión: Semicosmopolita (Paleártica: frecuente, Oriental:

Bali, y Neotropical)

-Hemileius (H.) laticlava Pérez-Íñigo y Baggio, 1991

DistriBuCIÓN: Brasil.

-Hemileius (H.) major (Mahunka, 1985) (Scheloribates)

DistRIBUCIÓN: Pequeñas Antillas.
-Hemileius (H.) microclava (Hammer, 1961) (Scheloribates)

DisTRIBUCIÓN: Neotropical (Perú y Panamá).

-Hemileius (H.) muscicola (Hammer, 1961) (Scheloribates)

DisTRIBUCIÓN: Perú.

-Hemileius (H.) nicki Denmark y Woodring, 1965

DisTRIBUCIÓN: U.S.A. (Florida).

-Hemileius (H.) proximus (Berlese, 1916) (Oribatula (H.))

DistriBuCión: Neotropical (Argentina y Paraguay).

-Hemileius (H.) quadripilis (Fitch, 1856) (Oribata)

(=Oribatula (Hemileius) comatus Berlese, 1920)

(=Notaspis pallida Ewing, 1909)

DISTRIBUCIÓN: Neártica.

-Hemileius (H.) rectus Lee, 1989

DisTRIBUCIÓN: Australia.

-Hemileius (H.) robustus Pérez-Íñigo, 1969

DistriBUCIÓN: Mediterránea occidental

-Hemileius (H.) suramericanus (Hammer, 1958) (Oribatula)

DISTRIBUCIÓN: Neotropical (frecuente) y U.S.A. (Kentucky).

-Hemileius (H.) tenuis Aoki, 1982

DISTRIBUCIÓN: Japón.

-Hemileius (H.) thujae Choy y Cho, 1995

DisTRIBUCIÓN: Corea.

-Hemileius (H.) translamellatus Karppinen, 1958

DISTRIBUCIÓN: Finlandia.

-Hemileius (H.) trichosus (Hammer, 1958) (Scheloribates)

DISTRIBUCIÓN: Bolivia.

Hemileius (Tuberemaeus) Sellnick, 1930 (31 spp. y 1 ssp.)

(=Anisochthodes Newell, 1957)

(=Setulobates Mahunka, 1984)

EsPECIE TIPO: Tuberemaeus singularis Sellnick, 1930

Distribución: Pantropical (excepto Neotropical) y subtropical

(Paleártica meridional).

-Hemileius (Tuberemaeus) areolatus (Balogh y Mahunka, 1967) (T.)

DisTRIBUCIÓN: Congo.

-Hemileius (Tuberemaeus) asiaticus (Yamamoto y Aoki, 2000) (T.)

DISTRIBUCIÓN: China suroriental.

-Hemileius (Tuberemaeus) bellissimus (Hammer, 1971) (T.)

DistribuCión: Melanesia (I. Fiji).

-Hemileius (Tuberemaeus) deletus (Hammer, 1979) (T.)

DistRIBUCión: Oriental (Java y Filipinas).

-Hemileius (Tuberemaeus) dispar (Balogh y Mahunka, 1974) (T.)

DisTRIBUCión: Península Malaya.

-Hemileius (Tuberemaeus) fissuratus (Balogh, 1970) (T.)

DistriBUCIÓN: Nueva Guinea y Filipinas.

-Hemileius (Tuberemaeus) formosanus (Tseng, 1984) (T.)

DisTRIBUCIÓN: Taiwan [Formosa].

-Hemileius (Tuberemaeus) foveolatus (Balogh, 1958) (Liebstadia)

DisTRIBUCIÓN: Tanzania.

-Hemileius (Tuberemaeus) foveolatus foveolatus (Balogh, 1958) DistribuCión: Tanzania.

-Hemileius (Tuberemaeus) foveolatus tridactylus (Balogh, 1959)

(Liebstadia)

DisTRIBUCión: Tanzania

-Hemileius (Tuberemaeus) glaber (Balogh, 1970) (T.)

DistRIBUCIÓN: Nueva Guinea.

-Hemileius (Tuberemaeus) heterotrichus (Mahunka, 1984) (Setulobates) DistRIBUCIÓN: Tanzania.

-Hemileius (Tuberemaeus) indentatus (Hammer, 1973) (T.)

Distribución: Polinesia (I. Samoa).

-Hemileius (Tuberemaeus) inornatus (Pérez-Íñigo, 1982) (T.)

Distribución: Guinea Ecuatorial: I. Pagalu [Annobón].

-Hemileius (Tuberemaeus) lagunensis (Corpuz-Raros, 1979) (T.)

DistRIBUCIÓN: Filipinas.

-Hemileius (Tuberemaeus) lineatus (Balogh, 1970) (T.)

Distribución: Nueva Guinea y Filipinas.

-Hemileius (Tuberemaeus) longisetosus (Balogh, 1970) (T.)

Distribución: Nueva Guinea.

-Hemileius (Tuberemaeus) luzonensis (Corpuz-Raros, 1979) (T.)

DisTRIBUCIÓN: Filipinas. 
-Hemileius (Tuberemaeus) nagaii (Mahunka, 1988) (T.)

DISTRIBUCIÓN: Borneo.

-Hemileius (Tuberemaeus) neonominatus nom. nov.

[=Tuberemaeus punctatus Balogh, 1970, "nom. praeoc.” por Hammer,

1961 (“hom. sec.")]

DistriBuCión: Nueva Guinea y Península Malaya.

-Hemileius (Tuberemaeus) papillifer (Newell, 1957) (Anisochthodes) DisTRIBUCión: Hawai y Filipinas.

-Hemileius (Tuberemaeus) perforatoides (Hammer, 1979) (T.)

DISTRIBUCIÓN: Oriental (Java y Filipinas)

-Hemileius (Tuberemaeus) perforatus (Willmann, 1931) (Liebstadia)

DisTRIBUCIÓN: Oriental (Java y Filipinas) y Australiana (Polinesia).

-Hemileius (Tuberemaeus) propinquus (Balogh y Mahunka, 1974) (T.) DistriBución: Península Malaya.

-Hemileius (Tuberemaeus) pseudoareolatus (Mahunka, 1977) (T.) DisTriBuCión: Kenia.

-Hemileius (Tuberemaeus) scrobinus (Berlese, 1916) (Oribatula (H.)) DisTRIBUCIÓN: Italia.

-Hemileius (Tuberemaeus) sculpturatus (Mahunka, 1987) (T.) DISTRIBUCIÓN: Vietnam.

-Hemileius (Tuberemaeus) similis (Balogh, 1970) (T.)

DistriBución: Ceilán [Sri Lanka].

-Hemileius (Tuberemaeus) singularis (Sellnick, 1930) (T.) DistriBuCión: Oriental (Sumatra y Filipinas), Corea y Hawai.

-Hemileius (Tuberemaeus) subareolatus (Mahunka, 1977) (T.) DistriBución: Kenia.

-Hemileius (Tuberemaeus) thienemanni (Willmann, 1931) (Liebstadia) DisTRIBUCIÓN: Oriental (Indonesia) y Polinesia (I. Samoa).

-Hemileius (Tuberemaeus) tuberculatus (Balogh, 1970) (T.) DisTRIBUCIÓN: Nueva Guinea y Filipinas.

-Hemileius (Tuberemaeus) vermiculatus (Balogh, 1970) (T.)

DistriBución: Nueva Guinea y Filipinas.

Hemileius (Turcibates) Ayyildiz y Luxton, 1989 (4 spp.) (=Tenuileius Lee, 1989 "sin. nov.")

EsPECIE TIPO: Turcibates parvus Ayyildiz y Luxton, 1989

DISTRIBUCIÓN: Paleártica meridional y Australiana.

-Hemileius (Turcibates) lanceolatus Bayartogtokh y Aoki, 1997 (H.) DISTRIBUCIÓN: Mongolia.

-Hemileius (Turcibates) minimus Lee, 1989 (H. (Tenuileius)) DisTRIBUCIÓN: Australia.

-Hemileius (Turcibates) paratenuis Lee, 1989 (H. (Tenuileius)) DISTRIBUCIÓN: Australia.

-Hemileius (Turcibates) parvus (Ayyildiz y Luxton, 1989) (T.)

(?=Dometorina rossica Bashkirova, 1958 "sp. inq.")

DisTRIBUCIÓN: Mediterránea.

Hemileius (Urubambates) Hammer, 1961 (6 spp.)

EsPECIE TIPO: Urubambates punctatus Hammer, 1961

DisTRIBUCIÓN: Paleártica meridional, Etiópica y Neotropical.

-Hemileius (Urubambates) calcaratus (Mahunka, 1984) (U.) DisTRIBUCIÓN: Sudáfrica.

-Hemileius (Urubambates) flagellatus (Mahunka, 1985) (U.) DisTriBuCión: Pequeñas Antillas.

-Hemileius (Urubambates) paraguayensis (Balogh y Mahunka, 1981) (U.)

DisTRIBUCión: Paraguay.

-Hemileius (Urubambates) perlongus (Vasiliu y Calugar, 1976) (U.)

DISTRIBUCIÓN: Rumania.

-Hemileius (Urubambates) punctatus (Hammer, 1961) (U.)

DisTRIBUCIÓN: Perú.

-Hemileius (Urubambates) romanicus (Vasiliu y Calugar, 1981) (U.)

DISTRIBUCIÓN: Rumania.

Heteroleius Balogh y Mahunka, 1966 (3 spp.)

EsPecie TIPO: Heteroleius longissimus Balogh y Mahunka, 1966

DisTRIBUCIÓN: Paleotropical y Paleártica meridional.

-Heteroleius longissimus Balogh y Mahunka, 1966

DisTRIBUCIÓN: Congo y Filipinas.
-Heteroleius navicula (Berlese, 1913) (Oribatula)

DistriBución: Java.

-Heteroleius planus (Aoki, 1984) (Symphauloppia)

DisTRIBUCIÓN: Este de Paleártica oriental.

Monoschelobates Balogh y Mahunka, 1969 (2 spp.)

ESPECIE TIPO: Monoschelobates parvus Balogh y Mahunka, 1969

DisTRIBUCIÓN: Neotropical.

-Monoschelobates parvus Balogh y Mahunka, 1969

DisTRIBUCIÓN: Brasil.

-Monoschelobates translamellatus Pérez-Íñigo y Baggio, 1991

DISTRIBUCIÓN: Brasil.

Mucrobates Balogh y Mahunka, 1979 (1 sp.)

EsPECIE TIPO: Mucrobates fissuratus Balogh y Mahunka, 1979

DISTRIBUCIÓN: Neotropical.

-Mucrobates fissuratus Balogh y Mahunka, 1979

DISTRIBUCIÓN: Cuba.

Nasozetes Sellnick, 1930 (4 spp.)

(=Kinabaluella Mahunka, 1996 "sin. nov.")

ESPECIE TIPO: Nasozetes sumatrensis Sellnick, 1930

DISTRIBUCIÓN: Australianooriental.

-Nasozetes choerognathus Willmann, 1931

DistriBUCiÓN: Oriental (Indonesia).

-Nasozetes porcella (Mahunka, 1996) (Kinabaluella)

DISTRIBUCIÓN: Borneo.

-Nasozetes stunkardi Sengbusch, 1957

DistRIBUCIÓN: Micronesia (I. Marianas) y Filipinas.

-Nasozetes sumatrensis Sellnick, 1930

DISTRIBUCION: Sumatra.

Paraleius Travé, 1960 (3 spp.)

EsPeCIE TIPO: Oribella leontonycha Berlese, 1910

DisTRIBUCIÓN: Paleártica y Oriental.

Paraleius (Paraleius) Travé, 1960 (1 sp.)

DisTRIBUCIÓN: Paleártica.

-Paraleius (P.) leontonychus (Berlese, 1910) (Oribella)

DisTRIBUCIÓN: Paleártica.

Paraleius (Metaleius) Travé, 1960 (1 sp.)

EsPeCiE TiPo: Metaleius strenzkei Travé, 1960

DisTRIBUCIÓN: Paleártica.

-Paraleius (Metaleius) strenzkei (Travé, 1960) (M.)

(?=Oribatula (Hemileius) sternalis Berlese, 1916 "sp. inq.")

DisTRIBUCIÓN: Mediterránea.

Paraleius (Wallworkiella) Hammer, 1979 (1 sp.)

ESPECIE TIPO: Wallworkiella nasalis Hammer, 1979

DisTRIBUCIÓN: Oriental.

-Paraleius (Wallworkiella) nasalis (Hammer, 1979) (W.)

DISTRIBUCIÓN: Java.

Plumobates Balogh y Mahunka, 1966 (1 sp.)

EsPeCIE TIPO: Plumobates decoratus Balogh y Mahunka, 1966

DisTRIBUCIÓN: Australiana.

-Plumobates decoratus Balogh y Mahunka, 1966

Distribución: Australia.

Siculobata Grandjean, 1953 (4 spp. y 1 ssp.)

EsPeCiE TIPO: Oppia tibialis sicula Berlese, 1892

DisTRIBUCIÓN: Pantropical (excepto Etiópica) y subtropical.

Siculobata (Siculobata) Grandjean, 1953 (3 spp. y 1 ssp.)

=Floribates Norton y Kethley, 1989 "sin. nov.")

DistRIBUCIÓN: Pantropical (excepto Etiópica) y subtropical.

-Siculobata (S.) florens (Berlese, 1908) (Oribatula)

DisTRIBUCIÓN: U.S.A. (Florida). 
-Siculobata (S.) malabarica Ramani y Haq, 1998

DisTRIBUCIÓN: India (Kerala).

-Siculobata (S.) sicula (Berlese, 1892) (Oppia tibialis s.)

Distribución: Semicosmopolita: Paleártica meridional (Medite-

rránea, Polonia e Irán), Islas del Pacífico y Argentina.

-Siculobata (S.) sicula sicula (Berlese, 1892)

DistriBuCión: Paleártica meridional (Mediterránea, Polonia e Irán) e Islas del Pacífico.

-Siculobata (S.) sicula platensis (Berlese, 1916) (Oribatula (Hemileius))

DISTRIBUCIÓN: Argentina

Siculobata (Vesiculobates) Hammer, 1979 (1 sp.)

EsPECIE TIPO: Vesiculobates silvaticus Hammer, 1979

DISTRIBUCIÓN: Oriental.

-Siculobata (Vesiculobates) silvatica (Hammer, 1979) (V.)

DISTRIBUCIÓN: Java

Simkinia Krivolutsky, 1966 (5 spp.)

EsPecie Tipo: Simkinia turanica Krivolutsky, 1966

DisTRIBUCIÓN: Paleártica.

-Simkinia elongata Krivolutsky, 1969

DisTRIBUCIÓN: Asia centooccidental (frecuente).

-Simkinia montana Krivolutsky y Grishina, 1970

Distribución: Paleártica: Cáucaso y Paleártica oriental (excepto Este).

-Simkinia schachtachtinskoi (Kulijev, 1961) (Oribatula)

Distribución: Paleártica meridional (Cáucaso y Sur de Paleártica oriental).

-Simkinia tianschanica Krivolutsky, 1971

DisTRIBUCIÓN: Paleártica meridional (Crimea y Asia centrooccidental).

-Simkinia turanica Krivolutsky, 1966

(=Hemileius ovalis Kulijev, 1968)

DisTRIBUCión: Paleártica meridional (Sureste de Europa y Sur de

Paleártica oriental: frecuente).

Zeascheloribates Luxton, 1982 (1 sp.)

EsPECIE TIPO: Zeascheloribates palustris Luxton, 1982

DisTRIBUCión: Australiana.

-Zeascheloribates palustris Luxton, 1982

DisTRIBUCIÓN: Nueva Zelanda.

Maudheimiidae J. y P. Balogh, 1984 (2 gen. y 4 spp.) DISTRIBUCIÓN: Antártica.

Maudheimia Dalenius, 1958 (3 spp.)

ESPECIE TIPO: Maudheimia wilsoni Dalenius, 1958

DISTRIBUCIÓN: Antártica.

-Maudheimia petronia Wallwork, 1962

DisTRIBUCIÓN: Antártida.

-Maudheimia tanngardenensis Coetzee, 1997

DISTRIBUCIÓN: Antártida.

-Maudheimia wilsoni Dalenius, 1958

DISTRIBUCIÓN: Antártida.

Multimaudheimia gen. nov. (1 sp.)

ESPECIE TIPO: Maudheimia marshalli Coetzee, 1997

DisTRIBUCIÓN: Antártica.

-Multimaudheimia marshalli (Coetzee, 1997) (Maudheimia)

DisTRIBUCIÓN: Antártida.

Liebstadiidae J. y P. Balogh, 1984 (10 gen. y 58 spp.) DisTRIBUCIÓN: Cosmopolita.

Angullozetes Hammer, 1967 (1 sp.)

ESPECIE TIPO: Angullozetes rostratus Hammer, 1967

DISTRIBUCIÓN: Australiana.

-Angullozetes rostratus Hammer, 1967

DistriBUCión: Nueva Zelanda.
Areozetes Hammer, 1961 (2 spp.)

EsPECIE TIPO: Areozetes altimontanus Hammer, 1961

DISTRIBUCIÓN: Tropical (Oriental y Neotropical).

-Areozetes altimontanus Hammer, 1961

Distribución: Perú.

-Areozetes incertus Balogh, 1970

DisTRIBUCIÓN: Ceilán [Sri Lanka].

Berndibula Mahunka, 2000 (1 sp.)

EsPeCIE TIPO: Berndibula bisculpturata Mahunka, 2000

DisTRIBUCIÓN: Oriental.

-Berndibula bisculpturata Mahunka, 2000

DisTRIBución: China (Hong-Kong).

Cordiozetes Mahunka, 2000 (2 spp.)

(=Mixoribatula Mahunka, 1987)

EsPeCIE TIPO: Cordiozetes clavisetus Mahunka, 1983

DISTRIBUCIÓN: Tropical (Oriental y Neotropical).

-Cordiozetes clavisetus Mahunka, 1983

DisTRIBUCIÓN: Brasil.

-Cordiozetes olahi Mahunka, 1987 (C. (Mixoribatula))

DISTRIBUCIÓN: Vietnam.

Haloribatula Schuster, 1957 (1 sp.)

EsPeCIE TIPO: Haloribatula tenareae Schuster, 1957

DisTRIBUCIÓN: Paleártica.

-Haloribatula tenareae Schuster, 1957

DISTRIBUCIÓN: Francia.

Liebstadia Oudemans, 1906 (15 spp.)

(=Irinobates Krivolutsky y Christov, 1970 "sin. nov.")

(=Rajskibates J. y P. Balogh, 1984)

EsPECIE TIPO: Notaspis similis Michael, 1888

DistribuCión: Semicosmopolita (Holártica, Oriental, "Etiópica" y Australiana).

-Libstadia divergens (Mihelcic, 1955) (Protoribates)

DisTRIBUCIÓN: Paleártica meridional (Europa meridional y Asia centrooccidental).

-Liebstadia gallardoi (Morell, 1987) (Protoribates)

DisTRIBUCIÓN: Mediterránea occidental.

-Liebstadia gratiosa (Vasiliu y Calugar, 1973) (Protoribates)

DisTRIBUCIÓN: Rumania.

-Liebstadia humerata Sellnick, 1928

DisTRIBUCIÓN: Holártica (Paleártica occidental: frecuente, y Neártica) y Vietnam.

-Liebstadia longior (Berlese, 1908) (Protoribates)

(=Protoribates badensis Sellnick, 1928)

DisTRIBUCIÓN: Holártica (Paleártica y Canadá).

-Liebstadia mongolica Bayartogtokh, 2001

(=Liebstadia elongata Bayartogtokh, 2001)

DisTRIBUCIÓN: Mongolia.

-Liebstadia neonominata nom. nov.

[=Oribata monodactyla Storkan, 1925, “nom. praeoc.” por Haller, 1884 ("hom. prim.")]

DistriBución: "Checoslovaquia".

-Liebstadia pannonica (Willmann, 1951) (Protoribates)

(=Oribatula alata Mihelcic, 1956)

(=Protoribates austriacus Willmann, 1953)

(=Protoribates micropterus Mihelcic, 1957)

(=Protoribates novus Willmann, 1953)

(=Protoribates variabilis Rajski, 1958)

DisTRIBUCIÓN: Holártica: Paleártica (frecuente) y U.S.A. (Carolina del Norte).

-Liebstadia parabadensis (Kulijev, 1968) (Protoribates)

DistriBución: Cáucaso.

-Liebstadia piligera Balogh, 1958 "sp. inq."

DisTRIBUCión: Angola.

-Liebstadia pilosa (Krivolutsky y Christov, 1970) (Irinobates)

DISTRIBUCIÓN: Asia centrooccidental. 
-Liebstadia saifulmalukensis (Hammer, 1977) (Gerloubia)

(=Liebstadia khugniensis Bayartogtokh, 2001)

Distribución: Paleártica meridional (Ibérica y Sur de Paleártica oriental).

-Liebstadia similis (Michael, 1888) (Notaspis)

(=Oribatula anomala Hull, 1916)

(=Protoribates serratomarginatus Mahunka, 1983)

(=Protoribates silesius Sellnick, 1925)

DistribuCión: Holártica (Paleártica y Neártica septentrional): frecuente y Nueva Zelanda.

-Liebstadia tenuis (Ayyildiz y Luxton, 1989) (Rajskibates) DisTRIBUCIÓN: Turquía.

-Liebstadia willmanni Miko y Weigmann, 1966

DisTRIBUCIÓN: Europa central.

Maculobates Hammer, 1962 (14 spp.)

(=Subulobates Hammer, 1972 "sin. nov.")

EsPeCIE TIPO: Maculobates longiporosus Hammer, 1962

DisTRIBUCIÓN: Australiana y austral (excepto Etiópica)

-Maculobates albulus (Hammer, 1972) (Subulobates)

DisTRIBUCIÓN: Polinesia (Tahití).

-Maculobates breviporosus Mahunka, 1980

Distribución: América austral (Tierra del Fuego).

-Maculobates dubius Hammer, 1971

Distribución: Australiana (Islas del Pacífico).

-Maculobates endroedyyoungai Mahunka, 1989

DISTRIBUCIÓN: Tasmania.

-Maculobates longipilosus Hammer, 1967

DistRIBUCIÓN: Nueva Zelanda.

-Maculobates longiporosus Hammer, 1962

DisTRIBUCIÓN: América austral.

-Maculobates longus Hammer, 1967

DisTRIBUCIÓN: Nueva Zelanda.

-Maculobates luteomarginatus Hammer, 1967

DisTRIBUCIÓN: Nueva Zelanda.

-Maculobates luteus Hammer, 1967

DisTRIBUCión: Nueva Zelanda.

-Maculobates magnus Hammer, 1967

DisTRIBUCIÓN: Nueva Zelanda.

-Maculobates minor Hammer, 1967

DisTRIBUCIÓN: Nueva Zelanda.

-Maculobates nordenskjoeldi (Trägardh, 1907) (Oribatula)

DistRiBución: Antártica (I. Shetland del Sur) y América austral (Tierra del Fuego)

-Maculobates ventroacutus Hammer, 1971

Distribución: Australiana (Islas de Pacífico).

-Maculobates vulgaris Hammer, 1967

DisTRIBUCIÓN: Nueva Zelanda.

Poroscheloribates Arillo, Gil-Martín y Subías, 1994 (1 sp.)

EsPECIE TIPO: Poroscheloribates canariensis Arillo, Gil-Martín y Subías, 1994

DISTRIBUCIÓN: Paleártica.

-Poroscheloribates canariensis Arillo, Gil-Martín y Subías, 1994 DISTRIBUCIÓN: I Canarias.

Reductobates Balogh y Mahunka, 1966 (5 spp.)

(=Ingella Hammer, 1967 "sin. nov.")

ESPECIE TIPO: Reductobates humeratus Balogh y Mahunka, 1966

DISTRIBUCIÓN: Australiana.

-Reductobates bicolor (Hammer, 1973) (Ingella)

Distribución: Polinesia (I. Tonga).

-Reductobates brassi Balogh, 1970

DisTRIBUCIÓN: Nueva Guinea.

-Reductobates bullager (Hammer, 1967) (Ingella)

DisTRIBUCIÓN: Australiana (Nueva Zelanda y Australia).

-Reductobates humeratus Balogh y Mahunka, 1966

DisTRIBUCIÓN: Australia.

-Reductobates latiohumeralis Hammer, 1972

Distribución: Polinesia (Tahití).
Totobates Hammer, 1961 (16 spp.)

ESPECIE TIPO: Totobates discifer Hammer, 1961

DistriBución: Austral (excepto Etiópica).

-Totobates acutissimus (Hammer, 1967) (Maculobates)

DistRIBUCión: Nueva Zelanda.

-Totobates anareensis (Dalenius, 1958) (Liebstadia)

DisTRIBUCIÓN: Antártica (I. Macquarie).

-Totobates antarcticus Wallwork, 1964 (T. elegans a.)

DisTRIBUCIÓN: Austral (Antártica y Nueva Zelanda).

-Totobates capita Hammer, 1968

DisTRIBUCIÓN: Nueva Zelanda.

-Totobates communis Hammer, 1967

DistriBUCIÓN: Nueva Zelanda.

-Totobates discifer Hammer, 1961

DisTRIBUCIÓN: Neotropical (Perú y Chile).

-Totobates elegans (Hammer, 1958) (Protoribates)

DistRiBución: Austral (Argentina y Antártica).

-Totobates latus Hammer, 1967

DisTRIBUCión: Nueva Zelanda.

-Totobates macroonyx Hammer, 1967

DistRIBUCIÓN: Nueva Zelanda.

-Totobates marionensis Pletzen y Kok, 1971

DISTRIBUCIÓN: Antártica.

-Totobates microseta Hammer, 1968

DisTRIBUCIÓN: Nueva Zelanda.

-Totobates minimus Hammer, 1967

DisTRIBUCIÓN: Nueva Zelanda.

-Totobates mollicoma Hammer, 1962

DISTRIBUCIÓN: Argentina.

-Totobates ovalis Hammer, 1967

DisTRIBUCIÓN: Nueva Zelanda.

-Totobates pterygoides Hammer, 1962

DisTRIBUCIÓN: Argentina.

-Totobates viviparus Hammer, 1962

Distribución: Chile.

Symbioribatidae Aoki, 1966 (2 gen. y 2 spp.) DisTRIBUCIÓN: Australianooriental.

Piffliella Hammer, 1979 (1 sp.)

EsPecie Tipo: Piffliella eduardi Hammer, 1979

DisTRIBUCIÓN: Oriental.

-Piffliella eduardi Hammer, 1979

DisTRIBUCIÓN: Java.

Symbioribates Aoki, 1966 (1 sp.)

EsPeCIE TIPO: Symbioribates papuensis Aoki, 1966

DISTRIBUCIÓN: Australiana.

-Symbioribates papuensis Aoki, 1966

DisTRIBUCIÓN: Nueva Guinea.

Scheloribatidae Jacot, 1935 (20 gen., 4 subg., 324 spp. y 18 sspp.) DISTRIBUCIÓN: Cosmopolita.

Annobonzetes Pérez-Íñigo, 1983 (3 spp.)

ESPECIE TIPO: Annobonzetes sphaericus Pérez-Íñigo, 1983

DisTRIBUCIÓN: Etiópica.

-Annobonzetes latissimus (Berlese, 1916) (Podoribates)

DisTRIBUCIÓN: "África oriental".

-Annobonzetes latus (Warburton, 1912) (Oribata)

DisTRIBUCIÓN: I. Seychelles.

-Annobonzetes sphaericus Pérez-Íñigo, 1983

Distribución: Guinea Ecuatorial: I. Pagalu [Annobón].

Coronibatula Mahunka, 1988 (1 sp.)

EsPeCIE TIPO: Coronibatula lienhardi Mahunka, 1988

DISTRIBUCIÓN: Oriental.

-Coronibatula lienhardi Mahunka, 1988

DISTRIBUCIÓN: Borneo. 
Cosmobates Balogh,1959 (1 sp.)

ESPECIE TIPO: Cosmobates tunicatus Balogh, 1959

DistribuCión: Etiópica.

-Cosmobates tunicatus Balogh, 1970

Distribución: Congo.

Euscheloribates Kunst, 1958 (7 spp.)

ESPECIE TIPO: Euscheloribates samsinaki Kunst, 1958

DisTRIBUCIÓN: Paleártica y Pantropical (excepto Neotropical).

Euscheloribates (Euscheloribates) Kunst, 1958 (5 spp.)

(=Corynobates J. y P. Balogh, 1992 "sin. nov.")

(=Tribates Mahunka, 1978 "sin. nov.")

DisTRIBUCIÓN: Paleártica y Pantropical (excepto Neotropical).

-Euscheloribates (E.) clavisetus Mahunka, 1988

DisTRIBUCIÓN: Malgache (I. Reunión).

-Euscheloribates (E.) cryptus (Mahunka, 1978) (Tribates)

DISTRIBUCIÓN: Malgache (I. Mauricio).

-Euscheloribates (E.) mixtus (Mahunka, 1978) (Tribates) DisTRIBUCIÓN: Malgache.

-Euscheloribates (E.) pembertoni (Jacot, 1934) (Protoschelobates) DISTRIBUCIÓN: Hawai.

-Euscheloribates (E.) samsinaki Kunst, 1958

DistRIBUCIÓN: Europa ("Checoslovaquia") y Oriental (India: Tripura, y Vietnam)

Euscheloribates (Nanobates) J. y P. Balogh,1984 (2 spp.)

EsPECIE TIPO: Birobates fenicheli Balogh, 1970

DisTRIBUCIÓN: Australianooriental.

-Euscheloribates (Nanobates) clavatus (Mahunka, 1988) (N.)

DISTRIBUCIÓN: Vietnam.

-Euscheloribates (Nanobates) fenicheli (Balogh, 1970) (Birobates)

DistriBUCIÓN: Nueva Guinea.

Fijibates Hammer, 1971 (4 spp.)

EsPECIE TIPO: Fijibates rostratus Hammer, 1971

DisTRIBUCIÓN: Pantropical (excepto Etiópica).

-Fijibates aelleni (Mahunka, 1988) (Scheloribates)

DisTRIBUCIÓN: Borneo.

-Fijibates anterostratus Corpuz-Raros, 1980

DisTRIBUCIÓN: Filipinas.

-Fijibates dlouhyi (Mahunka, 1984) (Scheloribates)

DisTRIBUCión: Neotropical (Paraguay y Panamá).

-Fijibates rostratus Hammer, 1971

DisTRIBUCión: Oriental (Java y Filipinas) y Australiana (Islas del Pacífico)

Fissurobates Balogh y Mahunka, 1969 (1 sp.)

ESPECIE TIPO: Fissurobates spectabilis Balogh y Mahunka, 1969

DisTRIBUCIÓN: Neotropical.

-Fissurobates spectabilis Balogh y Mahunka, 1969

DISTRIBUCIÓN: Bolivia.

Grandjeanobates Ramsay, 1967 (4 spp.)

(=Aellenobates Mahunka, 1978 "sin. nov")

ESPECIE TIPO: Grandjeanobates novazealandicus Hammer, 1967

DisTRIBUCIÓN: Pantropical.

-Grandjeanobates cryptacus (Mahunka, 1978) (Aellenobates) DisTRIBUCión: Malgache.

-Grandjeanobates laticlava (Hammer, 1961) (Scheloribates)

DisTRIBUCIÓN: Neotropical (Perú y Argentina) y Bután.

-Grandjeanobates novazealandicus Hammer, 1967

DisTRIBUCión: Nueva Zelanda.

-Grandjeanobates sculpturatus (Mahunka 1988) (Aellenobates)

DisTRIBUCIÓN: Malgache (I. Reunión).

Hammerabates Balogh, 1970 (6 spp.)

(=Otaheitea Hammer, 1972)

(=Yoronoribates Aoki, 1987 "sin. nov.")

EsPECIE TIPO: Hammerabates trisetosus Balogh, 1970
DisTRIBUCIÓn: Semicosmopolita: Pantropical (excepto Neotropical),

Paleártica meridional y subantártica.

-Hammerabates elongatus Tseng, 1984

DisTRIBUCIÓN: Taiwan [Formosa].

-Hammerabates minusculus (Aoki, 1987) (Yoronoribates)

DisTRIBUCIÓN: Japón.

-Hammerabates nasalis Mahunka, 1983

DisTRIBUCIÓN: Etiópica y subantártica (I. San Pablo y Amsterdam). -Hammerabates rotundus Mahunka, 1983

DistRIBUCIÓN: Tanzania.

-Hammerabates sulcatus (Hammer, 1972) (Otaheitea)

DisTRIBUCIÓN: Polinesia (Tahití)

-Hammerabates trisetosus Balogh, 1970

DistriBución: Oriental (India: Bengala Occidental) y Australiana

(Nueva Guinea y Polinesia: I. Tokelau).

Muliercula Coetzer, 1968 (7 spp.)

EsPECIE TIPO: Muliercula muliercula Coetzer, 1968

DisTRIBUCIÓN: Etiópica.

-Muliercula bilineata Mahunka, 1986

DisTRIBUCión: Tanzania.

-Muliercula discrepans (Balogh, 1959) (Scheloribates)

DisTRIBUCIÓN: Angola.

-Muliercula inexpectata Badejo, Woas y Beck, 2002

DisTRIBUCIÓN: Nigeria.

-Muliercula longisacculus (Mahunka, 1984) (Incabates)

DisTRIBUCIÓN: Tanzania.

-Muliercula muliercula Coetzer, 1968

DISTRIBUCIÓN: Sudáfrica.

-Muliercula ngoyensis Coetzer, 1968

DisTRIBUCIÓN: Sudáfrica.

-Muliercula spora Coetzer, 1968

DistRIBUCIÓN: Sudáfrica.

Nannerlia Coetzer, 1968 (3 spp.)

EsPeCIE TIPO: Nannerlia longinqua Coetzer, 1968

DISTRIBUCIÓN: Etiópica y Paleártica meridional.

-Nannerlia bombretensis Hammer, 1977

DISTRIBUCIÓN: Pakistán.

-Nannerlia elongatissima Mahunka, 1984

DisTRIBUCIÓN: Tanzania.

-Nannerlia longinqua Coetzer, 1968

DisTRIBUCIÓN: Sudáfrica.

Pachygena Hammer, 1872 (1 sp.)

EsPECIE TIPO: Pachygena falcata Hammer, 1972

DISTRIBUCIÓN: Australiana.

-Pachygena falcata Hammer, 1972

DisTRIBUCIÓN: Polinesia (Tahití)

Perscheloribates Hammer, 1973 (40 spp. y 1 ssp.)

EsPeCIE TIPO: Perscheloribates clavatus Hammer, 1973

DISTRIBUCIÓN: Pantropical y subtropical.

Perscheloribates (Perscheloribates) Hammer, 1973 (38 spp. y 1 ssp.)

(=Ischeloribates Corpuz-Raros, 1980 "sin. nov.")

(=Scheloribatella Mahunka, 1984 "sin. nov.")

DISTRIBUCIÓN: Pantropical y subtropical.

-Perscheloribates (P.) abbreviatus (Wallwork, 1977) (Scheloribates)

Distribución: I. Santa Helena.

-Perscheloribates (P.) agusanensis Corpuz-Raros, 1980

DistribuCión: Filipinas.

-Perscheloribates (P.) albialatus (Hammer, 1961) (Scheloribates)

Distribución: Perú e India (Bengala Occidental).

-Perscheloribates (P.) baluktotus Corpuz-Raros, 1980

DisTRIBUCIÓN: Filipinas.

-Perscheloribates (P.) benguetensis (Corpuz-Raros, 1980) (Ischelo-

ribates)

DISTRIBUCIÓN: Filipinas. 
-Perscheloribates (P.) brevialatus (Hammer, 1961) (Scheloribates) DisTRIBUCIÓN: Perú.

-Perscheloribates (P.) brevipterus (Hammer, 1962) (Scheloribates)

DisTRIBUCIÓN: Argentina.

-Perscheloribates (P.) cavernicolus (Corpuz-Raros, 1980) (Ischeloribates)

DisTRIBUCIÓN: Filipinas.

-Perscheloribates (P.) clavatus Hammer, 1973

Distribución: Oriental (Filipinas), Australiana (Polinesia) y

Paleártica meridional (Japón).

-Perscheloribates (P.) clavatus clavatus Hammer, 1973

DisTRIBUCión: Polinesia y Filipinas.

-Perscheloribates (P.) clavatus torquatus Aoki, 1984 DisTRIBUCIÓN: Japón.

-Perscheloribates (P.) conjuges (Hammer, 1967) (Scheloribates)

Distribución: Nueva Zelanda.

-Perscheloribates (P.) crassus Corpuz-Raros, 1980

DISTRIBUCIÓN: Filipinas.

-Perscheloribates (P.) curvirhynchus (Wallwork, 1977) (Scheloribates)

Distribución: I. Santa Helena.

-Perscheloribates (P.) ethiopicus (Mahunka, 1986) (Ischeloribates) DisTRIBUCIÓN: Tanzania.

-Perscheloribates (P.) evanescens (Wallwork, 1977) (Scheloribates)

DisTRIBUCIÓN: I. Santa Helena.

-Perscheloribates (P.) fissuratus (Hammer, 1961) (Scheloribates) Distribución: Perú.

-Perscheloribates (P.) keriensis (Hammer, 1967) (Scheloribates) Distribución: Nueva Zelanda.

-Perscheloribates (P.) lanceolatus (Aoki, 1984) (Ischeloribates)

DisTRIBUCIÓN: Japón.

-Perscheloribates (P.) latus (Hammer, 1958) (Scheloribates) Distribución: Bolivia.

-Perscheloribates (P.) luminosus (Hammer, 1961) (Scheloribates)

Distribución: Perú e India (Orissa).

-Perscheloribates (P.) lumotus Corpuz-Raros, 1980

DistRIBUCIÓN: Filipinas.

-Perscheloribates (P.) luteus (Hammer, 1962) (Scheloribates)

DisTRIBUCión: Neotropical (Chile y Venezuela).

-Perscheloribates (P.) luzonensis Corpuz-Raros, 1980

Distribución: Filipinas.

-Perscheloribates (P.) minimus Mahunka, 1992

DisTRIBUCión: Senegal.

-Perscheloribates (P.) minusculus (Hammer, 1961) (Scheloribates) DistriBución: Perú.

-Perscheloribates (P.) minutus (Pletzen, 1965) (Scheloribates) DistriBución: Sudáfrica y Filipinas.

-Perscheloribates (P.) monodactylus (Morell, 1987) (Scheloribates) (?=Scheloribates elongatus Mihelcic, 1963 "sp. inq.") DisTRIBUCIÓN: España.

-Perscheloribates (P.) monttensis (Hammer, 1962) (Scheloribates) DisTribución: Chile.

-Perscheloribates (P.) nodosus Corpuz-Raros, 1980

Distribución: Filipinas.

-Perscheloribates (P.) quezonensis (Corpuz-Raros, 1980) (Ischeloribates)

DisTRIBUCIÓN: Filipinas.

-Perscheloribates (P.) reiteratus nom. nov.

[=Scheloribates calcaratus Wallwork, 1977, "nom. praeoc." por Jacot, 1934 ("hom. prim.")]

Distribución: I. Santa Helena.

-Perscheloribates (P.) rostratus (Hammer, 1958) (Scheloribates) DisTRIBUCión: Neotropical (Argentina y Panamá).

-Perscheloribates (P.) rustenburgensis (Pletzen, 1963) (Scheloribates) Distribución: Sudáfrica.

-Perscheloribates (P.) setiger (P. Balogh, 1988) (Ischeloribates) DisTRIBUCIÓN: Ecuador.

-Perscheloribates (P.) shiraensis (Evans, 1953) (Protoribates) Distribución: Tanzania.

-Perscheloribates (P.) subtropicus (Hammer, 1961) (Scheloribates) Distribución: Neotropical.
-Perscheloribates (P.) surigaoensis Corpuz-Raros, 1980

DisTRIBUCIÓN: Filipinas.

-Perscheloribates (P.) tenuis Corpuz-Raros, 1980

DisTRIBUCIÓN: Filipinas.

-Perscheloribates (P.) tzitzikamaensis (Pletzen, 1963) (Scheloribates)

DisTRIBUCIÓN: Sudáfrica.

Perscheloribates (Makischeloribates) Corpuz-Raros, 1980 (1 sp.)

EsPECIE TIPO: Makischeloribates tripartitus Corpuz-Raros, 1980

DisTRIBUCIÓN: Oriental.

-Perscheloribates (Makischeloribates) tripartitus (Corpuz-Raros, 1980) (M.)

DISTRIBUCIÓN: Filipinas.

Perscheloribates (Oxyscheloribates) J. y P. Balogh, 1990 (1 sp.)

EsPECIE TIPO: Scheloribates aculeatus Hammer, 1961

DISTRIBUCIÓN: Neotropical.

-Perscheloribates (Oxyscheloribates) aculeatus (Hammer, 1961)

(Scheloribates)

DisTRIBUCIÓN: Perú.

Planobates Hammer, 1973 (1 sp.)

EsPeCIE TIPO: Planobates circumalatus Hammer, 1973

DISTRIBUCIÓN: Australiana.

-Planobates circumalatus Hammer, 1973

Distribución: Polinesia (I. Tonga).

Rhabdoribates Aoki, 1967 (1 sp.)

EsPECIE TIPO: Rhabdoribates siamensis Aoki, 1967

DISTRIBUCIÓN: Oriental.

-Rhabdoribates siamensis Aoki, 1967

DisTRIBUCIÓN: Oriental (Tailandia y Vietnam).

Samoabates Hammer, 1973 (1 sp.)

ESPECIE TIPO: Samoabates acutirostrum Hammer, 1973

DisTRIBUCIÓN: Australiana.

-Samoabates acutirostrum Hammer, 1973

Distribución: Polinesia (I. Samoa).

Scheloribates Berlese, 1908 (217 spp. y 17 sspp.)

EsPECIE TIPO: (Zetes latipes Koch, 1844) =Zetes pallidulus Koch, 1841

DisTRIBUCIÓN: Cosmopolita.

Scheloribates (Scheloribates) Berlese, 1908 (215 spp. y 17 sspp.)

(=Bischeloribates Mahunka, 1988)

(=Megascheloribates Lee y Pajak, 1990 "sin. nov.")

(=Paraschelobates Jacot, 1934)

(=Philoribates Corpuz-Raros, 1980 "sin. nov.")

=Protoschelobates Jacot, 1934)

(=Semischeloribates Hammer, 1973 "sin. nov.")

(=Storkania Jacot, 1929)

DISTRIBUCIÓN: Cosmopolita

-Scheloribates (S.) abraensis Corpuz-Raros, 1980

DisTRIBUCIÓN: Filipinas.

-Scheloribates (S.) acutirostris Calugar y Vasiliu, 1983

DistriBución: Cuba.

-Scheloribates (S.) aequalis Hammer, 1967

DistRIBUCión: Nueva Zelanda.

-Scheloribates (S.) aethiopicus Mahunka, 1982

Distribución: Etiopía e I. Canarias.

-Scheloribates (S.) agilis (Nicolet, 1855) (Oribata) "sp. inq."

Distribución: Mediterránea (Francia y Egipto)

-Scheloribates (S.) agricolus Corpuz-Raros, 1980

DISTRIBUCIÓN: Filipinas.

-Scheloribates (S.) alexandrinae Vasiliu e Ivan, 1995

DisTRIBUCIÓN: Egipto.

-Scheloribates (S.) angulatus Willmann, 1931

DistRIBUCIÓN: Sumatra.

-Scheloribates (S.) anzacensis Hammer, 1967

DistRIBUCión: Nueva Zelanda. 
-Scheloribates (S.) artigasi Pérez-Iñigo y Baggio, 1980 DisTRIBUCIÓN: Brasil.

-Scheloribates (S.) ascendens Weigmann y Wunderle, 1990 DisTRIBUCIÓN: Alemania.

-Scheloribates (S.) atahualpensis Hammer, 1961

DisTRIBUCIÓN: Neotropical (Perú y Argentina)

Scheloribates (S.) azovicus Subbotina, 1989 DisTRIBUCIÓN: Ucrania.

-Scheloribates (S.) badius (Ewing, 1908) (Oribata) DistriBución: U.S.A. (Ilinois).

-Scheloribates (S.) baloghi Kardar, 1976 DisTRIBUCIÓN: India (Uttar Pradesh)

-Scheloribates (S.) barbatulus Mihelcic, 1956 (=Scheloribates subsimilis Mihelcic, 1956) DISTRIBUCIÓN: Paleártica meridional.

-Scheloribates (S.) bhadurii Sanyal, 1992 DisTRIBUCIÓN: India (Bengala Occidental).

-Scheloribates (S.) bhutanensis Sanyal, 1988 DisTRIBUCIÓN: Bután.

-Scheloribates (S.) biarcualis Hammer, 1973 DistriBución: Polinesia.

-Scheloribates (S.) bicornis Mahunka, 199 DISTRIBUCIÓN: Macaronésica (Cabo Verde).

-Scheloribates (S.) bicuspidatus Kardar, 1976 DisTRIBUCIÓn: India (Uttar Pradesh).

-Scheloribates (S.) bidactylus Hammer, 1961 Distribución: Perú.

-Scheloribates (S.) bilineatus Balogh, 1958 "sp. inq." DisTRIBUCIÓN: Congo.

-Scheloribates (S.) biunguis (Berlese, 1920) (Protoribates (S.)) DisTRIBUCIÓN: Java.

-Scheloribates (S.) brachypterus Wallwork, 1977 Distribución: I. Santa Helena.

-Scheloribates (S.) brasilocompressus Badejo, Woas y Beck, 2002 DisTRIBUCIÓN: Brasil.

-Scheloribates (S.) brasilosphericus Badejo, Woas y Beck, 2002 DisTRIBUCIÓN: Brasil.

-Scheloribates (S.) brevipodus Lee y Pajak, 1990 DISTRIBUCIÓN: Australia.

-Scheloribates (S.) callipus (Berlese, 1908) (Protoribates (S.)) (=Protoschelobates seghetti Runkel y Kates, 1947) DisTRIBUCIÓN: U.S.A. nororiental.

-Scheloribates (S.) camtschaticus Subbotina, 1989 DisTRIBUCIÓN: Este de Rusia asiática.

-Scheloribates (S.) caprai Bernini, 1973 (=Scheloribates baloghi Pérez-Íñigo, 1982, "nom. praeoc." por Kardar, 1976) (=Scheloribates subsimilis Balogh, 1962, "nom. praeoc." por Mihelcic, 1956) DistriBución: Mediterránea occidental, Etiópica (Tanzania) y Oriental (India: Tripura).

-Scheloribates (S.) carneus (Hull, 1916) (Oribatula) "sp. inq." DISTRIBUCIÓN: Islas Británicas.

-Scheloribates (S.) castlei (Jacot, 1934) (Protoschelobates) DisTRIBUCIÓN: Hawai.

-Scheloribates (S.) chauhani Baker, 1945 DisTRIBUCIÓN: India (Uttar Pradesh).

-Scheloribates (S.) clavilanceolatus (Ewing, 1907) (Oribata) DistRIBUCIÓN: U.S.A. (excepto Sur).

-Scheloribates (S.) clavipectinatus (Ewing, 1907) (Oribata) DisTRIBUCIÓN: U.S.A. (Ilinois).

-Scheloribates (S.) concentricus Balogh, 1962 DisTRIBUCIÓN: Tanzania.

-Scheloribates (S.) confusia Coetzer, 1968 DisTRIBUCIÓN: Sudáfrica.

-Scheloribates (S.) conirostris Corpuz-Raros, 1980 DisTRIBUCIÓN: Filipinas.

-Scheloribates (S.) corpusculum Bayartogtokh, 2000 DisTRIBUCIÓN: Mongolia.

-Scheloribates (S.) crassipodus Lee y Pajak, 1990 DISTRIBUCIÓN: Australia.
-Scheloribates (S.) crassus Hammer, 1967

DistriBuCión: Nueva Zelanda.

-Scheloribates (S.) crimicus Subbotina, 1989

DisTRIBUCIÓN: Ucrania (Crimea).

-Scheloribates (S.) cruciseta Jeleva y Vu, 1987

DisTRIBUCIÓN: Vietnam.

-Scheloribates (S.) curvialatus Hammer, 1961

DistriBución: Perú e India (Bengala Occidental).

-Scheloribates (S.) cuyi Corpuz-Raros, 1980

DistribuCión: Filipinas.

Scheloribates (S.) dalawaeus Corpuz-Raros, 1980

DistRIBUCIÓN: Filipinas.

-Scheloribates (S.) danaus Subbotina, 1987

Distribución: Este de Rusia asiática.

-Scheloribates (S.) decarinatus Aoki, 1984

DisTRIBUCIÓN: Este de Paleártica oriental.

-Scheloribates (S.) dentatus Katsumata, 1988

DISTRIBUCIÓN: Japón.

-Scheloribates (S.) diforamenatus Lee y Pajak, 1990

DistriBución: Australia.

-Scheloribates (S.) dimentmani (Vasiliu e Ivan, 1995) (Hemileius)

DISTRIBUCIÓN: Israel.

-Scheloribates (S.) discifer Balogh, 1959

DisTRIBUCIÓN: Angola.

-Scheloribates (S.) distinctus Mihelcic, 1964

DisTRIBUCIÓN: Paleártica meridional (Europa meridional y Asia centrooccidental).

-Scheloribates (S.) elegans Hammer, 1958

DistriBUCIÓN: Tropical: Oriental (Filipinas e India: Kerala), Hawai y Bolivia.

-Scheloribates (S.) elegantulus Hammer, 1961

Distribución: Neotropical (Perú y Venezuela).

-Scheloribates (S.) elongatus (Warburton, 1912) (Oribata)

DistRIBUCIÓN: I. Seychelles.

-Scheloribates (S.) elsi Pletzen, 1965

DisTRIBUCIÓN: Sudáfrica.

-Scheloribates (S.) eusetosus Lee y Pajak, 1990

Distribución: Australia.

-Scheloribates (S.) exiguus Mahunka, 1992

DisTRIBUCIÓN: Senegal.

-Scheloribates (S.) feideri Calugar y Vasiliu, 1983

DisTriBución: Cuba.

-Scheloribates (S.) femoralis (Nicolet, 1855) (Oribata) "sp. inq." DisTRIBUCIÓN: Francia.

-Scheloribates (S.) femoratus Willmann, 1931

Distribución: Java.

-Scheloribates (S.) femoroserratus Pérez-Íñigo y Baggio, 1980

DISTRIBUCIÓN: Brasil.

-Scheloribates (S.) fijiensis Hammer, 1971 (S. praeincisus f.)

DisTRIBUCión: Islas de Pacífico y Filipinas.

-Scheloribates (S.) filipinus (Corpuz-Raros, 1980) (Philoribates) DisTRIBUCIÓN: Filipinas.

-Scheloribates (S.) fimbriatoides Hammer, 1977

Distribución: Melanesia (I. Fiji) e India (Tripura).

-Scheloribates (S.) fimbriatus Thor, 1930

DistriBuCIÓN: Cosmopolita (Paleártica meridional, Neártica y Pantropical).

-Scheloribates (S.) fimbriatus fimbriatus Thor, 1930

(?=Protoribates (Scheloribates) artifex Berlese, 1916 "sp. inq.") (?=Oribata helvina Ewing, 1909 "sp. inq.")

(?=Protoribates (Scheloribates) lanceoliger Berlese, 1908 "sp. inq.")

(=Scheloribates penicillatus Mihelcic, 1957)

(?=Galumna sylvicola Banks, 1909 “sp. inq.")

DisTRIBUCIÓN: Cosmopolita (Paleártica meridional: frecuente, Neártica y Pantropical).

-Scheloribates (S.) fimbriatus africanus Wallwork, 1964 DistriBuCIÓN: Chad y Arabia Saudita.

-Scheloribates (S.) fimbriatus calcaratus Jacot, 1934 DISTRIBUCIÓN: Hawai. 
-Scheloribates (S.) fimbriatus javensis Willmann, 1931

Distribución: Oriental e Islas del Pacífico.

-Scheloribates (S.) fimbriatus whitteni Jacot, 1934

DisTRIBUCión: Polinesia (I. Marquesas).

-Scheloribates (S.) flagellatus Wallwork, 1966

Distribución: Antártica (I. Campbell).

-Scheloribates (S.) fuscosensillus Corpuz-Raros, 1980

Distribución: Filipinas.

-Scheloribates (S.) giganteus Hammer, 1961

DisTRIBUCIÓN: Perú.

-Scheloribates (S.) gilvulus (Koch, 1841) (Zetes) "sp. inq."

Distribución: Alemania.

-Scheloribates (S.) grandis Subbotina, 1979

DisTRIBUCIÓN: Cáucaso.

-Scheloribates (S.) guhitanus Corpuz-Raros, 1980

DisTRIBUCIÓN: Filipinas.

-Scheloribates (S.) gunini Bayartogtokh, 2000

DisTRIBUCIÓN: Mongolia.

-Scheloribates (S.) helenensis Wallwork, 1977

Distribución: I. Santa Helena.

-Scheloribates (S.) heterodactylus (Mahunka, 1988) (Bischeloribates)

("non" Scheloribates heterodactylus Pletzen, 1963, ahora en Topobates)

DistriBución: Oriental: Borneo e India (Tripura).

-Scheloribates (S.) heterosetosus Lee y Pajak, 1990

DisTRIBUCIÓN: Australia

-Scheloribates (S.) heterotrichus Mahunka, 1984

DisTRIBUCIÓN: Tanzania.

-Scheloribates (S.) huancayensis Hammer, 196

DisTRIBUCión: Neotropical (Perú y Panamá) y Noreste de India.

-Scheloribates (S.) humeratus (Hull, 1916) (Oribatula) "sp. inq."

DistriBuCiÓN: Islas Británicas.

-Scheloribates (S.) imperfectus Hammer, 1972

DisTriBución: Polinesia.

-Scheloribates (S.) indicus (Oudemans, 1915) (Murcia)

DisTRIBUCión: Ceilán [Sri Lanka] e Islas del Pacífico.

-Scheloribates (S.) indicus indicus (Oudemans, 1915)

DistriBuCión: Ceilán [Sri Lanka] y Hawai.

-Scheloribates (S.) indicus marquesalis Jacot, 1934

Distribución: Polinesia (I. Marquesas).

-Scheloribates (S.) iteratus nom. nov.

[=Scheloribates microclava Balogh, 1962, "nom. praeoc." por Hammer,

1961 ("hom. prim.")]

DisTRIBUCIÓN: Tanzania.

-Scheloribates (S.) jucundior (Berlese, 1923) (Protoribates (S.) par-

vulus $j$.)

DisTRIBUCIÓN: India (Bengala Occidental)

-Scheloribates (S.) kraepelini (Berlese, 1908) (Protoribates (S.))

DisTRIBUCIÓN: Java.

-Scheloribates (S.) labyrinthicus Jeleva, 1962

DisTRIBUCIÓN: Mediterránea.

-Scheloribates (S.) labyrinthicus labyrinthicus Jeleva, 1962

DisTRIBUCIÓN: Mediterránea.

-Scheloribates (S.) labyrinthicus oscensis Pérez-Íñigo jr., Herrero y

Pérez-Íñigo, 1987

DisTRIBUCIÓN: España.

-Scheloribates (S.) laevigatus (Koch, 1835) (Zetes)

(=Scheloribates angustirostris Mihelcic, 1957)

$(=$ Oribata lucasi Nicolet, 1855$)$

(=Oribata michaeli Hull,1914)

(=Scheloribates robustus Mihelcic, 1969, "nom. praeoc." por Pletzen, 1963)

DisTRIBUCIÓN: Semicosmopolita (Holártica: frecuente, y

Paleotropical).

-Scheloribates (S.) laminatus (Ewing, 1909) (Oribata)

DisTRIBUCión: U.S.A. (Ilinois).

-Scheloribates (S.) latoincisus Hammer, 1973

Distribución: Polinesia (I. Tonga)

-Scheloribates (S.) leleupi Balogh, 1959

Distribución: Etiópica (Congo y Tanzania).

-Scheloribates (S.) lencoranicus Subbotina, 1981

DisTRIBUCIÓN: Cáucaso.
-Scheloribates (S.) longiporosus Kulijev, 1968

Distribución: Cáucaso.

-Scheloribates (S.) longisetosus Feider, Vasiliu y Calugar, 1973

DISTRIBUCIÓN: Rumania.

-Scheloribates (S.) longus Kulijev, 1968

Distribución: Paleártica (Sureste de Europa y Sur de Siberia).

-Scheloribates (S.) louwi Pletzen, 1965

DisTRIBUCIÓN: Sudáfrica.

-Scheloribates (S.) louwi louwi Pletzen, 1965

DisTRIBUCión: Sudáfrica.

-Scheloribates (S.) louwi nimirumi Coetzer, 1968

DisTRIBUCIÓN: Sudáfrica.

-Scheloribates (S.) lucasiformis (Trägardh, 1905) (Oribata) “sp. inq."

DisTRIBUCiÓN: Sudán.

-Scheloribates (S.) luchili Wharton, 1938

Distribución: Méjico.

-Scheloribates (S.) luciensis Mahunka, 1985

DisTRIBUCIÓN: Norte de Neotropical.

-Scheloribates (S.) luteomarginatus Hammer, 1958

DISTRIBUCIÓN: Argentina.

-Scheloribates (S.) maculatus Wallwork, 1977

Distribución: I. Santa Helena.

-Scheloribates (S.) magnus Abdel-Hamid, Al-Assiuty y Trrad, 1983 DisTRIBUCIÓN: Egipto.

-Scheloribates (S.) manoai Jacot, 1934

DisTRIBUCIÓN: Hawai.

-Scheloribates (S.) maoriensis Hammer, 1968

DistriBUCión: Nueva Zelanda.

-Scheloribates (S.) marginedentatus (Trägardh, 1931) (Chamobates)

"sp. inq."

DistriBución: Chile (I. Juan Fernández).

-Scheloribates (S.) matulisus Corpuz-Raros, 1980

DistriBUCIÓN: Filipinas.

-Scheloribates (S.) megalonyx (Berlese, 1916) (Protoribates (S.))

DisTRIBUCIÓN: Argentina.

-Scheloribates (S.) microsetosus (Lee y Pajak, 1990) (Megascheloribates)

("non" Scheloribates microsetosus Wallwork, 1977, ahora en Topobates) (=Megascheloribates pajaki J. y P. Balogh, 2002)

DistRIBUCIÓN: Australia.

-Scheloribates (S.) milleri Jacot, 1936

DisTRIBUCIÓN: U.S.A. oriental.

-Scheloribates (S.) minifimbriatus Mínguez, Subías yRuiz, 1986

(?=Protoribates (Scheloribates) exilior Berlese, 1916 "sp. inq.")

DisTRIBUCIÓN: Holártica meridional (Mediterránea y U.S.A.

Carolina del Norte), Guinea Ecuatorial y Panamá

-Scheloribates (S.) mochlosimilaris Badejo, Woas y Beck, 2002

DisTRIBUCIÓN: Nigeria.

-Scheloribates (S.) moestus (Banks, 1895) (Oribata)

DistriBUCión: U.S.A oriental.

-Scheloribates (S.) muiri Jacot, 1934

Distribución: Islas del Pacífico.

-Scheloribates (S.) muiricius Jacot, 1937

Distribución: U.S.A. (Carolina del Norte).

-Scheloribates (S.) multiiteratus nom. nov.

[=Oribata simplex Storkan, 1925, "nom. praeoc." por Gervais, 1849 ("hom. prim.")]

DistRIBUCIÓN: "Checoslovaquia".

-Scheloribates (S.) multirepetitus nom. nov.

[=Incabates striatus Corpuz-Raros, 1980, "nom. praeoc." por Hammer, 1958 ("hom. sec.")]

DistRIBUCIÓN: Filipinas.

-Scheloribates (S.) mumfordi Jacot, 1934 (S. (Paraschelobates))

Distribución: Polinesia (I. Marquesas).

-Scheloribates (S.) mumfordi mumfordi Jacot, 1934

Distribución: Polinesia (I. Marquesas).

-Scheloribates (S.) mumfordi uapoui Jacot, 1934 (S. (Parasche-

lobates))

DisTRIBUCIÓN: Polinesia (I. Marquesas). 
-Scheloribates (S.) nanus Lee y Pajak, 1980

DisTRIBUCIÓN: Australia.

-Scheloribates (S.) neonominatus nom. nov.

[=Scheloribates baloghi Calugar y Vasiliu, 1983, "nom. praeoc." por Kardar, 1976 ("hom. prim.")]

DisTRIBUCIÓN: Cuba.

-Scheloribates (S.) nigeriocompressus Badejo, Woas y Beck, 2002 DisTRIBUCIÓN: Nigeria.

-Scheloribates (S.) nigeriosphericus Badejo, Woas y Beck, 2002 DisTRIBUCIÓN: Nigeria.

-Scheloribates (S.) nudus Woodring, 1965

DisTRIBUCIÓN: U.S.A. (Luisiana).

-Scheloribates (S.) oahuensis Jacot, 1934

DisTRIBUCIÓN: Hawai.

-Scheloribates (S.) obesus Hammer, 1958

DisTRIBUCIÓN: Bolivia.

-Scheloribates (S.) obsessus nom. nov.

[=Scheloribates indicus Sanyal, 1992, "nom. praeoc." por Oudemans, 1915 ("hom. sec.")]

DisTRIBUCIÓN: India (Bengala Occidental).

-Scheloribates (S.) obtusus Pletzen, 1963

DistriBución: Sudáfrica.

-Scheloribates (S.) orixaensis Badejo, Woas y Beck, 2002

DisTRIBUCIÓN: Brasil.

-Scheloribates (S.) ornatus Balogh, 1959

DisTRIBUCIÓN: Congo.

-Scheloribates (S.) oryzae Wu, Xin y Aoki, 1986

DisTRIBUCIÓN: Este de Paleártica oriental.

-Scheloribates (S.) pacificus (Sellnick, 1959) (Styloribates)

DistriBución: Polinesia: I. Australes [I. Tubuai].

-Scheloribates (S.) pahabaeus (Corpuz-Raros, 1980) (Incabates)

DisTRIBUCIÓn: Filipinas.

-Scheloribates (S.) palawanus Corpuz-Raros, 1980

DisTRIBUCIÓN: Filipinas.

-Scheloribates (S.) pallidulus (Koch, 1841) (Zetes)

(=Scheloribates campestris Mihelcic, 1966)

(=Protoribates (Scheloribates) frigidus Berlese, 1908)

(=Zetes fuscomaculatus Koch, 1841)

(=Zetes latipes Koch, 1844)

Distribución: Cosmopolita (excepto Antártica; frecuente en Holártica).

-Scheloribates (S.) papillaris Tseng, 1984

DisTRIBUCIÓN: Taiwan [Formosa].

-Scheloribates (S.) parabilis Woodring, 1965

DisTRIBUCIÓN: U.S.A. (Luisiana).

-Scheloribates (S.) parabrevipodus Lee y Pajak, 1990

DisTRIBUCIÓN: Australia.

-Scheloribates (S.) parananus Lee y Pajak, 1990

Distribución: Australia.

-Scheloribates (S.) parvulus (Berlese, 1916) (Protoribates (S.))

DisTRIBUCIÓN: Somalia.

-Scheloribates (S.) parvus Pletzen, 1963

DisTRIBUCIÓN: Etiópica (Sudáfrica y Chad) e India (Assam).

-Scheloribates (S.) parvus parvus Pletzen, 1963

Distribución: Sudáfrica e India (Assam).

-Scheloribates (S.) parvus conglobatus Wallwork, 1964 Distribución: Chad.

-Scheloribates (S.) pauliensis Pérez-Íñigo y Baggio, 1980 DISTRIBUCIÓN: Brasil.

-Scheloribates (S.) peracutus Balogh, 1962

DisTRIBUCIÓN: Tanzania.

-Scheloribates (S.) perforatus Wallwork, 1964

Distribución: Chad.

-Scheloribates (S.) perisi Pérez-Íñigo, 1982

Distribución: Guinea Ecuatorial: I. Pagalu [Annobón].

-Scheloribates (S.) philippinensis Corpuz-Raros, 1980

DisTRIBUCIÓN: Filipinas.

-Scheloribates (S.) pilosus Sellnick, 1943 “sp. inq."

DISTRIBUCIÓN: Italia.
-Scheloribates (S.) polygonatus Balogh y Mahunka, 1974

Distribución: Cuba.

-Scheloribates (S.) ponticuliger (Berlese, 1916) (Protoribates (S.))

DISTRIBUCIÓN: Somalia.

-Scheloribates (S.) ponticus Subbotina, 1989

Distribución: Cáucaso.

-Scheloribates (S.) praeincisus (Berlese, 1910) (Protoribates (S.))

(=Murcia insularis Oudemans, 1915)

(=Scheloribates zaherii Abdel-Hamid, Al-Assiuty y Trrad, 1983)

DISTRIBUCIÓN: Pantropical (frecuente) y subtropical.

-Scheloribates praeincisus praeincisus (Berlese, 1910)

DistriBuCIÓN: Tropical (Oriental: frecuente, Islas del Pacífico: frecuente, y Panamá) y Holártica meridional (Egipto, Irán y U.S.A.: Tejas).

-Scheloribates (S.) praeincisus acuticlava Pérez-Íñigo y Baggio, 1986 DisTRIBUCIÓN: Neotropical (Brasil y Guatemala).

-Scheloribates (S.) praeincisus atlanticus Pérez-Iñigo, 1982 DistriBución: Guinea Ecuatorial: I. Pagalu [Annobón]

-Scheloribates (S.) praeincisus cubanus Scull, Jeleva y Cruz, 1984 DisTRIBUCIÓN: Cuba.

-Scheloribates (S.) praeincisus interruptus (Berlese, 1916) (Protoribates $(S)$.

DistRIBUCIÓn: Tropical (Oriental: frecuente, e Islas del Pacífico: frecuente)

-Scheloribates (S.) praeincisus sandvicensis (Jacot, 1934) (Protoschelobates insularis $s$.)

(=Scheloribates praeincisus rotundiclava Pérez-Íñigo y Baggio, 1986) Distribución: Hawai, India (Tripura), Brasil y U.S.A. (Tejas). -Scheloribates (S.) praeincisus tenuiseta Hammer, 1971

Distribución: Melanesia (I. Fiji) e India (Bengala Occidental).

-Scheloribates (S.) praelineatus Hammer, 1977

DisTRIBUCIÓN: Pakistán.

-Scheloribates (S.) praeoccupatissimus nom. nov.

[=Scheloribates minutus Mahunka, 1984, “nom. praeoc." por Pletzen, 1965 ("hom. prim.")]

DistribuCión: Tanzania.

-Scheloribates (S.) praeoccupatus nom. nov.

[=Megascheloribates calcaratus Lee y Pajak, 1990, "nom. praeoc." por Jacot, 1934 ("hom. sec.")]

DistriBuCIÓN: Australia.

-Scheloribates (S.) praestantissimus (Berlese, 1916) (Protoribates (S.))

DistribUCIÓN: África oriental.

-Scheloribates (S.) praestantissimus praestantissimus (Berlese, 1916) DisTRIBUCIÓN: Somalia.

-Scheloribates (S.) praestantissimus major (Berlese, 1916) (Protoribates $(S)$.

DistRIBUCIÓN: "África oriental".

-Scheloribates (S.) primoricus (Subbotina, 1987) (Semischeloribates)

DisTRIBUCIÓN: Este de Rusia asiática.

-Scheloribates (S.) pseudomochlosimilaris Badejo, Woas y Beck, 2002 DisTRIBUCIÓN: Nigeria.

-Scheloribates (S.) pseudoprincipalis Balogh, 1962

DisTRIBUCIÓN: Tanzania.

-Scheloribates (S.) pubescens Mahunka, 1984

DisTRIBUCIÓN: Sudáfrica.

-Scheloribates (S.) quintus Wunderle, Beck y Woas, 1990

DisTRIBUCIÓN: Alemania.

-Scheloribates (S.) rakhali Sanyal, 1992

DisTRIBUCIÓN: India (Bengala Occidental).

-Scheloribates (S.) rectus Hammer, 1958

Distribución: Pantropical (Bolivia, Sudáfrica e India: Bengala Occidental).

-Scheloribates (S.) repetitivus nom. nov.

[=Scheloribates pacificus Hammer, 1967, "nom. praeoc." por Sellnick, 1959 ("hom. sec.")]

DistrIBUCIÓN: Nueva Zelanda.

-Scheloribates (S.) repetitus nom. nov.

[=Scheloribates crassus Kulijev, 1979, "nom. praeoc.” por Hammer, 1967

("hom. prim.")]

DistRIBUCIÓN: Cáucaso. 
-Scheloribates (S.) rigidisetosus Willmann, 1951

Distribución: Paleártica meridional (Europa central y Este de Paleártica oriental).

-Scheloribates (S.) robustus Pletzen, 1963

DisTRIBUCIÓN: Sudáfrica.

-Scheloribates (S.) rostrodentatus Hammer, 1977

DisTRIBUCIÓN: Pakistán.

-Scheloribates (S.) rostropilosus Sanyal, 1988

DisTRIBUCIÓN: Bután.

-Scheloribates (S.) rotundatus Hammer, 1955

DisTRIBUCIÓN: Neártica septentrional.

-Scheloribates (S.) rufafulvus Kardar, 1976

DisTRIBUCIÓN: India (Uttar Pradesh).

-Scheloribates (S.) rugiceps Balogh, 1962

DisTRIBUCIÓN: Tanzania.

-Scheloribates (S.) rugosus Hammer, 1958

DisTRIBUCIÓN: Neotropical (Bolivia y Venezuela).

-Scheloribates (S.) sacsahuamanensis Hammer, 1961

DisTRIBUCIÓN: Perú.

-Scheloribates (S.) saswatii Dhali y Bhaduri, 1981

DisTRIBUCIÓN: Himalaya.

-Scheloribates (S.) saudicus Bayoumi y Al-Khalifa, 1985

Distribución: Arabia Saudita.

-Scheloribates (S.) saxicola (Halbert, 1920) (Oribatula)

DisTRIBUCión: Islas Británicas.

-Scheloribates (S.) schauenbergi Mahunka, 1988

DisTRIBUCIÓN: Malgache (I. Reunión).

-Scheloribates (S.) semidesertus Bulanova-Zachvatkina y Mahmudova, 1991

Distribución: Sur de Rusia europea.

-Scheloribates (S.) sergienkoae Subbotina, 1987

Distribución: Sureste de Europa.

-Scheloribates (S.) sikkimensis Dhali y Bhaduri, 1981

DisTriBución: Himalaya.

-Scheloribates (S.) simplex (Gervais, 1849) (Oribata) "sp. inq."

Distribución: Chile.

-Scheloribates (S.) sine Kulijev, 1979

DisTRIBUCIÓN: Cáucaso.

-Scheloribates (S.) sphaeroides Hammer, 1973

Distribución: Polinesia (I. Samoa).

-Scheloribates (S.) spirulatus Vasiliu y Calugar, 1973

DisTRIBUCIÓN: Rumania.

-Scheloribates (S.) striatus Hammer, 1958

DisTRIBUCión: Bolivia e India (Tripura)

-Scheloribates (S.) striolatus Balogh, 1961

DisTRIBUCIÓN: Madagascar.

-Scheloribates (S.) teldanicus Vasiliu e Ivan, 1995

DisTRIBUCIÓN: Israel.

-Scheloribates (S.) thermophilus Hammer, 1961

Distribución: Tropical (Perú, India: Bengala Occidental, e Islas del Pacífico).

-Scheloribates (S.) thermophilus thermophilus Hammer, 1961

DistriBución: Perú e India (Bengala Occidental).

-Scheloribates (S.) thermophilus corolevuensis Hammer, 1971

DistRIBUCión: Islas del Pacífico.

-Scheloribates (S.) translamellaris Kardar, 1976

DisTRIBUCIÓN: India (Uttar Pradesh).

-Scheloribates (S.) transplicatus Abdel-Hamid, Al-Assiuty y Trrad, 1983

DisTRIBUCIÓN: Egipto.

-Scheloribates (S.) tricarinatus Coetzer, 1968

DisTRIBUCIÓN: Sudáfrica.

-Scheloribates (S.) tuberculatus Pérez-Íñigo jr., Herrero y Pérez-Íñigo, 1987

DisTRIBUCIÓN: España.

-Scheloribates (S.) tubuaiensis Sellnick, 1959

Distribución: Polinesia y Paleártica occidental (Cáucaso).

-Scheloribates (S.) turcmensis Subbotina, 1987

DistriBución: Asia centrooccidental.

-Scheloribates (S.) uluguruensis Mahunka, 1983

DisTRIBUCIÓN: Tanzania.
-Scheloribates (S.) unisetosus Lee y Pajak, 1990

DistriBuCión: Australia.

-Scheloribates (S.) urenicus Subbotina, 1978

DISTRIBUCIÓN: Rusia europea central.

-Scheloribates (S.) vanzwaluwenburgi (Jacot, 1934) (Protoschelobates)

DisTRIBUCIÓN: Hawai.

-Scheloribates (S.) variabilis Pletzen, 1965

DisTRIBUCIÓN: Sudáfrica.

-Scheloribates (S.) viguerasis Scull, Jeleva y Cruz, 1984

DisTRIBUCIÓN: Cuba.

-Scheloribates (S.) volcanensis Hammer, 1962

DisTRIBUCIÓN: Chile.

-Scheloribates vulgaris Hammer, 1961

DistribuCión: Perú.

-Scheloribates (S.) willmanni Hammer, 1961

(=Scheloribates angulatus Hammer, 1958, "nom. praeoc." por Willmann, 1931)

DisTRIBUCIÓN: Neotropical.

-Scheloribates (S.) xylobatoides Mahunka, 1977

DisTRIBUCIÓN: Grecia.

-Scheloribates (S.) yezoensis Fujita y Fujikawa, 1987

DisTRIBUCIÓN: Japón.

-Scheloribates (S.) yorubaensis Badejo, Woas y Beck, 2002

DISTRIBUCIÓN: Nigeria.

-Scheloribates (S.) zealandicus Hammer, 1967

DistriBuCión: Nueva Zelanda e India (Andhra Pradesh).

Scheloribates (Andeszetes) Hammer, 1961 (2 spp.)

(=Neoscheloribates Hammer, 1973 "sin. nov.")

ESPECIE TIPO: Andeszetes diversidactylus Hammer, 1961

DisTRIBUCIÓN: Tropical (Neotropical y Australiana).

-Scheloribates (Andeszetes) diversidactylus (Hammer, 1961) (A.)

DisTRIBUCIÓN: Perú.

-Scheloribates (Andeszetes) grandiporosus (Hammer, 1973) (Neos-

cheloribates)

DisTRIBUCIÓN: Polinesia (I. Tonga).

Scheloribatoides Mahunka, 1988 (1sp.)

EsPECIE TIPO: Scheloribatoides lamellatus Mahunka, 1988

DisTRIBUCIÓN: Etiópica.

-Scheloribatoides lamellatus Mahunka, 1988

DisTRIBUCIÓN: Tanzania.

Similobates Mahunka, 1982 (2 spp.)

ESPECIE TIPO: Similobates demetororum Mahunka, 1982

DisTRIBUCIÓN: Etiópica.

-Similobates deficiens (Wallwork, 1977) (Scheloribates)

DisTRIBUCIÓN: I. Santa Helena.

-Similobates demetororum Mahunka, 1982

DISTRIBUCIÓN: Etiopía.

Striatobates Hammer, 1973 (1 sp.)

EsPECIE TIPO: Striatobates tuberculatus Hammer, 1973

DISTRIBUCIÓN: Australiana.

-Stiatobates tuberculatus Hammer, 1973

DisTRIBUCIÓN: Polinesia (I. Samoa).

Topobates Grandjean, 1958 (22 spp.)

(=Berndia Mahunka, 1988 "sin. nov.")

(=Flagellobates Mahunka, 1978 "sin. nov.")

(=Setobates Balogh, 1961)

ESPECIE TIPO: Topobates granifer Grandjean, 1958

DisTRIBUCIÓN: Cosmopolita (excepto Neotropical y Antártica).

-Topobates alisanicus (Tseng, 1984) (Scheloribates)

DisTRIBUCIÓN: Taiwan [Formosa].

-Topobates alvaradoi (Pérez-Íñigo, 1969) (Scheloribates)

DisTRIBUCión: Mediterránea occidental.

-Topobates berndhauseri (Mahunka, 1978) (Flagellobates)

DisTRIBUCión: Malgache (I. Mauricio).

-Topobates carpathicus (Weigmann y Miko, 1998) (Scheloribates (T.))

DisTRIBUCIÓN: Eslovaquia. 
-Topobates circumcarinatus (Weigmann y Miko, 1998) (Scheloribates (T.))

DISTRIBUCIÓN: Alemania.

-Topobates comatus (Pérez-Íñigo jr., Herrero y Pérez-Íñigo, 1987)

(Setobates)

DistriBución: Suroeste de Europa.

-Topobates coronopubes (Lee y Pajak, 1988) (Setobates)

DisTRIBUCión: Australia.

-Topobates granifer Grandjean, 1958

DISTRIBUCIÓN: Francia e "India".

-Topobates heterodactylus (Pletzen, 1963) (Scheloribates)

DISTRIBUCIÓN: Sudáfrica.

-Topobates holsaticus Weigmann, 1969

DISTRIBUCIÓN: Europa centromeridional.

-Topobates humeralis Subbotina, 1976

DisTRIBUCIÓN: Europa oriental.

-Topobates lanceolatus (Wallwork, 1977) (Scheloribates)

DisTRIBUCión: I. Santa Helena.

-Topobates magnus (Balogh, 1962) (Setobates)

DisTRIBUCIÓN: Tanzania y Egipto.

-Topobates medius (Hammer, 1967) (Setobates)

(=Setobates discors Hammer, 1967)

DisTRIBUCIÓN: Nueva Zelanda.

-Topobates microsetosus (Wallwork, 1977) (Scheloribates) DisTRIBUCión: I. Santa Helena.

-Topobates multiplisetus (Mahunka, 1977) (Scheloribates) DisTRIBUCIÓN: Kenia.

-Topobates scheloribatoides (Ramsay, 1966) (Multoribates)

(=Setobates minor Hammer, 1967)

DisTRIBUCión: Nueva Zelanda.

-Topobates setosus (Mahunka, 1988) (Berndia)

DistriBución: Malgache (I. Reunión)

-Topobates sphaeroides Subbotina, 1985

DISTRIBUCIÓN: Rusia europea central.

-Topobates tanzanicus Mahunka, 1993

DisTRIBUCIÓN: Tanzania.

-Topobates ultraforaminosus (Lee y Pajak, 1988) (Setobates) DistriBución: Australia.

-Topobates umbraili (Schweizer, 1956) (Hemileius)

DisTRIBUCIÓN: Suiza.

Oripodidae Jacot, 1925 (15 gen., 1 subg., 97 spp. y 2 sspp.) DistrIBUCIÓN: Cosmopolita.

Anoripoda Sellnick, 1959 (1 sp.)

EsPECIE TIPO: Anoripoda nasalis Sellnick, 1959

DISTRIBUCIÓN: Australiana.

-Anoripoda nasalis Sellnick, 1959

DistriBuCIÓN: Polinesia (I. Sociedad).

Benoibates Balogh, 1958 (16 spp.)

(=Exoripoda Woolley, 1961 "sin. nov.")

(=Haploripoda Balogh y Mahunka, 1967 "sin. nov.")

(=Reductoripoda Mahunka y Palacios-Vargas, 1996 "sin. nov.")

ESPECIE TIPO: Benoibates flagellifer Balogh, 1958

DisTRIBUCIÓN: Pantropical (excepto Oriental).

-Benoibates absolutus (Mahunka y Palacios-Vargas, 1996) (Reductoripoda)

DisTRIBUCIÓN: Méjico.

-Benoibates amazonicus Balogh y Mahunka, 1969

DisTRIBUCIÓN: Neotropical.

-Benoibates bolivianus Balogh y Mahunka, 1969

DISTRIBUCIÓN: Neotropical.

-Benoibates borhidii Balogh y Mahunka, 1980

Distribución: Cuba.

-Benoibates chacoensis Mahunka, 1984

DisTRIBUCión: Paraguay.

-Benoibates crinitus (Berlese, 1910) (Protoribates (Scheloribates)) DISTRIBUCIÓN: U.S.A. (Florida).
-Benoibates excavatus (Woolley, 1961) (Exoripoda)

Distribución: Centroamérica (frecuente).

-Benoibates flagellifer Balogh, 1958

DisTRIBUCión: Angola.

-Benoibates juglans (Jacot, 1938) (Exoribatula)

Distribución: U.S.A. (Florida).

-Benoibates marginatus (Hammer, 1973) (Exoribatula)

Distribución: Polinesia (I. Tonga).

-Benoibates minimus Mahunka, 1985

DisTRIBUCIÓN: Pequeñas Antillas.

-Benoibates muscicola Baranek, 1981

DisTRIBUCIÓN: Argentina.

-Benoibates plurisetus Mahunka, 1983

DisTRIBUCIÓN: Paraguay.

-Benoibates reductus (Balogh y Mahunka, 1967) (Haploripoda)

Distribución: Congo.

-Benoibates rugosus Mahunka, 2001

DISTRIBUCIÓN: Kenia.

-Benoibates suramericanus (Mahunka, 1983) (Exoripoda)

DISTRIBUCIÓN: Brasil.

Birobates Balogh, 1970 (6 spp.)

(=Trischeloribates Hammer, 1971)

EsPECIE TIPO: Birobates reductus Balogh, 1970

DISTRIBUCIÓN: Australianooriental.

-Birobates acutus (Hammer, 1971) (Trischeloribates)

DisTRIBUCIÓN: Melanesia (I. Fiji).

-Birobates latus (Hammer, 1971) (Trischeloribates)

Distribución: Melanesia (I. Fiji).

-Birobates makinisus Corpuz-Raros, 1979

DistRIBUCIÓN: Filipinas.

-Birobates payatosensillus Corpuz-Raros, 1979

DistRIBUCIÓN: Filipinas.

-Birobates reductus Balogh, 1970

DisTRIBUCIÓN: Nueva Guinea.

-Birobates rotundus (Hammer, 1971) (Trischeloribates)

DisTRIBUCIÓN: Melanesia (I. Fiji).

Brachyoripoda Balogh, 1970 (3 spp.)

EsPeCIE TIPO: Brachyoripoda foveolata Balogh, 1970

DisTRIBUCIÓN: Paleotropical y Paleártica meridional.

-Brachyoripoda foveolata Balogh, 1970

DistriBución: Ceilán [Sri Lanka].

-Brachyoripoda minima Mahunka, 1988

DisTRIBUCión: Malgache (I. Mauricio).

-Brachyoripoda punctata Ohkubo, 1980

DISTRIBUCIÓN: Japón.

Calobates Balogh, 1961 (10 spp.)

EsPeCIE TIPO: Oripoda ornatissima Balogh, 1959

DistriBuCIÓN: Pantropical y Paleártica meridional.

Calobates (Calobates) Balogh, 1961 (4 spp.)

DisTRIBUCIÓN: Tropical (Etiópica y Neotropical)

-Calobates (C.) antichthon Higgins, 1966

DisTRIBUCIÓn: Guyana [Guayana Británica].

-Calobates (C.) ornatissimus (Balogh, 1959) (Oripoda)

DisTRIBUCIÓN: Angola.

-Calobates (C.) ornatus (Mahunka, 1986) (Protoripoda)

Distribución: Kenia.

-Calobates (C.) tuberculatus (Mahunka, 1988) (Protoripoda)

DistribuCión: Malgache (I. Mauricio).

Calobates (Protoripoda) Balogh, 1970 (6 spp.)

(?=Frischia Oudemans, 1915 "gen. inq.")

EsPeCIE TIPO: Protoripoda woolleyi Balogh, 1970

DisTRIBUCIÓN: Pantropical (excepto Neotropical) y Paleártica meridional. -Calobates (Protoripoda) elongatus (Oudemans, 1915) (Frischia) "sp. inq."

Distribución: Ceilán [Sri Lanka]. 
-Calobates (Protoripoda) flagellatus (Choi, 1994) (P.)

DisTRIBUCIÓN: Corea.

-Calobates (Protoripoda) incurva (Berlese, 1916) (Oripoda)

DisTRIBUCIÓN: Somalia.

-Calobates (Protoripoda) insularis (Balogh, 1970) (P.)

Distribución: Ceilán [Sri Lanka].

-Calobates (Protoripoda) lineatus (Mahunka, 1988) (P.)

DistriBuCión: Malgache (I. Mauricio).

-Calobates (Protoripoda) woolleyi (Balogh, 1970) (P.)

DisTRIBUCIÓN: Nueva Guinea.

Campbellobates Wallwork, 1964 (5 spp. y 1 ssp.)

ESPECIE TIPO: Campbellobates acanthus Wallwork, 1964

DisTRIBUCIÓN: Oriental, Australiana y Antártica.

-Campbellobates acanthus Wallwork, 1964

DisTRIBUCIÓN: Antártica (I. Campbell) y Hawai.

-Campbellobates acanthus acanthus Wallwork, 1964

DisTRIBUCIÓN: Antártica (I. Campbell).

-Campbellobates acanthus hawaiiensis P. Balogh, 1985 DISTRIBUCIÓN: Hawai.

-Campbellobates aureus Hammer, 1967

DistRIBUCión: Nueva Zelanda.

-Campbellobates latohumeralis Hammer, 1967

DisTRIBUCIón: Nueva Zelanda.

-Campbellobates occultus Hammer, 1967

DisTRIBUCión: Nueva Zelanda.

-Campbellobates philippinensis Corpuz-Raros, 1979

DISTRIBUCIÓN: Filipinas.

Cosmopirnodus Balogh, 1970 (3 spp.)

EsPeCIE TIPO: Cosmopirnodus pulcherrimus Balogh, 1970 DisTRIBUCIÓN: Australianooriental y Paleártica meridional.

-Cosmopirnodus angulatus Ichisawa y Aoki, 1998

DisTRIBUCIÓN: Japón

-Cosmopirnodus pulcherrimus Balogh, 1970

DistriBUCIÓN: Nueva Guinea.

-Cosmopirnodus tridactylus Mahunka, 1988

DISTRIBUCIÓN: Vietnam.

Gymnobates Banks, 1902 (1 sp.)

ESPECIE TIPO: Gymnobates glaber Banks, 1902

DISTRIBUCIÓN: Neártica.

-Gymnobates glaber Banks, 1902

DisTRIBUCIÓN: U.S.A. (Washington D.C.).

Gymnobatoides Woolley, 1966 (1 sp.)

EsPECIE TIPO: Gymnobates longus Banks, 1909

DisTRIBUCIÓN: Neártica.

-Gymnobatoides longus (Ewing, 1909) (Gymnobates)

DisTRIBUCIÓN: U.S.A. (Ilinois).

Oripoda Banks, 1904 (33 spp.)

EsPeCIE TIPO: Oripoda elongata Banks y Pergande, 1904

DisTRIBUCIÓN: Cosmopolita (excepto Antártica).

-Oripoda alata (Balogh y Mahunka, 1980) (Gymnobates) DisTRIBUCIÓN: Cuba.

-Oripoda angolensis (Balogh, 1963) (Truncopes)

DISTRIBUCIÓN: Angola.

-Oripoda anguina (Balogh y Mahunka, 1969) (Truncopes) DistribuCión: Bolivia.

-Oripoda anomala Baranek, 1982

DISTRIBUCIÓN: Argentina.

-Oripoda araucariae C. y C., jr. Pérez-Íñigo, 1993

DisTRIBUCIÓN: Brasil.

-Oripoda auriculata Mahunka, 1996

DISTRIBUCIÓN: Madagascar.

-Oripoda australis Berlese, 1916

DisTRIBUCión: Argentina.

-Oripoda benegasi Fernández, 1999

DISTRIBUCIÓN: Argentina.
-Oripoda brasiliensis Pérez-Iñigo y Baggio, 1980

DisTRIBUCIÓN: Brasil.

-Oripoda canagaratnami (Balogh, 1970) (Truncopes)

DisTRIBUCión: Ceilán [Sri Lanka].

-Oripoda clavata Woolley, 1961

DistRIBUCIÓn: Norte de Neotropical.

-Oripoda cordobensis J. y P. Balogh, 1990

(=Truncopes australis Balogh y Mahunka, 1968, “nom. praeoc." por Berlese, 1916)

DISTRIBUCIÓN: Argentina.

-Oripoda corticola Baranek, 1981

DisTRIBUCIÓN: Argentina.

-Oripoda cubana (Balogh y Mahunka, 1980) (Truncopes)

DisTRIBUCIÓN: Cuba.

-Oripoda divergens (Balogh y Mahunka, 1969) (Truncopes)

DisTRIBUCIÓN: Bolivia.

-Oripoda elongata Banks y Pergande, 1904

DisTRIBUCIÓN: U.S.A. y Méjico.

-Oripoda excavata Mahunka, 1988

DISTRIBUCIÓN: Vietnam.

-Oripoda humerata (Balogh, 1959) (Benoibates)

DisTRIBUCIÓN: Angola.

-Oripoda lenkoi Balogh y Mahunka, 1978

DISTRIBUCIÓN: Brasil.

-Oripoda lobata Mahunka, 1985

DisTRIBUCIÓN: Norte de Neotropical.

-Oripoda longiseta Woolley, 1961

DisTRIBUCIÓN: Norte de Neotropical

-Oripoda luminosa (Hammer, 1979) (Truncopes)

DistRIBUCIÓN: Java.

-Oripoda magna (Balogh y Mahunka, 1969) (Truncopes)

DISTRIBUCIÓN: Bolivia.

-Oripoda maxensis Mahunka, 1984

DISTRIBUCIÓN: Paraguay.

-Oripoda moderata Berlese, 1916

DistRIBUCIÓN: U.S.A. (Misuri).

-Oripoda montana (Hammer, 1962) (Gymnobates)

DISTRIBUCIÓN: Perú

-Oripoda pinicola Aoki y Ohkubo, 1974

DisTRIBUCIÓN: Este de Paleártica oriental.

-Oripoda prominens $\mathrm{P}$. Balogh, 1985

DisTRIBUCIÓN: Hawai.

-Oripoda punctata Baranek, 1982

DISTRIBUCIÓN: Argentina.

-Oripoda sacculifera Mahunka, 2001

DistriBUCIÓN: Kenia.

-Oripoda scissurata (Balogh y Mahunka, 1980) (Truncopes)

DisTRIBUCIÓN: Cuba.

-Oripoda sumonyii Mahunka, 1985

DisTRIBUCIÓN: Sudáfrica.

-Oripoda trilabiata Hammer, 1961

DISTRIBUCIÓN: Perú y Corea.

Pseudopirnodus Baranek, 1985 (4 spp.)

EsPECIE TIPO: Pseudopirnodus persetosus Baranek, 1985

DistriBución: Pantropical (excepto Oriental).

-Pseudopirnodus domrowi (Balogh y Mahunka, 1978) (Pirnodus)

DISTRIBUCIÓN: Australia.

-Pseudopirnodus imitans (Balogh y Mahunka, 1968) (Pirnodus)

DistRIBUCIÓn: Argentina.

-Pseudopirnodus madegassus Mahunka, 1966

DisTRIBUCIÓN: Madagascar.

-Pseudopirnodus persetosus Baranek, 1985

DISTRIBUCIÓN: Argentina.

Pteroripoda Balogh y Mahunka, 1974 (2 spp.)

ESPECIE TIPO: Pteroripoda minutissima Balogh y Mahunka, 1974

DISTRIBUCIÓN: Tropical (Oriental y Neotropical).

-Pteroripoda minutissima Balogh y Mahunka, 1974

DisTRIBUCIÓN: Cuba. 
-Pteroripoda perennis Mahunka, 1996 DISTRIBUCIÓN: Borneo.

Scriptoripoda P. Balogh, 1985 (2 spp.)

EsPecie TIPO: Scriptoripoda excellens P. Balogh, 1985

DISTRIBUCIÓN: Australiana.

-Scriptoripoda excellens P. Balogh, 1985

DisTRIBUCIÓN: Hawai.

-Scriptoripoda tenorioae P. Balogh, 1985

DISTRIBUCIÓN: Hawai.

Subpirnodus Mahunka, 1988 (1 sp.)

ESPECIE TIPO: Subpirnodus mirabilis Mahunka, 1988

DisTRIBUCIÓN: Oriental.

-Subpirnodus mirabilis Mahunka, 1988

DISTRIBUCIÓN: Vietnam.

Truncopes Grandjean, 1956 (9 spp. y 1 ssp.)

EsPeCIE Tipo: Truncopes optatus Grandjean, 1956

DisTRIBUCIÓN: Paleártica meridional y Oriental.

-Truncopes fujikawae Aoki y Ohkubo, 1974

DisTRIBUCIÓN: Japón.

-Truncopes makilingensis Corpuz-Raros, 1979

DISTRIBUCIÓN: Filipinas.

-Truncopes moderatus Aoki y Ohkubo, 1974

Distribución: Este de Paleártica oriental.

-Truncopes montanus Wen y Chen, 1992

DisTRIBUCIÓN: China suroriental.

-Truncopes optatus Grandjean, 1956

DisTRIBUCIÓN: Paleártica meridional (Mediterránea occidental y

Japón).

-Truncopes optatus optatus Grandjean, 1956

DisTRIBUCIÓN: Mediterránea occidental.

-Truncopes optatus asiaticus Aoki y Ohkubo, 1974

DISTRIBUCIÓN: Japón.

-Truncopes orientalis Mahunka, 1987

DisTRIBUCIÓN: Vietnam.

-Truncopes pyriformis Hammer, 1981

DISTRIBUCIÓN: Java.

-Truncopes sinaraja Mahunka, 2001

DisTRIBUCIÓN: Ceilán [Sri Lanka].

-Truncopes yoshidai Aoki y Ohkubo, 1974

Distribución: Este de Paleártica oriental.

Pirnodidae Grandjean, 1956 (3 gen. y 6 spp.)

DisTRIBUCIÓN: Tropical (Australiana y Neotropical) y subtropical.

Cryptoribatula Jacot, 1934 (2 spp.)

(=Euaella Hammer, 1973)

EsPeCIE TIPO: Cryptoribatula taishanensis Jacot, 1934

DistriBuCión: Australiana y Paleártica meridional.

-Cryptoribatula euaensis Hammer, 1973

(=Euaella gitteae Hammer, 1973)

Distribución: Polinesia (I. Tonga).

-Cryptoribatula taishanensis Jacot, 1934

DisTRIBUCIÓN: Noreste de China.

Huarpescopes Fernández, Monetti y Martínez, 1995 (1 sp.)

EsPECIE TIPO: Pirnodus cryophilus Fernández, 1989

DISTRIBUCIÓN: Neotropical.

-Huarpescopes cryophilus (Fernández, 1989) (Pirnodus)

DISTRIBUCIÓN: Argentina.

Pirnodus Grandjean, 1956 (3 spp.)

EsPeCIE TIPO: Pirnodus detectidens Grandjean, 1956

DISTRIBUCIÓN: Paleártica meridional y Neotropical.

-Pirnodus andinus Baranek, 1985

DisTRIBUCIÓN: Argentina.

-Pirnodus detectidens Grandjean, 1956

Distribución: Suroeste de Europa.
-Pirnodus soyeri Travé, 1969

Distribución: Macaronésica (I. Salvajes).

Protoribatidae J. y P. Balogh, 1984 (9 gen., 4 subg., 69 spp. y 3 sspp.) DistriBución: Cosmopolita.

Cribrozetes Balogh, 1970 (1 sp.)

EsPecie Tipo: Cribrozetes multiareolatus Balogh, 1970

DISTRIBUCIÓN: Oriental.

Cribrozetes multiareolatus Balogh, 1970

DistRIBUCIÓN: Oriental: Ceilán [Sri Lanka] y Filipinas.

Perxylobates Hammer, 1972 (14 spp.)

(=Octodurozetes Mahunka, 1993 "sin. nov.")

EsPECIE TIPO: Xylobates vermiseta Balogh y Mahunka, 1968

DisTRIBUCIÓN: Pantropical y subtropical.

-Perxylobates barbatus Hammer, 1972

DisTRIBUCIÓN: Polinesia (Tahití) y Java.

-Perxylobates berndhauseri (Mahunka, 1993) (Octodurozetes)

DisTRIBUCIÓN: Suiza.

-Perxylobates brevisetus Mahunka, 1988

DisTRIBUCIÓN: Vietnam.

-Perxylobates coreanus Choi y Aoki, 1993

DISTRIBUCIÓN: Corea

-Perxylobates guehoi Mahunka, 1978

DisTRIBUCIÓN: Malgache.

-Perxylobates longissimus (Warburton, 1912) (Oribata)

Distribución: I. Seychelles.

-Perxylobates mahunkai Bayoumi, 1980

DisTRIBUCIÓN: Egipto.

-Perxylobates obesus Bayoumi, 1977

DisTRIBUCIÓN: Egipto.

-Perxylobates paravermiseta Mahunka, 1976

Distribución: China (Hong-Kong).

-Perxylobates sinensis Wen, Aoki y X. Wang, 1984

Distribución: Este de China.

-Perxylobates sinlimes (Hammer, 1971) (Xylobates)

Distribución: Melanesia (I. Fiji) y Pequeñas Antillas.

-Perxylobates taidinchani Mahunka, 1976

Distribución: China (Hong-Kong).

-Perxylobates vermiseta (Balogh y Mahunka, 1968) (Xylobates)

Distribución: Pantropical (Malgache, Oriental, Islas del Pacífico y Pequeñas Antillas).

-Perxylobates vietnamensis (Jeleva y Vu, 1987) (Xylobates)

DisTRIBUCIÓN: Vietnam.

Protoribates Berlese, 1908 (56 spp. y 3 sspp.)

EsPeCIE TiPo: Oribata dentata Berlese, 1883

DisTRIBUCIÓN: Cosmopolita.

Protoribates (Protoribates) Berlese, 1908 (38 spp. y 3 sspp.)

(=Alloribates Banks, 1947)

(=Propeschelobates Jacot, 1936)

(=Styloribates Jacot, 1934)

(=Xylobates Jacot, 1929)

DisTRIBUCIÓn: Cosmopolita.

-Protoribates (P.) albidus (Ewing, 1908) (Oribata)

DisTRIBUCIÓN: U.S.A. (Ilinois).

-Protoribates (P.) angulatus Choi, 1986

DisTRIBUCIÓN: Corea.

-Protoribates (P.) antillensis (Mahunka, 1985) (Xylobates)

Distribución: Centroamérica.

-Protoribates (P.) bayanicus Bayartogtokh, 2000

DisTRIBUCIÓN: Mongolia.

-Protoribates (P.) boraboraensis Sellnick, 1959

Distribución: Polinesia (I. Sociedad).

-Protoribates (P.) brevisetosus (Fujita, 1989) (Xylobates)

DisTRIBUCIÓN: Japón.

-Protoribates (P.) capucinus Berlese, 1908

DistriBución: Cosmopolita (excepto Antártica). 
-Protoribates (P.) capucinus capucinus Berlese, 1908 (=Xylobates capucinus angustior Jacot, 1937) (=Alloribates singularis Banks, 1947)

DisTRIBUCIÓN: Cosmopolita (excepto Antártica): frecuente en tropical (excepto Etiópica) y subtropical.

-Protoribates (P.) capucinus bonairensis Willmann, 1936 DisTRIBUCión: Pequeñas Antillas.

-Protoribates (P.) capucinus tentaculus Mihelcic, 1958 DisTRIBUCión: Austria.

-Protoribates (P.) clavatus Willmann, 1931

DisTRIBUCIÓN: Sumatra.

-Protoribates (P.) concretus (Kramer, 1898) (Oribata) "sp. inq."

Distribución: América austral (Tierra del Fuego).

-Protoribates (P.) crassisetiger Choi, 1986

DisTRIBUCIÓN: Este de Paleártica oriental.

-Protoribates (P.) crassisetiger crassisetiger Choi, 1986 DisTRIBUCIÓN: Corea.

-Protoribates (P.) crassisetiger nipponicus Fujita, 1989 DiSTRIBUCIÓN: Japón.

-Protoribates (P.) dentatus (Berlese, 1883) (Oribata)

(=Oribata monodactyla Haller, 1884)

(=Peloribates vastus Mihelcic, 1956)

DisTRIBUCIÓN: Holártica, Oriental (Filipinas y Vietnam) y Melanesia (I. Fiji).

-Protoribates (P.) diani (Mahunka, 1986) (Xylobates)

DisTRIBUCIÓN: Kenia.

-Protoribates (P.) geonjiensis Choi, 1994

DisTRIBUCIÓN: Corea.

-Protoribates (P.) gracilis (Aoki, 1982) (Xylobates)

DisTRIBUCión: Japón.

-Protoribates (P.) hakonensis Aoki, 1994

DisTRIBUCIÓN: Japón.

-Protoribates (P.) halleri (Michael, 1898) (Oribata) "sp. inq." (=Oribata simplex Haller, 1884, "nom. praeoc." por Gervais, 1849) Distribución: "Neotropical".

-Protoribates (P.) imperfectus (Banks, 1906) (Galumna)

DisTRIBUCIÓN: U.S.A. oriental.

-Protoribates (P.) iracemae Pérez-Íñigo y Baggio, 1994

DisTRIBUCIÓN: Brasil.

-Protoribates (P.) longiuscula (Koch, 1841) (Zetes) "sp. inq." DisTRIBUCión: Alemania.

-Protoribates (P.) lophothrichus (Berlese, 1904) (Oribata)

(=Styloribates pectinatus Jacot, 1934)

(=Xylobates prionotus Woolley, 1968)

Distribución: Semicosmopolita (Holártica: frecuente, Etiópica: Senegal e I. Santa Helena, Vietnam y Hawai).

-Protoribates (P.) madagascarensis (Balogh, 1961) (Scheloribates)

DistribuCión: Etiópica (Madagascar y Kenia).

-Protoribates (P.) magnus Aoki, 1982

Distribución: Japón y Este de China.

-Protoribates (P.) mollicoma (Hammer, 1973) (Xylobates)

Distribución: Polinesia (I. Tonga) e India (Bengala Occidental)

-Protoribates (P.) myrmecophilus Aoki e Ito, 1997

Distribución: Oriental.

-Protoribates (P.) nagaroensis (Fujita, 1989) (Xylobates)

DisTRIBUCIÓN: Japón.

-Protoribates (P.) natalensis (Pletzen, 1963) (Scheloribates)

Distribución: Sudáfrica e India (Bengala Occidental).

-Protoribates (P.) oblongus (Ewing, 1909) (Oribata)

(=Oribata longa Ewing, 1909)

$(=$ Xylobates longisetae Jacot, 1937)

DisTRIBUCión: Neártica (excepto Oeste).

-Protoribates (P.) paracapucinus (Mahunka, 1988) (Xylobates)

DisTRIBUCIÓN: Oriental (Borneo y Filipinas) y Paleártica oriental (Irán).

-Protoribates (P.) parvulus Willmann, 1931

DisTRIBUCIÓN: Sumatra.

-Protoribates (P.) pembertoni (Jacot, 1934) (Xylobates)

DisTRIBUCIÓN: Hawai.
-Protoribates (P.) robustior (Jacot, 1937) (Xylobates oblongus $r$.)

(=Xylobates capucinus robustior Jacot, 1937, “nom. praeoc.” por Jacot, 1937)

DistribuCión: U.S.A. (Carolina del Norte).

-Protoribates (P.) rodriguezi (Mahunka, 1988) (Xylobates)

DisTRIBUCIÓN: Oriental (Borneo y Filipinas).

-Protoribates (P.) rotundus (Aoki, 2002) (Xylobates)

DisTRIBUCIÓN: Japón.

-Protoribates (P.) souchnaiensis Abdel-Hamid,1964

DisTRIBUCIÓN: Egipto.

-Protoribates (P.) tenuis (Wen, Aoki y X. Wang, 1984) (Xylobates)

Distribución: Este de China.

-Protoribates (P.) triangularis (Hammer, 1971) (Xylobates)

Distribución: Islas del Pacífico.

-Protoribates (P.) varisetiger (Wen, Aoki y X. Wang, 1984) (Xylobates) DisTRIBUCión: Este de China.

-Protoribates (P.) yezoensis (Fujikawa, 1983) (Xylobates)

DISTRIBUCIÓN: Japón.

Protoribates (Triaungius) Kulijev, 1978 (18 spp.)

(=Brasilobates Pérez-Íñigo y Baggio, 1980 "sin. nov.")

(=Glaberoribates Tseng, 1984 "sin. nov.")

EsPeCIE TIPO: Scheloribates fallax Kulijev, 1968

DISTRIBUCIÓN: Pantropical y subtropical.

-Protoribates (Triaungius) acutus (Hammer, 1979) (Xylobates) DisTRIBUCIÓN: Java.

-Protoribates (Triaungius) bipilis (Hammer, 1972) (Xylobates)

Distribución: Polinesia.

-Protoribates (Triaungius) bisculpturatus (Mahunka, 1988) (Xylobates) DisTRIBUCIÓN: Borneo.

-Protoribates (Triaungius) duoseta (Hammer, 1979) (Xylobates)

DisTRIBUCIÓN: Oriental (Java y Borneo).

-Protoribates (Triaungius) durbanensis (Pletzen, 1963) (Scheloribates) DisTRIBUCIÓN: Sudáfrica.

-Protoribates (Triaungius) fallax (Kulijev, 1968) (Scheloribates)

DistribuCión: Cáucaso.

-Protoribates (Triaungius) longilamellatus Berlese, 1916 (P. (Scheloribates))

Distribución: “África oriental” y Java.

-Protoribates (Triaungius) luteus (Hammer, 1979) (Xylobates)

DisTRIBUCIÓN: Java.

-Protoribates (Triaungius) maigsius (Corpuz-Raros, 1979) (Xylobates)

DistRIBUCIÓN: Filipinas.

-Protoribates (Triaungius) maximus (Mahunka, 1988) (Brasilobates) DisTRIBUCIÓN: Vietnam.

-Protoribates (Triaungius) obtusus (Mihelcic, 1956) (Peloribates)

DistRIBUCióN: Paleártica meridional (Mediterránea e Irán).

-Protoribates (Triaungius) praeoccupatus nom. nov.

[=Brasilobates bipilis Pérez-Íñigo y Baggio, 1980, “nom. praeoc." por Hammer, 1972 ("hom. sec.")]

DisTRIBUCIÓN: Neotropical (Brasil y Panamá).

-Protoribates (Triaungius) punctatus (Grobler, 1991) (Brasilobates)

DistriBuCión: Sudáfrica.

-Protoribates (Triaungius) rhomboides (Hammer, 1972) (Xylobates) DistriBución: Polinesia (Tahití).

-Protoribates (Triaungius) sangumburiensis (Choi, 1996) (Brasilobates) DistRIBUCIÓN: Corea.

-Protoribates (Triaungius) seminudus (Hammer, 1971) (Xylobates)

Distribución: Islas del Pacífico e India.

-Protoribates (Triaungius) spinosus (Fujita, 1989) (Brasilobates)

DisTRIBUCIÓN: Japón.

-Protoribates (Triaungius) urbanlus (Tseng, 1984) (Glaberoribates)

Distribución: Taiwan [Formosa].

Setoxylobates Balogh y Mahunka, 1967 (6 spp.)

EsPECIE TIPO: Setoxylobates foveolatus Balogh y Mahunka, 1967

DistriBución: Pantropical (excepto Neotropical).

Setoxylobates (Setoxylobates) Balogh y Mahunka, 1967 (1 sp.) DISTRIBUCIÓN: Oriental. 
-Setoxylobates (S.) foveolatus Balogh y Mahunka, 1967

DistRIBUCIÓN: Oriental (Vietnam e India: Bengala Occidental).

Setoxylobates (Plenoxylobates) Hammer, 1979 (3 spp.)

EsPeCIE TIPO: Plenoxylobates ramosus Hammer, 1979

DisTRIBUCIÓN: Oriental.

-Setoxylobates (Plenoxylobates) curtiseta (Hammer, 1979) (P.) DisTRIBUCIÓN: Java.

-Setoxylobates (Plenoxylobates) mayuloeus (Corpuz-Raros, 1979)

(Perxylobates)

DISTRIBUCIÓN: Filipinas.

-Setoxylobates (Plenoxylobates) ramosus (Hammer, 1979) (P.)

DISTRIBUCIÓN: Java.

Setoxylobates (Polyxylobates) Hammer, 1973 (2 spp.)

EsPeCIE TIPO: Polyxylobates diversiporosus Hammer, 1973

DisTRIBUCIÓn: Australiana y Etiópica.

-Setoxylobates (Polyxylobates) diversiporosus (Hammer, 1973) (P.) DisTribución: Polinesia (I. Tonga).

-Setoxylobates (Polyxylobates) principalis (Berlese, 1916) (Protoribates)

DISTRIBUCIÓN: Somalia.

Sicaxylobates Luxton, 1985 (1 sp.)

EsPECIE TIPO: Xylobates sicafer Hammer, 1968

DisTRIBUCIÓN: Australiana.

-Sicaxylobates sicafer (Hammer, 1968) (Xylobates) DisTRIBUCIÓN: Nueva Zelanda.

Transoribates Pérez-Íñigo, 1992 (2 spp.)

(=Lagenobates Weigmann y Miko, 2002 "sin. nov.")

EsPeCIE TIPO: (Protoribates latus Mihelcic, 1965) =Oribata lagenula

Berlese, 1904

DisTRIBUCiÓN: Paleártica.

-Transoribates agricola (Nakamura y Aoki, 1989) (Protoribates)

Distribución: Este de Paleártica oriental.

-Transoribates lagenula (Berlese, 1904) (Oribata)

(=Protoribates latus Mihelcic, 1965)

(=Protoribates pupula Berlese, 1910)

DisTRIBUCIÓN: Paleártica (menos frecuente en el Norte).

Trixylobates Balogh y Mahunka, 1978 (1 sp.)

ESPECIE TIPO: Trixylobates bidactylus Balogh y Mahunka, 1978

DISTRIBUCIÓN: Neotropical.

-Trixylobates bidactylus Balogh y Mahunka, 1978 DISTRIBUCIÓN: Brasil.

Tuxenia Hammer, 1958 (3 spp.)

ESPECIE TIPO: Tuxenia complicata Hammer, 1958

DisTRIBUCIÓN: Neotropical austral.

-Tuxenia brevis Covarrubias, 1967

(=Tuxenia brevisetosa Mahunka, 1980)

DISTRIBUCIÓN: América austral.

-Tuxenia complicata Hammer, 1958

DISTRIBUCIÓN: Argentina.

-Tuxenia manantialis Hammer, 1962

DisTRIBUCIÓN: América austral.

Vilhenabates Balogh, 1963 (5 spp.)

EsPeCIE TIPO: Peloribates minutus Balogh, 1958

DistriBución: Paleotropical.

Vilhenabates (Vilhenabates) Balogh, 1963 (3 spp.)

DISTRIBUCIÓN: Paleotropical.

-Vilhenabates (V.) hyalinus Tseng, 1984

DISTRIBUCIÓN: Taiwan [Formosa].

-Vilhenatates (V.) minutus (Balogh, 1958) (Peloribates)

Distribución: Congo.

-Vilhenabates (V.) simplex Balogh, 1970

DistriBución: Ceilán [Sri Lanka].
Vilhenabates (Phalacrozetes) Aoki, 1965 (2 spp.)

EsPeCIE TIPO: Phalacrozetes sinatus Aoki, 1965

DisTRIBUCIÓN: Oriental.

-Vilhenabates (Phalacrozetes) similis (Mahunka, 1988) (P.)

DisTRIBUCIÓN: Borneo.

-Vilhenabates (Phalacrozetes) sinatus (Aoki, 1965) (V.)

DisTRIBUCIÓN: Oriental.

Haplozetidae Grandjean, 1936 (18 gen., 11 subg., 209 spp. y 5 sspp.) DistRIBUCIÓN: Cosmopolita.

Acutozetes Balogh, 1970 (5 spp.)

EsPECIE TIPO: Acutozetes rostratus Balogh, 1970

DISTRIBUCIÓN: Australianooriental.

-Acutozetes bornemisszai J. y P. Balogh, 1986

Distribución: Melanesia (I. Fiji).

-Acutozetes chibai Aoki, 1976

Distribución: Península Malaya.

-Acutozetes javensis Hammer, 1979

DisTRIBUCIÓN: Oriental (Indonesia).

-Acutozetes nadchatrami Balogh y Mahunka, 1974

DistriBución: Península Malaya.

- Acutozetes rostratus Balogh, 1970

Distribución: Nueva Guinea.

Afroleius Mahunka, 1984 (3 spp.)

EsPECIE TIPO: Afroleius deformis Mahunka, 1984

DISTRIBUCIÓN: Etiópica.

-Afroleius deformis Mahunka, 1984

DisTRIBUCIÓN: Sudáfrica.

-Afroleius minor Mahunka, 1984

DisTRIBUCIÓN: Sudáfrica.

-Afroleius simplex Mahunka, 1984

DisTRIBUCIÓN: Sudáfrica.

Baloghia Mahunka, 1994 (2 spp.)

EsPeCIE TIPO: Baloghia juditae Mahunka, 1994

DISTRIBUCIÓN: Etiópica.

-Baloghia juditae Mahunka, 1994

DisTRIBUCIÓN: Malgache (I. Comores).

-Baloghia spinifera Mahunka, 1994

DistRIBUCIÓN: Malgache (I. Comores).

Baloghiella Bulanova-Zachvatkina, 1960 (2 spp.)

EsPECIE TIPO: Baloghiella prima Bulanova-Zachvatkina, 1960

DisTRIBUCIÓN: Paleártica.

-Baloghiella granulata Bayartogtokh y Akrami, 2000

DISTRIBUCIÓN: Irán.

-Baloghiella prima Bulanova-Zachvatkina, 1960

DISTRIBUCIÓN: Asia centrooccidental.

Berlesiella Hammer, 1979 (2 spp.)

ESPECIE TIPO: Berlesiella scutata Hammer, 1979

Distribución: Paleotropical.

Berlesiella (Berlesiella) Hammer, 1979 (1 sp.)

DistriBuCIÓN: Oriental.

-Berlesiella (B.) scutata Hammer, 1979

Distribución: Java.

Berlesiella (Comororibula) Mahunka, 1994 (1 sp.)

EsPeCIE TIPO: Comororibula truncata Mahunka, 1994

DISTRIBUCIÓN: Etiópica.

Berlesiella (Comororibula) truncata (Mahunka, 1994) (C.)

Distribución: Malgache (I. Comores).

Bolkiah Mahunka, 1997 (1 sp.)

EsPECIE TIPO: Bolkiah hauseri Mahunka, 1997

DISTRIBUCIÓN: Oriental. 
-Bolkiah hauseri Mahunka, 1997

DisTRIBUCIÓN: Borneo.

Borneozetes Mahunka, 1997 (1 sp.)

EsPECIE TIPO: Borneozetes lanceolatus Mahunka, 1997

DistriBución: Oriental.

-Borneozetes lanceolatus Mahunka, 1997

DisTRIBUCIÓN: Borneo.

Cantharozetes Hammer, 1961 (1 sp.)

EsPeCIE TIPO: Cantharozetes lucens Hammer, 1961

DISTRIBUCIÓN: Neotropical.

-Cantharozetes lucens Hammer, 1961

DisTRIBUCIÓN: Perú

Conozetes Balogh y Mahunka, 1969 (1 sp.)

ESPECIE TIPO: Conozetes arcualis Balogh y Mahunka, 1969

DISTRIBUCIÓN: Neotropical.

-Conozetes arcualis Balogh y Mahunka, 1969

DistRIBUCIÓN: Brasil.

Indoribates Jacot, 1929 (27 spp. y 1 ssp.)

EsPECIE TIPO: Protoribates punctulatus Sellnick, 1925

DisTRIBUCIÓn: Cosmopolita (excepto Antártica).

Indoribates (Indoribates) Jacot, 1929 (8 spp.)

(=Nixozetes Mahunka, 1977)

(=Sundazetes Hammer, 1979)

DISTRIBUCIÓN: Australianooriental y Paleártica meridional.

-Indoribates (I.) crassisetiger (Fukuyama y Aoki, 2000) (Nixozetes)

DisTRIBUCIÓN: China suroriental.

-Indoribates (I.) gregoryi (Balogh, 1970) (Cosmobates)

DisTRIBUCIÓN: Nueva Guinea.

-Indoribates (I.) japonicus (Aoki, 1988) (Sundazetes)

DisTRIBUCIÓN: Japón.

-Indoribates (I.) javanus (Mahunka, 1977) (Nixozetes)

DisTRIBUCIÓN: Oriental (Indonesia y Filipinas).

-Indoribates (I.) multisetus (Wen y Zhao, 1994) (Sundazetes)

DisTRIBUCIÓN: China suroriental.

-Indoribates (I.) nobilis (Golosova, 1984) (Cosmobates)

DisTRIBUCIÓN: Vietnam.

-Indoribates (I.) panabokkei (Balogh, 1970) (Cosmobates)

(=Nixozetes apoensis Corpuz-Raros, 1979)

DistribuCión: Oriental (Ceilán [Sri Lanka] y Filipinas).

-Indoribates (I.) punctulatus (Sellnick, 1925) (Protoribates)

(=Sundazetes crispus Hammer, 1979)

DisTRIBUCIÓN: Java.

Indoribates (Haplozetes) Willmann, 1935 (11 spp. y 1 ssp.)

EsPECIE TIPO: Peloribates vindobonensis Willmann, 1935

DisTRIBUCIÓN: Cosmopolita (excepto Antártica).

-Indoribates (Haplozetes) brevisetosus (Bayartogtokh, 2000) (H.) DISTRIBUCIÓN: Mongolia.

-Indoribates (Haplozetes) brevisetus (Balogh, 1970) (Cosmobates) DisTRIBUCIÓN: Nueva Guinea.

-Indoribates (Haplozetes) carneus (Tseng, 1984) (Lauritzenia) DisTRIBUCIÓN: Taiwan [Formosa].

-Indoribates (Haplozetes) chiayiensis (Tseng, 1984) (Muliercula) DisTRIBUCIÓN: Taiwan [Formosa].

-Indoribates (Haplozetes) clavatus (Bayartogtokh, 2000) (H.) DisTRIBUCIÓN: Mongolia.

-Indoribates (Haplozetes) cuticulatus (Tseng, 1984) (Muliercula) DisTRIBUCión: Taiwan [Formosa].

-Indoribates (Haplozetes) minutus (Tseng, 1984) (Lauritzenia) DisTRIBUCión: Taiwan [Formosa].

-Indoribates (Haplozetes) nudus (Hammer, 1958) (Peloribates) DisTRIBUCIÓN: Neotropical.

-Indoribates (Haplozetes) quadripilus (Berlese, 1916) (Protoribates) DisTRIBUCIÓN: Java y Melanesia (I. Fiji).
-Indoribates (Haplozetes) ulykpani (Bayartogtokh y Aoki, 1998) (H.)

DisTRIBUCIÓN: Mongolia.

-Indoribates (Haplozetes) vindobonensis (Willmann, 1935) (Peloribates)

DistriBUCión: Paleártica y Yemen.

-Indoribates (Haplozetes) vindobonensis vindobonensis (Willmann, 1935)

DisTRIBUCIÓN: Paleártica (frecuente) y Yemen

-Indoribates (Haplozetes) vindobonensis curtipilis Kunst, 1977)

(Haplozetes)

DisTRIBUCIÓN: Europa central.

Indoribates (Mancoribates) Hammer, 1961 (4 spp.)

(=Minasbates Pérez-Iñigo y Baggio, 1996 "sin. nov.")

EsPeCIE TIPO: Mancoribates rostropilosus Hammer, 1961

DisTRIBUCIÓN: Tropical (Neotropical y Australiana).

-Indoribates (Mancoribates) acutirostrum (Hammer, 1968) (Lauritzenia)

DisTRIBUCIÓN: Nueva Zelanda.

-Indoribates (Mancoribates) rostropilosus (Hammer, 1961) (M.)

DisTRIBUCIÓN: Neotropical.

-Indoribates (Mancoribates) rotundirostrum (Hammer, 1968) (Lauritzenia)

DisTRIBUCIÓN: Nueva Zelanda.

-Indoribates (Mancoribates) sphaeroclava (Pérez-Iñigo y Baggio, 1996) (Minasbates)

DISTRIBUCIÓN: Brasil.

Indoribates (Philippizetes) Corpuz-Raros, 1979 (2 spp.)

EsPECIE TIPO: Nixozetes (Philippizetes) philippinensis Corpuz-Raros, 1979 DISTRIBUCIÓN: Oriental.

-Indoribates (Philippizetes) corpusrarosae (Mahunka, 1987) (Nixozetes (P.))

DISTRIBUCIÓN: Borneo.

-Indoribates (Philippizetes) philippinensis (Corpuz-Raros, 1979)

(Nixozetes (P.))

DISTRIBUCIÓN: Filipinas.

Indoribates (Triungulozetes) Subías, 2001 (2 spp.)

=Mixobates Subías y Gil-Martín, 1993, "nom. praeoc." por Thor, 1905)

EsPECIE TIPO: Haplozetes triungulatus Beck, 1964

DisTRIBUCIÓN: Neotropical y Paleártica meridional.

-Indoribates (Triungulozetes) fusifer (Berlese, 1908) (Protoribates

(Scheloribates))

(=Haplozetes (Mixobates) insularis Pérez-Íñigo y Peña, 1996)

DisTRIBUCIÓN: Mediterránea.

-Indoribates (Triungulozetes) triungulatus (Beck, 1964) (Haplozetes) DisTRIBUCIÓN: El Salvador.

Lauritzenia Hammer, 1958 (26 spp.)

EsPECIE TIPO: Lauritzenia longipluma Hammer, 1958

DisTRIBUCIÓN: Pantropical y subtropical.

Lauritzenia (Lauritzenia) Hammer, 1958 (6 spp.)

DisTRIBUCIÓN: Pantropical (excepto Australiana).

-Lauritzenia (L.) furtadoi (Balogh y Mahunka, 1974) (Haplozetes)

DistriBución: Península Malaya.

-Lauritzenia (L.) glabra Pérez-Íñigo y Baggio, 1996

DisTRIBUCIÓN: Brasil.

-Lauritzenia (L.) insignis (Balogh y Mahunka, 1966) (Haplozetes)

DisTRIBUCIÓN: Congo.

-Lauritzenia (L.) longipluma Hammer, 1958

DistRIBUCIÓN: Neotropical (Argentina y Perú) e India (Bengala Occidental)

-Lauritzenia (L.) loongchiensis (Tseng, 1984) (Haplozetes)

DistriBUCIÓN: Taiwan [Formosa].

-Lauritzenia (L.) minimicoma (Beck, 1964) (Haplozetes)

Distribución: Neotropical (Perú y Panamá) e India (Tripura). 
Lauritzenia (Incabates) Hammer, 1961 (18 spp.)

(=Canaribates Pérez-Íñigo y Peña, 1994 "sin. nov.")

EsPECIE TIPO: Incabates nudus Hammer, 1961

DisTRIBUCIÓN: Pantropical (excepto Etiópica) y subtropical

-Lauritzenia (Incabates) angusta (Hammer, 1971) (I.)

(=Incabates medius Hammer, 1971)

DISTRIBUCIÓN: Australiana, Filipinas y Japón.

-Lauritzenia (Incabates) aokii (Choi, 1985) (Incabates) DisTRIBUCIÓN: Corea.

-Lauritzenia (Incabates) atlantica Pérez-Íñigo y Peña, 1996 DisTRIBUCIÓN: I. Canarias.

-Lauritzenia (Incabates) barbata Choi y Kim, 2002 DisTRIBUCIÓN: Corea

-Lauritzenia (Incabates) canariensis Pérez-Íñigo y Peña, 1997 DisTRIBUCIÓN: I. Canarias.

-Lauritzenia (Incabates) depilis Pérez-Íñigo y Peña, 1996 DisTRIBUCIÓN: I. Canarias.

-Lauritzenia (Incabates) elegans (Kunst, 1977) (Haplozetes) DisTRIBUCIÓN: Holártica meridional (U.S.A. suroriental y Europa centromeridional).

-Lauritzenia (Incabates) elongata (Lee y Shepherd, 1990) (Magnobates) DisTRIBUCIÓN: Australia.

-Lauritzenia (Incabates) globula (Lee y Shepherd, 1990) (Magnobates) DISTRIBUCIÓN: Australia.

-Lauritzenia (Incabates) hispanica Morell, 1991 (L.) DisTRIBUCIÓN: Ibérica.

-Lauritzenia (Incabates) major (Aoki, 1970) (I.) DisTRIBUCIÓN: Japóny Taiwan [Formosa].

-Lauritzenia (Incabates) mucronata (Lee, 1993) (I.) DisTRIBUCIÓN: Australia.

-Lauritzenia (Incabates) nuda (Hammer, 1961) (I.) DisTRIBUCIón: Perú y Oriental: Filipinas e India (Bengala Occidental).

-Lauritzenia (Incabates) pallida (Mihelcic, 1956) (Peloribates) (=Protoribates meridionalis Mihelcic, 1965)

DISTRIBUCIÓN: Mediterránea.

-Lauritzenia (Incabates) punctata (Lee, 1993) (I.) DISTRIBUCIÓN: Australia.

-Lauritzenia (Incabates) similis Subías y Gil-Martín, 1995 DisTRIBUCIÓN: Portugal.

-Lauritzenia (Incabates) sinuata (Pérez-Iñigo jr., 1990) (Haplozetes) DisTRIBUCIÓN: España.

-Lauritzenia (Incabates) tenuifusus (Berlese, 1916) (Protoribates (Scheloribates))

(=Canaribates chamobatoides Pérez-Íñigo y Peña, 1994)

DISTRIBUCIÓN: Mediterránea.

Lauritzenia (Magnobates) Hammer, 1967 (2 spp.)

(=Gymnozetes Calugar y Vasiliu, 1983 "sin. nov")

EsPECIE TIPO: Magnobates flagellifer Hammer, 1967

Distribución: Pantropical (excepto Etiópica).

-Lauritzenia (Magnobates) flagellifer (Hammer, 1967) (M.)

DisTRIBUCIÓN: Nueva Zelanda y Vietnam.

-Lauritzenia (Magnobates) orghidani (Calugar y Vasiliu, 1983)

(Gymnozetes)

DisTRIBUCIÓN: Cuba.

Magyaria Balogh, 1963 (17 spp.)

EsPECIE TIPO: Scheloribates reticulatus Balogh, 1958

DisTRIBUCIÓn: Pantropical (excepto Neotropical)

-Magyaria agusana Corpuz-Raros, 1991

DISTRIBUCIÓN: Filipinas.

-Magyaria annobonica Pérez-Íñigo, 1982

DisTRIBUCIÓN: Guinea Ecuatorial: I. Pagalu [Annobón].

-Magyaria atlantica Pérez-Íñigo, 1983

DistriBución: Guinea Ecuatorial: I. Pagalu [Annobón].

-Magyaria breviseta Mahunka, 1978

DisTRIBUCIÓN: Malgache (I. Mauricio).

-Magyaria cancellata (Beck, 1964) (Haplozetes)

DistriBución: Sudán e India (Bengala Occidental).
-Magyaria fenestrata Balogh y Mahunka, 1974

DisTRIBUCión: Península Malaya.

-Magyaria filipina Corpuz-Raros, 1979

DISTRIBUCIÓN: Filipinas.

-Magyaria florida Mahunka, 1985

DISTRIBUCIÓN: Sudáfrica.

-Magyaria incisa Balogh y Mahunka, 1974

DisTRIBUCión: Península Malaya.

-Magyaria javensis Hammer, 1979

DISTRIBUCIÓN: Oriental.

-Magyaria luzonica Corpuz-Raros, 1991

DISTRIBUCIÓN: Filipinas.

-Magyaria mindanensis Corpuz-Raros, 1979

DisTRIBUCIÓN: Filipinas.

-Magyaria ornata Balogh, 1963

Distribución: Congo.

-Magyaria pulcherrima Balogh, 1970

DistriBUCIÓN: Ceilán [Sri Lanka].

-Magyaria reticulata (Balogh, 1958) (Scheloribates)

DISTRIBUCIÓN: Congo.

-Magyaria strinovichi Balogh, 1970

DisTRIBUCIÓN: Nueva Guinea.

-Magyaria triungulata Mahunka, 1988

DisTRIBUCIÓN: Borneo.

Paraxylobates Balogh y Mahunka, 1969 (1 sp.)

EsPeCIE TIPO: Paraxylobates imitans Balogh y Mahunka, 1969

DISTRIBUCIÓN: Neotropical.

-Paraxylobates imitans Balogh y Mahunka, 1969

DistriBuCión: Brasil.

Peloribates Berlese, 1908 (78 spp. y 2 sspp.)

EsPeCIE TIPO: Oribata peloptoides Berlese, 1888

DisTRIBUCIÓn: Cosmopolita (excepto Antártica).

Peloribates (Peloribates) Berlese, 1908 (76 spp. y 2 sspp.)

(=Capillozetes Balogh, 1943)

(=Euryparazetes Radford, 1950)

(=Indobates Pandit y Bhattacharya, 1999 "sin. nov.")

(=Parazetes Willmann, 1930, “nom. praeoc."por Sclater, 1879)

DisTRIBUCIÓN: Cosmopolita (excepto Antártica).

-Peloribates (P.) ababaeus Corpuz-Raros, 1979

DistribuCIÓN: Filipinas.

-Peloribates (P.) acutus Aoki, 1961

DisTRIBUCIÓN: Este de Paleártica oriental y Oriental (Tailandia).

-Peloribates (P.) acutus acutus Aoki, 1961

Distribución: Este de Paleártica oriental.

-Peloribates (P.) acutus curvisacculatus Aoki, 1967 DisTRIBUCIÓN: Tailandia.

-Peloribates (P.) alaskensis Hammer, 1955

DisTRIBUCIÓN: Alaska.

-Peloribates (P.) americanus Jacot, 1939 (P. europaeus a.)

DisTRIBUCIÓN: Neártica nororiental.

-Peloribates (P.) angulatus Bayartogtokh, 2000

Distribución: Mongolia.

-Peloribates (P.) asejugalis (Pandit y Bhattacharya, 1999) (Indobates)

DisTRIBUCIÓN: India (Bengala Occidental).

-Peloribates (P.) barbatus Aoki, 1977

DisTRIBUCIÓN: Japón.

-Peloribates (P.) buntotanus Corpuz-Raros, 1981

DistRIBUCIÓN: Filipinas.

-Peloribates (P.) canadensis Hammer, 1952

DisTRIBUCIÓN: Neártica septentrional (frecuente).

-Peloribates (P.) clavatus (Ewing, 1909) (Oribata)

DisTRIBUCIÓN: U.S.A. (Ilinois)

-Peloribates (P.) curtipilus Jacot, 1937

DistribuCión: U.S.A oriental.

-Peloribates (P.) decumanus (Berlese, 1908) (Protoribates (Scheloribates))

(=Peloribates anomalus Pérez-Íñigo y Baggio, 1980)

DisTRIBUCIÓN: Brasil 
-Peloribates (P.) dispersus Beck, 1964

DisTRIBUCIÓN: Perú.

-Peloribates (P.) europaeus Willmann, 1935

DisTRIBUCión: Holártica (Paleártica: frecuente, y U.S.A.: Nuevo Méjico).

-Peloribates (P.) floridensis Nevin, 1975

DistribuCión: U.S.A. (Florida).

-Peloribates (P.) formosus Nakatamari, 1985

Distribución: Japón.

-Peloribates (P.) fragilis Hammer, 1967

Distribución: Nueva Zelanda.

-Peloribates (P.) fuscosetosus Corpuz-Raros, 1981

DisTRIBUCIÓN: Filipinas.

-Peloribates (P.) genavensium Mahunka, 1983

Distribución: Méjico.

-Peloribates (P.) glaber Mihelcic, 1956

(=Peloribates alatus Mihelcic, 1957)

DistribuCión: Mediterránea.

-Peloribates (P.) grandis (Willmann, 1930) (Parazetes)

Distribución: Guatemala y Japón.

-Peloribates (P.) gressitti Balogh y Mahunka, 1967

DisTRIBUCión: Oriental (Vietnam y Filipinas).

-Peloribates (P.) guttatoides Hammer, 1979

DisTRIBUCIÓN: Oriental (Indonesia).

-Peloribates (P.) guttatus Hammer, 1979

DisTRIBUCIÓN: Java.

-Peloribates (P.) haramachiensis Aoki, 1999

DisTRIBUCIÓN: Japón.

-Peloribates (P.) hirsutus (Banks, 1895) (Oribata)

Distribución: U.S.A. (Nueva York).

-Peloribates (P.) hubbardi (Banks, 1904) (Galumna)

(=Peloribates histricinus Berlese, 1910)

(=Peloribates tillandsius Nevin, 1975)

DisTRIBUCIÓN: U.S.A. (Florida).

-Peloribates (P.) hungaricus (Balogh, 1943) (Capillozetes)

DistRibución: Tanzania.

-Peloribates (P.) hystrix (Berlese, 1916) (Protoribates (Scheloribates)) Distribución: U.S.A. (Misuri).

-Peloribates (P.) intermedius Mondal, 1984

Distribución: India (Bengala Occidental).

-Peloribates (P.) iowaensis Ewing, 1917

DisTRIBUCión: U.S.A. (Iowa).

-Peloribates (P.) juniperi (Ewing, 1913) (Oribata)

DisTRIBUCIÓN: Neártica septentrional.

-Peloribates (P.) kalboprodorsalis Corpuz-Raros, 1979

DisTRIBUCIÓN: Filipinas.

-Peloribates (P.) kaszabi Mahunka, 1988

DisTRIBUCIÓN: Vietnam.

-Peloribates (P.) levipunctatus Aoki, 1984

DisTRIBUCIÓN: Japón.

-Peloribates (P.) longicoma Hammer, 1958

DistRIBUCIÓN: Neotropical (Argentina y Brasil).

-Peloribates (P.) longipilosus Csiszár, 1962

Distribución: Sur de Europa.

-Peloribates (P.) longisetosus (Willmann, 1930) (Parazetes)

Distribución: Norte de Neotropical y Este de Paleártica oriental.

-Peloribates (P.) magkakaibaeus Corpuz-Raros, 1979

DisTRIBUCIÓN: Filipinas.

-Peloribates (P.) magnisetosus Hammer, 1967

DistribuCIÓN: Nueva Zelanda.

-Peloribates (P.) moderatus Aoki, 1984

DistriBuCIÓN: Japón.

-Peloribates (P.) muscicola Hammer, 1961

DisTRIBUCIÓN: Perú y Japón.

-Peloribates (P.) neonominatus nom. nov.

[=Peloribates hirsutus Mahunka, 1983, "nom. praeoc." por Banks, 1895 ("hom. sec.")]

DisTRIBUCIÓN: Tanzania.

-Peloribates (P.) nigeriensis Badejo, Woas y Beck, 2002

DisTRIBUCIÓN: Nigeria.
-Peloribates (P.) nishinoi Aoki, 1977

DisTRIBUCIÓN: Japón.

-Peloribates (P.) ominei Nakatamari, 1985

DisTRIBUCIÓN: Japón.

-Peloribates (P.) pakistanensis Hammer, 1977

Distribución: Sur de Paleártica oriental (Pakistán) y Norte de Oriental.

-Peloribates (P.) palawanus Corpuz-Raros, 1981

DisTRIBUCIÓN: Filipinas.

-Peloribates (P.) paraguayensis Balogh y Mahunka, 1981

DisTRIBUCIÓN: Paraguay.

-Peloribates (P.) peloptoides (Berlese, 1888) (Oribata)

(=Oribata banksi Ewing, 1909)

DisTRIBUCIÓN: Neotropical (Paraguay y Brasil) y U.S.A. (Ilinois).

-Peloribates (P.) perreti Mahunka, 1984

DistRIBUCIÓN: Neotropical (Paraguay y Uruguay).

-Peloribates (P.) pilipinus Corpuz-Raros, 1981

DisTRIBUCIÓN: Filipinas.

-Peloribates (P.) pilosus Hammer, 1952

DisTRIBUCIÓN: Holártica (menos frecuente en el Sur).

-Peloribates (P.) pinguisetus Mahunka, 1985

Distribución: Pequeñas Antillas y U.S.A. (Tejas).

-Peloribates (P.) plumosus P. Balogh, 1985

DisTRIBUCIÓN: Australia.

-Peloribates (P.) porosus Beck, 1964

Distribución: Perú.

-Peloribates $(P$.) praeoccupatus nom. nov.

[=Peloribates longisetosus Tseng, 1984, "nom. praeoc." por Willmann, 1930 (“hom. sec.")]

DisTRIBUCIÓN: Taiwan [Formosa].

-Peloribates (P.) pseudoporosus Balogh y Mahunka, 1967

DisTRIBUCIÓN: Vietnam.

-Peloribates (P.) rangiroaensis Hammer, 1972

DistribuCión: Este de Paleártica oriental y Polinesia.

-Peloribates (P.) rangiroaensis rangiroaensis Hammer, 1972 DisTRIBUCIÓN: Polinesia.

-Peloribates (P.) rangiroaensis asiaticus Aoki, 1974

DisTRIBUCIÓN: Este de Paleártica oriental.

-Peloribates (P.) ratubakensis Hammer, 1979

DisTRIBUCIÓN: Java.

-Peloribates (P.) repetitus nom. nov.

[=Peloribates minutus Aoki, 1967, “nom. praeoc." por Balogh, 1958 ("hom. prim.")]

DisTRIBUCIÓN: Tailandia.

-Peloribates (P.) reticulatus Balogh, 1970

Distribución: Nueva Guinea.

-Peloribates (P.) rigidicoma Hammer, 1958

DistribuCIÓN: Bolivia.

-Peloribates (P.) robustus Grishina, 1981

DistribuCión: Suroeste de Siberia.

-Peloribates (P.) ryukyuensis Aoki y Nakatamari, 1974

DistRIBUCIÓN: Japón.

-Peloribates (P.) serratoseta (Ewing, 1907) (Oribata)

DisTRIBUCIÓN: U.S.A. central.

-Peloribates (P.) sierramadrensis Corpuz-Raros, 1981

DisTRIBUCIÓN: Filipinas.

-Peloribates (P.) stellatus Balogh y Mahunka, 1967

DisTRIBUCIÓN: Vietnam.

-Peloribates (P.) tredecemsetosus Corpuz-Raros, 1981

DistribuCIÓN: Filipinas.

-Peloribates (P.) tripuraensis Sanyal y Saha, 1996

DistRIBUCIÓN: India (Tripura).

-Peloribates (P.) tunisiensis Mahunka, 1980

DisTRIBUCIÓN: Mediterránea occidental.

-Peloribates (P.) turgidus Wen y Zhao, 1994

Distribución: China suroriental.

-Peloribates (P.) uracasensis Aoki, 1984

DistribuCión: Micronesia (I. Marianas).

-Peloribates (P.) varisculptus Corpuz-Raros, 1981

DisTRIBUCIÓN: Filipinas. 
-Peloribates (P.) yezoensis Fujikawa, 1986

DisTRIBUCIÓN: Japón.

-Peloribates (P.) yunnanensis Wen, 1985

DisTRIBUCIÓN: China suroriental.

Peloribates (Aokibates) Mahunka, 1988 (1 sp.) EsPECIE TIPO: Aokibates yoshii Mahunka, 1988 DisTRIBUCIÓN: Oriental.

-Peloribates (Aokibates) yoshii (Mahunka, 1988) (A.) DISTRIBUCIÓN: Borneo.

Peloribates (Tentaculozetes) Balogh, 1970 (1 sp.) EsPeCIE TIPO: Tentaculozetes loksai Balogh, 1970 DisTRIBUCIÓN: Australiana.

-Peloribates (Tentaculozetes) loksai (Balogh, 1970) (T.) DisTRIBUCIÓN: Nueva Guinea.

Pilobatella Balogh y Mahunka, 1967 (7 spp.)

EsPecie TiPo: Pilobatella punctulata Balogh y Mahunka, 1967

DISTRIBUCIÓN: Pantropical (excepto Australiana).

-Pilobatella berlesei Bhattacharya y Banerjee, 1979

DistriBución: India (Bengala Occidental).

-Pilobatella maurensis Scull, 1985

Distribución: Cuba.

-Pilobatella monstruosa Mahunka, 1986

DisTRIBUCIÓN: Kenia.

-Pilobatella pseudovermiseta Corpuz-Raros, 1979

DisTRIBUCIÓN: Filipinas.

-Pilobatella punctulata Balogh y Mahunka, 1967

DisTRIBUCIÓN: Congo.

-Pilobatella schauenbergi Mahunka, 1977

DisTRIBUCIÓN: Oriental.

-Pilobatella xena Mahunka, 1977

DISTRIBUCIÓN: Kenia.

Pilobates Balogh, 1960 (3 spp.)

EsPeCIE TIPO: Protoribates pilosellus Balogh, 1958

DistriBuCión: Etiópica y Paleártica meridional.

Pilobates (Pilobates) Balogh, 1960 (2 spp.)

DisTRIBUCión: Etiópica y Paleártica meridional

-Pilobates (P.) carpetanus Pérez-Iñigo, 1969

(?=Protoribates elongatus Mihelcic, 1956 "sp. inq.")

(?=Protoribates longior Mihelcic, 1957 "sp. inq.")

DisTRIBUCIÓN: Mediterránea.

-Pilobates (P.) pilosellus (Balogh, 1958) (Protoribates)

DisTRIBUCIÓN: Angola y Cáucaso.

Pilobates (Italobates) Mahunka, 1994 (1 sp.)

EsPeCIE TIPO: Protoribates (Scheloribates) incisura Berlese, 1916

DISTRIBUCIÓN: Etiópica.

-Pilobates (Italobates) incisura (Berlese, 1916) (Protoribates (Sche-

loribates))

DISTRIBUCIÓN: Somalia.

Setincabates Lee, 1993 (1 sp.)

ESPECIE TIPO: Setincabates hypersetosus Lee, 1993

DISTRIBUCIÓN: Australiana.

-Setincabates hypersetosus Lee, 1993

DisTRIBUCIÓN: Australia.

Trachyoribates Berlese, 1908 (31 spp. y 2 sspp.)

EsPeCIE TIPO: Oribata ampulla Berlese, 1905

DISTRIBUCIÓN: Pantropical y subtropical.

Trachyoribates (Trachyoribates) Berlese, 1908 (2 spp.)

DISTRIBUCIÓN: Australianooriental.

-Trachyoribates (T.) ampulla (Berlese, 1905) (Oribata)

DisTRIBUCIÓN: Oriental (Indonesia) y Nueva Guinea.
-Trachyoribates (T.) chinensis Fukuyama y Aoki, 2000

DISTRIBUCIÓN: China suroriental.

Trachyoribates (Rostrozetes) Sellnick, 1925 (29 spp. y 2 sspp.)

(=Carabozetes Mihelcic, 1957)

(=Zaherizetes Yousef y Nasr, 1976 "sin. nov.")

ESPECIE TIPO: (Rostrozetes foveolatus Sellnick, 1925) =Trachyoribates

ovulum Berlese, 1908

DISTRIBUCIÓN: Pantropical y subtropical.

-Trachyoribates (Rostrozetes) aegypticus (Yousef y Nasr, 1976)

(Zaherizetes)

DISTRIBUCIÓN: Egipto.

-Trachyoribates (Rostrozetes) africanus (Yousef y Nasr, 1976)

(Zaherizetes)

DisTRIBUCIÓN: Egipto.

-Trachyoribates (Rostrozetes) angulifer (Balogh y Mahunka, 1979) (R.) DISTRIBUCIÓN: Cuba.

-Trachyoribates (Rostrozetes) bothulifer (Balogh y Mahunka, 1979) (R.) DisTRIBUCIÓN: Cuba.

-Trachyoribates (Rostrozetes) carinatus (Beck, 1965) (R.)

DisTRIBUCIÓN: Neotropical.

-Trachyoribates (Rostrozetes) cristatus (Balogh y Mahunka, 1969) (R.)

DisTRIBUCIÓN: Neotropical (Brasil y Venezuela).

-Trachyoribates (Rostrozetes) cubanus (Balogh y Mahunka, 1974) (R.) DisTRIBUCIÓN: Cuba.

-Trachyoribates (Rostrozetes) dimorphichaites (Higgins, 1996) (R.) DistribuCión: Neotropical: Guyana [Guayana Británica] y Venezuela.

-Trachyoribates (Rostrozetes) dispar (Balogh, 1958) (Peloribates) DisTRIBUCión: Angola.

-Trachyoribates (Rostrozetes) florens (Balogh, 1970) (R.)

DistriBución: Ceilán [Sri Lanka].

-Trachyoribates (Rostrozetes) geminosetosus (Balogh y Mahunka, 1978) (R.)

DISTRIBUCIÓN: Brasil.

-Trachyoribates (Rostrozetes) glaber (Beck, 1965) (R.)

DistRIBUCIÓN: Perú.

-Trachyoribates (Rostrozetes) heterotrichus (Pérez-Íñigo y Baggio, $1991(R$.

DisTRIBUCIÓN: Brasil.

-Trachyoribates (Rostrozetes) inornatus (Pérez-Íñigo y Baggio, 1991) (R.)

DisTRIBUCIÓN: Brasil.

-Trachyoribates (Rostrozetes) irregularis (Balogh y Mahunka, 1969 (R.)

DisTRIBUCIÓn: Bolivia y Vietnam.

-Trachyoribates (Rostrozetes) komodensis (Mahunka, 1977) (R.)

DisTRIBUCIÓN: Indonesia (Komodo).

-Trachyoribates (Rostrozetes) mammillatus (Warburton, 1912) (Ori-

bata)

DISTRIBUCIÓN: I. Seychelles.

-Trachyoribates (Rostrozetes) monstruosus (Balogh y Mahunka, 1969 (R.)

DistRIBUCIÓN: Neotropical (Brasil y Venezuela).

-Trachyoribates (Rostrozetes) nagaii (Mahunka, 1987) (R.)

DISTRIBUCIÓN: Borneo.

-Trachyoribates (Rostrozetes) ovulum Berlese, 1908 (T.)

DisTRIBUCIÓN: Pantropical y subtropical.

-Trachyoribates (Rostrozetes) ovulum ovulum Berlese, 1908

(=Peloribates areolatus Balogh, 1958)

(=Trachyoribates dorsalis Balogh, 1958)

(=Rostrozetes flavus Woodring, 1965)

(=Rostrozetes foveolatus Sellnick, 1925)

(=Rostrozetes geneuxi Mahunka, 1985)

(=Trachyoribates nodosus Hammer, 1958)

(=Rostrozetes pulcherrimus Balogh, 1961)

(=Peloribates punctulatus Balogh, 1958)

(=Rostrozetes punctulifer Balogh y Mahunka, 1979)

(=Rostrozetes trimorphus Balogh y Mahunka, 1979)

DisTRIBUCIÓN: Pantropical (frecuente) y subtropical. 
-Trachyoribates (Rostrozetes) ovulum appalachicola (Jacot, 1938)

(R. foveolatus a.)

Distribución: U.S.A. (Carolina del Norte).

-Trachyoribates (Rostrozetes) ovulum poensis (Mihelcic, 1957)

(Carabozetes p.)

(=Rostrozetes foveolatus nebulosus Beck, 1965)

DisTRIBUCIÓN: Guinea Ecuatorial: I. Bioco [Fernando Poo] y Perú.

-Trachyoribates (Rostrozetes) perezinigoi (P. Balogh, 1995) (R.)

DisTRIBUCIÓN: Brasil.

-Trachyoribates (Rostrozetes) pinguis (Balogh y Mahunka, 1978) (R.)

DisTRIBUCIÓN: Brasil.

-Trachyoribates (Rostrozetes) polygonatus (Balogh y Mahunka, 1969) (R.)

DisTRIBUCIÓN: Brasil.

-Trachyoribates (Rostrozetes) pseudofurcatus (Balogh y Mahunka, 1968) $(R$.)

DistRIBUCión: Neotropical (Argentina y Paraguay).

-Trachyoribates (Rostrozetes) punctatus (Karppinen, 1966) (R.)

DistriBución: Guinea e India (Bengala Occidental).

-Trachyoribates (Rostrozetes) rimachensis (Beck, 1965) (R.)

DisTRIBUCIÓN: Neotropical (Perú y Brasil).

-Trachyoribates (Rostrozetes) schalleri (Beck, 1965) (R.)

DisTRIBUCIÓN: Neotropical.

-Trachyoribates (Rostrozetes) sexclavatus (Sellnick, 1925) (R.)

DisTRIBUCIÓN: Brasil.

-Trachyoribates (Rostrozetes) shibai (Aoki, 1976) (R.)

DisTRIBUCIÓN: Península Malaya.

Nasobatidae Balogh, 1972 (1 gen. y 2 spp.)

DISTRIBUCIÓN: Neotropical.

Nasobates Woolley, 1966 (2 spp.)

EsPECIE TIPO: Nasobates spinosus Woolley, 1966

DisTRIBUCIÓN: Neotropical.

-Nasobates mirabilis Balogh y Mahunka, 1969

DisTRIBUCIÓN: Neotropical.

-Nasobates spinosus Woolley, 1966

(=Nasobates paraguayensis Mahunka, 1985)

DisTRIBUCIÓN: Neotropical: Honduras y Paraguay.

Tubulozetidae P. Balogh, 1989 (1 gen. y 1 sp.) DiSTRIBUCIÓN: Neotropical.

Tubulozetes P. Balogh, 1989 (1 sp.)

EsPecie TIPO: Tubulozetes rostratus P. Balogh, 1989

DISTRIBUCIÓN: Neotropical.

-Tubulozetes rostratus P. Balogh, 1989

DISTRIBUCIÓN: Ecuador.

\section{Galumnoidea Jacot, 1925}

Parakalummidae Grandjean, 1936 (3 gen., 3 subg., 37 spp. y 1 ssp.) DisTRIBUCIÓN: Cosmopolita.

Neoribates Berlese, 1914 (30 spp. y 1 ssp.)

ESPECIE TIPO: Oribata roubali Berlese, 1910

DisTRIBUCIÓn: Cosmopolita (excepto Antártica).

Neoribates (Neoribates) Berlese, 1914 (17 spp.)

Distribución: Cosmopolita (excepto Etiópica y Antártica).

-Neoribates (N.) aurantiacus (Oudemans, 1914) (Galumna)

DistRIBUCIÓN: Holártica (menos frecuente en el Sur) y Vietnam.

-Neoribates (N.) barbatus Hammer, 1968

DisTRIBUCIÓN: Nueva Zelanda.

-Neoribates (N.) erectus (Balogh y Mahunka, 1969) (Protokalumma) DisTRIBUCIÓN: Brasil.

-Neoribates (N.) formaminiferus Sellnick, 1923

DistribuCIÓN: Brasil.

-Neoribates (N.) fulvus Sellnick, 1923 (N. foraminiferus $f$.)

DisTRIBUCIÓN: Brasil.
-Neoribates (N.) gracilis Travé, 1972

Distribución: Sur de Europa.

-Neoribates (N.) macrosacculatus Aoki, 1966

DisTRIBUCIÓN: Japón.

-Neoribates (N.) neglectus Willmann, 1953

DISTRIBUCIÓN: Europa central.

-Neoribates (N.) oceanicus (Oudemans, 1915) (Galumna)

DistRiBuCión: Ceilán [Sri Lanka].

-Neoribates (N.) pallidus Aoki, 1988

DistRIBUCIÓN: Japón.

-Neoribates (N.) parvisetigera (Aoki, 1965) (Protokalumma)

Distribución: Este de Paleártica oriental (frecuente).

-Neoribates (N.) quadrisetosus (Ewing, 1917) (Oribata)

DisTRIBUCIÓN: U.S.A. nororiental (frecuente).

-Neoribates (N.) rimosus Suzuki, 1978

DisTRIBUCIÓN: Japón.

-Neoribates (N.) rotundus Aoki, 1982

DisTRIBUCIÓN: Japón y Taiwan [Formosa].

-Neoribates (N.) roubali (Berlese, 1910) (Oribata)

DistriBuCión: Paleártica.

-Neoribates (N.) setiger Balogh y Mahunka, 1978

DistRIBUCIÓN: Australia.

-Neoribates (N.) tuberculatus Willmann, 1956

DisTRIBUCIÓN: "Checoslovaquia".

Neoribates (Parakalumma) Jacot, 1929 (3 spp. y 1 ssp.)

EsPeCIE TIPO: Neoribates lydia Jacot, 1923

DisTRIBUCIÓN: Holártica y Australiana.

-Neoribates (Parakalumma) headlandi (Stary, 1997) (P.)

DisTRIBUCIÓN: Australia.

-Neoribates (Parakalumma) lydia Jacot, 1923 (N.)

Distribución: Este de Paleártica oriental.

-Neoribates (Parakalumma) robustus (Banks, 1895) (Oribata)

(=Oribata centropterus Berlese, 1908)

DisTRIBUCióN: Holártica (U.S.A. oriental y Japón).

-Neoribates (Parakalumma) robustus robustus (Banks, 1895)

DisTRIBUCIÓN: Holártica (U.S.A. oriental: frecuente, y Japón).

-Neoribates (Parakalumma) robustus floridanus (Jacot, 1935) (P.) DisTRIBUCIÓN: U.S.A. (Florida).

Neoribates (Protokalumma) Jacot, 1929 (10 spp.)

EsPeCIE TIPO: Oribata depressa Banks, 1895

DisTRIBUCIÓN: Neártica y Australianooriental.

- Neoribates (Protokalumma) corticis (Ewing, 1913) (Oribata) DistribuCión: U.S.A. (Michigan).

-Neoribates (Protokalumma) depressa (Banks, 1895) (Oribata)

DisTRIBUCIÓN: Neártica oriental (frecuente).

-Neoribates (Protokalumma) flagellum (Balogh, 1970) (P.)

DisTRIBUCIÓN: Nueva Guinea.

-Neoribates (Protokalumma) insignificans (Mahunka, 1995) (Parakalumma)

DISTRIBUCIÓN: Borneo.

-Neoribates (Protokalumma) jacoti (Balogh y Mahunka, 1967) (P.)

DisTRIBUCIÓN: Vietnam.

-Neoribates (Protokalumma) neonominatus nom. nov.

[=Protokalumma jacoti Norton y Kethley, 1989, "nom. praeoc." por Balogh y Mahunka, 1967 (“hom. prim.")]

DisTRIBUCión: U.S.A. (Misuri).

-Neoribates (Protokalumma) pterotus (Banks, 1910) (Galumna)

(=Oribata (Neoribates) fissuratus Berlese, 1914)

DisTRIBUCIÓN: U.S.A. centrooriental.

-Neoribates (Protokalumma) punctulatus (Balogh, 1970) (P.)

DisTRIBUCIÓN: Nueva Guinea.

-Neoribates (Protokalumma) salicis (Ewing, 1913) (Oribata)

DistribuCión: U.S.A. (Michigan).

-Neoribates (Protokalumma) szabadosi (Balogh, 1970) (P.)

DisTRIBUCIÓN: Nueva Guinea. 
Perezinigokalumma gen. nov. (4 spp.)

EsPecie TiPo: Protokalumma afrum Pérez-Íñigo, 1969

DisTRIBUCIÓN: Tropical (Etiópica y Neotropical).

-Perezinigokalumma afrum (Pérez-Íñigo, 1969) (Protokalumma)

DistRIBUCIÓN: Guinea Ecuatorial: I. Pagalu [Annobón].

-Perezinigokalumma foveolata (Balogh y Mahunka, 1969) (Parakalumma)

DisTRIBUCIÓN: Neotropical (Brasil y Surinám).

-Perezinigokalumma munizi (Balogh y Mahunka, 1974) (Parakalumma) DisTRIBUCIÓN: Cuba.

-Perezinigokalumma piton (Mahunka, 1998) (Parakalumma) DistriBución: Pequeñas Antillas.

Sandenia Oudemans, 1917 (3 spp.)

EsPECIE TIPO: Galumna georgiae Oudemans, 1914

DISTRIBUCIÓN: Antártica y Oriental.

Sandenia (Sandenia) Oudemans, 1917 (1 sp.)

DisTRIBUCIÓN: Antártica.

-Sandenia (S.) georgiae (Oudemans, 1914) (Galumna)

DisTRIBUCIÓN: Antártica (I. Georgia del Sur).

Sandenia (Porokalumma) Wallwork, 1966 (2 spp.)

(=Neokalumma Tseng, 1984 "sin. nov.")

EsPECIE TIPO: Sandenia rotunda Wallwork, 1963

DISTRIBUCIÓN: Antártica y Oriental.

-Sandenia (Porokalumma) laiae (Tseng, 1984) (Neokalumma)

Distribución: Taiwan [Formosa].

-Sandenia (Porokalumma) rotunda Wallwork, 1966 (S.)

DisTRIBUCIÓN: Antártica.

Galumnidae Jacot, 1925 (33 gen., 7 subg., 409 spp. y 30 sspp.) DisTRIBUCIÓN: Cosmopolita.

Acrogalumna Grandjean, 1956 (9 spp. y 1 ssp.)

EsPeCIE TIPO: Oribata elimata longipluma Berlese, 1904

DistriBuCIÓN: Cosmopolita (excepto Antártica)

-Acrogalumna abrupta Hammer, 1972

DisTRIBUCIÓN: Polinesia (Tahití).

-Acrogalumna bipartita Aoki y Hu, 1993

DisTRIBUCIÓN: China suroriental.

-Acrogalumna cubana (Balogh y Mahunka, 1979) (Allogalumna)

Distribución: Cuba.

-Acrogalumna longipluma (Berlese, 1904) (Oribata elimata l.)

DistRIBUCIÓN: Semicosmopolita (Holártica, Etiópica: Sudáfrica e

I. Santa Helena, India: Kerala, y Nueva Zelanda).

-Acrogalumna longipluma longipluma (Berlese, 1904)

(=Galumna filata Oudemans, 1913)

(=Galumna latipluma Mihelcic, 1952)

(=Galumna longiporus Mihelcic, 1952)

(=Oribata setiformis Hall, 1911)

Distribución: Semicosmopolita (Holártica: frecuente en

Europa, Etiópica: Sudáfrica e I. Santa Helena, India: Kerala, y Nueva Zelada)

-Acrogalumna longipluma confluentina (Willmann, 1953) (Allogalumna)

DisTRIBUCIÓN: Austria

-Acrogalumna machadoi Balogh, 1960

DisTRIBUCIÓN: Etiópica (Congo y Sudáfrica).

-Acrogalumna monttensis Hammer, 1972

DISTRIBUCIÓN: Chile.

-Acrogalumna pacifica (Trägardh, 1931) (Galumna)

Distribución: Chile (I. Juan Fernández).

-Acrogalumna pectinata (Jacot, 1937) (Zetes)

Distribución: U.S.A. (Carolina del Norte).

-Acrogalumna shogranensis Hammer, 1977

DisTRIBUCIÓN: Pakistán.
Aegyptogalumna Al-Assiuty, Abdel-Hamid, Seif y El-Deeb, 1985 (1 sp.) EsPECIE TIPO: Aegyptogalumna mastigophora Al-Assiuty, Abdel-Hamid, Seif y El-Deeb, 1985

DisTRIBUCIÓN: Paleártica.

-Aegyptogalumna mastigophora Al-Assiuty, Abdel-Hamid, Seif y El-Deeb, 1985

DISTRIBUCIÓN: Egipto.

Allogalumna Grandjean, 1936 (34 spp.)

=Ctenogalumna Balogh, 1961 "sin. nov.")

EsPeCIE TIPO: Galumna alamellae Jacot, 1935

DISTRIBUCIÓN: Cosmopolita (excepto Antártica).

-Allogalumna alamellae (Jacot, 1935) (Galumna)

(=Allogalumna alata transversa Mihelcic, 1957)

=Galumna (Pergalumna) diversiareata Mihelcic, 1956)

(=Allogalumna neerlandica Hammer, 1952)

DISTRIBUCIÓN: Europa centromeridional.

-Allogalumna alpha Pérez-Íñigo y Baggio, 1994

DisTRIBUCIÓN: Brasil.

-Allogalumna borhidii Balogh y Mahunka, 1979

DISTRIBUCIÓN: Norte de Neotropical.

-Allogalumna confluens Balogh, 1960

DisTRIBUCIÓN: Congo.

-Allogalumna costata Mahunka, 1996

DISTRIBUCIÓN: Madagascar.

-Allogalumna dilatata J. y P. Balogh, 1983

DisTRIBUCIÓn: Australia.

-Allogalumna exigua Popp, 1960

DisTRIBUCIÓN: Egipto.

-Allogalumna filiger Hammer, 1962

DisTRIBUCIÓN: Neotropical (Chile y Panamá).

-Allogalumna gedaii Mahunka, 1995

DisTRIBUCIÓN: Tailandia.

-Allogalumna hungarica Willmann,1938

DisTRIBUCIÓN: Hungría.

-Allogalumna hydrophila Hammer, 1962

DistriBuCión: Chile.

-Allogalumna incompleta Mahunka, 1988

DISTRIBUCIÓN: Borneo.

-Allogalumna insolita Mahunka, 1996

DISTRIBUCIÓN: Madagascar.

-Allogalumna integer (Berlese, 1904) (Oribata alata i.)

DISTRIBUCIÓN: Suroeste de Europa.

-Allogalumna italica (Jacot, 1935) (Galumna)

DistRIBUCión: Sur de Europa.

-Allogalumna leleupi Balogh, 1962

DISTRIBUCIÓN: Tanzania.

-Allogalumna madagascarensis (Balogh, 1961) (Ctenogalumna)

DISTRIBUCIÓN: Madagascar.

-Allogalumna margaritifera Balogh, 1960

Distribución: Congo.

-Allogalumna microporosa Mahunka, 1979

DisTRIBUCIÓN: Norte de Neotropical.

-Allogalumna moresonensis (Engelbrecht, 1972) (Ctenogalumna)

DisTRIBUCIÓN: Sudáfrica.

-Allogalumna multesima Grandjean, 1957

DisTRIBUCIÓN: Norte de Neotropical.

-Allogalumna novazealandica Hammer, 1968

DistRIBUCIÓN: Nueva Zelanda.

-Allogalumna parva (Berlese, 1916) (Oribata)

DISTRIBUCIÓN: Italia.

-Allogalumna pellucida Wallwork, 1965

DisTRIBUCIÓN: Chad.

-Allogalumna plowmanae J. y P. Balogh, 1983

Distribución: Australia.

-Allogalumna pocsi Mahunka, 1996

DISTRIBUCIÓN: Madagascar.

-Allogalumna quadrimaculata (Mahunka, 1988) (Pergalumna)

DISTRIBUCIÓN: Borneo. 
-Allogalumna rotundiceps Aoki, 1996

DisTRIBUCIÓN: Japón.

-Allogalumna scripta (Balogh y Mahunka, 1966) (Galumna)

DisTRIBUCIÓN: Congo.

-Allogalumna sinornata Mahunka, 1992

DisTRIBUCión: Senegal.

-Allogalumna superporosa Mahunka 1996

DisTRIBUCIÓN: Madagascar.

-Allogalumna triangulata Mahunka, 1978

DisTRIBUCIÓN: Malgache (I. Mauricio).

-Allogalumna upoluensis Hammer, 1973

Distribución: Polinesia (I. Samoa).

-Allogalumna vojnitsi Mahunka, 1993

DistriBución: Tanzania.

Carinogalumna Engelbrecht, 1973 (3 spp.)

(=Paracarinogalumna Mahunka, 1998 "sin. nov.")

(=Pseudogalumna Pérez-Ínigo y Baggio, 1994 "sin. nov.")

EsPECIE TIPO: Carinogalumna montana Engelbrecht, 1973

DisTRIBUCIÓN: Tropical (Etiópica y Neotropical).

-Carinogalumna clericata (Berlese, 1914) (Oribata)

DisTRIBUCIÓN: Neotropical.

-Carinogalumna genavensium (Mahunka, 1998) (Paracarinogalumna)

DisTRIBUCIÓN: Pequeñas Antillas.

-Carinogalumna montana Engelbrecht, 1973

DisTRIBUCIÓN: Sudáfrica.

Centroribates Berlese, 1914 (1 sp.)

EsPeCIE TIPO: Oribata mucronata G. y R. Canestrini, 1882

DisTRIBUCIÓN: Paleártica.

-Centroribates mucronatus (G. y R. Canestrini, 1882) (Oribata)

DistRIBUCIÓN: Suroeste de Europa.

Cryptogalumna Grandjean, 1957 (2 spp.)

ESPECIE TIPO: Cryptogalumna cryptodonta Grandjean, 1957

DISTRIBUCIÓN: Paleártica meridional y Oriental.

-Cryptogalumna cryptodonta Grandjean, 1957

DisTRIBUCión: Mediterránea occidental.

-Cryptogalumna grandjeani Balakrishnan y Haq, 1985

Distribución: India (Kerala).

Dicatozetes Grandjean, 1956 (2 spp.)

EsPECIE TIPO: Centroribates uropygium Grandjean, 1928

DISTRIBUCIÓN: Paleártica meridional.

-Dicatozetes numidicus Bernini, 1984

DisTRIBUCIÓN: Argelia.

-Dicatozetes uropygium (Grandjean, 1928) (Centroribates)

DisTRIBUCIÓN: Mediterránea occidental.

Didymonycha Mahunka, 1984 (1 sp.)

EsPECIE TIPO: Didymonycha hesperis Mahunka, 1984

DISTRIBUCIÓN: Etiópica.

-Didymonycha hesperis Mahunka, 1984

DisTRIBUCión: Tanzania.

Dimidiogalumna Engelbrecht, 1972 (3 spp.)

EsPECIE TIPO: Dimidiogalumna villiersensis Engelbrecht, 1972

DISTRIBUCIÓN: Etiópica y Paleártica meridional.

-Dimidiogalumna azumai Aoki, 1996

DisTRIBUCión: Japón.

-Dimidiogalumna comoroensis Mahunka, 1994

DistriBución: Malgache (I. Comores).

-Dimidiogalumna villiersensis Engelbrecht, 1972

DisTRIBUCIÓN: Sudáfrica.

Disparagalumna Hammer, 1973 (1 sp.)

EsPeCIE TIPO: Disparagalumna tongaensis Hammer, 1973

DisTRIBUCIÓN: Australiana.

-Disparagalumna tongaensis Hammer, 1973

Distribución: Polinesia (I. Tonga).
Flagellozetes Balogh, 1970 (1 sp. y 1 ssp.)

EsPeCIE TiPO: Flagellozetes porosus Balogh, 1970

DisTRIBUCIÓN: Oriental.

-Flagellozetes porosus Balogh, 1970

DistriBución: Oriental: Ceilán [Sri Lanka]e India (Kerala).

-Flagellozetes porosus porosus Balogh, 1970 DistriBUCión: Ceilán [Sri Lanka].

-Flagellozetes porosus indicus Balakrishnan y Haq, 1985 DistriBución: India (Kerala).

Galumna Heyden, 1826 (164 spp. y 9 sspp.)

EsPECIE TIPO: Notaspis alatus Hermann, 1804

DISTRIBUCIÓN: Cosmopolita.

Galumna (Galumna) Heyden, 1826 (151 spp. y 9 sspp.)

(=Holokalumma Jacot, 1929)

(=Holozetes Jacot, 1929)

(=Zetes Koch, 1836)

DISTRIBUCIÓN: Cosmopolita.

-Galumna (G.) aba Mahunka, 1989

DISTRIBUCIÓN: Vietnam.

-Galumna (G.) aegyptica Abdel-Hamid, Al-Assiuty y Trrad, 1982

DistribuCión: Egipto.

-Galumna (G.) agueroi P. Balogh, 1997

Distribución: Costa Rica.

-Galumna (G.) alata (Hermann, 1804) (Notaspis)

DisTRIBUCIÓN: Semicosmopolita (Paleártica occidental, U.S.A., I.

Seychelles, Hawai, Argentina y Antártica: I. Georgia del Sur).

-Galumna (G.) alata alata (Hermann, 1804)

$(=$ Galumna coleoptratrum occidentale Jacot, 1929)

(=Oribata octopunctata Ewing, 1909)

(=Zetes satellitius Koch, 1841)

DisTRIBUCIÓN: Semicosmopolita (Paleártica occidental: menos frecuente en el Norte, U.S.A.: frecuente, I. Seychelles, Hawai, Argentina y Antártica: I. Georgia del Sur).

-Galumna (G.) alata multiiterata nom. nov.

[=Galumna maxima Willmann, 1939, "nom. praeoc.”por Berlese, 1916 ("hom. sec.")]

DisTRIBUCIÓN: Macaronésica (Madeira)

-Galumna (G.) ambigua Wallwork, 1977

DisTRIBUCión: I. Santa Helena.

-Galumna (G.) angularis Jeleva, Scull y Cruz, 1984

(=Galumna hamifer Mahunka, 1985)

DisTRIBUCIÓN: Neotropical (Centroamérica: frecuente, y Brasil).

-Galumna (G.) ankaratra Mahunka, 1997

DISTRIBUCIÓN: Madagascar.

-Galumna (G.) antalata Banks, 1916

DisTRIBUCIÓN: Tasmania.

-Galumna (G.) appressala (Ewing, 1910) (Oribata) "sp. inq."

DisTRIBUCIÓN: India (Kerala).

-Galumna (G.) arabica Bayoumi y Al-Khalifa, 1984

DisTRIBUCión: Arbia Saudita.

-Galumna (G.) araujoi Pérez-Íñigo y Baggio, 1994

DisTRIBUCIÓN: Brasil.

-Galumna (G.) armatifera Mahunka, 1996

DisTRIBUCIÓN: Madagascar.

-Galumna (G.) arrugata Jeleva, Scull y Cruz, 1984

DisTRIBUCIÓN: Cuba.

-Galumna (G.) atomaria (Berlese, 1914) (Oribata)

DistriBUCIÓN: Java.

-Galumna (G.) australis (Berlese, 1914) (Oribata)

DistriBUCIÓN: Neotropical y Australiana: Hawai.

-Galumna (G.) australis australis (Berlese, 1914) DISTRIBUCIÓN: Neotropical.

-Galumna (G.) australis pembertoni (Jacot, 1934) (Zetes) DisTRIBUCIÓN: Hawai.

-Galumna (G.) azoreana Pérez-Íñigo, 1992

Distribución: I. Azores.

-Galumna (G.) baloghi Wallwork, 1965

DistriBuCIÓN: Chad. 
-Galumna (G.) banksi Jacot, 1929

DisTRIBUCIÓN: U.S.A. nororiental.

-Galumna (G.) barnardi (Jacot, 1940) (Holozetes)

DisTRIBUCIÓN: Sudáfrica.

-Galumna (G.) basilewskyi Balogh, 1962

DisTRIBUCIÓN: Tanzania.

-Galumna (G.) berlesei Oudemans, 1919

DISTRIBUCIÓN: Europa centromeridional

-Galumna (G.) betzaida Mahunka, 1992

DISTRIBUCIÓN: Israel.

-Galumna (G.) bimorpha Mahunka, 1987

DisTRIBUCIÓN: Hungría.

-Galumna (G.) bradleyi (Jacot, 1935) (Zetes)

DisTRIBUCIÓN: U.S.A. (Florida).

-Galumna (G.) brasiliensis Sellnick, 1923

DisTRIBUCIÓN: Brasil.

-Galumna (G.) californica (Hall, 1911) (Oribata alata c.)

DisTRIBUCIÓN: U.S.A. (California).

-Galumna (G.) calva Stary, 1997

DisTRIBUCIÓN: Australia.

-Galumna (G.) capensis Engelbrecht, 1969

DisTRIBUCIÓN: Sudáfrica.

-Galumna (G.) capensis capensis Engelbrecht, 1969 DisTRIBUCIÓN: Sudáfrica.

-Galumna (G.) capensis dissimilis Engelbrecht, 1969 DisTRIBUCIÓN: Sudáfrica.

-Galumna (G.) castanea (Canestrini, 1898) (Oribata) "sp. inq."

DisTRIBUCIÓN: Nueva Guinea.

-Galumna (G.) changchunensis Wen, 1987

DisTRIBUCIÓN: Noreste de China.

-Galumna (G.) chujoi Aoki, 1966

DisTRIBUCIÓN: Este de Paleártica oriental.

-Galumna (G.) circularis Hammer, 1958

DisTRIBUCIÓN: Bolivia.

-Galumna (G.) cirripilis (Canestrini, 1898) (Oribata) "sp. inq."

DISTRIBUCIÓN: Nueva Guinea.

-Galumna (G.) coloradensis (Jacot, 1929) (Holokalumma)

DiSTRIBUCIÓN: U.S.A. centromeridional.

-Galumna (G.) colossus Oudemans, 1915

Distribución: Ceilán [Sri Lanka].

-Galumna (G.) comparabilis Engelbrecht, 1972

DisTRIBUCIÓN: Sudáfrica.

-Galumna (G.) coreana Choi, 1986

DISTRIBUCIÓN: Corea.

-Galumna (G.) coronata Mahunka, 1992

DisTRIBUCIÓN: Senegal.

-Galumna (G.) costata Mahunka, 1978

Distribución: Malgache (I. Mauricio).

-Galumna (G.) crenata Deb y Raychaudhuri, 1975

DisTRIBUCIÓN: India (Bengala Occidental).

- Galumna (G.) cubana Jeleva, Scull y Cruz, 1984

DisTRIBUCIÓN: Cuba.

-Galumna (G.) cuneata Aoki, 1961

Distribución: Este de Paleártica oriental.

-Galumna (G.) delectum Pérez-Íñigo y Baggio, 1996

DISTRIBUCIÓN: Brasil.

-Galumna (G.) difficilis (Berlese, 1916) (Oribata) "sp. inq."

DisTRIBUCIÓN: Somalia.

-Galumna (G.) dimidiata Engelbrecht, 1969

DisTRIBUCIÓN: Sudáfrica.

-Galumna (G.) dimorpha Krivolutskaja, 1952

DistRIBUCIÓN: Paleártica centromeridional (excepto Este).

-Galumna (G.) discifera Balogh, 1960

(=Galumna engelbrechti J. y P. Balogh, 2002, "nom. praeoc." por

Mahunka, 1997)

DisTRIBUCIÓN: Etiópica (Congo y Sudáfrica)

-Galumna (G.) dispar Willmann, 1931

Distribución: Java.

-Galumna (G.) divergens Mahunka, 1995

DisTRIBUCIÓN: Borneo.
-Galumna (G.) dorsalis (Koch, 1835) (Zetes) "sp. inq."

DisTRIBUCIÓN: Alemania.

-Galumna (G.) dubia Mihelcic, 1953

DisTRIBUCIÓN: Austria.

-Galumna (G.) egregia Sellnick, 1923

DistribuCión: Neotropical (Brasil y Panamá).

-Galumna (G.) elegantula (Jacot, 1935) (Zetes)

DisTRIBUCIÓN: U.S.A. (Florida).

-Galumna (G.) elimata (Koch, 1841) (Oribata)

Distribución: Semicosmopolita (Paleártica, U.S.A.: California,

Sudáfrica, I. Santa Helena, Vietnam, Hawai y Neotropical).

-Galumna (G.) elimata elimata (Koch, 1841)

$(=$ Oribata obvius Berlese, 1914)

(=Oribata obvius norvegicus Berlese, 1914)

(=Dimidiogalumna wallworki J. y P. Balogh, 2002)

DisTRIBUCIÓN: Semicosmopolita (Paleártica: frecuente, U.S.A.: California, Sudáfrica, I. Santa Helena, Vietnam, Hawai y Neotropical).

-Galumna (G.) elimata sinensis Jacot, 1922 (G. obvia s.) DISTRIBUCIÓN: "China".

-Galumna (G.) engelbrechti Mahunka, 1997

DisTRIBUCIÓN: Madagascar.

-Galumna (G.) euaensis Hammer, 1973

Distribución: Polinesia (I. Tonga)

-Galumna (G.) europaea (Berlese, 1914) (Oribata emarginatus e.)

DisTRIBUCIÓN: Paleártica meridional (Europa centromeridional y

Asia centrooccidental).

-Galumna (G.) exigua Sellnick, 1925

DISTRIBUCIÓN: Sumatra.

-Galumna (G.) fijiensis Hammer, 1973

DistriBUCIÓN: Melanesia (I. Fiji)

-Galumna (G.) flabellifera Hammer, 1958

DisTRIBUCIÓN: Pantropical y subtropical.

-Galumna (G.) flabellifera flabellifera Hammer, 1958 (=Pergalumna pyri Elbadry y Nasr, 1975) DisTRIBUCIÓN: Pantropical (frecuente) y subtropical.

-Galumna (G.) flabellifera orientalis Aoki, 1965 DisTRIBUCIÓN: Oriental y Japón.

-Galumna (G.) flagellata Willmann, 1925

DisTRIBUCIÓN: Paleártica centromeridional (excepto Este).

-Galumna (G.) floridae (Jacot, 1929) (Holokalumma)

DisTRIBUCIÓN: U.S.A. (Florida).

-Galumna (G.) fordi (Jacot, 1934) (Zetes)

Distribución: Islas del Pacífico.

-Galumna (G.) gharbiensis Bayoumi, Al-Assiuty, Abdel-Hamid y El-

Shereef, 1983

DisTRIBUCIÓN: Egipto.

-Galumna (G.) gibbula Grandjean, 1956 (G. tarsipennata g.)

DisTRIBUCIÓN: Mediterránea.

-Galumna (G.) glabra Pérez-Íñigo y Baggio, 1991

DisTRIBUCIÓN: Brasil.

-Galumna (G.) globuloides (Tafner, 1905) (Oribata) "sp. inq."

DistriBución: Hungría.

-Galumna (G.) granalata Aoki, 1984

DisTRIBUCIÓN: Japón.

-Galumna (G.) grandjeani Balogh, 1962

DistriBuCIÓN: Tanzania.

-Galumna (G.) hammerae P. Balogh, 1985

Distribución: Australia.

-Galumna (G.) heros (Canestrini, 1897) (Oribata) "sp. inq."

DisTRIBUCIÓN: Nueva Guinea.

-Galumna (G.) hexagona Balogh, 1960

DistriBución: Angola.

-Galumna (G.) homodactyla (Karpelles, 1893) (Oribata) "sp. inq."

DisTRIBUCIÓN: Hungría.

-Galumna (G.) hudsoni Hammer, 1952

DisTRIBUCIÓN: Canadá.

-Galumna (G.) humida (Hall, 1911) (Oribata)

DisTRIBUCIÓN: U.S.A. (California). 
-Galumna (G.) imperfecta Hammer, 1972

Distribución: Polinesia (Tahití).

-Galumna (G.) incerta Pérez-Íñigo y Baggio, 1991

DISTRIBUCIÓN: Brasil.

-Galumna (G.) incisa Mahunka, 1982

DisTRIBUCIÓN: Etiopía.

-Galumna (G.) innexa Pérez-Iñigo y Baggio, 1986

DisTRIBUCIÓN: Brasil.

-Galumna (G.) iranensis Mahunka, 2001

DISTRIBUCIÓN: Irán

-Galumna (G.) irazu P. Balogh, 1997

Distribución: Costa Rica.

-Galumna (G.) iterata nom. nov.

[=Zetes banksi Jacot, 1929, “nom. praeoc.” por Jacot, 1929 ("hom. sec.”)]

(=Galumna jacoti Marshall, Reeves y Norton, 1987, "nom. praeoc." por

Wharton, 1938)

DisTRIBUCión: U.S.A. meridional.

-Galumna (G.) ithacensis (Jacot, 1929) (Zetes)

DisTRIBUCIÓN: U.S.A. centrooriental (frecuente).

-Galumna (G.) jacoti Wharton, 1938

DisTRIBUCIÓN: Méjico.

-Galumna (G.) karajica Mahunka, 2001

DISTRIBUCIÓN: Irán.

-Galumna (G.) kazakhstani Krivolutskaja, 1952

Distribución: Paleártica meridional (Mediterránea oriental y Asia centrooccidental).

-Galumna (G.) khoii Mahunka, 1989

DisTRIBUCIÓN: Vietnam.

-Galumna (G.) lanceata (Oudemans, 1900) (Notaspis)

(=Galumna maxima Mihelcic, 1956)

DistRiBución: Paleártica (frecuente) y Vietnam.

-Galumna (G.) laselvae P. Balogh, 1997

Distribución: Costa Rica.

-Galumna (G.) longiclava Pérez-Íñigo y Baggio, 1991

DisTRIBUCIÓN: Brasil.

-Galumna (G.) longiporosa Fujikawa, 1972

DisTRIBUCIÓN: Japón.

-Galumna (G.) louisianae (Jacot, 1929) (Zetes)

(=Galumna confusa Woodring, 1965)

DistriBuCIÓN: Sureste de U.S.A.

-Galumna (G.) lunaris Jeleva, Scull y Cruz, 1984

DisTRIBUCIÓN: Cuba.

-Galumna (G.) lyrica (Jacot, 1935) (Holokalumma)

DisTRIBUCIÓN: U.S.A. (Florida).

-Galumna (G.) macroptera (Ewing, 1909) (Oribata)

DisTRIBUCIÓN: U.S.A. meridional.

-Galumna (G.) macroptera macroptera (Ewing, 1909) DisTRIBUCIÓN: U.S.A. (Tejas).

-Galumna (G.) macroptera matecumbei (Jacot, 1935) (Zetes) DisTRIBUCIÓN: U.S.A. (Florida).

-Galumna (G.) major (Pearce, 1906) (Oribata alata m.) "sp. inq." DisTRIBUCIÓN: India (Sikkim).

-Galumna (G.) mariae Balogh, 1961

DistribuCión: Tanzania y Macaronésica (Cabo Verde).

-Galumna (G.) mauritii Mahunka, 1978

Distribución: Malgache (I. Mauricio).

-Galumna (G.) maxima (Berlese, 1916) (Oribata) "sp. inq."

DisTRIBUCIÓN: Etiópica ("África oriental” y Sudáfrica).

-Galumna (G.) maxima maxima (Berlese, 1916) "ssp. inq." DistribuCIÓN: "África oriental”.

-Galumna (G.) maxima lawrencei Jacot, 1940 DisTRIBUCIÓN: Sudáfrica.

-Galumna (G.) maxima natalensis Jacot, 1940 DISTRIBUCIÓN: Sudáfrica.

-Galumna (G.) media (Berlese, 1914) (Oribata)

DISTRIBUCIÓN: Java.

-Galumna (G.) microfissum Hammer, 1968

Distribución: Nueva Zelanda.

-Galumna (G.) minuta (Ewing, 1909) (Oribata)

DisTRIBUCIÓN: U.S.A. centrooriental (frecuente).
-Galumna (G.) mollis Kunst, 1958

DisTRIBUCIÓN: Bulgaria.

-Galumna (G.) monteithi Balogh y Mahunka, 1978

DisTRIBUCIÓN: Australia.

-Galumna (G.) monticola Hammer, 1977

DisTRIBUCIÓN: Pakistán.

-Galumna (G.) mystax C. y C. jr., Pérez-Íñigo, 1993

DisTRIBUCIÓN: Brasil.

-Galumna (G.) nigra (Ewing, 1909) (Oribata)

DisTRIBUCIÓN: Neártica nororiental (frecuente).

-Galumna (G.) nilgiria (Ewing, 1910) (Oribata)

Distribución: India (Kerala).

-Galumna (G.) niliaca Al-Assiuty, Abdel-Hamid,Seif y El-Deeb, 1985

DisTRIBUCIÓN: Egipto.

-Galumna (G.) nodula Nevin, 1974

DisTRIBUCIÓN: U.S.A. (Florida).

-Galumna (G.) nonoensis P. Balogh, 1988

DisTRIBUCIÓN: Ecuador.

-Galumna (G.) nuda Engelbrecht, 1972

DisTRIBUCIÓN: Sudáfrica.

-Galumna (G.) ovata (Berlese, 1916) (Oribata) "sp. inq."

DisTRIBUCIÓN: "África oriental".

-Galumna (G.) pallida Hammer, 1958

DisTRIBUCIÓN: Neotropical (Argentina y Panamá).

-Galumna (G.) parascaber Deb y Raychaudhuri, 1975

Distribución: India (Bengala Occidental).

-Galumna (G.) parva Woodring, 1965

Distribución: U.S.A. (Luisiana).

-Galumna (G.) parviporosa J. y P. Balogh, 1983

DisTRIBUCIÓN: Australia.

-Galumna (G.) perezi Pérez-Íñigo y Baggio, 1994

DistRIBUCIÓN: Brasil.

-Galumna (G.) planiclava Hammer, 1973

Distribución: Polinesia (I. Tonga) y Japón.

-Galumna (G.) planiclava planiclava Hammer, 1973

Distribución: Polinesia (I. Tonga).

-Galumna (G.) planiclava ishigakiensis Aoki, 1982

DISTRIBUCIÓN: Japón.

-Galumna (G.) polyporus Mihelcic, 1952

DistribuCión: Austria.

-Galumna (G.) pusilla Sellnick, 1923

DisTRIBUCIÓN: Brasil.

-Galumna (G.) reiterata nom. nov.

[=Galumna longiporosa Choi, 1986, "nom. praeoc." por Fujikawa, 1972

("hom. prim.")]

DisTRIBUCIÓN: Corea.

-Galumna (G.) reticulata Hammer, 1958

DisTRIBUCIÓN: Neotropical (Argentina y Panamá).

-Galumna (G.) rossica Sellnick, 1926

Distribución: Paleártica (excepto Oeste): frecuente.

-Galumna (G.) rostrata Sellnick, 1922

DistRiBuCión: I. Célebes [Sulawesi].

-Galumna (G.) rugosa Hammer, 1968

DisTRIBUCIÓN: Nueva Zelanda.

-Galumna (G.) sabahna Mahunka, 1995

DISTRIBUCIÓN: Borneo.

-Galumna (G.) saboori Mahunka, 2001

DISTRIBUCIÓN: Irán.

-Galumna (G.) samoaensis Jacot, 1924

Distribución: Polinesia (I. Samoa).

-Galumna (G.) scaber Hammer, 1968

DisTRIBUCIÓN: Nueva Zelanda.

-Galumna (G.) sequoiae (Jacot, 1929) (Zetes)

DisTRIBUCIÓN: U.S.A. (California).

-Galumna (G.) setigera Mihelcic, 1956

(=Galumna (Pergalumna) crassicostata Mihelcic, 1956)

$(=$ Galumna distincta Mihelcic, 1956)

(=Galumna (Pergalumna) fusigera Mihelcic, 1956)

(=Galumna sicca Mihelcic, 1957)

DISTRIBUCIÓN: Mediterránea occidental. 
-Galumna (G.) similis Pérez-Iñigo y Baggio, 1980

DistriBuCión: Neotropical (Brasil y Uruguay) y U.S.A. (Tejas).

-Galumna (G.) sinuofrons Jacot, 1922

DisTRIBUCIÓN: "China"

-Galumna (G.) strinovichi J. y P. Balogh, 1983

DisTRIBUCIÓN: Australia.

-Galumna (G.) swezeyi (Jacot, 1928) (Zetes)

DisTRIBUCIÓN: Hawai.

-Galumna (G.) szentivanyorum J. y P. Balogh, 1983

DisTRIBUCIÓN: Australia.

-Galumna (G.) tarsipennata Oudemans, 1913

(=Galumna (Pergalumna) lata Mihelcic, 1956)

(=Galumna nova Mihelcic, 1957)

(=Galumna parvula Mihelcic, 1956)

DistriBución: Paleártica meridional (Paleártica occidental: frecuente en el Sur, y Asia centrooccidental) y Brasil.

-Galumna (G.) tessellata (Ewing, 1910) (Oribata)

DisTRIBUCIÓN: India (Kerala).

-Galumna (G.) texana Banks, 1906

DisTRIBUCIÓN: U.S.A. (Tejas)

-Galumna (G.) tokyoensis Aoki, 1966

DistriBuCión: Este de Paleártica oriental

-Galumna (G.) tricuspidata Engelbrecht, 1969

DisTRIBUCIÓN: Sudáfrica.

-Galumna (G.) triops Balogh, 1960

DisTRIBUCIÓN: Angola.

-Galumna (G.) triquetra Aoki, 1965

DisTRIBUCIÓN: Oriental (Tailandia y Vietnam) y Australia.

-Galumna (G.) tuberculata Mahunka, 1997

DisTRIBUCIÓN: Madagascar.

-Galumna (G.) unica Sellnick, 1923

DISTRIBUCIÓN: Brasil.

-Galumna (G.) valida Aoki, 1994

DisTRIBUCIÓN: Micronesia (I. Marianas).

-Galumna (G.) varia Mahunka, 1995

DisTRIBUCIÓN: Tailandia.

-Galumna (G.) virginiensis Jacot, 1929

DisTRIBUCIÓN: U.S.A. centrooriental (frecuente).

-Galumna (G.) weni Aoki y Hu, 1993

DistriBUCIÓN: China suroriental.

-Galumna (G.) zachvatkini Grishina, 1982

Distribución: Paleártica meridional (Sureste de Europa y Sur de Paleártica oriental).

Galumna (Angulogalumna) Grishina, 1981 (1 sp.)

ESPECIE TIPO: Angulogalumna asiatica Grishina, 1981

DISTRIBUCIÓN: Paleártica.

-Galumna (Angulogalumna) asiatica (Girshina, 1981) (A.)

DISTRIBUCIÓN: Rusia asiática.

Galumna (Cosmogalumna) Aoki, 1988 (3 spp.)

(=Variogalumna Mahunka, 1995 "sin. nov.")

EsPeCIE TIPO: Cosmogalumna ornata Aoki, 1988

DISTRIBUCIÓN: Oriental y Paleártica meridional.

-Galumna (Cosmogalumna) ornata (Aoki, 1988) (C.)

DISTRIBUCIÓN: Japón.

-Galumna (Cosmogalumna) praeoccupata nom. nov.

[=Cosmogalumna imperfecta Aoki y Hu, 1993, "nom. praeoc." por Hammer, 1972 ("hom. sec.")]

DisTRIBUCIÓN: China suroriental

-Galumna (Cosmogalumna) singularis (Mahunka, 1995) (Variogalumna)

DISTRIBUCIÓN: Borneo.

Galumna (Erogalumna) Grandjean, 1964 (1 sp.)

EsPeCIE TIPO: Erogalumna zeucta Grandjean, 1964

DISTRIBUCIÓN: Neotropical.

-Galumna (Erogalumna) zeucta (Grandjean, 1964) (E.)

DisTRIBUCIÓN: Venezuela.
Galumna (Indogalumna) Balakrishnan, 1985 (7 spp.)

ESPECIE TIPO: Indogalumna microsulcata Balakrishnan, 1985

DisTRIBUCIÓN: Pantropical y subtropical (Paleártica meridional).

-Galumna (Indogalumna) microsulcata (Balakrishnan, 1985) (I.)

DisTRIBUCIÓN: India (Kerala).

-Galumna (Indogalumna) monticola (Balakrishnan, 1985) (I.)

DisTRIBUCIÓN: India (Kerala).

- Galumna (Indogalumna) neonominata nom. nov.

[=Galumna clavata Pérez-Íñigo y Baggio, 1991, "nom. praeoc." por

Sellnick, 1931 (+) (“hom. prim.”)]

DisTRIBUCIÓN: Brasil.

-Galumna (Indogalumna) pterolineata Hammer, 1972 (G.)

DisTRIBUCIÓN: Polinesia (Tahití).

-Galumna (Indogalumna) rasilis Pérez-Íñigo, 1987 (G.)

(=Galumna rugosa Wallwork, 1977, "nom. praeoc.” por Hammer, 1968)

(=Galumna sanctaehelenae J. y P. Balogh, 2002)

Distribución: I. Azores e I. Santa Helena.

-Galumna (Indogalumna) undulata (Balakrishnan, 1985) (I.)

DisTRIBUCIÓN: India (Kerala)

Galumna (Kabylogalumna) Bernini, 1984 (1 sp.)

EsPeCIE TIPO: Kabylogalumna rhinoceros Bernini, 1984

DisTRIBUCIÓN: Paleártica.

-Galumna (Kabylogalumna) rhinoceros (Bernini, 1984) (K.)

DISTRIBUCIÓN: Argelia.

Globogalumna J. y P. Balogh, 1990 (1 sp.)

ESPECIE TIPO: Allogalumna globulifera Balogh y Mahunka, 1978

DISTRIBUCIÓN: Neotropical.

-Globogalumna globulifera (Balogh y Mahunka, 1978) (Allogalumna)

DISTRIBUCIÓN: Brasil.

Heterogalumna Balogh, 1960 (3 spp.)

EsPeCIE TIPO: Heterogalumna lineolata Balogh, 1960

DISTRIBUCIÓN: Etiópica.

-Heterogalumna lineolata Balogh, 1960

DistRIBUCIÓN: Congo.

-Heterogalumna monticola Balogh, 1962

DisTRIBUCIÓN: Tanzania.

-Heterogalumna pygmaea (Balogh, 1958) (Galumna)

DisTRIBUCIÓN: Angola.

Kinezogalumna Aoki y Hu, 1993 (1 sp.)

ESPECIE TIPO: Kinezogalumna calcicola Aoki y Hu, 1993

DISTRIBUCIÓN: Oriental

-Kinezogalumna calcicola Aoki y Hu, 1993

DisTRIBUCIÓN: China suroriental.

Leptogalumna Balogh, 1960 (2 spp.)

EsPECIE TIPO: Leptogalumna ciliata Balogh, 1960

DistriBUCión: Pantropical (excepto Oriental).

-Leptogalumna ciliata Balogh, 1960

DisTRIBUCIÓN: Angola, Polinesia (Tahití) y Cuba.

-Leptogalumna reducta Mahunka, 1996

DISTRIBUCIÓN: Madagascar.

Neogalumna Hammer, 1973 (2 spp.)

ESPECIE TIPO: Neogalumna antenniger Hammer, 1973

DISTRIBUCIÓN: Australianooriental.

-Neogalumna antenniger Hammer, 1973

DisTRIBUCIÓN: Polinesia (I. Samoa)

-Neogalumna curviporosa Balakrishnan, 1986

DISTRIBUCIÓN: India (Kerala).

Notogalumna Sellnick, 1959 (4 spp.)

EsPeCIE TIPO: Notogalumna praetiosa Sellnick, 1959

DISTRIBUCIÓN: Pantropical (excepto Neotropical).

-Notogalumna africana Mahunka, 1988

DISTRIBUCION: Tanzania. 
-Notogalumna nortoni Ramani y Haq, 1990

DisTRIBUCIÓN: India (Kerala).

-Notogalumna praetiosa Sellnick, 1959

DisTRIBUCIÓN: Polinesia: I. Australes [I. Tubuai] y Borneo.

-Notogalumna truncata (Warburton, 1912) (Oribata)

DisTRIBUCión: I. Seychelles.

Orthogalumna Balogh, 1961 (3 spp.)

(=Mirogalumna Mahunka, 1993 "sin. nov.")

EsPeCIE TIPO: Orthogalumna saeva Balogh, 1961

DISTRIBUCIÓN: Pantropical (excepto Australiana) y subtropical (Holártica meridional).

-Orthogalumna saeva Balogh, 1961

DisTRIBUCIÓN: Etiópica (Madagascar y Sudáfrica), India (Tripura) y Japón.

-Orthogalumna terebrantis Wallwork, 1965

DisTRIBUCIÓN: Uruguay y U.S.A. (Florida).

-Orthogalumna xena (Mahunka, 1993) (Mirogalumna)

DISTRIBUCIÓN: Ruanda.

Pergalumna Grandjean, 1936 (103 spp. y 14 sspp.)

(?=Neorizetes Jacot, 1933 "gen. inq.")

EsPeCIE TIPO: Oribata nervosa Berlese, 1914

DISTRIBUCIÓN: Cosmopolita (excepto Antártica)

-Pergalumna aegra Pérez-Íñigo y Baggio, 1986

Distribución: Brasil.

-Pergalumna aequalis (Sellnick, 1923) (Stictozetes)

Distribución: Neotropical (Brasil y Venezuela).

-Pergalumna akitaensis Aoki, 1961

Distribución: Este de Paleártica oriental.

-Pergalumna altera (Oudemans, 1915) (Galumna)

(=Galumna (Pergalumna) diversa Mihelcic, 1956)

(=Pergalumna harunaensis Aoki, 1961)

DistRIBUCIÓN: Semicosmopolita (Holártica: frecuente en Europa,

Sudáfrica, Oriental: Vietnam y China suroriental, y Micronesia: I.

Marianas).

-Pergalumna amamiensis Aoki, 1984

DisTRIBUCIÓN: Japón.

-Pergalumna andasibe Mahunka, 1996

Distribución: Madagascar.

-Pergalumna andhraense Raju, Appalanaidu y Rao, 1981

Distribución: India (Andhra Pradesh).

-Pergalumna andicola Hammer, 1961

Distribución: Perú.

-Pergalumna anellata Hammer, 1961

DisTRIBUCIÓN: Perú.

-Pergalumna annulata Mahunka, 1995

DisTRIBUCIÓN: Borneo.

-Pergalumna aokii Nakatamari, 1982

DisTRIBUCIÓN: Japón.

-Pergalumna arborea (Jacot, 1929) (Zetes)

Distribución: U.S.A. (Connecticut).

-Pergalumna australis (Pérez-Íñigo y Baggio, 1980) $(P$.) DisTRIBUCión: Brasil.

-Pergalumna bellesii Pérez-Íñigo y Baggio, 1997

Distribución: Brasil.

-Pergalumna bifissurata Hammer, 1972

DistriBución: Polinesia (Tahití).

-Pergalumna bimaculata Hammer, 1973

Distribución: Polinesia (I. Tonga).

-Pergalumna bryani (Jacot, 1934) (Zetes)

Distribución: Islas del Pacífico.

-Pergalumna bryani bryani (Jacot, 1934)

Distribución: Islas del Pacífico.

-Pergalumna bryani marquesi (Jacot, 1934) (Zetes) Distribución: Polinesia.

- Pergalumna bryani uapoui (Jacot, 1934) (Zetes)

Distribución: Polinesia (I. Marquesas).

-Pergalumna californiae (Jacot, 1929) (Zetes)

Distribución: U.S.A. (California).
-Pergalumna cardosensis Pérez-Iñigo y Baggio, 1986

DisTRIBUCión: Neotropical (Brasil y Perú)

-Pergalumna circula (Jacot, 1935) (Galumna flagellifera c.)

DisTRIBUCIÓN: U.S.A. (Florida).

-Pergalumna comparandus (Berlese, 1920) (Oribata emarginatus c.)

DisTRIBUCIÓN: Argentina.

-Pergalumna complicata Balogh y Mahunka, 1978

DisTRIBUCIÓN: Brasil.

-Pergalumna conspicua Balogh, 1962

DistRIBUCIÓN: Tanzania.

-Pergalumna corniculata (Berlese, 1905) (Oribata elimatus c.)

DistRiBución: Java.

-Pergalumna corolevuensis Hammer, 1971

Distribución: Melanesia (I. Fiji).

-Pergalumna corrugis (Jacot, 1929) (Zetes)

Distribución: U.S.A. nororiental.

-Pergalumna corrugis corrugis (Jacot, 1929)

DisTRIBUCIÓN: U.S.A. (Masachuset).

-Pergalumna corrugis milleri (Jacot, 1935) (Zetes) DISTRIBUCIÓN: U.S.A. nororiental.

-Pergalumna crassipora Mahunka, 1995

Distribución: Borneo.

-Pergalumna cribriger (Berlese, 1916) (Oribata)

(=Galumna flagelliferum Jacot, 1929)

Distribución: U.S.A.

-Pergalumna cucheae Mahunka, 1998

DistribuCión: Pequeñas Antillas.

-Pergalumna curva (Ewing, 1907) (Oribata)

Distribución: Pantropical (Oriental: Indonesia, Polinesia y

Neotropical: Bolivia y Perú) y Holártica.

-Pergalumna curva curva (Ewing, 1907) (=Oribata tantillus Berlese, 1908)

DISTRIBUCIÓN: Holártica (U.S.A. centrooriental: frecuente, y Paleártica: Sureste de Europa y Este de Paleártica oriental) y Java.

-Pergalumna curva ventralis (Willmann, 1931) (Galumna v.) (=Galumna duplicata Hammer, 1958) DisTRIBUCIÓN: Pantropical (Sumatra, Polinesia y Neotropical: Bolivia y Perú) y "Holártica".

-Pergalumna decorata Balogh y Mahunka, 1977

DisTRIBUCIÓN: Bolivia.

-Pergalumna decoratissima Pérez-Iñigo y Baggio, 1986

DisTRIBUCIÓN: Neotropical (Brasil y Venezuela).

-Pergalumna dodsoni Nevin, 1979

DisTRIBUCIÓN: Neártica septentrional.

-Pergalumna dubitanda Hammer, 1972

DisTRIBUCión: Polinesia (Tahití).

-Pergalumna elongata Engelbrecht, 1972

DisTRIBUCIÓN: Sudáfrica.

-Pergalumna emarginata (Banks, 1895) (Oribata)

DISTRIBUCIÓN: Neártica.

-Pergalumna emarginata emarginata (Banks, 1895) (=Zetes emarginatus bidens Jacot, 1935)

(=Oribata emarginatus columbianus Berlese, 1916) (=Pergalumna omnifagous Rockett y Woodring, 1966) DisTRIBUCIÓN: Neártica: frecuente.

-Pergalumna emarginata coscobensis (Jacot, 1935) (Zetes) DisTRIBUCIÓN: U.S.A. (Conéticut).

-Pergalumna) emarginata laevis (Jacot, 1935) (Zetes) DisTRIBUCIÓN: U.S.A. (Florida).

-Pergalumna fastigata Mahunka 1996

DISTRIBUCIÓN: Madagascar.

-Pergalumna filifera Mahunka, 1978

DistRIBUCión: Malgache (I. Mauricio) y Australia

-Pergalumna formicaria (Berlese, 1914) (Oribata)

DisTRIBUCIÓN: Holártica (Paleártica occidental y Neártica septentrional).

-Pergalumna foveolata Hammer, 1973

Distribución: Polinesia (I. Tonga)

-Pergalumna frater Balogh, 1960

DistriBución: Congo. 
-Pergalumna fusca (Berlese, 1916) (Oribata (Stictozetes)) DisTRIBUCIÓn: Somalia.

-Pergalumna graminetum (Jacot, 1935) (Zetes)

DisTRIBUCIÓN: U.S.A. nororiental.

-Pergalumna granulata Balogh y Mahunka, 1967

DisTRIBUCIÓN: Vietnam.

-Pergalumna hastata Aoki, 1987

DisTRIBUCIÓN: Japón.

-Pergalumna hauseri Mahunka, 1995

DisTRIBUCIÓN: Borneo.

-Pergalumna hawaiiensis (Jacot, 1934) (Galumna)

Distribución: Islas del Pacífico.

-Pergalumna hawaiiensis hawaiiensis (Jacot, 1934) Distribución: Islas del Pacífico.

-Pergalumna hawaiiensis marquesana (Jacot, 1934) (Galumna) Distribución: Polinesia.

-Pergalumna heroica (Willmann, 1931) (Galumna)

DistriBución: Java.

-Pergalumna horvathorum P. Balogh, 1997

DisTRIBUCión: Costa Rica.

-Pergalumna imadatei Aoki y Hu, 1993

Distribución: China suroriental.

-Pergalumna incomperta Engelbrecht, 1972

DISTRIBUCIÓN: Sudáfrica.

-Pergalumna indivisa Mahunka, 1995

DisTRIBUCIÓN: Borneo.

-Pergalumna intermedia Aoki, 1963

Distribución: Este de Paleártica oriental y China.

-Pergalumna intermedia intermedia Aoki, 1963

DistRIBUCIÓN: Este de Paleártica oriental y China.

-Pergalumna intermedia retroversa Aoki y Hu, 1993 DISTRIBUCIÓN: China suroriental.

-Pergalumna irregularis Wallwork, 1977

Distribución: I. Santa Helena.

-Pergalumna jongkyui Choi, 1986

DisTRIBUCIÓN: Corea.

-Pergalumna kotschyi Mahunka, 1989

DisTRIBUCIÓN: Vietnam.

-Pergalumna longiporosa Fujita y Fujikawa, 1987

DistriBución: Japón.

-Pergalumna longisetosa Balogh, 1960

Distribución: África central.

-Pergalumna magnipora (Hammer, 1961) (Galumna)

DistRIBUCión: Pantropical (Perú, Sudáfrica y Oriental) y subtropical (Este de Paleártica oriental).

-Pergalumna magnipora magnipora (Hammer, 1961) DistribuCión: Perú.

-Pergalumna magnipora capensis Engelbrecht, 1972 DisTRIBUCión: Sudáfrica e India (Tripura).

-Pergalumna magnipora capillaris Aoki, 1961 (P. c.)

Distribución: Este de Paleártica oriental y Oriental (Vietnam).

-Pergalumna magnipora xishuangbanna Aoki y Hu, 1993 DisTRIBUCIÓN: China suroriental.

-Pergalumna margaritata Mahunka, 1989

DisTRIBUCIÓN: Vietnam.

-Pergalumna mauritii Mahunka, 1978

DISTRIBUCIÓN: Malgache (I. Mauricio) y Micronesia (I. Marianas)

-Pergalumna medialis (Sellnick, 1925) (Stictozetes)

DISTRIBUCIÓN: Sumatra.

-Pergalumna melloi Pérez-Íñigo y Baggio, 1994

DISTRIBUCIÓN: Brasil.

-Pergalumna menglunensis Aoki y Hu, 1993

Distribución: China suroriental.

-Pergalumna minor (Willmann, 1928) (Galumna)

DisTRIBUCión: Holártica (Europa centromeridional y U.S.A.: Nueva York)

-Pergalumna minoricana Pérez-Íñigo jr., 1991

Distribución: España (I. Baleares).

-Pergalumna montana Hammer, 1961

DistRIBUCIÓN: Neotropical (Perú y Venezuela).
-Pergalumna myrmophila (Berlese, 1914) (Oribata longiplumus m.)

(=Galumna lineata Oudemans, 1914)

(=Galumna longior Willmann, 1928)

(=Galumna sexareata Mihelcic, 1956)

(=Allogalumna subaequalis Mihelcic, 1956)

(=Allogalumna subaequelis fusca Mihelcic, 1956)

DisTRIBUCIÓN: Paleártica meridional (Paleártica occidental: frecuente en Mediterránea, y Pakistán) y Etiópica: Guinea

Ecuatorial: Bioco [Fernando Poo] y Tanzania (Zanzíbar).

-Pergalumna nasica Pérez-Íñigo y Baggio, 1980

DisTRIBUCIÓN: Brasil.

-Pergalumna nervosa (Berlese, 1914) (Oribata)

DistRIBUCión: Holártica (Paleártica y U.S.A.) y Sudáfrica.

-Pergalumna nervosa nervosa (Berlese, 1914)

(=Galumna retalata Oudemans, 1915)

Distribución: Holártica (Paleártica: frecuente, y U.S.A.) y Sudáfrica.

-Pergalumna nervosa punctata (Mihelcic, 1957) (Galumna (P.)) DisTRIBUCIÓN: Mediterránea occidental.

-Pergalumna nuda Balogh, 1960

DistriBución: Angola.

-Pergalumna numerosa (Sellnick, 1923) (Galumna)

DistriBución: Brasil.

-Pergalumna obsessa nom. nov.

[=Galumna pallida Tseng, 1984, “nom. praeoc." por Hammer, 1958 ("hom. prim.")]

Distribución: Taiwan [Formosa].

-Pergalumna operata Tseng, 1984

DisTRIBUCIÓn: Taiwan [Formosa].

-Pergalumna parva Pérez-Íñigo y Baggio, 1986

DisTRIBUCIÓN: Brasil.

-Pergalumna passimpunctata Balogh y Mahunka, 1969

Distribución: Brasil.

-Pergalumna paucisetosa (Jacot, 1929) (Galumna)

Distribución: "China".

-Pergalumna pauliensis Pérez-Íñigo y Baggio, 1991

DistriBución: Brasil.

-Pergalumna pertrichosa Mahunka, 1995

DISTRIBUCIÓN: Borneo.

-Pergalumna plumata Pérez-Íñigo y Baggio, 1986

DisTRIBUCIÓN: Neotropical.

-Pergalumna pocsi Mahunka, 1984

DisTRIBUCIÓN: Tanzania.

-Pergalumna pseudomargaritata Mahunka, 1994

DisTRIBUCIÓN: Tailandia.

-Pergalumna pterinervis (Canestrini, 1898) (Oribata)

DisTRIBUCIÓN: Nueva Guinea y Oriental (Indonesia).

-Pergalumna punctulata Balogh y Mahunka, 1967

DisTRIBUCIÓN: Vietnam.

-Pergalumna pyramidalis (Tseng, 1984) (Galumna)

Distribución: Taiwan [Formosa].

-Pergalumna remota (Hammer, 1968) (Allogalumna)

DisTRIBUCIÓN: Nueva Zelanda.

-Pergalumna reniformis Hammer, 1958

Distribución: Nueva Zelanda.

-Pergalumna rugosala (Ewing, 1909) (Oribata) "sp. inq."

DisTRIBUCIÓN: Noreste de U.S.A.

-Pergalumna semistriata Pérez-Íñigo jr., 1990

DisTRIBUCIÓN: España.

-Pergalumna semistriata semistriata Pérez-Íñigo jr., 1990 DisTRIBUCIÓN: España.

-Pergalumna semistriata matritensis Arillo y Subías, 1993 DisTRIBUCIÓN: España.

-Pergalumna silvatica Hammer, 1961

Distribución: Neotropical (Perú y Panamá).

-Pergalumna silvestris Hammer, 1968

Distribución: Nueva Zelanda.

-Pergalumna somalica (Berlese, 1916) (Oribata ovatus s.)

DisTRIBUCIÓn: Somalia. 
-Pergalumna striata (Pérez-Iñigo y Baggio, 1980) (Allogalumna) DistriBución: Brasil.

-Pergalumna strigulata Mahunka, 1978

DisTRIBUCIÓN: Malgache (I. Mauricio).

-Pergalumna sulcatomarginata Mahunka, 1986

Distribución: Kenya.

-Pergalumna sura P. Balogh, 1987

Distribución: Costa Rica.

-Pergalumna tahitiensis J. y P. Balogh, 2002

(=Pergalumna montana Hammer, 1972, "nom. praeoc." por Hammer, 1961) Distribución: Polinesia (Tahití).

-Pergalumna tanzanica Mahunka, 1984

Distribución: Tanzania.

-Pergalumna taprobanica P. Balogh, 1988

DisTRIBUCión: Ceilán [Sri Lanka].

-Pergalumna tsavoensis Mahunka, 1986 DisTribución: Kenia.

-Pergalumna variosculpturata Mahunka y Mahunka-Papp, 1999 DisTRIBUCIÓN: Hungría.

-Pergalumna weberi (Jacot, 1935) (Zetes)

DistRIBUCIÓN: U.S.A. (Florida).

-Pergalumna weberi weberi (Jacot, 1935)

Distribución: U.S.A. (Florida).

-Pergalumna weberi plumalae (Jacot, 1935) (Zetes) DisTRIBUCIÓN: U.S.A. (Florida).

-Pergalumna willmanni (Zachvatkin, 1953) (Galumna)

Distribución: Paleártica (Europa central y Suroeste de Siberia).

Pilizetes Sellnick, 1937 (16 spp.)

EsPECIE TIPO: Pilizetes africanus Sellnick, 1937

DISTRIBUCIÓN: Pantropical (excepto Australiana).

Pilizetes (Pilizetes) Sellnick, 1937 (13 spp.)

DISTRIBUCIÓN: Etiópica.

-Pilizetes (P.) africanus Sellnick, 1937

DistribuCión: Tanzania.

-Pilizetes (P.) australis Balogh y Mahunka, 1966

DistribuCión: Sudáfrica.

-Pilizetes (P.) basilewskyi Balogh, 1958

DistribuCión: Tanzania.

-Pilizetes (P.) brevisetus Mahunka, 1984

Distribución: Tanzania.

-Pilizetes (P.) csoengeyi Mahunka, 1983

Distribución: Tanzania.

-Pilizetes (P.) curtipilus Balogh, 1960

Distribución: Congo.

-Pilizetes (P.) denticulatus Mahunka, 1986

DistribuCión: Kenia.

-Pilizetes (P.) dudichi Balogh, 1966

Distribución: Chad.

-Pilizetes (P.) saskai Mahunka, 1969

Distribución: Tanzania.

-Pilizetes (P.) sellnicki Balogh, 1958

Distribución: Angola.

-Pilizetes (P.) subglaber Balogh, 1962

DisTRIBUCIÓN: Tanzania.

-Pilizetes (P.) subsimilis Mahunka, 1984

DisTRIBUCIÓN: Tanzania.

-Pilizetes (P.) tuberculatus Mahunka, 1994

Distribución: Malgache (I. Comores).

Pilizetes (Neopilizetes) J. y P. Balogh, 1990 (2 spp.)

(?=Kratzensteinia Oudemans, 1919 "gen. inq.")

EsPECIE TIPO: Pilizetes neotropicus Balogh y Mahunka, 1978

DisTRIBUCIÓN: Neotropical.

-Pilizetes (Neopilizetes) neotropicus Balogh y Mahunka, 1978 (P.) DisTRIBUCIÓN: Brasil.

-Pilizetes (Neopilizetes) rugifrons (Stoll, 1891) (Oribata) “sp. inq." DisTRIBUCIÓN: América central.
Pilizetes (Sarawakiella) Mahunka, 1966 (1 sp.)

ESPECIE TIPO: Sarawakiella longipilosa Mahunka, 1966

DisTRIBUCIÓN: Oriental

-Pilizetes (Sarawakiella) longipilosa (Mahunka, 1966) (S.)

DisTRIBUCIÓN: Borneo.

Pilogalumna Grandjean, 1956 (13 spp. y 5 sspp.)

ESPECIE TIPO: Pilogalumna ornatula Grandjean, 1956

DistriBución: Cosmopolita (excepto Australiana y Antártica).

-Pilogalumna antillensis Mahunka, 1998

DistriBución: Pequeñas Antillas.

-Pilogalumna arabica Bayoumi y Al-Khalifa, 1986

DisTRIBUCIÓN: Arabia Saudita y Yemen.

-Pilogalumna binadalares (Jacot, 1929) (Galumna areolata b.)

DisTRIBUCIÓN: Noreste de Neártica.

-Pilogalumna bloemfonteinensis Engelbrecht, 1972

DisTRIBUCIÓN: Sudáfrica.

-Pilogalumna boevi (Krivolutskaja, 1952) (Allogalumna)

DISTRIBUCIÓN: Paleártica centromeridional (excepto extremos Este y Oeste).

-Pilogalumna cozadensis Nevin, 1976

Distribución: U.S.A. (Nebraska).

-Pilogalumna crassiclava (Berlese, 1914) (Allogalumna)

DistRIBUCIÓN: Paleártica centromeridional.

-Pilogalumna crassiclava crassiclava (Berlese, 1914) (=Galumna allifera Oudemans, 1919)

DisTRIBUCIÓN: Paleártica centromeridional (frecuente).

-Pilogalumna crassiclava longiareata Pérez-Íñigo, 1976 (P. allifera l.)

DISTRIBUCIÓN: Mediterránea occidental.

-Pilogalumna crassiclava montana Grishina y Sergienko, 1978 (P. allifera $m$.)

DISTRIBUCIÓN: Ucrania.

-Pilogalumna kimberleyensis Engelbrecht, 1972

DisTRIBUCIÓN: Sudáfrica.

-Pilogalumna ornatula Grandjean, 1956

DisTRIBUCIÓN: Mediterránea.

-Pilogalumna ornatula ornatula Grandjean, 1956

(=Galumna adareata Mihelcic, 1957)

(=Galumna decipiens Mihelcic, 1956)

(=Allogalumna molensis Mihelcic, 1957)

(=Allogalumna pterata Mihelcic, 1957)

DisTRIBUCIÓN: Mediterránea.

-Pilogalumna ornatula fayoumensis Abdel-Hamid, 1964 DISTRIBUCIÓN: Egipto

-Pilogalumna recta (Mihelcic, 1957) (Allogalumna)

DisTRIBUCIÓN: España.

-Pilogalumna steinmanni Aoki, 1975

DisTRIBUCiÓN: Corea.

-Pilogalumna tenuiclava (Berlese, 1908) (Oribata)

DISTRIBUCIÓN: Holártica.

-Pilogalumna tenuiclava tenuiclava (Berlese, 1908)

(?=Acarus alatus Schrank, 1803 "sp. inq.")

(?=Acarus aquaticus marginatus De Geer, 1778 "sp. inq.")

(=Galumna areolata Willmann, 1923)

(=Allogalumna atra Mihelcic, 1957)

(=Galumna radiata Sellnick, 1928)

DISTRIBUCIÓN: Holártica (frecuente en Europa).

-Pilogalumna tenuiclava halli (Jacot, 1929) (Galumna areolata h.) DisTRIBUCIÓN: U.S.A. (California).

-Pilogalumna tenuiclava hispida (Jacot, 1935) (Galumna alata h.) DisTRIBUCIÓN: U.S.A. (Florida).

-Pilogalumna variabilis Engelbrecht, 1972

DISTRIBUCIÓN: Sudáfrica e India (Tripura).

Psammogalumna Balogh, 1943 (2 spp.)

EsPECIE TIPO: Stictozetes hungaricus Sellnick, 1925

DistriBUCión: Paleártica.

-Psammogalumna hungarica (Sellnick, 1925) (Stictozetes)

DisTRIBUCIÓN: Paleártica meridional (excepto extremos Este y Oeste). 
-Psammogalumna thysanura (Krivolutskaja, 1952) (Allogalumna) DisTRIBUCIÓN: Sur de Paleártica oriental.

Rostrogalumna Engelbrecht, 1973 (1 sp.)

ESPECIE TIPO: Galumna rostrata Engelbrecht, 1969

DisTRIBUCIÓN: Etiópica.

-Rostrogalumna rostrata (Engelbrecht, 1969) (Galumna) DisTRIBUCIÓN: Sudáfrica.

Sacculogalumna Engelbrecht, 1973 (1 sp.)

ESPECIE TIPO: Sacculogalumna saccularis Engelbrecht, 1973 DistribuCIÓN: Etiópical.

-Sacculogalumna saccularis Engelbrecht, 1973 DISTRIBUCIÓN: Sudáfrica.

Setogalumna P. Balogh, 1985 (2 spp.)

EsPECIE TIPO: Setogalumna excellens P. Balogh, 1985

DISTRIBUCIÓN: Australiana y Paleártica meridional.

-Setogalumna diminuta Arillo y Subías, 1993

DisTRIBUCIÓN: España.

-Setogalumna excellens P. Balogh, 1985

DisTRIBUCIÓN: Australia.

Sphaerogalumna Balogh, 1961 (1 sp.)

EsPECIE TIPO: Pergalumna index Balogh, 1960

DisTRIBUCIÓN: Etiópica.

-Sphaerogalumna index (Balogh, 1960) (Pergalumna)

DisTRIBUCIÓN: Congo.

Stictozetes Berlese, 1916 (5 spp.)

(=Strabogalumna Mahunka, 1995 "sin. nov.")

(=Trachygalumna Balogh, 1960 "sin. nov.")

EsPeCIE TIPO: Oribata (Stictozetes) scaber Berlese, 1916

DistriBuCión: Paleotropical.

-Stictozetes bisulcatus (Balogh, 1960) (Trachygalumna) DisTRIBUCIÓn: Congo.

-Stictozetes margaritae (Mahunka, 1993) (Trachygalumna) DisTRIBUCIÓN: Tanzania.

-Stictozetes microporosus (Mahunka, 1986) (Trachygalumna) DisTRIBUCIÓN: Kenia.

-Stictozetes psadilliferus (Mahunka, 1995) (Strabogalumna) DisTRIBUCIÓN: Borneo.

-Stictozetes scaber (Berlese, 1916) (Oribata (S.))

DISTRIBUCIÓN: Somalia.

Taeniogalumna Balogh, 1962 (2 spp.)

EsPeCIE TIPO: Taeniogalumna sphaerula Balogh, 1962

DisTRIBUCIÓN: Etiópica.

-Taeniogalumna sphaerula Balogh, 1962

DisTRIBUCIÓN: Tanzania.

-Taeniogalumna tanzanica Mahunka, 1983

DISTRIBUCIÓN: Tanzania.

Trichogalumna Balogh, 1960 (19 spp.)

EsPeCIE TIPO: Pilogalumna lunai Balogh, 1958

DisTRIBUCión: Pantropical (excepto Neotropical) y subtropical.

-Trichogalumna arborea Ohkubo, 1984

DisTRIBUCIÓN: Japón.

-Trichogalumna chimaera Ohkubo, 1984

DISTRIBUCIÓN: Japón.

-Trichogalumna chitralensis Hammer, 1977 DisTRIBUCIÓN: Pakistán.

-Trichogalumna duoporosa Hammer, 1971

DisTRIBUCIÓN: Melanesia (I. Fiji).

-Trichogalumna granuliala Ohkubo, 1984 DISTRIBUCIÓN: Japón.

-Trichogalumna hygrophila Ohkubo, 1984 DisTRIBUCIÓN: Japón.

-Trichogalumna imperfecta Ohkubo, 1984 DISTRIBUCIÓN: Japón.
-Trichogalumna lineata Ohkubo, 1984

DisTRIBUCIÓN: Japón.

-Trichogalumna lunai (Balogh, 1958) (Pilogalumna)

DisTRIBUCIÓN: Etiópica (Angola y Chad).

-Trichogalumna madagassica Mahunka, 1996

DisTRIBUCIÓN: Madagascar.

-Trichogalumna microseta Wallwork, 1965

DisTRIBUCIÓN: Chad.

-Trichogalumna montana Balogh, 1962

DisTRIBUCIÓN: Tanzania.

-Trichogalumna nipponica (Aoki, 1966) (Pergalumna duplicata n.) DisTRIBUCIÓN: Este de Paleártica oriental (frecuente) y Noreste de Oriental (China)

-Trichogalumna pumularis Engelbrecht, 1972

DISTRIBUCIÓN: Sudáfrica.

-Trichogalumna punctata Engelbrecht, 1972

DISTRIBUCIÓN: Sudáfrica.

-Trichogalumna seminuda Balogh, 1960

DistRIBUCIÓN: Angola e India (Tripura).

-Trichogalumna subnuda Balogh y Mahunka, 1967

DisTRIBUCIÓN: Vietnam.

-Trichogalumna taeniata Hammer, 1971

DisTRIBUCIÓN: Melanesia (I. Fiji).

-Trichogalumna vietnamica Mahunka, 1987

DISTRIBUCIÓN: Vietnam.

Trichogalumnella Mahunka, 1992 (1 sp.)

EsPECIE TIPO: Trichogalumnella hauseri Mahunka, 1992

DISTRIBUCIÓN: Etiópica.

-Trichogalumnella hauseri Mahunka, 1992

DISTRIBUCIÓN: Senegal.

Vaghia Oudemans, 1919 (5 spp.)

EsPeCIE TIPO: Oribata (Stictozetes) stupendus Berlese, 1916

DisTRIBUCIÓN: Paleártica y Oriental.

-Vaghia blascoi Travé, 1981

DisTRIBUCIÓN: India (Kerala).

-Vaghia carinata (Travé, 1956) (Galumna)

DISTRIBUCIÓN: Suroeste de Europa.

-Vaghia simplex Travé, 1957

DISTRIBUCIÓN: Mediterránea occidental.

- Vaghia stupenda (Berlese, 1916) (Oribata (Stictozetes))

DISTRIBUCIÓN: Suroeste de Europa.

-Vaghia uniporosa Arillo y Subías, 1993

DISTRIBUCIÓN: España.

Xenogalumna Balogh, 1961 (1 sp.)

EsPECIE TIPO: Xenogalumna longula Balogh, 1961

DISTRIBUCIÓN: Etiópica

-Xenogalumna longula Balogh, 1961

DISTRIBUCIÓN: Madagascar.

Galumnellidae Piffl, 1970 (5 gen., 2 subg. y 32 spp.) DistRIBUCIÓN: Pantropical y subtropical.

Galumnella Berlese, 1916 (15 spp.)

EsPECIE TIPO: Galumnella paradoxa Berlese, 1916

DISTRIBUCIÓN: Pantropical (excepto Neotropical) y subtropical (Paleártica meridional).

Gallumnella (Galumnella) Berlese, 1916 (14 spp.)

DisTRIBUCIÓN: Pantropical (excepto Neotropical) y subtropical (Paleártica meridional).

-Galumnella (G.) apiculata Mahunka, 1992

Distribución: Senegal.

-Galumnella (G.) areolata Balogh, 1960

DistriBUCIÓN: Etiópica: Angola y Rodesia [Zimbabue].

-Galumnella (G.) cellularis Balogh y Mahunka, 1967

DISTRIBUCION: Vietnam. 
-Galumnella (G.) geographica Mahunka, 1995

DisTRIBUCIÓN: Borneo.

-Galumnella (G.) nipponica Suzuki y Aoki, 1970

(=Galumnella angustifrons Aoki, 1970)

DisTRIBUCIÓN: Este de Paleártica oriental y Oriental: India (Kerala)

y Taiwan [Formosa].

-Galumnella (G.) paradoxa Berlese, 1916

DisTRIBUCIÓN: Somalia.

-Galumnella (G.) pauliani Balogh, 1961

DisTRIBUCIÓN: Madagascar.

-Galumnella (G.) pilosa Engelbrecht, 1972

DisTRIBUCIÓN: Sudáfrica.

-Galumnella (G.) punctipennis Balogh, 1960

DistriBución: Congo.

-Galumnella (G.) rugosa Balogh, 1960

DistriBución: Congo.

-Galumnella (G.) rugosula Balogh, 1960

DisTRIBUCIÓN: Congo.

-Galumnella (G.) subareolata Mahunka, 1969

DistRIBUCiÓN: Etiópica (Tanzania y Senegal).

-Galumnella (G.) tanzanica Mahunka, 1984

DisTRIBUCión: Tanzania.

-Galumnella (G.) woschitzi Balogh, 1970

Distribución: Nueva Guinea.

Galumnella (Bigalumnella) Mahunka, 1994 (1 sp.)

ESPECIE TIPO: Bigalumnella csavasorum Mahunka, 1994 DISTRIBUCIÓN: Oriental.

-Galumnella (Bigalumnella) csavasorum (Mahunka, 1994) (B.)

DisTRIBUCIÓN: Tailandia.

Galumnopsis Grandjean, 1931 (13 spp.)

EsPECIE TIPO: Galumnopsis holoscripta Grandejan, 1931

DisTRIBUCIÓN: Pantropical.

Galumnopsis (Galumnopsis) Grandjean, 1931 (8 spp.)

DISTRIBUCIÓN: Pantropical (excepto Oriental).

-Galumnopsis (G.) clavata Mahunka, 1983

DisTRIBUCIÓN: Tanzania.

-Galumnopsis (G.) holoscripta Grandjean, 1931

DisTRIBUCión: Panamá.

-Galumnopsis (G.) longisetus Mahunka, 1983

DisTRIBUCIÓN: Tanzania.
-Galumnopsis (G.) rostrata Balogh, 1962

DisTRIBUCIÓN: Tanzania.

-Galumnopsis (G.) ruginervis Balogh, 1962

DISTRIBUCIÓN: Tanzania.

-Galumnopsis (G.) sagitta Balogh, 1970

DistriBuCIÓN: Nueva Guinea.

-Galumnopsis (G.) secunda (Sellnick, 1923) (Galumnella)

DistrIBUCIÓN: Neotropical (Brasil y Panamá).

-Galumnopsis (G.) sellnicki Balogh, 1960

DISTRIBUCIÓN: Etiópica.

Galumnopsis (Porogalumnella) Balogh, 1968 (5 spp.)

EsPeCIE TIPO: Porogalumnella quadriporosa Balogh, 1968

DistriBución: Pantropical (excepto Neotropical).

-Galumnopsis (Porogalumnella) africana (Mahunka, 1978) (P.)

DISTRIBUCIÓN: Malgache (I. Mauricio).

-Galumnopsis (Porogalumnella) pulchella (Aoki y Hu, 1993) (P.)

DisTRIBUCIÓN: China suroriental.

-Galumnopsis (Porogalumnella) quadriporosa (Balogh, 1968) (P.)

DisTRIBUCIÓN: Nueva Guinea.

-Galumnopsis (Porogalumnella) reducta (Mahunka, 1995) (P.)

DISTRIBUCIÓN: Borneo.

-Galumnopsis (Porogalumnella) setosa (Balakrishnan y Haq, 1982) (P.)

DisTRIBUCIÓN: India (Kerala).

Iberogalumnella Arillo y Subías, 1993 (1 sp.)

EsPECIE TIPO: Iberogalumnella alandalusica Arillo y Subías, 1993

DISTRIBUCIÓN: Paleártica meridional.

-Iberogalumnella alandalusica Arillo y Subías, 1993

DISTRIBUCIÓN: Mediterránea occidental.

Monogalumnella Mahunka, 1986 (1 sp.)

EsPECIE TIPO: Monogalumnella neotricha Mahunka, 1986

DISTRIBUCIÓN: Etiópica.

-Monogalumnella neotricha Mahunka, 1986

DisTRIBUCIÓN: Tanzania.

Trypogalumnella Mahunka, 1995 (2 spp.)

ESPECIE TIPO: Trypogalumnella poronota Mahunka, 1995

DISTRIBUCIÓN: Oriental.

-Trypogalumnella densoporosa Mahunka, 1995

DISTRIBUCIÓN: Borneo.

-Trypogalumnella poronota Mahunka, 1995

DISTRIBUCIÓN: Borneo. 


\section{Comentarios al listado}

De las más de 10.000 especies descritas hasta ahora, y recopiladas en el listado previo, alrededor de 1.300 son consideradas como sinónimas, por lo que el número de especies (y subespecies) válidas en la actualidad se aproxima a las 9.000 (8.946 concretamente, 8.631 especies y 315 subespecies), aunque el número actual real sea seguramente algo menor ya que bastantes de ellas o son de difícil identificación, por lo que figuran como "species inquiriendae" (250), o posiblemente sean sinónimas de otras previamente descritas. Respecto a los géneros (y subgéneros) destacar el que el número de relativo sinonimias es todavía superior al de especies ya que se han descrito 1.564 de los que sólo 1.204 son válidos: 1.005 géneros y 199 subgéneros (es decir que cerca de la cuarta parte son considerados como sinónimos), los cuales se agrupan en 169 familias pertenecientes a 51 superfamilias.

De los tres grandes grupos de oribátidos existentes, los inferiores (que agruparía los Palaeosomata, Parhyposomata, Enarthronota, Mixonomata y Holosomata), los superiores (Brachypylina) picnonóticos y los superiores poronóticos, el más abundante es el de los superiores picnonóticos que agrupa alrededor de la mitad de los géneros y especies descritos: 542 géneros (y 92 subgéneros) y 3.913 especies (y 138 subespecies), de los que más de la cuarta parte son oppioideos (197 géneros y subgéneros, y 1.095 especies y subespecies). De los otros dos grupos destacar el hecho de que, aunque los oribátidos superiores poronóticos, teóricamente los más evolucionados, incluyen muchos más géneros y especies (321 géneros, 69 subgéneros, 2.747 especies y 109 subespecies) que los inferiores (142 géneros, 38 subgéneros, 1.972 especies y 68 subespecies), teóricamente los más primitivos, con el número de familias y superfamilias ocurre lo contrario (19 superfamilias y 38 familias de inferiores frente a 10 superfamilias y 44 familias de superiores poronóticos). Además muchas de las familias son de distribución cosmopolita o están presentes en varias regiones biogeográficas, y lo mismo se puede decir respecto a bastantes géneros, sobre todo los más ricos en especies, entre las que tampoco es raro encontrarlas de distribución cosmopolita, semicosmopolita o distribuidas en más de una región biogeográfica, sobre todo las pertenecientes a los oribátidos inferiores. Todo esto concuerda, desde el punto de vista evolutivo, con lo ya expuesto por HAMMER \& WALLWORK (1979) sobre la persistencia en la actualidad de un elevado número de géneros de distribución cosmopolita ya presentes en la Pangea (siendo originada la distribución actual de los oribátidos, en gran medida, por la deriva continental) y que han perdurado hasta nuestros días, y por lo comentado por WALLWORK (1984) que considera que un $30 \%$ de los géneros de oribátidos inferiores tienen una distribución cosmopolita, mientras que este número desciende a un $15 \%$ en los oribátidos superiores. Es decir que la mayoría de las familias de oribátidos inferiores, que ya debían de estar presentes en la Pangea, han llegado hasta nuestros días, motivo por el cual presentan una distribución cosmopolita, y lo mismo que ocurre, aunque en menor medida, con los géneros (y todavía en menor medida con las especies) en ellas incluidos; pero como ocurre con otras faunas relictas, la diversidad de géneros dentro de cada familia es menor que en en las familias de oribátidos superiores cuya evolución ha sido posterior; es por dicho motivo por el que, además, en las familias de oribátidos superiores es menor la proporción de géneros ( $\mathrm{y}$ menor todavía la de especies) cosmopolitas o ampliamente distribuidas, distribución que también podría deberse a otros factores distintos a los meramente evolutivos, como ponen de manifiesto SuBÍAs et al. (1992): dispersión por el aire, el agua, los animales o por la actividad humana.

Como es natural, debido a que es donde históricamente se ha prestado mayor atención a esta fauna, la región mejor conocida es la Paleártica con alrededor de 3.200 especies válidas, siendo seguida (en números redondos) por la Etiópica (1.500 especies), Neotropical (1.500 especies), Oriental (1.400 especies), Neártica (1.200 especies), Australiana (1.000 especies) y Antártica (100 especies). Es de destacar lo poco conocida que está la región Neártica ya que era de esperar un número de especies similar al de la Paleártica y lo presenta muy inferior; en cambio era esperable que eso sucediese así con las restantes regiones biogeográficas (excepto la Antártica que presenta un número muy reducido de especies).

En resumen, y para finalizar, a pesar de la utilidad del presente trabajo para futuros estudios biogeográficos y sobre biodiversidad para evaluar impactos ambientales y considerar espacios naturales a preservar, los número anteriores nos muestran la necesidad de incrementar el número de estudios taxonómicos y faunísticos sobre los ácaros oribáti- 
dos para poder ir aproximándonos al conocimiento real de dicha fauna que, con todos los riesgos que implica hacer prospectiva de futuro sobre un grupo todavía tan desconocido, posiblemente duplique el número real de especies al actualmente conocido aproximándose a las 20.000 , siendo probable que en cada región biogeográfica existan alrededor de 5.000 especies diferentes.

\section{AGRADECIMIENTOS}

Quisiera agradecer a los revisores de este trabajo, los Dres. Gonzalo Giribet y Wojciech Niedbala, sus sugerencias y comentarios, que sin duda han mejorado la redacción original. Asimismo, quiero hacer extensivo mi agradecimiento a la Dra. Marian Ramos, editora de Graellsia, por su apoyo en la edición del mansucrito y a Pepe Fernández por la maquetación del mismo.

\section{Referencias}

Abdel-Hamid, M. E., Bayoumi, B. M. \& Mohamad, A. I., 1980. Check-list of the oribatid mites (Acari: Oribatei) of Egypt. Bulletin of the Faculty of Science, Assiut University, 9: 139-157.

Alvarado, R., 1976. Código internacional de nomenclatura zoológica. H. Blume. Madrid.

AoKI, J., 1965-1967. Oribatiden (Acarina) Thailands. III. Nature and Life in Southeast Asia, 4(I, 1965): 129-193, 5(II, 1967): 189-207.

AoKI, J., 1995. Oribatid Mites of High Altitude Forests of Taiwan II. Mt. Nan-hu-ta Shan. Special Bulletin of the Japanese Society of Coleopterology, 4: 123-130.

Aoki, J., TAKAKU, G. \& ITO, F., 1994. Aribatidae, a new myrmecophilous mite family from Java. International Journal of Acarology, 20(1): 3-10.

Aoki, J., Yамамото, Y., Wen, Z., Wang, H. \& Hu, S., 1997. [A checklist of oribatid mites of China (Acari: Oribatida). First Report.] Bulletin of the Institute of Environmental Science and Technology, 23: 63-80. (en japonés).

BAKer, E. W. \& Wharton, G. W., 1952. An Introduction to Acarology. The Macmillan Company. New York. $465 \mathrm{pp}$.

BAlogh, J., 1958. Oribatides nouvelles de l'Afrique tropicale. Revue Zoologique et Botanique Africaine, 58: $1-34$.

BALOGH, J., 1962. Mission zoologique de l'I.R.S.A.C. en Afrique orientale. LXXV. Acari oribates. Annales $d u$ Musée Royal de l'Afrique Centrale. Zoologie, 110: 90-131.

BAlogh, J., 1968-1970. New oribatids (Acari) from New Guinea. (I-II). Acta Zoologica Academiae Scientiarum Hungaricae, 14(I, 1968): 259-285, 16(II, 1970): 291344.
BAlogh, J., 1972. The oribatid genera of the world. Akadémiai Kiadó. Budapest. 188 pp. + 71 láms.

BAlogh, J., 1987. A critique of Steffen Woas: Beitrag zur Revision der Oppioidea sensu Balogh, 1972 (Acari, Oribatei). Folia Entomologica Hungarica, 48: 17-20.

Balogh, J. \& BAlogh, P., 1984. A review of the Oribatuloidea Thor, 1929 (Acari: Oribatei). Acta Zoologica Hungarica, 30(3-4): 257-313.

Balogh, J. \& Balogh, P., 1987. A new outline of the family Lohmanniidae Berlese, 1916 (Acari, Oribatei). Acta Zoologica Hungarica, 33: 327-398.

Balogh, J. \& BAlogh, P., 1988-1990. The soil mites of the world 2-3. Oribatid mites of the Neotropical Region. Elsevier. Amsterdam-Oxford-New YorkTokio. I(2, 1988): 335 pp., II(3, 1990): 333 pp.

Balogh, J. \& Balogh, P., 1992. The oribatid mites genera of the world. Hungarian Natural History Museum. Budapest. I: 263 pp., II: 375 pp.

Balogh, J. \& Balogh, P., 1998. On the family Micreremidae Grandjean, 1954 (Acari, Oribatei). Opuscula Zoologica Budapest, 31: 17-23.

BAlogh, J. \& BAlogh, P., 1999. The extra-holarctic species of the Oripodidae Jacot, 1925 (Acari: Oribatei). Folia Entomologica Hungarica, 60: 21-43.

BAlogh, J. \& BAlogh, P., 2002. Identification keys to the oribatid mites of the extra-holarctic regions. Well-Press Publishing Limited. Miskolc. I: 453 pp., II: 504 pp.

Balogh, J. \& MahunKa, S., 1983. The soil mites of the world 1. Primitive oribatids of the Palaearctic Region. Elsevier. Amsterdam-Oxford-New York. 372 pp.

BAyoumi, B. M. \& Al-KhalifA, M. S., 1985. Oribatid mites (Acari) of Saudi Arabia. Fauna of Saudi Arabia, 7: 66-92.

Behan-Pelletier, V., 1993. Eremaeidae (Acari: Oribatida) of North America. Memoirs of the Entomological Society of Canada, 168: 1-193.

Behan-Pelletier, V., 1997a. The semiaquatic genus Tegeocranellus (Acari: Oribatida: Ameronothroidea) of North and Central America. The Canadian Entomologist, 129: 537-577.

Behan-Pelletier, V., 1997b. Oribatid mites of the Yukon. In: Danks, H. V. Insects of the Yukon. Biological Survey of Canada (Terrestrial Arthropods). Ottawa: 115-149.

Behan-Pelletier, V., 2001. Phylogenetic relationships of Hypozetes (Acari: Tegoribatidae). Acarology: Proccedings of the 10th International Congress. CSIRO Publishing, Melbourne: 50-57.

Behan-Pelletier, V., Paoletti, M. G., Bissett, B. \& StInNER, B. R., 1993. Oribatid mites of forest habitats in northern Venezuela. Tropical Zoology, Special Issue 1: 39-54.

Bernini, F., Carnevale, G., Bagnoli, G. \& Stouge, S., 2002. An early ordovician oribatid mite from the 
islands of Öland, Sweden. En: F. Bernini, R. Nannelli, Nuzzaci y E. de Lillo (eds.), Acarid phylogeny and evolution: Adapatation in mites and ticks. Kluwer Academic Publishers. Dordrecht: 45-47.

Bernini, F., Castagnoli, M. \& Nannelli, R., 1995. Check-list della specie della Fauna Italiana. 24. Arachnida. Acari. Edizioni Calderini. Bologna. 131 pp.

Buitendisk, A. M., 1945. Voorloopige Catalogus van de Acari in de Collectie-Oudemans. Zoologische Mededeelingen, 24: 281-391.

CHOI, S.-S., 1997. [Checklist of oribatid mites of Kore.] Korean Arachnology, 13: 83-104 (en coreano).

Colloff, M. J., 1993. A taxonomic revision of the oribatid mites genus Camisia. Journal of Natural History, 27: 1325-1408.

Colloff, M. J. \& Halliday, R. B., 1998. Oribatid mites. A catalogue of australian genera and species. Monographs on Invertebrate Taxonomy, 6: 1-223.

Corpuz-Raros, L. A., 1979. Philippine Oribatei (Acarina). I Preliminary list of species and description of forty new species. The Philippine Agriculturist, 62: 1-82.

Corpuz-Raros, L. A., 1992. Oribatid mites (Acari: Oribatida) from the Visayas and Palawan, Philippines. Asia Life Sciences, 1: 75-109.

ENGELMANN, H.-D., 1972-1988. Bibliographia oribatologica Nr. 5-21. Abhandlungen und Berichte des Naturkundemuseums Görlitz, 47(5, 1972): 15 pp., 48(6, 1973): 19 pp., 48(7, 1973): 20 pp., 49(8, 1975): 18 pp., 50(9, 1976): 19 pp., 51(10, 1977): 19 pp., 52(11, 1978): 23 pp., 53(12, 1979): 19 pp., 54(13, 1980): 28 pp., 55(14, 1981): 24 pp., 56(15, 1983): 27 pp., 57(16, 1983): 16 pp., 58(17, 1984): 37 pp., 59(18, 1985): 21 pp., 60(19, 1986): 27 pp., 61(20, 1987): 23 pp., 62(21, 1988): 31 pp.

EngelmanN, H.-D. \& Schwalbe, T., 1991. Bibliographia oribatologica 22. Abhandlungen und Berichte des Naturkundemuseums Görlitz, 64: 1-51.

Flogaitis, E., 1992. Catalogue of oribatid mites of Greece (Acari: Oribatida). Biologia Gallo-hellenica, 19: 29-54.

FUJIKAWA, T., 1991. [List of oribatid families and genera of the world.] Edaphologia, 46: 1-130 (en japonés).

Fujikawa, T., Fujita, M. \& AOKI, J., 1993. [Checklist of oribatid mites of Japan (Acari: Oribatida).] The Acarological Society of Japan, 2(1): 1-121 (en japonés).

Ghilyarov, M. S. \& Krivolutsky, D. A., 1975. [Claves de identificación de ácaros edáficos. Sarcoptiformes.] Nauka. Moscú. 492 pp. (en ruso).

GJELSTRUP, P., 1978. Oversigt over Danmarks pansermider (Acarina, Oribatei). Entomologiske Meddelelser, 46: 109-121.

Gjelstrup, P. \& Solhoy, T., 1994. Oribatid mites (Acari). In: Ingólfsson, A. (ed.). The Zoology of
Iceland. III, 57. Steenstrupia, Zoological Museum, University of Copenhagen. Copenhagen. 78 pp.

Golosova, L., Karppinen, E. \& Krivolutsky, D. A., 1983. List of oribatid mites (Acarina, Oribatei) of northern Palaearctic Region. II. Siberia and the Far East. Acta Entomologica Fennica, 43: 1-14.

Grandjean, F., 1954. Essai de classification des Oribates (Acariens). Bulletin de la Société Zoologique de France, [1953], 78(5-6): 421-446.

HAMMEN, L. VAN DER, 1952. The Oribatei (Acari) of the Netherlands. Zoologische Verhandelingen, 17: 1-139.

HAMmer, M., 1944. Studies on the oribatids and collemboles of Greenland. Meddelelser om Gronland, 141: $1-210$.

HAMmer, M., 1971. On some oribatids from Viti Levu, the Fiji Islands. Det Kongelige Danske Videnskabernes Selskab Biologiske Skrifter, 16(6): 1$60+35$ láms.

HAMmer, M., 1972. Tahiti. Investigation on the oribatid fauna of Tahiti, and on some oribatids found on the Atoll Rangiora. Det Kongelige Danske Videnskabernes Selskab biologiske Skrifter, 19(3): 1$65+26$ láms.

Hammer, M., 1973. Oribatids from Tongatapu and Eua, the Tonga Islands, and from Upolu, Western Samoa. Det Kongelige Danske Videnskabernes Selskab Biologiske Skrifter, 20(3): 1-70 + 29 láms.

HAMMER, M., 1977. Investigations on the oribatid fauna of Norh-West Pakistan. Det Kongelige Danske Videnskabernes Selskab Biologiske Skrifter, 21(4): 1$72+34$ láms.

HAMmer, M., 1979. Investigations on the oribatid fauna of Java. Det Kongelige Danske Videnskabernes Selskab Biologiske Skrifter, 22(9): 1-79 + 47 láms.

Hammer, M. \& Wallwork, J. A., 1979. A Review of the World Distribution of Oribatid Mites (Acari: Cryptostigmata) in Relation to Continental Drift. Det Kongelige Danske Videnskabernes Selskab Biologiske Skrifter, 22(4): 1-31.

Karppinen, E. \& Krivolutsky, D. A., 1982. List of oribatid mites (Acarina, Oribatei) of northern Palaearctic Region. I. Europe. Acta Entomologica Fennica, 41: 1-18.

Karppinen, E., Krivolutsky, D. A. \& Poltavskaja, M. P., 1986. List of oribatid mites (Acarina, Oribatei) of northern Palaearctic Region. III. Arid lands. Annales Entomologici Fennici, 52: 81-94.

Karppinen, E., Krivolutsky, D. A., TARba, Z. M., Shtanchaeva, U. Y. \& Gordeeva, E. W., 1987. List of oribatid mites (Acarina, Oribatei) of northern Palaearctic Region. IV. Caucasus and Crimea. Annales Entomologici Fennici, 53: 119-137.

Karppinen, E., Melamud, V. V., Miko, L. \& KrivoLUTSKY, D. A., 1992. Further information onthe oribatid fauna (Acarina, Oribatei) of the northern 
Palaearctic Region: Ukraina and Czechoslovakia. Entomologica Fennica, 3: 41-56.

Kunst, M., 1971. [Oribatei.] [In: Claves para la fauna de Checoslovaquia, vol. 4.] Klic zvireny CSSR. Praha. pp. 531-580 (en checo).

Luxton, M., 1985. Cryptostigmata (Arachnida: Acari) A concise review. Fauna of New Zealand, 7: 1-106.

LuXTON, M., 1987. The oribatid mites (Acari: Cryptostigmata) of J. E. Hull. Journal of Natural History, 21 : 1273-1291.

MahunKA, S., 1986-1987. A survey of the family Carabodidae C. L. Koch, 1836 (Acari: Oribatida). (III). Acta Zoologica Hungarica, 32(I, 1986): 73-135, 33(II, 1987): 399-434.

MahunKA, S., 1994. Further notes, additions and redescriptions of the Oribatid species preserved in the Berlese Collection (Acari: Oribatida), II. Folia Entomologica Hungarica, 55: 233-261.

MahunKA, S., 2002. A survey of the Oribatida fauna of Madagascar (Acari: Oribatida). Folia Entomologica Hungarica, 63: 5-16.

MahunKA, S. \& MAhunKA-PAPP, L., 1995. The oribatid species described by Berlese (Acari). Hungarian Natural History Museum. Budapest. 325 pp.

MahunKa, S. \& Mahunka-Papp, L, 2000. Checklist of the oribatid mites of Hungary (Acari: Oribatida). Folia Entomologica Hungarica, 61: 27-53.

Marshall,V. G., Reeves, R. M. \& Norton, R. A., 1987. Catalogue of the Oribatida (Acari) of continental United States and Canada. Memoirs of the Entomological Society of Canada, 139: 1-418.

MartíneZ, P. A. \& Velis, G. J., 2000. Listado de los oribátidos (Acari: Oribatida) de la República Argentina. Revista de la Sociedad Entomológica Argentina, 59: 119-134.

Michael, A. D., 1898. Oribatidae. Das Tierreich. 3. Acarina. Berlin. 93 pp.

NiedBala, W., 1992. Phthiracaroidea (Acari, Oribatida). Systematic Studies. PWN-Polish Scientific Publishers, Warszawa \& Elsevier, Amsterdam-OxfordNew York-Tokyo. 612 pp.

NiedBala, W., 1998. Ptytimous mites of the Pacific Islands. Recent konwledge, origin, descriptions, redescriptions, diagnoses and zoogeography (Acari: Oribatida). Genus, 9: 431-558.

Niedbala, W., 2000. The ptyctimous mites fauna of the Oriental and Austalian regions and their centres of origin (Acari: Oribatida). Genus (supplement). Wroclaw. 493 pp.

Niedbala, W., 2001. Study on the diversity of Ptyctimous mites (Acari, Oribatida) and quest for centres of its origin: the fauna of the Ethiopian region. Monographs of the Upper Silesian Museum, 3: $1-245$.
Niedbala, W., 2002. Ptyctimous mites (Acari, Oribatida) of the Nearctic region. Monographs of the Upper Silesian Museum, 4: 1-261.

Niemi, R., Karppinen, E. \& Uusitalo, M., 1997. Catalogue of the Oribatida (Acari) of Finland. Acta Zoologica Fennica, 207: 1-39.

Norton, R. A., Bonamo, P. M., Grierson, J. D. \& SHEAR, W. A., 1988. Oribatid mite fossils froma a terrestrial devonian deposit near Gilboa, New York. Journal of Paleontology, 62(2): 259-269.

Olszanowski, Z., Rajski, A. \& Niedbala, W., 1996. [Oribatida.] In: Catalogus faunae Poloniae. XXXIV, 9, Acari. Polska Akademia Nauk. Instytut Zoologii. Poznan. 241 pp. (en polaco).

Oudemans, A. C., 1927. Notizen über Acari. 27. Reihe (Oribatidae). Archiv für Naturgeschichte, 91: 120-147.

Oudemans, A. C., 1937. Oribatei Dugès X 1833. Kritisch Historisch Overzicht der Acarologie. Leiden. F: 2521-2735.

Paulitchenko, P. G., 1994. [A guide to the ceratozetoid mites (Oribatei, Ceratozetoidea) of Ukraine.] Shmalhausen Institute of Zoology. Kiev. 143 pp. (en ruso).

Pérez-Íñigo, C., 1993. Acari, Oribatei, Poronota. In: Ramos, M. A. et al. (eds.). Fauna Ibérica, vol. 3. Museo Nacional de Ciencias Naturales, CSIC. Madrid. 320 pp.

Pérez-ÍÑIGO, C., 1997. Acari, Oribatei, Gymnonota I. In: Ramos, M. A. et al. (eds.). Fauna Ibérica, vol. 9. Museo Nacional de Ciencias Naturales, CSIC. Madrid. 374 pp.

SANYAL, A. K., 1992. Oribatid mites (Acari). Fauna of West Bengal, 3. State Fauna Series, 3: 213-356.

SANYAL, A. K., 1995. Oribatid mites (Acari: Cryptostigmata). Fauna of Himalaya, 2. State Fauna Series, 4: 51-91.

SANYAL, A. K. \& BhadURI, A. K., 1986. Check list of oribatid mites (Acari) of India. Records of the Zoological Survey of India. Miscellaneous publication. Occasional paper, 83: 1-79.

Schatz, H., 1983. Oribatei, Hornmilben. In: Catalogus Faunae Austriae. 9 i: U.-Ordn. Österreichischen Akademie der Wissenschaften. Wien. 115 pp.

ScHATZ, H., 1988. Oribatid mites of the Galapagos Islands - faunistics, ecology and speciation. Experimental and Applied Acarology, 22: 373-409.

SchATZ, H., 2001. The genus Eremaeozetes (Acari: Oribatida) on the Galápagos Islands. Acarologia, 41(4): 475-493.

SchWALBE, T., 1992-2001. Bibliographia oribatologica Nr. 23-31. Abhandlungen und Berichte des Naturkundemuseums Görlitz, 66(23, 1992): 22 pp., 67(24, 1993): 31 pp., 68(25, 1994): 28 pp., 68(26, 1995): 32 pp., 69(27, 1996): 24 pp., 70(28, 1997): 19 pp., 70(29, 1998): 48 pp., 71(30, 1999): 28 pp. у 72(31, 2001): $31 \mathrm{pp}$. 
Schwalbe, T., 2001. Oribatologica Nr. 32. Acari Bibliographia Acarologica. Oribatida, 1(2): 1-22.

SchwalBe, T. \& Franke, K., 2002. Oribatologica Nr. 33. Acari - Bibliographia Acarologica. Oribatida, 2(2): 5-19.

Schwalbe, T. \& Franke, K., 2003. Oribatida Nr. 34 Acari - Bibliographia Acarologica. Oribatida, 3(2): $1-24$.

SEllnick, M., 1960. Oribatei. Die Tierwelt Mitteleuropas. Quelle \& Meyer. Leipzig. III, 4: 45134.

SErgienKo, G. D., 1994. [Oribátidos Inferiores.] [In: Fauna de Ucrania, 25(21).] Academia de Ciencias de Ucrania. Kiev. 203 pp. (en ruso).

Shtanchaeva, U. Y., 2001. Catalog of oribatid mites (Acariformes, Oribatida) of the Caucasus. Acarina, 9(2): 177-221.

Stary, J. \& Block, W., 1998. Distribution and biogeography of oribatid mites (Acari: Oribatida) in Antarctica, the subantarctic islands andnearby land areas. Journal of Natural History, 32: 861-894.

SubÍAs, L. S. \& Arillo, A., 2001. Acari, Oribatei, Gymnonota II. In: Ramos, M. A. et al. (eds.). Fauna Ibérica, vol. 15. Museo Nacional de Ciencias Naturales, CSIC. Madrid. 289 pp.

Subías, L. S. \& Arillo, A., 2002. Oribatid mite fossils from the Upper Devonian of South Mountain, New York and the Lower Carboniferous of County Antrim, Northern Ireland (Acariformes, Oribatida). Estudios del Museo de Ciencias Naturales de Álava, 17: 93-106.

Subías, L. S., Arillo, A. \& Gil-Martín, J., 1992. Consideraciones biogeográficas sobre los oribátidos (Acari, Oribatida) de Marruecos y Sahara Occidental. Historia Natural'91, Palma de Mallorca, 1: 189-202.

Subías, L. S., Arillo, A. \& Gil-Martín, J., 1994. Oribátidos de Marruecos y Sahara Occidental. II. Listado de especies (Acari, Oribatida). Boletín de la Real Sociedad Española de Historia Natural (Sección Biológica), 91: 129-134.

SubíAs, L. S. \& BAlogh, P., 1989. Identification keys to the genera of Oppiidae Grandjean, 1951 (Acari: Oribatei). Acta Zoologica Hungarica, 35: 355-412.

SubíAs, L. S. \& Gil-Martín, J., 1997. Systematic and biogeographic checklist of oribatids from Western Mediterranean (Acari, Oribatida). Annali del Museo Civico di Storia Naturale "G. Doria”, 91: 459-498.
Subías, L. S., Zaballos, J. P., Banda, E., FontalCazalla, F. \& Nieves-Aldrey, J. L., 2004. Ácaros oribátidos (Acari: Oribatei) del Parque Nacional de la isla de Coiba, Panamá. Revista de Biología Tropical, 52(1): en prensa.

Swift, S. F. \& Norton, R. A., 1998. Preliminary report on oribatid mite (Acari: Oribatida) diversity inthe Hawaiian Islands. Bishop Museum. Occasional Papers, 57: 1-44.

Travé, J., 1960. Contribution a l'etude de la faune de la Massane (3e note) Oribates (Acariens) 2e partie (I). Vie et Milieu, 11(2): 209-232.

Travé, J., André, H. M., Taberly, G. \& Bernini, F., 1996. Les Acariens Oribates. Agar \& Sialf. Wavre. $110 \mathrm{pp}$.

TsenG, Y.-H., 1982-4. Taxonomical study of oribatid mites from Taiwan (Acarina: Astigmata) (I-II). Chinese Journal of Entomology, 2(I, 1982): 53-106, 4(II, 1984): 27-74.

VASILIU, N. \& IVAN, O., 1995. Oribatid mites from Israel. In: Soil Fauna of Israel, vol. 1. Academiei Române. Bucuresti: 69-86.

VAsiliu, N., IVAN, O. \& VAsiliU, M., 1993. [The faunistic synopsis oribatids (Acarina: Oribatida) from Romania.] Suceava. Anuarul Muzeului Bucovinei, 12: 1-72 (en rumano).

Vu, Q. M. \& VuOng, T. H., 1995. [The list of oribatid mites (Acari: Oribatei) from Vietnam.] Vietman National Centre for Natural Science and Technology. Journal of Biology, 17: 49-55 (en vietnamita).

WALlWORK, J. A., 1961. An annotated check-list of the oribatid mites of Ghana (Acari: Oribatei). Revue de Zoologie et Botanique Africaine, 64: 49-64.

WAllwork, J. A., 1984. Perspectives in acarine biogeography. Acarology, 6(1): 63-70.

Zoological Record, 1952-1974. Arachnida. The Zoological Society of London, 87-107 (12) (19501970). 
Nacional de Ciencias Naturales y

Consejo Superior de Investigaciones Científicas Licencia Creative Commons 3.0 España (by-nc) 


\section{ÍNDICE ALFABÉTICO DE NOMBRES TAXONÓMICOS}

En este apartado se presentan todos los géneros (y subgéneros), así como las especies (y subespecies), conocidos, ordenados alfabéticamente (con autor y año de descripción) para su mejor localización en el texto e indicando, en su caso, si son considerados sinónimos y de quien. En un primer apartado se relacionan los géneros y subgéneros diferenciándose estos últimos porque entre el nombre del taxon y el del autor figura entre paréntesis, y con distinto tipo de letra, el del género al que pertenece. Por lo que respecta al apartado de especies, tras el epíteto específico figura, entre paréntesis, el género o subgénero en el que se encuentra actualmente adscrito, y en el caso de subespecies tras el género (o subgénero) figura el epíteto de la especie a la que se subordina. Para una mayor utilidad de este segundo apartado se ha optado por expresar con distintos tipos de letra las diferentes distribuciones biogeográficas básicas, a saber: "redonda" para los táxones Paleárticos, “cursiva" para los táxones Neárticos, "negrita" para los táxones Etiópicos, "redonda subrayada" para los táxones Orientales, "negrita subrayada” para los táxones Australianos, "cursiva subrayada" para los táxones Antárticos, "negrita cursiva" para los táxones Neotropicales y "negrita cursiva subrayada" en el caso de sinonimias. En este último caso la especie figura en el género en el que se encuentra incluida en la actualidad, mientras que en el listado viene con el nombre del género en el que fue descrita. Como hay solapamientos entre distintas de las distribuciones biogeográficas se ha optado por considerar toda China y el Himalaya como región Oriental, al igual que las islas subantárticas de San Pablo y Ámsterdam; la Florida de U.S.A. se incluye en la Neotropical, así como las islas subantárticas de Las Malvinas (I. Falkland) y la Tierra del Fuego, y lo mismo se ha hecho con las Islas de Tristan de Cunha, incluidas en la región Etiópica.

\section{ÍNDICE ALFABÉTICO DE GÉNEROS (Y SUBGÉNEROS) DE ORIBÁTIDOS}

\begin{tabular}{|c|c|}
\hline $\begin{array}{l}\text { Abchasiella Gordeeva y Tarba, } 1990 \ldots \ldots . . . . .19,124 \\
\text { Acanthobelba Enami y Aoki, } 1993 \ldots \ldots \ldots . .16,77\end{array}$ & $\begin{array}{l}\text { trhypochthonius Balogh, } 1958 \\
\text { (=Eohypochthonius) }\end{array}$ \\
\hline canthozetes Balogh, $1958 \ldots \ldots \ldots \ldots \ldots \ldots \ldots . . . . . .16,87$ & s Wallwork, 1965 \\
\hline caroceras Grandjean, $1936 \ldots \ldots \ldots \ldots \ldots \ldots \ldots . . . . . . . . . . . . .16,87$ & $(=$ Farchacarus $) \ldots \ldots \ldots . .$. \\
\hline ychus Grandjean, 1932 & Africoribates Evans, 1953 \\
\hline $1885 \ldots \ldots \ldots \ldots \ldots \ldots . . . . . .10,22$, & Afroleius Mahunka, 1984 \\
\hline chipterina Berlese, 1916 & Afroliochthonius (Liochthonius) \\
\hline ) Subías y & 13,31 \\
\hline Rodr & hrus Wallwork, 1961 ......... \\
\hline an, 1956 & Afrotoc \\
\hline 916 (=Crotonia) $\ldots 15$, & Afrotriti \\
\hline 28 & Afroze \\
\hline ...... 20, & Akans \\
\hline 45 & $\mathrm{Akr}$ \\
\hline & \\
\hline & .. 21,156 \\
\hline …......... 18, & Albo \\
\hline ......... 25 & $(=\mathbf{T}$ \\
\hline 7 ........ 1 & Alciop \\
\hline 1932 & Aleur \\
\hline ..... 21 & Alisn \\
\hline 967 & $1 \ldots \ldots .1$ \\
\hline ...... 1 & $\ldots \ldots \ldots \ldots \ldots . . .1$ \\
\hline $84 \ldots \ldots \ldots \ldots \ldots \ldots . . .15,75$ & Allog \\
\hline an, 1932 & ..... 23, 182 \\
\hline & Allo \\
\hline $\begin{array}{l}\text { egyptogalumna Al-Assiuty, Abdel-Hamid, Seif, } \\
\text { El-Deeb, } 1985\end{array}$ & \\
\hline ka, 1978 & Allori \\
\hline$(=\mathrm{C}$ & \\
\hline …................. 112 & Allosuctobelba Moritz, 1970 \\
\hline Aethioppia Balogh, 1983 . & Allozetes Berlese, 1913 ......... \\
\hline & \\
\hline$(=$ Triteremella $)$ & (=Kalyptrazetes) .. \\
\hline
\end{tabular}

Allozetes Feider, Vasiliu y Calugar, 1971

(=Feiderzetes) (................................... 23, 182

Alphypochthonius Schweizer, 1956

(=Melanozetes) ….......................... 23, 176

Alpizetes Mahunka, 2001 .................... 23, 182

Altrhypochthonius Weigmann, 1997

(=Mainothrus) …............................. 15, 59

Amazoppia Balogh y Mahunka, 1969 ...... 17, 93

Amerigloboppia (Neoamerioppia)

Subías, 1989 ..................................... 19, 114

Amerioppia Hammer, 1961 .................... 18, 113

Amerobelba Berlese, 1908 ..................... 17, 101

Ameronothrus Berlese, 1896 ................... 21, 156

Amerus Berlese, 1896 .......................... 18, 104

Ametroproctus Higgins y

Woolley, 1968 .............................. 7, 21, 159

Amnemochthonius Grandjean, 1949 ......... 13, 34

Amolops Hull, 1916 (=Ramusella) ........ 19, 118

Ampullobates Grandjean, 1962 ............... 15, 70

Amuracarus Lange, 1975 .......................... 13, 27

Anachipteria Grandjean, 1932 ............... 22, 168

Anakingia Hammer, 1961 ...................... 16, 87

Anarea Dalenius, 1958 (=Halozetes) $\quad \ldots . .21,156$

Ancestroppia (Neotrichoppia)

Subías y Rodríguez, 1986 .................. 19, 127

Andacarus Grandjean, 1958

(=Stomacarus) ….............................. 13, 27

Anderemaeus Hammer, 1958 .................. 18, 105

Andesamerus Hammer, 1962 ................. 18, 105

Andesperuviella Paschoal, 1989

(=Pheroliodes) …............................... 15, 73

Andeszetes (Scheloribates) Hammer,

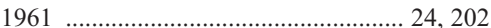

Anellozetes Hammer, 1962 .................... 23, 180

Angelia Berlese, 1885 (=Nothrus) .......... 15, 63 
Angullozetes Hammer, 1967 .................. 24, 195 Angulogalumna (Galumna) Grishina,

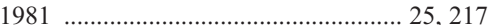

Anisochthodes Newell, 1957

(=Tuberemaeus)

Annobonzetes Pérez-Íñigo, 1983 ............ 24, 196

Anomaloppia Subías, 1978 ……………. 19, 116

Anoplozetes Lee y Pajak, 1987 .............. 23, 184

Anoribatella Kunst, 1962

(=Tectoribates) $\ldots . . \ldots \ldots \ldots . . .22,172$

Anoripoda Sellnick, $1959 \quad$....................... 24, 203

Antarcticola Wallwork, 1967 .................. 21, 156

Antarctoplophora Mahunka, 1980

(=Neophthiracarus) ……………….... 14, 56

Antarctozetes Balogh, 1961

(=Humerobates) …………………..... 23, 181

Antennoppia (Lasiobelba)

Mahunka, 1983

18,113

Antillobodes Mahunka, 1985

(=Phyllocarabodes) ……................... 21, 151

Antilloppia Mahunka, 1985 (=Neoppia) .. 18,109

Aokibates (Peloribates) Mahunka, 1988 .. 25, 211

Aokiella (Odontocepheus) Balogh y

Mahunka, 1967

21,153

Aphelacarus Grandjean, 1932 .................. 13, 28

Apograptoppia (Graptoppia) Subías y

Rodríguez, $1985 \ldots \ldots \ldots \ldots \ldots \ldots \ldots \ldots \ldots \ldots . . .19,116$

Apolohmannia (Perlohmannia)

Aoki, 1960

13,36

Apoplophora Aoki, 1980 .......................... 14, 41

Apotomocepheus Aoki, 1965 ................... 21, 148

Apotritia Norton y Lions, 1992 ................. 14, 47

Aquanothrus Engelbrecht, 1975 .............. 21, 156

Arborichthonius Norton, 1982 .................. 13, 29

Arceremaeus Hammer, 1961 ................... 18, 108

Archegocepheus Aoki, 1965 .................. 21, 148

Archegotocepheus Mahunka, 1988

(=Megalotocepheus) ………………... 21, 147

Archegozetes Grandjean, 1931 ................ 14, 59

Archeonothrus Trägardh, 1906 .................. 13, 27

Archeremella Balogh y Mahunka, 1974 .. 18, 106

Archiphthiracarella Mahunka, 1996

(=Archiphthiracarus) …………….... 14, 54

Archiphthiracarus (Phthiracarus) Balogh y

Mahunka, 1979 .................................... 14, 54

Archoplophora Hammen, 1959 ................ 14, 41

Arcoppia Hammer, 1977 ........................ 19, 120

Arcozetes Hammer, 1958 ........................ 22, 172

Arenozetes Krivolutsky, 1971 .................. 16, 87

Areozetes Hammer, 1961 ......................... 24, 195

Argentinobates (Hydrozetes)

Fernández, 1984

... 21,156

Argentinovertex Fernández y

Cleva,2001(=Arthrovertex) $\quad$............... 21, 160

Aribates Aoki, Takaku e Ito, 1994 ........... 17, 101

Arotrobates Luxton, $1992 \quad$....................... 21, 157

Arphthicarus Niedbala, 1994

(=Notophthiracarus)

14,50

Arthrhoplophora Berlese, 1910 ................. 13, 35

Arthrochthonius Ewing, 1917

(=Hypochthoniella)

13, 29
Arthrodamaeus Grandjean, 1954

Arthronothrus Trägardh, 1910

(=Eulohmannia)

Arthrovertex Balogh, 1970

Asiacarus Krivolutsky, 197

(=Thamnacarus) ................................. 14, 41

Asperemaeus Behan-Pelletier, $1982 \quad$........ 17, 99

Astegistes Hull, 1916 ............................... 17, 92

Atalotegaeus Luxton, 1988 ...................... 16, 84

Atopochthonius Grandjean, 1949 ............ 13, 35

Atropacarus Ewing, 1917 .......................... 14, 48

Ausoribula Lee, 1992 ............................. 24, 191

Austracarus J. y P. Balogh, 1983

(=Ozacarus)

14,40

Austracarus Mahunka, 1984

(=Ululohmannia)

Austrachipteria Balogh y

Mahunka, 1966

Austrocarabodes Hammer, 1966 ............ 21, 148

Austroceratobates Mahunka, 1985

(=Limnozetella)

22,165

Austroceratoppia Hammer, 1979 ............... 17, 93

Austrodamaeus Balogh y Mahunka, 1981 .. 15, 76

Austroeremulus Mahunka, 1985 ............ 17, 102

Austrogneta Balogh y Csiszár, 1963 ...... 18, 108

Austronothrus Hammer, 1966 ................... 15, 64

Austrophthiracarus Balogh y Mahunka, 1978

(=Neophthiracarus) ………………..... 14, 56

Austroppia Balogh, 1983 ........................ 19, 121

Austrotritia Sellnick, 1959 ........................ 14, 42

Austrozetes Balogh y Mahunka, 1969

(=Rhopalozetes)

17,90

Autogneta Hull, 1916 ...................... 18, 108

Autoppia Golosova y Karppinen, 1983 .. 19, 124

Baioppia (Lanceoppia) Luxton, 1985 .... 18, 110

Bakobodes Mahunka, 1996

(=Pseudocarabodes)

21,153

Balazsella Mahunka, 1983 ........................ 24, 192

Baloghacarus Mahunka, 1983 .................. 15, 70

Baloghia Mahunka, 1994 ......................... 25, 207

Baloghiella Bulanova-Zachvatkina,

1960 ………………………….........25, 207

Baloghobates Hammer, 1967

(=Humerobates) ................................ 23, 181

Baloghodes (Austrocarabodes)

Mahunka, 1986 ................................ 21, 149

Baloghoppia Mahunka, 1983 ................. 19, 128

Balzania Jacot, 1929 (=Conoppia) .......... 16, 83

Banksia Oudemans y Voigts, 1905

(=Xenillus)

. 17, 97

Banksinoma Oudemans, 1930 ................ 18, 109

Banksinus Jacot, 1938 (=Humerobates) .. 23, 181

Baobabula Mahunka, 1975 …..............24, 192

Basiceramerus Corpuz-Raros, 1979 ......... 20, 146

Basidoppia Mahunka, 1983 …................ 19, 121

Basilobelba Balogh, 1958 ...................... 18, 104

Basiloppia Balogh, 1983 …………….... 18, 110

Bathocepheus Aoki, $1978 \quad$......................... 21, 149

Beckiella Grandjean, 1964 ..................... 20, 140

Beklemishevia Zachvatkin, 1945 .............. 13, 27

Belba Heyden, $1826 \ldots \ldots \ldots \ldots \ldots \ldots \ldots \ldots \ldots \ldots . . .16,77$

Belbodamaeus Bulanova-Zachvatkina,

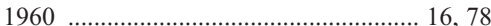

Belemacarus Pérez-Íñigo y Baggio,

1997 ................................................ 22, 172

Belloppia Hammer, 1968 ....................... 19, 125

Belorchestes Grandjean, 1951 ................. 17, 91

Benoibates Balogh, 1958 ........................ 24, 203

Berlesezetes Mahunka, 1980 .................. 16, 87

Berlesiella Hammer, 1979 ..................... 25, 207

Berndamerus Mahunka, 1977 ............... 17, 101

Berndbella Mahunka, 1985

(=Caveremulus) …............................ 17, 102

Berndia Mahunka, 1988 (=Topobates) .. 24, 202

Berndibula Mahunka, 2000 ................... 24, 195

Berndobodes (Yoshiobodes)

Mahunka, 1986 ............................... 21, 154

Berndotritia Mahunka, 1987

(=Oribotritia) ..................................... 14, 44

Berniniella Balogh, 1983 ..................... 19, 125

Besuchetacarus (Notophthiracarus) Mahunka,

1985 ............................................. 14, 52

Bicristoppia (Lanceoppia) Subías, 1989 .. 18, 111

Bicyrthermannia Hammer, 1979 ............. 15, 67

Bigalumnella (Galumnella)

Mahunka, 1994 ............................... 25, 222

Bioppia Mahunka y Paoletti, 1984

(=Ramusella) …................................. 19, 118

Bipassalozetes Mihelcic, 1957 ........ 22, 161, 162

Birobates Balogh, 1970 ......................... 24, 203

Birotegaeus Luxton, 1988 ....................... 16, 85

Birsteinius Krivolutsky, 1965 ................ 17, 95

Bischeloribates Mahunka, 1988

(=Scheloribates) ….......................... 24, 198

Bolkiah Mahunka, 1997 ......................... 25, 207

Boreozetes Hammer, 1955

(=Neogymnobates) .......................... 23, 177

Borhidia Balogh y Mahunka, 1974 ........ 20, 133

Bornebuschia Hammer, 1966 .............. 7, 16, 84

Bornemiszaella P. Balogh, 1994 ........... 20, 131

Borneozetes Mahunka, 1997 ................. 25, 208

Borneremaeus Mahunka, 1991 ............... 20, 141

Brachioppia Hammer, 1961 ................... 19, 122

Brachioppiella Hammer, 1962 ............... 19, 122

Brachychochthonius Jacot, 1938

(=Brachychthonius) ........................... 13, 29

Brachychthonius Berlese, 1910 ............... 13, 29

Brachyoripoda Balogh, 1970 ................... 24, 203

Brasiliotritia (Euphthiracarus)

Märkel, 1964 ...................................... 14, 47

Brasilobates Pérez-Íñigo y Baggio, 1980

(=Triaungius) ….............................. 25, 206

Brasiloppia Pérez-Íñigo y Baggio, 1986 .. 20, 132

Brassiella Balogh, 1970 ....................... 24, 192

Brassoppia Balogh, 1983 ..................... 19, 122

Brazilozetes Balogh y Mahunka, 1969 .... 16, 88

Bruneibelba Mahunka, 2001

(=Ussuribata)

20,138

Bruneiella Mahunka, 1997

(=Sacculobates)

15,71

Bukitritia Mahunka, 1990 ....................... 14, 46

Bulbocepheus Mahunka, 1988 ................ 20, 141

Bulleremaeus Hammer, 1966 ................. 21, 158

Bursoplophora Subías y Pérez-Íñigo,

1978

13,35 
Caenobelba (Belba) Norton, 1980 ............ 16, 78 Caenosamerus Higgins y Woolley, 1969 .. 18, 105 Caleremaeus Berlese, 1910

Calhoplophora Berlese, 1923

(=Tropacarus)

Calipteremaeus Paschoal, 1987 ............... 15, 73

Calobates Balogh, 1961

Caloppia Balogh, 1958

(=Chaunoproctus)

24, 203

Calozetes Balogh y Mahunka, 1969 ........ 16, 88

Calugarella J. y P. Balogh, 1992 ............ 23, 185

Calvoppia Jacot, 1934 (=Phauloppia) .. 23, 190

Calyptophthiracarus (Notophthiracarus)

Aoki, 1980 ......................................... 14, 52

Calyptozetes (Mycobates) Thor, $1930 \quad$.. 23, 183

Camisia Heyden, 1826 ............................. 15, 64

Campachipteria Aoki, 1995 .............. 10, 22, 168

Campbellobates Wallwork, 1964 ............ 24, 204

Campbelloppia (Solenoppia) Luxton,

1985 ............................................... 19, 124

Canaloppia (Berniniella) Mahunka y Palacios-

Vargas, 1998 ...................................... 19, 125

Canaribates Pérez-Íñigo y Peña, 1994

(=Incabates)

25,209

Cantharozetes Hammer, 1961 ............... 25, 208

Capillibates Hammer, 1966 .................... 21, 156

Capillonothrus (Heminothrus) Kunst, 1971

.. 15,66

Capilloppia Balogh y Mahunka, 1966 .... 23, 186 Capillozetes Balogh, 1943

(=Peloribates) ….............................. 25, 209

Carabocepheus Berlese, $1910 \quad$................ 21, 154

Carabodella Mahunka, 1986

(=Hardybodes)

21,152

Carabodes Koch, 1835 .................... 10, 21, 149

Carabodoides Jacot, 1937

(=Epieremulus) ............

Carabozetes Mihelcic, 1957

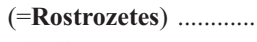

18,106

Cardioribates Jacot, 1934

(=Podoribates) $\quad$................................... 23, 186

Carinabella Hammer, 1977 ..................... 17, 100

Carinogalumna Engelbrecht, 1973 ......... 25, 214

Carolohmannia (Lohmannia) Norton, Metz y

Sharma, 1978 ..................................... 14, 39

Cassioppia Poltavskaya, 1994

(=Lalmoppia) …................................. 19, 130

Caucasiozetes Shtanchaeva, 1984 ........... 16, 88

Caudamaeolus P. Balogh, 1988 ............. 18, 102

Caveremulus Mahunka, 1983 ............... 17, 102

Cavernella (Oribatella) Bernini, 1975 .. 22, 172

Cavernocarabodes Mahunka, 1974 ........ 21, 151

Cavernocepheus Balogh y Mahunka,

1969

20,141

Cavernozetes Mahunka, 1984 ................. 16, 88

Celaeno Koch, 1835 (=Eupelops) .......... 22, 162

Centroribates Berlese, 1914 ................... 25, 214

Cepheus Koch, 1835 ............................. 16, 82

Cerachipteria Grandjean, 1935 .............. 22, 168

Ceratobates Balogh y Mahunka, 1969 .... 22, 166

Ceratokalumma Balogh, 1970

(=Achipterina)

22,172
Ceratoppia Berlese, 1908

Ceratoppiella Hammer, 1977

17,93

Ceratotenuiala Aoki y Maruyama, 1983 .... 17, 99

Ceratozetella Shaldybina, 1966 .............. 22, 173

Ceratozetes Berlese, 1908 .................... 22, 174

Ceratozetoides Shaldybina, 1966

(=Ceratozetella)

22,173

Ceresella Paulitchenko, 1993

(=Ceratozetella)

22,173

Cerocepheus Trägardh, 1931 ............... 7, 16, 84

Ceroribatula Lee y Birchby, 1989

(=Oribatula)

23, 187

Cerostocepheus Mahunka, 1973 ............ 21, 146

Ceylobodes J. y P. Balogh, 1992

(=Trichocarabodes)

21,153

Chamobates Hull, 1916 .......................... 23, 179

Chamozetes Sellnick, 1974

(=Ocesobates)

23,180

Charassobates Grandjean, 1929 ............... 16, 86

Chaunoproctellus Mahunka, 1992 .......... 24, 192

Chaunoproctus Pearce, 1906 ................. 24, 192

Chavinia Hammer, 1961 ......................... 18, 112

Cheloppia Hammer, 1971 ..................... 20, 130

Christovizetes Krivolutsky, 1975 ............. 16, 88

Chudalupia Wallwork, 1981 .................. 21, 156

Chuoppia Tseng, 1982 (=Arcoppia) ...... 19, 120

Cilioppia Balogh, 1983 (=Lasiobelba) .... 18, 113

Circellobates Luxton, 1992 .................. 21, 157

Clavazetes Hammer, 1966

(=Papillocepheus)

Claviceps Canestrini y Fanzago, 1877

(=Murcia) …..................................... 23, 177

Coartobelba (Novosuctobelba)

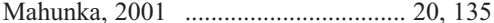

Coggiella Berlese, 1916 (=Joelia) ......... 22, 170

Collohmannia Sellnick, 1922 ................... 14, 36

Comeremaeus Hammer, 1962 .................. 17, 93

Comororibula (Berlesiella) Mahunka, 1994

25,207

Comorozetes Mahunka, 1994 ................. 16, 88

Compactozetes Hammer, 1966 ................ 16, 83

Conchogneta Grandjean, 1963 ............... 18, 108

Condylobelba Mahunka, 2001 ................ 20, 134

Condyloppia Balogh, 1983 ..................... 19, 116

Confinoppia (Neotrichoppia) Subías y

Rodríguez, 1986 ................................. 19, 127

Congocepheus Balogh, 1958 .................... 21, 151

Congoppia Balogh, 1983 ....................... 19, 123

Conoppia Berlese, 1908 ........................... 16, 83

Conozetes Balogh y Mahunka, 1969 ...... 25, 208

Constrictobates Balogh y Mahunka, 1966

24, 192

Constrictocepheus (Pseudotocepheus) Grobler,

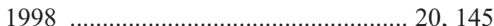

Convergoppia (Lanceoppia) Balogh,

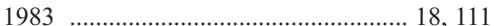

Cordiozetes Mahunka, 1983 ................. 24, 195

Cordylobates (Humerobates) Luxton, 1995

23,182

Coronibatula Mahunka, 1988 ................. 24, 196

Coronoquadroppia (Quadroppia)

Ohkubo, 1995
Coropoculia (Ametroproctus) Aoki y Fujikawa,

1972 ........................................ 7, 21, 159

Corynobates J. y P. Balogh, 1992

(=Euscheloribates) …....................... 24, 197

Corynoppia Balogh, 1983 ..................... 20, 130

Cosmobates Balogh, 1959 ..................... 24, 197

Cosmochthonius Berlese, 1910 ............... 13, 32

Cosmogalumna (Galumna) Aoki, 1988 .. 25, 217

Cosmogneta Grandjean, 1960 ............... 18, 109

Cosmohermannia Aoki y Yoshida, 1970 .... 15, 67

Cosmopirnodus Balogh, 1970 ............... 24, 204

Cosmoppia Balogh, 1983 (=Dissorhina) .. 19, 125

Cosmozetes Balogh y Mahunka, 1969 .... 16, 88

Costeremus Aoki, 1970 ........................ 18, 103

Crassoribatula Hammer, 1967 ................ 23, 186

Cretoppia Mahunka, 1986

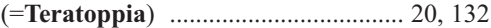

Cribrozetes Balogh, 1970 ........................ 25, 205

Cristamerus Hammer, 1977 ................... 18, 105

Cristeremaeus Balogh y Csiszár, 1963 .. 18, 106

Cristonothrus (Malaconothrus) subgen.

nov. (........................................ 7, 15, 61

Crotonia Thorell, 1876 .............................. 15, 66

Cryptacarus Grandjean, 1950 .................... 14, 38

Cryptobothria Wallwork, 1963 ............... 23, 182

Cryptogalumna Grandjean, 1957 ........... 25, 214

Cryptoplophora Grandjean, 1932 ............. 13, 35

Cryptoppia Csiszár, 1961 ...................... 19, 116

Cryptoribatula Jacot, 1934 ...................... 24, 205

Cryptozetes Hammer, 1962

(=Dometorina) …............................. 24, 192

Csibiplophora Mahunka, 1984

(=Bursoplophora) .............................. 13, 35

Ctenacarus Grandjean, 1939 .................. 13, 28

Ctenamerus J. y P. Balogh, 1992 ............ 18, 105

Ctenobelba Balogh, 1943 ...................... 17, 101

Ctenogalumna Balogh, 1961

(=Allogalumna) .............................. 25, 213

Ctenoppia Balogh, 1983 ........................... 19, 122

Ctenoppiella Gordeeva y Karppinen, 1988

(=Lauroppia) …................................ 19, 126

Cubabodes Balogh y Mahunka, 1974 .... 21, 151

Cubacepheus Balogh y Mahunka,1979

(=Reticulocepheus) ............................ 16, 84

Cubachipteria Balogh y Mahunka, 1979 .. 22, 169

Cubaoppia Balogh, 1983 ..................... 19, 116

Cultrobates Willmann, 1930 ................. 22, 172

Cultroribella Mahunka, 1985 ................... 17, 92

Cultroribula Berlese, 1908 ....................... 17, 92

Cultrozetes Sellnick, 1922 (=Astegistes) .. 17, 92

Cuneoppia Balogh y Mahunka, 1969 .... 20, 134

Cuspidozetes Hammer, 1962 ................. 22, 170

Cuspitegula Hammer, 1966 ..................... 16, 88

Cycloppia Balogh, 1983 ......................... 18, 110

Cylindroppia (Discoppia) Subías y Rodríguez,

1986 .............................................. 19, 123

Cymbaeremaeus Berlese, 1896 .............. 21, 158

Cyrthermannia Balogh, 1958 .................. 15, 67

Cyrtozetes (Ceratozetella) Behan-Pelletier,

1985 ............................................ 22, 174

Daedaloppia Hauser y Mahunka, 1983

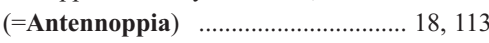


Damaeolus Paoli, 1908

Damaeoppia Ohkubo, 1995

(=Quinquoppia)

Damaeus Koch, 1835 ............................. 16, 78

Dameobelba Sellnick, 1928 ..................... 16, 80

Dameosoma Berlese, 1892 (=Oppia) ... 19, 114

Dampfiella Sellnick, 1931 ..................... 20, 141

Danobates Gjelstrup, 1978

(=Ocesobates)

23,180

Darthvaderum Hunt, 1996 ....................... 15, 73

Dasybelba Woolley y Higgins, 1979

(=Nododamaeus)

16,81

Decoribatula Lee y Birchby, 1989

(=Reticuloppia) ..........

Demisalto Coetzee, 1993 ....................... 23, 184

Dendracarus Balogh, 1961 ..................... 14, 38

Dendrohermannia P. Balogh, 1985 ......... 15, 68

Dendrozetes Aoki, 1970 .......................... 17, 93

Dentachipteria Nevin, 1974 .................... 22, 169

Dentizetes Hammer, 1952 ...................... 22, 175

Dentoppia Gordeeva, 2001

(=Moritzoppiella)

19,127

Diapterobates Grandjean, 1936 .............. 23, 181

Dicastribates J. y P. Balogh, 1988 ............ 15, 70

Dicatozetes Grandjean, 1956 ................. 25, 214

Dicondyla Aoki, 1965

(=Dolicheremaeus) ............................ 20, 141

Dicrotegaeus Luxton, 1988 ................. 7, 16, 84

Didierotocepheus (Afrotocepheus) Mahunka,

1994

20,141

Didymonycha Mahunka, 1984 ............... 25, 214

Dimidiogalumna Engelbrecht, 1972 ...... 25, 214

Dinoxenillus Pérez-Íñigo y Baggio, 1980

(=Xenillus)

.. 17, 97

Dinozetes Balogh, 1962 .......................... 16, 88

Diodontocepheus Mihelcic, 1958

(=Eupterotegaeus) …........................ 16, 83

Diorchestes Grandjean, 1951

(=Microzetorchestes) ........................ 17, 91

Diphauloppia J. y P. Balogh, 1984 .......... 23, 186

Diplobodes Aoki, 1958 ........................ 21, 152

Discoppia Balogh, 1983 ......................... 19, 123

Discosuctobelba Hammer, 1979

(=Ussuribata)

20,138

Disparagalumna Hammer, 1973 ............. 25, 214

Dissorhina Hull, 1916 ........................... 19, 125

Dividoppia (Subiasella) Mahunka, 1987 .. 19, 130

Dolicheremaeus Jacot, 1938 .................. 20, 141

Dometorina Grandjean, 1951 ................ 24, 192

Dorycranosus (Liacarus) Woolley, 1969 .... 17, 96

Drepanoppia Balogh, 1983

Drukoppia Gordeeva, 2001

(=Moritzoppiella)

18,110

Drymobates Grandjean, 1930

19,127

23, 185

Drymobatoides Jacot, 1936 ................... 23, 185

Dudichella Balogh, 1970 ......................... 16, 85

Dudichoplophora Mahunka, 1982 ........... 14, 42

Dynatozetes Grandjean, 1960 ................ 23, 185

Dyobelba (Subbelba) Norton, 1979 ........ 16, 82

Dysarcoppia (Mimoppia) Mahunka y Palacios-

Vargas, 1998
Dzarogneta (Oxyoppia) Kulijev, 1978 .. 19, 129

Edwardzetes Berlese, 1914 .................. 22, 175

Elapheremaeus Grandjean, 1944

(=Ctenobelba) .

Elaphoppia Balogh, $1983 \quad 19,126$

Ellipsozetes Bernini, 1980 .................... 23, 182

Elliptochthonius Norton, 1975 ................. 13, 28

Enantioppia Balogh y Mahunka, 1969 .. 20, 131

Eniochthonius Grandjean, 1933

(=Hypochthoniella)

.. 13, 29

Enisella Ayyildiz y Luxton, 1989 ............ 20, 131

Ensicamisia (Camisia) Kunst, 1971 ........ 15, 65

Entomotritia Märkel, 1964 (=Mesotritia) _. 14,43

Eobrachychthonius Jacot, 1936 ............... 13, 30

Eohypochthonius Jacot, 1938 .................. 13, 28

Epactozetes Grandjean, 1930 ................. 22, 173

Epidamaeus (Damaeus) Bulanova-Zachvatkina,

1957 .............................................. 16, 79

Epieremulus Berlese, 1916 .................. 18, 106

Epilohmannia Berlese, 1910 ................... 14, 36

Epilohmannoides Jacot, $1936 \quad$.................. 14, 37

Epimerella Kulijev, 1967 ....................... 20, 131

Eporibatula Sellnick, 1928

(=Phauloppia)

…................... 23, 190

Eremaeozetes Berlese, 1913 ................. 22, 165

Eremaeus Koch, 1835 ........................... 17, 100

Eremella Berlese, 1913

Eremobelba Berlese, 1908 ...................... 18, 103

18,106

Eremobodes Jacot, 1937 ...................... 18, 109

Eremulus Berlese, 1908 ......................... 17, 102

Erioppia Balogh, 1983 ........................ 18, 113

Erogalumna (Galumna) Grandjean, 1964

Ethiovertex Mahunka, 1982 .................. 22, 160

... 25, 217

Euaella Hammer, 1973

(=Cryptoribatula)

24,205

Eudamaeus (Damaeus) Pérez-Íñigo, 1987 .. 16, 80

Eueremaeus Mihelcic, 1963 .................. 17, 100

Eulohmannia Berlese, 1910 ..................... 14, 36

Eupelops Ewing, 1917 ........................... 22, 162

Euphthiracarus Ewing, 1917 .................... 14, 46

Eupterotegaeus Berlese, 1916 .................. 16, 83

Eupunctoribates Hammer, 1977

(=Zachvatkinibates)

23, 184

Eurostocepheus Aoki, 1965 .................. 21, 146

Euryacarus (Javacarus) Woolley, 1966 .. 14, 38

Euryparazetes Radford, 1948

(=Peloribates)

25,209

Euscheloribates Kunst, 1958 .................. 24, 197

Eutegaeus Berlese, 1916

.. 16, 85

Euzetes Berlese, 1908

$10,22,175$

Ewingozetes Hammer, 1952 (=Pelopsis) .. 23, 183

Exanthoppia J. y P. Balogh, 1983 ......... 18, 113

Exochocepheus Woolley y Higgins, 1968

22,161

Exoribatula Jacot, 1936 ......................... 24, 193

Exoripoda Woolley, 1961 (=Benoibates) .. 24, 203

Falsolobozetes Tseng, 1984

(=Podoribates)

23,186

Farchacarus Wallwork, 1967 .................. 22, 173

Feiderzetes Subías, 1977 ........... 23, 182

Fenestrella Mahunka, 1987 .................... 17, 102
Fenestrobates (Oribatella) Balogh y Mahunka,

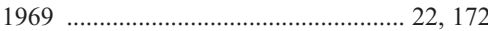

Fenestrobelba Balogh, 1970 ................... 20, 134

Fenichelia Balogh, 1970 ..................... 21, 159

Fernandocepheus Mahunka, 1982 .......... 20, 144

Ferolocella Grabowski, 1971 ................ 22, 170

Fijibates Hammer, 1971 ......................... 24, 197

Fijirella Hammer, 1971 (=Gressittolus) .... 18, 103

Fineoppia Khanbekyan y Gordeeva, 1991

(=Dzarogneta) .................................. 19, 129

Fissicepheus Balogh y Mahunka, 1967 .. 20, 144

Fissurobates Balogh y Mahunka, 1969 .. 24, 197

Flabellobelba Pérez-Íñigo, 1995 .............. 15, 75

Flagellobates Mahunka, 1978

(=Topobates) …................................. 24, 202

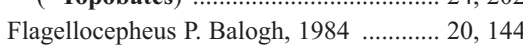

Flagellozetes Balogh, 1970 ................... 25, 214

Flagrosuctobelba (Suctobelbella)

Hammer, 1979 .................................. 20, 138

Flammaeremaeus Balogh, 1968 ................. 15, 73

Flexa (Carabodes) Kulijev, 1977 .......... 21, 150

Floribates Norton y Kethley, 1989

(=Siculobata) …................................ 24, 194

Foraminoppia Subías y Arillo, 1998 ...... 19, 128

Fortuynia Hammen, 1960 .................... 21, 157

Fosseremus Grandjean, 1954 .................. 18, 103

Fossonothrus (Tyrphonothrus) Hammer,

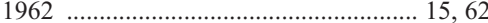

Fossoppia Mahunka, 1994 .................... 19, 128

Foveolatoppia Mahunka, 1988 ............... 19, 128

Fovoribatula Lee y Birchby, 1989

(=Zygoribatula)

23,188

Frischia Oudemans, 1915

(?=Protoripoda)

24,203

Frondoppia Mahunka, 1983

(=Graptoppia)

19,116

Fuegoplophora Mahunka, 1980

(=Neophthiracarus) .......................... 14, 56

Fuerteventuria Pérez-Íñigo y Peña, 1996

(=Gerloubia) ..................................... 24, 191

Furcobates Sellnick, 1959 ....................... 22, 175

Furcodamaeus Pérez-Íñigo y Baggio,

1980 ............................................... 18, 104

Furcoppia Balogh y Mahunka, 1966 ........ 17, 92

Furcoribula Balogh, 1943 ...................... 17, 92

Furculoppia (Multioppia) Balogh, 1983 .. 19, 117

Fuscozetes Sellnick, 1928 ..................... 23, 175

Fusozetes Balogh, 1972 ......................... 16, 88

Fusuloppia Balogh, 1983 ...................... 18, 113

Galapagacarus P. Balogh, 1985 .............. 15, 68

Galumna Heyden, 1826 ....................... 25, 214

Galumnella Berlese, 1916 .................... 25, 221

Galumnopsis Grandjean, 1931 ............... 25, 222

Gamerozetes (Edwardzetes) J. y P. Balogh,

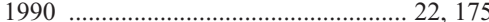

Gehypochthonius Jacot, 1936 ................. 13, 28

Geminoppia J. y P. Balogh, 1983 ............ 18, 110

Geminozetes Balogh y Csiszár, 1963 .... 23, 176

Gemmazetes Fujikawa, 1979

(=Oribellopsis) $\quad$................................. 18, 107

Genavensia Mahunka, 1983 .................. 22, 173

Gendzella Kulijev, 1977 (=Ferolocella) .. 22, 170 
Geocepheus Sitnikova, 1979

(=Reticulocepheus) ..........

Gephyrazetes Hirauchi, 1999

Gerloubia Coetzer, 1968 ...................... 24, 191

Ghilarovizetes Shaldybina, 1969 ............ 23, 176

Ghilarovus Krivolutsky, 1966 ............... 23, 184

Gibbibodes (Gibbicepheus) Mahunka,

1986 ................................................ 21, 152

Gibbicepheus Balogh, 1958 ................... 21, 152

Gilarovella Lange, 1974 ......................... 13, 28

Ginglymacarus Ewing, 1917

(=Phthiracarus)

14,53

Gittella Hammer, 1961 .......... 19, 122

Glaberoribates Tseng, 1984

(=Triaungius) …................................ 25, 206

Glabroppia (Karenella) Subías y Rodríguez,

1986 .......................................... 20, 130

Glanderemaeus Balogh y Csiszár, 1963 .. 21, 158

Globogalumna J. y P. Balogh, 1990 ........ 25, 217

Globonothrus Tseng, 1982 (=Eupelops) .. 22, 162

Globoppia Hammer, 1962 ….................. 18, 110

Globozetes Sellnick, 1928 .................... 23, 180

Gobiella Balogh y Mahunka, 1965

(=Kaszabobates)

18,107

Goyoppia Balogh, 1983 ...................... 18, 113

Gozmanyina Balogh y Mahunka, 1983 .... 13, 33

Gradjeanacarus Zachvatkin, 1958

(=Ctenacarus)

13,28

Gradjeanella Balogh, 1961

(=Grandjeania)

Grandjeania Balogh, 1963 .................... 23, 186

Grandjeanobates Ramsay, 1967 ............. 24, 197

Grandjeanoplophora Balogh y Mahunka, 1979

Granizetes Hammer, 1961 ..................... 22, 166

Granuloppia Balogh, 1958 .................... 20, 131

Granuloteratoppia P. Balogh, 1988 ........ 20, 132

Graptoppia Balogh, 1983 ...................... 19, 116

Gredosella Gil-Martín, Arillo y Subías,

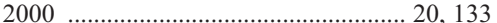

Gressittolus Balogh, 1970 .................... 18, 103

Gressittoppia (Brachioppiella) Balogh,

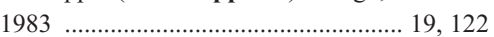

Grypoceramerus Suzuki y Aoki, 1970 .... 18, 108

Guaranozetes Balogh y Mahunka, 1981 .. 22, 173

Guatemalozetes (Scotiazetes) Mahunka,

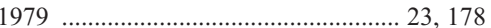

Guineobodes (Pasocepheus) Mahunka,

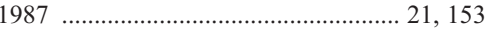

Gustavia Kramer, 1879 ......................... 17, 95

Gymnobates Banks, 1902 ..................... 24, 204

Gymnobatoides Woolley, 1966 .............. 24, 204

Gymnobodes Balogh, 1965 ................... 21, 152

Gymnodamaeus Kulczynski, 1902 ......... 15, 76

Gymnodampia Jacot, 1937

(?=Defectamerus) ….......................... 18, 105

Gymnonothrus Ewing, 1917 (=Nothrus) .... 15, 63

Gymnozetes Calugar y Vasiliu, 1983

(=Magnobates)

Gymnozetes P. Balogh, 1984

(=Sturmozetes)

17,91

Hafenferrefia Jacot, 1939
Hafenrefferia Oudemans, 1906

Hafenrefferiella Sellnick, 1952

(=Hafenferrefia)

Haloribatula Schuster, 1957

Halozetes Berlese, 1916

Hammation Grandjean, 1959

(=Basilobelba) .................................. 18, 104

Hammerabates Balogh, 1970 .................. 24, 197

Hammerella Balogh, 1983 ................... 20, 132

Hammeria Sellnick, 1944 (=Propelops) .. 22, 164

Hammeriella Paschoal, 1989

(=Paschoalia)

$7,15,73$

Hamobates Hammer, 1962

(=Zetomimus)

23,179

Hamoppia (Lanceoppia) Hammer, 1968 .. 18, 111

Hamotegeus Balogh y Mahunka, 1969

(=Sadocepheus) …............................. 16, 84

Haplacarus Wallwork, 1962 .................... 14, 38

Haplamerus J. y P. Balogh, 1992 ............ 18, 105

Haplobelba Balogh y Mahunka, 1969 .... 18, 104

Haplochthonius Willmann, 1930 ............. 13, 34

Haploripoda Balogh y Mahunka, 1967

(=Benoibates)

24,203

Haplozetes (Indoribates) Willmann,

1935

Hardybodes

Mahunka, 1980

(=Pseudoceratoppia) ….................... 17, 94

Hauserophthiracarus Mahunka, 1982

(=Calyptophthiracarus) ..................... 14, 52

Hauseroplophora Mahunka, 1977 ............ 13, 35

Hauserozetes Mahunka, 1980 ................. 16, 88

Helioppia Balogh, 1983 ....................... 19, 116

Hellenamerus Mahunka, 1974 ............... 17, 101

Heloribates (Hydrozetes) Grandjean, 1966.

Helvetacarus Mahunka, 1993

(=Atropacarus)

.. 21, 156

Helvetobelba Mahunka y Mahunka-Papp

1999 ............................................... 20, 135

Hemileius Berlese, 1916 ....................... 24, 193

Heminothrus Berlese, 1913 ..................... 15, 65

Heptacarus Piffl, 1963 .......................... 14, 38

Hermannia Nicolet, 1855 .................. 15, 68, 69

Hermanniella Berlese, 1908 .................... 15, 70

Hermannobates Hammer, 1961 ............... 15, 71

Heterobelba Berlese, 1913 ..................... 18, 104

Heterochthonius Berlese, 1910 ................ 13, 33

Heterodamaeus Ewing, 1917

(=Gymnodamaeus)

Heterodamaeus Woolley, 1957

(=Adrodamaeus)

Heterodamaeus Mihelcic, 1964

(=Parabelbella)

Heterogalumna Balogh, 1960

Heterohermannia (Hermannia) Woas,

1992

Heteroleius Balogh y Mahunka, 1966

Heteroppia Balogh, 1970 ..................... 18, 113

Heterozetes Willmann, 1917 ................ 22, 173

17, 99 Hexachaetoniella Paschoal, 1987 .............. 15, 74
Hexatocepheus (Acrotocepheus) Wen,

1993 ................................................. 20, 146

Hexoppia Balogh, 1958 ......................... 20, 133

Himalacarus Sheals, 1965

(=Zachvatkinella)

13,27

Hoffmanacarus Mahunka, 1995 ............ 22, 169

Hokkachipteria Balogh y Mahunka, 1979

(=Izuachipteria) ….......................... 22, 168

Holokalumma Jacot, 1929 (=Galumna) .. 25, 214

Hololohmannia Kubota y Aoki, 1998 ...... 13, 36

Holonothrus Wallwork, 1963 .................. 15, 67

Holozetes Jacot, 1929 (=Galumna) ...... 25, 214

Hoploderma Michael, 1898

$$
\text { (=Phthiracarus) .......... }
$$

oplophora Koch, 1836

(=Phthiracarus) ….............................. 14, 53

Hoplophorella Berlese, 1923 .................... 14, 48

Hoplophthiracarus Jacot, 1933 .................. 14, 50

Huarpescopes Fernández, Monetti y Martínez, 1995 .............................................. 25, 205

Huilicheremaeus Fernández, Marcangeli y

Eguaras, 1997 ................................ 21, 160

Humerobates Sellnick, 1928 ................. 23, 181

Hummelia Oudemans, 1916

(?=Euphthiracarus) .......................... 14, 46

Hungarobelba Balogh, 1943 ................ 18, 103

Hungaromotrichus Mahunka, 1993

(=Mabulatrichus) …......................... 23, 184

Hydroecocepheus Corpuz-Raros, 1979 .. 20, 144 Hydronothrus Aoki, 1964

(=Trhypochthoniellus) ….................. 15, 60

Hydrozetes Berlese, 1902 ...................... 21, 155

Hygroribates Jacot, 1934

(=Ameronothrus) ….......................... 21, 156

Hymenobelba Balogh, 1962 ................... 18, 105

Hymenozetes Balogh, 1962 .................... 16, 88

Hypocepheus Krivolusky, 1971 ............... 16, 83

Hypochthoniella Berlese, 1910 ................ 13, 29

Hypochthonius Koch, 1835 .................... 13, 29

Hypodamaeus Bulanova-Zachvatkina, 1957

(=Damaeus) …...................................... 16, 78

Hypogeoppia Subías, 1981 .................... 19, 126

Hypovertex Krivolutsky, 1969 ................ 22, 161

Hypozetes Balogh, 1959 ....................... 22, 166

Iberogalumnella Arillo y Subías, 1993 .... 25, 222

Iberoppia Pérez-Íñigo, 1986 .................. 18, 108

Iburidania Aoki, 1959

(=Epilohmannoides) .......................... 14, 37

Idiodamaeus Paschoal, 1984

(=Austrodamaeus) …......................... 15, 76

Idiozetes Aoki, 1976 ............................ 22, 165

Ikarotocepheus Mahunka, 1988 ............. 21, 146

Imparatoppia Jacot, 1934

(=Phauloppia) …............................. 24, 190

Incabates (Lauritzenia) Hammer,

1961 ................................................. 25, 209

Incudozetes J. y P. Balogh, 2002

(=Sphaerozetes) …......................... 23, 178

Indobates Pandit y Bhattacharya, 1999

(=Peloribates) …............................... 25, 209

Indogalumna (Galumna) Balakrishnan,

1985

25,217 
Indoribates Jacot, 1929 .......................... 25, 208

Indotocepheus (Odontocepheus) Mondal y

Kundu,1999 ……………………....2 21, 153

Indotritia Jacot, 1929 ................................. 14, 43

Infernobates Karppinen y Poltavskaja,

1990 ............................................. 18, 107

Ingella Hammer, 1967 (=Reductobates) .. 24, 196

Inigozetes Subías, 2000 .......................... 23, 182

Insculptoppia (Ramusella) Subías, 1980 .. 19, 118

Insculptoppiella (Ramusella) Subías y

Rodríguez, 1986 ................................ 19, 119

Intermedioppia Subías y Rodríguez,

$1987 \ldots \ldots \ldots \ldots \ldots \ldots \ldots \ldots \ldots \ldots \ldots \ldots \ldots \ldots \ldots \ldots \ldots \ldots \ldots \ldots . . .19,116$

Interoppia Mahunka, 1987

(=Hammerella) ................................. 20, 132

Irinobates Krivolutsky y Christov, 1970

(=Liebstadia) ................................... 24, 195

Ischeloribates Corpuz-Raros, 1980

(=Perscheloribates) $\quad$........................... 24, 197

Issaniella Grandjean, 1962 ........................ 15, 71

Italobates (Pilobates) Mahunka, 1994 .... 25, 211

Iugoribates Sellnick, 1944 ...................... 23, 180

Ivarsia Karppinen y Krivolutsky, 1987

(=Ensicamisia) ….............. 15, 65

Izuachipteria (Achipteria) Balogh y Mahunka,

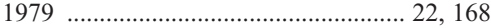

Jacotella Banks, 1947 ................................ 15, 76

Javacarus Balogh, 1961 ........................... 14, 38

Javalohmannia Hammer, 1979

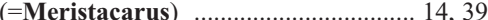

Javieroppia Mínguez y Subías, 1986 ...... 19, 117 Jeannelia Dalenius, 1958

(=Humerobates) ………………….... 23, 181

Jermyia Mahunka, 2002

(=Gressittoppia)

19,122

Joboppia Ruiz, Mínguez y Subías, 1988 .. 18, 109

Joelia Oudemans, 1906 .......................... 22, 170

Johnstonella Paschoal, 1983

(=Gymnodamaeus) …........................... 15, 76

Jornadia Wallwork y Weems, 1984 ........ 23, 187

Joshuella Wallwork, 1972 ......................... 15, 76

Jugatala Ewing, 1913 ............................... 23, 176

Jurabates Jacot, 1929 (=Minunthozetes) .. 23, 182

Kabylogalumna (Galumna) Bernini,

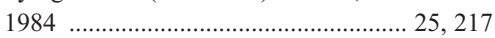

Kakophthiracarus (Hoplophorella) Mahunka,

1992 ......................................................... 49

Kalayaan Corpuz-Raros, 1998 …............ 21, 146

Kalloia (Diplobodes) Mahunka, 1985 .... 21, 152

Kalyptrazetes Balogh, 1972 ….................. 16, 89

Karenella Hammer, 1962 ........................ 20, 130

Kartoeremaeus Higgins, 1979

(=Eueremaeus)

17,100

Kaszabobates Balogh, 1972 ..................... 18, 107

Kaszabozetes Mahunka, 1988 .................. 16, 89

Keralotrichus (Zetomotrichus) Mahunka,

1985

Kilimabates Mahunka, 1984

(=Anellozetes)

23,185

23, 180

Kinabaluella Mahunka, 1996

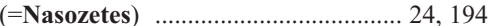

Kinezogalumna Aoki y Hu, 1993 .......... 25, 217
Klapperiches (Carabodes) Mahunka, 1979

Kochia Oudemans, 1900 (=Xenillus) $\quad$...... 17, 97

$10,21,150$

Kodiakella Hammer, 1967 ....................... 17, 99

Kokoppia Balogh, 1983 ......................... 19, 123

Koreoribates Choi, 1994 ...................... 22, 164

Koreozetes (Neogymnobates) Aoki,

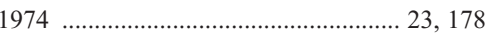
Kratzensteinia Oudemans, 1919

25,220

Krivolutskiella Gordeeva, 1980 ............... 13, 33

Kulievia Vasiliu e Ivan, 1999 ................. 19, 126

Kunoppia Mahunka, 1987

$$
\text { (=Medioppia) }
$$

19,123

Kunstella Krivolutsky, 1974

Labiogena Hunt, 1996

(=Novazelandiella)

Lagenobates Weigmann y Miko, 2002

(=Transoribates)

19,123
22,170

Lalmoppia (Subiasel 1986

Lamellarea Kok, 1

amellobates Hammer, 1958

Lamellocepheus Balogh, 1961

Lamellovertex Bernini, 1976

Lamellozetes Covarrubias, 1967

Laminize 1986

Laminoppia Hammer, 1968

Lancelalmoppia (Lanceoppia) Subías, 1989

Lanceoppia Hammer, 1962 ..................... 18, 110

Lanibelba (Belbodamaeus) Norton, 1980 .. 16, 78

Laroppia Subías, 1989 ........................... 18, 113

Lasiobelba Aoki, 1959 ......................... 18, 113

Latilamellobates (Viracochiella) Shaldybina,

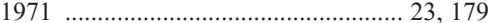

Latovertex Mahunka, 1987

(=Exochocepheus) …........................ 22, 161

Lauritzenia Hammer, 1958 ....................... 25, 208

Lauroppia Subías y Mínguez, 1986 ........ 19, 126

Lawrencoppia Jacot, 1936

(=Phyllhermannia)

Leebates Balogh y Mahunka, 1996

(=Viracochiella)

15,69

Leiosoma Nicolet, 1855 (=Liacarus) $\quad$...... 17, 95

Lemurobates Mahunka, 1997 ................. 22, 169

Lemuroppia Mahunka, 1944 ................. 18, 114

Leobodes Aoki, 1965 ............................ 21, 154

Leoppia Pérez-Íñigo, 1983 ..................... 20, 132

Lepidacarus Csiszár, 1961 ........................ 14, 38

Lepidoribates Sellnick, 1920

(=Tegoribates) .

22,169

Lepidozetes Berlese, 1910 ....................... 22, 169

Leptogalumna Balogh, 1960 ................. 25, 217

Leptoppia Mahunka, 1997 ....................... 19, 123

Leptorchistis Canestrini y Berlese, 1885

17,91

Leptotocepheus Balogh, 1961 ................ 20, 144

Lesseria Oudemans, 1917

(=Epilohmannia) ….......................... 14, 36

Leuroxenillus Woolley y Higgins, 1966

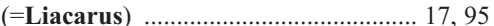

Liacaroppia Subías y Rodríguez, 1986 .. 19, 126

Liacarus Michael, 1898 .................... 10, 17, 95

Licneremaeus Paoli, 1908 ..................... 21, 160

Licnobelba Grandjean, 1931 .................. 15, 75

Licnocepheus Woolley, 1969 ............. 8, 18, 106

Licnodamaeolus Covarrubias, 1998 .......... 15, 74

Licnodamaeus Grandjean, 1931 ............... 15, 74

Licnoliodes Grandjean, 1931 .................. 15, 73

Licnozetes Balogh y Mahunka, 1969 ...... 16, 89

Liebstadia Oudemans, 1906 .................. 24, 195

Limnozetella Willmann, 1931 ............... 22, 165

Limnozetes Hull, 1916 ............................ 22, 165

Lineoppia J. y P. Balogh, 1983 ............. 19, 128

Linothrus Tseng, 1982 (=Cyrthermannia) .. 15, 67

Liochthonius Hammen, 1959 .................. 13, 30

Liodes Heyden, 1826 (=Neoliodes) .... 11, 15, 71

Litholestes Grandjean, 1951 ................... 17, 91

Loboppia Balogh, 1983 ......................... 18, 111

Lobozetes Hammer, 1958 ..................... 23, 176

Loftacarus Lee, 1981 ............................... 13, 27

Lohmannia Michael, 1898 ........................ 14, 38

Longocepheus Balogh y Mahunka,

1966 .............................................. 20, 144

Lopheremaeus Paschoal, 1988 .................. 15, 73

Lopholiodes Passchoal, 1987 ................... 15, 73

Lophotocepheus J. y P. Balogh, 1983 .... 20, 144

Lophozetes P. Balogh, 1985

(=Macrogena) …............................... 23, 176

Lucioppia (Subiasella) Mahunka, 1985 .. 20, 130

Lucoppia Berlese, 1908 .......................... 23, 187

Lunoribatula Mahunka, 1982 ................. 23, 187

Luxtoneremaeus J. y P. Balogh, 1992 .... 18, 106

Luxtonia Mahunka, 2001 ....................... 20, 131

Lyrifissiella Paschoal, 1989 ..................... 15, 73

Lyroppia Balogh, 1961 ............................ 20, 131

Mabulatrichus Coetzee, 1993 ................. 23, 184

Macarotritia Pérez-Íñigo, 1986

(=Austrotritia) …................................ 14, 42

Machadobelba Balogh, 1958 ...................... 18, 106

Machadocepheus Balogh, 1958 ............. 21, 152

Machuella Hammer, 1961 ..................... 20, 133

Macquariella Wallwork, 1963

(=Macquarioppia) …......................... 17, 94

Macquarioppia Wallwork, 1964 ............... 17, 94

Macrogena Wallwork, 1966 .................... 23, 176

Macrosoma Hammer, 1979

(=Senectoppia) .................................. 20, 132

Maculobates Hammer, 1962 ................. 24, 196

Maerkelotritia Hammer, 1967 ................. 14, 43

Magellozetes (Ceratozetes) Hammer,

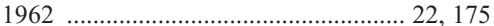

Magnobates (Lauritzenia) Hammer,

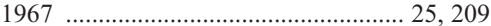

Magoebazetes (Cosmozetes) Engelbrecht,

1972 ........................................... 16, 88

Magyaria Balaogh, 1963 ....................... 25, 209

Mahnertella Mahunka, 1997

(=Rugoppia) …................................ 20, 130

Mahunkaia Schatz, 2002 ....................... 22, 165

Mahunkazetes J. y P. Balogh, 1992 ........ 23, 185

Mahunkella Balogh, 1983 .................... 19, 129

Mahunkobates Calugar, 1989 ..................... 15, 71 
Mainothrus Choi, 1996 Makischeloribates (Perscheloribates) Corpuz-

Raros, 1980

15, 59 Minguezetes Subías, Kahwash y Ruiz,

Malacoangelia Berlese, 1913 ................... 13, 29

Malaconothrus Berlese, 1904 .............. 7, 15, 60

Malgasodes Mahunka, $2000 \quad$................. 21, 152

Malgoceras (Acaroceras) Mahunka, 1993 .. 16, 87

Mancoribates (Indoribates) Hammer,

$1961 \ldots \ldots . . .25,208$

Mantigueracarus (Rhacaplacarus) Balogh y

Mahunka, 1992 ................................ 14, 58

Maorizetes Hammer, 1966 ....................... 17, 93

Marshallia Gordeeva, 1980

(=Gozmanyina)

Masthermannia Berlese, 1913 ................. 15, 68

Maudheimia Dalenius, 1958 ............. 7, 24, 195

Mauribodes J. y P. Balogh, 1992

(=Kalloia) ….................................... 21, 152

Medioppia Subías y Mínguez, 1985 ...... 19, 123

Medioxyoppia Subías, 1989 ................... 19, 124

Megalotocepheus Aoki, 1965 .................. 21, 147

Megascheloribates Lee y Pajak, 1990

(=Scheloribates)

Megatrichobates Grobler, 2000 ….......... 23, 187

Megazetes (Microzetes) Balogh, 1959 .... 16, 89

Megeremaeus Higgins y Woolley, 1965 .... 17, 99

Melanozetes Hull, 1916 ........................... 23, 176

Membranoppia Hammer, 1968 ............... 18, 111

Meriocepheus Aoki, 1973 ..................... 21, 153

Meristacarus Grandjean, 1934 ................. 14, 39

Meristolohmannia Balogh y Mahunka, 1966

Mesoplophora Berlese, 1904 .................... 14, 42

Mesotritia Forsslund, 1963 ..................... 14, 43

Metabelba Grandjean, 1936 .................... 16, 80

Metabelbella Bulanova-Zachvatkina,

1957

Metaleius (Paraleius) Travé, 1960 ........ 24, 194

Metaphthiracarus (Phthiracarus) Aoki,

Metapyroppia Woolley, 1969 .................... 17, 94

Metrioppia Grandjean, 1931 ................... 17, 94

Mexiceremus J. y P. Balogh, 1998 .......... 21, 159

Mexicoppia (Furcoppia) Mahunka, 1983 .. 17, 92

Michaelia Haller, 1884 (=Lohmannia) .... 14, 38

Micreremus Berlese, 1908 21, 159

Microchthonius Kahwash, Subías y Ruiz, 1989

(=Nanochthonius) ............................ 13, 33

Microlamellarea Coetzee, 1987 .............. 21, 160

Microphthiracarus Mahunka, 1982

(=Archiphthiracarus)

Microppia Balogh, 1983

Microtegeus Berlese, 1916 ...................... 16, 86

Microtritia Märkel, 1964 ......................... 14, 47

Microzetes Berlese, 1913 ......................... 16, 89

Microzetorchestes Balogh, 1943 .............. 17, 91

Mikizetes Hammer, 1958 ........................ 23, 184

Millotacarus (Paulianacarus) Balogh, 1961

Mimoppia Balogh, 1983 ...................... 19, 121

Minasbates Pérez-Íñigo y Baggio, 1996

(=Mancoribates)
13,33

24, 198

14, 39

16,81

14,56

14,54

19,124

1990

Minoricoppia Pérez-Íñigo Jr., 1991

(=Pseudoamerioppia)

Minunthozetes Hull, 1916 ........ 23,182

Mirabilozetes Mahunka, 1977 ................. 16, 89

Miracarus Kunst, 1959 ............................ 16, 89

Mirobelba Pérez-Íñigo y Peña, 1994

(=Parabelbella)

Mirogalumna Mahunka, 1993

(=Orthogalumna)

Miroppia Hammer, 1968 ........................ 19, 124

Mirus Kulijev, 1967 (=Spinozetes) ........ 19, 108

Mixacarus Balogh, 1958 ........................ 14, 39

Mixobates Gil-Martín y Subías, 1993

(=Triungulozetes) .............................. 25, 208

Mixochthonius Niedbala, 1972 ............... 13, 31

Mixoribatula Mahunka, 1987

(=Cordiozetes)

Mixozetes (Ceratozetes) J. y P. Balogh,

1990

Mochloribatula Mahunka, 1978 ............. 23, 185

Mochlozetes Grandjean, 1930 ................ 23, 185

Mongaillardia Grandjean, 1961 .............. 17, 101

Monofurcoppia Pérez-Íñigo y Sarasola, 1995

17,93

Monogalumnella Mahunka, 1986 ......... 25, 222

Monophauloppia (Paraphauloppia) P. Balogh, 1988

23, 190

(=Paraphysobates)

Monoschelobates Balogh y Mahunka, 1969

22,169

Montizetes Kunst, 1971 ...................... 18, 107

Moritzichthonius Pereda e Iturrondobeitia, 1990 (=Neoliochthonius) ............................. 13, 3

Moritziella Balogh, 1983

(=Moritzoppia) .................................. 19, 126

Moritzoppia Subías y Rodríguez, 1988 .... 19, 126

Moritzoppiella (Moritzoppia) Gordeeva,

2000 ....................................... 19, 127

Mucrobates Balogh y Mahunka, 1979 .... 24, 194

Mucronothrus Trägardh, 1931 ................. 15, 59

Muliercula Coetzer, 1968 ....................... 24, 197

Multilanceoppia (Multioppia) Subías,

....... 19, 117

Multimaudheimia gen. nov. ............... 7, 24, 195

Multimedioppia Subías, 1991 ................ 19, 124

Multioppia Hammer, 1961 ..................... 19, 117

Multipulchroppia Subías, 1989 _............. 19, 120

Multoribatella (Oribatella) subgen.

nov.

Multoribates (Exoribatula) Hammer,

1961 ............... 24, 193

Multoribula Balogh y Mahunka, 1966 ...... 17, 93

Murcia Koch, 1835 ......................... 10, 23, 177

Mycobates Hull, 1916 ........................... 23, 182

Mycozetes Spain, 1968 ........................ 23, 183

Mystacozetes Balogh, 1962 .................... 16, 90

Mysterozetes Hammer, 1961 ................... 17, 90

25, 208 Mystroppia Balogh, 1959 ....................... 20, 130
Monophysobates J. y P. Balogh, 1992
Nacunansella Fernández y Cleva,1998 .... 15, 74

Naiazetes (Geminozetes) Behan-Pelletier,

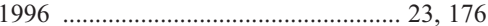

Nanhermannia Berlese, 1913 .................. 15, 68

Nannerlia Coetzer, 1968 ........................ 24, 197

Nanobates (Euscheloribates) J. y P. Balogh, 1984

24, 197

Nanochthonius (Cosmochthonius) Subías y Gil-

Martín, $1995 \ldots \ldots \ldots \ldots \ldots \ldots \ldots \ldots \ldots \ldots \ldots \ldots . . . . .13,33$

Nasobates Woolley, 1966 ....................... 25, 212

Nasozetes Sellnick, $1930 \ldots \ldots \ldots \ldots \ldots \ldots \ldots . .24,194$

Neamerus (Amerus) Willmann, 1939 .... 18, 105

Negroppia Vasiliu y Calugar, 1977 .......... 17, 94

Nehypochthonius Norton y Metz, 1980 .... 13, 36

Nellacaroides Mahunka, 1998

(=Microzetes) .....................

Nellacarus Grandjean, 1936

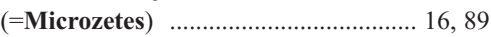

Nemacepheus Aoki, 1968 ...................... 16, 85

Neoamerioppia Subías, 1989 .................. 19, 114

Neoatrichosus (Eohypochthonius) Fernández,

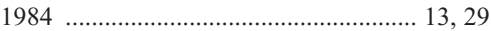

Neobelba (Metabelba) Bulanova-Zachvatkina, 1967 .............................................. 16, 81

Neobrachychthonius Moritz, 1976 ........... 13, 31

Neocarabodes (Diplobodes) Balogh y Mahunka,

1969 ........................................ 21, 152

Neocepheus Willmann, 1936

(=Carabodes) $\quad$..................................... 21, 149

Neochthonius Karppinen, 1984 ............... 13, 34

Neoctenacarus Moritz, 1974 .................. 13, 28

Neoepilohmannia Bolen y McDaniel, 89

(=Epilohmannia) …............................ 14, 36

Neoeutegaeus Aoki, 1964 ...................... 16, 85

Neogalumna Hammer, 1973 ................... 25, 217

Neogymnobates Ewing, 1917 ............... 23, 177

Neohermannia Bayoumi y Mahunka,

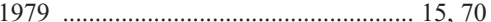

Neokalumma Tseng, 1984

(=Porokalumma) $\quad$............................... 25, 213

Neolepidozetes Hammer, 1977

(=Scutozetes) …................................. 22, 169

Neoliochthonius Lee, $1982 \ldots \ldots \ldots \ldots \ldots \ldots . . . . . .13,31$

Neoliodes Berlese, 1888 .................... 11, 15, 71

Neolohmannia Bulanova-Zachvatkina, 1960

(=Perlohmannia) …......................... 13, 36

Neolucoppia Tseng, 1984 ....................... 23, 187

Neomycobates Wallwork, 1963 ............. 23, 183

Neonooliodes Hunt, 1996 ....................... 15, 73

Neonothrus Forsslund, 1955

(=Platynothrus)

Neophthiracarus (Phthiracarus) Balogh y Csiszár, 1963 ...................................... 14, 56

Neophysobates Luxton, 1987 ................. 22, 169

Neopilizetes (Pilizetes) J. y P. Balogh, 1990 ................................................. 25, 220

Neoppia Bhattacharya y Banerjee, 1981 .... 18, 109 Neoprotophthiracarus Mahunka, 1980

(=Neophthiracarus)

Neoprototritia Shereef, 1978

(=Arthrhoplophora)

14,56

Neoribates Berlese, 1914

13,35 
Neoribatula Ewing, 1917

(=Zygoribatula)

Neoscheloribates Hammer, 1973

(=Andeszetes)

Neoscutovertex Mihelcic, 1957

(=Scutovertex) (................................ 22, 161

Neosteganacarus Balogh y Mahunka, 1992

(=Rafacarus)

Neostrinatina Mahunka, 1979 ............... 19, 127

Neosuctobelba Balogh y Mahunka,

1969 20, 135

Neotocepheus (Plenotocepheus) Hammer,

1966 ................................................ 20, 145

Neotrichacarus Hammer, 1973

(=Heptacarus)

14,38

Neotrichocepheus Hammer, 1973 .......... 20, 144

Neotrichoppia Subías e Iturrondobeitia, 1980

19,127

Neotrichozetes Travé, 1961 ................... 23, 186

Neozetes Berlese, 1885 (=Gustavia) ........ 17, 95

Neseutegaeus Woolley, 1965 ................... 16, 85

Nesiacarus Csiszár, 1961 .......................... 14, 40

Nesiotizetes Jacot, 1934 ......................... 23, 186

Nesopelops Hammer, 1973 .................... 22, 164

Nesoppia Luxton, 1985

(=Membranoppia)

Nesoribatula Aoki, 1964

(=Exoribatula)

18,111

Nesotocepheus Hammer, 1972

(=Pseudotocepheus)

24,193

20,145

Nesozetes Hammer, 1971 ....................... 24, 191

Ngorongobodes J. y P. Balogh, 1992

(=Uluguroides) …............................. 21, 149

Niedbalaia Mahunka, 1999

(=Euphthiracarus)

Niloppia Balogh, 1983

............... 19, 114

Niphocepheus Balogh, 1943 .................. 16, 84

Nippobodes Aoki, 1959 ......................... 21, 154

Nippohermannia (Nanhermannia) P. Balogh,

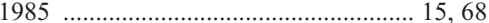

Nipponiella Gordeeva, 1980 .................... 13, 33

Nixozetes Mahunka, 1977

(=Indoribates) ..................................... 25, 208

Nodocepheus Hammer, 1958 ................... 16, 85

Nododamaeus (Parabelbella) Hammer,

16,81

Nooliodes Paschoal, 1989 ....................... 15, 73

Nortonacarus Balogh y Mahunka, 1992

(=Rafacarus) ….................................. 14, 58

Nortonbelba Bernini, 1980 ....................... 16, 81

Nortonella Paschoal, 1982 ...................... 15, 76

Nosybea Mahunka, 1993

(=Lamellocepheus) .....

1994 .................. 20, 134

Notaspis Hermann, 1804 (?=Ceratoppia) .. 17, 93

Nothrolohmannia Balogh, 1968 ............... 13, 29

Nothrus Koch, 1836 ............................... 15, 63

Notogalumna Sellnick, 1959 ................. 25, 217

Notohermannia P. Balogh, 1985 ............. 15, 68

Notophthiracarus Ramsay, 1966 .............. 14, 50

Notophthiracarus Balogh y Mahunka, 1967

(=Protophthiracarus)
Notoppia Balogh y Mahunka, 1966 ......

Novazelandiella Paschoal, 1989

Novonothrus Hammer, 1966

Novoribatella Engelbrecht, 1986 ..........

ovosuctobelba Hammer, 1977 ............... 20, 135

Ocellotocepheus Mahunka, 1989 ............ 21, 147

Ocesobates Aoki, 1965 ........................... 23, 180

Octodurozetes Mahunka, 1993

(=Perxylobates)

Octoliodes Paschoal, 1987

(=Lopholiodes)

25,205

15,73

Octoppia Balogh y Mahunka, 1969 ........ 19, 117

Odontocepheus Berlese, 1913 ............... 21, 153

Odontodamaeus Paschoal, 1982

(=Gymnodamaeus)

15,76

Oglasacarus (Mikizetes) Bernini, 1979 .. 23, 184

Oligoppia Balogh, 1983 ......................... 19, 114

Ommatocepheus Berlese, 1913 ................ 16, 83

Onazetes Bugrov, 1991 (=Lepidozetes) .. 22, 169

Onychobates Hammer, 1967 ................. 23, 178

Operculoppia Hammer, 1968 .................. 18, 111

Ophidiotrichus Grandjean, 1953 ........... 22, 170

Opisthocepheus Aoki, 1976

(=Pasocepheus)

21,153

Oppia Koch, 1836

19,114

Oppiella Jacot, 1937

19,128

Opsioristes Woolley, 1967 ....................... 17, 97

Orbiculobates Grandjean, 1961 ............... 15, 71

Oribata Latreille, 1802

(?=Phauloppia)

Oribatella Banks, 1895

Oribatodes Banks, 1895

Oribatula Berlese, 1896

Oribella Berlese, 1908

Oribellopsis Kunst, 1971

Oribotritia Jacot, 1924

Oripoda Banks, 1904

$1,24,190$

Oromurcia Thor, 1930

Orthogalumna Balogh, 196

Orthozetes Balogh, 1962

Otaheitea Hammer, 1972

(=Hammerabates)

$7,22,170$

Otocepheus Berlese, 1905

... 16,83

... 23,187

Otoppia Balogh, 1983

Ovobates Mahunka, 1994

18,107

Ovochthonius Rjabinin, 1977

... 18, 107

Ovonothrus Kunst, 1971

(=Capillonothrus)

Oxyamerus Aoki, 1965

.14, 44

Oxyoppia Balogh y Mahunka, 1969 .......... 19, 129

Oxyoppiella (Oxyoppia) Subías y Rodríguez, 1986

19,129

Oxyoppioides Subías y Mínguez, 1985 .. 19, 129

Oxyscheloribates (Perscheloribates) J. y P.

Balogh, 1990 ................................... 24, 198

Oxyzetes Balogh, 1958 .......................... 17, 90

Ozacarus Colloff y Halliday, 1998 ............ 14, 40

Pabulozetes Tseng, 1982

(=Chaunoproctus) $\quad$........................... 24, 192

Pachygena Hammer, 1972 ..................... 24, 197

Paenoppia Woolley y Higgins, 1965 ......... 17, 95
Palaeacaroides Lange, 1972

Palaeacarus Trägardh, 1932 .................... 13, 27

Pallidacarus Krivolutsky, 1975 ............. 23, 184

Palmitalia Pérez-Íñigo y Peña, 1997 ...... 22, 167

Pantelozetes Grandjean, 1953 ............... 18, 107

Papillacarus Kunst, 1959 ......................... 14, 40

Papillocepheus Balogh y Mahunka, 1966 ................................................... 20, 144

Papillochthonius Gil-Martín, Subías

y Arillo, 1992 ..................................... 13, 31

Papillonotus Wallwork, 1961 ................. 20, 133

Papuacepheus Balogh, 1968 ................ 21, 147

Papuazetes Balogh, 1968 ......................... 17, 90

Parabelbella Bulanova-Zachvatkina, 1967 .. 16, 81

Paracamisia Olszanowski y Norton, 2002

(=Capillonothrus) ............................. 15, 66

Paracarinogalumna Mahunka, 1998

(=Carinogalumna) ........................... 25, 214

Paraceratoppia Rjabinin, 1982 ................. 17, 94

Parachipteria Hammen, 1952 ........... 10, 22, 169

Paradamaeus (Damaeus) Bulanova-Zachvatkina, 1957 ................................................... 16, 80

Paradolicheremaeus Tseng, 1982 ............ 20, 144

Parafurcobates (Furcobates) Hammer,

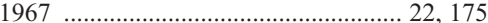

Paragloboppia (Taiwanoppia) Subías,

1989

19,115

Parahypozetes Hammer, 1967

(=Austrachipteria) ........................... 22, 166

Parakalumma (Neoribates) Jacot, 1929 .. 25, 212

Paralamellobates (Lamellobates) Bhaduri y

Raychaudhuri, 1968 .......................... 22, 167

Paraleius Travé, 1960 ............................. 24, 194

Paraliochthonius Moritz, 1976

(=Neoliochthonius) ............................. 13, 31

Paraliodes Hall, 1911 (=Phauloppia) .... 24, 190

Paralobozetes Tseng, 1984 .................... 23, 186

Paralopheremaeus Paschoal, 1987 ........... 15, 73

Paralycus Womersley, 1944 .................... 13, 34

Paramedioppia Mahunka y Mahunka-Papp,

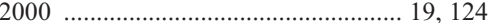

Parametabelba (Metabelba) Mihelcic,

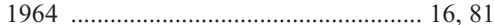

Parapelops Jacot, 1938 (=Pelopsis) ........ 23, 183

Paraphauloppia Hammer, 1967 _............. 23, 190

Paraphthiracarus Aoki, 1980

(=Archiphthiracarus) …................... 14, 54

Paraphysobates Mahunka, 1985 ................ 22, 169

Paraphysobates Luxton, 1985

(=Neophysobates) ............................ 22, 169

Parapirnodus Balogh y Mahunka, 1968 .. 24, 191

Parapyroppia Pérez-Íñigo y Subías,

1979 .............................................. 17, 94

Pararamusella Mahunka y Palacios-Vargas,

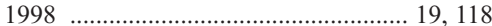

Pararectoppia Mahunka, 1987

(=Lalmoppia) ................................. 19, 130

Paraschelobates Jacot, 1934

(=Scheloribates) …........................... 24, 198

Parasuctobelba (Fenestrobelba) Hammer,

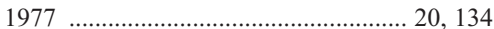

Parasynoppia Aoki, 1983

(=Elaphoppia) …............................ 19, 126 
Paratritia Moritz, 1976 Parautogneta Golosova, 1974 ................ 18, 109 Paraxylobates Balogh y Mahunka, 1969 .. 25, 209 Parazetes Willmann, 1930

(=Peloribates) 25,209 Pareutegaeus Woolley, 1965 ..................... 16, 85 Parhypochthonius Berlese, 1904 .............. 13, 28 Parisuctobelba Higgins y Woolley, 1976 .. 20, 135 Paroppia Hammer, 1968 .......................... 19, 115 Parplophora (Mesoplophora) Niedbala, 1985

14,42

Paschoalia nom. nov. ……………….... 7, 15, 73

Pasocepheus Aoki, 1976 ........................ 21, 153

Passalobates (Bipassalozetes) Pérez-Íñigo y

Peña, 1996 ...................................... 22, 162

Passalomonia (Passalozetes) Mahunka,

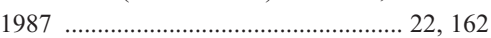

Passalozetes Grandjean, 1932 ................ 22, 162

Patagonozetes J. y P. Balogh, 1990 ......... 22, 167

Paternoppia Gil-Martín, Subías y Arillo,

2000 .......... 18, 109

Paulianacarus Balogh, 1961 ...................... 14, 40

Paulonothrus Kunst, 1971

(=Heminothrus) .................................. 15, 65

Pectinoppia Subías y Rodríguez, 1986

(=Dzarogneta) .................................... 19, 129

Pediculochelus Lavoipierre, 1946

(=Paralycus) ……............................. 13, 34

Pedrocortesella Hammer, 1961 ................. 15, 74

Pedrocortesia Hammer, 1958

(=Pheroliodes)

(2)

Pedunculozetes Hammer, $1962 \ldots 23,180$

Pelokylla Adolph y Haq, 1982

(=Drymobatoides)

23,185

Peloppia Sellnick, 1931 (=Metrioppia) .... 17, 94

Pelops Koch, 1835 (=Eupelops) ............ 22, 163

Pelopsis Hall, 1911 ................................... 23, 183

Peloptoribula Mahunka, 1984 .................. 17, 93

Peloptulus Berlese, 1908 …………….... 22, 164

Peloribates Berlese, 1908 ........................ 25, 209

Peltenuiala Norton, 1983 .......................... 17, 99

Pentabodes P. Balogh, 1984

(=Phyllocarabodes)

21,151

Pentazetes J. y P. Balogh, 1983 ............... 23, 178

Perezinigokalumma gen. nov. ........... 7, 25, 213

Pergalumna Grandjean, 1936 .................. 25, 218

Peridromotritia Jacot, 1923

(=Phthiracarus) …………………..... 14, 53

Perlohmannia Berlese, 1916 ..................... 13, 36

Permycobates Strenzke, 1954

(=Calyptozetes) …………………..... 23, 183

Perscheloribates Hammer, 1973 ............... 24, 197

Perspicuoppia (Oppiella) Pérez-Íñigo,

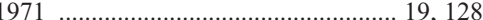

Persuctobelba Mahunka, 2000 ................ 20, 135

Pertorgunia Dalenius, 1958

(=Halozetes) ……………………..... 21, 156

Perutritia (Mesotritia) Märkel, 1964 ........ 14, 44

Perxylobates Hammer, 1972 …................ 25, 205

Petrozetes Sitnikova, 1968

(=Antarcticola)
Phalacrozetes (Vilhenabates) Aoki, 1965

.

Phauloppiella Subías, 1977 .................... 24, 191

Phenopelops Petrunkevitch, 1955

$$
\text { (=Eupelops) }
$$

Pheroliodes Grandjean, 1931

Philippizetes (Indoribates) Corpuz-Raros, 1979

Philippobodes J. y P. Balogh, 1992

(=Bathocepheus)

15,73

Philippotocepheus J. y P. Balogh, 1992 .. 21, 147

Philoribates Corpuz-Raros, 1980

(=Scheloribates) …............................ 24, 198

Philotritia Mahunka, 1988 (=Oribotritia) .. 14, 44

Phrathicarus Niedbala, 1994

(=Calyptophthiracarus)

Phthiracarulus Berlese, 1920

(=Mesoplophora)

.................... 14, 52

.

Phthiracarus Perty, 1841 ...................... 14, 53

Phthirarica (Notophthiracarus) Mahunka,

1982 ..................................................... 14, 53

Phylacozetes Grandjean, 1936 ................. 17, 90

Phyllhermannia Berlese, 1916 ................. 15, 69

Phyllocarabodes Balogh y Mahunka, 1969 ............................................... 21, 151

Phyllochthonius Travé, 1967 ................... 13, 35

Phyllolohmannia (Mixacarus) J. y P. Balogh, 1987

Phyllonothrus Sellnick, 1959

(=Masthermannia)

Phyllorchestes Mahunka, 1983

(=Zetorchestes)

Phyllotegeus Berlese, 1913 (=Conoppia) .. 16, 83

Phyllozetes Gordeeva, 1978 ..................... 13, 33

Physobates Hammer, 1962 ...................... 22, 169

Piffliella Hammer, 1979 ......................... 24, 196

Pilizetes Sellnick, 1939 ........................ 25, 220

Pilobatella Balogh y Mahunka, 1967 ..... 25, 211

Pilobates Balogh, 1960 .......................... 25, 211

Pilocepheus Pérez-Íñigo, 1992 ................... 16, 83

Pilogalumna Grandjean, 1956 ............... 25, 220

Pirnodus Grandjean, 1956 .................... 25, 205

Plaesioppia (Brassoppia) Balogh, 1983 .. 19, 122

Plakoribates Popp, 1960 ........................ 22, 169

Planobates Hammer, 1973 ....................... 24, 198

Planoristes Iturrondobeitia y Subías, 1978

Plasmobates Grandjean, 1929 ................. 15, 71

Plateremaeus Berlese, 1908 ..................... 15, 73

Platyamerus J. y P. Balogh, 1983 ............ 18, 105

Platyliodes Berlese, 1916 ........................ 15, 72

Platynothrus (Heminothrus) Berlese,

$$
1913
$$

ene Hammer, 1966 ............... 20, 144

Plenoxylobates (Setoxylobates) Hammer,

1979 ................................................ 25, 207

Pleodamaeus Paschoal, 1983

(=Gymnodamaeus) ...

15,76

Plesiodamaeus Grandjean, 1954 .............. 15, 77

Plesiotritia Walker, 1965 (=Oribotritia) .... 14, 44

Pletzenoppia Balogh, 1983 ................... 19, 123
Plonaphacarus Niedbala, 1986

(=Rhacaplacarus) …........................... 14, 57

Plumobates Balogh y Mahunka, 1966 .... 24, 194

Plumozetes Balogh, 1972 ....................... 17, 90

Pluritrichoppia Subías y Arillo, 1989 ...... 19, 115

Pocsia (Euphthiracarus) Mahunka, 1983 .. 14, 47

Pocsoppia Mahunka, 1984

(=Granuloppia) …............................ 20, 131

Podacarus Grandjean, 1955 ................... 21, 157

Podopterotegaeus Aoki, 1969 ................. 16, 82

Podoribates Berlese, 1908 ..................... 23, 186

Poecilochthonius Balogh, 1943 .............. 13, 31

Polyoppia Hammer, 1968 ....................... 18, 112

Polypterozetes Berlese, 1916 ..................... 16, 82

Polyxylobates (Setoxylobates) Hammer,

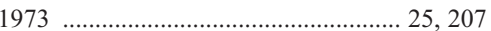

Pontiobates Luxton, 1989 ..................... 24, 191

Porallozetes (Pentazetes) J. y P. Balogh,

1992 ................................................. 23, 178

Porobelba Grandjean, 1936 ..................... 16, 81

Porofenichelia Mahunka, 1985

(=Fenichelia) ..................................... 21, 159

Porogalumnella (Galumnopsis) Balogh,

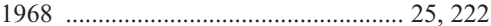

Porokalumma (Sandenia) Wallwork,

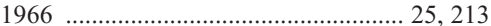

Poroliodes Grandjean, 1934 ...................... 15, 72

Poroscheloribates Arillo, Gil-Martín y Subías,

1994 ................................................ 24, 196

Porozetes (Sphaerozetes) Hammer

1962 ........................................... 23, 178

Porrhoppia Balogh, 1970 ...................... 19, 121

Porrhotegaeus Balogh y Mahunka, 1966 .... 16, 85

Posthermannia Grandjean, 1954

$(=$ Masthermannia $)$............................ 15, 68

Pravoppia (Membranoppia) Luxton,

1985 ......................................... 18, 111

Prionoribatella Aoki, 1975 .................... 22, 172

Processoppia Balogh, 1983 .................. 18, 112

Procorynetes (Liacarus) Woolley, 1967 .... 17, 97

Propelops Jacot, 1937 ........................... 22, 164

Propeschelobates Jacot, 1936

(=Protoribates) …........... 25, 205

Proteremaeus Piffl, 1965 ......................... 18, 107

Proteremella Balogh, 1959 (=Eremella) .. 18, 106

Protobelba (Belbodamaeus) Norton,

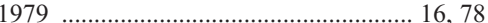

Protocepheus Jacot, 1928 ......................... 16, 83

Protokalumma (Neoribates) Jacot, 1929 .. 25, 212

Protophthiracarus (Notophthiracarus) Balogh,

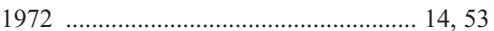

Protoplophora Berlese, 1910 ................... 13, 35

Protoribates Berlese, 1908 ..................... 25, 205

Protoribotritia Jacot, $1938 \ldots \ldots \ldots \ldots \ldots \ldots \ldots \ldots . .14,45$

Protoripoda (Calobates) Balogh, 1970 .. 24, 203

Protoschelobates Jacot, 1934

(=Scheloribates) …......................... 24, 198

Prototritia Berlese, 1910 ......................... 13, 35

Protozetes Balogh, 1962 ....................... 17, 90

Protozetomimus (Zetomimus) Pérez-Íñigo,

1990 ........................................... 23, 179

Provertex Mihelcic, 1959 ....................... 22, 161 
Psammocepheus (Fissicepheus) Aoki, 1970

............ 25, 220

Psednobates Luxton, 1992 ...................... 21, 157

Pseudachipteria Travé, 1960

(=Parachipteria) ........................ 10, 22, 169

Pseudantarcticola Balogh, 1970 ............. 21, 157

Pseuderemulus Balogh y Mahunka, 1968

17,102

Pseudoamerioppia Subías, 1989 .............. 19, 118

Pseudobrachioppiella Tseng, 1982 .......... 19, 128

Pseudocarabodes Mahunka, 1991 ......... 21, 153

Pseudocepheus Jacot, 1928 (=Xenillus) .... 17, 97

Pseudoceratoppia Hammer, 1962 ............. 17, 94

Pseudocryptacarus McDaniel, Norton y Bolen,

1979 (=Heptacarus) ........................... 14, 38

Pseudogalumna Pérez-Íñigo y Baggio, 1994

(=Carinogalumna) ........................... 25, 214

Pseudogeminozetes (Furcobates) Tseng,

1984 .................................................. 22, 175

Pseudonothrus (Allonothrus) Balogh, 1958 14,59

seudophysobates Fujikawa, 1991

(=Neophysobates)

22,169

Pseudopirnodus Baranek, 1985 .............. 24, 204

Pseudoppia Pérez-Íñigo, 1966 ............... 24, 191

Pseudopyroppia Rjabinin, 1987 ............... 17, 94

Pseudotectoribates Subías, 1977 ........... 22, 172

Pseudotocepheus Balogh, 1961 .............. 20, 145

Pseudotritia Willmann, 1919

(=Euphthiracarus)

14,46

Pteramerus Balogh, 1964 ....................... 18, 105

Pterobates Balogh y Mahunka, 1977 ........ 16, 85

Pterochthonius Berlese, 1913 ................... 13, 35

Pteroripoda Balogh y Mahunka, 1974 .... 24, 204

Pterozetes Hammer, 1966 ....................... 16, 85

Ptiloppia Balogh, 1983 ........................... 19, 128

Pulchroppia Hammer, 1979 ................... 19, 120

Pulchroppiella Balogh, 1983 ................. 19, 118

Punctizetes Hammer, 1971 ..................... 22, 167

Punctoribates Berlese, 1908 ................... 23, 183

Pustuloppia Mahunka, 1994 .................. 18, 112

Pyroppia Hammer, 1955 ......................... 17, 94

Quadroppia Jacot, 1939 ......................... 20, 133

Quadroribula J. y P. Balogh, 1992 ............ 17, 93

Quatrobelba (Subbelba) Norton, 1980 .... 16, 82

Quinquoppia Tseng, 1982 ...................... 19, 115

Radamoppia Mahunka, 1994

(=Lancelalmoppia)

18,111

Rafacarus (Steganacarus) Niedbala,

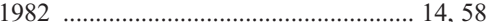

Rajskibates J. y P. Balogh, 1984

(=Liebstadia) …................................ 24, 195

Ramogneta Karppinen, 1966 ................. 18, 107

Ramonoppia Morell, 1990 ..................... 19, 118

Ramsayellus Spain y Luxton, 1970 ........ 23, 182

Ramuloppia Balogh, 1961 ..................... 19, 124

Ramusella Hammer, 1962 ..................... 19, 118

Ramuselloppia Subías y Rodríguez,

1986 ............................................... 19, 119

Rastellobata Grandjean, 1961 ............... 17, 102

Rectoppia (Ramusella) Subías, 1980 .... 19, 119

Reductobates Balogh y Mahunka, 1966 .. 24, 196
Reductoppia (Similoppia) P. Balogh,

1984

Reductoripoda Mahunka y Palacios-Vargas, 1996

(=Benoibates) .................................... 24, 203

Reptacarus Pérez-Íñigo y Peña, 1995 ...... 14, 41

Reteremuloides Mahunka, 1989 _........... 17, 102

Reteremulus Balogh y Mahunka, 1966 .... 17, 102

Reticobella Hammer, 1962

(=Suctobelbila)

20,139

Reticulocepheus Vasiliu y Calugar, 1977 .. 16, 84

Reticuloppia Balogh y Mahunka, 1966 .. 24, 191

Rhabdoribates Aoki, 1967 ................... 24, 198

Rhabdozetes Hammer, 1962 .................. 17, 90

Rhacaplacarus Niedbala, 1986 ................ 14, 57

Rhaphidosus (Liacarus) Woolley, 1969 .... 17, 97

Rhaphigneta (Autogneta) Grandjean,

$1960 \quad 18,108$

Rhaphoppia Balogh, 1983

(=Processoppia)

18,112

Rhinoppia Balogh, 1983 ...................... 19, 124

Rhinosuctobelba Woolley y Higgins,

. 20, 135

Rhopalozetes Balogh, 1962 .................... 17, 90

Rhynchobelba Willmann, 1953 .............. 20, 135

Rhynchobella Hammer, 196

(=Suctobelbila)

20,139

Rhynchoppia Balogh, 1968 .................. 20, 135

Rhynchoribates Grandjean, 1929 ............ 20, 139

Rhysotritia Märkel y Meyer, 1959

(=Acrotritia)

Rimandocepheus Corpuz-Raros, 1998 .... 21, 147

Rioppia Balogh y Mahunka, 1977 .......... 18, 107

Rohria Balogh y Mahunka, 1977 ........... 23, 185

Romanobates Feider, Vasiliu y Calugar, 1970

(=Lucoppia) …................................ 23, 187

Rostrogalumna Engelbrecht, 1973 ......... 25, 221

Rostrozetes (Trachyoribates) Sellnick,

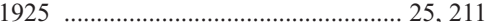

Rugoppia Mahunka, 1986 ..................... 20, 130

Rugozetes Balogh, $1960 \ldots \ldots \ldots \ldots \ldots \ldots \ldots \ldots . . . . .17,90$

Rykella Balogh, 1962 ............................ 23, 185

Sabacarus Ramsay y Sheals, 1969 ............ 14, 45

Sabahoppia (Ramusella) Mahunka,

1987

Sabahtritia Mahunka, 1987 .................... 14, 47

Sacculobates Grandjean, 1962 ................ 15, 71

Sacculogalumna Engelbrecht, 1973 ........ 25, 221

Sacculoppia Balogh y Mahunka, 1968 .. 19, 129

Sacculozetes Behan-Pelletier y Rjabinin,

199

22,167

Sadocepheus Aoli, $1965 \ldots 16,84$

Safrobates (Macrogena) Mahunka, 1989

Sagittabodes J. y P. Balogh, 1992 ......... 21, 153

Sagittazetes J. y P. Balogh, 1983 22, 167

Salpasozetes Mahunka, 1977

(=Bipassalozetes)

22,162

Saltatrichus (Demisalto) Coetzee, 1993 .. 23, 184

Samarocepheus Corpuz-Raros, 1990 ....2 21, 147

Samoabates Hammer, 1973 ................... 24, 198

Sandenia Oudemans, 1917 ...................... 25, 213

Sarawakiella (Pilizetes) Mahunka, 1996 .. 25, 220
Saxicolestes Grandjean, 1951

17,91

Scapheremaeus Berlese, 1910 ............... 21, 158

Scapuleremaeus Behan-Pelletier, 1989

$7,21,159$

Schalleria Balogh, 1962 ......................... 17, 90

Schalleriella Engelbrecht, 1972 ............... 17, 90

Scheloribatella Mahunka, 1984

(=Perscheloribates)

24,197

Scheloribates Berlese, 1908 ................ 4, 24, 198

Scheloribatoides Mahunka, 1988 ............ 24, 202

Schizozetes Balogh, 1962 ...................... 17, 91

Schusteria Grandjean, 1968 ................... 21, 157

Schweizerzetes Mahunka, 2001

(=Zachvatkinibates) $\ldots \ldots \ldots \ldots \ldots \ldots \ldots \ldots . . . . . . . . . . .23,184$

Scotiazetes Wallwork, 1966 ................... 23, 178

Scriptoripoda P. Balogh, 1985 ................. 24, 205

Sculptozetes Mahunka, 1984

(=Melanozetes) (.................................. 23, 176

Scutovertex Michael, 1879 .................... 22, 161

Scutoverticosus Kok, 1968 ................... 22, 161

Scutozetes Hammer, 1952 ..................... 22, 169

Seboetocepheus Mahunka, 1985 ............ 20, 145

Selenoribates Strenzke, 1961 ................. 21, 157

Sellnickia Oudemans, 1927 ..................... 24, 191

Sellnickochthonius Krivolutsky, 1964 ...... 13, 31

Selvazetes Behan-Pelletier, 1998

(=Naiazetes) …................................ 23, 176

Semipunctoribates Mahunka, 1987 ........ 23, 184

Semischeloribates Hammer, 1973

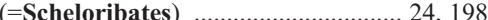

Senectoppia Aoki, 1976 ....................... 20, 132

Senilochthonius (Haplochthonius)

Mahunka, 1992 ................................... 13, 34

Senoribula Mahunka, 1975 ................... 24, 191

Separatoppia Mahunka, 1983 ................. 19, 129

Serrarius Michael, 1883 (=Gustavia) ...... 17, 95

Serratobelba Mahunka, 1984 ................ 20, 135

Serratoppia Subías y Mínguez, 1985 ...... 19, 124

Seteremaeozetes P. Balogh, 1988 ........... 22, 165

Seteremaeus Hammer, 1971 .................. 21, 159

Setincabates Lee, 1993 .......................... 25, 211

Setobates Balogh, 1961 (=Topobates) .. 24, 202

Setogalumna P. Balogh, 1985 ............... 25, 221

Setoppia Balogh, 1983 .......................... 18, 112

Setoxylobates Balogh y Mahunka, 1967 .. 25, 206

Setulobates Mahunka, 1984

(=Tuberemaeus) …............................. 24, 193

Setuloppia Balogh, 1983 ...................... 19, 123

Seychellozetes Mahunka, 1984

(=Drymobatoides) …...................... 23, 185

Sibiremaeus Rjabinin y Krivolutsky, 1975

(=Proteremaeus) ............................... 18, 107

Sicaxylobates Luxton, 1985 ................... 25, 207

Siciliophora (Prototritia) Bernini, 1983 .... 13, 36

Siciliotrichus Bernini, 1973 .................. 22, 172

Siculobata Grandjean, 1953 ................... 24, 194

Sigmonothrus Chakrabarti y Kundu, 1978

(=Platynothrus) ….............................. 15, 66

Similobates Mahunka, 1982 ................... 24, 202

Similochthonius Mahunka, 1985 .............. 13, 34

Similoppia Mahunka, 1983 .................. 19, 121

Simkinia Krivolutsky, 1966 .................. 24, 195 
Singabodes Mahunka, 1998

Sinolohmannia Balogh y Mahunka, 1979

(=Epilohmannia)

Sinozetes Mahunka, 2000

(=Kaszabozetes)

Solenozetes Grandjean, $1931 \ldots$ 15, 71

Spathulocepheus Balogh y Mahunka,

1969

Spatiodamaeus Bulanova-Zachvatkina,

1957

Sphaerobates Sellnick, 1928

(=Podoribates)

Sphaerochochthonius Mahunka, 1985

(=Similochthonius) .............................. 13, 34

Sphaerochthoniella Mahunka, 1985

(=Sphaerochthonius) ......................... 13, 34

Sphaerochthonius Berlese, 1910 .............. 13, 34

Sphaerogalumna Balogh, 1961 ............. 25, 221

Sphaerozetella Jacot, 1929

(=Sphaerozetes) …........................... 23, 178

Sphaerozetes Berlese, 1885 ................... 23, 178

Sphagnoppia J. y P. Balogh, 1986 ......... 19, 115

Sphodrocepheus Woolley y Higgins,

1963

16,84

Spinoppia Higgins y Woolley, $1966 \quad$...... 24, 191

Spinotocepheus Hammer, 1981 .............. 20, 146

Spinozetes Piffl, 1966 ......................... 18, 108

Stachyoppia Balogh, 1961 .................... 20, 131

Stakarenoppia (Karenella) Subías y Rodríguez,

1986 ............................................ 20, 130

Staurobates Grandjean, 1966 ................. 18, 105

Stauroma Grandjean, 1966 .................... 18, 105

Steganacarellus (Hoplophorella) Mahunka,

1986 .............................................. 14, 50

Steganacarus Ewing, 1917 ....................... 14, 58

Stelechobates Grandjean, 1965 ............. 24, 192

Stenoppia (Graptoppia) Balogh, 1983 .... 19, 116

Stenoxenillus Woolley y Higgins, 1966

(=Liacarus) …..................................... 17, 95

Sternoppia Balogh y Mahunka, 1968 .... 20, 132

Stictozetes Berlese, 1916 ....................... 25, 221

Stomacarus Grandjean, 1952 ................... 13, 27

Stonyxenillus (Xenillus) Woolley y Higgins,

1966 ................................................. 17, 99

Storkania Jacot, 1929 (=Scheloribates) .. 24, 198

Strabogalumna Mahunka, 1995

(=Stictozetes) ….............................. 25, 221

Strenzkea Travé, 1967 ............................. 17, 91

Striatobates Hammer, 1973 ................... 24, 202

Striatoppia Balogh, 1958 ....................... 20, 131

Strinatacarus Mahunka, 1974 ................... 14, 41

Sturmacarus P. Balogh, 1984

(=Neophthiracarus) …...................... 14, 56

Sturmozetes J. y P. Balogh, 1992 ............. 17, 91

Styloribates Jacot, 1934

(=Protoribates)

25,205

Stylozetes (Microzetes) Balogh y Mahunka,

Subbelba Bulanova-Zachvatkina, 1967 .... 16, 82

Subiasella Balogh, 1983 ........................ 19, 130

Subphauloppia Hammer, 1967 ................ 24, 191
Subpirnodus Mahunka, 1988

Subulobates Hammer, 1972

$$
\text { (=Maculobates) }
$$

Sucteremaeus Golosova y Krivolutsky,

$$
1975 \text {............................................... 20, } 135
$$

Suctobelba Paoli, 1908 .......................... 20, 135

Suctobelbata Gordeeva, 1991 ................ 20, 136

Suctobelbella Jacot, 1937 ....................... 20, 136

Suctobelbila Jacot, 1937 ........................ 20, 139

Suctobelbiloides Mahunka, 1988 ............ 20, 139

Suctoppia Balogh, 1958

(=Suctobelbila) ...

20,139

Suctoribates Balogh, 1963

Suctotegeus Mahunka, 1987

20,140

Sulcoribula Hammer, 1971 ....................... 17, 93

Sumatrotritia Mahunka, 1989 ................. 14, 47

Sundazetes Hammer, 1979

(=Indoribates) .

25,208

Svalbardia Thor, $1930 \quad \ldots \ldots \ldots \ldots \ldots \ldots \ldots \ldots . . . . . . . . . . . . .23,182$

Symbioribates Aoki, 1966 .................... 24, 196

Symphauloppia Balogh, 1972

$$
\text { (=Pseudoppia) ... }
$$

24, 191

Synchthonius Hammen, 1952

.. 13,32

Synichotritia Walker, 1965 ...................... 14, 47

Synoppia Balogh y Mahunka, 1969

$$
\text { (=Sternoppia) }
$$

Szentivanyella Balogh y Mahunka, 1969 .... 17, 91

Taeniogalumna Balogh, 1962 ................. 25, 221

Taiwanoppia Tseng, 1982 ...................... 19, 115

Talpacarus Grandjean, 1939

(=Torpacarus)

14,41

Tansocepheus Mahunka, 1983 ............... 21, 153

Tanzoppia Mahunka, 1988 ..................... 19, 115

Tauroplophora (Grandjeanoplophora) Gordeeva,

Niemi y Petrova-Nikitina, 1998 ............ 13, 35

Tecteremaeus Hammer, 1961 .................. 18, 108

Tectocarabodes Mahunka, 1988 .............. 21, 153

Tectocepheus Berlese, 1896 ..................... 21, 154

Tectodamaeus (Parabelbella) Aoki, 1984 .. 16, 81

Tectopelops Jacot, 1929 (=Eupelops) .... 22, 163

Tectoppia Wallwork, 1961 ...................... 19, 115

Tectoppiella Mahunka, 1984

(=Setoppia)

18,112

Tectoribates Berlese, 1910 ....................... 22, 172

Tegeocranellus Berlese, 1913 ........... 8, 21, 155

Tegeocranus Nicolet, 1855 (=Cepheus) .... 16, 82

Tegeozetes Berlese, 1913 ...................... 21, 155

Tegoribates Ewing, 1917 ...................... 22, 169

Teleioliodes Grandjean, 1934 ................... 15, 72

Temburongia Mahunka, 1990 .................... 14, 48

Tentaculozetes (Peloribates) Balogh,

$$
1970 \text {............................................. 25, } 211
$$

Tenuelamellarea Subías e Iturrondobeitia, ..........

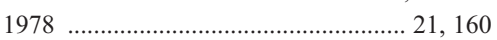
Tenuiala Ewing, 1913 .............................. 17, 99 Tenuialoides Woolley y Higgins, 1966 .... 17, 99 Tenuileius Lee, 1989 (=Turcibates) ….. 24, 194 Teraja Mahunka, 1995 (=Caucasiozetes) .... 16, 88 Teratoppia Balogh, 1959 ....................... 20, 132 Teratoppiella (Teratoppia) Balogh,

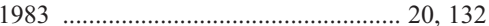

Tereticepheus S. y F. Bernini, 1990 .......... 16, 84

Terratritia Ramsay y Sheals, 1969 ............ 14, 45
Terrazetes Jacot, 1929 (=Unguizetes) .... 23, 186

Tessacarus Grandjean, 1962

(=Lamellocepheus) ............................. 16, 86

Tetracondyla Newell, 1956

(=Dolicheremaeus) ........................... 20, 141

Tetrochthonius Hammer, 1958

(=Haplochthonius)

13,34

Tetroppia Gordeeva, 1999

(=Moritzoppia) ….............................. 19, 126

Thalassozetes Schuster, 1963 .................. 21, 157

Thamnacarus Grandjean, 1950 ............... 14, 41

Thyrisoma Grandjean, 1953

(=Banksinoma) ….............................. 18, 109

Tikizetes Hammer, 1967 .............................. 16, 84

Tokunocepheus Aoki, 1966 ................... 21, 148

Tongacarus Hammer, 1973 (=Javacarus) .. 14, 38

Topalia Balogh y Csiszár, 1963 ............... 16, 86

Topobates Grandjean, 1958 ..................... 24, 202

Torpacarus Grandjean, 1950 ................... 14, 41

Totobates Hammer, 1961 ....................... 24, 196

Trachygalumna Balogh, 1960

$(=$ Stictozetes $) \quad$................................... 25, 221

Trachyhoplophora Berlese, 1923

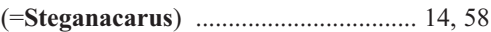

Trachyoribates Berlese, 1908 ................ 25, 211

Traegardhacarus Zachvatkin, 1945

(=Palaeacarus) ….............................. 13, 27

Transoribates Pérez-Íñigo, 1992 ........... 25, 207

Trapezoppia Balogh y Mahunka, 1969 .. 19, 123

Trematoppia Balogh, 1964 .................... 18, 112

Trhypochthoniellus Willmann, 1928 ....... 15, 60

Trhypochthonius Berlese, 1904 ................ 15, 60

Triaungius (Protoribates) Kulijev, 1978 .. 25, 206

Tribates Mahunka, 1978

(=Euscheloribates) ........................... 24, 197

Trichacaroceras (Acaroceras) Mahunka,

1991 ............................................. 16, 87

Tricheremaeus Berlese, 1908 ................ 17, 101

Trichocarabodes Balogh, 1961 ............... 21, 153

Trichocepheus Balogh y Mahunka, 1966 .. 20, 146

Trichocondyla J. y P. Balogh, 1986 ........ 20, 146

Trichodamaeus (Aleurodamaeus) Mahunka,

1984 ............................................. 15, 77

Trichogalumna Balogh, 1960 ................. 25, 221

Trichogalumnella Mahunka, 1992 ......... 25, 221

Trichonothrus Mahunka, 1986 ................. 15, 64

Trichoppia Balogh, 1961 ........................ 17, 94

Trichoribatella Mahunka, 1983

(=Latilamellobates)

23,179

Trichoribates Berlese, 1910

(=Murcia)

$10,23,177$

Trichoribatula Balogh, 1961

(=Phauloppia) ................. 24, 190

Trichotocepheus Aoki, 1965 ................ 21, 147

Trichozetes Balogh y Mahunka, 1980 ...... 17, 91

Trichthonius Hammer, 1961 ..................... 13, 33

Trihumerozetes Sellnick, 1959

(=Humerobates)

23,181

Trilohmannia Willmann, 1923

(=Trhypochthonius) ...........

.. 15,60

Trimalaconothrus Berlese, 1916 ......... 7, 15, 61

Tripiloppia Hammer, 1968 ...................... 19, 128 
Triplophora Mahunka, 1977

(=Arthrhoplophora) .....

Trischeloribates Hammer, 1971

(=Birobates)

Tritegeus Berlese, 1913

ritia Berlese, 1883 (=Oribotritia)

Triungulozetes (Indoribates) Subías,

2001

Trixylobates Balogh y Mahunka, 1978 .. 25, 207

Trizetes Berlese, 1904 ......................... 20, 140

Tropacarus (Steganacarus) Ewing, 1917 .... 14, 59

Truncopes Grandjean, 1956 .................... 24, 205

Truncozetes Balogh y Mahunka, 1969 .. 22, 173

Trypogalumnella Mahunka, 1995 .......... 25, 222

Tuberemaeus (Hemileius) Sellnick,

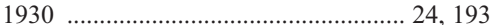

Tuberocepheus Balogh y Mahunka,

1969

Tuberoppia Golosova, 1974 ................... 19, 128

Tubulozetes P. Balogh, 1989 .................. 25, 212

Tumerozetes Hammer, 1966 ................... 16, 85

Tumidalvus Ewing, 1908

(=Trhypochthonius)

Tuparezetes Spain, 1969 ....................... 20, 133

Turcibates (Hemileius) Ayyildiz y Luxton,

1989

24, 194

Tutorozetes Hammer, 1967

23,178

Tuxenia Hammer, 1958 ......................... 25, 207

Tyrphonothrus (Trimalaconothrus) Knülle,

1957 ........................................... 7, 15, 62

Udetaliodes Jacot, 1929 (=Neoliodes) ...... 15, 71

Ugandoppia Mahunka, 1988

$(=$ Brachioppiella $)$............................. 19, 122

Uluguroides (Austrocarabodes) Mahunka, 1983

1,149

Ulugurozetes Mahunka, 1984

(=Ramsayellus)

23,182

Ululohmannia Mahunka, 1987

14,41

Umbellozetes Krivolutsky, 1969 ............ 22, 169

Unduloribates Balogh, 1943 ................. 22, 165

Undulozetes (Licnozetes) Balogh y Mahunka,

1969
Unguizetes Sellnick, 1925

Unicobelba Mahunka y

(=Suctobelbata) ......................20, 136

Uracrobates Balogh y Mahunka, 1967 .. 23, 186

Urobates Hammer, 1973

(=Uracrobates) …............................. 23, 186

Uronothrus Berlese, 1913 (=Camisia) .... 15, 64

Uroppia Balogh, 1983 .......................... 19, 119

Urubambates (Hemileius) Hammer,

1961 ............................................... 24, 194

Ussuribata (Suctobelbella) Rjabinin,

1975 ............................................... 20, 138

Vaghia Oudemans, 1919 ...................... 25, 221

Variogalumna Mahunka, 1995

(=Cosmogalumna)

Varioppia Mahunka, 1985

25,217

Veloppia Hammer, 1955 .................. 18, 106

Vepracarus Aoki, 1965 (=Papillacarus) .... 14, 40

Verachthonius Moritz, 1976 ..................... 13, 32

Vermacarus Balogh y Mahunka, 1980 ...... 17, 91

Vesiculobates (Siculobata) Hammer,

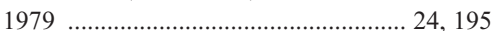

Vicinebates Paulitchenko, 1991

(=Latilamellobates)

Vietobates Mahunka, 1987

(=Limnozetes)

23,179

Vietoppia Mahunka, 1988

(=Taiwanoppia)

22,165

Vigilomicrozetes Tseng, 1982

$$
\text { (=Nothrus) }
$$

15,63

Vilhenabates Balogh, 1963 ................... 25, 207

Viracochiella Hammer, 1961 .......... 23, 178, 179

Wallworkella Balogh, 1983

(=Wallworkoppia)

Wallworkiella (Paraleius) Hammer,

1979

Wallworkodes J. y P. Balogh, 2002

Wallworkoppia Subías, 1989

Westwoodia Cambridge, 1875

(=Crotonia)

Williamsia Hammer, 1958

(=Williamszetes) ................................ 22, 170

Williamszetes Hammer, 1961
Woasella J. y P. Balogh, 2002

(=Hammerella) 20,132

Woolleybates J. y P. Balogh, 1984

(=Jornadia) ...................................... 23, 187

Xenilloides Pérez-Íñigo y Baggio, 1989 .... 17, 97

Xenillus Robineau-Desvoidy, 1839 ......... 17, 97

Xenogalumna Balogh, 1961 ................... 25, 221

Xenolohmannia Balogh y Mahunka, 1969

Xenoppia Mahunka, 1982 ..................... 19, 116

Xiphobates (Chamobates) Paulitchenko, 1994

23,180

Xiphobelba Csiszár, 1961 ................... 18, 104

Xylobates Jacot, 1929 (=Protoribates) .. 25, 205

Yambaramerus Aoki, 1996 .................... 17, 102

Yemenobodes Mahunka, 2000

(=Pseudocarabodes) …..................... 21, 153

Yoronoribates Aoki, 1987

(=Hammerabates) …....................... 24, 197

Yoshiobodes Mahunka, 1986 ................... 21, 154

Yungaseremaeus Balogh y Mahunka, 1969

18,106

Zachvatkinella Lange, 1954 ................... 13, 27

Zachvatkinibates Shaldybina, 1973 ........ 23, 184

Zaherizetes Yousef y Nasr, 1976

(=Rostrozetes) …....................

Zealandobates Hammer, 1967

(=Ramsayellus)

Zeanothrus Hammer, 1966

(=Trimalaconothrus)

Zeaotritia (Indotritia) Mahunka, 1988 .... 14, 43

Zeascheloribates Luxton, 1982 ............... 24, 195

Zeasuctobelba Hammer, 1966 ................ 20, 139

Zetes Koch, 1836 (=Galumna) .............. 25, 214

Zetobelba Hull, 1916 (=Zygoribatula) .. 23, 188

Zetomimus Hull, 1916 ..................... 23, 179

Zetomotrichus Grandjean, 1934 ............. 23, 185

Zetorchella Berlese, 1916

(=Chaunoproctus) …........................ 24, 192

Zetorchestes Berlese, 1888

Zygachipteria Mihelcic, 1956

(=Cerachipteria) .............................. 22, 168

Zygoribatula (Oribatula) Berlese, 1916 .. 23, 188 


\section{ÍNDICE ALFABÉTICO Y BIOGEOGRÁFICO DE ESPECIES (Y SUBESPECIES) DE ORIBÁTIDOS}

aba (Galumna) …………………………..... 214

ababaeus (Peloribates) ….............................. 209

abalai (Chaunoproctus) …........................... 192

abarkouhiensis [R. (Insculptoppia)]

(=elliptica) ............................................... 118

abbreviatus (Perscheloribates) ................... 197

abchasica [L. (Antennoppia)] ........................ 113

abchasica (Ceratoppia) ................................... 93

abchasica [Q. (Coronoquadroppia)] .............. 133

abchasicus (Papillacarus) .............................. 40

abchasicus [H. (Platynothrus)] (=peltifer) .... 66

abdita (Aeroppia) ......................................... 112

abditus (Paraphysobates) ......................... 169

abdominalis [L. (Dorycranosus)] ................. 96

abdosensilla (Camisia) ................................... 64

abeloosi (Miracarus) (=similis) ...................... 90

aberrans (Belba) (=sculpta) …..................... 77

aberrans (Protoribotritia) ..................................... 45

aberrata (Medioppia) (=subpectinata) ........ 124

aborigensis [D. (Epidamaeus)] ........................ 79

aborigensis (Niphocepheus) ........................... 84

abraensis (Scheloribates) ….......................... 198

abresi (Stomacarus) ................................... 27

abrupta (Acrogalumna) ............................... 213

absalom (Eupelops) sp. inq. ....................... 163

abscondita (Cryptoplophora) .......................... 35

abscondita (Cryptoplophora) .......................... 35

abscondita (Cryptoplophora) ...................... 35

abscondita (Cryptoplophora) ……................. 35

abscondita (Cryptoplophora) ........................ 35

abscondita [M. (Parplophora)] ...................... 42

absimilis (Atropacarus) .................................. 48

absolon (Dolicheremaeus) ........................... 141

absolutus (Benoibates) ............................... 203

abstemius (Notophthiracarus) ...................... 50

abstemius (Phthiracarus) …........................... 53

abulensis (Montizetes) ................................ 107

acanthophora (Indotritia) (=krakatauensis) .... 43

acanthus (Campbellobates) ......................... 204

acarinus (Aphelacarus) .................................... 28

acarinus (Aphelacarus) .................................. 28

acarinus (Aphelacarus) ................................. 28

acarinus (Aphelacarus) .................................. 28

acaroides (Murcia) sp. inq. ............................ 177

achalensis (Pheroliodes) ................................. 73

achipteroides (Anachipteria) …..................... 168

achipteroides (Anachipteria) ........................ 168

aciculatus (Papillacarus) .................................. 40

aciculatus (Papillacarus) .................................. 40

aciculatus (Reteremulus) ............................. 102

acostulatus (Eueremaeus) ........................... 100

acromios (Eupelops) ...................................... 163

acromios (Eupelops) ……………………..... 163

acromios (Eupelops) ...................................... 163

acruciata (Eremaeus) (=hepaticus) …........ 100

actirostrata (Medioxyoppia) .......................... 124

aculeata [M. (Allobelba)] ................................ 80

aculeatisetae (Neoliodes) hawaiiensis ......... 72

aculeatus [P. (Neophthiracarus)] ................ 56 aculeatus (Notophthiracarus)

aculeatus [R. (Mantigueracarus)] ....................58

aculeatus [P. (Oxyscheloribates)] ............... 198

acuminata (Ceratoppia) Koch, 1841 (=bipilis)

cuminata (Ceratoppia) Gol., 1981 ............... 93

acuminata (Ceratoppia) Gol., 1981 ................ 93

acuminata (Lauroppia) maritima ................. 126

acuminata (Lauroppia) maritima .................. 126

acuminata [O. (Zygoribatula)] ................. 188

acuminatus [L. (Rhaphidosus)] ...................... 97

acuminatus [L. (Rhaphidosus)] ..................... 97

acuspidatus (Mycobates) ............................ 182

acuspidatus (Planoristes) ............................... 97

acuta (Achipteria) .................................. 10, 167

acuta (Achipteria) ................................ 10, 167

acuta (Anachipteria) .................................... 168

acuta (Beckiella) ........................................ 140

acuta (Graptoppia) sundensis ...................... 116

acuta (Hafenrefferia) ...................................... 99

acuta (Karenella) ......................................... 130

acuta (Medioxyoppia) ................................. 124

acuticlava (Scheloribates) praeincisus ........ 201

acutidens [L. (Dorycranosus)] ......................... 96

acutidens [L. (Dorycranosus)] ....................... 96

acutidens (Suctobelbella) ............................... 136

acutidens (Suctobelbella) ............................... 136

acutidens (Suctobelbella) .......................... 136

acutinotata (Paraphauloppia) ................... 190

acutipes (Separatoppia) .............................. 129

acutirostris [Z. (Protozetomimus)] ................ 179

acutirostris (Medioppia) (=ordunensis) ...... 123

acutirostris (Scheloribates) ........................ 198

acutirostrum [I. (Mancoribates)] ...............2 208

acutirostrum (Plasmobates) …..................... 71

acutirostrum (Samoabates) ….................... 198

acutisetosa (Nanhermannia) ........................68

acutissima (Suctobelbella) sp. inq. ............ 136

acutissimus (Totobates) ............................. 196

acutodentata [S. (Ussuribata)] ..................... 138

acutus (Birobates) ........................................ 203

acutus (Cavernocepheus) ........................... 141

acutus [L. (Dorycranosus)] .............................. 97

acutus (Eremaeozetes) ................................. 165

acutus (Grypoceramerus) .............................. 108

acutus (Peloribates) …............................... 209

acutus (Peloribates) …................................... 209

acutus (Rhynchoribates) ……..................... 139

acutus [P. (Triaungius)] …............................ 206

adami (Eremulus) ........................................ 102

adamsoni (Nesiotizetes) ............................. 186

adanata [N. (Confinoppia)] .......................... 127

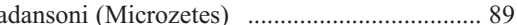

adareata (Pilogalumna) (=ornatula) ......... 220

adelae (Xenillus) ......................................... 97

adelaidae (Oribella) ......................................... 107

adjacens (Aeroppia) ....................................... 112

adjecta (Phauloppia) ..................................... 190

adjuncta (Lanceoppia) adminensis (Licnoliodes) ............................... 73

admirabilis (Notophthiracarus) (=obsessus) .... 51 admirabilis [N. (Protophthiracarus)] ............ 53

adpressus (Gymnodamaeus) ............................ 76

adpressus (Gymnodamaeus) ........................... 76

aduncus (Rhacaplacarus) …........................ 57

aegra (Pergalumna) ................................... 218

aegrota (Belba) ............................................... 77

aegyptiaca (Anachipteria) …......................... 168

aegyptiaca (Oppiella) (=nova) ...................... 128

aegyptica (Epilohmannia) pallida ……........... 37

aegyptica (Galumna) ................................... 214

aegypticus [P. (Archiphthiracarus)] ................ 54

aegypticus (Berlesezetes) .............................. 87

aegypticus (Lamellobates) molecula ............ 167

aegypticus [T. (Rostrozetes)] ........................ 211

aelleni (Ceratozetella) (=thienemanni) ........ 173

aelleni (Fijibates) ............................................. 197

aelleni [S. (Guatemalozetes)] ...................... 178

aelleni (Neoamerioppia) ............................ 114

aelleni (Strinatacarus) ….............................. 41

aenigmaticus (Xenilloides) ............................ 97

aenus [P. (Neophthiracarus)] ....................... 56

aeoliana (Berniniella) .................................... 125

aeoliana (Mongaillardia) ............................. 101

aepyornis [R. (Insculptoppiella)] .............. 119

aequalis (Melanozetes) sp. inq. ................... 176

aequalis (Papillacarus) .................................... 40

aequalis (Pergalumna) ............................... 218

aequalis (Scheloribates) ................................ 198

aequalis [O. (Zygoribatula)]

$(?=$ arcuatissima) ...................................... 188

aequidentatus [L. (Dorycranosus)] ................ 97

aequipunctatus (Euphthiracarus)

(=monodactylus) ........................................ 46

aequiseta (Ramonoppia) .............................. 118

aequivoca (Arcoppia) .................................. 120

aethiopica (Basilobelba) ............................. 104

aethiopica (Gustavia) .................................... 95

aethiopicus (Scheloribates) .......................... 198

aethiopicus (Scheloribates) …....................... 198

affinis (Acrotritia) ardua .................................. 45

affinis (Carabodes) ....................................... 149

affinis [D. (Epidamaeus)] ............................... 79

affinis (Eupelops) (=plicatus) ...................... 163

affinis [S. (Flagrosuctobelba)] ...................... 138

affinis (Mochlobates) .................................... 185

affinis (Oribatula) (=tibialis) ..................... 188

affinis (Phthiracarus) ..................................... 53

africana (Archeremella) .............................. 106

africana (Basilobelba) ............................... 104

africana (Beckiella) .................................... 140

africana (Beklemishevia) ............................. 27

africana (Eremella) ..................................... 106

africana (Heterobelba) ................................ 104

africana (Hoplophorella) (=vitrina) .............. 49

africana (Indotritia) ....................................... 43

africana (Lasiobelba) (=neonominata) ........ 113

africana (Machuella) (=draconis) ….......... 133 
africana (Mesoplophora) ………................... 42

africana (Neoamerioppia) ......................... 114

africana (Neoamerioppia) ............................... 114

africana (Notogalumna) ............................. 217

africana (Oribotritia) ........................................ 44

africana (Pedrocortesella) ............................. 74

africana [H. (Phyllhermannia)] ..................... 69

africana [G. (Porogalumnella)] ................. 222

africana (Protoribotritia) ................................. 45

africana (Prototritia) ……............................. 35

africana (Senoribula) .................................. 191

africana (Separatoppia) ............................. 129

africana [G. (Stenoppia)] ........................... 116

africana (Szentivanyella) ............................ 91

africana (Topalia) ............................................ 86

africanus (Allozetes) .................................... 166

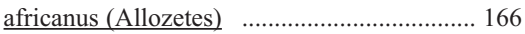

africanus (Annectacarus) ............................... 37

africanus (Annectacarus) ............................. 37

africanus (Berlesezetes) (=ornatissimus) ......87

africanus (Brachychthonius) $s p$. inq. ......... 29

africanus (Diplobodes) .............................. 152

africanus (Dolicheremaeus) ....................... 141

africanus (Eohypochthonius) ...................... 28

africanus (Eremulus) sp. inq. ..................... 102

africanus (Fosseremus) (=laciniatus) $\quad$........ 103

africanus (Ghilarovizetes) .......................... 176

africanus (Hoplophthiracarus) (=kugohi) .... 50

africanus (Humerobates) ........................... 181

africanus (Hypovertex) .............................. 161

africanus (Liochthonius) ............................. 30

africanus (Mahunkazetes) .......................... 185

africanus (Meristacarus) ............................... 39

africanus (Micreremus) .............................. 159

africanus (Neoeutegaeus) ............................... 85

africanus (Parhypochethonius) $s p$. inq. ........ 28

africanus (Passalozetes) ........................ 12, 162

africanus (Passalozetes) ....................... 12, 162

africanus (Pheroliodes) ................................ 73

africanus (Pilizetes) ..................................... 220

africanus (Plasmobates) sp. inq. .................. 71

africanus (Plenotocepheus) ....................... 144

africanus [E. (Pocsia)] ................................ 47

africanus (Podoribates) javensis ................ 186

africanus [T. (Rostrozetes)] .......................... 211

africanus (Scheloribates) fimbriatus ........... 199

africanus (Scheloribates) fimbriatus ........ 199

africanus (Tectoribates) .............................. 172

africanus (Tegeocranellus) (=knysnaensis) .. 155

africanus (Trhypochthonius) $s p$. inq. .......... 60

africanus [A. (Trichacaroceras)] ..................8 87

africanus [A. (Trichodamaeus)] …............... 77

africanus (Trimalaconothrus) $s p$. inq . .......... 61

africanus (Uracrobates) .............................. 186

afrum (Perezinigokalumma) ................. 7, 213

agalawatta (Austrocarabodes) ….................. 148

agathis (Condylobelba) …........................... 134

agaveae [O. (Zygoribatula)] ......................... 188

agenjoi [C. (Klapperiches)] ....................... 150

agenjoi (Parachipteria) ................................... 169

aggenitalis (Euphthiracarus) ............................ 46

agilis (Scheloribates) sp. inq. ........................ 198 agressor (Austrocarabodes) ….................... 148

agressor (Sagittazetes) .............................. 167

agricola [D. (Cylindroppia)] (=cylindrica) .... 123

agricola (Passalozetes) ................................ 162

agricola (Transoribates) ................................ 207

agricolus (Scheloribates) ….......................... 198

agrosticula (Joshuella) .................................... 76

agueroi (Galumna) ................................... 214

agusana (Magyaria) ...................................... 209

agusanensis (Perscheloribates) ..................... 197

ahumerata (Gerloubia) (=hispanica) ......... 191

ainu (Damaeus) ............................................... 78

akhtyamovi (Banksinoma) ............................. 109

akidosus [X. (Stonyxenillus)] ......................... 99

akimovi (Papillacarus) ..................................... 40

akitaensis (Nothrus) ....................................... 63

akitaensis (Pergalumna) ................................. 218

akitaensis (Pergalumna) ................................ 218

akusiensis (Uroppia) ...................................... 119

akusiensis (Uroppia) ....................................... 119

alachua [D. (Epidamaeus)] (=floridus) ....... 79

alamellae (Allogalumna) ............................. 213

alamellata (Ramusella)(=clavipectinata) ... 118

alandalusica (Iberogalumnella) .............. 12, 222

alas (Allozetes) ............................................. 166

alas (Tegeocranellus) ....................................... 155

alaskensis (Hololohmannia) ............................ 36

alaskensis (Maerkelotritia) (=kishidai) .......... 43

alaskensis (Peloribates) ............................. 209

alaskensis (Propelops) .................................. 164

alata (Galumna) .......................................... 214

alata (Galumna) ............................................ 214

alata (Galumna) ...................................... 214

alata (Galumna) ….................................... 214

alata (Galumna) .......................................... 214

alata (Galumna) ....................................... 214

alata [R. (Insculptoppia)] .......................... 118

alata (Liebstadia) (=pannonica) ................... 195

alata (Oribatula) (=allifera) $\ldots \ldots \ldots \ldots \ldots \ldots . . . . . . . . . . . .188$

alata (Oripoda) ............................................ 204

alata (Pilogalumna) (?=tenuiclava) ............ 220

alatus [L. (Dorycranosus)] (=splendens) ..... 97

alatus (Ikarotocepheus) ............................... 146

alatus (Neoliodes) ................................................. 71

alatus (Peloribates) (=glaber) ...................... 210

alatus (Phyllozetes) ......................................... 33

alatus (Tectocepheus) .................................... 154

alazon (Euphthiracarus) ................................ 46

albialatus (Perscheloribates) .......................... 197

albialatus (Perscheloribates) ........................ 197

albidus (Austrocarabodes) …..... 148

albidus (Protoribates) .................................. 205

albulus (Maculobates) 196

albulus (Trimalaconothrus) (=obsessus) ......62

albulus [T. (Tyrphonothrus)] ........................ 62

alces (Berlesezetes) ......................................... 87

alces (Ramusella) (=clavipectinata)

aleinikovae [D. (Epidamaeus)] ....................... 79

alejnicovae [R. (Insculptoppiella)] ................ 119

alestensis (Licnobelba) (=latiflabellata) ........ 75

alexandrinae (Scheloribates) ........................ 198

alfonsii [R. (Insculptoppiella)] ...................... 119 algarvensis [V. (Latilamellobates)] .............. 179

algicola (Autoppia) ...................................... 124

aliena (Suctobelba) ......................................... 135

alienus (Damaeus) (=riparius) ..................... 78

alienus (Notophthiracarus) …..................... 50

aligarhiensis (Lauroppia) (=fallax) ............ 126

aliquantus [P. (Archiphthiracarus)] ................ 54

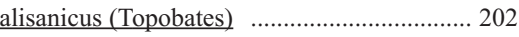

alius (Liochthonius) ........................................ 30

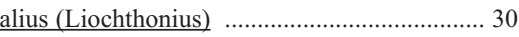

allaudi (Crotonia) ........................................... 66

alleganiensis [B. (Caenobelba)] ..................... 78

allifera (Oribatula) tibialis ........................... 188

allifera (Pilogalumna) (=crassiclava) .......... 220

alloenasuta (Suctobelbella) ................... 11, 136

alloenasuta (Suctobelbella) ................... 11, 136

almagrensis (Trimalaconothrus) ................. 61

almagulae (Cultroribula) ................................. 92

almeriensis (Flabellobelba) ………………...... 75

almerodai (Dolicheremaeus)

alpestris [A. (Izuachipteria)] ....................... 168

alpestris (Liochthonius) .................................. 30

alpestris (Montizetes) ..................................... 107

alpestris (Montizetes) ...................................... 107

alpha (Allogalumna) .................................... 213

alpina (Anachipteria) (?=deficiens) $\ldots \ldots \ldots . . . .168$

alpina (Belba) ............................................. 77

alpina (Murcia) ............................................. 177

alpina (Oribatula) ........................................ 187

alpinus [M. (Calyptozetes)] .......................... 183

alpinus (Chamobates) .................................. 179

alpinus (Euphthiracarus) reticulatus ................ 46

alpinus (Melanozetes) Halb., 15

(=stagnatilis) …....................................... 176

alpinus (Melanozetes) Schweiz., 56 sp. inq. .. 176

alpinus (Notophthiracarus) ……................... 50

alpinus (Scutovertex) ................................... 161

altaica (Moritzoppia) myrmophila ................ 127

altaica (Perlohmannia) ..................................... 36

altaica (Rhynchobelba) ................................ 135

altaicus [L. (Dorycranosus)] .......................... 97

altaicus [L. (Dorycranosus)] ......................... 97

altaicus (Melanozetes) ................................... 176

altera (Pergalumna) ........................................ 218

altera (Pergalumna) ...................................... 218

altera (Pergalumna) .................................. 218

altera (Pergalumna) …................................. 218

altera (Pergalumna) .................................. 218

alticola (Dolicheremaeus) ............................. 141

alticola (Protozetes) digitifer ......................... 90

alticola [L. (Rhaphidosus)] ............................ 97

altimontana (Paraphauloppia) ................... 190

altimontanoides (Paraphauloppia) .............. 190

altimontanus (Areozetes) .............................. 195

altimontanus (Diapterobates) ........................ 181

altimontanus [H. (Platynothrus)] ................... 66

altimonticola (Liochthonius) ......................... 30

altimonticola (Liochthonius) ........................ 30

altimonticola (Podopterotegaeus) …................. 82

altissima (Microtritia) (=hauseri) .................. 47

altissimus (Diapterobates) variabilis ........... 181

altissimus (Trimalaconothrus) ….................... 61 
altitudinis (Litholestes) ..................................... 91 altitudinis [S. (Tropacarus)] (=desmeti) ........ 59 altus [M. (Calyptozetes)] …………………... 183 altus (Liochthonius) ………………………..... 30 altus (Liochthonius) ...…………………….... 30

altvateri (Suctobelba) ........................................ 135

alvaradoi (Topobates) ……………………...... 202

alvarezi (Intermedioppia) ………………..... 116

alvarezi (Microtegeus) …………………….... 86

alvarezius (Scapheremaeus) ........................ 158

alveolatus (Austrocarabodes) …………..... 148

alveolatus (Dolicheremaeus) ………………... 141

alveolatus (Scapheremaeus) ......................... 158

alvordensis (Eueremaeus) ………………..... 100

alzanii (Tegoribates) (=latirostris) …........... 169

amabilis [F. (Psammocepheus)] ..................... 144

amamiensis (Dolicheremaeus) infrequens .... 142

amamiensis (Pergalumna) ………………...... 218

amamiensis (Trichotocepheus) ....................... 147

amazonensis [M. (Perutritia)] .......................... 44

amazonensis (Xenillus) .................................. 98

amazonica (Acroppia) ................................. 128

amazonica (Multipulchroppia) ..................... 120

amazonicus (Belemacarus) ......................... 172

amazonicus (Benoibates) ............................ 203

amazonicus (Dolicheremaeus) ..................... 141

amazonicus (Rhynchoribates) ........................ 139

amazonicus (Spathulocepheus) ................. 153

amazonicus (Xenillus) ..................................... 98

ambigua (Dampfiella) ………………………... 141

ambigua (Galumna) …………………..... 214

ambiguus (Ceratozetes) ................................ 174

ambiguus (Rhacaplacarus) ............................. 57

ambitus (Lamellocepheus) (=personatus) …8 86

amblyptera (Oribatula) (=tibialis) ………...... 188

americana (Acrotritia) (=ardua) .................... 45

americana (Crotonia) ..................................... 67

americana (Cheloppia) ................................. 130

americana (Epilohmannia) pallida ............... 37

americana (Furcoppia) ................................. 92

americana (Joelia) sp. inq. .......................... 170

americanus (Fosseremus) ............................... 103

americanus (Kalyptrazetes) .............................. 89

americanus (Peloptulus) ……………………... 164

americanus (Peloptulus) …………………... 164

americanus (Peloptulus) …………………...... 164

americanus (Peloribates) ............................... 209

americanus (Sellnickochthonius) ..................... 31

americanus (Tectocepheus) ............................ 154

americanus (Tegoribates) ................................ 169

americanus (Tegoribates) .............................. 169

americanus (Trhypochthonius) ......................... 60

amica (Autogneta) ……………………....... 108

amicabilis (Brachychthonius) ........................... 29

amictus (Camisia) sp. inq. ............................. 64

amicus [P. (Archiphthiracarus)]

(=anonymus

amicus (Camisia) biurus ...................................... 65

ammonoosuci (Adoristes) ................................ 95

amnicus (Limnozetes) .................................... 165

amnicus (Limnozetes) .................................... 165

amoenus (Rhacaplacarus) amonstruosus (Pseudotocepheus)

amonstruosus (Pseudotocepheus) ................... 14

monstruosus (Pseudotocepheus) ................ 145

amparoae (Ramonoppia) ............................... 118

ampla (Oribotritia) …………………….......... 44

amplus (Dynatozetes) ………………………... 185

ampulla (Trachyoribates) …………............... 211

ampulla (Trachyoribates) ………….......... 211

amudariensis (Hydrozetes) ………………..... 155

amurica (Suctobelbella) ……………………... 136

amurica (Suctobelbella) ………………….... 136

amygdaliformis (Epilohmannia) .................... 36

anakolosus [X. (Stonyxenillus)] ...................... 99

ananthakrishni [R. (Insculptoppia)] .............. 118

anareensis (Austroppia) (=crozetensis) $\quad$....... 122

anareensis (Totobates) .................................. 196

anareolata (Lyroppia) ................................... 131

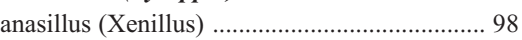

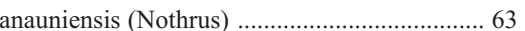

anauniensis (Nothrus) ..................................... 63

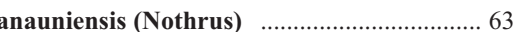

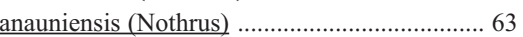

anauniensis (Nothrus) …………………........ 63

anauniensis (Nothrus) ....................................... 63

anceps (Oribotritia) ………………………...... 44

anchiseta (Acrotritia) (=vestita) .................... 46

andalusicabulensis (Paternoppia) ………….... 109

andasibe [C. (Klapperiches)] …………….... 150

andasibe (Pergalumna) ………………….... 218

andhraense (Pergalumna) ………………....... 218

andicola (Edwardzetes) ………………........ 175

andicola (Epieremulus) granulatus ............ 106

andicola (Pergalumna) ............................... 218

andina (Borhidia) ……................................ 133

andinus (Liochthonius) ................................. 30

andinus (Pedunculozetes) ............................ 180

andinus (Pirnodus) ..................................... 205

andinus [L. (Procorynetes)] ……………...... 97

andinus [N. (Protophthiracarus)] …………... 53

andinus (Pseudotocepheus) ....................... 145

andrassyi [S. (Ussuribata)] ........................... 138

andrei (Hoplophorella) (=vitrina) ................... 49

andrei (Licnoliodes) ......................................... 73

andrewi (Liochthonius) …............................... 30

andrianovae [O. (Zygoribatula)] .................... 188

andulau (Corynoppia) ……………………..... 130

andulauensis (Dolicheremaeus) ...................... 141

anellata (Pergalumna) …............................ 218

angarensis (Proteremaeus) ....................... 107

angelus (Pterochthonius) …………………..... 35

angelus (Pterochthonius) …………………... 35

angelus (Pterochthonius) …………………...... 35

angelus (Pterochthonius) ………………….... 35

angelus (Pterochthonius) ............................... 35

anglica (Achipteria) (=acuta) ....................... 167

angolensis (Basidoppia) ……..................... 121

angolensis (Hoplophorella) ........................... 48

angolensis (Lamellobates) (=molecula) ...... 167

angolensis (Metabelba) (?=glabriseta) .......... 80

angolensis (Nothrus) $s p$. inq. ........................ 63

angolensis (Oripoda) …………………...... 204

angolensis (Phthiracarus) (=lentulus) ........... 54 angolensis (Tegeozetes) $s p$. inq. .................. 155

anguina (Oripoda) ........................................ 204

angularis (Galumna) ..................................... 214

angulata (Camisia) (=amictus) ......................... 64

angulata (Cultroribula) …………………….... 92

angulata [H. (Phyllhermannia)] .................. 69

angulata [O. (Zygoribatula)] $(=$ glabra) $\quad$....... 189

angulatus (Austrocarabodes) $s p$. inq. ........ 148

angulatus [M. (Calyptozetes)] ...................... 183

angulatus (Chamobates) (?=confusus) …... 179

angulatus (Cosmopirnodus) ........................... 204

angulatus [D. (Epidamaeus)] ……………….... 79

angulatus (Malaconothrus) ........................... 60

angulatus (Papillacarus) ……………........... 40

angulatus (Peloribates) ................................... 209

angulatus (Protoribates) ................................. 205

angulatus (Punctoribates) …………………... 183

angulatus [S. (Rafacarus)] ................................ 58

angulatus (Scheloribates) Will., 31 ............... 198

angulatus (Scheloribates) Ham., 58

(=willmanni) ………………………….... 202

angulatus (Tectocepheus) (=sarekensis) ...... 155

angulatus (Trimalaconothrus) ............................ 61

angulatus (Trimalaconothrus) .......................... 61

angulifer [T. (Rostrozetes)] ……………….... 211

angulosa (Oribatella) ...................................... 170

angusta (Dampfiella) ……………………........ 141

angusta [L. (Incabates)] ................................. 209

angusta [L. (Incabates)] ……......................... 209

angusta [L. (Incabates)] ………………......... 209

angusta (Oribotritia) ............................................... 44

angusta [G. (Stenoppia)] ............................. 116

angustata (Murcia) ………………………..... 177

angustatus [L. (Dorycranosus)] ....................... 97

angustatus (Hoplophthiracarus) ....................... 50

angustifrons (Galumnella) (=nipponica) .... 222

angustior (Protoribates) (=capucinus) ........ 206

angustipes (Damaeus) .................................... 78

angustirostratus [D. (Epidamaeus)] ................. 79

angustirostris (Scheloribates) (=laevigatus) .. 200

angustirostrum [T. (Tyrphonothrus)] .......... 62

angustissima [V. (Latilamellobates)]

(=latilamellata) …………………………. 179

angustolamellata (Oribatula) …….................. 187

angustopili (Setoppia) ..................................... 112

angustus (Ceratozetes) .................................... 174

angustus (Dolicheremaeus) ……………....... 142

angustus (Fuscozetes) ……………………... 175

angustus (Neseutegaeus) ………………….... 85

anica (Pedrocortesella) …………………….... 74

ankae (Pseudoamerioppia) ……………...... 118

ankaratra (Galumna) .................................. 214

annamariae (Tereticepheus) .............................. 84

annobonensis (Meristacarus) africanus ...... 39

annobonica (Magyaria) ............................... 209

annulata (Fenestrobelba) …………………..... 134

annulata (Granuloteratoppia) ........................ 132

annulata (Pergalumna) ……………………...... 218

annulus (Haplamerus) …………………… 105

anomala (Liebstadia) (=similis) .................. 196

anomala (Oripoda) ..................................... 204

anomala (Ramuselloppia) ................................ 119 
anomaloides (Steganacarus) (=magnus) …...58 anomalus (Peloribates) $(=$ decumanus) $\quad$...... 209 anomalus (Steganacarus) (=magnus) .......... 58 anomia (Camisia) …………………………..... 65 anomola (Oribatella) .................................... 170 anonymus [P. (Archiphthiracarus)] ................. 54 anonymus [P. (Archiphthiracarus)] ............ 54 anonymus [P. (Archiphthiracarus)] ………..... 54 anonymus [P. (Archiphthiracarus)] ............ 54 anonymus [P. (Archiphthiracarus)] .............. 54 anonymus (Sellnickochthonius) ..................... 31 anoporosus (Tecteremaeus) ............................ 108 anosculpturatus [P. (Neophthiracarus)] ...... 56 ansata (Camisia) (=spinifer) ............................65 ansifera (Geminoppia) …………………..... 110 antalata (Galumna) .................................... 214 antarcticus (Alaskozetes) …………………..... 156 antarcticus (Alaskozetes) ................................. 156 antarcticus (Haplochthonius) ........................... 34 antarcticus [C. (Magellozetes)] ..................... 175 antarcticus [C. (Magellozetes)] …………..... 175 antarcticus (Totobates) ………………......... 196 antarcticus (Totobates) …………………..... 196 antennata (Setoppia) …………………….... 112 antenniger (Neogalumna) ……………….... 217 anteriosetosus (Plateremaeus) ...................... 73 anterostratus (Fijibates) ……………............. 197 antetriheterodactyla (Acrotritia) (=ardua) .... 45 anthelionus (Sphodrocepheus) ......................... 84 antichthon (Calobates) …………………....... 203 antillensis (Acroppia) .................................. 128 antillensis (Licneremaeus) (=discoidalis) .... 160 antillensis [O. (Oxyoppiella)] ....................... 129 antillensis (Pilogalumna) ........................... 220 antillensis (Protoribates) ............................. 205 antipai (Euphthiracarus) …………………....... 46 antis (Eremulus) ............................................. 102 antonii (Gehypochthonius) sp. inq. ................ 28 antsiranana (Lemurobates) .......................... 169 anulatus (Leobodes) ……………………...... 154 anuncata [R. (Insculptoppia)] ......................... 118 anzacensis (Scheloribates) ……………….... 198 aokii (Acrotritia) ............................................. 45 aokii (Acrotritia) ............................................... 45 aokii (Annectacarus) ……………………….... 37 aokii (Ceratozetella) ......................................... 173 aokii (Diplobodes) .......................................... 152 aokii (Dolicheremaeus) …………………...... 142 aokii [D. (Epidamaeus)] ................................... 79 aokii [L. (Incabates)] ..................................... 209 aokii [P. (Neophthiracarus)] ……………..... 56 aokii (Notophthiracarus) ............................... 50 aokii (Oribotritia) …………………………….... 44 aokii (Oxyamerus) ………………………….... 140 aokii (Oxyamerus) ……………………….... 140 aokii (Pergalumna) …………………………... 218 aokii (Pseudotocepheus) ............................... 145 aokii (Sellnickochthonius) …………………..... 31 aokii (Tripiloppia) ……………………...... 128 aokii (Yoshiobodes) ………………………... 154 aokii (Zetorchestes) ……………………….... 91 aokii (Zetorchestes) ………............................. 91 aotearoana [I. (Zeaotritia)] ............................ 43 aoutii (Phyllochthonius) ………………….... 35 apathyi (Chamobates) $s p$. inq _............... 179 apatomorpha (Ctenobelba) ........................... 101 apenninica [V. (Latilamellobates)]

$$
\text { (Eincisella) }
$$$$
\text { aperta [S. (Dividoppia)] }
$$

aphelesa (Metabelba)

aphidinus (Parhypochthonius)

aphidinus (Parhypochthonius)

aphidinus (Parhypochthonius)

aphidinus (Parhypochthonius)

aphidinus (Parhypochthonius)

apicalis (Epieremulus)

apicalis (Eupelops)

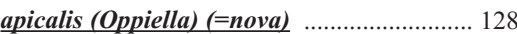

apiculata (Galumnella) .............................. 221

apiculatus (Phthiracarus) (=longulus) .......... 54

apletosa (Lucoppia) (=burrowsi) ................ 187

apoensis (Indoribates) (=panabokkei) ........ 208

apophysiger (Heminothrus) ............................... 65

appalachicola (Berlesezetes) ornatissimus .... 87

appalachicola [T. (Rostrozetes)] ovulum ...... 212

appalachicus (Damaeus) ................................ 78

appalachicus (Eremaeus) ............................... 100

appalachicus (Palaeacarus) (=hystricinus) $\quad . .27$

applicatus (Steganacarus) ……………………... 58

appressala (Galumna) ……………………..... 214

approximata (Suctobelbila) ......................... 139

approximatus (Eupelops) $(=$ plicatus) .......... 163

approximatus (Liochthonius) ...…………….... 30

aprofundata (Jacotella) $(?=$ =glabra) ………... 76

apuanicus (Carabodes) …………………........ 149

apunctatus (Licnoliodes) ................................. 73

\section{aquariorum (Trhypochthoniellus)}

\section{(=longisetus)}

aquaticus (Heterozetes) ................................ 173

aquaticus (Heterozetes) …………………..... 173

aquaticus (Mainothrus) ....................................... 59

aquilinus (Eurostocepheus) ........................... 146

aquilus (Notophthiracarus) ........................... 50

arabica (Galumna) ……………………........ 214

arabica (Lasiobelba) .................................... 113

arabica (Pilogalumna) …………………….... 220

arabica (Pilogalumna) ................................ 220

aragonensis (Scotiazetes) ....................... 12, 178

araios (Notophthiracarus) ............................. 50

araneola (Ctenacarus) ...................................... 28

araneola (Ctenacarus) _.................................. 28

araneola (Ctenacarus) ……………………... 28

araneola (Ctenacarus) …………………........... 28

araneola (Ctenacarus) ……………………..... 28

araneola (Ctenacarus) _.................................. 28

araucana (Eremaeozetes) ............................. 165

araucariae (Oripoda) .................................... 204

araujoi (Galumna) ………………………….... 214

arborea (Pergalumna) .................................. 218

arborea (Trichogalumna) ................................ 221

arboreus (Afronothrus) ……………………....... 59

arboreus (Eremaeozetes) ................................. 165

arboreus (Humerobates)

(?=rostrolamellatus) arboreus (Scapheremaeus) ............................ 158

arboricola (Brassiella) …………………..... 192

arboricola (Oribatula) …………………….... 187

arboriseta (Papillacarus) …………………....... 40

arcana (Suctobelbella) ………………..... 11, 136

arcana (Suctobelbella) ........................... 11, 136

arcanus (Scutoverticosus) ……………….... 161

arcidiaconoae (Lasiobelba) .............................. 113

arcidiaconoae (Lasiobelba) …………….......... 113

arcta (Beckiella) ......................................... 140

arctata (Acrotritia) (=ardua) .......................... 45

arctica (Ceratoppia) (=quadridentata) .......... 93

arctica (Cultroribula) …………………......... 92

arctica (Oribatella) ........................................... 170

arctica (Oribatella) ……………………...... 170

arctica (Pyroppia) ............................................. 94

arcticola [D. (Epidamaeus)] ………………….. 79

arcticola [D. (Epidamaeus)] _.......................... 79

arcticus [L. (Dorycranosus)] Banks, 1899 sp.

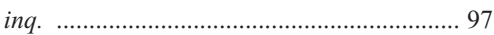

arcticus [L. (Dorycranosus)] Gri., 1984

(=neonominatus) ....................................... 97

arcticus (Montizetes) ....................................... 107

arcticus (Sellnickochthonius)

(=immaculatus) ……………………….... 32

arcticus (Sphaerozetes) …………………...... 178

arcticus (Sphaerozetes) .................................. 178

arctosus (Cosmochthonius) (=lanatus) ….... 33

arcualis (Arcoppia) ………………………... 120

arcualis (Conozetes) _.............................. 208

arcualis (Hypovertex) .................................... 161

arcualis (Hypovertex) ……………………..... 161

arcuata (Camisia) .......................................... 65

arcuata [S. (Flagrosuctobelba)] ...................... 138

arcuata [S. (Lalmoppia)] ………………..... 130

arcuata (Microppia) ……………………….... 124

arcuata (Zeasuctobelba) .............................. 139

arcuata [O. (Zygoribatula)] ……………….... 188

arcuatissima [O. (Zygoribatula)] .................. 188

arcuatissima [O. (Zygoribatula)] ………….... 188

arcuatum (Banksinoma) ............................... 109

arcuatus (Scapheremaeus) ........................... 158

arcuatus (Yambaramerus) …………............... 102

arcuatus (Yoshiobodes) …........................... 154

ardala (Crotonia) ……...................................67 67

ardua (Acrotritia) ............................................. 45

ardua (Acrotritia) ............................................... 45

ardua (Acrotritia) ………............................ 45

ardua (Acrotritia) ……................................. 45

ardua (Acrotritia) …………………………. 45

ardua (Acrotritia) ......................................... 45

ardua (Lamellarea) ....................................... 160

ardua [T. (Teratoppiella) ............................... 132

arduinii (Carabodes) ....................................... 149

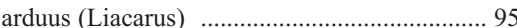

arenaria (Graptoppia) ....................................... 116

arenarius (Berlesezetes) ……………………. 87

areolata (Epilohmannia) pallida ...................... 37

areolata (Galumnella) _............................ 221

areolata [H. (Phyllhermannia)] ........................ 69

areolata (Nanhermannia) (=elegantula) .......68

areolata (Pilogalumna) (=tenuiclava) $\ldots . . . . . .220$ 


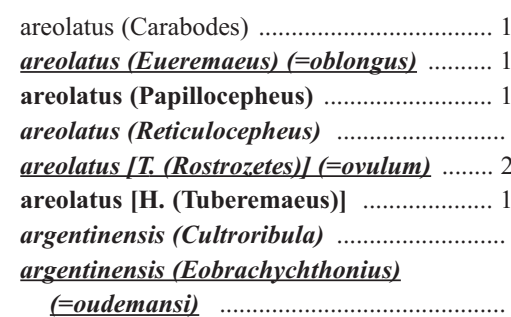

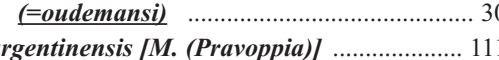

argentinensis (Scapheremaeus) ................... 158

argentinensis (Tenuelamellarea) ................. 160

argentinensis (Xenillus) ............................... 98

argentinensis (Zetomimus) (=furcatus) ...... 179

aridulus (Eueremaeus) .................................. 100

aridus (Ametroproctus) .................................... 159

arilloi (Xenillus) …………………………….... 98

aristosa (Hermanniella) ...................................... 70

aristosa (Hermanniella) ……………….......... 70

armadillo (Prototritia) ................................... 35

armata (Achipteria) ……………………...... 167

armata [S. (Dyobelba)] ……………………... 82

armata (Suctobelbella) …………………....... 136

armata [P. (Tectodamaeus) ] .............................. 81

armata [P. (Tectodamaeus)] …………........... 81

armatifera (Galumna) ……………............ 214

armatus [P. (Archiphthiracarus)] ................. 54

armatus (Ceratozetes) ……………………....... 174

armatus (Cuspidozetes) .................................. 170

armatus (Edwardzetes) ................................. 175

armatus (Eupterotegaeus) ………………………... 83

armatus (Suctobelbiloides) ………………...... 139

armatus (Vermacarus) ……………………... 91

armipes (Punctoribates) ................................. 183

arnoldii (Diapterobates) ……………………. 181

arnoldii (Thamnacarus) sp. inq. ....................... 41

arrecta $($ Hermanniella $)(=$ picea $)$..................... 70

arrogans (Austrocarabodes) ........................... 148

arrugata (Galumna) ...................................... 214

articristata (Lauroppia) …………………...... 126

artifex (Scheloribates) (?=fimbriatus) ….... 199

artigasi (Scheloribates) ................................. 199

artilamellatus (Podoribates) ........................ 186

artiodactylus (Atopochthonius) ........................ 35

artiodactylus (Atopochthonius) ....................... 35

artiodactylus (Atopochthonius) …………….... 35

artus (Holonothrus) ………………………..... 67

artus (Holonothrus) ............................................ 67

arvernensis (Damaeus) ……………………...... 78

ascendens (Scheloribates) …………………... 199

asculpturatus (Mochlozetes) ......................... 185

asejugalis (Peloribates) …………………….... 209

asensillata [M. (Perutritia)] ............................ 44

aseta (Pletzenoppia) ……………………….. 123

asetosa (Pseudoceratoppia) ........................... 94

ashoroensis (Nothrus) ......................................... 63

ashoroensis (Trhypochthoniellus) ....................60 60

asiatica [G. (Angulogalumna)] ....................... 217

asiatica (Paraceratoppia) ………………….... 94

asiatica (Collohmannia) ……………………... 36

asiatica (Cryptoplophora) …………………..... 35

asiatica (Neoamerioppia) asiatica (Neoamerioppia) …........................... 114

asiatica (Oribatella) ................................... 170

asiatica (Oribotritia) …………………………..... 44

asiatica (Rykella) …………………………...... 185

asiaticus (Cosmochthonius) …………………. 32

asiaticus [M. (Cristonothrus)] ……………...... 6

asiaticus (Eohypochthonius) ……………….... 28

asiaticus (Microtegeus) ……………….......... 86

asiaticus (Microzetes) ……………………….... 89

asiaticus (Neogymnobates) luteus ................. 178

asiaticus (Nothrus) ………………………….... 63

asiaticus (Nothrus) ……………………………. 63

asiaticus (Parhypochthonius) ……………….... 28

asiaticus (Peloribates) rangiroaensis $\quad$............ 210

asiaticus (Plasmobates) ……………………..... 71

asiaticus (Plasmobates) …………………….... 71

asiaticus (Truncopes) optatus ......................... 205

asiaticus [H. (Tuberemaeus)] ......................... 193

asinus (Suctobelbella) .................................. 136

askewi (Terratritia) ………………………........ 45

asotthalomensis (Liochthonius) ....................... 30

asper (Liochthonius) …………………...... 11, 30

asper (Liochthonius) ................................ 11, 30

asper (Liochthonius) .................................... 11, 30

asper [B. (Passalobates)] …………………... 162

aspera (Indotritia) …………………………....... 43

asperatus (Damaeolus) ..................................... 103

asperatus (Damaeolus) ............................... 103

asperula (Phauloppia) (=lucorum) .............. 190

asperulus (Chaunoproctus) ………………....... 192

asperulus (Gymnodamaeus) $(=$ bicostatus).. .76

aspidioti (Nothrus) (=ovivorus) ........................ 64

assamensis (Cosmochthonius) ……………...... 32

assamensis [M. (Cristonothrus)] ..................... 61

assamica (Malacoangelia) …………………...... 29

assimilis [P. (Archiphthiracarus)] ………….... 54

assimilis (Ramusella) $(=$ clavipectinata)

assimiloides (Ramusella) .............................. 118

astatus (Papillochthonius) ………………….... 31

astrachanicus (Semipunctoribates) ................. 184

asymmetrica (Aeroppia) ………………...... 112

asymmetricus (Caucasiozetes) ………………... 88

asymmetricus (Pseudotocepheus) ............... 145

atahualpensis (Scheloribates) ....................... 199

atavisticus (Comorozetes) ............................ 88

ater (Notophthiracarus) ………………….... 50

aterrimus (Euzetes) $s p$. inq. ........................... 175

atimonensis (Basilobelba) ……………….... 104

atlantica [L. (Incabates)] ………………….... 209

atlantica (Magyaria) ………………………... 209

atlantica (Metrioppia) ................................... 94

atlanticus [P. (Archiphthiracarus)] ................... 54

atlanticus (Damaeus) (=australis) ………..... 78

atlanticus (Scheloribates) praeincisus ...... 201

atmetos (Limnozetes) ..................................... 165

atomaria (Galumna) ……………………....... 214

atomaria (Suctobelba) …………………...... 135

atomus (Minunthozetes) (?=quadriareatus) .. 182

atra (Pilogalumna) (=tenuiclava) ................ 220

atraktus (Liacarus) ........................................ 95

atratus [P. (Archiphthiracarus)] …………... 54

atrichosus [C. (Klapperiches)] …………..... 150 attenuata (Oribotritia) ....................................... 44

attenuatus (Liochthonius) .................................. 30

atuelanus (Malaconothrus) ........................... 60

atypica (Ramuloppia) ramiseta ................... 124

atypicus (Huilicheremaeus) ........................ 160

atypicus (Mochlozetes) …………………..... 185

atypicus (Notophthiracarus) (=inelegans) .... 51

auberti (Podacarus) ……………………........ 157

augusta (Pedrocortesella) ............................... 75

aurantiacus (Neoribates) ................................ 212

aurantiacus (Neoribates) ............................... 212

aurantiacus (Neoribates) ………………….... 212

aurata (Belba) …………………………..... 77

auratus (Saxicolestes) …………………………... 91

aureonotata [G. (Tauroplophora)] ................... 35

aureopunctatus (Trimalaconothrus) ................. 61

aureus (Campbellobates) ............................. 204

aureus [P. (Neophthiracarus)] ........................ 56

auriculata (Oripoda) …………………….... 204

auriculatus (Anellozetes) ............................. 180

auriculatus (Carabodes) .................................. 149

auriculus (Trichocepheus) ............................ 146

aurita (Hafenrefferia) (=gilvipes) .................. 99

auritus (Damaeus) ...………………………... 78

auritus (Damaeus) ………………………….... 78

auritus (Dolicheremaeus) ………………….... 142

auritus (Eupelops) (=plicatus) ……............ 163

australica (Epilohmannia) pallida ………... 37

australicus (Cosmochthonius) ...................... 32

australiensis (Anderemaeus) ....................... 105

australis (Africoribates) …………………..... 180

australis (Anachipteria) (=signata) ............ 168

australis [P. (Archiphthiracarus)] ..................... 54

australis (Austrocarabodes) ........................ 148

australis (Austrodamaeus) ............................... 76

australis (Baloghacarus) .................................. 70

australis (Camisia) ………………………............ 65

australis [L. (Convergoppia)] …………...... 111

australis (Damaeus) ......................................... 78

australis (Damaeus) ...................................... 78

australis (Defectamerus) crassisetiger ........... 105

australis (Edwardzetes) …............................ 175

australis (Eremulus) ……….......................... 102

australis (Galumna) ……............................. 214

australis (Liochthonius) .................................. 30

australis (Longocepheus) .............................. 144

australis (Mesotritia) ........................................ 43

australis (Multioppia) ................................. 117

australis (Nesiacarus) ..................................... 40

australis (Notophthiracarus) ......................... 51

australis (Oripoda) Berl., 16 ......................... 204

australis (Oripoda) B. у M., 68

(=cordobensis) ………………………….... 204

australis (Paraphauloppia) .......................... 190

australis (Paschoalia) …………………….... 73

australis (Paschoalia) ...................................... 73

australis (Pergalumna) ................................ 218

australis (Pilizetes) …………………………... 220

australis (Pseudotocepheus) ........................ 145

australis (Similochthonius) .................................. 34

australis (Solenozetes) ..................................... 71

australis [T. (Tyrphonothrus)] _...................... 62 
australoides (Anachipteria) .......................... 168

austriaca (Jacotella) ......................................... 76

austriaca (Liebstadia) (=pannonica) …....... 195

austroafricanus (Notophthiracarus) ............. 51

austroafricanus (Trichonothrus) ................... 64

austroamericana (Monofurcoppia) .............. 93

austroamericana (Murcia) ........................... 177

austroamericanus (Gibbicepheus) .............. 152

austroamericanus (Mycobates) .................... 182

auxiliaris (Berlesezetes) (=ornatissimus) ......87

avenifer (Eremulus) .................................. 102

avenifer (Eremulus) …................................. 102

avenifer (Eremulus) ................................... 102

aveniferus (Zetomimus) (?=furcartus) ........ 179

aviculus (Oribatella) ..................................... 170

aviger (Hypozetes) ......................................... 166

aysineep (Eueremaeus) ................................ 100

azaisi (Rhynchoppia) ................................... 135

azaleos [M. (Calyptozetes)] ......................... 183

azerbeidjanica (Berniniella) ......................... 125

azerbeidjanica (Oribatula) tibialis ............... 188

azoreana (Galumna) .................................... 214

azorensis (Nothrus) palustris .......................... 64

azorensis (Steganacarus) hirsutus ................... 58

azorensis (Xenillus) discrepans ..................... 98

azoricus (Carabodes) ................................... 149

azoricus (Melanozetes) ………….................. 176

azoricus (Pilocepheus) …………………........ 83

azovicus (Scheloribates) ................................ 199

azumaensis (Trimalaconothrus) ...................... 61

azumai (Dimidiogalumna) ........................... 214

babalus (Basiceramerus) ….......................... 146

baburini (Oppiella) ....................................... 128

baccanensis (Gibbicepheus) …...................... 152

baccettii (Licnodamaeus) ............................... 74

bacillatus [P. (Metaphthiracarus)]

(=setosus)

bacillatus [L. (Rhaphidosus)]

bacillifera (Schalleria) ................................ 90

bacilliger (Austrocarabodes) ....................... 148

bacilliger (Pseudotocepheus) ....................... 145

bacilligera (Aethioppia) ............................. 112

bacilligera (Arcoppia) ……........................ 12

bacilliseta (Multioppia) ............................... 117

bacillum [D. (Epidamaeus)] ............................. 79

backstroemi (Neoliodes) .................................. 71

baculifera (Suctobelbella) ............................ 136

badensis [N. (Amerigloboppia)] ..................... 114

badensis (Liebstadia) (=longior) ................... 195

baderi [B. (Gressittoppia)] ......................... 122

baderi (Suctobelbila) ................................... 139

baderi (Xenillus) (=discrepans)

badghysi [L. (Dorycranosus)] ......................... 97

badia (Ceratoppia) (=bipilis) .......................... 93

badius (Mainothrus) ........................................... 59

badius (Mainothrus) ......................................... 59

badius (Scheloribates) ................................. 199

baetica (Lauroppia) ...................................... 126

baggioi [R. (Mantigueracarus)] ….................. 58

baggioi (Xenillus) ........................................... 98

bagnalli [R. (Insculptoppia)] ........................ 118

bagnalli (Oribotritia) ......................................... 44 bahamensis (Mochloribatula)

aichengensis [R (Insculptoppiella)] ....... 119

bajau (Otocepheus) ........................................ 147

bakeri [D. (Epidamaeus)] …............................. 79

bakeri [D. (Epidamaeus)] ............................ 79

bako (Pasocepheus)

balazsi (Eremobelba) ................................... 103

balazsi [H. (Kakophthiracarus)] ................... 49

balazsi (Scapheremaeus) ............................. 158

balcanicus [S. (Tropacarus)] ........................... 59

balearica (Pseudoamerioppia) ...................... 118

balearicus (Steganacarus) ................................. 58

baliensis (Eremulus) .................................... 102

baliensis [O. (Oxyoppiella)] ........................... 129

baliensis [S. (Ussuribata)] ........................... 138

baloghi [P. (Archiphthiracarus)] F. y S., 57 .... 54

baloghi [P. (Archiphthiracarus)] M., 82

(=neonominatus)

baloghi (Arcoppia) (=viperea) ….................. 121

baloghi (Arthrovertex) ................................... 160

baloghi (Ceratorchestes) .................................. 93

baloghi (Dolicheremaesu)

baloghi (Dolicheremaeus) ............................ 142

baloghi [S. (Flagrosuctobelba)] .................... 138

baloghi (Galumna) ..................................... 214

baloghi (Hungarobelba) ................................ 103

baloghi [V. (Latilamellobates)] .................... 179

baloghi [V. (Latilamellobates)] ................... 179

baloghi (Machadobelba) 106

baloghi (Mabulatrichus) ............................... 184

baloghi (Microzetes) ................................... 89

baloghi [P. (Neophthiracarus)] .................... 56

baloghi (Niphocepheus) nivalis ..................... 84

baloghi (Nodocepheus) ................................. 85

baloghi (Paratritia) .............................................. 45

baloghi (Polyoppia) ……............................. 112

baloghi (Scheloribates) Kardar, 76 ................ 199

baloghi (Scheloribates) P.-Í., 82 (=caprai) .. 199

baloghi (Scheloribates) C. y V., 83

(=neonominatus) ....................................... 20

baloghi (Striatoppia) ................................. 131

baloghi [T. (Teratoppiella)]

(=pluripectinata)

baloghi [O. (Zygoribatula)

baloghius (Scapheremaeus) ................... 158

baloghorum (Cyrthermannia) ........................ 67

baloghorum [P. (Archiphthiracarus)] (=bulbiferus)

baltazarae (Basilobelba)

baluktotus (Perscheloribates)

bandae (Odontocepheus)

banksi (Galumna) Jac., 29

banksi (Galumna) (Jac.

banksi [L. (Lancelalmoppia)]

banksi (Lohmannia) ......................................... 38

banksi (Oribotritia) .......................................... 44

banksi (Peloribates) (=peloptoides)

banksi (Phauloppia) ....................................... 190

banksi (Phthiracarus) …............................. 53

banksi [H. (Platynothrus)] ............................. 66

banksi [H. (Platynothrus)] ................................ 66 bannisteri (Pedrocortesella) …….................. 75

baobab [H. (Senilochthonius)] ...................... 34

barbarae (Adhaesozetes) ........................... 158

barbarae (Tegeocranellus) ............................ 155

barbarae (Tegeocranellus) .............................. 155

barbata (Basilobelba) ...................................... 104

barbata (Beklemishevia) ............................... 27

barbata (Belba) ................................................. 77

barbata (Hermanniella) ...................................... 70

barbata (Heterobelba) .................................. 104

barbata [L. (Incabates)] ............................... 209

barbata (Oribatella) ..................................... 170

barbata (Oxybrachioppia) ............................. 129

barbata (Xiphobelba) .................................. 104

barbatis (Multioppia) (?=wilsoni) ................ 117

barbatula (Dolicheremaeus) vilhenarum .. 143

barbatula (Epilohmannia) sp. inq. ............... 36

barbatulus (Scheloribates) ............................. 199

barbatus (Costeremus) .................................. 103

barbatus (Neoribates) …............................ 212

barbatus (Nodocepheus) dentatus ……......... 85

barbatus (Nodocepheus) dentatus ……...... 85

barbatus (Peloribates) ……………………..... 209

barbatus (Perxylobates) ................................. 205

barbatus (Perxylobates) …......................... 205

barbatus (Trimalaconothrus) ........................... 61

barborae (Steganacarus) (=spinosus) ............ 58

barguzini (Campachipteria) .......................... 168

barlowi (Paulianacarus) ............................... 40

barnardi (Galumna) ……........................... 215

barrancensis (Pseudoamerioppia) .................. 118

barrancensis (Pseudoamerioppia) .............. 118

barrancensis [T. (Tyrphonothrus)] ................ 62

barringtonensis (Pheroliodes) ….................. 73

bartkei (Dolicheremaeus) .............................. 142

bartosi (Belba) (=patelloides) ……................. 77

basidii (Basidoppia) .................................... 121

basilewskyi (Carabodes) sp. inq. ................ 149

basilewskyi (Chaunoproctus) .................... 192

basilewskyi (Galumna) ............................... 215

basilewskyi (Nothrus) ...................................... 63

basilewskyi (Pilizetes) .................................. 220

bataviensis (Neoliodes) ……........................... 71

bataviensis (Neoliodes) ................................. 71

bathamae (Halozetes)

baudi (Charassobates) ...................................... 86

bayanicus (Protoribates) .................................. 205

bayoumii (Neoppia) ....................................... 109

beccus (Oribatula) …............................. 187

becki (Acaroceras) .......................................... 87

becki (Eohypochthonius) ............................... 28

becki [L. (Lancelalmoppia)] ….................. 111

becki (Nothrus) ............................................ 63

becki [H. (Phyllhermannia)] ......................... 69

beemanensis (Belloppia) ............................... 125

behenae (Alpizetes) ...................................... 182

beijingensis (Odontocepheus) ….................. 153

belbiformis (Zachvatkinella) ......................... 27

belgicae (Halozetes) .......................................... 156

bella (Campachipteria) .................................. 168

bella (Campachipteria) ............................... 168

bella (Eremobelba) ........................................ 103 
bella [S. (Flagrosuctobelba)] (?=nasalis) .... 138 bellesii (Pergalumna) ................................... 218 bellicosa (Eremobelba) ................................... 103 bellicosus (Austrocarabodes) ......................... 148 bellingeri (Indotritia) ......................................... 43 bellissimus [H. (Tuberemaeus)] …................ 193 bellula [Q. (Coronoquadroppia)

(=galaica)

bellus (Carabodes)

bellus (Pseudotectoribates) (=subsimilis) .... 172

benegasi (Oripoda) .................................... 204

bengalensis (Cosmochthonius) ......................... 33

bengalensis (Dolicheremaeus) ...................... 142

bengalensis (Haplacarus) foliatus .................. 38

bengalensis (Hoplophthiracarus) …................. 50

bengalensis [L. (Paralamellobates)] .............. 167

bengalensis (Sphaerochthonius) …................... 34

benguetensis (Perscheloribates) …................. 197

benoiti (Drymobatoides) ............................. 185

benoiti (Hoplophorella) (=stilifera) .............. 49

benoiti (Metabelba) ......................................... 80

benoiti (Phthiracarus) sp. inq. ...................... 53

bentonensis (Achipteria) ............................. 167

bentoni (Notophthiracarus) ……................... 5

benyovszkyi (Leptoppia) ............................. 123

beringiana (Suctobelba) ................................... 135

beringianus [M. (Calyptozetes)] ................... 183

beringianus [A. (Coropoculia)] …………..... 159

berlesei (Arthrhoplophora) (=vulpes) ............ 35

berlesei (Brachychthonius) ................................ 29

berlesei (Brachychthonius) ........................... 29

berlesei (Camisia) (=biverrucata) ...................6 65

berlesei (Dolicheremaeus) (=damoeoides) .. 142

berlesei [D. (Epidamaeus)] ………................... 79

berlesei (Eremulus) …................................ 102

berlesei (Galumna) ........................................... 215

berlesei [L. (Lancelalmoppia)] .................. 111

berlesei (Murcia) (=trimaculata) ................ 177

berlesei (Oribatella) ........................................ 170

berlesei (Oribotritia) .............................................. 44

berlesei (Oribotritia) ............................................ 44

berlesei (Pantelozetes) .................................... 107

berlesei (Eupelops) sp. inq. .......................... 163

berlesei (Phauloppia) (=lucorum)

berlesei [H. (Phyllhermannia)] ........................ 69

berlesei (Pilobatella) ..................................... 211

berlesei (Plateremaeus) ................................. 73

berlesei (Rhacaplacarus) ..............................5 57

berlesei (Steganacarus) (=applicatus) .......... 58

berndhauseri (Bipassalozetes) .................... 162

berndhauseri (Multipulchroppia) .................. 120

berndhauseri (Multipulchroppia) .............. 120

berndhauseri (Otocepheus) .............................. 147

berndhauseri (Perxylobates) ........................... 205

berndhauseri (Topobates) …....................... 202

berndi (Didierotocepheus) ............................ 141

berninii (Hoplophorella) ................................. 48

berninii [N. (Ancestroppia)] .......................... 127

berninii (Berniniella) ..................................... 125

berninii (Carabodes) ...................................... 149

berninii [R. (Insculptoppia)] ........................... 118

berninii (Oribatella) bertheti (Lanceoppia)

beskidyensis (Medioppia) ............................ 123

besucheti (Acrotocepheus) …......................... 146

besucheti [S. (Flagrosuctobelba)] ................... 138

besucheti (Oppiella) ....................................... 128

betzaida (Galumna) …….............................. 215

bhadurii (Haplacarus) ………………................ 38

bhadurii (Scheloribates) …........................... 199

bhutanensis (Cosmochthonius) …………....... 33

bhutanensis (Scheloribates) ......................... 199

bhutanicus (Euphthiracarus) ............................ 46

biangulata (Suctobelbella) ............................ 136

biangulatus [H. (Platynothrus)] ................... 66

biarcualis (Scheloribates) ........................... 199

biarcuata [S. (Flagrosuctobelba)] .................. 138

biarea (Murcia) .............................................. 177

bicarinata (Achipteria) ................................... 167

bicarinata (Berniniella) .................................... 125

bicarinata (Berniniella) .................................... 125

bicarinata (Camisia) (=segnis) ........................6 65

bicarinata (Ctenobelba) (=pectinigera) ...... 101

bicarinata (Oribotritia) (=africana) ................ 44

bicarinatus [N. (Besuchetacarus)] ................ 52

bicarinatus [H. (Platynothrus)] ..................... 66

bicarinatus [E. (Pocsia)] ................................ 47

bicarinatus (Steganacarus) _........ 58

bicaudata (Grandjeania) ........................... 187

bicentenaria (Joshuella) ................................. 76

bichei (Atropacarus) .......................................... 48

biciliatus (Nothrus) sp. inq. ............................. 63

bicillata [R. (Insculptoppiella)] .................... 119

biclavata [S. (Dyobelba)] ................................... 82

biclavata [S. (Dyobelba)] .................................. 82

biclavulus (Hemileius) ................................ 193

bicolor (Carabodes) sp. inq. ........................ 149

bicolor (Nothrus) sp. inq. ............................... 63

bicolor (Reductobates) .............................. 196

bicornicula (Cyrthermannia) .......................... 67

bicornis (Astegistes) (=pilosus) ...................... 92

bicornis [M. (Calyptozetes)] …..................... 183

bicornis (Ceratozetes) …............................ 174

bicornis (Gustavia) (=microcephala) ............ 95

bicornis (Minunthozetes) (=semirufus) ...... 182

bicornis (Scheloribates)

bicornuta (Bicyrthermannia) ...........................6 67

bicornutus (Scapheremaeus) ….................. 158

bicostatus (Berndamerus) .............................. 101

bicostatus (Gymnodamaeus) .......................... 76

bicostatus (Gymnodamaeus) ............................. 76

bicristata [L. (Bicristoppia)] ........................ 111

bicristatus (Arthrodamaeus) ........................... 76

bicristatus [H. (Platynothrus] (=peltifer) ...... 66

bicultrata (Cultroribula) ......................... 92

bicultrata (Cultroribula) ................................ 92

bicultrata (Cultroribula) .................................. 92

bicuspidata (Allosuctobelba) .......................... 134

bicuspidata (Cultroribula) ........................... 92

bicuspidata (Furcoribula) (=furcillata) ........ 92

bicuspidata (Gerloubia) ................................ 19

bicuspidata (Oromurcia) ................................ 178

bicuspidata (Oromurcia) ............................... 178

bicuspidatus (Anellozetes) bicuspidatus (Arcozetes) .............................. 172

bicuspidatus (Scheloribates) .......................... 199

bidactyla (Austrachipteria) ........................ 166

bidactylus (Bipassalozetes) ......................... 162

bidactylus (Ethiovertex) ............................. 160

bidactylus (Scheloribates) ........................... 199

bidactylus (Trixylobates) .............................. 207

bidens [C. (Flexa)] ...................................... 150

bidens (Pergalumna) (=emarginata) ............. 218

bidens (Scotiazetes) …................................. 178

bidentata (Allosuctobelba) ............................ 134

bidentata (Arcoppia) ...................................... 120

bidentata (Austrachipteria) ........................ 166

bidentatus (Fuscozetes) ................................ 175

bidentatus (Liacarus) ...................................... 95

bidentatus (Pelopsis) .................................... 183

bidentatus (Zetomotrichus) ........................... 185

bifida [D. (Cylindroppia)] (=cylindrica) ...... 123

bifidatus (Xenillus) (=tegeocranus) .............. 99

bifidus (Dolicheremaeus) .............................. 142

bifilis (Arcoppia) sp. inq. .......................... 120

bifissurata (Pergalumna) ........................... 218

biflagellata (Arcoppia) .............................. 120

biflagellata (Sphagnoppia) ......................... 115

bifoliata (Lohmannia) ................................... 38

bifoveolata [S. (Ussuribata)] ........................ 138

bifurca (Austrotritia) ................................... 42

bifurcata (Acrotritia) (=vestita) ........................ 46

bifurcata (Banksinoma) lanceolata ............. 109

bifurcata (Brachioppiella) ......................... 122

bifurcata (Fenestrella) …............................ 102

bifurcata (Machadobelba) …......................... 106

bifurcata (Nanhermannia) .............................. 68

bifurcatus (Furcodamaeus) ....................... 104

bifurcatus (Liochthonius) ................................ 30

bifurcatus (Pelopsis) ...................................... 183

bifurcatus (Pelopsis) .................................. 183

bifurcatus (Reteremuloides) …...................... 102

bifurcatus (Tumerozetes) ............................. 86

bilamellata (Furcoribula) .............................. 92

bilineata (Machuella) ventrisetosa ................ 133

bilineata (Muliercula) ................................... 197

bilineatus (Ameronothrus) ................................ 156

bilineatus (Ameronothrus) ............................ 156

bilineatus (Scheloribates) sp. inq. .............. 199

bilobatus (Lobozetes) ................................... 176

bilobus (Eupelops) ....................................... 163

bilobus (Eupelops) ....................................... 163

bilunatifer (Eremaeozetes) .......................... 165

bimaculata (Pergalumna) .......................... 218

bimaculata [H. (Phyllhermannia)] .................. 69

bimaculatus (Brachychthonius) ...................... 29

bimaculatus (Brachychthonius) ...................... 29

bimaculatus (Diapterobates) (=notatus) ...... 181

bimorpha (Galumna) ..................................... 215

binadalares (Pilogalumna) ............................. 220

binodosa [L. (Bicristoppia)] ....................... 111

binodosa (Bornebuschia) ............................ 84

biovatus (Eutegaeus) …............................... 85

bipartita (Acrogalumna) .............................. 213

bipartita (Acrotritia) ................................... 45

bipartita (Oribotritia) ........................................... 44 


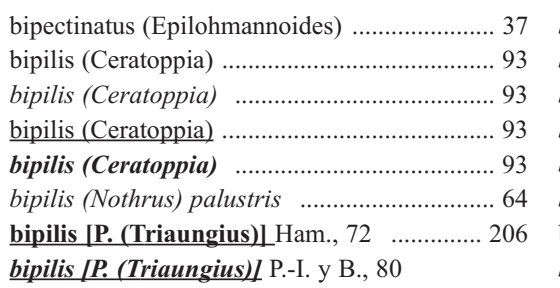

(=praeoccupatus) ………………............. 206

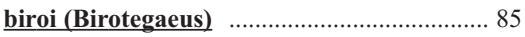

biroi (Fenichelia) ........................................ 159

biroi (Meristacarus) ....................................... 39

birulai (Globozetes) ..................................... 180

bisculpturata (Berndibula) ............................ 195

bisculpturatus (Scapheremaeus) ................. 158

bisculpturatus [P. (Triaungius)] .................... 206

biseriata (Brachioppiella) .......................... 122

bisetus (Podopterotegaeus) .................................. 82

bisignatus (Tectocepheus) (=minor) ............ 154

bispina (Cubachipteria) ............................... 169

bispinatus (Arthrovertex) .......................... 160

bispinosus (Chamobates) .............................. 179

bispinosus (Nothrus) sp. inq. ......................... 63

bistilus (Nothrus) silvestris ............................. 64

bistriata (Camisia) (=horrida) ..........................6 65

bistriatus [H. (Platynothrus)] ........................... 66

bisulcata (Eremobelba) sp. inq. .................. 103

bisulcatus (Stictozetes) ................................. 221

bisulcatus (Tritegeus) ..................................... 84

bisulcus (Hoplophthiracarus) ….................. 50

bithongabela (Pedrocortesella) …................75

bituberculata (Mahunkaia) ........................ 165

bituberculata [O. (Oxyoppiella)] ................ 129

bituberculata [O. (Oxyoppiella)] …............... 129

bituberculatus [D. (Epidamaeus)] .................. 79

biundata (Exoribatula) ................................. 193

biunguiculata (Eulohmannia) (=ribagai) ... 36

biunguis (Scheloribates) ................................ 199

biurus (Camisia) ................................................ 65

biurus (Camisia) .................................................. 65

biurus (Camisia) ............................................... 65

bivaginata (Bursoplophora) ............................ 35

biverrucata (Camisia) ......................................... 65

biverrucata (Camisia) ...................................... 65

biverrucata (Camisia) ...................................... 65

bivittata [S. (Ussuribata)] ............................ 138

bizygata (Oribatula) …............................... 187

blancus [T. (Tyrphonothrus)] ........................ 62

blascoi (Vaghia) …........................................ 221

blattarum [R. (Sabahoppia)] ......................... 119

blocki (Laminoppia) …............................... 110

blocki (Oribatella) ......................................... 170

bloemfonteinensis (Pilogalumna) .............. 220

bloszyki (Beckiella) ....................................... 140

boedvarssoni (Ocesobates) ............................ 180

boevi (Pilogalumna) ........................................ 220

bogduulensis (Diapterobates) ......................... 18

bogorensis (Meristacarus) ……...................... 39

bogorensis (Tecteremaeus) …........................ 108

bolei (Dissorhina) .......................................... 125

boletorum (Murcia) .................................... 177

boletorum (Phauloppia) bolivari (Mesoplophora)

boliviana (Sternoppia)

bolivianus (Benoibates)

bolivianus (Dolicheremaeus) ..................... 142

bolivianus (Tegeocranellus) .......................... 155

bolivianus (Xenillus) ...................................... 98

bombretensis (Nannerlia) ............................... 197

bonairensis (Protoribates) capucinus .......... 206

bonairensis [O. (Zygoribatula)] .............. 12, 188

bonairensis [O. (Zygoribatula)] …...... 12, 188

bonairensis [O. (Zygoribatula)] ............ 12, 188

bonifacioi (Kalayaan) .................................. 147

boninensis (Austrocarabodes) ...................... 148

boninensis [S. (Lalmoppia)] .......................... 130

boothianus (Zetomimus) …........................... 179

boraboraensis (Protoribates) ....................... 205

boraha (Rugoppia) .................................... 131

borbora (Crotonia) ........................................... 67

borealis (Achipteria) Banks, 1899 sp. inq. .. 167

borealis (Achipteria) Ewing, 1918

(=bentonensis)

borealis [P. (Archiphthiracarus)]

borealis [P. (Archiphthiracarus)]

borealis (Banksinoma)

borealis (Camisia) (=horrida) ........................ 65

borealis (Ceratozetes) .................................. 174

borealis (Chamobates) (=pusillus) .............. 180

borealis (Eobrachychthonius) ......................... 30

borealis (Eobrachychthonius) ........................ 30

borealis (Eremaeus) Strenz., 52

$$
\text { (=translamellatus) }
$$

borealis (Eremaeus) Wen, 88

$$
\text { (=neonominatus }
$$

borealis (Exochocepheus)

borealis (Hypochthoniella) (=crosbyi) .......... 29

borealis (Liacarus) ……................................... 95

borealis (Limnozetes) ................................... 165

borealis (Sellnickochthonius) .......................... 31

boreomontanus (Eremaeus) .......................... 100

boresetosus (Phthiracarus) .............................. 53

boresetosus (Phthiracarus) ............................ 53

boresetosus (Phthiracarus) ............................... 53

boresetosus (Phthiracarus) ........................... 53

boreus (Spatiodamaeus) ……………………….... 82

borgesi (Peloptulus) ....................................... 164

borhidii (Allogalumna) ................................. 213

borhidii (Beckiella) ........................................ 140

borhidii (Benoibates) ..................................... 203

borhidii [B. (Canaloppia)]

borhidii [C. (Klapperiches)] ....................... 15

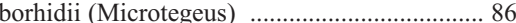

borhidii (Microtegeus) ................................... 86

borhidii (Microtegeus)

borhidii (Rhynchoribates) .......................... 140

borneensis (Anakingia) …............................... 87

bornemisszai (Acutozetes) ........................... 207

bornemisszai (Setoppia) ............................. 112

bornemisszai (Viracochiella) ..................... 179

borussicus (Nothrus) ..................................... 63

borussicus (Nothrus) ...................................... 63

borussicus (Nothrus) ...................................... 63

borussicus (Nothrus) borussicus (Ophidiotrichus) (=tectus) ........ 170

boschmai (Synchthonius) (=crenulatus) ..... 32

bosniae (Tegeocranellus) ................................. 155

bostocki (Eutegaeus) ….............................. 85

bostocki (Eutegaeus) …................................. 85

botari (Lamellobates) ................................... 166

bothulifer [T. (Rostrozetes)] ......................... 211

botschi (Parhypochthonius) sp. inq. ................ 28

botulus (Kaszabozetes) ...................................... 89

boulfekhari [S. (Tropacarus)] .......................... 59

bouvetoyaensis (Alaskozetes) …………….... 156

brachiatus (Cepheus) ............................................ 82

brachyodon (Suctobelbella) (=acutidens) .... 136

brachypterus (Scheloribates) ..................... 199

brachyramosa (Arcoppia) ............................... 120

brachyrostrum (Crotonia) (=cophinaria) ......67

brachythrix (Mesotritia) (=nuda) ..................... 44

brachytrichinus (Oppia) nitens ................... 115

bradleyi (Galumna) ....................................... 215

brasilensis (Cultroribula) ............................. 92

brasilensis (Nothrus) ....................................... 63

brasilensis (Trhypochthoniellus) longisetus .... 60

brasiliana (Acrotritia) …………………....... 45

brasiliana (Acrotritia) ....................................... 45

brasiliana (Acrotritia) .................................... 45

brasiliana (Dometorina) plantivaga ............ 192

brasilianus (Dolicheremaeus) ...................... 142

brasilianus (Xenillus) .................................... 98

brasiliensis [E. (Brasiliotritia)] ...................... 47

brasiliensis (Eremulus) ................................. 102

brasiliensis (Galumna) ............................... 215

brasiliensis (Mesotritia) ...................................... 43

brasiliensis (Hoplophorella) ........................... 48

brasiliensis (Oripoda) .................................. 204

brasiliensis [N. (Protophthiracarus)] ............. 53

brasiliensis (Rhynchoribates) ..................... 140

brasiliensis (Sternoppia) ............................... 132

brasiliensis (Teratoppia) ............................... 132

brasiliensis (Unguizetes) (=setiger)

brasilocompressus (Scheloribates) .............. 199

brasilosphericus (Scheloribates) .................. 199

brassi (Brassoppia) ..................................... 122

brassi (Reductobates) …............................. 196

brassicae (Crotonia) .......................................... 67

brauni [O. (Zygoribatula)] (= glabra) .......... 189

brazilianus (Rhopalozetes) ............................ 90

brazilianus (Stelechobates) .......................... 192

braziliensis (Epieremulus) ........................... 106

braziliensis (Epieremulus) .......................... 106

brazilozetoides (Berlesezetes) ...................... 87

bregetovae (Ceratozetella) ............................ 173

bregetovae (Damaeolus) ............................... 103

bregetovae (Ocesobates) .............................. 180

brevialatus (Perscheloribates) ...................... 198

breviclava [C. (Flexa) ] .................................. 150

breviclava (Mainothrus) .................................. 59

breviclava (Membranoppia) ........................ 111

breviclava (Striatoppia) ............................ 131

breviclava (Tegeozetes) tunicatus ................ 155

breviclavata (Cultroribula) .............................. 92

breviclavatus [L. (Procorynetes)] .................... 97

brevicornuta (Crotonia) ................................. 67 
brevicornuta (Oribatella)

brevicornuta (Oribatella) ................................ 170 brevicuspidata (Oribatella) ............................ 170

brevicuspidatus (Punctoribates) ..................... 183

brevicuspidis (Oribatella) ………………….... 170

brevicuspis (Ceratoppia) $(=$ spinipes)

brevicuspis (Eupelops) .................................... 163

brevicuspis (Murcia) ……………………...... 177

brevifer (Eueremaeus) ............................ 11, 100

brevilamellata (Oribatella) sp. inq. ............ 170

brevilamellatus [M. (Calyptozetes)] ............ 183

brevilamellatus (Hemileius) ............................ 193

brevilamellatus (Liacarus) ................................ 95

brevipectinata [B. (Gressittoppia)]

pepitensis ............................................ 122

brevipectinata [M. (Multilanceoppia)] .........117

brevipectinata [M. (Multilanceoppia)] ........... 117

brevipectinata [T. (Teratoppiella)] ………... 132

brevipes (Ameronothrus)

(=nigrofemoratus) ………………………... 156

brevipes (Micreremus) ………………………... 159

brevipes (Mixacarus) ...…………………….... 39

brevipes (Mixacarus) ........................................ 39

brevipila (Oribatella) ...…………………... 170

brevipila (Xenoppia) ……………………….... 116

brevipilis (Halozetes) belgicae ...................... 156

brevipilis (Hoplophorella) ............................... 48

brevipilosa (Ctenobelba) ............................... 101

brevipilosa [I. (Zeaotritia)] ............................... 43

brevipilus (Eupelops) .................................... 163

brevipilus [S. (Tropacarus)] .............................. 59

brevipodus (Scheloribates) ......................... 199

breviporosus (Maculobates) ......................... 196

brevipterus (Perscheloribates) .................... 198

brevirostris (Nothrus) sp. inq. ......................... 63

brevis (Berlesezetes) ……………………….... 87

brevis (Berlesezetes) ...................................... 87

brevis (Carabodes) ........................................ 149

brevis (Liochthonius) .......................................... 30

brevis (Liochthonius) ...................................... 30

brevis (Liochthonius) ..................................... 30

brevis (Oribotritia) ………………………... 44

brevis (Tuxenia) ............................................... 207

brevis (Zetomimus) ……………………..... 179

breviseta (Austrachipteria) .......................... 166

breviseta (Eremobelba) ………………….... 103

breviseta (Indotritia) …………………………. 43

breviseta (Lauroppia) ………………………... 126

breviseta (Liochthonius) ................................ 30

breviseta (Magyaria) ………………………. 209

breviseta (Mesotritia) ........................................... 43

breviseta (Paroppia) ...................................... 115

breviseta (Sadocepheus) ................................ 84

breviseta [P. (Tectodamaeus)] ............................ 81

breviseta [I. (Zeaotritia)] (=brevisetosa) ........ 43

brevisetae [P. (Archiphthiracarus)] ................. 55

brevisetae [P. (Archiphthiracarus)] ............... 55

brevisetiger (Cryptoppia) …………………..... 116

brevisetiger (Trimalaconothrus) ....................... 61

brevisetiger (Nippobodes) .............................. 154

brevisetosa (Tuxenia) (=brevis) ..................... 207

brevisetosa [I. (Zeaotritia)] brevisetosa [O. (Zygoribatula)] Ewing, 09 .. 188 brevisetosa [O. (Zygoribatula)] L. y B., 91 (=obsessa)

brevisetosus [D. (Epidamaeus)] ........................ 79

brevisetosus [I. (Haplozetes)] ………………... 208

brevisetosus (Hypochthonius) rufulus (=rufulus)

brevisetosus [H. (Platynothrus)] ................... 66

brevisetosus (Protoribates) ............................. 205

brevisetosus (Rimandocepheus) ……………... 147

brevisetosus (Unduloribates) ……………...... 165

brevisetus [I. (Haplozetes)] ............................ 208

brevisetus (Hypozetes) ................................... 166

brevisetus (Megalotocepheus) ………………. 147

brevisetus (Perxylobates) ………………….... 205

brevisetus (Pilizetes) ..................................... 220

brevisetus (Rhacaplacarus) ............................. 57

brevisetus (Tritegeus) ………………………..... 84

brevispathulata (Eremobelba) ........................ 103

brevitarsus (Eremaeus) ................................... 100

brevitibialis [D. (Epidamaeus)] ....................... 79

brevitutorium [E. (Gamerozetes)] ………..... 175

brinoni (Globoppia) …………………….... 110

bromeliarum [O. (Multoribatella)] .......... 7, 172

bruneiana (Karenella) ....................................... 130

bruneiensis (Condylobelba) .......................... 134

bruneiensis (Dolicheremaeus) ....................... 142

bryani (Indotritia) $s p$. inq. ............................. 43

bryani (Pergalumna) ……………………..... 218

brvani (Phauloppia) ……………………..... 190

bryobius [P. (Archiphthiracarus)] ...................... 55

bryobius [P. (Archiphthiracarus)] .................. 55

bryobius [P. (Archiphthiracarus)] ………….... 55

bryophilus (Tegoribates) .............................. 169

bucephala (Beckiella) ................................... 140

bucephalus (Acrotocepheus) ……………..... 146

bulanovae [Z. (Protozetomimus)] ................. 179

bulanovae (Medioppia) (=subpectinata) ...... 124

bulanovae (Oribatella) ................................... 170

bulanovae (Oribatula) (=interrupta) .......... 187

bulanovae (Ptiloppia) ................................... 128

bulbifer (Oribotritia) .......................................... 44

bulbiferus [P. (Archiphthiracarus)] ............. 55

bulbipedata (Damaeus) sp. inq. ...................... 78

bulbipes (Belba) (=corynopus) ...................... 77

bulbofemoralis (Damaeus) ............................... 78

bulganensis (Dissorhina) …………………... 125

bulgarica (Furculoppia) (=furcata) ............ 117

bulgaricus (Hypozetes) .................................... 166

bulgaricus (Hypozetes) ………………………... 166

bulgaricus (Scutovertex) …………………... 161

bullager (Reductobates) ................................ 196

bullocki (Suctobelbella) …………………...... 136

buntotanus (Peloribates) ................................... 209

bunya (Hexachaetoniella) …......................... 74

burckhardti (Acrotocepheus) ………………... 146

burckhardti (Pulchroppia) ……………........ 120

buresi [T. (Tyrphonothrus)] …………………... 62

burrowsi (Lucoppia) ……………………….... 187

burrowsi (Lucoppia) ....................................... 187

burrowsi (Lucoppia) .................................. 187

butantaniensis (Xenillus) ................................. 98 butiae (Austrocarabodes) ........................... 148

byzovae (Heterochthonius) ............................. 33

byzovae (Oribatella) ....................................... 170

cachoeirensis (Tecteremaeus) ...................... 108

cadeti (Austrocarabodes) ........................... 148

cadizi [P. (Archiphthiracarus)] ......................... 55

caelatus (Lamellovertex) .............................. 161

caelestis (Steganacarus) …….......................... 58

caesarea (Licnobelba) ....................................... 75

cagayana (Bicyrthermannia) ..........................67 67

cajamarcensis (Brachioppia) ..................... 122

cajamarcensis [T. (Tyrphonothrus)] .................6 62

cajamarcensis [T. (Tyrphonothrus)] .............. 62

calcarata (Fossoppia) ................................ 128

calcarata (Multioppia) (=tremblevi) ............ 117

calcarata (Nothrolohmannia) ….................. 29

calcarata (Oribatella) ..................................... 171

calcarata (Oribatella) .................................. 171

calcarata (Teratoppia) ............................... 132

calcaratus (Farchacarus) ................................ 173

calcaratus (Farchacarus) ............................ 173

calcaratus (Hardybodes) ........................... 152

calcaratus (Perscheloribates) (=reiteratus) .. 198

calcaratus [C. (Phyllocarabodes)] ............... 151

calcaratus (Scheloribates) fimbriatus

Jac., 34 .......................................

calcaratus (Scheloribates) L. y P., 90

(=praeoccupatus) …................................ 201

calcaratus [H. (Urubambates)] …….......... 194

calcehtokensis (Malaconothrus) .....................6 60

calcicola (Kinezogalumna) ............................ 217

california [C. (Klapperiches)] ...................... 151

californiae (Pergalumna) ............................... 218

californica (Galumna) ................................. 215

californica [B. (Protobelba)] ........................... 78

californicus (Passalozetes) ............................ 162

californicus (Passalozetes) .......................... 162

californiensis (Eremaeus) .............................. 100

californiensis (Hydrozetes) ............................ 155

caliginosus (Notophthiracarus) (=australis) .... 51

caliptera (Oribatula) $(?=$ allifera) $\ldots \ldots \ldots \ldots \ldots . . . . .188$

callainii [S. (Tropacarus)] .............................. 59

callipus (Scheloribates) ................................ 199

callipygis [C. (Xiphobates)] …....................... 180

callipygus (Torpacarus) ................................. 41

callitarsus (Pedrocortesella) ….................... 75

callitoca (Mongaillardia) ............................... 101

callosus (Plateremaeus) …….......................... 73

calmorum (Pedrocortesella) ….................... 75

calugari (Notophthiracarus) ....................... 51

calva (Galumna) ........................................ 215

calva [T. (Paragloboppia)] .......................... 115

calycifera (Mochloribatula) ......................... 185

cambricus (Mycobates) …............................ 182

camelus (Crotonia) ........................................ 67

campbellensis [M. (Pravoppia)] .................. 111

campbellensis (Stomacarus) ............................ 27

campestris (Ceratozetes) ............................... 174

campestris [N. (Coartobelba)] ...................... 135

campestris (Scheloribates) (=pallidulus) ... 201

campinaranensis (Arcoppia) ....................... 120

camtschaticus (Scheloribates) ...................... 199 
canadaris (Protoribotritia) ................................ 45 canadensis (Banksinoma) lanceolata ............ 109 canadensis (Ceratoppia) (=spinipes) …........ 93 canadensis [D. (Epidamaeus)] ........................ 79 canadensis (Limnozetes) (=ciliatus) ............ 165 canadensis (Peloribates) ............................. 209 canadensis (Propelops) .................................. 164 canadensis (Propelops) .............................. 164 canadensis (Trhypochthoniellus) longisetus .... 60 canagaratnami (Oripoda) ............................. 204 canagaratnami (Rhopalozetes) ........................ 90 canaliculatus (Neoliodes) sp. inq. ................... 71 canariensis (Anomaloppia) ........................... 116 canariensis [N. (Calyptophthiracarus)] .......... 52 canariensis (Hermannia) ................................... 69 canariensis [L. (Incabates)] .......................... 209 canariensis (Multioppia) .............................. 117 canariensis (Oribella) ....................................... 107 canariensis (Poroscheloribates) …................. 196 canariensis (Xenillus) discrepans .................... 98 canariensis [O. (Zygoribatula)] (=glabra) .. 189 cancellata (Magyaria) …........................... 209 cancellata (Magyaria) .................................. 209 cancellatus (Chaunoproctus) …..................... 192 candidulus [N. (Calyptophthiracarus)] ............ 52 canestrinii (Acrotritia) (=ardua) .................... 45 canestrinii (Hermanniella) $s p$. inq. ................... 70 canningsi [A. (Coropoculia)] ......................... 159 canopeus [T. (Tyrphonothrus)] ...................... 62 capense (Oppia) ............................................ 114 capense (Oppia) ..................................... 114 capensis (Afrozetes) magoebaensis .............. 87 capensis (Eremaeozetes) ............................. 165 capensis (Eupelops) …............................... 163 capensis (Galumna) ….............................. 215 capensis (Hydrozetes) ………………….... 155 capensis (Neoliodes) $s p$. inq. ......................... 72 capensis (Pergalumna) magnipora ............ 219 capensis (Pergalumna) magnipora.................. 219 capensis (Rhopalozetes) ............................... 90 capillaris (Pergalumna) magnipora ............... 219 capillaris (Pergalumna) magnipora ................ 219 capillata (Capilloppia) ............................... 186 capillata (Rhynchoppia) ............................. 135 capillata (Xenolohmannia) ............................. 41 capillatus [H. (Capillonothrus)] ........................66 66 capillatus [H. (Capillonothrus)] ...................... 66 capillatus (Dolicheremaeus) ........................ 142 capillatus (Notophthiracarus) ...................... 51 capillatus (Trichocarabodes) ….................... 153 capilligera [L. (Antennoppia)] .................... 113 capistrata (Crotonia) ..................................67 67 capita (Totobates) ......................................... 196 capitanea (Oribotritia) ........................................ 44 capitata (Eremobelba) ….............................. 103 capitata (Eremobelba) …............................ 103 capitata (Gredosella) …............................... 133 capitata [O. (Zygoribatula)] ....................... 188 capitatus (Anderemaeus) ............................. 105 capitatus (Neogymnobates) capitatus (Phthiracarus) sp. inq. .................... 53 capitatus (Xenillus) capitulum (Beckiella)

capitulum (Protozetes) .................................... 90 caporiacci (Multioppia) (?=laniseta) …...... 117

caprai (Scheloribates) ................................. 199

caprai (Scheloribates) ............................... 199

caprai (Scheloribates) ….............................. 199

capreolatus (Dolicheremaeus) .................... 142

captator (Dissorhina) (=ornata) .................. 125

capucinus [O. (Fenestrobates)] .................... 172

capucinus (Protoribates) ................................. 205

capucinus (Protoribates) ............................. 205

capucinus (Protoribates) .............................. 205

capucinus (Protoribates) ................................ 205

capucinus (Protoribates) ............................ 205

capucinus (Protoribates) .............................. 205

carbonarius [L. (Rhaphidosus)] ...................... 97

carboneli (Plasmobates) ................................. 71

carcharodon (Suctobelbella) ........................... 136

carcharodon (Suctobelbella) .......................... 136

cardosensis (Microtegeus) .............................. 86

cardosensis (Pergalumna) ............................ 218

carinata (Austrotritia) ……............................. 42

carinata (Beckiella) ..................................... 140

carinata (Suctobelbella) ............................... 136

carinata (Vaghia) ......................................... 221

carinatissima (Berniniella) ............. 125

carinatus (Carabodes) (=subcarinatus) ...... 150

carinatus (Dolicheremaeus) .......................... 142

carinatus (Eueremaeus) .............................. 100

carinatus [H. (Platynothrus)] Kramer, 1897

(=punctatus)

carinatus [H. (Platynothrus)] Banks, 1910

carinatus [T. (Rostrozetes)] .......................... 211

carinatus (Scapheremaeus) ........................ 158

carinatus (Solenozetes) ................................ 71

carinatus (Suctoribates) ............................... 140

carinatus [S. (Tropacarus)] .............................. 59

carinatus [S. (Tropacarus)] ............................... 59

carinatus (Wallworkodes) .......................... 146

carinthiaca (Lauroppia) maritima ................. 126

carinulatus (Pheroliodes) ............................... 73

carli (Mycobates) ........................................... 182

carlosi (Steganacarus) …............................... 58

carneus [I. (Haplozetes)] .............................. 208

carneus (Scheloribates) sp. inq. ................... 199

carniolica (Lauroppia) ................................. 126

carolensis [L. (Carolohmannia)] ..................... 39

caroli (Synichotritia) ...................................... 47

carolina (Ferolocella) (=tessellata) …........ 170

carolinae (Oribotritia) ......................................... 44

carolinae (Multioppia) (?=wilsoni) .............. 117

carolinae (Nothrus) (=monodactylus) ..........6 64

carolinensis [S. (Dyobelba)] ............................. 82

carolinensis [L. (Rhaphidosus)] ..................... 97

carolinicus (Hypochthonius) rufulus .............. 29

carpathicus (Topobates) .............................. 202

carpatica (Dissorhina) longipilosa ................ 125

carpatica [M. (Multilanceoppia)] .................. 117

carpetanus (Pilobates) .................................... 211

carrolli (Camisia) ........................................... 65

cartalinicus (Liacarus) (=brevilamellatus) ....95

carusoi (Steganacarus) ....................................... 58 casabranquensis (Pheroliodes) ...................... 74

cassolai (Cosmogneta) ................................ 109 castagnoliae (Senectoppia) (=complicatum) .. 132

castanea (Banksinoma) sp. inq. ................... 109

castanea (Galumna) $s p$. inq. ....................... 215

castaneus (Comeremaeus) ........................... 93

castaneus (Liacarus) ..................................... 95

castaneus (Sphaerozetes) .............................. 178

castaneus [H. (Platynothrus)] ........................ 66

castlei (Scheloribates) .................................. 199

castriensis (Cultroribula) ............................ 92

castrii [C. (Klapperiches)] (=minusculus) .. 151

castrii (Microzetes) ........................................... 89

casuarina [D. (Cylindroppia)]

(=cylindrica $)$.............................................. 123

cataracta [S. (Rafacarus)] (=rafalskii) .......... 59

catarinensis (Ceratozetes) ............................. 174

cathayanus (Euphthiracarus) …...................... 46

catskillensis (Achipteria) ............................... 167

caucasica (Mesoplophora) (=michaeliana) .. 42

caucasica (Murcia) ....................................... 177

caucasica (Zachvatkinella) .............................. 27

caucasicus (Cepheus) ......................................... 82

caucasicus (Chamobates) .............................. 179

caucasicus (Eupelops) .................................. 163

caucasicus (Heterochthonius) ........................... 34

caucasicus (Microzetes) .................................. 89

caucasicus (Palaeacarus) ……….................... 27

caucasicus (Pantelozetes) (=paolii) ............ 107

caudalis (Crotonia) .......................................... 67

caudata (Phauloppia) ................................. 190

caudata (Sellnickia) Berl., 1910 (=caudata

Mich.) ...................................................... 191

caudata (Sellnickia) Mich., 1898 .................. 191

caudata (Sellnickia) Mich., 1898 ................. 191

caudatus (Acrotocepheus) ............................ 146

caudatus (Dendrozetes) .................................. 94

caudatus (Nesopelops) ................................ 164

caudatus (Notophthiracarus) ........................ 51

caudatus (Poroliodes) (=farinosus) ….......... 72

cavaticus (Oribellopsis) .................................. 107

cavaticus (Oribellopsis) …............................ 107

cavernalis [R. (Insculptoppia)] ........................ 118

$\underline{\text { cavernicolus (Perscheloribates) }}$...................... 198

cavernicolus (Samarocepheus)

cavernosa (Hoplophorella) (=neonominata) .... 49

cavernosa [H. (Kakophthiracarus)] ............ 49

cavernosus (Acaroceras) .............................. 87

cavernosus [P. (Archiphthiracarus)] ............ 55

cavernosus (Charassobates) ......................... 86

cayugensis (Heterozetes) (=minnesotensis) .. 173

cazanicus (Hoplophthiracarus) ....................... 50

cejansis (Beckiella) ...................................... 140

celisi (Liacarus) sp. inq. .............................. 95

celisi (Trichocarabodes) .............................. 154

cellularis (Austrocarabodes) ....................... 148

cellularis (Galumnella) ................................... 221

cellulatifer (Scapheremaeus) …................... 158

cellulatus (Damaeolus) ................................... 103

cellulosa (Eremobelba) ................................. 103

cendanai (Basilobelba) ................................. 104

centenarius (Kalayaan) …….......................... 147 


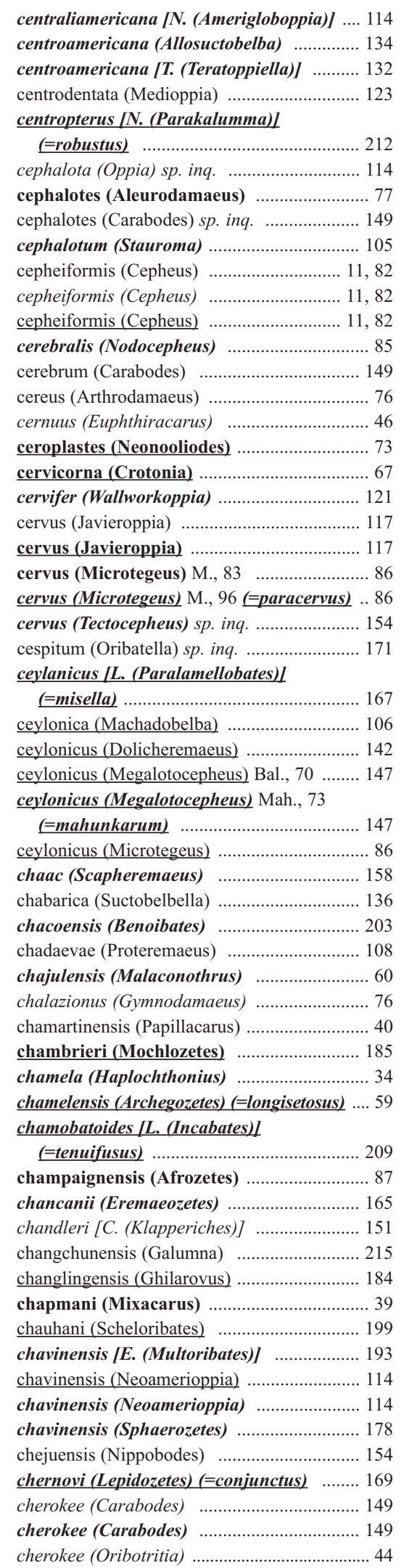

cherubin (Polypterozetes)

chiatous (Eueremaeus) .................................... 100

chiayiensis [I. (Haplozetes)] ............................ 208

chibai (Acutozetes) ………............................. 207

chichijimensis (Oribotritia) ............................... 44

chiebunensis (Liacarus) ................................... 95

chilensis (Anderemaeus) .............................. 105

chilensis (Dometorina) ................................. 192

chilensis (Lamellozetes) ............................... 93

chilensis (Neoamerioppia) .............................. 114

chilensis [N. (Protophthiracarus)] ................ 53

chilensis [O. (Zygoribatula)] bonairensis .... 188

chiloensis (Crotonia) ...................................... 67

chimaera (Trichogalumna) ............................ 221

chimalae (Zachvatkinella) ............................... 27

chinensis (Fissicepheus) …………………...... 144

chinensis (Gerloubia) ……………………..... 191

chinensis (Gibbicepheus) ............................... 152

chinensis (Meristolohmannia) …………….... 39

chinensis (Trachyoribates) ……………......... 211

chirstlus (Carabodes) ……………………........ 149

chistyakovi (Oppiella) (=nova) ……............ 128

chitinofincta (Anomaloppia) ........................ 116

chitralensis [S. (Flagrosuctobelba)] ………... 138

chitralensis (Trichogalumna) ………………. 22

choerognathus (Nasozetes) ............................. 194

christineae (Tuparezetes) …………………... 133

christovi (Arenozetes) 87

chroniosus (Liacarus) ....................................... 95

chujoi (Galumna) ………………………….... 215

chukchi [D. (Epidamaeus)] …………………... 79

chulumaniensis (Ramusella) ........................... 118

chulumaniensis (Ramusella) ......................... 118

cicatricosus (Dolicheremaeus) ...................... 142

cidarus (Liacarus) ………………………...... 95

ciliata (Leptogalumna) ................................. 217

ciliata (Leptogalumna) ……………………... 217

ciliata (Leptogalumna) …………………..... 217

ciliata (Teratoppia) ……………………….... 132

ciliatus (Limnozetes) …............................... 165

ciliatus (Limnozetes) ………………………... 165

ciliosus (Atropacarus) ……………………..... 48

ciliosus (Stomacarus) ……………………...... 27

cincinnatus [D. (Epidamaeus)] ......................... 79

cincta (Banksinoma) ......................................... 109

cinctus (Torpacarus) ...................................... 4

cingulatus (Eremulus) …………………...... 102

circula (Pergalumna) ................................... 218

circularis (Galumna) ................................... 215

circularis (Tumerozetes) …………………...... 86

circulus (Epieremulus) ................................... 106

circumalatus (Planobates) ........................... 198

circumcarinatus (Topobates)

circumciliata (Pseudoamerioppia) ............. 118

circumita [Q. (Coronoquadroppia)] ............... 133

circumita [Q. (Coronoquadroppia)] ............. 133

circumita [Q. (Coronoquadroppia)] ............... 133

circumita [Q. (Coronoquadroppia)] ........... 133

circumita [Q. (Coronoquadroppia)] ............ 133

circumvallatus (Neoliodes) (=theleproctus) .... 72

cirripilis (Galumna) $s p$. inq. ........................ 215

cirrosus [H. (Platynothrus)] (=peltifer) ........ 66 cisalpina (Ceratozetella) ................................. 173

cisalpina [C. (Ceratozetella)] ........................ 173

citelli (Infernobates) ........................................ 107

cladonicola (Trhypochthonius) .............................. 60

clara (Hermannia) _.................................... 69

clara (Mesotritia) ……………………………...... 43

clarencei (Achipteria) ...................................... 167

clathratus (Sphaerozetes) ……………......... 178

claudelionsi [R. (Insculptoppia)] ................. 118

clavasensilla (Belba) ...................................... 77

clavasetosa (Hermanniella) ……………...... 70

clavasetosa (Pseudoceratoppia) .................... 94

clavata [O. (Aciculoppia)] ………………...... 129

clavata (Acrotritia) …………………....... 11, 45

clavata (Acrotritia) .................................. 11, 45

clavata (Beckiella) ....................................... 140

clavata (Galumnopsis) ................................ 222

clavata [G. (Indogalumna)]

(=neonominata) ………………………..... 217

clavata (Indotritia) ……………………….... 43

clavata [V. (Latilamellobates)] ........................ 179

clavata (Oripoda) ........................................... 204

clavata (Topalia) …………………………….... 86

clavata [S. (Ussuribata)] ................................. 138

clavata [H. (Phyllhermannia)] ........................ 69

clavata [O. (Zygoribatula)] ……………....... 188

clavatoflagellatus (Eremulus) sp. inq. .......... 102

clavatopilus (Xenillus) .................................... 98

clavatotrichobothria [O. (Zygoribatula)]

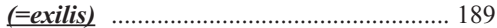

clavatum (Aeroppia) .................................. 112

clavatus [P. (Archiphthiracarus)] ……………... 55

clavatus (Atropacarus) …………………......... 48

clavatus (Atropacarus) .................................. 48

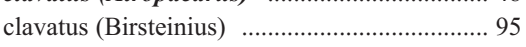

clavatus (Carabodes) Warb., 12 sp. inq. .... 149

clavatus (Carabodes) Jac., 38

(=neonominatus) ……………………….... 150

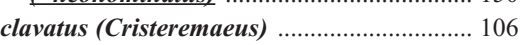

clavatus (Fissicepheus) ................................... 144

clavatus (Fissicepheus) …………………….... 144

clavatus (Haplochthonius) ................................ 34

clavatus (Haplochthonius) ................................ 34

clavatus (Haplochthonius) .............................. 34

clavatus [I. (Haplozetes)] .................................. 208

clavatus (Hemileius) ......................................... 193

clavatus (Liochthonius) ……………………..... 30

clavatus [E. (Nanobates)] …………………..... 197

clavatus (Ommatocepheus) ………………..... 83

clavatus (Oribellopsis) .................................... 107

clavatus (Peloribates) ................................. 209

clavatus (Perscheloribates) ………................. 198

clavatus (Perscheloribates) ……................. 198

clavatus [L. (Procorynetes)] …………………... 97

clavatus (Protoribates) ………………............ 206

clavatus (Protozetes) ........................................ 90

clavatus (Tectocepheus) velatus ............... 12, 155

clavatus (Tectocepheus) velatus .............. 12, 155

clavatus [A. (Uluguroides)] pentatrichus .. 149

clavatus (Unguizetes) ……………………...... 186

clavatus (Unguizetes) .................................... 186

clavellatus (Hoplophthiracarus) ........................ 50 
clavifer (Phthiracarus) .................................... 53

clavifer (Scapheremaeus) ........................... 158 claviger (Dolicheremaeus) ............................ 142 claviger [L. (Dorycranosus)] (=acutus) ....... 97 claviger (Eupelops) .................................... 163 claviger (Notophthiracarus) …....................51 claviger (Phthiracarus) sp. inq. ....................... 53 clavigera (Hermanniella) ................................ 70 clavigera (Hermanniella) ................................. 70 clavigera (Metabelbella) .................................. 81 clavigera (Moritzoppia) unicarinata .............. 127 clavigera (Moritzoppia) unicarinata ............ 127 clavigerus (Atropacarus) ................................ 48 clavigerus (Oribellopsis) .............................. 107 clavigerus (Pseudotocepheus) ..................... 145 clavilanceolatus (Scheloribates) ................. 199 clavimera (Setoppia) ..................................... 112 claviopsis (Fissicepheus) ............................... 144 clavipectinata (Ramusella) ............................. 118 clavipectinata (Ramusella) ........................... 118 clavipectinata (Ramusella) .......................... 118 clavipectinata (Ramusella) .......................... 118 clavipectinata (Ramusella) ......................... 118 clavipectinatus (Scheloribates) .................... 199 clavipes [D. (Paradamaeus)] .......................... 80 clavisensillata (Ceratoppia) ............................ 93 claviseta (Suctobelbella) ...................... 11, 136 claviseta (Suctobelbella) ........................ 11, 136 claviseta (Suctobelbella) ...................... 11, 136 clavisetosus (Chaunoproctus) (=latior) ...... 192 clavisetus (Cordiozetes) ................................ 195 clavisetus (Euscheloribates) .......................... 197 clavisetus (Scapheremaeus) ......................... 158 clemens (Phthiracarus) ................................... 53 clemens (Phthiracarus) ……............................53 clericata (Carinogalumna) .......................... 214 clypeator (Xenillus) ........................................ 98 clypeator (Xenillus) ......................................... 98 clypeatus (Ceratozetes) sp. inq. ..................... 174 clypeatus (Scutovertex) sp. inq. .................... 161 coarctata (Hymenobelba) ............................. 105 coarctatus [P. (Constrictocepheus)] .......... 145 cochleaformis (Carabodes) .......................... 149 cochlearia (Hoplophorella) (=hamata) ........ 49 cochlearium (Globoppia) ............................. 110 cocuyana (Neoamerioppia) .......................... 114 coggii (Oribatula) (=tibialis) ......................... 188 cognata [O. (Oxyoppiella)] bituberculata .. 129 cognata [O. (Zygoribatula)] ......................... 188 cognatus [P. (Archiphthiracarus)] .................. 55 cohici (Glabroppia) ................................... 130 coiffaiti (Perlohmannia) ................................... 36 coineaui (Arthrovertex) ................................. 160 coineaui (Phauloppia) ................................... 190 colchica (Oribatella) ...................................... 171 coleoptrata (Achipteria) .................................. 167 coleoptrata (Achipteria) ................................. 167 coleoptrata (Achipteria) .............................. 167 coleoptrata (Achipteria) .................................. 167 collaris (Hoplophorella) ................................. 48 colliger [P. (Neotocepheus)] …..................... 145 colloffi [H. (Phyllhermannia)] .......................... 69 colloffi [I. (Zeaotritia)] (=brevisetosa) ............ 43 colobanthi (Halozetes) (=crozetensis) ........... 156 coloradensis (Galumna) ................................. 215 coloradensis (Galumna) ............................... 215 coloradensis (Oppia) ................................... 115 colorado (Carabodes) .................................. 149 colossus (Galumna) …................................. 215 columbiana (Aeroppia) (=magnipilosa) ...... 112 columbiana (Hermanniella) (=subnigra) ...... 71 columbiana (Pergalumna) (=emarginata) .. 218 columbianus [L. (Dorycranosus)] ................... 97 columbianus [L. (Dorycranosus)] .................. 97 columbianus (Eueremaeus) ......................... 100 columbianus (Xenillus) .................................. 98 columbreti [V. (Latilamellobates)] ................ 179 comas (Carabodes) ........................................ 149 comatus [P. (Archiphthiracarus)] .................... 55 comatus [N. (Calyptophthiracarus)] ............ 52 comatus (Hemileius) (=quadripilis) ............ 193 comatus (Topobates) ............................. 12, 203 comelicensis (Sphaerozetes) …..................... 178 comitalis (Nanhermannia) ................................. 68 comitalis (Nanhermannia) ............................... 68 comitalis (Nanhermannia) ............................. 68 communis (Totobates) .............................. 196 commutabilis (Phthiracarus) _......................... 53 commutata (Lauroppia) ............................... 126 commutata (Oribatula) ............................ 187 comorensis (Sellnickochthonius) .................. 31 comoroensis (Dimidiogalumna) ................... 214 comosa (Xenolohmannia) ............................ 41 comosus [P. (Neophthiracarus)] ....................... 56 compacta [I. (Afrotritia)] ................................ 43 compacta [ F. (Parasuctobelba)] …............... 134 comparabilis (Galumna) ........................... 215 comparabilis [H. (Phyllhermannia)] ............ 69 comparandus (Pergalumna) ....................... 218 comparativus (Notophthiracarus) …...........51 complanatus (Pheroliodes) ............................ 74 complanatus (Plateremaeus) ........................ 73 completa (Indotritia) (=javensis) ..................... 43 completus (Nesiacarus) …................................ 40 complexa [S. (Usssuribata)] ........................... 138 complexa [S. (Ussuribata)] .......................... 138 complicata (Oxyoppia) ................................ 129 complicata (Pergalumna) ............................ 218 complicata (Tuxenia) ..................................... 207 complicatum (Senectoppia) ......................... 132 complicatus (Steganacarus) (=vestitus) compositocarinata (Lauroppia) ................... 126 compressa (Setoppia) ................................. 112 compressus (Phthiracarus) .............................. 53 compressus (Phthiracarus) ................... 53 compressus (Phthiracarus) .............................. 53 compta (Belba) ................................................ 77 comteae (Acrotritia) (=vestita) ….................. 46 comteae (Eremobelba) ................................. 103 comteae (Euphthiracarus) ........................... 46 comteae (Rioppia) ....................................... 107 concava (Camisia) (=spinifer) ........................65 concavus (Bathocepheus) .............................. 149 concavus (Cosmochthonius) ….................... 33 concavus (Holonothrus) ................................... 67

concavus (Mixochthonius) ............................... 31

concavus (Mixochthonius) ............................... 31

concavus (Pheroliodes) ….............................. 74 concavus (Tegeocranellus) ............................ 155

concentricus (Neoliodes) ............................... 72 concentricus (Neoliodes) ................................. 72 concentricus (Scheloribates) ....................... 199 concinna [O. (Zygoribatula)] (=connexa) .. 188 concinuus (Hoplophthiracarus) ..................... 50 concolor [N. (Calyptophthiracarus)] .............. 52 concolor (Oppia) sp. inq. .............................. 115 concolor [O. (Zygoribatula)] ........................ 188 concretus (Protoribates) sp. inq. .................. 206 concurvatus (Comorozetes) .......................... 88 concurvatus (Tectocepheus) .......................... 154 condylifer (Condyloppia) .............................. 116 confertus (Cubabodes) ................................. 151 confervae (Hydrozetes) ................................... 155 confervae (Hydrozetes) ................................... 155 confinis [N. (Confinoppia)] ......................... 127 confinis (Cultroribula) ..................................... 92 conflata (Granuloppia) ............................ 132 confluens (Allogalumna) ............................. 213 confluens (Plakoribates) ................................ 169 confluentina (Acrogalumna) longipluma ...... 213 conformis (Defectamerus) ............................. 105 conformis (Phauloppia) (=lucorum) .......... 190 conformis (Phthiracarus) (=incertus) ......... 54 confundatus (Hemileius) (=initialis) .......... 193 confusa (Arcoppia) (=robusta) .................... 121 confusa (Galumna) (=louisianae) .............. 216 confusa (Ramusella) ..................................... 118 confusia (Scheloribates) ............................. 199 confusus (Chamobates) .................................. 179 confusus (Chamobates) ............................... 179 confusus (Xenillus) ........................................ 98 conglobatus (Scheloribates) parvus .......... 201 congoensis (Granuloppia) .......................... 132 congoensis (Hermanniella) ......................... 70 congregator (Trhypochthonius) tectorum ........ 60 congruus (Verachthonius) ................................ 32 conica (Suctobelbella) ….............................. 136 conicus (Malaconothrus) ................................. 60 coniferinus (Parapirnodus) ......................... 191 coniferus (Nothrus) .......................................... 63 conirostris (Scheloribates) ……...................... 199 conitus [M. (Calyptozetes)] ........................... 183 coniunctus (Steganacarus) ................................ 58 coniunctus (Tectocepheus) ............................ 154 conjuges (Perscheloribates) ........................ 198 conjuncta (Berniniella) .................................. 125 conjuncta (Phauloppia) (=lucorum) ............ 190 conjunctus (Ceratozetes) ............................. 174 conjunctus (Dolicheremaeus) ........................ 142 conjunctus (Lepidozetes) ............................. 169 conjunctus (Liacarus) ....................................... 95 conjunctus (Minguezetes) .................... 12, 182 conjunctus (Zachvatkinibates) ...................... 184 connexa (Gustavia) (=magnilamellata) ........95 connexa [O. (Zygoribatula)] .......................... 188 connexa [O. (Zygoribatula)] ….................. 188 


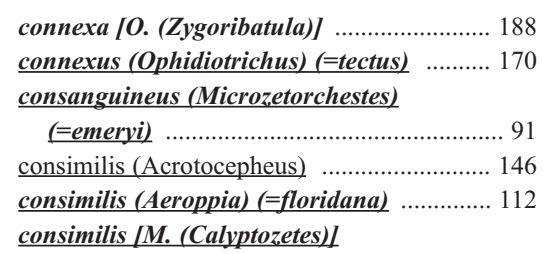
consimilis [M. (Calyptozetes)]

(=sarekensis) 183 consimilis (Indotritia) .......................................... 43 consimilis [L. (Lancelalmoppia)] ................ 111 consimilis (Neseutegaeus) ............................ 85 consimilis (Notophthiracarus) ...................... 51 consimilis (Suctobelba) ................................. 135 conspectus (Trhypochthonius) ........................ 60 conspicua (Pergalumna) ……………….... 218 conspicuus (Notophthiracarus) ................... 51 conspicuus (Ramsayellus) .......................... 182 conspicuus (Tricheremaeus) ............................ 101 contaminatus (Eupelops) .............................. 163 contigua (Hexachaetoniella) …................... 74 contiguus (Ceratozetes) (=conjunctus) ........ 174 contiguus (Exochocepheus) ........................ 161

contiguus (Liacarus) ....................................... 95 continua (Nanhermannia) .................................. 68 contortula (Oribotritia) .................................. 44 contortulus (Notophthiracarus) ……….......51 contracta [O. (Zygoribatula)] …................. 188 contractilis (Phthiracarus) (=laevigatus) ...... 54 contractus (Pseudotocepheus) .................... 145 contraria (Microtritia) ................................... 47 contraria (Oribotritia) ……........................... 44 contrarius (Rhacaplacarus) ............................... 57 controversus (Atropacarus) ........................... 48 conundrum (Pedrocortesella) …….............. 75 convexa (Hermannia) ..................................... 69 convexa (Hermannia) ....................................... 69 convexa (Hermannia) ......................................69 69 convexa (Novazelandiella) ............................. 74 convexus (Scapheremaeus) …...................... 158 convexus (Tegeocranellus) ............................. 155 copectus (Hemileius) cophinaria (Crotonia) ….................................. 67 cophinaria (Crotonia) ..................................... 67 copiosus (Pheroliodes) .................................. 74 copperminensis (Murcia) ............................... 177 copperminensis (Murcia) .............................. 177 copulata (Furcoribula) (=furcillata) ........... 92 coracinus (Liacarus) ......................................... 95 coracinus (Liacarus) ...................................... 95 corae (Cepheus) ................................................. 82 coralgablensis (Allodamaeus) ........................ 72 corallifera [B. (Gressittoppia)] ….............. 122 corallium (Lohmannia) ..................................... 38 cordiformis (Eremaeus) (=hepaticus) .......... 100 cordisetus (Caveremulus) .......................... 102 cordisetus [M. (Cristonothrus)] …............... 61 cordobensis (Oripoda) ................................... 204 cordobensis (Pheroliodes) .............................. 74 cordobensis (Ramusella) ............................... 118 cordobensis (Staurobates) schusteri ............ 105 cordylinosa (Paraphauloppia) ...................... 190 coreana (Galumna) ........................................ 215 coreana (Lohmannia) ...................................... 38

coreanus (Defectamerus) crassisetiger .......... 105 coreanus (Dolicheremaeus) infrequens ........ 142 coreanus [D. (Epidamaeus)] ........................... 79 coreanus (Liacarus) gammatus ........................ 96 coreanus (Perxylobates) ............................... 205 coriaceus (Alaskozetes) .................................. 156 coriaceus (Alaskozetes) ……........................ 156 coriaceus (Carabodes) ................................. 149 corletti (Acrotritia) ......................................... 45 corneri (Sabacarus) .............................................. 45 corneri (Sabacarus) …....................................... 45 corneri (Sabacarus) ............................................ 45 corniculata (Dissorhina) ornata .................... 125 corniculata [R. (Insculptoppia)] P.-I. y

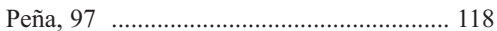

corniculata [R. (Insculptoppia)] I. y V., 99

(=neonominata) ........................................... 119

corniculata (Pergalumna) ............................. 218

corniculifera (Arcoppia) ........................... 120

corniger (Scapheremaeus) ........................... 158

cornigera (Suctobelbella) (?=acutidens) … 136

cornuta (Belba) ............................................... 77

cornuta (Ceratoppia) (=bipilis) …….............. 93

cornuta (Furcoppia) ……............................. 92

cornuta (Furcoppia) .................. 92

cornuta [H. (Kakophthiracarus)]

(=multirugosa) .................um.

cornuta (Negroppia) .................................... 94

cornuta (Parapyroppia) ................................... 94

cornuta (Pedrocortesella) ............................ 75

cornuta (Suctobelbila) ................................. 139

cornuta [S. (Ussuribata)] ........................... 138

cornutus (Ceratorchestes) ............................. 93

cornutus (Costeremus) ................................ 103

cornutus [M. (Cristonothrus) ........................ 61

cornutus (Eueremaeus) sp. inq. .................... 100

cornutus (Humerobates) ............................ 181

cornutus (Iugoribates) .................................. 180

cornutus [C. (Magoebazetes)] ….................. 88

cornutus (Microtegeus)

cornutus (Papillacarus) ……………………...... 40

cornutus (Scapheremaeus) .......................... 158

cornutus (Tecteremaeus) …….......................... 108

cornutus (Tecteremaeus) .............................. 108

corolevuensis (Pergalumna) ....................... 218

corolevuensis (Scheloribates)

thermophilus …........................................ 202

coronarius (Dolicheremaeus) ....................... 142

coronarius (Fissicepheus) .............................. 144

coronarius (Fissicepheus) .............................. 144

coronata (Berniniella) (=hauseri) ….......... 125

coronata (Eremobelba) ............................... 103

coronata (Galumna) ................................. 215

coronata (Nanheremannia) (=dorsalis) ........ 68

coronata [H. (Phyllhermannia)] .......................69 69

coronatus [L. (Dorycranosus)] ........................ 97

coronatus [C. (Klapperiches)] .................... 151

coronatus (Seboetocepheus) ........................ 145

coronopubes (Topobates) …........................ 203

corporaali (Oribotritia) ....................................... 44

corpulentus (Austrocarabodes) sp. inq. ...... 148 corpuscula (Anachipteria) (=howardi) ........ 168

corpusculum (Scheloribates) ....................... 199

corpusrarosae [I. (Philippizetes)] ................... 208

corrugata (Oppiella) (=nova) …................. 128 corrugatum (Senectoppia) (=complicatum) .. 132 corrugatus (Ameronothrus) (=lineatus) ...... 156 corrugatus (Comorozetes) ........................... 88 corrugis (Pergalumna) ................................ 218 corsicanus (Ophidiotrichus) ......................... 170

corsicanus (Saxicolestes) ................................. 91 corticalis (Nothrus) sp. inq. ............................ 63 corticalis (Phauloppia) (?=lucorum) corticeus (Mycobates) ................................ 182 corticis [N. (Protokalumma)] ........................ 212 corticola (Oripoda) ....................................... 204 corynopus (Belba) ........................................... 77 corynopus (Belba) ........................................... 77 corynopus (Belba) ……..................................... 77 coscobensis (Pergalumna) emarginata ........ 218 costai [N. (Calyptophthiracarus)] .................... 52 costanotus (Damaeus) …............................... 78 costata (Allogalumna) …........................... 213

costata (Galumna) ..................................... 215 costula (Licnodamaeus) .................................... 74 costulata (Tectoppia) .................................. 115 costulatus (Austrocarabodes) …................. 148 costulatus (Eremaeozetes) …........................ 165 costulatus (Licneremaeus) …...................... 160 costulatus (Microzetes) ................................. 89 costulatus (Plateremaeus) ............................. 73 costulatus (Trichocarabodes) ...................... 154 costulifera [N. (Amerigloboppia)] ............... 114 covarrubiasi (Loboppia) …........................ 111 covarrubiasi (Novonothrus) ......................... 64 coweetaensis (Carabodes) ............................ 149 coxalis [D. (Epidamaeus)] .............................. 79 coxalis [D. (Epidamaeus)] ............................. 79 cozadensis (Pilogalumna) ............................ 220 craigheadi [D. (Epidamaeus)] ......................... 79 crassa (Macrogena) …................................ 176 crassiclava (Pilogalumna) ............................ 220 crassicostata (Galumna) (=setigera) ............ 216 crassiori (Austrotritia) lebronneci .............. 42 crassipes (Hermannia) (=gibba) .................... 69 crassipes (Oribatula) (=tibialis) .................. 188 crassipilosa (Oribatella) ................................ 171 crassipodus (Scheloribates) ........................ 199 crassipora (Pergalumna) ............................... 218 crassirostris (Punctoribates) sellnicki ............ 184 crassisensillatus [D. (Epidamaeus)] ................ 79 crassiseta (Acutoppia) ............................... 109 crassiseta (Ceratoppia) ……........................... 93 crassisetae (Euphthiracarus) .......................... 46 crassisetiger (Defectamerus) ......................... 105 crassisetiger (Eohypochthonius) …................. 28 crassisetiger (Eohypochthonius) …................2 28 crassisetiger (Eohypochthonius) .................. 28 crassisetiger (Indoribates) ............................ 208 crassisetiger (Protoribates) ............................ 206 crassisetosa (Belba) .......................................... 77 crassisetosa (Heterobelba) .......................... 104 crassisetosa (Mesoplophora) …….................. 42 
crassisetosus (Malaconothrus) ………………...60

crassisetosus (Haplochthonius) …………….... 34 crassisetosus (Ommatocepheus) ........................ 83 crassisetosus (Steganacarus) (?= carusoi) .... 58 crassisetosus (Suctoribates) …………….... 140 crassisetosus [T. (Tyrphonothrus)] .................... 62 crassisetus (Nothrus) ………………………... 63 crassispinosus (Spatiodamaeus) ........................... 82 crassus [P. (Archiphthiracarus)] ……………... 55 crassus (Liochthonius) ……………………..... 30 crassus (Nesopelops) ……………………....... 164 crassus (Perscheloribates) ………………….. 198 crassus (Scapheremaeus) …………………... 158 crassus (Scheloribates) Ham., 67 .................. 199 crassus (Scheloribatres) Kul., 79

(=repetitus) ... 201 crassus (Trhypochthoniellus) (=longisetus) .... 60 craterifer (Hoplophorella) ............................. 48 craterifer (Plesiodamaeus) ................................ 77 cremersi (Metabelba) (=papillipes) ................ 80 crenata [Q. (Coronoquadroppia)] ............... 133 crenata (Galumna) ………………………..... 215 crenata [R. (Insculptoppia)] …………....... 118 crenatosetosa (Parabelbella) .............................. 81 crenellatus (Austrocarabodes) ……………... 148 crenophilus (Phthiracarus) ............................... 53 crenulata (Tenuiala) ………………………….... 99 crenulatus (Oribatodes) …………………....... 83 crenulatus (Synchthonius) …………………..... 32 crenulatus (Synchthonius) _........................... 32 creta (Teratoppia) ……………………….... 132 cretensis [N. (Calyptophthiracarus)]

cribelliger [M. (Calyptozetes)] _.... 183

cribraria (Ferolocella) ……………………..... 170

cribrarius (Euphthiracarus) ................................ 46

cribrarius (Euphthiracarus) ............................ 46

cribrarius (Euphthiracarus) ………………….... 46

cribratus (Solenozetes) …………………….... 71

cribriger (Pergalumna) ………………….... 218

cribriger (Pergalumna) ................................. 218

cricoides (Sellnickochthonius) .......................... 31

cricoides (Sellnickochthonius) ......................... 31

cricoides (Sellnickochthonius) ........................ 31

crimicus (Scheloribates) ................................. 199

crinita (Oribella) (=pectinata) ....................... 107

crinitosimilis [P. (Archiphthiracarus)]

(Ebryobius)

crinitus (Austrocarabodes) sp. inq. .............. 148

crinitus (Benoibates) ...................................... 203

crinitus [H. (Capillonothrus)] (=thori) 66

crinitus (Chaunoproctus) (=pedestris) ........ 192

crinitus (Diapterobates) .................................. 181

crinitus (Megalotocepheus) ........................... 147

crinitus (Megalotocepheus) ……………….... 147

crinitus (Nothrus) ……………………………... 63

crinitus (Phthiracarus) ………… 53

crinitus (Plenotocepheus) …………………..... 145

crinitus (Trimalaconothrus) ............................... 62

crispatus (Damaeus) …………………………. 78

crisposetosa (Suctobelbella) ............................ 136

crispus [P. (Archiphthiracarus)] ........................ 55 crispus [P. (Archiphthiracarus)] ................... 55

crispus (Dolicheremaeus) .............................. 142 crispus (Eremulus) .................................... 102

crispus (Indoribates) (=punctulatus) .......... 208 crispus (Trhypochthoniellus) (=longisetus) .... 60 crispus (Trimalaconothrus) ……………........ 62 crispus (Trimalaconothrus) ............................ 62 cristata (Apoplophora) ……………………...... 41 cristata [O. (Dzarogneta)] .............................. 129 cristata (Moritzoppia) unicarinata ................ 127 cristata (Quadroppia) .................................. 133 cristata [G. (Stenoppia)] ................................... 116 cristata (Ululohmannia) ................................... 41 cristatus (Hoplophthiracarus) ............................ 50 cristatus (Licneremaeus) ……................... 160 cristatus (Otocepheus) .................................... 147

cristatus (Otocepheus) ……………………... 147 cristatus [T. (Rostrozetes)] ............................ 211 cristatus (Tecteremaeus) ............................... 108 cristatus (Zetomimus) ..................................... 179 cristipes (Trematoppia) ………………….... 112 cronus (Arcoppia) ……………………..... 120 crosbyi (Hypochthoniella) ................................ 29 crosbyi (Oribellopsis) …............................... 107 crosbyi (Oribellopsis) ...................................... 107 crossleyi (Melanozetes) …........................... 176 crozetensis (Austroppia) .................................. 122 crozetensis (Austroppia) ............................. 122 crozetensis (Halozetes) .................................. 156 crozetensis (Halozetes) ……………………... 156 crozetensis (Halozetes) ................................. 156 crozetensis (Humerobates) ............................. 181 cruciata (Schalleria) ..................................... 90 cruciseta (Scheloribates) ………………....... 199 crux (Porrhoppia) ………………………....... 121 cruzae (Papillacarus) ....................................... 40 cryophilus (Huarpescopes) ........................... 205 cryptacus (Grandjeanobates) …………...... 197 cryptodonta (Cryptogalumna) ........................ 214 cryptomeriae (Arcoppia) ………………….. 120 cryptonota (Lyrifissiella) ................................. 73 cryptopa (Maerkelotritia) ................................... 43 cryptoreticulata (Pedrocortesella) ……....... 75 cryptus (Euscheloribates) ...………………... 197 crystallina (Dometorina) ….......................... 192 csavasorum [G. (Bigalumnella)] ………….... 222 csiszarae (Atropacarus) …………………........ 48 csiszarae (Ctenobelba) ........................................ 101 csiszarae (Lanceoppia) ................................ 110 csoengeyi (Pilizetes) ..................................... 220 ctenifera (Oxybrachioppia) ........................... 129 cuauhtemoctzini (Fuscozetes) ....................... 175 cubana (Acrogalumna) ................................ 213 cubana (Beckiella) ........................................ 140 cubana (Borhidia) _..................................... 133 cubana (Galumna) ................................... 215 cubana (Mesoplophora) ………………….... 42 cubana (Oripoda) ...…………………....... 204 cubana [O. (Oxyoppiella)] ............................ 129 cubanus (Arceremaeus) ............................... 108 cubanus (Cosmozetes) ..................................... 88 cubanus (Hermannobates) .............................. 71 cubanus (Licneremaeus) ............................... 160

cubanus [T. (Rostrozetes)] ……………….... 211 cubanus (Scheloribates) praeincisus .......... 201 cucheae (Pergalumna) .............................. 218 cucheana [L. (Bicristoppia)] ....................... 111 cucullata (Achipteria) .................................... 167 cucullata (Hoplophorella) ................................. 48 cucullata (Hoplophorella) .............................. 48 cucullata (Hoplophorella) ............................ 48 cucullata (Hoplophorella) ................................ 48 cucullata (Hoplophorella) ........................... 48 cucullata (Hoplophorella) ................................ 48 cuculloides (Hoplophorella) (=cucullata) .... 48 cucundus [P. (Neophthiracarus)] (=diaze) .... 56 cuii [P. (Tectodamaeus)] .................................... 81 culterisetosus [D. (Epidamaeus)] ..................... 79 cultrata (Epilohmannia) ................................. 36 cuneata (Galumna) ......................................... 215 cuneiseta (Hoplophorella) (=hamata) .......... 49 cuneocostulata (Moritzoppia) ......................... 126 cupulata (Crotonia) ........................................ 67 curarii (Varioppia) $(=$ radiata) ....................... 120 curassensis (Hoplophorella) cucullata .......... 48 curiosus [P. (Archiphthiracarus)] ………..... 55 curiosus (Notophthiracarus) ………….......... 51 curta (Achipteria) ............................................ 167

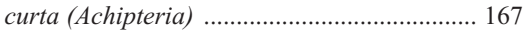
curta (Achipteria) ......................................... 167 curta (Oromurcia) (=lucens) ......................... 178 curticephala (Acrotritia) ................................ 45 curticephala (Acrotritia) …………………...... 45 curticephala (Acrotritia) ………………….... 45 curticephala (Acrotritia) ................................. 45 curtipetata (Eremobelba) ………................... 103 curtipila (Arcoppia) ……………………..... 120 curtipilis (Ceratoppia) bipilis ......................... 93 curtipilis [L. (Dorycranosus)] (=splendens) .... 97 curtipilis [I. (Haplozetes)] vindobonensis .... 208 curtipilis (Melanozetes) sp. inq. ..................... 176 curtipilus (Eupelops) .................................... 163 curtipilus (Peloribates) ................................ 209 curtipilus (Pilizetes) ..................................... 220 curtipilus (Pseudotocepheus) sp. inq. .......... 145 curtipilus (Ramusella) …………………........ 118 curtipilus (Ramusella) ……………………... 118 curtipilus (Ramusella) ................................... 118 curtiramosa [R. (Rectoppia)] strinatii ............. 119 curtiseta (Arcoppia) arcualis ...................... 120 curtiseta [P. (Constrictocepheus)] ……….... 145 curtiseta (Odontocepheus) ............................. 153 curtiseta [S. (Plenoxylobates)] ……………..... 207 curtispinosa (Basidoppia) ................................ 121 curtulus [P. (Archiphthiracarus)] ..................... 55 curtus (Liochthonius) rigidisetosus ............... 31 curva (Pergalumna) _................................... 218 curva (Pergalumna) _.................................. 218 curva (Pergalumna) …………………......... 218 curva (Pergalumna) …………………….... 218 curvatus (Granizetes) ……………………... 166 curvialatus (Scheloribates) …………………... 199 curvialatus (Scheloribates) .......................... 199 curviclavata (Globoppia) .............................. 110 


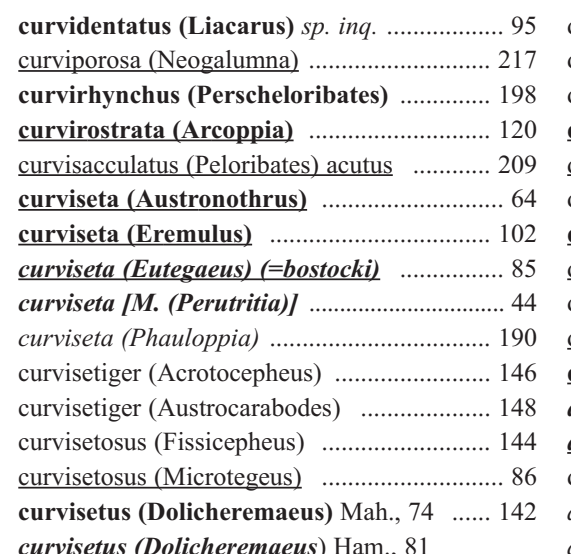

curvisetus (Dolicheremaeus) Ham., 81

(Epraeoccupatus) ………………………... 143

curvisetus (Malgasodes) …………………..... 152

curvispina (Acroppia) .................................. 128

curypterus (Unguizetes) ................................. 186

cuscensis (Brachioppia) …………………..... 122

cuscensis (Brachioppia) …………………...... 122

cuscensis (Brachioppia) ............................. 122

cuscensis (Podoribates) ................................. 186

cuspidatiformis (Chamobates) ....................... 179

cuspidatus (Berlesezetes) .................................. 87

cuspidatus (Ceratozetes) ................................. 174

cuspidatus (Chamobates) ................................ 179

cuspidatus (Chamobates) ................................ 179

cuspidatus (Chamobates) …………………..... 179

cuspidatus (Diapterobates) pusillus ............... 181

cuspidatus (Dolicheremaeus) ........................ 142

cuspidatus (Heterozetes) …………………..... 173

cuspidatus (Liacarus) (=xylariae) .................. 96

cuspidatus [F. (Parafurcobates)] ................. 175

cuspidatus (Podoribates) …………………... 186

cuspidatus (Scapheremaeus) ........................ 158

cuspidatus (Scotiazetes) .................................. 178

cuspidentatus (Tectocepheus) .......................... 154

cuspidentatus (Tectocepheus) ......................... 154

cuspidodenticulata (Ceratozetella) .................. 173

cuticulatus [I. (Haplozetes)] ............................ 208

cuyi (Scheloribates) ....................................... 199

cyclonota (Phauloppia) sp. inq. .................. 190

cyclops (Scapheremaeus) ………………....... 158

cyclosoma (Oppia) (=denticulata) ................. 115

cyclosporosa [O. (Zygoribatula)] ……….... 188

cylindrica [D. (Cylindroppia)] ........................ 123

cylindrica [D. (Cylindroppia)] ....................... 123

cylindrica (Epilohmannia) ................................. 36

cylindrica (Epilohmannia) ............................... 36

cylindrica (Epilohmannia) ………………...... 36

cylindrica (Epilohmannia) ............................... 36

cylindrica (Epilohmannia) .............................. 36

cylindrica (Epilohmannia) ………………..... 36

cylindricus (Torpacarus) ................................. 4

cymba (Cymbaeremaeus) ............................... 158

cymba (Cymbaeremaeus) …………………….. 158

cynocephalus (Carabodes) (=cephalotes) .... 149

dactylaris [O. (Zygoribatula)] …………….... 188

dactyloscopica (Jornadia) ............................. 187

dactyloscopicus (Notophthiracarus) ............. 51 dactyloscopicus [C. (Xiphobates)] ………….. 180

daghestanica (Belba) ………………………... 77

daghestanica (Epilohmannia) ............................ 36

daimonios [N. (Protophthiracarus)] ............. 53

dalawaeus (Scheloribates) ….......................... 199

dalecarlica (Conchogneta) ............................ 108

dalenii (Tripiloppia) ..................................... 128

daliensis (Ghilarovus) ……………............... 184

dallaii (Mystroppia) ……………………….... 130

damoeoides (Dolicheremaeus) ……………..... 142

damoeoides (Dolicheremaeus) ..................... 142

damoeoides (Dolicheremaeus) .................... 142

danae (Steganacarus) (=coniunctus) ............ 58

danaus (Scheloribates) ..................................... 199

danos (Eueremaeus) ......................................... 100

danos [S. (Guatemalozetes)] .......................... 178

danubialis (Sellnickochthonius) ........................ 31

danubianus (Phthiracarus) ............................... 53

dargoltsiana (Suctobelbella) ............................ 136

darwini (Eremaeozetes) .................................. 165

darwini [P. (Neophthiracarus)] ....................... 56

dashidorzsi (Lepidozetes) ................................. 169

dashidorzsi (Lepidozetes) ................................. 169

dasypus (Phthiracarus) sp. inq. ....................... 53

davisi (Austrocarabodes) ............................... 148

davisorum (Xenillus) ...................................... 98

debililamellata [R. (Rectoppia)] ..................... 119

debilis (Eueremaeus) (=quadrilamellatus) .... 101

debilis (Mycobates) ………………………..... 182

debilitranslamellata [O. (Zygoribatula)] ...... 188

deboissezoni (Congoppia) .......................... 123

decarinatus (Scheloribates) ........................... 199

decedens (Amerobelba) ................................... 101

decempectinata (Lauroppia) ............................ 126

decemsetiger (Adrodamaeus) ............................ 75

decemsetosa (Neoamerioppia) ..................... 114

dechambrieri (Acaroceras) .............................. 87

dechambrieri (Oribatella) .............................. 171

dechambrierorum (Arcoppia) ....................... 120

decipiens (Atropacarus) ..................................... 48

decipiens (Oxyoppioides) ................................. 129

decipiens (Pilogalumna) $(=$ ornatula) .......... 220

decorata (Pergalumna) ............................... 218

decorata (Suctobelbella) ............................... 136

decoratissima (Pergalumna) ........................ 218

decoratus (Papillocepheus) .......................... 144

decoratus (Plumobates) ................................ 194

decoratus (Similochthonius) …………………... 34

decorus (Papillocepheus) ............................. 144

decoui (Reticulocepheus) ................................ 84

decui (Lasiobelba) ....................................... 113

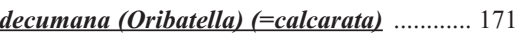

decumana (Oribotritia) $s p$. inq. ......................... 44

decumanus (Peloribates) .............................. 209

defectuosa (Ramusella) .................................. 118

deficiens (Anachipteria) .................................... 168

deficiens (Beckiella) ................................. 140

deficiens (Neoamerioppia) ............................ 114

deficiens (Papillocepheus) ........................... 144

deficiens (Similobates) ................................. 202

deformatus (Xenillus) .................................... 98

deformis (Afroleius) ....................................... 207 degradatus (Meristacarus) ………………….... 39

delamarei (Niphocepheus) nivalis ………….... 84

delamarei (Provertex) …................................. 161

delamellatus (Montizetes) .............................. 107

delectum (Galumna) _.......................... 215

deleoni (Chaunoproctus) ............................... 192

delessei (Suctobelbella) …………………..... 136

deletus [H. (Tuberemaeus)] ............................ 193

delicata (Lyroppia) ………………………..... 131

delicata (Suctobelbella) ................................ 136

delicatissimus (Plenotocepheus) ................. 145

deliciosa (Brachioppia) ................................. 122

deliensis (Murcia) sp. inq. .............................. 177

delticus (Pheroliodes) ……………………….... 74

demeteri (Basidoppia) ................................... 121

demeteri (Beklemishevia) ............................. 27

demeteri [P. (Passalomonia)] ....................... 162

demeteri (Scapheremaeus) ............................ 158

demetororum (Similobates) ......................... 202

demetrii (Gilarovella) ........................................ 28

denaliensis (Cyrtozetes) ................................... 174

denaliensis (Cyrtozetes) ................................. 174

dendricola (Kokoppia) ..................................... 123

dendrisetosus (Cryptacarus) ............................... 38

dendroetus [C. (Klapperiches)] (=radiatus) .. 151

dendropectinata (Mimoppia) .......................... 121

denigratus (Pontiobates) ………………....... 192

densefoveolatus (Dolicheremaeus) .............. 142

densoporosa (Trypogalumnella) ...................... 222

densus (Eremulus) ………………………...... 102

dentata (Abchasiella) ....................................... 124

dentata (Austroceratoppia) ............................... 93

dentata (Austrotritia) .......................................... 42

dentata (Baloghoppia) ................................... 128

dentata (Lauroppia) ……………………....... 126

dentata [F. (Mexicoppia)] .................................. 92

dentata [F. (Mexicoppia)] ……......................... 92

dentata [F. (Mexicoppia)] ............................... 92

dentata (Murcia) nova ................................... 177

dentata (Oribatula) Mih., 1969

(=neonominata $)$......................................... 187

dentata (Oribatula) G. y S., 2000

(=praeoccupata) ...................................... 187

dentata [H. (Phyllhermannia)] ....................... 69

dentata (Pyroppia) …………………………... 94

dentata (Pyroppia) ......................................... 94

dentata (Suctobelbila) ....................................... 139

dentata (Suctobelbila) ……………………..... 139

dentata (Suctobelbila) …………………....... 139

dentata (Suctobelbila) .................................... 139

dentata [O. (Zygoribatula)] sp. inq. ........... 188

dentaticuspis (Oribatella) ............................. 171

dentatus (Cepheus) ……………………………..... 82

dentatus (Chamobates) .................................... 179

dentatus (Eupterotegaeus) .................................. 83

dentatus (Humerobates)

(=rostrolamellatus) ……………………. 181

dentatus (Mabulatrichus) ………………….... 184

dentatus (Mirabilozetes) …………………..... 89

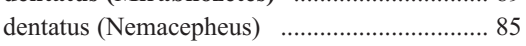

dentatus (Nodocepheus) ................................ 85

dentatus (Pheroliodes) ……………………....... 74 
dentatus (Pantelozetes) (=paolii) …............. 107

dentatus (Plenotocepheus) ............................ 145

dentatus (Protoribates) ................................... 206

dentatus (Protoribates) .................................. 206

dentatus (Protoribates) ….............................. 206

dentatus (Protoribates) ................................ 206

dentatus (Pseudotocepheus) ...................... 145

dentatus (Scheloribates) ................................. 199

dentatus (Sphodrocepheus) ............................ 84

denticulata (Lauroppia) ............................... 126

denticulata (Oppia) ........................................ 115

denticulata (Oppia) ...................................... 115

denticulatus (Neseutegaeus) .............................. 85

denticulatus (Pilizetes) ................................. 220

denticuspidatus (Peloptulus) ......................... 164

dentifer (Edwardzetes) .................................. 175

dentifer (Edwardzetes) .................................. 175

dentifera [M. (Moritzoppiella)] ..................... 127

dentilamellata (Prionoribatella) .................... 172

dentilamellatus (Eremaeus) (=hepaticus) .... 100

dentipes (Hermannia) sp. inq. ...................... 69

dentissima (Novosuctobelba) ........................ 135

dentissima (Novosuctobelba) ........................ 135

dentonyx (Subphauloppia) …..................... 191

dentotutorii (Chamobates) ........................... 179

depauperatus [C. (Xiphobates)] ............ 12, 180

depilata (Strenzkea) ......................................... 91

depilatus (Africoribates) ............................ 180

depilatus (Eupelops) (=curtipilus) $\quad$............... 163

depilatus [C. (Klapperiches)] …................... 151

depilatus (Orthozetes) .................................90

depilis [L. (Incabates)] ................................... 209

depilis (Mochloribatula) .............................. 185

depressa [N. (Protokalumma)] ....................... 212

depressa [N. (Protokalumma)] .................... 212

depressculus (Euphthiracarus) ......................... 46

depressus (Tectocepheus) ............................ 154

desaussurei (Cosmochthonius) ....................... 33

desaussurei (Kalyptrazetes) ............................ 89

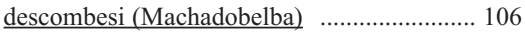

deserticola (Tectoribates) ................................ 172

deserticola (Thamnacarus) ............................... 41

deserticus (Bipassalozetes) ..................... 12, 162

deserticus (Bipassalozetes) ..................... 12, 162

desmeti [S. (Tropacarus)] .................................. 59

detectidens (Pirnodus) .................................. 205

detosus (Liacarus) ......................................... 95

devilliersi (Halozetes) marinus ....................... 157

devilliersi (Halozetes) marinus ..................... 157

diamantei (Lopholiodes) ............................... 73

diamantensis (Mikizetes) .............................. 184

diani (Protoribates) ..................................... 206

diaphanus (Atropacarus) (=striculus) .......... 48

diaphora [S. (Campbelloppia)] ................... 124

diaphoros (Acrotritia) ...................................... 45

diaphoros (Hoplophorella) ............................. 48

diaphoros (Rhacaplacarus) ……..................5 57

diazae [P. (Neophthiracarus)] ........................ 56

dicella (Crotonia) ............................................ 67

diceros (Zeasuctobelba) ............................... 139

dicerosa (Rhinosuctobelba) ......................... 135

dichosa (Joboppia) dichosa (Joboppia)

[L. (Dorycranosus)] ....................... 97

dickinsoni [C. (Klapperiches)] ...................... 151

dicornus (Eupelops) eximius ....................... 163

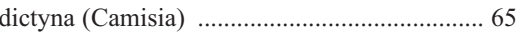

dictyna (Camisia) ............................................... 65

didyma (Oribotritia) .......................................... 44

diehli (Acrotocepheus) ................................... 146

differens (Anomaloppia) ............................. 116

differens (Eupelops) ....................................... 163

difficilis (Galumna) sp. inq. ....................... 215

diffissa (Suctobelbella) (=italica) …............. 136

diforamenatus (Scheloribates) ….............. 199

digita (Cerachipteria) ..................................... 168

digitata (Lamellarea) .................................. 160

digitifer (Protozetes) ..................................... 90

digna (Hoplophorella) .................................... 48

dikra (Acrotritia) .............................................. 45

dikra (Acrotritia) ........................................... 45

dikros (Rhacaplacarus) …..........................57

dilatata (Allogalumna) ............................... 213

dilatatus (Rhynchoribates) ......................... 140

dilucidus [P. (Neophthiracarus)] .................. 56

dilutus (Liochthonius) .................................... 30

dilutus (Sphaerochthonius) .............................. 34

dimentmani (Scheloribates) ......................... 199

dimera (Neoliodes) (=theleproctus) ……....... 72

dimidiata (Galumna) ............................... 215

diminuta (Nacunansella) .............................. 74

diminuta (Setogalumna) .............................. 221

dimorpha (Epilohmannia) ............................ 36

dimorpha (Galumna) ................................... 215

dimorpha (Kodiakella) .................................... 99

dimorphichaites [T. (Rostrozetes)] ............. 211

dimorphus (Hydrozetes) ............................... 155

dinghuensis [H. (Phyllhermannia)] ................69 69

dinota (Acrotritia) .............................................. 45

dipankari (Malaconothrus) ..............................6 60

discifer (Anellozetes) …............................. 180

discifer (Nothrus) ........................................... 63

discifer (Scheloribates) ................................. 199

discifer (Totobates) ....................................... 196

discifera (Galumna) ...................................... 215

discoidalis (Beckiella) ................................ 140

discoidalis (Licneremaeus) ........................ 160

discors (Topobates) (=medius) ..................... 203

discrepans (Acrotocepheus) duplicornutus .. 146

discrepans (Miracarus)

discrepans (Muliercula) .............................. 197

discrepans (Plenotocepheus) ....................... 145

discrepans [M. (Stylozetes)] ......................... 89

discrepans (Suctobelba) ....................... 135

discrepans (Xenillus) ........................................ 98

discrepans (Xenolohmannia) ........................ 41

discrepus (Rhacaplacarus) ............................. 57

discreta (Apoplophora) (=pantotrema)

discreta (Neoppia) ......................................... 109

disjuncta (Pararamusella) ............................ 118

disjuncta [M. (Pravoppia)] ......................... 111

disjunctus (Xenillus) ................................... 98

dispar (Galumna) ........................................ 215 dispar (Machadobelba) …........................... 106

dispar (Orthozetes) . ...................................... 90

dispar (Rhacaplacarus) .................................... 57

dispar [P. (Porallozetes)] …...................... 178

dispar [T. (Rostrozetes)] ............................. 211

dispar [H. (Tuberemaeus)] ............................ 193

disparilis [E. (Pocsia)] ................................... 47

dispariseta (Anomaloppia) .......................... 116

dispersa (Hexachaetoniella) .......................... 74

dispersosetosa (Suctobelbella) ...................... 136

dispersus (Peloribates) .............................. 210

dissimilis (Arcoppia) .................................... 120

dissimilis (Galumna) capensis ................... 215

dissimilis (Hoplophorella) ............................. 48

dissimilis [C. (Klapperiches)] ....................... 151

dissimilis (Mesotritia) .......................................... 43

dissimilis (Perlohmannia) ................................. 36

dissimilis (Perlohmannia) ............................. 36

dissimiloides (Arcoppia) ............................ 120

dissonus [P. (Archiphthiracarus)] .................... 55

dissonus [P. (Neophthiracarus)]

(=praeoccupatus) …................................... 57

distenta (Epimerella) .................................... 131

distentus (Neseutegaeus) ............................... 85

distincta (Campachipteria) ............................ 168

distincta (Campachipteria) ........................... 168

distincta (Ceratoppia) (=bipilis) ................... 93

distincta (Galumna) (=setigera) …................ 216

distincta (Lauroppia) .................................. 126

distincta (Oppiella) (=neonominata) .......... 128

distincta (Phauloppia) sp. inq. ..................... 190

distinctus (Dolicheremaeus) .......................... 142

distinctus (Eupelops) ................................ 163

distinctus (Notophthiracarus) ...................... 51

distinctus (Scheloribates) .............................. 199

distinguenda (Oromurcia) (?=sudetica) ...... 178

ditrichosus (Megeremaeus) ............................ 99

divergens (Cultroribula) .................................. 92

divergens (Galumna) .................................... 215

divergens (Liebstadia) .................................. 195

divergens (Oripoda) ......................................... 204

diversa (Cultroribula) ....................................... 92

diversa (Pergalumna) (=altera) .................. 218

diversa (Pseudoceratoppia) .......................... 94

diversa [O. (Zygoribatula)] (=cognata) ...... 188

diversiareata (Allogalumna) (=alamellae) .. 213

diversidactylus [S. (Andeszetes)] .................. 202

diversipes [S. (Dyobelba)] (=carolinensis) $\ldots .82$

diversipilis (Spatiodamaeus) ….......................... 82

diversipilus (Eupelops) (=plicatus) .............. 163

diversiporosus [S. (Polyxylobates)] ............. 207

diversiseta (Cosmochthonius) lanatus ............ 33

diversiseta [T. (Paragloboppia)] ................ 115

diversisetosa (Hermanniella) ....................... 70

diversisetosa (Hymenobelba) ...................... 105

diversisetosus (Xenillus) ................................ 98

diversosetosa [S. (Flagrosuctobelba)] .......... 138

diversus [L. (Dorycranosus)] (=splendens) .... 97

diversus [D. (Epidamaeus)] tatricus ................ 80

diversus (Eupelops) (=plicatus) .................. 163

diversus (Verachthonius) ............................... 32

divida (Acrotritia) ........................................... 45 
divida (Oribatula)

dividipeltatus (Eremaeozetes) ...................... 165

divisa (Persuctobelba) ................................ 135

divisus (Caleremaeus) ................................ 106

dixa (Acrotritia) ............................................... 45

djaparidzae (Ceratozetella) ............................. 173

dlouhyi (Fijibates) ........................................ 197

dlouhyorum [E. (Brasiliotritia)] .................... 47

doderleini (Platyliodes) .................................... 72

dodsoni (Pergalumna) .................................. 218

dogmai (Cuneoppia) ...................................... 134

doliaris (Hermannia) sp. inq. .......................... 69

dolosa (Epilohmannia) .................................. 36

dolosa (Hermanniella) ..................................... 70

dolosa (Hermanniella) ...................................... 70

dolosus (Sellnickochthonius) ........................... 32

domahidyi (Hymenobelba) ......................... 105

domesticus (Cosmochthonius) sp. inq. .......... 33

dominae (Chamobates) (=cuspidatus) ........ 179

dominiaki [P. (Archiphthiracarus)] .................. 55

domrowi (Nanhermannia) .............................6 68

domrowi (Pseudopirnodus) ....................... 204

donatoi (Steganacarus) .................................... 58

donghaksaensis (Neogymnobates) ................. 177

doratosa (Metapyroppia) ................................ 94

doris (Lauroppia) ........................................... 126

dorni (Dolicheremaeus) .................................. 142

dorsalis (Carabodes) tsushimaensis .............. 150

dorsalis (Galumna) sp. inq. .......................... 215

dorsalis (Nanhermannia) ................................ 68

dorsalis (Nanhermannia) ................................ 68

dorsalis (Nanhermannia) ............................ 68

dorsalis [T. (Rostrozetes)] (=ovulum) ........... 211

dorsata (Banksinoma) (?=lanceolata) ….... 109

dorsatus (Neoliodes) (=theleproctus) ............ 72

dorsofoveolatus (Malaconothrus) ...................... 60

doryphoros (Liacaroppia) ......................... 126

doryura (Zachvatkinella) ................................. 27

douglasensis (Passalozetes) ........................ 162

douhereti (Meristacarus) ............................. 39

doumandji (Steganacarus) ............................... 58

draco (Pteramerus) ..................................... 105

draconis (Machuella) ..................................... 133

draconis (Machuella) ................................... 133

dryas [M. (Calyptozetes)] ........................... 183

dubia (Achipteria) ......................................... 167

dubia (Dampfiella) ................................... 14

dubia [O. (Dzarogneta)] ................................ 129

dubia (Galumna) ........................................... 215

dubia (Hoplophorella) .................................... 48

dubia (Joelia) .................................................... 170

dubia (Lauroppia) (=fallax) ...................... 126

dubinini (Ameronothrus) …………………..... 156

dubinini (Belba) ................................................ 77

dubinini (Diapterobates) ................................. 181

dubinini (Hermanniella) .................................... 70

dubinini (Hermanniella) ................................... 70

dubinini (Phthiracarus) ...................................... 53

dubiosus (Parhypochthonius) sp. inq. ............ 28

dubita [O. (Zygoribatula)] (=undulata) ...... 190

dubitanda (Pergalumna) ............................ 218

dubius (Ceratozetes) (=mediocris) .............. 174 dubius [C. (Flexa)] …................................... 150

dubius (Maculobates) .................................. 196

dubius [E. (Pocsia)] ........................................ 47

dubius (Sadocepheus) ..................................... 84

dudichi (Carabodes) ...................................... 149

dudichi (Kokoppia) .................................. 123

dudichi (Oribatella) .................................... 171

dudichi (Phthiracarus) (=boresetosus) .......... 53

dudichi (Pilizetes) ........................................ 220

duffyi (Serratoppia) ...................................... 124

dugesi (Adrodamaeus) (=femoratus) ............ 75

dungeri (Hypogeoppia) .................................. 126

duodentata (Bicyrthermannia) ........................6 67

duoporosa (Trichogalumna) ...................... 221

duoseta [P. (Triaungius)] .............................. 206

duplex (Eupelops) (=torulosus) .................. 164

duplex (Oribotritia) .............................................. 44

duplex (Suctobelbella) .................................... 136

duplicarinatus (Dolicheremaeus) .................. 142

duplicata (Acrotritia) ...................................... 45

duplicata (Acrotritia) …………........................ 45

duplicata (Beckiella) .................................... 140

duplicata (Pergalumna) (=ventralis) ......... 218

duplicatus (Dolicheremaeus) ......................... 142

duplicornutus (Acrotocepheus) ................... 146

durbanensis [P. (Triaungius)] .................... 206

durhamensis (Sphagnoppia) .......................... 115

durian (Otocepheus)

dusan (Microtritia) tropica .............................. 47

dzaparidzei (Phauloppia) (=lucorum) ........ 190

echigoensis (Ceratotenuiala) ......................... 99

echinata (Camisia) (=spinifer) .......................6 65

echinatus (Papillacarus) ………..................... 40

echinodiscus (Atropacarus) ............................. 48

echinopus (Damaeus) .................................... 78

echinus (Notophthiracarus) ......................... 51

eckeri [M. (Megazetes)] ................................8 89

ecphyla (Crotonia) ......................................... 67

ecphylus (Rhacaplacarus) ............................. 57

ecuadorensis (Xenillus) ................................. 98

ecuadoriensis (Cosmozetes) ........................... 88

ecuadoriensis (Eupelops) ........................... 163

ecuadoriensis [C. (Klapperiches)] ................ 151

ecuadoriensis (Rhynchoribates) ................. 140

edentatus (Rhynchoribates) ....................... 140

edentulus (Hydrozetes) …………………….... 155

editae (Lauroppia) .......................................... 126

eduardi (Piffliella) ....................................... 196

eduardoi [R. (Rectoppia)] ............................. 119

edwardensis (Halozetes) ................................ 157

edwardsi (Edwardzetes)

edwardsi (Edwardzetes) ............................... 175

egelida [O. (Zygoribatula)] ........................... 188

egenus (Chamobates) .................................. 179

egregia (Galumna) …............................. 215

egregius (Carabodes) .................................... 149

egregius (Malaconothrus) .......................... 11, 60

egregius (Malaconothrus) ...................... 11, 60

egregius [P. (Neophthiracarus)] ….............. 56

egypticus (Papillacarus) ................................... 40

eichhornicus (Trimalaconothrus) ................... 62

ejuncida (Acrotritia) ....................................... 45 ksteeni (Annectacarus) ……….................... 37

elamellata (Fortuynia) …........................... 157

elamellata (Rykella) .................................... 185

elastica (Mesotritia) (=nuda) …....................... 44

elegans (Achipteria) ..................................... 167

elegans (Austrocarabodes) …....................... 148

elegans (Bipassalozetes) ................................. 162

elegans (Cryptoppia) (=mahunkai) .............. 116

elegans (Eremulus) ........................................ 102

elegans (Fissicepheus) …............................ 144

elegans (Ghilarovus) .................................. 184

elegans (Hypochthonius) ................................. 29

elegans [L. (Incabates)] .......................... 12, 209

elegans [L. (Incabates)] ......................... 12, 209

elegans (Oppia) sp. inq. ............................... 115

elegans (Pheroliodes) ..................................... 74

elegans (Pulchroppia) ................................... 120

elegans (Scheloribates) …….......................... 199

elegans (Scheloribates) …........................... 199

elegans (Scheloribates) ............................... 199

elegans (Sphaerochthonius) Berl., 10

(=gemma) ….......................................... 34

elegans (Sphaerochthonius) Berl., 16

(=gemma) …….................................... 34

elegans (Sumatrotritia) ….............................. 47

elegans (Synchthonius) …............................... 32

elegans (Synchthonius) .................................... 32

elegans (Synchthonius) …............................... 32

elegans (Tectocepheus) ................................. 154

elegans (Torpacarus) ...................................... 41

elegans (Totobates) ..................................... 196

elegans (Totobates) ..................................... 196

elegans (Trimalaconothrus) ..........................62

elegantis [D. (Epidamaeus)] …......................... 79

elegantissima (Nanhermannia) .....................6 68

elegantissima (Oribatula) ............................. 187

elegantissima (Suctobelbella) ........................ 136

elegantula [S. (Flagrosuctobelba)] .......... 11, 138

elegantula [S. (Flagrosuctobelba)] ........ 11, 138

elegantula [S. (Flagrosuctobelba)] ......... 11, 138

elegantula [S. (Flagrosuctobelba)] ........ 11, 138

elegantula (Galumna) ................................. 215

elegantula (Lanceoppia) ................................ 110

elegantula (Mesotritia) ...................................... 43

elegantula (Nanhermannia) .............................. 68

elegantula (Nanhermannia) ...........................68

elegantulus (Austrodamaeus) ......................... 76

elegantulus (Scheloribates) .......................... 199

elegantulus (Trhypochthonius) $s p$. inq. .......... 60

elevatus (Gibbicepheus) .............................. 152

elevatus (Sadocepheus) ….............................. 84

elimata (Galumna) ......................................... 215

elimata (Galumna) ....................................... 215

elimata (Galumna) ....................................... 215

elimata (Galumna) ..................................... 215

elimata (Galumna) …................................. 215

elimata (Galumna) .................................... 215

elisabethae (Dolicheremaeus) …................. 142

elisabethae (Parabelbella) ................................ 81

elisabethae (Sellnickochthonius) ................... 32

elisae (Ethiovertex) .................................... 160

elisae (Subbelba) .............................................. 82 


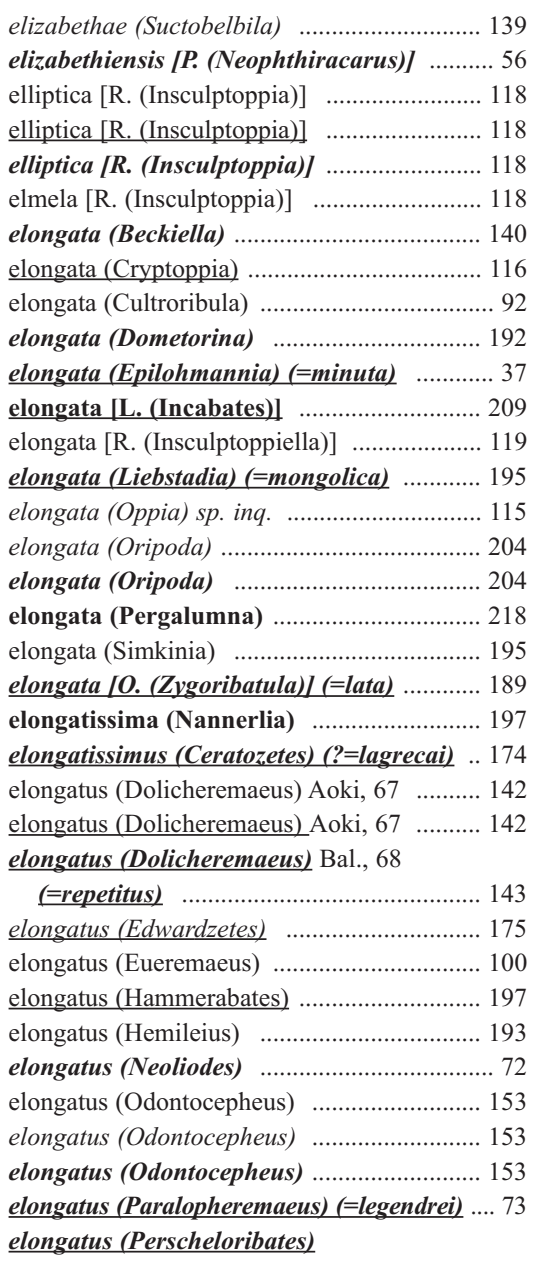

(?=monodactylus) 198

elongatus (Pilobates) (?=carpetanus) .......... 211

elongatus (Proteremaeus) ............................... 108

elongatus [C. (Protoripoda)] sp. inq. ............ 203

elongatus (Scheloribates) ............................. 199

elongatus (Thamnacarus) ................................. 41

elongatus (Thamnacarus) ............................. 41

elsi (Scheloribates) ....................................... 199

elsosneadensis (Sellnickochthonius) ............... 32

elsosneadensis (Sellnickochthonius) …........... 32

elsosneadensis (Sellnickochthonius) ............. 32

elsosneadensis (Sellnickochthonius) ............ 32

elsosneadensis (Williamszetes) ..................... 170

emarginata (Pergalumna) ............................. 218

emarginata (Pergalumna) ............................ 218

emarginatus (Scapheremaeus) ….............. 158

embeyisztini (Licneremaeus) ........................ 160

embrionalis (Lohmannia) ........................... 39

emeiensis [A. (Hexatocepheus)] .................. 146

emeiensis (Liacarus) .......................................... 95

emeryi (Microzetorchestes) ............................ 91

emeryi (Microzetorchestes) ............................ 91

emmae (Phyllozetes) …................................... 33

emmae (Phyllozetes) ........................................... 33

emmae (Phyllozetes) ……………………...... 33

emmae (Phyllozetes) ……….......................... 33 emmae (Phyllozetes) ..................................... 33

encantadae (Heptacarus) ............................. 38

endroedyi [P. (Archiphthiracarus)] ............ 55

endroedyi (Limnozetella) ........................... 165

endroedyi (Subphauloppia) ....................... 191

endroedyi [O. (Zygoribatula)] .................... 188

endroedyyoungai (Maculobates) …............ 196

endroedyyoungai (Notophthiracarus) ........51

endroedyyoungai (Suctobelbila) ................ 139

engelbrechti (Demisalto) ........................... 184

engelbrechti (Eupelops) ............................... 163

engelbrechti (Galumna) Mah., 1997 .......... 215

engelbrechti (Galumna) J. y P. B., 2002

(=discoidalis) ........................................... 215

engelbrechti (Lamellobates) ...................... 166

engelbrechti [C. (Magoebazetes)] .................8 88

engelbrechti (Microlamellarea) ................... 160

engelbrechti [H. (Phyllhermannia)] .............69 69

engelmanni (Multioppia) ............................. 117

enghoffi (Arcoppia) arcualis ........................ 120

enigma (Pheroliodes) ..................................... 74

enigmatica [H. (Kakophthiracarus)] ................ 49

ennarpi (Lepidacarus) ..................................... 38

enodis (Ceratozetes) …............................. 174

enoplura (Jacotella) ........................................ 76

enoplura (Jacotella)

ensifer (Austrocarabodes) ............................. 148

ensifer [M. (Cristonothrus)] ….................... 61

ensifer (Exoribatula) ................................. 193

ensifer (Gibbicepheus) sp. inq. .................... 152

ensifer (Liochthonius) (=furcillatus) ............ 30

ensifer (Phylacozetes) .................................... 90

ensifer (Protoribotritia) ....................................... 45

ensifera (Eremella) ..................................... 106

ensifera (Hoplophorella) ................................ 48

eoeryi (Punctoribates) ............................. 183

ephippiata (Murcia) sp. inq. ........................ 177

ephippiger (Eremaeozetes) ............................. 165

ephippiger (Eremaeozetes) …..................... 165

ephippigera (Hoplophorella) ......................... 48

epiphytos (Zachvatkinibates) ........................ 184

eques (Neoliodes) $s p$. inq. ............................... 72

equestris (Zetorchestes) ................................. 91

equisetosus [P. (Neophthiracarus)] ................ 56

erabuensis (Trichotocepheus) ........................ 147

erabuensis (Trichotocepheus) ....................... 147

erbanensis (Xenillus) …................................ 98

erectus (Austrocarabodes) ........................... 148

erectus (Carabodes) ................................... 149

erectus (Idiozetes) ........................................ 165

erectus (Idiozetes) .........n..... 165

erectus (Neoribates) ..................................... 212

eremaeozetoides (Pasocepheus) ...... 153

eremitus (Exochocepheus) ............................. 161

eremuloides (Berndamerus) ........................... 101

ericius [M. (Parametabelba)] ........................... 81

erinaceus (Eupelops) .................................. 163

erinaceus [N. (Calyptophthiracarus)]

(=olivaceus)

erosus (Sellnickochthonius)

(=immaculatus)

escobari (Hydrozetes) escotata (Moritzoppia) ….............................. 126

espatulatus (Odontocepheus) ........................ 153

espeletiae [P. (Neophthiracarus)] .................... 56

espeletiae [L. (Procorynetes)] ......................... 97

espeletiae [S. (Reductoppia)] ........................ 121

espeletiarum [N. (Amerigloboppia)] ............. 114

espinarensis (Nothrus) .................................. 63

esposi (Eremobelba) .................................... 103

espozi (Oxyoppia) (=spinosa) ………........... 129

esulcatus (Epilohmannoides) .......................... 37

esulcatus (Epilohmannoides) …...................... 37

ethiopica (Bursoplophora) .............................. 35

ethiopica (Microtritia) (=tropica) .................. 47

ethiopicus (Perscheloribates) ....................... 198

etruscus (Oribellopsis) .................................. 107

euaensis (Cryptoribatula) .......................... 205

euaensis (Dampfiella) ................................ 141

euaensis (Dolicheremaeus) ........................... 142

euaensis (Galumna) ….............................. 215

eucalla [O. (Zygoribatula)] ............................. 188

eugenius (Scapheremaeus) .......................... 158

eupalineus [P. (Archiphthiracarus)] ................ 55

eupectinata (Ctenoppia) ............................ 122

euramosa () ................................................... 123

eurhostus (Opsioristes) ................................. 97

europaea (Allosuctobelba) grandis ................ 134

europaea (Galumna) .................................... 215

europaea [S. (Lalmoppia)] ............................ 130

europaea (Suctobelbila) dentata .................... 139

europaeus (Hypochthonius) rufulus ................ 29

europaeus (Peloribates) ................................ 210

europaeus (Peloribates) ................................ 210

eusetosa [H. (Phyllhermannia)] .....................69

eusetosus (Scheloribates) …......................... 199

eutricha (Oribatella) .................................... 171

evanescens (Perscheloribates) .................... 198

evansi (Africoribates) ................................. 180

evansi (Belloppia) ........................................ 125

evansi (Liochthonius) .............................. 11, 30

evansi (Liochthonius) ............................. 11, 30

evansi (Scutovertex) ...................................... 161

eveana (Mongaillardia) ................................ 101

evergladensis (Hoplophorella) ....................... 49

evexus (Notophthiracarus) .............................. 51

evidens [H. (Heterohermannia)] ....................... 69

ewingi (Allodamaeus) ..................................... 72

ewingi (Allodamaeus) ....................................... 72

ewingi (Campachipteria) .............................. 168

ewingi (Campachipteria) ............................. 168

ewingi [L. (Convergoppia)] ....................... 111

ewingi (Oppia) ................................................ 115

exastus (Ophidiotrichus) ............................. 170

exaggeratus (Heminothrus) ………………...... 65

exarata [O. (Zygoribatula)] .......................... 189

excavata (Oripoda) ........................................ 204

excavata (Suctobelbila) ................................ 139

excavata [O. (Zygoribatula)] (=connexa) .... 188

excavatus (Benoibates) ............................... 203

excavatus (Cavernozetes) .............................. 88

excavatus (Machadocepheus) .................... 152

excavatus (Plateremaeus) ………………….... 73

excavatus [S. (Tropacarus)] ............................ 59 
excavatus (Trhypochthoniellus)

(=longisetus) ............................................. 60 excellens [N. (Calyptophthiracarus)] ............. 52 excellens [C. (Klapperiches)] ...................... 151 excellens (Scriptoripoda) ……...................... 205

excellens (Setogalumna) ............................. 221

excelsior (Rhynchoribates) …..................... 140

excelsus (Acrotocepheus) ............................. 146

excisa (Camisia) (=segnis) .............................. 65

excisa (Multioppia) (=neglecta) .................. 117

excrescens (Brachioppia) ............................ 122

excultus (Euphthiracarus) .................................. 46

exempta (Hypogeoppia) ................................ 126

exigua (Allogalumna) ..................................... 213

exigua (Galumna) ………………................... 215

exigua (Graptoppia) ....................................... 116

exigua (Subiasella) ………......................... 130

exigua (Subiasella) ..................................... 130

exigualis (Mycobates) ................................. 182

exigualis (Mycobates) .................................. 182

exiguus (Scheloribates) ……...................... 199

exilicornis (Oribatella) ................................... 171

exilior (Nothrus) silvestris ............................. 64

exilior (Parabelbella) ..................................... 81

exilior (Scheloribates) (?=minifimbriatus) .. 200

exilis (Hermannia) $s p$. inq. ............................ 69

exilis (Mixacarus) ............................................... 39

exilis (Mixacarus) ….......................................... 39

exilis (Oribotritia) ............................................... 44

exilis [O. (Zygoribatula)] ................................ 189

exilis [O. (Zygoribatula)] ............................ 189

exilis [O. (Zygoribatula)] ……..................... 189

eximius (Eupelops) ...................................... 163

eximius (Rhacaplacarus) ….......................... 57

exobothridialis (Banksinoma) ….................. 109

exobothridialis (Melanozetes) ...................... 176

exobothridialis (Mesotritia) ............................... 43

exobothridialis [H. (Phyllhermannia)] ......... 69

exornata [H. (Phyllhermannia)] ................... 69

exornatus (Licneremaeus) ......................... 160

exornatus (Patagonozetes) .......................... 167

expansa [Q. (Coronoquadroppia)] ................ 134

expansum (Joboppia) ..................................... 109

expansus (Megeremaeus) .................................. 99

expansus [H. (Platynothrus)] skoettsbergi ... 66

expansus (Tectocepheus) velatus ................. 155

expansus (Tectocepheus) velatus ................ 155

expolitus (Liochthonius) (?=sellnicki) ......... 31

exserta [P. (Tectodamaeus)] .............................. 81

exspinosa [P. (Tectodamaeus)] ........................... 81

exsudans (Oribatula) ..................................... 187

extensa (Oribatella) ...................................... 171

extensa (Oribatella) ........................................ 171

externus (Liacarus) ........................................... 96

extraneus (Adoristes) (=ovatus) ………........ 95

extraordinarius (Phthiracarus) ......................... 53

extrema [N. (Amerigloboppia)] ..................... 114

extrema (Congoppia) (=deboissezoni) ........ 123

extrema (Granuloppia) .............................. 132

extrema (Masthermannia) ............................. 68

extremodentata [O. (Zygoribatula)]

(=exilis) extrudens (Berniniella)

exuvialis (Camisia) (=biurus) ...........................6 65

ezoensis (Mycobates) ..................................... 183

ezoensis (Nothrus) .......................................... 63

ezzati (Cyrthermannia) ........................................ 67

fabulosus (Rhynchoribates) ....................... 140

facetus [P. (Neophthiracarus)] …............... 56

facula (Chamobates) (?=subglobulus) ….... 180

faeroensis (Mesotritia) ....................................... 43

fageti (Spatiodamaeus) ……………………..... 82

falcata (Cultroribula) (=bicultrata) .............. 92

falcata (Lauroppia) ...................................... 126

falcata (Pachygena) …................................ 197

falcata (Suctobelbella) (=longicuspis) ........ 137

falcatus (Austrocarabodes) ........................ 148

falcatus (Carabodes) .................................... 149

falcatus [P. (Metaphthiracarus)] (=setosus) .... 56

falciformis [P. (Archiphthiracarus)] ................ 55

falklandica [H. (Phyllhermannia)] ................. 69

fallax (Lauroppia) ........................................... 126

fallax (Lauroppia) ………………………... 126

fallax (Lauroppia) ….................................... 126

fallax (Lauroppia) ....................................... 126

fallax (Lauroppia) ..................................... 126

fallax (Ramsayellus) .................................... 182

fallax [P. (Triaungius)] ................................... 206

falxa (Drepanoppia) ……............................ 110

falzonii (Zetorchestes) (=micronychus) ........ 91

fanzagoi (Campachipteria) ............................. 168

farinosus [D. (Epidamaeus)] …...................... 79

farinosus (Eupelops) ..................................... 163

farinosus (Poroliodes) ………....................... 72

fasciata [R. (Rectoppia)] ............................ 119

fasciata [R. (Rectoppia)] ............................. 119

fasciata [R. (Rectoppia)] ........................... 119

fasciata [R. (Rectoppia)] ........................... 119

fasciata [R. (Rectoppia)] ............................. 119

fasciatus (Gephyrazetes) ….......................... 185

fastigata (Pergalumna) .............................. 218

fatidicus (Notophthiracarus) ........................ 51

faviger (Micreremus) ….............................. 160

fayoumensis (Pilogalumna) ornatula ........... 220

fazendae (Xenillus) ....................................... 98

fecundus (Notophthiracarus)

fecundus (Xenillus) ...................................... 98

feideri (Acaroceras) ....................................... 87

feideri [L. (Bicristoppia)] .............................. 111

feideri (Cepheus) ................................................ 82

feideri (Lauroppia) ....................................... 126

feideri (Notophthiracarus) ............................ 5

feideri (Phthiracarus) (=zicmani) ................. 54

feideri (Rhacaplacarus) ................................. 57

feideri (Scheloribates) .................................. 199

femoralis (Carabodes)

femoralis (Neoliodes) (=striatus) …............... 72

femoralis (Scheloribates) sp. inq. .................. 199

femoratus (Adrodamaeus) .............................. 75

femoratus (Scheloribates) …........................... 199

femoroserratus (Scheloribates) .................... 199

fenestralis (Arcoppia) .................................. 120

189 fenestralis (Gibbicepheus) …........................ 152 enestrata (Ctenobelba) (=pectinigera) ........ 101

fenestrata (Magyaria) ................................. 209

fenestratus (Austrocarabodes) …............... 148

fenicheli [E. (Nanobates)] ........................ 197

fenneri (Nanhermannia) ….............................. 68

fenneri (Nanhermannia) ............................ 68

ennica (Oribotritia) ……………….................... 44

fernandoi (Xenillus) ...................................... 98

ferox (Maorizetes) ....................................... 93

ferrugineus (Phthiracarus) .............................. 53

ferrumequina [Q. (Coronoquadroppia)]

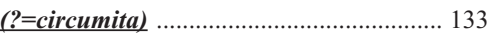

festoneata (Hypogeoppia) (=terricola) ........ 126

feuerborni (Limnozetes) ................................ 166

figuratus (Ceratozetes) ................................. 174

figuratus (Eueremaeus) (=oblongus) .......... 100

fijiensis (Dolicheremaeus) …........................ 142

fijiensis (Galumna) ...................................... 215

fijiensis [F. (Parasuctobelba)] ................... 134

fijiensis (Scheloribates) ……........................ 199

fijiensis (Scheloribates) …........................ 199

fijiensis [T. (Tyrphonothrus)] crassisetosus .. 63

filamentosa [R. (Insculptoppiella)] ........... 119

filata (Acrogalumna) (=longipluma) .......... 213

filifera (Pergalumna) ................................ 218

filifera (Pergalumna) .................................. 218

filiferus (Machadocepheus) ....................... 152

filiferus [P. (Metaphthiracarus)] (=setosus) .... 56

filiformis (Parapyroppia) ............................. 94

filiger (Allogalumna) ..................................... 213

filigera (Ramusella) …............................ 118

filipina (Magyaria) ......................................... 209

filipinus (Scheloribates) ............................... 199

filippovae (Metabelba) ……............................ 80

filosus (Sacculozetes) ................................... 167

fimbriata [T. (Teratoppiella)] …................ 132

fimbriatissimus (Liochthonius) .................... 30

fimbriatissimus (Liochthonius) ........................ 30

fimbriatissimus (Liochthonius) ...................... 30

fimbriatoides (Scheloribates) ........................ 199

fimbriatoides (Scheloribates) …................ 199

fimbriatus (Caucasiozetes) .............................. 88

fimbriatus (Liochthonius) Jac., 36 .................. 30

fimbriatus (Liochthonius) Ham., 58

(=fimbriatissimus) ..................................... 30

fimbriatus (Scapheremaeus) .......................... 158

fimbriatus (Scheloribates) ............................ 199

fimbriatus (Scheloribates) ........................... 199

fimbriatus (Scheloribates) ........................... 199

fimbriatus (Scheloribates) …....................... 199

fimbriatus (Scheloribates) ……................... 199

fimbriatus (Scheloribates) ........................... 199

finitima (Hoplophorella) .................................. 49

finlayi (Suctobelbella) .................................. 136

fiorii (Joelia) .................................................. 170

firmus [D. (Adamaeus)] ................................... 79

firthensis (Sphaerozetes) .............................. 178

fischeri (Camisia) (=biverrucata) ...................65

fisheri (Scapheremaeus) ............................... 158

fisheri (Scapheremaeus) ............................ 158

fissurata (Banksinoma) spinifera ............... 109

fissurata (Berniniella) .................................... 125 
fissurata (Lauroppia) (=maritima) .............. 126 fissurata (Microtritia) ........................................ 47 fissurata (Suctobelbila) ……………………... 139 fissuratus [L. (Dorycranosus)] (=nitidus) $\quad . . .97$ fissuratus (Licnodamaeus) ………………….... 74 fissuratus (Mucrobates) ................................ 194 fissuratus (Perscheloribates) ........................ 198 fissuratus [N. (Protokalumma)] (=pterotus) .. 212 fissuratus [H. (Tuberemaeus)] ....................... 193 fissuratus [H. (Tuberemaeus)] ..................... 193 fixa (Moritzoppia) (=unicarinata) .............. 127 fjellbergi (Ceratozetella) ................................... 173 fjellbergi (Ceratozetella) …………………... 173 flabella [S. (Flagrosuctobelba)] ……………... 138 flabellatus (Hardybodes) ............................... 152 flabellatus (Licnozetes) ................................... 89 flabellifer (Austrocarabodes) ....................... 148 flabellifera (Galumna) ............................. 12, 215

flabellifera (Galumna) ............................ 12, 215

flabellifera (Galumna) .......................... 12, 215

flabellifera (Galumna) …....................... 12, 215

flabellifera (Galumna) ......................... 12, 215

flabellifera (Galumna) ........................ 12, 215

flabellifera (Suctobelbella) $s p$. inq. ............ 136

flabelliger (Mycobates) ................................. 183

flabrarius (Zetorchestes) .................................... 91

flagellaris (Eremobelba) (=gracilior) ........... 103

flagellaris [R. (Insculptoppiella)] ................... 119

flagellata [M. (Akrodamaeus)] ……............... 81

flagellata (Arcoppia) ……………………...... 120

flagellata (Belba) …………………………...... 77

flagellata (Camisia) ………………………...... 65

flagellata (Crotonia) ......................................... 67

flagellata (Galumna) ……………………….... 215

flagellata (Gittella) ……………………….... 122

flagellata (Hauseroplophora) ......................... 35

flagellata (Mesotritia) (=maerkeli) ................... 43

flagellata (Neoamerioppia) ............................. 114

flagellata (Paroppia) ……………………...... 115

flagellata [A. (Rhaphigneta)] ......................... 108

flagellata (Suctobelbella) ............................. 136

flagellatissimus (Steganacarus) …………….... 58

flagellatus [P. (Archiphthiracarus)] ……..... 55

flagellatus (Brazilozetes) ................................ 88

flagellatus (Ctenamerus) …………………..... 105

flagellatus (Damaeus) ……………………….... 78

flagellatus [P. (Neophthiracarus)]

(Eshiptoni)

flagellatus (Novonothrus)

flagellatus [C. (Protoripoda)]

flagellatus (Pseudotocepheus)

flagellems (Scheloribates

flagellatus (Solenozetes) …....................... 7

flagellatus [H. (Urubambates)] …………….. 194

flagelliapex (Neoamerioppia) ………........... 114

flagellifer (Benoibates) ................................... 203

flagellifer (Carabodes) (?=quadrangulus) .. 149

flagellifer (Caucasiozetes) …………................ 88

flagellifer (Damaeus) ……………………….... 78

flagellifer (Epilohmannia) ……………........... 36

flagellifer (Eremulus) …………………......... 102

flagellifer (Eremulus) flagellifer (Eremulus)

flagellifer (Eremulus)

flagellifer (Leptotocepheus)

flagellifer [L. (Magnobates)

flagellifer [L. (Magnobates)]

flagellifer [M. (Megazetes)

flagellifer (Mikizetes)

flagellifer (Solenozetes)

flagellifera (Oppia)

flagellifera (Pergalumna) $(=$ cribriger)

flagelliformis (Mesotritia) ............................... 43

flagelliformis (Mesotritia) ................................ 43

flagelliformis (Trimalaconothrus) ................... 62

flagelliseta (Hermannobates) .......................... 71

flagelliseta (Metabelba) .................................. 80

flagelloides (Austrotritia) lebronneci ........... 42

flagelloides [D. (Epidamaeus)] ....................... 79

flagellum (Nothrus) …….................................. 63

flagellum [N. (Protokalumma)] ................... 212

flagrus (Notophthiracarus) ............................. 51

flamiferus (Scapheremaeus) ......................... 158

flammeisetosa (Belba) ……………………..... 77

flammeus (Liacarus) …………………………. 96

flammula (Oribatella) $(?=q u a d r i c o r n u t a)$

flatus (Mochlozetes) ……………………... 185

flavida [M. (Parplophora)] ............................... 42

flavipes (Banksinoma) (?=lanceolata) ........ 109

flavus (Euphthiracarus) .................................. 46

flavus (Eupterotegaeus) ..................................... 83

flavus [T. (Rostrozetes)] (=ovulum) …….... 211

flechtmanni (Brasiloppia) ............................. 132

flechtmanni (Humerobates) ........................ 181

flexiloquus (Notophthiracarus) ..................... 51

flexipilus [P. (Archiphthiracarus)] .................. 55

flexisetosa (Hymenobelba) ............................. 105

flexisetosus (Phthiracarus) (=longulus) ........ 54

flexispinosus [D. (Epidamaeus)] ……………... 79

flexuosa (Epilohmannia) ………………….... 36

flexuosa (Eremobelba) .................................... 103

flexus [D. (Epidamaeus)] ................................. 79

floccosus [D. (Epidamaeus)] .......................... 79

floralis (Pseudoamerioppia) ........................... 118

florens [O. (Aokiella)] …............................... 153

florens (Cyrthermannia) ................................... 67

florens (Micreremus) ................................... 160

florens [T. (Rostrozetes)] ………………….... 21

florens (Siculobata) ....................................... 194

floresiana (Parachipteria) ............................... 169

floresianus (Melanozetes) azoricus ............... 176

florida (Magyaria) ……………………….... 209

floridae (Fuscozetes) ..................................... 175

floridae (Galumna) ..................................... 215

floridae (Hoplophorella) (=hamata) ............... 49

floridana (Aeroppia) ........................................ 112

floridana (Aeroppia) .................................... 112

floridana (Mochloribatula) ............................ 185

floridana [O. Zygoribatula)] ........................ 189

floridanus [N. (Parakalumma)] robustus .... 212

floridensis [L. (Dorycranosus)] ...................... 97

floridensis (Neoliodes) .................................... 72

floridensis (Peloribates) ............................ 210

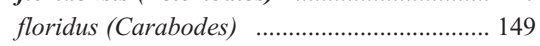

floridus (Carabodes) ................................... 149

floridus (Diplobodes) …………………….... 152

floridus [D. (Epidamaeus)] ............................ 79

floridus [D. (Epidamaeus)] ………………..... 79

flumengalei (Autogneta) ................................ 108

fluviatilis [H. (Capillonothrus)] ……………..... 66

foliaceisetus (Austrocarabodes) ..................... 148

foliata (Basilobelba) ………………………..... 104

foliata (Bicyrthermannia) ................................ 67

foliata (Corynoppia) ....................................... 130

foliata (Ctenobelba) ………………………..... 101

foliata (Dampfiella) ........................................ 141

foliata (Eremobelba) ...................................... 103

foliata (Machadobelba) …………………….... 107

foliata (Oribatella) ………............................. 171

foliata [H. (Phyllhermannia)] ....................... 69

foliatifer (Sellnickochthonius) …………….... 32

foliatoides (Corynoppia) ............................... 130

foliatus (Carabodes) (=chirstlus)

foliatus (Cosmochthonius) ................................ 33

foliatus [M. (Cristonothrus) ……………...... 61

foliatus (Cymbaeremaeus) ............................ 158

foliatus (Haplacarus) ……………………… 38

foliatus (Haplacarus) ………………………...... 38

foliatus (Holonothrus) ………………………... 67

foliatus (Javacarus) ………………………….... 38

foliatus (Javacarus) ……………………….... 38

foliatus (Koreoribates) .................................. 165

foliatus (Paulianacarus) Mond. y Chakr., 82 .. 40

foliatus (Paulianacarus) Sark. y Sub., 84

(=sarbias) ……………………………….... 40

foliatus (Sellnickochthonius) ...…………..... 32

foliatus (Suctoribates) …………………….... 140

foliatus (Torpacarus) ...................................... 41

foliifer [M. (Phyllolohmannia)] ....................... 40

foliisetosus (Ctenacarus) ………………….... 28

foliosa (Striatoppia) ...................................... 131

foliosa [S. (Ussuribata)] ................................. 138

folium [D. (Epidamaeus)] …………………….. 79

fonseciai (Hoplophorella) ............................... 49

fonticula (Suctobelbila) .................................. 139

foraminiferus (Neoribates) .......................... 212

forceps (Lamellarea) ................................... 160

forceps (Schalleria) ........................................ 90

forceps (Xenillus) _........................................ 98

fordi (Galumna) …………………………........ 215

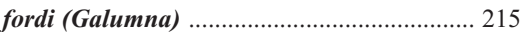

forficula (Paenoppia) ..................................... 95

formica (Damaeus) sp. inq. ........................... 78

formicaria (Pergalumna) ................................. 218

formicaria (Pergalumna) ............................... 218

formosa (Berniniella) ..................................... 125

formosa (Moritzoppia) (=unicarinata) ......... 127

formosa (Murcia) ........................................... 177

formosana (Cyrthermannia) .............................. 67

formosana (Heterobelba) stellifera ................. 104

formosana (Multioppia) …………………….... 117

formosana (Quinquoppia) …………………..... 115

formosanus [H. (Tuberemaeus)] …………... 193

formosus (Peloribates) ................................... 210

formosus (Sellnickochthonius) ......................... 32

fornerisae (Ceratobates) ................................. 166 
fornicarius (Notophthiracarus) fornicatus (Megalotocepheus) foroliviensis (Damaeus) sp. inq. .................... 78 forsslundi (Carabodes) (=ornatus) .............. 150 forsslundi (Eupelops) ................................. 163 forsslundi (Eupelops) ................................... 163 forsslundi [S. (Flagrosuctobelba)] ................. 138 forsslundi (Liochthonius) Ham., 52 ................ 30 forsslundi (Liochthonius) Mah., 69 (=asotthalomensis) forsslundi (Nanhermannia) forsslundi (Oribellopsis) .................................. 107 forsslundi (Provertex) ...................................... 161 forsslundi (Tripiloppia) ............................. 128

forsslundi (Rhacaplacarus) …....................... 57

forsteri (Luxtoneremaeus) .......................... 106

forsteri [H. (Phyllhermannia)] ................... 69

fortis (Setoppia) ............................................ 112

fortisensillus [D. (Epidamaeus)] ……............ 79

fortispinosus [D. (Epidamaeus)] …………..... 79

fortispinosus [D. (Epidamaeus)] .................... 79

fortispinosus [V. (Laminizetes)] .................... 179

fossatus [H. (Platynothrus)] sp. inq. ............. 66

fossatus (Scutovertex)

fossulatus (Eueremaeus)

(=quadrilamellatus)

fournieri (Bornemiszaella)

foveiventris (Selenoribates)

foveolata [G. (Apograptoppia)] .............. 11, 116

foveolata [G. (Apograptoppia)] .............. 11, 116

foveolata (Beckiella) ................................... 140

foveolata (Brachyoripoda) ……..................... 203

foveolata (Camisia) ........................................ 65

foveolata (Camisia) ......................................... 65

foveolata (Foveolatoppia) ........................... 128

foveolata (Kunstella) .................................... 170

foveolata (Neoamerioppia) ......................... 114

foveolata (Perezinigokalumma) .................. 213

foveolata (Pergalumna) .............................. 218

foveolata [H. (Phyllhermannia)] .................... 69

foveolata (Sabahtritia) .................................... 47

foveolata [K. (Stakarenoppia)] .................. 130

foveolatus (Allonothrus) ................................ 59

foveolatus [P. (Archiphthiracarus)]

\section{(=pygmaeus)}

foveolatus (Cosmochthonius) lanatus

foveolatus (Cosmochthonius) lanatus .......... 33

foveolatus (Eremulus) .................................. 102

foveolatus (Eueremaeus) ................................ 100

foveolatus (Eueremaeus) ............................... 100

foveolatus (Eupelops) ................................... 163

foveolatus (Euphthiracarus) ............................ 46

foveolatus (Hoplophthiracarus) ……………... 50

foveolatus [D. (Kalloia)] ............................... 152

foveolatus (Limnozetes) ciliatus …............... 166

foveolatus (Microtegeus) …............................. 86

foveolatus (Microtegeus) ……....................... 86

foveolatus (Mixacarus) exilis ........................... 39

foveolatus (Peloptulus) ................................. 164

foveolatus (Podoribates) .............................. 186

foveolatus (Pseudotocepheus) ………......... 145

foveolatus [T. (Rostrozetes)] (=ovulum) ...... 211 foveolatus (Sadocepheus)

oveolatus (Scapheremaeus) .......................... 158

foveolatus (Setoxylobates) …......................... 207

foveolatus (Spinotocepheus)

foveolatus (Torpacarus)

foveolatus (Trimalaconothrus)

oveolatus (Trimalaconothrus)

foveolatus [H. (Tuberemaeus)] …................ 193

foveoreticulatus [P. (Neophthiracarus)] ........ 56

fraenzlei (Oribatula) .................................. 187

fragilis [H. (Cordylobates)] …..................... 182

fragilis [D. (Epidamaeus)] ................................ 79

fragilis [F. (Parasuctobelba)] ..................... 134

fragilis (Peloribates) ..................................... 210

francisi (Zetomimus) .................................... 179

franconia (Eupelops) major ......................... 163

franzi (Cerachipteria) ...................................... 168

franzi (Fernandocepheus) ........................... 144

franzi (Nooliodes) ............................................ 73

franzi (Sadocepheus) ....................................... 84

frater (Hoplophthiracarus) ........................... 50

frater (Pergalumna) .................................. 218

fratercula (Beckiella) ................................ 140

fraterculus (Gymnobodes) .......................... 152

fraternalis (Gredosella) ................................ 133

fraternus [P. (Archiphthiracarus)] ...............55

frigidus (Scheloribates) (=pallidulus) .......... 201

frisiae [O. (Zygoribatula)] ............................ 189

frisiae [O. (Zygoribatula)] ........................... 189

frondeus (Jacotella) ……................................ 76

frondifer (Gehypochthonius) .......................... 28

frondosa (Cosmohermannia) ........................... 67

frondosa (Suctobelbella) .............................. 136

frondosa (Suctobelbella) ……...................... 136

frondosus (Gibbicepheus) ........................... 152

frondosus (Gibbicepheus) ……..................... 152

frondosus (Gibbicepheus) …..................... 152

frontatus (Epieremulus) ............................... 106

frontinalis [L. (Dorycranosus)] ...................... 97

frothinghami (Suctobelbella) ....................... 136

frouini (Lineoppia) ..................................... 129

fucicolum (Ameronothrus) (=marinus) ..... 156

fueginus (Eutegaeus) ................................... 85

fuentesi (Sellnickochthonius) ............................ 32

fuerteventurae (Eupelops) claviger ............. 163

fuerteventurae (Subbelba) elisae ...................... 82

fujianensis (Taiwanoppia) ............................ 115

fujikawae (Dolicheremaeus) ....................... 142

fujikawae (Oribella)

fujikawae (Truncopes) ................................... 205

fujinitaensis (Trhypochthonius) ...................... 60

fukushiraensis (Hypochthoniella) .................. 29

fuligineus (Eupelops) (=plicatus) …............. 163

fulvus (Euphthiracarus) ................................. 46

fulvus (Halozetes) …………………………...... 157

fulvus (Neoribates) .................................. 212

fulvus (Notophthiracarus) …........................ 5

fumigata (Murcia) sp. inq. ........................... 177

funafutiensis (Neoliodes) ……....................... 72

fungifer (Hermannia) ...................................... 69

fungifer (Sphaerochthonius) ........................ 34

fungisetosus (Scapheremaeus) ..................... 158 fungorum (Humerobates) sp. inq. ................ 181

furcata (Acrotritia) …................................... 45

furcata (Austrachipteria) ............................. 166

furcata (Camisia) (=biurus) .......................... 65

furcata [M. (Furculoppia)] ............................ 117

furcata (Heterobelba) ................................ 104

furcata [R. (Insculptoppia)] ......................... 118

furcata (Murcia) …...................................... 177

furcata (Sabahtritia) ……................................ 47

furcata [P. (Tectodamaeus)] .............................. 81

furcatus (Acaroceras) .................................. 87

furcatus (Cavernocepheus) ........................... 141

furcatus (Dolicheremaeus) ........................... 142

furcatus (Notophthiracarus) ............................ 51

furcatus (Phthiracarus) sp. inq. ..................... 53

furcatus [H. (Platynothrus)] (=banksi) ....... 66

furcatus (Sellnickochthonius) ........................... 32

furcatus (Zetomimus) ..................................... 179

furcatus (Zetomimus) ................................ 179

furcillata (Furcoribula) .................................... 92

furcillata (Furcoribula) ................................. 92

furcillatus (Dolicheremaeus)

furcillatus (Liochthonius) .................................. 30

furcula (Dolicheremaeus) .............................. 142

furtadoi (Lauritzenia) .................................... 208

furugelma (Multioppia) ............................... 117

furvus [P. (Archiphthiracarus)] ……................. 55

fusa (Microtritia) ……................................ 47

fusca (Pedrocortesella) .................................. 75

fusca (Pergalumna) Berl., 16 ...................... 219

fusca (Pergalumna) Mih., 57

(=myrmophila) ……................................. 219

fusca [O. (Zygoribatula)] .............................. 189

fuscipes (Fuscozetes) .................................. 175

fuscipes (Fuscozetes) ................................. 175

fuscipes (Fuscozetes) …………………....... 175

fuscomaculatus (Scheloribates)

(=pallidulus) …........................................ 201

fuscosensillus (Scheloribates) …................... 200

fuscosetosus (Peloribates) ........................... 210

fuscus (Defectamerus) ................................... 105

fuscus (Liacarus) (=xylariae) ........................ 96

fuscus (Umbellozetes) .................................. 170

fusifer (Cavernocepheus) ............................. 141

fusifer (Gustavia) .......................................... 95

fusifer [I. (Triungulozetes)] .......................... 208

fusifer [I. (Triungulozetes)] …..................... 208

fusifer (Xenillus) ........................................... 98

fusiformis (Anellozetes) ................................ 181

fusiformis [L. (Dorycranosus)] ..................... 97

fusiformis [R. (Insculptoppia)] .................. 119

fusiformis (Tenuialoides) ................................ 99

fusiger (Brazilozetes) ...................................... 88

fusiger (Cerachipteria) (?=pyrenaica) ........ 168

fusiger (Ceratozetes) (=gracilis) .................. 174

fusiger (Eupelops) (=plicatus)

fusiger (Fusozetes) .................................... 88

fusiger (Minunthozetes) (=semirufus) ........ 182

fusigera (Galumna) (=setigera) …............... 216

fusisetosa (Cubaoppia) ................................ 116

fusticulus (Euphthiracarus) ......................... 46

fusticulus (Notophthiracarus) …................... 51 
fusuligera (Fusuloppia) ............................... 113

fusulus (Euphthiracarus) ............................... 46 fusulus (Euphthiracarus) .............................. 46 galaica [Q. (Coronoquadroppia)] .................. 134 galaica [Q. (Coronoquadroppia)] ................ 134 galapagensis (Torpacarus) omittens .............. 41 galapagoensis (Acaroceras) ......................... 87 galba (Liochthonius) ..................................... 30 galeata (Hoplophorella) (=subsellata) ......... 49 galeodula (Beklemishevia) ............................ 27 galeodula (Beklemishevia) ............................. 27 galerulata (Heterobelba) ................................ 104 gallardoi (Liebstadia) ..................................... 195 gallonae (Solenozetes) …............................. 71 galula [O. (Zygoribatula)] ........................... 189 gammatus (Liacarus) ..................................... 96 gapsaensis (Multioppia) .............................. 117 garciai (Beckiella) ......................................... 140 gaussi (Ceratozetes) ..................................... 174 gaveae (Mesoplophora) .................................. 42 gaveae (Mesoplophora) .................................. 42 gebennicus (Belorchestes) ............................... 91 gedaii (Allogalumna) …............................. 213 gelasinus (Xenillus) ...................................... 98 geminatus [P. (Constrictocepheus)] ............ 145 geminatus (Papuacepheus) ……................. 147 geminosetosus [T. (Rostrozetes)] ................. 211 geminus (Chamobates) .................................. 179 geminus [M. (Cristonothrus)] …....................... 61 geminus [M. (Cristonothrus)] ….................. 61 geminus (Dolicheremaeus) .......................... 142 geminus (Eupelops) .................................... 163 gemma (Sphaerochthonius) ............................ 34 gemma (Sphaerochthonius) .......................... 34 gemma (Sphaerochthonius) ........................... 34 gemma (Sphaerochthonius) .......................... 34 gemmula (Ceratozetes) ................................. 174 genavensis (Atropacarus) ................................ 48 genavensis (Lamellocepheus) ….................. 86 genavensium [O. (Aciculoppia)] .................. 129 genavensium (Bursoplophora) ....................... 35 genavensium (Carinogalumna) .................. 214 genavensium (Peloribates) ......................... 210 genavensium (Rhynchoribates) ................... 140 geneuxi [T. (Rostrozetes)] (=ovulum) .......... 211 geniculata (Phauloppia) (?=lucorum) ........ 190 geniculosus [D. (Adamaeus)] (=onustus) ...... 79 geographica (Eremobelba) ........................... 103 geographica (Galumnella) ............................ 222 geometrica (Suctobelbella) ............................ 136 geometricus (Epieremulus) ............................ 106 geonjiensis (Protoribates) ............................. 206 georgensis (Rhopalozetes) …......................... 90 georgiae (Antarcticola) ................................. 156 georgiae (Sandenia) ...................................... 213 georgii (Dolicheremaeus) .............................. 142 gephyrus [X. (Stonyxenillus)] …………….... 99 germaineae (Neotrichozetes) spinulosus .... 186 germanicus (Parhypochthonius)

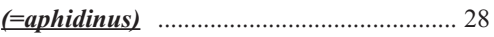
gessneri (Phauloppia) .................................. 190 getica (Lauroppia) ghanensis (Dolicheremaeus) magnus hanensis [A. (Pseudonothrus)] (=hirtus ghanemsis (Teleioliodes) gharbiensis (Galumna)

ghardaqensis (Selenoribates)

ghilarovi (Punctoribates)

ghiljarovi (Multioppia)

gibba (Globoppia)

gibba (Hermannia)

gibba (Hermannia)

gibba (Hermannia)

gibba (Hermannia)

gibba [M. (Parplophora)] (=leviseta) ........... 42

gibber [P. (Archiphthiracarus)] ........................ 55

gibber [N. (Confinoppia)] ............................ 127

gibber (Hermanniella)

gibbera (Maerkelotritia) (=cryptopa) ..............

gibbiceps (Carabodes) ................................. 149

gibbofemoratus [D. (Epidamaeus)] ................ 79

gibbosa (Lasiobelba)

12,215

gibbula (Galumna)

12,215

gibbus (Aleurodamaeus) (=setosus) .............. 77

gibbus (Adrodamaeus) (=femoratus) ........... 75

gibbus (Eupelops) .................................... 163

gibbus (Heterochthonius) ............................... 34

gibbus (Peloptulus) ....................................... 164

gigantea (Allosuctobelba) ............................ 134

gigantea [P. (Apolohmannia)] ....................... 36

gigantea [P. (Apolohmannia)] ........................ 36

gigantea (Austrachipteria) ......................... 166

gigantea (Collohmannia) ................................ 36

gigantea (Epilohmannia) ................................. 36

gigantea (Hermannia) ....................................... 69

gigantea (Murcia) latincisa ........................ 177

gigantea (Oribatella) ................................... 171

giganteus (Humerobates) rostrolamellatus .... 181

giganteus (Scheloribates) ......................... 200

giganticus (Allonothrus) .................................. 59

giganticus (Dolicheremaeus) ...................... 142

gigas (Oribotritia) ................................................. 44

gigas (Oribotritia) .............................................. 44

gildersleeveae (Nortonella) ............................. 76

gilmartinoi (Multioppia) ............................... 117

gilva (Arcoppia) (=tripartita) ....................... 121

gilvipes (Hafenrefferia) ................................. 99

gilvulus (Scheloribates) sp. inq. ................... 200

gilyarovi [D. (Epidamaeus)] ............................ 79

gisini (Liochthonius) ...................................... 30

gitteae (Cryptoribatula) (=euaensis) ............ 205

giustii (Licneremaeus) .................................. 160

glaber (Berlesezetes) ...................................... 87

glaber [L. (Dorycranosus)] (=nitidus) ......... 97

glaber (Eupelops) (?=subexutus) ................ 164

glaber (Gymnobates) .................................... 204

glaber (Heminothrus) .................................. 65

glaber (Nooliodes) ......................................... 73

glaber (Peloribates) ....................................... 210

glaber (Podoribates) ………......................... 186

glaber [T. (Rostrozetes)] ............................... 211 glaber (Scapheremaeus) …......................... 158

glaber (Scutovertex) ................................... 161

glaber [T. (Tyrphonothrus)] .............................. 63

glaber [T. (Tyrphonothrus)] ............................. 63

glaber [H. (Tuberemaeus)] …...................... 193

glabra (Brachioppia) ................................. 122

glabra (Eobrachychthonius)

(=latior) ……............................................ 30

glabra (Galumna) ........................................ 215

glabra (Jacotella) ............................................ 76

glabra (Lauritzenia) ................................... 208

glabra (Mesotritia) .............................................. 43

glabra (Multioppia) ........................................ 117

glabra (Phauloppiella) ................................. 191

glabra [H. (Phyllhermannia)] (=dentata) ...... 69

glabra (Subphauloppia) .............................. 191

glabra [O. (Zygoribatula)] ........................... 189

glabrata (Austrotritia) ....................................... 42

glabrata (Mestotritia) ....................................... 43

glabrata (Mesotritia) ......................................... 43

glabrata (Microtritia) Nied., 93 ................... 47

glabrata (Microtritia) Sta., 93

(=neonominata) …................................. 47

glabratus (Africoribates) …….................... 180

glabriseta (Metabelba) ................................. 80

glabriseta (Oxybrachioppia) …….................. 129

glabrisetus (Meristacarus) heterotrichus ........ 39

glabrisetus (Spatiodamaeus) ............................... 82

glabroclava (Suctobelbella) (=duplex) ........ 136

glabrus (Austrocarabodes) ......................... 148

glabrus (Austroeremulus) .......................... 102

gladiata (Nanhermannia) .............................. 68

gladiata [H. (Phyllhermannia)] ......................... 69

gladiator [M. (Parametabelba)]

(=romandiolae) ……..................................... 81

gladiator (Pseuderemulus) ........................ 102

gladiator (Rugozetes) ...................................... 90

glandulosus (Scutovertex) ............................. 161

glauca (Hoplophorella) (=hamata) ................ 49

globata (Paraphauloppia) .......................... 190

globiceps (Belba) sp. inq. ................................. 77

globifer [P. (Archiphthiracarus)] .................. 55

globifer [D. (Epidamaeus)] ............................ 79

globifer (Microtegeus) ..................................... 86

globifer [L. (Procorynetes)] ............................ 97

globifer [L. (Procorynetes)] ........................... 97

globifera (Globoppia) ................................... 110

globiger [C. (Klapperiches)] ....................... 151

globiger (Malaconothrus) .................................. 60

globigera (Heteroppia) .................................. 113

globipes (Metabelba) (=papillipes) ................ 80

globosa (Dissorhina) ornata ......................... 125

globosa (Dissorhina) ornata ........................ 125

globosa (Medioppia) (=subpectinata) .......... 124

globosa [O. (Zygoribatula)] ......................... 189

globosus [P. (Archiphthiracarus)] .................... 55

globosus [P. (Archiphthiracarus)] .................. 55

globosus [P. (Archiphthiracarus)] …................ 55

globosus [P. (Archiphthiracarus)] ................ 55

globosus (Ceratorchestes) ............................. 93

globosus (Liacarus) (=tremellae) .................. 96

globosus (Longocepheus) ........................... 144 
globosus (Neoliodes)

globularis (Sphaerozetes) sp. inq. .................. 178

globulifera (Globogalumna) .......................... 217

globulifera (Suctobelbila) ……………….... 139

globuliferus (Neoliochthonius) ……………..... 31

globuloides (Galumna) sp. inq. .................... 215

globulus (Euzetes) ……………………….... 175

globus [P. (Archiphthiracarus)] (=globosus) .. 55

glomerata (Prototritia) ……………………..... 35

glomerata (Prototritia) …………………..... 35

glycyphagoides (Damaeus) .............................. 78

gobiensis [P. (Archiphthiracarus)]

(=bryobius) …………………………......... 55

gobiensis (Bipassalozetes) ............................. 162

gobletus [P. (Constrictocepheus)] ................... 145

golosovae [D. (Epidamaeus)] ……………….... 79

golosovae (Gozmanyina) ................................ 33

golosovae [R. (Insculptoppia)] ....................... 119

golosovae (Parautogneta) ................................ 109

gomerae (Oribatella) …………………....... 171

gomozovae (Medioppia) ………………….... 123

gorodkovi (Nanhermannia) ............................ 68

gozmanyi [O. (Zygoribatula)]

(=arcuatissima $)$....................................... 188

gracilata [O. (Zygoribatula)] ....................... 189

gracile (Acrotritia) ………………………….... 45

gracilior (Eremobelba) …………………...... 103

gracilior (Eremobelba) ................................... 103

gracilior (Eremobelba) ................................. 103

gracilior (Micreremus) $(=$ brevipes $) \quad$............. 159

gracilipes (Cepheus) $s p$. inq. ......................... 82

gracilipes (Damaeus) (=auritus) ...................... 78

gracilis (Acrotocepheus) …………………... 146

gracilis (Ceratozetes) ……………………..... 174

gracilis (Ceratozetes) .................................... 174

gracilis (Ceratozetes) …………………....... 174

gracilis (Ceratozetes) ……………………..... 174

gracilis (Ceratozetes) ……........................... 174

gracilis (Ceratozetes) .................................... 174

gracilis (Ceratozetes) ………………………... 174

gracilis (Eohypochthonius) ………………..... 28

gracilis (Eohypochthonius) ............................. 28

gracilis (Eohypochthonius) ………………...... 28

gracilis (Eohypochthonius) ……….............. 28

gracilis (Eohypochthonius) ........................... 28

gracilis (Eremaeus) ……............................... 100

gracilis (Gehypochthonius) …………………... 28

gracilis (Hammerella) ……………………...... 132

gracilis [H. (Heterohermannia)] ........................ 69

gracilis (Holonothrus) …………………….... 67

gracilis (Iugoribates) .................................... 180

gracilis (Kokoppia) ...................................... 123

gracilis [S. (Lalmoppia)] …………………..... 130

gracilis [S. (Lalmoppia)] ............................. 130

gracilis (Liacarus) (=subterraneus) .............. 96

gracilis (Mahunkaia) .................................. 165

gracilis (Malaconothrus) (=monodactylus) .... 61

gracilis (Multioppia) ……………………........ 117

gracilis (Multioppia) ……............................ 117

gracilis (Neoribates) ……………………...... 212

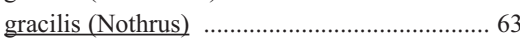

gracilis (Nothrus) ………………………....... 63

gracilis (Paraphauloppia) ............................ 190

gracilis (Phauloppia) ................................... 190

gracilis (Protoribates) …………………….... 206

gracilis (Sellnickochthonius) ……………….... 32

gracilis (Separatoppia) ………………........ 129

graciosa (Eremobelba) ………………….. 103

graeca $($ Mesoplophora $)(=$ michaeliana $)$........ 42

graeca (Multipulchroppia) ............................ 120

graecus (Haplochthonius) ................................. 34

graecus (Platyliodes) ……………………….... 72

grafatus (Dolicheremaeus) ............................ 142

grahamius (Scapheremaeus) ...................... 158

graminetum (Pergalumna) ............................ 219

gramineus (Torpacarus) ................................... 41

graminosus (Heptacarus) ................................. 38

granalata (Galumna) ........................................ 215

grancanariae (Ctenobelba) ............................. 101

grancanariae (Parabelbella) ............................. 81

grancanariae (Subbelba) elisae ……………..... 82

grandiporosus [S. (Andeszetes)] ................. 202

grandis (Acrotocepheus) …………………... 146

grandis (Adoristes) $(=$ ovatus) $\quad$......................... 95

grandis (Allosuctobelba) …………………... 134

grandis (Allosuctobelba) .............................. 134

grandis (Allosuctobelba) …………............... 134

grandis (Anachipteria) …............................ 168

grandis (Austrachipteria) ……………........ 166

grandis (Berniniella) ……............................ 125

grandis (Cepheus) (=dentatus) ......................... 82

grandis (Ceratozetes) $(=$ gracilis) .................... 174

grandis [L. (Dorycranosus)] (=acutus) .......... 97

grandis (Eobrachychthonius)

(=latior)

grandis (Eremaeus) …………………………. 100

grandis (Eremaeus) ……………………........ 100

grandis (Eupelops) ………………………….... 163

grandis (Hermannia) (=convexa) .................. 69

grandis (Hermanniella) .................................... 70

grandis (Oppia) (=denticulata) ..................... 115

grandis (Paschoalia) ..................................... 73

grandis (Peloribates) ...................................... 210

grandis (Peloribates) ................................... 210

grandis (Ramsavellus) …………………..... 182

grandis (Reticulocepheus) ............................. 84

grandis (Rhynchoribates) ............................. 140

grandis (Scheloribates) .................................... 200

grandis (Trimalaconothrus) .............................. 62

grandis [A. (Uluguroides)] .......................... 149

granditrichosus (Annectacarus) ................... 37

grandjeani (Acaronychus) …………….......... 27

grandjeani (Alaskozetes) antarcticus ............ 156

grandjeani (Berlesezetes) ................................ 87

grandjeani (Carabodes) …………………....... 150

grandieani (Cryptogalumna) ...................... 214

grandjeani [D. (Epidamaeus)]

(=puritanicus)

grandjeani (Galumna) ........................................ 215

grandjeani (Hoplophorella)

grandjeani (Hypochthoniella)

(=minutissimua $) \quad$..................................... 29

grandjeani [R. (Mantigueracarus)] ……..... 58 grandjeani (Masthermannia) _....................... 68

grandjeani (Mesotritia) ..................................... 43

grandjeani (Mesotritia) ................................. 43

grandjeani (Mochloribatula) ........................ 185

grandjeani (Mongaillardia) ............................. 101

grandjeani (Nanhermannia) .......................... 68

grandjeani (Niphocepheus) nivalis ................... 84

grandjeani [H. (Platynothrus)] (=peltifer) .... 66

grandjeani (Protoplophora) (=palpalis) ........ 35

grandjeani (Prototritia) ................................... 36

grandjeani (Prototritia) …………………..... 36

grandjeani (Rugozetes) ……......................... 90

grandjeani (Solenoppia) …………………..... 124

grandjeani (Steganacarus) …………………...... 58

grandjeani (Tricheremaeus) ........................... 101

grandjeani (Zetorchestes) .................................. 91

grandjeani [O. (Zygoribatula)] …………….... 189

grandjeanica (Porobelba) ................................... 82

granifer (Topobates) ……………………….... 203

granifer (Topobates) ……………………...... 203

granosus [C. (Klapperiches)] ....................... 151

granulata (Acrotritia) …………………….... 45

granulata [L. (Antennoppia)] …………..... 113

granulata (Baloghiella) ................................... 207

granulata (Hermanniella) ................................... 70

granulata (Hermanniella) ............................... 70

granulata (Pergalumna) …………………...... 219

granulata (Pulchroppia) ………………….... 120

granulata (Suctobelba) …………………….... 135

granulata (Topalia) ……………………….... 86

granulata (Wallworkoppia) (=machadoi) .... 121

granulata [O. (Zygoribatula)] …………........ 189

granulatus (Arotrobates) …………………….... 157

granulatus (Bipassalozetes) (=perforatus) .. 162

granulatus (Carabodes) ................................ 150

granulatus (Carabodes) ................................... 150

granulatus (Damaeus) ………………………... 78

granulatus (Dolicheremaeus) ……………...... 142

granulatus [D. (Epidamaeus)] ………….......... 79

granulatus (Epieremulus) ............................ 106

granulatus (Eueremaeus) (=oblongus) ….. 100

granulatus (Javacarus) ……………………..... 38

granulatus (Javacarus) …………………....... 38

granulatus (Liacarus) sp. inq. ........................... 96

granulatus (Licnodamaeus) .......................... 74

granulatus (Micreremus) .............................. 160

granulatus (Microtegeus) ………………….... 86

granulatus [P. (Millotacarus)] …………….... 41

granulatus (Nesiacarus) .................................. 40

granulatus (Nesiacarus) …………………….... 40

granulatus (Nesiacarus) ............................... 40

granulatus (Passalozetes)

(=neonominatus) ………………………. 162

granulatus (Pseudotocepheus) ...................... 145

granulatus (Rhacaplacarus) ............................. 57

granulatus (Sadocepheus) ................................. 84

granulatus (Scutovertex) (=sculptus) .......... 161

granulatus (Tectocepheus) velatus ................. 155

granulatus (Trimalaconothrus) ......................... 62

granulatus [L. (Undulozetes)] ……………..... 89

granuliala (Trichogalumna) ……………....... 221

granulosa [K. (Stakarenoppia)] .................... 130 
granulosus (Cepheus) …………………………... 83 granulosus [M. (Cristonothrus)] .................... 61 granulosus (Papillonotus) ........................... 133 gratiosa (Liebstadia) ...................................... 195 gratiosa (Metabelbella) .................................... 81 gratiosa [O. (Zygoribatula)] .......................... 189 gratus (Podoribates) (=longipes) ……......... 186 gravouwensis (Schalleriella) ........................ 91 greensladeae (Darthvaderum) …………...... 73 gregarius (Gymnodamaeus) ............................ 76 gregorioi (Carabodes) …................................. 150 gregoryi (Indoribates) ................................ 208 gressitti (Apotomocepheus) ........................ 148 gressitti (Austrocarabodes) …................... 148 gressitti (Flammaeremaeus) …..................... 73 gressitti (Hemileius) .................................... 193 gressitti [L. (Lancelalmoppia)] ................... 111 gressitti (Notophthiracarus) ….................... 51 gressitti (Peloribates) .................................. 210 griseus (Atropacarus) …………..................... 48 griseus (Sellnickochthonius) ......................... 32 grishinae (Punctoribates) .............................. 183 grobleri (Paulianacarus) .............................. 40 groenlandicus (Ameronothrus)

(=maculatus) …........................................... 156 groenlandicus [D. (Epidamaeus)] .................... 79 groenlandicus [D. (Epidamaeus)] .................. 79 groenlandicus (Propelops) ............................ 164 groenlandicus (Propelops) ........................... 164 grossmani (Damaeus) ...................................... 78 grossmani (Damaeus) ................................. 78 grossmani (Hoplophthiracarus) sp. inq. ........ 50 grucheti (Arcoppia) .................................. 120 grymae (Camisia) …....................................6 65 guadarramica [O. (Zygoribatula)] ................ 189 guadarramicus (Carabodes) .......................... 150 guadarramicus (Ceratozetes) ....................... 174 guadarramicus (Ghilarovus) hispanicus ........ 184 guadarramicus (Humerobates)

rostrolamellatus ….................................... 181

guadarramicus (Niphocepheus) ...................... 84 guadeloupensis (Cyrthermannia) ...................67 67 guanarteme (Steganacarus) ............................ 58 guanicola (Serratoppia) ............................... 124 guanophilus (Sellnickochthonius) .................. 32 guarani (Brachioppia) (=deliciosa) ............ 122 guarani (Epilohmannia) ............................... 37 guehoi (Perxylobates) .................................. 205 guelticola [R. (Rectoppia)] (=mihelcici) ...... 119 guerini (Scapheremaeus) ............................... 158 gueyeae (Hydrozetes) ..................................... 155 gueyeae (Papillacarus) .................................. 40 guhitanus (Scheloribates) ............................... 200 guilfordidei (Gymnodamaeus) ........................ 76 guineana (Arcoppia) gumista [O. (Coronoquadroppia)]

(=michaeli) gunini (Scheloribates) .................................. 200 gunjina (Pedrocortesella) ….......................... 75 guttatoides (Peloribates) ................................. 210 guttatus (Peloribates) .................................... 210 guyi (Limnozetes) guzhangensis (Lohmannia) gygeri (Sellnickochthonius) (=immaculatus) gymnonota (Pedrocortesella) gyoergyi (Lamellobates) gyoergyi (Multipulchroppia) gyrata (Ramusella) haarlovi (Lanceoppia) hachijoensis (Dolicheremaeus) infrequens .142 hachijoensis (Dolicheremaeus) infrequens .. 142 hailongensis [O. (Zygoribatula)] .................. 189 hainanensis (Annectacarus) …....................... 37 hainanensis (Megalotocepheus) ................... 147 hainardorum (Ramusella) ......................... 118 hakonensis (Protoribates) ............................... 206 hakonensis (Trimalaconothrus) ....................... 62 hallaensis (Astegistes) .................................. 92 hallasanensis (Acrotritia) .................................. 45 halleri (Protoribates) sp. inq. ...................... 206 halli (Pilogalumna) tenuiclava ................... 220 hallidayi [P. (Neophthiracarus)] ................. 56 halophilus (Eupelops) .................................... 163 halterata (Similoppia) ….......................... 121 hamanni [M. (Phyllolohmannia)] .................... 40 hamanni (Xiphobelba) ................................. 104 hamata (Eremobelba) .................................. 103

hamata (Hoplophorella) .................................... 49

hamata (Hoplophorella) .................................. 49

hamata (Hoplophorella) ................................ 49 hamata (Hoplophorella) …............................. 49

hamata (Hoplophorella) ............................... 49

hamata (Hoplophorella)

hamata (Moritzoppia) sp. inq. ..................... 126

hamata (Suctobelbella) ........................... 11, 136

hamata (Suctobelbella) ......................... 11, 136

hamatus (Hoplophthiracarus) ........................... 50

hamatus (Hoplophthiracarus) ...................... 50

hamatus (Hoplohthiracarus) ........................ 50

hamidi (Neoamerioppia) ................................ 114

hamidi (Notophthiracarus) ......................... 51

hamifer (Acaroceras) .................................... 87

hamifer (Galumna) (=angularis) …............ 214

hamifera (Suctobelbella) ............................ 136

hamiltoni (Globoppia) ............................... 110

hammeni [P. (Archiphthiracarus)]

(=peristomaticus)

hammeni (Notophthiracarus) …….............. 51

hammeni (Striatoppia) ................................. 13

hammeni (Striatoppia)

hammerae (Aeroppia)

hammerae (Anderemaeus)

hammerae (Arcoppia)

hammerae (Austrachipteria) J. y P. Bal.,

\section{2}

hammerae (Austrachipteria) M., 97

(=hammerae J.,P.B.,92)

hammerae [M. (Calyptozetes)]

hammerae (Chamobates) (=javensis) .......... 180

hammerae [D. (Epidamaeus)] ……................ 79

hammerae (Galumna) .............................. 215

hammerae (Glanderemaeus) ........................ 158

hammerae (Hermannobates) ........................ 71 hammerae (Nodocepheus) ……………….... 85

hammerae (Notophthiracarus) …............... 51

hammerae (Pseudotocepheus) …................... 145

hammerae (Quadroppia) .............................. 133

hammerae (Quadroppia) ............................ 133

hammerae (Quadroppia) ........................... 133

hammerae (Quadroppia) ............................. 133

hammerae (Scapheremaeus) (=patella) ...... 159

hammerae (Sellnickochthonius) (=lydiae) .... 32

hammerae (Senectoppia) (=multisulcatum) .. 132

hammerae (Suctobelbella) ........................... 136

hammerae (Suctobelbella) ............................. 136

hammerae (Trhypochthonius) sp. inq. ............ 60

hammerae (Xenillus) .................................... 98

hamobatoides (Ceratozetes) ….................... 174

hamulifera (Camisia) ......................................6 65

hamulifera (Camisia) .................................. 65

hangayi (Pedrocortesella) …….................... 75

hannecarti (Brachioppiella) ...................... 122

hanswursti (Eremaeozetes) ............................ 165

hanyensis (Sellnickochthonius) ....................... 32

haradai (Adrodamaeus) ................................. 75

haradai (Austrocarabodes) ........................... 148

haradai [F. (Psammocepheus)] ....................... 144

haramachiensis (Peloribates) ....................... 210

hardyi (Pedrocortesella) ................................... 75

hardyi (Pedrocortesella) .............................. 75

harioti (Ameronothrus) sp. inq. ..................... 156

harpezus (Kalyptrazetes) .............................. 89

harteni (Suctobelbella) ................................... 136

hartensteini [B. (Gressittoppia)] ............... 122

harunaensis (Pergalumna) (=altera) ......... 219

hastata (Pergalumna) ...................................... 219

hastata (Suctobelbella) .................................. 136

hastatus (Compactozetes) ……........................ 83

hastatus [D. (Epidamaeus)] ........................... 79

hastatus (Eremulus) ................................... 102

hastatus (Furcobates) .................................. 175

hasticeps (Achipteria) sp. inq. ...................... 167

hastilis (Neoctenacarus) ................................. 28

hatanoensis (Nothrus) ....................................... 63

hauseri (Acrotritia) (=hyeroglyphica) ......... 45

hauseri (Allosuctobelba) .............................. 134

hauseri [P. (Archiphthiracarus)]

(=anonymus) …......................................... 54

hauseri (Arthrovertex) .................................. 160

hauseri (Baloghacarus) .................................. 70

hauseri [Y. (Berndobodes)] ......................... 154

hauseri (Berniniella) serratirostris ................ 125

hauseri [N. (Besuchetacarus)] ....................... 52

hauseri (Bolkiah) ........................................ 208

hauseri (Borneremaeus) .............................. 141

hauseri (Chamobates) ..................................... 179

hauseri [N. (Confinoppia)] ............................ 127

hauseri (Congocepheus) ................................ 151

hauseri [M. (Cristonothrus)] .......................... 61

hauseri [M. (Cristonothrus)] ......................... 61

hauseri (Issaniella) mograbin .......................... 71

hauseri (Lamellobates) (=molecula) ............. 167

hauseri [S. (Lucioppia)] .............................. 130

hauseri (Luxtonia) ....................................... 131

hauseri (Mesoplophora) ................................ 42 
hauseri [F. (Mexicoppia)] hauseri (Microtritia) ....................................... 47 hauseri (Nothrus) ............................................. 63 hauseri (Oribotritia) .............................................. 44 hauseri (Otocepheus) .................................... 147 hauseri (Papillonotus) ..................................... 133 hauseri (Passalozetes) .................................... 162 hauseri (Pergalumna) ..................................... 219 hauseri (Pseudotocepheus) ......................... 145 hauseri (Rhynchoppia) .................................. 135 hauseri [R. (Sabahoppia)] (=blattarum) ...... 119 hauseri (Sabahtritia) ....................................... 47 hauseri (Suctobelbila) …............................. 139

hauseri (Tecteremaeus) ................................. 108

hauseri (Trichogalumnella) ........................ 221

hauserorum (Brachychthonius) ...................... 29

hauserorum (Bulbocepheus) ........................... 141

hauserorum (Oxyamerus) ............................. 140

hawaiiensis (Campbellobates) acanthus .... 204

hawaiiensis (Microtritia) .............................. 47

hawaiiensis (Neoliodes) ….......................... 72

hawaiiensis (Oribotritia) ................................ 44

hawaiiensis (Paroppia) …............................ 115

hawaiiensis (Pergalumna) ......................... 219

hawaiiensis (Tenuelamellarea) ….............. 160

haydeni (Hemileius) ........................................ 193

headlandi [N. (Parakalumma)] .................. 212

hebes (Unduloribates) ................................... 165

hebes (Unduloribates) ….............................. 165

hectorperezius (Scapheremaeus) ............... 158

heegi (Eupelops) sp. inq. ............................. 163

heimi (Carabodes) (=labyrinthicus) ............ 150

heintoogensis (Campachipteria) .................. 168

heleios (Heterozetes) ................................... 173

helenae [O. (Cavernella)] ……........................ 172

helenae (Ceratozetella) .................................... 173

helenensis (Scheloribates) ........................... 200

hellenica (Machuella) (=ventrisetosa) ........ 133

hellenicus (Berndamerus) ............................... 101

hellenicus (Microzetes) .................................... 89

helleri (Lemuroppia) ................................ 114

helleri [A. (Malgoceras)] ……........................ 87

helluonis [P. (Neophthiracarus)] …............... 56

helvetica (Belba) ………………………….... 77

helvetica (Metrioppia) ...................................... 94

helvetica (Metrioppia) .................................... 94

helvetica (Nortonella) ...................................... 76

helvetica (Paramedioppia) ............................ 124

helveticus (Brachychthonius)

(=bimaculatus) …..................................... 29

helveticus (Hypocepheus) ............................. 83

helveticus (Kaszabobates) ............................ 107

helvinus (Scheloribates) (?=fimbriatus) ...... 199

henicos (Oribotritia) .......................................... 44

hepaticus (Eremaeus) ...................................... 100

hepaticus (Eremaeus) ................................... 100

herculeana (Ceratoppia) (=bipilis) ................93

herculeanus (Steganacarus) ............................ 58

herenessica (Austrotritia) ................................ 42

hericius (Protocepheus) ................................ 83

hermanni (Oribotritia) hermannioides (Melanozetes) sp. inq. .......... 176 heveae (Sellnickia) (=caudata) .................... 191

herminae (Porrhotegaeus) …...................... 85 hexagona (Galumna) …........................... 215

heroica (Pergalumna) ….............................. 219 hexagonalis (Cubabodes) ........................... 152

heros (Galumna) sp. inq. …...................... 215 hexagonus (Minguezetes) ...................... 12, 182

herzegowinensis (Autogneta) willmanni …... 108 hexagonus (Minguezetes) ........................12, 182

hesperidiana (Lasiobelba) ............................ 113 hexagonus (Minguezetes) ....................... 12, 182

hesperis (Didymonycha) …........................ 214 hexapili (Lanceoppia) ….............................. 110

hessei (Capilloppia) ................................. 186 hexapilis (Neoamerioppia) $\quad$.......................... 114

heterocuspis (Ceratozetella) ......................... 173 hexaporosus (Parapirnodus) ….................... 191

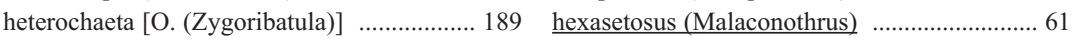

heterodactylus (Cultrobates) ...................... 172 hexasetosus (Malaconothrus) …................... 61

heterodactylus (Scheloribates) …............ 12, 200 hexatricha (Basiloppia) .............................. 110

heterodactylus (Topobates) ....................... 203 hidasii (Anderemaeus) ............................... 106

heterodentata (Oribatella) ............................. 171 hieroglyphicus (Scapheremaeus) .................. 158

heteropoda (Metabelba) sp. inq. ….............. 80 hierrensis (Hemileius) ～................................... 193

heteroporosa (Murcia) …........................... 177 higginsi (Brachioppiella) ........................... 122

heteroporosa [O. (Zygoribatula)] higginsi (Eueremaeus) $\quad$................................ 100

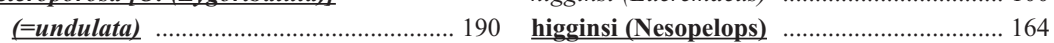

heterosa [L. (Antennoppia)] ..................... 113 higginsi [C. (Klapperiches)] ........................ 151

heterosetiger (Otocepheus) ........................... 147 highlandensis (Dentachipteria) .................... 169

heterosetiger (Xenillus) …............................ 98 himalayensis (Dolicheremaeus) …............... 142

heterosetosa (Acanthobelba) ........................ 77 himalayensis (Eremaeozetes) ....................... 165

heterosetosa [H. (Steganacarellus)] …....... 50 himalayensis (Eremobelba) ….................... 103

heterosetosus (Cepheus) .................................... 83 himalayensis [O. (Indotocepheus)] ............. 153

heterosetosus (Scheloribates) ..................... 200 himalayensis (Megalotocepheus) ................. 147

heterospinifer (Camisia) ................................ 65 himalayensis (Nanhermannia) ....................... 68

heterotricha (Apoplophora) .......................... 41 hippy (Machuella) (=ventrisetosa) ............. 133

heterotricha (Apoplophora) ........................ 41 hippy (Ramusella) …................................. 118

heterotricha (Epilohmannia) ......................... 37 hirashimai (Microtegeus) …........................ 86

heterotricha (Eremobelba) …....................... 103 hirsuta (Masthermannia) ............................... 68

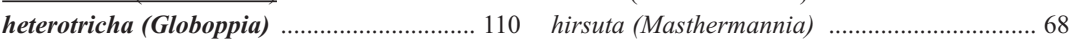

heterotricha (Hermannia) ............................... 69 hirsuta (Masthermannia) ............................... 68

heterotricha (Hexoppia) …......................... 133 hirsutus (Dolicheremaeus) ........................ 142

heterotricha (Hoplophorella) ....................... 49 hirsutus (Eupelops) (=acromios) …........... 163

heterotricha (Indotritia) (=krakatauensis) $\ldots .43$ hirsutus (Heptacarus) …............................ 38

heterotricha (Medioppia) …........................ 123 hirsutus (Heptacarus) …............................... 38

heterotricha (Oribotritia) ….............................. 44 hirsutus (Papillacarus) ................................... 40

heterotricha [H. (Steganacarellus)] …........ 50 hirsutus (Papillacarus) .................................. 40

heterotricha [G. (Stenoppia)] (=italica) ….. 116 hirsutus (Papillacarus) …............................... 40

heterotricha [O. (Zygoribatula)l hirsutus (Papillacarus) ................................ 40

(=bonairensis) ……................................. 188 hirsutus (Peloribates) Banks, 1895 ............. 210

heterotrichosus [A. (Baloghodes)] .............. 149 hirsutus (Peloribates) Mah., 1983

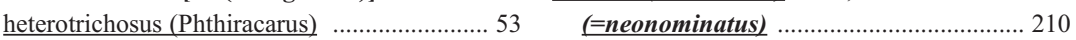

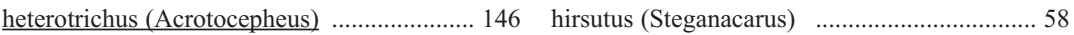

heterotrichus (Calyptophthiracarus) .................5 52 hirtus (Brachychthonius) ................................. 29

heterotrichus (Congocepheus) .................. 151 hirtus (Eupelops) (=major) ........................ 163

heterotrichus [M. (Cristonothrus)] .............. 61 hirtus (Murcia) sp. inq. .................................. 177

heterotrichus (Dicastribates) ........................ 70 hirtus [P. (Neophthiracarus)] ......................... 56

heterotrichus (Dolicheremaeus) …........... 142 hirtus [A. (Pseudonothrus)] …...................... 59

heterotrichus (Eurostocepheus) …................ 146 hispanica (Gerloubia) .................................... 191

heterotrichus (Meristacarus) …........................ 39 hispanica [L. (Incabates)] ........................ 12, 209

heterotrichus (Meristacarus) …................... 39 hispanica (Tenuelamellarea) ........................ 160

heterotrichus (Papillocepheus) ................. 144 hispanica (Tenuelamellarea) ...................... 160

heterotrichus (Phyllozetes) (=emmae) $\quad$.......... 33 hispanica (Tenuelamellarea) ........................ 160

heterotrichus [E. (Pocsia)] ........................... 47 hispanica [O. (Zygoribatula)] ....................... 189

heterotrichus [T. (Rostrozetes)] ….............. 211 hispanicus (Adrodamaeus) .......................... 75

heterotrichus (Scheloribates) ..................... 200 hispanicus (Carabodes) ............................... 150

heterotrichus (Sellnickochthonius) ….......... 32 hispanicus (Ghilarovus) …........................... 184

heterotrichus (Trimalaconothrus) ................ 62 hispanicus (Paralopheremaeus) …................. 73

heterotrichus [H. (Tuberemaeus)] .............. 193 hispanicus (Passalozetes) ............................... 162

heterotrichus (Xenillus) …......................... 98 hispaniola (Beklemishevia) .......................... 27

hettigei (Trichocarabodes) …....................... 154 hispaniola (Lohmannia) (=turcmenica) ........ 39 


\begin{tabular}{|c|c|c|}
\hline spida (Pilogalumna) tenuiclava ................. 220 & & \\
\hline stricinus (Hoplophthiracarus) ....................... 50 & umeratus (Cristeremaeus) ............................ 106 & ypoquercus (Phyllozetes) \\
\hline stricinus (Hoplophthiracarus) ..................... 50 & umeratus (Epieremulus) ................................ 106 & yrcanica (Hafenferrefia) ................ \\
\hline stricinus (Peloribates) (=hubbardi) .......... 210 & 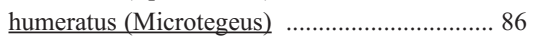 & ystricinus (Liochthonius) .............. \\
\hline vaoae (Crotonia) nukuhivae (=nukuhivae) .. 67 & umeratus (Microtegeus) & iystricinus (Liochthonius) \\
\hline 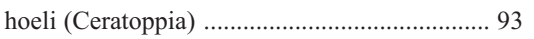 & umeratus (Pheroliodes) .... & nystricinus (Palaeacarus) . \\
\hline eli (Ceratoppia) ..... & lumeratus (Reductobates). & iystricinus (Palaeacarus) \\
\hline 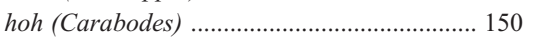 & emaeus) ….................. 158 & hystricinus (Palaeacarus) \\
\hline kkaidensis (Hermannia) ............................... 69 & umeratus (Scheloribates) sp. inq. ................. 200 & iystrix (Peloribates) .......... \\
\hline kkaidensis (Hermannia) ............................... 69 & $1 ., 97 \ldots .63$ & erica (Bursoplophora) .......... \\
\hline kkaiensis (Cepheus) .................. & & ibericus [L. (Dorycranosus)] (=zachvatkini) .. \\
\hline ensis (Eueremaeus) ….......................... 100 & (= humeratus $\mathrm{PB}, 97)$ & ibericus [D. (Epidamaeus)] (=pyrenaicus) ... \\
\hline onensis (Achipteria) ............................... 167 & humeratus (Yoshiobodes) (?=nakatamarii) .. 154 & ibericus (Tectocepheus) (=sarekensis) . \\
\hline ta (Galumnopsis) & ...... 184 & \\
\hline ... 203 & toppia)] & noppia)] (=quadriseta). \\
\hline cepheus) ..... & ...... 66 & 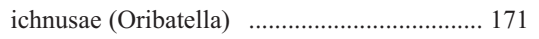 \\
\hline cepheus) ...... & .......... 66 & \\
\hline umna) sp. inq. ................... 215 & ......... 215 & \\
\hline ochthonius) …...................... 32 & ......... 154 & iracarus) \\
\hline obates) variabilis . & ......... 70 & ignota (Belba) (=sculpta) .. \\
\hline us)] & ........ 150 & ignota (Mesoplophora) ................... \\
\hline$\ldots .146$ & .... 213 & eus) (=siculus) \\
\hline 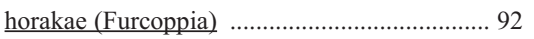 & ..... 125 & \\
\hline eudoceratoppia) ............ & ....... 117 & ilha \\
\hline a) $]$ & 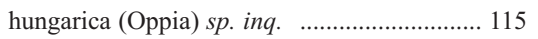 & \\
\hline (Sacculobates) ........................ 71 & ) & \\
\hline .......... 65 & ...... 220 & illinoisensis (Cham \\
\hline .............65 65 & ....... 77 & illino \\
\hline & & illinoisensis $\left(\mathrm{Ho}_{1}\right.$ \\
\hline$a$ (Camisia) & (=bryobius) & illinoisensis $(Q u$ \\
\hline & ... 150 & illun \\
\hline & 1 & \\
\hline sp. inq. ....... &. .153 & pheus) \\
\hline .... 136 & $\ldots 210$ & $\underline{\mathrm{ima}}$ \\
\hline abodes) $\ldots \ldots \ldots . .$. & ..... 32 & \\
\hline (ermannobates) .............. & chthonius) & \\
\hline iius) (................................... 30 & ...... 184 & imit \\
\hline hortobagyensis (Phthiracarus) (=longulus) .... 54 & $\ldots . . .173$ & \\
\hline & .... 39 & imit \\
\hline$(=$ glabra $)$ & 153 & s) . \\
\hline orvathorum (Pergalumna) ............. & ...... 158 & 12,16 \\
\hline. .168 & $\ldots 115$ & \\
\hline. .168 & ...... 89 & 15) .. \\
\hline loribates) ......... & 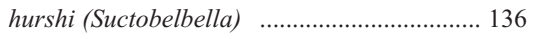 & imm \\
\hline. .200 & ..... 130 & ius) \\
\hline losuctobelba). & ............. 140 & ntocepheus) .. \\
\hline .115 & ...... 71 & \\
\hline$\ldots 210$ & .... 207 & $\ldots 17$ \\
\hline$\ldots 215$ & 146 & pia) .......... \\
\hline ... 50 & $\ldots 213$ & \\
\hline. .182 & ..... 45 & tesella) \\
\hline (Diapterobates) …......................... 181 &. .45 & 15 \\
\hline lis (Diapterobates) & .... 45 & \\
\hline (Diapterobates) .... & .... 45 & tella) ... \\
\hline & $\ldots 45$ & \\
\hline. .193 & .... 123 & \\
\hline 203 & nna) …........................ 221 & \\
\hline 92 & 163 & \\
\hline ... 195 & uezetes) (=hexagonus) .. 182 & nipteria)] . \\
\hline$\ldots 195$ & 183 & \\
\hline & ....... 99 & rabodes) \\
\hline umerata (Notoppia) & hypersetosus (Setincabates). & imperfectus (Cosmochthonius) . . \\
\hline
\end{tabular}


imperfectus (Passalozetes)

imperfectus (Protoribates) ............................... 206

imperfectus (Scheloribates) .......................... 200

imperialis (Hafenferrefia) sp. inq. ................. 99

impolita (Baobabula) ...................................... 192

impressus (Brachychthonius) ............................. 29

improvisus (Notophthiracarus) ........................ 51

improvisus (Pseudocarabodes) ................... 153

imreorum (Epilohmannia) .............................. 37

inacessus [P. (Archiphthiracarus)] ………... 55

inaequalis (Dolicheremaeus) ......................... 142

inaequalis (Licnodamaeus) ................................ 74

inaequipes [M. (Akrodamaeus)] …………..... 81

inaequirostris (Arcoppia) .............................. 120

inaequus (Notophthiracarus) ....................... 51

inaestimabilis (Steganacarus) ………............... 58

inauditus (Notophthiracarus) ......................... 51

inca (Beckiella) ............................................ 140

inca (Pheroliodes) ......................................... 74

incaensis (Arceremaeus) .............................. 108

incerta (Arcoppia) ……………………....... 120

incerta (Galumna) ……………………….... 216

incerta (Oribatula) ………………………....... 187

incertus (Areozetes) ………………………….. 195

incertus (Phthiracarus) ..................................... 54

incertus (Phthiracarus) .................................. 54

incertus (Pterobates) ……………………..... 85

incertus (Unguizetes) ...................................... 186

incipatus (Charassobates) ............................... 86

incisa (Ceratoppia) …………………………..... 93

incisa (Galumna) ……………………........ 216

incisa (Magyaria) ………………………....... 209

incisa (Microtritia) ………………………..... 47

incisa [M. (Moritzoppiella)] ........................... 127

incisa (Oribatella) ………………………….... 171

incisa (Sternoppia) ....................................... 132

incisella [V. (Latilamellobates)] ...................... 179

incisirostra (Oppia) ........................................ 115

incisiva [R. (Rectoppia)] .............................. 119

incisivus (Afronothrus) …………………....... 59

incisivus (Afronothrus) …………………….... 59

incisivus (Afronothrus) .................................. 59

incisivus (Afronothrus) .................................. 59

incisura [P. (Italobates)] ………………........ 211

incisus (Cepheus) …………………………….. 83

incisus (Chamobates) (=pusillus) ................ 180

incisus [H. (Heloribates)] (=thienemanni) .. 156

incisus (Iugoribates) (=cornutus) ................. 180

inclinata (Pletzenoppia) …………………... 123

incognita (Oribotritia) ..................................... 44

incognitus (Steganacarus) …………………..... 58

incomparabilis (Notophthiracarus) ............ 51

incomperta (Pergalumna) ........................... 219

incomperta (Phauloppia) ............................... 190

incompleta (Allogalumna) ............................... 213

incompleta (Oribatula) …………………..... 187

incompletus (Eupelops) ……………………... 163

incompletus (Papillacarus) ............................. 40

incompletus (Tecteremaeus) .......................... 108

incomptus (Steganacarus) ………………….... 58

incondita (Hermanniella) ................................... 70 inconditus (Atropacarus) …………………....... 48

incrassatus [P. (Neophthiracarus)] ................. 56

ncredibilis (Euphthiracarus) ....................... 46

incredibilis [R. (Mantigueracarus)] ………... 58

incredibilis (Phthiracarus) .............................. 54

incrustata (Oribatula) …………………..... 187

incrustatus [A. (Uluguroides)] ……………... 149

inculpatus (Atropacarus) …………………...... 48

incurva (Campachipteria) distincta ……....... 168

incurva (Ceratozetella) ………………………... 173

incurva [S. (Lalmoppia)] ………………….... 130

incurva [S. (Lalmoppia)] ................................ 130

incurva (Oribatula) (=tibialis) $\quad$...................... 188

incurva [C. (Protoripoda)] ........................... 204

incurvata (Oribatella) ................................... 171

incurvata (Phauloppia) (=lucorum) ............ 190

incurvata (Schalleria) ..................................... 90

incurvatus [M. (Calyptozetes)] ....................... 183

incurvatus [M. (Calyptozetes)] ..................... 183

indentatus [L. (Dorycranosus)] ....................... 97

indentatus [H. (Tuberemaeus)] .................... 193

index (Acaroceras) ……………………….... 87

indes (Sphaerogalumna) ............................. 221

indica (Apoplophora) (=pantotrema) ............. 41

indica (Basilobelba) ....................................... 104

indica (Epilohmannia) pallida ………............. 37

indica (Eremobelba) …………………............ 103

indica [S. (Flagrosuctobelba)] semiplumosa .. 138

indica (Malacoangelia) remigera ……............. 29

indica (Mesotritia) …………………………......... 43

indica (Multioppia) …………………….......... 117

indicator (Hydrozetes) .................................. 155

indicus (Allonothrus) ………………................ 59

indicus (Archegozetes) (=longisetosus) ….... 59

indicus (Dolicheremaeus) ................................. 142

indicus (Flagellozetes) porosus ..................... 214

indicus (Hoplophthiracarus) (=repetitus) $\quad . . .50$

indicus [P. (Millotacarus)] ................................ 41

indicus (Notophthiracarus) .............................. 51

indicus (Papillacarus) ……………………….... 40

indicus (Scheloribates) Oud., 15 ................... 200

indicus (Scheloribates) Oud., 15 ................ 200

indicus (Scheloribates) San., 92

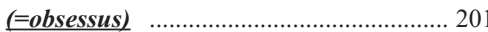

indicus (Uracrobates) ……………………...... 186

indifferens [M. (Cristonothrus)] .................... 61

indiligens (Notophthiracarus) ......................... 51

indistinctus (Tumerozetes) …………….......... 86

indivisa (Pergalumna) .................................... 219

indubiatus (Notophthiracarus) ...................... 51

indusiatus (Trimalaconothrus) .......................... 62

induta (Eremella) ........................................... 106

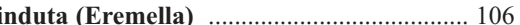

induta (Eremella) …………………………...... 106

induta (Eremella) ………………………...... 106

inelegans (Notophthiracarus) ......................... 51

inelegans (Notophthiracarus) ........................ 51

inenarrabilis (Notophthiracarus) ..................... 51

inenodabilis (Fenestrobelba) ………............. 134

ineptus (Notophthiracarus) …………………..... 51

ineptus (Notophthiracarus) ........................... 51

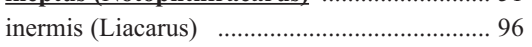

inermis (Liacarus) ………………………….... 96

nermis (Unguizetes) ………………………... 186

inexpectata (Epilohmannia) .............................. 37

inexpectata (Epilohmannia) ……………….... 37

inexpectata (Muliercula) ...………………... 197

inexpectata (Rhynchobelba) ............................. 135

inexpectatus (Javacarus) ............................... 38

inexpectatus (Phthiracarus) ………………….... 54

inexspectatus (Spinozetes) ............................ 108

infissus [L. (Dorycranosus)] (=acutus) ........ 97

inflata (Dometorina) ..................................... 192

inflatus [N. (Calyptophthiracarus)] ............. 53

inflatus (Rhacaplacarus) ………………….... 57

inflexa (Oribatella) ............................................ 171

inflexus (Tectocepheus) (=sarekensis) ........ 155

infrequens (Dolicheremaeus) ......................... 142

infusionem [H. (Platynothrus)] (?=peltifer) .... 66

inglisi (Euphthiracarus) .................................. 46

inhambanensis (Fortuynia) .......................... 157

initialis (Hemileius) ........................................ 193

initialis (Hemileius) ……………………….... 193

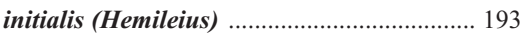

inlenticulatus (Passalozetes) ............................ 162

inlenticulatus (Scutovertex) ............................ 161

innexa (Galumna) ...………………………. 216

inopinatus (Calyptophthiracarus) pavidus ....... 53

inopinatus (Dolicheremaeus) ......................... 142

inopinatus [C. (Phyllocarabodes)] .............. 151

inopinatus [E. (Pocsia)] ……………………... 47

inornata (Baloghoppia) .................................. 128

inornata (Berniniella) ........................................ 125

inornatus [P. (Archiphthiracarus)] ................... 55

inornatus [D. (Epidamaeus)] ............................ 79

inornatus [T. (Rostrozetes)] ………………... 211

inornatus [H. (Tuberemaeus)] ..................... 193

inoueae (Hoplophthiracarus) ……………….... 50

insculpta [R. (Insculptoppia)] ........................ 119

insculpta [R. (Insculptoppia)] .......................... 119

insculptus (Annectacarus) .............................. 37

insertus (Eremaeus) ……………………....... 100

insignificans [N. (Protokalumma)] ................. 212

insignipes (Epilohmannia) ............................. 37

insignis (Achipterina) .................................... 172

insignis (Achipterina) .................................... 172

insignis [L. (Antennoppia)] .......................... 113

insignis (Banksinoma) .................................... 109

insignis (Ceratozetes) …………………….... 174

insignis (Lauritzenia) .................................... 208

insignis (Minguezetes) ................................... 182

insignis (Minguezetes) …………………….... 182

insignis (Minguezetes) ................................. 182

insignis [P. (Neophthiracarus)] ...................... 56

insignis (Papuazetes) …………………......... 90

insignis (Perlohmannia) …………………….... 36

insignis (Rhynchoribates) ............................. 140

insignis (Rykella) ........................................... 185

insignitus (Ocesobates) (=boodvardssoni) .. 180

insignitus (Rhacaplacarus) …………………...... 57

insolens (Hoplophorella) ................................. 49

insolens (Rhacaplacarus) …………………... 57

insolita (Allogalumna) …………………..... 213

insolita (Multioppia) Mih., 56 (?=laniseta) .... 117 
insolita (Multioppia) I. y V., 99 ........................ 117

insolita (Pluritrichoppia) ................................ 115

insolitus (Nippobodes) ................................... 154

insolitus [C. (Phyllocarabodes)] .................. 151

insperatus (Scutoverticosus) ........................... 161

insulana [S. (Flagrosuctobelba)] …............... 138

insulana [S. (Flagrosuctobelba)] ................ 138

insulana (Multioppia) ............................ 11, 117

insulana (Multioppia) ........................... 11, 117

insulana (Multioppia) ......................... 11, 117

insulanus (Steganacarus) .................................. 58

insularis (Aeroppia) ...................................... 112

insularis (Ameronothrus) (=maculatus) ...... 156

insularis [P. (Archiphthiracarus)]

(=bulbiferus)

.... 55

insularis (Atropacarus) striculus .................... 48

insularis (Basilobelba) ................................. 104

insularis (Basilobelba) .................................. 104

insularis (Basilobelba) ............................... 104

insularis (Bursoplophora) ................................. 35

insularis (Dometorina) plantivaga ................ 192

insularis (Gittella) ..................................... 122

insularis [R. (Insculptoppia)] (=insculpta) ... 119

insularis (Mesoplophora) (=africana) .......... 42

insularis [M. (Multilanceoppia)] ................ 117

insularis (Parachipteria) ................................. 169

insularis (Phthiracarus) ….............................5 54

insularis [C. (Protoripoda)] .......................... 204

insularis (Scapheremaeus) ......................... 158

insularis (Scheloribates) (=praeincisus) ...... 201

insularis [I. (Triungulozetes)] (=fusifer) .... 208 insularis [O. (Zygoribatula)] (=frisiae) ...... 189

insulata (Lasiobelba) .................................... 113

integer (Allogalumna) ................................. 213

integer (Mycobates) ..................................... 183

integra (Multioppia) ....................................... 117

integrus (Mixacarus) ..................................... 39

interiunctus (Acaroceras) .............................. 87

interlamellaris (Beckiella) ............................ 140

interlamellaris (Belba) .................................... 77

interlamellaris (Heminothrus) .......................... 65

interlamellaris (Metabelbella) ......................... 81

intermedia (Achipteria) (=coleoptrata) ......... 167

intermedia (Autogneta) longilamellata ........ 108

intermedia [O. (Dzarogneta)] ........................ 129

intermedia (Globoppia) .............................. 110

intermedia (Globoppia) ................................... 110

intermedia [H. (Heterohermannia)] ................ 69

intermedia (Oribatula) ................................. 187

intermedia (Pergalumna) .............................. 219

intermedia (Pergalumna) .............................. 219

intermedia (Serratoppia) ............................... 124

intermedia (Suctobelbella) (=subtrigona) .. 137

intermedius (Alaskozetes) antarcticus .......... 156

intermedius (Alaskozetes) antarcticus .......... 156

intermedius (Anellozetes) …....................... 181

intermedius (Austrocarabodes) …................ 148

intermedius (Bipassalozetes) ........................ 162

intermedius (Eueremaeus) (=oblongus) ...... 100

intermedius (Euphthiracarus) (=reticulatus) .. 46

intermedius [C. (Flexa)] ................................ 150

intermedius (Fuscozetes) .............................. 175 intermedius (Halozetes) .................................. 157

intermedius (Haplochthonius) ......................... 34

intermedius (Hermannobates) ...................... 71

intermedius (Lamellobates) .......................... 166

intermedius (Liochthonius) ............................ 30

intermedius (Liochthonius) ............................. 30

intermedius [C. (Mixozetes)] ........................ 175

intermedius (Nesopelops) ............................. 164

intermedius (Peloribates) ............................... 210

intermedius (Pheroliodes) ............................. 74

intermedius [T. (Tyrphonothrus)] (=maior) .... 63 internodentatus (Liacarus)

(=brevilamellatus) ...................................... 95

interpositus (Chamobates) ............................ 180

interrogata (Neoamerioppia) ........................ 114

interrupta (Arcoppia) ................................... 120

interrupta (Lohmannia) javana ......................... 39

interrupta (Oribatula) ..................................... 187

interrupta (Oribatula) ................................. 187

interrupta (Pseudoppia) ................................. 191

interruptus (Carabodes) ................................ 150

interruptus (Cavernozetes) .......................... 88

interruptus (Melanozetes) .............................. 176

interruptus (Scheloribates) praeincisus ........ 201

interruptus (Scheloribates) praeincisus .... 201

interruptus (Scheloribates) praeincisus ...... 201

intralamellata (Oppiella) (=nova) ................ 128

intrudens (Berniniella) . 125

inundata (Autogneta) .................................... 108

inupiaq (Ceratozetes) ..................................... 174

inurbanus (Steganacarus) ........................... 58

inusitata (Sumatrotritia) ................................... 47

inusitatus [N. (Calyptophthiracarus)] ............... 53

invenusta (Camisia) ....................................... 65

invenustus [P. (Archiphthiracarus)] ................ 55

invisitata (Mesoplophora) ........................... 42

invisitata (Mesoplophora) .............................. 42

involutus (Congocepheus) ............................ 151

ionicus (Hellenamerus) ................................. 101

ionicus (Neoliodes) ........................................ 72

ionthadosus (Xenillus) .................................... 98

iowaensis (Peloribates) …............................ 210

iracemae (Protoribates) ................................. 206

iranensis (Galumna) ........................................ 216

iranica (Anomaloppia) ................................. 116

irazu (Galumna) ....................................... 216

irenae (Eremaeozetes) .................................. 165

iriomotensis [M. (Cristonothrus)] ...................61

irmayi (Beckiella) ........................................ 140

irmayi (Octoppia) .......................................... 117

irmayi (Yoshiobodes) ..................................... 154

irmayi (Yoshiobodes) ................................... 154

irregularis (Pergalumna)

irregularis [T. (Rostrozetes)] ….................... 211

irregularis [T. (Rostrozetes)] …................. 211

irregularis (Xenillus) ....................................... 98

irreprehensus (Phthiracarus) ......................... 54

ishigakiensis (Austrotritia) (=saraburiensis) .... 42

ishigakiensis (Galumna) planiclava ............. 216

ishikariensis (Nothrus) ......................................... 63

ishikawai (Hoplophthiracarus) ........................ 50

islandica (Banksinoma) (=lanceolata) ......... 109 islandica [C. (Ensicamisia)] (=solhoevi) ..... 65

smalia (Xiphobelba) …................................ 104

issanielloides (Hermanniella) ............................ 70

italica (Achipteria) ........................................ 167

italica (Allogalumna) ................................... 213

italica (Nortonbelba) …................................ 81

italica [M. (Parametabelba)] ............................. 81

italica [G. (Stenoppia)] ............................... 116

italica (Suctobelbella) ............................. 11, 136

italicus (Adrodamaeus) .................................. 75

italicus (Microzetorchestes) ........................... 91

italicus (Phthiracarus) ........................................ 54

italicus (Phthiracarus) .................................... 54

italicus (Poecilochthonius) ............................... 31

italicus (Pocilochthonius) ............................... 31

italiensis (Sphaerozetes) (=orbicularis) ...... 178

itatiaiae [T. (Tyrphonothrus)] P. B., 97 .......... 63

itatiaiae [T. (Tyrphonothrus)] J.,P.B.,02

(=itatiaiae P.B.,97) ...................................... 63

iterata (Galumna) ........................................... 216

iterata (Galumna) ......................................... 216

iteratus (Dolicheremaeus) magnus ............ 143

iteratus (Scheloribates) …......................... 200

iteratus (Trimalaconothrus) .............................. 62

iteratus (Trimalaconothrus) ........................6 62

ithacensis (Galumna) ................................... 216

itoi (Yambaramerus) ....................................... 102

iturrondobeitiai (Foraminoppia) .................... 128

izabalensis (Torpacarus) .............................. 41

izquierdoae [L. (Antennoppia)] .................... 113

izuensis (Diapterobates) ................................. 181

jaccoudi (Apoplophora) (=pantotrema) ........ 41

jaccoudi (Cosmozetes) ................................... 88

jacoti (Belba) …................................................ 77

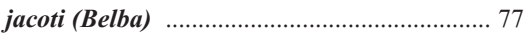

jacoti (Epilohmannoides) ................................ 37

jacoti (Epilohmannoides) ............................... 37

jacoti (Galumna) Whar., 38 .......................... 216

jacoti (Galumna) M. et al., 87 (=iterata) .... 216

jacoti (Indotritia) ................................................. 43

jacoti (Indotritia) ................................................. 43

jacoti (Lanceoppia) ..................................... 110

jacoti (Mesotritia) ................................................ 43

jacoti (Phthiracarus) ........................................... 54

jacoti [N. (Protokalumma)] B. y M., 67 ...... 212

jacoti [N. (Protokalumma)] N. y K., 89

(=neonominatus) ...................................... 212

jacoti (Rhacaplacarus) ................................. 57

jacoti (Sellnickochthonius) ...................... 11, 32

jacoti (Sellnickochthonius) ....................... 11, 32

jahnae (Berniniella) ...................................... 125

jaliscoensis (Nothrus) .................................... 63

jamaicaensis [C. (Klapperiches)] ................. 151

jamalica (Moritzoppia) ................................. 126

jamiesoni (Anoplozetes) .............................. 184

janae (Metabelbella) ........................................ 81

jandai (Dampfiella) ................................... 141

jandiae (Multioppia) ..................................... 117

janetscheki (Liacarus) .................................... 96

japonensis (Eupelops) .................................. 163

japonensis [H. (Platynothrus)] peltifer ............ 66

japonica (Austroceratoppia) ........................... 93 


japonica (Austroceratoppia)
japonica (Autogneta) ..................................................

jonasi (Proteremaeus) …………………….... 108

jongkyui (Pergalumna) ................................. 219

joonsooi (Trimalaconothrus) ........................... 62

jordanai (Liacarus) ……………………………. 96

jornoti (Lohmannia) ……………………...... 39

jucunda (Oribatella) ....................................... 171

jucundior (Scheloribates) .............................. 200

juditae (Baloghia) ………………………..... 207

jugata (Cerachipteria) digita ........................... 168

jugatus (Brachychthonius) sp. inq. ................. 29

juglans (Benoibates) .................................... 203

jugorum [O. (Zygoribatula)] (=exilis) …..... 189

juliae (Lohmannia) ....................................... 39

jumbongiensis [P. (Metaphthiracarus)] ……... 56

juncta (Cultroribula) .......................................... 92

juncta (Cultroribula) ...................................... 92

juniperi (Peloribates) .................................... 210

junonis (Ramusella) ....................................... 118

junonis (Ramusella) ..................................... 118

juradae (Melanozetes) sp. inq. ...................... 176

juvenalis (Phthiracarus) (=ferrugineus) …... 53

juvenalis [C. (Nanochthonius)] ....................... 33

kadazan (Otocepheus) …………………….... 147

kaindicola (Arcoppia) ……………………... 120

kaisilai (Autogneta) ……………………….... 108

kalalao [L. (Bicristoppia)] ............................ 111

kalboprodorsalis (Peloribates) ....................... 210

kalimantanensis (Arcoppia) (=aequivoca) .. 120

kaliurangensis [S. (Flagrosuctobelba)] .......... 138

kamaensis [D. (Epidamaeus)] ……………….. 79

kamenskii (Palaeacarus) ………………………. 27

kamerunensis (Granuloppia) .................... 132

kamillae (Notophthiracarus) ......................... 51

kananaskis (Ceratozetes) ............................. 174

kananaskis (Eremaeus) .................................. 100

kananaskis (Veloppia) ................................. 106

kanangra (Pedrocortesella) ........................... 75

kanekoi (Diplobodes) ..................................... 152

kanekoi (Gibbicepheus) ................................. 152

kanekoi (Liacarus) ……………………......... 96

kanoi [H. (Phyllhermannia)] …………............ 69

kanoi [H. (Phyllhermannia)] ........................... 69

karajica (Galumna) ........................................ 216

karamani (Steganacarus) (=magnus) .............58

karelicus [D. (Epidamaeus)] ………………….... 79

kargi (Cosmogneta) ……………………….... 109

karinae (Setoppia) …………………......... 112

karpellesi (Euphthiracarus) sp. inq. ................ 46

karppineni (Membranoppia) (=krivoluzkyi) .. 111

karppineni (Passalozetes) …………………..... 162

karubei (Diplobodes) …............................... 152

kaszabi (Achipterina) .................................. 172

kaszabi (Bipassalozetes) (=striatus) ............ 162

kaszabi (Kaszabobates) ................................. 107

kaszabi (Peloribates) .................................... 210

kaszabi (Phthiracarus) $(=$ boresetosus) ........... 53

kaszabi (Rhacaplacarus) ……………………... 57

kaszabi (Triteremella) ....................................... 106

kazakhstani (Galumna) ................................... 216

kazakhstanicus (Gymnodamaeus) ..................... 76

kazakovi (Berniniella) ……………………... 125

keewatin (Megeremaeus) ................................. 99 keilbachi (Moritzoppia) unicarinata .............. 127

kelbadjarica [O. (Zygoribatula)] …............... 189

kellyi (Novazelandiella) ………………….... 74

keningau (Otocepheus) …………………..... 147

kenyaensis (Uroppia) .................................... 119

keralensis (Dolicheremaeus) ……………...... 142

keralensis (Haplacarus) ……………………... 38

keralensis [O. (Zygoribatula)] ......................... 189

kerangas (Senectoppia) …………………..... 132

keretinus [L. (Dorycranosus)] ........................... 97

keriensis [M. (Cristonothrus)] ……………....... 61

keriensis [M. (Cristonothrus)] ....................... 61

keriensis (Perscheloribates) .......................... 198

kethleyi (Novonothrus) ..................................... 64

kethleyi (Tegeocranellus) .................................. 155

kevani (Eremaeus) ........................................ 100

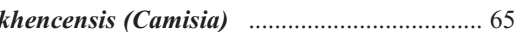

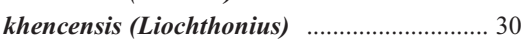

khoii (Galumna) ………………………….... 216

khosrovica [O. (Dzarogneta)] ......................... 129

khugniensis (Liebstadia)

(=saifulmalukensis) ………………….... 195

khustaiensis [D. (Epidamaeus)] ........................ 79

kieviensis [C. (Xiphobates)] ………………..... 180

kiiensis [M. (Cristonothrus)] ............................. 61

kikonaiensis [H. (Capillonothrus)] capillatus .... 66

kilchini (Liacarus) ………………………....... 96

kimberleyensis (Pilogalumna) ..................... 220

kinabalu (Dampfiella) ……………………..... 141

kinabaluensis (Austrotritia) …………….......... 42

kingi (Berlesezetes) …………………………..... 87

kingi (Damaeus) (=angustipes) ...................... 78

kirai (Pasocepheus) ……………………….... 153

kirghisicus (Liochthonius) ................................ 30

kirghisicus (Liochthonius) ............................... 30

kirgisicus [Z. (Protozetomimus)] ................... 179

kishidai (Epilohmannoides) (=bipectinatus) .... 37

kishidai (Maerkelotritia) ....................................... 43

kishidai (Maerkelotritia) ..................................... 43

kittenbergeri (Pseudotectoribates) ................. 172

kittenbergeri (Pseudotectoribates) ............. 172

kivuensis (Eupelops) sp. inq. ....................... 163

klabati (Euphthiracarus) .................................... 46

klabati (Euphthiracarus) ............................... 46

knighti [O. (Zygoribatula)] ............................. 189

knoepffleri (Phauloppia) ................................. 190

knowltoni (Gymnodamaeus) …………………... 76

knuellei (Lanceoppia) …………………….... 110

knuellei (Tectocepheus) .................................. 154

knysnaensis (Tegeocranellus) ........................ 155

kobauensis (Scapuleremaeus) ..................... 159

kochi (Camisia) (=biurus) ................................. 65

kochi (Eupelops) (=acromios) ....................... 163

kochi [P. (Neophthiracarus)] .......................... 56

kodiakensis [D. (Epidamaeus)] ........................ 79

kodiakensis [D. (Epidamaeus)] ....................... 79

koeszegiensis (Liacarus) ................................ 96

koki (Brachioppia) ………………………..... 122

koki (Drepanoppia) ………………………. 110

koki [O. (Zygoribatula)] ………………..... 189

komareki (Nanhermannia) .............................. 68

komodensis [T. (Rostrozetes)] ....................... 211 
kootenai (Megeremaeus) ………………….... 99

koreanus (Papillacarus) ................................... 40 koreense (Oribotritia) (=asiatica) ....................... 44

koreensis (Acrotritia) ..................................... 45

koreensis (Fissicepheus) coronarius .............. 144

kornhuberi (Neoliodes) ................................... 72

kornhuberi (Neoliodes) ............................... 72

kosarovi (Corynoppia) .................................. 130

kosarovi (Corynoppia) .................................... 130

kosarovi (Metabelbella) .................................. 81

kotschyi (Pergalumna) ................................... 219

koumantanosi (Phthiracarus) ........................... 54

kovacsi (Lanceoppia) .................................... 110

koyukon [D. (Epidamaeus)] ........................... 79

krachan (Annectacarus) .................................... 37

kraepelini (Scheloribates) .............................. 200

krakatauensis (Indotritia) ..................................... 43

krakatauensis (Indotritia) ................................ 43

krakatauensis (Indotritia) .................................. 43

krakatauensis (Indotritia) ….......................... 43

krakatauensis (Indotritia) ................................. 43

krantzi (Dolicheremaeus) ............................ 142

krivolutskyi (Birsteinius) ............................... 95

krivolutskyi (Hypocepheus) ............................. 83

krivolutskyi [R. (Insculptoppia)] .................. 119

krivolutskyi [S. (Lalmoppia)] ......................... 130

krivolutskyi (Maerkelotritia) .............................. 43

krivolutskyi (Metrioppia) ............................ 94

krivolutskyi (Oribatella) ............................... 171

krivoluzkyi (Membranoppia) …................. 111

krygeri (Oppiella) (=nova) …....................... 128

kuehnelti (Eueremaeus) (=oblongus) .......... 100

kuehnelti (Javacarus) ...................................... 38

kuehnelti (Javacarus) ….................................. 38

kuehnelti (Javacarus) ................................... 38

kuehnelti (Lasiobelba) ........................... 11, 113

kuehnelti (Lasiobelba) ........................ 11, 113

kuehnelti (Provertex) ...................................... 161

kugohi (Hoplophthiracarus) ............................. 50

kugohi (Hoplophthiracarus) ......................... 50

kugohi (Hoplophthiracarus) ............................ 50

kugohi (Hoplophthiracarus) ........................50

kugohi (Hoplophthiracarus) ........................... 50

kulaginae (Epilohmannia) ............................... 37

kulczynskii (Damaeus) (=flagellifer) ............ 78

kulczynskii (Hoplophorella) .......................... 49

kumadai (Ocesobates) .................................. 180

kummeri (Dolicheremaeus) ........................ 142

kunsti (Operculoppia) .............................. 112

kunsti (Oribatella) ....................................... 171

kunsti (Pocsia) ................................................... 47

kurchevi [O. (Multoribatella)] ….................. 172

kurikii (Trimalaconothrus) ............................... 62

kurilica (Suctobelbella) ................................. 136

kurosawai (Cepheus) ............................................. 83

kurozumii (Eremaeozetes) .......................... 165

kurti (Tenuiala) .......................................... 99

kushiroensis [S. (Dyobelba)] ............................. 82

kushiroensis (Oribellopsis) ............................. 107

kusseri [C. (Klapperiches)] …..................... 151

kutchin (Ceratozetes) ................................... 174

kyushuensis (Phthiracarus) (=clemens) ........ 53 labradorica [C. (Ensicamisia)] (=lapponica)

labyrinthicus (Carabodes) .......................... 150

labyrinthicus (Carabodes) ............................. 150

labyrinthicus (Euphthiracarus) …..................... 46

abyrinthicus (Microtegeus) …....................... 86

labyrinthicus (Scheloribates) ........................ 200

lacandonicus (Allozetes) .............................. 166

laccosis (Hydrozetes) ..................................... 155

laciniatus (Fosseremus) …............................. 103

laciniatus (Fosseremus) ............................. 103

laciniatus (Fosseremus) ……......................... 103

laciniatus (Fosseremus) ............................. 103

laciniatus (Fosseremus) ............................. 103

lacrimans (Zetomotrichus) ........................... 185

lacrimans (Zetomotrichus) ....................... 185

lacrimans (Zetomotrichus) …....................... 185

lacunosus (Liochthonius) ................................. 30

lacustris (Hydrozetes) ....................................... 155

lacustris (Hydrozetes) ..................................... 155

laensis (Nesopelops) ................................... 164

laetabilis (Rhacaplacarus) ............................. 57

laetepictus (Liochthonius) sp. inq. .................. 30

laetus (Malaconothrus) ...................................... 61

laevigatus (Eupelops) (=plicatus) ................ 163

laevigatus (Phthiracarus) ................................. 54

laevigatus (Phthiracarus) …........ 54

laevigatus (Scheloribates) …......................... 200

laevigatus (Scheloribates) ..................... 200

laevigatus (Scheloribates) .......................... 200

laevigatus (Scheloribates) …......................... 200

laevis (Archoplophora) (=rostralis) .............. 42

laevis (Brachychthonius) sp. inq. .................... 29

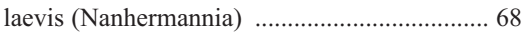

laevis (Paulianacarus) …............................. 40

laevis (Pergalumna) emarginata ............... 218

laevis (Suctobelbella) ................................... 136

laevis (Suctobelbella) .................................... 136

laevis (Tegeocranellus) ................................... 155

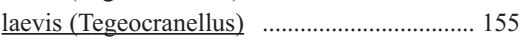

laevis (Tegeocranellus) .................................. 155

laeviusculus (Odontocepheus) sp. inq. .......... 153

lagenula (Transoribates) ................................ 207

lagrecai (Ceratozetes) ............................. 12, 174

lagrecai (Eutegaeus) ........................................ 85

lagunensis [H. (Tuberemaeus)] …................. 193

laiae [S. (Porokalumma)] .............................. 213

laiae (Suctobelbella) ...................................... 136

lamellata (Austrachipteria) ........................ 166

lamellata (Beckiella) …......................... 140

lamellata (Brassoppia) ................................ 122

lamellata (Limnozetella) ....................... 165

lamellata (Medioppia) ................................. 123

lamellata (Moritzoppia) (=unicarinata) ...... 127

lamellata (Multioppia) (?=laniseta) ............ 117

lamellata (Murcia) (=obesa)

lamellata (Parapyroppia) (=transitoria) ........ 94

lamellata (Pseudoamerioppia) ................... 118

lamellata (Subphauloppia) ........................ 191

lamellata [R. (Insculptoppia)] (=elliptica) .. 118

lamellatus [A. (Coropoculia)] ....................... 159

lamellatus (Caucasiozetes) ............................. 88

lamellatus (Geminozetes) ............................. 176 amellatus (Neoliodes) …….......................... 72

lamellatus (Oribatodes) (=mirabilis) $\quad$.............83

lamellatus (Scheloribatoides) .................... 202

lamellatus (Trichocepheus) ....................... 146

lamellatus (Xenillus) _...................................... 98

lamellicornis (Rugoppia) ........................... 131

laminatus (Scheloribates) .............................. 200

lamingtoni [P. (Neophthiracarus)] ..............56

laminipes (Lopheremaeus) .............................. 73

laminipes (Scutovertex) ............................... 161

lamottei (Ramogneta) .................................. 107

lanatus [P. (Archiphthiracarus)]

(=bryobius) …........................................... 55

lanatus (Cosmochthonius) ............................... 33

lanatus (Cosmochthonius) ................................ 33

lanatus (Cosmochthonius) ............................. 33

lanatus (Cosmochthonius) ............................. 33

lanatus (Cosmochthonius) ............................. 33

lancearia (Lanceoppia) ............................... 110

lanceata (Galumna) ........................................ 216

lanceata (Galumna) …................................ 216

lanceocrinus (Eremulus) sp. inq. ................ 102

lanceolata (Austrotritia) ................................... 42

lanceolata (Banksinoma) ............................... 109

lanceolata (Banksinoma) ........................... 109

lanceolata (Crotonia) .....................................6 67

lanceolata (Lohmannia) ….............................. 39

lanceolata (Lohmannia) ................................ 39

lanceolata (Neoamerioppia) ........................ 114

lanceolata [M. (Parametabelba)] ..................... 81

lanceolata (Pyroppia) ..................................... 94

lanceolata (Pyroppia) ...................................... 94

lanceolata (Striatoppia) ............................... 131

lanceolata (Striatoppia) ............................... 131

lanceolata (Striatoppia) ............................... 131

lanceolata (Suctobelbella) longicuspis ........ 136

lanceolata (Suctobelbila) spicata .................. 139

lanceolatus (Arotrobates) …….......................... 157

$\underline{\text { lanceolatus (Borneozetes) }}$............................. 208

lanceolatus (Perscheloribates) ..................... 198

lanceolatus (Pseudotocepheus) ................... 145

lanceolatus (Scutozetes) ................................. 169

lanceolatus (Scutozetes) ................................ 169

lanceolatus (Scutozetes) ................................ 169

lanceolatus (Topobates) .............................. 203

lanceolatus [H. (Turcibates)] ....................... 194

lanceoliger (Scheloribates) (?=fimbriatus) .. 199

lanceoseta (Hoplophorella) ............................ 49

lanceoseta (Karenella) ................................ 130

lanceosetoides (Hoplophorella) .................... 49

lanceosetoides (Karenella) ............................ 130

lanceosetoides (Karenella) ........................ 130

lanceosetosus (Neoliodes) .............................. 72

laneus (Podoribates) …................................. 186

languida (Achipteria) sp. inq. ........................ 167

laniseta (Multioppia) wilsoni ........................ 117

laniseta (Multioppia) wilsoni ....................... 117

laniseta (Multioppia) wilsoni ..................... 117

lapelerii (Elaphoppia) …........................... 126

lapidaria (Suctobelba) .................................. 135

lapidarius (Chamobates) sp. inq. .................. 180

lapponica [C. (Ensicamisia)] ......................... 65 
lapponica [C. (Ensicamisia)] lapponica [C. (Ensicamisia)] ….......................6 65 lapponicus (Ameronothrus) ………………...... 156 lapponicus (Edwardzetes) (=edwardsi) ........ 175 lapponicus (Liochthonius) ................................ 30 lapponicus (Liochthonius) ................................ 30 lapshovi (Palaeacarus) ……………………..... 27 largus [P. (Archiphthiracarus)] .......................... 55 largus [P. (Neophthiracarus)] (=repetitus) ...... 57 larreae (Jornadia) ……………………….... 187 laselvae (Galumna) ……………………..... 216 lasithiensis [S. (Tropacarus)] ……………….... 59 lata (Cultroribula) ………………………...... 92 lata (Cultroribula) …………………………….... 92

lata (Cultroribula) ……………………......... 92 lata (Dometorina) …………………………... 192

lata (Galumna) (=tarsipennata) …………... 217

lata (Hafenferrefia) ............................................. 99

lata [O. (Zygoribatula)] .................................. 189

lata [O. (Zygoribatula)] ................................ 189

latebrosus (Notophthiracarus) ....................... 51

latens (Microzetes) (=septentrionalis) …...... 89

laterodentatus (Nodocepheus) ...................... 85

laterospinosus (Rhacaplacarus) …………....5 57

laterostris (Liacarus) ......................................... 96

laticephalus (Neamerus) (?=lundbladi) ...... 105

laticeps (Cuneoppia) ...................................... 134

laticeps (Epieremulus) ……………………... 106

laticeps (Verachthonius) ..................................... 32

laticlava (Grandjeanobates) ……………........ 197

laticlava (Grandjeanobates) ......................... 197

laticlava (Hemileius) ……………………... 193

laticuspidata (Sulcoribula) ………………..... 93

laticuspidatus (Ceratozetes) ........................... 174

laticuspidatus (Peloptulus) ............................ 164

laticuspis (Cultroribula) …………………... 92

laticuspis (Exochocepheus) ……………….... 161

latidens (Berniniella) ……………………...... 125

latifasciata (Globoppia) …………………....... 110

latiflabellata (Licnobelba) …………………..... 75

latifolius (Phyllozetes) ..................................... 33

latifolius (Phyllozetes) ………………………. 33

latilamellaris (Tectocepheus) ......................... 154

latilamellata [V. (Latilamellobates)] ............... 179

latilamellata (Palmitalia) ............................... 167

latilamellata (Schalleria) ................................ 90

latilamellata (Szentivanyella) ......................... 91

latilamellatus (Congocepheus) ................... 15

latilamellatus (Liacarus) ..................................... 96

latilamellatus (Limnozetes) ........................... 166

latilamellaltus (Peloptulus) ………………....... 164

latilamellatus (Xenillus) ..................................... 98

latilamellatus (Zachvatkinibates) ................... 184

latilobatus (Punctoribates) .............................. 183

latilobatus (Punctoribates) ………………..... 183

latincisa (Murcia) .......................................... 177

latiohumeralis (Reductobates) …………... 196

latior (Carabocepheus) lounsburyi ............. 154

latior (Chaunoproctus) ………………........... 192

latior (Eobrachychthonius) ................................ 30

latior (Eobrachychthonius) ............................. 30

latior (Eobrachychthonius) ............................. 30 latior (Notophthiracarus) ................................. 51

atior (Notophthiracarus) …………………...... 51

latior (Notophthiracarus) .............................. 51

latipes (Scheloribates) (=pallidulus) ............. 201

latipilosa (Fenichelia) ................................. 159

latipilosus (Eupelops) ………………………... 163

latipilosus (Eupelops) .................................... 163

latipilosus (Lepidozetes) ………………….... 169

latipilosus (Lepidozetes) ................................ 169

latipluma (Acrogalumna) (=longipluma) .... 213

latirostris (Beckiella) …………………........ 141

latirostris (Hypochthonius) sp. inq. ................ 29

latirostris (Murcia) ………………………….... 177

latirostris (Oribatula) .................................... 187

latirostris (Oxyamerus) …………………..... 140

latirostris (Peloptulus) …………………....... 164

latirostris (Scapheremaeus) ........................ 158

latirostris (Suctobelbella) ............................... 136

latirostris (Suctobelbella) ................................ 136

latirostris (Tegoribates) ….............................. 169

latisetosus (Neochthonius) ................................. 34

latissimus (Annobonzetes) ............................ 196

latissimus (Austrocarabodes) …………… 148

latissimus (Dinozetes) ...................................... 88

latisternalis [O. (Dzarogneta)] ..................... 129

latisternum (Cycloppia) .............................. 110

latitecta (Anachipteria) $(=$ howardi) ............. 168

latiusculus [L. (Dorycranosus)] …………….... 97

lativentris (Liacarus) (=subterraneus) .......... 96

latoclava (Lyrifissiella) …………………….... 73

latodentata [S. (Ussuribata)] .......................... 138

latohumeralis (Campbellobates) ................ 204

latohumeralis (Gibbicepheus) …………….... 152

latoincisus (Scheloribates) ……………….... 200

latolamellata (Gustavia) ……………………... 95

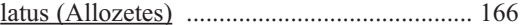

latus (Annobonzetes) ………………………... 196

latus (Birobates) …………………………..... 203

latus (Cavernozetes) ……………………….... 88

latus (Cepheus) ……………………………..... 83

latus (Cepheus) ……………………………..... 83

latus (Cepheus) ………………………………...... 83

latus (Feiderzetes) ………………………..... 182

latus (Liacarus) Ewing, 09 .............................. 96

latus (Liacarus) Schweiz., 56 (=schweizeri) .... 96

latus (Liochthonius) Jac., 36

latus (Liochthonius) Mah., 82

(=neonominatus)

atus (Megalotocepheus)

atus (Neseutegaeus) ……………………..... 85

latus (Nippobodes) ………………………...... 154

latus (Perscheloribates) ................................ 198

latus (Plateremaeus) …………………………... 73

latus (Scapheremaeus) …………………… 158

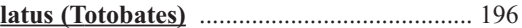

latus (Transoribates) (=lagenula) ................. 207

latus [T. (Tyrphonothrus)] .............................. 63

latus (Xenillus) $(=$ clypeator) ........................... 98

laubieri [O. (Zygoribatula)] (=glabra) ........ 189

laurisilvae (Hermanniella) .............................. 70

lautus (Phthiracarus) …………………………. 54

lavoipierrei (Paralycus) .................................. 34 avoipierrei (Paralycus) ……………………... 34

lawariensis (Mixacarus) sp. inq. .................... 39

lawariensis (Proteremaeus) .............................. 108

lawrencei (Galumna) maxima …………… 216

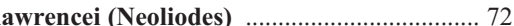

lawrencei (Xenillus) …………………….... 98

laxtoni (Suctobelbella) (?=subcornigera) .... 137

laysanensis (Hypozetes) .................................. 166

laysanensis (Hypozetes) ............................... 166

lazitanicus (Steganacarus) (=patruelis) ........ 58

lebanonensis (Xenillus) ……………………... 98

lebedevi (Lauroppia) ..................................... 126

lebronneci (Austrotritia) .................................... 42

lebronneci (Austrotritia) ............................... 42

lebruni (Paroppia) ........................................ 115

lectronus (Liacarus) ......................................... 96

ledensis (Dentizetes) ....................................... 175

leei (Notophthiracarus) ................................. 51

leei (Pedrocortesella) ………………………... 75

leei (Stomacarus) ………………………........ 27

legendrei (Paralopheremaeus) ....................... 73

legendrei (Paralopheremaeus) ………………. 73

leleupi (Allogalumna) ……………………… 213

leleupi (Heminothrus) ……………………..... 65

leleupi (Liacarus) sp. inq. ............................... 96

leleupi (Nothrus) ............................................... 63

leleupi (Oppia) sp. inq. ................................. 115

leleupi (Scheloribates) ................................. 200

lemnae (Hydrozetes) …………………………. 155

lemnae (Hydrozetes) ……………………....... 155

lemnae (Hydrozetes) ……………………..... 155

lemnae (Hydrozetes) ……………………....... 155

lemuria (Hoplophorella) ……………………... 49

lemuria (Lasiobelba) ................................... 113

lencoranicus (Liacarus) .................................... 96

lencoranicus (Scheloribates) …...................... 200

lengersdorfi (Damaeus) ................................... 78

lenicomus (Mycobates) (=parmeliae) .......... 183

lenis [M. (Multilanceoppia)] brevipectinata .... 117

lenkoi (Epilohmannia) ................................... 37

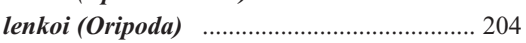

lenticulata [O. (Zygoribatula)] ................ 12, 189

lenticulata [O. (Zygoribatula)] .............. 12, 189

lenticulatus (Hypovertex) …………………..... 161

lentula (Acrotritia) ………………………….... 45

lentulus (Phthiracarus) ……………………….... 54

lentulus (Phthiracarus) ……………………... 54

lentulus (Phthiracarus) ……………………... 54

lentus (Liochthonius) ....................................... 30

leoi (Rimandocepheus) ……………………..... 147

leontonychus (Paraleius) ............................. 194

leowae (Archeremella) …………………….... 106

lepidus (Austrocarabodes) .............................. 148

leporoides (Eremobelba) .............................. 103

leporosa (Eremobelba) ................................. 103

leptaleus (Liochthonius) ………………………... 30

leslevae [O. (Coronoquadroppia)]

(=michaeli) …………………………….... 134

leucaena (Arthrovertex) …………………...... 160

leurolomasus (Gymnodamaeus) ......................... 76

leurolomasus (Gymnodamaeus) ...................... 76

levigata [O. (Zygoribatula)] ........................... 189 
levipunctatus (Peloribates)

levis (Allozetes) ............................................... 166 levis (Liochthonius) (=evansi) leviseta [M. (Parplophora)] …....................... 42 leviseta [M. (Parplophora)] …….................... 42 leviseta [M. (Parplophora)] …....................... 42 lewissmithi (Furcobates) ............................ 175 liber (Punctoribates) .................................... 183 licnophora (Hoplophorella) (=cucullata) ...... 48 licnophorus (Licneremaeus) ................... 12, 160 licnophorus (Licneremaeus) .................. 12, 160 lichenis (Allomycobates) ................................ 182 lienhardi (Acrotocepheus) ............................ 146 lienhardi [N. (Besuchetacarus)] ...................... 52 lienhardi (Coronibatula) ................................. 196 lienhardi (Hoplophorella) (=vitrina) ............49 lienhardi (Papillacarus) ……............................. 40 lienhardi (Passalozetes) ............................... 162 lienhardi (Pseudotocepheus) ...................... 145 lienhardi (Ptiloppia) ...................................... 128 lienhardi (Sabahtritia) ….................................. 47 lienhardi (Suctobelbella) ............................ 137 lienhardi (Zetomotrichus) .............................. 185 lienhardorum (Otocepheus) ........................... 147 ligamentifer (Stomacaus) …......................... 27 ligiangensis (Leobodes) …............................ 154 ligneus [P. (Archiphthiracarus)] ....................... 55 ligneus [P. (Archiphthiracarus)] .................... 55 lignivora (Dissorhina) (=ornata) ................ 125 ligulata (Cultroribula) ..................................... 92 ligulifera (Hoplophorella) (=hamata) ..........49 limae (Discoppia) …..................................... 123 limasetosa (Belba) ......................................... 77 limbata (Acrotritia) duplicata .......................... 45 limbulatus (Eupelops) sp. inq. ...................... 163 limburgiensis (Oribella) (=pectinata) .......... 107 limpida (Dometorina) ................................... 192 lindquisti (Eueremaeus) ................................. 100 lindsayae (Pheroliodes) ................................ 74 lineaporosa [O. (Zygoribatula)] ................ 189 linearis [B. (Passalobates)] ............................. 162 linearis [B. (Passalobates)] ......................... 162 linearis (Zetomotrichus) ................................ 185 lineata (Apoplophora) (=pantotrema) .......... 41 lineata (Cultroribella) ................................ 92 lineata [S. (Flagrosuctobelba)] ….............. 138 lineata (Machuella) ......................................... 133 lineata (Oribatula) (=repetita) …................ 188 lineata (Pergalumna) (=myrmophila) ........ 219 lineata (Trichogalumna) .................................. 22 lineata [O. (Zygoribatula)] ........................... 189

lineatus (Ameronothrus) ................................ 156 lineatus (Ameronothrus) ................................. 156 lineatus (Brachychthonius) ........................... 29 lineatus (Dolicheremaeus) ............................ 142 lineatus (Eremaeozetes) …........................... 165 lineatus (Eremaeozetes) .............................. 165 lineatus (Geminozetes) .............................. 176 lineatus [C. (Protoripoda)] …..................... 204 lineatus [H. (Tuberemaeus)] ........................... 193 lineatus [H. (Tuberemaeus)] ....................... 193 lineola [O. (Zygoribatula)] (=exilis) lineolata (Heterogalumna)

lineolatus (Bipassalozetes)

lineolatus (Constrictobates)

lineolatus (Dolicheremaeus) Bal. y Mah., 67

lineolatus (Dolicheremaeus) Ham., 81 (=obsessus)

lineolatus (Notophthiracarus) ......................

lineolatus (Trimalaconothrus) ..........

lingulatus [L. (Dorycranosus)] …................ 97

linieatus (Licneremaeus) …........................... 160

linjiangensis (Oribatella) ............................. 171

lionsi [L. (Hamoppia)] ................................ 111

lionsi (Notophthiracarus) ............................ 51

liparus [P. (Archiphthiracarus)] ....................... 55

lischanni (Melanozetes) sp. inq. .................... 176

lisosetosus [T. (Tyrphonothrus)] ..................... 63

litoralis (Mabulatrichus) ................................ 184

litoralis (Oribatella) arctica .......................... 170

litosus (Caenosamerus) ................................. 105

littlewoodi (Pulchroppiella) ........................... 118

littoralis (Halozetes) ……................................ 157

littorea (Schusteria) ...................................... 157

littoristicus [C. (Klapperiches)]

lividus (Rhacaplacarus)

lobata (Austrachipteria) Ham., 67

lobata (Austrachipteria) J. y P. B., 83

(=hammerae)

lobata (Karenella) ........................................ 130

lobata (Oripoda) ........................................... 204

lobata (Suctobelbella) acutidens ................. 136

lobatus (Cepheus) sp. inq.

lobatus (Reticulocepheus) (=decoui) ........... 84

lobatus (Torpacarus) ..................................... 41

lobatus (Zachvatkinibates) .......................... 184

lobodentata (Suctobelba) ............................. 135

loebli (Acrotocepheus) .................................. 146

loebli (Lohmannia) (=paradoxa) .................. 39

loebli (Rhacaplacarus) .................................... 57

lokobensis (Rhopalozetes) ........................... 90

loksai (Medioppia) .......................................... 123

loksai (Megalotocepheus) ............................ 147

loksai (Suctobelbella) ................................ 137

loksai [P. (Tentaculozetes)] ........................... 211

longesensillus (Achipteria) ............................. 167

longesensillus (Melanozetes) ......................... 176

longiareata (Pilogalumna) crassiclava .......... 220

longibulbula (Crotonia) ................................6 67

longicarinatus (Epieremulus) ...................... 106

longicaudatus (Loftacarus) ........................... 27

longicaulis (Anellozetes) ............................... 18

longiceps (Pheroliodes) .................................. 74

longiclava (Galumna) 216

longiclava (Neoamerioppia) ........................ 114

longiclava (Neoamerioppia)

longiclava [S. (Ussuribata)] ........................ 138

longicoma (Leoppia) .................................... 132

longicoma (Neoamerioppia) ........................ 114

longicoma (Peloribates) .............................. 210

longicornis (Gustavia) ................................... 95

longicornis (Gustavia) …............................... 95

longicornis (Protozetes) ................................... 90 longicornutus (Furcobates) (=hastatus) ...... 175

longicurva (Suctobelbella) .................. 11, 137

longicuspis (Mahunkazetes) ...................... 185

longicuspis (Scapheremaeus) ..................... 158

longicuspis (Suctobelbella) ......................... 137

longicuspis (Suctobelbella) ......................... 137

longicuspis (Suctobelbella) ........................ 137

longicuspis (Suctobelbella) ......................... 137

longicuspis [O. (Zygoribatula)] ……........... 189

longifilis (Ceratoppia) $s p$. inq. ..................... 93

longifissus (Eupelops) (=subuliger) ............ 164

longifolius (Phyllozetes) ….............................. 33

longilamellata (Autogneta) ............................ 108

longilamellata (Autogneta) .......................... 108

longilamellata (Moritzoppia) ......................... 126

longilamellata (Oribatula) Schwei., 56 ....... 187

longilamellata (Oribatula) Sub., 77

(=macrostega) ……................................... 187

longilamellatus (Liacarus) .............................. 96

longilamellatus (Scapheremaeus) ................ 158

longilamellatus [P. (Triaungius)] ................ 206

longilamellatus [P. (Triaungius)] .................. 206

longilinea [O. (Zygoribatula)] ..................... 189

longinqua (Nannerlia) ................................. 197

longior (Liebstadia) ............................. 12, 195

longior (Liebstadia) .............................. 12, 195

longior [E. (Multoribates)] .......................... 193

longior (Otocepheus) …................................ 147

longior (Otocepheus) .................................. 147

longior (Pergalumna) (=myrmophila) ........ 219

longior (Pilobates) (?=carpetanus) .............. 211

longipectinata (Trapezoppia) ...................... 123

longipedus [D. (Epidamaeus)] ......................... 79

longipes (Damaeus) Will., 41 .......................... 78

longipes (Damaeus) Mih., 57 (=flagellifer) .... 78

longipes (Neoliodes) $s p$. inq . .......................... 72

longipes (Podoribates) .................................. 186

longipes (Podoribates) .................................. 186

longipes (Trhypochthonius) $s p$. inq. ............ 60

longipes (Xenillus) ....................................... 98

longipila (Synichotritia) ................................... 48

longipilis (Chamobates) ............................... 180

longipilis (Jornadia) .................................... 187

longipilis (Liacarus) ...................................... 96

longipilis (Murcia) Willm., 51 ..................... 177

longipilis (Murcia) Mih., 58 (=brevicuspis) .. 177

longipilis (Zachvatkinella) ............................. 27

longipilosa (Dissorhina) ............................... 125

longipilosa [M. (Pravoppia)] (=loxolineata) .. 111

longipilosa [P. (Sarawakiella)] ....................... 220

longipilosus (Chaunoproctus) .................... 192

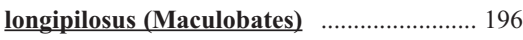

longipilosus (Peloribates) .............................. 210

longipilus (Asperemaeus) ................................. 99

longipilus (Dolicheremaeus) ....................... 142

longipilus (Globozetes) ................................. 180

longipilus (Liochthonius) ............................. 30

longipilus (Liochthonius) .............................. 30

longipilus [P. (Neotocepheus)] …................. 145

longipilus (Nothrus) Berl., 10 ....................... 63

longipilus (Nothrus) borussicus Mi., 59

(=neonominatus) ...................................... 63 


\begin{tabular}{|c|c|c|}
\hline gipilus (Oribatula) (=pannonica) & tosa (Epimerella) smirnovi & ngispinosus [D. (Epidamaeus)] \\
\hline 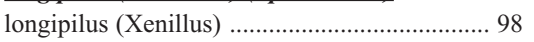 & ngisetosa (Eremobelba) ............................... 103 & longissima (Goyoppia) …................................ 113 \\
\hline longipluma (Acrogalumna) & ongisetosa (Hermanniella) …........................ 70 & ongissimus (Heteroleius) .............. \\
\hline longipluma (Acrogalumna) & 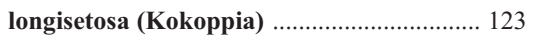 & longissimus (Heteroleius) ... \\
\hline longipluma (Acrogalumna) & ongisetosa (Mesoplophora) & longissimus (Perxylobates) \\
\hline longipluma (Acrogalumna) .......... & longisetosa (Microppia) minus ...................... 124 & longissimus (Vermacarus) ...................... \\
\hline longipluma (Acrogalumna) ....... & longisetosa (Microppia) minus .................... 124 & longitarsalis [D. (Epidamaeus)] ............ \\
\hline longipluma (Lauritzenia) …........................... 208 & longisetosa (Multioppia) ............................. 117 & longitarsalis [D. (Epidamaeus)] \\
\hline longipluma (Lauritzenia) .............................. 208 & longisetosa (Oribatella) superbula Ham., 77 .. 172 & longitudinalis (Suctobelbila) ................ \\
\hline longiporosa (Galumna) Fuji., 72 & longisetosa (Oribatella) F. у A., 78 & longiuscula (Protoribates) $s p$. inq. ........ \\
\hline & 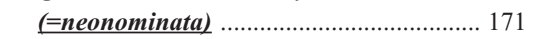 & longocuspidatus (Ceratozetes) ...... \\
\hline (=reiterata $)$ & longisetosa (Pergalumna) .................... & longohisterosoma (Moritzoppia) \\
\hline longiporosa (Pergalumna) ........... & sa (Quadroppia) .................... & galumna) ............... \\
\hline longiporosa (Phauloppia) Kulij., 68 & longisetosa (Setoppia) .... & longulus (Austrocarabodes) sp. inq. \\
\hline (=lucorum) & longisetosa (Suctobelbella) ........ & 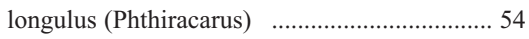 \\
\hline longiporosa (Phauloppia) P.-I., 76 & longisetosa (Tectoppia) ................ & longulus (Phthiracarus) ..... \\
\hline (=lucorum) & longisetosus (Annectacarus). & longus (Gymnobatoides) \\
\hline longiporosa (Phauloppia) Mah., 82 & longisetosus (Archegozetes) …............... & longus (Longocepheus) .......... \\
\hline (=lucorum) & longisetosus (Archegozetes) ............... & 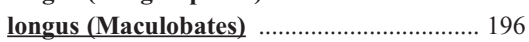 \\
\hline longiporosa (Phauloppia) Djap., 85 & longisetosus (Archegozetes) .... & longus (Parapirnodus) \\
\hline (=lucorum) & Carabodes) tenuis ...................... 150 & longus (Protoribates) (=oblongus) \\
\hline longiporosa [O. (Zygoribatula)] & Chaunoproctus) …................... 192 & \\
\hline 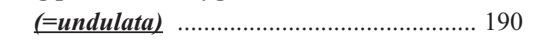 & longisetosus (Eobrachychthonius) ................... 30 & \\
\hline longiporosus (Maculobates) ........................ 196 & longisetosus [D. (Epidamaeus)] ……............... 79 & longus (Sch \\
\hline longiporosus (Minguezetes) ....................... 182 & sus (Eupelops) …............................. 163 & 154 \\
\hline longiporosus (Minguezetes) .......................... 182 & us (Ghilarovizetes) .......................... 176 & loongchiensis \\
\hline longiporosus (Scheloribates) ......................... 200 & longisetosus (Heminothrus) .............................. 65 & toribates) .. \\
\hline us (Unguizetes) …........................ 186 & 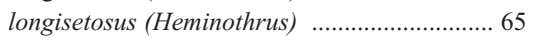 & .206 \\
\hline longiporus (Acrogalumna) (=longipluma) .. 213 & 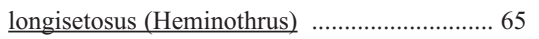 & .206 \\
\hline longiramosa (Wallworkoppia) (=cervifer) $\quad . .121$ & osus (Megatrichobates) ......... & toribates) ....... \\
\hline longirostralis (Euphthiracarus) ........................ 46 & zetes) …..................... 176 & rotoribates) \\
\hline stralis (Paralobozetes) .......................... 186 & tacarus) …........................ 39 & Pheroliodes) ................ \\
\hline stris (Gustavia) (=maior) .................... 95 & es) Will., $30 \quad \ldots \ldots \ldots \ldots . . . .210$ & inq. ...................... \\
\hline pelbella) …................ 11, 137 & longisetosus (Peloribates) Will., 30 .............. 210 & hermannia)] ........... \\
\hline$\ldots \ldots \ldots \ldots \ldots . .11,137$ & longisetosus (Peloribates) Will., $30 \quad \ldots \ldots \ldots \ldots . . .210$ & umna) ....................... \\
\hline laconothrus) Willm., 29 .... 62 & longisetosus (Peloribates) Tseng, 84 & 216 \\
\hline (Trimalaconothrus) H., 66 & (=praeoccupatus) & cepheus) \\
\hline 62 & es) & .200 \\
\hline cula) …........................ 197 & ndocepheus) ....................... 147 & ta [M. (Pravoppia)] ............... \\
\hline goribatula)] .................. 189 & oribates) …......................... 200 & lucasi (Scheloribates) (=laevigatus) ... \\
\hline oppia) …...................... 126 & longisetosus (Steganacarus) (=herculeanus) .. 58 & iformis (Scheloribates) $s p$. inq. ... \\
\hline elbella) ...................... 137 & arus) ............................. 41 & ... 208 \\
\hline [rodamaeus)] ........................ 81 & longisetosus (Trimalaconothrus) .......... &. .178 \\
\hline ) pilosella $\ldots . . .$. & sus [H. (Tuberemaeus)] ...... & 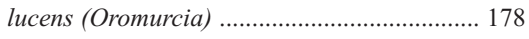 \\
\hline eremaeus) magnus .......... 143 & sus (Xenillus) ................................... 98 & 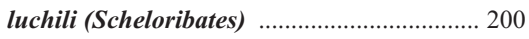 \\
\hline (Epieremulus) ........................... 106 & ius (Yungaseremaeus) .................. 106 & lucianus (Microtegeus) \\
\hline 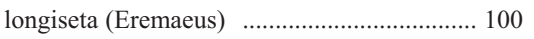 & s (Galumnopsis) .......................... & crotritia) (=curticephala) ....... \\
\hline (Genavensia) ..................................... 173 & 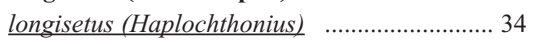 & lucida (Phauloppia) (=rauschenensis) \\
\hline longiseta (Globoppia) intermedia $\ldots . . . \ldots \ldots . . . . . .110$ & ..... 160 & 142 \\
\hline longiseta (Globoppia) intermedia ............... 110 & racarus) .......... & .... 96 \\
\hline 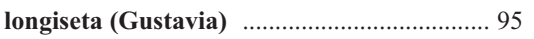 & longisetus (Oribatodes) (=mirabilis) & lucidus (Phthiracarus) (=piger) \\
\hline longiseta (Mesoplophora) $(=$ cubana) ............ 42 & longisetus (Pheroliodes) .................... & 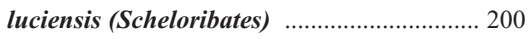 \\
\hline 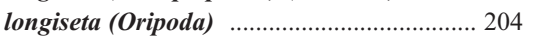 & longisetus (Sphaerochthonius) & lucifer (Chamobates) (=cuspidatus) ... \\
\hline longiseta (Sadocepheus) & longisetus (Sturmozetes) ................................ 91 & lucorum (Phauloppia) .... \\
\hline 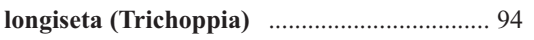 & ochthoniellus) ....................... 60 & $\ldots 190$ \\
\hline longiseta [O. (Zygoribatula)] ........................ 189 & longisetus (Trhypochthoniellus) ......................... 60 & luculenta (Neolucoppia) \\
\hline longisetae (Halozetes) belgicae ..................... 156 & s (Trhypochthoniellus) .................. 60 & ....... 63 \\
\hline longisetae (Protoribates) (=oblongus) $\ldots . . . . .206$ & longisetus (Trhypochthoniellus) & us) sp. inq. . \\
\hline longisetosa (Arcoppia) .................................. 121 & longisetus (Trhypochthoniellus) ...................... 60 & .................. 171 \\
\hline longisetosa (Arcoppia) ............................... 121 & longispina (Ceratozetella) ............................. 173 & .. 131 \\
\hline longisetosa (Banksinoma) & longispina (Ceratozetes) . & luisiae (Rugoppia) .. \\
\hline longisetosa (Ctenobelba) ……......................... 101 & longispina (Oribatella) ……............................. 171 & luisiae (Triteremella) \\
\hline
\end{tabular}


luminosa (Cyrthermannia) …………............67 67

luminosa (Diphauloppia) ............................. 186

luminosa (Oripoda) ……………………...... 204

luminosus (Perscheloribates) ......................... 198

luminosus (Perscheloribates) ..................... 198

lumotus (Perscheloribates) ………………....... 198

lunai (Trichogalumna) ................................. 221

lunare (Banksinoma) lanceolata ...................... 109

lunaris (Austrocarabodes) ........................... 148

lunaris (Berniniella) ………………………... 125

lunaris (Galumna) ………………………..... 216

lunaris (Microzetes) ………………………….... 89

lundbladi (Neamerus) ...................................... 105

lupitae (Kalyptrazetes) ..................................... 89

luridus (Phthiracarus) ...………………….... 54

luscus (Liacarus) …………………………...... 96

lustrum (Limnozetes) ..................................... 166

lutea (Ceratoppiella) .......................................... 94

lutea (Kodiakella) ............................................. 99

luteoauratus [C. (Klapperiches)] …........... 151

luteomarginatus (Lopholiodes) ..................... 73

luteomarginatus (Maculobates) ……….... 196

luteomarginatus (Scheloribates) ................... 200

luteus (Anellozetes) ………………………..... 181

luteus (Hypochthonius) ………………………. 29

luteus (Hypochthonius) ................................... 29

luteus (Hypochthonius) ……………………..... 29

luteus (Hypochthonius) ………………….... 29

luteus (Maculobates) ……………………...... 196

luteus (Neogymnobates) .................................. 177

luteus (Perscheloribates) ................................ 198

luteus [P. (Triaungius)] ................................... 206

Iutulentus (Atropacarus) (=clavigerus) …..... 48

luxtoni [L. (Baioppia)] ………………......... 110

luxtoni (Dolicheremaeus) .............................. 142

luxtoni [R. (Insculptoppia)] ………………... 119

luzonensis (Perscheloribates) ………………. 198

luzonensis [H. (Tuberemaeus)] ……….......... 193

luzonica (Dampfiella) ..................................... 141

luzonica (Magyaria) ……………………….... 209

lydia [N. (Parakalumma)] ............................... 212

lydia [N. (Parakalumma)] ………………….... 212

lydiae (Sellnickochthonius) ............................ 32

lydiae (Sellnickochthonius) ............................ 32

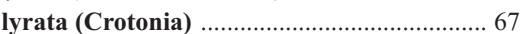

lyrica (Galumna) .......................................... 216

lyroseta (Ramusella) ..................................... 118

mabar [O. (Zygoribatula)] ………………... 189

macedi (Nothrus) ............................................. 63

macedonicus (Passalozetes) ............................. 162

macerochaeta (Metabelbella) ............................ 81

macfarlanei (Africoribates) ......................... 180

macfarlani (Stomacarus) ................................. 27

macfarlani (Stomacarus) …………………... 27

machadoi (Acrogalumna) ........................... 213

machadoi (Dolicheremaeus) ........................ 142

machadoi (Eremaeozetes) ........................... 165

machadoi (Metabelba) ........................................ 80

machadoi (Phthiracarus) ................................ 54

machadoi (Plasmobates) sp. inq. .................. 71

machadoi (Rhacaplacarus) ………………... 57

machadoi (Rhynchobelba) machadoi (Striatoppia)

machadoi (Striatoppia)

machadoi (Wallworkoppia)

mackenziensis [D. (Epidamaeus)]

macleani (Proteremaeus)

macquariensis (Halozetes) D. $58(A$.

$$
\text { (=marinus) }
$$

macrodentata (Austrachipteria)

macrodentata (Suctobelbella)

macrodentatus (Acrotocepheus)

macrodon (Sucobelbella) (=subcornigera)

macrofissura (Micreremus)

macrofoliatus (Archegocepheus)

………..... 14

174

macromucronatus (Leptotocepheus) ........ 144

macroonvx (Totobates) ……..................... 196

macropoda (Belba) …………………………….... 77

macroporosus (Eupelops) torulosus ............... 164

macroprionus (Platyliodes) ............................. 72

macroprionus (Platyliodes) ............................. 72

macroptera (Galumna) .................................. 216

macropunctinatum (Lopholiodes) ................ 73

macrorostrum (Parhypochthonius) sp. inq. .... 28

macrosacculatus (Neoribates) ...………….... 212

macrosculpturatus (Atropacarus) ……………... 48

macroseta [S. (Flagrosuctobelba)] ................. 138

macrosetosus (Cosmochthonius) ...…………. 33

macrosetosus (Ethiovertex) ......................... 161

macrostega (Oribatula) ……………………..... 187

maculata (Fortuynia) ……………………. 157

maculata [S. (Lalmoppia)] …………........... 130

maculata [S. (Lalmoppia)] ……………….... 130

maculata [S. (Lalmoppia)] ………………... 130

maculatus (Ameronothrus) ………………..... 156

maculatus (Ameronothrus) ............................ 156

maculatus (Ameronothrus) ............................ 156

maculatus (Austrocarabodes) ……………... 148

maculatus (Notophthiracarus) ....................... 51

maculatus (Papillonotus) ............................. 133

maculatus (Scheloribates) ........................... 200

maculosa (Crassoribatula) ........................... 186

maculosa (Ctenobelba) (?=pectinigera) ....... 101

maculosus (Atropacarus) ................................ 48

maculosus (Eremaeozetes) ………………….... 165

madagascarensis (Allogalumna) ................. 213

madagascarensis (Hermanniella) ................ 70

madagascarensis (Lanceoppia) ................... 110

madagascarensis (Meristacarus) ................... 39

madagascarensis (Nothrus) ............................. 64

madagascarensis (Oribatella) ..................... 171

madagascarensis (Protoribates) ................ 206

madagascarensis (Striatoppia) …………... 131

madagassica (Bursoplophora) ........................ 35

madagassica (Pustuloppia) ......................... 112

madagassica (Trichogalumna) ..................... 221

madecassus (Rhopalozetes) ......................... 90

madegassus (Pseudopirnodus) ................... 204

madeirensis (Anomaloppia) ……................... 116

madeirensis (Liacarus) ……………………...... 96

madeirensis (Scapheremaeus) (=corniger) .. 158

madininensis (Teleioliodes) ............................. 72 maerkeli (Lanceoppia) …………………...... 110

maerkeli (Mesotritia) ……………………….... 43

maerkeli (Mesotritia) ......................................... 43

magdalenae (Scapheremaeus) ..................... 158

magellanis (Anderemaeus) .......................... 106

magellanis (Austroppia) (=crozetensis) ...... 122

maghrebinus [S. (Tropacarus)] ......................... 59

magkakaibaeus (Peloribates) ……………...... 210

magna (Ceratozetella) imperatoria ................. 173

magna (Furcoribula) (=furcillata) ................ 92

magna [B. (Gressittoppia)] ……................. 122

magna (Heterobelba) ...................................... 104

magna (Hoplophorella) $(=$ sculptilis) $\quad$............ 49

magna (Mongaillardia) …………………..... 101

magna (Oribotritia) ........................................... 44

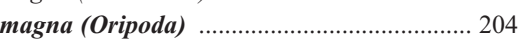

magna (Parachipteria) _................................. 169

magna (Parapyroppia) (=cornuta) ................ 94

magna [O. (Zygoribatula)] (=connexa) …... 188

magnifera [S. (Flagrosuctobelba)] ............. 138

magnifica (Cultroribula) ................................. 92

magnifica (Gustavia) ....................................... 95

magnificus (Zetomimus) (=furcatus) .......... 179

magnilamellata (Anachipteria) .................... 168

magnilamellata (Gustavia) .............................. 95

magnilamellatus [T. (Tyrphonothrus)] ............. 63

magnipilosa (Aeroppia) ................................. 112

magnipora (Pergalumna) .............................. 219

magniporosa (Paraphauloppia) .................. 190

magniporosus (Uracrobates) ……………....... 186

magniporus (Eueremaeus) .............................. 100

magniserrata (Spinoppia) ........................... 191

magniseta (Oribatella) (=quadridentata) .... 171

magnisetosus (Adrodamaeus) .......................... 75

magnisetosus (Peloribates) ………………... 210

magnisetosus [T. (Tyrphonothrus)] ………... 63

magnodentata [S. (Ussuribata)] ...................... 139

magnum (Polyoppia) ..................................... 112

magnus (Archegozetes) .................................. 59

magnus (Archegozetes) ……………………..... 59

magnus (Archegozetes) …………………….... 59

magnus (Archegozetes) .................................. 59

magnus (Carabodes) ........................................ 150

magnus (Damaeolus) ........................................ 103

magnus (Dolicheremaeus) …………........... 142

magnus (Dynatozetes) .................................... 185

magnus (Eohypochthonius) ………………..... 28

magnus (Eohypochthonius) ……………….... 28

magnus (Eohypochthonius) ......................... 28

magnus (Eremaeus) (=hepaticus) ................. 100

magnus (Humerobates)

(?=guadarramicus) ……………………... 181

magnus (Maculobates) …………………….... 196

magnus (Neobrachychthonius) .......................... 31

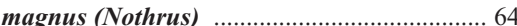

magnus (Protoribates) ……………………….. 206

magnus (Protoribates) …………………….... 206

magnus [D. (Saltatrichus)] ………………..... 184

magnus (Scheloribates) …………………...... 200

magnus (Steganacarus) ..................................... 58

magnus (Steganacarus) …………………..... 58

magnus (Steganacarus) …………………….... 58 


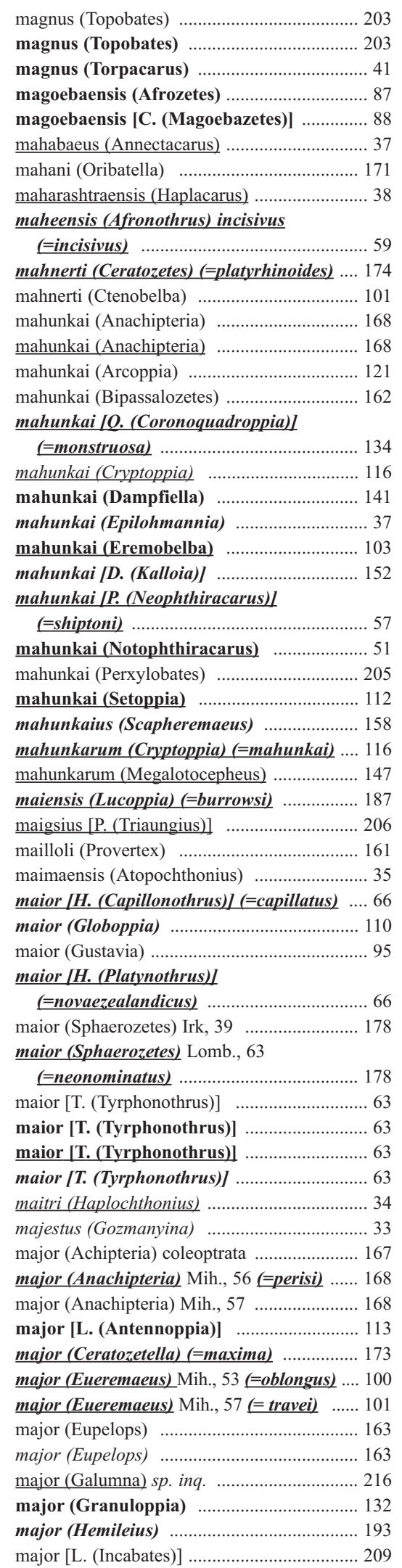

major [L. (Incabates)] ..................................... 209 major (Liacarus) (=brevilamellatus) $\quad$............ 95 major (Minunthozetes) (=semirufus) .......... 182 major (Oribatula) $(=$ saxicola) ........................ 187 major (Prototritia) ....................................... 36 major (Scheloribates) praestantissimus .... 201 major (Tritegeus) ……................................... 84 makarcevae (Sadocepheus) ………………..... 84 makartzevi (Sucteremaeus) ……………….... 135 makilingensis (Basiceramerus) ……………... 146 makilingensis (Truncopes) ............................ 205 makinisus (Birobates) ………………………... 203 malabarica (Siculobata) ………………….... 195 malabaricus (Drymobatoides) ....................... 185 malagensis (Phthiracarus) …………………... 54 malakipinae (Rhynchoppia) .......................... 135 malangensis (Dolicheremaeus) …................. 143 malapectinata (Pulchroppia) ………................ 120 malatestai [S. (Tropacarus)] ………………...... 59 malaya (Apoplophora) ………........................ 41 malaya (Oribatella) …………………….......... 171 malleolus (Neoliodes) (=concentricus) .......... 72 mammillaris (Masthermannia) ......................... 68 mammillaris (Masthermannia) _.....................68 68 mammillaris (Masthermannia) ........................... 68 mammillaris (Masthermannia) ..................... 68 mammillaris (Masthermannia) ........................ 68 mammillatus [T. (Rostrozetes)] ………….... 211 manantialis (Tuxenia) ................................. 207 mandjeliae (Pentazetes) …………………... 178 manganellii (Steganacarus) ............................5 58 manganoi [C. (Klapperiches)] ....................... 151 manguiati (Bathocepheus) ............................. 149 maniculatus (Trimalaconothrus) ...................... 62 manifera (Anomaloppia) …………………..... 116 manifera (Anomaloppia) .............................. 116 manifera (Dolicheremaeus) ……………….... 143 manifera [C. (Klapperiches)] ……………...... 151 manipurensis (Hoplophorella) ......................... 49 manoai (Scheloribates) ............................... 200 manzanoensis (Minguezetes) (=insignis) .... 182 maoershanensis (Caenosamerus) spatiosus .... 105 maoershanensis (Oribellopsis) crosbyi ........ 107 maoriensis (Scheloribates) ............................ 200 marcuardi (Apoplophora) ................................. 41 marcuzzii (Ctenobelba) ……………………... 101 margaritae (Stictozetes) …………………... 22 margaritata (Pergalumna) ………………….... 219 margaritata (Striatoppia) ……………….... 131 margaritata (Suctobelbila) ........................... 139 margaritatus [L. (Undulozetes)] ……......... 89 margaritifera (Allogalumna) ....................... 213 margaritifera (Striatoppia) _........................ 131 margatensis (Eupelops) ............................... 163 marginalis (Scapheremaeus) ........................ 158 marginata (Pilogalumna) (?=tenuiclava) .... 220 marginatus (Belbodamaeus) ………………..... 78 marginatus (Benoibates) ………………...... 203 marginatus (Carabodes) ................................ 150 marginatus (Gressittolus) ................................. 103 marginatus (Gressittolus) ……………….... 103 marginatus (Javacarus) $s p$. inq. …….......... 38 marginatus (Liacarus) $s p$. inq. ....................... 96 marginatus (Malaconothrus) .............................61 marginatus (Neobrachychthonius) ................... 31 marginatus (Notophthiracarus) …………..... 51 marginatus (Scutovertex) ………………... 161 marginatus (Sturmozetes) .............................. 91 marginedentata (Lauroppia) falcata .............. 126 marginedentatus (Scheloribates) sp. inq. .... 200 marginepunctatus (Carabodes)

(=labyrinthicus) ………………………..... 150 margosetosa (Xiphobelba) (=hamanni) ...... 104 mariae (Galumna) ............................................ 216 mariae (Galumna) ……………………..... 216 mariana [O. (Zygoribatula)] acuminata .. 188 mariannae (Pseudoceratoppia) .................... 94 marianoi (Gehypochthonius) ........................ 28 marianus (Hoplophthiracarus) ..................... 50 marieae (Austrachipteria) (=hammerae) .... 166 mariehammerae (Aeroppia) ........................ 112 mariehammerae (Dolicheremaeus) ............... 143 mariehammerae [O. (Zygoribatula)] ............. 189 marilynae (Neogymnobates) …………….... 178 marina (Fortuynia) ……………………...... 157 marina [O. (Zygoribatula)] ............................. 189 marinus (Ameronothrus) …………………….. 156 marinus (Ameronothrus) ................................ 156 marinus (Halozetes) ………………………....... 157 marinus (Halozetes) ………………………..... 157 marinus (Halozetes) …………………........... 157 marionensis (Dometorina) ………………..... 192 marionensis (Halozetes) ………………......... 157 marionensis (Totobates) .................................. 196 maritalis (Quadroppia) ……………………... 133 maritima (Corynoppia) ……………………... 130 maritima (Lauroppia) …………………….... 126 maritima (Lauroppia) ................................... 126 maritimus (Hoplophthiracarus) …………….... 50 maritimus (Zachvatkinibates) .......................... 184 maritimus (Zachvatkinibates) .......................... 184 markusi (Dolicheremaeus) ............................. 143 marlenae (Crotonia) ......................................... 67 marmoratus (Scapheremaeus) .................... 158 maroccanus (Cosmochthonius) …………....... 33 maroccanus (Sellnickochthonius) …............... 32 marplatensis (Neoliodes) ................................ 72 marquesalis (Scheloribates) indicus ……... 200 marquesana (Pergalumna) hawaiiensis .... 219 marquesi (Pergalumna) bryani ................... 218 marshalli (Eueremaeus) ................................ 100 marshalli (Multimaudheimia) ................. 7, 195 marshalli [D. (Saltatrichus)] …………….... 184 martii (Schalleria) ......................................... 90 maruyamai (Ghilarovizetes) ………………..... 176 masahitoi (Autogneta) ................................... 108 masahitoi (Autogneta) …………………........ 108 masinasin (Eueremaeus) ................................ 100 mastax (Lineoppia) ...................................... 129 mastigophora (Aegyptogalumna) .................... 213 mastigophora (Medioxyoppia) ....................... 124 mastyx (Hermanniella) …………………..... 70 matecumbei (Galumna) macroptera ............ 216 matildebellae (Eremella) ............................... 106 
matritensis (Corynoppia) kosarovi ................ 130 matritensis (Oribella) ................................... 107 matritensis (Pergalumna) semistritata .......... 219 matritensis [O. (Zygoribatula)] (=glabra) .. 189 matshabelii (Liacarus) ................................... 96 matskasii (Xenillus) ...................................... 98 matulisus (Scheloribates) ............................. 200 maurensis (Pilobatella) ............................... 211 maurii (Epilohmannia) .................................. 37 mauritanica (Grandjeanoplophora) ................. 35 mauritii (Dolicheremaeus) .......................... 143 mauritii (Galumna) ..................................... 216 mauritii [H. (Phyllhermannia)] Jac., 36 ...... 69 mauritii [H. (Phyllhermannia)] Mah., 78

(=neonominata) ………………................... 69

mauritii (Pergalumna) ................................ 219

mauritii (Pergalumna) ............................... 219

mauritii (Suctobelbella) claviseta ............... 136

mauritius (Drymobatoides) ........................ 185

mauritius (Humerobates)

rostrolamellatus ........................................ 182

mauritius (Neoliodes) .................................... 72

mauritius (Unguizetes) ............................... 186

maurus (Notophthiracarus) ……................... 51

mausiae (Hauserozetes) ................................8 88

maxensis (Oripoda) ...................................... 204

maxima (Amerobelba) (=decedens) $\quad$............. 101

maxima (Austrachipteria) .......................... 166

maxima (Ceratozetella) Berl., 08 .................. 173

maxima (Ceratozetella) Will., 53

(=maxima Ber.)

maxima (Galumna) Berl., $16 s p$. inq _... 216

maxima (Galumna) alata Willm., 39

(=multiiterata)

maxima (Galumna) Mih., 56 (=lanceata) .. 216

maxima (Gittella) ......................................... 122

maxima (Maerkelotritia) (=cryptopa) ............ 43

maximus (Africoribates) ............................. 180

maximus (Damaeus) ......................................... 78

maximus (Mochlozetes) ................................. 185

maximus (Rhopalozetes) ................................. 90

maximus (Steganacarus) sp. inq. .................... 58

maximus [P. (Triaungius)] ............................ 206

mayeriana (Achipteria) .................................. 167

mayottei (Notophthiracarus) ......................... 51

mayuloeus [S. (Plenoxylobates)] .................. 207

mcadami (Arcoppia) .................................... 121

mcadami (Arcoppia) .................................. 121

meakanensis [H. (Capillonothrus)] ................. 66

meakanensis (Nothrus) .................................... 64

media [Q. (Coronoquadroppia)] .................... 134

media (Epilohmannia) cylindrica ................ 36

media (Galumna) ......................................... 216

media [L. (Incabates)] (=angusta) .............. 209

media (Medioppia) ......................................... 123

media (Medioppia) ..................................... 123

media (Suctobelba) ......................................... 135

medialis (Liacarus) ...................................... 96

medialis (Pergalumna) ................................... 219

medialis (Tenuialoides) ................................. 99

medialis (Tenuialoides) ….............................. 99

medialis [S. (Ussuribata)] .............................. 139 mediocris [P. (Archiphthiracarus)] .................. 55

mediocris (Ceratozetes) ................................ 174

mediocris (Ceratozetes) ................................ 174

mediocris (Ceratozetes) ............................... 174

mediocris (Ceratozetes) …......................... 174

mediocris (Oribatella) ............................... 171

mediocris (Pseudoppia) ............................... 191

mediolamellatus (Tegeocranellus) ................ 155

mediosetosus (Archegozetes) magnus .......... 59

mediosetosus (Archegozetes) magnus ......... 59

mediterraneus (Arthrodamaeus) ...................... 76

mediterraneus (Selenoribates) ...................... 157

medius (Melanozetes) sp. inq. ...................... 176

medius (Pseudotocepheus) .......................... 145

medius (Topobates) ......................................... 203

medusa (Unduloribates) ................................ 165

megacephalum (Granuloppia)

(=neonominata) ......................................... 132

megacephalum (Hydrozetes) sp. inq. ............ 155

megaforamina (Oribatula) ........................ 187

megale (Oribotritia) .......................................... 44

megalonyx (Scheloribates) .......................... 200

megalotrichus (Stelechobates) ................... 192

meghalayensis (Euphthiracarus) ..................... 46

megistos (Eremaeus) .................................. 100

melanesiae (Crotonia) (=nukuhivae)

melanomerus (Schusteria) ......................... 157

melisi (Medioppia) ......................................... 123

melloi (Pergalumna) .................................... 219

membranifer (Phthiracarus) ............................ 54

membranifer (Phthiracarus) …...................... 54

membraniger (Eutegaeus) ……................... 85

membranigera (Dudichella) …....................... 85

membranigera (Dudichella) ........................ 85

membranulifer (Phylacozetes) ..................... 90

memorabilis [S. (Flagrosuctobelba)] ............ 138

menglunensis (Allosuctobelba) ................... 134

menglunensis (Pergalumna) .......................... 219

menkei [L. (Baioppia)] ................................ 111

mensarosi (Atalotegaeus) ............................. 85

mercedesae [T. (Paragloboppia)] ................... 115

meridiana [M. (Akrodamaeus)] ..................... 81

meridianus (Melanozetes) ............................ 176

meridianus (Melanozetes) ............................. 176

meridianus (Punctoribates) ............................ 183

meridionalis (Belba) ......................................... 77

meridionalis (Bursoplophora) ......................... 35

meridionalis [N. (Calyptophthiracarus)] ........ 53

meridionalis (Chudalupia) ........................... 156

meridionalis (Eupelops) .............................. 163

meridionalis [S. (Flagrosuctobelba)] ............ 138

meridionalis [L. (Incabates)] (=pallida) ...... 209

meridionalis [V. (Latilamellobates)]

(=incisella) .............................................. 179 meridionalis (Oribatella) (=superbula) ...... 172

meridionalis (Paraceratoppia) ...................... 94 meridionalis (Sellnickochthonius) .................. 32 meridionalis [O. (Zygoribatula)] (=glabra) .. 189 merimna [R. (Insculptoppia)] ..................... 119 meristacaroides (Meristolohmannia) .......... 39

meruensis (Amerioppia) ............................. 113

meruensis [O. (Zygoribatula)] .................... 189 mesosetosa (Oribatula) …........................ 187

messneri (Suctobelbella) ............................ 137

meszarosi [H. (Kakophthiracarus)]

(=tuberculosissima $)$................................... 50

metulifera [M. (Moritzoppiella)] .................. 127

metzi (Mochloribatula) ................................. 185

mexicana (Balazsella) ................................. 192

mexicana (Schalleria) .................................. 90

mexicana [M. (Dysarcoppia)] ...................... 121

mexicanus (Microtegeus) ............................. 86

meyeri (Antarcticola) ..................................... 156

michaeli (Cepheus) (=cepheiformis) ............... 82

michaeli [Q. (Coronoquadroppia)] ................ 134

michaeli [D. (Epidamaeus)] ........................... 79

michaeli [D. (Epidamaeus)] ............................ 79

michaeli (Eueremaeus) ................................. 100

michaeli (Metabelba) (=papillipes) ................. 80

michaeli (Notophthiracarus) ......................... 51

michaeli (Scheloribates) (=laevigatus) ........ 200

michaeli (Steganacarus) ................................... 58

michaeliana (Mesoplophora) ......................... 42

michaeliana (Mesoplophora) ......................... 42

michaii (Huilicheremaeus) ......................... 160

micheli (Gibbicepheus) ............................... 152

mickii (Halozetes) belgicae ........................... 156

microcephala (Gustavia) ............................... 95

microcephala (Gustavia) ……....................... 95

microcephala (Gustavia) ................................ 95

microchaetus (Birsteinius) ............................. 95

microclava [N. (Coartobelba)] ..................... 135

microclava (Hemileius) .............................. 193

microclava (Heminothrus) ............................. 65

microclava (Scheloribates) (=iteratus) ........ 200

microdentata [F. (Mexicoppia)] ….................. 92

microdentata [M. (Moritzoppiella)] .............. 127

microdentata (Suctobelbella) ...................... 137

microfissum (Galumna) .............................. 216

microfoveolata (Oribatella) .......................... 171

microfoveolata (Oribatella) .......................... 171

microlaminatus (Austrocarabodes) .......... 148

microlancearia (Lanceoppia) ...................... 110

micromorpha (Fortuynia) elamellata ........ 157

micronychus (Zetorchestes) ............................ 91

microporosa (Allogalumna) ....................... 213

microporosa [O. (Zygoribatula)] .................. 189

microporosus (Stictozetes) ......................... 221

microptera (Conoppia) Berl., 85

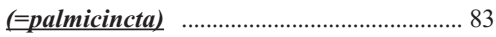

microptera (Conoppia) Can., 86

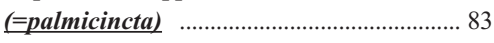

microptera (Liebstadia) (=pannonica) ........ 195

micropterus [M. (Megazetes)] ….................. 89

micropunctinatum (Lopholiodes) ................... 73

microsculptratus (Bipassalozetes) ................ 162

microseta (Beckiella) .................................... 141

microseta (Metrioppia) (=helvetica) .............. 94

microseta (Neoamerioppia) longiclava ...... 114

microseta (Pocsia) .......................................... 47

microseta (Totobates) .................................... 196

microseta (Trichogalumna) ...................... 221

microsetosa (Hermanniella) ......................... 70

microsetosa (Pseudoceratoppia) .................. 94 


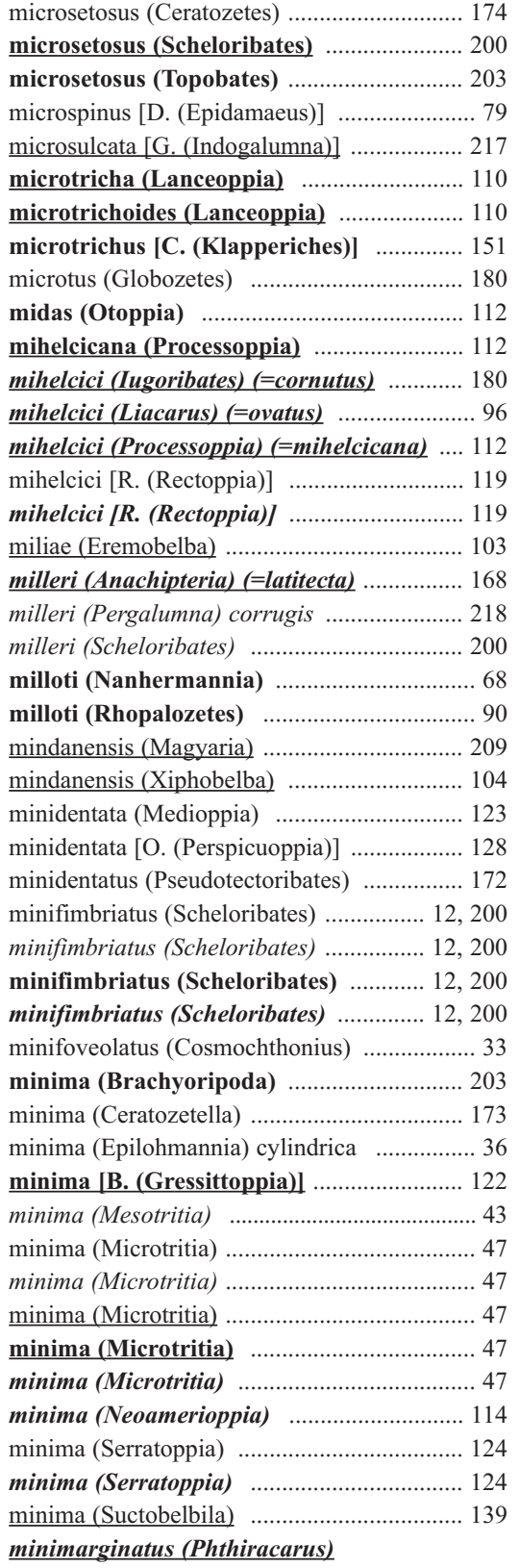

(=boresetosus) ….........................................53

minimedia (Medioppia) ................................. 123

minimicoma (Lauritzenia) ........................... 208

minimicoma (Lauritzenia) .......................... 208

minimus (Benoibates) ................................ 203

minimus (Charassobates) ............................... 86

minimus (Micreremus) (=brevipes) ........... 160

minimus (Nodocepheus) ............................... 85

minimus (Nothrus) sp. inq. ............................. 64

minimus (Perscheloribates) ....................... 198

minimus (Punctoribates) .............................. 183

minimus (Totobates) ................................... 196

minimus [H. (Turcibates)] ........................... 194

miniporus [M. (Safrobates)] ….................. 176

minisetosa (Hoplophorella) minitricha [A. (Uluguroides)]

minnesotensis (Heterozetes) ........................... 173

minnesotensis (Propelops) ........................... 164

minor (Afroleius) ....................................... 207

minor [L. (Antennoppia)] .......................... 113

minor [P. (Archiphthiracarus)]

$$
\text { (=bulbiferus) }
$$

minor (Belba) sp. inq. …............................... 77

minor (Ceratozetes) gracilis .......................... 174

minor (Chaunoproctus) ............................. 192

minor (Chaunoproctus)

minor (Cyrtozetes) denaliensis ...................... 174

minor (Dolicheremaeus) Berl., 13 ............... 143

minor (Dolicheremaeus) capill. W., 62

(=neonominatus)

minor (Dolicheremaeus) magnus W., 62

(=iteratus

minor (Eupelops) acromios ........................... 163

minor (Globoppia) ........................................ 110

minor (Halozetes) marinus ............................. 157

minor (Heminothrus) ........................................ 65

minor (Hoplophthiracarus) (=kugohi) .......... 50

minor (Jacotella) (=quadricaudicula) ..........76

minor (Maculobates) …........................... 196

minor (Mycobates) ......................................... 183

minor (Pergalumna) ....................................... 219

minor (Pergalumna) ..................................... 219

minor (Plasmobates) $s p$. inq

minor (Sternoppia) ........................................ 133

minor (Suctobelbella) ................................... 137

minor (Tectocepheus) ................................... 154

minor (Tectocepheus) .................................... 154

minor (Tectocepheus) .................................. 154

minor (Tectocepheus) ................................ 154

minor (Teratoppia) .................................... 132

minor (Topobates) (=scheloribatoides) ........ 203

minoricana (Pergalumna) .............................. 219

minus (Hoplophthiracarus) (=histricinus) .... 50

minus (Microppia) ........................................ 124

minus (Microppia) ....................................... 124

minus (Microppia) ......................................... 124

minus (Microppia) ........................................ 124

minus (Microppia) ..................................... 124

minus (Microppia) .......................................... 124

minus [D. (Saltatrichus)] ........................... 184

minuscula (Cerachipteria) ............................ 168

minusculus [C. (Klapperiches)] ..................... 151

minusculus (Perscheloribates) 198

minusculus (Podoribates) ............................ 186

minusculus (Hammerabates) ......................... 197

minuta (Achipteria) sp. inq. ........................ 167

minuta (Belba) ................................................... 77

minuta (Berniniella) ......................... 125

minuta (Epilohmannia) ................................... 37

minuta (Eremobelba) …................................. 103

minuta (Galumna) ........................................ 216

minuta (Galumna) ........................................ 216

minuta [H. (Heterohermannia)] ....................... 69

minuta (Microtritia) (=minima) ..................... 47

minuta [M. (Moritzoppiella)] ........................ 127

minuta (Neoppia) .......................................... 109

minuta (Oppia) (=nitens) .............................. 115 minuta (Oribatella) ..................................... 171

minuta (Oribatula) (=tibialis) ..................... 188

minuta (Pseudoamerioppia) ........................... 118

minutipes (Micreremus) (=brevipes)

minutisetarum (Novoribatella) .................. 170

minutisetosa [K. (Glabroppia)] .................... 130

minutissima (Dameobelba) ............................ 80

minutissima (Hypochthoniella) ...................... 29

minutissima (Hypochthoniella) ...................... 29

minutissima (Hypochthoniella) .................... 29

minutissima (Hypochthoniella) ..................... 29

minutissima (Hypochthoniella) .................... 29

minutissima (Hypochthoniella) .................... 29

minutissima (Microppia) (=minus) ................ 124

minutissima (Pteroripoda) ......................... 204

minutissimus [P. (Archiphthiracarus)]

(=pygmaeus) ……............................... 56

minutissimus (Ceratozetes) Willm., 1951 .... 174

minutissimus (Ceratozetes) Sub. y Ari.,

2000 (=nanus) .......................................... 174

minutus (Eupelops) .................................. 163

minutus [I. (Haplozetes)] .............................. 208

minutus (Pedunculozetes) ........................ 180

minutus (Peloribates) (=repetitus) .............. 210

minutus (Perscheloribates) ....................... 198

minutus (Perscheloribates) ............................ 198

minutus (Pheroliodes) .................................... 74

minutus (Scutovertex) …............................... 161

minutus (Scheloribates)

(=praeoccupatissimus) $\quad$.............................. 201

minutus (Vilhenabates) ……....................... 207

mirabilis (Atropacarus) ..................................... 48

mirabilis (Cerocepheus) ............................... 84

mirabilis (Dicrotegaeus) …........................... 84

mirabilis (Dinozetes) .................................... 88

mirabilis (Gerloubia) (=hispanica) .............. 191

mirabilis (Hammerella) ................................ 132

mirabilis (Hardybodes) .................................. 152

mirabilis (Hardybodes) ............................. 152

mirabilis (Hymenozetes) …......................... 88

mirabilis (Hypocepheus) .................................. 83

mirabilis (Hypovertex) ................................. 161

mirabilis (Leobodes)

mirabilis (Lopheremaeus) ………..................... 73

mirabilis (Nasobates) ................................... 212

mirabilis (Nothrus) .............................................. 64

mirabilis (Oribatodes) .................................... 83

mirabilis (Pheroliodes) ……………………. 74

mirabilis (Sabahtritia) ................................... 47

mirabilis [D. (Spatiodamaeus)]

(=verticillipes) ……………………............. 82

mirabilis (Sternoppia) ................................. 133

mirabilis (Subpirnodus) ............................... 205

mirabilis (Suctobelbella) ............................ 137

mirabilis (Trimalaconothrus) ….......................6 62

mirabiloides (Cepheus) ....................................... 83

mirandus (Microzetes) …........................... 89

mirandus (Hoplophthiracarus) ....................... 50

mirandus (Rhacaplacarus) ............................ 57

mirena (Multimedioppia) .............................. 124

mirus (Berlesezetes) ornatissimus ……...........8 87

mirus (Notophthiracarus) ............................... 51 
mirus (Rhynchoribates)

misella [L. (Paralamellobates)] .................... 167

misella [L. (Paralamellobates)] …............... 167

misella [L. (Paralamellobates)] .................... 167

misella [L. (Paralamellobates)] ….............. 167

mississippii (Tegeocranellus) ....................... 155

missouri (Indotritia) .......................................... 43

misumaiensis (Trhypochthonius) .....................60 60

mitakensis (Phauloppia) ............................... 191

mitis (Fissicepheus) ..................................... 144

mitis (Fissicepheus) ...................................... 144

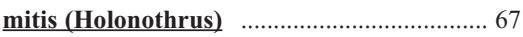

mitlsensillus [D. (Epidamaeus)] ..................... 79

mitratus [N. (Calyptophthiracarus)] ................ 53

mitratus (Fissicepheus) ................................. 144

mitratus (Sphodrocepheus) ............................... 84

mitrofanovi (Serratoppia) ............................. 124

mixoppia (Neostrinatina) ............................. 127

mixta (Achipteria) (=acuta) ….................... 167

mixtus (Austrocarabodes) ............................ 148

mixtus (Euphthiracarus) (=monodactylus) .... 46

mixtus (Euscheloribates) .......................... 197

miyauchii (Sellnickochthonius) ...................... 32

mizusawai (Tokunocepheus) ....................... 148

mizusawai (Tokunocepheus)

mochlosimilaris (Scheloribates) ……......... 200

moderata (Oripoda) ..................................... 204

moderatior (Achipteria) sp. inq. .................... 167

moderatus (Peloribates) ............................... 210

moderatus (Truncopes) ................................... 205

modesta (Phauloppia) Banks, 04

(=lucorum)

190

modesta (Phauloppia) Banks, 10

(elucorum)

190

modesta [H. (Phyllhermannia)] ..................... 69

modesta (Striatoppia) .................................... 131

modestior (Neoliodes) $s p$. inq. ...................... 72

modestus [P. (Archiphthiracarus)] .................. 55

modestus (Eremulus) (=flagellifer) ............ 102

modestus (Microtegeus) ……........................... 86

modestus (Trichotocepheus) erabuensis ........ 148

modicus (Notophthiracarus) ......................... 51

moestus (Scheloribates) .................................. 200

mograbin (Issaniella) .......................................... 71

mograbin (Issaniella) ................................... 71

moldavicus (Punctoribates) …..................... 183

molecula (Lamellobates) ..................... 166, 167

molecula (Lamellobates) ................... 166, 167

molecula (Lamellobates) .................... 166, 167

molecula (Lamellobates) ................... 166, 167

molecula (Lamellobates) ..................... 166, 167

molensis (Pilogalumna) (=ornatula) .......... 220

mollicoma (Hydrozetes) (=lemnae) ............... 155

mollicoma (Plenotocepheus) ….................. 145

mollicoma (Protoribates) ............................. 206

mollicoma (Protoribates) ................................ 206

mollicoma (Totobates) .................................. 196

mollicomus (Melanozetes) ............................ 176

mollicomus (Melanozetes) ............................. 176

mollis (Galumna) ........................................ 216

mollis (Gressittolus) (=marginatus) ............ 103

mollis (Indotritia) (=javensis) mollis (Liochthonius) ……............................... 30

mollis (Liochthonius) ................................. 30

mollis [H. (Phvllhermannia)] ..........................6 69

mollisetosus (Malaconothrus)

$$
\text { (=monodactylus) }
$$

mollisimilis (Melanozetes) ............................. 176

molodovi (Oribatella) ...................................... 171

monegrensis (Cosmochthonius) ....................... 33

monensis (Allonothrus) …............................... 59

mongolica (Belba) ........................................ 77

mongolica (Liebstadia) ................................... 195

mongolica (Maerkelotritia) krivolutskyi .......... 43

mongolica (Nortonella) .................................. 76

mongolicus (Bipassalozetes) ....................... 162

mongolicus (Birsteinius) ................................ 95

mongolicus [D. (Epidamaeus)] ....................... 79

mongolicus (Eupelops) ................................. 163

mongolicus (Montizetes) ................................ 107

mongolicus (Proteremaeus) ........................... 108

mongolicus (Tectoribates) ............................. 172

monicai (Pedrocortesella) ............................... 75

moniles (Bipassalozetes) .............................. 162

monilipeda (Metabelba) ................................... 80

monilipes (Caleremaeus) …………………..... 106

monoceros (Banksinoma) ........................... 109

monoceros (Schalleria) ............................. 90

monocerus (Heterochthonius) ......................... 34

monocornis [M. (Calyptozetes)] .................. 183

monodactyla (Acrotritia) ................................. 45

monodactyla (Cryptobothria) ........................ 182

monodactyla (Liebstadia) (=neonominata) .. 195

monodactyla (Macrogena) ........ 176

monodactyla (Parapyroppia) (=cornuta) ...... 94

monodactylus (Allonothrus) ........................ 59

monodactylus (Allonothrus) ............................ 59

monodactylus (Euphthiracarus) ...................... 46

monodactylus (Euphthiracarus) ...................... 46

monodactylus (Euphthiracarus) .................. 46

monodactylus (Malaconothrus) .......................61 61

monodactylus (Malaconothrus) …...................61 61

monodactylus (Malaconothrus) .................... 61

monodactylus (Mycobates) .......................... 183

monodactylus (Neophysobates) ................. 169

monodactylus (Nesopelops) ........................ 164

monodactylus (Nothrus) ................................ 64

monodactylus (Perscheloribates) ................... 198

monodactylus (Protoribates) (=dentatus) .... 206

monodactylus (Trhypochthonius)

(=tectorum)

monospicus (Oribatella)

monozygata (Oribatula) 187

monster (Persuctobelba) ............................ 135

monstrosus (Eremulus)

monstruosa (Acroppia) …......................... 128

monstruosa [Q. (Coronoquadroppia)] .......... 134

monstruosa [Q. (Coronoquadroppia)] .......... 134

monstruosa [Q. (Coronoquadroppia)] .......... 134

monstruosa (Dendrohermannia) ................. 68

monstruosa [F. (Parasuctobelba)] ............. 134

monstruosa (Pilobatella) ............................. 211

monstruosetosa (Basilobelba) ................... 104

monstruosus (Cavernocepheus) ..................... 141 monstruosus (Hermannobates) ..................... 71

monstruosus (Leobodes) …......................... 154

monstruosus [T. (Rostrozetes)] ..................... 211

monstruosus (Tectocarabodes) .................. 153

montana (Carinogalumna) ....................... 214

montana (Ceratoppia) (=spinipes) ............... 93

montana (Licnobelba) (=latiflabellata) ........ 75

montana [M. (Moritzoppiella)] .................... 127

montana (Murcia) Irk, 39 (=monticola) ...... 177

montana (Murcia) Ham., 61 ........................ 177

montana (Oripoda) ....................................... 204

montana (Pergalumna) Ham., 61 ................ 219

montana (Pergalumna) Ham., 72

(=tahitiensis) …....................................... 220

montana (Pilogalumna) crassiclava .......... 220

montana [S. (Ouatrobelba)]

(=neonominata) …................................... 82

montana (Simkinia) ....................................... 195

montana (Subbelba) ………………………….... 82

montana (Trichogalumna) ......................... 221

montanus (Aquanothrus) ............................. 156

montanus [P. (Archiphthiracarus)] ................. 55

montanus (Carabodes) ................................. 150

montanus (Dolicheremaeus) .......................... 143

montanus (Liacarus) ......................................... 96

montanus (Megeremaeus) ............................. 99

montanus (Neophysobates) ......................... 169

montanus (Peloptulus) .................................. 164

montanus (Platyliodes) ................................. 72

montanus (Rhynchoribates) ….................... 140

montanus (Trhypochthonius) .....................6 60

montanus (Trimalaconothrus) ...................... 62

montanus (Truncopes) …............................. 205

montanus (Verachthonius) ............................. 32

montanus (Verachthonius) ........................... 32

monteithi [P. (Constrictocepheus)] …........ 145

monteithi (Galumna) ............................... 216

monteithi (Neseutegaeus) .............................. 85

monteithi (Pheroliodes) .................................. 74

monticola (Anderemaeus) .............................. 106

monticola (Calozetes) ..................................... 88

monticola (Cavernocepheus) ...................... 141

monticola (Ceratozetes) .................................. 174

monticola (Galumna) ....................................... 216

monticola (Heterogalumna) ......................... 217

monticola [G. (Indogalumna)] ..................... 217

monticola (Murcia) ......................................... 177

monticola [P. (Nododamaeus)] ........................ 81

monticola (Nothrus) ….............................. 64

monticola (Nothrus) ...................................... 64

monticola (Sellnickochthonius) .................... 32

monticolus (Birsteinius) …............................. 95

monticolus (Eremaeus) ................................... 100

monticolus (Propelops) ............................. 164

montigenus (Rhacaplacarus) ........................ 57

montis (Pedrocortesella) ................................. 75

montium (Phthiracarus) (=longulus) ............ 54

montivagus (Diapterobates) sp. inq. ............ 181

monttensis (Acrogalumna) .......................... 213

monttensis (Perscheloribates) ...................... 198

monyx (Euphthiracarus) ................................. 46

moonsani (Xenillus) ........................................ 98 
mooseri (Eobrachychthonius) moraviacus [L. (Dorycranosus)]

(=splendens)

morenoi (Paraphauloppia) ........................... 190

morenoi (Scapheremaeus) ......................... 158

moresonensis (Allogalumna) ...................... 213

moresonensis [B. (Gressittoppia)] .............. 122

moribei (Thamnacarus) (=elongatus)

moritzi [L. (Baioppia)] .............................. 111

moritzi [S. (Flagrosuctobelba)] .................... 138

moritzi (Liochthonius) ............................. 11, 30

moritzi (Mixochthonius) …............................. 31

moritzi (Multioppia) ...................................... 117

moritzi (Verachthonius) .................................. 32

morticina (Oribatella) (=cespitum) .............. 171

morulisensillatus (Scapheremaeus) ........... 158

motasi (Lauroppia) .......................................... 126

moyae (Xenillus) ............................................. 98

muara (Sellnickochthonius) ........................... 32

mucronata [L. (Incabates)] ........................ 209

mucronatus (Annectacarus) ........................ 37

mucronatus (Centroribates) …....................... 214

mucronatus (Liacarus) .............................. 11, 96

mucronatus (Liacarus) ........................... 11, 96

mucronatus (Truncozetes) ........................... 173

muiri (Scheloribates) ................................. 200

muiricius (Scheloribates) .............................. 200

muliercula (Muliercula) ............................. 197

multesima (Allogalumna) ............................ 213

multiareolatus (Cribrozetes) .......................... 205

multiareolatus (Licnozetes) ........................... 89

multicorrugata [G. (Stenoppia)] .................. 116

multicuspidatus (Plakoribates) ...................... 169

multidentata (Serratobelba) ...................... 135

multidentatus (Pantelozetes) (=paolii) ........ 107

multiiterata (Galumna) alata ....................... 214

multiiteratus (Scheloribates) ........................ 200

multilineata (Striatoppia) ............................. 131

multipilosa (Austrogneta) .......................... 108

multipilosa (Austrogneta) ............................ 108

multipilosus (Neogymnobates) ...................... 178

multipilosus (Trimalaconothrus) .................. 62

multiplisetus (Topobates) .............................. 203

multiplumosa [S. (Flagrosuctobelba)] .......... 138

multipora (Hermanniella) ................................ 70

multiporosa (Mochloribatula) ....................... 185

multipunctata (Multoribula) ........................ 93

multirepetitus (Scheloribates) …................. 200

multirugosa [H. (Kakophthiracarus)] ........ 49

multisetosa (Epilohmannia) .......................... 37

multisetosa (Trichocondyla) ..................... 146

multisetosus (Atropacarus) ............................... 48

multisetosus [E. (Multoribates)] …................. 193

multisetosus [P. (Neophthiracarus)] ............ 56

multisetosus (Xenillus) .................................... 98

multisetus (Indoribates) ............................... 208

multisulcatum (Senectoppia) ....................... 132

multisulcatus (Trhypochthonius) $s p$. inq. ........ 60

multituberculata (Enantioppia) ................. 131

multituberculata [H. (Kakophthiracarus)] .... 49

$\underline{\text { multituberculata (Suctobelbila) }}$ multituberculata [S. (Ussuribata)

mumfordi (Scheloribates) …...................... 200

mundus (Phthiracarus) (=longulus) .............. 54

mundus (Punctoribates) ............................. 183

munizi (Perezinigokalumma) ...................... 213

muraiae (Bursoplophora) ............................... 35

murciensis (Atropacarus) ….............................. 48

murcioides (Mixacarus) .................................. 39

murotensis (Liacarus) ....................................... 96

murphyi [P. (Archiphthiracarus)]

=montanus) …................................... 55

murphyi (Dolicheremaeus) ............................. 143

murphyi (Sumatrotritia) ................................... 47

musci (Adrodamaeus) ...................................... 75

musci (Phauloppia) (?=lucorum) …............ 190

muscicola (Anellozetes) ................................ 181

muscicola (Benoibates) .................................. 203

muscicola (Haplochthonius) ............................. 34

muscicola (Hemileius) .................................... 193

muscicola (Peloribates) ................................ 210

muscicola (Peloribates) .................................. 210

muscicola (Stachyoppia) .............................. 131

muscicola (Stachyoppia) ............................ 131

muscorum (Liochthonius) ................................ 30

muscorum (Tegeocranellus) ............................ 155

muscorum (Tegeocranellus) 155

mussardi (Baobabula) ................................. 192

mussardi (Graptoppia) ............................... 116

mussardi (Liochthonius) ............................... 30

mutabilis (Dolicheremaeus) …..................... 143

mutabilis (Hoplophthiracarus) ...................... 50

mutabilis [P. (Neophthiracarus)] …............5 56

mutabilis (Xenillus) ....................................... 98

mutilus (Camisia) (=horrida) .......................... 65

mutsalpina (Trhypochthonius) (=tectorum) .... 60

mwali [H. (Kakophthiracarus)] .................. 49

myrica (Murcia) ........................................... 177

mvrmecophila (Oppia) (=nitens) ................. 115

myrmecophilus (Protoribates) ….................. 206

myrmobates (Ceratozetes)

(?=laticuspidatus) .................................... 174

myrmophila (Moritzoppia) ............................. 127

myrmophila (Pergalumna) ...................... 12, 219

myrmophila (Pergalumna) ................. 12, 219

mystax (Galumna) ....................................... 216

mystax (Nothrus) ……………………….......... 64

nadchatrami (Acutozetes) ............................... 207

nadiji (Mycobates) (=parmeliae) .................. 183

nagaii [T. (Rostrozetes)] ................................ 211

nagaii [H. (Tuberemaeus)] ............................ 194

naganoensis (Protoribates) …................ 206

nagaroorica (Eremobelba) ........................... 103

naginata [S. (Flagrosuctobelba)

(=elegantula $)$........................................... 138

nagoyae (Medioxyoppia) ............... 124

nagyi (Neoamerioppia) .............................. 114

nahani (Eueremaeus) ................................. 100

naias (Zetomimus) ..................................... 179

naisselinei (Achipterina) ........................... 172

nakanei (Fissicepheus) ................................ 144

nakatamarii (Yoshiobodes) .......................... 154

nakatamarii (Yoshiobodes) ........................... 154 naltschicki [V. (Latilamellobates)] ................ 179

nana (Berniniella) ........................................ 125

nana [Q. (Coronoquadroppia)] ....................... 134

nana [M. (Moritzoppiella)] ........................... 127

nana (Nanhermannia) ..................................... 68

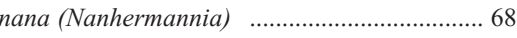

nana (Nanhermannia) …............................. 68

nana (Nanhermannia) ..................................... 68

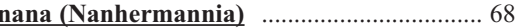

nana (Nanhermannia) ................................. 68

nana (Ramusella) (=curtipilus) .................... 118

nantahalaensis (Carabodes) ........................ 150

nanus (Ceratozetes) ....................................... 174

nanus (Scheloribates) .................................. 201

naranensis (Suctobelbella) ............................ 137

nasalis (Aeroppia) ........................................ 112

nasalis (Anoripoda) .................................. 203

nasalis (Brachioppiella) ............................. 122

nasalis [Q. (Coronoquadroppia)] ................. 134

nasalis (Dolicheremaeus) .............................. 143

nasalis (Eupterotegaeus) ................................... 83

nasalis [S. (Flagrosuctobelba)] ...................... 138

nasalis [S. (Flagrosuctobelba)] ….............. 138

nasalis (Hammerabates) …....................... 197

nasalis (Hammerabates) ............................... 197

nasalis (Hoplophthiracarus) …......................... 50

nasalis (Mucronothrus) ..................................... 60

nasalis (Mucronothrus) ….............................. 60

nasalis (Mucronothrus) ............................... 60

nasalis (Mucronothrus) .................................. 60

nasalis [P. (Wallworkiella)] ......................... 194

nasata (Nanhermannia) …............................ 68

nashiroi (Scapheremaeus) ............................ 158

nasica (Pergalumna) ................................... 219

naskreckii (Holonothrus) ..............................6 67

nasuta (Perlohmannia) ................................... 36

nasuta (Rhinoppia) ...................................... 124

nasutus (Ceratozetes) .............................. 12, 174

nasutus (Chamobates) (=subglobulus) ........ 180

nasutus [D. (Epidamaeus)] ........................... 79

nasutus (Euphthiracarus)

natalensis (Afrozetes) .................................... 87

natalensis (Archeonothrus) .......................... 27

natalensis (Berlesezetes) .................................. 87

natalensis (Galumna) maxima ................. 216

natalensis [C. (Magoebazetes)] …................ 88

natalensis (Paraphysobates) ….................. 169

natalensis [H. (Phyllhermannia)] ................6 69

nathanaeli [H. (Phyllhermannia)] ............... 69

natalensis (Protoribates) ............................ 206

natalensis (Protoribates) ................................ 206

nativa (Oribatula) …..................................... 187

navarrensis (Atropacarus) (=wandae) .......... 48

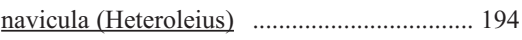

nayoroensis (Cosmochthonius) ...................... 33

nayoroensis (Diapterobates) ........................... 181

nayoroensis (Oribatula) ............................... 187

nayoroensis (Suctobelbella) ......................... 137

nebaikini (Proteremaeus) .............................. 108

nebulosa (Dampfiella) .................................. 141

nebulosus [T. (Rostrozetes)] (=poensis) ...... 212

necrophagus (Halozetes) ................................. 157 


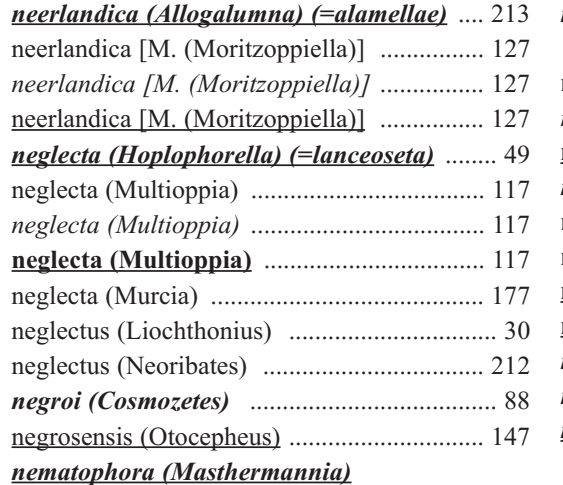
nematophora (Masthermannia)

(=mammillaris)

nemoralis (Eueremaeus) .

nemoralis (Phauloppia) (?=coineaui) ….... 190

nemoralis (Zachvatkinibates) ......................... 184

nemoricultricis (Pheroliodes) ......................... 74

nemorosus (Steganacarus) ................................. 58

nemossensis (Tricheremaeus) ........................... 101

neomexicanus (Passalozetes) ......................... 162

neomexicanus (Passalozetes) ...................... 162

neonominata (Brassiella) ................................ 192

neonominata (Brassiella) ............................. 192

neonominata [L. (Convergoppia)] ............... 111

neonominata (Cultroribula) …………............ 92

neonominata (Cultroribula) ............................. 92

neonominata (Fusuloppia) ……………….... 113

neonominata (Granuloppia) ……………........ 132

neonominata (Graptoppia) ............................ 116

neonominata (Graptoppia) ......................... 116

neonominata (Hoplophorella) ....................... 49

neonominata /G. (Indogalumna)] ............... 217

neonominata [R. (Insculptoppia)] ................ 119

neonominata (Jacotella) .................................... 76

neonominata (Lasiobelba) .............................. 113

neonominata (Lasiobelba) .......................... 113

neonominata (Lasiobelba) ............................. 113

neonominata (Liebstadia) ................................. 195

neonominata (Microtritia) .............................. 47

neonominata (Oppia) ……………………..... 115

neonominata (Oppiella) .................................. 128

neonominata (Oribatella) .............................. 171

neonominata (Oribatula) ................................ 187

neonominata (Pedrocortesella) ........................ 75

neonominata [H. (Phyllhermannia)] ............. 69

neonominata [S. (Quatrobelba)] ...................... 82

neonominata (Suctobelbella) ....................... 137

neonominata (Suctobelbila) ............................. 139

neonominatus (Anellozetes) ......................... 181

neonominatus [P. (Archiphthiracarus)] .......55

neonominatus (Carabodes) .......................... 150

neonominatus (Carabodes) ............................ 150

neonominatus (Ceratozetes) ……………….... 174

neonominatus (Dolicheremaeus) ................. 142

neonominatus [L. (Dorycranosus)] ……......... 97

neonominatus (Eremaeus) ............................. 100

neonominatus (Liochthonius) ....................... 30

neonominatus (Malaconothrus) ......................... 61

neonominatus (Nothrus) borussicus ................. 63 neonominatus (Passalozetes)

neomexicanus

neonominatus (Peloribates)

neonominatus [N. (Protokalumma)] ............ 212

neonominatus (Pseudotocepheus) ................ 145

neonominatus (Scheloribates) ………......... 201

neonominatus (Scutovertex) ……………... 161

neonominatus (Sphaerozetes) globularis .. 178

neonominatus [H. (Tuberemaeus)] ............ 194

neonominatus [H. (Tuberemaeus)] ……..... 194

neonominatus (Xenillus) ................................. 98

neoplumosus [M. (Cristonothrus)] ................ 61

neosimplex (Neobrachychthonius)

(=marginatus) …………………………... 31

neosota (Eremobelba) (= gracilior) ............... 103

neotricha (Epilohmannia) ............................. 37

neotricha (Hermannia) ………………………... 69

neotricha (Monogalumnella) ........................ 222

neotrichus [N. (Protophthiracarus)] ………... 53

neotrichus (Trichozetes) ............................... 91

neotropica (Lyroppia) ................................. 131

neotropica (Machadobelba) ......................... 107

neotropica [H. (Phyllhermannia)] ................ 69

neotropicalis (Acrotritia) ardua .................... 45

neotropicalis (Campachipteria) .................. 168

neotropicalis (Dissorhina) ......................... 125

neotropicus (Afronothrus) (=incisivus) ........ 59

neotropicus (Allonothrus) ............................... 59

neotropicus (Heptacarus) .............................. 38

neotropicus (Mixacarus) ................................ 39

neotropicus [P. (Neopilizetes)] .................... 220

neotropicus (Papillocepheus) ...................... 144

neotropicus (Plakoribates) ........................... 169

neotropicus (Suctoribates) _......................... 140

nepalensis (Dolicheremaeus) ......................... 143

nepalensis (Hoplophthiracarus) ...................... 50

nepalensis (Oribotritia) ........................................ 44

nepalensis (Provertex) ……………………..... 161

nepenthes (Otocepheus) ……........................ 147

nepos (Carabodes) (=coriaceus) ................... 149

nepotulus (Eupelops) ...................................... 163

nervosa (Eremobelba) (=gracilior) ............... 103

nervosa (Pergalumna) .................................... 219

nervosa (Pergalumna) _................................ 219

nervosa (Pergalumna) _.............................. 219

nervosus (Mystacozetes) ................................. 90

nesebarensis (Passalozetes) .......................... 162

neuquinus (Pheroliodes) .................................. 74

nevadensis [H. (Capillonothrus)] ...................... 66

nevesi (Hafenferrefia) ..................................... 99

newelli (Setuloppia) _...................................... 123

ngongi (Rhacaplacarus) ................................. 57

ngoyensis (Muliercula) .................................. 197

nicki (Hemileius) .......................................... 193

nicoleti (Achipteria) $(=$ coleoptrata) $\quad$............. 167

nicoleti (Camisia) (=biverrucata) ...................65

nicoleti (Notophthiracarus) ........................... 51

nicora (Lucoppia) ............................................ 187

nidicola (Ameronothrus) …………………….... 156

nidicola (Damaeus) ........................................ 78

nidicola (Globoppia) ……………………….... 110

nidicola (Onychobates) …………................ 178 niedbalai (Acrotritia) $(=$ vestita) $\quad$...................... 46

niedbalai [S. (Rafacarus)] ………………....... 58

niger (Carabodes) ........................................ 150

niger (Compactozetes) …………………...... 83

niger (Notophthiracarus) (=schizocomus) .... 52

niger (Passalozetes) ………………………... 162

niger (Scutovertex) ………………………..... 161

niger (Xenillus) ................................................. 98

nigeriensis (Hoplophorella) .......................... 49

nigeriensis (Peloribates) ............................... 210

nigeriocompressus (Scheloribates) ............ 201

nigeriosphericus (Scheloribates) ................. 201

nigerrimus (Chamobates) (=subglobulus) ... 180

nigerrimus (Notophthiracarus) ....................... 51

nigerrimus [L. (Procorynetes)] ……………. 97

nigra (Galumna) ........................................... 216

nigra [O. (Multoribatella)] ............................. 172

nigrescens (Liacarus) ....................................... 96

nigricans (Neoliodes) ……………………..... 72

nigricans (Tectoppia) ……............................. 116

nigricans (Trhypochthonius) ............................. 60

nigriclavatus (Ampullobates) ......................... 70

nigrisetosus (Ceratozetes) .............................. 174

nigrisetosus (Eremulus) …………………….... 102

nigrisetosus (Eremulus) ............................... 102

nigroclava (Novazelandiella) ......................... 74

nigrofemoratus (Ameronothrus) ...................... 156

nigrofemoratus (Ameronothrus) ..................... 156

nigrolobatus (Megalotocepheus) ………….... 147

nigromaculatus (Spinotocepheus) …………. 146

nigrosetosus [C. (Klapperiches)] ………….... 151

nikolskii [M. (Moritzoppiella)] ..................... 127

nilamburicus (Phyllozetes) …………………..... 33

nilgiria (Galumna) ……………………….... 216

niliaca (Galumna) ………………………....... 216

niliaca (Striatoppia) ……………………..... 131

niliaca (Striatoppia) ………………………..... 131

niliaca [O. (Zygoribatula)] ………………….... 189

niliacus (Sellnickochthonius) ........................... 32

niloticus (Hydrozetes) sp. inq. ....................... 155

nimbus (Dolicheremaeus) ............................ 143

nimirumi (Scheloribates) louwi .................. 200

nipponica (Galumnella) ................................ 222

nipponica (Galumnella) .................................. 222

nipponica (Suctobelbella) claviseta ............... 136

nipponica (Trichogalumna) ........................... 221

nipponica (Trichogalumna) ........................... 221

nipponica (Zachvatkinella) ................................ 27

nipponicus (Cultrobates) ………………….... 172

nipponicus (Protoribates) crassisetiger ........ 206

nipponicus (Trimalaconothrus) ……………... 62

nishinoi (Peloribates) ..................................... 210

nitens (Achipteria) Nic., 1855 (=acuta) .. 10, 167

nitens (Achipteria) L. Koch, 1879 (=acuta) .. 167

nitens (Achipteria) Kis., 1930 (=acuta) $\ldots . . . .167$

nitens (Carabodes) sp. inq. ............................. 150

nitens (Euzetes) $(=$ globulus) .................. 10, 175

nitens (Liacarus) ……………………………....... 96

nitens (Liacarus) ...……………………....... 10

nitens [M. (Moritzoppiella)] ........................... 127

nitens (Oppia) ……………………………. 115

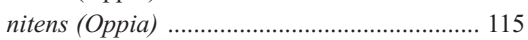




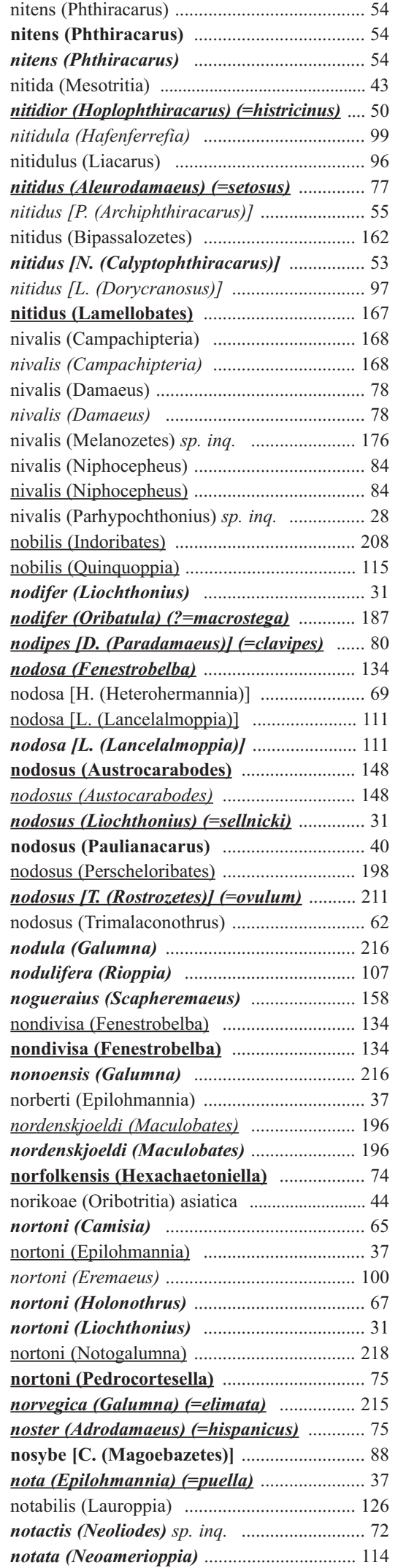

notatus (Diapterobates) …............................ 181

notatus (Diapterobates) .............................. 18

notatus (Notophthiracarus) ............................ 51

notialis (Belba) (=sasakawai) ........................ 77

notoapodematus (Gymnodamaeus) ................ 76

notogastralis [H. (Phyllhermannia)] ............ 69

notoneotrichus (Heptacarus) ……………...... 38

notoneotrichus (Heptacarus) ........................ 38

nova (Allosuctobelba) .................................. 134

nova (Camisia) .............................................. 65

nova (Camisia) ............................................... 65

nova (Collohmannia) (=gigantea) ................ 36

nova (Galumna) (=tarsipennata) ................ 217

nova (Liebstadia) (=pannonica) ................. 195

nova (Mesotritia) ................................................ 43

nova (Murcia) ................................................ 177

nova (Murcia) .................................................. 177

nova (Oppiella) ................................................. 128

nova (Oppiella) ............................................ 128

nova (Oppiella) …………............................ 128

nova (Oppiella) …....................................... 128

nova (Oppiella) …....................................... 128

nova (Oppiella) ........................................... 128

nova (Suctobelbata) ..................................... 136

nova (Trapezoppia) .................................... 123

novaecaledoniae (Holonothrus) ..................... 67

novaeguineae (Arcoppia) arcualis ............. 120

novaeguineae (Licneremaeus) .................... 160

novaeguineae (Licneremaeus) ..................... 160

novaeguineae (Licneremaeus) ................... 160

novaeguineae (Licneremaeus) ................... 160

novaezealandiae (Andesamerus) ................ 105

novaezealandiae (Arcoppia) (=curtisetosa) .. 120

novaezealandiae [T. (Tyrphonothrus)] ........ 63

novaezealandicus [H. (Platynothrus)] ….... 66

novaguineanus (Zetorchestes) ..................... 91

novazealandica (Allogalumna) ................. 213

novazealandica (Paraphauloppia) …........ 190

novazealandica [O. (Zygoribatula)] .......... 189

novazealandicus (Edwardzetes) .............. 175

novazealandicus (Grandjeanobates) .......... 197

novazealandicus (Pterozetes) ........................ 85

novazealandicus (Sellnickochthonius) ........ 32

novemsetosus (Plateremaeus) ……................ 73

novus (Cosmochthonius) .................................. 33

novus (Fuscozetes) ......................................... 175

novus (Gibbicepheus) ................................ 152

novus (Licneremaeus) .................................. 160

novus (Tectocepheus) (=minor) ................. 154

novus [(T. (Tyrphonothrus)] (=maior) .......... 63

nuciferosa (Scapheremaeus) .......................... 158

nuda (Achipteria) sp. inq. ........................... 167

nuda (Galumna) ........................................... 216

nuda (Granuloppia) ................................. 132

nuda [L. (Incabates)] ................................... 209

nuda [L. (Incabates)] .................................... 209

nuda (Indotritia) .......................................... 43

nuda (Mesotritia) .................................................. 44

nuda (Mesotritia) .................................................. 44

nuda (Pergalumna) ..................................... 219

nuda (Tenuiala) ............................................ 99

nudiuscula (Pelopsis) (=bifurcatus) ............ 183 nudus (Guaranozetes) ................................. 173

nudus [I. (Haplozetes)] .............................. 208

nudus (Humerobates) ..................................... 181

nudus (Humerobates)

nudus (Humerobates) …............................ 181

nudus (Neophysobates) …........................... 169

nudus (Scheloribates) .................................... 201

nugarica (Oribatella) .................................. 171

nukuhivae (Crotonia) ……............................. 67

nukusia (Graptoppia) ................................... 116

numatai [H. (Capillonothrus)] ......................... 66

numerosa (Pergalumna) .............................. 219

numerosus (Diapterobates) (=humeralis) .. 181

numidiana [A. (Rhaphigneta)] ..................... 108

numidicus (Dicatozetes) ................................ 214

oauensis (Scheloribates) ............................... 201

oaxacanus (Acaroceras) ................................ 87

obesa (Murcia) ………...................................... 177

obesa (Murcia) ............................................... 177

obesa (Pedrocortesella) …….......................... 75

obesus (Atropacarus) ...................................... 48

obesus (Dynatozetes) ..................................... 185

obesus (Perxylobates) ................................... 205

obesus (Scheloribates) ................................... 201

oblectatoria (Ceratoppia) ……...................... 93

obliqus [F. (Pseudogeminozetes)] ................ 175

obliquus (Cavernocepheus) ......................... 141

oblitera (Hermanniella) .................................. 70

obliteratus (Scapheremaeus) ........................ 159

oblonga (Antarcticola) (=meyeri) ................. 156

oblonga (Hoplophorella) ............................... 49

oblonga [O. (Zygoribatula)] (=lata) ............ 189

oblongarea (Murcia) ....................................... 177

oblongata (Medioppia) .................................. 123

oblongus (Ameronothrus) .................................. 156

oblongus (Diapterobates) .............................. 181

oblongus (Eueremaeus) ................................ 100

oblongus (Eueremaeus) ................................ 100

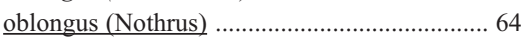

oblongus (Nothrus) ...................................... 64

oblongus (Odontocepheus) Karp., 1893

(=elongatus) …........................................ 153

oblongus (Odontocepheus) Banks, 1895 ....... 153

oblongus (Odontocepheus) Banks, 1895 .... 153

oblongus (Protoribates) ................................ 206

oblongus (Steganacarus) (=magnus) .............58

obscura (Eremobelba) sp. inq. .................... 103

obscura (Lauroppia) ..................................... 126

obscura (Metabelba) (=papillipes) ................8 80

obscurus [P. (Archiphthiracarus)] .................... 55

obscurus [P. (Archiphthiracarus)] .............. 55

obscurus (Meristacarus) madagascarensis ...... 39

obscurus (Sellnickochthonius)

(=immaculatus) ……….............................. 32

obsessa (Pergalumna) .................................... 219

obsessa [O. (Zygoribatula)] .......................... 189

obsessus (Dolicheremaeus) …….................. 143

obsessus [P. (Neophthiracarus)] ….............. 56

obsessus (Notophthiracarus) …………….... 51

obsessus (Scheloribates) …............................. 201

obsessus (Trimalaconothrus) .......................... 62

obsoleta (Ctenobelba) sp. inq. ...................... 101 
obsoleta (Medioppia) ...................................... 123

obsoleta (Medioppia) ................................... 123

obsoleta (Medioppia) .................................... 123

obsoleta (Quadroppia) ................................. 133

obsoletior (Hoplophorella) (=hamata) .......... 49

obsoletus (Austrocarabodes) ....................... 148

obsoletus (Nothrus) sp. inq. ............................. 64

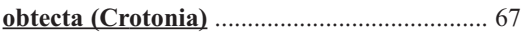

obtecta (Crotonia) ........................................... 67

obtectus (Seteremaeozetes) …...................... 165

obtusa (Allosuctobelba) ............................... 134

obtusa (Arcoppia) .......................................... 121

obtusa (Metabelba) ........................................... 80

obtusa (Metabelba) ….................................... 80

obtusa (Notohermannia) ................................ 68

obtusinotata (Paraphauloppia) ................. 190

obtusisetus (Dolicheremaeus) …................. 143

obtusus (Ceratozetes) (=campestris) ............ 174

obtusus (Ghilarovizetes) ................................. 176

obtusus (Rhynchoribates) ........................... 140

obtusus (Scheloribates) ............................... 201

obtusus [P. (Triaungius)] ............................... 206

obvia (Galumna) (=elimata) ........................ 215

occidentale (Galumna) (=alata) ................... 214

occidentalis (Ameronothrus) (=lineatus) ... 156

occidentalis [M. (Calyptozetes)] ................... 183

occidentalis [C. (Klapperiches)] pulcher ...... 151

occidentalis (Eremaeus) ............................... 100

occidentalis (Hermanniella) ............................ 70

occidentalis (Karenella) lanceoseta ............ 130

occidentalis (Podacarus) auberti .................. 157

occultus [P. (Archiphthiracarus)] ..................... 55

occultus (Campbellobates) ............................ 204

occultus (Eupelops) ..................................... 163

occultus (Neoliochthonius) ............................ 31

occultus (Xenillus) ......................................... 98

oceana [O. (Zygoribatula)] …..................... 189

oceanica (Gustavia) ......................................... 95

oceanicus (Liochthonius) ............................. 31

oceanicus (Neoribates) ................................. 212

oceanicus (Nothrus) ……................................... 64

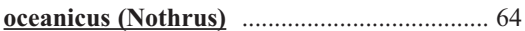

oceanicus (Nothrus) ....................................... 64

ocellatus (Austrocarabodes) ........................ 148

ocellatus (Damaeolus) ................................ 103

ocellatus (Liacarus) ......................................... 96

ocellatus (Liochthonius) (=hystricinus) ........ 30

ocellatus (Neoliodes) ....................................... 72

ocellatus (Ommatocepheus) ............................. 83

ocellatus (Ommatocepheus) ............................ 83

ochraceus (Atropacarus) .................................. 48

ochthus [P. (Archiphthiracarus)] ……........ 55

ocotlicus (Murcia) ......................................... 177

octo (Fenestrobelba) ..................................... 134

octocoma (Oligoppia) ................................. 114

octofilamentis (Parhypochthonius)

(=aphidinus) ….......................................... 28

octogonalis [C. (Phyllocarabodes)] ............... 151

octomaculatus (Eremaeozetes) ...................... 165

octomaculatus (Eremaeozetes) ...................... 165

octomaculatus (Eremaeozetes) …............... 165

octopunctata (Galumna) (=alata) octosetosus (Arthrodamaeus) .......................... 76

octosetosus (Hydrozetes) ................................. 155

ocularis (Lamellobates) …….......................... 167

odontotus (Acaroceras) ................................... 87

oenipontanus [N. (Calyptophthiracarus)] ........ 53

oesziae (Sellnickochthonius) .......................... 32

officiosus (Mochlozetes) ............................. 185

ogawai (Papillacarus) ……............................. 40

ogilviensis (Murcia) ..................................... 177

oginoi (Dolicheremaeus) ............................. 143

oglasae (Odontocepheus) .............................. 153

oglasae [M. (Oglasacarus)] ........................... 184

oglasae (Ophidiotrichus) ............................. 170

oglasicola (Protoplophora) ............................... 35

ohat (Phauloppia) (=lucorum) .................... 190

ohnishi (Liochthonius) .................................... 31

okinawa (Eremobelba) ................................... 103

okuyamai (Mesotritia) ........................................... 44

olahi (Cordiozetes) ....................................... 195

olbiopolitanus (Kaszabobates) ...................... 107

oleariae (Mycozetes) .................................... 183

oligochaeta (Aethioppia) ............................ 112

oligotricha (Protoribotritia) ............................... 45

oligotricha (Protoribotritia) ................................ 45

olitor [D. (Epidamaeus)] ............................... 79

olivaceus [N. (Calyptophthiracarus)] …….... 53

olivaceus (Liacarus) sp. inq. .......................... 96

ombinatus (Umbellozetes) …......................... 170

ominei (Peloribates) .................................... 210

omissus (Chamobates) (=subglobulus) ........ 180

omittens (Torpacarus) ............................... 41

omittens (Torpacarus) ................................... 41

omniphagous (Pergalumna) (=emarginata) .. 218

omo (Carabodes) (=granulatus) .................. 150

omodeoi [Q. (Coronoquadroppia)] ................ 134

ondriasi (Papillacarus) .................................... 40

ondriasi (Papillacarus) ……............................. 40

onondaga (Limnozetes) ................................. 166

onondaga (Limnozetes) ................................ 166

onubensis (Passalozetes) ............................. 162

onustus [D. (Adamaeus)] ................................. 79

onustus [D. (Adamaeus)] ............................... 79

opacus [P. (Archiphthiracarus)] ...................... 55

opcus (Tegeocranellus) …………………......... 155

operata (Pergalumna) .................................... 219

opilioides (Damaeus) ....................................... 78

opipara (Oribotritia) ........................................... 44

opisthoseta (Trimalaconothrus) …..............6. 62

opistodentata (Suctobelbella) .......................... 137

oppiana (Nosybelba) ..................................... 134

opposita (Beckiella) ............. 141

oppositus (Trimalaconothrus) P. Bal., 97 ...... 62

oppositus (Trimalaconothrus) J. y P. Bal., 02

(=oppositus P. Bal., 97) ............................. 62 optabilis (Austrotritia) (=saraburiensis) ........ 42 optatus [T. (Tyrphonothrus)] (?=saxosus) .... 63

optatus (Truncopes) ..................................... 205

optivus (Rhacaplacarus) .............................. 57

opuntiseta (Striatoppia) ................................ 131

opuntiseta (Striatoppia) ............................... 131

opuntiseta (Striatoppia) …............................. 131

opuntiseta (Striatoppia) ................................ 131 orangutan (Otocepheus) …............................ 147

orangutan (Pseudocarabodes) ........................ 153

orbicularis (Sphaerozetes) ............................. 178

orbicularius (Gymnodamaeus) ........................ 76

orbiculata (Hermanniella) ................................ 70

orbiculata (Peltenuiala) ................................... 99

orbiculatus (Chaunoproctus) ......................... 192

orbiculus (Orbiculobates) ............................ 71

ordunensis (Medioppia) ................................ 123

oregonae (Camisia) ......................................... 65

oregonensis (Achipteria) .............................. 168

oregonensis (Metrioppia) ................................ 94

oregonensis (Oribotritia) ..................................... 44

oreia (Moritzoppia) ....................................... 127

oresbios (Ametroproctus) ............................... 159

oresbios (Ceratozetes) ................................... 174

oresbios (Eremaeus) ........................................ 100

orghidani [L. (Magnobates)] ......................... 209

oribatelloides (Achipterina) ......................... 172

oribatelloides (Liacarus) .................................. 96

orientalis (Arcoppia) fenestralis ................... 120

orientalis (Arcoppia) fenestralis ............... 120

orientalis [N. (Besuchetacarus)] ..................... 52

orientalis (Congocepheus) ............................. 151

orientalis [P. (Constrictocepheus)] ................ 145

orientalis (Dolicheremaeus) ......................... 143

orientalis (Eremaeus) ................................... 100

orientalis (Galumna) flabellifera .................. 215

orientalis (Galumna) flabellifera ................. 215

orientalis (Lamellobates) ................................ 167

orientalis (Lamellobates) ............................. 167

orientalis (Lauroppia) ................................... 126

orientalis (Leptotocepheus) ........................... 144

orientalis (Lucoppia) .................................... 187

orientalis (Melanozetes) .................................. 176

orientalis (Metabelba) ……............................. 80

orientalis [P. (Millotacarus)] ............................ 41

orientalis (Oppia) ........................................... 115

orientalis (Oribatella) ...................................... 171

orientalis (Palaeacarus) ….............................. 27

orientalis (Passalozetes) californicus .......... 162

orientalis (Pseudopyroppia) ............................ 94

orientalis (Rhynchoribates) $\ldots \ldots \ldots \ldots \ldots \ldots \ldots . . . . . . . .140$

orientalis (Truncopes) .................................... 205

orientata (Oppiella) (=nova) ........................ 128

oriformis (Podoribates) ............................. 186

orixaensis (Scheloribates) ........................... 201

orkneyensis (Berlesezetes) ornatissimus ........87

orknevensis [B. (Gressittoppia)] .................. 122

ornata (Acrotritia) ........................................... 46

ornata (Acrotritia) ............................................. 46

ornata (Apoplophora) .................................... 41

ornata [G. (Cosmogalumna)] ......................... 217

ornata (Dissorhina) ...................................... 125

ornata (Dissorhina) ...................................... 125

ornata (Dissorhina) ...................................... 125

ornata (Dissorhina) ..................................... 125

ornata (Epilohmannia) ................................... 37

ornata (Eremobelba) ..................................... 103

ornata (Jacotella) B. y C., 63 ......................... 76

ornata (Jacotella) B. у C., 63 ....................... 76

ornata (Jacotella) P.-I., 72 (=neonominata) .. 76 
ornata (Jacotella) Mah., 79 (Efrondeus) _..... 76 ornata (Lucoppia) …………………………... 187 ornata (Magyaria) ………………………..... 209 ornata (Oribatella) ……............................... 171 ornata (Suctobelbella) Kriv., 66 ..................... 137 ornata (Suctobelbella) B. y M., 69

(=neonominata)

ornata (Suctobelbila) (=neonominata) ........ 139 ornatissima (Apoplophora) (=pantotrema) .. 41 ornatissima (Exanthoppia) ......................... 113 ornatissima (Masthermannia) ...................... 68 ornatissima (Suctobelbella) ............................ 137 ornatissimus (Berlesezetes) .............................. 87 ornatissimus (Berlesezetes) ………………... 87 ornatissimus (Berlesezetes) ………………...... 87 ornatissimus (Berlesezetes) …………........... 87 ornatissimus (Berlesezetes) ............................. 87 ornatissimus (Calobates) ............................... 203 ornatissimus (Damaeolus) _.......................... 103 ornatissimus (Eupterotegaeus) ............................. 83 ornatissimus (Eupterotegaeus) ........................... 83 ornatissimus (Heminothrus) ............................... 65 ornatissimus (Heminothrus) ............................ 65 ornatissimus [C. (Klapperiches)] …............ 151 ornatissimus (Lepidacarus) …………………... 38 ornatissimus (Plateremaeus) ............................ 73

ornatula (Pilogalumna) ……………………..... 220 ornatus (Africoribates) ………………..... 180 ornatus [P. (Archiphthiracarus)] …………........ 55 ornatus(Calobates) ………………………... 203 ornatus (Carabodes) ………………………... 150 ornatus (Charassobates) ................................. 86 ornatus (Congocepheus) …………………... 151 ornatus (Costeremus) ……………………...... 103 ornatus (Damaeus) (=torquisetosus) ……..... 78 ornatus (Dolicheremaeus) …………………... 143 ornatus (Eueremaeus) (=oblongus) ............ 100 ornatus (Fosseremus) (=laciniatus) ............. 103 ornatus (Gymnodamaeus) .............................. 76 ornatus (Heptacarus) ………………………... 38 ornatus (Mystacozetes) ..................................... 90 ornatus /C. (Phyllocarabodes)] ..................... 151 ornatus (Porrhotegaeus) ............................... 85 ornatus (Scapheremaeus) .............................. 159 ornatus (Scutovertex) .................................... 161 ornatus (Scheloribates) …............................ 201 ornatus (Tectoribates) ..................................... 172 ornatus (Tectoribates) ............................... 172

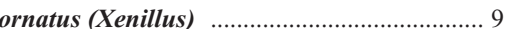
ornatus (Yoshiobodes) ……………………..... 154 ornatus (Zetorchestes) ……………………...... 91 ornithorhyncha (Rhynchobelba) ..................... 135 ornithorhynchus (Fissicepheus) ..................... 144 oromii (Heminothrus) ......................................... 65 oromurcii (Diapterobates) ………………...... 18 orthodactyla (Heteroppia) ................................. 113 orthogonia (Camisia) ........................................ 65 orthogonios (Liacarus) ....................................... 96 orthogonios (Liacarus) ...................................... 96 ortizi (Damaeus) …………………………….... 78 ortizi (Rhacaplacarus) ....................................57 oryzae (Scheloribates) oryzae (Scheloribates)

oscensis (Berniniella) serratirostris .............. 125

oscensis (Scheloribates) labyrinthicus ........... 200

osithchnjukovi (Phyllozetes) ............................ 33

osornensis [H. (Capillonothrus)] .................... 66

osoyoosensis (Eueremaeus) ........................... 100

otagoensis (Halozetes) …………………....... 157

oudemansi (Achipteria) Jacot, 29 sp. inq. .... 168 oudemansi (Achipteria) Ham., 52

(Epraeoccupata) ………………………. 168

otaheitensis (Acrotritia) …………………….... 46

otaheitensis (Acrotritia) .................................... 46

oudemansi (Banksinoma) lanceolata ............. 109

oudemansi (Eobrachychthonius) ..................... 30

oudemansi (Eobrachychthonius) ..................... 30

oudemansi (Eobrachychthonius) ……............. 30

oudemansi (Eobrachychthonius) ................... 30

oudemansi (Eobrachyththonius) …................. 30

oudemansi (Eupelops) .................................... 163

oudemansi (Processoppia) .......................... 112

ovalis (Achipteria) $(=$ coleoptrata) ................ 167

ovalis (Dampfiella) ........................................... 141

ovalis (Epilohmannia) ................................... 37

ovalis (Eremaeus) (=hepaticus) ….............. 100

ovalis (Liacarus) (=brevilamellatus) ............... 95

ovalis (Parachipteria) sp. inq. ........................ 169

ovalis (Scutovertex) (?=sculptus) ................. 16

ovalis (Scutozetes) ………………………..... 169

ovalis (Simkinia) (=turanica) ....................... 195

ovalis (Totobates) ……................................. 196

ovata (Banksinoma) ……………………...... 109

ovata (Crotonia) …………………………........ 67

ovata (Epilohmannia) …………………………..... 37

ovata (Epilohmannia) ………………………... 37

ovata (Galumna) sp. inq. ........................... 216

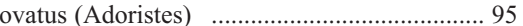

ovatus (Christovizetes) ……………………….... 88

ovatus [L. (Dorycranosus)] (=splendens) ...... 97

ovatus (Liacarus) ……………………………... 96

ovatus [H. (Platynothrus)] ................................ 66

ovatus (Sphaerochthonius) …………………...... 34

ovidianus (Ceratozetes) .................................. 174

ovivorus (Nothrus) sp. inq. ............................... 64

ovivorus (Nothrus) sp. inq. .............................. 64

ovulum [T. (Rostrozetes)] ................................ 211

ovulum [T. (Rostrozetes)] .............................. 211

ovulum [T. (Rostrozetes)] ………………...... 211

ovulum [T. (Rostrozetes)] ................................ 211

ovulum [T. (Rostrozetes)] ............................. 211

ovulum [T. (Rostrozetes)] ………………...... 211

oxapampensis (Heterobelba) ......................... 104

oxyptera [V. (Latilamellobates)]

?=incisella) 179

oxyrhinus (Trimalaconothrus) …………...... 62

oxyrhinus (Trimalaconothrus) …_.

ozkani (Anomaloppia) ……………………... 116

pachypilus [M. (Cristonothrus)] .................. 61

pachyseta (Berlesezetes) (=ornatissimus) .......87

pachytrichosus (Damaeus) (=flagellifer) ...... 78

pacifica (Acrogalumna) ............................... 213

pacifica (Basilobelba) …………………....... 104

pacifica (Epilohmannia) pallida ……………..... 37 pacifica (Epilohmannia) pallida ……………... 37

pacifica (Epilohmannia) pallida ………….... 37

pacifica (Epilohmannia) pallida ..................... 37

pacifica (Erioppia) problematica ………... 113

pacifica (Exoribatula) …………………..... 193

pacifica (Furcoribula) ……………………...... 92

pacifica (Peltenuiala) .................................... 99

pacifica [T. (Paragloboppia)] ....................... 115

pacifica [H. (Phyllhermannia)] ……………... 69

pacificus (Brachychthonius) …………….... 29

pacificus (Ceratozetes) .................................. 174

pacificus (Otocepheus) sp. inq. .................... 147

pacificus (Palaeacaroides) .............................. 27

pacificus (Palaeacaroides) ………………….... 27

pacificus (Propelops) ………………………... 164

pacificus (Scheloribates) Sell., 59 ............... 201

pacificus (Scheloribates) Ham., 67

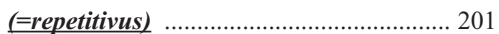

pacificus (Zetorchestes) ................................... 91

pacificus (Zetorchestes) ................................ 91

paeneminima (Microtritia) (=simplex) .......... 47

pagoda (Plasmobates) ……………………….... 71

pagoda (Plasmobates) …………………….... 71

pahabaeus (Scheloribates) ………………...... 201

paillei [R. (Insculptoppiella)] ......................... 119

pairathi (Haplacarus) …………..... 38

pajaki (Scheloribates) (=microsetosus)

pakistanensis (Euphthiracarus) ........................ 46

pakistanensis (Hoplophthiracarus) ………….... 50

pakistanensis (Hoplophthiracarus) ................... 50

pakistanensis (Multioppia) ………………….... 117

pakistanensis (Peloribates) ............................ 210

pakistanensis (Peloribates) ............................. 210

palaciosi (Prototritia) …………………….... 36

palaciosi (Scapheremaeus) ............................. 159

palawanus (Peloribates) ................................. 210

palawanus (Scheloribates) ………................. 201

pallens (Hoplophorella) .................................. 49

pallens (Hoplophorella) ................................. 49

pallens (Hoplophorella) ................................ 49

pallens (Nothrus) (=palustris) ......................... 64

palliatus [H. (Platynothrus)] [=peltifer) ........ 66

pallida (Epilohmannia) ………………….... 37

pallida (Epilohmannia) ...................................... 37

pallida (Epilohmannia) ................................... 37

pallida (Galumna) .......................................... 216

pallida [L. (Incabates)] …………………….... 209

pallida (Oribatula) (=tibialis) ……………... 188

pallida (Pergalumna) (=obsessa) ................ 219

pallidulus (Hypochthonius) (=rufulus) ........ 29

pallidulus (Scheloribates) ........................ 12, 201

pallidulus (Scheloribates) ..................... 12, 201

pallidulus (Scheloribates) ………......... 12, 201

pallidulus (Scheloribates) ........................ 12, 201

pallidulus (Scheloribates) …............... 12, 201

pallidulus (Scheloribates) ...................... 12, 201

pallidus (Cosmochthonius) sp. inq. ................ 33

pallidus (Dolicheremaeus) (=damoeoides) .. 142

pallidus (Hemileius) (=quadripilis) ............ 193

pallidus (Neoribates) …………..................... 212

pallidus (Phthiracarus) (=compressus) ....... 53

pallidus (Sphaerochthonius) .............................. 34 
pallidus (Trimalaconothrus) ......................... 62

palmaria (Arcoppia) ….............................. 121

palmata (Brachioppia) ................................ 122

palmerae (Limnozetes) .................................. 166

palmicincta (Conoppia) ..................................... 83

palmicincta (Conoppia) ................................... 83

palmicincta (Conoppia) ……............................ 83

palmicincta (Conoppia)

palmifer (Carabodes) .............................. 12, 150

palmifer (Carabodes) ............................. 12, 150

palmifer (Carabodes) ............................12, 150

palmifer (Carabodes) ............................. 12, 150

palpalis (Protoplophora) ................................... 35

palpalis (Protoplophora) ................................ 35

palpalis (Protoplophora) ................................ 35

paludicola (Hypochthoniella) .......................... 29

paludicola (Svalbardia) ............................... 182

paludicola (Svalbardia) ................................. 182

paludis (Hoplophthiracarus) (=illinoisensis) .. 50

palustris (Heterozetes) ................................... 173

palustris (Lamellobates) (=molecula) .......... 167

palustris (Nothrus) .......................................... 64

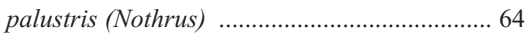

palustris (Nothrus) .............................................. 64

palustris (Nothrus) ............................................ 64

palustris (Oppiella) (=nova) ......................... 128

palustris (Oribatella) .................................... 171

palustris (Punctoribates) .............................. 183

palustris (Scapheremaeus) ............................ 159

palustris (Scapheremaeus) ............................ 159

palustris (Scapheremaeus) .......................... 159

palustris (Suctobelbella) ................................. 137

palustris (Suctobelbella) ............................. 137

palustris (Zeascheloribates) ........................ 195

panabokkei (Indoribates) ............................. 208

panabokkei (Rhopalozetes) ............................. 90

pandanus (Haplacarus) ............................... 38

pangasuganensis (Acrotocepheus) ................ 146

pankovi [M. (Multilanceoppia)] ……............. 117

pannonica (Liebstadia) ........................... 12, 195

pannonica (Liebstadia) ......................... 12, 195

pannonica (Oribatula) .................................... 187

pannonicus (Pantelozetes) ............................ 107

pannonicus (Punctoribates) .......................... 183

pannonicus (Scutovertex) .............................. 161

pannosus (Dolicheremaeus) ......................... 143

pantotrema (Apoplophora) .............................. 41

pantotrema (Apoplophora) .............................. 41

pantotrema (Apoplophora) .......................... 41

paoliana (Medioppia) (=subpectinata) ........ 124

paolianus (Heminothrus) ................................. 65

paolianus (Heminothrus) .................................. 65

paoliensis [L. (Dorycranosus)] ....................... 97

paolii [G. (Apograptoppia)] (=foveolata) .... 116

paolii [O. (Coronoquadroppia)]

(=monstuosa) …........................................... 134

paolii (Globozetes) (?=longipilus) ................. 180

paolii [R. (Insculptoppia)] ............................ 119

paolii (Pantelozetes) ...................................... 107

paolii (Pantelozetes) ........................................ 107

paolii (Pantelozetes) ..................................... 107

papillaris (Scheloribates) .............................. 201 papillaris (Taiwanoppia)

papillata (Striatoppia)

papillata (Striatoppia)

papillata (Striatoppia)

papillatus (Atropacarus) (=platakisi

papillatus (Chaunoproctus) (=pedestris)

papillifer [H. (Tuberemaeus)]

papillifer [H. (Tuberemaeus)]

papillipes (Metabelba)

papillipes (Metabelba)

papillisetigera (Corynoppia)

papillosus (Carabodes) (=coriaceus) …...... 149

papillosus (Microtegeus) .............................. 86

papineaui (Geminoppia) …....................... 110

papua (Acroppia) (=processigera) .............. 128

papua (Arcoppia) cronus ........................... 120

papua (Holonothrus) …................................. 67

papuana (Cryptobothria) ….................... 182

papuana (Dampfiella) ….......................... 141

papuana (Neoamerioppia) ….................... 114

papuana [S. (Ussuribata)] ......................... 139

papuanus [P. (Guineobodes)] …............... 153

papuanus (Humerobates) …....................... 181

papuanus (Orthozetes) ................................. 90

papuanus (Reteremulus) aciculatus .......... 102

papuanus (Yoshiobodes) .......................... 154

papuensis (Dolicheremaeus) ….................. 143

papuensis (Eutegaeus) ………………….... 85

papuensis (Novonothrus) ............................... 64

papuensis (Symbioribates) …...................... 196

paraanalis (Graptoppia) …............................ 116

paraaokii (Oribotritia) ……….............................. 44

parabadensis (Liebstadia) .............................. 195

parabaloghi [P. (Archiphthiracarus)] .......... 55

parabilis (Scheloribates) ............................. 201

parabotrichus [P. (Archiphthiracarus)] ............ 55

parabrevipodus (Scheloribates) …............ 201

paracapucinus (Protoribates) …................ 206

paracapucinus (Protoribates) ….................... 206

paracervus (Microtegeus) ……..................... 86

paraclavatus (Atropacarus) ............................ 48

paracorynopus (Belba) ..................................... 77

paracuriosus (Notophthiracarus) ……........ 51

paracutidens (Suctobelbella) ........................ 137

paradecipiens (Kulievia) ............................ 126

paradoxa (Arthrhoplophora) ............................. 35

paradoxa (Chavinia) .................................... 112

paradoxa (Galumnella) ............................... 222

paradoxa (Graptoppia) ................................ 116

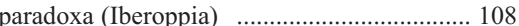

paradoxa (Lohmannia) .................................... 39

paradoxa (Tuberoppia) …........... 128

paraensis (Hoplophorella) ........................... 49

parafoliata (Ctenobelba) ................................. 101

parafoveolatus (Hoplophthiracarus) ................ 50

parafusulus (Euphthiracarus) ........................ 46

paragaveae (Mesoplophora) ......................... 42

paraglobosus [P. (Archiphthiracarus)] ............ 55

paraguayensis (Afronothrus) (=incisivus) ....59

paraguayensis (Nasobates) (=spinosus) ...... 212

paraguayensis (Peloribates) ...................... 210 paraguayensis (Pseudoamerioppia) .............. 118

paraguayensis (Pseudoamerioppia) ........... 118

paraguayensis (Torpacarus) omittens ............ 41

paraguayensis [H. (Urubambates)] ............ 194

parahirtus (Brachychthonius) .......................... 29

paraitalica (Metabelba) ................................... 80

paraleviseta [M. (Parplophora)] ....................... 42

paraligneus (Phthiracarus) (=ferrugineus) .... 53

parallela [O. (Coronoquadroppia)]

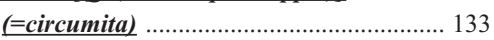

parallela [N. (Nippohermannia)] ....................6 68

parallela [N. (Nippohermannia)] .....................6 68

parallelodentata [S. (Flagrosuctobelba)] ...... 138

parallelus (Annectacarus) ............................ 37

parallelus [L. (Dorycranosus)] .......................... 97

parallelus [L. (Dorycranosus)] ..................... 97

parallelus (Poecilochthonius) …................. 31

parallelus (Pseudotocepheus) …................ 145

parallelus (Tumerozetes) …........................ 86

parananus (Scheloribates) ......................... 201

paranasalis (Brachioppiella) …................. 122

paraparvula [H. (Steganacarellus)] ............50

parapectinata (Medioppia) ......................... 123

parapinnata (Suctobelbella) ....................... 137

paraplanus (Sellnickochthonius) .................... 32

parapocsi [P. (Archiphthiracarus)] .............. 55

parapulverosa (Metabelba) ............................. 80

parareductus (Notophthiracarus) ................ 52

parareticulata (Acrotritia) ............................. 46

parareticulatus (Euphthiracarus) ……............ 46

pararusseolus (Allonothrus) ............................59

parascaber (Galumna) …............................ 216

parasensillus (Wallworkoppia) ….............. 121

parasentus (Notophthiracarus) …..................... 52

parasigma (Berniniella) .............................. 125

parasomalicus (Notophthiracarus) .............. 52

paraspinosa (Oribotritia) ................................. 44

paraspinosus (Carabodes) ............................... 150

paraspinosus [D. (Epidamaeus)] ..................... 80

parasummersi (Notophthiracarus) .............. 52

paratenuis [H. (Turcibates)] …................... 194

paratina (Dampfiella) ................................. 141

paratubulus [P. (Archiphthiracarus)] .............. 55

paravariolosus (Notophthiracarus) .............. 52

paravermiseta (Perxylobates) ….................... 205

pardinus (Notophthiracarus) ..................... 52

parilloi (Notophthiracarus) .......................... 52

paripilis (Neoamerioppia) ........................... 114

parisi (Oribatula) ........................................... 187

parisiensis (Hydrozetes) ................................... 155

paritractus (Ceratozetes) ................................. 174

parki (Porobelba) ............................................. 82

parmata (Basilobelba) .................................. 104

parmatus [P. (Archiphthiracarus)] .................. 55

parmeliae (Mycobates) ................................. 183

parmeliae (Mycobates) ................................. 183

partiocrispa (Subbelba) ........................................ 82

partita (Pelopsis) (=bifurcata) ..................... 183

parva (Allogalumna) .................................... 213

parva (Autogneta) ........................................ 108

parva (Furcoppia) .......................................... 92

parva (Galumna) .......................................... 216 


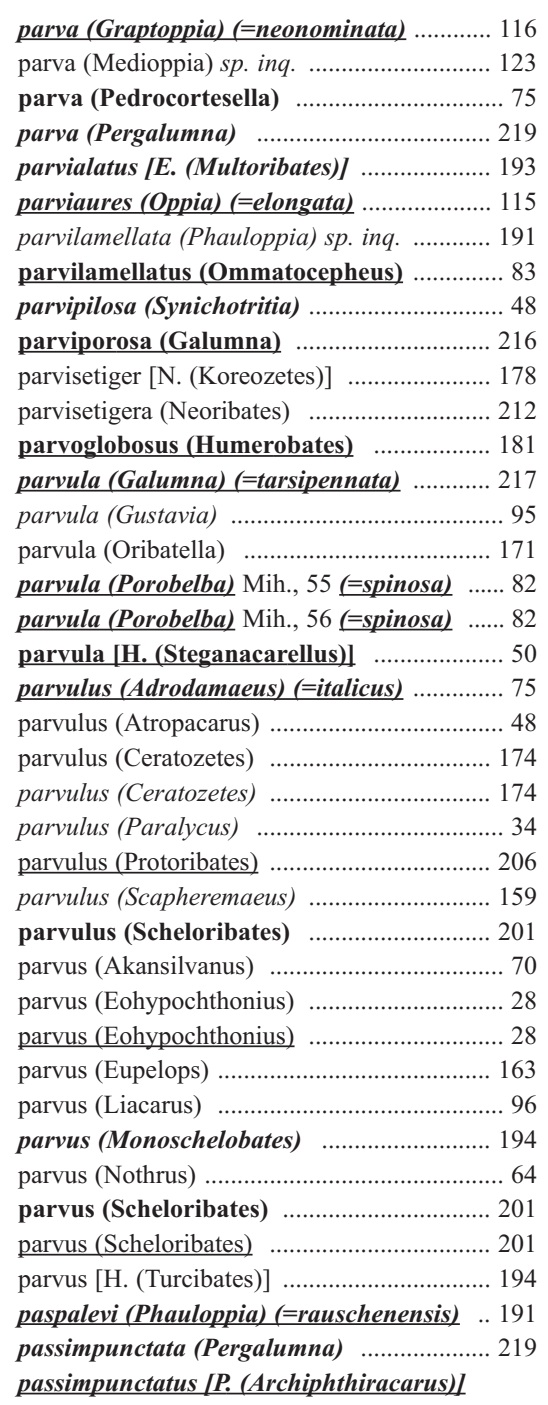

(=pygmaeus) ……........................................... 56

patagonica [M. (Pravoppia)] ……................. 111

patagoniensis (Liochthonius) ........................ 31

patavina (Campachipteria) ........................... 168

patchlorum (Heterozetes) (=aquaticus) ...... 173

patella (Scapheremaeus) ................................ 159

patella (Scapheremaeus) …...................... 159

patelloides (Belba) ......................................... 77

patoi (Temburongia) …................................. 48

patrius [M. (Calyptozetes)] .......................... 183

patruelis (Steganacarus) ……………................ 58

paucesculptus (Passalozetes) ....................... 162

pauciareolatus [M. (Cristonothrus)] ............ 7, 61

paucipectinatus (Hypochthonius) rufulus ..... 29

pauciramosa (Multioppia) .......................... 117

pauciseta (Heteroppia) …............................... 113

pauciseta (Heteroppia) ............................. 113

paucisetosa (Pergalumna) .............................. 219

paucituberculata [S. (Dyobelba)] ........................ 82

paucus [P. (Archiphthiracarus)] …….............. 55

paucus [P. (Archiphthiracarus)] ………...... 55

paucus [P. (Archiphthiracarus)] ..................... 55 pauliani (Brachychthonius) .......................... 29

pauliani (Galumnella) ….......................... 222

pauliani (Machadocepheus) ........................ 152

pauliani (Notophthiracarus) ....................... 52

pauliani [H. (Phyllhermannia)] …................ 70

pauliani (Pseudotocepheus) ....................... 145

pauliensis (Pergalumna) .............................. 219

pauliensis (Pseudotocepheus) ..................... 145

pauliensis (Scheloribates) ............................ 201

paulista (Hydrozetes) ...................................... 155

paulyi (Indotritia) ............................................. 43

pauper [N. (Coartobelba)] ............................ 135

pauropelor (Crotonia) …………………........ 67

pavidus [N. (Calyptophthiracarus)] ................ 53

pavlovskii [D. (Epidamaeus)] ........................ 80

pavlovskii (Papillacarus) ................................ 40

payatosensillus (Birobates) ......................... 203

pearsei (Gymnodamaeus) ................................ 76

pectigera (Neoamerioppia) ........................... 114

pectinata (Acrogalumna) ............................... 213

pectinata (Acrotritia) (=ardua) ...................... 45

pectinata (Kokoppia) ................................. 123

pectinata (Kokoppia) ................................. 123

pectinata (Lucoppia) (=burrowsi) ................ 187

pectinata (Mesoplophora) (=michaeliana) .... 42

pectinata (Multipulchroppia) Bal. y

Mah., 67 .....

pectinata (Multipulchroppia) Aoki, 67

$$
\text { (=siamensis) }
$$

pectinata (Nanhermannia)

pectinata (Nanhermannia) .............................6 68

pectinata (Oribella) ........................................ 107

pectinata (Oribella) ...................................... 107

pectinata (Senectoppia) …............................ 132

pectinata (Senectoppia) .............................. 132

pectinata [T. (Teratoppiella)] Bal., 61 ........ 132

pectinata [T. (Teratoppiella)] B. у M., 69

(=pluripectinata)

pectinatus (Bipassalozetes) ......................... 162

pectinatus (Dolicheremaeus) ........................ 143

pectinatus (Eremobodes) ............................... 109

pectinatus (Eremulus) ..................................... 102

pectinatus (Protoribates)

(?=lophothrichus) …….............................. 206

pectinatus (Spinozetes) .................................. 108

pectinifera (Belba) sp. inq. .......................... 77

pectiniger (Oxyzetes) ................................... 90

pectinigera (Ctenobelba) .............................. 101

peculiaris (Andesamerus) …...................... 105

peculiaris (Bornebuschia) ........................... 84

peculiaris (Platyamerus) …........................ 105

pedemontanus (Chamobates) sp. inq. .......... 180

pedestris (Chaunoproctus) ........................ 192

peduncularius (Liochthonius) .......................... 31

pegazzanoae (Cepheus) ………………………. 83

pehuen (Gozmanyina) .................................. 33

pellitus (Pheroliodes) ....................................... 74

pellucida (Allogalumna) ............................. 213

pellucidus [P. (Archiphthiracarus)] …......... 55

peloponnesiaca (Dissorhina) ornata .............. 125

peloptoides (Peloribates) .............................. 210

peloptoides (Peloribates) ................................ 210 peltifer [H. (Platynothrus)] ............................... 66

peltifer [H. (Platynothrus)] ............................ 66

peltifer [H. (Platynothrus)] .......................... 66

peltifer [H. (Platynothrus)] …............................66

peltifer [H. (Platynothrus)] …....................... 66

peltifer [H. (Platynothrus)] ............................ 66

peltiferinus [H. (Capillonothrus)] capillatus .... 66

pembertoni (Euscheloribates) …................ 197

pembertoni (Galumna) australis ............... 214

pembertoni (Protoribates) .......................... 206

pendula (Pulchroppia) ................................ 120

penetrabilis (Mochlozetes) ............................. 185

penetrabilis (Mochlozetes) .......................... 185

penicillata (Acrotritia) P.-I., 69

(=hyeroglyphica) ...................................... 45

penicillata (Acrotritia) Mah., 82

(=koreensis) ….......................................... 45

penicillata [S. (Flagrosuctobelba)] ............. 138

penicillata [S. (Flagrosuctobelba)] .............. 138

penicillatus (Hardybodes) ............................ 152

penicillatus [C. (Klapperiches)] ................ 151

penicillatus (Scheloribates) (?=fimbriatus) .. 199

penicillifer (Brassiella) ............................. 192

penicillifer (Punctizetes) ….......................... 167

penicillifer (Punctizetes) .............................. 167

penicilliger (Xenillus) .................................... 98

penicillum (Autogneta) .................................. 108

penicillus [C. (Klapperiches)] ....................... 151

penicillus (Liochthonius) ................................ 31

peniculatus (Carabodes) (=palmifer) .......... 150

pennata [O. (Zygoribatula)] ...................... 189

pennatus (Rhabdozetes) ............................... 90

pentasetarum [C. (Magoebazetes)] ............... 88

pentasetarum (Novoribatella) .................... 170

pentasetata [D. (Cylindroppia)] ................... 123

pentasetosus (Carabodes) ............................ 150

pentatrichus [A. (Uluguroides)] ................. 149

pepitensis [B. (Gressittoppia)] ...................... 122

pepitensis [B. (Gressittoppia)] ...................... 122

pepitensis (Liochthonius) ............................... 31

peracuta [S. (Flagrosuctobelba)] ................ 138

peracutus (Scheloribates) .......................... 201

peracutus (Steganacarus) (=sol) ..................... 58

perarmata (Suctobelbella) ............................ 137

perarmatus (Eupelops) (=major) ................ 163

perates (Mycobates) ...................................... 183

perdentata (Suctobelbella) .......................... 137

peregovitsi (Anomaloppia) .......................... 116

peregrinus (Meriocepheus) ............................. 153

perelegans (Liochthonius) .............................. 31

perennis (Pteroripoda) ................................... 205

perexigua (Mesoplophora) (=michaeliana) .... 42

perezi (Galumna) .......................................... 216

perezinigoi (Annectacarus) ............................. 37

perezinigoi (Arcoppia) .................................. 121

perezinigoi (Carabodes) ................................. 150

perezinigoi (Chamobates) ............................ 180

perezinigoi (Cosmochthonius) ......................... 33

perezinigoi (Ctenobelba) .............................. 101

perezinigoi (Hypogeoppia) ............................ 126

perezinigoi (Jacotella) (=frondeus) .............. 76

perezinigoi [L. (Lancelalmoppia)] ............... 111 
perezinigoi (Liacarus) ..................................... 96 perezinigoi [R. (Mantigueracarus)] .............. 58 perezinigoi (Nothrus) ................................ 11, 64 perezinigoi (Nothrus) ............................. 11, 64 perezinigoi (Notophthiracarus) ……............. 52 perezinigoi [T. (Rostrozetes)] ....................... 212 perezinigoi (Sphaerozetes) ........................... 178 perezinigoi (Steganacarus) (=hirsutus) ........58 perfecta (Multioppia) ……............................. 117 perfecta (Murcia) ........................................ 177 perfectus [S. (Tropacarus)] (=brevipilus) ...... 59 perforata (Crotonia) ......................................... 67 perforata (Suctobelbella) ............................... 137 perforatoides [H. (Tuberemaeus)] ................ 194 perforatulus (Scutovertex) ............................ 161 perforatus (Bipassalozetes) ........................... 162 perforatus (Scheloribates) ........................... 201 perforatus (Scutovertex) (=neonominatus) .. 161 perforatus [H. (Tuberemaeus)] ....................... 194 perforatus [H. (Tuberemaeus)] ………….... 194 perfusorius (Liochthonius) ............................... 31 pergeli (Arcoppia) (=rugosa) ..................... 121 pergrata (Hoplophorella) .............................. 49 periculosa (Brachioppiella) ......................... 122 perinfamis (Cyrthermannia) sp. inq. ............... 68 perisi (Anachipteria) ....................................... 168 perisi (Arcoppia) ......................................... 121 perisi (Dolicheremaeus) ............................... 143 perisi (Hoplophorella) .................................... 49 perisi (Scheloribates) ................................... 201 peristomaticus [P. (Archiphthiracarus)] .......... 55 peritus (Ceratozetes) ....................................... 174 peritus (Ceratozetes) ................................... 174 perkinsi (Humerobates) .............................. 181 perkinsi (Phauloppia) ................................. 191 perlongoides (Birsteinius) .............................. 95 perlongum (Murcia) ...................................... 177 perlongus (Birsteinius) ...................................... 95 perlongus [H. (Urubambates)] ....................... 194 perlongus (Zachvatkinibates) ........................ 184 perlucidus (Phthiracarus) ............................. 54 perlucunda [H. (Steganacarellus)] .............. 50 permirus (Hoplophorella) .............................. 49 permixta [S. (Ussuribata)] (=papuana) ...... 139 permixtus (Bipassalozetes) ............................ 162 permixtus (Xenillus) (=clypeator) .................. 98 permodica (Mesoplophora) ............................ 42 permodica (Mesoplophora) ............................ 42 pernettyae [S. (Campbelloppia)] ................... 124 perolata (Oppia) (=nitens) ............................ 115 perona [P. (Nododamaeus)] ............................ 81 perparvus (Phthiracarus) ................................ 54 perpendiculata (Suctobelbella)

(=acutidens) ............................................ 136
perpropinqus [P. (Neophthiracarus)] …...... 56 perproxima (Campachipteria) ....................... 168 perpusillus (Liochthonius) (=brevis) ……..... 30 perreti (Cavernocarabodes) ....................... 151 perreti (Dolicheremaeus) ............................ 143 perreti (Peloribates) ..................................... 210 perrugosa (Eremobelba) ................................. 103 persetosus (Pseudopirnodus) persimilis [P. (Archiphthiracarus)]

persimilis (Murcia) persimilis (Rhacaplacarus)

persimplex (Phthiracarus) personatus (Lamellocepheus) personatus (Steganacarus) perspicua [O. (Perspicuoppia)] pertenuis [M. (Parplophora)] pertenuis [M. (Parplophora)] perti (Notophthiracarus) pertineata [L. (Lancelalmoppia)] pertrichosa (Pergalumna) ............................. 219 pertusus (Cepheus) sp. inq. ………………….... 83 peruensis (Acrotritia) ........................................ 46 peruensis (Acrotritia) ................................... 46 peruensis (Aeroppia) .................................... 112 peruensis (Berlesezetes) ……............................ 87 peruensis (Berlesezetes) peruensis [M. (Cristonothrus)] peruensis [M. (Cristonothrus)] peruensis (Nothrus) peruensis (Suctobelbila) peruensis (Xenillus) perversus (Atropacarus) pervicax (Hoplophorella) pervigens (Rhacaplacarus) peseki (Dampfiella) ..... petalus (Caudamaeolus) petalus [J. (Euryacarus)] petiolata (Laroppia) petiti (Campachipteria) petri (Ceratozetes) (=simulator) petrinjensis (Globozetes) ........ petrocoriensis (Microzetes) petrohuensis (Austroppia) .............................. 122 petronia (Maudheimia) ................................. 195 petrophagus (Scapheremaeus) ..................... 159 petrosus (Scapheremaeus) ............................. 159 petrovae (Umbellozetes) ............................... 170 petrovi (Ceratozetes) .................................... 174 petrunkevitchi (Hydrozetes) ........................... 155 peullaensis [B. (Plaesioppia)] ....................... 122 phaeonotus (Peloptulus) ................................ 164 phalangioides (Metabelbella) .......................... 81 phalerata (Apoplophora) ............................... 41 phaleratus [P. (Neophthiracarus)] ................ 57 phaseolus (Brazilozetes) ................................ 88 philippica (Dampfiella) ............................... 141 philippinensis (Acrotocepheus) ................... 146 philippinensis (Allosuctobelba) grandis ........ 134 philippinensis (Campbellobates) ….............. 204 philippinensis (Dolicheremaeus) ................. 143 philippinensis (Farchacarus) ….................... 173 philippinensis (Nesiacarus) …...................... 40 philippinensis [I. (Philippizetes)] .................. 208 philippinensis (Ramusella) …...................... 118 philippinensis (Scheloribates) ….................. 201 philippinensis (Suctotegeus) ……….............. 86 philodendrus (Tuparezetes) ………............. 133 phitosi (Hauseroplophora) ............................... 35 phitosi (Liochthonius) ……............................. 31 phitosi (Microzetes) ......................................... 89 phoretica (Neoamerioppia) ........................ 114 phryxothrixus (Xenillus) (=occultus) ............ 98 phylliferus (Zetorchestes) ........................... 91 phylliformis (Carabodes) ............................. 150 phylliformis [H. (Phyllhermannia)] ............ 70 phyllophora (Oribatella) .............................. 171 phyllophora [H. (Phyllhermannia)] …........ 70 phyllophorus (Atropacarus) ............................ 48 phyllophorus (Atropacarus) …........................ 48 phyllophorus (Machadocepheus) .............. 152 phyllophorus (Neoeutegaeus) …………...... 85 phyllophorus (Sellnickochthonius) ................. 32 phyllophorus (Sphaerochthonius) ................ 34 phyllosetus (Zetorchestes) ............................. 91 phyllotrichus (Dolicheremaeus) ............... 143 physoseta [M. (Stylozetes)] ............................ 89 phytophilus (Eupelops) (=acromios) .......... 163 picea (Hermanniella) ....................................... 70 picea (Hermanniella) ....................................... 70 picea (Hermanniella) ....................................... 70 picipes (Diapterobates) (=humeralis) .......... 181 picturatus (Austrocarabodes) .................... 148 pictus (Scutovertex) .................................... 161 piffli (Arcoppia) ............................................. 121 piffli (Eremobelba) ................................... 104

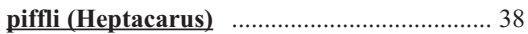
piffli (Lanceoppia) ….............................. 110 piffli (Mesotritia) (=nuda) ................................ 44 piger (Phthiracarus) ....................................... 54 piger (Phthiracarus) ........................................ 54 pigerrima (Camisia) (=biurus) ……...............6 65 pileiformis (Nothrus) sp. inq. .......................... 64 pilifera [S. (Flagrosuctobelba)] .................. 138 piligera (Liebstadia) sp. inq. ...................... 195 pilipes (Trimalaconothrus) ............................ 62 pilipinus (Eremulus) ……............................... 102 pilipinus (Peloribates) …............................. 210 pilosa (Epilohmannia) ................................... 37 pilosa (Galumnella) …................................ 222 pilosa (Liebstadia) ......................................... 195 pilosa (Murcia) (=brevicuspis) .................... 177 pilosa [O. (Oxyoppiella)] (=polynesia) ........ 129 pilosa (Phauloppia) Koch, 1841 ..................... 191 pilosa (Phauloppia) Koch, 1841 ................... 191 pilosa (Phauloppia) Banks, 1895 (=banksi) .. 190 pilosella (Ctenobelba) .................................. 101 pilosella (Condyloppia) ............................... 116 pilosellus (Malaconothrus) ............................ 61 pilosellus (Pilobates) .................................... 211 pilosellus (Pilobates) .................................... 211 pilosetosus (Scutovertex) ............................... 161 pilososetosa (Balazsella) .............................. 192 pilososetosus (Mixochthonius) .......................... 31 pilososetosus (Mixochthonius) ........................ 31 pilosus (Africoribates) ................................. 180 pilosus [P. (Archipththiracarus)]

(=bryobius) ……….................................... 55 pilosus (Astegistes) .................................... 92 pilosus [J. (Euryacarus)] ................................ 38 pilosus [P. (Neophthiracarus)] .................... 57 pilosus (Parhypochthonius) ............................ 28 pilosus (Peloribates) ...................................... 210 
pilosus (Peloribates)

piluliferus (Neoliochthonius) .......... 31

piluliferus (Neoliochthonius) ............................ 31

pinea [M. (Moritzoppiella)] ………………..... 127

pinguis [D. (Epidamaeus)] ……………………... 80

pinguis [T. (Rostrozetes)] ........................... 212

pinguisetus (Peloribates) ........................ 12, 210

pinguisetus (Peloribates) ........................ 12, 210

pini (Fuscozetes) …………………………... 176

pini [B. (Lanibelba)] ……………………...... 78

pini [B. (Lanibelba)] _....................................... 78

pinicola (Oripoda) ………………………….... 204

pinicus (Propelops) ………………………... 164

pinicus (Propelops) ………………………... 164

pinifera (Ramusella) ........................................ 118

pinnatifilis (Hammerella) $s p$. inq. .............. 132

pinnata (Phauloppia) sp. inq. ...................... 191

pinnatus (Austrocarabodes) …………….... 148

pinnatus (Eutegaeus) ……………………...... 85

pinnigera (Lohmannia) …………………..... 39

pinnigera (Suctobelbella) ............................. 137

pinsapi (Medioppia) ……………………….... 123

piramidalis (Odontocepheus) .......................... 153

pirata (Fossoppia) ……………………...... 128

pirenaicus (Carabodes) ................................... 150

piriformis (Belba) …………………………….... 77

piriformis (Sphaerozetes) ………………….... 178

piriformis (Trichoppia) …………………...... 94

pirinensis (Carabodes) ………………............ 150

pisacensis (Oribatula) ................................... 187

pisacensis (Scapheremaeus) ........................ 159

pisgahi (Euphthiracarus) (=pulchrum) ........ 46

pistillifer (Fosseremus) (=laciniatus) .......... 103

pitentzin [T. (Tyrphonothrus)] ....................... 63

piton (Perezinigokalumma) ........................... 213

pius (Brachychthonius) .................................. 29

pius (Brachychthonius) .................................. 29

planatus (Belorchestes) ……………………..... 91

planiclava (Galumna) ................................... 216

planicornis (Eupelops) $s p$. inq. ....................... 163

planissima [P. (Monophauloppia)] .............. 190

plantivaga (Dometorina) …………………...... 192

plantivaga (Dometorina) ............................... 192

plantivaga (Dometorina) ............................. 192

plantivaga (Dometorina) ………………….. 192

plantivaga (Dometorina) ............................... 192

planus (Heteroleius) ………………………....... 194

planus (Phthiracarus) ………………………... 54

planus (Sellnickochthonius) ............................... 32

planus (Sellnickochthonius) ............................. 32

planus (Trimalaconothrus) $s p$. inq. ............... 62

platakisi (Atropacarus) ..................................... 48

platensis (Hydrozetes) sp. inq. ...................... 155

platensis (Podoribates) ................................ 186

platensis (Siculobata) sicula ......................... 195

platynotus [M. (Parametabelba)] ……………... 81

platypterus (Acanthozetes) …………………... 87

platyrhinoides (Ceratozetes) ......................... 174

platyrhinus (Ceratozetes) ............................. 174

platyrhinus (Trimalaconothrus) .................... 62

platyrhinus (Trimalaconothrus) plenus [P. (Archiphthiracarus)] …............... 55

pletzenae [L. (Convergoppia)]

(Eneonominata)

pletzenae (Pheroliodes) (=africanus)

pletzenae (Pletzenoppia) .............................. 123

plicata (Machuella) ventrisetosa ………….... 133

plicata (Suctobelbella) ……………………..... 137

plicatus (Eupelops) …………………………... 163

plicatus (Eupelops) ……………………….... 163

plokosus (Gymnodamaeus) ............................. 76

plowmanae (Allogalumna) ........................... 213

plumalae (Pergalumna) weberi .................. 220

plumata [S. (Flagrosuctobelba)]

(=praeoccupata) …………………….... 138

plumata (Medioppia) ...................................... 123

plumata (Pergalumna) ………………….... 219

plumata (Suctobelbella) ................................. 137

plumatus (Cosmochthonius) .............................. 33

plumatus (Cosmochthonius) .............................. 33

plumatus (Cosmochthonius) ............................ 33

plumifer (Plumozetes) .................................... 90

plumiger (Spathulocepheus) ......................... 153

plummeri (Oribatella) …………………....... 171

plumosulus (Yoshiobodes) .............................. 154

plumosus (Chaunoproctus) ………………...... 192

plumosus [M. (Cristonothrus)] ……………....... 6

plumosus [D. (Epidamaeus)] ............................ 80

plumosus (Eremaeus) ...................................... 100

plumosus (Halozetes) ………………………..... 157

plumosus (Heptacarus) …………………...... 38

plumosus [Z. (Keralotrichus)] ...................... 185

plumosus (Liochthonius) Mah., 69 ................. 31

plumosus (Liochthonius) Chin. y Aoki, 72

$(=$ alius $)$.................................................. 30

plumosus (Otocepheus) …………………..... 147

plumosus (Peloribates) …………………….... 210

plumosus (Sellnickochthonius) ……………... 32

plumosus (Yoshiobodes) ................................ 154

pluripectinata [O. (Dzarogneta)] ………... 129

pluripectinata [T. (Teratoppiella)] ................. 132

pluriseta (Nanhermannia) Mah., 84 ............ 68

pluriseta (Nanhermannia) Mah., 88

(=sabahensis)

plurisetosa (Pulchroppiella) ……………........ 118

plurisetus (Benoibates) ................................ 203

plurisetus (Dolicheremaeus) ........................ 143

pocsi (Allogalumna) ..................................... 213

pocsi [P. (Archiphthiracarus)] (=anonymus) ....54

pocsi (Eupelops) ………………………....... 164

pocsi [B. (Gressittoppia)] ……………….... 122

pocsi [R. (Insculptoppiella)] …………............ 119

pocsi [C. (Klapperiches)] ………………..... 15

pocsi [M. (Megazetes)] …………………….... 89

pocsi (Pergalumna) ...................................... 219

pocsi [H. (Phyllhermannia)] sp. inq. ............. 70

pocsi (Rhynchoribates) ……………........... 140

pocsi (Suctobelbila) ........................................ 139

pocsorum (Basidoppia) ............................... 121

poensis [T. (Rostrozetes)] ovulum .............. 212

poensis [T. (Rostrozetes)] ovulum ................ 212

poggii [C. (Klapperiches)] .............................. 151 polaris (Murcia) ...………………………..... 177

polita (Oxyoppia) ..................................... 129

polita [M. (Parplophora)] ................................. 42

polita [M. (Parplophora)] ………………...... 42

politus (Eueremaeus) (=columbianus) ........ 100

politus (Liacarus) $(=$ coracinus) $\quad$..................... 95

politus (Rhacaplacarus) (=eximius) ............... 57

pollinivorus (Saxicolestes) ............................... 91

polonicus (Amerus) ………………………... 104

polpaicoensis [Z. (Protozetomimus)] .......... 179

polychothomus [L. (Dorycranosus)] ………... 97

polychothomus [L. (Dorycranosus)] .............. 97

polygonalis (Licneremaeus) ……………...... 160

polygonalis [S. (Porozetes)] ……................... 178

polygonalis [S. (Porozetes)] ……………….... 178

polygonata (Lunoribatula) ………………... 187

polygonata (Lunoribatula) ............................ 187

polygonata (Neoamerioppia) ........................ 114

polygonatus (Africoribates) .......................... 180

polygonatus [T. (Rostrozetes)] ………........... 212

polygonatus (Scheloribates) .......................... 201

polygrammus (Nortonella) .............................. 76

polynesia [O. (Oxyoppiella)] ......................... 129

polvnesia [O. (Oxyoppiella)] ....................... 129

polynesia [O. (Oxyoppiella)] ....................... 129

polynesica (Striatoppia) similis .................. 131

polynesius (Nesopelops) …………………..... 164

polyphyllos (Adhaesozetes) ......................... 158

polyporetes (Carabodes) ............................... 150

polyporus (Galumna) ………………………... 216

polyptychus (Megalotocepheus) ………....... 147

polysetosa (Ctenobelba) …………………...... 101

polysetosus (Neoliodes) ……………………..... 72

polysetosus (Scapheremaeus) .......................... 159

polystriata [H. (Heterohermannia)] ............... 69

polytretos (Euphthiracarus) (=crassisetae) .... 46

polytricha (Camisia) ………………................ 65

polytrichus (Austrocarabodes) …………... 148

pomboi [D. (Eudamaeus)] ……………………... 80

pomboi (Humerobates) ................................... 181

ponticula [S. (Flagrosuctobelba)] ................... 138

ponticula [S. (Flagrosuctobelba)] ………... 138

ponticuliger (Scheloribates) ......................... 201

ponticus (Cosmochthonius) ............................. 33

ponticus (Scheloribates) ........................................ 201

pontiger (Carabodes) (=marginatus) ……... 150

pontiger (Ceratobates) .................................... 166

pontigera [N. (Coartobelba)] ………………... 135

poppei (Adoristes) ………………………..... 95

poppei (Adoristes) ………………………...... 95

poppi (Lanceoppia) ……………………...... 110

porcella (Eremobelba) ……………………... 104

porcella (Nasozetes) ……………………….... 194

porcellus (Neoliodes) sp. inq. ........................ 72

porcinolus (Dolicheremaeus) .......................... 143

porcula (Meristacarus) ……………………….... 39

porcula (Meristacarus) ……………………..... 39

porcula (Meristacarus) .................................... 39

pori (Lasiobelba) ………………………….... 113

porifera (Arcoppia) ...................................... 121

poronota (Trypogalumnella) ......................... 222

porosa (Fenichelia) ………………………..... 159 


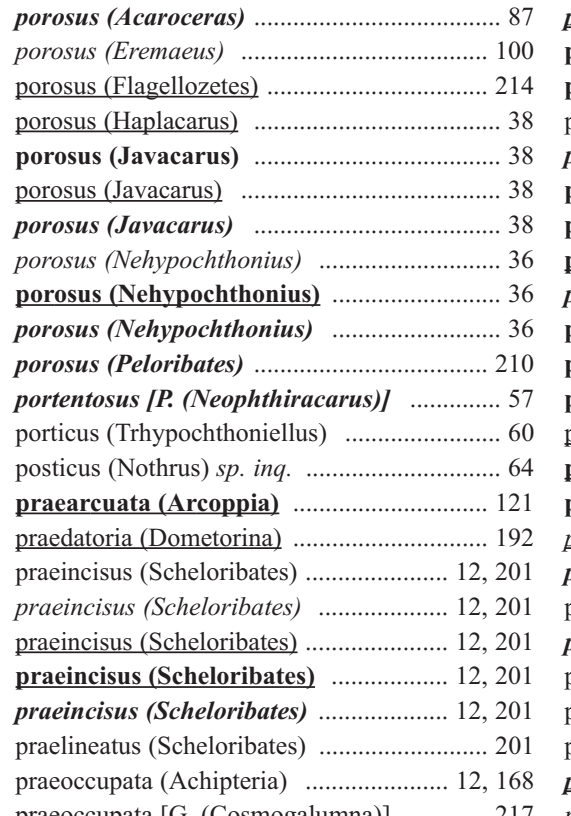

praeoccupata [G. (Cosmogalumna)] ............ 217

praeoccupata [S. (Flagrosuctobelba)] ............. 138 praeoccupata (Hoplophorella) ....................... 49

praeoccupata (Oribatula) ............................. 187

praeoccupatissimus (Scheloribates) ......... 201

praeoccupatus (Dolicheremaeus) .................. 143

praeoccupatus (Malaconothrus) .................. 61

praeoccupatus [P. (Neophthiracarus)] ........ 57

praeoccupatus (Nothrus) ……......................... 64

praeoccupatus (Peloribates) ……….............. 210

praeoccupatus [H. (Platynothrus)] ...................6 66

praeoccupatus (Scheloribates) ….............. 201

praeoccupatus [P. (Triaungius)] ................... 206

praestans [M. (Moritzoppiella)] ..................... 127

praestantissimus (Scheloribates) ................ 201

praetiosa (Notogalumna) .............................. 218

praetiosa (Notogalumna) ............................ 218

praetritia (Epilohmannia) ............................... 37

pragensis (Punctoribates) .............................. 183

prahuensis (Trimalaconothrus) ……...................62 62

prasadi (Belba) .................................................. 77

prasadi (Christovizetes) …….......................... 88

pratensis (Anachipteria) .............................. 168

pratensis (Mesoplophora) cubana ................ 42

pratensis (Nothrus) ........................................... 64

pratensis (Nothrus) .......................................... 64

pratensis (Podoribates) ................................ 186

prelli (Suctobelbata) ....................................... 136

prelli (Suctobelbata) ........................................ 136

presbytis [C. (Ensicamisia)] .............................. 65

pretiosa (Banksinoma) ................................. 109

prima (Baloghiella) ...................................... 207

primorica (Oppiella) ...................................... 128

primoricus (Scheloribates) ............................. 201

princeps (Heminothrus) (=targionii) .............66

principalis (Diapterobates) (=reticulatus) .. 181

principalis [S. (Polyxylobates)] .................. 207

prionotus (Protoribates) (=lophothrichus) .. 206

prior (Liochthonius) ......................................... 31 prior (Phthiracarus) (=longulus) .................. 54

prismaticus [M. (Cristonothrus)] ................ 61

problematica (Erioppia) ................................ 113

problematica (Moritzoppia) .......................... 127

problematica (Topalia) ................................. 86

problematicus (Ceratozetes) ....................... 174

problematicus [C. (Klapperiches)] ............ 151

probus (Phthiracarus) …............................. 54

procera (Dampfiella) .................................... 141

procera (Leptoppia) ................................... 123

procerus (Notophthiracarus) ........................ 52

processigera (Acroppia) ............................... 128

processigera (Acroppia) ………..................... 128

processigera (Acroppia) ............................. 128

processus (Anellozetes) ................................. 181

processus [C. (Magellozetes)] ....................... 175

processus /C. (Magellozetes) .......................... 175

processus (Malaconothrus) ................................. 61

processus [M. (Stylozetes)] ........................... 89

prodigiosus (Licneremaeus) ........................... 160

prodorsissima [O. (Zygoribatula)] ................ 189

producta (Medioppia) .................................... 124

producta (Oribatella) (=ornata) .................. 171

profundus (Elliptochthonius) .......................... 28

prolongata (Oribatella) ................................ 171

promecus (Cryptacarus) ................................... 38

promecus (Cryptacarus) ............................... 38

prominens (Hoplophorella) ........................... 49

prominens (Oribatula) ................................ 187

prominens (Oripoda) .................................... 204

prominens (Passalozetes) ........................... 162

prominens (Suctobelbella) .............................. 137

promissus (Pseudotocepheus) ..................... 145

propexa (Metabelba) ..................................... 80

propinqua (Indotritia) ........................................ 43

propinqua (Metabelba) ................................... 80

propinqua (Oppiella) ................................... 128

propinqua (Pedrocortesella) …...................... 75

propinqua [O. (Zygoribatula)] (=glabra) .... 189

propinquus (Bipassalozetes) (=perforatus) .. 162

propinquus (Licneremaeus) $s p$. inq. .......... 160

propinquus (Liochthonius) .............................. 31

propinquus (Phthiracarus) .............................. 54

propinquus [H. (Tuberemaeus)] ..................... 194

prosopis (Parapirnodus) ............................... 191

prostrata (Dampfiella) ................................ 141

proximus (Acaronychus) ................................... 27

proximus (Eueremaeus) ................................ 100

proximus (Hemileius) ................................... 193

proximus (Hoplophthiracarus) ........................ 50

proximus (Hoplophthiracarus) …................5 50

proximus (Hoplophthiracarus) ...................... 50

proximus (Tectoribates)

prudens (Murcia) (?=angustata) …............ 177

prunum (Carabodes) ....................................... 150

psadilliferus (Stictozetes) ............................... 221

psammophylus (Brachychthonius) .................. 29

psammophylus (Ceratozetes) ......................... 174

pseudoaciculatus (Papillacarus) ....................... 40

pseudoaciculatus (Papillacarus) ….............. 40

pseudoareolatus (Lamellozetes) ................... 93

pseudoareolatus [H. (Tuberemaeus)] ......... 194 pseudoauritus (Damaeus) ................................. 78

pseudoborussicus (Nothrus) (=anauniensis) $\quad$.. 63

pseudocallipygus (Torpacarus) ...................... 41

pseudocarinatus [S. (Tropacarus)] .................. 59

pseudocircumita [Q. (Coronoquadroppia)] .... 134

pseucocircumita [Q. (Coronoquadroppia)] .. 134

pseudocircumita [Q. (Coronoquadroppia)] .. 134

pseudoconfinis (Neotrichoppia) .................... 127

pseudocontiguus (Liacarus) ............................. 96

pseudocorrugata [M. (Pravoppia)] .............. 111

pseudocorynopus (Belba) (=patelloides) ...... 77

pseudocostulata (Brachioppia) (=deliciosa) .. 122

pseudofurcatus (Acaroceras) ....................... 87

pseudofurcatus (Dolicheremaeus) ............... 143

pseudofurcatus [T. (Rostrozetes)] ................. 212

pseudofusiger (Minunthozetes) .................... 182

pseudohigginsi [B. (Gressittoppia)] .......... 122

pseudohystricinus (Liochthonius) .................. 31

pseudoimmaculatus (Brachychthonius) .......... 29

pseudoitalica (Metabelba) .............................. 80

pseudolamellatus (Malaconothrus) ................... 61

pseudolaticeps (Verachthonius) ...................... 32

pseudomaculatus (Ameronothrus)

(=maculatus) …............................................ 156

pseudomargaritata (Pergalumna) …............. 219

pseudomochlosimilaris (Scheloribates) .... 201

pseudonodosa [H. (Heterohermannia)] .......... 69

pseudopapillipes [M. (Neobelba)] .................. 81

pseudoporosus (Peloribates) …...................... 210

pseudoprincipalis (Scheloribates) .............. 201

pseudoreticulatus (Austrocarabodes) .......... 148

pseudoreticulatus (Scapheremaeus) .......... 159

pseudornata (Suctobelbella) ........................ 137

pseudornatissima (Suctobelbella) ................. 137

pseudosculptus (Eupterotegaeus) sp. inq. ........ 83

pseudosetosus (Fuscozetes) .................... 12, 176

pseudosetosus (Fuscozetes) ................... 12, 176

pseudotatricus [D. (Epidamaeus)] …………... 80

pseudovermiseta (Pilobatella) ..................... 211

psyla (Basidoppia) ....................................... 121

pterata (Pilogalumna) (=ornatula) .............. 220

pterinervis (Pergalumna) .............................. 219

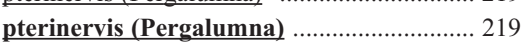

pterolineata [G. (Indogalumna)] ................ 217

pterota (Hafenrefferia) (=gilvipes) ................ 99

pterotus [N. (Protokalumma)] ....................... 212

pterygoides (Totobates) ................................ 196

pubescens (Krivolutskiella) ........................... 33

pubescens (Scheloribates) .......................... 201

pudicus (Phthiracarus) ................................. 54

puella (Epilohmannia) .................................. 37

puertomonttensis (Oribatella) ...................... 171

puertomonttensis (Ramusella) ....................... 118

puertomonttensis (Ramusella) ..................... 118

puertomonttensis (Ramusella) ..................... 118

puertomonttensis (Ramusella) .................... 118

pugio (Acaroceras) ....................................... 87

pulchella (Eremella) ...................................... 106

pulchella (Eremobelba) ............................... 104

pulchella [H. (Heterohermannia)] .................. 69

pulchella (Phylloribatula) ........................... 160

pulchella [G. (Porogalumnella)] .................... 222 


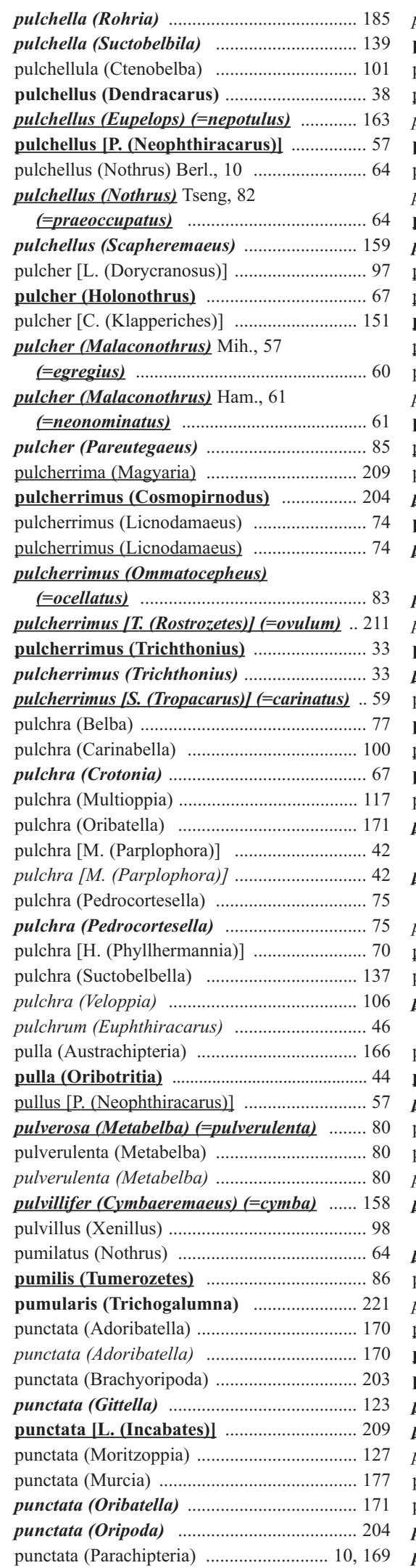

punctata (Parachipteria) ....................... 10, 169

punctata (Parachipteria) .................... 10, 169

punctata (Pergalumna) nervosa .................... 219

punctata [H. (Phyllhermannia)] ...................... 70

punctata (Suctobelbella) ............................. 137

punctata (Trichogalumna) ........................... 221

punctatus [M. (Calyptozetes)] ........................ 183

punctatus [M. (Calyptozetes)] ...................... 183

punctatus [P. (Constrictocepheus)] …......... 145

punctatus [P. (Constrictocepheus)] .............. 145

punctatus (Dolicheremaeus) ........................... 143

punctatus (Hoplophthiracarus) ........................ 50

punctatus (Nesopelops)

punctatus (Papillacarus) .................................. 40

punctatus [H. (Platynothrus)] ............................ 66

punctatus [H. (Platynothrus)] .......................... 66

punctatus [T. (Rostrozetes)] ...................... 212

punctatus [T. (Rostrozetes)] ........................... 212

punctatus (Scutovertex) ............................... 161

punctatus (Tectoribates) (=proximus) .......... 172

punctatus [P. (Triaungius)] ........................ 206

punctatus [H. (Tuberemaeus)]

(=neonominatus

punctatus [H. (Urubambates)]

punctillata (Suctobelbila) .............................. 139

punctipennis (Galumnella) ........................ 222

punctulata (Hermanniella) (=picea) .............. 70

punctulata (Multipulchroppia) schauenbergi .. 120

punctulata (Oribatella) sp. inq. .................. 171

punctulata (Pergalumna) ................................ 219

punctulata (Pilobatella) ............................ 21

punctulatus (Atropacarus)

punctulatus [L. (Dorycranosus)]

(=splendens)

punctulatus (Euphthiracarus) Berl., 13

$=$ cribrarius

punctulatus (Euphthiracarus) Jac., 30 .............. 46

punctulatus (Indoribates) .............................. 208

punctulatus (Malaconothrus) Ham., 52 .......... 61

punctulatus (Malaconothrus) Bal., 58

(=praeoccupatus) ...................................... 61

punctulatus (Proteremaeus) ........................... 108

punctulatus [N. (Protokalumma)] .............. 212

punctulatus [T. (Rostrozetes)] (=ovulum) .... 211

punctulatus (Steganacarus) (=spinosus) .......... 58

punctulatus (Tectocepheus) .......................... 154

punctulatus (Xenillus) Banks, 1895 ................ 98

punctulatus (Xenillus) J. у P. B., 1984

(=neonominatus)

punctum (Punctoribates) _............. 183

punctum (Punctoribates) ............................. 183

punctum (Punctoribates) ............................. 183

punctum (Punctoribates) .......................... 183

pundamiliaensis (Scapheremaeus) ............ 159

puniceus (Trhypochthoniellus) (=longisetus) .. 60

pupula (Transoribates) (=lagenula) ............ 207

puritanicus [D. (Epidamaeus)] ...................... 80

purpurarius [C. (Klapperiches)] ..................... 151

purvisi (Malaconothrus) ....................................... 61

pusilla (Galumna) ..................................... 216

pusilla (Mesoplophora) .................................... 42 pusilla (Oribatella) ......................................... 171

pusillus (Adrodamaeus) …............................... 75

pusillus (Allozetes) .......................................... 166

pusillus (Chamobates) ................................. 180

pusillus (Diapterobates) .................................. 181

pusillus (Liochthonius) ...................................... 31

pusillus (Phthiracarus) .................................... 54

pusillus (Phthiracarus) ................................. 54

pustulata [O. (Dzarogneta)] ........................ 129

pustulata (Hoplophorella) ............................ 49

pustulata (Reticuloppia) ................................. 191

pustulatus (Cepheus) ........................................ 83

pustulatus (Dolicheremaeus)

pustulatus (Limnozetes) ................................. 166

putinglupa (Dampfiella) .................................. 141

puyeue (Novonothrus) .................................... 64

puylaerti [P. (Archiphthiracarus)] .............. 55

puzanovi (Epimerella) ................................... 131

pygiseta (Uracrobates) …............................ 186

pygmaea (Heterogalumna) ........................ 217

pygmaeus [P. (Archiphthiracarus)] ................... 56

pygmaeus [P. (Archiphthiracarus)] ............... 56

pygmaeus [P. (Archiphthiracarus)] ............ 56

pygmaeus [P. (Archiphthiracarus)] ................... 56

pygmaeus [P. (Archiphthiracarus)] …........ 56

pygmaeus [P. (Archiphthiracarus)] .............. 56

pygmaeus (Malaconothrus) ...................... 11, 61

pygmaeus (Malaconothrus) ..................... 11, 61

pygmaeus (Malaconothrus) ..................... 11, 61

pygmaeus (Pseudotocepheus) .................... 145

pyramidalis (Neoliodes) ................................ 72

pyramidalis (Pergalumna) ............................. 219

pyramidalis (Trizetes) ..................................... 140

pyrenaica (Cerachipteria) digita ................... 168

pyrenaica (Hungarobelba) ............................. 103

pyrenaicus [D. (Epidamaeus)] ….................... 80

pyrenaicus (Microzetes) .................................. 89

pyri (Galumna) (=flabellifera) .................... 215

pyriformis (Allonothrus) …............................. 59

pyriformis (Machuella) ventrisetosa ….... 133

pyriformis (Truncopes) ….............................. 205

pyrigerum (Adoristes) (=ovatus) .................... 95

pyrigerus (Paralycus) ...................................... 34

pyrostigmata [O. (Zygoribatula)] (=frisiae) .. 189

quadracantha (Camisia) (=spinifer) .............. 65

quadrangulus (Carabodes) ........................... 150

quadrata [S. (Flagrosuctobelba)] .............. 138

quadrata (Lamellarea) ............................... 160

quadriareatus (Minunthozetes) ...................... 182

quadricarina [S. (Flagrosuctobelba)]

(=elegantula) ............................................. 138

quadricarinata (Austrotritia) ..................... 42

quadricarinata (Quadroppia) ........................ 133

quadricarinata (Quadroppia) ........................ 133

quadricarinata (Quadroppia) ................... 133

quadricarinata (Quadroppia) ........................ 133

quadricaudicula (Jacotella) ............................ 76

quadricornis (Lamellobates) ........................ 167

quadricornuta (Cyrthermannia) …................... 68

quadricornuta (Oribatella) ............................. 171

quadricornuta (Oribatella) ......................... 171

quadricornutus (Hymenozetes) ...................... 88 
quadricuspidatus (Cultrobates) quadricuspis (Oribatella)

(?=quadricornuta) ................................... 171

quadridentata (Achipteria) ........................... 168

quadridentata (Austrachipteria) ................ 166

quadridentata (Austrogneta) ...................... 108

quadridentata (Ceratoppia) ............................. 93

quadridentata (Ceratoppia) ............................ 93

quadridentata (Nanhermannia) ........................ 68

quadridentata (Nanhermannia) ..................6 68

quadridentata (Oribatella) ............................. 171

quadridentata (Oribatella) ............................. 171

quadridentatus (Phylacozetes) ...................... 90

quadrifasciatus (Mixacarus) ............................. 39

quadrihastatus (Spatiodamaeus)

(=verticillipes) …….......................................... 82

quadrilamellatus (Eueremaeus) .................... 101

quadrilamellatus (Eueremaeus) ..................... 101

quadrilineata (Striatoppia) .......................... 131

quadrilineatus (Scapheremaeus) ................ 159

quadrilineatus (Schizozetes) .......................... 91

quadrilobatus [S. (Porozetes)] ....................... 178

quadrilobatus [S. (Porozetes)] ...................... 178

quadrimaculata (Allogalumna) ....................... 213

quadrimaculata [S. (Lalmoppia)] .................. 130

quadrimucronata (Oribatella) (=eutricha) .. 171

quadripertitus (Fosseremus) (=laciniatus) .... 103

quadripilis (Hemileius) ............................... 193

quadripilosa (Elaphoppia) ......................... 126

quadripilus [I. (Haplozetes)] ........................ 208

quadripilus [I. (Haplozetes)] ..................... 208

quadripilus (Nothrus) ..................................... 64

quadriporosa [G. (Porogalumnella)] ........ 222

quadrirotunda [H. (Phyllhermannia)] .............. 70

quadriseriata [H. (Heterohermannia)]

(=reticulata)

quadriseriatus (Steganacarus) (=applicatus) .. 58

quadriseta [L. (Antennoppia)] ..................... 113

quadriseta (Gymnodamaeus) ........................... 76

quadriseta (Sternoppia) ............................... 133

quadrisetosa (Metrioppia) ............................... 94

quadrisetosa (Oppia) sp. inq. ....................... 115

quadrisetosa (Oribatula) ............................... 188

quadrisetosa (Quadroribula) ........................... 93

quadrisetosa (Quadroribula) ........................93

quadrisetosus (Microtegeus) …...................... 86

quadrisetosus (Microtegeus) .......................... 86

quadrisetosus (Neoribates) ......................... 212

quadrispinata (Oribatella) ........................... 171

quadristriatus (Microtegeus) ........................... 86

quadristriatus [H. (Platynothrus)] Ham., 58 .... 66

quadristriatus [H.(Platynothrus)] C.K.78

(=praeoccupatus)

quadrituberculata (Lauroppia) ....................... 126

quadrituberculata (Rugoppia) .................. 131

quadrituberculatus [D. (Epidamaeus)] ............. 80

quadrivertex (Zachvatkinibates) .................... 184

quadrivertex (Zachvatkinibates) ................. 184

quagesetosa (Ausoribula) ............................. 191

quateorum (Acrotocepheus) ….................. 146

quathlambae [G. (Stenoppia)] …................ 116

quattuor (Setoppia) quatuour (Fenestrobelba)

queenslandica (Hoplophorella)

(=singularis)

quezonensis (Perscheloribates) …................ 198

quietus (Notophthiracarus) …..................... 52

quinquedentata [S. (Flagrosuctobelba)] ........ 138

quinquenodosa (Suctobelbila) ..................... 139

quinquenodosa (Suctobelbila) .................... 139

quinquenodosa (Zeasuctobelba) ............... 139

quinquepilosa (Graptoppia) (=paraanalis) .... 116

quinquesetosa (Cultroribula) …..................99

quintus (Scheloribates) .............................. 201

rabori (Epilohmannoides) ............................... 37

raczi (Microzetes) ............................................. 89

radiata (Arcoppia) ..................................... 121

radiata (Multioppia) ...................................... 117

radiata (Multioppia) ..................................... 117

radiata (Pilogalumna) (=tenuiclava) .......... 220

radiata (Varioppia) ..................................... 120

radiata [R. (Rectoppia)] ............................. 119

radiata [R. (Rectoppia)] ................................. 119

radiatus (Cubabodes) ................................. 152

radiatus (Eutegaeus) ………………............ 85

radiatus [C. (Klapperiches)] ....................... 151

radiatus [P. (Neophthiracarus)] ................ 57

radiatus (Pseudotocepheus) ....................... 145

radula (Rhynchoribates)

rafaelinus (Mexiceremus) ........................... 159

rafalskii [P. (Archiphthiracarus)]

(=cognatus)

rafalskii (Notophthiracarus)

rafalskii (Berniniella) (=hauseri) ................ 125

rafalskii (Hoplophorella) ............................. 49

rafalskii (Hoplophthiracarus) .....................50

rafalskii (Kokoppia) ................................. 123

rafalskii (Oribotritia) ........................................... 44

rafalskii [S. (Rafacarus)] .............................. 59

rafalskii (Rhacaplacarus) ……........................ 57

rafalskii (Trimalaconothrus) ........................... 62

rafflesiae (Otocepheus) ….............................. 147

rajskii (Brachioppiella) ............................. 122

rakhali (Scheloribates)

ramensis [M. (Cristonothrus)] .......................... 61

rameus (Megeremaeus) ………...................... 99

ramifera (Pulchroppia) ................................ 120

ramirezae (Papillacarus) ................................... 40

ramirezi (Bornemiszaella) ............................ 131

ramiseta (Ramuloppia) .......................... 124

ramisetosa (Congoppia) ............................... 123

ramosa (Brachioppiella) ........................122

ramosa (Pseudobrachioppiella) ................... 128

ramosa (Schalleria) 90

ramosa (Suctobelbella) ................................ 137

ramosus (Neoliodes)

ramosus (Papillacarus) ..................................... 40

ramosus [S. (Plenoxylobates)] ...................... 207

ramosus (Trhypochthoniellus) .......................60 60

ramsayi (Austrotritia) ...................................... 42

ramsayi (Lanceoppia) ................................. 110

ramsayi (Notophthiracarus) ....................... 52

ramulifera [M. (Furculoppia)] (=furcata) ... 117 ramulosa (Hymenobelba) ........................... 105

rangifer (Arcoppia) ….............................. 121

rangifera (Multioppia) .................................. 117

rangiroaensis (Hoplophorella) ..................... 49

rangiroaensis (Peloribates) …................... 210

ranokaoensis (Sabacarus) (=corneri) ............. 45

rapaensis (Eupelops) .................................. 164

rapax (Phthiracarus) …............................. 54

rapoporti (Poecilochthonius)

(=spiciger) ……........................................ 31

rara (Metabelba) ............................................ 80

rara [O. (Perspicuoppia)] .............................. 128

rarisetosa (Pedrocortesella) ........................... 75

rarisetosus (Montizetes) .................................. 107

rarus (Singabodes) …...................................... 153

rasile (Acrotritia) (=sinensis) .........................46

rasilis [G. (Indogalumna)] .......................... 217

rasilis [G. (Indogalumna)] ........................... 217

rastelligera (Rastellobata) .............................. 102

rastratus [C. (Xiphobates)] ............................. 180

rattura [L. (Lancelalmoppia)] .................... 111

ratubakensis (Peloribates) ........................... 210

raulti (Paralycus) ......................................... 34

rauschenensis (Phauloppia) .......................... 191

rausensis (Murcia) ....................................... 177

ravenala [L. (Lancelalmoppia)] .................. 111

raychaudhuri (Hoplophorella) (=vitrina) ...... 49

recasensis (Damaeus) ...................................... 78

recta (Beckiella) .......................................... 141

recta (Pilogalumna) ...................................... 220

rectangularis (Cyrtozetes) .......................... 174

rectisetosus [P. (Archiphthiracarus)]

(=montanus) ……........................................... 55 rectosetosus (Hypochthonius) (=luteus) ........ 29

rectus (Hemileius) ........................................ 193

rectus (Podoribates) ....................................... 186

rectus (Scheloribates) .................................. 201

rectus (Scheloribates) …….............................. 201

rectus (Scheloribates) .................................. 201

redempta (Ceratozetella) ............................... 173

reducta (Hoplophorella) (=repetita) ............... 49

reducta (Leptogalumna) ............................ 217

reducta (Oribatella) …............................... 171

reducta [G. (Porogalumnella)] ….................. 222

reducta [H. (Steganacarellus)] .................... 50

reducta (Teratoppia) ................................. 132

reductus [L. (Afroliochthonius)] .................. 31

reductus (Benoibates) .................................. 203

reductus (Birobates) .................................... 203

reductus (Notophthiracarus)

(=parareductus) ........................................... 52

reductus (Ozacarus) …................................ 40

reductus (Papillocepheus) ........................... 144

reductus (Phthiracarus) ................................. 54

reductus [H. (Platynothrus)] ..........................66 66

redux (Achipteria) (=coleoptrata) ................ 167

reevesi [S. (Dyobelba)] ...................................... 82

reevesi (Eueremaeus) (=columbianus) ........ 100

reevesi [G. (Naiazetes)] ................................. 176

reevesi [G. (Naiazetes)] .............................. 176

refracta (Acrotritia) ....................................... 46

refracta (Acrotritia) ……............................. 46 


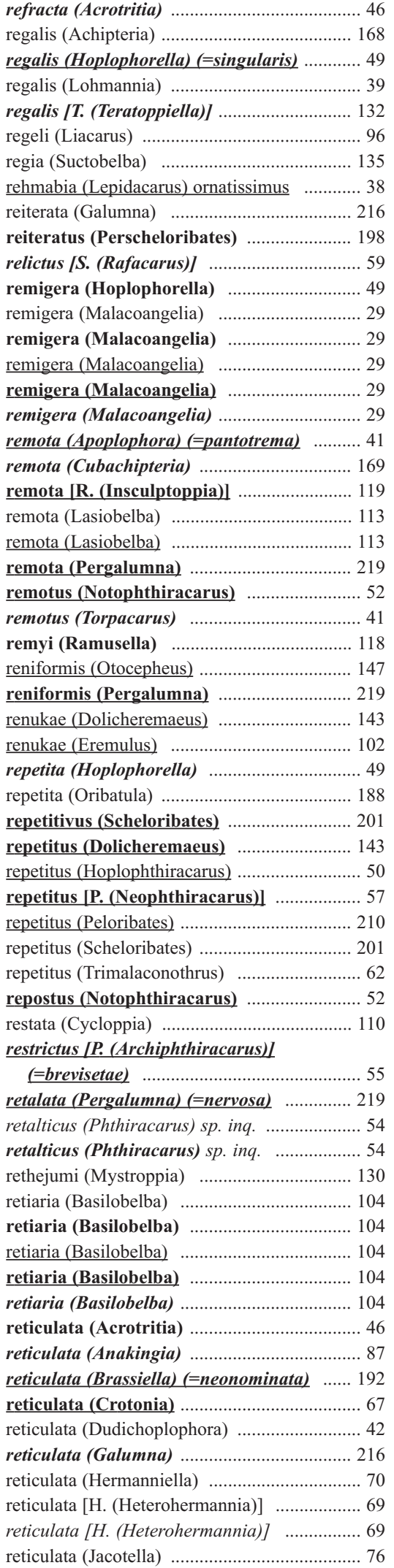

reticulata (Lohmannia) ..................................... 39

reticulata (Lucoppia) ................................. 187

reticulata (Lucoppia) ..................................... 187

reticulata (Magyaria) .................................. 209

reticulata (Oribatella) .................................. 171

reticulata (Oribatella) ................................ 171

reticulata (Pedrocortesella) Warb., 12 .......... 75

reticulata (Pedrocortesella) Rjab., 86

(=neonominata) …………………............. 75

reticulata (Reticuloppia) ........................... 191

reticulata (Sternoppia) ................................. 133

reticulata (Suctobelba) ................................... 136

reticulata [S. (Ussuribata)] ............................. 139

reticulatus (Alismobates) ............................. 157

reticulatus (Allonothrus) russeolus ................. 59

reticulatus (Allonothrus) russeolus .............. 59

reticulatus (Arthrodamaeus) ............................. 76

reticulatus (Bipassalozetes) ........................... 162

reticulatus (Bulleremaeus) ......................... 158

reticulatus (Carabodes) .................................. 150

reticulatus (Chaunoproctus) ......................... 192

reticulatus [A. (Coropoculia)] ...................... 159

reticulatus [A. (Coropoculia)] ...................... 159

reticulatus (Cosmochthonius) (=lanatus) ...... 33

reticulatus (Diapterobates) ............................. 181

reticulatus (Diapterobates) ............................. 18

reticulatus (Eremaeozetes) .......................... 165

reticulatus (Eupelops) ................................... 164

reticulatus (Euphthiracarus) ............................ 46

reticulatus (Heptacarus) .............................. 38

reticulatus (Heptacarus) …........................ 38

reticulatus (Hymenozetes) ............................ 88

reticulatus (Inigozetes) ................................. 182

reticulatus (Javacarus) ….............................. 38

reticulatus (Lamellobates) ........................... 167

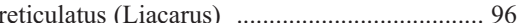

reticulatus (Licnocepheus) ............................ 106

reticulatus [C. (Magoebazetes)] …................. 88

reticulatus (Microtegeus) ................................. 86

reticulatus (Microtegeus) ................................. 86

reticulatus (Nesiacarus)

reticulatus (Nothrus) ........................................... 64

reticulatus (Ommatocepheus) .......................... 83

reticulatus (Peloptulus) ................................... 164

reticulatus (Peloribates) .............................. 210

reticulatus (Reticulocepheus) .......................8 84

reticulatus (Scapheremaeus) (=guerini) ...... 158

reticulatus (Trhypochthoniellus) …................6 60

reticulatus (Trimalaconothrus) Bal., 58

sp. inq.

reticulatus (Trimalaconothrus) Yam., 77

(=repetitus) ….......................................... 62

reticulatus (Unguizetes)

reticulatus (Unguizetes) ............................... 186

reticulatus (Zetorchestes) ................................. 91

reticulofemorata (Beckiella) ......................... 141

reticuloides (Oribatella) ................................. 171

retractus (Caleremaeus) ................................ 106

retroversa (Pergalumna) intermedia .............. 219

retusa (Indotritia) .............................................. 43

reunionensis (Nothrus) ................................. 64

rhadamanthus (Gehypochthonius) ................... 28 rhadamanthus (Gehypochthonius) .................. 28

rhadamanthus (Gehypochthonius) ................. 28

rhadamanthus (Gehypochthonius) ............... 28

rhadamanthus (Gehypochthonius) ................ 28

rhamphosus (Eupterotegaeus) ........................... 83

rhinina [R. (Rectoppia)] ................................ 119

rhinoceros [G. (Kabylogalumna)] ................ 217

rhodopeia [M. (Parametabelba)] ................... 81

rhomboides [P. (Triaungius)] …................... 206

ribagai (Eulohmannia) ..................................... 36

ribagai (Eulohmannia) .................................. 36

ribagai (Eulohmannia) ...................................... 36

ridiculus [N. (Phthirarica)] ............................ 53

riezuae (Acrotritia) (=sextiana) .................... 45

rigida [L. (Antennoppia)] ............................... 113

rigida (Hermanniella) subnigra ....................... 71

rigidicoma (Peloribates) .............................. 210

rigidiseta (Lanceoppia) …........................... 110

rigidisetosus (Liochthonius) ........................... 31

rigidisetosus (Scheloribates) …..................... 202

rigidisetosus (Scheloribates) ....................... 202

rigidisetus (Eremulus) ................................ 102

rileyi (Mycobates) sp. inq. ........................... 183

rimachensis [T. (Rostrozetes)] ...................... 212

rimosus (Austrocarabodes) ........................ 148

rimosus (Austrodamaeus) (=elegantulus) ... 76

rimosus (Carabodes) ....................................... 150

rimosus (Carabodes) ..................................... 150

rimosus (Eremulus) ................................... 102

rimosus (Hoplophthiracarus) (=kugohi) ..... 50

rimosus (Microtegeus) ….............................. 86

rimosus (Neoribates) ..................................... 212

ringueleti [H. (Argentinobates)] ................... 156

ringwoodensis (Dentachipteria) .................... 169

riparius (Damaeus) ............................................ 78

riparius (Thalassozetes) …............................. 157

ritza [O. (Coronoquadroppia)]

(=monstruosa) …….................................... 134

rizali (Kalayaan) ……................................... 147

rjabinini (Pedrocortesella) ............................... 75

robertsi [P. (Archiphthiracarus)] ....................... 56

robinsoni (Rhynchoribates) ........................ 140

roblensis (Pheroliodes) ................................... 74

robusta (Arcoppia) ........................................ 121

robusta (Austrotritia) ..................................... 42

robusta (Austrotritia) .................................... 42

robusta (Berniniella) ...................................... 125

robusta (Cosmohermannia) ..........................67

robusta (Hermanniella) .................................. 71

robusta (Machuella) ventrisetosa ............... 133

robusta (Oribatula) …................................ 188

robusta (Porobelba) ............................................ 82

robusta (Separatoppia) …........................... 129

robusta [O. (Zygoribatula)] ......................... 189

robustia (Arcoppia) ..................................... 121

robustior (Hoplophthiracarus)

(=histricinus) .............................................. 50

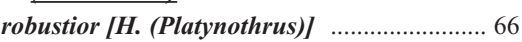

robustior (Protoribates) Ja., 37 (X

oblongus r.) ............................................. 206

robustior (Protoribates) Ja., 37 (X. capucinus r.)

(=robustior) …….................................. 206 
robustus [M. (Cristonothrus)] ……................. 61

robustus (Dolicheremaeus) ............................ 143

robustus (Hemileius) …………………......... 193

robustus (Holonothrus) ...................................67

robustus (Liacarus) Ew., 18 ........................... 96

robustus (Liacarus) Mih., 54 (=chroniosus) ....96

robustus (Lopholiodes) ............................... 73

robustus (Nothrus) truncatus .......................... 64

robustus [N. (Parakalumma)] ......................... 212

robustus [N. (Parakalumma)] ..................... 212

robustus [N. (Parakalumma)] ...................... 212

robustus (Peloribates) .................................... 210

robustus [D. (Saltatrichus)] ........................ 184

robustus (Scheloribates) P., 63 ................... 202

robustus (Scheloribates) Mih., 69

(=laevigatus) …....................................... 200

rodriguezi (Protoribates) .............................. 206

roguini (Eremaeozetes) ................................ 165

rohdendorfi (Metabelba) ................................. 80

rohri (Cosmozetes) ....................................... 88

rohri [M. (Cristonothrus)] P. Bal., 97 ............ 61

rohri [M. (Cristonothrus] J. y P. Bal., 02

(=rohri P. B., 97)

rohri (Xenillus)

roigi (Suctobelbella) ................................... 137

roissi (Eremaeus) ........................................... 100

romandiolae [M. (Parametabelba)] ................ 81

romaniae [D. (Epidamaeus)] tecticola ............ 80

romanicus [H. (Urubambates)] ...................... 194

romeroi (Hydroecocepheus) .......................... 144

ropalus (Atropacarus) (=phyllophorus) ........ 48

rosarius (Arthrodamaeus) .................................. 76

rossica (Belba) ................................................ 77

rossica (Belba) ……........................................ 77

rossica (Berniniella) .................................... 125

rossica (Galumna) ................................... 216

rossica (Oppiella) (=nova) ........................... 128

rossicus (Adrodamaeus) ..................................... 75

rossicus (Aphelacarus) (=acarinus) .............. 28

rossicus [O. (Fenestrobates)] ....................... 172

rossicus (Ovochthonius) .................................... 34

rossicus (Propelops) ....................................... 164

rossicus [H. (Turcibates)] (?=parvus) ....... 194

rostralis (Archoplophora) .................................. 42

rostralis (Archoplophora) .............................. 42

rostralis (Archoplophora) ............................... 42

rostralis (Archoplophora) ............................. 42

rostralis (Diapterobates) ............................... 181

rostralis (Ghilarovizetes) .............................. 176

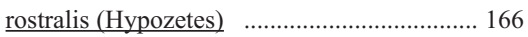

rostrata (Camisia) (=segnis) ...........................6 65

rostrata (Dometorina) ….............................. 192

rostrata (Galumna) ...................................... 216

rostrata (Galumnopsis) …......................... 222

rostrata (Heterobelba) .................................... 104

rostrata [M. (Parplophora)] …..................... 42

rostrata (Rostrogalumna) ......................... 221

rostrata [O. (Zygoribatula)] (=fusca) .......... 189

rostratus (Acutozetes) …............................ 207

rostratus (Angullozetes) .............................. 195

rostratus (Eupterotegaeus) ................................ 83

rostratus (Fijibates) ........................................ 197 rostratus (Fiiibates)

ostratus (Mucronothrus) (=nasalis) …........ 60

rostratus (Perscheloribates) ....................... 198

rostratus (Rhynchoribates) ......................... 140

rostratus (Sellnickochthonius) ........................ 32

rostratus (Sellnickochthonius) ......................... 32

rostratus (Sphaerozetes) ............................. 178

rostratus (Tubulozetes) …........................... 212

rostrodentatus (Scheloribates) ...................... 202

rostroincisa [D. (Cylindroppia)] cylindrica .... 123

rostroincisa [D. (Cylindroppia)] cylindrica .. 123

rostrolamellatus (Humerobates) ...................... 181

rostrolamellatus (Humerobates) ..................... 181

rostrolamellatus (Humerobates) ................ 181

rostrolamellatus (Humerobates) ................ 181

rostrolamellatus (Humerobates) .................. 181

rostropilosus [M. (Cristonothrus)] ..................61 61

rostropilosus [I. (Mancoribates)] ................ 208

rostropilosus (Scheloribates) …..................... 202

rostropterus (Nesozetes) .............................. 191

rostroreticulata (Hammerella) ...................... 132

rostrorugosa (Apoplophora) (=pantotrema) .... 4

rostroserratus (Ceratozetes) ..................... 174

rostrotuberculatus (Diapterobates) ................. 181

rostroundulatus (Ceratozetes) ........................ 175

rothschildi (Crotonia)

rotoruensis (Compactozetes) ........................... 83

rotoruensis (Lopholiodes) …........................ 73

rotunda (Arcoppia) .................................... 121

rotunda (Ceratozetella) .................................. 173

rotunda (Exoribatula) ............................... 193

rotunda (Fortuynia) .................................... 157

rotunda (Murcia) ........................................ 177

rotunda (Neoamerioppia) ........................... 114

rotunda (Porokalumma) .............................. 213

rotunda (Pseudopyroppia) ............................. 94

rotundata (Cultroribula) (=bicultrata) .......... 92

rotundata [V. (Latilamellobates)] incisella .... 179

rotundata (Oribatula) sp. inq. ....................... 188

rotundata (Tuberoppia) ................................. 128

rotundatus (Fuscozetes) ................................. 176

rotundatus (Lamellobates) (=molecula) ...... 167

rotundatus (Liacarus) ................................... 96

rotundatus (Pheroliodes) ................................ 74

rotundatus (Scheloribates) ............................ 202

rotundatus (Sellnickochthonius) .................. 32

rotundiceps (Allogalumna) ............................. 214

rotundiclava (Scheloribates)

(=sandvicensis)

201

rotundigranulatus (Gymnodamaeus) 76

rotundirostrum [I. (Mancoribates)] .......... 208

rotundocuspidatus (Diapterobates) ................ 181

rotundocuspidatus (Diapterobates) ............... 181

rotundus (Alismobates) ............................... 157

rotundus [O. (Aokiella)] ................................ 153

rotundus (Birobates) …............................. 203

rotundus (Hammerabates) .......................... 197

rotundus (Neoribates) ................................... 212

rotundus (Neoribates) ….............................. 212

rotundus (Phthiracarus) Ewing, 08

(=globosus) rotundus (Phthiracarus) Berl., 23

(=lentulus) …....................................... 54

rotundus (Protoribates) ................................... 206

rotundus (Steganacarus) (=magnus) ............58

roubali (Neoribates) ..................................... 212

roubali (Neoribates) ..................................... 212

roubali (Phthiracarus) (=nitens) …….......... 54

royi (Mycobates) ....................................... 183

rubeni (Epimerella) ......................................... 131

rubens (Fuscozetes) (?=intermedius) .......... 175

ruber (Murcia) sp. inq. ................................. 177

ruber (Odontocepheus) .................................... 153

rubescens (Meristacarus) …………………...... 39

rubescens (Meristacarus) .............................. 39

rubida (Lasiobelba) .................................... 113

rubra [H. (Phyllhermannia)] ......................... 70

rubripedes (Dolicheremaeus) ...................... 143

rubrus (Humerobates) (?=rostrolamellatus) .. 181

ruchljadevi [O. (Zygoribatula)] .................... 189

rudentiger (Dentizetes) .................................. 175

rudentiger (Macrogena) .............................. 176

rudentigera (Amerioppia) ............................ 113

ruderalis (Passalozetes) .............................. 162

rufafulvus (Scheloribates) ............................. 202

rufulus (Hypochthonius) ............................... 29

rufulus (Hypochthonius) .................................. 29

rufulus (Hypochthonius) ............................... 29

rufulus (Hypochthonius) …….......................... 29

rufulus (Hypochthonius) ............................... 29

rugiceps (Scheloribates) .............................. 202

rugifrons [O. (Zygoribatula)] (=exarata) .... 189

rugifrons [P. (Neopilizetes)] ........................ 220

ruginervis (Galumnopsis) ......................... 222

rugosa (Arcoppia) ........................................ 121

rugosa (Autogneta) (=parva) $\ldots \ldots \ldots \ldots \ldots \ldots \ldots . . . . . . . . . .108$

rugosa (Epilohmannia) pallida ........................ 37

rugosa (Galumna) ..................................... 216

rugosa (Galumnella) ..................................... 222

rugosa (Gustavia) sp. inq. ............................. 95

rugosa (Hermannia) (=convexa) .................. 69

rugosa /G. (Indogalumna)](=rasilis) .......... 217

rugosa (Senectoppia) Aoki, 76 ...................... 132

rugosa (Senectoppia) Ham., 79

(=multisulcatum) …................................. 132

rugosa (Serratobelba) ............................... 135

rugosa [ O. (Zygoribatula)] (=exarata) ..... 189

rugosala (Pergalumna) sp. inq. .................... 219

rugosior (Carabodes) ..................................... 150

rugosior (Carabodes) ................................... 150

rugosula (Galumnella) ................................. 222

rugosula (Oribatella) .................................... 171

rugosus (Austrocarabodes) ....................... 148

rugosus (Benoibates) ….............................. 203

rugosus (Bipassalozetes) ............................. 162

rugosus (Chaunoproctellus) ..................... 192

rugosus (Gibbicepheus) ................................ 152

rugosus (Haplacarus) …................................. 38

rugosus (Limnozetes) .................................... 166

rugosus (Neoliodes) ...................................... 72

rugosus [M. (Megazetes)] ................................ 89

rugosus (Microtegeus) ................................. 86

rugosus (Paulianacarus) ................................ 40 


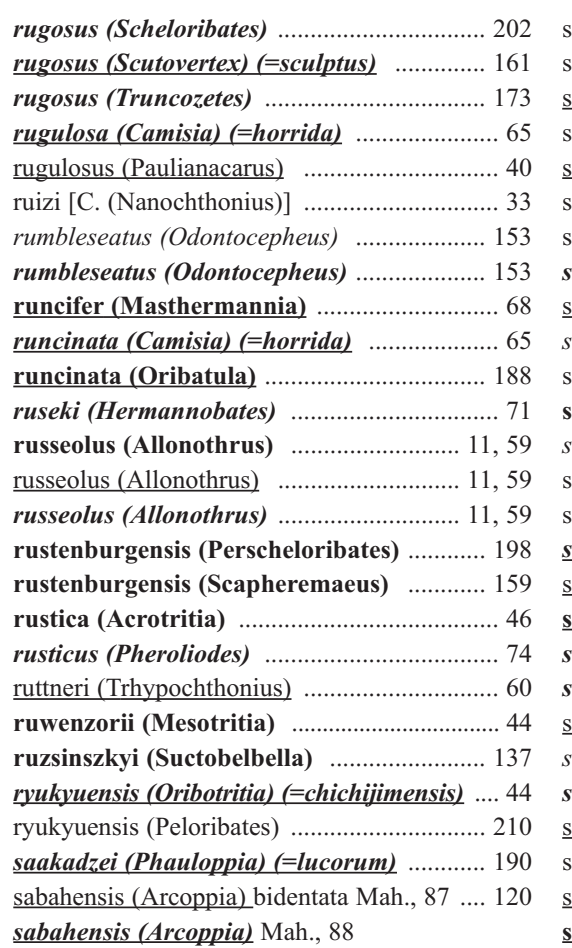

(=aequivoca) …………………………... 120

sabahensis (Nanhermannia) ….........................6 68

sabahensis (Suctobelbella) …………............... 137

sabahna (Austroceratoppia) ………………...... 93

sabahna (Calugarella) ………………….......... 185

sabahna (Galumna) …………………………...... 216

sabahna (Hoplophorella) …………………..... 49

sabahnus (Dolicheremaeus) ................... 12, 143

sabahnus (Microtegeus) ……………………... 86

sabahnus (Unguizetes) ………………………... 186

sabinae (Eremaeozetes) ………………...... 165

saboori (Galumna) ………………………..... 216

sabulosa [O. (Zygoribatula)] ........................ 189

sabulosus [B. (Passalobates)] ……….............. 162

sacchareus (Tegeocranellus) ......................... 155

saccharomycetoides (Epieremulus)

(Egeometricus) ………………………..... 106

saccularis (Sacculogalumna) ...................... 221

sacculifera (Oripoda) ……………………... 204

sacculiferus (Steganacarus) (=grandjeani) .... 58

sacculus (Trimalaconothrus) ........................... 62

sacsahuamanensis (Scheloribates) .............. 202

sacyae (Notophthiracarus) ………………….. 52

sadbinia [O. (Dzarogneta)] ………………....... 129

sadoensis (Nothrus) .......................................... 64

saetosa (Suctobelba) (=granulata) .............. 135

saeva (Orthogalumna) …………………..... 218

saeva (Orthogalumna) ……………………... 218

saeva (Orthogalumna) …………………...... 218

sagami (Goyoppia) ………………………….... 113

sagatus (Reptacarus) ………………………….... 41

sagitta (Galumnopsis) …………………..... 222

sagitta (Sagittabodes) …………………….... 153

sagittatus (Flagellocepheus) ........................ 144

sahariensis (Aphelacarus) acarinus ................. 28 sahariensis [R. (Rectoppia)] fasciata ............ 119

saifulmalukensis (Liebstadia) ......................... 196

sajisei (Eurostocepheus) ................................. 146

sakamorii (Oribatula) …………………...... 188

sakamorii (Oribatula) …................................ 188

sakeni (Berniniella) …………………………. 125

salamoni (Xenillus) ……………………….... 98

salasi (Bornemiszaella) ............................... 13

salicifolius (Eohypochthonius) ……….............. 29

salicis [N. (Protokalumma)] ........................ 212

salijanica (Oribatula) ……………………..... 188

salina [O. (Zygoribatula)] …………………. 189

salish (Eremaeus) ........................................ 100

salmanticensis (Hypogeoppia) terricola ........ 126

salonae (Foraminoppia) ................................ 128

saltaensis (Fosseremus) (=laciniatus) $\quad$......... 103

saltaensis (Liochthonius) ................................. 31

saltaensis (Liochthonius) ............................... 31

saltaensis (Liochthonius) ............................... 31

saltaensis (Mochlozetes) ............................... 185

saltator (Zetorchestes) ……………………….... 91

saltuensis (Gymnodamaeus) ............................. 76

salvadoriensis [N. (Amerigloboppia)] ……... 114

samadi (Oppia) …………………………….... 115

samaina (Medioppia) ………………………. 124

samarensis (Dolicheremaeus) ......................... 143

samoaensis (Galumna) ................................. 216

samoaensis (Nesopelops) ……..................... 164

samoaensis (Oribotritia) ………………….... 44

samoensis [C. (Klapperiches)] …………....... 151

samsinaki (Euscheloribates) ........................... 197

samsinaki (Euscheloribates) ........................... 197

sanctaehelenae [G. (Indogalumna)]

(=rasilis) …………………………....... 217

sanctaeluciae (Haplochthonius) ........................ 34

sanctaeluciae (Haplochthonius) ………….... 34

sanctaeluciae (Haplochthonius) ..................... 34

sanctaemariae (Melanozetes) azoricus ........... 176

sanctipauli [E. (Brasiliotritia)] dlouhyorum .... 47

sanctipauli (Xenillus) ................................... 98

sandvicensis (Scheloribates) praeincisus .. 12, 201

sandvicensis (Scheloribates) praeincisus .. 12, 201

sandvicensis (Scheloribates)

praeincisus

12,201

sandvicensis (Scheloribates)

praeincisus ..................................... 12, 201

sangumburiensis [P. (Archiphthiracarus)] ...... 56

sangumburiensis [P. (Triaungius)] ................. 206

sanremensis (Lucoppia) (=burrowsi) .......... 187

sanvicensis [P. (Archiphthiracarus)] …………. 56

saraburiensis (Austrotritia) …………………... 42

saraburiensis (Austrotritia) ............................... 42

saraburiensis (Austrotritia) … 42

sarahae (Phthiracarus) ................................. 54

sarawak (Sabahtritia) ……………………….... 47

sarawaki [E. (Pocsia)] ………………………... 47

sarawaki (Rhacaplacarus) ………………......... 57

sarbias (Paulianacarus) …………………………. 40

sardoa (Oribatella) ………………………....... 171

sardoa (Oribatula) (=tibialis) ……................ 188

sarekensis [M. (Calyptozetes)] …………….... 183

sarekensis [M. (Calyptozetes)] …………....... 183 sarekensis (Suctobelbella) ............................. 137

sarekensis (Suctobelbella) ............................ 137

sarekensis (Tectocepheus) velatus ................. 155

sarekensis (Tectocepheus) velatus ................. 155

sarekensis (Tectocepheus) velatus ............... 155

sarekensis (Tectocepheus) velatus ................. 155

sarekensis (Tectocepheus) velatus ............... 155

sarekensis (Tectocepheus) velatus .............. 155

sarkari (Paulianacarus) (=sarbias) ………... 40

sarvari (Belba) ……………………………..... 77

sasakawai (Belba) …………………………....... 77

sasakawai (Belba) …………………………..... 77

saskai [O. (Oxyoppiella)] ………………….... 129

saskai (Pilizetes) ………………………..... 220

saswatii (Scheloribates) ................................. 202

satellitius (Galumna) $(=$ alata) ........................ 214

saucius (Rhacaplacarus) .................................. 57

saudicus (Hypozetes) translamellatus ........... 166

saudicus (Scheloribates) ................................... 202

savagei (Campachipteria) ……………….... 168

saxicola (Dometorina) plantivaga ................. 192

saxicola (Ghilarovus) ……………………........ 184

saxicola (Hypovertex) …………………….... 161

saxicola (Oribatula) interrupta ....................... 187

saxicola (Phauloppia) ....................................... 191

saxicola (Scheloribates) ……………………... 202

saxosus [T. (Tyrphonothrus)] .............................. 63

sayedi [O. (Zygoribatula)] ………………….... 189

scaber (Galumna) ………………………....... 216

scaber (Stictozetes) ………………………...... 221

scabra [H. (Heterohermannia)] ........................... 69

scabra [H. (Heterohermannia)] ....................... 69

scalaris (Liochthonius) (=sellnicki) ............... 31

scalifera [O. (Oxyoppiella)] ........................... 129

scalifera [O. (Oxyoppiella)] ……………...... 129

scaliger (Platyliodes) ………………………... 72

scaliger (Platyliodes) ......................................... 72

scalpellata (Suctobelba) …………………..... 136

scapellata (Hoplophorella) (=vitrina) ............ 49

scapulatus (Mysterozetes) .............................. 90

schachtachtinskoi (Simkinia) ......................... 195

schalleri (Acaroceras) .................................... 87

schalleri [T. (Rostrozetes)] …………........... 212

schatzi (Galapagacarus) …………………….... 68

schatzi [C. (Klapperiches)] .............................. 151

schatzi (Notophthiracarus) .............................. 52

schatzi (Scapheremaeus) ................................ 159

schatzi (Torpacarus) ……………………..... 41

schauenbergi [P. (Archiphthiracarus)] ........ 56

schauenbergi (Austrocarabodes) ................. 149

schauenbergi (Cryptacarus) ............................. 38

schauenbergi (Cryptacarus) ……………...... 38

schauenbergi (Hoplophorella) (=hamata) .... 49

schauenbergi (Multipulchroppia) ............... 120

schauenbergi (Neoppia) ............................... 109

schauenbergi (Pilobatella) ...................... 12, 211

schauenbergi (Scheloribates) ....................... 202

schauenbergi [O. (Zygoribatula)] .............. 189

scheloribatoides (Topobates) ....................... 203

schizocomus (Notophthiracarus) .................. 52

schneideri (Ameronothrus) ............................... 156

schneideri (Phauloppia) (=lucorum) 
scholzi (Passalozetes) schoutedeni [L. (Paralamellobates)]

$$
\text { (=misella) }
$$

schubarti (Plasmobates) …….......................... 71

schuetzi (Chamobates) ................................... 180

schuetzi (Chamobates) ................................ 180

schuilingi (Allonothrus) ................................... 59

schuilingi (Allonothrus) ……............................ 59

schuilingi (Allonothrus) …...........................59

schusteri (Ameronothrus) .............................. 156

schusteri [L. (Convergoppia)] ..................... 111

schusteri (Epilohmannia) .............................. 37

schusteri [H. (Heterohermannia)] ..................... 69

schusteri (Microtritia) ...................................... 47

schusteri (Nesiacarus) .................................... 40

schusteri (Notophthiracarus) $\ldots \ldots \ldots \ldots \ldots \ldots . . . . . .52$

schusteri (Staurobates) ............................... 105

schusteri (Zetorchestes) ................................... 91

schwartzi (Austrocarabodes) ........................ 149

schweizeri [L. (Hamoppia)] ........................ 111

schweizeri (Liacarus) Forss., 63 …................. 96

schweizeri (Liacarus) Wooll., 68

(=schweizeri)

scimitarum (Trimalacon

scissurata (Oripoda) 204

scitus [P. (Archiphthiracarus)] ......................... 56

sclerotrichus (Trhypochthonius) sp. inq. ........ 60

scoparius (Hermannobates) .......................... 71

scopoli [P. (Neophthiracarus)] ….................. 57

scopulae [C. (Flexa)] ................................... 150

scotiae (Halozetes) sp. inq. ............................. 157

scotiae [M. (Pravoppia)] …......................... 111

scotti (Acrotritia) ............................................ 46

scotti (Nothrus) (=seychellensis) ...................... 64

scripta (Allogalumna) ………..................... 214

scriptus [M. (Megazetes)] ........................... 89

scrobinus [H. (Tuberemaeus)] ....................... 194

scrofa (Suctobelbella) .................................. 137

scrupeus (Rhacaplacarus) ................................ 57

sculperens (Ethiovertex) ..................... 12, 161

sculpta (Belba) ............................................. 77

sculpta (Lasiobelba) ..................................... 113

sculptilis (Hoplophorella) ............................. 49

sculptilis (Oppiella) (=nova) ....................... 128

sculptrata (Pedrocortesella) ............................ 75

sculptrata (Pedrocortesella) .............................. 75

sculptrus (Xenillus) (=discrepans) ................98

sculpturata (Aeroppia) ................................. 112

sculpturata (Condylobelba) .......................... 134

sculpturata (Epilohmannia) ......................... 37

sculpturata (Oribatella) ................................ 171

sculpturata [H. (Phyllhermannia)] .............. 70

sculpturata [O. (Zygoribatula)] ................ 189

sculpturatus (Fosseremus) ......................... 103

sculpturatus (Grandjeanobates) ................ 197

sculpturatus [H. (Tuberemaeus)] …............... 194

sculptus (Adrodamaeus) (=hispanicus) ........75

sculptus (Scutovertex) ................................. 161

sculptus (Scutovertex) …............................. 161

sculptus (Scutovertex) .............................. 161

sculptus [T. (Tyrphonothrus)] ............................ 63 sculptus (Tritegeus) ……………………....... 84

scutata (Berlesiella) …................................. 207

scutata (Suctobelbila) ................................... 139

scutata (Suctobelbila) …........................... 139

scutatus (Malaconothrus) ……......................... 61

scutatus (Plakoribates) …............................. 169

scutatus (Trimalaconothrus) ............................. 62

scutigera (Lyroppia) .................................. 131

scutigera (Lyroppia) .................................... 131

scymus (Carabodes) (=labyrinthicus) .......... 150

secata (Arcoppia) ..................................... 121

seconda (Terratritia) …...................................... 45

secta (Suctobelba) ........................................ 136

sectus (Belorchestes) ....................................... 91

secunda (Galumnopsis) ................................ 222

secundus [A. (Baloghodes)] ........................ 149

secundus [E. (Pocsia)] …............................... 47

sedlaceki (Rhynchoppia) ............................ 135

segestris (Neoliodes) ......................................... 72

seghetti (Scheloribates) (=callipus) ............ 199

segmentatus (Arthrovertex) ....................... 160

segnis (Camisia) ................................................ 65

segnis (Camisia) .................................................. 65

segnis (Camisia) …................................... 65

segnis (Camisia) ….......................................... 65

segnoides (Camisia) (=segnis) 65

sejugatus (Annectacarus) ............................. 37

sejugatus (Chaunoproctus) ........................... 192

sejunctus (Liacarus) (=koeszegiensis) ......... 96

selgae (Damaeus) ............................................ 78

selgae (Punctoribates) …............................ 183

selgae (Xenillus) .............................................. 98

sellnicki (Achipteria) ..................................... 168

sellnicki (Adelphacarus) .................................. 28

sellnicki [P. (Archiphthiracarus)] (=ligneus) .. 55

sellnicki (Beckiella) ..................................... 141

sellnicki (Belba) ................................................ 77

sellnicki (Belba) ............................................... 77

sellnicki (Ceratozetella) ............................... 173

sellnicki [L. (Convergoppia)] ...................... 111

sellnicki (Galumnopsis) .............................. 222

sellnicki (Indotritia) (=krakatauensis) .......... 43

sellnicki (Liochthonius) .................................. 31

sellnicki (Liochthonius) ................................. 31

sellnicki (Liochthonius) ................................. 31

sellnicki (Maerkelotritia) (=kishidai) .............. 43

sellnicki (Melanozetes) ................................. 176

sellnicki (Melanozetes) ................................... 176

sellnicki (Mystroppia) .................................. 130

sellnicki (Nanhermannia) ...................................6 68

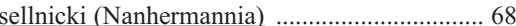

sellnicki [P. (Neophthiracarus)] (=obsessus) .... 56

sellnicki (Pilizetes) ......... 220

sellnicki (Punctoribates) ................................. 184

semengok (Gymnobodes) ............................... 152

semiareolatus (Cosmochthonius) ................ 33

semiareolatus (Licneremaeus) .................... 160

semibarbulata (Lohmannia) ............................ 39

semicapillatus (Dolicheremaeus) ................. 143

semiconvexus (Scapheremaeus) .................... 159

semicostulata (Arcoppia) ........................... 121

semidentata (Suctobelbella) .......................... 137 semidesertus (Scheloribates) ….................... 202

semifoveolatus (Cosmochthonius) ................... 33

semilunatus (Dolicheremaeus) ….................. 143

seminuda (Oppia) .................................... 115

seminuda (Trichogalumna) ........................ 221

seminuda (Trichogalumna) ............................ 221

seminudus [P. (Triaungius)] .......................... 206

seminudus [P. (Triaungius)] ........................ 206

seminulum (Euzetes) $s p$. inq. ....................... 175

semiornatus (Scapheremaeus) ….................. 159

semiornatus (Sellnickochthonius)

(=immaculatus) ............................................ 32

semiplumosa [S. (Flagrosuctobelba)] ............ 138

semiplumosa [S. (Flagrosuctobelba)] ........ 138

semireticulata (Pedrocortesella) .................. 75

semirufus (Minunthozetes) ............................ 182

semistriata (Pergalumna) ................................ 219

senecionis [N. (Amerigloboppia)] ................. 114

senegalensis (Nothrus) …………..................... 64

senegalensis [T. (Paragloboppia)] …........... 115

senensis (Miracarus) ........................................ 89

senex (Atropacarus) (=striculus) …............... 48

sengbuschi (Ramusella) ................................ 118

sengbuschi (Ramusella) .......................... 118

sengbuschi (Ramusella) ............................ 118

sengbuschi (Ramusella) ............................. 118

sensiclavata [R. (Insculptoppia)] .................. 119

sensilla [B. (Gressittoppia)] ........................ 122

sentus (Notophthiracarus) …........................ 52

separata [S. (Ussuribata)] .............................. 139

sepilok (Dampfiella) .................................... 141

septatus (Nothrus) ........................................ 64

septemtuberculatus (Pseudotocepheus)

(=amonstruosus) …................................ 145

septenia (Parisuctobelba) ............................. 135

septentrionalis (Eupelops) ............................ 164

septentrionalis (Eupelops) ............................ 164

septentrionalis (Hermanniella) ......................... 71

septentrionalis (Hermanniella) ....................... 71

septentrionalis (Indotritia) (=krakatauensis) .. 43

septentrionalis (Microzetes) ............................. 89

septentrionalis (Trhypochthonius) .................. 60

septentrionalis [H. (Capillonothrus)]

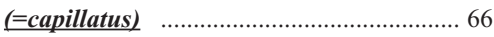

sequoiae (Galumna) .................................... 216

serapi (Austroceratoppia) ................................93

sergienkae [C. (Xiphobates)] ........................ 180

sergienkoae [V. (Latilamellobates)] .............. 179

sergienkoae (Scheloribates) .......................... 202

serrata (Achipteria) ........................................ 168

serrata (Ctenobelba) ...................................... 101

serrata (Hermanniella) ................................... 71

serrata (Lohmannia) ......................................... 39

serrata (Machadobelba) ............................... 107

serrata (Metrioppia) .......................................... 94

serrata (Metrioppia) ....................................... 94

serrata (Murcia) ........................................ 177

serrata (Oribatella) ..................................... 171

serrata (Oribotritia) ............................................ 44

serrata [H. (Phyllhermannia)] ...................... 70

serrata (Serratoppia) .................................... 124

serrata (Serratoppia) .................................... 124 


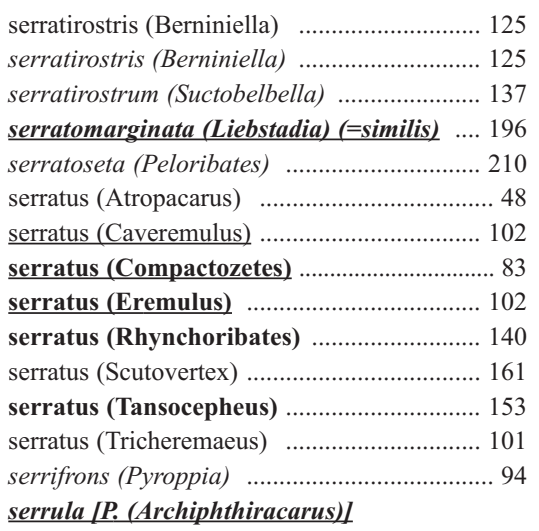

serrula [P. (Archiphthiracarus)]

(Epygmaeus) …….....................................5 56

serrula (Oribatella) ....................................... 171

serrula (Oribotritia) ........................................... 44

serrulata (Arcoppia) .................................... 121

serrulatus (Phthiracarus) ………….................. 54

servadeii (Belba) sp. inq. ................................. 77

sestai (Microzetes) ........................................... 89

setacea (Hermanniella) subnigra ................... 71

setantis (Trhypochthonius) ................................6 60

setanus (Phthiracarus) .................................. 54

setanus (Phthiracarus) ................................. 54

setatus (Defectamerus) .................................. 105

setiformis (Acrogalumna) (=longipluma) .. 213

setiformis (Conoppia) ........................................ 83

setiformis [H. (Phyllhermannia)] ……........ 70

setiger [D. (Epidamaeus)] .............................. 80

setiger (Eremaeus) (=hepaticus) .................. 100

setiger (Murcia) ............................................... 177

setiger (Neoribates) ................................... 212

setiger (Perscheloribates) ............................ 198

setiger (Pseudotocepheus) ........................... 145

setiger (Sadocepheus) undulatus ................... 84

setiger (Stomacarus) ....................................... 27

setiger (Unguizetes) ...................................... 186

setiger (Xenillus) ........................................... 98

setigera (Galumna) ......................................... 216

setilonga (Berniniella) .................................. 125

setosa (Banksinoma) .................................... 109

setosa (Banksinoma) ................................... 109

setosa (Dampfiella) ...................................... 141

setosa [S. (Flagrosuctobelba)] ..................... 138

setosa [G. (Porogalumnella)] ......................... 222

setosa (Xiphobelba) …............................... 104

setosa [O. (Zygoribatula)] ……................... 189

setosellus (Phthiracarus) (=longulus) .......... 54

setosoclavata (Suctobelbella) ......................... 137

setosus (Aleurodamaeus) ................................ 77

setosus (Ceratorchestes) ................................ 93

setosus (Epactozetes) .................................... 173

setosus (Fuscozetes) ...................................... 176

setosus (Fuscozetes) ...................................... 176

setosus (Fuscozetes) ……………................... 176

setosus (Humerobates) ............................... 182

setosus (Megalotocepheus) japonicus .......... 147

setosus [P. (Metaphthiracarus)] ...................... 56

setosus [P. (Metaphthiracarus)] ...................... 56

setosus [P. (Metaphthiracarus)] …………..... 56 setosus (Topobates) ...................................... 203

setosus (Trhypochthoniellus) (=longisetus) .... 60

setosus (Zetomimus) ..................................... 179

setulosa (Achipteria) ................................... 168

setus (Eobrachychthonius) .............................. 30

sexareata (Pergalumna) (=myrmophila) ... 219

sexclavatus [T. (Rostrozetes)] ...................... 212

sexcornuta (Schalleria) ................................. 90

sexdentata (Oribatella) .................................... 17

sexdentata (Oribatella) ................................ 17

sexdentata (Suctobelbella) ............................ 137

sexdentatus (Pseudotocepheus) sp. inq. ...... 145

sexidimorphus (Pseudotocepheus) .............. 145

sexmaculata (Multioppia) (?=laniseta) ........ 117

sexnodosa (Suctobelbila) ............................. 139

sexnodosa (Suctobelbila) ............................ 139

sexnotatus (Eobrachychthonius) (=latior) .... 30

sexpilosa (Ceratoppia) ..................................... 93

sexpilosa (Ceratoppia) .................................... 93

sexpilosa (Goyoppia) .................................. 113

sexpilosa (Hexachaetoniella) ….................... 74

sexpilosus [D. (Neocarabodes)] ..................... 152

sexpilosus [D. (Neocarabodes)] ................. 152

sexsetosa (Pseudoceratoppia) ....................... 94

sexsetosa [S. (Ussuribata)] ............................ 139

sextiana (Acrotritia) clavata .............................. 45

seychellensis (Dolicheremaeus) .................. 143

seychellensis (Nothrus) ................................... 64

seychellensis (Ramsayellus) ....................... 182

seydi (Lanceoppia) ................................... 110

shagdarsureni (Eupelops) ............................. 164

shaldybinae [R. (Insculptoppia)]

(=insculpta) ……................................... 119

shaldybinae (Oribatella) ................................ 171

sharipovi (Moritzoppia) ............................... 127

shauni (Pseudotocepheus) .......................... 145

shealsi (Austrotritia) (=saraburiensis) .......... 42

shealsi (Belloppia) ........................................ 125

shealsi (Notophthiracarus) …..................... 52

sheshanensis [R. (Insculptoppia)] ................ 119

shibai (Fortuynia) elamellata ........................ 157

shibai [T. (Rostrozetes)] ................................ 212

shillongensis (Eremobelba) …..................... 104

shinanoensis (Multioppia) .............................. 117

shiptoni [P. (Neophthiracarus)] .................... 57

shiraensis (Perscheloribates) ...................... 198

shirakamiensis (Caenosamerus) .................... 105

shirakamiensis (Tectocepheus) ...................... 154

shiranensis (Cyrtozetes) ............................. 174

shogranensis (Acrogalumna) ........................ 213

shogranensis (Euphthiracarus) ......................... 46

shogranensis (Novosuctobelba) ..................... 135

shogranensis (Sphaerozetes) …........... 178

shtanchaevae (Epilohmannia) ......................... 37

shukuminensis (Cultroribula) .......................... 92

siamensis (Dolicheremaeus) .......................... 143

siamensis (Hoplophthiracarus) (=kugohi) .... 50

siamensis (Multipulchroppia) ........................ 120

siamensis (Rhabdoribates) ............................ 198

sibirica (Campachipteria) ............................. 168

sibirica [C. (Ensicamisia)] …........................... 65

sibirica (Pyroppia) .......................................... 94 sibiricus [H. (Platynothrus)] ............................. 66

sibiricus [H. (Platynothrus)] ......................... 66

sibiricus [H. (Platynothrus)] …........................... 66

sibiricus (Punctoribates) …............................ 184

sicafer (Sicaxylobates) …........................... 207

sicarius (Pheroliodes) .................................. 74

sicata (Dometorina) ..................................... 192

sicca (Galumna) (=setigera) ........................ 216

siccatus (Diapterobates)

(=rotundocuspidatus)

sicilicomus (Notophthiracarus) .................... 52

sicilifera [S. (Flagrosuctobelba)] ................ 138

sicula [P. (Siciliophora)] ................................. 36

sicula (Siculobata) ........................................ 195

sicula (Siculobata) .................................... 195

sicula (Siculobata) ......................................... 195

siculus (Adrodamaeus) ..................................... 75

siculus (Eupelops) (=plicatus) ..................... 163

siculus (Scutovertex) (=minutus) ................ 161

siculus (Siciliotrichus) ................................ 172

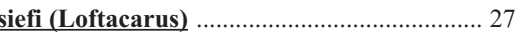

sierramadrensis (Peloribates) ........................ 210

sierriensis (Euphthiracarus) tanythrix ............ 46

sigma (Berniniella) ......................................... 125

sigmella (Subiasella) ................................... 130

signata (Anachipteria) .................................. 168

signata (Dissorhina) ..................................... 125

signatus (Cosmochthonius) ............................ 33

sikkimensis (Scheloribates) ......................... 202

silesius (Liebstadia) (=similis) .................... 196

silva (Cymbaeremaeus) ................................ 158

silvai (Beckiella) .......................................... 141

silvanus [G. (Naiazetes)] ............................. 176

silvatica (Berniniella) .................................. 125

silvatica (Parautogneta) ................................ 109

silvatica (Pergalumna) ................................ 219

silvatica [S. (Vesiculobates)] ....................... 195

silvaticus (Malaconothrus) ............................ 61

silvestris (Eueremaeus) (=oblongus) $\ldots . . . \ldots 100$

silvestris (Eupelops) ..................................... 164

silvestris (Neoliodes) ........................................ 72

silvestris (Nothrus) ............................................. 64

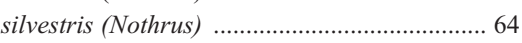

silvestris (Nothrus) .......................................... 64

silvestris (Nothrus) ......................................... 64

silvestris (Nothrus) ........................................64 64

silvestris (Pergalumna) .............................. 219

silvestris (Trhypochthonius) .............................6 60

silvicola (Drymobates) .................................... 185

silvicola (Limnozetes) ................................... 166

silvicola (Limnozetes) .................................. 166

silvicola (Neoeutegaeus) .................................. 85

silvicola (Striatoppia) .................................. 131

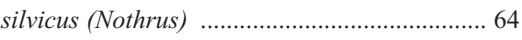

simile (Acrotritia) .............................................. 46

similesuperbula (Oribatella) (=willmanni) .. 172

similidentata (Suctobelbella) ....................... 137

similifallax (Lauroppia) .............................. 126

similis (Acaroceras) ........................................ 87

similis [P. (archiphthiracarus)] ......................... 56

similis (Austrocarabodes) .......................... 149

similis [E. (Brasiliotritia)] ............................ 47 
similis (Ceratozetella) ……......................... 173

similis (Ceratozetes) ..................................... 174

similis (Chavinia) ........................................... 112

similis (Dampfiella) …................................ 141

similis (Dampfiella) ….............................. 141

similis (Eobrachychthonius) .............................. 30

similis (Eupelops) (=ureaceus) .................... 164

similis (Galumna) ................................. 12, 217

similis (Galumna) ............................... 12, 217

similis [G. (Gibbibodes)] …...................... 152

similis (Heminothrus) ..................................... 65

similis (Hermanniella) .................................... 71

similis (Hoplophthiracarus) .............................50

similis [L. (Incabates)] ................................ 209

similis [C. (Klapperiches)] .............................. 151

similis (Liacarus) (=coracinus) ...................... 95

similis (Licneremaeus) .................................. 160

similis (Liebstadia) ........................................... 196

similis (Liebstadia) .......................................... 196

similis (Liebstadia) ......................................... 196

similis (Liebstadia) ................................... 196

similis (Limnozetes) ...................................... 166

similis (Liochthonius) ................................... 31

similis (Lohmannia) ....................................... 39

similis (Lyroppia) ........................................ 131

similis (Machadobelba) ………...................... 107

similis (Malacoangelia) ................................... 29

similis (Mesotritia) ............................................... 44

similis (Microtegeus) ..................................... 86

similis (Miracarus) .............................................. 90

similis (Moritzoppia) ................................... 127

similis (Multioppia) .................................... 117

similis [P. (Multipulchroppia)] ..................... 120

similis (Neoamerioppia) ............................. 114

similis [P. (Neophthiracarus)] (=radiatus) ....57

similis (Notophthiracarus) ............................ 52

similis (Oribatella) ........................................ 171

similis (Pareutegaeus) ..................................... 85

similis [V. (Phalacrozetes)] ......................... 207

similis [H. (Phyllhermannia)] .......................... 70

similis (Schizozetes) ….................................. 91

similis (Sellnickochthonius) .......................... 32

similis (Sellnickochthonius) .......................... 32

similis (Spatiodamaeus) ..................................... 82

similis (Steganacarus) ……............................ 58

similis (Striatoppia) ................................... 131

similis (Suctobelbella) .................................. 137

similis (Trimalaconothrus) sp. inq. .............. 62

similis [H. (Tuberemaeus)] ......................... 194

similis (Unguizetes) ................................... 186

simillima [S. (Flagrosuctobelba)]

(=semiplumosa) ……............................... 138

similoides (Dampfiella) …….......................... 141

simonettae (Steganacarus) .............................. 58

simplex (Afroleius) ........................................ 207

simplex (Berniniella) (=inornata) ................ 125

simplex (Ctenobelba) ..................................... 101

simplex (Cycloppia) (=restata) .................... 110

simplex (Cyrthermannia) .............................. 68

simplex (Charassobates) ................................. 86

simplex (Dolicheremaeus) ........................... 143

simplex (Eupelops) (=major) simplex [S. (Flagrosuctobelba)]

semiplumosa ….................................. 138

simplex (Fusuloppia) (=neonominata) ........ 113

simplex (Globoppia) ................................. 110

simplex (Haplobelba) .................................. 104

simplex (Haplochthonius) ............................... 34

simplex (Haplochthonius) .............................. 34

simplex (Haplochthonius) ............................ 34

simplex (Haplochthonius) …........................... 34

simplex (Liacarus) coracinus .......................... 95

simplex (Liochthonius) .......................... 11, 31

simplex (Liochthonius) ............................ 11, 31

simplex (Liochthonius) $\ldots \ldots \ldots \ldots \ldots \ldots \ldots \ldots . . . . . . . . . . .11,31$

simplex (Liochthonius) ......................... 11, 31

simplex (Lophotocepheus) .......................... 144

simplex (Machadobelba) .............................. 107

simplex (Microppia) minus (=minus) .......... 124

simplex (Microtritia) ........................................ 47

simplex (Microtritia) ........................................ 47

simplex (Nipponiella) ....................................... 33

simplex (Protoribates) (=halleri) ............... 206

simplex (Pseudotocepheus)

(=amonstruosus)

simplex (Rhynchobelba)

simplex (Scapheremaeus) ............................ 159

simplex (Scheloribates) Ger., 1849 sp. inq. .. 202

simplex (Scheloribates) Stor., 1925

=multiiteratus) ................ 200

simplex (Trimalaconothrus) ............................ 62

simplex (Vaghia) .......................................... 221

simplex (Vilhenabates) ................................ 207

simplirostratus (Papillacarus) …….................... 40

simpliseta [D. (Kalloia)] ............................ 152

simplisetosus (Paulianacarus) …………….... 40

simplisetus (Microzetes) ................................ 89

simplissima [S. (Lalmoppia)] ......................... 130

simplitricha (Multioppia) ............................ 117

simulator (Ceratozetes) ................................ 175

sinaraja (Truncopes) ....................................... 205

sinarmatus (Afrotocepheus) ...................... 141

sinatus [V. (Phalacrozetes)] .......................... 207

sine (Scheloribates) ….................................... 202

sinensis (Acrotritia) .......................................... 46

sinensis (Acrotritia) ……….............................. 46

sinensis (Acrotritia) ........................................ 46

sinensis (Acrotritia) ..................................... 46

sinensis (Arcoppia) fenestralis ...................... 120

sinensis (Fortuynia) ..................................... 157

sinensis (Galumna) elimata .......................... 215

sinensis (Nesiacarus) ...................................... 40

sinensis (Perxylobates)

singaporensis (Dolicheremaeus) …............. 143

singularis [G. (Cosmogalumna)] ................... 217

singularis (Hoplophorella) ............................ 49

singularis (Hoplophorella)

singularis (Hoplophorella) ............................. 49

singularis (Hoplophorella) ............................ 49

singularis (Lepidozetes) ................................ 169

singularis (Lepidozetes) ................................. 169

singularis (Mahunkobates) ........................... 71

singularis (Megalotocepheus) ...............12, 147

singularis (Metabelba) ..................................... 80 singularis (Protoribates) (=capucinus) ........ 206

singularis (Sacculoppia) ............................. 129

singularis (Suctobelbella) ............................... 137

singularis (Suctobelbella) ............................. 137

singularis [H. (Tuberemaeus)] ...................... 194

singularis [H. (Tuberemaeus)] ...................... 194

singularis[H. (Tuberemaeus)] .................... 194

singularis (Xenillus) ...................................... 98

sinica (Banksinoma) ...................................... 109

sinica (Fenestrella) ...................................... 102

sinica (Suctobelbella) ................................... 137

sinicus (Allonothrus) ….................................. 59

siniloanensis (Philippotocepheus) ............... 147

sinlimes (Perxylobates) .............................. 205

sinlimes (Perxylobates) ................................ 205

sinornata (Allogalumna) ............................. 214

sinuata (Camisia) (=horrida) ........................6 65

sinuata [S. (Flagrosuctobelba)] ............. 11, 138

sinuata [S. (Flagrosuctobelba)] ........... 11, 138

sinuata [L. (Incabates)] ................................. 209

sinuofrons (Galumna) ….............................. 217

sinuosociliatus (Austrocarabodes) ............ 149

sinuosus (Notophthiracarus) ....................... 52

sinuosus (Scapheremaeus) ........................... 159

sisiri (Gibbicepheus) ................................... 152

sitnikovae [D. (Cylindroppia)]

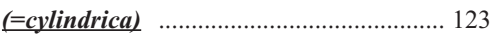

sitnikovae (Diapterobates) ........................... 181

sitnikovae (Diapterobates) ........................... 181

sitnikovae (Fissicepheus) ............................... 144

sitnikovae (Membranoppia) ....................... 111

sitnikovae (Moritzoppia) …......................... 127

sitnikovae (Oribatella) ................................. 171

sitnikovae (Oribatula) .................................. 188

skoettsbergi [H. (Platynothrus)] ..................66 66

skoettsbergi [H. (Platynothrus)] ..................... 66

skookumchucki (Ouadroppia)

(=quadricarinata) .................................... 133

skrjabini (Perlohmannia) sp. inq. ..................... 36

skrjabini [O. (Zygoribatula)] ........................ 189

slossonae (Fuscozetes) (=fuscipes) .............. 175

smirnovi (Damaeus) ......................................... 78

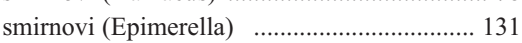

smirnovi (Thamnacarus) …................................ 41

smirnovi [O. (Zygoribatula)] ........................ 189

smithersi (Capilloppia) .............................. 186

snowdonensis (Campachipteria) …............... 168

socia [O. (Zygoribatula)] (=cognata) .......... 188

socotrensis [O. (Zygoribatula)] ................... 189

sol (Gustavia) (=microcephala) …................ 95

sol (Helioppia) ................................................. 116

sol (Steganacarus) ....................................... 58

solhoeyi [C. (Ensicamisia)] ............................. 65

solitaria (Oribotritia) ..................................... 44

solitarius (Notophthiracarus) ...................... 52

sollertia (Basilobelba) (=retiaria) ................. 104

soloduchi (Ctenobelba) ............................... 101

solomonensis (Apoplophora) ….................... 41

solomonensis (Notophthiracarus) ……......... 52

somalica (Pergalumna) ............................. 219

somalicus (Eupelops) ................................ 164

somalicus (Notophthiracarus) ..................... 52 


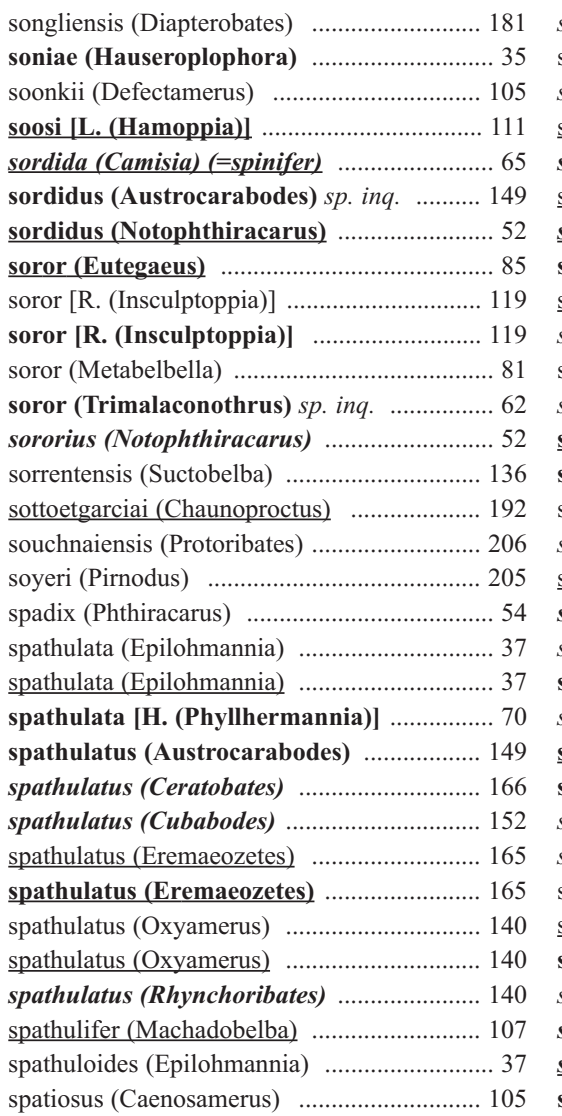

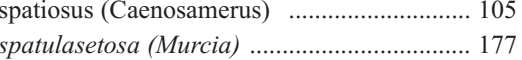

spatulata (Hoplophorella) (=hamata) ............ 49

spatulatus (Otocepheus) …………………...... 147

speciosa (Oppia) ………………………….... 115

speciosus (Dolicheremaeus) sp. inq. ............ 143

speciosus (Hydrozetes) (=lacustris) ............... 155

spectabilis (Fissurobates) ............................. 197

spectabilis (Rhynchoribates) ........................ 140

spectabilis (Sphaerochthonius) ........................... 34

sphaerica (Ceratoppia) ………………………... 93

sphaerica (Ceratoppia) ................................... 93

sphaericus (Annobonzetes) ...……………... 196

sphaericus (Liacarus) ........................................ 96

sphaericus (Punctoribates) ............................ 184

sphaeroclava [I. (Mancoribates)] ................. 208

sphaerodendron (Sternoppia) ........................ 133

sphaeroides (Scheloribates) .......................... 202

sphaeroides (Topobates) ……………………. 203

sphaeroideus (Austrocarabodes) ................ 149

sphaerula (Austrocarabodes) ……………...... 149

sphaerula (Taeniogalumna) .......................... 221

sphaerula (Unguizetes) ..................................... 186

sphaerulus (Ellipsozetes) ………………….... 182

sphaerulus (Phthiracarus) (=globosus) ….... 55

sphagni (Limnozetes) (=ciliatus) ………..... 165

sphagni [M. (Parametabelba)] ………………... 81

sphagnicola (Processoppia) ……………...... 112

sphagnicola (Trhypochthonius) ..................... 60

sphagnicola [T. (Tyrphonothrus)] (=maior) .... 63

spherisensilla [O. (Zygoribatula)] …….......... 189 spicata (Suctobelbila)

spiciger (Poelcilochthonius) _.................... 31

spiciger (Poecilochthonius) …………………... 31

spiciger (Poecilochthonius) ............................ 31

spiculifer [Y. (Berndobodes)] ......................... 154

spiculifera (Acrotritia) (=sinensis) ................ 46

spiculifera [O. (Oxyoppiella)] ...................... 129

spiculigera (Suctobelbella) sp. inq. .............. 137

spilotus $[X .($ Stonyxenillus)] ………….............. 99

spina (Joelia) …………………………........ 170

spinatus (Eupterotegaeus) .................................. 83

spinatus (Neseutegaeus) …………………..... 85

spinatus (Rhacaplacarus) …………………..... 57

spinifer (Camisia) .............................................. 65

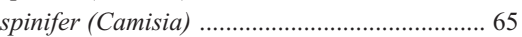

spinifer (Camisia) …………………………............. 65

spinifer (Camisia) ............................................. 65

spinifer (Eremulus) ....................................... 102

spinifera (Baloghia) ……………………...... 207

spinifera (Banksinoma) ................................ 109

spinifera (Multioppia) …………………..... 117

spiniferus (Rhacaplacarus) ........................... 57

spiniformis (Carabodes) ................................. 150

spiniger (Liacarus) ......................................... 96

spiniger (Rhacaplacarus) ................................ 57

spiniger [P. (Tectodamaeus)] ………………........ 81

spinipes (Aethioppia) ……………………... 112

spinipes (Ceratoppia) bipilis .......................... 93

spinipes (Ceratoppia) bipilis .......................... 93

spinipes (Ceratozetella) (?=maxima) .......... 173

spinipes (Otoppia) .......................................... 112

spinipes (Physobates) …………………….... 169

spinipes (Scutovertex) $s p$. inq. .................... 161

spinipes (Tikizetes) ………………………...... 84

spinitecta (Heterobelba) .................................. 104

spinogenuala (Murcia) .................................... 177

spinosa (Achipteria) armata ......................... 167

spinosa (Apoplophora) ……………………….... 41

spinosa (Lohmannia) sp. inq. .......................... 39

spinosa (Mesotritia) (=maerkeli) .................... 43

spinosa (Oribotritia) …………………………... 44

spinosa (Oxyoppia) ........................................ 129

spinosa (Porobelba) …………………………….... 82

spinosa (Porobelba) ………............................... 82

spinosissima (Heterobelba) ........................... 104

spinosissima (Lucoppia) (=burrowsi) .......... 187

spinosus (Carabodes) ……………………….. 150

spinosus (Cosmochthonius) .............................. 33

spinosus (Cristamerus) ………………………... 105

spinosus (Eupelops) (=acromios) ................. 163

spinosus (Megeremaeus) ……………………... 99

spinosus (Nasobates) ………………………... 212

spinosus (Papillacarus) .................................... 40

spinosus (Seteremaeus) ………………….... 159

spinosus (Steganacarus) ……………………..... 58

spinosus [M. (Stylozetes)] .............................. 89

spinosus (Tectocepheus) ……………………... 154

spinosus [P. (Triaungius)] ............................... 206

spinosus (Trhypochthonius) tectorum .............60 60

spinosus [C. (Xiphobates)] (=rastratus) ...... 180

spinosus (Zetomimus) .................................... 179 spinulosa (Peloptoribula) ............................... 93

spinulosa (Synichotritia) ............................... 48

spinulosus (Neotrichozetes) .......................... 186

spinus (Euphthiracarus) ............................... 46

spirochaeta (Suctobelbella) ......................... 137

spirofilus [H. (Platynothrus)] (=peltifer) ...... 66

spirulatus (Scheloribates) ............................... 202

spitsbergensis (Ceratozetes) .............................. 175

spitsbergensis (Ceratozetes) ........................... 175

splendens [L. (Dorycranosus)] .......................... 97

splendens (Moritzoppia) sp. inq. ................... 127

splendidus (Sphaerochthonius) ……………..... 34

splendidus (Sphaerochthonius) ....................... 34

splendidus (Sphaerochthonius) ........................ 34

splendidus (Sphaerochthonius) ..................... 34

splendidus (Sphaerochthonius) ..................... 34

spongiosus (Eupelops) .................................. 164

spoofi (Ameronothrus) (=marinus) ............... 156

spora (Muliercula) ………………………..... 197

springsmythi (Nothrus) ..................................... 64

springthorpei (Pheroliodes) ........................... 74

spumosa (Heterobelba) ………………….... 104

spurcus (Notophthiracarus) ………………... 52

sqamaceus (Umbellozetes) ........................... 170

squamiger (Neoliodes) $s p$. inq. ...................... 72

squamiseta (Suctobelbella) ……………....... 137

squamosa (Suctobelbila) ………………....... 139

squamosa (Suctobelbila) .............................. 139

squamosus (Austrocarabodes) imperfectus .. 148

squarrosa (Oppiella) (=nova) …………….... 128

stabelchodi (Parhypochthonius) sp. inq. ........ 28

stagaardi (Capillibates) ................................... 156

stagnatilis (Melanozetes) ………………........ 176

starki (Adrodamaeus) ......................................... 75

stebaevae (Licnodamaeus) ............................... 74

steinboecki (Eupterotegaeus)

(=ornatissimus) …………………………... 83

steineri (Scutovertex) (=sculptus) ................ 161

steinmanni (Fissicepheus) ............................. 144

steinmanni (Pilogalumna) …………............. 220

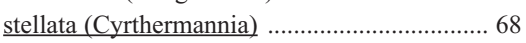

stellata (Cyrthermannia) …………………..... 68

stellata (Pedrocortesella) ................................. 75

stellatus (Peloribates) ……………………….... 210

stellifer (Cuspitegula) ……………………….... 88

stellifer (Hymenozetes) .................................... 89

stellifer (Passalozetes) ………………....... 12, 162

stellifera (Heterobelba) ..................................... 104

stellifera (Heterobelba) ................................... 104

stellifera (Multioppia) ………………….......... 117

stellifera (Multioppia) .................................. 117

stenolamellatus (Exochocepheus) ………...... 161

stenotus (Notophthiracarus) …......................... 52

stenotus (Phthiracarus) ……………………..... 54

stepensis (Xenillus) ……………………….... 98

stepposa $($ Jacotella $)$ (=austriaca)

stercus (Trhypochthonius) ............................... 60

sterigma (Acrotritia) ………………………..... 46

sternalis [P. (Metaleius)] (?=strenzkei) ........ 194

sticta (Niloppia) …………………………....... 114

stigmata (Granuloppia) ................................. 132

stigmosus (Hoplophthiracarus) ……………... 50 


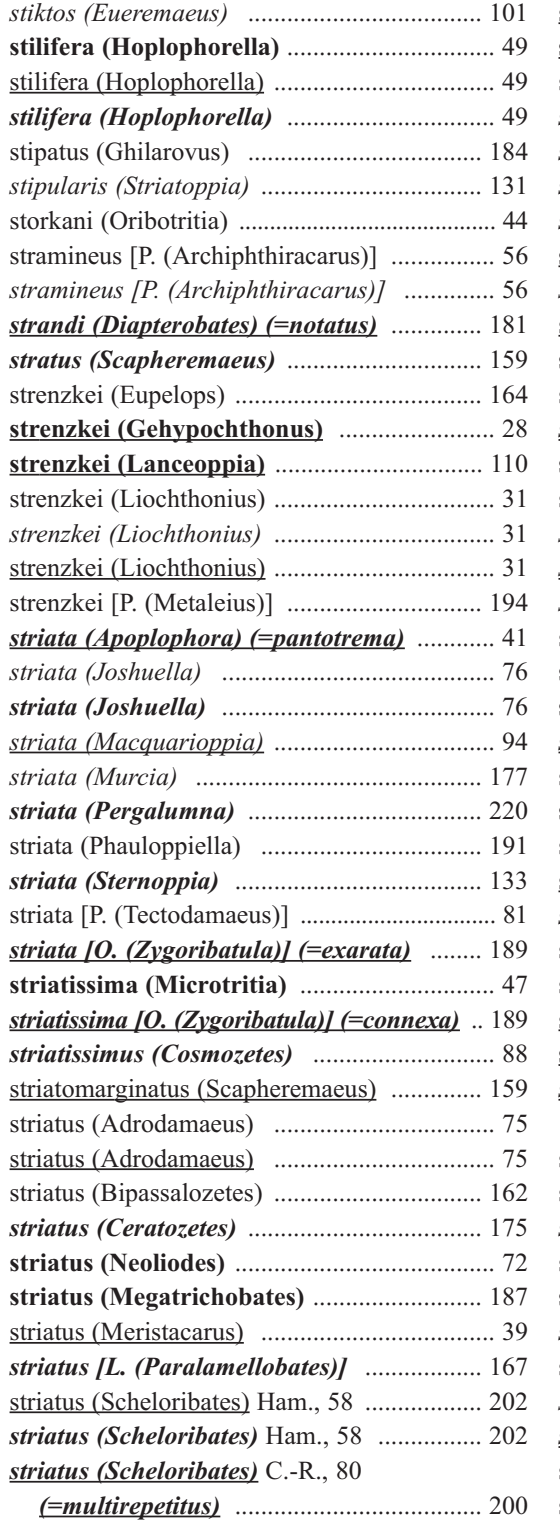

striatus (Sellnickochthonius)

(=immaculatus) ………………………..... 32

strictior (Eobrachychthonius) latior ssp. inq. .. 30

striculus (Atropacarus) ……………………….... 48

striculus (Atropacarus) ...................................... 48

striculus (Atropacarus) ……………………..... 48

striculus (Atropacarus) ……….................... 48

strigosus [N. (Protophthiracarus)] ………..... 53

strigulata (Pergalumna) …………………... 220

strinatii (Oribatella) ........................................ 172

strinatii [R. (Rectoppia)] ………………….... 119

strinatii (Sphaerochthonius) .......................... 34

strinovichi (Galumna) ………………........ 217

strinovichi [C. (Klapperiches)] .................... 151

strinovichi (Magyaria) ................................. 209

strinovichi (Setoppia) ……………………..... 112

striolatus (Scheloribates) ………………..... 202

struthio [O. (Oxyoppiella)] ………………... 129 stunkardi (Nasozetes) ..................................... 194

stunkardi (Nasozetes) ……………………..... 194

stupenda (Vaghia) .............................................. 221

sturmi (Anderemaeus) ...................................... 106

sturmi (Neoamerioppia) ............................... 114

sturmi (Pseudotocepheus) ............................ 145

sturmi (Truncozetes) ................................... 173

stylesi (Eutegaeus) ………………………....... 85

styosetosus (Arborichthonius) ......................... 29

styosetosus (Arborichthonius) ………………. 29

styphelos (Rhacaplacarus) ………………..... 57

styriaca (Epilohmannia) .................................... 37

subaequalis (Pergalumna) (=myrmophila) .. 219

subaequalis (Rhynchoribates) .................... 140

subalpinus (Carabodes) ................................ 150

subalpinus (Gymnodamaeus) ............................ 76

subantarctica [O. (Zvgoribatula)] ………..... 189

subaquila (Ceratozetes) ................................. 175

subarcticus (Carabodes) …………………….... 150

subareolata (Galumnella) ……………….... 222

subareolatus [H. (Tuberemaeus)] ………... 194

subborealis (Propelops) (=monticolus) ......... 164

subcarinatus (Carabodes) .............................. 150

subciliata (Hoplophorella) ............................... 49

subclavatus (Fissicepheus) .............................. 144

subcomplexa [F. (Parasuctobelba)] ……….... 134

subcomplexa [F. (Parasuctobelba)] ............ 134

subcorniger (Scapheremaeus) ....................... 159

subcornigera (Suctobelbella) ......................... 137

subcornigera (Suctobelbella) ………………... 137

subcornigera (Suctobelbella) ....................... 137

subcricoides (Brachychthonius)

(=impressus)

subdolus [P. (Archiphthiracarus)]

subexutus (Eupelops) .................................... 164

subfallax [M. (Moritzoppiella)] …………..... 127

subglaber (Micreremus) …………………….... 160

subglaber (Pilizetes) ……………………….... 220

subglaber (Scapheremaeus) .......................... 159

subglabra [H. (Heterohermannia)] .................... 69

subglabra [H. (Heterohermannia)] ................ 69

subglobosus (Phthiracarus) (=globosus) ...... 55

subglobulus (Chamobates) ............................. 180

subiasi [P. (Archiphthiracarus)] ........................ 56

subiasi [D. (Epidamaeus)] ............................... 80

subiasi [R. (Insculptoppia)] .......................... 119

subiasi [S. (Lalmoppia)] …………………..... 130

subiasi (Tripiloppia) …………………….... 128

subinconspicuus (Ceratozetes) ....................... 175

subintectus (Sphaerozetes) sp. inq. ………... 178

subita (Hoplophorella) .......................... 49

sublanatus (Cosmochthonius) ………….......... 33

sublestus (Mixacarus) ………………………… 39

submolesta (Oribotritia) ....................................... 44

subniger (Sadocepheus) ................................... 84

subniger (Tegoribates) ..................................... 169

subnigra (Hermanniella) ................................. 71

subnigra (Hermanniella) ................................. 71

subnitida [L. (Antennoppia)] ....................... 113

subnitidus (Ovobates) ………………………. 190

subnuda (Trichogalumna) ............................... 221

subnudus (Gymnobodes) .............................. 152 subnudus (Xenillus) _...……………………... 99

subpectinata (Medioppia) ............................... 124

subpectinata (Medioppia) ............................ 124

subpectinata (Medioppia) ........................... 124

subrasus [M. (Cristonothrus)] .......................... 61

subsellata (Hoplophorella) ............................ 49

subseminulum (Ceratozetella) (?=maxima) .. 173

subsimilis (Dolicheremaeus) ………........... 143

subsimilis (Pilizetes) ……………………... 220

subsimilis (Pseudotectoribates) .................... 172

subsimilis (Scheloribates) Mih., 56$$
\text { (=barbatulus) }
$$

subsimilis (Scheloribates) Bal., 62

(=caprai) …………................................ 199

subspinipes (Scutovertex) ……………….... 161

substriata [O. (Zygoribatula)] connexa .... 188

substrictus (Atropacarus) ................................ 48

subterraneus (Liacarus) …………………….... 96

subterraneus (Liacarus) ............................... 96

subtilis (Belba) $s p$. inq. .................................... 78

subtilis [M. (Parplophora)] …………………... 42

subtilis [M. (Parplophora)] .......................... 42

subtilis [M. (Parplophora)] ............................ 42

subtrigona (Suctobelbella) ....................... 11, 137

subtrigona (Suctobelbella) ...................... 11, 137

subtropica (Taiwanoppia) …………......... 115

subtropicus (Perscheloribates) ..................... 198

subula (Pedrocortesella) ................................. 75

subulatus (Eupelops) sp. inq. ......................... 164

subuliger (Eupelops) ……....................... 164

subuligera (Lasiobelba) ................................... 113

subverticillipes (Spatiodamaeus) ....................... 82

succinctus (Rhacaplacarus) …………………... 57

succinta (Oribotritia) ...................................... 44

suchetae (Lasiobelba) ……………………..... 113

suciui [R. (Insculptoppia)] .......................... 119

suctobelboides (Suctobelbila) ………............. 139

suctorius (Suctoribates) ................................. 140

sudamericana [R. (Rectoppia)] fasciata ...... 119

sudamericanus [P. (Archiphthiracarus)] ….. 56

sudetica (Oromurcia) ..................................... 178

suecicus (Sellnickochthonius) ……………........ 32

suecicus (Sellnickochthonius) ......................... 32

suecicus (Sellnickochthonius) ……………….. 32

suecicus (Sellnickochthonius) …………….... 32

sufflata (Hammerella) ................................. 132

sufflexa (Belba) (=corynopus) ……………... 77

sulcatomarginata (Pergalumna) ................. 220

sulcatus (Afronothrus) (=incisivus) .............. 59

sulcatus (Dolicheremaeus) .............................. 143

sulcatus (Hammerabates) …………………. 197

sultan (Sacculobates) ……………………….... 71

sumatrana (Hypochthoniella) …………….... 29

sumatrana (Hypochthoniella) ............................ 29

sumatranus (Dolicheremaeus) ....................... 143

sumatranus (Notophthiracarus) …………….... 52

sumatrensis (Achipteria) ………………….... 168

sumatrensis (Nasozetes) …………………….... 194

sumatrensis (Oppiella) (=nova) …………... 128

summersi (Notophthiracarus) ....................... 52

sumonyii (Oripoda) ………………………. 204

sundaicus (Trimalaconothrus) foveolatus ...... 62 
sundarbanensis (Hoplophorella)

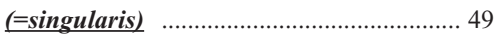

sundensis (Graptoppia) …............................. 116

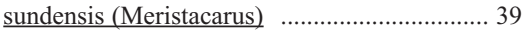

sungai (Caucasiozetes) ……............................ 88

sungohi (Defectamerus) ................................. 105

suoxiensis (Mixacarus) …….............................. 40

superba (Camisia) (=spinifer) ........................ 65

superbula (Oribatella) .................................... 172

superbula (Oribatella) ................................... 172

superbus (Diplobodes) ................................. 152

superbus (Xenillus) ........................................ 99

superporosa (Allogalumna) ......................... 214

supertrichus (Heptacarus) .................................. 38

sura (Pergalumna) ..................................... 220

suramericana (Multoribula) ......................... 93

suramericana [O. (Oxyoppiella)] ................. 129

suramericana [O. (Oxyoppiella)] ............... 129

suramericana [O. (Oxyoppiella)] ................ 129

suramericanus (Benoibates) ........................ 203

suramericanus (Cosmochthonius) plumatus ...... 33

suramericanus (Cosmochthonius) plumatus ...... 33

suramericanus (Cosmochthonius) plumatus .. 33

suramericanus (Eupelops) .................... 12, 164

suramericanus (Eupelops) .................. 12, 164

suramericanus (Hemileius) .......................... 193

suramericanus (Hemileius) .......................... 193

suramericanus (Nothrus) ............................. 64

surigaoensis (Acrotocepheus) ……….......... 146

surigaoensis (Perscheloribates) …................ 198

suzukii (Sphaerochthonius) ........................... 34

suzukii (Sphaerochthonius) …….................. 34

swezeyi (Galumna) ……............................ 217

swezeyi (Neoliodes) ...................................... 72

swiftae (Phthiracarus) ................................ 54

sylvicola (Scheloribates) (?=fimbriatus) ...... 199

symmetrica (Basilobelba) retiaria ............. 104

symmetrica (Machadobelba) ...................... 107

symmetrica (Machadobelba) ….................... 107

symmetrica (Malacoangelia) (=remigera) .... 29

synlamellata (Beckiella) ............................... 141

szabadosi [N. (Protokalumma)] ................. 212

szaboi (Oribatella) ..................................... 172

szaboi (Setoppia) ............................................. 112

szanisloi (Epilohmannia) (=cylindrica) ........ 36

szemmelveiszi (Liochthonius) .................... 31

szentirmayi (Cycloppia) ............................ 110

szentivanyi (Austrocarabodes) ...................... 149

szentivanyi (Austrocarabodes) ....................... 149

szentivanyi (Cultroribula) (=bicultrata) ........ 92

szentivanyi (Dolicheremaeus) …................ 143

szentivanyi (Szentivanyella) ….................... 91

szentivanyorum (Galumna) ......................... 217

szentivanyorum (Pseudotocepheus) .......... 145

szunyoghyi (Oribatella) .............................. 172

taberlyi (Solenoppia) ............................... 124

tablasotus (Trhypochthonius) sp. inq. .......... 60

tabulatus (Paradolicheremaeus)

tadrosi [O. (Zygoribatula)] (=undulata) ...... 190

taeda (Epilohmannia) ...................................... 37

taedaceus (Gymnodamaeus) ........................... 76

taeniata (Trichogalumna) .......................... 22 taeniophorus (Amnemochthonius) .................. 34

aeniophorus (Amnemochthonius) .............. 34

tahitiana (Austrotritia) lebronneci …........... 42

tahitiensis (Eupelops) ............................... 164

tahitiensis [S. (Flagrosuctobelba)]

$$
\text { semiplumosa }
$$

hitiensis [S. (F]agr

semiplumosa ......................................... 138

tahitiensis (Ozacarus) …................................ 40

tahitiensis (Pergalumna) ........................... 220

aidinchani (Dolicheremaeus) ….................. 143

taidinchani (Perxylobates) ............................ 205

taisetsuensis (Nothrus) ...................................... 64

taishanensis (Cryptoribatula) ........................ 205

taiwanensis (Humerobates) ......................... 182

taiwanica (Dometorina) ............................... 192

taiwanica (Epilohmannia) .............................. 37

taiwanus (Dolicheremaeus) infrequens ....... 142

tajikistanica (Pyroppia) .................................... 94

takahashii (Euphthiracarus) ............................ 46

takasago (Cepheus) ………………………........ 83

talacrensis [L. (Baioppia)] ........................... 111

talis [O. (Zygoribatula)] ................................ 189

talischica (Conchogneta) (=dalecarlica) .... 108

tamarae [H. (Heloribates)] .............................. 156

tamdao (Multioppia) ...................................... 117

tameyei [O. (Zygoribatula)] .......................... 190

taminae (Ramusella) (=clavipectinata) ........ 118

tamlaensis (Nippobodes) ............................. 154

tamurai (Dolicheremaeus) …........................ 143

tanaitica (Graptoppia) ................................. 116

tanana (Melanozetes) .................................... 176

tanganyikensis (Metabelba) sp. inq. ............ 80

tanngardenensis (Maudheimia) …............... 195

tantilla (Pergalumna) (=curva) .................... 218

tanythrix (Euphthiracarus) ............................. 46

tanytrichosus (Peloptulus) ............................ 164

tanzanica (Galumnella) ............................... 222

tanzanica (Pergalumna) ............................... 220

tanzanica (Taeniogalumna) ....................... 221

tanzanicus (Liochthonius) .............................. 3

tanzanicus (Topobates) ................................. 203

tanzica (Machadobelba) ............................... 107

tanzicus (Liacarus) ............................................ 96

tanzicus [M. (Megazetes)] ……..................... 89

tanzicus (Rhacaplacarus) .............................. 57

taprobanae (Tenuelamellarea) ...................... 160

taprobanica (Pergalumna) .............................. 220

taprobanicus (Machadocepheus) …...............152

tarandus (Austrocarabodes) ...................... 149

tardus (Eupelops)

tardus (Phthiracarus) (=longulus) ................ 54

tardus (Trimalaconothrus) ....................... 11, 62

tardus (Trimalaconothrus) ...................... 11, 62

tardus (Trimalaconothrus) ....................... 11, 62

targionii (Heminothrus) ..................................... 66

targionii (Heminothrus) . .

targionii (Heminothrus) ................................... 66

tarmani (Minunthozetes) ............................... 182

tarraswahlbergi (Tripiloppia) .................... 128

tarsipennata (Galumna) ............................... 217

tarsipennata (Galumna) .............................. 217 tasetata (Ramusella) .................................... 118

tasmanica [L. (Lancelalmoppia)] .............. 111

tatarica (Suctobelbella) ................................... 137

tatrica (Camisia) ............................................... 65

tatricus [D. (Epidamaeus)] …........................... 80

tatricus [D. (Epidamaeus)] ........................... 80

tatricus (Fuscozetes) ...................................... 176

tauricus (Damaeus) ......................................... 78

tauricus (Phyllozetes) ....................................... 33

taurina (Camisia) (=spinifer) ........................6 65

taurus (Acaroceras) …............................. 87

taurus (Congocepheus) …............................ 151

taurus (Cosmochthonius) ................................. 33

taurus [O. (Dzarogneta)] ............................... 129

taurus (Scapheremaeus) ….............................. 159

taurus (Scapheremaeus) .............................. 159

tavruei (Phauloppia) ...................................... 191

tecticola [D. (Epidamaeus)] ............................. 80

tectopedacuta (Murcia) sp. inq. .................... 177

tectopediosa [S. (Dyobelba)] ............................. 82

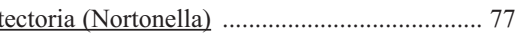

tectorum (Trhypochthonius) ..............................60 60

tectorum (Trhypochthonius) ........................... 60

tectorum (Trhypochthonius) .............................. 60

tectorum (Trhypochthonius) ...........................60 60

tectus (Ophidiotrichus) ................................... 170

tectus (Podopterotegaeus) ..................................... 82

tegeocranus (Xenillus) ………….................... 99

tegeocranus (Xenillus) ………........................ 99

teldanicus (Scheloribates) …......................... 202

temperata (Pedrocortesella) …..................... 75

templadoi (Medioppia) (=media) …............. 123

tenareae (Haloribatula) ..................................... 195

tenella (Oribatula) (=pannonica) …............. 187

tenerifensis [C. (Klapperiches)] ..................... 151

tenerifensis (Oribatella) ............................... 172

tenerifensis (Steganacarus) ............................... 58

tenorioae (Scriptoripoda) …....................... 205

tentaculus (Protoribates) capucinus ............... 206

tenue (Multioppia) ......................................... 117

tenuelamellata [O. (Zygoribatula)]

(=frisiae) ……........................................ 189

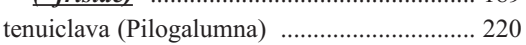

tenuiclava (Pilogalumna) ............................... 220

tenuiclava [H. (Platynothrus)] .......................66

tenuicoma (Brachioppiella) ....................... 122

tenuicoma (Nanhermannia) ...........................6 68

tenuifusus [L. (Incabates)] ............................ 209

tenuilamellatus [L. (Dorycranosus)] ............... 97

tenuipectinata (Lauroppia) ............................ 126

tenuipes [D. (Epidamaeus)] ............................ 80

tenuipes [D. (Epidamaeus)] .......................... 80

tenuis (Arthrodamaeus) (?=rosarius) ............ 76

tenuis (Berniniella) ....................................... 125

tenuis (Carabodes) ......................................... 150

tenuis [D. (Cylindroppia)] ............................ 123

tenuis (Eremulus) …..................................... 102

tenuis (Hemileius) .......................................... 193

tenuis (Liebstadia) ......................................... 196

tenuis (Oribatella) ........................................... 172

tenuis (Perscheloribates) …........................... 198

tenuis (Phthiracarus) (=boresetosus) ............. 53 
tenuis (Protoribates) ………………................ 206

tenuis (Tectocepheus) .................................... 155

tenuiseta [N. (Confinoppia)] confinis .......... 127

tenuiseta [P. (Constrictocepheus)] ............ 145

tenuiseta (Hoplophthiracarus) ….................50

tenuiseta (Mimoppia) ................................. 121

tenuiseta [H. (Phyllhermannia)] ....................... 70

tenuiseta (Scheloribates) praeincisus ............ 201

tenuiseta (Scheloribates) praeincisus ........ 201

tenuiseta [O. (Zygoribatula)] ........................ 190

tenuiseta [O. (Zygoribatula)] ........................ 190

tenuisetiger (Eremaeus) ................................... 100

tenuisetosa (Belba) ......................................... 78

tenuisetosa (Nanhermannia) ........................6 68

tenuisetosus (Dicastribates) ............................ 70

tenuisetosus [D. (Epidamaeus)] ..................... 80

tenuisetus (Cosmochthonius) ............................ 33

tenuissimus [D. (Epidamaeus)] ....................... 80

tenuitibialis (Damaeus) .................................... 78

tepetlensis (Murcia) ....................................... 177

tepoztecus (Trhypochthonius) ......................... 60

tequila (Berniniella) ................................... 125

teraja (Arcoppia) ......................................... 121

terebrantis (Orthogalumna) ......................... 218

teretis (Oribotritia) ............................................ 45

terminalis (Eupelops) ..................................... 164

terminalis (Nothrus) (=monodactylus) .......... 64

terrae (Epilohmannoides) .............................. 37

terrae (Epilohmannoides) .............................. 37

terrapene (Atropacarus) .................................... 48

terrestris (Hydrozetes) (=confervae) ............. 155

terrestris (Neoliodes) …..................................... 72

terrestris (Neoliodes) ….................................. 72

terrestris (Neoliodes) …..................................... 72

terrestris (Neoliodes) ........................................ 72

terricola (Hypogeoppia) .................................. 126

terricola (Hypogeoppia) ................................ 126

terricola (Hypogeoppia) .............................. 126

terricola [R. (Insculptoppia)] ........................ 119

terricola [O. (Zygoribatula)] (=connexa) .... 188

tessellata (Ferolocella) ................................ 170

tessellata (Galumna) ....................................... 217

testacea (Mesotritia) (=flagelliformis) ............ 43

testudineus (Phthiracarus) sp. inq. .................. 54

tetrasklerosis (Zachvatkinibates) ................... 184

tetrosus (Eueremaeus) ................................ 101

texana (Galumna) ........................................ 217

texana (Lohmannia) ......................................... 39

texana (Mochloribatula) ...................... 185

texanus [N. (Calyptophthiracarus)] ................ 53

thabazimbiensis (Eupelops) curtipilus ...... 163

thaiensis (Nanhermannia) ................................6 68

thalassophila [O. (Zygoribatula)] ................... 190

thamdrupi [L. (Hamoppia)] ........................ 111

thani (Hermanniella) ...................................... 71

theleproctus (Neoliodes) .................................. 72

theleproctus (Neoliodes) …............................. 72

theleproctus (Neoliodes) .................................72

theliis (Gymnodamaeus) ................................... 76

thermophilus (Scheloribates) ......................... 202

thermophilus (Scheloribates) ..................... 202

thermophilus (Tutorozetes) thienemanni (Ceratozetella)

ienemanni (Ceratozetella) …................... 173

thienemanni [H. (Heloribates)] ....................... 156

thienemanni [H. (Heloribates)] ..................... 156

thienemanni [H. (Tuberemaeus)] .................. 194

thienemanni [H. (Tuberemaeus)] ….......... 194

thoreaui (Rhacaplacarus) ............................... 58

thori [H. (Capillonothrus)] ............................... 66

thori [H. (Capillonothrus)] ............................ 66

thori [H. (Capillonothrus)] ............................... 66

thori [L. (Lancelalmoppia)] ........................ 111

thujae (Hemileius) ......................................... 193

thysanura (Psammogalumna) ....................... 221

tianmuensis (Mixacarus) .................................. 40

tianmuensis (Sabahtritia) …..............................47

tianschanica (Simkinia) ................................. 195

tianschanicus (Megalotocepheus) ................ 147

tianshanensis (Murcia) ….............................. 177

tianshanensis (Oribellopsis) ........................... 107

tibialis (Bipassalozetes) $s p$. inq. .................... 162

tibialis (Eupelops) ........................................ 164

tibialis (Oribatula) ........................................ 188

tibialis (Oribatula) .................................. 188

tibialis (Oribatula) ....................................... 188

tibialis (Oribatula) 188

tichomirovae (Berniniella) ............................ 125

tichomirovi (Pallidacarus) ..................... 184

tichonravovi (Metabelbella) .............................. 81

tillandsiae (Scapheremaeus)

tillandsiophilus (Scapheremaeus) ................ 159

tillandsius (Peloribates) (=hubbardi) .......... 210

timah (Bukitritia) ............................................ 46

timah (Bukitritia) .............................................. 46

tinctus (Notophthiracarus) ……................... 52

titanius (Tectocepheus) ................................ 155

tiwi (Oribotritia) ............................................. 45

tjanshanica (Murcia) ........................................ 177

tjibodensis (Spinotocepheus) ......................... 146

tobaicus (Hydrozetes) ..................................... 155

toeglyesii (Suctobelbella) .............................. 137

toeroeki (Setoppia) ...................................... 112

tohivea (Notophthiracarus) .......................... 52

tohokuensis (Suctobelbella) ............................ 137

tokara (Allosuctobelba) tricuspidata ............ 134

tokara (Nanhermannia) ................................. 68

tokaraensis (Nippobodes) .............................. 154

tokukoae (Oribotritia) ………………………….... 45

tokyoensis (Galumna) ............................... 217

tokyoensis (Ramusella) (=sengbuschi) ........ 118

tolanaro (Pseudotocepheus) ............... 145

toletana (Serratoppia) (?=intermedia) ........ 124

tonatiuh (Scapheremaeus) .......................... 159

tongaensis (Disparagalumna) .................... 214

tongaensis (Neotrichocepheus) ….............. 144

tongatapuensis (Nesopelops) ....................... 164

tonkini (Trimalaconothrus) ........................... 62

topali (Phauloppia) ....................................... 191

topali [L. (Undulozetes)] ................................ 89

torosus [P. (Archiphthiracarus)] ....................... 56

torquatus (Perscheloribates) clavatus ............ 198

torquisetosus (Damaeus) .................................. 78 torrijosi (Oribatula) ....................................... 188

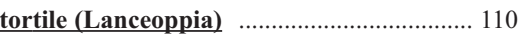

tortilis [O. (Zygoribatula)] ............................. 190

tortilis [O. (Zygoribatula)] ............................ 190

tortuosa (Acanthobelba) ..................................... 77

torulosus (Eupelops) ......................................... 164

torvus (Spatiodamaeus) (?=verticillipes) ........ 82

toxotes (Setoppia) ….................................. 112

traegardhi (Acaronychus) ................................... 27

traegardhi (Acaronychus) ............................... 27

traegardhi (Acaronychus) ................................ 27

traegardhi (Acaronychus) ............................... 27

traegardhi (Conchogneta) .............................. 109

traegardhi (Conchogneta) ............................ 109

traegardhi [N. (Phthirarica)] .......................... 53

traegardhi (Tripiloppia) ............................. 128

transcripta (Novoribatella) ......................... 170

transitans (Oribatula) ................................... 188

transitans (Oribatula) .................................... 188

transitans (Steganacarus) (=magnus) .......... 58

transitoria (Fenestrobelba) ........................... 134

transitoria (Mahunkella) ............................. 129

transitoria (Neosuctobelba) .......................... 135

transitoria (Parapyroppia) ............................ 94

transitus (Nortonella) ........................................ 77

translamellaris (Multioppia) ..................... 117

translamellaris (Scheloribates) ….................. 202

translamellaris (Tectocepheus) …................... 155

translamellata (Acroppia) ......................... 128

translamellata (Ceratozetella) ......................... 173

translamellata (Ctenobelba) ........................... 101

translamellata [M. (Moritzoppiella)]

(=neerlandica) ............................................ 127

translamellata (Ramusella) ............................ 118

translamellata (Teratoppia) ....................... 132

translamellatus (Allozetes) ............................. 166

translamellatus (Allozetes) …..................... 166

translamellatus (Allozetes) ........................... 166

translamellatus (Anellozetes) Shald., 71 ...... 181

translamellatus (Anellozetes) Mah., 85

(=neonominatus) ..................................... 181

translamellatus [M. (Cristonothrus)] ...............6 61

translamellatus [M. (Cristonothrus)] ............. 61

translamellatus (Eremaeus) …......................... 100

translamellatus (Eremaeus) ......................... 100

translamellatus (Eremulus) ....................... 102

translamellatus (Hemileius) ........................... 193

translamellatus (Hypozetes) ....................... 166

translamellatus [C. (Klapperiches)]

(=similis) .................................................... 151

translamellatus (Liacarus) (=xylariae) .......... 96

translamellatus (Monoschelobates) ........... 194

translamellatus (Tectocepheus) ..................... 155

translamellatus (Tenuialoides) (=fusiformis) .. 99

translineata (Oribatula) .............................. 188

translucens (Lanceoppia) ............................. 110

transplicatus (Scheloribates) _....................... 202

transrugosa (Suctobelbila) .......................... 139

transvaalensis [H. (Phyllhermannia)] .......... 70

transvaalensis (Zetorchestes) ....................... 91

transvectus (Orbiculobates) .......................... 71

transversa (Allogalumna) (=alamellae) ....... 213 


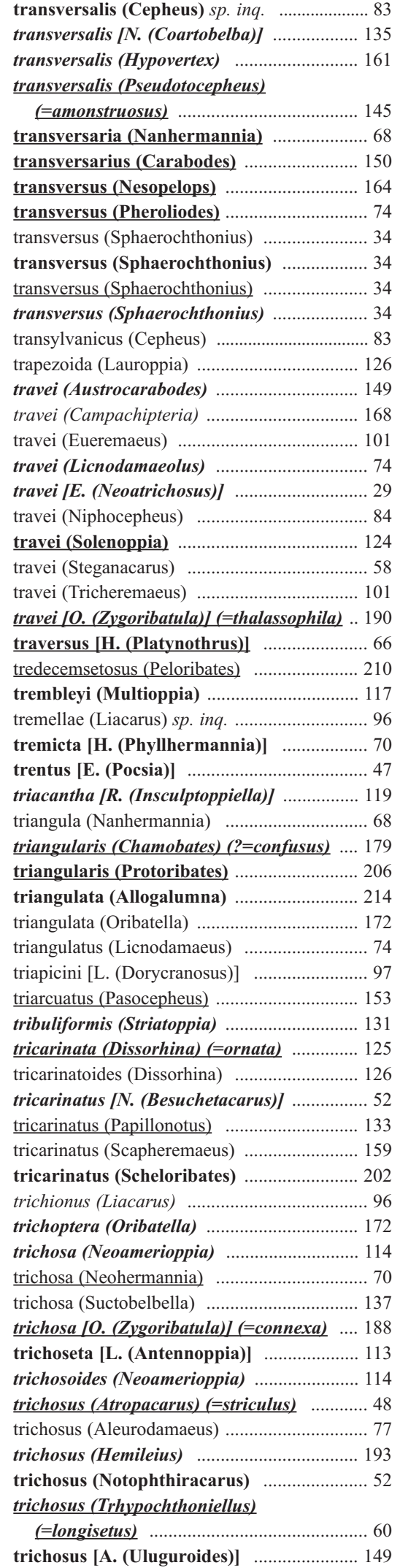

trichotos [T. (Paragloboppia)] ...................... 115

triconica (Berniniella) (=bicarinata)

tricornis (Mahunkaia) ................................. 165

tricornuta (Furcoppia) ................................. 92

tricornuta [S. (Ussuribata)] ….................... 139

tricornutus (Dolicheremaeus) .................... 143

tricuspidata (Allosuctobelba) ........................ 134

tricuspidata (Amazoppia) ............................ 93

tricuspidata (Galumna) .............................. 217

tricuspidata (Metrioppia) ............................... 94

tricuspidatus (Globozetes) (=birulai) .......... 180

tricuspidatus (Pantelozetes) ........................... 107

tricuspidatus (Sphaerozetes) .......................... 178

tridactyla (Oribatella) .................................... 172

tridactylus [M. (Calyptozetes)] ..................... 183

tridactylus (Cosmopirnodus)

tridactylus (Hydrozetes) .................................... 156

tridactylus (Sphodrocepheus) .......................... 84

tridactylus [H. (Tuberemaeus)] foveolatus .. 193

tridentata (Cultroribula) Mih., 58 ................... 92

tridentata (Cultroribula) Aoki, 65

\section{(=neonominata}

ridentata (Furcoribula)

tridentata (Medioppia) …....................... 12

tridentata (Moritzoppia) ................................. 127

tridentata (Oppia) (=neonominata) .............. 115

tridentatus (Mycobates) ................................. 183

tridentatus (Neomvcobates) ......................... 183

trifolius (Lepidozetes) ................................... 169

trifolius (Lepidozetes) .................................... 169

triforata (Paraphauloppia) ....................... 190

trifurcata (Cultroribula) (=bicultrata) .......... 92

trifurcata (Schalleria) ................................. 90

trifurcus (Eremaeozetes)

triglavensis (Eueremaeus) ............................ 101

triglochin (Brachioppia) ............................. 122

trigona (Suctobelba) ...................................... 136

trigonalis [D. (Epidamaeus)] .......................... 80

trigonella [O. (Zygoribatula)] ........................ 190

trigonosternum (Cavernocarabodes) ............ 151

triheterodactylis (Acrotritia)

(=hyeroglyphica) …................................... 45

trilabiata (Oripoda) ......................................... 204

trilabiata (Oripoda) .......................................... 204

trilobata (Medioppia) .................................... 124

trilobus (Edwardzetes) …................................. 175

trimaculata (Murcia) ................................. 10, 177

trimaculata (Murcia) ............................ 10, 177

trimaculata (Oromurcia) (=lucens) ............. 178

trimorphus [T. (Rostrozetes)] (=ovulum) .... 211

trimucronata (Wallworkoppia) .................. 12

trimucronatus (Birsteinius) …......................... 95

trimucronatus (Dolicheremaeus) …............. 143

trimucronatus (Leptotocepheus) ................ 144

trinacriae (Peloptulus) .................................. 164

trinebulosa (Hermannia) sp. inq. ..................... 69

trinodosa (Zeasuctobelba) .......................... 139

trionus (Eueremaeus) ................................... 10

triops (Galumna) ……................................. 217

tripartita (Arcoppia) ................................. 12

tripartita (Arcoppia) ................................... 121

tripartita (Hoplophorella) ............................... 49 tripartita (Indotritia) ....................................... 43

tripartita (Suctobelbila) ............................... 139

tripartitus [P. (Makischeloribates)] …............. 198

tripartitus (Notophthiracarus) …................ 52

triplicatulus (Unguizetes) (=sphaerula) ...... 186

triplicicornutus (Acrotocepheus) ….............. 146

tripuraensis (Peloribates) …........................... 210

tripurensis (Striatoppia) …........................... 131

triquetra (Galumna) ..................................... 217

triquetra (Galumna) ................................. 217

triramosa (Arcoppia) .................................... 121

triramosa (Brachioppia) .............................. 122

triramosa (Brachioppiella) ......................... 122

trirostrata (Oribatula) ............................... 188

trirugis (Scapheremaeus) ............................ 159

triseta (Apoplophora) ….................................. 41

triseta (Tanzoppia) ....................................... 115

trisetosa (Oribotritia) ........................................... 45

trisetosus (Austrodamaeus) (=elegantulus) .. 76

trisetosus (Cerostocepheus) ......................... 146

trisetosus (Hammerabates) ............................. 197

trisetosus (Hammerabates) ….................... 197

tristani (Stomacarus) ….................................. 27

tristis (Phthiracarus) ......................................... 54

tristius (Pedrocortesella) ............................... 75

tritici [O. (Zygoribatula)] ............................... 190

trituberculatus (Zetorchestes) sp. inq. ........ 91

tritylos [D. (Epidamaeus)] ............................... 80

tritylos [D. (Epidamaeus)] …......................... 80

triungulata (Magyaria) ................................... 209

triungulatus [I. (Triungulozetes)] ................ 208

trivandricus (Annectacarus) .............................. 37

trivialis (Cosmochthonius) ............................... 33

troisi (Amerus) ............................................. 104

tropica (Cultroribula) ……............................ 92

tropica (Dometorina) ................................. 193

tropica (Indotritia) (=krakatauensis) ............... 43

tropica (Microtritia) ........................................... 47

tropica (Microtritia) ....................................... 47

tropica (Microtritia) ......................................... 47

tropica (Microtritia) ........................................ 47

tropica (Microtritia) .......................................... 47

tropica (Pseudantarcticola) ............................. 157

tropicalis (Brachioppia) ............................ 122

tropicus (Hoplophthiracarus) .......................... 50

tropicus (Sellnickochthonius) ......................... 32

truncata (Campachipteria) ............................ 168

truncata [B. (Comororibula)] …………..... 207

truncata (Eremobelba) ............................. 104

truncata (Macrogena) …............................. 176

truncata (Medioppia) .................................... 124

truncata (Membranoppia) ........................... 111

truncata (Notogalumna) ............................ 218

truncata (Pedrocortesella) ............................ 75

truncata [H. (Phyllhermannia)] …............... 70

truncata [O. (Zygoribatula)] ........................... 190

truncata [O. (Zygoribatula)] ........................... 190

truncatum (Adoristes) (=ovatus) .................... 95

truncatus (Eremulus)

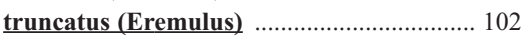

truncatus (Nothrus) ....................................... 64

truncatus (Oxyamerus) 
truncicola (Suctobelbata) ............................. 136 trupchumi (Melanozetes) sp. inq. .................. 176 tryjanowskii (Crotonia) ................................. 67 tryphosa (Camisia) .................................... 65 tsavoensis (Eremaeozetes) .......................... 165 tsavoensis (Pergalumna) ............................. 220 tschabovskyi (Suctobelbella) ......................... 137 tshendsureni [L. (Procorynetes)] .................... 97 tsushimaensis (Carabodes) ............................ 150 tuberculata (Carinabella) .............................. 100 tuberculata (Crotonia) .................................... 67 tuberculata (Ctenobelba) (=pilosella) ........... 101 tuberculata (Cyrthermannia) ......................... 68 tuberculata (Cyrthermannia) .......................6 68 tuberculata (Cyrthermannia) ……………........ 68 tuberculata (Dometorina) ............................... 193 tuberculata (Eremobelba) ........................... 104 tuberculata (Galumna) .................................. 217 tuberculata (Jacotella) (=ornata) ................... 76 tuberculata (Machadobelba) ........................... 107 tuberculata (Medioppia) ................................. 124 tuberculata [H. (Phyllhermannia)] ................ 70 tuberculata (Ramusella) (=sengbuschi) ...... 118 tuberculata (Suctobelbella) ............................ 137 tuberculata (Suctobelbila) ............................ 139 tuberculata (Triteremella) (?=kaszabi) ........ 106 tuberculata (Viracochiella) .......................... 179 tuberculatus (Archegozetes) (=magnus) ........ 59 tuberculatus (Belbodamaeus) ......................... 78 tuberculatus (Bulleremaeus) …................... 158 tuberculatus (Calobates) ........................... 203 tuberculatus (Caucasiozetes) …....................... 88 tuberculatus (Cryptacarus) ……....................... 38 tuberculatus (Eremaeozetes) ……................ 165 tuberculatus (Eremaeozetes) ...................... 165 tuberculatus (Eremaeozetes) ....................... 165 tuberculatus (Exochocepheus) ....................... 161 tuberculatus (Gibbicepheus) .......................... 152 tuberculatus (Gibbicepheus) ..................... 152 tuberculatus (Liochthonius) ........................ 31 tuberculatus [P. (Neophthiracarus)] ................. 57 tuberculatus (Neoribates) ............................... 212 tuberculatus (Ocellotocepheus) …................. 147 tuberculatus (Papillocepheus) ..................... 144 tuberculatus (Pilizetes) ............................... 220 tuberculatus (Scheloribates) .......................... 202 tuberculatus (Sphodrocepheus) …………....... 84 tuberculatus (Striatobates) tuberculatus [H. (Tuberemaeus)] ................... 194 tuberculatus [H. (Tuberemaeus)] .............. 194 tuberculatus (Xenillus) .................................... 99 tuberculosissima [H. (Kakophthiracarus)] .... 50 tuberculosus (Ametroproctus) ...................... 159 tuberculosus (Cepheus) ......................................... 83 tuberculosus (Machadocepheus) ................ 152 tuberosa (Arcoppia) ...................................... 121 tuberosa (Hoplophorella) (=praeoccupata) .... 49 tuberosa (Jugatala) ............................................ 176 tuberosa (Jugatala) ....................................... 176 tuberosa [H. (Kakophthiracarus)]

(=tuberculosissima) tuberosa (Phauloppia) tuberosa (Setoppia)

tuberosa (Suctobelbella) (=acutidens) ........ 136 tuberosa [S. (Ussuribata] ................................ 139

tuberosus (Charassobates) ……………….... 86

tuberosus (Eremaeus) ..................................... 100

tubifer (Liacarus) .......................................... 96

tubuaiensis (Scheloribates) ............................ 202

tubuaiensis (Scheloribates) ….................... 202

tubulus [P. (Archiphthiracarus)] .................. 56

tuloyus (Meristacarus) …............................... 39

tumescitus (Suctotegeus) …….......................... 86

tumida (Microtritia) ....................................... 47

tumidisetosus (Chamobates) ....................... 180

tumulata (Suctobelba) .................................. 136

tunicatus (Cosmobates) ............................... 197

tunicatus (Lopheremaeus) sp. inq. ............... 73

tunicatus (Tegeozetes) .................................... 155

tunicatus (Tegeozetes) …........................... 155

tunicatus (Tegeozetes) ….............................. 155

tunicatus (Tegeozetes) ................................ 155

tunicatus (Tegeozetes) ................................... 155

tunisica (Dissorhina) ornata .......................... 125

tunisiensis (Peloribates) ......................... 12, 210

tupasae (Acrotocepheus) ............................... 146

tupi (Dampfiella) ......................................... 141

turanica (Simkinia) .......................................... 195

turbulentus (Ramsayellus) ........................... 182

turcica (Enisella) ......................................... 131

turcmenica (Lohmannia) ................................. 39

turcmenica (Lohmannia) ................................ 39

turcmenica (Lohmannia) ............................. 39

turcmenicus (Ghilarovus) ............................... 184

turcmensis (Scheloribates) ............................. 202

turgidus (Peloribates) …................................ 210

turgidus (Phthiracarus) ................................... 54

turgidus (Podoribates) .................................. 186

turgiseta (Karenella) ................................... 130

turki [L. (Hamoppia)] ................................. 111

tuxeni (Liochthonius) Fors., 57 ....................... 31

tuxeni (Liochthonius) Chin., 74 (=moritzi) .... 30

tuxeni (Membranoppia) ............................... 111

tuxeni (Pantelozetes) (=paolii) .................... 107

tuxtlasensis (Allonothrus) .............................. 59

tyrrhenica (Bursoplophora) ............................. 35

tyrrhenica (Oribatella) .................................. 172

tyrrhenicus (Liochthonius) .............................. 31

tzanoudakisi [P. (Archipththiracarus)]

(=montanus) tzitzikamaensis (Perscheloribates) …....... 198 uapoui (Pergalumna) bryani ...................... 218 uapoui (Scheloribates) mumfordi ............... 200 ubali (Edwardzetes) ....................................... 175 uberabensis (Hydrozetes) 156 ucrainica [O. (Zygoribatula)] connexa .......... 188 uenoi (Campachipteria) .............................. 168 uenoi [D. (Epidamaeus)] ……....................... 80 ugamaensis (Cosmochthonius) ........................ 33 ugraseni (Schusteria) ................................. 157 uherkovichi (Moritzoppia) ............................. 127 uliginosa (Oppiella) (=nova) ....................... 128 ulneungensis (Pyroppia) .................................. 94 ultraciliata [L. (Antennoppia)] .................. 114 ultraforaminosus (Topobates) …................ 203

uluguruensis (Scheloribates) ..................... 202

ulykpani [I. (Haplozetes)] ............................ 208

umbellatus (Lepidozetes) ............................. 169

umbraili (Topobates) _..................................... 203

umbraticus (Gymnodamaeus) ........................ 76

umbratilis (Camisia) sp. inq. .......................... 65

uncinata [O. (Dzarogneta)] ........................... 129

uncinatus (Notophthiracarus) ….................. 52

uncinulus (Notophthiracarus) ...................... 52

uncunguis (Psednobates) .............................. 157

undatus (Plenotocepheus) ............................ 145

undirostratus (Papillacarus) ............................ 40

undulata [G. (Indogalumna)] ........................ 217

undulata (Indotritia) ............................................... 43

undulata (Oribatella) ................................... 172

undulata (Suctobelbila) ............................... 139

undulata [O. (Zygoribatula)] Berl., 16 ........... 190

undulata [O. (Zygoribatula)] Berl., 16 ........ 190

undulata [O. (Zygoribatula)] Berl., 16 ...... 190

undulata [O. (Zygoribatula)] Berl., 16 .......... 190

undulata [O. (Zygoribatula)] Berl., 16 ...... 190

undulata [O. (Zygoribatula)] Bal., 66

(=baloghi) ................................................ 188

undulatus (Africoribates) .......................... 180

undulatus (Cavernocepheus) ...................... 141

undulatus (Ceratozetes) ................................ 175

undulatus (Eremaeozetes) ............................. 165

undulatus (Licnodamaeus) ............................. 74

undulatus (Licnodamaeus) ............................... 74

undulatus (Megalotocepheus) ….................. 147

undulatus (Microtegeus) ............................... 86

undulatus (Sadocepheus) ................................. 84

undulatus (Tereticepheus) ................................ 84

undulatus [T. (Tyrphonothrus)] ....................... 63

undulatus (Unduloribates) ........................... 165

undulatus (Unduloribates) ........................... 165

unguifera (Crotonia) ..................................... 67

unica (Galumna) ........................................... 217

unica (Oribatula) ........................................ 188

unicarinata (Austrotritia) ................................. 42

unicarinata (Austrotritia) ........................... 42

unicarinata (Moritzoppia) .............................. 127

unicarinata (Moritzoppia) ........................... 127

unicarinata (Moritzoppia) .......................... 127

unicarinata (Moritzoppia) ........................... 127

unicarinatoides (Moritzoppia) unicarinata .... 127

unicarinatus (Notophthiracarus) ............... 52

unicorniculatus (Zetorchestes) micronychus

ssp. inq.

unicornis (Belba)

unilateralis (Annectacarus) .............................. 37

unilateralis (Annectacarus) .......................... 37

unilateralis (Annectacarus) ......................... 37

unilateralis (Liochthonius) ............................ 31

unimaculatus (Diapterobates) ...................... 181

uniporosa (Vaghia) ....................................... 221

unisetosus (Scheloribates) …...................... 202

unispinata (Oribatella) ................................. 172

unispinata (Oribatella) ................................. 172

universitatum (Tectocepheus) velatus .......... 155

unjangensis (Liacarus) .................................... 96 


\begin{tabular}{l} 
unqus (Hoplophorella) \\
\hline upelbensis (Basiceramerus)
\end{tabular}

variabilis (Pilogalumna) ................................. 220

variabilis (Scheloribates) ............................. 202

variabilis (Sellnickochthonius) ........................ 32

variabilis (Suctobelbella) ................................ 137

variabilis (Xenillus) ....................................... 99

variabilis [O. (Zygoribatula)] (=frisiae) ...... 189

varians (Hoplophorella) ................................ 49

varians [R. (Insculptoppiella)] ....................... 119

varians [R. (Insculptoppiella)] ................... 119

variaporosa (Oribatula) ……………………... 188

variatepictus (Bipassalozetes)

(=intermedius) …………………………... 162

variatus (Eupelops) ………………………........ 164

variesetosus (Sphaerochthonius) …………… 35

variolatus (Dolicheremaeus) ……................. 143

variolobatus (Dolicheremaeus) .................... 143

variolosa (Cultroribula) ................................. 92

variolosus (Eupelops) (=torulosus) ............... 164

variolosus (Notophthiracarus) ...................... 52

variolosus (Xenillus) ...................................... 99

variopectinata (Ctenoppia)........................... 122

variosculpturata (Pergalumna) .................. 220

variosetosa [S. (Ussuribata)] …………….... 139

variosetosa [S. (Ussuribata)] ……………….... 139

variosetosa [S. (Ussuribata)] ........................ 139

variosetosa [S. (Ussuribata)] ........................ 139

variosetosus [M. (Cristonothrus)] ................. 6

varisculptus (Peloribates) ………………...... 210

varisetiger (Protoribates) ………………….... 206

varius (Humerobates) ……………………….... 182

vartismithi (Chamobates) sp. inq. ................. 180

vastus (Liacarus) (=brevilamellatus) ............ 95

vastus (Protoribates) (=dentatus) ................. 206

vaucheri (Austrocarabodes) ........................ 149

vegeta [O. (Zygoribatula)] ............................. 190

velata (Geminoppia) …………………….... 110

velata (Topalia) …………………………….... 86

velatus (Congocepheus) ………………….... 151

velatus (Kaszabozetes) ……………………….... 89

velatus (Tectocepheus) ……………………..... 155

velatus (Tectocepheus) ................................... 155

velatus (Tectocepheus) …………………....... 155

velatus (Tectocepheus) ….................................. 155

velatus (Tectocepheus) …………………….... 155

velatus (Tectocepheus) ................................... 155

venetiolanus (Holonothrus) ........................... 67

venezuelanus (Xenillus) ................................ 99

venosus (Platyliodes) (?=doderleini) …........ 72

ventosus [H. (Protophthiracarus)] ................... 53

ventosus [H. (Protophthiracarus)] …………. 53

ventralis (Pergalumna) curva ....................... 218

ventralis (Pergalumna) curva ……………...... 218

ventralis (Pergalumna) curva .................... 218

ventralis (Pergalumna) curva ...................... 218

ventricosa (Camisia) (=segnis) ........................ 65

ventricosus (Hypochthonius) $s p$. inq. .......... 29

ventrisetosa (Machuella) ……………………... 133

ventrisetosa (Machuella) …………………...... 133

ventrisetosa (Machuella) ............................. 133

ventrisetosa (Machuella) ............................... 133

ventroacutus (Maculobates) ...................... 196

ventrolaminata [M. (Pravoppia)] .................. 111 ventronodosa [S. (Lalmoppia)]

(=maculata) ………………………….... 130

ventrosquamosa (Neoamerioppia) ................. 114

ventrosquamosa (Neoamerioppia) ................. 114

ventrosquamosa (Neoamerioppia) ……......114

ventrostriatus (Gibbicepheus) …………….... 152

venusta (Ceratozetella) ……………………... 173

venusta (Oribatula) (=tibialis) ..................... 188

venusta (Hoplophorella) ………………....... 49

venustus (Circellobates) …………………...... 157

vera (Medioppia) …………………………... 124

vera (Oribatula) ……………………………... 188

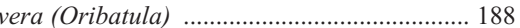

vera (Suctobelbella) ......................................... 137

veracruzensis (Archegozetes) (=magnus) ...... 59

verai (Eremaeozetes) ....................................... 165

veriornatus (Gymnodamaeus) ......................... 76

vermicularis (Eohypochthonius) ........................ 29

vermicularis (Pheroliodes) ............................. 74

vermiculatus (Carabodes) (=labyrinthicus) .. 150

vermiculatus (Cosmozetes) ............................. 88

vermiculatus (Neoliodes) ……......................... 72

vermiculatus [H. (Tuberemaeus)] …………... 194

vermiculatus [H. (Tuberemaeus)] ............... 194

vermiseta (Perxylobates) …………………. 205

vermiseta (Perxylobates) …………………... 205

vermiseta (Perxylobates) …………………... 205

vermiseta (Perxylobates) .............................. 205

vernaculus (Steganacarus) ................................. 58

verrucatus (Austrocarabodes) ........................ 149

verrucatus (Cubabodes) ……………………. 152

verrucatus [D. (Epidamaeus)] ………………... 80

verrucosa (Achipteria) ………………………... 168

verrucosa (Belba) ...……………………….... 78

verrucosa (Belba) ………………………….... 78

verrucosa (Epilohmannia) $(=$ cylindrica)

verrucosa [H. (Kakophthiracarus)]

(=tuberculosissima $)$....................................... 50

verrucosa (Setoppia) ................................... 112

verrucosus (Cepheus) ........................................... 83

verrucosus (Otocepheus) ……........................ 147

verrucosus (Plenotocepheus) ....................... 145

verticillatus (Hymenozetes) ............................. 89

verticillipes (Spatiodamaeus) .............................. 82

verticillipes (Spatiodamaeus) ............................. 82

vesca (Mesoplophora) ……………………...... 42

vestita (Acrotritia) ………………………….... 46

vestita (Acrotritia) ………………………….... 46

vestita (Acrotritia) ………………………….... 46

vestita (Acrotritia) ………………………...... 46

vestita (Eremella) .......................................... 106

vestita (Lyrifissisella) ....................................... 73

vestitus (Steganacarus) …............................ 58

veteratorius (Notophthiracarus) ………….... 52

vetula (Dissorhina) (=ornata) ....................... 125

vibrissa (Wallworkoppia) ………………..... 121

vicarius (Hydrozetes) sp. inq. ....................... 156

vicarius (Pseudotocepheus) ………………... 145

vicarius (Tectocepheus) …………………... 155

vicina (Neoamerioppia) ............................... 114

vicina (Neoamerioppia) …………………... 114

vicina (Sternoppia) ……………………….... 133 


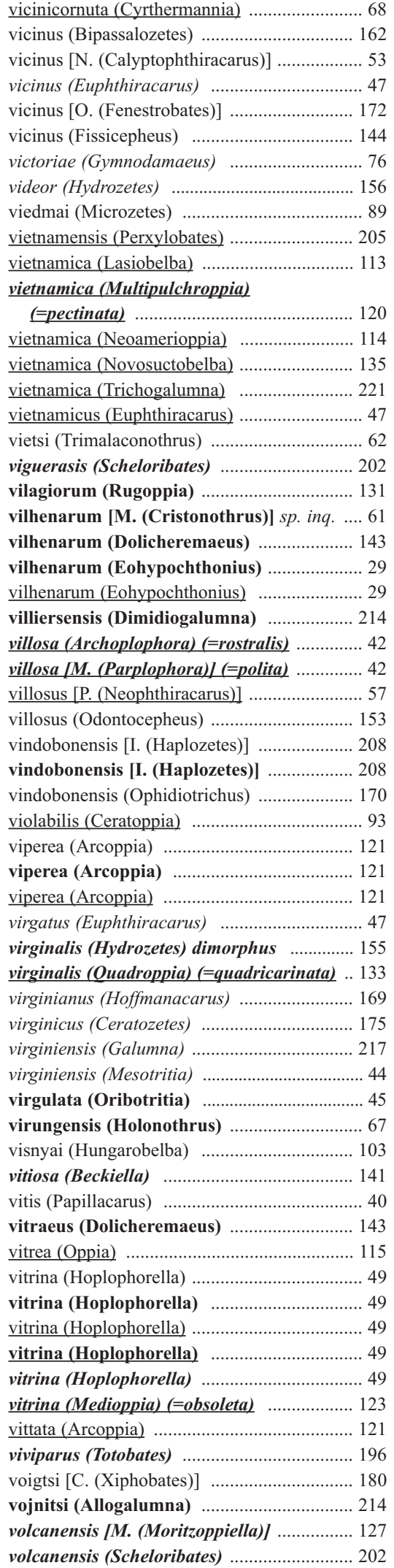

volcanicus (Scapheremaeus) ....................... 159

volgini (Ceratozetes) .................................. 175

volgini (Zachvatkinibates) ............................ 184

vombi (Liacarus) (=coracinus) ..................... 95

voskresenskii (Amuracarus) ............................ 27

vtorovi [F. (Mexicoppia)] ................................ 92

vtorovi [F. (Mexicoppia)] ……........................ 92

vtorovi [O. (Oxyoppiella)] ............................ 129

vulcania (Lohmannia) ..................................... 39

vulgaris (Maculobates) ................................ 196

vulgaris (Pheroliodes) ................................... 74

vulgaris (Scheloribates) ................................ 202

vulgaris (Xenillus) (=tegeocranus) ................ 99

vulgaris [O. (Zygoribatula)] ........................... 190

vulpes (Arthrhoplophora) ............................ 35

wakensis (Neoliodes)

walkeri (Apotritia) ............................................ 47

walkeri (Brachioppiella) …........................ 122

wallacei (Acrotocepheus) .............................. 146

wallacei (Dolicheremaeus) ............................. 143

wallworki (Acrotritia) ................................... 46

wallworki [P. (Archiphthiracarus)] ............ 56

wallworki (Belloppia) ................................ 125

wallworki (Cosmochthonius) (=lanatus) ...... 33

wallworki (Dolicheremaeus) ..................... 143

wallworki (Epilohmannoides) ....................... 37

wallworki (Galumna) (=elimata) …............. 215

wallworki [P. (Neophthiracarus)] ……......... 57

wallworki [M. (Pravoppia)] .......................... 111

wallworki (Sphaerochthonius) (=gemma) ... 34

wallworki (Steganacarus) …...................... 58

wallworki (Trimalaconothrus) ...................... 62

walteri (Eremaeus) .................................... 100

walteri (Novazelandiella) .............................. 74

wandae (Atropacarus) .................................... 48

wangi (Dolicheremaeus) ............................... 143

washburni (Oppiella) (=nova) ...................... 128

watanabei (Banksinoma) .............................. 109

waterhousei (Arcoppia) …......................... 121

waterhousei (Gibbicepheus) ..................... 152

waterhouseri (Suctobelbila) ….................... 139

watertonensis (Ceratozetes) .......................... 175

watsoni (Stomacarus) ....................................... 27

weberi (Pergalumna) ...................................... 220

wehnckei (Pheroliodes) ................................. 74

weigmanni (Campachipteria) ........................ 168

weigmanni (Notophthiracarus) .................... 52

weni (Galumna) ............................................ 217

werneri (Basilobelba) ............................. 104

werneri (Steganacarus) ................................ 58

wettsteini (Carabodes) ................................... 150

whartoni (Jacotella) ........................................ 76

whitteni (Scheloribates) fimbriatus …...... 200

widagdoi (Rhynchoppia) ............................. 135

williamsae (Anakingia)

willmanni (Autogneta) ................................... 108

willmanni (Campachipteria) (=fanzagoi) .... 168

willmanni [C. (Klapperiches)] ....................... 151

willmanni [C. (Klapperiches)] …................... 151

willmanni (Lanceoppia) ............................. 110

willmanni (Liacarus) ..................................... 96

willmanni (Liebstadia) ................................ 196 willmanni (Mucronothrus) ............................. 60

willmanni [P. (Neophthiracarus)] ................ 57

willmanni (Nothrus) ..................................... 64

willmanni (Oppia) (=denticulata) ................ 115

willmanni (Oribatella) ................................. 172

willmanni (Pergalumna) ................................ 220

willmanni (Scheloribates) ............................ 202

wilsoni (Maudheimia) …............................. 195

wilsoni (Multioppia) ....................................... 117

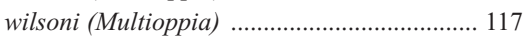

wilsoni (Multioppia) ..................................... 117

wilsoni (Multioppia) ..................................... 117

wilsoni (Multioppia) ..................................... 117

winchesteri (Sphaerozetes) ........................... 178

winkleri (Arcoppia) cronus ......................... 120

witfonteinensis (Rhopalozetes) ................... 90

wittmeri (Eremobelba) ................................... 104

wittmeri (Hoplophthiracarus) (=kugohi) ..... 50

woasi [H. (Heterohermannia)] ........................ 69

woelkei (Eremaeozetes) ................................. 165

wolwekransensis (Bipassalozetes) .............. 162

womersleyi [S. (Ussuribata)] ........................ 139

womersleyi [S. (Ussuribata)] ...................... 139

wonalancetanus (Carabodes) ......................... 150

wongi (Caucasiozetes) ................................... 88

woodringi (Lanceoppia) .............................. 110

woolleyi (Eueremaeus) .................................. 101

woolleyi (Neoamerioppia) .......................... 114

woolleyi [C. (Protoripoda)] ........................ 204

woonhahi (Adrodamaeus) ................................ 75

woschitzi (Galumnella) ............................. 222

wulongensis [P. (Tectodamaeus)] ……............. 81

wuyanensis [T. (Tyrphonothrus)] .....................63

xanthoparmeliae (Phylloribatula) ............. 160

xarifae (Gehypochthonius) .............................. 28

xena (Arthrovertex) .................................. 160

xena (Epilohmannoides) ................................ 37

xena (Neosuctobelba) ................................... 135

xena (Orthogalumna) ................................... 218

xena (Pilobatella) ....................................... 211

xenus (Pseudocarabodes) ............................. 153

xilofila (Microtritia) (=minima) .................... 47

xinjiangensis (Ceratozetes) …....................... 175

xinjiangensis (Multioppia) ……....................... 117

xinjiangensis (Phauloppia) .......................... 191

xishuangbanna (Pergalumna) magnipora ..... 219

xizangensis (Eupterotegaeus) ............................. 83

xylariae (Liacarus) .......................................... 96

xylobatoides (Scheloribates) ........................ 202

yachidairaensis (Trimalaconothrus) ................ 62

yaginumai (Calipteremaeus) ......................... 73

yamamotoi [M. (Cristonothrus)] .................... 61

yamasakii [H. (Capillonothrus)] ....................... 66

yamasakii [H. (Capillonothrus)] ...................... 66

yamashitai (Scapheremaeus) ........................ 159

yanoi (Nehypochthonius) ................................. 36

yaoi [P. (Tectodamaeus)] ................................... 81

yasumai (Hermanniella) .................................. 71

yayeyamensis (Liacarus) ................................. 96

yayeyamensis (Liacarus) …............................ 96

ybarrai (Xenillus) ............................................ 99

yemeniticus (Unguizetes) ........................... 186 
yepesensis [O. (Dzarogneta)] ...... yezoensis (Ceratozetella) yezoensis (Costeremus) yezoensis (Diapterobates) variabilis yezoensis [L. (Dorycranosus)] yezoensis (Peloribates) yezoensis (Protoribates) yezoensis (Scheloribates) yezoensis (Suctobelbella) yinae (Leobodes) yinae (Malaconothrus) yinae [M. (Phyllolohmannia)]

yodai (Lasiobelba) (=kuehnelti) yorubaensis (Scheloribates)

yoshidai (Truncopes)

yoshii [L. (Antennoppia)] yoshii [P. (Aokibates)] yoshii (Dolicheremaeus) voshii (Hoplophthiracarus) (=kugohi) youngai (Longocepheus) ypsilon (Hymenobelba) ypsilon (Hymenobelba) ypsilonsignata (Suctobelbata) yukonensis (Eueremaeus) yukonensis (Mycobates) yunkeri (Fortuynia)
129

173

103

181

... 97

211

206

202

138

154

... 61

40

113

202

205

114

211

143

50

144

105

105

136

101

183

157 yunnanensis (Cristamerus)

yunnanensis (Dolicheremaeus)

yunnanensis [D. (Epidamaeus)

yunnanensis (Eremobelba)

yunnanensis (Lasiobelba)

yunnanensis (Peloribates)

yunnanensis [T. (Tyrphonothrus)]

yuwana (Medioxyoppia)

yuwanensis (Nippobodes)

zaballosi (Odontocepheus)

zachtvatkini (Arthrhoplophora)

zachvatkini (Beklemishevia)

zachvatkini [L. (Dorycranosus)]

zachvatkini (Galumna)

zachvatkini (Medioppia) (=subpectinata)

zachvatkini (Metabelbella)

zachvatkini (Perlohmannia)

zachvatkini (Semipunctoribates)

zaherii (Scheloribates) (=praeincisus)

zangherii (Camisia) $s p$. inq.

zangherii (Indotritia)

zealandica (Miroppia)

zealandicus [M. (Cristonothrus)]

zealandicus (Scheloribates)

zealandicus (Scheloribates)

zebra (Notophthiracarus) zehntneri (Machuella) ventrisetosa

zehntneri (Machuella) ventrisetosa ….......... 133

zelawaiensis (Sellnickochthonius) .................. 32

zelawaiensis (Sellnickochthonius) ................... 32

zelawaiensis (Sellnickochthonius) .................. 32

zellwegeri (Dampfiella) ............................... 141

3 zeteki (Ceratozetes) ....................................... 175

zeucta [G. (Erogalumna)] ……...................... 217

zeugus (Compactozetes) …………………...... 83

zeyensis [S. (Lalmoppia)] ........................... 130

zhuzhikovi (Mixacarus) ................................. 40

zicmani (Phthiracarus) .................................... 54

zicsica [L. (Lancelalmoppia)] ..................... 111

zicsii (Cultroribula) ....................................... 92

zicsii (Eremobelba) ...................................... 104

zicsii (Rhacaplacarus) ................................... 58

zicsii [O. (Zygoribatula)] ............................ 190

zikani (Heterobelba) .................................. 104

zikani (Teleioliodes) ........................................ 72

zimmermani (Neoliodes) …......................... 72

zimmermani (Neoliodes) ……...................... 72

zlotini (Metrioppia) ...................................... 94

zsuzsankae [O. (Oxyoppiella)] ..................... 129

zumpti (Humerobates) ................................. 182

zushi [N. (Confinoppia)] ........................... 127

zwarti (Epilohmannia) .................................. 37 